\title{
Nuclear ground-state masses and deformations: FRDM(2012)
}

\author{
P. Möller ${ }^{\mathrm{a}, *}$, A. J. Sierk ${ }^{\mathrm{a}}$, T. Ichikawa ${ }^{\mathrm{b}}$, H. Sagawa ${ }^{\mathrm{c}, \mathrm{d}}$ \\ ${ }^{a}$ Theoretical Division, Los Alamos National Laboratory, Los Alamos, NM 87545, United States \\ ${ }^{b}$ Yukawa Institute for Theoretical Physics, Kyoto University, Kyoto 606-8502, Japan \\ ${ }^{c}$ RIKEN Nishina Center, Wako 351-0198, Japan \\ ${ }^{d}$ Center for Mathematics and Physics University of Aizu, Aizu Wakamatsu, Fukushima 965-0001, Japan
}

September 30, 2015

\begin{abstract}
We tabulate the atomic mass excesses and binding energies, ground-state shell-plus-pairing corrections, ground-state microscopic corrections, and nuclear ground-state deformations of 9318 nuclei ranging from ${ }^{16} \mathrm{O}$ to $A=339$. The calculations are based on the finite-range droplet macroscopic and the folded-Yukawa single-particle microscopic nuclear-structure models, which are completely specified. Relative to our FRDM(1992) mass table in ATOMIC DATA AND NuClear DATA TABles [59 185 (1995)], the results are obtained in the same model, but with considerably improved treatment of deformation and fewer of the approximations that were necessary earlier, due to limitations in computer power. The more accurate execution of the model and the more extensive and more accurate experimental mass data base now available allows us to determine one additional macroscopic-model parameter, the densitysymmetry coefficient $L$, which was not varied in the previous calculation, but set to zero. Because we now realize that the FRDM is inaccurate for some highly deformed shapes occurring in fission, because some effects are derived in terms of perturbations around a sphere, we only adjust its macroscopic parameters to ground-state masses.

The values of ten constants are determined directly from an optimization to fit ground-state masses of 2149 nuclei ranging from ${ }^{16} \mathrm{O}$ to ${ }_{106}^{265} \mathrm{Sg}$ and ${ }_{108}^{264} \mathrm{Hs}$. The error of the mass model is $0.5595 \mathrm{MeV}$ for the entire region of nuclei included in the adjustment, but is only $0.3549 \mathrm{MeV}$ for the region $N \geq 65$.

We also provide masses in the FRLDM, which in the more accurate treatments now has an error of $0.6618 \mathrm{MeV}$, with $0.5181 \mathrm{MeV}$ for nuclei with $N \geq 65$, both somewhat larger than in the FRDM. But in contrast to the FRDM, it is suitable for studies of fission and has been extensively so applied elsewhere, with FRLDM(2002) constants. The FRLDM(2012) fits 31 fission-barrier heights from ${ }^{70} \mathrm{Se}$ to ${ }^{252} \mathrm{Cf}$ with a root-mean-square deviation of $1.052 \mathrm{MeV}$.
\end{abstract}

${ }^{*}$ Corresponding author.

Email address: E-mail: moller@lanl.gov (P. Möller) 
This page is intentionally left blank to optimize the layout for double-sided printing. 


\section{Contents}

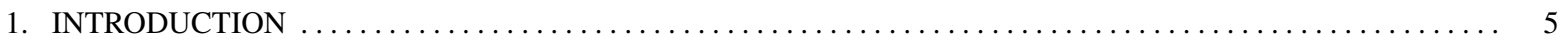

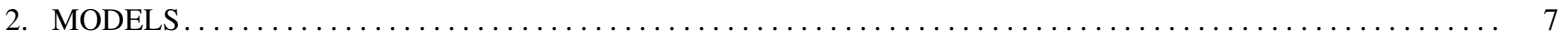

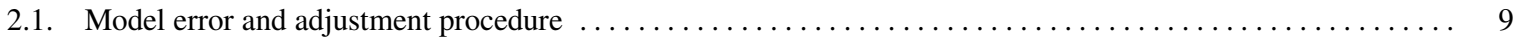

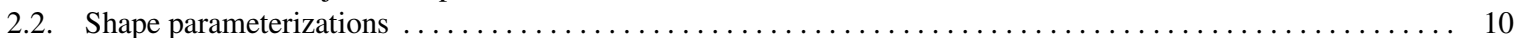

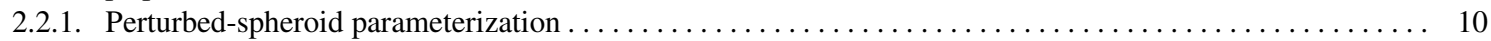

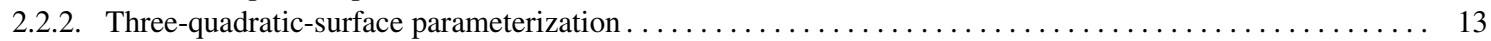

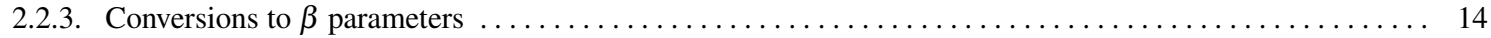

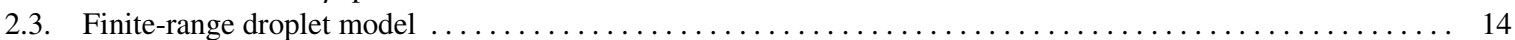

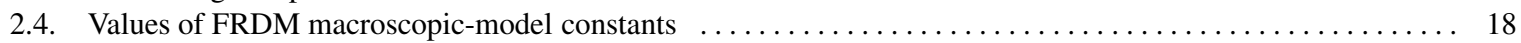

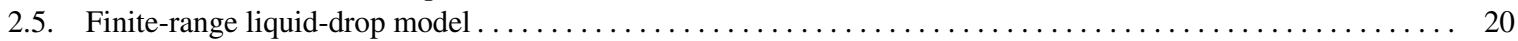

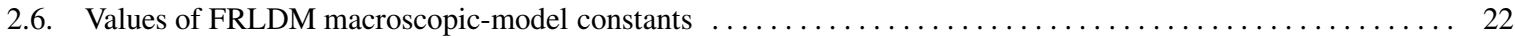

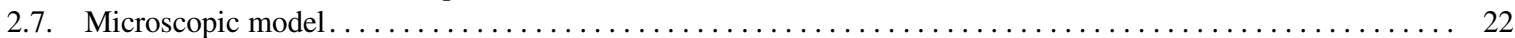

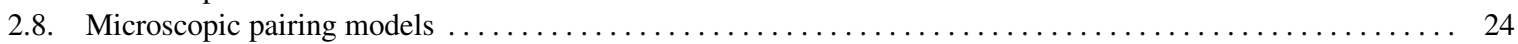

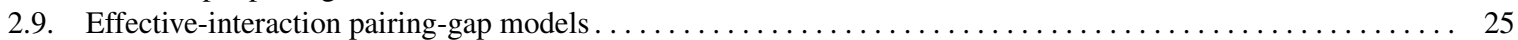

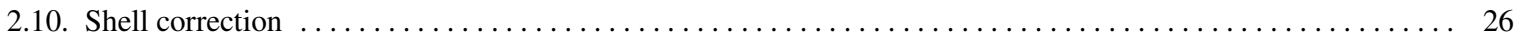

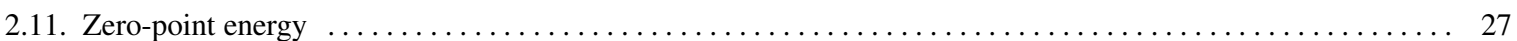

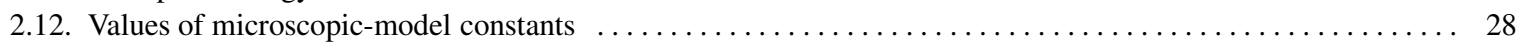

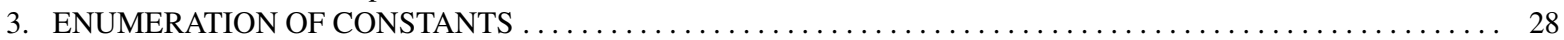

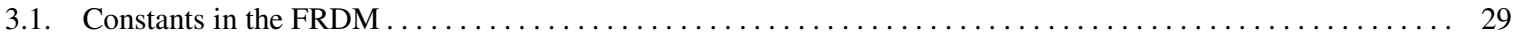

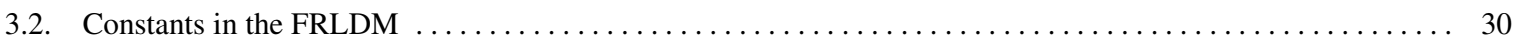

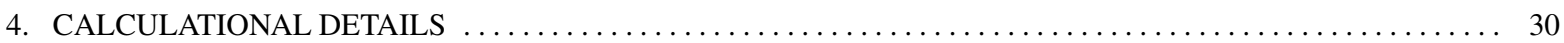

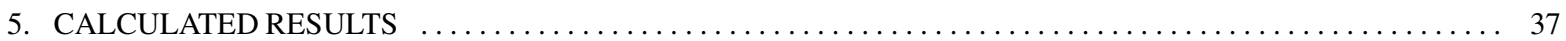

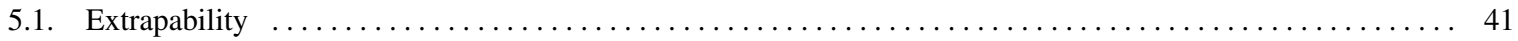

5.2. Detailed comparisons of masses and deformations in the FRDM(1992) and FRDM(2012) ........... 49

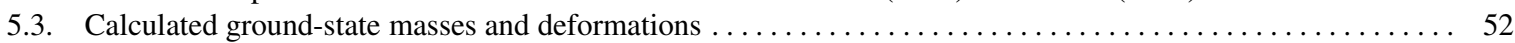

5.3.1. Do magic numbers really disappear for some exotic nuclei? .................... 53

5.3.2. Dependence of model accuracy with nucleon number $A \ldots \ldots$

6. SOME ADDITIONAL STUDIES AND DISCUSSION $\ldots \ldots \ldots \ldots \ldots$

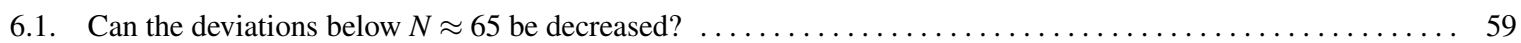

6.1.1. Improved choice of spin-orbit and single-particle potential diffuseness constants . . . . . . . . . . . 60

6.1.2. Improved determination of zero-point energies $\ldots \ldots \ldots \ldots \ldots$

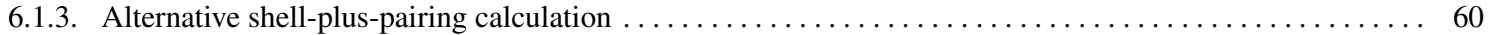

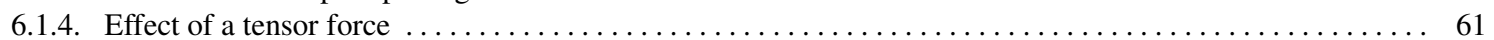

6.2. Comments on general nuclear-structure results in the FRDM/FRLDM and other nuclear-structure models ... . 62

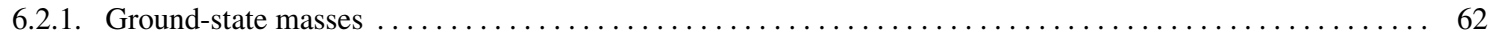

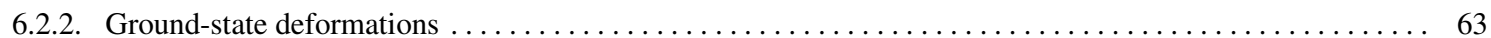

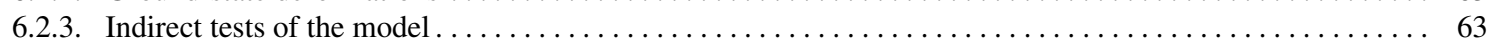

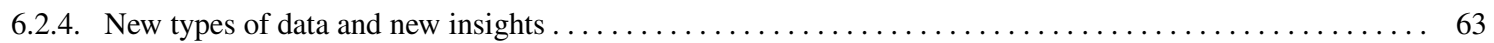

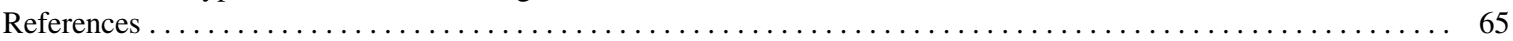

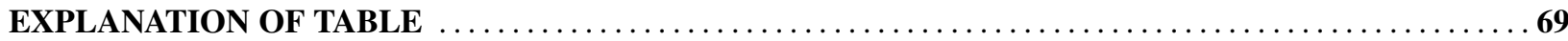

TABLE Calculated Nuclear Ground-State Masses and Deformations, Compared to the AME2003 Evaluation where available 
This page is intentionally left blank to optimize the layout for double-sided printing. 


\section{INTRODUCTION}

We presented our first macroscopic-microscopic global nuclear mass calculation about 35 years ago [1, 2]. That calculation, which was based on a finite-range liquid-drop model for the macroscopic energy and a folded-Yukawa single-particle potential for the microscopic corrections, was somewhat limited in scope. With only 4023 nuclei included, it did not extend to the proton or neutron drip lines or to the region of superheavy nuclei. Also, the quantities tabulated were limited to ground-state masses, $Q_{2}$ and $Q_{4}$ moments, and microscopic corrections.

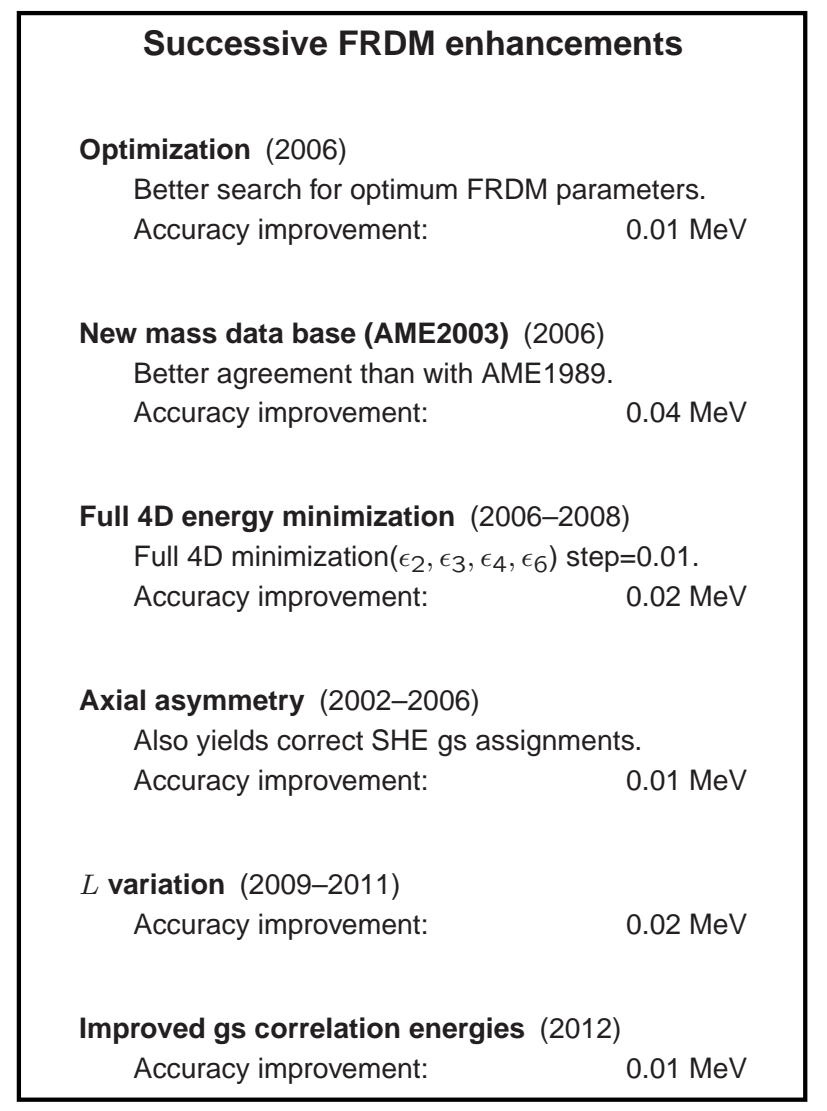

Fig. 1: Successive enhancements to $\operatorname{FRDM}(1992)$ with $\sigma_{\mathrm{th}}=0.669 \mathrm{MeV}$ and their impact, leading to $\operatorname{FRDM}(2012)$ with $\sigma_{\mathrm{th}}=0.5595 \mathrm{MeV}$. The years when the different effects were investigated are given in parentheses. These steps will be discussed in Sect. 4

Our next publication of calculated nuclear masses occurred in 1988 [3, 4]. In these calculations new pairing models had been incorporated and two different macroscopic models were investigated, namely the finite-range liquid-drop model (FRLDM) [3] and the finite-range droplet model (FRDM) [4]. These abbreviations are also used to designate the full macroscopic-microscopic nuclear structure models based on the respective macroscopic models. The former is the macroscopic model used in the 1981 [1,2] calculations and the latter is an improved version [5] of the droplet model $[6,7,8]$. Because there were several unresolved issues in the 1988 calculations $[3,4]$ these tables should be regarded as interim progress reports.

Over the next few years those issues were resolved. Their resolution led to the mass tables FRDM(1992) and FRLDM(1992) [9], presenting results on nuclear ground-state masses and deformations for 8979 nuclei ranging from ${ }^{16} \mathrm{O}$ to ${ }^{339} 136$ and extending from the proton drip line to the neutron drip line [9]. The calculation was based on the macroscopic-microscopic approach. The shell corrections were obtained from single-particle levels calculated in a folded-Yukawa single-particle potential [10] by use of the Strutinsky method [11,12]. Residual pairing corrections were calculated in the Lipkin-Nogami approximation [13, 14, 15, 16]. Two 1992 mass tables were provided, both based on these shell-plus-pairing corrections, but with the macroscopic contribution to the total potential energy obtained in two different liquid-drop-type models, namely the finite-range droplet model, and the finite-range liquid-drop model. We refer to this previous macroscopic-microscopic mass model in which the total potential energy is calculated as a sum of shell-plus-pairing corrections from folded-Yukawa single-particle levels and a macroscopic energy term from the finite-range droplet model as $\operatorname{FRDM}(1992)$. The year in parentheses refers to the year the constants of the macroscopic model were determined and frozen. The potential-energy model in which the macroscopic term is given by the finite-range liquid-drop model is referred to as FRLDM(1992). Although these mass models were published in 1995, we refer to them as FRDM(1992) and FRLDM(1992), because the mass models were finalized in September 1992 and widely distributed at that time. Also, we could not predict at manuscript submission when the manuscript would appear in print.

Subsequent comparisons of predictions of $\operatorname{FRDM}(1992)$ [9] with nuclear masses measured after the calculations were published showed that the model would reliably predict masses of nuclei that were not included in the deter- 
mination of model constants. In fact, with a properly defined model error, that is, a definition different from the root-mean-square error, which includes contributions from experimental errors, see Refs. [3, 9], we find that for 529 new masses in AME2003 [17] that were not known when the FRDM(1992) constants were determined, the error is only $0.462 \mathrm{MeV}$, compared to $0.669 \mathrm{MeV}$ with respect to the AME1989 data base [18] used in the determination of the FRDM(1992) constants. Furthermore, there was no systematic increase in the model error with distance from $\beta$ stability. It has also been established that these mass-model results agree very well with $Q_{\alpha}$ values observed in the decay of subsequently discovered superheavy elements [19, 20, 21, 22, 23, 24, 25, 26].

Many other nuclear-structure properties were successfully modeled, for example a special result of the 1981 mass calculation was the interpretation of certain spectroscopic results in terms of an intrinsic octupole deformation of nuclei in their ground state $[1,27,28,29]$.

We present results of our new calculations of nuclear ground-state masses and deformations, namely FRDM(2012) and FRLDM(2012). A summary description of the steps leading to the improved model are given in Fig. 1. These steps will be discussed in detail in section 4 .

Because in the macroscopic-microscopic approach we calculate single-particle energies and wave functions, it is possible to calculate a large number of nuclear-structure properties in addition to nuclear ground-state masses. These include the following:

\section{Ground-state deformation multipoles:}

Quadrupole $\varepsilon$ deformation

Octupole $\varepsilon$ deformation

Hexadecapole $\varepsilon$ deformation

Hexacontatetrapole $\varepsilon$ deformation

Related quadrupole $\beta$ deformation

Related octupole $\beta$ deformation

Related hexadecapole $\beta$ deformation

$\beta_{3}$

Related hexacontatetrapole $\beta$ deformation

$\beta_{4}$

Beta-decay properties:

$Q$ value of the $\beta$ decay

$\beta$-decay half-life

$\beta$-delayed one-neutron emission probability

$\beta$-delayed two-neutron emission probability

$\beta_{6}$

$\beta$-delayed three-neutron emission probability

\section{Lipkin-Nogami pairing quantities:}

Neutron pairing gap

$\Delta_{\mathrm{n}}$

Proton pairing gap

$\Delta_{\mathrm{p}}$

Neutron number-fluctuation constant

Proton number-fluctuation constant

\section{Odd-particle spins:}

Projection of the odd-neutron angular momentum along the symmetry axis

\section{Alpha-decay properties: \\ $Q$ value of the $\alpha$ decay \\ $\alpha$-decay half-life}

\section{FRDM mass-related quantities:}

Spherical macroscopic energy

$E_{\mathrm{mac}}^{\mathrm{sph}}$

Microscopic correction

Calculated mass excess

Discrepancy

Calculated binding energy

\section{FRLDM mass-related quantities:}

Finite-range liquid-drop model microscopic correction

Finite-range liquid-drop model mass excess

$E_{\text {mic }}^{\mathrm{FL}}$

$M_{\mathrm{th}}^{\mathrm{FL}}$ 
Folded-Yukawa finite-range single-particle related quantities:

Shell correction

Pairing correction

\section{Neutron and proton separation energies:}

One-neutron separation energy

Two-neutron separation energy

Three-neutron separation energy

One-proton separation energy

Two-proton separation energy

As mentioned above, we present in the Table the calculated ground-state masses and deformations and some related quantities. Some of the other quantities will be published later.

In the next section we specify the macroscopic-microscopic finite-range droplet model in some detail. We repeat some of the model details found in Ref. [9] for several reasons. First, we wish to correct the very few misprints that we and our colleagues found. Second, to provide in what is probably our final nuclear mass-table publication a complete specification of the model in one location. Third, the retrievable manuscript file on the ADNDT web site of the FRDM(1992) manuscript is as of this writing of poor quality and not searchable.

We discuss in particular the constants of the model, paying special attention to how to count the number of constants of a model. We present a summary of all constants in the model, including both those constants that have been determined from a least-squares adjustment to ground-state masses and fission-barrier heights and those that have been determined from other considerations. In addition we count what are considered "natural constants", such as $\hbar$. After our model has been specified, we discuss how it has been applied in the current calculation.

\section{MODELS}

In the macroscopic-microscopic method the total potential energy, which is calculated as a function of shape, proton number $Z$, and neutron number $N$, is the sum of a macroscopic term and a microscopic term representing the shell-plus-pairing correction. Thus, the total nuclear potential energy can be written as

$$
E_{\mathrm{pot}}(Z, N, \text { shape })=E_{\mathrm{mac}}(Z, N, \text { shape })+E_{\mathrm{s}+\mathrm{p}}(Z, N, \text { shape })
$$

We study two alternative models for $E_{\mathrm{mac}}$, given by Eqs. (40) and (62). The shell-plus-pairing correction is given by Eqs. (76) and (77).

It is practical to define an additional energy, the microscopic correction $E_{\text {mic }}$, which is different from the shellplus-pairing correction $E_{\mathrm{s}+\mathrm{p}}$. For a specific deformation $\varepsilon_{\mathrm{a}}$, the latter is determined solely from the single-particle level spectrum at this deformation by use of Strutinsky's shell-correction method [11, 12] and a pairing model. In contrast, the microscopic correction is given by

$$
E_{\mathrm{mic}}\left(\varepsilon_{\mathrm{a}}\right)=E_{\mathrm{S}+\mathrm{p}}\left(\varepsilon_{\mathrm{a}}\right)+E_{\mathrm{mac}}\left(\varepsilon_{\mathrm{a}}\right)-E_{\mathrm{mac}}\left(\varepsilon_{\mathrm{sphere}}\right)
$$

This definition has the desirable consequence that the potential energy $E_{\mathrm{pot}}$ of a nucleus at a certain deformation, for example the ground-state deformation $\varepsilon_{\mathrm{gs}}$, is simply

$$
E_{\text {pot }}\left(\varepsilon_{\mathrm{gs}}\right)=E_{\mathrm{mic}}\left(\varepsilon_{\mathrm{gs}}\right)+E_{\mathrm{mac}}\left(\varepsilon_{\text {sphere }}\right)
$$

However, the reader should note that in the literature the term microscopic correction is sometimes used instead for shell-plus-pairing correction. When results are presented it is usually $E_{\text {mic }}$ that is tabulated, because it represents all additional effects over and above the spherical macroscopic energy. In practical calculations it is $E_{\mathrm{s}+\mathrm{p}}$ that is calculated. To obtain the total energy a deformed macroscopic energy term is then added to $E_{\mathrm{s}+\mathrm{p}}$. These concepts are illustrated in Fig. 2. There exist several different models for both the macroscopic and microscopic terms. Most of the initial studies following the advent of Strutinsky's shell-correction method used the liquid-drop model [30, 31] as the macroscopic model.

The preferred model in the current calculations has its origin in a 1981 nuclear mass model [1,2], which utilized the folded-Yukawa single-particle potential developed in 1972 [10, 32]. The macroscopic model used in the 1981 calculation was a finite-range liquid-drop model, which contained a modified surface-energy term to account for the finite range of the nuclear force and the diffuseness of the nuclear surface. The modified surface-energy term was given by the Yukawa-plus-exponential finite-range model [33]. The macroscopic part in this formulation does not describe such features as nuclear compressibility and corresponding variations in the proton and neutron radii.

The droplet model $[6,7,8]$, an extension of the liquid-drop model $[30,31]$ that includes higher-order terms in $A^{-1 / 3}$ and $(N-Z) / A$, does describe such features. However, in its original formulation the droplet model was very inaccurate for nuclei far from stability. These deficiencies led Myers to suggest that the surface-energy terms of the droplet model also be generalized to account for the finite range of the nuclear force, and to more accurately 


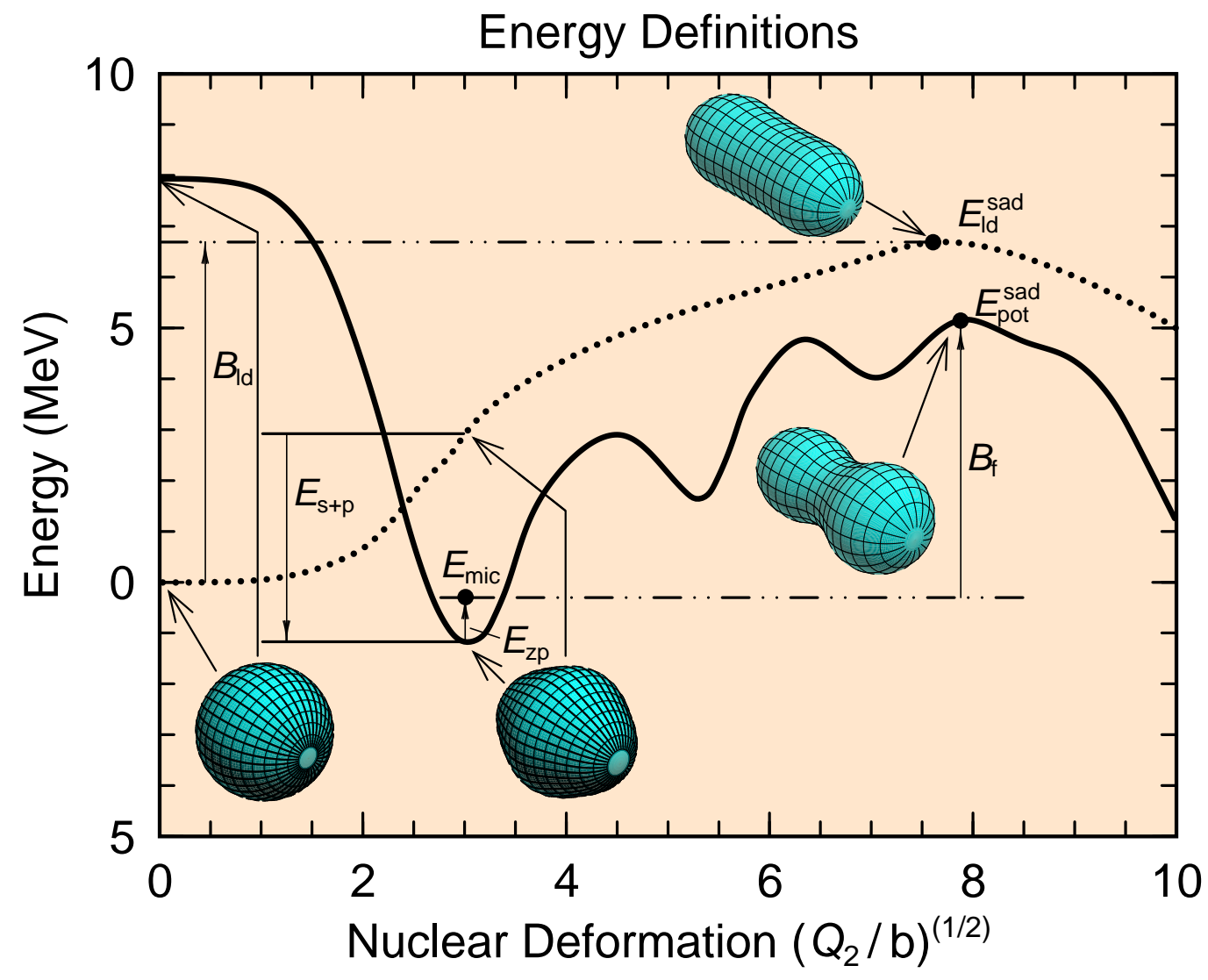

Fig. 2: Various energy concepts used in macroscopic-microscopic potential-energy calculations. The dotted line is the macroscopic "liquid-drop" (FRLDM) energy along a specified path; the solid line is the total macroscopic-microscopic energy along a partially different shape sequence. So that the various energy concepts can be illustrated, the shapes for which the energies have been calculated are: At $Q_{2}=0$ the energies are calculated for a spherical shape. For the shapes from the sphere to the ground-state shape, the shapes are the same for both curves and chosen so that they evolve continuously from the sphere to the calculated macroscopic-microscopic ground-state shape. From the ground state towards larger deformations, the total-energy curve is along the optimal fission path that includes all minima and saddle points identified along this path in the five-dimensional deformation space; the liquid-drop-energy curve joins smoothly the macroscopic energy for the shape at the macroscopic-microscopic ground-state (which is not the lowest macroscopic energy at this value of $Q_{2}$ ) to the FRLDM saddle point. The energies are calculated for ${ }^{232}$ Th. $B_{\mathrm{f}}$ is the fission-barrier height, $B_{\mathrm{ld}}$ is the calculated macroscopic barrier height, $E_{\mathrm{mic}}$ is defined in eq. $2, E_{\mathrm{s}+\mathrm{p}}$ is the shell-plus-pairing correction, and $E_{\mathrm{zp}}$ is the zero-point energy discussed in Sect. 2.11.

account for to the diffuseness of the nuclear surface. Thus, the Yukawa-plus-exponential model for the surface tension was incorporated into the droplet model. During this work it also became apparent that the description of nuclear compressibility in the original droplet model was unsatisfactory, since the squeezing of the central density of light nuclei was overpredicted. The deficiency was serious because it starts to become important by about $A=120$ and becomes even more pronounced for lighter nuclei. To account for compressibility effects for light nuclei and for other higher-order effects an empirical exponential term was added. The final modifications leading to the FRDM was the addition of a charge-asymmetry term and a constant $[5,4]$. The charge-asymmetry term and the constant were originally proposed and included in the 1981 mass model [1].

The additions of these effects and terms to the droplet model [5] resulted in dramatic improvements in its predictive properties, as summarized in the discussion of Table A in Ref. [4]. Mass calculations based on both the FRLDM [3] and the FRDM [4] were presented in the 1988 review of mass models in ATOMIC DATA AND NUCLEAR DATA TABLES. These calculations also used an improved pairing model relative to that used in the 1981 work. In the 1988 results the error in the FRDM was $8 \%$ lower than that in the FRLDM.

However, there were two major unresolved issues in the 1988 calculations. First, there existed some deficiencies in the pairing model and the values of the constants that were used. Second, $\varepsilon_{3}$ and $\varepsilon_{6}$ shape degrees of freedom were still not included, so deviations between calculated and measured masses due to the omission of these shape degrees of freedom were still present. Extensive investigations of pairing models and their constants have now been completed and resulted in an improved formulation of the pairing model [16]. In the $\operatorname{FRDM}(1992)$ we also minimized the potential energy with respect to $\varepsilon_{3}$ and $\varepsilon_{6}$ shape degrees of freedom in an approximate fashion. In the FRDM(2012) calculation we have improved the determination of ground-state shapes very significantly and also made other improvements which will be discussed after the model specification.

Although the FRDM is now our preferred model of ground-state masses, we also present results for the FRLDM because we are now aware that the FRDM cannot be applied to the very deformed shapes occurring in fission. The FRLDM can also be used in studies that assume constant nuclear density. We therefore specify below both models. Because several of the model constants are determined by minimization of the model error, we start by defining a proper way to determine model error, which unlike a root-mean-square "rms" definition, does not contain contributions 
from the experimental statistical uncertainties.

\subsection{Model error and adjustment procedure}

In many studies the model error has been defined as simply the rms deviation, which as usual is given by

$$
\mathrm{rms}=\left[\frac{1}{n} \sum_{i=1}^{n}\left(M_{\mathrm{exp}}^{i}-M_{\mathrm{th}}^{i}\right)^{2}\right]^{\frac{1}{2}}
$$

Here $M_{\mathrm{th}}^{i}$ is the calculated mass for a particular value of the proton number $Z$ and neutron number $N$, and $M_{\mathrm{exp}}^{i}$ is the corresponding measured quantity. There are $n$ such measurements for different $N$ and $Z$. The choice (4) is a reasonable definition when all the errors $\sigma_{\exp }^{i}$ associated with the measurements are small compared to the model error. However, for large $\sigma_{\exp }^{i}$ the above definition is unsatisfactory, since both the theoretical and experimental errors contribute to the rms deviation. The definition (4) will therefore always overestimate the intrinsic model error.

When the experimental errors are large, it is necessary to use an approach that "decouples" the theoretical and experimental errors from one another. This can be accomplished by observing that the calculated masses are distributed around the true masses with a standard deviation $\sigma_{\mathrm{th}}$. There exist powerful statistical methods for determining the intrinsic model error $\sigma_{\mathrm{th}}$. The model error obtained in this way contains no contributions from the experimental uncertainties $\sigma_{\text {exp }}^{i}$. To introduce such an error concept, a new set of equations for determining model parameters and error was derived [3] by use of statistical arguments and the maximum-likelihood (ML) method. Here we generalize from the original assumption [3] $e_{\mathrm{th}}^{i} \in \mathrm{N}\left(0, \sigma_{\mathrm{th}}\right)$ that the theoretical error term $e_{\mathrm{th}}^{i}$ is normally distributed with zero mean deviation from the true mass to $e_{\mathrm{th}}^{i} \in \mathrm{N}\left(\mu_{\mathrm{th}}, \sigma_{\mathrm{th}}\right)$ to allow for an error with a mean $\mu_{\mathrm{th}}$ that is different from zero and a standard deviation $\sigma_{\text {th }}$ around this mean [34]. Normally, if the model is adjusted only to a specific type of data, such as masses, the mean is very close to zero for the data to which the model constants were adjusted but may be significantly different for new masses that were not included when the model constants were determined [9, 24]. We are led to the generalized equations

$$
\begin{gathered}
\sum_{i=1}^{n} \frac{\left[M_{\mathrm{exp}}^{i}-\left(M_{\mathrm{th}}^{i}+\mu_{\mathrm{th}}{ }^{*}\right)\right]}{\sigma_{\exp }^{i}+\sigma_{\mathrm{th}}{ }^{2 *}} \frac{\partial M_{\mathrm{th}}^{i}}{\partial p_{v}}=0, \quad v=1,2, \ldots, m \\
\sum_{i=1}^{n} \frac{\left[M_{\mathrm{exp}}^{i}-\left(M_{\mathrm{th}}^{i}+\mu_{\mathrm{th}}{ }^{*}\right)\right]^{2}-\left({\sigma_{\mathrm{exp}}^{i}}^{2}+\sigma_{\mathrm{th}}{ }^{2 *}\right)}{\left({\sigma_{\mathrm{exp}}^{i}}^{2}+\sigma_{\mathrm{th}}{ }^{2}\right)^{2}}=0 \\
\sum_{i=1}^{n} \frac{\left[M_{\mathrm{exp}}^{i}-\left(M_{\mathrm{th}}^{i}+\mu_{\mathrm{th}}{ }^{*}\right)\right]}{\left(\sigma_{\exp }^{i}+\sigma_{\mathrm{th}}{ }^{2 *}\right)}=0
\end{gathered}
$$

where $p_{v}$ are the unknown parameters of the model. The notation $\sigma_{\mathrm{th}}{ }^{2}$ means that by solving Eqs. (6) and (7) we obtain the estimate $\sigma_{\mathrm{th}}{ }^{2 *}$ of the true $\sigma_{\mathrm{th}}{ }^{2}$. Equation (5) is equivalent to minimizing $S$ with respect to $p_{v}$, where

$$
S=\sum_{i=1}^{n} \frac{\left[M_{\mathrm{exp}}^{i}-\left(M_{\mathrm{th}}^{i}+\mu_{\mathrm{th}}^{*}\right)\right]^{2}}{\sigma_{\exp }^{i}+\sigma_{\mathrm{th}}^{2 *}}
$$

Thus, we are led to two additional equations relative to the usual least-squares equations that arise when model parameters are estimated by adjustments to experimental data under the assumption of a perfect theory with $\sigma_{\text {th }}=0$ and $\mu_{\mathrm{th}}=0$. For the FRLDM the least-squares equations (5) are linear, whereas for the FRDM they are non-linear.

When the model contains a term $a_{0} A^{0}$ that is strictly constant, Eq. (7) is identical to the member in Eq. (5) that corresponds to the derivative with respect to this constant. Thus, one should in this case put $\mu_{\mathrm{th}}{ }^{*}=0$ and solve only the remaining $m+1$ equations. One may therefore in this case characterize the error of the model in the region where the parameters were adjusted solely by the quantity $\sigma_{\text {th }}$. In other cases one should solve the full set of equations. If $\mu_{\mathrm{th}}{ }^{*}$ is significantly different from zero the theory needs modification. Even if $\mu_{\mathrm{th}}=0$ in the original data region, it is entirely possible (although undesirable) that one obtains a mean error $\mu_{\mathrm{th}}{ }^{*}$ that is substantially different from zero when one analyzes model results for new data points to which the parameters were not adjusted. In this case the most complete characterization of the theoretical error requires both its mean $\mu_{\mathrm{th}}$ and its standard deviation $\sigma_{\mathrm{th}}$ around this mean.

To allow for a single error measure that is similar to an rms deviation between the data and model we later also calculate the square root of the second central moment of the error term, $\sigma_{\mathrm{th} ; \mu=0}$, in our studies of model behavior in new regions of nuclei. This quantity is obtained by setting $\mu_{\mathrm{th}}{ }^{*}=0$ when solving Eq. (6). In contrast to the rms measure, it has the advantage that it has no contributions from the experimental errors.

Equations (5)-(7) constitute a system of $m+2$ equations that are to be solved together. It is instructive to rewrite Eqs. (6) and (7) as

$$
\sigma_{\mathrm{th}}{ }^{2 *}=\frac{1}{\sum_{i=1}^{n} w_{i} k_{\sigma}} \sum_{i=1}^{n} w_{i}^{k_{\sigma}}\left[\left(M_{\mathrm{exp}}^{i}-M_{\mathrm{th}}^{i}-\mu_{\mathrm{th}}{ }^{*}\right)^{2}-\sigma_{\mathrm{exp}}^{i}{ }^{2}\right]
$$




$$
\mu_{\mathrm{th}}^{*}=\frac{1}{\sum_{i=1}^{n} w_{i}^{k_{\mu}}} \sum_{i=1}^{n} w_{i}^{k_{\mu}}\left[\left(M_{\mathrm{exp}}^{i}-M_{\mathrm{th}}^{i}\right)\right]
$$

where

$$
\begin{gathered}
w_{i}^{k}=\frac{1}{\left(\sigma_{\exp }^{i}{ }^{2}+\sigma_{\mathrm{th}^{2 *}}\right)^{k}} \\
k_{\sigma}=2 \\
k_{\mu}=1
\end{gathered}
$$

The unknowns $\mu_{\mathrm{th}}{ }^{*}$ and $\sigma_{\mathrm{th}}{ }^{2 *}$ can easily be determined from Eqs. (9) and (10) by an iterative procedure whose convergence is extremely rapid, requiring only about four iterations. An interpretation, not a proof, of Eq. (9) is that the experimental error is "subtracted out" from the difference between the experimental and calculated masses.

A common misconception is that one has to "throw away" data points that have errors that are equal to or larger than the error of the model whose parameters are determined. When a proper statistical approach, such as the one above, is used, this is no longer necessary, as is further illustrated by simulations in Ref. [3].

We will see below that the discrepancy between our mass calculations and measured masses systematically increases as the size of the nuclear system decreases. It is therefore of interest to consider that the mass-model error is a function of mass number $A$. A simple function to investigate is

$$
\sigma_{\mathrm{th}}=\frac{c}{A^{\alpha}}
$$

where $c$ and $\alpha$ are two parameters to be determined. Whereas under the assumption of a constant model error one determines this single error constant from Eq. (9), we find that the ML method for the error assumption in Eq. (14), with two unknowns, and assuming $\mu_{\text {th }}=0$, yields the equations

$$
\begin{aligned}
& \sum_{i=1}^{n} \frac{\left(M_{\exp }^{i}-M_{\mathrm{th}}^{i}\right)^{2}-\left[\sigma_{\exp }^{i}{ }^{2}+\left(\frac{c^{*}}{A_{i}^{\alpha^{*}}}\right)^{2}\right]}{\left[\sigma_{\exp }^{i}{ }^{2}+\left(\frac{c^{*}}{A_{i}^{\alpha^{*}}}\right)^{2}\right]^{2} A_{i}^{\alpha^{*}}}=0 \\
& \sum_{i=1}^{n} \frac{\left(M_{\exp }^{i}-M_{\mathrm{th}}^{i}\right)^{2}-\left[\sigma_{\exp }^{i}+\left(\frac{c^{*}}{A_{i}^{\alpha^{*}}}\right)^{2}\right]}{\left[\sigma_{\exp }^{i}+\left(\frac{c^{*}}{A_{i}^{\alpha^{*}}}\right)^{2}\right]^{2} A_{i}^{\alpha^{*}+1}}=0
\end{aligned}
$$

These equations are considerably more complicated to solve than Eq. (9). Solutions were obtained for the FRDM(1992) in Ref. [9]. We have also studied the error versus $A$ in the FRDM(2012), see Sect. 5.3.2.

\subsection{Shape parameterizations}

The original parameterization of the folded-Yukawa single-particle model was the three-quadratic-surface parameterization [35, 10]. It was designed to allow great flexibility in describing shapes late in the fission process. However, it is less suitable for describing ground-state shapes.

To allow a better description of ground-state shapes and to allow close comparison with results of Nilsson modifiedoscillator calculations, we incorporated the Nilsson perturbed-spheroid parameterization, or $\varepsilon$ parameterization, into the folded-Yukawa single-particle computer code in 1973 [36, 32, 37].

In our work we use the $\varepsilon$ parameterization for all calculations related to ground-state properties. In our adjustment of macroscopic constants of the FRLDM we also include 31 outer saddle-point heights of fission barriers. The shapes of these saddle points were obtained in a modern barrier calculation based on several million different shapes in the three-quadratic-surface parameterization [38, 39].

\subsubsection{Perturbed-spheroid parameterization}

The $\varepsilon$ parameterization was originally used by Nilsson [40] in the modified-oscillator single-particle potential. It was introduced to limit the dimensions of the matrices from which the single-particle energies and wave functions are obtained by diagonalization. This requirement leads to somewhat complex expressions for the nuclear shape. Here we employ its extension to higher-multipole distortions. In contrast to the FRDM(1992) mass table we now also consider axially asymmetric shapes $[41,42,43]$. Note that a factor $\frac{1}{2} \sqrt{\frac{4 \pi}{9}}$ is missing in front of the $V_{4}(\gamma)$ function in Eq. (3) of Ref. [43]. Some misprints in the equations presented in Ref. [9] are corrected below. Some studies of the effect of axial asymmetry and octupole degrees of freedom on nuclear masses were presented in Refs. [44, 45]. Consideration of axial asymmetry is needed to study shape coexistence. We presented earlier studies of shape coexistence throughout the nuclear chart in Refs. [25, 46]. 
As the first step in defining the $\varepsilon$ parameterization a "stretched" representation is introduced. The stretched coordinates $\xi, \eta$, and $\zeta$ are defined by

$$
\begin{aligned}
\xi & =\left\{\frac{m \omega_{0}}{\hbar}\left[1-\frac{2}{3} \varepsilon_{2} \cos \left(\gamma+\frac{2}{3} \pi\right)\right]\right\}^{1 / 2} x \\
\eta & =\left\{\frac{m \omega_{0}}{\hbar}\left[1-\frac{2}{3} \varepsilon_{2} \cos \left(\gamma-\frac{2}{3} \pi\right)\right]\right\}^{1 / 2} y \\
\zeta & =\left\{\frac{m \omega_{0}}{\hbar}\left[1-\frac{2}{3} \varepsilon_{2} \cos \gamma\right]\right\}^{1 / 2} z
\end{aligned}
$$

where $\hbar \omega_{0}$ is the oscillator energy, $\varepsilon_{2}$ the ellipsoidal deformation parameter, and $\gamma$ the non-axiality angle. It is then convenient to define a "stretched" radius vector $\rho_{\mathrm{t}}$ by

$$
\rho_{\mathrm{t}}=\left(\xi^{2}+\eta^{2}+\zeta^{2}\right)^{1 / 2}
$$

a stretched polar angle $\theta_{t}$ by

$$
u=\cos \theta_{t}=\frac{\zeta}{\rho_{\mathrm{t}}}=\left[\frac{1-\frac{2}{3} \varepsilon_{2} \cos \gamma}{1-\frac{1}{3} \varepsilon_{2} \cos \gamma\left(3 \cos ^{2} \theta-1\right)+\left(\frac{1}{3}\right)^{1 / 2} \varepsilon_{2} \sin \gamma \sin ^{2} \theta \cos 2 \phi}\right]^{1 / 2} \cos \theta
$$

and a stretched azimuthal angle $\phi_{\mathrm{t}}$ by

$$
v=\cos 2 \phi_{\mathrm{t}}=\frac{\xi^{2}-\eta^{2}}{\xi^{2}+\eta^{2}}=\frac{\left[1+\frac{1}{3} \varepsilon_{2} \cos \gamma\right] \cos 2 \phi+\left(\frac{1}{3}\right)^{1 / 2} \varepsilon_{2} \sin \gamma}{1+\frac{1}{3} \varepsilon_{2} \cos \gamma+\left(\frac{1}{3}\right)^{1 / 2} \varepsilon_{2} \sin \gamma \cos 2 \phi}
$$

In the folded-Yukawa model the single-particle potential is very different from that in the Nilsson modifiedoscillator model. However, the definition of the $\varepsilon$ parameterization will be most clear if we follow the steps in the Nilsson model. The implementation in the folded-Yukawa model will then be simple. The Nilsson modified-oscillator potential is defined by

$$
\begin{aligned}
V=\frac{1}{2} & \hbar \omega_{0} \rho_{\mathrm{t}}^{2}\left\{1+2 \varepsilon_{1} P_{1}\left(\cos \theta_{\mathrm{t}}\right)\right. \\
& -\frac{2}{3} \varepsilon_{2} \cos \gamma P_{2}\left(\cos \theta_{\mathrm{t}}\right)+\frac{1}{3} \varepsilon_{2} \sin \gamma\left(\frac{8}{5} \pi\right)^{1 / 2}\left[Y_{2}^{2}\left(\theta_{\mathrm{t}}, \phi_{\mathrm{t}}\right)+Y_{2}^{-2}\left(\theta_{\mathrm{t}}, \phi_{\mathrm{t}}\right)\right] \\
& \left.+2 \varepsilon_{3} P_{3}\left(\cos \theta_{\mathrm{t}}\right)+2 \varepsilon_{4} V_{4}\left(\cos \theta_{\mathrm{t}}, \cos 2 \phi_{\mathrm{t}}\right)+2 \varepsilon_{5} P_{5}\left(\cos \theta_{\mathrm{t}}\right)+2 \varepsilon_{6} P_{6}\left(\cos \theta_{\mathrm{t}}\right)\right\} \\
& -\kappa \hbar \stackrel{\circ}{\stackrel{\omega}{\omega}_{0}}\left[2 \vec{l}_{\mathrm{t}} \cdot \vec{s}+\mu\left(\vec{l}_{\mathrm{t}}^{2}-<\vec{l}_{\mathrm{t}}^{2}>\right)\right]
\end{aligned}
$$

where $\vec{l}_{\mathrm{t}}$ is the angular-momentum operator in the stretched coordinate system, $\vec{s}$ is the spin operator [40], and

$$
V_{4}(u, v)=a_{40} P_{4}+\sqrt{\frac{4 \pi}{9}}\left[a_{42}\left(Y_{4}^{2}+Y_{4}^{-2}\right)+a_{44}\left(Y_{4}^{4}+Y_{4}^{-4}\right)\right]
$$

Here the hexadecapole potential $V_{4}(u, v)$ is made dependent on $\gamma$ in such a way that axial symmetry is maintained when $\gamma=0,60^{\circ},-120^{\circ}$, and $-60^{\circ}$, for mass-symmetric shapes and for $\varepsilon_{6}=0$. This is accomplished by choosing the coefficients $a_{4 i}$ so that they have the transformation properties of a hexadecapole tensor. However, this is achieved only for mass-symmetric shapes and for $\varepsilon_{6}=0$. The $\varepsilon$ parameterization has not been generalized to a more general case. Thus [43]

$$
\begin{aligned}
& a_{40}=\frac{1}{6}\left(5 \cos ^{2} \gamma+1\right) \\
& a_{42}=-\frac{1}{12} \sqrt{30} \sin 2 \gamma \\
& a_{44}=\frac{1}{12} \sqrt{70} \sin ^{2} \gamma
\end{aligned}
$$


It is customary to now assume that the shape of the nuclear surface is equal to the shape of an equipotential surface given by Eq. (21). By neglecting the $\vec{l}_{\mathrm{t}} \cdot \vec{s}$ and $\vec{l}_{\mathrm{t}}^{2}$ terms and solving for $\rho_{\mathrm{t}}$ and then using Eqs. (17)-(20) to derive an expression for $r$ in the non-stretched laboratory system we obtain

$$
\begin{aligned}
r(\theta, \phi)=\frac{R_{0}}{\omega_{0} / \stackrel{\circ}{\omega}_{0}}\left\{\left[1-\frac{2}{3} \varepsilon_{2} \cos \left(\gamma+\frac{2}{3} \pi\right)\right]\left[1-\frac{2}{3} \varepsilon_{2} \cos \left(\gamma-\frac{2}{3} \pi\right)\right]\left[1-\frac{2}{3} \varepsilon_{2} \cos \gamma\right]\right\}^{-1 / 2} \\
\times\left[1-\frac{1}{3} \varepsilon_{2} \cos \gamma-\frac{2}{9} \varepsilon_{2}^{2} \cos ^{2} \gamma+\varepsilon_{2}\left(\cos \gamma+\frac{1}{3} \varepsilon_{2} \cos 2 \gamma\right) u^{2}\right. \\
\times\left[1-\frac{2}{3} \varepsilon_{2} \cos \gamma \frac{1}{2}\left(3 u^{2}-1\right)+\left(\frac{1}{3}\right)^{1 / 2} \varepsilon_{2} \sin \gamma\left(1-\frac{2}{3} \varepsilon_{2} \cos \gamma\right)\left(1-u^{2}\right) v\right]^{1 / 2}\left(1-u^{2}\right) v \\
\times \quad\left[2 \varepsilon_{1} P_{1}(u)+2 \varepsilon_{3} P_{3}(u)+2 \varepsilon_{4} V_{4}(u, v)+2 \varepsilon_{5} P_{5}(u)+2 \varepsilon_{6} P_{6}(u)\right]^{-1 / 2}
\end{aligned}
$$

In the Nilsson model the starting point is to define the potential. After the potential has been generated the shape of the nuclear surface is deduced by the above argument. In the folded-Yukawa model the starting point is different. There, the equation for the nuclear surface, given by Eq. (24) in the case of the $\varepsilon$ parameterization, is specified in the initial step. Once the shape of the surface is known, the single-particle potential may be generated as described in later sections.

The quantity $\omega_{0} / \stackrel{\circ}{\omega}_{0}$ is determined by requiring that the volume remain constant with deformation, which gives

$$
\begin{aligned}
\left(\frac{\omega_{0}}{\omega_{0}}\right)^{3}= & \frac{1}{4 \pi}\left\{\left[1-\frac{2}{3} \varepsilon_{2} \cos \left(\gamma+\frac{2}{3} \pi\right)\right]\left[1-\frac{2}{3} \varepsilon_{2} \cos \left(\gamma-\frac{2}{3} \pi\right)\right]\left[1-\frac{2}{3} \varepsilon_{2} \cos \gamma\right]\right\}^{-1 / 2} \\
& \times \int_{0}^{\pi} d \theta_{\mathrm{t}} \int_{0}^{2 \pi} d \phi_{\mathrm{t}} \sin \theta_{\mathrm{t}}\left[1-\frac{2}{3} \varepsilon_{2} \cos \gamma P_{2}(u)+\varepsilon_{2} \sin \gamma\left(\frac{8 \pi}{45}\right)^{1 / 2}\left(Y_{2}^{2}+Y_{2}^{-2}\right)\right. \\
+ & \left.2 \varepsilon_{1} P_{1}(u)+2 \varepsilon_{3} P_{3}(u)+2 \varepsilon_{4} V_{4}(u, v)+2 \varepsilon_{5} P_{5}(u)+2 \varepsilon_{6} P_{6}(u)\right]^{-3 / 2}
\end{aligned}
$$

The above equation is derived by determining the volume inside the nuclear surface given by Eq. (24), with the integral $\int d^{3} r$ inside the surface evaluated in terms of the "non-stretched" coordinates $\theta$ and $\phi$. After a variable substitution one arrives at the expression in Eq. (25).

The Legendre polynomials $P_{l}$ occurring in the definitions of the $\varepsilon$ parameterization are defined by

$$
P_{l}(u)=\frac{1}{2^{l} l !} \frac{d^{l}}{d u^{l}}\left(u^{2}-1\right)^{l}, \quad l=0,1,2, \ldots, \infty
$$

The first six Legendre polynomials are

$$
\begin{aligned}
& P_{0}(u)=1 \\
& P_{1}(u)=u \\
& P_{2}(u)=\frac{1}{2}\left(3 u^{2}-1\right) \\
& P_{3}(u)=\frac{1}{2}\left(5 u^{3}-3 u\right) \\
& P_{4}(u)=\frac{1}{8}\left(35 u^{4}-30 u^{2}+3\right) \\
& P_{5}(u)=\frac{1}{8}\left(63 u^{5}-70 u^{3}+15 u\right) \\
& P_{6}(u)=\frac{1}{16}\left(231 u^{6}-315 u^{4}+105 u^{2}-5\right)
\end{aligned}
$$

The associated Legendre functions $P_{l}^{m}$ are defined by

$$
P_{l}^{m}(u)=\frac{\left(1-u^{2}\right)^{m / 2}}{2^{l} l !} \frac{d^{l+m}}{d u^{l+m}}\left(u^{2}-1\right)^{l}, \quad l=0,1,2, \ldots, \infty ; \quad m=0,1,2, \ldots, l
$$


The spherical harmonics are then determined from the relations

$$
\begin{gathered}
Y_{l}^{m}(\theta, \phi)=(-)^{m}\left[\frac{(2 l+1)}{4 \pi} \frac{(l-m) !}{(l+m) !}\right]^{1 / 2} P_{l}^{m}(\cos \theta) e^{i m \phi}, \quad m \geq 0 \\
Y_{l}^{m *}(\theta, \phi)=(-)^{m} Y_{l}^{-m}(\theta, \phi)
\end{gathered}
$$

which yield for the functions used here

$$
\begin{aligned}
Y_{2}^{2}(\theta, \phi) & =\sqrt{\frac{15}{32 \pi}} \sin ^{2} \theta e^{2 i \phi} \\
Y_{2}^{-2}(\theta, \phi) & =\sqrt{\frac{15}{32 \pi}} \sin ^{2} \theta e^{-2 i \phi} \\
Y_{4}^{4}(\theta, \phi) & =\sqrt{\frac{315}{512 \pi}} \sin ^{4} \theta e^{4 i \phi} \\
Y_{4}^{-4}(\theta, \phi) & =\sqrt{\frac{315}{512 \pi}} \sin ^{4} \theta e^{-4 i \phi} \\
Y_{4}^{2}(\theta, \phi) & =\sqrt{\frac{45}{128 \pi}} \sin ^{2} \theta\left(7 \cos ^{2} \theta-1\right) e^{2 i \phi} \\
Y_{4}^{-2}(\theta, \phi) & =\sqrt{\frac{45}{128 \pi}} \sin ^{2} \theta\left(7 \cos ^{2} \theta-1\right) e^{-2 i \phi}
\end{aligned}
$$

The sums

$$
\begin{aligned}
& S Y_{22}=Y_{2}^{2}(\theta, \phi)+Y_{2}^{-2}(\theta, \phi) \\
& S Y_{44}=Y_{4}^{4}(\theta, \phi)+Y_{4}^{-4}(\theta, \phi) \\
& S Y_{42}=Y_{4}^{2}(\theta, \phi)+Y_{4}^{-2}(\theta, \phi)
\end{aligned}
$$

are required in the expression for the single-particle potential and in the corresponding equation for the nuclear surface. When the arguments of the spherical harmonics are the stretched angles $\theta_{\mathrm{t}}$ and $\phi_{\mathrm{t}}$ we obtain

$$
\begin{aligned}
& S Y_{22}=\sqrt{\frac{15}{8 \pi}} \sin ^{2} \theta_{\mathrm{t}} \cos 2 \phi_{\mathrm{t}}=\sqrt{\frac{15}{8 \pi}}\left(1-u^{2}\right) v \\
& S Y_{44}=\sqrt{\frac{315}{128 \pi}} \sin ^{4} \theta \cos 4 \phi=\sqrt{\frac{315}{128 \pi}}\left(1-u^{2}\right)^{2}\left(2 v^{2}-1\right) \\
& S Y_{42}=\sqrt{\frac{45}{32 \pi}} \sin ^{2} \theta_{\mathrm{t}}\left(7 \cos ^{2} \theta_{\mathrm{t}}-1\right) \cos 2 \phi_{\mathrm{t}}=\sqrt{\frac{45}{32 \pi}}\left(1-u^{2}\right)\left(7 u^{2}-1\right) v
\end{aligned}
$$

\subsubsection{Three-quadratic-surface parameterization}

In the three-quadratic-surface parameterization the shape of the nuclear surface is defined in terms of three smoothly joined portions of quadratic surfaces of revolution. They are completely specified by [35, 36, 32],

$$
\rho^{2}= \begin{cases}a_{1}^{2}-\frac{a_{1}^{2}}{c_{1}^{2}}\left(z-l_{1}\right)^{2}, & l_{1}-c_{1} \leq z \leq z_{1} \\ a_{2}^{2}-\frac{a_{2}^{2}}{c_{2}^{2}}\left(z-l_{2}\right)^{2}, & z_{2} \leq z \leq l_{2}+c_{2} \\ a_{3}^{2}-\frac{a_{3}^{2}}{c_{3}^{2}}\left(z-l_{3}\right)^{2}, & z_{1} \leq z \leq z_{2}\end{cases}
$$

The left-hand surface is denoted by the subscript 1 , the right-hand one by 2 , and the middle one by 3 . Each surface is specified by the position $l_{i}$ of its center, its transverse semiaxis $a_{i}$, and its semi-symmetry axis $c_{i}$. At the left and right intersections of the middle surface with the end surfaces the value of $z$ is $z_{1}$ and $z_{2}$, respectively.

There are nine numbers required to specify the expressions in Eq. (34) but three numbers are eliminated by the conditions of constancy of the volume and continuous first derivatives at $z_{1}$ and $z_{2}$. The introduction of an auxiliary unit of distance $u$ through

$$
u=\left[\frac{1}{2}\left(a_{1}^{2}+a_{2}^{2}\right)\right]^{\frac{1}{2}}
$$


permits the definition of three mass-symmetric coordinates $\sigma_{i}$ and three mass-asymmetric coordinates $\alpha_{i}$ by

$$
\begin{aligned}
\sigma_{1} & =\frac{\left(l_{2}-l_{1}\right)}{u} \\
\sigma_{2} & =\frac{a_{3}^{2}}{c_{3}^{2}} \\
\sigma_{3} & =\frac{1}{2}\left(\frac{a_{1}^{2}}{c_{1}^{2}}+\frac{a_{2}^{2}}{c_{2}^{2}}\right) \\
\alpha_{1} & =\frac{1}{2} \frac{\left(l_{1}+l_{2}\right)}{u} \\
\alpha_{2} & =\frac{\left(a_{1}^{2}-a_{2}^{2}\right)}{u^{2}} \\
\alpha_{3} & =\frac{a_{1}^{2}}{c_{1}^{2}}-\frac{a_{2}^{2}}{c_{2}^{2}}
\end{aligned}
$$

The coordinate $\alpha_{1}$ is not varied freely but is instead determined by the requirement that the center of mass be at the origin. These shape coordinates were historically used for about 30 years [35, 10, 47, 48, 49, 50, 1, 2, 51, 52]. However when we started to explore the full five-dimensional shape space we realized that an intuitive interpretation of calculations based on these coordinates is difficult and have introduced instead five alternative shape coordinates:(1) elongation, expressed in terms of the charge quadrupole moment $Q_{2}$, (2) neck diameter $d$, (3) left nascent-fragment deformation $\varepsilon_{\mathrm{f} 1}$, (4) right nascent-fragment deformation $\varepsilon_{\mathrm{f} 2}$, and (5) mass asymmetry $\alpha_{\mathrm{g}}$. The transformations from these coordinates to the precise shape given by Eq. (34) are lengthy and as regards the neck diameter highly nonlinear so we refer to Ref. [39] for details. These deformation variables have been used exclusively in our fission studies since 1999, the more important ones being $[53,54,55,38,56,57,58,39,59,60,61]$. However, the actual shapes generated by the expressions in Eq. (34) are the same, regardless of what primary "deformation" coordinates we use, it is just the interpretation of the calculated fission potential-energy surfaces that is facilitated by our more recent choices. One should also note that in our recent studies where we calculate potential-energy surfaces for more than 5 million shapes, we actually study (on a discrete, densely spaced grid) all shapes accessible to the parameterization, which would have been an impossible task some decades ago.

\subsubsection{Conversions to $\beta$ parameters}

A common parameterization, which we do not use here, is the $\beta$ parameterization. However, since we want to present some of our results in terms of $\beta$ shape parameters, we introduce the parameterization and a scheme to express shapes generated in other parameterizations in terms of $\beta$ deformation parameters. In the $\beta$ parameterization the radius vector $r$ is defined by

$$
r(\theta, \phi)=R_{0}\left(1+\sum_{l=1}^{\infty} \sum_{m=-l}^{l} \beta_{l m} Y_{l}^{m}\right)
$$

where $R_{0}$ is deformation dependent so as to conserve the volume inside the nuclear surface. When only axially symmetric shapes are considered the notation $\beta_{l}$ is normally used for $\beta_{l 0}$. Since the spherical harmonics $Y_{l}^{m}$ are orthogonal, one may determine the $\beta$ parameters corresponding to a specific shape in the $\varepsilon$ parameterization by use of

$$
\beta_{l m}=\sqrt{4 \pi} \frac{\int r(\theta, \phi) Y_{l}^{m}(\theta, \phi) d \Omega}{\int r(\theta, \phi) Y_{0}^{0}(\theta, \phi) d \Omega}
$$

where $r$ is now the radius vector in the $\varepsilon$ parameterization, given by Eq. (24). This conversion equation is in fact valid for a radius vector $r(\theta, \phi)$ defined by any parameterization.

When the $\beta$ parameters corresponding to a specific shape in the $\varepsilon$ parameterization are determined, one should observe that higher-order $\beta$ parameters may be non-zero even if higher-order $\varepsilon$ parameters are identically zero. For this reason, the nuclear ground-state shape is not completely specified by the $\beta$ parameters in the Table, whereas the shape is completely defined by the $\varepsilon$ parameters.

\subsection{Finite-range droplet model}

The finite-range droplet model, developed in 1984 [5], combines the finite-range effects of the FRLDM [62, 63, 33] with the higher-order terms in the droplet model. In addition, the finite-range droplet model contains an exponential term

$$
-C A e^{-\gamma A^{1 / 3} \bar{\varepsilon}}
$$


where $C$ and $\gamma$ specify the strength and range, respectively, of this contribution to the energy and the quantity $\bar{\varepsilon}$ is a dilatation variable given by Eq. (49). The exponential term leads to an improved description of compressibility effects. As in the original mass model [1] we have also added a constant $A^{0}$ term (whose coefficient accidentally came out to be zero in the FRDM(1992) mass table) and a charge asymmetry term, see Eqs. $(40,62)$. All these terms turn out to be crucial to the substantially improved results obtained in the finite-range droplet model relative to the original droplet model. These empirical terms will be further discussed below.

Most of our results are based on the finite-range droplet model for the macroscopic term. Relative to the formulation given in Ref. [5], which unfortunately has numerous misprints, we use a new model for the average neutron and proton pairing gaps. The complete expression for the contribution to the atomic mass excess from the FRDM macroscopic energy is obtained after minimization with respect to variations in $\bar{\varepsilon}$ and $\bar{\delta}$, where $\bar{\delta}$ is the average bulk relative neutron excess given by Eq. (47). One then obtains

$$
\begin{aligned}
& E_{\text {mac }}(Z, N, \text { shape })= \\
& M_{\mathrm{H}} Z+M_{\mathrm{n}} N \quad \text { mass excesses of } Z \text { hydrogen atoms and } N \text { neutrons } \\
& +\left(-a_{1}+J \bar{\delta}^{2}-\frac{1}{2} K \bar{\varepsilon}^{2}\right) A \quad \text { volume energy } \\
& +\left(a_{2} B_{1}+\frac{9}{4} \frac{J^{2}}{Q} \bar{\delta}^{2} \frac{B_{\mathrm{s}}{ }^{2}}{B_{1}}\right) A^{2 / 3} \quad \text { surface energy } \\
& +a_{3} A^{1 / 3} B_{\mathrm{k}} \quad \text { curvature energy } \\
& +a_{0} A^{0} \quad A^{0} \text { energy } \\
& +c_{1} \frac{Z^{2}}{A^{1 / 3}} B_{3} \quad \text { Coulomb energy } \\
& -c_{2} Z^{2} A^{1 / 3} B_{\mathrm{r}} \quad \text { volume redistribution energy } \\
& -c_{4} \frac{Z^{4 / 3}}{A^{1 / 3}} \quad \text { Coulomb exchange correction } \\
& -c_{5} Z^{2} \frac{B_{\mathrm{w}} B_{\mathrm{s}}}{B_{1}} \quad \text { surface redistribution energy } \\
& +f_{0} \frac{Z^{2}}{A} \quad \text { proton form-factor correction to the Coulomb energy } \\
& -c_{\mathrm{a}}(N-Z) \quad \text { charge-asymmetry energy } \\
& +W\left(|I|+\left\{\begin{array}{ll}
1 / A, & Z \text { and } N \text { odd and equal } \\
0, & \text { otherwise }
\end{array}\right) \quad\right. \text { Wigner energy } \\
& + \begin{cases}+\bar{\Delta}_{\mathrm{p}}+\bar{\Delta}_{\mathrm{n}}-\delta_{\mathrm{np}}, & Z \text { and } N \text { odd } \\
+\bar{\Delta}_{\mathrm{p}}, & Z \text { odd and } N \text { even } \\
+\bar{\Delta}_{\mathrm{n}}, & Z \text { even and } N \text { odd } \\
+0, & Z \text { and } N \text { even }\end{cases} \\
& -a_{\mathrm{el}} Z^{2.39} \quad \text { energy of bound electrons }
\end{aligned}
$$

where $A=Z+N$ is the mass number and $I=(N-Z) / A$ is the relative neutron excess. This expression differs from the corresponding one used in our earlier calculations [5] only in the form of the average pairing energy appearing in the next-to-last term. One should note that after minimization the exponential term [Eq. (39)] is present only implicitly in Eq. (40) through its presence in Eq. (49). For the average neutron pairing gap $\bar{\Delta}_{\mathrm{n}}$, average proton pairing gap $\bar{\Delta}_{\mathrm{p}}$, 
and average neutron-proton interaction energy $\delta_{\text {np }}$ we now use $[16,64,65]$

$$
\begin{aligned}
& \bar{\Delta}_{\mathrm{n}}=\frac{r_{\mathrm{mac}} B_{\mathrm{s}}}{N^{1 / 3}} \\
& \bar{\Delta}_{\mathrm{p}}=\frac{r_{\mathrm{mac}} B_{\mathrm{s}}}{Z^{1 / 3}} \\
& \delta_{\mathrm{np}}=\frac{h}{B_{\mathrm{s}} A^{2 / 3}}
\end{aligned}
$$

These expressions contain only two adjustable constants $r_{\text {mac }}$ and $h$, which are further discussed in Sect. 2.4. The zero reference point for the pairing energy now corresponds to even-even nuclei rather than to halfway between even-even and odd-odd nuclei as was sometimes done earlier [1,2].

The quantities $c_{1}, c_{2}, c_{4}$, and $c_{5}$ are defined by

$$
\begin{aligned}
& c_{1}=\frac{3}{5} \frac{e^{2}}{r_{0}} \\
& c_{2}=\frac{1}{336}\left(\frac{1}{J}+\frac{18}{K}\right) c_{1}^{2} \\
& c_{4}=\frac{5}{4}\left(\frac{3}{2 \pi}\right)^{2 / 3} c_{1} \\
& c_{5}=\frac{1}{64 Q} c_{1}^{2}
\end{aligned}
$$

In Eq. (40) we have kept only the first term in the expression for the proton form-factor correction to the Coulomb energy, so that $f_{0}$ is given by

$$
f_{0}=-\frac{1}{8}\left(\frac{145}{48}\right) \frac{r_{\mathrm{p}}^{2} e^{2}}{r_{0}^{3}}
$$

The bulk nuclear asymmetry $\delta$ is defined in terms of the neutron density $\rho_{\mathrm{n}}$ and proton density $\rho_{\mathrm{p}}$ by

$$
\delta=\frac{\rho_{\mathrm{n}}-\rho_{\mathrm{p}}}{\rho_{\text {bulk }}}
$$

and the average bulk nuclear asymmetry is given by

$$
\bar{\delta}=\left(I+\frac{3}{16} \frac{c_{1}}{Q} \frac{Z}{A^{2 / 3}} \frac{B_{\mathrm{v}} B_{\mathrm{s}}}{B_{1}}\right) /\left(1+\frac{9}{4} \frac{J}{Q} \frac{1}{A^{1 / 3}} \frac{B_{\mathrm{s}}{ }^{2}}{B_{1}}\right)
$$

The relative deviation in the bulk of the density $\rho$ from its nuclear matter value $\rho_{0}$ is defined by

$$
\varepsilon=-\frac{1}{3} \frac{\rho-\rho_{0}}{\rho_{0}}
$$

and the average relative deviation in the bulk of the density is given by

$$
\bar{\varepsilon}=\left(C e^{-\gamma A^{1 / 3}}-2 a_{2} \frac{B_{2}}{A^{1 / 3}}+L \bar{\delta}^{2}+c_{1} \frac{Z^{2}}{A^{4 / 3}} B_{4}\right) / K
$$

The quantity $B_{1}$ is the relative generalized surface or nuclear energy in a model that accounts for the effect of the finite range of the nuclear force. It is given by

$$
B_{1}=\frac{A^{-2 / 3}}{8 \pi^{2} r_{0}^{2} a^{4}} \iint_{V}\left(2-\frac{\left|\mathbf{r}-\mathbf{r}^{\prime}\right|}{a}\right) \frac{e^{-\left|\mathbf{r}-\mathbf{r}^{\prime}\right| / a}}{\left|\mathbf{r}-\mathbf{r}^{\prime}\right| / a} d^{3} r d^{3} r^{\prime}
$$

where the integration is over the specified sharp-surface deformed generating shape of volume $V$. Since the volume of the generating shape is conserved during deformation we have

$$
V=\frac{4 \pi}{3} R_{0}^{3}
$$


where $R_{0}$ is the radius of the spherical shape. The relative Coulomb energy $B_{3}$ is given by

$$
B_{3}=\frac{15}{32 \pi^{2}} \frac{A^{-5 / 3}}{r_{0}^{5}} \iint_{V} \frac{d^{3} r d^{3} r^{\prime}}{\left|\mathbf{r}-\mathbf{r}^{\prime}\right|}\left[1-\left(1+\frac{1}{2} \frac{\left|\mathbf{r}-\mathbf{r}^{\prime}\right|}{a_{\mathrm{den}}}\right) e^{-\left|\mathbf{r}-\mathbf{r}^{\prime}\right| / a_{\mathrm{den}}}\right]
$$

The quantities $B_{1}$ and $B_{3}$ are evaluated for $R_{0}=r_{0} A^{1 / 3}$. However, in the FRDM the equilibrium value $R_{\text {den }}$ of the equivalent-sharp-surface radius corresponding to the nuclear density is given by the expression

$$
R_{\mathrm{den}}=r_{0} A^{1 / 3}(1+\bar{\varepsilon})
$$

Thus, the actual value of the nuclear radius is determined by the balance between Coulomb, compressibility, and surface-tension effects as expressed by Eq. (49). To calculate this balance it is necessary to know the response of the surface-energy and Coulomb-energy terms $B_{1}$ and $B_{3}$ to size changes. To account for this response we introduce the quantities $B_{2}$ and $B_{4}$, which are related to the derivatives of $B_{1}$ and $B_{3}$. These derivatives are evaluated numerically and during this evaluation the radius $R$ of the generating shape is varied around the value $r_{0} A^{1 / 3}$.

The quantity $B_{2}$, which as mentioned above is related to the derivative of the relative generalized surface energy $B_{1}$, is defined by

$$
B_{2}=\frac{1}{2 x_{0}}\left[\frac{d}{d x}\left(x^{2} B_{1}\right)\right]_{x=x_{0}}
$$

with

$$
x=\frac{R}{a} \text { and } x_{0}=\frac{r_{0} A^{1 / 3}}{a}
$$

The quantity $B_{4}$ is related to the derivative of the relative Coulomb energy $B_{3}$ and is defined by

$$
B_{4}=-y_{0}^{2}\left[\frac{d}{d y}\left(\frac{B_{3}}{y}\right)\right]_{y=y_{0}}
$$

with

$$
y=\frac{R}{a_{\mathrm{den}}} \text { and } y_{0}=\frac{r_{0} A^{1 / 3}}{a_{\mathrm{den}}}
$$

For spherical shapes the quantities $B_{1}, B_{2}, B_{3}$, and $B_{4}$ can be evaluated analytically. One obtains

$$
\begin{aligned}
& B_{1}^{(0)}=1-\frac{3}{x_{0}^{2}}+\left(1+x_{0}\right)\left(2+\frac{3}{x_{0}}+\frac{3}{x_{0}^{2}}\right) e^{-2 x_{0}} \\
& B_{2}^{(0)}=1-\left(1+2 x_{0}+2 x_{0}^{2}\right) e^{-2 x_{0}} \\
& B_{3}^{(0)}=1-\frac{5}{y_{0}^{2}}\left[1-\frac{15}{8 y_{0}}+\frac{21}{8 y_{0}^{3}}-\frac{3}{4}\left(1+\frac{9}{2 y_{0}}+\frac{7}{y_{0}^{2}}+\frac{7}{2 y_{0}^{3}}\right) e^{-2 y_{0}}\right] \\
& B_{4}^{(0)}=1+5\left[-\frac{3}{y_{0}^{2}}+\frac{15}{2 y_{0}^{3}}-\frac{63}{4 y_{0}^{5}}+\frac{3}{4}\left(\frac{2}{y_{0}}+\frac{12}{y_{0}^{2}}+\frac{32}{y_{0}^{3}}+\frac{42}{y_{0}^{4}}+\frac{21}{y_{0}^{5}}\right) e^{\left.-2 y_{0}\right]}\right.
\end{aligned}
$$

The expression $B_{3}$ for the relative Coulomb energy yields the energy for an arbitrarily shaped, homogeneously charged, diffuse-surface nucleus to all orders in the diffuseness constant $a_{\mathrm{den}}$. The constants in front of the integrals for $B_{1}$ and $B_{3}$ are chosen so that $B_{1}$ and $B_{3}$ are 1 for a sphere in the limit in which the range constant $a$ and the diffuseness constant $a_{\mathrm{den}}$ are zero, in analogy with the definition of the quantities $B_{\mathrm{s}}$ and $B_{\mathrm{C}}$ in the standard liquid-drop and droplet models. The quantities $B_{2}$ and $B_{4}$, which are related to the derivatives of $B_{1}$ and $B_{3}$, respectively, were introduced above to treat the response of the nucleus to a change in size, resulting from a finite compressibility. The 
shape-dependent quantities $B_{\mathrm{s}}, B_{\mathrm{v}}, B_{\mathrm{w}}, B_{\mathrm{k}}$, and $B_{\mathrm{r}}$, which are defined [7] in the standard droplet model, are given by

$$
\begin{array}{lr}
B_{\mathrm{s}}=\frac{A^{-2 / 3}}{4 \pi r_{0}^{2}} \int_{S} d S & \text { surface energy } \\
B_{\mathrm{v}}=-\frac{15 A^{-4 / 3}}{16 \pi^{2} r_{0}{ }^{4}} \int_{S} \widetilde{W}(\mathbf{r}) d S & \text { neutron skin energy } \\
B_{\mathrm{W}}=\frac{225 A^{-2}}{64 \pi^{3} r_{0}^{6}} \int_{S}[\widetilde{W}(\mathbf{r})]^{2} d S & \text { surface redistribution energy } \\
B_{\mathrm{k}}=\frac{A^{-1 / 3}}{8 \pi r_{0}} \int_{S}\left(\frac{1}{R_{1}}+\frac{1}{R_{2}}\right) d S & \text { curvature energy } \\
B_{\mathrm{r}}=\frac{1575 A^{-7 / 3}}{64 \pi^{3} r_{0}{ }^{7}} \int_{V}[\widetilde{W}(\mathbf{r})]^{2} d^{3} r & \text { volume redistribution energy }
\end{array}
$$

where

$$
\begin{array}{r}
W(\mathbf{r})=\int_{V} \frac{1}{\left|\mathbf{r}-\mathbf{r}^{\prime}\right|} d^{3} r^{\prime} \\
\bar{W}=\frac{3 A^{-1}}{4 \pi r_{0}^{3}} \int_{V} W(\mathbf{r}) d^{3} r \\
\widetilde{W}(\mathbf{r})=W(\mathbf{r})-\bar{W}
\end{array}
$$

and $R_{1}$ and $R_{2}$ are the principal radii of curvature.

\subsection{Values of FRDM macroscopic-model constants}

The constants appearing in the expression for the finite-range droplet macroscopic model fall into four categories. The first category, which represents fundamental constants, includes [1, 2]

$$
\begin{aligned}
& M_{\mathrm{H}}=7.289034 \mathrm{MeV} \quad \text { hydrogen-atom mass excess } \\
& M_{\mathrm{n}}=8.071431 \mathrm{MeV} \quad \text { neutron mass excess } \\
& e^{2}=1.4399764 \mathrm{MeV} \text { fm electronic charge squared }
\end{aligned}
$$

One should note that for consistency we continue to use the same values for the fundamental constants as in our 1981 mass calculation [1, 2]. Results of a more recent evaluation of the fundamental constants appear in Refs. [66].

The second category, which represents constants that have been determined from considerations other than nuclear masses, includes $[1,2,3,4]$

$$
\begin{array}{rrrl}
a_{\mathrm{el}} & = & 1.433 \times 10^{-5} \mathrm{MeV} & \text { electronic-binding constant } \\
K & = & 240 \mathrm{MeV} & \text { nuclear compressibility constant } \\
r_{\mathrm{p}} & = & 0.80 \mathrm{fm} & \text { proton root-mean-square radius } \\
r_{0}= & 1.16 \mathrm{fm} & \text { nuclear-radius constant } \\
a & = & 0.68 \mathrm{fm} & \text { range of Yukawa-plus-exponential potential } \\
a_{\mathrm{den}} & = & 0.70 \mathrm{fm} & \begin{array}{r}
\text { range of Yukawa function used to } \\
\text { generate nuclear charge distribution }
\end{array}
\end{array}
$$

The third category, representing those constants whose values were obtained from consideration of odd-even mass differences $[64,65,16]$ and other mass-like quantities, are

$$
\begin{array}{rrrl}
r_{\text {mac }} & =4.80 \mathrm{MeV} & \text { average pairing-gap constant } \\
h & =6.6 \mathrm{MeV} & \text { neutron-proton interaction constant } \\
W & =\quad 30 \mathrm{MeV} & \text { Wigner constant } \\
a_{3}= & 0 \mathrm{MeV} & \text { curvature-energy constant }
\end{array}
$$

It should be noted that the final calculated mass excess is strictly independent of the value used for $r_{\text {mac }}$. This constant affects only the division of the mass excess between a macroscopic part and the remaining microscopic correction. We will therefore not include $r_{\text {mac }}$ when we later count the number of constants in our mass model. It is the pairing constant $r_{\text {mic }}$ which enters the microscopic model that affects the mass excess. It will be discussed below. 


\subsection{Finite-range liquid-drop model}

In the present version of our model the contribution to the atomic mass excess from the FRLDM macroscopic energy is given by

$$
\begin{aligned}
& E_{\text {mac }}^{\mathrm{FL}}(Z, N, \text { shape })= \\
& M_{\mathrm{H}} Z+M_{\mathrm{n}} N \quad \text { mass excesses of } Z \text { hydrogen atoms and } N \text { neutrons } \\
& \text { - } a_{\mathrm{v}}\left(1-\kappa_{\mathrm{v}} I^{2}\right) A \quad \text { volume energy } \\
& +a_{\mathrm{s}}\left(1-\kappa_{\mathrm{s}} I^{2}\right) B_{1} A^{2 / 3} \quad \text { surface energy } \\
& +a_{0} A^{0} B_{\mathrm{W}} \quad A^{0} \text { energy } \\
& +c_{1} \frac{Z^{2}}{A^{1 / 3}} B_{3} \quad \text { Coulomb energy } \\
& \text { - } c_{4} \frac{Z^{4 / 3}}{A^{1 / 3}} \quad \text { Coulomb exchange correction } \\
& +f\left(k_{\mathrm{f}} r_{\mathrm{p}}\right) \frac{Z^{2}}{A} \quad \text { proton form-factor correction to the Coulomb energy } \\
& \text { - } c_{\mathrm{a}}(N-Z) \quad \text { charge-asymmetry energy } \\
& +W\left(|I| B_{\mathrm{W}}+\left\{\begin{array}{ll}
1 / A, & Z \text { and } N \text { odd and equal } \\
0, & \text { otherwise }
\end{array}\right) \quad\right. \text { Wigner energy } \\
& + \begin{cases}+\bar{\Delta}_{\mathrm{p}}+\bar{\Delta}_{\mathrm{n}}-\delta_{\mathrm{np}}, & Z \text { and } N \text { odd } \\
+\bar{\Delta}_{\mathrm{p}}, & Z \text { odd and } N \text { even } \\
+\bar{\Delta}_{\mathrm{n}}, & Z \text { even and } N \text { odd } \\
+0, & Z \text { and } N \text { even }\end{cases} \\
& -a_{\mathrm{el}} Z^{2.39} \quad \text { energy of bound electrons }
\end{aligned}
$$

This expression differs from the corresponding one used in our earlier FRLDM(1992) [9] only through the introduction of the shape-dependent factor $B_{\mathrm{W}}$ in the $A^{0}$ and Wigner terms.

For the average neutron pairing gap $\bar{\Delta}_{\mathrm{n}}$, average proton pairing gap $\bar{\Delta}_{\mathrm{p}}$, and average neutron-proton interaction energy $\delta_{\text {np }}$ we use $[64,65,16]$

$$
\begin{gathered}
\bar{\Delta}_{\mathrm{n}}=\frac{r_{\mathrm{mac}} B_{\mathrm{s}}}{N^{1 / 3}} \\
\bar{\Delta}_{\mathrm{p}}=\frac{r_{\mathrm{mac}} B_{\mathrm{s}}}{Z^{1 / 3}} \\
\delta_{\mathrm{np}}=\frac{h}{B_{\mathrm{s}} A^{2 / 3}}
\end{gathered}
$$

The zero reference point for the pairing energy corresponds to even-even nuclei rather than to halfway between eveneven and odd-odd nuclei as has sometimes been the case previously [1,2].

In the above expressions the quantities $c_{1}$ and $c_{4}$ are defined in terms of the electronic charge $e$ and the nuclearradius constant $r_{0}$ by

$$
\begin{aligned}
& c_{1}=\frac{3}{5} \frac{e^{2}}{r_{0}} \\
& c_{4}=\frac{5}{4}\left(\frac{3}{2 \pi}\right)^{2 / 3} c_{1}
\end{aligned}
$$

The quantity $f$ appearing in the proton form-factor correction to the Coulomb energy is given by

$$
f\left(k_{\mathrm{F}} r_{\mathrm{p}}\right)=-\frac{1}{8} \frac{r_{\mathrm{p}}^{2} e^{2}}{r_{0}{ }^{3}}\left[\frac{145}{48}-\frac{327}{2880}\left(k_{\mathrm{F}} r_{\mathrm{p}}\right)^{2}+\frac{1527}{1209600}\left(k_{\mathrm{F}} r_{\mathrm{p}}\right)^{4}\right]
$$


where the Fermi wave number is

$$
k_{\mathrm{F}}=\left(\frac{9 \pi Z}{4 A}\right)^{1 / 3} \frac{1}{r_{0}}
$$

The relative neutron excess $I$ is

$$
I=\frac{N-Z}{N+Z}=\frac{N-Z}{A}
$$

The relative surface energy $B_{\mathrm{s}}$, which is the ratio of the surface area of the nucleus at the actual shape to the surface area of the nucleus at the spherical shape, is given by

$$
B_{\mathrm{s}}=\frac{A^{-2 / 3}}{4 \pi r_{0}^{2}} \int_{S} d S
$$

The quantity $B_{1}$ is the relative generalized surface or nuclear energy in a model that accounts for the effect of the finite range of the nuclear force. It is given by

$$
B_{1}=\frac{A^{-2 / 3}}{8 \pi^{2} r_{0}^{2} a^{4}} \iint_{V}\left(2-\frac{\left|\mathbf{r}-\mathbf{r}^{\prime}\right|}{a}\right) \frac{e^{-\left|\mathbf{r}-\mathbf{r}^{\prime}\right| / a}}{\left|\mathbf{r}-\mathbf{r}^{\prime}\right| / a} d^{3} r d^{3} r^{\prime}
$$

The relative Coulomb energy $B_{3}$ is given by

$$
B_{3}=\frac{15}{32 \pi^{2}} \frac{A^{-5 / 3}}{r_{0}^{5}} \iint_{V} \frac{d^{3} r d^{3} r^{\prime}}{\left|\mathbf{r}-\mathbf{r}^{\prime}\right|}\left[1-\left(1+\frac{1}{2} \frac{\left|\mathbf{r}-\mathbf{r}^{\prime}\right|}{a_{\mathrm{den}}}\right) e^{-\left|\mathbf{r}-\mathbf{r}^{\prime}\right| / a_{\mathrm{den}}}\right]
$$

For spherical shapes the quantities $B_{1}$ and $B_{3}$ can be evaluated analytically. With

$$
x_{0}=\frac{r_{0} A^{1 / 3}}{a} \text { and } y_{0}=\frac{r_{0} A^{1 / 3}}{a_{\mathrm{den}}}
$$

one obtains

$$
\begin{aligned}
& B_{1}^{(0)}=1-\frac{3}{x_{0}^{2}}+\left(1+x_{0}\right)\left(2+\frac{3}{x_{0}}+\frac{3}{x_{0}^{2}}\right) e^{-2 x_{0}} \\
& B_{3}^{(0)}=1-\frac{5}{y_{0}^{2}}\left[1-\frac{15}{8 y_{0}}+\frac{21}{8 y_{0}^{3}}-\frac{3}{4}\left(1+\frac{9}{2 y_{0}}+\frac{7}{y_{0}^{2}}+\frac{7}{2 y_{0}^{3}}\right) e^{-2 y_{0}}\right]
\end{aligned}
$$

The expression $B_{3}$ for the relative Coulomb energy yields the energy for an arbitrarily shaped, homogeneously charged, diffuse-surface nucleus to all orders in the diffuseness constant $a_{\mathrm{den}}$. The constants in front of the integrals for $B_{1}$ and $B_{3}$ have been chosen so that $B_{1}$ and $B_{3}$ are 1 for a sphere in the limit in which the range $a$ and diffuseness $a_{\mathrm{den}}$ are zero, in analogy with the definition of the quantities $B_{\mathrm{s}}$ and $B_{\mathrm{C}}$ in the standard liquid-drop model.

Relative to the FRLDM(1992) model specification in Ref. [9] we have here introduced a shape-dependent factor $B_{\mathrm{W}}$ for the $A^{0}$ and Wigner terms. We have earlier pointed out that such a shape dependence is necessary to obtain continuity of the FRLDM potential energy at scission. For example, if in symmetric fission of ${ }^{264} \mathrm{Fm}$ we treat the touching configuration of two symmetric spheres as a single deformed ${ }^{264} \mathrm{Fm}$ system or as two touching ${ }^{132} \mathrm{Sn}$ nuclei a shape dependence is necessary to obtain continuity. This is discussed in detail in Refs. [52, 57, 70]. To discuss here the postulated shape dependence for the Wigner term that we have used since 1989 [52] we follow closely the discussion there. We note that in an extensive discussion of the Wigner term [8], it was pointed out that if a system is broken up into $n$ identical pieces, then the Wigner term must be evaluated separately for each piece, with the result that it simply jumps to $n$ times its original value. For symmetric fission into two identical fragments this simple argument would imply a shape dependence corresponding to a step function at scission. In reality one would expect that the step function is washed out over some range of shapes in the scission region. Obviously, if the area of a cross section in the neck region is very small then there is hardly any communication between the two fragments and we have essentially the two-system configuration. For cylinder-like shapes and those with even bulgier midsections, that is for shapes (in the three-quadratic-surface parameterizations) with $\sigma_{2} \geq 0$, we clearly have a one-system configuration. How close we are to one or the other situation is related to the amount of communication through the neck. If the area of a cross section through the neck is $S_{3}$ and the area of the maximum cross section of the smaller one of the end bodies, that is a cross section through the center of the end surface of revolution, is $S_{1}$, then we may relate the amount of communication to the dimensionless quantity $S_{3} / S_{1}$. As a simple ansatz we propose the shape dependence

$$
B_{\mathrm{W}}= \begin{cases}\left(1-\frac{S_{3}}{S_{1}}\right)^{2} a_{\mathrm{d}}+1 & , \quad \sigma_{2} \leq 0 \\ 1 & , \quad \sigma_{2} \geq 0\end{cases}
$$

Suppose $a_{\mathrm{d}}=1.0$. Then, with the above shape dependence we would find that for scission shapes we have a Wigner term that is precisely two times the Wigner term for a single system. For cylinder-like configurations and for shapes 
with thicker neck regions we would have a Wigner term that is equal to the term for a single shape. Thus, with the above shape dependence we obtain the desired values in the two limiting cases. However, at scission there is still some communication between the two fragments. This can be illustrated by considering the shell correction calculated by use of the Strutinsky method, for which we for symmetric configurations have a well-defined prescription, regardless of shape. For two touching ${ }^{132} \mathrm{Sn}$ nuclei we obtain a shell correction whose magnitude is about $10 \%$ lower than for two well-separated nuclei. This leads us to chose a value of $a_{\mathrm{d}}=0.9$ for the damping coefficient. We have actually calculated potential-energy surfaces and investigated their structure for other choices of the parameter $a_{\mathrm{d}}$, which also occurs in the shape dependence of the $A^{0}$ term. From such studies it has turned out that the above value leads to potential-energy surfaces that when used in studies of 1) fission half-lives [52, 39], 2) fission-barrier heights across the nuclear chart, [57], 3) bimodal fission [52, 38], and 4) fission-fragment mass distributions [59] are in good agreement with experimental data for The uncertainty in the estimate of $a_{\mathrm{d}}$ from these studies is about 0.1 . For the $A^{0}$ term we postulate the same shape dependence [52].

\subsection{Values of FRLDM macroscopic-model constants}

The constants appearing in the expression for the finite-range liquid-drop macroscopic model fall into four categories. The first category, which represents fundamental constants, includes [1, 2]

$$
\begin{aligned}
& M_{\mathrm{H}}=7.289034 \mathrm{MeV} \quad \text { hydrogen-atom mass excess } \\
& M_{\mathrm{n}}=8.071431 \mathrm{MeV} \quad \text { neutron mass excess } \\
& e^{2}=1.4399764 \mathrm{MeV} \mathrm{fm} \quad \text { electronic charge squared }
\end{aligned}
$$

The second category, which represents constants that have been determined from considerations other than nuclear masses, includes $[1,2]$

$\begin{aligned} a_{\mathrm{el}} & = & 1.433 \times 10^{-5} \mathrm{MeV} \\ r_{\mathrm{p}} & = & 0.80 \mathrm{fm} \\ r_{0} & = & 1.16 \mathrm{fm} \\ a & = & 0.68 \mathrm{fm} \\ a_{\mathrm{den}} & = & 0.70 \mathrm{fm}\end{aligned}$

electronic-binding constant

proton root-mean-square radius

nuclear-radius constant

range of Yukawa-plus-exponential potential

range of Yukawa function used to generate nuclear charge distribution

The third category, representing those constants whose values were obtained from consideration of odd-even mass differences $[64,65,16]$ and other mass-like quantities, are

$\begin{aligned} r_{\mathrm{mac}} & =4.80 \mathrm{MeV} \\ h & =6.6 \mathrm{MeV} \\ W & =30 \mathrm{MeV} \\ a_{\mathrm{d}} & =0.9\end{aligned}$

average pairing-gap constant neutron-proton interaction constant Wigner constant Wigner damping constant

It should be noted that the final calculated mass excess is strictly independent of the value used for $r_{\text {mac }}$. This constant affects only the division of the mass excess between the macroscopic part and the remaining microscopic correction. We therefore do not include $r_{\text {mac }}$ when we later count the number of constants in our mass model. It is the pairing constant $r_{\text {mic }}$ which enters the microscopic model that affects the mass excess. It will be discussed below.

Since $\mu_{\mathrm{th}}=0$ in our case, Eqs. (6) and (8) can be solved with the experimental data set of 2149 masses with $Z \geq 8$ and $N \geq 8$ [17] and 31 fission-barrier heights to determine the remaining macroscopic constants and the error of our model. To present all the macroscopic model constants together we list them here but discuss their adjustment later. These constants are

$$
\begin{array}{rlrl}
a_{\mathrm{v}} & =16.022835 \mathrm{MeV} & & \text { volume-energy constant } \\
\kappa_{\mathrm{v}} & =1.927910 \mathrm{MeV} & \text { volume-asymmetry constant } \\
a_{\mathrm{s}}=21.269461 \mathrm{MeV} & \text { surface-energy constant } \\
\kappa_{\mathrm{s}}=2.388587 \mathrm{MeV} & \text { surface-asymmetry constant } \\
a_{0}=2.649971 \mathrm{MeV} & A^{0} \text { constant } \\
c_{\mathrm{a}}= & =055673 \mathrm{MeV} & \text { charge-asymmetry constant }
\end{array}
$$

The resulting error in the FRLDM is $\sigma_{\text {th }}=0.6618 \mathrm{MeV}$. We note that the constants have not changed very much, except possibly the charge-asymmetry constant which decreased to about half its value in the previous version in FRLDM(1992) [9] and in the FRLDM(2002) [57].

\subsection{Microscopic model}

The shell-plus-pairing correction $E_{\mathrm{s}+\mathrm{p}}(Z, N$, shape $)$ is the sum of the proton shell-plus-pairing correction and the neutron shell-plus-pairing correction, namely

$$
E_{\mathrm{s}+\mathrm{p}}(Z, N, \text { shape })=E_{\mathrm{s}+\mathrm{p}}^{\mathrm{prot}}(Z, \text { shape })+E_{\mathrm{s}+\mathrm{p}}^{\text {neut }}(N, \text { shape })
$$


We give here the equations for the neutron shell-plus-pairing correction. Completely analogous expressions hold for protons. We have

$$
E_{\mathrm{s}+\mathrm{p}}^{\text {neut }}(N, \text { shape })=E_{\text {shell }}^{\text {neut }}(N, \text { shape })+E_{\text {pair }}^{\text {neut }}(N, \text { shape })
$$

Both terms are evaluated from a set of calculated single-particle levels. As before, the shell correction is calculated by use of Strutinsky's method $[11,12]$. Thus

$$
E_{\text {shell }}^{\text {neut }}(N, \text { shape })=\sum_{i=1}^{N} e_{i}-\widetilde{E}^{\text {neut }}(N, \text { shape })
$$

where $e_{i}$ are calculated single-particle energies and $\widetilde{E}^{\text {neut }}(N$, shape $)$ is the smooth single-particle energy sum calculated in the Strutinsky method. The pairing correction is the difference between the pairing correlation energy and the average pairing correlation energy, namely

$$
E_{\text {pair }}^{\text {neut }}(N, \text { shape })=E_{\text {p.c. }}^{\text {neut }}(N, \text { shape })-\widetilde{E}_{\text {p.c. }}^{\text {neut }}(N, \text { shape })
$$

where $E_{\text {p.c. }}^{\text {neut }}(N$, shape $)$ is given by Eq. (101) and $\widetilde{E}_{\text {p.c. }}^{\text {neut }}(N$, shape $)$ is given by Eq. (108). For the pairing correction we now use the Lipkin-Nogami $[13,14,15]$ version of the BCS method, which takes into account the lowest-order correction to the total energy of the system associated with particle-number fluctuation.

The single-particle potential felt by a nucleon is given by

$$
V=V_{1}+V_{\text {s.o. }}+V_{\mathrm{C}}
$$

The first term is the spin-independent nuclear part of the potential, which is calculated in terms of the folded-Yukawa potential

$$
V_{1}(\mathbf{r})=-\frac{V_{0}}{4 \pi a_{\mathrm{pot}^{3}}} \int_{\mathrm{V}} \frac{e^{-\left|\mathbf{r}-\mathbf{r}^{\prime}\right| / a_{\mathrm{pot}}}}{\left|\mathbf{r}-\mathbf{r}^{\prime}\right| / a_{\mathrm{pot}}} d^{3} r^{\prime}
$$

where the integration is over the volume of the generating shape, whose volume is held fixed at $\frac{4}{3} \pi R_{\text {pot }}{ }^{3}$ as the shape is deformed. The potential radius $R_{\text {pot }}$ is given by

$$
R_{\text {pot }}=R_{\text {den }}+A_{\text {den }}-B_{\text {den }} / R_{\text {den }}
$$

with

$$
R_{\mathrm{den}}=r_{0} A^{1 / 3}(1+\bar{\varepsilon})
$$

Values of the model constants $A_{\mathrm{den}}$ and $B_{\mathrm{den}}$ will be given later. The potential depths $V_{\mathrm{p}}$ for protons and $V_{\mathrm{n}}$ for neutrons are given by

$$
\begin{aligned}
& V_{\mathrm{p}}=V_{\mathrm{s}}+V_{\mathrm{a}} \bar{\delta} \\
& V_{\mathrm{n}}=V_{\mathrm{s}}-V_{\mathrm{a}} \bar{\delta}
\end{aligned}
$$

The average bulk nuclear asymmetry $\bar{\delta}$ appearing in Eqs. (84) and (85) and average relative deviation $\bar{\varepsilon}$ in the bulk of the density appearing in Eq. (83) are given by the droplet model and thus depend on the values of the droplet-model constants. The FRDM macroscopic constants are determined in a nonlinear least-squares adjustment, which requires between 1000 and 10000 steps to find the optimum constants. In principle, these constants should then be used in the determination of the single-particle potential, the potential-energy surfaces should be recalculated with the new constants, a new mass calculation should be performed, and a new set of macroscopic constants should be determined, with this iteration repeated until convergence. Any change of the single-particle potential would also make necessary a redetermination of the spin-orbit strength and the diffuseness. Because the calculation of potential-energy surfaces and other aspects of these steps would be very time-consuming, only one iteration has been performed. In our current mass calculation we have found additional evidence that the above form (and constants) of the single-particle potential are very satisfactory and we will comment further when we discuss the calculated results.

Furthermore, in determining the single-particle potential we have used the following early forms [71] of the droplet model expressions for $\bar{\delta}$ and $\bar{\varepsilon}$ :

$$
\begin{gathered}
\bar{\delta}=\left(I+\frac{3}{8} \frac{c_{1}}{Q} \frac{Z^{2}}{A^{5 / 3}}\right) /\left(1+\frac{9}{4} \frac{J}{Q} \frac{1}{A^{1 / 3}}\right) \\
\bar{\varepsilon}=\left(-\frac{2 a_{2}}{A^{1 / 3}}+L \bar{\delta}^{2}+c_{1} \frac{Z^{2}}{A^{4 / 3}}\right) / K
\end{gathered}
$$

The range $a_{\text {pot }}$ of the Yukawa function in Eq. (81) has been determined from an adjustment of calculated singleparticle levels to experimental data in the rare-earth and actinide regions [37]. It is kept constant for nuclei throughout the periodic system. 
The spin-orbit potential is given by the expression

$$
V_{\text {s.o. }}=-\lambda\left(\frac{\hbar}{2 m_{\text {nuc }} c}\right)^{2} \frac{\sigma \cdot \nabla V_{1} \times p}{\hbar}
$$

where $\lambda$ is the spin-orbit interaction strength, $m_{\text {nuc }}$ is the nucleon mass, $\sigma$ represents the Pauli spin matrices, and $p$ is the nucleon momentum.

The spin-orbit strength has been determined from adjustments to experimental levels in the rare-earth and actinide regions. It has been shown $[37,1,72]$ that many nuclear properties throughout the periodic system are well reproduced with $\lambda$ given by a function linear in $A$ through the values determined in these two regions. This gives

$$
\lambda_{\mathrm{p}}=6.0\left(\frac{A}{240}\right)+28.0=0.025 A+28.0=k_{\mathrm{p}} A+l_{\mathrm{p}}
$$

for protons and

$$
\lambda_{\mathrm{n}}=4.5\left(\frac{A}{240}\right)+31.5=0.01875 A+31.5=k_{\mathrm{n}} A+l_{\mathrm{n}}
$$

for neutrons.

Finally, the Coulomb potential for protons is given by

$$
V_{\mathrm{C}}(\mathbf{r})=e \rho_{\mathrm{c}} \int_{\mathrm{V}} \frac{d^{3} r^{\prime}}{\left|\mathbf{r}-\mathbf{r}^{\prime}\right|}
$$

where the charge density $\rho_{\mathrm{c}}$ is given by

$$
\rho_{\mathrm{c}}=\frac{Z e}{\frac{4}{3} \pi A r_{0}^{3}}
$$

The basis functions used to generate the matrix elements of the single-particle Hamiltonian is a set of deformed, axially symmetric, harmonic-oscillator eigenfunctions, specifically all those that for a given shape have an energy less than or equal to $\left(N_{\text {bas }}+0.5\right) \hbar \omega_{0}$. The overall curvature of the basis functions is chosen to yield

$$
\hbar \omega_{0}=C_{\text {cur }} / A^{1 / 3}
$$

\subsection{Microscopic pairing models}

Because of its basic simplicity, the BCS pairing model $[73,74,75,76]$ has been the pairing model of choice in most previous nuclear-structure calculations [77, 10, 1, 2]. However, a well-known deficiency of the BCS model is that for large spacings between the single-particle levels at the Fermi surface, no non-trivial solutions exist. In practical applications, these situations occur not only at magic numbers, but also, for example, for deformed actinide nuclei at neutron numbers $N=142$ and 152. By taking into account effects associated with particle-number fluctuations, the Lipkin-Nogami approximation $[13,14,15]$ goes beyond the BCS approximation and avoids such collapses.

In solving the pairing equations for neutrons or protons in either the BCS or Lipkin-Nogami model, we consider a constant pairing interaction $G$ acting between $N_{2}-N_{1}+1$ doubly degenerate single-particle levels, which are occupied by $N_{\text {int }}$ nucleons. This interaction interval starts at level $N_{1}$, located below the Fermi surface, and ends at level $N_{2}$, located above the Fermi surface. With the definitions we use here, the levels are numbered consecutively starting with number 1 for the level at the bottom of the well. Thus, for even particle numbers, the last occupied levels in the neutron and proton wells are $N / 2$ and $Z / 2$, respectively.

The level pairs included in the pairing calculation are often chosen symmetrically around the Fermi surface. However, for spherical nuclei it is more reasonable to require that degenerate spherical states have equal occupation probability. This condition cannot generally be satisfied simultaneously with a symmetric choice of levels in the interaction region. We therefore derive the pairing equations below for the more general case of arbitrary $N_{1}$ and $N_{2}$.

In the Lipkin-Nogami pairing model $[13,14,15]$ the pairing gap $\Delta$, Fermi energy $\lambda$, number-fluctuation constant $\lambda_{2}$, occupation probabilities $v_{k}^{2}$, and shifted single-particle energies $\varepsilon_{k}$ are determined from the $2\left(N_{2}-N_{1}\right)+5$ coupled nonlinear equations

$$
\begin{gathered}
N_{\mathrm{tot}}=2 \sum_{k=N_{1}}^{N_{2}} v_{k}^{2}+2\left(N_{1}-1\right) \\
\frac{2}{G}=\sum_{k=N_{1}}^{N_{2}} \frac{1}{\sqrt{\left(\varepsilon_{k}-\lambda\right)^{2}+\Delta^{2}}} \\
v_{k}^{2}=\frac{1}{2}\left[1-\frac{\varepsilon_{k}-\lambda}{\sqrt{\left(\varepsilon_{k}-\lambda\right)^{2}+\Delta^{2}}}\right], k=N_{1}, N_{1}+1, \ldots, N_{2} \\
\varepsilon_{k}=e_{k}+\left(4 \lambda_{2}-G\right) v_{k}^{2}, \quad k=N_{1}, N_{1}+1, \ldots, N_{2}
\end{gathered}
$$




$$
\lambda_{2}=\frac{G}{4}\left[\frac{\left(\sum_{k=N_{1}}^{N_{2}} u_{k}^{3} v_{k}\right)\left(\sum_{k=N_{1}}^{N_{2}} u_{k} v_{k}{ }^{3}\right)-\sum_{k=N_{1}}^{N_{2}} u_{k}{ }^{4} v_{k}{ }^{4}}{\left(\sum_{k=N_{1}}^{N_{2}} u_{k}{ }^{2} v_{k}^{2}\right)^{2}-\sum_{k=N_{1}}^{N_{2}} u_{k}{ }^{4} v_{k}{ }^{4}}\right]
$$

where

$$
u_{k}^{2}=1-v_{k}^{2}, k=N_{1}, N_{1}+1, \ldots, N_{2}
$$

The quasi-particle energies $E_{k}$ of the odd nucleon in an odd- $A$ nucleus are now given by [14]

$$
E_{k}=\left[\left(\varepsilon_{k}-\lambda\right)^{2}+\Delta^{2}\right]^{1 / 2}+\lambda_{2}, \quad k=N_{1}, N_{1}+1, \ldots, N_{2}
$$

In the Lipkin-Nogami model it is the sum $\Delta+\lambda_{2}$ that is identified with odd-even mass differences [14]. We denote this sum by $\Delta_{\mathrm{LN}}$.

The pairing-correlation energy plus quasi-particle energy in the Lipkin-Nogami model is given by

$$
E_{\text {p.c. }}=\sum_{k=N_{1}}^{N_{2}}\left(2 v_{k}^{2}-n_{k}\right) e_{k}-\frac{\Delta^{2}}{G}-\frac{G}{2} \sum_{k=N_{1}}^{N_{2}}\left(2 v_{k}^{4}-n_{k}\right)-4 \lambda_{2} \sum_{k=N_{1}}^{N_{2}} u_{k}^{2} v_{k}^{2}+E_{i} \theta_{\mathrm{odd}, N_{\mathrm{tot}}}
$$

where $e_{k}$ are the single-particle energies and $n_{k}$, with values 2,1 , or 0 , specify the sharp distribution of particles in the absence of pairing. The quasi-particle energy $E_{i}$ for the odd particle occupying level $i$ is given by Eq. (100), and $\theta_{\text {odd, }, N_{\text {tot }}}$ is unity if $N_{\text {tot }}$ is odd and zero if $N_{\text {tot }}$ is even.

\subsection{Effective-interaction pairing-gap models}

In microscopic pairing calculations, the pairing strength $G$ for neutrons and protons can be obtained from effectiveinteraction pairing gaps $\Delta_{G_{\mathrm{n}}}$ and $\Delta_{G_{\mathrm{p}}}$ given by [16]

$$
\begin{aligned}
& \Delta_{G_{\mathrm{n}}}=\frac{r_{\mathrm{mic}} B_{\mathrm{s}}}{N^{1 / 3}} \\
& \Delta_{G_{\mathrm{p}}}=\frac{r_{\mathrm{mic}} B_{\mathrm{s}}}{Z^{1 / 3}}
\end{aligned}
$$

The dependence of the pairing strength $G$ on the corresponding effective-interaction pairing gap $\Delta_{G}$ is obtained from the microscopic equations by assuming a constant level density for the average nucleus in the vicinity of the Fermi surface. This allows the sums in the equations to be replaced by integrals. The average level density of doubly degenerate levels is taken to be

$$
\widetilde{\rho}=\frac{1}{2} \widetilde{g}(\widetilde{\lambda})
$$

where $\widetilde{g}$ is the smooth level density that is obtained in Strutinsky's shell-correction method and $\tilde{\lambda}$ is the Fermi energy of the smoothed single-particle energy [10,78]. Thus, we can make the substitution

$$
\sum_{k=N_{1}}^{N_{2}} f\left(e_{k}-\lambda\right) \Longrightarrow \widetilde{\rho} \int_{y_{1}}^{y_{2}} f(x) d x
$$

where

$$
\begin{aligned}
& y_{1}=\frac{-\frac{1}{2} N_{\mathrm{tot}}+N_{1}-1}{\widetilde{\rho}} \\
& y_{2}=\frac{-\frac{1}{2} N_{\mathrm{tot}}+N_{2}}{\widetilde{\rho}}
\end{aligned}
$$

The gap equation (95) can now be evaluated for an average nucleus, with the result

$$
\begin{aligned}
\frac{1}{G} & =\frac{1}{2} \widetilde{\rho} \int_{y_{1}}^{y_{2}} \frac{d x}{\sqrt{x^{2}+\Delta_{G}^{2}}} \\
& =\frac{1}{2} \widetilde{\rho}\left[\ln \left(\sqrt{y_{2}^{2}+\Delta_{G}^{2}}+y_{2}\right)-\ln \left(\sqrt{y_{1}^{2}+\Delta_{G}^{2}}+y_{1}\right)\right]
\end{aligned}
$$

From this expression, the pairing strength $G$ in the BCS model can be determined in any region of the nuclear chart.

The same expression may also be used in the Lipkin-Nogami case, but some reinterpretations are necessary. It is now the energies $\varepsilon_{k}$ occurring in Eq. (95) that are assumed to be equally spaced. These are not precisely the singleparticle energies $e_{k}$ but are related to them by Eq. (97). Thus, in order for $\varepsilon_{k}$ to be equally spaced, the single-particle 
energies $e_{k}$ must be shifted downward by the amounts $\left(4 \lambda_{2}-G\right) v_{k}^{2}$. Since the occupation probability $v_{k}^{2}$ is approximately unity far below the Fermi surface and zero far above, the corresponding single-particle energy distribution is approximately uniform far above and far below the Fermi surface but spread apart by the additional amount $4 \lambda_{2}-G$ close to the Fermi surface. Although this decrease in level density near the Fermi surface is accidental, it is in approximate accord with the ground-state structure of real nuclei, since the increased stability associated with ground-state configurations is due to low level densities near the Fermi surface [78, 65].

In the Lipkin-Nogami model, it is the quantity $\Delta+\lambda_{2}$ that is associated with odd-even mass differences, whereas in the BCS model it is $\Delta$ only that should be directly compared to the experimental data. This leads to the expectation that there is a related difference between $\Delta_{G}^{\mathrm{LN}}$ and $\Delta_{G}^{\mathrm{BCS}}$, the effective-interaction pairing gaps associated with the LN and BCS models, respectively. Since we determine the constants of the model for $\Delta_{G}^{\mathrm{LN}}$ directly from least-squares minimization, it is not necessary to specify exactly such a relationship. However, the above observation is of value as a rough rule of thumb, and to remind us to expect that the effective-interaction pairing gaps in the BCS and LN models will be of somewhat different magnitude.

The expression for the average pairing correlation energy plus quasi-particle energy $\widetilde{E}_{\text {p.c. }}$ in the Lipkin-Nogami model is obtained in a similar manner as the expression for the pairing matrix element $G$. For the average pairing correlation energy plus quasi-particle energy in the Lipkin-Nogami model we then obtain

$$
\begin{aligned}
\widetilde{E}_{\text {p.c. }}= & \frac{1}{2} \widetilde{\rho}\left[\left(y_{2}-G\right)\left(y_{2}-\sqrt{y_{2}^{2}+\Delta_{G}^{2}}\right)+\left(y_{1}-G\right)\left(y_{1}+\sqrt{y_{1}^{2}+\Delta_{G}^{2}}\right)\right] \\
& +\frac{1}{4}\left(G-4 \widetilde{\lambda}_{2}\right) \widetilde{\rho} \Delta_{G}\left[\tan ^{-1}\left(\frac{y_{2}}{\Delta_{G}}\right)-\tan ^{-1}\left(\frac{y_{1}}{\Delta_{G}}\right)\right]+\bar{\Delta} \theta_{\text {odd }, N_{\text {tot }}}
\end{aligned}
$$

where the average pairing gap $\bar{\Delta}$ is given by Eqs. (41) and (42) or Eqs. (63) and (64).

The expression for $\widetilde{\lambda}_{2}$ for an average nucleus is fairly lengthy, being given by

$$
\tilde{\lambda}_{2}=\frac{G}{4}\left(\frac{A-C}{B-C}\right)
$$

where

$$
\begin{aligned}
& A=\left(\frac{\widetilde{\rho} \Delta_{G}}{4}\right)^{2}\left\{\left(\frac{2}{G \widetilde{\rho}}\right)^{2}-\left[\ln \left(\frac{\sqrt{y_{2}^{2}+\Delta_{G}^{2}}}{\sqrt{y_{1}^{2}+\Delta_{G}^{2}}}\right)\right]^{2}\right\} \\
& B=\frac{\Delta_{G}^{2} \widetilde{\rho}^{2}}{16}\left[\tan ^{-1}\left(\frac{y_{2}}{\Delta_{G}}\right)-\tan ^{-1}\left(\frac{y_{1}}{\Delta_{G}}\right)\right]^{2} \\
& C=\frac{\widetilde{\rho} \Delta_{G}}{32}\left[\Delta_{G}\left(\frac{y_{2}}{y_{2}{ }^{2}+\Delta_{G}{ }^{2}}-\frac{y_{1}}{y_{1}{ }^{2}+\Delta_{G}{ }^{2}}\right)+\tan ^{-1}\left(\frac{y_{2}}{\Delta_{G}}\right)-\tan ^{-1}\left(\frac{y_{1}}{\Delta_{G}}\right)\right]
\end{aligned}
$$

One should note that the pairing strength $G$ depends on the interval $\left(N_{1}, N_{2}\right)$ over which the pairing force is active. However, in our formulation we do not use $G$ as a primary constant. Instead, we use the effective-interaction pairing gaps $\Delta_{G_{\mathrm{n}}}$ and $\Delta_{G_{\mathrm{p}}}$, which are independent of the choice of interaction interval $\left(N_{1}, N_{2}\right)$. We choose the pairing interaction interval so that at least all levels up to $5 \mathrm{MeV}$ above the Fermi surface are included. It has sometimes been asked whether particles scattered into the continuum by the pairing force would escape from the nucleus if the interaction interval includes unbound states. Of course not! The superfluid state is the most bound configuration. The single-particle picture does not give the true nuclear ground or excited states; it only serves as the set of basis functions for the pairing calculation. Instead, the quasi-particle energies obtained in the pairing calculation represent a subset of all possible excited states. If, in an excited nucleus, the quasi-particle energies are lower than the particle separation energies, no nucleons escape.

\subsection{Shell correction}

The Strutinsky shell-correction method $[11,12]$ requires two additional constants, the order $p$ and the range $\gamma_{S}$. The shell correction should be insensitive to these quantities within a certain range of values. Their values can therefore be determined in principle by requiring this "plateau condition" to be fulfilled, that is that the shell correction is constant for a range of these quantities $[11,12]$. We have found that for heavy nuclei this condition is indeed fulfilled, with the shell correction for nuclear ground-state shapes being insensitive to the values of these two constants. However, for light nuclei this is no longer the case. Here the shell correction may vary by several MeV for a reasonable range of values of the range $\gamma_{S}$. Moreover, the shell correction often does not exhibit any plateau. This probably indicates a gradual breakdown of the shell-correction method as one approaches the very lightest region of nuclei, where the number of single-particle levels is small, as was also discussed earlier, see Ref. [10] and references therein. 
In the present calculation we retain the same values of the order in the Strutinsky shell-correction method and the range $\gamma_{S}$ as in Ref. [9]. The range is expressed as

$$
\gamma_{\mathrm{S}}=C_{\mathrm{S}} \hbar \omega_{0} B_{\mathrm{s}}
$$

with $B_{\mathrm{s}}$ given by Eq. (70).

The version of the Strutinsky method $[11,12]$ that we use here was originally proposed for infinite single-particle wells. For finite wells the calculated shell correction diverges to $+\infty$ as the number of basis functions approaches $+\infty$. This difficulty is avoided by using only a limited number of basis functions. It has been found that the calculated shell correction is approximately independent of $N_{\text {bas }}$ in the range $8 \lesssim N_{\text {bas }} \lesssim 13$ [10].

One may expect the Strutinsky method to be less accurate for light nuclei than for heavy nuclei because the smooth, average quantities calculated in the Strutinsky method are less accurately determined from the few levels occurring in light nuclei. One could also ask if the method is less accurate near the drip lines than close to $\beta$ stability because the truncated single-particle level spectrum that we use deviates more from a realistic single-particle spectrum near the drip lines than near $\beta$-stable nuclei. Below, where we study the reliability of the model for light nuclei and for nuclei far from $\beta$ stability, we find that the model error does indeed grow as the size of the nuclear system decreases. However, we find no obvious increase in the model error for today's known nuclei that are the furthest from $\beta$ stability. The reliability of the Strutinsky method for the folded-Yukawa single-particle potential is further discussed in the appendix of Ref. [10].

\subsection{Zero-point energy}

As a final step in the calculation of nuclear ground-state masses, a zero-point energy is added to the calculated potential energy at the ground-state shape. In the FRDM(1992) calculation, only a contribution from zero-point motion in the $\varepsilon_{2}$ (fission) direction was added because we could not calculate the potential versus the axial asymmetry direction at that time. Since we now have that capability we also consider zero-point motion in the axial-asymmetry $\gamma$ variable.

In the harmonic approximation this zero-point energy $E_{\mathrm{zp}}$ is given by

$$
E_{0, \lambda}=\frac{1}{2} \hbar \omega_{\lambda}
$$

where

$$
\omega_{\lambda}=\left(C_{\lambda} / B_{\lambda}\right)^{1 / 2}
$$

Here $C_{\lambda}$ is the potential-energy stiffness constant and $B_{\lambda}$ is the inertia associated with motion in the $\lambda$-direction. We assume here that the inertia $B_{\lambda}$ is proportional to the incompressible irrotational flow, with the same proportionality factor for both $\varepsilon$ - and $\gamma$-vibrations. We write this relationship in the form

$$
B_{\lambda}=B_{\lambda}^{\mathrm{irr}} / \mathscr{K}^{2}
$$

so that

$$
\omega_{\lambda}=\mathscr{K} \omega_{\lambda}^{\mathrm{irr}}
$$

Since a realistic inertia is larger than the irrotational flow inertia we determine $\mathscr{K}$ in our adjustment of the other FRDM parameters to ground-state masses. We then use the same value of $\mathscr{K}$ in the FRLDM model.

The incompressible-flow values of the inertias for axially symmetric shapes are given by [1]:

$$
\begin{aligned}
& B_{\varepsilon_{2}}^{\mathrm{irr}}=\frac{2}{15} \frac{\left(1+\frac{2}{9} \varepsilon_{2}^{2}\right)}{\left(1-\frac{2}{3} \varepsilon_{2}\right)^{2}}\left(1-\frac{1}{3} \varepsilon_{2}^{2}-\frac{2}{27} \varepsilon_{2}^{3}\right)^{-4 / 3} M_{0} R_{0}{ }^{2} \\
& B_{\gamma}^{\mathrm{irr}}=\frac{2}{15}\left(\frac{1-\frac{2}{3} \varepsilon_{2}}{1+\frac{1}{3} \varepsilon_{2}}\right)^{2 / 3}\left[\ln \left(\frac{1+\frac{1}{3} \varepsilon_{2}}{1-\frac{2}{3} \varepsilon_{2}}\right)\right]^{2} M_{0} R_{0}{ }^{2}
\end{aligned}
$$

The stiffness constants $C_{\lambda}$ are determined from the curvatures with respect to $\varepsilon_{2}$ and $\gamma$

$$
C_{\gamma}=\left.\frac{\partial^{2} E}{\partial \gamma^{2}}\right|_{\mathrm{gs}} \quad C_{\varepsilon_{2}}=\left.\frac{\partial^{2} E}{\partial \varepsilon_{2}^{2}}\right|_{\mathrm{gs}}
$$

At the ground state we obtain the harmonic approximation to the potential energy by fitting a second-degree polynomial to the potential. Earlier we used only three points, the ground-state and one point on either side [9]. Now, in the $\varepsilon$ parameterization we use potential energies at $\varepsilon_{2}^{\mathrm{gs}}, \varepsilon_{2}^{\mathrm{gs}} \pm 0.05, \varepsilon_{2}^{\mathrm{gs}} \pm 0.10$, and $\varepsilon_{2}^{\mathrm{gs}} \pm 0.15$, that is, seven points to do a least-squares fit of a second-degree polynomial to these points. In the $\gamma$ direction we use $\gamma^{\mathrm{gs}}, \gamma^{\mathrm{gs}} \pm 5, \gamma^{\mathrm{gs}} \pm 10$, and $\gamma^{\mathrm{gs}} \pm 15$. For the 746 cases when the ground state is tri-axial, we obtain the inertias by interpolation between the their values on the prolate and oblate axes. As discussed in Ref. [1], as $\varepsilon_{2}$ goes to zero the $\varepsilon_{2}$ and $\gamma$ modes become identical. Also, for small distances away from the spherical shape, it is numerically difficult to calculate $\Delta E_{\gamma}$, so for ground states with $\varepsilon_{2}<0.17$ we put $\Delta E_{\gamma}=\Delta E_{\varepsilon_{2}}$. 


\subsection{Values of microscopic-model constants}

The constants appearing in the expressions occurring in the microscopic shell-plus-pairing calculation fall into four categories. The first category, which represents fundamental constants, includes

$$
\begin{aligned}
& m_{\text {nuc }}=938.90595 \mathrm{MeV} \quad \text { nucleon mass } \\
& \hbar c=197.32891 \mathrm{MeV} \text { fm Planck's constant multiplied } \\
& e^{2}=1.4399764 \mathrm{MeV} f \mathrm{fm} \quad \text { electronic charge squared }
\end{aligned}
$$

The electronic charge squared has already been counted among the macroscopic constants.

The second category, which represents constants that have been determined from considerations other than nuclear masses, includes $[1,2,10]$

$\begin{array}{rlrl}C_{\text {cur }} & = & 41 \mathrm{MeV} & \text { basis curvature constant } \\ V_{\mathrm{s}} & = & 52.5 \mathrm{MeV} & \text { symmetric potential-depth constant } \\ V_{\mathrm{a}} & = & 48.7 \mathrm{MeV} & \text { asymmetric potential-depth constant } \\ A_{\mathrm{den}} & = & 0.82 \mathrm{fm} & \text { potential radius correction constant } \\ B_{\mathrm{den}} & = & 0.56 \mathrm{fm}^{2} & \text { potential radius curvature-correction constant } \\ a_{\mathrm{pot}} & = & 0.8 \mathrm{fm} & \text { potential diffuseness constant } \\ k_{\mathrm{p}} & = & 0.025 & \text { proton spin-orbit } A \text { coefficient } \\ l_{\mathrm{p}} & = & 28.0 & \text { proton spin-orbit constant } \\ k_{\mathrm{n}} & =0.01875 & \text { neutron spin-orbit } A \text { coefficient } \\ l_{\mathrm{n}} & =\quad 31.5 & \text { neutron spin-orbit constant }\end{array}$

The third category, representing those constants whose values were obtained from consideration of mass-like quantities, are

$$
\begin{array}{rrrl}
N_{\text {bas }} & =12 & & \text { number of basis functions } \\
p & = & 8 & \text { order of Strutinsky shell correction } \\
C_{\mathrm{S}} & =1.0 & & \text { Strutinsky range coefficient }
\end{array}
$$

The fourth category, representing those constants whose values were obtained from a least-squares adjustment simultaneously with the macroscopic constants of the FRDM, are

$$
\begin{array}{rlrl}
r_{\text {mic }} & = & 3.2 \mathrm{MeV} & \text { LN effective-interaction pairing-gap constant } \\
\mathscr{K} & =0.2475 & \text { Zero-point energy constant }
\end{array}
$$

The constant $r_{\text {mic }}$ was determined during the development of FRDM(1992) [9] and we have retained the value determined there.

In addition, the following droplet-model constants, which have been determined in an earlier study [71], are used in the expressions for the average bulk nuclear asymmetry $\bar{\delta}$ and average relative deviation $\bar{\varepsilon}$ in the bulk density that are used to calculate $V_{\mathrm{p}}, V_{\mathrm{n}}$, and $R_{\text {den }}$ in Eqs. (84), (85), and (83), respectively:

$$
\begin{array}{rll}
a_{2} & =22.00 \mathrm{MeV} & \text { surface-energy constant } \\
J & =35 \mathrm{MeV} & \text { symmetry-energy constant } \\
L & =99 \mathrm{MeV} & \text { density-symmetry constant } \\
Q & =25 \mathrm{MeV} & \text { effective surface-stiffness constant } \\
K & =300 \mathrm{MeV} & \text { compressibility constant } \\
r_{0} & =1.16 \mathrm{fm} & \text { nuclear-radius constant }
\end{array}
$$

Insertion of these values and the value of $e^{2}$ on which $c_{1}$ depends in Eqs. (86) and (87) leads to

$$
\begin{gathered}
\bar{\delta}=\frac{(N-Z) / A+0.0112 Z^{2} / A^{5 / 3}}{1+3.15 / A^{1 / 3}} \\
\bar{\varepsilon}=-\frac{0.147}{A^{1 / 3}}+0.330 \bar{\delta}^{2}+\frac{0.00248 Z^{2}}{A^{4 / 3}}
\end{gathered}
$$

\section{ENUMERATION OF CONSTANTS}

It is always of interest to have a clear picture of exactly what constants enter a model. Naturally, anyone who sets out to verify a calculation by others or uses a model for new applications needs a complete specification of the model, for which a full specification of the constants and their values is an essential part. Also, when different models are compared it is highly valuable to fully understand exactly what constants enter the models. Unfortunately, discussions of model constants are often incomplete, misleading, and/or erroneous. For example, in Table A of Ref. [79] the 
Table A

Constants in the FRDM. The third column gives the number of constants adjusted to nuclear masses or mass-like quantities such as odd-even mass differences or fission-barrier heights. The fourth column gives the number of constants determined from other considerations.

\begin{tabular}{|c|c|c|c|}
\hline Constants & Comment & Mass-like & Other \\
\hline$M_{\mathrm{H}}, M_{\mathrm{n}}, e^{2}$ & Macroscopic fundamental constants & 0 & 3 \\
\hline $\begin{array}{l}a_{\mathrm{el}}, r_{0}, r_{\mathrm{p}} \\
a, a_{\mathrm{den}}, K\end{array}$ & $\begin{array}{l}\text { Macroscopic constants from considerations } \\
\text { other than mass-like data }\end{array}$ & 0 & 6 \\
\hline$a_{3}, W, h$ & $\begin{array}{l}\text { Macroscopic constants obtained } \\
\text { in prior adjustments to mass-like data }\end{array}$ & 3 & 0 \\
\hline $\begin{array}{l}a_{1}, a_{2}, J, Q, a_{0} \\
L, C, \gamma, c_{\mathrm{a}}\end{array}$ & $\begin{array}{l}\text { Macroscopic constants determined by } \\
\text { current least-squares adjustments }\end{array}$ & 9 & 0 \\
\hline$\hbar c, m_{\text {nuc }}$ & Microscopic fundamental constants & 0 & 2 \\
\hline $\begin{array}{l}V_{\mathrm{s}}, V_{\mathrm{a}}, A_{\mathrm{den}}, B_{\mathrm{den}}, C_{\mathrm{cur}} \\
k_{\mathrm{p}}, l_{\mathrm{p}}, k_{\mathrm{n}}, l_{\mathrm{n}}, a_{\mathrm{pot}}\end{array}$ & Microscopic constants & 0 & 10 \\
\hline$N_{\text {bas }}, p, C_{\mathrm{S}}$ & $\begin{array}{l}\text { Microscopic constants determined } \\
\text { from considerations of mass-like quantities }\end{array}$ & 3 & 0 \\
\hline$r_{\text {mic }}$ & $\begin{array}{l}\text { Microscopic constant determined by } \\
\text { previous least-squares adjustments }\end{array}$ & 1 & 0 \\
\hline $\mathscr{K}$ & $\begin{array}{l}\text { Microscopic constant determined by } \\
\text { current least-squares adjustments }\end{array}$ & 1 & 0 \\
\hline$a_{1}, a_{2}, J, K, L, Q$ & $\begin{array}{l}\text { Droplet-model constants that enter the single- } \\
\text { particle potential (see discussion in text) }\end{array}$ & 0 & 0 \\
\hline Subtotals & & 17 & 21 \\
\hline Total & & & 38 \\
\hline
\end{tabular}

number of parameters of the mass model of Spanier and Johansson [80] is listed as 12. However, in the article [80] by Spanier and Johansson, the authors themselves list in their Table A 30 parameters plus 5 magic numbers that are not calculated within the mass model and must therefore be considered parameters, for a total of at least 35 parameters.

We specify here all the constants that enter our model, rather than just those that in the final step are adjusted to experimental data by a least-squares procedure. We also include such constants as the number of basis functions used and fundamental constants like the electronic charge and Planck's constant.

\subsection{Constants in the FRDM}

The discussion in the previous section allows us to enumerate the constants in the FRDM model in Table A. From this list we see that the macroscopic-microscopic method requires relatively few constants. One feature of the model gives rise to a small complication when counting the number of constants. Droplet-model constants occur also in the determination of the single-particle potential. However, a different set of constants is used here because, as discussed above, one does not know what the optimum values are until the calculation has been completed. In principle, the calculation should be repeated with the new droplet-model constants defining the single-particle potential until convergence is obtained. In Table A we have counted the number of constants as if this procedure had been carried out.

However, since the droplet-model constants used in the present calculations are different in the microscopic part and in the macroscopic part, different counting schemes could also be employed. Since the droplet-model constants used in the microscopic expressions are obtained from four primary constants [71] and nuclear masses were used only to give rough estimates of these constants, one may not wish to regard them as determined from mass-like quantities. One of the four primary constants is the nuclear radius constant $r_{0}$, which has the same value as we use in our macroscopic model. Therefore, only three remain that could be considered as additional FRDM constants. With this classification scheme the number of constants adjusted to mass-like quantities remains 17 and the total number of constants in the model increases from 38 to 41 . Alternatively, if we do count the three primary constants as adjusted to nuclear masses, the total number of FRDM constants is 41 , while the number adjusted to mass-like quantities increases from 17 to 20 . 
Table B

Constants in the FRLDM. The third column gives the number of constants adjusted to nuclear masses or mass-like quantities such as odd-even mass differences or fission-barrier heights. The fourth column gives the number of constants determined from other considerations.

\begin{tabular}{|c|c|c|c|}
\hline Constants & Comment & Mass-like & Other \\
\hline$M_{\mathrm{H}}, M_{\mathrm{n}}, e^{2}$ & Macroscopic fundamental constants & 0 & 3 \\
\hline $\begin{array}{l}a_{\mathrm{el}}, r_{0}, r_{\mathrm{p}} \\
a, a_{\mathrm{den}}\end{array}$ & $\begin{array}{l}\text { Macroscopic constants from considerations } \\
\text { other than mass-like data }\end{array}$ & 0 & 5 \\
\hline$W, h$ & $\begin{array}{l}\text { Macroscopic constants obtained } \\
\text { in prior adjustments to mass-like data }\end{array}$ & 2 & 0 \\
\hline $\begin{array}{l}a_{\mathrm{v}}, \kappa_{\mathrm{V}}, a_{\mathrm{s}}, \kappa_{\mathrm{s}} \\
a_{0}, c_{\mathrm{a}}\end{array}$ & $\begin{array}{l}\text { Macroscopic constants determined by } \\
\text { current least-squares adjustments }\end{array}$ & 6 & 0 \\
\hline$\hbar c, m_{\mathrm{nuc}}$ & Microscopic fundamental constants & 0 & 2 \\
\hline $\begin{array}{l}V_{\mathrm{s}}, V_{\mathrm{a}}, A_{\mathrm{den}}, B_{\mathrm{den}}, C_{\mathrm{cur}} \\
k_{\mathrm{p}}, l_{\mathrm{p}}, k_{\mathrm{n}}, l_{\mathrm{n}}, a_{\mathrm{pot}}\end{array}$ & Microscopic constants & 0 & 10 \\
\hline$N_{\text {bas }}, p, C_{\mathrm{S}}, r_{\text {mic }}, \mathscr{K}$ & $\begin{array}{l}\text { Microscopic constants determined } \\
\text { from considerations of mass-like quantities }\end{array}$ & 4 & 0 \\
\hline$a_{1}, a_{2}, J, K, L, Q$ & $\begin{array}{l}\text { Droplet-model constants that enter the single- } \\
\text { particle potential (see discussion in text) }\end{array}$ & 3 & 0 \\
\hline Subtotals & & 16 & 20 \\
\hline Total & & & 36 \\
\hline
\end{tabular}

\subsection{Constants in the FRLDM}

The constants in the FRLDM, which are either identical to or similar to the constants in the FRDM, are enumerated in Table B. We mentioned in the discussion of the FRDM constants that the six constants in the last line of Table A would converge to the values of the same constants listed earlier in the table after a sufficient number of iterations. In the FRDM these constants therefore need not be regarded as additional constants. In contrast, in the FRLDM they must be regarded as constants obtained from adjustments to mass-like quantities. However, as mentioned in the discussion of the FRDM constants, these constants are all obtained from three primary constants, so we only include three in this category.

\section{CALCUlational DETAILS}

Our mass tabulation includes all nuclei in the FRDM(1992) and 339 additional nuclei requested by astrophysicists: below $N=82$ we have added nuclei on the neutron-rich side, up to about 20 in each isotope sequence. For example, the most neutron-rich chromium nucleus in the previous table was ${ }_{24}^{86} \mathrm{Cr}_{62}$, in the current table it is ${ }_{24}^{103} \mathrm{Cr}_{79}$. We refer below to this "new" neutron-rich region as NNR.

The adjustment of constants in the macroscopic model is simplified enormously because the ground-state shape and fission saddle-point shape are to high accuracy independent of the precise values of these constants when they are varied within a reasonable range [82]. We therefore calculate the ground-state deformation with one set of constants and subsequently determine the various shape-dependent terms in the mass expression at this deformation. The constants of the macroscopic model can then be adjusted, with the nuclear shapes remaining fixed. The ground-state shapes are always determined in the FRLDM.

A significant advantage of this approach is that the effect of new features can often be investigated without repeating the entire calculation from the beginning. With the more elaborate searches for ground-state minima relative to the FRDM(1992) (see below) and the consideration of axial-asymmetry effects that we now undertake, this would take around $50000 \mathrm{CPU}$ hours, of which about $40000 \mathrm{CPU}$ hours comes from the consideration of axial-asymmetry degrees of freedom. Our determination of mass-model constants and ground-state nuclear masses involves several steps that were summarized in Fig 1. We discuss these steps and then continue with a presentation and discussion of our results.

1. We found, when we could profit from vastly increased computer power, that in the optimization of the FRDM(1992) macroscopic constants we had not quite found the optimum values. We had started with about 20 different starting values for these constants and we always converged on the same solution. We later found, as discussed in Ref. [83], that when we investigated a larger set of starting values, several hundred sets, about 5\% would lead to a different, lower- $\sigma_{\text {th }}$ solution, namely $\sigma_{\text {th }}=0.6614 \mathrm{MeV}$. It is interesting to note that when we compare 


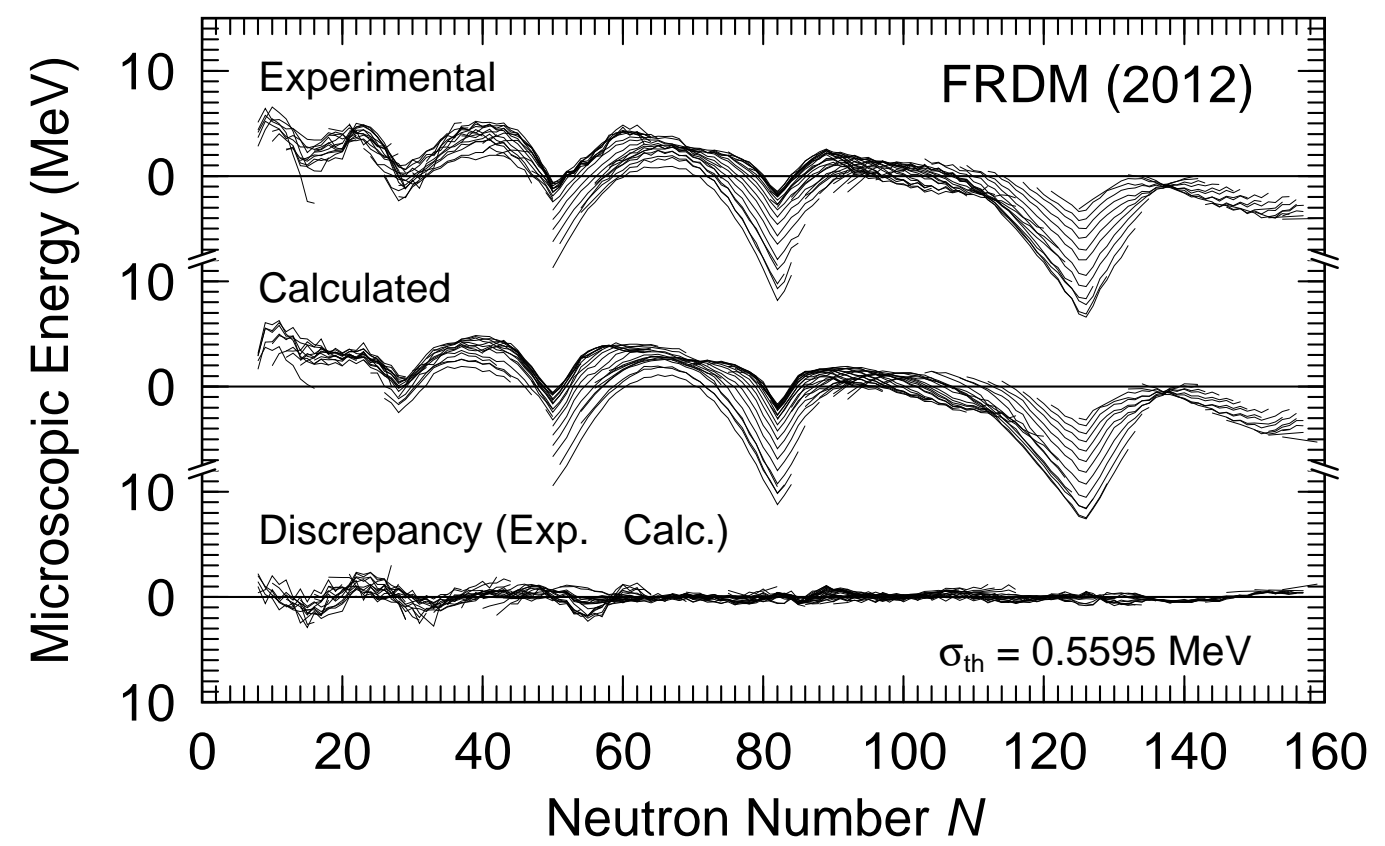

Fig. 3: Comparison of experimental to calculated microscopic energies $E_{\text {mic }}$ for 2149 nuclei, for a macroscopic model corresponding to the FRDM. The bottom part showing the difference between these two quantities is equivalent to the difference between measured and calculated ground-state masses. There are almost no systematic errors remaining for nuclei with $N \geq 65$, for which region the error is only $0.355 \mathrm{MeV}$. The results shown in this figure represent our new mass model. The lines are drawn through isotope chains.

the published FRDM(1992) to the masses that were new in AME2003 relative to AME1989 (529 data points, we found that in this "extrapolated" region the model error was quite low $\sigma_{\text {th }}=0.4617 \mathrm{MeV}$. With the more optimized model constants one would perhaps expect poorer extrapolation properties, since conventional wisdom is that a model that is extremely tightly bound to known data will do more poorly when applied to new regions. However, we found that the model with the better determined constants reproduced the masses in the new region with a $\sigma_{\mathrm{th}}=0.4208 \mathrm{MeV}$ accuracy! These investigations are discussed in slightly more detail in Ref. [83]. Since we had by then realized that the FRDM should not be applied to fission-barrier calculations we also investigated the impact of excluding them from our optimization of model constants. This led to a very minimal decrease in the model deviations, we obtained $\sigma_{\mathrm{th}}=0.6591 \mathrm{MeV}$. We also investigated the results

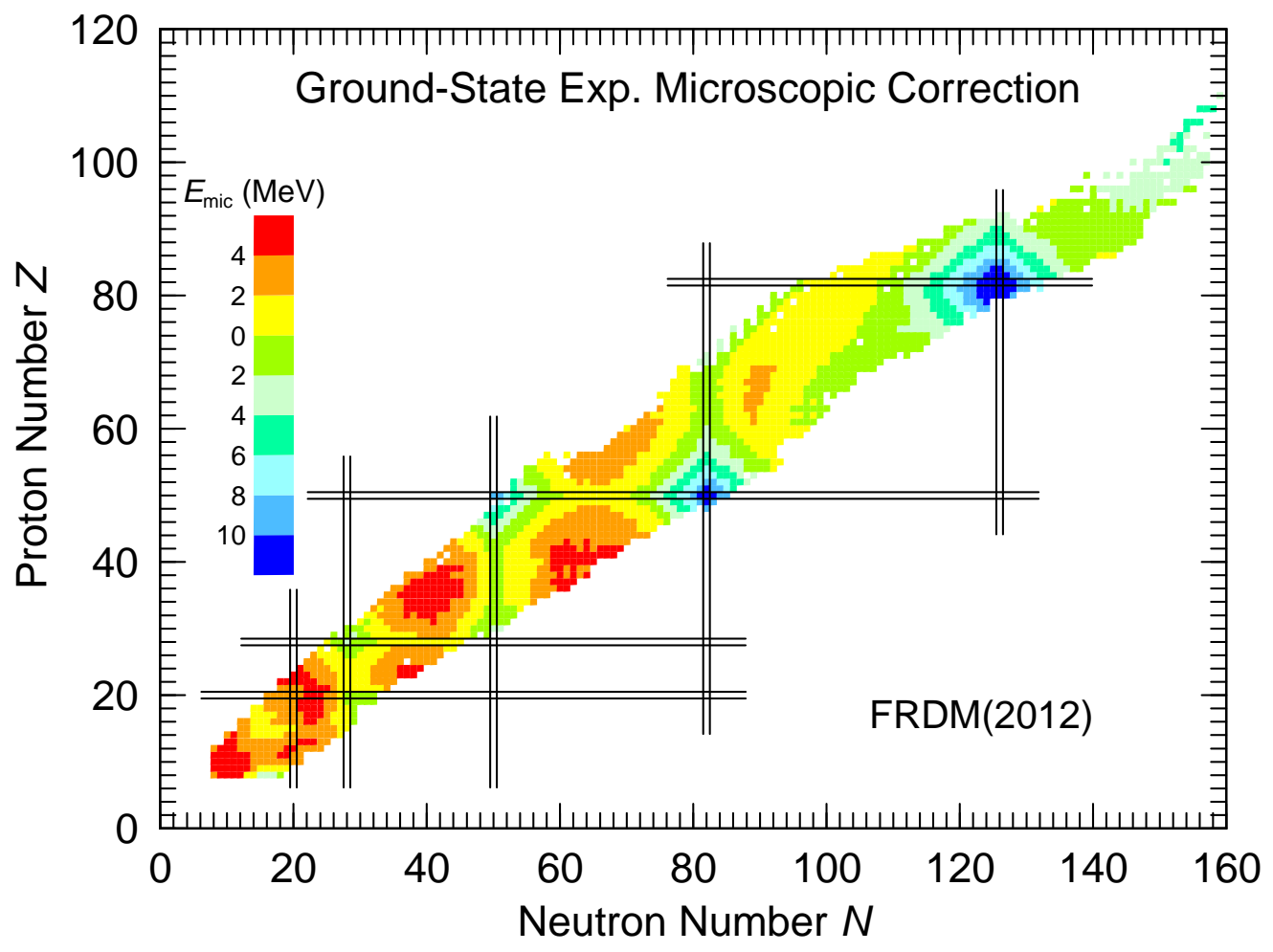

Fig. 4: Experimental microscopic correction corresponding to the top section in Fig. 3. 


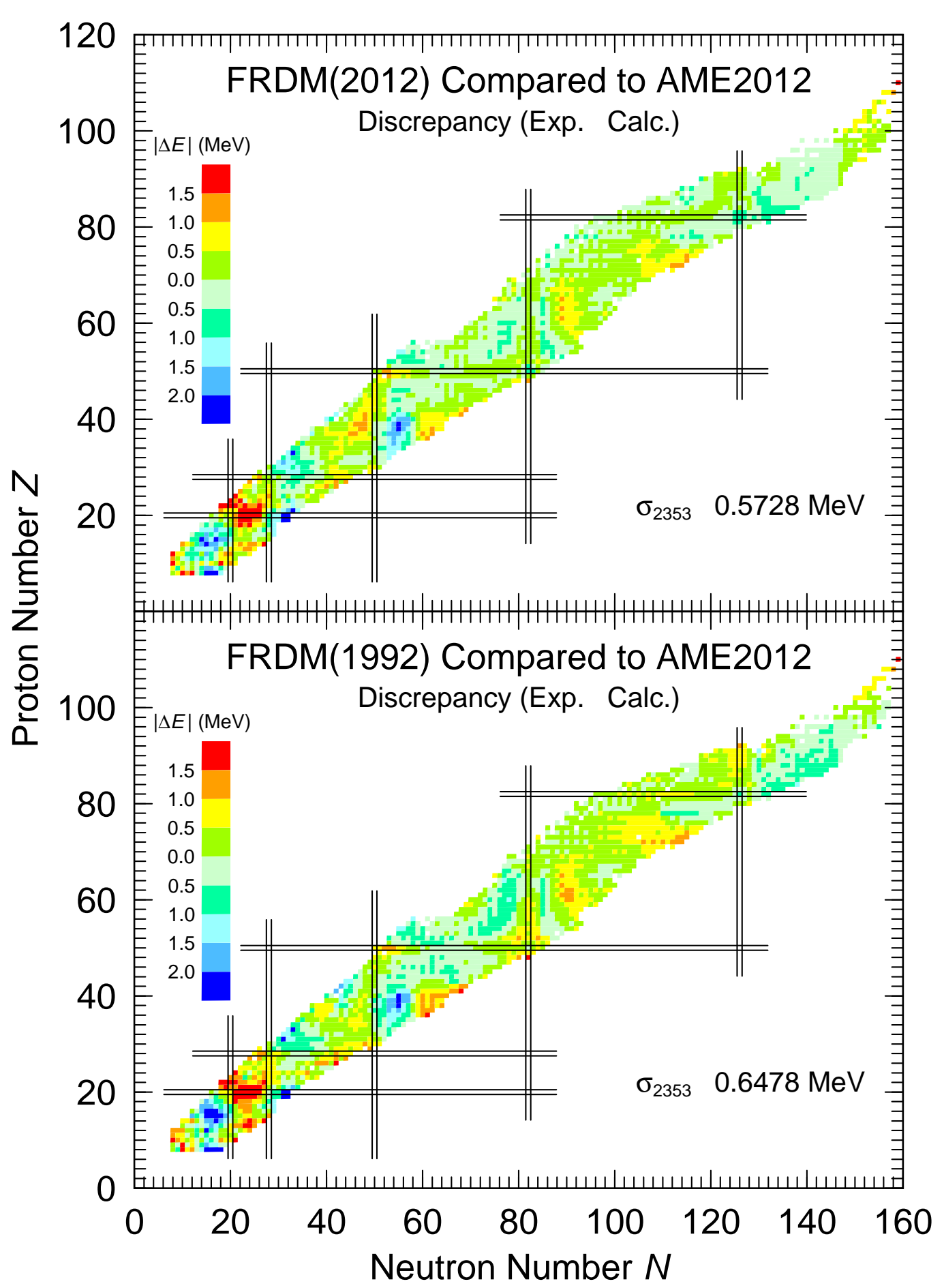

Fig. 5: Top Panel: Difference between measured masses and masses calculated in our current FRDM(2012). The model is adjusted to the AME2003 experimental evaluation [17] but we compare to the AME2012 evaluation [81]. Bottom panel: The previous FRDM(1992) is compared to the same data evaluation. The figure is discussed further in the text.

obtained when we adjusted to the AME2003 data base (which we also now do here) and obtained a model error $\sigma_{\text {th }}=0.6140 \mathrm{MeV}$. Thus, as indicated in the summary Fig. 1 we obtained an improvement of (about) $0.05 \mathrm{MeV}$ from these two enhancements. We now proceed to discuss the remaining steps.

2. As a first step, potential-energy surfaces are calculated versus $\varepsilon_{2}$, $\varepsilon_{4}$, and $\gamma$. In this calculation, which was actually performed in 2006, the FRLDM as defined in Ref. [9], with macroscopic constants as given in Ref. [57], was used. From these potential-energy surfaces a first estimate of the ground-state $\varepsilon_{2}, \varepsilon_{4}$ and $\gamma$ deformations are obtained. The grid-point distances are $\Delta \varepsilon_{2}=0.025, \Delta \varepsilon_{4}=0.02$, and $\Delta \gamma=2.5$. Details of these calculations are in Refs. [44, 45, 39, 25]. A large number of calculated potential-energy surfaces and discussions focused on shape isomers are in Ref. [46]. Calculated potential-energy surfaces in .pdf format can be accessed at URL [84]. They should be accessed by following the link "here" at the top of this page. Two sets of surfaces exist. One set is limited in deformations to $0 \leq \varepsilon_{2} \leq 0.45$ and $31 \leq A \leq 290$ "near-ground-state" potential-energy surfaces; the other to $0 \leq \varepsilon_{2} \leq 0.75$ and $171 \leq A \leq 330$, so called "fission" potential-energy surfaces. Axial asymmetry was not considered for NNR nuclei, because the request for additional nuclei came 5 years after we had concluded 


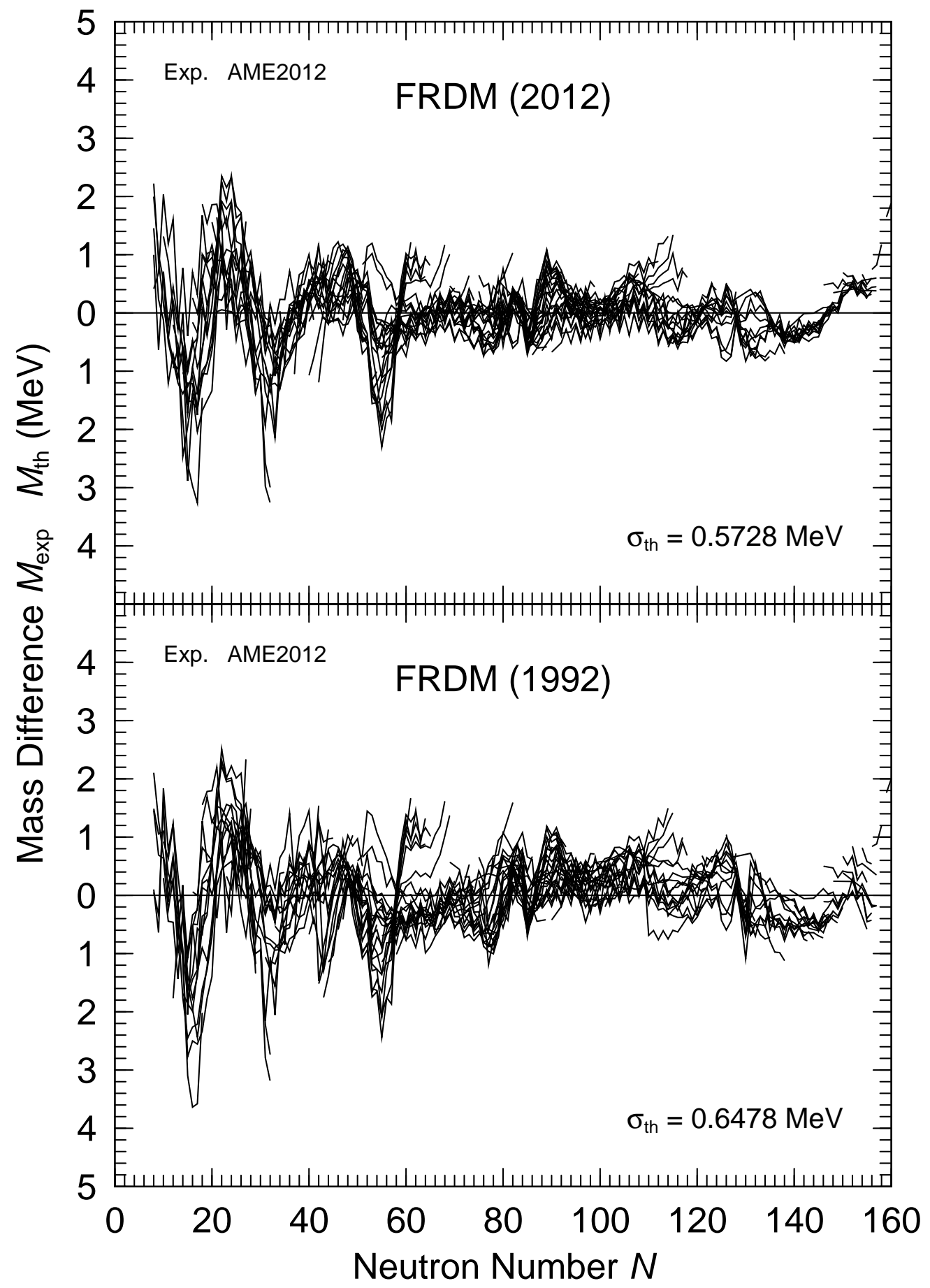

Fig. 6: The most recent mass evaluation AME2012 compared to FRDM(1992) and FRDM(2012). The improvements are particularly noticeable near the magic numbers $N=82$ and $N=126$ and in the shape-coexistence region near $N=40$.

the axial-asymmetry studies. But since deformations in the NNR are usually near spherical and because axial asymmetry is minor for lighter and neutron-rich nuclei (see Fig. 2 in Ref. [44]) we expect little effect on masses from this omission.

3. In our implementation of axial asymmetry we can only study axial asymmetry together with two other multipoles, namely quadrupole $\left(\varepsilon_{2}\right)$ and hexadecapole $\left(\varepsilon_{4}\right)$ multipoles. The largest effect on the ground state mass that we find in that study is about $0.8 \mathrm{MeV}$. Only about $10 \%$ of the nuclei are affected, usually to a much smaller degree, see Refs. [44, 45]. To calculate more accurate ground-state shapes and masses for axially symmetric nuclei we proceed as follows. We minimize the energy with respect to $\varepsilon_{2}, \varepsilon_{3}, \varepsilon_{4}$, and $\varepsilon_{6}$. We do a discrete minimization with a step size of 0.01 in each variable. We feel that a determination of the ground-state shapes to an accuracy of 0.01 in each of the four multipoles is quite sufficient, so that little would be gained by implementing cumbersome interpolation schemes. For each nucleus we do a minimization using several different starting points. One group of starting points are all the minima on the oblate and prolate axes that we found in the 3D calculation. We also start from the five locations $\left(\varepsilon_{2}=-0.25, \varepsilon_{3}=0, \varepsilon_{4}=-0.04, \varepsilon_{6}=0\right),\left(\varepsilon_{2}=-0.25, \varepsilon_{3}=\right.$ 


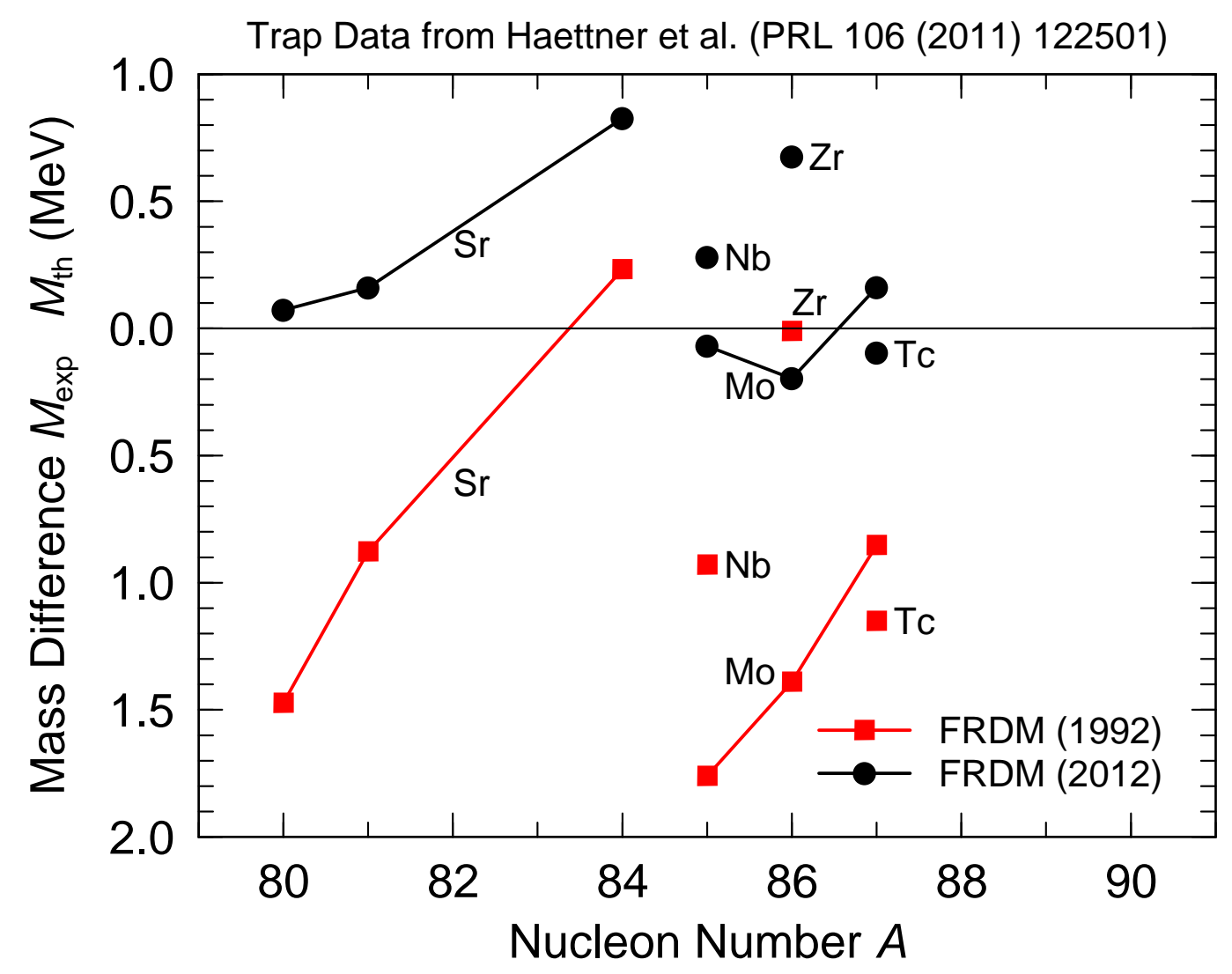

Fig. 7: Recent trap measurements [85] of 9 masses compared to FRDM(1992) and FRDM(2012). The new, more elaborate and accurate calculations have led to much better agreement with experimental masses in this region of shape coexistence.

$\left.0, \varepsilon_{4}=+0.04, \varepsilon_{6}=0\right),\left(\varepsilon_{2}=+0.25, \varepsilon_{3}=0, \varepsilon_{4}=-0.04, \varepsilon_{6}=0\right),\left(\varepsilon_{2}=+0.25, \varepsilon_{3}=0, \varepsilon_{4}=+0.04, \varepsilon_{6}=0\right)$, and $\left(\varepsilon_{2}=+0, \varepsilon_{3}=0, \varepsilon_{4}=0, \varepsilon_{6}=0\right)$. During these studies we were surprised to discover that in a few rare cases ( 10 or 20 or so) that there could be a minimum with $\varepsilon_{3} \neq 0$ separated from a minimum at $\varepsilon_{3}=0$ by a saddle in this $4 \mathrm{D}$ deformation space. What is also interesting is that we observed that if the octupole-deformed minimum was the lower of the two it would also correspond to a theoretical mass that agreed better with experiment. One example for which this occurs is ${ }^{228} \mathrm{Th}$. Typically these situations occur in the transition regions between octupole-asymmetric regions and octupole-symmetric regions on the heavy side of the octupole-asymmetric regions. We therefore also did minimizations with all of the above starting points but with $\varepsilon_{3}=0.10$ at the starting location.

4. Once the ground-state shapes in the 4D axially symmetric calculation have been determined, the various shapedependent functions occurring in the macroscopic energy are calculated for these shapes and stored. The shellplus-pairing corrections are also stored. Because we cannot calculate the FRDM shape-dependent parameters in the $\gamma$ plane we account for the axial asymmetry effects on the ground-state mass in the following manner. All the tabulated quantities are for the (lowest) minima in the axially symmetric space, and we account for the effect of axial asymmetry by modifying the calculated shell corrections by the difference between the potential energy at the ground-state minimum in the $\left(\varepsilon_{2}, \varepsilon_{3}, \varepsilon_{4}, \varepsilon_{6}\right)$ space and the minimum in the $\left(\varepsilon_{2}, \varepsilon_{4}, \gamma\right)$ if the axially asymmetric minimum is the lower one. The zero-point energies are now also calculated as described above and stored as separate entries.

5. The above rule that we select as the ground state the lowest minimum has to be modified for heavy nuclei. Simply expressed, for a nuclide with a high proton number the "fission-isomer" minimum can be lower than a less deformed "ground state" but the fission isomer minimum can have a much lower barrier with respect to fission than does the less deformed minimum, so the fission-isomer minimum is not a minimum that is sufficiently stable to be observed. We therefore need the auxiliary rule that we check the barrier with respect to fission and select as the ground state the minimum with the highest barrier with respect to fission. This consideration only leads to a different selection for the ground state than the much simpler rule to pick the lowest minimum for a few nuclei near ${ }^{228} \mathrm{Fm}$ and for some heavier nuclei, in particular those with $Z>114$ and $N>184$. These issues are discussed in detail and pedagogically illustrated in section III:F "Identifying the ground-state" in Ref. [39] and in the discussion of Figs. 6 and 8 in Ref. [61].

6. The constants of the FRDM are now determined by the optimization procedure described in Sec. 2.1. We assume the mean $\mu_{\mathrm{th}}$ is zero; thus we need only Eqs. 8, 9, 11, and 12. With all the shape-dependent macroscopic functions calculated and tabulated, and with the ground-state shell-plus-pairing energies and zero-point energies also tabulated, the actual optimization takes only 10 seconds or so to determine the optimum macroscopic constants. Once the constants are known (and all the tabulated quantities available) the FRDM(2012) is obtained 


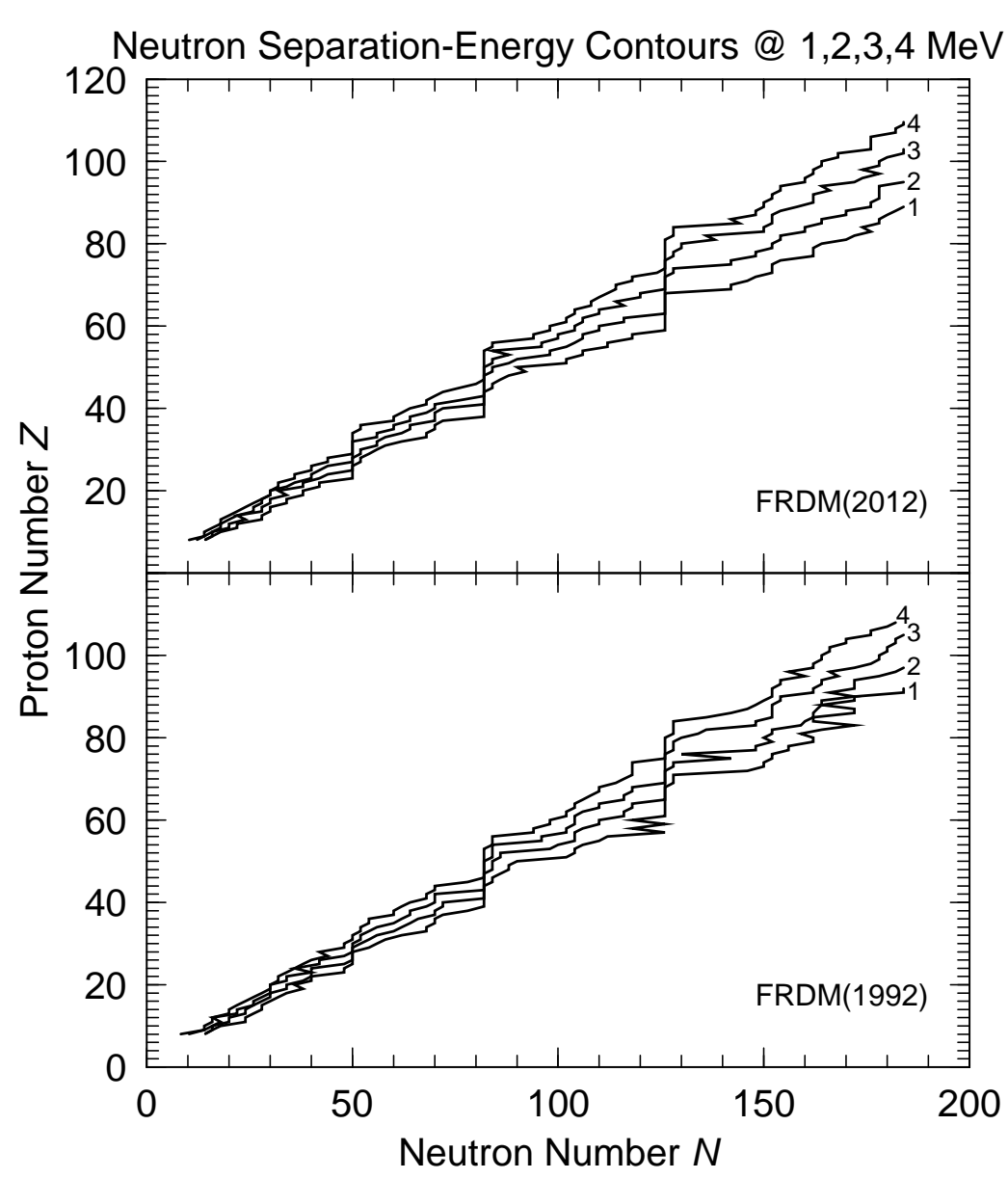

Fig. 8: Neutron separation-energy contours with $S_{\mathrm{n}}=1,2,3$, and $4 \mathrm{MeV}$ in the FRDM(1992) and FRDM(2012). Most of the staggering in the contour lines seen for FRDM(1992) are absent in the FRDM(2012) results.

in less than 10 seconds in the final computational step.

7. In the FRLDM we can calculate fission barriers. Therefore we determine the parameters by minimizing a weighted mean of the rms mass deviation and barrier rms deviations. with the weight 0.888 on the mass rms deviation and 0.111 on the barrier rms deviation. In case of multiple-humped fission barriers we only adjust to the outermost peak. In case of a triple-humped barrier we select the higher of the two outer peaks.

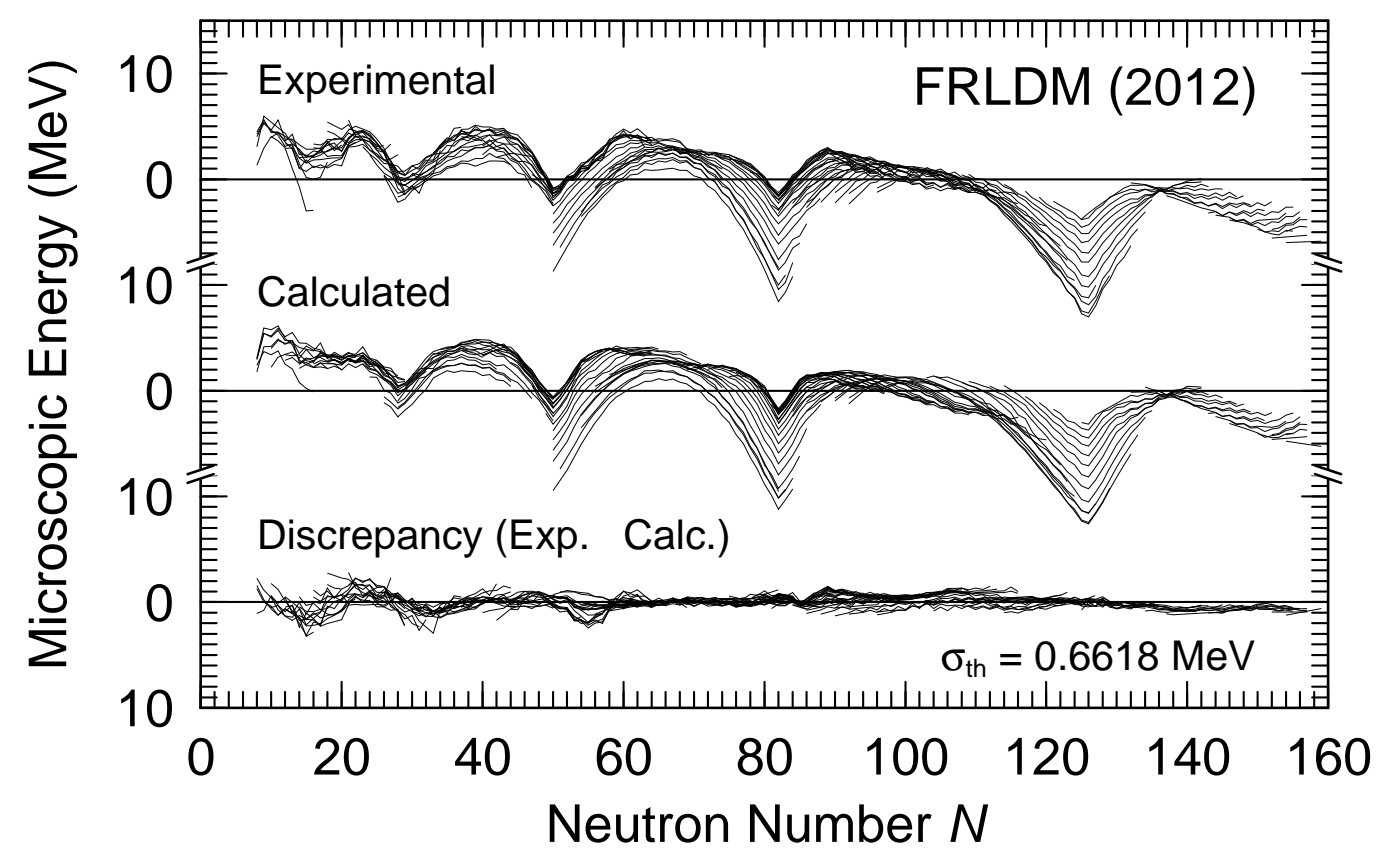

Fig. 9: Analogous to Fig. 3, but for the FRLDM, which contains no Coulomb redistribution terms. This leads to the systematic negative deviations for proton-rich nuclei in the heavy region, which indicate that these calculated masses are systematically too high. 


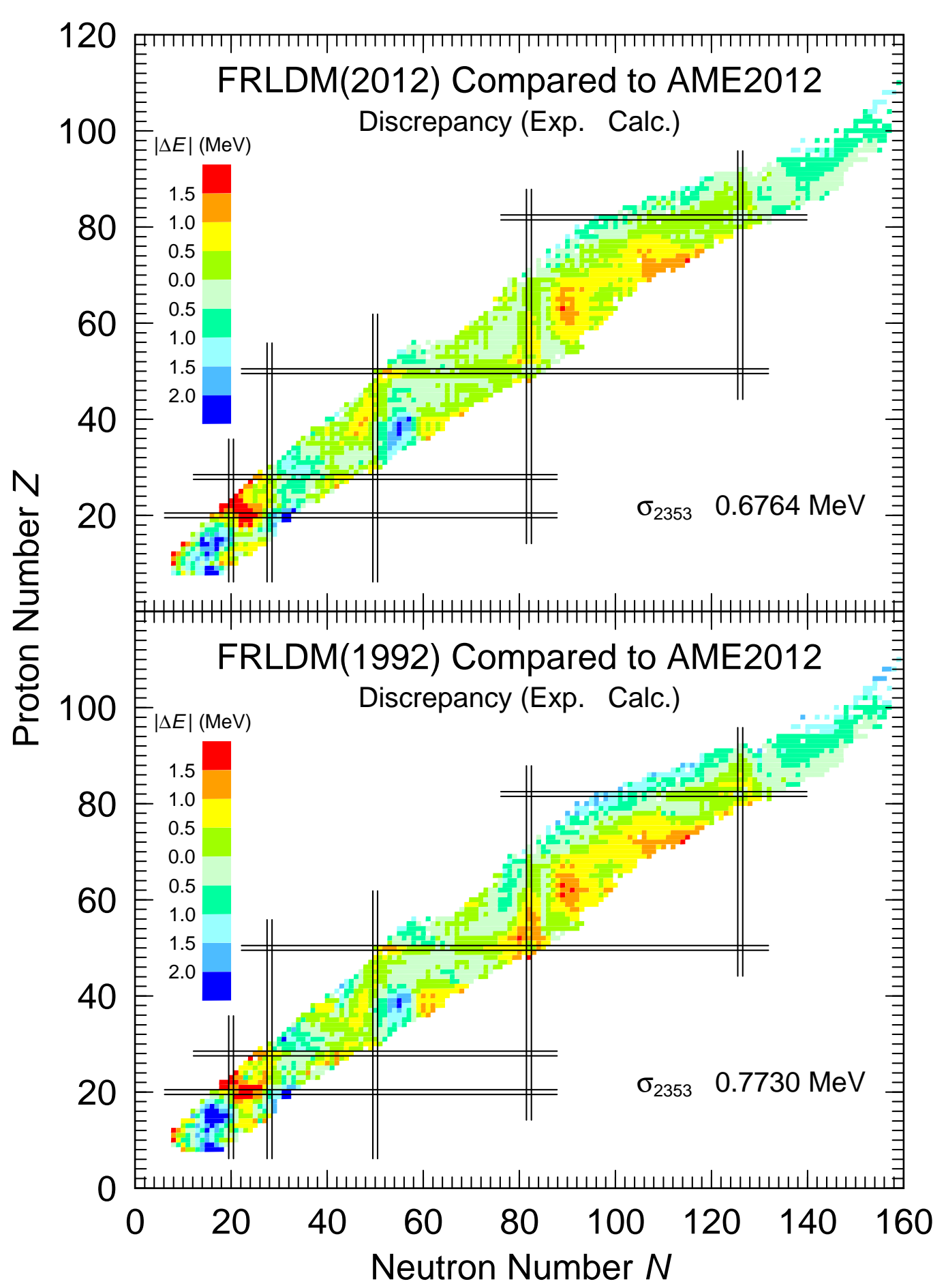

Fig. 10: Top panel: Difference between experimental masses from the AME2012 evaluation and masses calculated in the FRLDM(2012). Bottom panel: We compare here the previous FRLDM(1992) to the same experimental data evaluation.

8. The FRLDM(2012) mass table is generated as well a barrier table with barrier heights for the 31 nuclei that we included in the barrier adjustment. 


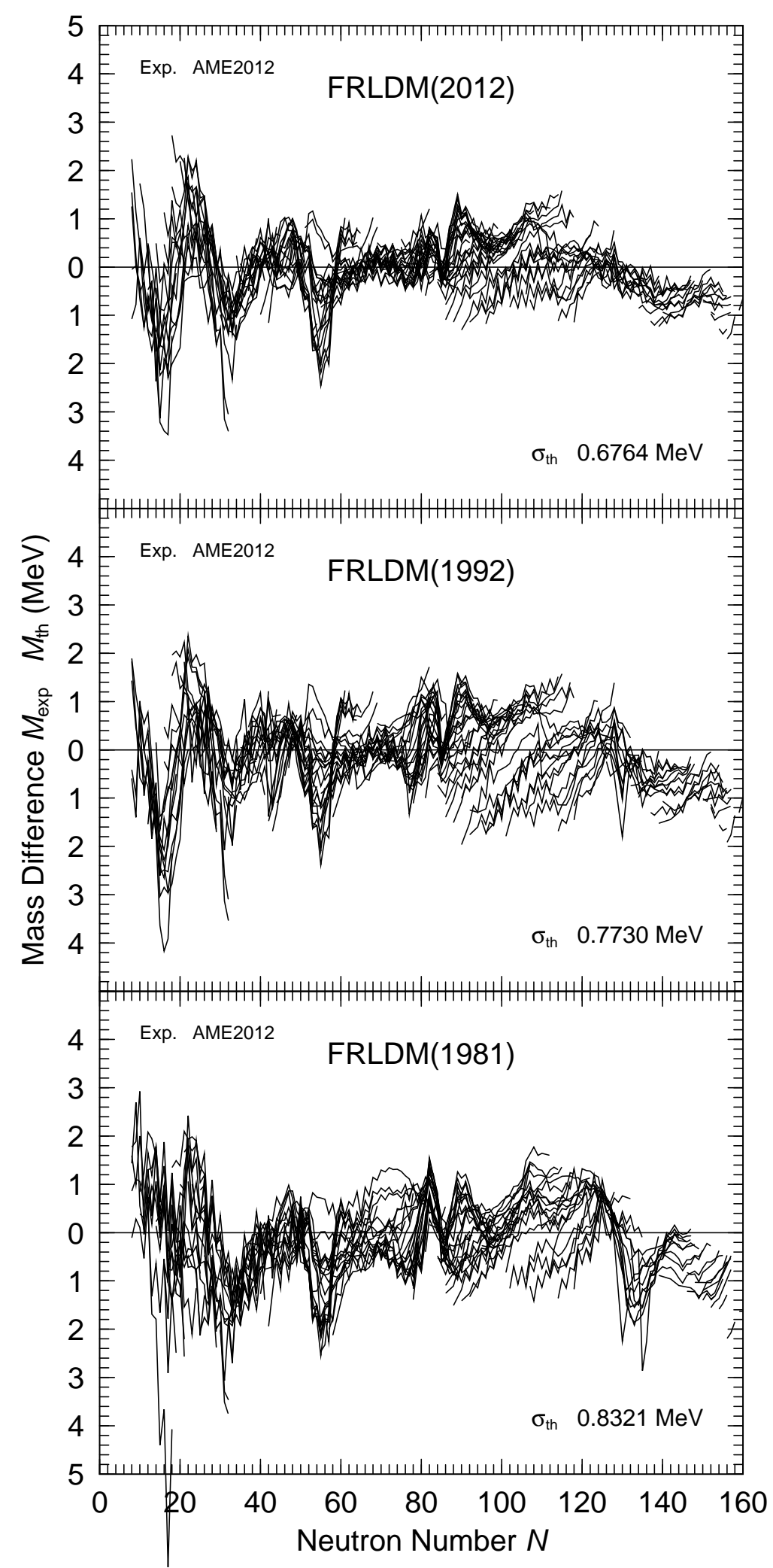

Fig. 11: Difference between measured masses from the AME2012 evaluation and masses calculated in the FRLDM(2012) (top panel) and masses calculated in the FRLDM(1992) (middle panel). The regions of improvement resemble those of the FRDM, namely we find improvements in the regions near $N=40, N=82$, and $N=126$. The deviations from experiment are larger than in the FRDM in the heavy region. In the bottom panel we compare our first mass model, the FRLDM(1981) to the same mass evaluation.

\section{CALCULATED RESUltS}

Figure 3 shows the results of the FRDM calculation. As usual $[8,1,9]$, the top part shows the differences between measured masses and the calculated spherical macroscopic FRDM masses plotted against the neutron number $N$, with isotopes of a particular element connected by a line. These differences have customarily been called "experimental" microscopic corrections and can be compared with the calculated microscopic corrections plotted in the middle part of the figure. It should be noted that despite the designation "experimental microscopic corrections" these do depend 
Table C

Comparison of Fission Barriers Calculated in the FRLDM(2012) and Experimental Barriers for 31 Nuclei

\begin{tabular}{rrrrrrrrrrrr}
\hline$Z$ & $N$ & $A$ & $\begin{array}{c}E_{\text {exp }} \\
(\mathrm{MeV})\end{array}$ & $\begin{array}{c}E_{\text {th }} \\
(\mathrm{MeV})\end{array}$ & $\begin{array}{c}\Delta E \\
(\mathrm{MeV})\end{array}$ & & $N$ & $A$ & $\begin{array}{c}E_{\text {exp }} \\
(\mathrm{MeV})\end{array}$ & $\begin{array}{c}E_{\text {th }} \\
(\mathrm{MeV})\end{array}$ & $\begin{array}{c}\Delta E \\
(\mathrm{MeV})\end{array}$ \\
\hline 34 & 36 & 70 & 39.40 & 37.83 & 1.57 & 92 & 146 & 238 & 5.50 & 5.74 & -0.24 \\
34 & 42 & 76 & 44.50 & 44.08 & 0.42 & 92 & 148 & 240 & 5.50 & 6.46 & -0.96 \\
42 & 48 & 90 & 40.92 & 41.07 & -0.15 & 94 & 142 & 236 & 4.50 & 4.57 & -0.07 \\
42 & 52 & 94 & 44.68 & 44.39 & 0.29 & 94 & 144 & 238 & 5.00 & 4.71 & 0.29 \\
42 & 56 & 98 & 45.84 & 47.06 & -1.22 & 94 & 146 & 240 & 5.15 & 5.05 & 0.10 \\
80 & 118 & 198 & 20.40 & 21.60 & -1.20 & 94 & 148 & 242 & 5.05 & 5.82 & -0.77 \\
84 & 126 & 210 & 21.40 & 22.16 & -0.76 & 94 & 150 & 244 & 5.00 & 6.59 & -1.59 \\
84 & 128 & 212 & 19.50 & 20.19 & -0.69 & 94 & 152 & 246 & 5.30 & 7.19 & -1.89 \\
88 & 140 & 228 & 8.10 & 7.59 & 0.51 & 96 & 146 & 242 & 5.00 & 4.61 & 0.39 \\
90 & 138 & 228 & 6.50 & 6.59 & -0.09 & 96 & 148 & 244 & 5.10 & 5.22 & -0.12 \\
90 & 140 & 230 & 7.00 & 5.66 & 1.34 & 96 & 150 & 246 & 4.80 & 6.01 & -1.21 \\
90 & 142 & 232 & 6.20 & 5.53 & 0.67 & 96 & 152 & 248 & 4.80 & 6.65 & -1.85 \\
90 & 144 & 234 & 6.50 & 5.49 & 1.01 & 96 & 154 & 250 & 4.40 & 6.33 & -1.93 \\
92 & 140 & 232 & 5.40 & 4.84 & 0.56 & 98 & 152 & 250 & 3.60 & 6.02 & -2.42 \\
92 & 142 & 234 & 5.50 & 5.10 & 0.40 & 98 & 154 & 252 & 4.80 & 5.78 & -0.98 \\
92 & 144 & 236 & 5.67 & 5.18 & 0.49 & & & & & & \\
\hline
\end{tabular}

on the macroscopic model used. Please also note that "microscopic corrections" and shell-plus-pairing corrections are different concepts, as elaborated on in the discussion of Fig. 2. In Fig. 4 we plot the experimental microscopic correction in nuclear-chart style. The doubly magic numbers stand out particularly well in the heavy region. In 1936 Bethe and Bacher had the idea that gaps in calculated single-particle level schemes might correspond to large deviations between experimental masses and masses obtained in the semiempirical mass model [86]. At the time there was little mass data available for heavy nuclei so he focused on, nucleon number $20\left({ }^{40} \mathrm{Ca}\right)$, but failed to find clear deviations. He concluded that it could be due to inaccurate experimental mass data. But in Fig. 4 we can see that there is no strong effect on masses for $Z=20, N=20$.

When the macroscopic and microscopic parts of the mass calculation are added to obtain the calculated mass excess and this sum is subtracted from the measured masses, the deviations in the bottom part of the figure remain. We have also plotted this deviation in "nuclear-chart style" format in Fig. 5. The trends of the error in the heavy region and with neutron number indicate that this mass model should be quite reliable for nuclei beyond the current end of the periodic system and towards the drip lines, as has also been our experience with the FRDM(1992) [24, 83, 87]. This is further verified by the studies and simulations discussed in Sec. 5.1 on extrapability. Because the FRDM(2012) was finalized on September 6, 2012 and the AME2012 appeared in December 2012 the FRDM(2012) is adjusted to the AME2003 data base [17]. Therefore 219 masses in Fig. 5 situated along the upper and lower borders of the colored region were not included in the adjustment of the model constants. But we see no tendency to increasing deviations in these regions.

In Fig. 6 we study the improvement with respect to experimental data in the FRDM(2012) relative to the FRDM(1992). We compare both models to the AME2012 [81]. In Ref. [9] the FRDM(1992) was compared to the AME1989 data set[18]. We obtained a model error $\sigma_{\text {th }}=0.669 \mathrm{MeV}$, but with respect to this new data set the deviation is decreased to $\sigma_{\mathrm{th}}=0.6314 \mathrm{MeV}$. The reason is that some measured masses were removed from in the new evaluation, others were revised, and also many of the new masses are in the heavier region where the FRDM(1992) is more accurate. In the new mass calculation the deviations are now smaller, $\sigma_{\mathrm{th}}=0.5595 \mathrm{MeV}$, an $11 \%$ reduction of the deviations. Particularly we notice that the large fluctuations near the magic neutron number $N=126$ are gone. Also at $N=82$ the deviations are considerably reduced, in particular when going into the magic shell. These improvements are partly due to the improved calculation of the zero-point energies as discussed in Sect. 2.11.

We also notice that a group of correlated large deviations just beyond $N=40$ have almost entirely disappeared. The improvements in this region $(N \approx 40)$ of shape coexistence is due to the more accurate execution of our calculations in the current version, made possible by vastly increased computer power. In the FRDM(1992) calculation, to obtain the potential energy at a specific deformation, we started by calculating a set of single-particle levels at this deformation for a single-particle potential with parameters (that is radius and depth) corresponding to a $\beta$-stable nucleus at the nucleon number $A$ under consideration. Then we calculated the shell-plus-pairing corrections for all nuclei with this A value from the proton drip line to the neutron drip line, using this same set of calculated single-particle levels. Then the macroscopic energy was calculated for each individual nuclide with correct parameters and correct $Z$ and $A$, that is no approximation here. By repeating this procedure for different shapes potential-energy surfaces were obtained. We then located all minima and selected the deepest minimum as the ground-state (with consideration of stability with respect to fission as discussed above). At this minimum we then calculated the single-particle levels with the single-particle potential parameters appropriate for this nucleus to obtain more accurate shell-plus-pairing corrections. We also calculated the effect of $\varepsilon_{3}$ and $\varepsilon_{6}$ shape variations at this minimum. However we now find that in cases of 


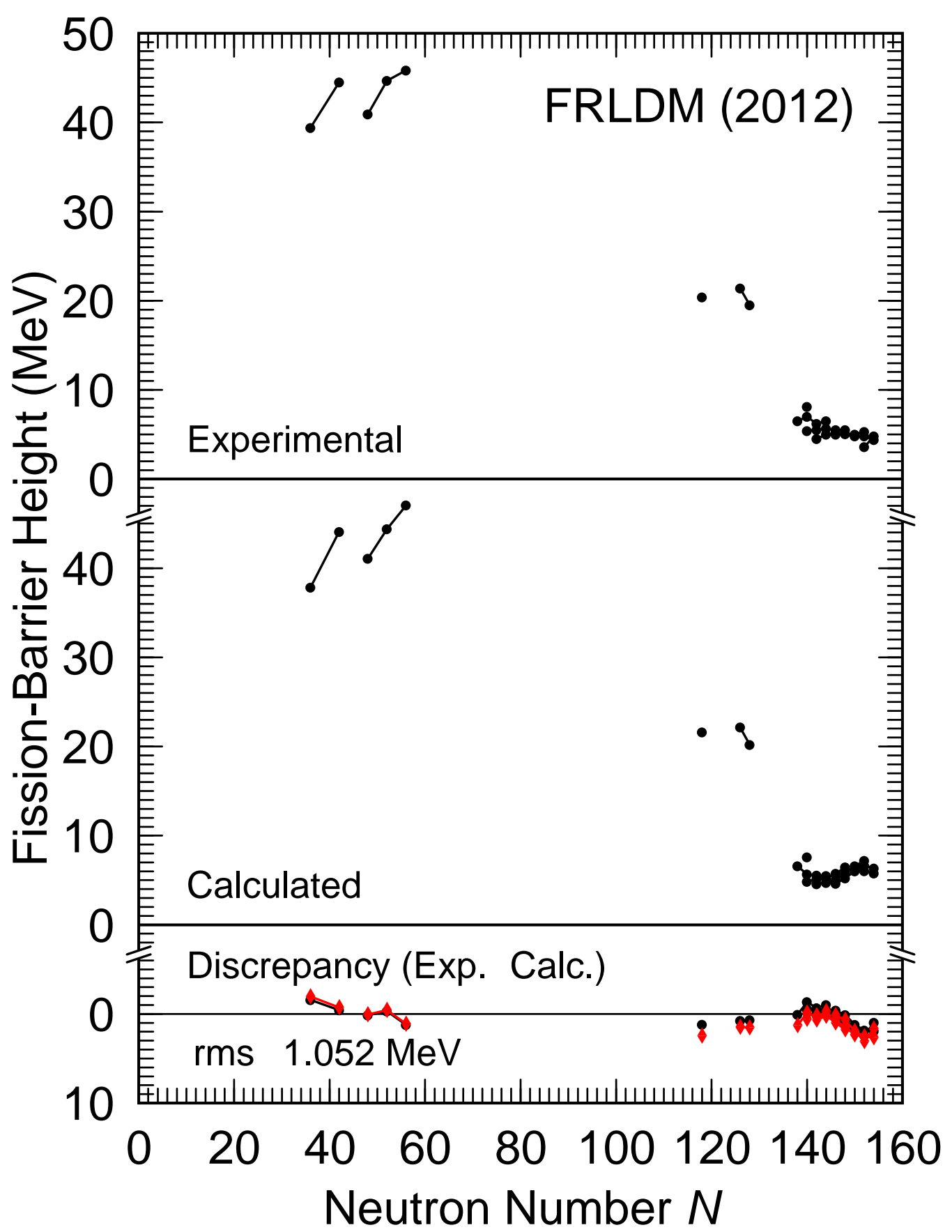

Fig. 12: Comparison of calculated and experimental fission-barrier heights for nuclei throughout the periodic table, after a readjustment of the macroscopic model constants. Experimental barrier heights are well reproduced by the calculations, the rms error is only $1.052 \mathrm{MeV}$ for 31 nuclei. In the actinide region it is the outer of the two peaks in the "double-humped" barrier that is compared to experimental data. In case of a triplehumped barrier we compare to the higher of the outer two peaks. The (red) diamond symbols indicate the barrier-height differences we obtain when the FRLDM is adjusted only to ground-state masses.

shape coexistence the other minimum in some cases would have been the lower minimum had these additional step been taken also at that minimum. However, at the time we were limited by computer power and the approximations made resulted in significant inaccuracies in only a few cases. The origin of these results is the dependence of the single-particle radii and depths on $N-Z$, in addition to their $A$ dependence. We find it interesting that our more accurate treatment here gives better agreement with experimental masses. This is a strong indication that we have implemented a realistic isospin dependence for the single-particle potential. We compare in Fig. 7 the FRDM(1992) and $\operatorname{FRDM}(2012)$ masses to new experiments [85] in the shape-coexistence region [25, 46] near $N=40$. In the new calculation the agreement with the measured masses is much improved.

Our nuclear-structure model framework allows us to calculate not just masses, but also other quantities such as $\beta$ decay half-lives and $\beta$-delayed neutron emission probabilities, and ground-state spins. We are currently in the process of calculating such quantities, which will be submitted for consideration for an ATOMIC DATA AND NUCLEAR DATA TABLES issue with an astrophysical emphasis, in analogy with our previous publication Ref. [24]. But as one example of improvements in the FRDM(2012) relative to FRDM(1992), of interest in r-process calculations we show in Fig. 8 results for the one-neutron separation energy $S_{1 \mathrm{n}}$. Displayed are contour lines representing the locations of the 


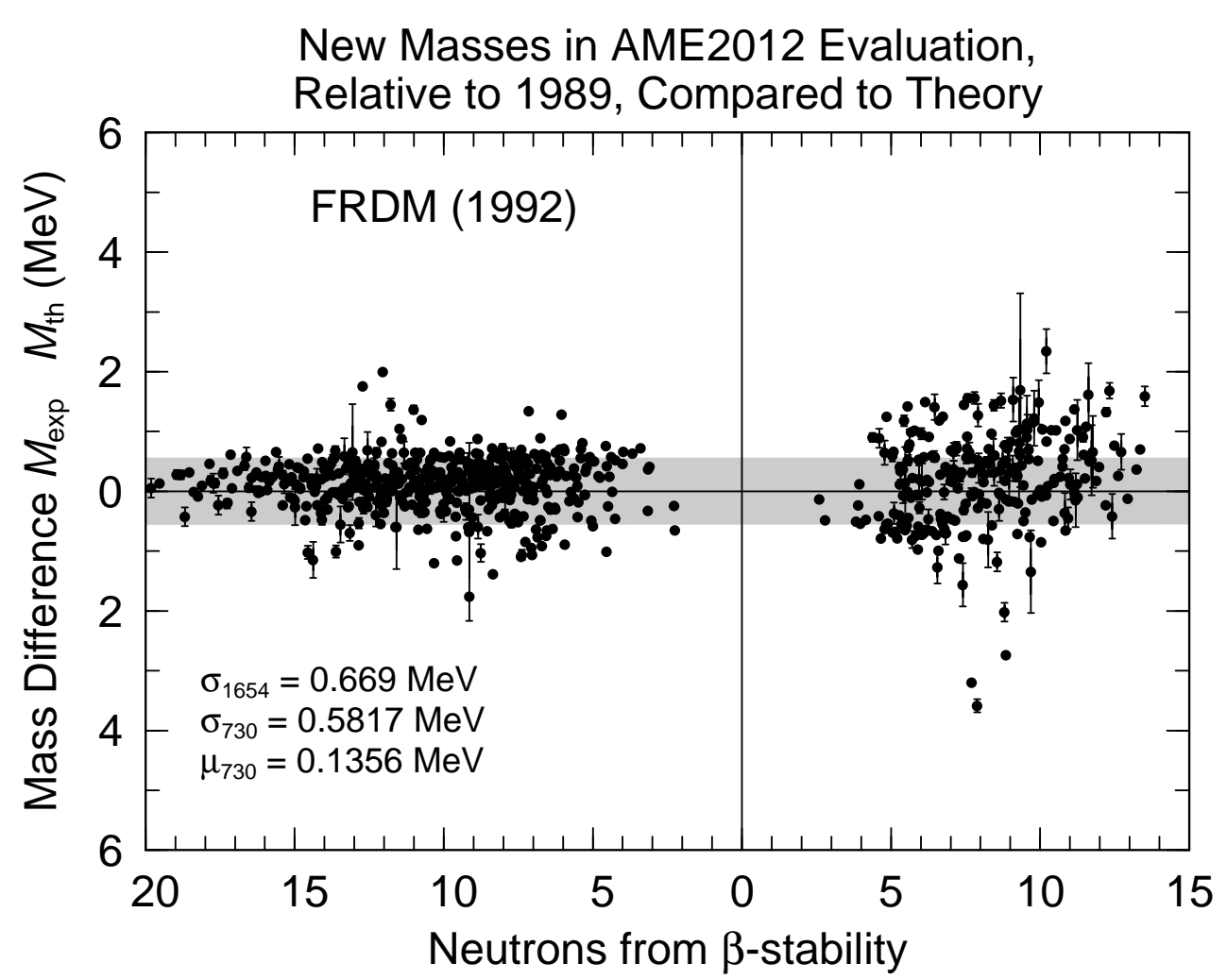

Fig. 13: The previous mass model, the FRDM(1992) compared to masses that are new in AME2012 relative to the data base AME1989. These new masses were not considered in the adjustment of the model constants. There are 730 such new masses. It is gratifying that the deviations are smaller $(0.5818 \mathrm{MeV})$ for these new masses than in the region where the model parameters were adjusted.

$S_{1 \mathrm{n}}=1,2,3$, and $4 \mathrm{MeV}$ contours for the two models. For FRDM(1992) there is pronounced staggering in some locations, which are essentially absent in the FRDM(2012). These improvements are mainly due to the more accurate ground-state shape deformations and the improved calculations of the ground-state correlation ("zero-point") energies.

The FRLDM(2012), which does not treat Coulomb redistribution effects, is somewhat less accurate than the

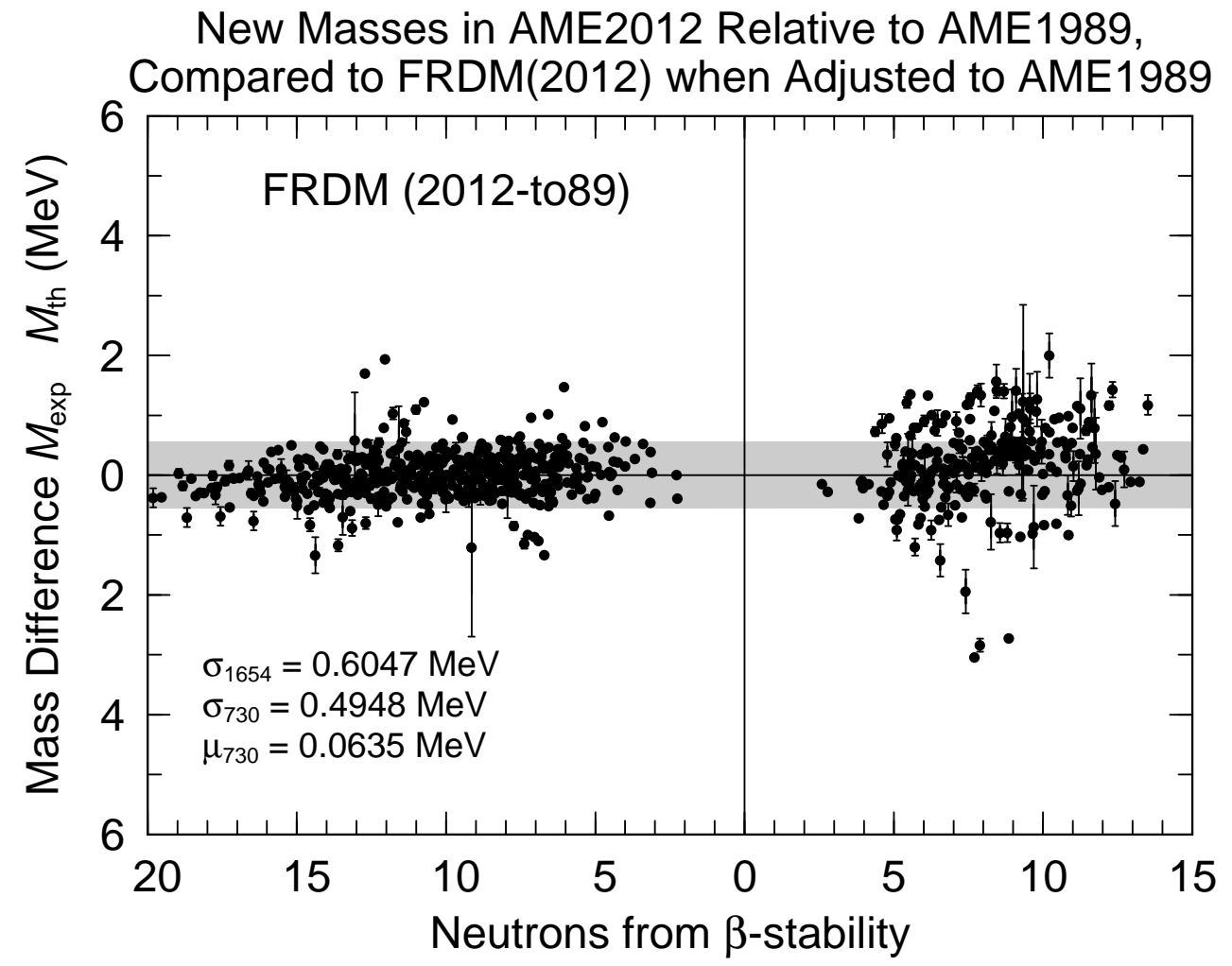

Fig. 14: We have adjusted our current mass model (with all the improvements discussed in the text included) to the older AME1989 experimental evaluation to test the extrapability of the model. It agrees better with the AME1989 data base than FRDM(1992), due to improvements in the calculations, $0.6047 \mathrm{MeV}$ versus $0.669 \mathrm{MeV}$ for the previous FRDM(1992). But it also extrapolates much better $0.4948 \mathrm{MeV}$ for the new nuclei, versus 0.5817 for the previous FRDM(1992). 


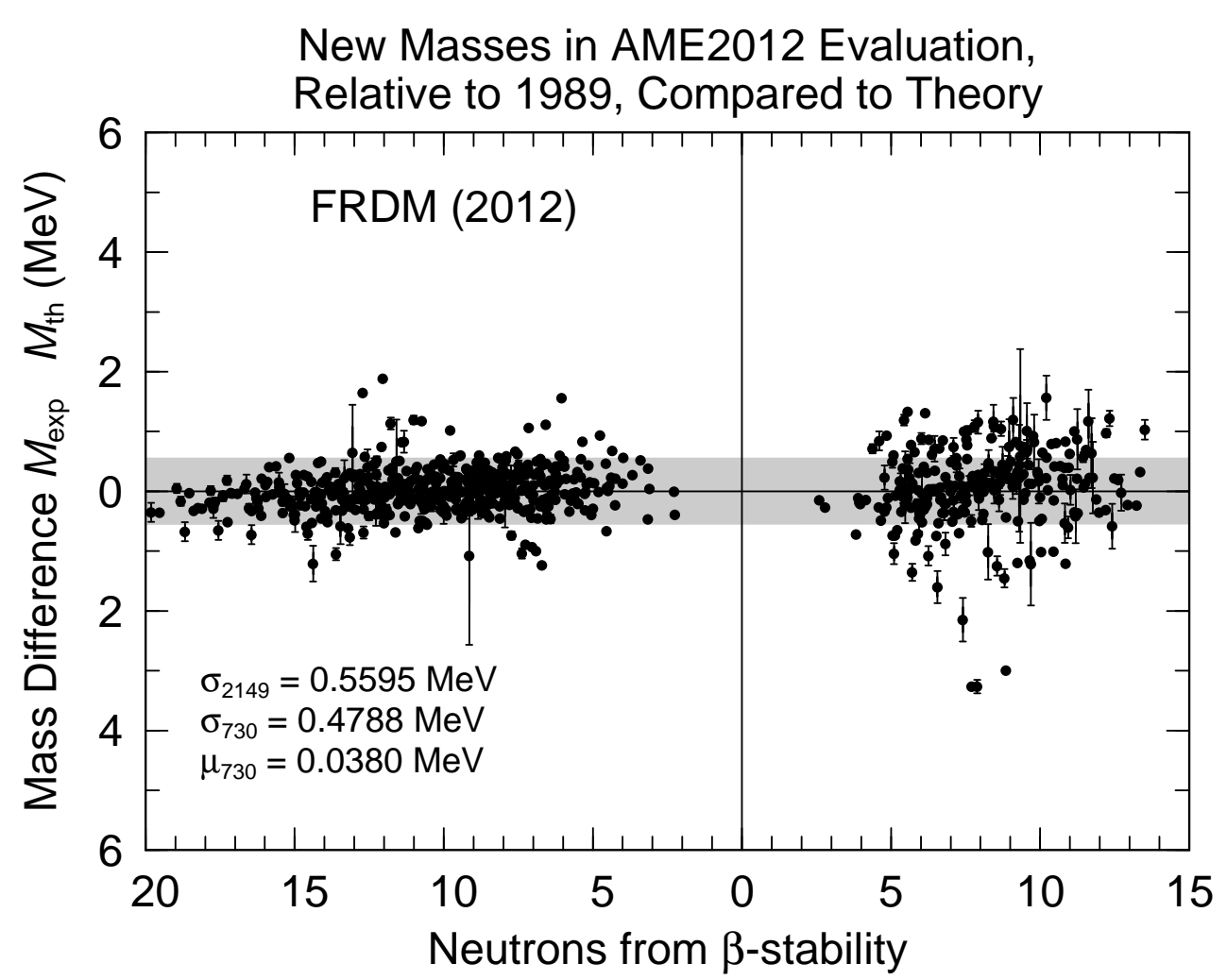

Fig. 15: Masses in FRDM(2012) compared to masses in AME2012 that were not in AME1989. The FRDM(2012) is adjusted to AME2003, so 513 masses shown here were included in this data set. But the accuracy increased only by $0.0160 \mathrm{MeV}$ a $3 \%$ change. This indicates that the model extrapolates quite satisfactorily.

$\operatorname{FRDM}(2012)$, with an $18 \%$ larger $\sigma_{\mathrm{th}}$, as is seen in Fig. 9. format, in Fig. 10. It is particularly in the heavy region that the FRLDM(2012) extends farther away from the zero deviation line, than does the FRDM(2012). There is also a systematic isospin effect on the differences, an effect which is absent in the $\operatorname{FRDM}(2012)$, which is especially clear in Fig. 11. This is a sign that the Coulomb redistribution effect is not treated in the FRLDM, which results in too low binding energies for heavy proton-rich nuclides [88]. We will further illustrate this issue in Sect. 5.1.

But, in contrast to the FRDM, we can calculate fission barriers in the FRLDM. We have recently published a calculation of fission-barrier heights for 5239 nuclides for all nuclei between the proton and neutron drip lines for the region $171 \leq A \leq 339$ [61]. This calculation was carried out exactly like here with the minor differences that 1) we have now improved the calculation of the ground-state correlation ("zero-point") energies and readjusted the macroscopic parameter set. That is, the shape space for the ground-state and fission saddle-point determinations are the same in the published barrier study as here. We include axial asymmetry corrections at the ground state in both calculations. We expect a negligible effect on barrier heights if they were calculated in the precise current model version. We have checked this for ${ }_{80}^{180} \mathrm{Hg}_{100}$, for which we tabulated in Ref. [89] a barrier height $9.81 \mathrm{MeV}$, with the current parameter set and the other features here we obtain a barrier height $9.65 \mathrm{MeV}$. We use the same experimental barrier data set as in Ref. [57] in our adjustment to barrier heights. We show in Table C and in Fig. 12 a comparison of the calculated barriers to the experimental data set.

Conventional wisdom has usually assumed that because the Coulomb and surface-energy terms in the macroscopic energy contribute with the same sign one cannot accurately determine the surface-energy constants from an adjustment to masses alone Rather one would need to also adjust the model parameters to fission-barrier heights because the terms contribute to the barrier heights with different signs. Obviously, if we were dealing with a completely accurate model this would not be necessary. We have tested this conventional wisdom by adjusting the FRLDM macroscopic constants (the usual 6 of them) considering only the AME2003 data set of 2149 masses and excluding fission barriers. In such an adjustment we obtain $\sigma_{\text {th }}=0.6364 \mathrm{MeV}$ for the FRLDM. It is somewhat remarkable that the agreement with experimental fission-barrier evaluations does not deteriorate greatly; we in this case obtain an rms deviation of 1.475 $\mathrm{MeV}$ with respect to the 31 barriers, which probably indicates the robust character of our mass models. We plot these deviations as (red) diamonds in Fig. 12.

\subsection{Extrapability}

One test of the reliability of a nuclear mass model is to compare differences between measured and calculated masses in new regions of nuclei that were not considered when the constants of the model were determined. It is common to characterize a mass model error (or accuracy) in a certain region of nuclear masses by the rms deviation. However, as we pointed out in Sect. 2.1 this is an unsuitable measure since it is a sum of the model error and the experimental error. Above we discussed a more correct measure, which we introduced in 1988 [3] and normally use ever since. The difference between these two numbers can be substantial, in particular in regions of new nuclei 


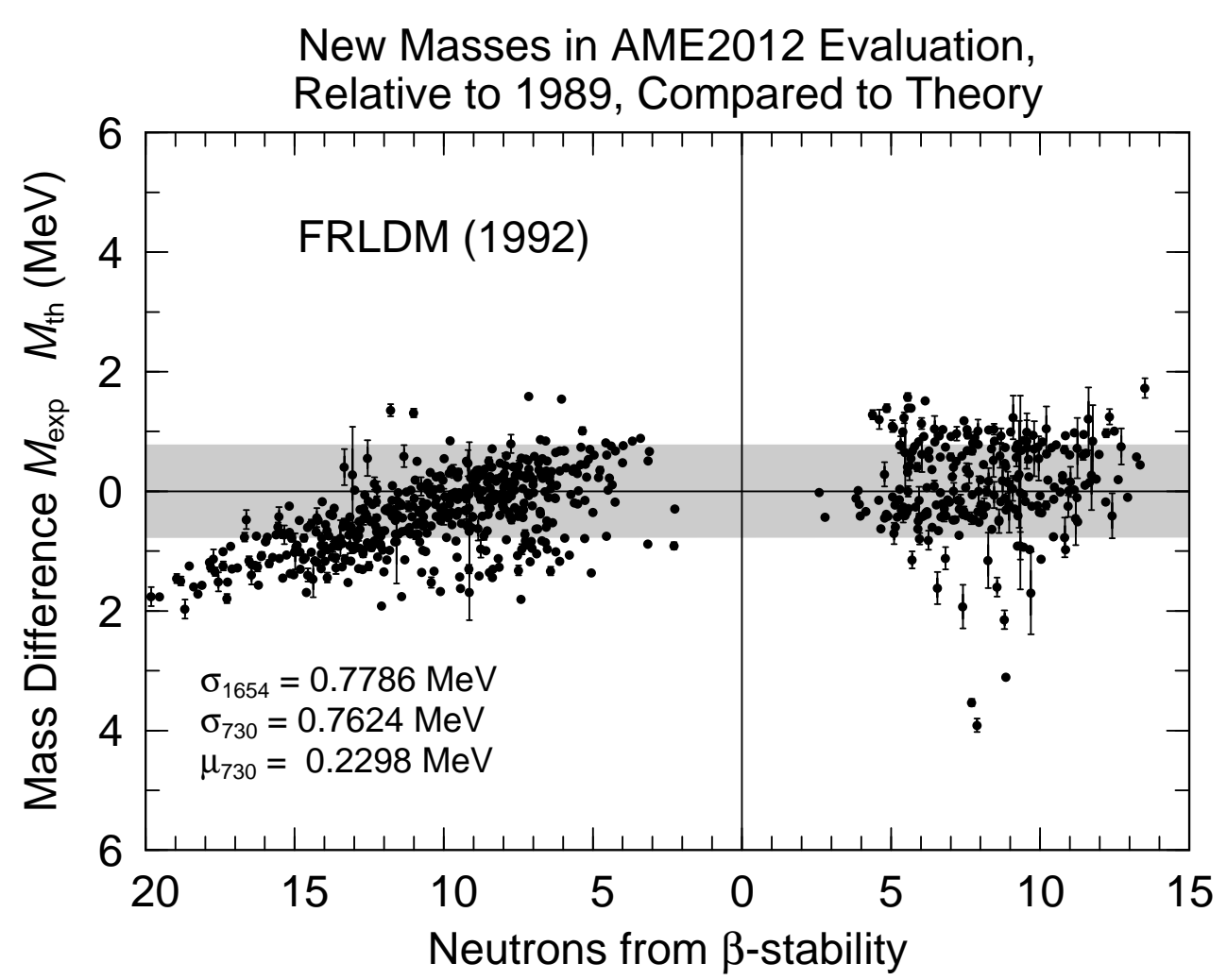

Fig. 16: The FRLDM(1992) compared to masses that are new in AME2012 relative to the data base AME1989. These new masses were not considered in the adjustment of the model constants. There are 730 such new masses. The error has not diverged in this region of new masses, but there is a systematic deviation towards proton drip, which is not present in the FRDM.

where previously the experimental uncertainties could be sufficiently large to give a noticeable contribution to the rms number. For example, in Ref. [24] we compared (in Table D, first entry) the FRDM(1992) which was adjusted to AME1989 to new measurements in AME1993 that were not included in AME1989; there were 217 such new masses.

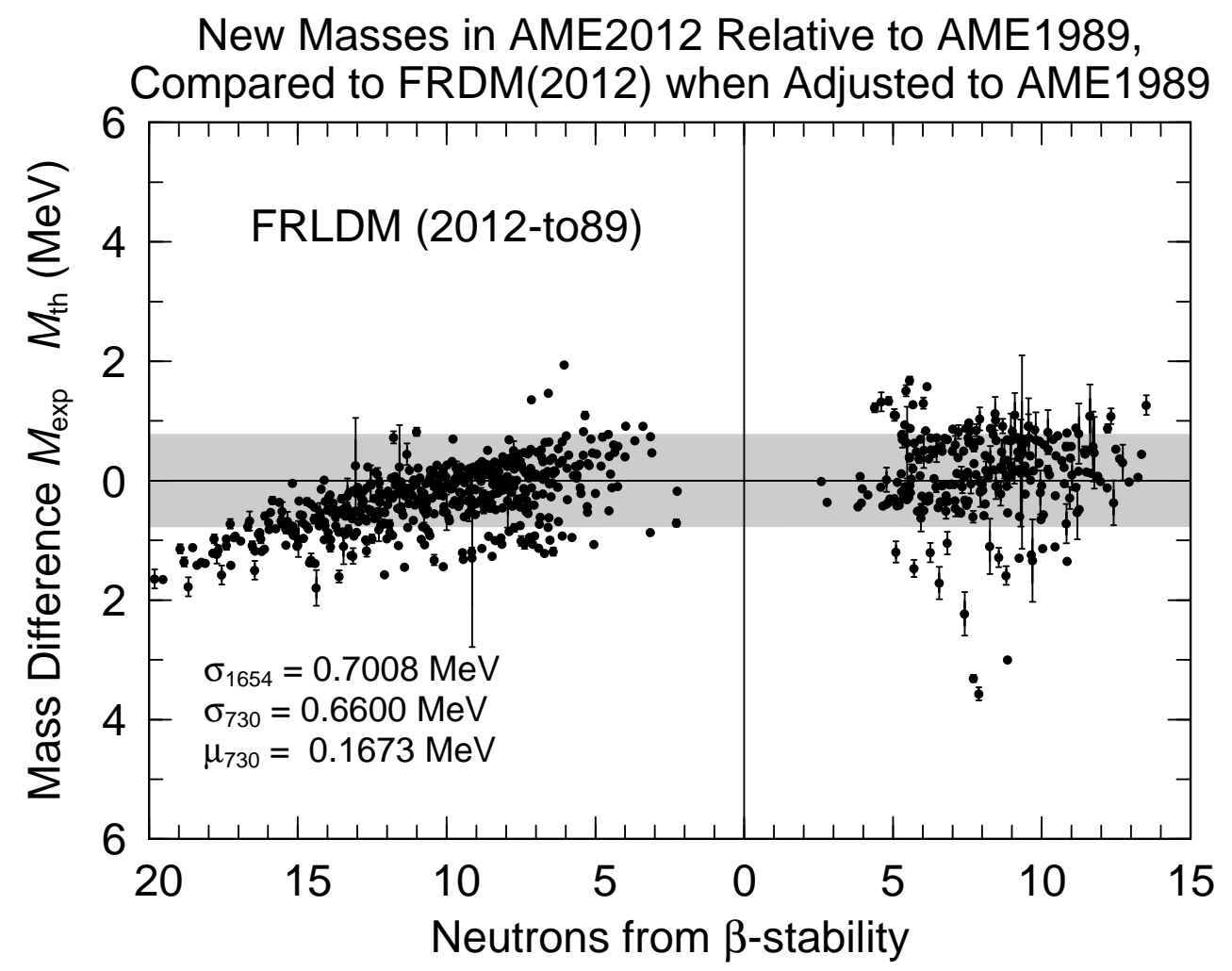

Fig. 17: We have adjusted the current FRLDM(2012) (with all the improvements discussed in the text included) to the older AME1989 experimental evaluation to test the extrapability of the model. It agrees better with the AME1989 data base than FRLDM(1992), due to improvements in the calculations, $0.7008 \mathrm{MeV}$ versus $0.7786 \mathrm{MeV}$ for the previous FRLDM(1992). But it also extrapolates considerably better $0.6600 \mathrm{MeV}$ for the new nuclei, versus $0.7624 \mathrm{MeV}$ for the previous FRDM(1992), although these 730 new nuclei were not taken into account in the adjustment. 


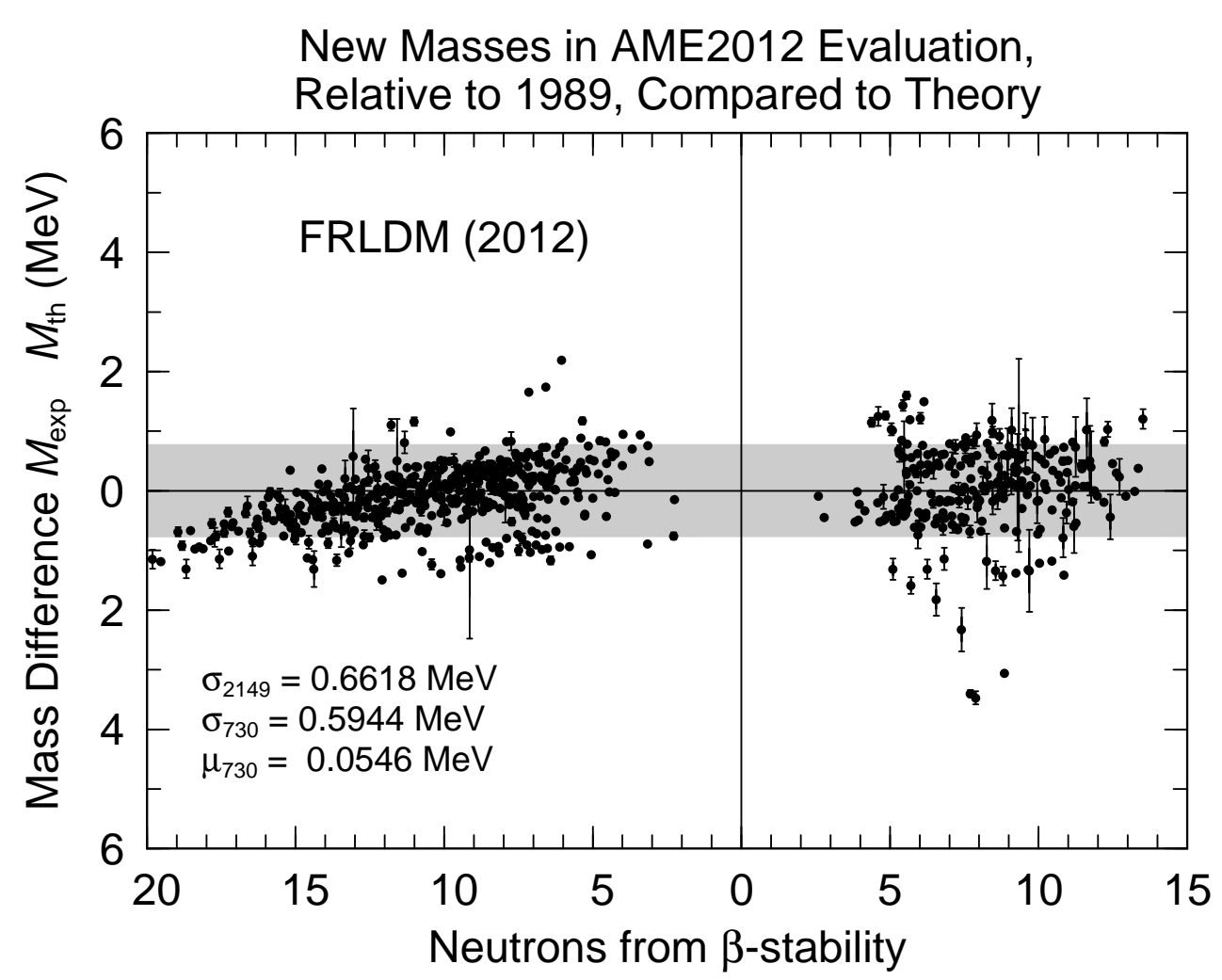

Fig. 18: Masses in FRLDM(2012) compared to masses in AME2012 that were not in AME1989. The FRLDM(2012) is adjusted to AME2003, 513 masses shown here were included in this data set. The inclusion of these masses increased the accuracy from $0.6600 \mathrm{MeV}$ to $0.5944 \mathrm{MeV}$, a $10 \%$ change. This indicates that the model extrapolates somewhat less well than the FRDM to new nuclei.

For the 1654 nuclei in AME1989 to which the FRDM(1992) was adjusted the model error $\sigma_{\text {th }}$ was $0.669 \mathrm{MeV}$, the rms deviation $0.681 \mathrm{MeV}$. For the new nuclei we found the model error $\sigma_{\mathrm{th}}=0.642 \mathrm{MeV}$ but the rms deviation is $0.730 \mathrm{MeV}$. Thus if the rms measure is used one finds that the "error" diverges in the new region of nuclei because the error is $100 \times 0.730 / 0.681-100$ percent $(7.2 \%)$ larger in the new region of nuclei. With the more appropriate error measure we find instead that error is $4 \%$ smaller in the new region of nuclei.

We are now in a position to compare the FRDM(1992) to a much larger data set of new mass measurements. In the AME2012 evaluation there are 730 masses that were not in the AME1989 data set. In Fig. 13 we show the differences between the new masses and masses tabulated in FRDM(1992). As is our custom we plot the differences as a function of neutrons from stability where for the position of the line of $\beta$-stability we use Green's approximation [90]:

$$
N-Z=\frac{0.4 A^{2}}{A+200}
$$

We observe that the accuracy of the previous (FRDM(1992)) mass model in this new region of nuclei has improved by $[1-(0.5817 / 0.669)] \times 100=13.0 \%$. The reasons are several. One is that this is not a double-blind test, the experimentalists were aware of our mass model and one can assume that if results strongly deviant from our mass model were obtained the results would be reevaluated (when masses are determined from $\alpha$ and $\beta$ decay chains it is non-trivial to reach iron-clad conclusions). An illustration of this possibility can be seen in the comparison of Figs. 1 and 2 and the associated discussion in [83]. Another reason might be that in the group of new nuclei most (533) correspond to the region with $N>65$ where the model error is lower than for $N \leq 65$, where our sample only contains 197 nuclides. The systematic deviation (mean deviation, see Sect. 2.1 for definitions) is $0.1356 \mathrm{MeV}$ indicating that the model masses are underbound by this amount on the average. However, as discussed below we have now found that in 1992 we had not determined the optimum set of constants, with better optimized constants the mean deviation is much smaller, see Table D.

Our current mass model FRDM(2012) is adjusted to a recent data base, AME2003. We could compare it to the masses that are new in AME2012 relative to AME2003 but we proceed slightly differently for two reasons. First the number of new nuclei would be somewhat limited, and, second, we want to compare how FRDM(2012) performs relative to FRDM(1992). Therefore we have adjusted our current model to AME1989 and obtain FRDM(2012-to89). It means that all the technical improvements and "new physics" outlined in Fig. 1 are included, but we adjust the macroscopic parameters to the earlier experimental data set. Thus we can make a consistent comparison of the two versions, because no feature or quantity of the subsequently new masses have entered into the development of FRDM(2012). We show in Fig. 14 the result of this comparison. First, we notice that we agree better with the AME1989 data base than does the FRDM(1992). Since we now adjust two more parameters to the experimental data base, namely the density-symmetry parameter $L$ (see Refs. [87, 68] and a renormalization constant related to the inertia used in the 


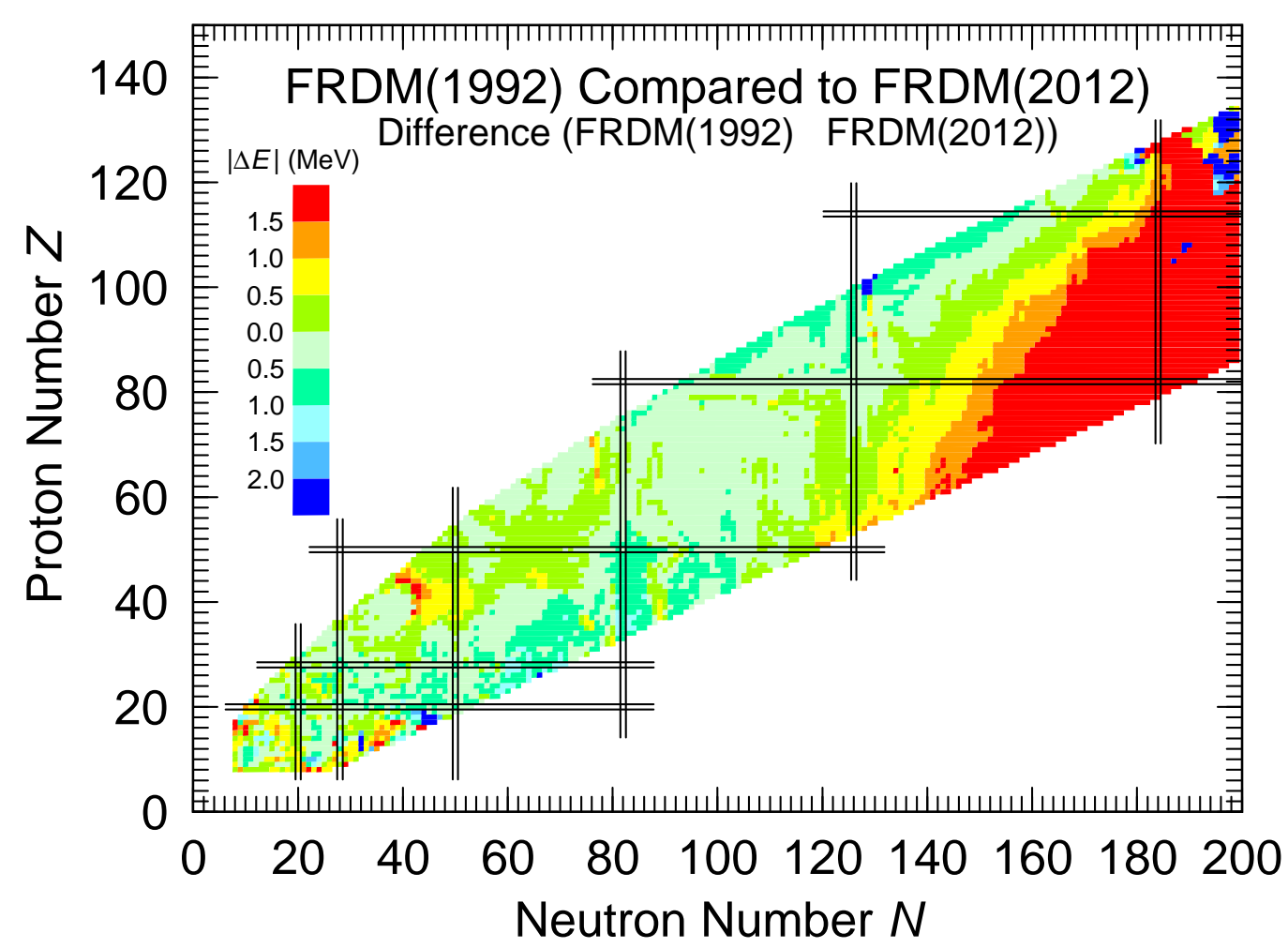

Fig. 19: Difference between masses in the FRDM(1992) and FRDM(2012). The main differences are towards neutron drip in the heavy region, in regions of shape coexistence and of axial asymmetry which are all discussed in more detail in the text.

zero-point energy calculation (see Sect. 2.11), one could have the valid concern that the better accuracy is just a consequence of the two additional variable parameters. However, we note that when this mass table (FRDM(2012-to89)) is compared to the new data set that was not taken into account in the adjustment the accuracy is much better than in the FRDM(1992), $0.4949 \mathrm{MeV}$ compared to $0.5817 \mathrm{MeV}$, that is a $15 \%$ improvement, which at this level of accuracy cannot be achieved with addition of some type of arbitrary new terms. Also, normally if more parameters are introduced to fit a model to known regions, and consequently bind the model more tightly to known data, then it is quite common

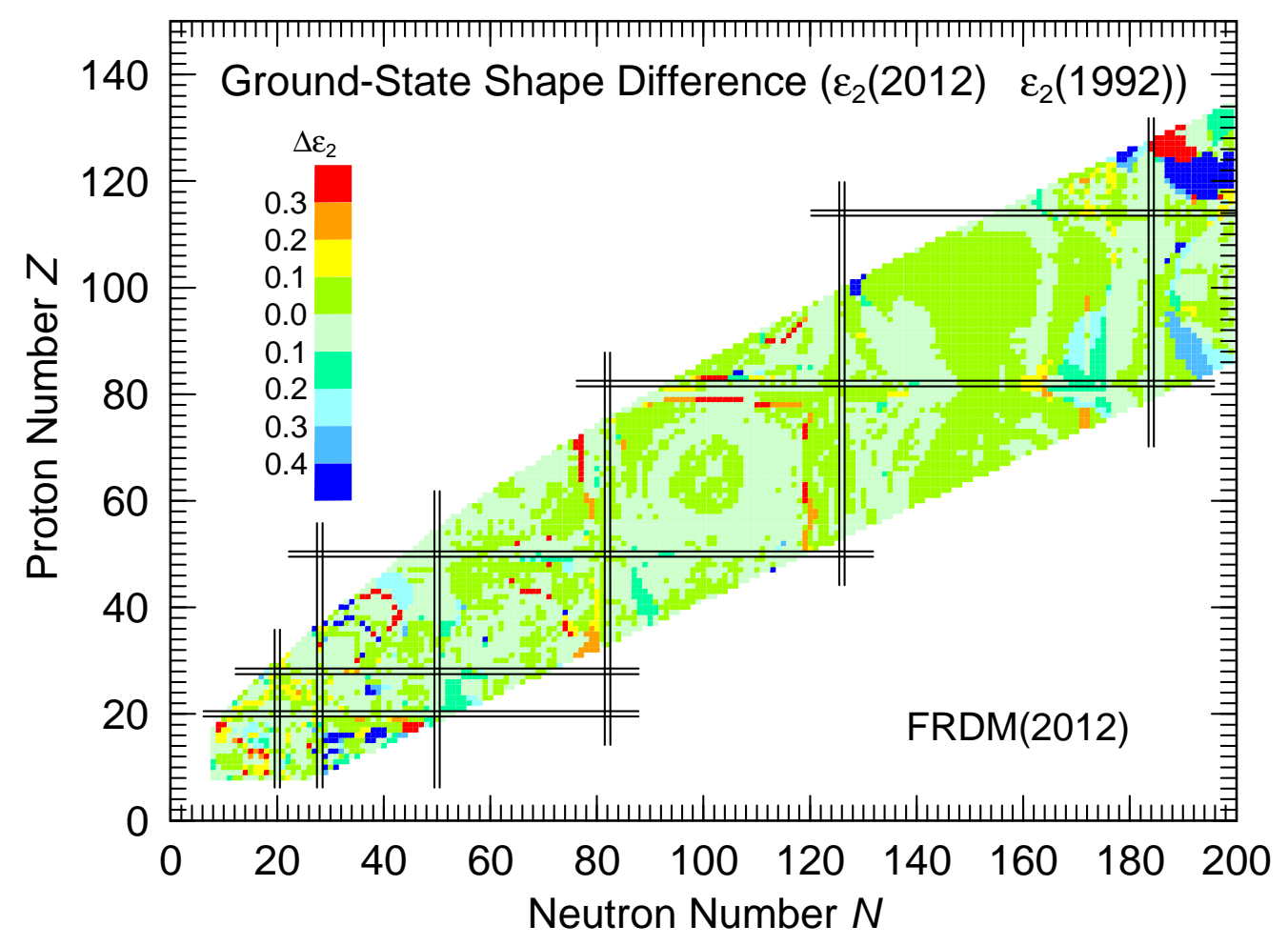

Fig. 20: Difference between the value of the ground-state shape parameter $\varepsilon_{2}$ obtained in the current model and the value obtained in FRDM(1992). The differences are largest in areas of shape coexistence, axial asymmetry, and going into magic numbers. 


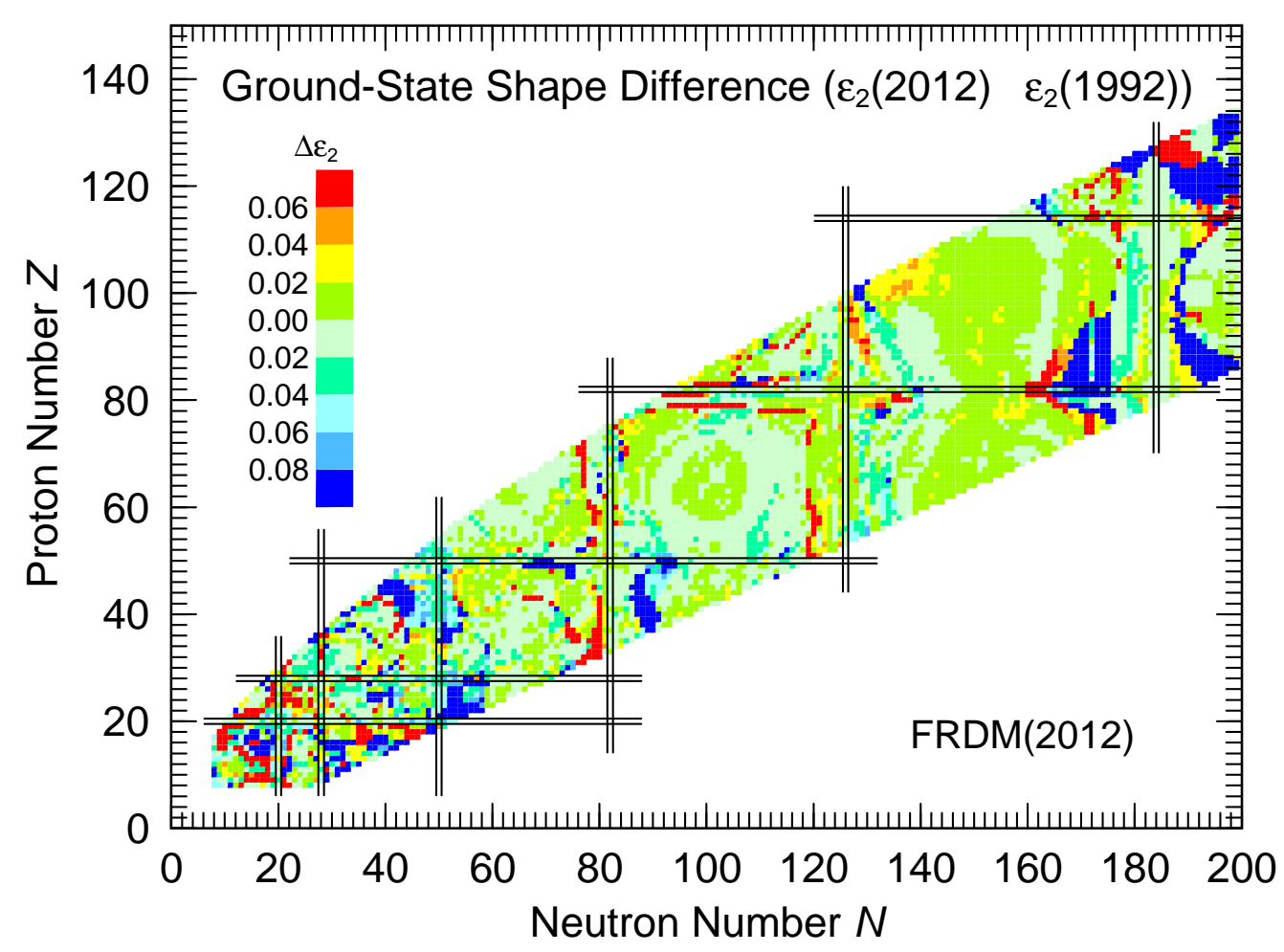

Fig. 21: A more detailed look at difference between the value of the ground-state shape parameter $\varepsilon_{2}$ obtained in the current model and the value obtained in FRDM(1992). In the well-deformed rare-earth and actinide regions there is little difference.

that this leads to an increase in the divergence of the model outside the region of adjustment. Since the opposite occurs here, and our test data set is substantial, we feel that this is a clear suggestion that the new constants varied represent the addition of realistic new features to the mass model.

In Fig. 15 we compare the current FRDM(2012) to the same data set as in the previous two figures. Most of these nuclei were included in the adjustment, namely 519 out of the total of 730 . However, the error only decreased by a small amount, from $0.4948 \mathrm{MeV}$ to $0.4788 \mathrm{MeV}$, a decrease by $0.0160 \mathrm{MeV}$, that is by $3.2 \%$.

The three nuclei with differences near $-3 \mathrm{MeV}$ are ${ }_{8}^{25} \mathrm{O}_{17},{ }_{19}^{51} \mathrm{~K}_{17}$, and ${ }_{20}^{52} \mathrm{Ca}_{32}$, with differences $-3.264,-2.996$,

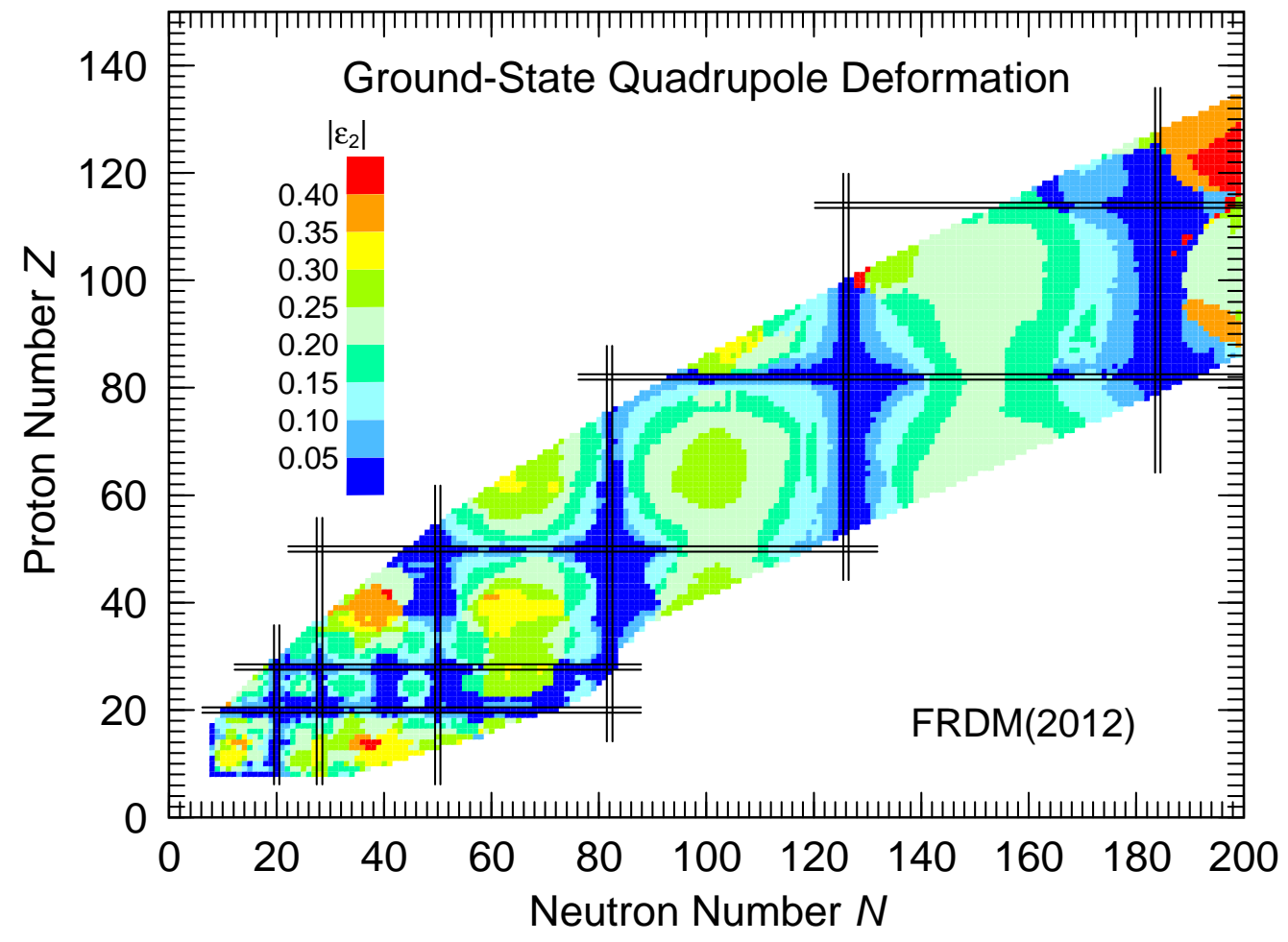

Fig. 22: Calculated values of $\left|\varepsilon_{2}\right|$ for nuclei with $N<200$ 


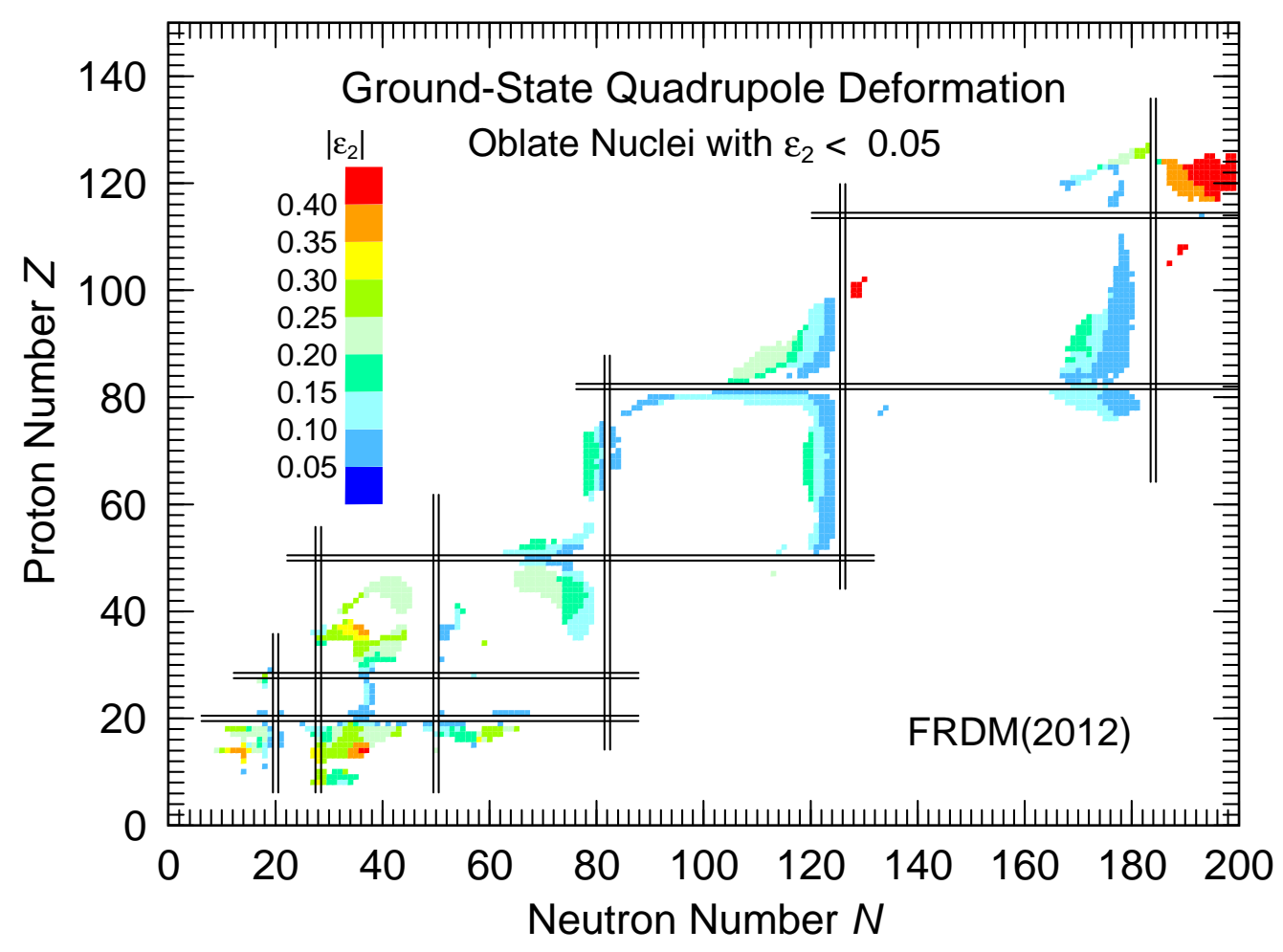

Fig. 23: Calculated values of $\left|\varepsilon_{2}\right|$ for nuclei with oblate ground-state shapes

and $-3.264 \mathrm{MeV}$, respectively. The two latter are in region of seemingly localized deviations that stands out in Fig. 5. The deviations occur in a type of region of localized deviations that occur in the chart below $N=65$, so the large deviations here near $Z=20$ and $N=30$ do not necessarily signal a collapse of the model in the neutron-rich region. For ${ }_{8}^{25} \mathrm{O}_{17}$ we observe that this is an extreme strain on our mean-field model with only 4 proton orbitals occupied and more than twice as many neutrons as protons. It is actually surprising to us that we do not obtain larger deviations considering that some (neutron-proton) asymmetry terms are only treated in first order. And, surprisingly perhaps, the deviation for the even more neutron-rich nuclide ${ }_{8}^{26} \mathrm{O}_{18}$ has decreased substantially to only $-1.454 \mathrm{MeV}$. The point with a deviation slightly below $-2 \mathrm{MeV}$ is ${ }_{21}^{54} \mathrm{Sc}_{33}$ situated in the same region of localized deviations.

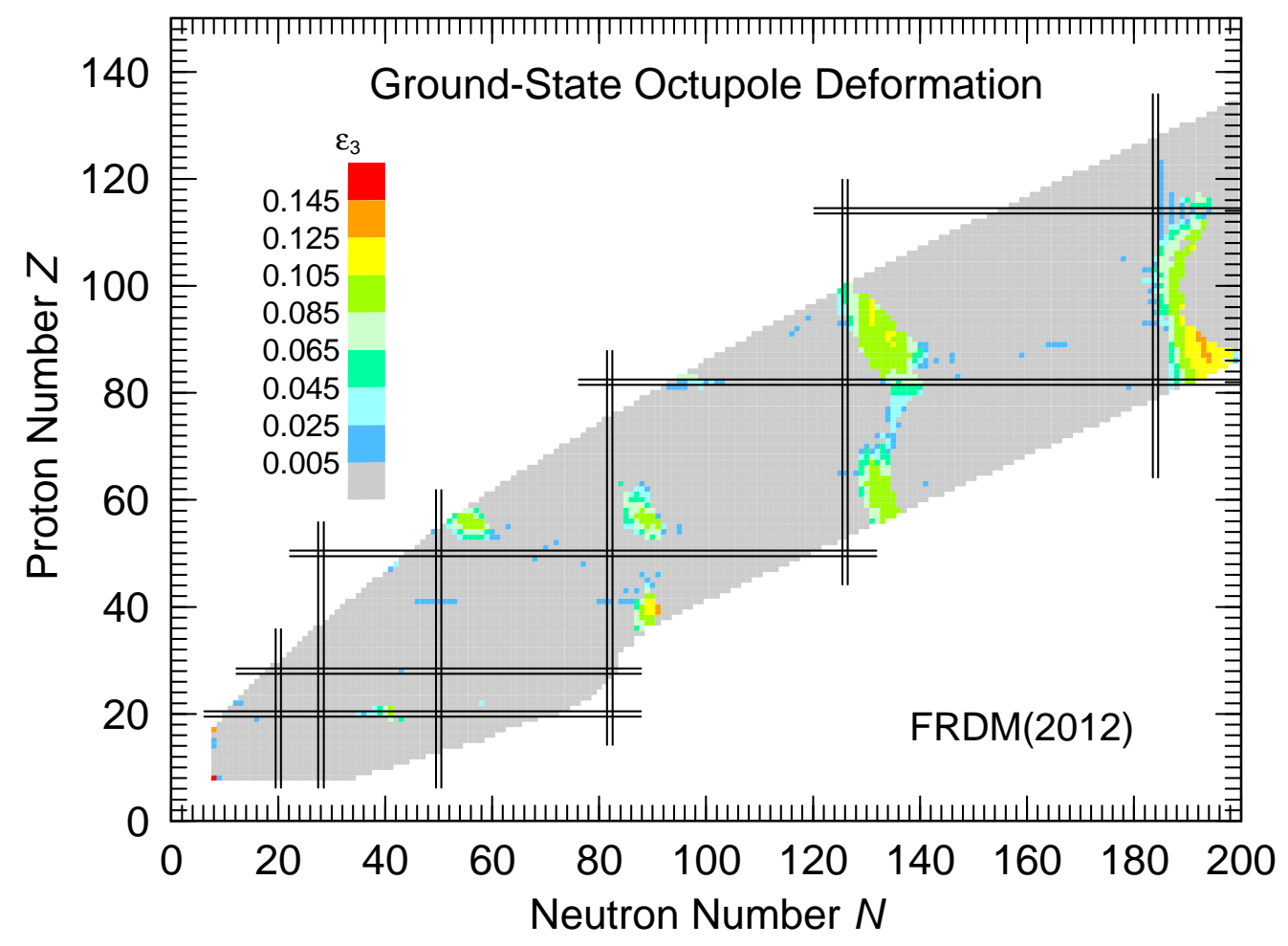

Fig. 24: Calculated values of $\varepsilon_{3}$. 


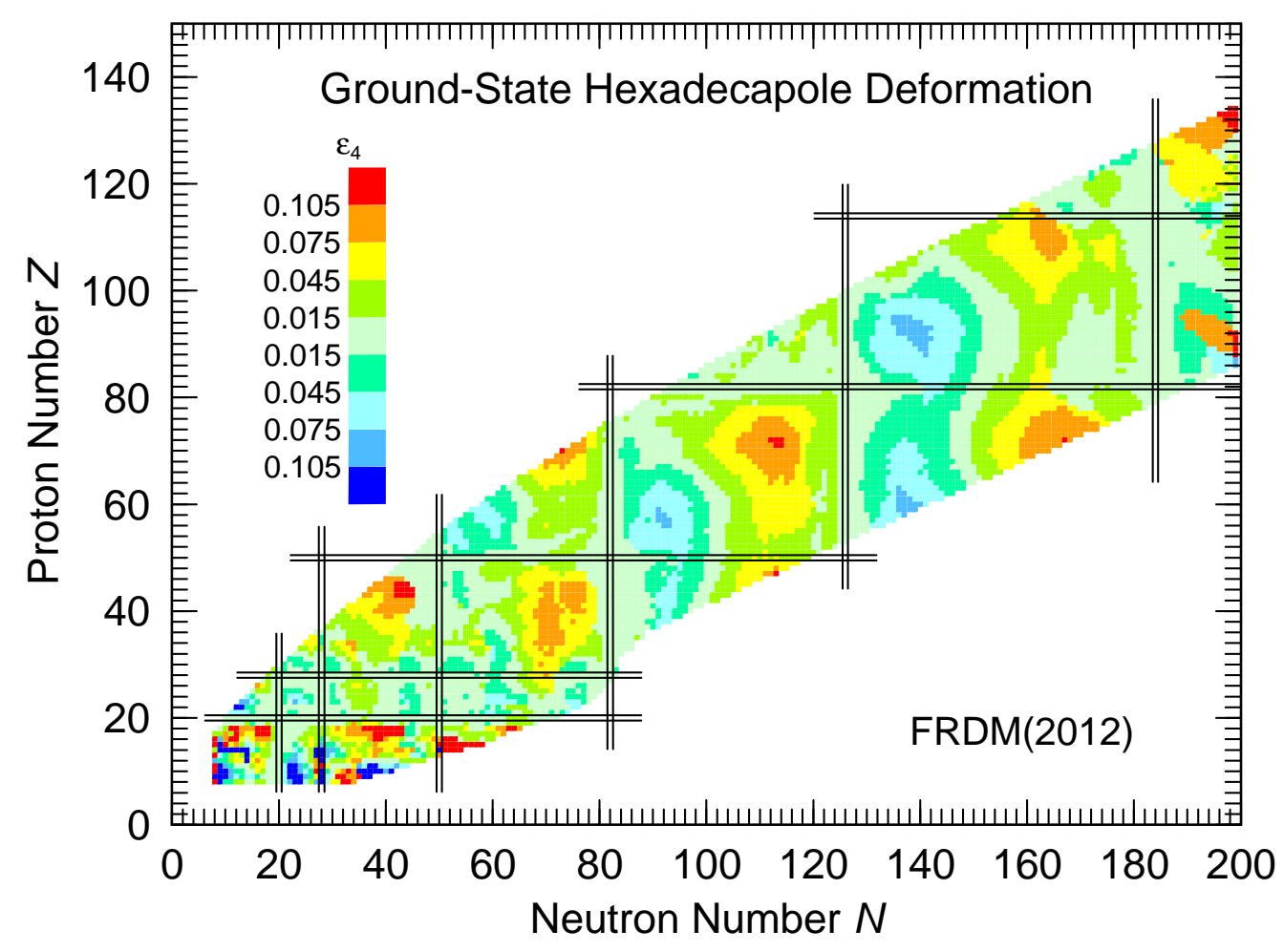

Fig. 25: Calculated values of $\varepsilon_{4}$.

We now do similar studies for the FRLDM as in the previous 3 figures for the FRDM. In Fig. 16 we show how the previous FRLDM(1992) predicts the 730 masses that were measured in the time frame 1989-2012. For the FRDM the error (see Fig. 13) is substantially less than in the region of adjustment. Not so for the FRLDM; the error is about the same as in the region of adjustment. The main reason for these deviations is the systematic increase in the deviations towards the proton drip line. We have discussed above and elsewhere (for example in Refs. [88, 91]) that this behavior has its roots in the lack of accounting for Coulomb redistribution effects. For proton-rich heavy nuclei there is a tendency for the charge to deviate from the assumption of a constant charge density and redistribute towards the surface, thus increasing the binding energy slightly. The sign of the deviations in Fig. 16 is consistent with this

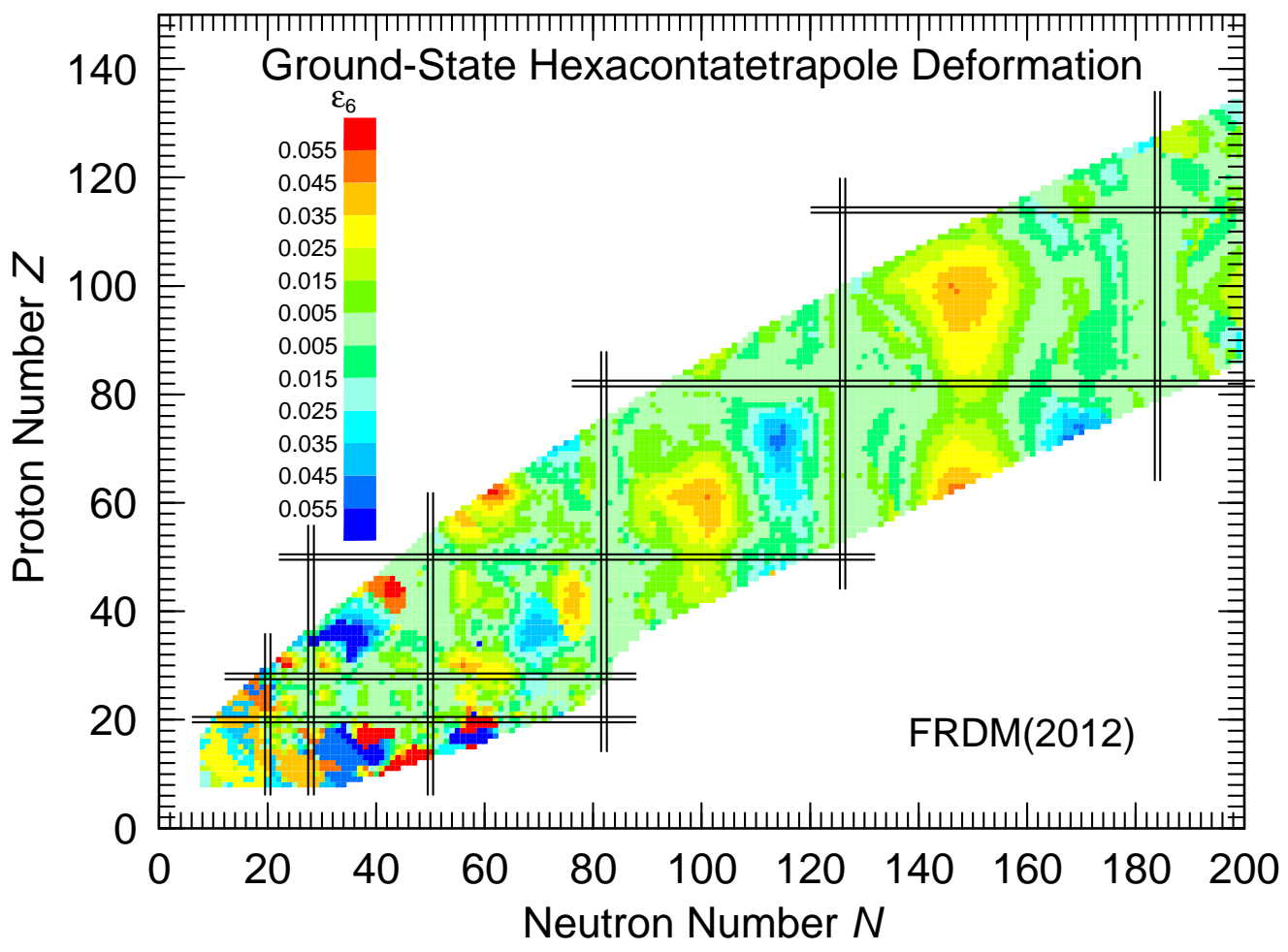

Fig. 26: Calculated values of $\varepsilon_{6}$. 


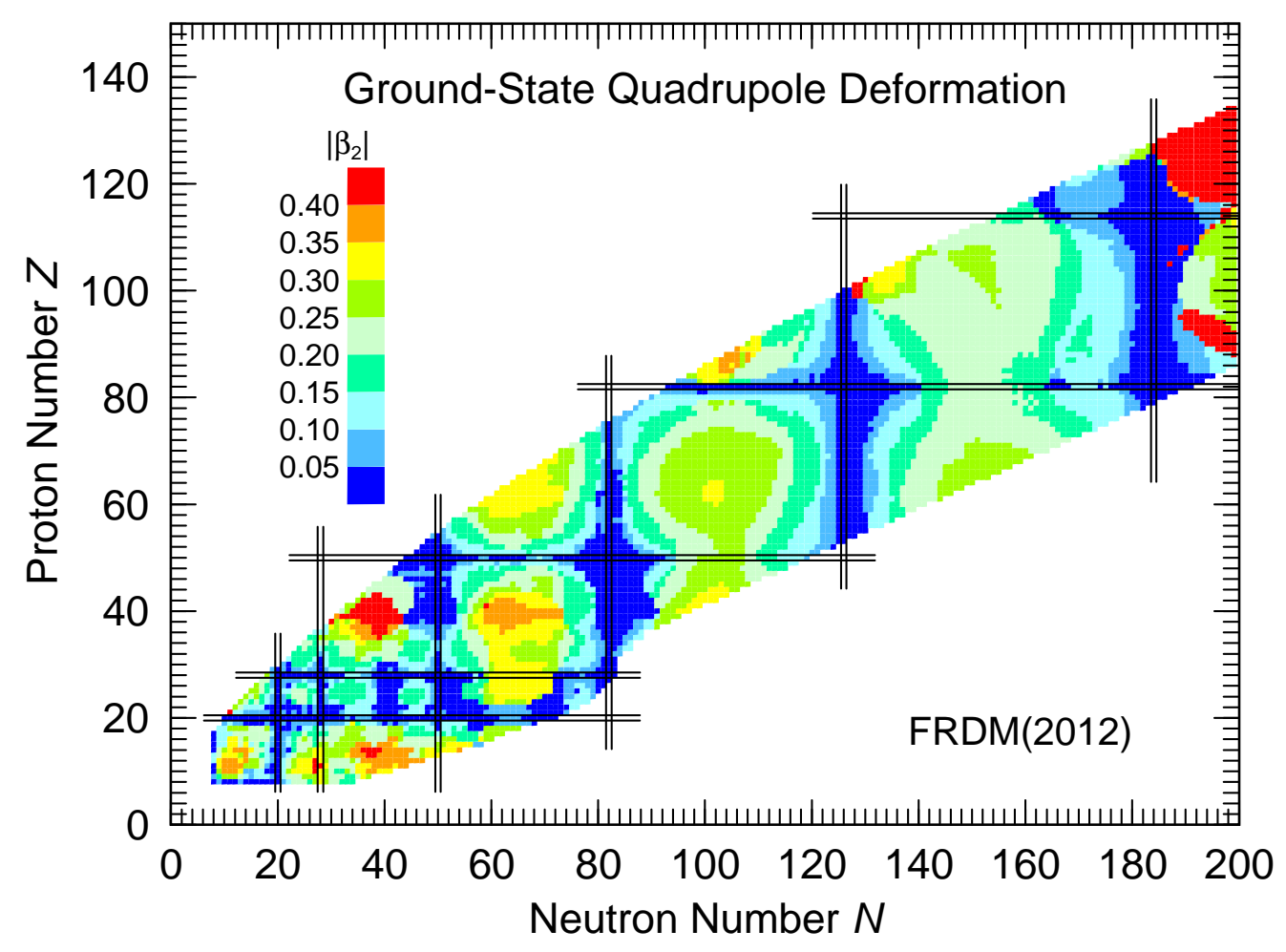

Fig. 27: Values of the shape parameter $\left|\beta_{2}\right|$ corresponding to calculated ground-state shapes, obtained by using the relation in Eq. (38).

interpretation. When the new version $\operatorname{FRLDM}(2012)$ is adjusted to the same region of experimental masses as the FRLDM(1992) the error decreases to $0.7008 \mathrm{MeV}$ (see Fig. 17), that is a decrease by $10.0 \%$ (compared to the $13.0 \%$ ) decrease for the FRDM). It extrapolates better to the 730 new nuclei; the error is now down from $0.7624 \mathrm{MeV}$ to $0.6600 \mathrm{MeV}$. This represents a $13.4 \%$ decrease in the error when we extrapolate to the new, "unknown" nuclei (versus $15.0 \%$ for the FRDM). In Fig. 18 we compare the precise FRLDM(2012) to these 730 nuclei; in this case 519 of them were taken into account in the adjustment of the model constants. The agreement is now better but the systematic deviations towards the proton drip line remain, although to a lesser degree. Now that most nuclei in this region were

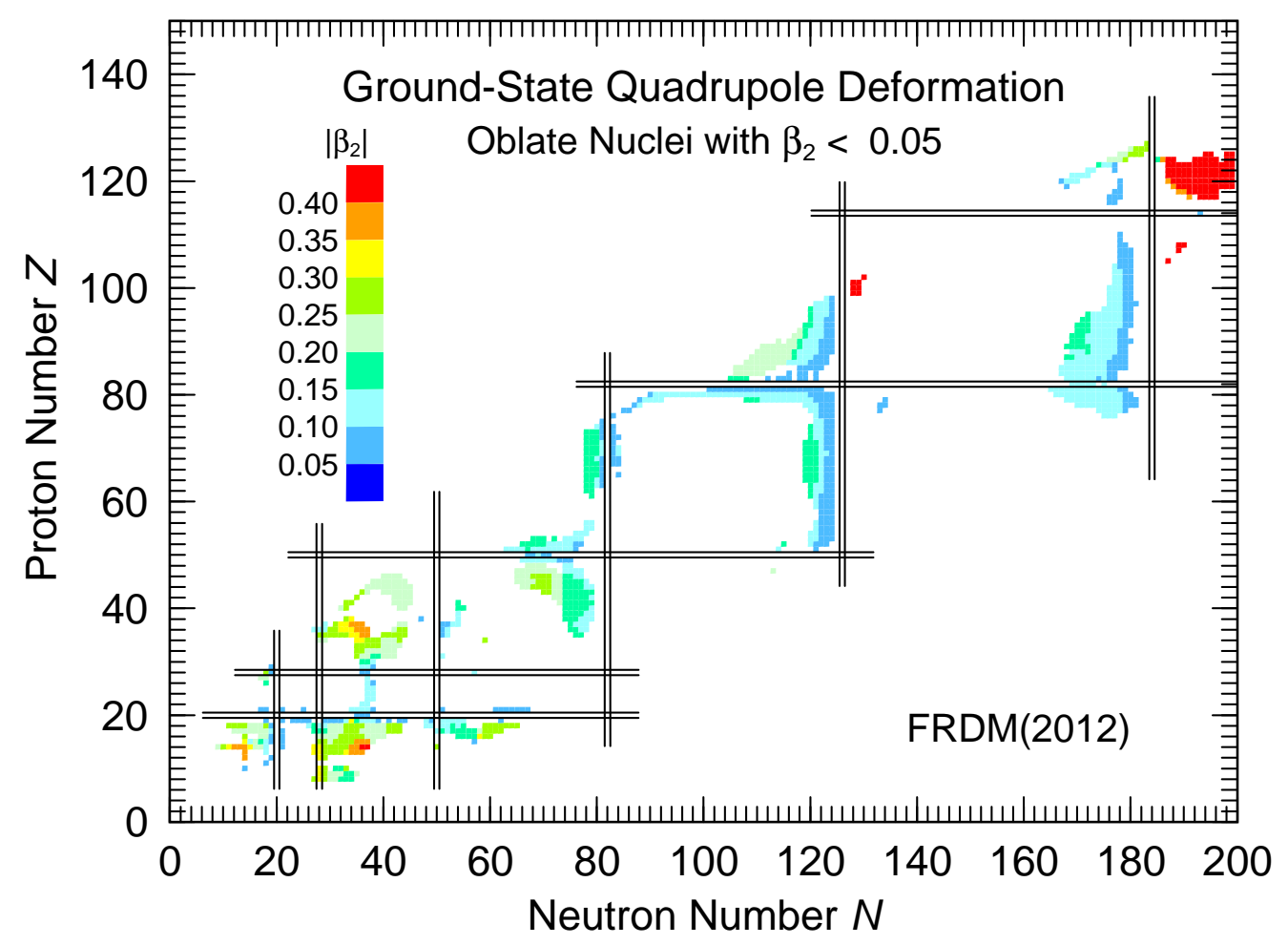

Fig. 28: Values of the shape parameter $\left|\beta_{2}\right|$ corresponding to calculated ground-state shapes, for nuclei with oblate ground-state shapes, obtained by using the relation in Eq. (38). 


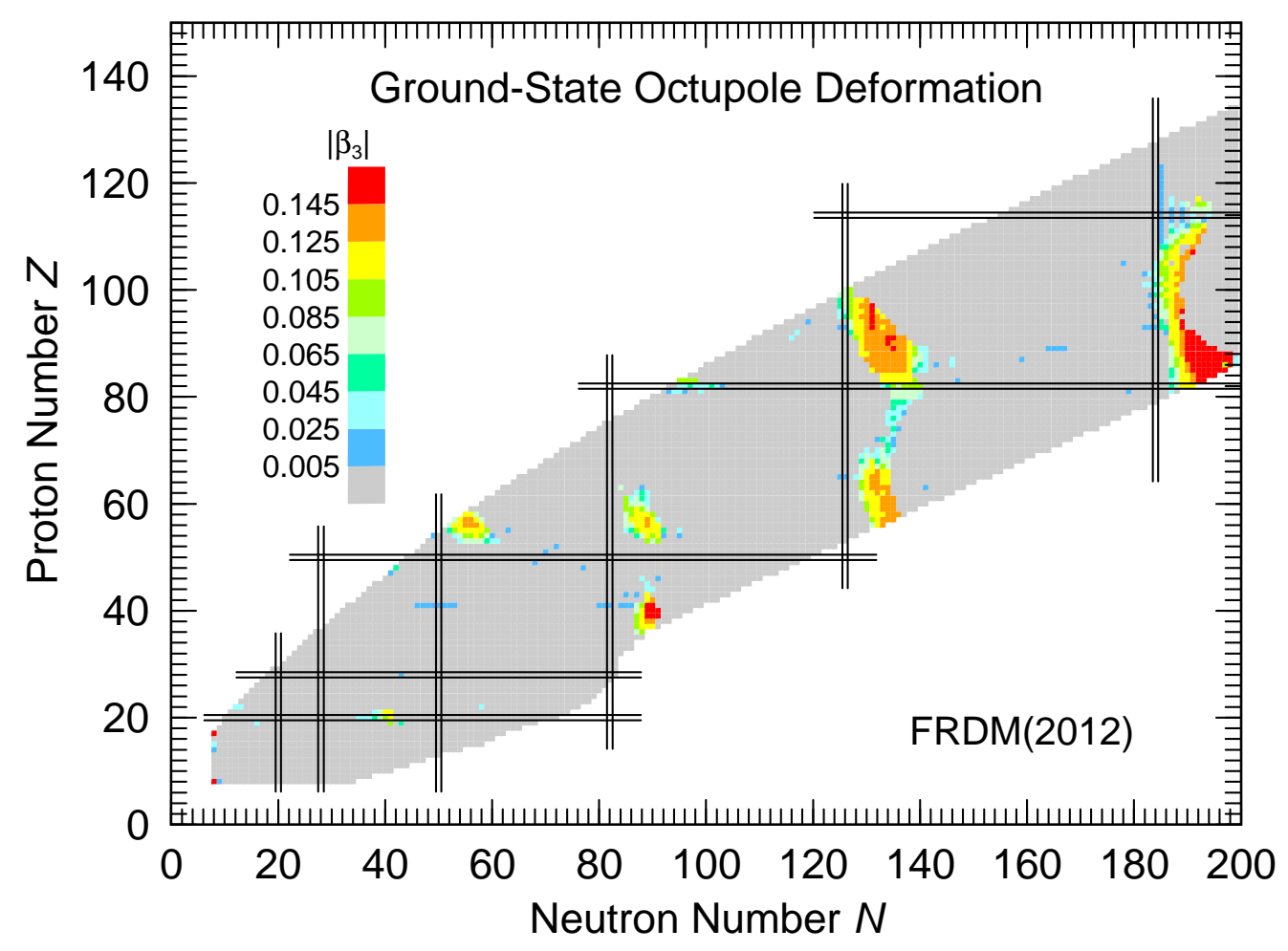

Fig. 29: Values of the shape parameter $\beta_{3}$ corresponding to calculated ground-state shapes, obtained by using the relation in Eq. (38).

included in the adjustment the error dropped from $0.6600 \mathrm{MeV}$ to $0.5944 \mathrm{MeV}$, a $9.9 \%$ drop. In the comparable study the FRDM only dropped much less, only by $3.2 \%$. This probably indicates the FRDM is considerably more reliable in applications to regions of nuclei that were not yet available when the model parameters were determined.

\subsection{Detailed comparisons of masses and deformations in the FRDM(1992) and FRDM(2012)}

In Fig. 19 we show the difference between the masses calculated in the FRDM(1992) and FRDM(2012). In most of the regions of known nuclei there is little difference, normally it is in the range $-0.5 \mathrm{MeV}$ to $0.5 \mathrm{MeV}$. A standard explanation of such results is: "of course, both models are adjusted to this data". But the parameters that are adjusted

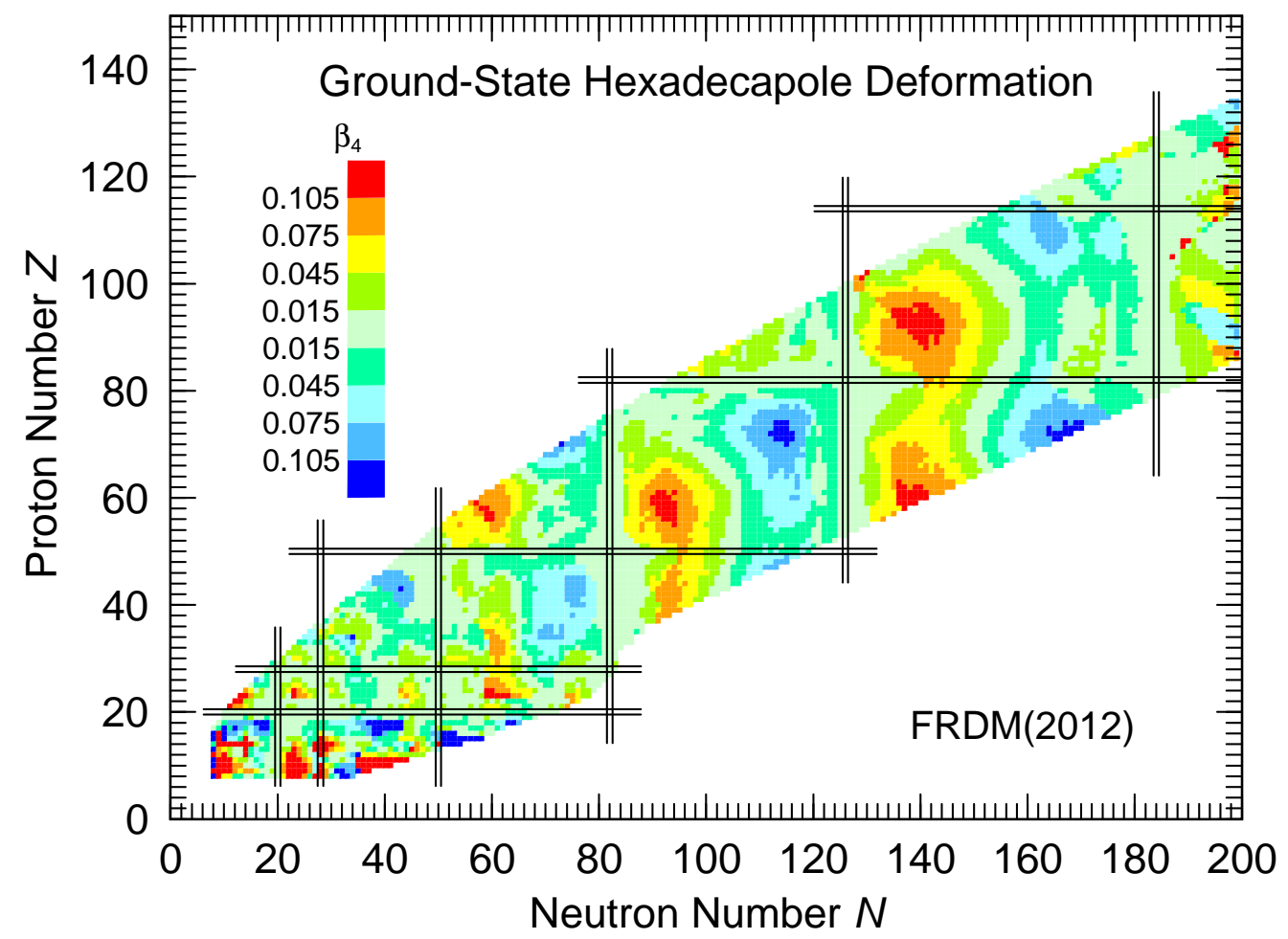

Fig. 30: Values of the shape parameter $\beta_{4}$ corresponding to calculated ground-state shapes, obtained by using the relation in Eq. (38). 


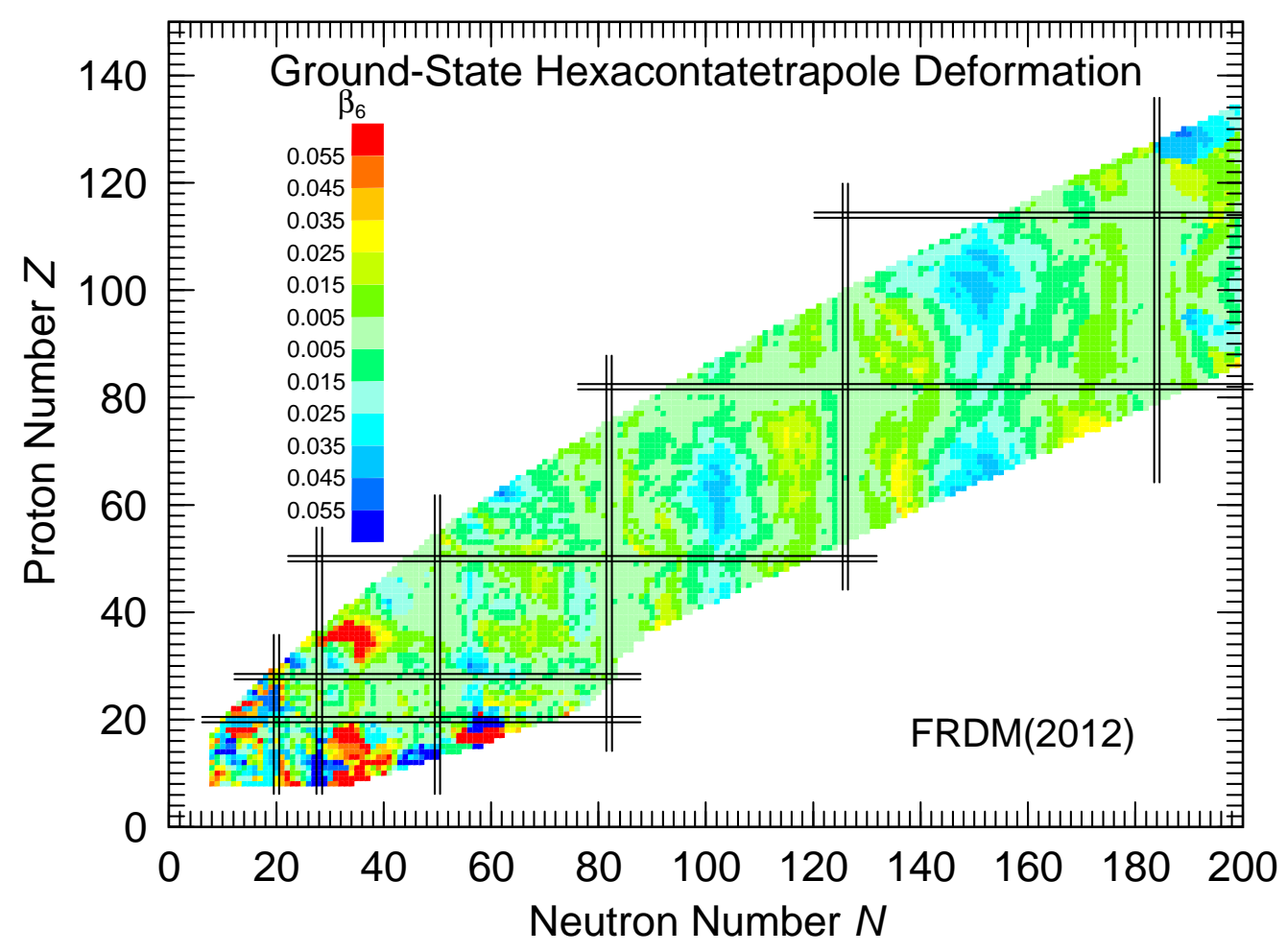

Fig. 31: Values of the shape parameter $\beta_{6}$ corresponding to calculated ground-state shapes, obtained by using the relation in Eq. (38).

cannot make the model adapt to the rapid fluctuations in the observed masses that are due to microscopic effects. They are substantial, in the range -12 to $+5 \mathrm{MeV}$ or so. Rather the reason for the limited differences is that the previous model was fairly well executed. But the improvements that have been implemented in the $\operatorname{FRDM}(2012)$ do sometimes lead to large changes in some localized regions of known nuclei. Furthermore, the changes lead to improved agreement with the calculated masses. For example, near $Z \approx 40, N \approx 40$ and $Z \approx 40, N \approx 65$ the changes occur because of aspects of our new calculations which impact shape-coexisting nuclei, as discussed above. The differences near $N=76$

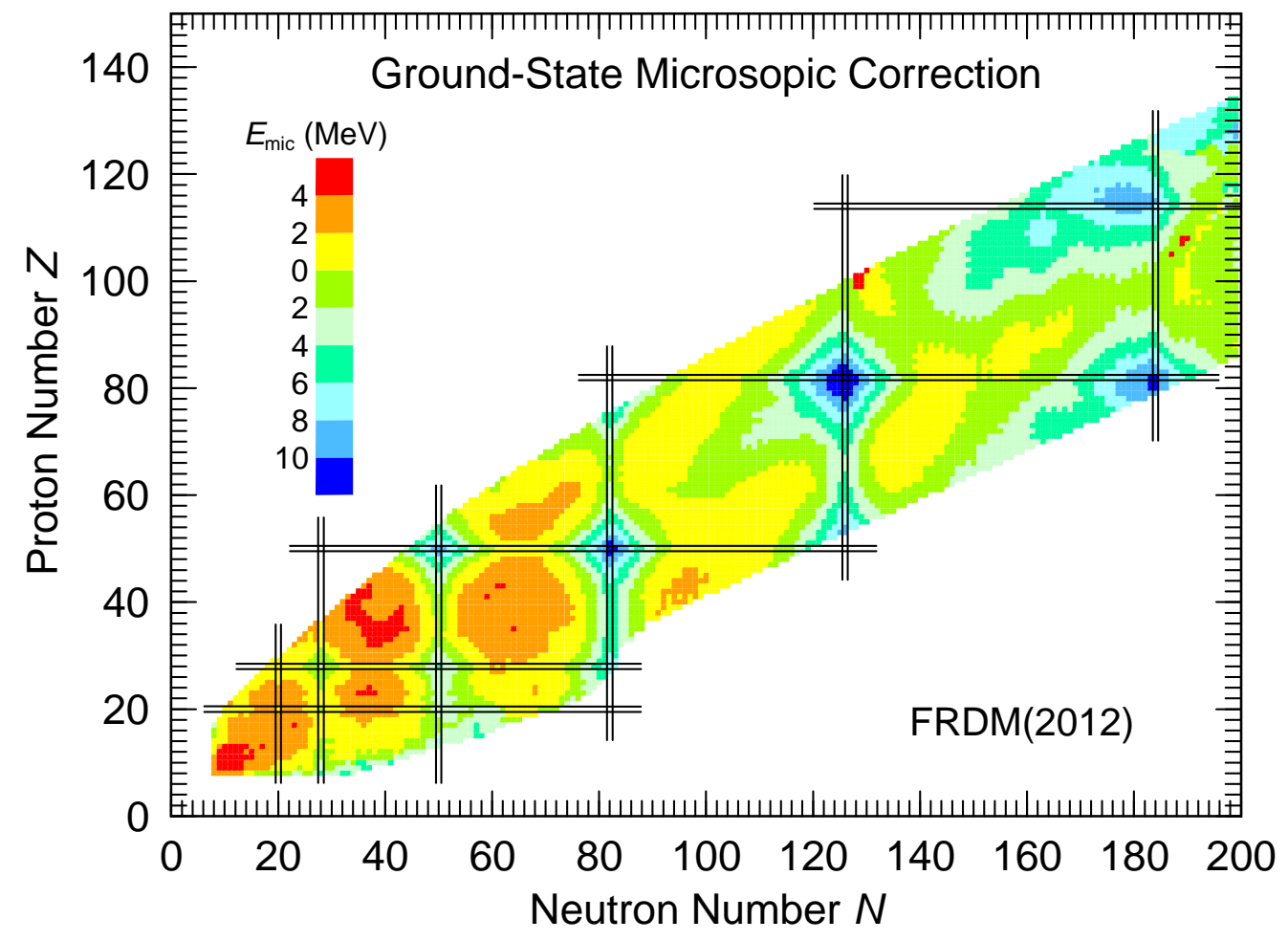

Fig. 32: Calculated ground-state microscopic corrections. The effect at magic numbers generally increases towards heavier nuclei. Large gaps in calculated level spectra at deformed ground-state shapes give enhanced stability, as is by now well-established experimentally, away from doubly magic nucleon numbers near ${ }_{108}^{270} \mathrm{Hs}_{162}$. This effect could already be seen 40 years ago in our early work [37]. More global studies appeared a little later in Refs. [2, 72]. 


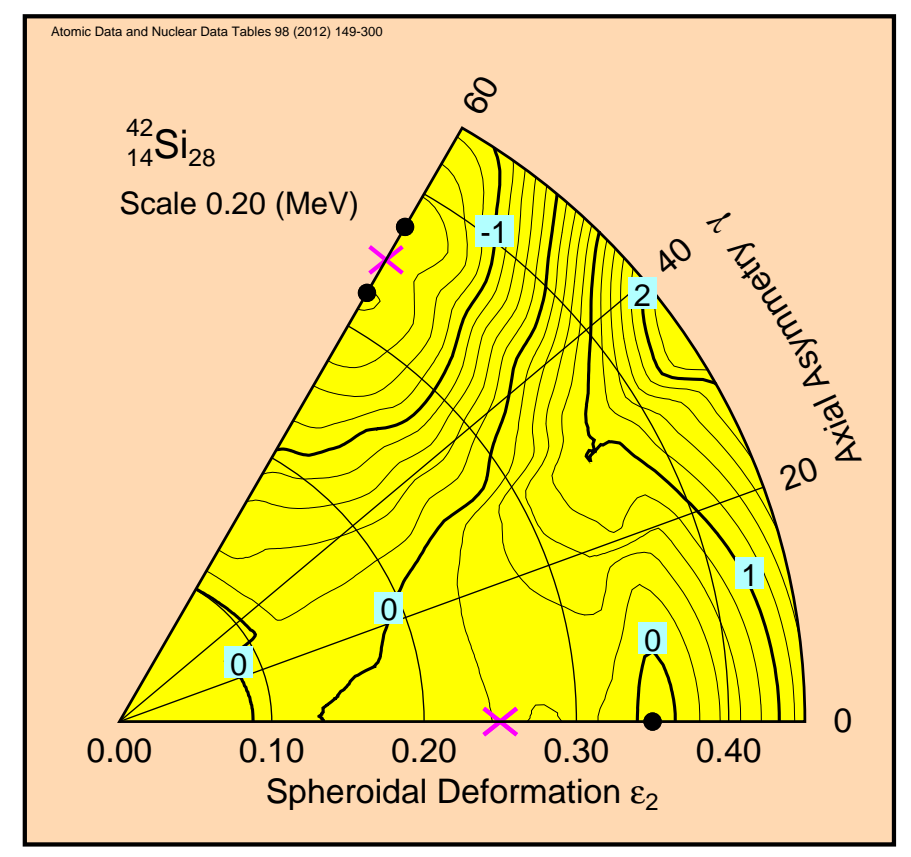

Fig. 33: Calculated potential-energy surface for ${ }_{14}^{42} \mathrm{Si}_{28}$. Although the neutron number is magic, $N=28$, the calculated ground-state shape is quite deformed (oblate). See further discussion in the text.

from about $Z=60$ to $Z=70$ is due to the consideration of axially asymmetric ground-state shapes, which also impacts some neutron-deficient nuclei just below $Z=82$, for example the ground state of ${ }^{192} \mathrm{Pt}$ is lowered by $0.30 \mathrm{MeV}$ by axial asymmetry [45].

The large differences in masses near the proton drip line in the heavy-element region are due to a more exact implementation of the rule to select as the ground state the minimum with the highest fission barrier that we now have, due to our calculation of potential-energy surfaces in the axial-asymmetry shape space. Often when we see the large differences, such as those near ${ }^{228} \mathrm{Fm}$, in the region $Z>114, N>184$, and near ${ }_{108}^{298} \mathrm{Hs}_{190}$ the fission barriers are very low, about one $\mathrm{MeV}$ only [61]. Therefore the seemingly pathological results with very sudden, discontinuous jumps in the model differences are not due to any mistake, they are an artifact of very low fission barriers and multiple minima in the calculated potential-energy surface. In the specific case of ${ }_{108}^{298} \mathrm{Hs}_{190}$ we find in the $\operatorname{FRDM}(2012)$ a ground-state deformation $\varepsilon_{2}=-0.64$ and mass excess $216.029 \mathrm{MeV}$, whereas in the FRDM(1992) the values tabulated are $\varepsilon_{2}=0.0$ and mass excess $212.97 \mathrm{MeV}$. As explained above, we did also consider fission stability in the previous calculations although it was unfortunately not stated in the paper. But, because we could not calculate the potential-energy surface in the axial-asymmetry plane we always assumed that minima on the oblate axis that were higher in energy than prolate minima would always be unstable in the $\gamma$ plane.

In the case of astrophysical applications, for example to the r-process, one may need to investigate if some other rule should be used in selecting the "ground state". For example in calculations of neutron-capture rates it would perhaps be more correct to select minima in the two nuclei involved in the capture process that have similar deformations. The same holds true in $\beta$-decay processes.

The systematic increase in the mass differences towards the neutron drip line in the heavy region is very gradual and may be untestable. For example for our most neutron-rich $\mathrm{Pb}$ nuclide we found in FRDM(1992) a mass excess 360.04 MeV, in the FRDM(2012) we obtain the mass excess 353.629 MeV. However, in the FRDM(1992) we have $S_{1 \mathrm{n}}=-1.58 \mathrm{MeV}$ and $Q_{\beta}=19.12 \mathrm{MeV}$. In the $\operatorname{FRDM}(2012)$ we find $S_{1 \mathrm{n}}=-1.41 \mathrm{MeV}$ and $Q_{\beta}=18.88 \mathrm{MeV}$. Thus, the differences are smaller than the accuracy stated in Ref. [24] for $S_{1 \mathrm{n}}(0.526 \mathrm{MeV})$ and $Q_{\beta^{-}}(0.647 \mathrm{MeV})$, so the effect on r-process calculations may be much less severe than the fairly large differences in the mass excesses seem to indicate.

Also of interest are the differences in ground-state deformations. We show in Fig. 20 the difference between the quadrupole deformation $\varepsilon_{2}$ obtained in the $\operatorname{FRDM}(2012)$ and $\operatorname{FRDM}(1992)$. The same quantity is plotted in a more detailed scale in Fig. 21. Above $N=160$ there are a substantial number of differences. They occur because there are multiple minima in the calculated potential-energy surfaces. Because we now study the energy with inclusion of axial asymmetry we can more correctly determine which of these minima has the highest barrier with respect to fission. So in many cases we now select a different minimum than was chosen in FRDM(1992) as the most stable minimum. For lighter nuclides most differences occur in the regions of shape coexistence and axial asymmetry. In the transition regions between deformed and spherical nuclei the calculated potential-energy surfaces are very flat and small effects can change the locations of the very shallow minima on such surfaces, therefore we also have differences in those regions. Also in the more detailed scale in Fig. 21 we see little difference between the two calculations in the traditional deformed rare-earth and actinide regions. 

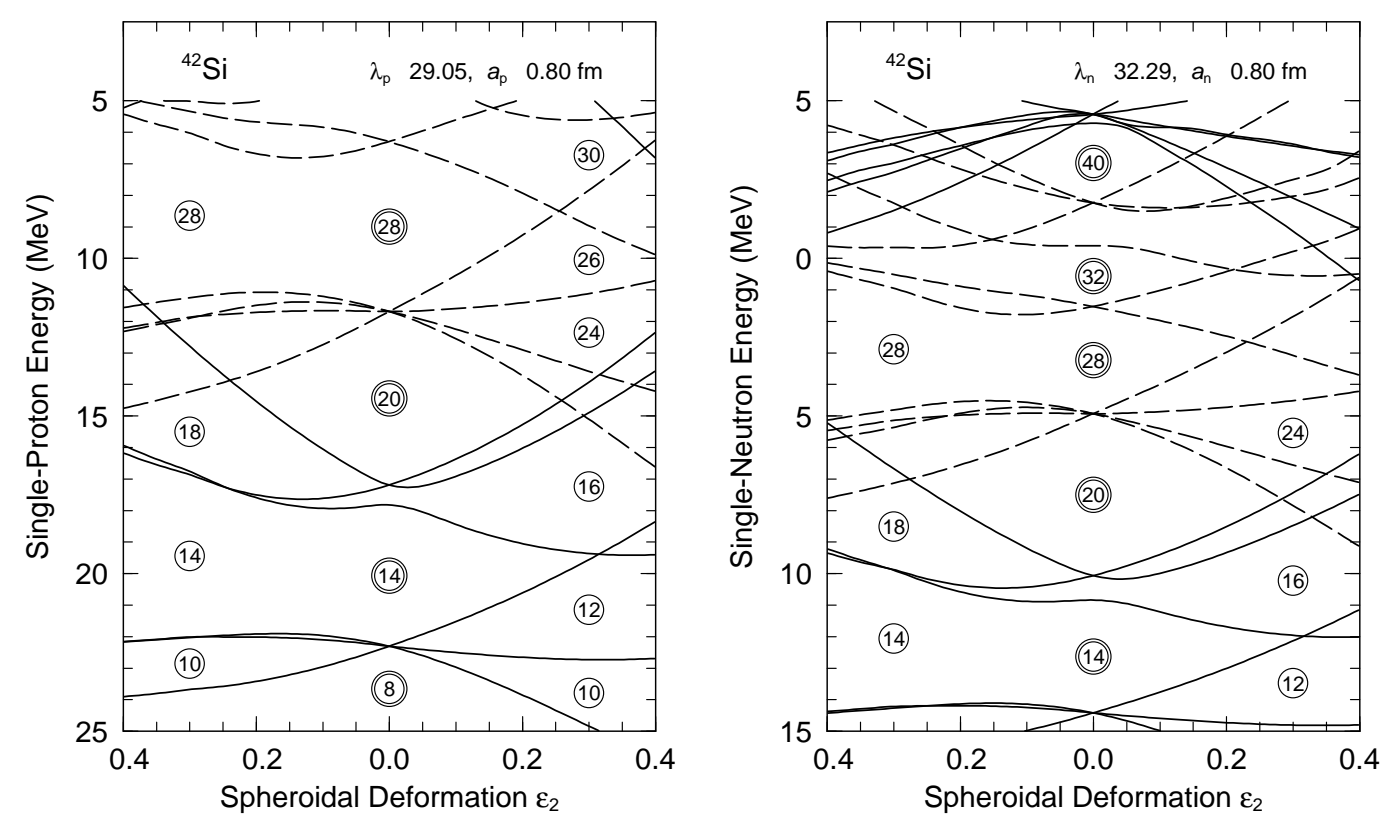

Fig. 34: Calculated proton and neutron single-particle levels for ${ }_{14}^{42} \mathrm{Si}_{28}$.

\subsection{Calculated ground-state masses and deformations}

We tabulate the new $\operatorname{FRDM}(2012)$ and $\operatorname{FRLDM}(2012)$ "mass tables" in the Table. As before we also tabulate the calculated shape parameters, both the calculated $\varepsilon$ shape parameters and their corresponding $\beta$ parameters, as well as the microscopic corrections. As explained above the microscopic corrections are different from the shell-pluspairing corrections and do depend on the macroscopic model used, therefore there are two "microscopic-correction" numbers for each nuclide, one corresponding to the FRDM(2012) and one to the FRLDM(2012). We also include the experimental masses that we used in our adjustment of the model parameters and the quoted errors, from Ref. [17]. We have added one new data set relative to what was tabulated for the $\operatorname{FRDM}(1992)$ in Ref. [9], namely the ground-state shell-plus-pairing corrections. These depend only on the single-particle level spectrum and are thus identical in the FRDM and FRLDM, so there is only one column of these data. Finally, we give ground-state masses in the FRDM expressed as total binding energies. It is a new column, but not a new data set because the total binding energy can be obtained from the mass excess through the relation

$$
E_{\text {bind }}=Z M_{\mathrm{H}}+N M_{\mathrm{n}}-M(Z, N),
$$

where $M_{\mathrm{H}}$ is the hydrogen-atom mass excess and $M_{\mathrm{n}}$ is the neutron mass excess. This total binding energy is physically the sum of the masses of $Z$ hydrogen atoms and $N$ neutrons at infinity minus the mass of the "assembled" atom. In the present context, total binding energy is used to mean the nuclear binding energy plus the difference between the binding energy of the $Z$ electrons comprising the atom, which we approximate by $a_{\mathrm{el}} Z^{2.39}$, with $a_{\mathrm{el}}=1.433 \times 10^{-5} \mathrm{MeV}$, and the binding energy of $Z$ hydrogen atoms. For consistency, the values $M_{\mathrm{H}}=7.289034 \mathrm{MeV}$ and $M_{\mathrm{n}}=8.071431 \mathrm{MeV}$ should be used here for these quantities [9], although more recent evaluations give slightly different results. An alternative possibility would have been to define total binding energy as the difference between the sum of the masses of all constituent particles (consisting of $Z$ protons, $Z$ electrons, and $N$ neutrons) at infinity minus the mass of the atom. This alternative definition of total binding energy differs from the one that has been used historically and that we have adopted here by the binding energy of $Z$ hydrogen atoms, which is numerically equal to $Z$ times $13.6056981 \mathrm{eV}$.

We show in graphical nuclear-chart style in Fig. 22 the calculated values of $\left|\varepsilon_{2}\right|$, in Fig. 23 the calculated values of $\left|\varepsilon_{2}\right|$ for those nuclei with $\varepsilon_{2}<-0.05$, in Fig. 24 the calculated values of $\varepsilon_{3}$, in Fig. 25 the calculated values of $\varepsilon_{4}$, and in Fig. 26 the calculated values of $\varepsilon_{6}$. The corresponding $\beta$ shape parameters are plotted in Figs. 27, 28, 29, 30, and 31. The results for the spheroidal deformation $\varepsilon_{2}$ in Fig. 22 show the by now well known regular behavior. The deformation increases by about 0.05 with each deformed region as we go toward lighter nuclei. Oblate deformations occur mainly in transition regions from deformed nuclei to magic numbers, on the heavy side of the deformed regions. The microscopic reason for the large prevalence of prolate nuclei has been discussed in Ref. [92]. Lighter nuclei show a more irregular behavior, possibly because a single nucleon orbital here has a (much) larger fractional effect on the system.

Nuclei that are calculated to be octupole deformed in the ground state are relatively rare as is shown in Fig. 24 . The most well-known region is around ${ }^{222} \mathrm{Ra}$. In our model, consideration of octupole shapes leads to significantly improved ground-state masses. The octupole effects were noticed in our first global nuclear mass calculation the FRLDM(1981) [1] and were studied in greater detail subsequently, see for example [27, 28, 29, 9, 45]. Axial asymmetry effects also affect a few nuclides, an in-depth discussion of these results is in Refs. [44, 45].

The hexadecapole deformation $\varepsilon_{4}$ behaves in a very regular fashion throughout most of the chart, except the very lightest region, see Fig. 25. It is minimum, near -0.10 , in the beginning of deformed regions and +0.10 at the end 


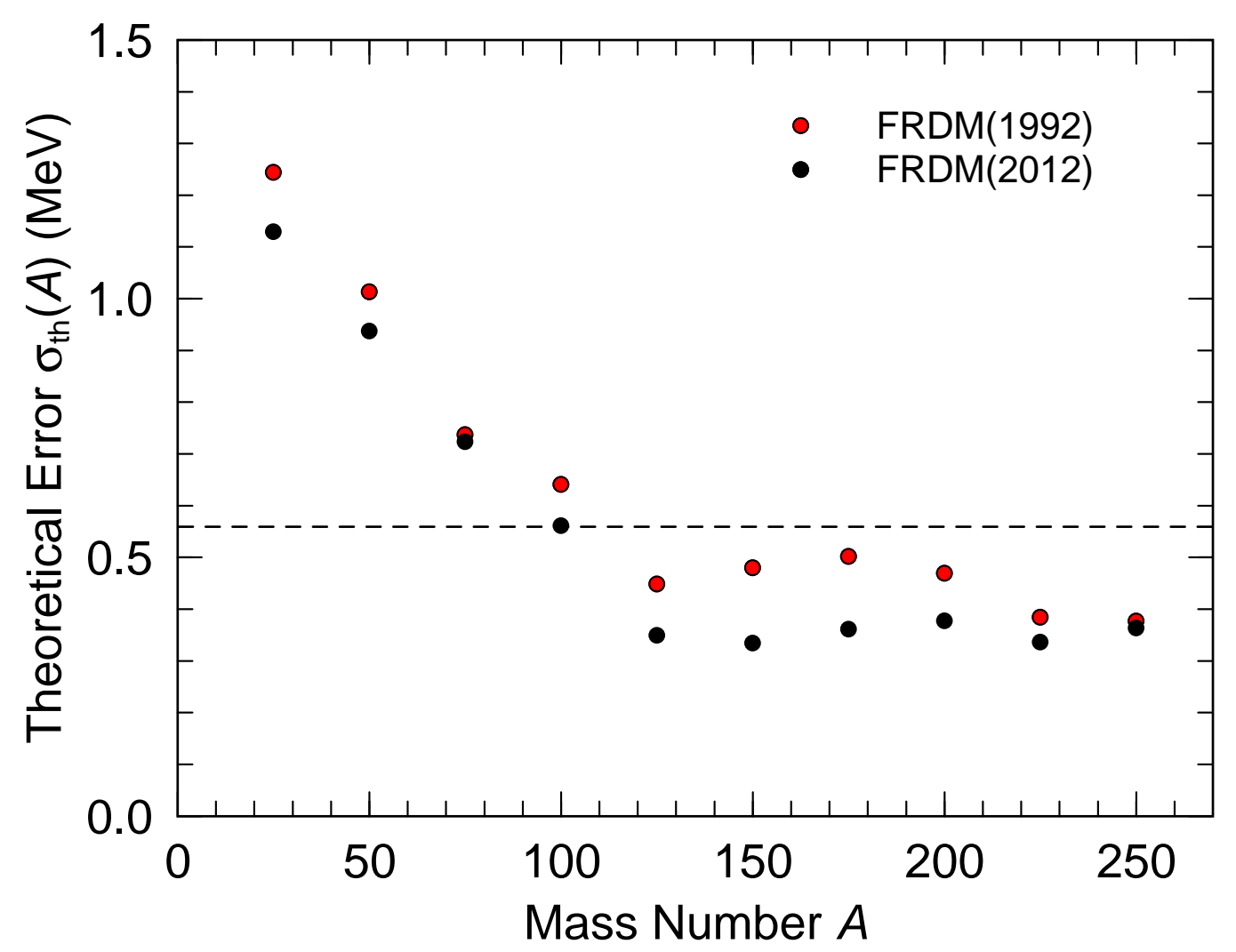

Fig. 35: Error in the mass FRDM(1992) and FRDM(2012) calculations as functions of $A$. The error is consistently smaller in the FRDM(2012) calculation than in the FRDM(1992) calculation for all regions of $A$.

of the deformed regions. Since the first experimental studies became available calculations have reproduced well this systematic behavior $[93,77,94]$.

\subsubsection{Do magic numbers really disappear for some exotic nuclei?}

Both in the light and heavy region there are nuclei with either the proton or neutron number "magic" but which are anyway calculated to be deformed. We give one example of this type of result, for ${ }_{14}^{42} \mathrm{Si}_{28}$ in Fig. 33. We find for this nucleus that the calculated ground-state deformation is $\varepsilon_{2}=-0.31, \varepsilon_{3}=0.00, \varepsilon_{4}=-0.12$, and $\varepsilon_{6}=-0.05$. When it is experimentally found that a nuclide with either a magic neutron number or a magic proton number is found to be deformed this is sometimes presented as a mystical new phenomenon for "exotic" nuclei, and taken as evidence that a long accepted magic number has "disappeared". But this is not necessarily the case. First let us recall what conventional magic numbers are. They are specific numbers that correspond to large gaps in calculated spherical level spectra for neutrons and protons. Large gaps are associated with increased stability at these specific numbers, which is also observed experimentally. We show in Fig. 34 calculated proton and neutron single-particle levels versus deformation for ${ }_{14}^{42} \mathrm{Si}_{28}$. This figure shows that the conventional magic numbers 8,20 , and 28 still exist for spherical shape. However, in our example the gaps at $Z=14$ and $N=28$ are somewhat larger near the oblate shape $\varepsilon_{2}=-0.3$ than at spherical shape so this leads to an oblate shape being the most stable configuration for this nuclide, although the normal spherical level gap at neutron number $N=28$ has not "disappeared".

\subsubsection{Dependence of model accuracy with nucleon number $A$}

Figure 3 shows that the error increases with decreasing nucleon number $A$ in a somewhat systematic fashion. To show this more clearly we have determined the model error for limited regions of nuclei by use of Eq. (9). We select $A=25(25) 250$ as centerpoints of the regions and define each region to extend from $A_{\text {center }}-24$ to $A_{\text {center }}+25$. the errors in these restricted regions are shown as black dots in Fig. 35. The analogous deviations in FRDM(1992) are plotted as black circles with a red interior. The FRDM(2012) errors are always smaller than the FRDM(1992) errors, vary almost completely linearly from $A=25$ to $A=125$ and are almost constant from $A=125$ to $A=250$. There are 1628 known masses in this region (from $A=101$ to $A=270$ ); for these nuclei together we find $\sigma_{\text {th }}=0.362 \mathrm{MeV}$. For the limited regions in this range we find that the smallest error is $\sigma_{\mathrm{th}}=0.335 \mathrm{MeV}$ for $A_{\text {center }}=150$ and the largest error is $\sigma_{\mathrm{th}}=0.378 \mathrm{MeV}$ for $A_{\text {center }}=200$.

\section{SOME ADDITIONAL STUDIES AND DISCUSSION}

It is natural to ask how sensitive our results, (for example extrapability and parameter values) are to various model assumptions we have made (such as setting the compressibility $K$ to $240 \mathrm{MeV}$ and to the data sets used) in the 
determination of model parameters. We performed some studies on how well the model performs in new regions of masses that were not used in the adjustment of model parameters in Sect. 5.1. We present the results of the studies of how well the model applies to new regions of nuclei and some sensitivity studies in Table D. First we review the steps that led to FRDM(2012); some of the first steps were discussed in Refs. [83] and [87]. So that we can refer to specific locations in the table, we give a line number in column 1. In column 2 a model designation is given; most of these "models" were just stages on the path to the current FRDM(2012). Differences between "models" can be of different types, namely new or different physics, different subset of constants varied, or the region of masses used in the adjustment of model parameters. Once a "model" has been developed we can, without changing the model, compare the mass table generated to different mass regions. In such comparisons no values of the constants are given in the table, they are in this case the same as the constant values given immediately above. In column 3 the first number ("A") refers to the data set to which the model was adjusted. The second number refers to the data set to which the model was compared to, that is what data set was used to calculate the model mean deviation $\mu_{\text {th }}$ and model error $\sigma_{\mathrm{th} ; \mu=0}$. There are three mass evaluations and various sets of "new" masses that we use in our adjustments and tests. The numbers and corresponding evaluated mass data bases are

1. This data set is the AME1989 mass evaluation [18]. The FRDM(1992) was adjusted to this data set. 1654 nuclei

2. This data set is the AME2003 mass evaluation [18]. The FRDM(2012) is adjusted to this data set.

2149 nuclei

3. This data set are masses that are in the interim AME2011 evaluation [95] but are not in the AME2003 evaluation. In some previous investigations we used this data set to represent "new" masses that were not used in the determination of model parameters (since the most recent AME2012 [81] evaluation was not available at that time).

4. This data set is the AME2012 mass in the evaluation [81].

154 nuclei

5. This data set is the masses that are in the AME2012 evaluation that are not in the AME2003 evaluation.

219 nuclei

6. This data set is the masses in the AME2012 evaluation that are not in the AME1989 evaluation.

730 nuclei

The number of nuclei we indicate are only those with $Z \geq 8$ and $N \geq 8$; we do not consider lighter nuclei in our calculations. When we perform an adjustment to find optimum constants we always show the error for the same region of nuclei used in the adjustment. Therefore, when a line contains a set of constant values the " $A$ " and " $C$ " regions are always the same.

In line 1 of the table we show the previous FRDM(1992) mass model. Its agreement with the 730 new masses in AME2012 is shown on the second line. We later found that we could optimize the parameters better, and this solution yields $\sigma_{\mathrm{th}}=0.6614 \mathrm{MeV}$ [83]. We also removed consideration of fission barriers and then arrive at model (92)-b. The mass $\sigma_{\text {th }}$ only decreases by a small amount, to $0.6591 \mathrm{MeV}$. This represents step 1 in Fig. 1 . On line 4 we show how this better optimized model agrees with masses that are new in AME2012. It is remarkable that when we more tightly bind the original model to the AME1989 it reproduces new masses better, in particular the mean ("systematic") error $\mu_{\mathrm{th}}$ is now much closer to zero. When a model with adjustable parameters is more tightly bound to known data one often finds that its performance outside this region has become poorer, but this is not the case here. On line 5 we compare the FRDM(1992) now in its incarnation (92)-b (with its better optimized parameters and no barriers included in the fit) to (the entire) AME2003, and find the error is now about $0.04 \mathrm{MeV}$ smaller. So the model agrees better with this new data set. This represents step 2 in Fig. 1. If we adjust the model to AME2003, rather than to AME1989 as was done for the model version (92)-b, the error decreases by only a small amount $0.0017 \mathrm{MeV}$ and the constants change by only a little (line 6), see also Ref. [83]. In model (07)-b in line 7 we have implemented the results of a full 4D search for the ground-state in a densely spaced grid, step 3 in Fig. 1 and gain $0.02 \mathrm{MeV}$ in accuracy, first reported at OMEG-7 in Sapporo [96]. Line 8, model (11)-b shows the improvement in accuracy, 0.01 MeV, of taking into account the effect of axial asymmetry on the ground state [44, 83, 45, 87]. corresponding to step 4 in Fig. 1 leading to an accuracy gain of $0.01 \mathrm{MeV}$. The next line shows how well the model at this stage "predicts" the masses that are new in AME2011 relative to AME2003. At this time, in 2011, we realized that the density-symmetry constant $L$ which had been kept at zero in FRDM(1992) (because of a very flat surface " $S$ ", see Eq. 8) could now be determined due to several developments: 1) our model is more accurate, 2) the experimental masses are more accurate, and 3) we adjust to a $30 \%$ larger data base. Accounting for the density-symmetry effect leads to a further improvement in accuracy by about $0.02 \mathrm{MeV}$, corresponding to step 5 in Fig. 1 and line 10 in Table D. The next line shows how this stage (11)-a extrapolates to the new region "3", see Ref. [87]. To get some estimate of uncertainties we adjust the model to the smaller data set AME1989 with $L$ fixed at 0 (line 12) and with also $L$ varying (line 13 ). Also with this data set we get an improvement in accuracy of about $0.02 \mathrm{MeV}$ and an uncertainty estimate for the density symmetry coefficient $L=70 \pm 15 \mathrm{MeV}$, and the symmetry energy coefficient $J=32.5 \pm 0.5 \mathrm{MeV}$, see Ref. [87].

As discussed in Sect. 4 we have now implemented the final step leading to FRDM(2012), namely an improved calculation of ground-state correlation energies, resulting in a further $0.01 \mathrm{MeV}$ improvement in accuracy entered as line 14 in Table D and step 6 in Fig. 1. Due to space limitations sideways in the table, we have not entered the values of 


\begin{tabular}{|c|c|c|c|c|c|c|c|c|c|c|c|c|c|c|}
\hline $\begin{array}{l}\text { Line } \\
\text { No }\end{array}$ & Model & $\mathrm{A} / \mathrm{C}$ & $\begin{array}{c}a_{1} \\
(\mathrm{MeV})\end{array}$ & $\begin{array}{c}a_{2} \\
(\mathrm{MeV})\end{array}$ & $\begin{array}{c}J \\
(\mathrm{MeV})\end{array}$ & $\begin{array}{c}Q \\
(\mathrm{MeV})\end{array}$ & $\begin{array}{c}K \\
(\mathrm{MeV})\end{array}$ & $\begin{array}{r}L \\
(\mathrm{MeV})\end{array}$ & $\begin{array}{r}a_{0} \\
(\mathrm{MeV})\end{array}$ & $\begin{array}{c}c_{\mathrm{a}} \\
(\mathrm{MeV})\end{array}$ & $\begin{array}{c}C \\
(\mathrm{MeV})\end{array}$ & $\gamma$ & $\begin{array}{c}\mu_{\mathrm{th}} \\
(\mathrm{MeV})\end{array}$ & $\begin{array}{l}\sigma_{\mathrm{th} ; \mu=0} \\
(\mathrm{MeV})\end{array}$ \\
\hline 1 & FRDM(1992) & $1 / 1$ & 16.247 & 22.92 & 32.73 & 29.21 & 240 & 0 & 0.00 & 0.436 & 60 & 0.831 & 0.0156 & 0.6688 \\
\hline 2 & FRDM(1992) & $1 / 6$ & & & & & & & & & & & 0.1356 & 0.5817 \\
\hline 3 & (92)-b & $1 / 1$ & 16.286 & 23.37 & 32.34 & 30.51 & 240 & 0 & -5.21 & 0.468 & 179 & 1.027 & 0.0000 & 0.6591 \\
\hline 4 & (92)-b & $1 / 6$ & & & & & & & & & & & -0.0243 & 0.5506 \\
\hline 5 & (92)-b & $1 / 2$ & & & & & & & & & & & 0.0076 & 0.6157 \\
\hline 6 & (06)-a & $2 / 2$ & 16.274 & 23.27 & 32.19 & 30.64 & 240 & 0 & -5.00 & 0.450 & 169 & 1.000 & 0.0000 & 0.6140 \\
\hline 7 & (07)-b & $2 / 2$ & 16.231 & 22.96 & 32.11 & 30.83 & 240 & 0 & -3.33 & 0.460 & 119 & 0.907 & 0.0000 & 0.5964 \\
\hline 8 & (11)-b & $2 / 2$ & 16.231 & 22.95 & 32.10 & 30.78 & 240 & 0 & -3.14 & 0.456 & 113 & 0.896 & 0.0001 & 0.5863 \\
\hline 9 & (11)-b & $2 / 3$ & & & & & & & & & & & -0.0850 & 0.6212 \\
\hline 10 & (11)-a & $2 / 2$ & 16.147 & 22.44 & 32.51 & 28.54 & 240 & 70.84 & -2.96 & 0.531 & 150 & 0.880 & -0.0004 & 0.5700 \\
\hline 11 & (11)-a & $2 / 3$ & & & & & & & & & & & -0.0516 & 0.5618 \\
\hline 12 & (11)-c & $1 / 1$ & 16.251 & 23.10 & 32.31 & 30.49 & 240 & 0 & -3.43 & 0.471 & 123 & 0.935 & -0.0003 & 0.6300 \\
\hline 13 & (11)-d & $1 / 1$ & 16.142 & 22.39 & 32.98 & 27.58 & 240 & 85.95 & -2.64 & 0.548 & 138 & 0.853 & 0.0000 & 0.6092 \\
\hline 14 & FRDM(2012) & $2 / 2$ & 16.195 & 22.76 & 32.30 & 28.72 & 240 & $\mathbf{5 3 . 5 0}$ & -4.00 & 0.489 & 205 & 0.988 & -0.0007 & 0.5595 \\
\hline 15 & FRDM(2012) & $2 / 5$ & & & & & & & & & & & 0.0642 & 0.6440 \\
\hline 16 & FRDM(2012) & $2 / 4$ & & & & & & & & & & & 0.0094 & 0.5728 \\
\hline 17 & $(12)-b$ & $4 / 4$ & 16.175 & 22.64 & 32.40 & 28.51 & 240 & 67.77 & -3.74 & 0.513 & 206 & 0.974 & 0.0000 & 0.5711 \\
\hline 18 & (12)-c & $1 / 1$ & 16.211 & 22.87 & 32.70 & 27.95 & 240 & 59.77 & -4.25 & 0.509 & 205 & 0.996 & 0.0000 & 0.6047 \\
\hline 19 & (12)-c & $1 / 4$ & & & & & & & & & & & 0.0307 & 0.5764 \\
\hline 20 & (12)-c & $1 / 6$ & & & & & & & & & & & 0.0635 & 0.4948 \\
\hline 21 & (12)-d & $4 / 4$ & 16.268 & 23.23 & 32.13 & 30.53 & 240 & 0 & -4.89 & 0.439 & 179 & 1.007 & 0.0000 & 0.5905 \\
\hline 22 & (12)-e & $1 / 1$ & 16.288 & 23.39 & 32.34 & 30.39 & 240 & 0 & -5.56 & 0.465 & 218 & 1.065 & -0.0002 & 0.6147 \\
\hline 23 & (12)-e & $1 / 4$ & & & & & & & & & & & 0.0161 & 0.5949 \\
\hline 24 & (12)-e & $1 / 6$ & & & & & & & & & & & -0.0197 & 0.5306 \\
\hline 25 & (12-Kfix-1) & $2 / 2$ & 16.319 & 23.93 & 32.45 & 28.41 & 100 & 23.54 & -8.45 & 0.384 & 127 & 0.823 & 0.0000 & 0.6025 \\
\hline 26 & (12-Kfix-2) & $2 / 2$ & 16.242 & 23.22 & 32.32 & 28.55 & 150 & 35.47 & -5.82 & 0.444 & 151 & 0.886 & 0.0000 & 0.5694 \\
\hline 27 & (12-Kfix-3) & $2 / 2$ & 16.212 & 22.92 & 32.31 & 28.64 & 200 & 46.11 & -4.65 & 0.474 & 180 & 0.944 & 0.0000 & 0.5612 \\
\hline 28 & (12-Kvar) & $2 / 2$ & 16.193 & 22.74 & 32.31 & 28.73 & 256 & 56.16 & -3.91 & 0.494 & 217 & 1.007 & 0.0000 & 0.5593 \\
\hline 29 & (12-Kfix-4) & $2 / 2$ & 16.165 & 22.47 & 32.33 & 28.96 & 400 & 76.96 & -2.84 & 0.521 & 334 & 1.147 & 0.0000 & 0.5619 \\
\hline 30 & $(12-K f i x-5)$ & $2 / 2$ & 16.141 & 22.26 & 32.36 & 29.21 & 600 & 101.28 & -1.98 & 0.541 & 515 & 1.286 & 0.0001 & 0.5671 \\
\hline 31 & $(12-K f i x-6)$ & $2 / 2$ & 16.123 & 22.12 & 32.38 & 29.42 & 800 & 124.79 & -1.39 & 0.554 & 623 & 1.350 & 0.0000 & 0.5715 \\
\hline 32 & (12-Kfix-7) & $2 / 2$ & 16.114 & 22.04 & 32.39 & 29.58 & 1000 & 143.45 & -1.09 & 0.561 & 825 & 1.439 & 0.0000 & 0.5750 \\
\hline 33 & (FY1970) & $2 / 2$ & 15.949 & 21.10 & 31.37 & 32.49 & 240 & 0 & 1.76 & 0.543 & 78 & 0.589 & -0.0001 & 0.6909 \\
\hline 34 & (FY1970)-L & $2 / 2$ & 15.935 & 21.01 & 31.37 & 31.96 & 240 & 39.03 & 2.30 & 0.543 & 106 & 0.668 & -0.0003 & 0.6876 \\
\hline
\end{tabular}

Table D

FRDM (1992) and successive enhancements. Adjustments have been performed for up to 9 macroscopic constants, i.e, the volume-energy $\left(a_{1}\right)$, the surface-energy $\left(a_{2}\right)$, the symmetry-energy $(J)$, the effective surface-stiffness $(Q)$, the density-symmetry $(L)$, the $A^{0}\left(a_{0}\right)$, the charge-asymmetry $\left(c_{\mathrm{a}}\right)$, the pre-exponential compressibility-term constant $(C)$ and the exponential compressibility-term range $(\gamma)$ constants. In one case the compressibility constant $(K)$ is also varied, in a few other "sensitivity" studies it is fixed at values different from $240 \mathrm{MeV}$. These results are in lines 25-32. The second column indicates a model designation and the third is to which data set (denoted by numbers " 1 " through "6") the model was Adjusted/Compared "(A/C)". The last two columns are the mean deviation (with sign) $\mu_{\text {th }}$ and the model error $\sigma_{\text {th } ; \mu=0}$, both defined in Sect. 2.1, with respect to the data set specified in the "C" column. In column three, "1" stands for AME1989, "2" for AME2003, "3" for masses in AME2011 that were not in AME2003, "4" for AME2012, "5" for masses in AME2012 that are not in AME2003, and "6" for masses in AME2012 that are not in AME1989. The top line gives the original model constants [9]. When no values are given, the set on the line just above is used. The value " 0 " in the $L$ column indicates $L$ was fixed at zero. See the text for additional discussions.

the constants to the precision we recommend in actual use of the model; the more accurate values given above should be used. When we investigate the extrapability of the model by calculating the accuracy for the 219 new masses in data set 5 we seemingly find a noticeable divergence from 0.5595 to 0.6440 , an increase of $15 \%$, see line 15 . However this increase is due to two outliers, ${ }_{8}^{25} \mathrm{O}_{17}$, and ${ }_{19}^{51} \mathrm{~K}_{17}{ }_{20}^{52} \mathrm{Ca}_{32}$ is not in set 5, it was measured earlier. But in AME2003 it is given as $-32.51 \pm 0.70 \mathrm{MeV}$ whereas in AME2012 it is given as $-34.26 \pm 0.06 \mathrm{MeV}($ !). ) discussed in Sect. 5.1 in connection with Fig. 15. If these two nuclei are removed from the 219 nuclei in data set 5 we obtain $\sigma_{\text {th }}=0.5706$ $\mathrm{MeV}$. So the $15 \%$ increase when we compare to this limited set of new nuclei does not prove a divergence of the mass model away from the region of adjustment. It is highly likely it is just a statistical fluctuation; see also the more extensive tests we discuss next.

We continue with some sensitivity studies. Line 16 shows the agreement of FRDM(2012) with the entire data set AME2012, of which 219 masses were not used in the determination of the model parameters. The error is 0.5728 $\mathrm{MeV}$ in this region. When the whole data set is used in the determination of model parameters the error decreases only very marginally, to $0.5711 \mathrm{MeV}$, line 17 . In line 18 we adjust the model to the more limited data set AME1989. 


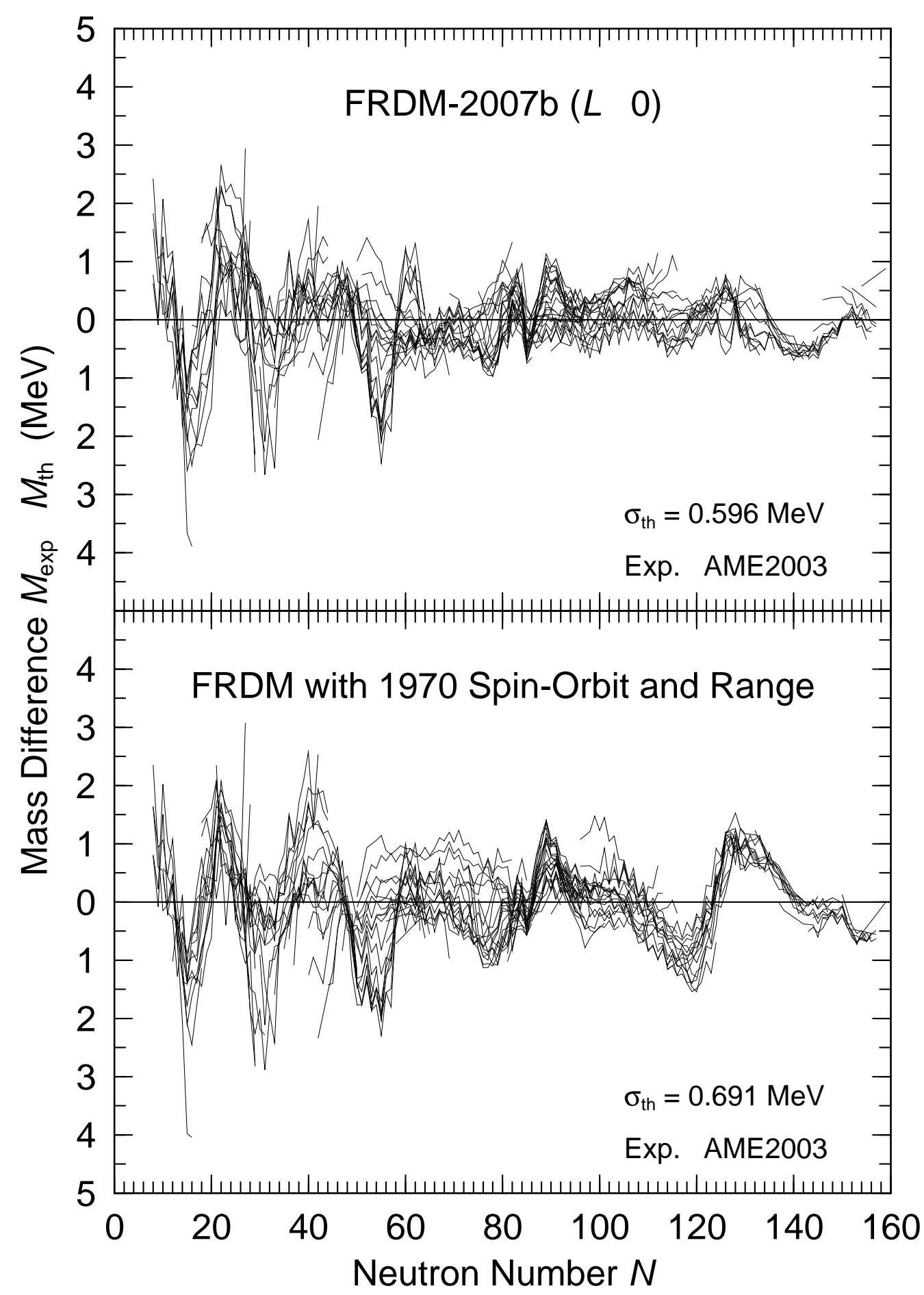

Fig. 36: Differences between experimental masses and FRDM masses for two different single-particle spin-orbit strengths and two different diffuseness parameters. See text for further discussion. The figure was originally published in Ref. [91].

When we compare this mass table to AME2012 we obtain the error $0.5764 \mathrm{MeV}$, line 18 . Although 730 nuclei in this evaluation were not used in the determination of the parameters of model (12)-c the error for the entire region is only $0.5764-0.5711=0.0053 \mathrm{MeV}$ larger than when all nuclei in AME2012 were included in the determination of the parameters (line 17). We therefore conclude that the model is very reliable (so far) when applied to nuclei outside the region of adjustment. How (12)-c extrapolates to a region that just contains new nuclei is on line 20, see Fig. 14 for a graphical illustration.

In lines 21-24 we do equivalent studies as in lines 17-20, but with $L=0$. By comparing lines 23 and 21 we note that also with $L=0$ the model extrapolates extremely well. But we again observe that the inclusion of densitysymmetry effects improve accuracy by about $0.02 \mathrm{MeV}$ (compare lines 17 and 21).

We have in our discussion above fixed the compressibility constant to $K=240 \mathrm{MeV}$. It is of interest to study (as was done in Ref. [9])) how the model accuracy and the values of the model constants depend on $K$. Lines $25-32$ in Table D show the results of such a study when $K$ is fixed at different values. Line 28 shows the value $K=256 \mathrm{MeV}$ is obtained when $\mathrm{K}$ is varied freely together with the other nine macroscopic constants. 


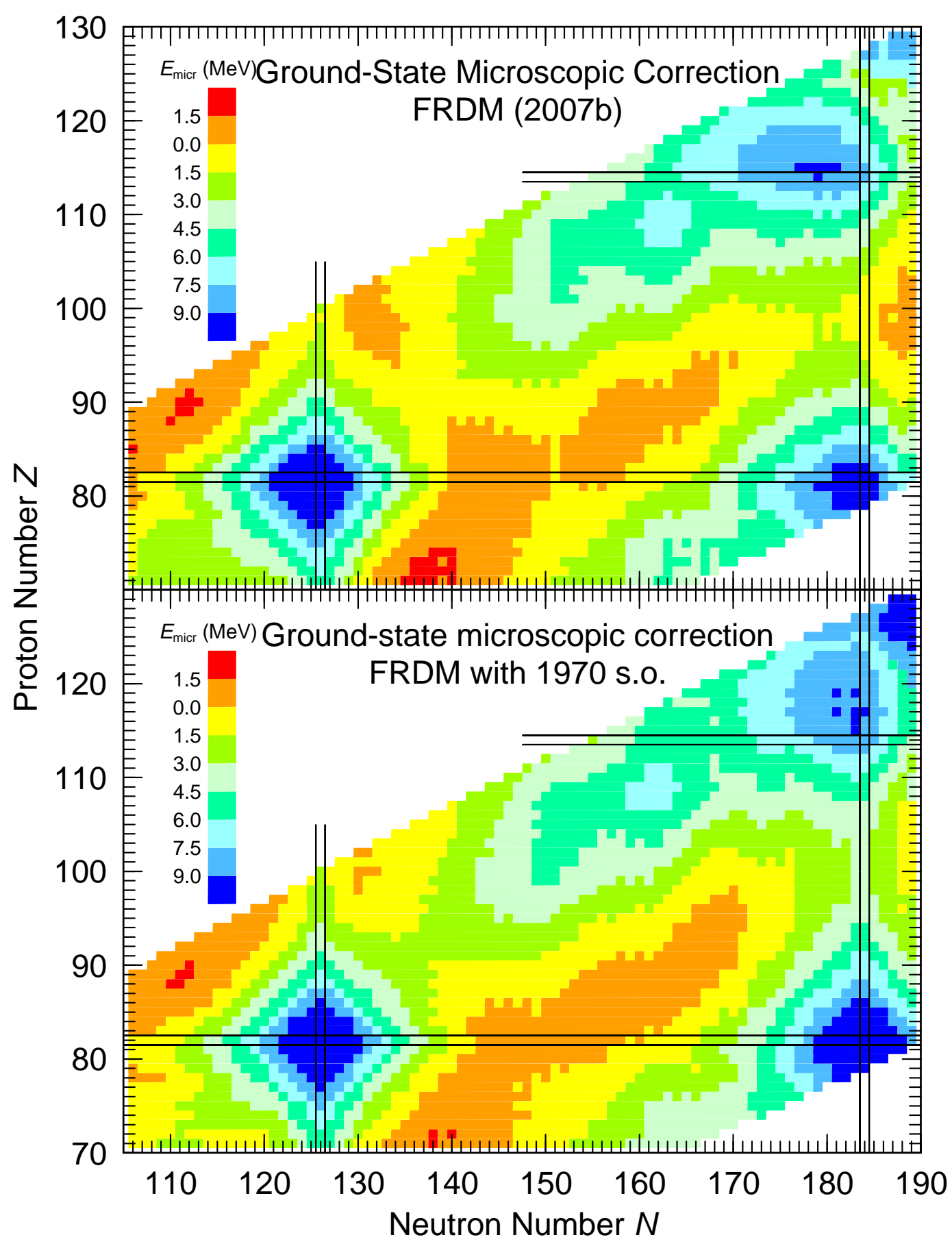

Fig. 37: Calculated microscopic corrections in FRDM models with two different single-particle parameter sets. It is somewhat remarkable that quite different single-particle potentials yield very similar stability properties in the heavy-element region, in particular the stability of the by now well-known region of deformed heavy elements in the vicinity of ${ }_{108}^{270} \mathrm{Hs}$ is clearly manifested in both results. This figure was originally published in Ref. [91].

We have also investigated the sensitivity of the mass model to some essential single-particle model parameters, namely the spin-orbit strength $\lambda$ and potential diffuseness constant $a_{\text {pot. }}$ Traditionally in single-particle models these are determined by comparing calculated and experimental single-particle levels. The process is somewhat ambiguous because observed nuclear levels are not single-particle levels. In the folded-Yukawa single-particle model the spinorbit strengths and diffuseness constants originally used were $\lambda_{\mathrm{p}}=\lambda_{\mathrm{n}}=32.0$ and $a_{\mathrm{pot}}=0.9 \mathrm{fm}$ [10]. These parameters were determined mainly by adjusting to levels in ${ }^{208} \mathrm{~Pb}$, see Ref. [37]. In 1973, during an extended visit to Los Alamos by PM and Sven-Gösta Nilsson, it was observed that this original choice led to a poor description of levels in deformed nuclei $[37,91]$ and new parameters were determined for the actinide region and for the rare-earth region [37]. Somewhat later, see Ref. [1], these studies served as a basis for a global prescription for the spin-orbit strength and diffuseness constant leading to Eqs. 89 and 90 for the proton and neutron spin-orbit strengths and to the value $a_{\text {pot }}=0.8 \mathrm{fm}$ for the potential diffuseness constant, see Sect. 2.12. At the time when we studied the sensitivity of mass model results to the spin-orbit and diffuseness constants, we had developed the model through the third step in Fig. 1, corresponding to line 7 in Table D. We changed the spin-orbit and diffuseness constants to the values used 


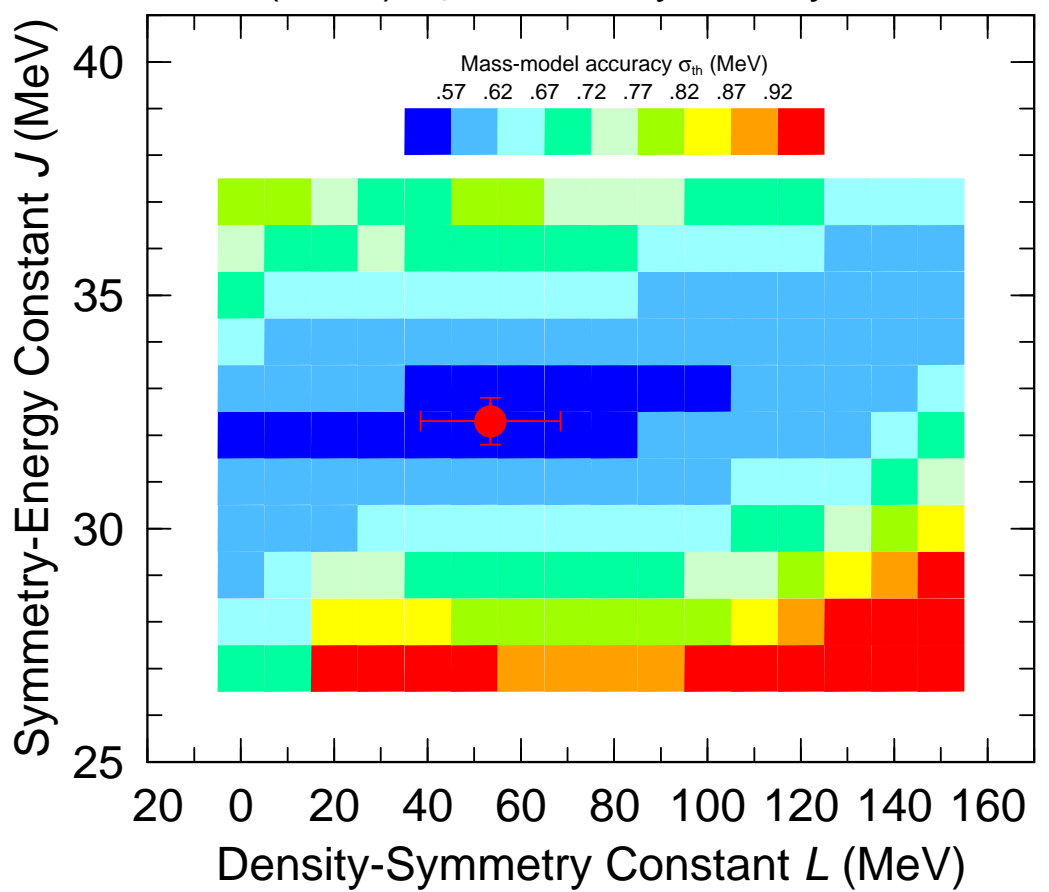

Fig. 38: Calculated mass model accuracy for different combinations of $L$ and $J$. The best accuracy, is obtained for the $L$ and $J$ in the FRDM(2012), line 14 in Table $\mathrm{D}$, and is indicated with a red dot with uncertainty bars.

originally and performed a full-fledged mass calculation that included a recalculation of all ground-state shapes in the four-dimensional deformation space discussed in step 3 in Sect. 4. We then, following the standard procedure detailed above, adjusted the macroscopic parameters to optimize agreement with AME2003. In Fig. 36 bottom panel we show the difference between experimental and calculated masses versus neutron number that we obtained. The results are also given as line 33 in Table D. In the top panel we give the corresponding results with the model (07)-b. It is clear that with the original single-particle model parameters, the calculated masses agree less well with experimental masses than with the current choice of spin-orbit strength and potential diffuseness constant. In fact the calculation is even less accurate than the results with the previous $\operatorname{FRDM}(1992)$. A particularly interesting observation is that the current spin-orbit and diffuseness strengths were chosen without any consideration of nuclear masses; in their determination

$\operatorname{FRDM}(2012) a_{1}$ versus Symmetry Constants

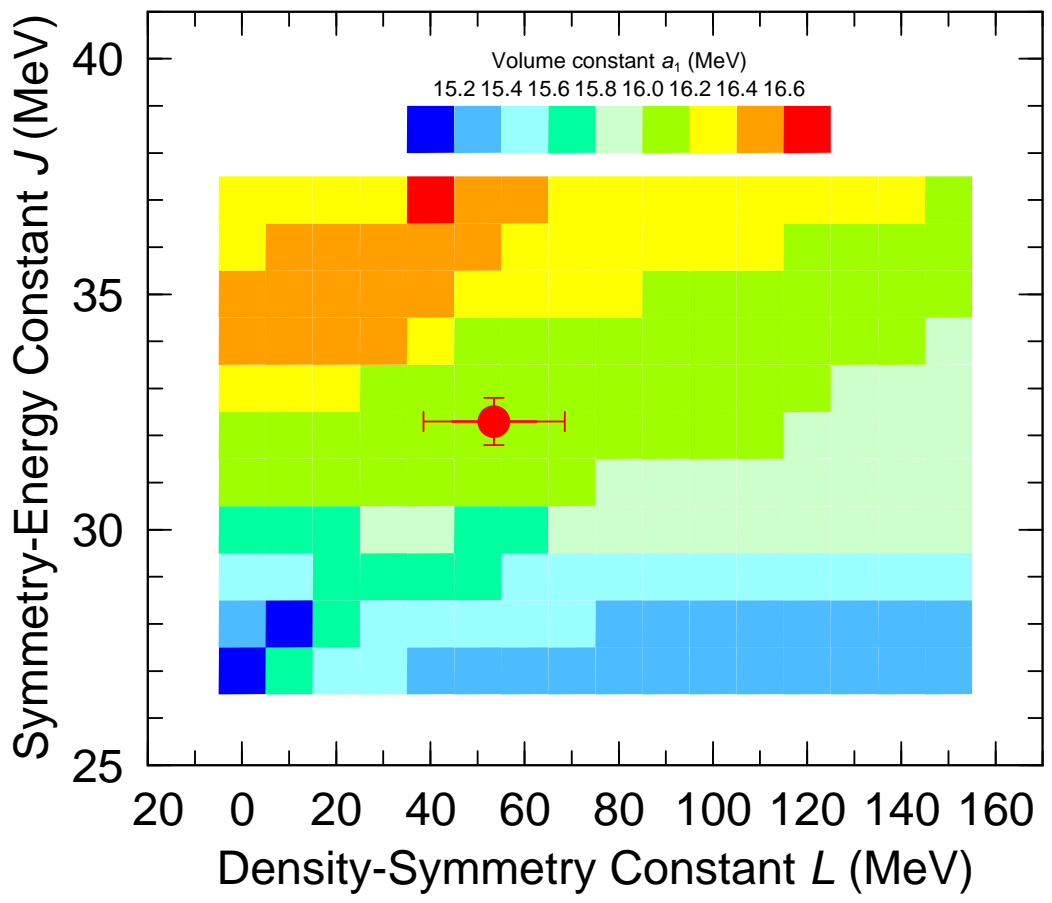

Fig. 39: Values of the volume constant that is obtained when the mass model is optimized with respect to seven macroscopic constants for 176 different value combinations or $L$ and $J$. The values of these constants in the $\operatorname{FRDM}(2012)$ is indicated by a red dot with uncertainty bars. 
only levels were considered [37]. This result shows that the model is working as a model should; if the model is enhanced so that better agreement with one type of experimental data is obtained, then better agreement with other types of data automatically follows and the model describes many different types of data in a consistent fashion.

We also investigate how the two calculations differ in the superheavy-element region. In Fig. 37 we show calculated microscopic corrections for nuclei from the $\mathrm{Pb}$ region to the $\mathrm{SHE}$ region calculated with the two different parameter sets. In this type of plot both calculations seem to give very similar results. In particular they both show large negative shell corrections centered around ${ }_{108}^{270} \mathrm{Hs}_{162}$. This is a result that is quite insensitive to macroscopic-microscopic model formulations within a very large parameter space. Macroscopic-microscopic calculations based on the Woods-Saxon model obtain results very similar to those in Fig. 37, see for example the review in Ref. [97] which again shows how robust these results are in reasonably realistic nuclear-structure models.

In the study with the original single-particle parameters we have also investigated the effect of varying $L$, see line 34 in Table D. The effect is very small, which shows that this formulation (non-optimum spin-orbit and potential diffuseness) has the consequence that the model is too inaccurate to allow clear manifestations of density-symmetry effects.

One may ask how correlated the values of $J$ and $L$ are in the $\operatorname{FRDM}(2012)$. To investigate this we have optimized the mass model with respect to seven other macroscopic constants ( $K$ is kept fixed in this investigation) for different combinations of $J$ and $L$. Specifically we consider $L=0(10) 150$ and $J=27(1) 37$ in units of MeV, for a total of 176 grid points. For each combination we start the minimization at 1440 different starting combinations of the seven parameters that are varied. Sometimes several minima are obtained; in Fig. 38 we show the lowest minimum $\sigma_{\text {th }}$ obtained at each gridpoint. In Fig. 39 we show corresponding values of the volume constant $a_{1}$. This constant is normally assumed to be close to $16 \mathrm{MeV}$. However, some distance from the values of $L$ and $J$ that optimize the mass model accuracy the value of $a_{1}$ becomes unrealistic. It is very satisfactory that the values of macroscopic parameters that optimize the mass-model accuracy (when $J$ and $L$ are also varied) are all within a realistic range.

The optimal values of the asymmetry variables $J$ and $L$ that we obtained from the mass model FRDM (2012) study are

$$
\begin{aligned}
& J=32.3 \pm 0.5 \mathrm{MeV} \\
& L=53.5 \pm 15 \mathrm{MeV}
\end{aligned}
$$

The above optimal $L$ value is somewhat smaller than the value in Ref. [98], because we have implemented a more accurate calculation of the zero-point fluctuation effect, see Sect. 2.11. These symmetry energy coefficients have been extensively studied by various experimental and theoretical methods because of their strong impact on astrophysical observables such as the neutron star mass and radius and also simulations of supernovae explosions [68]. The experimental and theoretical methods adopted to extract these values are: mass-fragmentation studies of heavy-ion collisions [99, 100], pigmy dipole resonances (PDR) [101, 102], dipole polarizability in ${ }^{208} \mathrm{~Pb}$ [103, 104], anti-analogue giant dipole resonances [105, 106], isospin dependence of giant monopole resonances [107, 108, 109], isobaric analogue states [110], constraints from observations of masses and sizes of neutron stars [111, 112], chiral effective field theories [113], and quantum Monte-Carlo simulations [114]. Compared with the constraints from these studies, our optimal values for $J$ and $L$ are very consistent with those from neutron star studies, PDR and dipole polarizability.

\subsection{Can the deviations below $N \approx 65$ be decreased?}

In a model of the relative conceptual simplicity of the FRDM(2012), although execution of actual calculations does involve substantial effort, one must expect some limit to how accurate it can eventually become. In our case we have managed to find remedies that removed various types of correlated deviations. In the 1981 mass model we noted that this type of correlated deviations in regions near ${ }^{222} \mathrm{Ra}$ and ${ }^{252} \mathrm{Fm}$ could be removed by searching for ground-state minima in a more general deformation space that included the four shape variables we explore accurately here; earlier somewhat less complete calculations are in $[1,27,9]$. In particular, minimizations with respect to $\varepsilon_{3}$ reduced many of the deviations near ${ }^{222} \mathrm{Ra}$ and minimizations with respect to $\varepsilon_{6}$, those near ${ }^{252} \mathrm{Fm} \mathrm{[1,9].} \mathrm{The} \mathrm{deviations} \mathrm{in} \mathrm{the} \mathrm{light}$ region in the current calculation look correlated and that could possibly hint that a remedy can be found. We have investigated several ideas, but they all were unsuccessful in removing the deviations. If they had been successful we would obviously have included the methods in our calculations of masses. But, although the ideas were unsuccessful we feel it is useful to give a brief discussion of these investigations. We looked at four different possibilities, namely

1. Possibly more optimum spin-orbit strength and potential diffuseness constants could be found.

2. The zero-point energy calculations might be improved if we instead of using a phenomenological renormalized irrotational-flow inertia used a more microscopic cranking-model inertia.

3. One could have some concerns about the particular version of the Strutinsky normalization we use, which is the original version, and how it would perform for light nuclei in particular, so we have investigated an alternative formulation proposed by Kruppa.

4. Some deviations are clearly outside the current model, such as the deviations near $Z=40$ and $N=56$ which we commented on above. We investigated if a tensor force could improve the accuracy in the light region.

In some of these studies we used masses in the $\mathrm{Ca}$ isotope chain to test the ideas for improvement for two reasons. First, these nuclei are all calculated to be spherical in shape. We assumed that also with new features implemented 


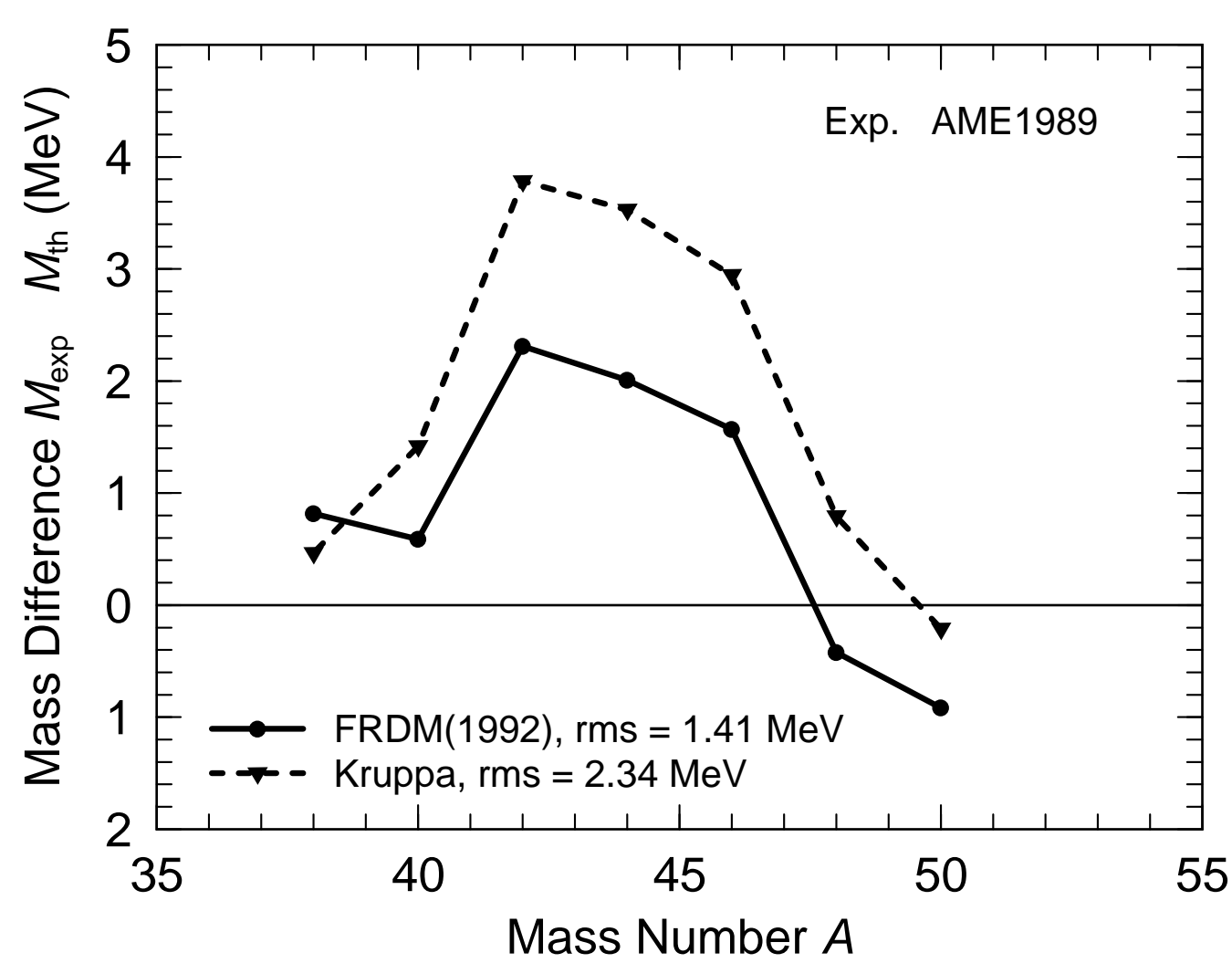

Fig. 40: Deviations between measured and calculated masses for the Ca isotope chain calculated with two models for the shell+pairing corrections. We use these isotopes as a testbed for ideas for improving the mass model in the light region of the nuclear chart. One of the methods is the Strutinsky shell-correction method, the other the Kruppa model.

they would remain spherical so we would only have to do calculations for this one shape for each of the isotopes with known masses (now ${ }^{36} \mathrm{Ca}-52 \mathrm{Ca}$ ). Second, the deviations are large and highly variable across the isotope chain, with an rms deviation of about one and a half MeV in both the FRDM(1992) and FRDM(2012) so it is a good test to investigate if a new model feature can significantly decrease these deviations. The deviations are very similar in both FRDM(1992) and FRDM(2012), because all shapes are spherical and it is mainly shell-plus-pairing corrections for identical shapes that determine the fluctuations in the deviations.

\subsubsection{Improved choice of spin-orbit and single-particle potential diffuseness constants}

To study the possibility that a different choice of proton and neutron spin-orbit strengths and a different choice of the diffuseness constants would improve the calculated masses we calculated masses along the $\mathrm{Ca}$ isotope chain for a four-dimensional grid in these constants. The maximum improvement in the calculated masses were less than 15\% so we do not consider this possibility to be a viable cure for the deviations in the low- $A$ region. Furthermore these constants would not give a globally improved model, and not even locally do we obtain significantly better results.

\subsubsection{Improved determination of zero-point energies}

We limited this study to zero-point motion in the $\varepsilon_{2}$ direction. Rather than using the phenomenological inertia in Eq, 116 we calculated the cranking-model inertia in the $\varepsilon_{2}$ direction at each calculated ground-state shape and calculated the zero-point energy using this inertia. We renormalized the cranking-model inertia by a constant (same for all nuclei) so as to obtain optimum agreement between all calculated masses and experimental data. We found this approach did not perform well. The main reason was that the zero-point energies could vary by a factor of three between neighboring isotopes also in cases where the potential surface stiffness parameters were almost identical. The main reason was the well-known cranking-model feature that at level crossings the cranking-model inertia is very sensitive to small details of the level crossing. Slightly better results might have been obtained by varying the groundstate deformation and minimizing the sum of the potential energy and zero-point energy. This would have been a massive effort with limited chances of success so we did not investigate this possibility.

\subsubsection{Alternative shell-plus-pairing calculation}

Here we investigate the alternative shell-correction model put forward in Ref. [115, 116]. But the masses calculated with this method show very similar fluctuations with respect to experimental data for the isotopes along the Ca chain, see Fig. 40. And, we recall that very early on it has been pointed out that one can expect decreasing accuracy of Strutinsky-type calculations with decreasing nucleon number $A[117,10]$. 


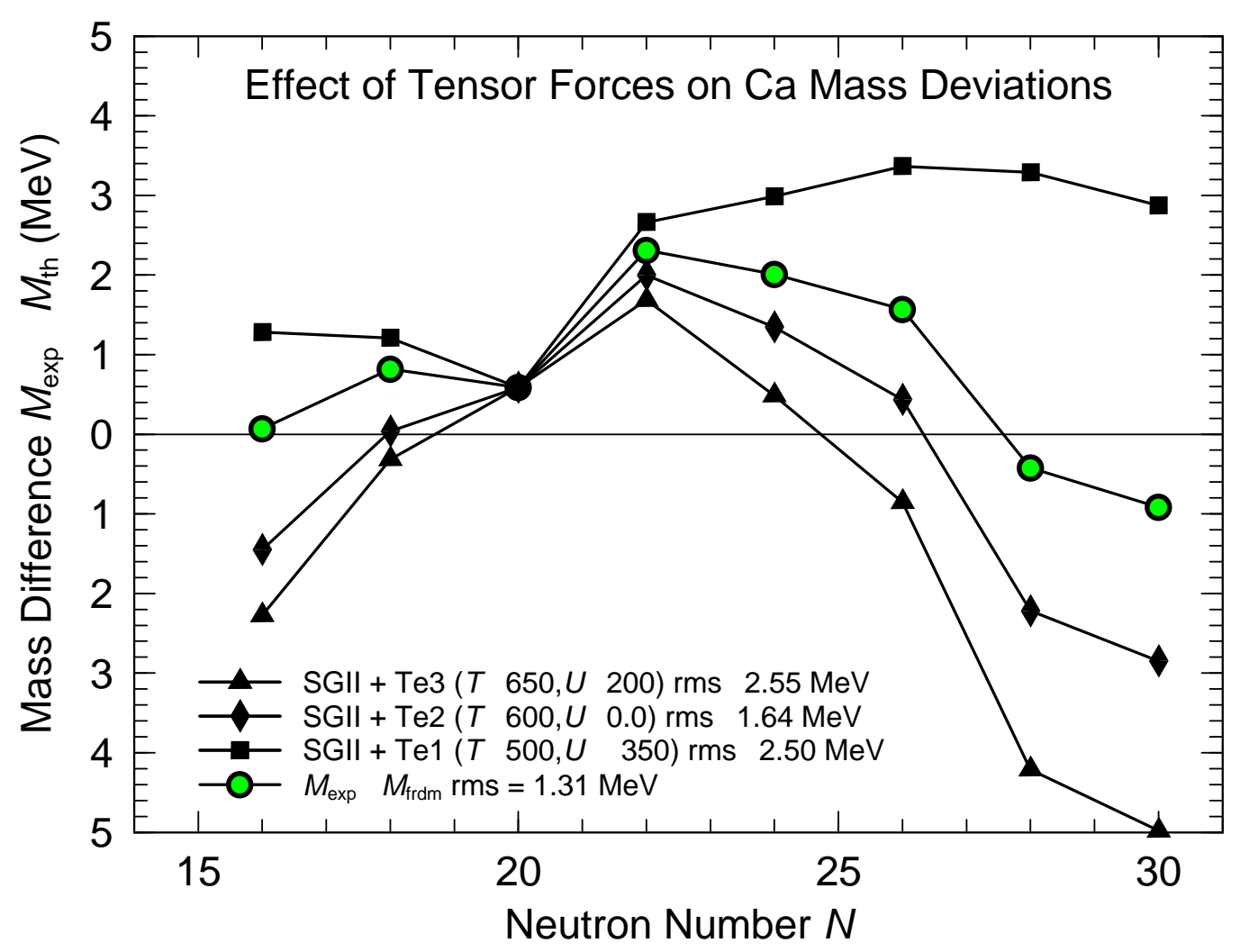

Fig. 41: Effect of tensor force on Ca-isotope mass deviations. The large filled circles show the mass deviations in the FRDM(2012) mass model. The three other curves show how these are modified due to the effect of different tensor forces. It seems that the oscillatory behavior of the deviations cannot be eliminated by these tensor forces.

\subsubsection{Effect of a tensor force}

In this study we have not incorporated a tensor force in the macroscopic-microscopic model which would be a monumental effort. Rather, to get a rough idea of the possible benefits of a tensor force, we study its possible impact indirectly. We calculate masses in an HFB approach with several different Skyrme forces without and with tensor terms. We use a specific case to illustrate our strategy. For example we calculate binding energy of ${ }^{40} \mathrm{Ca}$ and ${ }^{42} \mathrm{Ca}$ in the HFB approach without a tensor force. Then we calculate the binding energies for the same nuclei with the tensor force. We then argue that the effect of the tensor force is the difference in the change in the binding energy between the two calculations. We repeat this for other Ca isotopes. We then modify the errors calculated in the FRDM with the effect of the tensor force determined in this way.

For our calculations we use the Skyrme-type tensor interaction [118], which is the sum of the triplet-even and triplet-odd zero-range tensor parts,

$$
\begin{aligned}
v_{T} & =\frac{T}{2}\left\{\left[\left(\vec{\sigma}_{1} \cdot k^{\prime}\right)\left(\vec{\sigma}_{2} \cdot k^{\prime}\right)-\frac{1}{3}\left(\vec{\sigma}_{1} \cdot \vec{\sigma}_{2}\right) k^{\prime 2}\right] \delta\left(\vec{r}_{1}-\vec{r}_{2}\right)\right. \\
& \left.+\delta\left(\vec{r}_{1}-\vec{r}_{2}\right)\left[\left(\vec{\sigma}_{1} \cdot k\right)\left(\vec{\sigma}_{2} \cdot k\right)-\frac{1}{3}\left(\vec{\sigma}_{1} \cdot \vec{\sigma}_{2}\right) k^{2}\right]\right\} \\
& +U\left\{\left(\vec{\sigma}_{1} \cdot k^{\prime}\right) \delta\left(\vec{r}_{1}-\vec{r}_{2}\right)\left(\vec{\sigma}_{2} \cdot k\right)-\frac{1}{3}\left(\vec{\sigma}_{1} \cdot \vec{\sigma}_{2}\right)\left[k^{\prime} \cdot \delta\left(\vec{r}_{1}-\vec{r}_{2}\right) k\right]\right\},
\end{aligned}
$$

where the operator $k=\left(\vec{\nabla}_{1}-\vec{\nabla}_{2}\right) / 2 i$ acts on the right and $k^{\prime}=-\left(\overleftarrow{\nabla}_{1}-\overleftarrow{\nabla}_{2}\right) / 2 i$ on the left. The coupling constants $T$ and $U$ denote the strength of the triplet-even and triplet-odd tensor interactions, respectively. The tensor terms (123) give contributions to the binding energy and to the spin-orbit splitting that are proportional to the spin-orbit density $\vec{J}$. In spherical nuclei only the radial component of this vector does not vanish and is

$$
J_{q}(r)=\frac{1}{4 \pi r^{3}} \sum_{i \in q} v_{i}^{2}\left(2 j_{i}+1\right)\left[j_{i}\left(j_{i}+1\right)-l_{i}\left(l_{i}+1\right)-\frac{3}{4}\right] R_{i}^{2}(r),
$$

where $i=n, l, j$ runs over all states and $q=0(1)$ stands for neutrons (protons). The quantity $v_{i}^{2}$ is the occupation probability of each orbit and $R_{i}(r)$ is the radial part of the HF single-particle wave function. Furthermore, we observed that the exchange part of the central Skyrme interaction gives the same kind of contributions to the total energy density. The tensor contributions give extra terms to the energy density that read

$$
\delta E=\frac{1}{2} \alpha\left(J_{n}^{2}+J_{p}^{2}\right)+\beta J_{n} J_{p} .
$$


where $\alpha=\alpha_{C}+\alpha_{T}$ and $\beta=\beta_{C}+\beta_{T}$. The central exchange contributions are given by

$$
\alpha_{C}=\frac{1}{8}\left(t_{1}-t_{2}\right)-\frac{1}{8}\left(t_{1} x_{1}+t_{2} x_{2}\right), \quad \beta_{C}=-\frac{1}{8}\left(t_{1} x_{1}+t_{2} x_{2}\right),
$$

in terms of the parameters of the Skyrme force as defined in Ref. [119] and the tensor part reads

$$
\alpha_{T}=\frac{5}{12} U, \quad \beta_{T}=\frac{5}{24}(T+U)
$$

in terms of the triplet-even and triplet-odd terms appearing in Eq. (123).

In Fig. 41 we have plotted the mass deviations in the $\operatorname{FRDM(1992)~along~the~Ca~isotopes~and~also~the~deviations~}$ after the calculated masses have been modified with the tensor effect calculated as described above. The energy contribution of the tensor force increases or decreases monotonically, depending on the details of the adopted tensor interactions, from $A=40$ to $A=48$ and it therefore seems unlikely that any implementation of this type of tensor force can remedy the type of fluctuating deviations with respect to experiment that are present in the FRDM(1992) (and FRDM(2012)) along this isotope chain.

\subsection{Comments on general nuclear-structure results in the FRDM/FRLDM and other nuclear-structure models}

We have presented calculations of nuclear ground-state masses and deformations based on a global, general nuclear-structure model. It is natural to ask how the current mass calculations in this general model compare to other published nuclear-mass calculations. However, to present in a limited space such a comparison and associated discussion is nearly impossible, because few other "mass models" have their origin in what is generally agreed upon is a viable physical model or theory, in contrast to our work. We illustrate some of these issues below, and refer to comparisons to other models and experimental data already available in the literature, but plan a more extensive discussion in a future publication, since such a review is outside the scope of this paper.

To set the basis for our brief comments let us recall that it is generally accepted that a proper physical model should, apart from describing the core data that was the inspiration for the model, also 1) describe new measurements of similar data, in this case new mass measurements, 2) describe new types of data, and 3) lead to new insight.

The original Bethe-Weizäcker semi-empirical mass model [86] is an excellent example of a highly useful, proper physical model. Initially, it was instrumental in interpreting experimentally studied element transmutations. A problem in interpreting decays following neutron bombardment of uranium, erroneously ascribed to complex chains of $\beta^{-}$and $\alpha$-decays between nuclides in the region of the compound nucleus formed, was eventually resolved when the products of the reactions were identified as being light products such as barium with 56 protons [120]. The semi-empirical mass model, which had assumed spherical nuclei, was immediately generalized to include nuclear deformations, so that it could describe the splitting of the atom into two daughter nuclei of "roughly" similar size [121, 122], without the introduction of any new parameters.

\subsubsection{Ground-state masses}

In a recent review of the literature [26] we found no global macroscopic-microscopic calculations of ground-state masses with both a proper treatment of the deformation dependence of the macroscopic energy and a calculation of the shell-plus-pairing corrections based on calculated deformed single-particle spectra, except the early work of Seeger and Howard [123, 124], which we have discussed in Refs. [34, 98].

In the self-consistent Hartree-Fock treatments there is no obvious clear, straight progression towards models that describe nuclear-structure properties as accurately and generally in a consistent fashion as do our FRDM and FRLDM approaches. For example, there are more than 250 different forces or parameter sets in use [67]. Most fully selfconsistent mass calculations are only as accurate (with an rms deviation in the range 3 to $5 \mathrm{MeV}$ ) as a simple liquiddrop model. To some there may seem to be exceptions; for example it is stated in Ref. [125]: For the first time purely microscopic, self-consistent models of the Hartree-Fock Bogoliubov series (here HFB21) show about equal or even better accuracy than the widely used macroscopic-microscopic models. The calculation referred to is Ref. [126], but in this work two purely phenomenological corrections (Eqs. (5) and (6)) with 9 adjustable constants are introduced. Therefore this calculation cannot be considered self-consistent.

Studies of how well models predict new masses that were not included in the determination of model parameters may reveal interesting model issues and give clues to model improvements. A problem with addressing this question is that it may be necessary to wait for several years before such a question can be addressed, unless one performs simulations like those we have undertaken in relation to Figs. 13-18, until sufficiently many new mass data become available. In 1992 we undertook such an investigation in Ref. [34], in which we compared the ratios of the model error with respect to about 350 new nuclei, which were not known when the models were published, to the model error in the region of nuclei to which the model parameters were adjusted. Eight different models were studied. For "non-divergent" models, this ratio should be close to one. For the Hilf/von Groote models, which were developed with the aim to be useful in r-process modeling, the ratios were 1.72 and 1.87 for the two versions of the model $[127,128]$, with increasingly severe divergences as one considers more neutron-rich nuclei. Three other investigated models have ratios ranging from 2.67 to 12.33 . The FRDM and FRLDM models have ratios of 1.10 and 1.06 respectively. The Seeger-Howard nuclear-structure model [123], which, apart from the FRDM and FRLDM is at this time the only other global mass model which obtains the microscopic corrections by solving a Schrödinger equation, has the ratio 1.36. 
In Ref. [24] we compared several models to newly measured masses. We also looked at $\alpha$-decay $Q$-values $Q_{\alpha}$ for the heaviest observed decay chains. In Ref. [39] we have further commented on and compared several models to the $Q_{\alpha}$ values for the decay of ${ }^{278} 113$ and discussed why HFB models, as so far implemented, have significant difficulties in determining correct ground-state masses and related quantities in the heavy-element region. Additional Dubna $Q_{\alpha}$ data is discussed in Ref. [91]. The FRDM and FRLDM compare extremely well to this new data which are about 40 nucleons distant from nuclei to which the models were adjusted.

\subsubsection{Ground-state deformations}

Intrinsic nuclear deformations are more difficult to determine experimentally than are nuclear masses; considerable model-dependence exists in the inference of deformations from data. Therefore, it is also somewhat difficult to compare calculated deformation multipoles to experimental "data". The gold standard for extracting deformations from experiments and for a systematic comparison to various model calculations is probably the work by Raman and collaborators [129], to which we refer for an extensive discussion. Although our FRDM(2012) obtained different deformations for a small number of nuclei, its calculated quadrupole deformations are sufficiently similar to those from FRDM(1992) that the Raman review of the FRDM(1992) is also relevant to our current results.

It has been well-known for a long time that calculated hexadecapole deformations $\beta_{4}$ are in good agreement with experimental results (although the values deduced from experiment are quite model-dependent); see Refs. [93, 94, 48]. More recently, we have also demonstrated that subbarrier fusion cross sections are well described when our calculated nuclear deformations of the target and projectiles are taken into account, including higher-multipole moments such as $\beta_{4}$, relative to treatments where the ground-state shape is assumed to be spherical [130].

We have studied the occurrence of octupole and axially asymmetric shapes in the current model, comparing with various experimental observations in Refs. [44, 45] and finding excellent agreement. For example, we obtain the largest effect of octupole deformation for ${ }^{222} \mathrm{Ra}$ which is the nuclide for which the lowest-lying negative-parity band has been observed. No other global nuclear mass calculations take these symmetry-breaking shapes into account.

We have also also studied in the current model shape coexistence across the nuclear chart in Refs. [25, 46], where detailed comparisons with experimental data can be found. Our calculated regions of shape coexistence agree well with the experimentally observed locations.

Another method to investigate how realistic the calculated ground-state deformations are is to study ground-state spins of odd- $A$ nuclei, because level ordering, and consequently ground-state spins. depend sensitively on deformation [37]. If the shape, even the higher multipole contributions to the shape, is not correct, then the ground-state spin will be incorrect. We found in Ref. [24] that we agreed to $62 \%$ with experimentally observed odd- $A$ spins in the previous FRDM(1992) calculations. In our current calculation we obtain agreement in $60 \%$ of the cases if we exclude nuclei with calculated axial and reflection asymmetry. More details about these studies will be forthcoming. Bonneau and collaborators reviewed several models in Ref. [131]. If nuclei in shape-transition, axially-asymmetric, octupole, and shape-coexistence regions were excluded, they found for the $\operatorname{FRDM}(1992)$ spin agreement in $75.4 \%$ of the cases, better than any of the three other models (SIII, SkM* and Sly4) they investigated.

\subsubsection{Indirect tests of the model}

The rapid neutron-capture process (r-process) has been extensively studied over the years. Our first entry into this field with nuclear masses and $\beta$-decay properties calculated in a consistent nuclear-structure model framework was through a 1993 study [132]. In a recent study we performed r-process simulations with nuclear masses provided by the $\operatorname{FRDM}(2012)$ and the $\beta$-decay properties obtained in a calculation (not yet published) based on the ground-state shapes provided here. The new data base led to an obviously better reproduction of the rare-earth abundance peak (without resorting to "fission recycling") than is obtained in calculations based on FRDM(1992) nuclear data [133].

New results on the cooling of the neutron star crust were recently obtained based on implementation of detailed networks of electron capture and $\beta^{-}$decay spectra from our general nuclear-structure model [134].

The values we obtain for the symmetry-energy constant $J$ and density symmetry constant $L$ in our parameter adjustment to nuclear masses are in excellent agreement with values obtained by other methods [68].

\subsubsection{New types of data and new insights}

We stated above that it is desirable for a proposed model to not only describe new data measurements, but also to be generalizable to description of new types of data.

We mentioned that the model was successfully applied to modeling subbarrier fusion experiments [130]. It has recently been extensively applied to the calculation of fission-fragment yields $[59,135,60]$ New calculations predict a new region of asymmetric fission in the neutron-deficient $\mathrm{Pb}$ region [89]; this prediction can be subjected to experimental tests in the near term.

We have in the FRLDM calculated fission-barrier heights for more than 5000 nuclides, in excellent agreement with what can be deduced from experiment, from $A \approx 70$ to $A \approx 290$, see Refs. [39, 61]. No other model reproduces barrier data over such an extended region of nuclei as accurately as this work.

Finally, the FRDM/FRLDM results were instrumental in understanding the stability of elements above $Z=106$ as having its origin in shell gaps for deformed nuclei [136, 24, 137, 138, 139]. 


\section{Acknowledgments}

We are grateful to G. Carlsson, T. Kawano, and P. Tamagno for pointing out misprints in equations in previous work, in particular in Ref. [9]. We wish to note that Ragnar Bengtsson was very closely involved in implementing axial asymmetry in the folded-Yukawa model. Ang Li provided us with her results on the effect of a tensor force. Discussion with K.-L Kratz, W. D. Myers, S. Reddy, and J. Stone are appreciated.

This work profited from extensive comments by and collaborations with Japanese colleagues made possible by numerous and generous travel grants for P.M. to JUSTIPEN (Japan-U.S. Theory Institute for Physics with Exotic Nuclei) under grant number DE-FG02-06ER41407 (U. Tennessee). This work was carried out under the auspices of the NNSA of the U.S. Department of Energy at Los Alamos National Laboratory under Contract No. DE-AC52-06NA25396. TI was supported in part by MEXT SPIRE and JICFuS and Grant no. 25287065. 
[1] P. Möller and J. R. Nix, Nucl. Phys. A361 (1981) 117.

[2] P. Möller and J. R. Nix, Atomic Data Nucl. Data Tables 26 (1981) 165.

[3] P. Möller and J. R. Nix, Atomic Data Nucl. Data Tables 39 (1988) 213.

[4] P. Möller, W. D. Myers, W. J. Swiatecki, and J. Treiner, Atomic Data Nucl. Data Tables 39 (1988) 225.

[5] P. Möller, W. D. Myers, W. J. Swiatecki, and J. Treiner, Proc. 7th Int. Conf. on nuclear masses and fundamental constants, Darmstadt-Seeheim, 1984 (Lehrdruckerei, Darmstadt, 1984) p. 457.

[6] W. D. Myers and W. J. Swiatecki, Ann. Phys. (N. Y.) 55 (1969) 395.

[7] W. D. Myers and W. J. Swiatecki, Ann. Phys. (N. Y.) 84 (1974) 186.

[8] W. D. Myers, Droplet model of atomic nuclei (IFI/Plenum, New York, 1977).

[9] P. Möller, J. R. Nix, W. D. Myers, and W. J. Swiatecki, Atomic Data Nucl. Data Tables 59 (1995) 185.

[10] M. Bolsterli, E. O. Fiset, J. R. Nix, and J. L. Norton, Phys. Rev. C 5 (1972) 1050.

[11] V. M. Strutinsky, Nucl. Phys. A95 (1967) 420.

[12] V. M. Strutinsky, Nucl. Phys. A122 (1968) 1.

[13] H. J. Lipkin, Ann. Phys. (N. Y.) 9 (1960) 272.

[14] Y. Nogami, Phys. Rev. 134 (1964) B313.

[15] H. C. Pradhan, Y. Nogami, and J. Law, Nucl. Phys. A201 (1973) 357.

[16] P. Möller and J. R. Nix, Nucl. Phys. A536 (1992) 20.

[17] G. Audi, A. H. Wapstra, and C. Thibault, Nucl. Phys. A729 (2003) 337.

[18] G. Audi, Midstream atomic mass evaluation, private communication (1989), with four revisions.

[19] S. Hofmann, V. Ninov, F. P. Heßberger, P. Armbruster, H. Folger, G. Münzenberg, H. J. Schött, A. G. Popeko, A. V. Yeremin, A. N. Andreyev, S. Saro, R. Janik, and M. Leino, Z. Phys. A350 (1995) 277.

[20] S. Hofmann, V. Ninov, F. P. Heßberger, P. Armbruster, H. Folger, G. Münzenberg, H. J. Schött, A. G. Popeko, A. V. Yeremin, A. N. Andreyev, S. Saro, R. Janik, and M. Leino, Z. Phys. A350 (1995) 281.

[21] S. Hofmann, V. Ninov, F. P. Heßberger, P. Armbruster, H. Folger, G. Münzenberg, H. J. Schött, A. G. Popeko, A. V. Yeremin, S. Saro, R. Janik, and M. Leino, Z. Phys. A354 (1996) 229.

[22] Yu. Ts. Oganessian, J. Phys. G: Nucl. Part. Phys. 34 (2007) R165.

[23] Yu. Ts. Oganessian, F. Sh. Abdullin, P. D. Bailey, D. E. Benker, M. E. Bennett, S. N. Dmitriev, J. G. Ezold, J. H. Hamilton, R. A. Henderson, M. G. Itkis, Yu.V. Lobanov, A. N. Mezentsev, K. J. Moody, S. L. Nelson, A. N. Polyakov, C. E. Porter, A.V. Ramayya, F. D. Riley, J. B. Roberto, M. A. Ryabinin, K. P. Rykaczewski, R. N. Sagaidak, D. A. Shaughnessy, I.V. Shirokovsky, M. A. Stoyer, V. G. Subbotin, R. Sudowe, A. M. Sukhov, Yu. S. Tsyganov, V. K. Utyonkov, A. A. Voinov, G. K. Vostokin, and P. A. Wilk, Phys. Rev. Lett. 104 (2010) 142502.

[24] P. Möller, J. R. Nix, and K.-L. Kratz, Atomic Data Nucl. Data Tables 66 (1997) 131.

[25] P. Möller, A. J. Sierk, R. Bengtsson, H. Sagawa, and T. Ichikawa, Phys. Rev. Lett. 103 (2009) 212501.

[26] P. Möller and A. J. Sierk, Int. J. Mass Spectrom. 349-350 (2013) 19.

[27] G. A. Leander, R. K. Sheline, P. Möller, P. Olanders, I. Ragnarsson, and A. J. Sierk, Nucl. Phys. A388 (1982) 452.

[28] W. Nazarewicz, P. Olanders, I. Ragnarsson, J. Dudek, G. A. Leander, P. Möller, and E. Ruchowska, Nucl. Phys. A429 (1984) 269.

[29] G. A. Leander and Y. S. Chen, Phys. Rev. C 37 (1988) 2744.

[30] W. D. Myers and W. J. Swiatecki, Nucl. Phys. 81 (1966) 1.

[31] W. D. Myers and W. J. Swiatecki, Ark. Fys. 36 (1967) 343.

[32] P. Möller and J. R. Nix, Nucl. Phys. A229 (1974) 269.

[33] H. J. Krappe, J. R. Nix, and A. J. Sierk, Phys. Rev. C 20 (1979) 992.

[34] P. Möller and J. R. Nix, Proc. 6th Int. Conf. on nuclei far from stability and 9th Int. Conf. on nuclear masses and fundamental constants, Bernkastel-Kues, 1992 (IOP Publishing, Bristol, 1993) p. 43.

[35] J. R. Nix, Nucl. Phys. A130 (1969) 241.

[36] P. Möller and J. R. Nix, Proc. Third IAEA Symp. on the physics and chemistry of fission, Rochester, 1973, vol. I (IAEA, Vienna, 1974) p. 103.

[37] P. Möller, S. G. Nilsson, and J. R. Nix, Nucl. Phys. A229 (1974) 292.

[38] P. Möller, D. G. Madland, A. J. Sierk, and A. Iwamoto, Nature 409 (2001) 785. 
[39] P. Möller, A. J. Sierk, T. Ichikawa, A. Iwamoto, R. Bengtsson, H. Uhrenholt, and S. Åberg, Phys. Rev. C 79 (2009) 064304.

[40] S. G. Nilsson, Kgl. Danske Videnskab. Selskab. Mat.-Fys. Medd. 29:No. 16 (1955).

[41] S. E. Larsson, I. Ragnarsson, and S. G. Nilsson, Phys. Lett. 38B (1972) 269.

[42] S. E. Larsson, Phys. Scr. 8 (1973) 17.

[43] T. Bengtsson and I. Ragnarsson, Nucl. Phys. A436 (1985) 14.

[44] P. Möller, R. Bengtsson, B. G. Carlsson, P. Olivius, and T. Ichikawa, Phys. Rev. Lett. 97 (2006) 162502.

[45] P. Möller, R. Bengtsson, B. G. Carlsson, P. Olivius, T. Ichikawa, H. Sagawa, and A. Iwamoto ATOMIC DATA AND NuClear Data TABles 94 (2008) 758.

[46] P. Möller, A. J. Sierk, R. Bengtssson, H. Sagawa, and T. Ichikawa, Atomic Data AND Nuclear DatA TABLES, 98 (2012) 149.

[47] E. O. Fiset and J. R. Nix, Nucl. Phys. A193 (1972) 647.

[48] P. Möller and J. R. Nix, Nucl. Phys. A272 (1976) 502.

[49] P. Möller and J. R. Nix, Phys. Rev. Lett. 37 (1976) 1461.

[50] P. Möller and J. R. Nix, Nucl. Phys. A281 (1977) 354.

[51] P. Möller, J. R. Nix, and W. J. Swiatecki, Nucl. Phys. A469 (1987) 1.

[52] P. Möller, J. R. Nix, and W. J. Swiatecki, Nucl. Phys. A492 (1989) 349.

[53] P. Möller and A. Iwamoto, Proc. Conf. on Nuclear Shapes and Motions. Symposium in Honor of Ray Nix, 25-27 Oct. 1998, Sante Fe, NM, USA Acta Physica Hungarica, New Series, 10 (1999) 241.

[54] P. Möller and A. Iwamoto, Phys. Rev. C 61 (2000) 047602.

[55] P. Möller, D. G. Madland, A. J. Sierk, and A. Iwamoto, Tours 2000, Tours Symposium on Nuclear Physics IV, Tours, France September 4-7, 2000, and AIP Conference Proceedings 561 (2001) p. 455.

[56] P. Möller, D. G. Madland, A. J. Sierk, and A. Iwamoto, Proc. International Conference on Nuclear Data for Science and Technology (ND2001), October 7-12, Tsukuba, Japan, Journal of Nuclear Science and Technology, Supplement 2, (2002) pp. 703-708.

[57] P. Möller, A. J. Sierk, and A. Iwamoto, Phys. Rev. Lett. 92 (2004) 072501.

[58] T. Ichikawa, A. Iwamoto, P. Möller, and A. J. Sierk, Phys. Rev. C71 (2005) 044608.

[59] J. Randrup and P. Möller, Phys. Rev. Lett. 106 (2011) 132503.

[60] J. Randrup and P. Möller, Phys. Rev. C 88 (2013) 064606.

[61] P. Möller, A. J. Sierk, T. Ichikawa, A. Iwamoto, and M. Mumpower, Phys. Rev. C 91 (2015) 024310.

[62] H. J. Krappe and J. R. Nix, Proc. Third IAEA Symp. on the physics and chemistry of fission, Rochester, 1973, vol. I (IAEA, Vienna, 1974) p. 159.

[63] K. T. R. Davies, A. J. Sierk, and J. R. Nix, Phys. Rev. C 13 (1976) 2385.

[64] D. G. Madland and J. R. Nix, Bull. Am. Phys. Soc. 31 (1986) 799.

[65] D. G. Madland and J. R. Nix, Nucl. Phys. A476 (1988) 1.

[66] P. J. Mohr, B. N. Taylor, and D. B. Newell, Rev. Mod. Phys. 84 (2012) 1527.

[67] M. Dutra, O. Lourenço, J. S. Sá Martins, A. Delfino, J. R. Stone, and P. S. Stevenson, Phys. Rev. C 85 (2012) 035201.

[68] M.B. Tsang, J. R. Stone, F. Camera, P. Danielewicz, S. Gandolfi, K. Hebeler, C. J. Horowitz, Jenny Lee, W.G. Lynch, Z. Kohley, R. Lemmon, P. Möller, T. Murakami, S. Riordan, X. Roca-Maza, F. Sammarruca, A. W. Steiner, I. Vidaa, S.J. Yennello, Phys. Rev. C 86 (2012) 015803.

[69] http://physics.nist.gov/.

[70] P. Möller et. al., Atomic Data And Nuclear Data TAbles, to be published.

[71] W. D. Myers, Nucl. Phys. 145 (1970) 387.

[72] R. Bengtsson, P. Möller, J. R. Nix, and Jing-ye Zhang, Phys. Scr. 29 (1984) 402.

[73] Å. Bohr, B. R. Mottelson, and D. Pines, Phys. Rev. 110 (1958) 936.

[74] S. T. Belyaev, Kgl. Danske Videnskab. Selskab. Mat.-Fys. Medd. 31:No. 11 (1959).

[75] S. G. Nilsson and O. Prior, Kgl. Danske Videnskab. Selskab. Mat.-Fys. Medd. 32:No. 16 (1961).

[76] W. Ogle, S. Wahlborn, R. Piepenbring, and S. Fredriksson, Rev. Mod. Phys. 43 (1971) 424. 
[77] S. G. Nilsson, C. F. Tsang, A. Sobiczewski, Z. Szymański, S. Wycech, C. Gustafson, I.-L. Lamm, P. Möller, and B. Nilsson, Nucl. Phys. A131 (1969) 1.

[78] J. R. Nix, Ann. Rev. Nucl. Sci. 22 (1972) 65.

[79] P. E. Haustein, Atomic Data Nucl. Data Tables 39 (1988) 185.

[80] L. Spanier and S. A. E. Johansson, Atomic Data Nucl. Data Tables 39 (1988) 259.

[81] M. Wang, G. Audi, A. H. Wapstra, F. G. Kondev, M. MacCormick, X. Xu, and B. Pfeiffer, Chin. Phys. C36 (2012) 1603.

[82] P. Möller, Proc. 4th IAEA Symp. on physics and chemistry of fission, Jülich, 1979, vol. I (IAEA, Vienna, 1980) p. 283.

[83] P. Möller, R. Bengtsson, K.-L. Kratz, and H. Sagawa, Proc. International Conference on Nuclear Data and Technology, April 22-27, 2007, Nice, France, (EDP Sciences, (2008) p. 69, ISBN 978-2-7598-0090-2), and http://t2.lanl.gov/nis/molleretal/publications/nd2007.html.

[84] URL:http://t2.lanl.gov/nis/molleretal.

[85] E. Haettner, D. Ackermann, G. Audi, K. Blaum, M. Block, S. Eliseev, T. Fleckenstein, F. Herfurth, F. P. Heßberger, S. Hofmann, J. Ketelaer, J. Ketter, H.-J. Kluge, G. Marx, M. Mazzocco, Yu. N. Novikov, W. R. Plaß, S. Rahaman, T. Rauscher, W. R. Rodríguez, H. Schatz, C. Scheidenberger, L. Schweikhard, B. Sun, P. G. Thirolf, G. Vorobjev, M. Wang, and C. Weber, Phys. Rev. Lett. 106 (2011) 122501.

[86] H. A. Bethe and R. F. Bacher, Rev. Mod. Phys. 8 (1936) 82.

[87] P. Möller, W. D. Myers, H. Sagawa, and S. Yoshida, Phys. Rev. Lett. 108 (2012) 052501.

[88] P. Möller, J. R. Nix, W. D. Myers, and W. J. Swiatecki, Nucl. Phys. A536 (1992) 61.

[89] P. Möller and J. Randrup, Phys. Rev. C 91 (2015) 044316.

[90] A. E. S. Green, Nuclear physics (McGraw-Hill, New York, 1955) pp. 185, 250.

[91] P. Möller, Int. J. Mod. Phys. E-Nucl. Phys. 19 (2010) 575.

[92] I. Hamamoto and B. R. Mottelson, Phys. Rev. C 79 (2009) 034317.

[93] P. Möller, B. Nilsson, S. G. Nilsson, A. Sobiczewski, Z. Szymański, and S. Wycech, Phys. Lett. 26B (1968) 418.

[94] P. Möller, Nucl. Phys. A142 (1970) 1.

[95] G. Audi and W. Meng, Private Communication, April 2011.

[96] P. Möller, A. J. Sierk, R. Bengtsson, T. Ichikawa, A. Iwamoto, The 10th Int. Symp. on Origin of Matter and Evolution of Galaxies - From the Dawn of Universe to the Formation of Solar System - OMEG07 - December 4-7, Hokkaido University Sapporo, Japan, AIP Conference Proceedings, 1016 (2008) 150.

[97] P. Möller and J. R. Nix, J. Phys. G 20 (1994) 1681.

[98] P. Möller, J. Randrup, and A. J. Sierk, Phys. Rev. C 85 (2012) 024306.

[99] P. Danielewicz, R. Lacey, and W. G. Lynch, Science 298 (2002) 1592.

[100] Bao-An Li, Lie-Wen Chen, and Che Ming Ko, Phys. Rep. 464 (2008) 113.

[101] A. Carbone, G. Colò, A. Bracco, Li-Gang Cao, P. F. Bortignon, F. Camera, and O. Wieland Phys. Rev. C 81 (2010) 041301(R).

[102] A. Klimkiewicz, N. Paar, P. Adrich, M. Fallot, K. Boretzky, T. Aumann, D. Cortina-Gil, U. Datta Pramanik, Th. W. Elze, H. Emling, H. Geissel, M. Hellström, K. L. Jones, J. V. Kratz, R. Kulessa, C. Nociforo, R. Palit, H. Simon, G. Surówka, K. Sümmerer, D. Vretenar, and W. Waluś (LAND Collaboration), Phys. Rev. C 76 (2007) 051603(R).

[103] A. Tamii, I. Poltoratska, P. von Neumann-Cosel, Y. Fujita, T. Adachi, C. A. Bertulani, J. Carter, M. Dozono, H. Fujita, K. Fujita, K. Hatanaka, D. Ishikawa, M. Itoh, T. Kawabata, Y. Kalmykov, A. M. Krumbholz, E. Litvinova, H. Matsubara, K. Nakanishi, R. Neveling, H. Okamura, H. J. Ong, B. Özel-Tashenov, V. Yu. Ponomarev, A. Richter, B. Rubio, H. Sakaguchi, Y. Sakemi, Y. Sasamoto, Y. Shimbara, Y. Shimizu, F. D. Smit, T. Suzuki, Y. Tameshige, J. Wambach, R. Yamada, M. Yosoi, and J. Zenihiro, Phys. Rev. Lett. 107 (2011) 062502.

[104] X. Roca-Maza, M. Brenna, G. Colò, M. Centelles, X. Viñas, B. K. Agrawal, N. Paar, D. Vretenar, and J. Piekarewicz, Phys. Rev. C 88 (2013) 024316.

[105] Li-Gang Cao, X. Roca-Maza, G. Colò, and H.Sagawa, arXiv:1504.07166(2015).

[106] J. Yasuda, T. Wakasa, M. Okamoto, M. Dozono, K. Hatanaka, M. Ichimura, S. Kuroita, Y. Maeda, T. Noro, Y. Sakemi, M. Sasano, and K. Yako, Prog. Theor. Exp. Phys. 063D02 (2013).

[107] Li-Gang Cao, H. Sagawa, and G. Colò, Phys. Rev. C 86 (2012) 054313.

[108] D. Patel, U. Garg, M. Fujiwara, H. Akimune, G.P.A. Berg, M.N. Harakeh, M. Itoh, T. Kawabata, K. Kawase, 
B.K. Nayak, T. Ohta, H. Ouchi, J. Piekarewicz, M. Uchida, H.P. Yoshida, M. Yosoi, Phys. Lett. B 718 (2012) 447.

[109] T. Li, U. Garg, Y. Liu, R. Marks, B. K. Nayak, and P. V. Madhusudhana Rao, M. Fujiwara, H. Hashimoto, K. Nakanishi, S. Okumura, and M. Yosoi, M. Ichikawa, M. Itoh, R. Matsuo, and T. Terazono, M. Uchida, Y. Iwao, T. Kawabata, T. Murakami, H. Sakaguchi, S. Terashima, Y. Yasuda, and J. Zenihiro, H. Akimune, K. Kawase, and M. N. Harakeh, Phys. Rev. C 81 (2010) 034309.

[110] P. Danielewicz and J. Lee, Nucl. Phys. A 818 (2009) 36.

[111] A. W. Steiner and S. Gandolfi, Phys. Rev. Lett. 108 (2012) 081102.

[112] J. M. Lattimer and Y. Lim, Astrophys. J. 771, (2013) 51.

[113] K. Hebeler, J. M. Lattimer, C. J. Pethick, and A. Schwenk, Phys. Rev. Lett. 105 (2010) 161102.

[114] S. Gandolfi, J. Carlson, and S. Reddy, Phys. Rev. C 85 (2012) 032801(R).

[115] A. T. Kruppa, Phys. Lett. B 431 (1998) 237.

[116] P. Salamon, A. T. Kruppa, and T. Vertse, Phys. Rev. C 81 (2010) 064322.

[117] V. A. Ramamurthy, S. S. Kapoor, and S. K. Kataria, Phys. Rev. Lett. 25, (1970) 386.

[118] Sagawa and G. Colò, Progress in Particle and Nuclear Physics (edited by A. Faessler), 76 (2014) 76, and references therein.

[119] D. Vautherin and D. M. Brink, Phys. Rev. C 5 (1972) 626.

[120] O. Hahn and F. Strassmann, Naturwiss. 27 (1939) 11.

[121] L. Meitner and O. R. Frisch, Nature 143 (1939) 239.

[122] N. Bohr and J. A. Wheeler, Phys. Rev. 56 (1939) 426.

[123] P. A. Seeger and W. M. Howard, Nucl. Phys. A238 (1975) 491.

[124] P. A. Seeger and W. M. Howard, Atomic Data Nucl. Data Tables 17 (1976) 428.

[125] A. Sobiczewski and Y. A. Litvinov, Phys. Rev. C 89 (2014) 024311.

[126] S. Goriely, N. Chamel, and J. M. Pearson,, Phys. Rev. C 82 (2010) 035804.

[127] E. R. Hilf, H. von Groote, and K. Takahashi, Proc. 3rd Int. Conf. on nuclei far from stability, Cargèse, Corsica, France, 1976, CERN Report CERN 76-13 (1976) p. 142.

[128] H. von Groote, E. R. Hilf, and K. Takahashi, Atomic Data Nucl. Data Tables 17 (1976) 418.

[129] S. Raman, C. W. Nestor, Jr, and P. Tikkanen, Atomic Data NuCl. Data Tables 78 (2001) 1.

[130] A. Iwamoto, P. Möller, J. R. Nix, and H. Sagawa, Nucl. Phys. A596 (1996) 329.

[131] L Bonneau, P. Quentin, and P. Möller, Phys. Rev. C 76 (2007) 024320.

[132] K.-L. Kratz, J.-P. Bitouzet, F.-K. Thielemann, P. Möller, and B. Pfeiffer, Astrophys. J. 403 (1993) 216.

[133] K.-L. Kratz, K. Farouqi, and P. Möller, Astrophys. J. 792 (2014) 6.

[134] H. Schatz, S. Gupta, P. Möller, M. Beard, E. F. Brown, A. T. Deibel, L. R. Gasques, W. R. Hix, L. Keek, R. Lau, A. W. Steiner, and M. Wiescher, Nature 505 (2014) 62.

[135] J. Randrup, P. Möller, and A. J. Sierk, Phys. Rev. C 84 (2011) 034613.

[136] P. Armbruster, Ann. Rev. Nucl. Part. Sci. 35 (1985) 135.

[137] P. Armbruster and G. Münzenberg. Eur. Phys. J. H 37 (2012) 237.

[138] S. Hofmann, Radiochim. Acta 99 (2011) 405.

[139] S. Hofmann, Phys. Unserer Zeit 43 (2012) 30. 
Z Proton number. The mass table is ordered by increasing proton number. The corresponding chemical symbol of each named element is given in parentheses.

$N \quad$ Neutron number.

A Mass Number.

$\varepsilon_{2} \quad$ Calculated ground-state quadrupole deformation in the Nilsson perturbed-spheroid parameterization.

$\varepsilon_{3} \quad$ Calculated ground-state octupole deformation in the Nilsson perturbed-spheroid parameterization.

$\varepsilon_{4} \quad$ Calculated ground-state hexadecapole deformation in the Nilsson perturbed-spheroid parameterization.

$\varepsilon_{6} \quad$ Calculated ground-state hexacontatetrapole deformation in the Nilsson perturbed-spheroid parameterization.

$\beta_{2} \quad$ Calculated quadrupole deformation of the nuclear ground-state expressed in a spherical-harmonics expansion. The exact definition is given by Eq. (38).

$\beta_{3} \quad$ Calculated octupole deformation of the nuclear ground-state expressed in a spherical-harmonics expansion.

$\beta_{4} \quad$ Calculated hexadecapole deformation of the nuclear ground-state expressed in a spherical-harmonics expansion.

$\beta_{6} \quad$ Calculated hexacontatetrapole deformation of the nuclear ground-state expressed in a sphericalharmonics expansion.

$E_{\mathrm{s}+\mathrm{p}} \quad$ Calculated ground-state shell-plus-pairing correction. For a specific deformation this number is independent of the macroscopic model and depends only on the single-particle model.

$E_{\text {mic }} \quad$ Calculated ground-state microscopic energy, given by the difference between the calculated groundstate atomic mass excess and the spherical macroscopic energy calculated in in our preferred mass model, the FRDM (2012).

$E_{\text {bind }} \quad$ Calculated ground-state binding energy, calculated in in our preferred mass model, the FRDM (2012).

$M_{\text {th }} \quad$ Calculated ground-state atomic mass excess, in our preferred mass model, the FRDM (2012).

$M_{\text {exp }} \quad$ Experimental ground-state atomic mass excess in the AME2003 evaluation (Nucl. Phys. A 729 (2003) 337).

$\sigma_{\exp } \quad$ Experimental error associated with the ground-state atomic mass excess in the AME2003 evaluation (Nucl. Phys. A 729 (2003) 337).

$E_{\text {mic }}^{\mathrm{FL}} \quad$ Calculated ground-state microscopic energy, given by the difference between the calculated groundstate atomic mass excess and the spherical macroscopic energy calculated in the FRLDM (2012).

$M_{\mathrm{th}}^{\mathrm{FL}} \quad$ Calculated ground-state atomic mass excess, in the FRLDM (2012).

We note again that in the table effects of axial asymmetry on the calculated energy quantities are included; only a few nuclei are affected. However, for reasons of space, the listed deformations refer to the ground-state shape obtained when axial asymmetry is not considered. As discussed in Sect. 4, item 2, these details are available in previous publications. 


\begin{tabular}{|c|c|c|c|c|c|c|c|c|c|c|c|c|c|c|c|c|}
\hline \multicolumn{17}{|c|}{$Z=8(O)$} \\
\hline $8 \quad 16$ & -0.03 & 0.20 & 0.12 & -0.02 & -0.010 & -0.258 & -0.122 & 0.047 & -0.62 & 2.42 & 128.03 & -5.15 & -4.74 & 0.000 & 2.40 & -3.66 \\
\hline 917 & 0.05 & 0.01 & -0.12 & -0.02 & 0.061 & -0.014 & 0.152 & 0.035 & 1.82 & 3.74 & 132.45 & -1.49 & -0.81 & 0.000 & 3.71 & -0.04 \\
\hline 1018 & 0.01 & 0.00 & -0.04 & 0.02 & 0.010 & 0.000 & 0.048 & -0.019 & 1.60 & 3.42 & 141.84 & -2.81 & -0.78 & 0.001 & 3.42 & -1.39 \\
\hline 1119 & -0.01 & 0.00 & -0.04 & 0.02 & -0.010 & 0.000 & 0.047 & -0.020 & 2.23 & 3.78 & 144.95 & 2.15 & 3.34 & 0.003 & 3.78 & 3.46 \\
\hline 1220 & 0.01 & 0.00 & 0.02 & -0.02 & 0.010 & 0.000 & -0.024 & 0.020 & 1.10 & 2.52 & 152.98 & 2.19 & 3.80 & 0.001 & 2.52 & 3.36 \\
\hline 1321 & 0.09 & 0.00 & 0.03 & -0.03 & 0.096 & 0.000 & -0.034 & 0.027 & 0.37 & 2.02 & 155.15 & 8.09 & 8.06 & 0.012 & 2.05 & 9.11 \\
\hline 1422 & 0.00 & 0.00 & -0.01 & 0.03 & 0.000 & 0.000 & 0.012 & -0.030 & -1.14 & 0.73 & 161.39 & 9.92 & 9.28 & 0.057 & 0.76 & 10.75 \\
\hline 1523 & 0.00 & 0.00 & 0.00 & 0.03 & 0.000 & 0.000 & 0.000 & -0.029 & -1.89 & 0.18 & 162.16 & 17.22 & 14.61 & 0.122 & 0.22 & 17.83 \\
\hline 1624 & 0.00 & 0.00 & 0.02 & 0.03 & 0.001 & 0.000 & -0.023 & -0.029 & -2.09 & -0.17 & 165.98 & 21.48 & 19.07 & 0.236 & -0.10 & 21.90 \\
\hline 1725 & 0.04 & 0.00 & 0.02 & 0.03 & 0.043 & 0.000 & -0.022 & -0.030 & -2.03 & -0.06 & 164.91 & 30.61 & & & 0.02 & 30.82 \\
\hline 1826 & 0.00 & 0.00 & 0.00 & 0.03 & 0.000 & 0.000 & 0.000 & -0.029 & -2.06 & -0.33 & 167.41 & 36.19 & & & -0.25 & 36.16 \\
\hline 1927 & -0.02 & 0.00 & 0.00 & 0.03 & -0.021 & 0.000 & 0.000 & -0.029 & -2.57 & -0.35 & 165.50 & 46.17 & & & -0.26 & 45.93 \\
\hline $20 \quad 28$ & 0.00 & 0.00 & 0.00 & -0.03 & 0.000 & 0.000 & 0.000 & 0.030 & -2.87 & -0.48 & 166.82 & 52.92 & & & -0.37 & 52.47 \\
\hline 2129 & 0.08 & 0.00 & -0.05 & -0.03 & 0.087 & 0.000 & 0.064 & 0.037 & -2.10 & -0.34 & 163.92 & 63.89 & & & 0.05 & 63.50 \\
\hline 2230 & 0.10 & 0.00 & -0.10 & -0.03 & 0.114 & 0.000 & 0.131 & 0.049 & -1.84 & -1.22 & 165.12 & 70.76 & & & -0.00 & 70.99 \\
\hline $23 \quad 31$ & 0.18 & 0.00 & -0.12 & -0.04 & 0.205 & 0.000 & 0.170 & 0.081 & -2.15 & -2.01 & 162.45 & 81.50 & & & 0.30 & 82.64 \\
\hline $24 \quad 32$ & 0.19 & 0.00 & -0.12 & -0.04 & 0.216 & 0.000 & 0.172 & 0.083 & -2.01 & -2.40 & 162.41 & 89.61 & & & 0.22 & 90.88 \\
\hline 2533 & 0.22 & 0.00 & -0.05 & 0.00 & 0.238 & 0.000 & 0.083 & 0.017 & -1.64 & -0.50 & 156.45 & 103.64 & & & -0.03 & 102.60 \\
\hline 2634 & 0.17 & 0.00 & 0.04 & 0.04 & 0.187 & 0.000 & -0.032 & -0.046 & -1.39 & -0.57 & 155.44 & 112.72 & & & 0.10 & 111.74 \\
\hline 2735 & -0.28 & 0.00 & -0.10 & 0.04 & -0.284 & 0.000 & 0.137 & -0.068 & -2.62 & -3.28 & 153.57 & 122.67 & & & -0.70 & 123.47 \\
\hline $28 \quad 36$ & -0.32 & 0.00 & -0.12 & 0.04 & -0.322 & 0.000 & 0.167 & -0.078 & -3.40 & -4.18 & 152.82 & 131.49 & & & -0.39 & 133.39 \\
\hline 2937 & -0.27 & 0.00 & -0.06 & 0.04 & -0.276 & 0.000 & 0.090 & -0.056 & -3.07 & -2.51 & 146.10 & 146.28 & & & -0.82 & 145.99 \\
\hline $30 \quad 38$ & -0.20 & 0.00 & 0.05 & -0.04 & -0.208 & 0.000 & -0.038 & 0.047 & -2.61 & -2.15 & 143.61 & 156.84 & & & -1.14 & 155.81 \\
\hline 3139 & -0.20 & 0.00 & 0.06 & -0.04 & -0.209 & 0.000 & -0.049 & 0.049 & -3.07 & -2.80 & 138.81 & 169.71 & & & -1.59 & 168.83 \\
\hline 3240 & -0.15 & 0.00 & 0.12 & -0.04 & -0.158 & 0.000 & -0.124 & 0.060 & -3.12 & -4.78 & 138.21 & 178.38 & & & -1.85 & 179.20 \\
\hline 3341 & -0.15 & 0.00 & 0.12 & -0.05 & -0.159 & 0.000 & -0.124 & 0.069 & -3.26 & -5.55 & 133.19 & 191.48 & & & -2.12 & 192.79 \\
\hline $34 \quad 42$ & -0.17 & 0.00 & 0.08 & -0.05 & -0.179 & 0.000 & -0.076 & 0.062 & -2.66 & -3.74 & 128.40 & 204.34 & & & -1.49 & 204.49 \\
\hline \multicolumn{17}{|c|}{$Z=9(\mathbf{F})$} \\
\hline $\begin{array}{ll}8 & 17\end{array}$ & 0.05 & 0.00 & -0.12 & -0.02 & 0.061 & 0.000 & 0.152 & 0.035 & 1.24 & 3.14 & 129.22 & 0.95 & 1.95 & 0.000 & 3.11 & 2.01 \\
\hline 918 & 0.16 & 0.00 & -0.12 & -0.02 & 0.180 & 0.000 & 0.165 & 0.054 & 2.93 & 6.08 & 136.73 & 1.51 & 0.87 & 0.001 & 5.93 & 0.85 \\
\hline 1019 & 0.24 & 0.00 & -0.12 & 0.02 & 0.262 & 0.000 & 0.180 & 0.025 & 2.43 & 5.87 & 148.51 & -2.19 & -1.49 & 0.000 & 5.77 & -1.12 \\
\hline 1120 & 0.26 & 0.00 & -0.10 & 0.02 & 0.283 & 0.000 & 0.159 & 0.021 & 2.83 & 6.25 & 154.18 & 0.20 & -0.02 & 0.000 & 6.14 & 1.23 \\
\hline 1221 & 0.25 & 0.00 & -0.05 & 0.03 & 0.270 & 0.000 & 0.092 & -0.010 & 2.15 & 4.98 & 162.95 & -0.49 & -0.05 & 0.002 & 4.96 & 0.55 \\
\hline 1322 & 0.21 & 0.00 & -0.03 & 0.03 & 0.226 & 0.000 & 0.058 & -0.020 & 2.04 & 4.39 & 167.59 & 2.93 & 2.79 & 0.012 & 4.36 & 3.87 \\
\hline 1423 & 0.11 & 0.00 & -0.06 & 0.03 & 0.117 & 0.000 & 0.079 & -0.021 & 1.07 & 2.85 & 174.70 & 3.90 & 3.33 & 0.080 & 2.91 & 4.77 \\
\hline 1524 & 0.09 & 0.00 & -0.06 & 0.03 & 0.095 & 0.000 & 0.077 & -0.023 & 0.52 & 2.36 & 177.60 & 9.07 & 7.56 & 0.072 & 2.43 & 9.80 \\
\hline 1625 & 0.11 & 0.00 & -0.04 & -0.03 & 0.119 & 0.000 & 0.054 & 0.037 & 0.19 & 2.10 & 181.85 & 12.89 & 11.27 & 0.098 & 2.20 & 13.47 \\
\hline 1726 & 0.11 & 0.00 & 0.00 & -0.03 & 0.118 & 0.000 & 0.004 & 0.030 & 0.19 & 2.21 & 182.80 & 20.01 & 18.27 & 0.167 & 2.26 & 20.35 \\
\hline $18 \quad 27$ & 0.11 & 0.00 & 0.00 & 0.03 & 0.118 & 0.000 & 0.007 & -0.030 & 0.10 & 2.05 & 185.65 & 25.24 & 24.93 & 0.377 & 2.12 & 25.40 \\
\hline 1928 & 0.08 & 0.00 & -0.01 & 0.03 & 0.085 & 0.000 & 0.016 & -0.029 & -0.25 & 1.73 & 185.90 & 33.06 & & & 1.81 & 33.03 \\
\hline $20 \quad 29$ & -0.03 & 0.00 & -0.01 & -0.03 & -0.031 & 0.000 & 0.013 & 0.029 & -0.56 & 1.39 & 187.81 & 39.21 & & & 1.48 & 39.00 \\
\hline 2130 & 0.10 & 0.00 & -0.07 & -0.03 & 0.111 & 0.000 & 0.091 & 0.042 & -0.29 & 1.05 & 187.13 & 47.97 & & & 1.54 & 47.96 \\
\hline 2231 & 0.18 & 0.00 & -0.12 & -0.04 & 0.205 & 0.000 & 0.170 & 0.081 & -1.00 & 0.15 & 188.68 & 54.49 & & & 1.87 & 55.52 \\
\hline $23 \quad 32$ & 0.21 & 0.00 & -0.12 & 0.02 & 0.229 & 0.000 & 0.173 & 0.019 & -1.22 & 0.46 & 186.52 & 64.72 & & & 1.61 & 65.00 \\
\hline 2433 & 0.23 & 0.00 & -0.12 & 0.04 & 0.249 & 0.000 & 0.178 & 0.001 & -1.43 & -0.18 & 187.00 & 72.31 & & & 1.17 & 72.61 \\
\hline $25 \quad 34$ & 0.26 & 0.00 & -0.08 & 0.04 & 0.281 & 0.000 & 0.133 & -0.009 & -1.36 & 0.31 & 183.96 & 83.42 & & & 1.13 & 83.03 \\
\hline 2635 & 0.24 & 0.00 & -0.04 & 0.04 & 0.259 & 0.000 & 0.078 & -0.025 & -0.94 & 0.48 & 182.94 & 92.52 & & & 1.00 & 91.68 \\
\hline 2736 & 0.26 & 0.00 & 0.07 & 0.04 & 0.292 & 0.000 & -0.049 & -0.057 & -1.32 & -0.15 & 180.41 & 103.11 & & & 0.80 & 102.58 \\
\hline $28 \quad 37$ & -0.30 & 0.00 & -0.12 & 0.04 & -0.302 & 0.000 & 0.163 & -0.076 & -2.50 & -3.00 & 181.80 & 109.79 & & & 0.07 & 111.26 \\
\hline 2938 & -0.27 & 0.00 & -0.07 & 0.04 & -0.275 & 0.000 & 0.102 & -0.059 & -2.14 & -1.77 & 176.87 & 122.80 & & & -0.13 & 122.74 \\
\hline $30 \quad 39$ & -0.20 & 0.00 & 0.00 & 0.04 & -0.206 & 0.000 & 0.013 & -0.038 & -1.43 & -0.94 & 174.05 & 133.69 & & & -0.41 & 132.44 \\
\hline 3140 & -0.20 & 0.00 & 0.12 & -0.04 & -0.210 & 0.000 & -0.115 & 0.064 & -2.12 & -2.90 & 171.84 & 143.98 & & & -0.46 & 144.57 \\
\hline 3241 & -0.20 & 0.00 & 0.12 & -0.05 & -0.210 & 0.000 & -0.115 & 0.073 & -1.93 & -3.23 & 169.69 & 154.19 & & & -0.25 & 155.27 \\
\hline $33 \quad 42$ & -0.17 & 0.00 & 0.08 & -0.05 & -0.179 & 0.000 & -0.076 & 0.062 & -1.76 & -2.59 & 164.46 & 167.49 & & & -0.71 & 167.45 \\
\hline $34 \quad 43$ & -0.17 & 0.00 & 0.08 & -0.05 & -0.179 & 0.000 & -0.076 & 0.062 & -1.37 & -2.25 & 161.23 & 178.80 & & & -0.26 & 178.86 \\
\hline $35 \quad 44$ & -0.20 & 0.00 & 0.12 & -0.05 & -0.210 & 0.000 & -0.115 & 0.073 & -1.64 & -3.41 & 157.42 & 190.67 & & & -0.05 & 192.12 \\
\hline 3645 & 0.28 & 0.00 & -0.12 & -0.05 & 0.320 & 0.000 & 0.195 & 0.115 & -2.06 & -5.45 & 156.19 & 199.98 & & & 0.10 & 203.64 \\
\hline
\end{tabular}


$Z=9(\mathbf{F})$

\section{$Z=\mathbf{1 0}(\mathrm{Ne})$}

$-2.08$

$\begin{array}{lll}-5.16 & 150.59 & 213.65\end{array}$

$0.00 \quad 216.97$

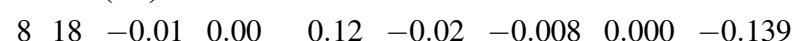

$\begin{array}{llllll}10 & 20 & 0.33 & 0.00 & -0.12 & 0.02\end{array}$

11

12

$\begin{array}{llllll}13 & 23 & 0.29 & 0.00 & -0.03\end{array}$

$\begin{array}{lllll}14 & 24 & -0.06 & 0.00 & -0.01\end{array}$

$\begin{array}{lllll}15 & 25 & 0.05 & 0.00 & 0.00\end{array}$

$\begin{array}{lllll}16 & 26 & 0.11 & 0.00 & 0.05\end{array}$

$\begin{array}{lllll}17 & 27 & 0.11 & 0.00 & 0.02\end{array}$

$\begin{array}{llllll}18 & 28 & -0.02 & 0.00 & 0.00\end{array}$

$\begin{array}{lllll}19 & 29 & -0.03 & 0.00 & 0.00\end{array}$

$\begin{array}{llllll}20 & 30 & 0.00 & 0.00 & 0.00 & 0.03\end{array}$

$\begin{array}{lllllll}21 & 31 & 0.10 & 0.00 & -0.06 & -0.04\end{array}$

$\begin{array}{lllllll}22 & 32 & 0.23 & 0.00 & -0.12 & -0.03\end{array}$

$\begin{array}{lllllll}23 & 33 & 0.28 & 0.00 & -0.11 & 0.04\end{array}$

$\begin{array}{llllll}24 & 34 & 0.29 & 0.00 & -0.10\end{array}$

$\begin{array}{llllll}25 & 35 & 0.28 & 0.00 & -0.07\end{array}$

0.04

2636

$\begin{array}{llll}0.29 & 0.00 & -0.03\end{array}$

$27 \quad 37$

$\begin{array}{llll}0.35 & 0.00 & 0.11\end{array}$

0.04

0.04

$\begin{array}{lllll}28 & 38 & -0.30 & 0.00 & -0.12\end{array}$

0.04

$0.364 \quad 0.000$

0.182

0.070

0.92

1.98

$\begin{array}{ll}2.89 & 134.37\end{array}$

$0.372 \quad 0.000$

$0.207 \quad 0.046$

0.80

$\begin{array}{ll}5.53 & 144.67\end{array}$

3.10

1.50
0.94

5.14160 .81

$\begin{array}{ll}5.81 & 166.96\end{array}$

0.87

$4.77 \quad 177.97$

$\begin{array}{ll}0.096 & -0.007 \\ 0.078 & -0.014\end{array}$

1.78

$\begin{array}{lll}4.77 & 182.69\end{array}$

$\begin{array}{llllll}0.03 & -0.063 & 0.000 & 0.013 & -0.030\end{array}$

1.61

$2.68 \quad 195.62$

$-0.052-0.035$

1.16

$\begin{array}{ll}2.59 & 203.38\end{array}$

1.07

$\begin{array}{lll}2.06 & 208.55\end{array}$

$\begin{array}{ll}-0.031 & 0.000\end{array}$

$\begin{array}{lll}0.000 & -0.029\end{array}$

$0.000 \quad 0.000$

$\begin{array}{lll}0.000 & -0.029\end{array}$

0.61

$2.26 \quad 208.71$

$\begin{array}{ll}0.111 & 0.000\end{array}$

$0.000-0.029$

0.01

$1.94 \quad 212.44$

$0.259 \quad 0.000$

$\begin{array}{llll}0.180 & 0.080 & -1.11\end{array}$

$\begin{array}{ll}1.40 & 212.33\end{array}$

$0.81 \quad 215.27$

$-7.21$

$\begin{array}{ll}21 & -7.04 \\ 5.29 & -5.73\end{array}$

5.32

0.000

0.000

$2.93 \quad 3.77$

$\begin{array}{lllll}0.304 & 0.000 & 0.178 & 0.008 & -1.80\end{array}$

$\begin{array}{lll}0.64 & 213.91 \quad 44.62\end{array}$

$0.315 \quad 0.000$

$\begin{array}{lll}0.168 & 0.005 & -2.15\end{array}$

$\begin{array}{lll}0.18 & 215.81 & 50.79\end{array}$

$\begin{array}{lllll}0.303 & 0.000 & 0.126 & -0.009 & -1.77\end{array}$

$\begin{array}{lll}0.28 & 213.44 & 61.23\end{array}$

$0.316 \quad 0.000$

$0.079-0.024-1.56$

$0.39 \quad 213.97$

68.77

$\begin{array}{llllllll}0.403 & 0.000 & -0.068 & -0.075 & -2.61 & -1.09 & 212.52 & 78.30\end{array}$

$\begin{array}{lllll}29 & 39 & -0.27 & 0.00 & -0.03\end{array}$

$\begin{array}{lll}0.04 & -0.302 & 0.000\end{array}$

$\begin{array}{llllll}0.163 & -0.076 & -2.49 & -2.38 & 213.76 & 85.12\end{array}$

$\begin{array}{lllll}30 & 40 & 0.23 & 0.00 & 0.00\end{array}$

$\begin{array}{lll}0.02 & -0.276 & 0.000\end{array}$

$\begin{array}{llllll}0.059 & -0.030 & -1.79 & -0.43 & 208.31 & 98.65\end{array}$

$\begin{array}{lllll}31 & 41 & -0.17 & 0.00\end{array}$

$0.05-0.05-0.178-0.000$

0.043

$\begin{array}{llll}-1.34 & -0.72 & 207.94 & 107.09\end{array}$

$\begin{array}{llll}32 & 42 & -0.17 & 0.00\end{array}$

$\begin{array}{llllll}0.08 & -0.05 & -0.179 & 0.000 & -0.076\end{array}$

$\begin{array}{lllll}0.056 & -1.36 & -1.43 & 204.63 & 118.47\end{array}$

$\begin{array}{llll}33 & 43 & -0.28 & 0.00\end{array}$

$\begin{array}{llllll}0.11 & -0.05 & -0.292 & 0.000 & -0.086\end{array}$

$\begin{array}{llllll}0.062 & -1.13 & -1.56 & 203.55 & 127.62\end{array}$

$\begin{array}{llllll}34 & 44 & 0.21 & 0.00 & 0.08 & -0.05\end{array}$

$\begin{array}{llllll}35 & 45 & 0.28 & 0.00 & -0.12 & -0.05\end{array}$

$\begin{array}{llllll}36 & 46 & 0.28 & 0.00 & -0.12 & -0.04\end{array}$

$\begin{array}{llll}0.231 & 0.000 & -0.083\end{array}$

$\begin{array}{lllll}0.075 & -1.57 & -2.36 & 199.89 & 139.35\end{array}$

$\begin{array}{lll}0.320 & 0.000 & 0.195\end{array}$

$\begin{array}{llllll}0.031 & -1.03 & -0.72 & 196.56 & 150.76\end{array}$

$\begin{array}{lll}0.318 & 0.000 & 0.194\end{array}$

$\begin{array}{lllll}0.115 & -2.53 & -4.50 & 195.47 & 159.92\end{array}$

$37 \quad 47$

$\begin{array}{lllll}0.32 & 0.00 & -0.12 & 0.02\end{array}$

0.3520 .000

0.204

$\begin{array}{lllll}0.103 & -2.32 & -4.09 & 192.93 & 170.53\end{array}$

$\begin{array}{llllll}38 & 48 & 0.32 & 0.00 & -0.12 & 0.03\end{array}$

$\begin{array}{lll}0.351 & 0.000 & 0.204\end{array}$

$\begin{array}{llllll}0.044 & -2.49 & -2.72 & 186.34 & 185.19\end{array}$

$\begin{array}{llllll}39 & 49 & 0.32 & 0.00 & -0.12 & 0.05\end{array}$

$0.348 \quad 0.000$

0.204

$\begin{array}{lllll}0.033 & -2.25 & -2.71 & 183.80 & 195.80\end{array}$

$\begin{array}{llllll}40 & 50 & 0.30 & 0.00 & -0.10 & -0.01\end{array}$

$\begin{array}{lll}0.332 & 0.000 & 0.169\end{array}$

$\begin{array}{llllll}0.011 & -2.45 & -3.34 & 178.88 & 208.79\end{array}$

$\begin{array}{llllll}41 & 51 & 0.32 & 0.00 & -0.12 & 0.06\end{array}$ $\begin{array}{llllll}0.062 & -2.10 & -3.12 & 175.78 & 219.96\end{array}$

$\begin{array}{llllll}0.000 & -2.63 & -4.21 & 171.04 & 232.78\end{array}$
0.000

0.000

0.000

0.000

0.000

0.026

0.027
0.110

0.147
0.269

0.571$$
\text { (1) }
$$

(n)

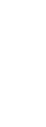

$5.43 \quad 1.53$

$5.08-6.39$

$5.69-4.49$

$4.75 \quad-7.34$

$\begin{array}{ll}4.74 & -4.04\end{array}$

$2.70-5.93$

$2.69-0.98$

$2.23 \quad 0.41$

$2.64 \quad 7.18$

$\begin{array}{ll}2.10 & 9.91\end{array}$

$2.32 \quad 17.67$

$2.01 \quad 21.85$

$\begin{array}{ll}1.83 & 30.23\end{array}$

$2.28 \quad 36.23$

$1.16 \quad 51.01$

$\begin{array}{ll}0.93 & 60.97\end{array}$

$\begin{array}{ll}0.86 & 68.18\end{array}$

$0.39 \quad 78.58$

$\begin{array}{ll}0.38 & 86.57\end{array}$

$0.09 \quad 97.74$

$\begin{array}{ll}-0.24 & 106.06\end{array}$

$-0.38 \quad 117.92$

$0.09 \quad 127.60$

$\begin{array}{ll}0.21 & 140.21\end{array}$

$\begin{array}{ll}0.44 & 150.17\end{array}$

$\begin{array}{ll}0.38 & 163.04\end{array}$

$\begin{array}{ll}0.45 & 173.32\end{array}$

$-0.16 \quad 186.03$

$\begin{array}{ll}-0.14 & 196.69\end{array}$

$-0.74 \quad 209.76$

$-0.40 \quad 221.14$
-1.34

$-1.34 \quad 234.20$
$1.59 \quad 44.97$
$0.157 \quad 0.016$$$
\begin{array}{lll}
2.15 & 202.25 & -0 .
\end{array}
$$

\section{$Z=11$ (Na)}

$\begin{array}{lllll}8 & 19 & -0.01 & 0.00 & 0.12\end{array}$

$\begin{array}{llllll}9 & 20 & 0.29 & 0.00 & -0.11\end{array}$

$\begin{array}{lllll}0.02 & -0.005 & 0.000 & -0.138 & -0.014\end{array}$

$\begin{array}{lllll}0.02 & 0.317 & 0.000 & 0.180 & 0.031\end{array}$

1.25

1.70

$2.92 \quad 133.28$

11.47

0.92

$\begin{array}{llllll}11 & 22 & 0.35 & 0.00 & -0.04\end{array}$

$\begin{array}{lll}0.03 & 0.372 & 0.000\end{array}$

$\begin{array}{ll}0.183 & 0.027\end{array}$

0.92
1.72

$\begin{array}{ll}5.55 & 146.20\end{array}$

6.62

$\begin{array}{lll}5.30 & 162.95 & -2.06\end{array}$

$\begin{array}{lll}6.08 & 172.93 & -3.97\end{array}$

$\begin{array}{ll}0.384 & 0.000 \\ 0.386 & 0.000\end{array}$

$\begin{array}{ll}0.109 & -0.002 \\ 0.059 & -0.021\end{array}$

1.21

$5.20 \quad 185.85$

$-3.97-5.18$

$\begin{array}{llllll}12 & 23 & 0.35 & 0.00 & 0.00\end{array}$

0.03

$\begin{array}{ll}0.353 & 0.000\end{array}$

$0.038-0.027$

2.33

$\begin{array}{llll}5.62 & 192.42 & -7.31 & -8.42\end{array}$

$\begin{array}{lllllll}14 & 25 & 0.26 & 0.00 & -0.01 & 0.02\end{array}$

$\begin{array}{llllll}15 & 26 & 0.24 & 0.00 & 0.02 & 0.03\end{array}$

$\begin{array}{llllll}16 & 27 & 0.25 & 0.00 & 0.06 & 0.03\end{array}$

$\begin{array}{ll}0.283 & 0.000\end{array}$

$0.044-0.013$

2.09

$\begin{array}{llll}4.57 & 201.94 & -8.76 & -9.36\end{array}$

$\begin{array}{lllll}0.263 & 0.000 & 0.004 & -0.033\end{array}$

1.51

$\begin{array}{llll}4.52 & 207.09 & -5.84 & -6.86\end{array}$

$\begin{array}{llllll}17 & 28 & 0.21 & 0.00 & 0.05 & -0.03\end{array}$

2.09

$\begin{array}{llll}3.97 & 214.29 & -4.97 & -5.52\end{array}$

$\begin{array}{lllll}3.85 & 217.95 & -0.56 & -0.99\end{array}$

2.42

$\begin{array}{llll}18 & 29 & -0.06 & 0.00\end{array}$

$\begin{array}{lllll}19 & 30 & -0.04 & 0.00\end{array}$

$\begin{array}{lllllll}0.01 & 0.03 & -0.063 & 0.000 & -0.011 & -0.028\end{array}$

2.07

$\begin{array}{ll}3.14 & 223.78\end{array}$

3.09229 .86

$\begin{array}{rrrrrr}20 & 31 & 0.00 & 0.00 & 0.00 & 0.04\end{array}$

$\begin{array}{lllllll}21 & 32 & 0.11 & 0.00 & -0.05 & -0.04\end{array}$

$\begin{array}{llllll}22 & 33 & 0.23 & 0.00 & -0.12 & 0.04\end{array}$

$\begin{array}{ll}0.000 & 0.000\end{array}$

$-0.011-0.029$

1.38

$\begin{array}{ll}0.121 & 0.000\end{array}$

$0.067 \quad 0.050$

1.72

$\begin{array}{ll}2.78 & 231.24\end{array}$

$2.47 \quad 234.25$

1.68

7.69
11.75

2.66

8.36
12.65

$18.44 \quad 19.06$

$23.49 \quad 24.89$

$\begin{array}{lll}1.90 & 234.92 & 30.90\end{array}$

$\begin{array}{llllll}0.293 & 0.000 & 0.175 & 0.005 & -0.97\end{array}$

$\begin{array}{ll}1.49 & 237.09\end{array}$

36.80

$\begin{array}{lll}1.48 & 236.34 & 45.62\end{array}$

$\begin{array}{lll}1.25 & 237.49 & 52.54\end{array}$

$\begin{array}{lllllll}25 & 36 & 0.29 & 0.00 & -0.04 & 0.04\end{array}$

$\begin{array}{llllll}0.04 & 0.315 & 0.000 & 0.091 & -0.020 & -1.12 \\ 0.04 & 0.329 & 0.000 & 0.045 & -0.034 & -1.10\end{array}$

$\begin{array}{lllllll}0.04 & 0.315 & 0.000 & 0.091 & -0.020 & -1.12 \\ 0.04 & 0.329 & 0.000 & 0.045 & -0.034 & -1.10\end{array}$

0.012 


\begin{tabular}{|c|c|c|c|c|c|c|c|c|c|c|c|c|c|c|c|c|c|}
\hline \multicolumn{18}{|c|}{$Z=11$ (Na) } \\
\hline 27 & & 0.35 & 0.00 & 0.10 & 0.04 & 0.401 & 0.000 & -0.056 & -0.071 & -2.40 & -0.13 & 237.37 & 60.74 & & & 0.93 & 60.87 \\
\hline 28 & 39 & 0.35 & 0.00 & 0.11 & 0.04 & 0.403 & 0.000 & -0.068 & -0.075 & -2.49 & -0.61 & 238.04 & 68.14 & & & 0.74 & 68.44 \\
\hline 29 & 40 & 0.28 & 0.00 & 0.05 & 0.04 & 0.312 & 0.000 & -0.020 & -0.052 & -1.20 & -0.22 & 235.49 & 78.76 & & & 0.41 & 78.22 \\
\hline 30 & 41 & 0.22 & 0.00 & 0.01 & 0.05 & 0.240 & 0.000 & 0.014 & -0.050 & -0.69 & 0.17 & 234.64 & 87.68 & & & 0.79 & 87.04 \\
\hline 31 & 42 & 0.22 & 0.00 & 0.01 & 0.05 & 0.240 & 0.000 & 0.014 & -0.050 & -0.72 & 0.15 & 231.93 & 98.46 & & & 0.79 & 97.75 \\
\hline 32 & 43 & 0.22 & 0.00 & 0.00 & 0.05 & 0.239 & 0.000 & 0.026 & -0.048 & -0.54 & 0.20 & 230.84 & 107.62 & & & 0.86 & 106.86 \\
\hline 33 & 44 & 0.22 & 0.00 & 0.01 & 0.05 & 0.240 & 0.000 & 0.014 & -0.050 & -0.57 & 0.19 & 227.60 & 118.93 & & & 0.91 & 118.16 \\
\hline 34 & 45 & 0.22 & 0.00 & 0.06 & -0.05 & 0.241 & 0.000 & -0.057 & 0.035 & -0.31 & 0.49 & 225.76 & 128.85 & & & 1.29 & 128.12 \\
\hline 35 & 46 & 0.31 & 0.00 & -0.08 & -0.05 & 0.347 & 0.000 & 0.145 & 0.097 & -1.68 & -1.67 & 224.21 & 138.46 & & & 1.25 & 139.82 \\
\hline 36 & 47 & 0.31 & 0.00 & -0.07 & -0.05 & 0.346 & 0.000 & 0.131 & 0.091 & -1.46 & -1.49 & 222.02 & 148.73 & & & 1.27 & 149.92 \\
\hline 37 & 48 & 0.31 & 0.00 & -0.12 & 0.03 & 0.339 & 0.000 & 0.200 & 0.030 & -1.84 & -1.35 & 217.76 & 161.06 & & & 0.77 & 161.60 \\
\hline 38 & 49 & 0.33 & 0.00 & -0.12 & 0.05 & 0.359 & 0.000 & 0.208 & 0.014 & -1.81 & -1.75 & 215.72 & 171.17 & & & 0.53 & 171.89 \\
\hline 39 & 50 & 0.34 & 0.00 & -0.05 & -0.02 & 0.375 & 0.000 & 0.114 & 0.053 & -1.74 & -0.72 & 210.20 & 184.76 & & & 0.50 & 184.45 \\
\hline 40 & 51 & 0.34 & 0.00 & -0.05 & 0.00 & 0.374 & 0.000 & 0.115 & 0.032 & -1.61 & -0.42 & 207.10 & 195.94 & & & 0.38 & 195.26 \\
\hline 41 & 52 & 0.33 & 0.00 & -0.06 & 0.02 & 0.361 & 0.000 & 0.127 & 0.015 & -1.96 & -0.87 & 202.72 & 208.38 & & & -0.09 & 207.76 \\
\hline 42 & 53 & 0.31 & 0.00 & -0.09 & 0.06 & 0.336 & 0.000 & 0.162 & -0.016 & -1.89 & -2.23 & 200.93 & 218.25 & & & -0.25 & 218.91 \\
\hline 43 & 54 & 0.33 & 0.00 & -0.06 & 0.06 & 0.359 & 0.000 & 0.131 & -0.026 & -2.17 & -2.27 & 195.83 & 231.42 & & & -0.78 & 231.69 \\
\hline 44 & 55 & 0.33 & 0.00 & -0.05 & 0.06 & 0.359 & 0.000 & 0.118 & -0.030 & -2.01 & -2.21 & 192.31 & 243.01 & & & -0.75 & 243.37 \\
\hline \multicolumn{18}{|c|}{$Z=12(\mathrm{Mg})$} \\
\hline 8 & 20 & 0.11 & 0.00 & -0.09 & -0.02 & 0.122 & 0.000 & 0.118 & 0.037 & 0.21 & 1.65 & 136.48 & 15.56 & 17.57 & 0.027 & 1.78 & 15.32 \\
\hline 9 & 21 & 0.29 & 0.00 & -0.03 & 0.03 & 0.316 & 0.000 & 0.078 & -0.014 & 1.34 & 4.21 & 150.19 & 9.92 & 10.91 & 0.016 & 4.15 & 9.78 \\
\hline 10 & 22 & 0.35 & 0.00 & -0.04 & 0.03 & 0.384 & 0.000 & 0.109 & -0.002 & 0.57 & 4.24 & 169.14 & -0.96 & -0.40 & 0.001 & 4.20 & -0.85 \\
\hline 11 & 23 & 0.35 & 0.00 & 0.02 & 0.03 & 0.388 & 0.000 & 0.035 & -0.029 & 1.23 & 4.99 & 181.22 & -4.97 & -5.47 & 0.001 & 4.89 & -4.74 \\
\hline 12 & 24 & 0.35 & 0.00 & 0.06 & 0.03 & 0.393 & 0.000 & -0.012 & -0.046 & 0.78 & 4.44 & 197.38 & -13.06 & -13.93 & 0.000 & 4.39 & -12.65 \\
\hline 13 & 25 & 0.31 & 0.00 & 0.05 & 0.03 & 0.346 & 0.000 & -0.013 & -0.042 & 1.81 & 4.83 & 204.63 & -12.23 & -13.19 & 0.000 & 4.76 & -11.76 \\
\hline 14 & 26 & -0.35 & 0.00 & -0.11 & 0.03 & -0.351 & 0.000 & 0.162 & -0.070 & 0.79 & 3.42 & 216.69 & -16.22 & -16.22 & 0.000 & 3.49 & -15.57 \\
\hline 15 & 27 & 0.25 & 0.00 & 0.05 & -0.03 & 0.275 & 0.000 & -0.037 & 0.017 & 1.90 & 4.25 & 221.54 & -13.00 & -14.59 & 0.000 & 4.23 & -12.45 \\
\hline 16 & 28 & 0.25 & 0.00 & 0.08 & -0.03 & 0.277 & 0.000 & -0.073 & 0.008 & 1.26 & 3.77 & 230.70 & -14.09 & -15.02 & 0.002 & 3.82 & -13.50 \\
\hline 17 & 29 & 0.21 & 0.00 & 0.06 & -0.03 & 0.230 & 0.000 & -0.057 & 0.016 & 1.96 & 3.68 & 234.84 & -10.16 & -10.62 & 0.014 & 3.70 & -9.64 \\
\hline 18 & 30 & 0.11 & 0.00 & 0.01 & 0.03 & 0.119 & 0.000 & -0.005 & -0.031 & 2.21 & 3.38 & 242.17 & -9.42 & -8.91 & 0.008 & 3.41 & -8.97 \\
\hline 19 & 31 & -0.05 & 0.00 & 0.01 & 0.04 & -0.052 & 0.000 & -0.011 & -0.038 & 2.10 & 3.24 & 24 & -4.14 & -3.22 & 0. & 3.30 & -3.77 \\
\hline 20 & 32 & 0.00 & 0.00 & 0.00 & 0.04 & 0.000 & 0.000 & 0.000 & -0.039 & 1.39 & 3.04 & 250.78 & -1.89 & -0.95 & 0.018 & 3.11 & -1.60 \\
\hline 21 & 33 & 0.11 & 0.00 & -0.04 & -0.04 & 0.120 & 0.000 & 0.054 & 0.048 & 1.80 & 3.05 & 252.24 & 4.72 & 4.89 & 0.020 & 3.24 & 5.01 \\
\hline 22 & 34 & 0.20 & 0.00 & -0.09 & 0.00 & 0.218 & 0.000 & 0.131 & 0.028 & 0.69 & 2.76 & 256.93 & 8.11 & 8.81 & 0.231 & 3.17 & 8.49 \\
\hline 23 & 35 & 0.25 & 0.00 & -0.09 & 0.04 & 0.270 & 0.000 & 0.144 & -0.006 & -0.35 & 2.26 & 257.88 & 15.23 & & & 2.72 & 15.53 \\
\hline 24 & 36 & 0.28 & 0.00 & -0.05 & 0.04 & 0.304 & 0.000 & 0.101 & -0.017 & -0.90 & 1.85 & 261.62 & 19.56 & & & 2.20 & 19.61 \\
\hline 25 & 37 & 0.28 & 0.00 & 0.00 & 0.04 & 0.306 & 0.000 & 0.039 & -0.035 & -0.95 & 1.66 & 261.37 & 27.89 & & & 1.92 & 27.72 \\
\hline 26 & 38 & 0.29 & 0.00 & 0.03 & 0.04 & 0.321 & 0.000 & 0.006 & -0.045 & -1.18 & 1.15 & 264.29 & 33.03 & & & 1.54 & 32.87 \\
\hline 27 & 39 & 0.35 & 0.00 & 0.10 & 0.04 & 0.401 & 0.000 & -0.056 & -0.071 & -2.70 & -0.04 & 264.25 & 41.14 & & & 0.93 & 41.43 \\
\hline 28 & 40 & -0.31 & 0.00 & -0.12 & 0.04 & -0.312 & 0.000 & 0.165 & -0.077 & -2.71 & -1.31 & 267.12 & 46.34 & & & 0.81 & 47.67 \\
\hline 29 & 41 & -0.27 & 0.00 & -0.06 & 0.04 & -0.276 & 0.000 & 0.090 & -0.056 & -1.62 & -0.08 & 263.97 & 57.56 & & & 0.91 & 57.64 \\
\hline 30 & 42 & 0.22 & 0.00 & 0.02 & 0.05 & 0.241 & 0.000 & 0.002 & -0.053 & -0.82 & -0.12 & 264.89 & 64.72 & & & 0.49 & 64.31 \\
\hline 31 & 43 & 0.22 & 0.00 & 0.03 & 0.05 & 0.242 & 0.000 & -0.010 & -0.055 & -0.77 & 0.09 & 262.16 & 75.52 & & & 0.77 & 75.10 \\
\hline 32 & 44 & 0.22 & 0.00 & 0.03 & 0.05 & 0.242 & 0.000 & -0.010 & -0.055 & -0.56 & 0.31 & 262.17 & 83.58 & & & 1.06 & 83.15 \\
\hline 33 & 45 & 0.22 & 0.00 & 0.06 & -0.05 & 0.241 & 0.000 & -0.057 & 0.035 & -0.57 & 0.39 & 259.02 & 94.80 & & & 1.08 & 94.24 \\
\hline 34 & 46 & 0.23 & 0.00 & 0.10 & -0.05 & 0.255 & 0.000 & -0.104 & 0.024 & -0.55 & 0.17 & 258.89 & 103.00 & & & 1.32 & 102.83 \\
\hline 35 & 47 & 0.22 & 0.00 & 0.10 & -0.05 & 0.244 & 0.000 & -0.106 & 0.025 & -0.40 & -0.09 & 255.60 & 114.36 & & & 1.11 & 114.21 \\
\hline 36 & 48 & 0.33 & 0.00 & -0.01 & -0.05 & 0.365 & 0.000 & 0.056 & 0.062 & -0.92 & 0.19 & 254.46 & & & & 1.43 & 123.43 \\
\hline 37 & 49 & 0.35 & 0.00 & -0.01 & -0.05 & 0.389 & 0.000 & 0.062 & 0.064 & -1.36 & -0.07 & 250.72 & 135.39 & & & 1.24 & 135.29 \\
\hline 38 & 50 & 0.35 & 0.00 & 0.00 & -0.05 & 0.389 & 0.000 & 0.049 & 0.058 & -1.26 & 0.05 & 249.28 & 144.90 & & & 1.27 & 144.71 \\
\hline 39 & 51 & 0.35 & 0.00 & 0.01 & -0.06 & 0.389 & 0.000 & 0.035 & 0.064 & -1.64 & -0.59 & 245.53 & 156.72 & & & 0.88 & 156.79 \\
\hline 40 & 52 & 0.35 & 0.00 & 0.01 & -0.06 & 0.389 & 0.000 & 0.035 & 0.064 & -1.56 & -0.80 & 243.98 & 166.34 & & & 0.77 & 166.53 \\
\hline 41 & 53 & 0.34 & 0.00 & 0.00 & -0.04 & 0.376 & 0.000 & 0.047 & 0.048 & -1.77 & -0.47 & 238.90 & 179.49 & & & 0.40 & 179.03 \\
\hline 42 & 54 & 0.34 & 0.00 & 0.00 & -0.01 & 0.375 & 0.000 & 0.050 & 0.018 & -1.58 & 0.18 & 236.12 & 190.34 & & & 0.37 & 189.27 \\
\hline 43 & 55 & 0.35 & 0.00 & 0.03 & 0.06 & 0.391 & 0.000 & 0.029 & -0.062 & -2.07 & -1.91 & 233.13 & 201.40 & & & -0.31 & 201.82 \\
\hline 44 & 56 & 0.35 & 0.00 & 0.04 & 0.06 & 0.392 & 0.000 & 0.017 & -0.066 & -2.05 & -2.06 & 230.79 & 211.82 & & & -0.22 & 212.56 \\
\hline 45 & 57 & 0.35 & 0.00 & 0.04 & 0.06 & 0.392 & 0.000 & 0.017 & -0.066 & -2.55 & -2.60 & 225.96 & 224.71 & & & -0.76 & 225.57 \\
\hline
\end{tabular}




\section{$Z=12(\mathrm{Mg})$}

$\begin{array}{llllll}46 & 58 & 0.35 & 0.00 & 0.04 & 0.06\end{array}$

\section{$Z=13(\mathrm{Al})$}

$\begin{array}{llllll}9 & 22 & 0.21 & 0.00 & -0.05\end{array}$

$\begin{array}{llllll}10 & 23 & 0.28 & 0.00 & -0.02 & 0.0\end{array}$

$\begin{array}{llllll}11 & 24 & 0.30 & 0.00 & 0.02 & 0.03\end{array}$

$\begin{array}{llllll}12 & 25 & 0.31 & 0.00 & 0.05 & 0.03\end{array}$

$\begin{array}{lllllll}13 & 26 & 0.25 & 0.00 & 0.02 & 0.03\end{array}$

$\begin{array}{lllllll}14 & 27 & -0.40 & 0.00 & -0.12 & -0.03\end{array}$

$\begin{array}{llllll}15 & 28 & 0.20 & 0.00 & 0.03 & -0.02\end{array}$

$\begin{array}{llllll}16 & 29 & 0.21 & 0.00 & 0.05 & -0.03\end{array}$

$\begin{array}{lllllll}17 & 30 & 0.14 & 0.00 & 0.03 & -0.03\end{array}$

$\begin{array}{lllllll}18 & 31 & -0.12 & 0.00 & 0.03 & 0.04 & -0.124\end{array}$

$\begin{array}{llllllll}19 & 32 & 0.05 & 0.00 & 0.01 & 0.04 & 0.054\end{array}$

$\begin{array}{llllll}20 & 33 & 0.03 & 0.00 & 0.00 & 0.04\end{array}$

$\begin{array}{lllllll}21 & 34 & 0.09 & 0.00 & -0.02 & -0.04\end{array}$

$\begin{array}{llllll}22 & 35 & 0.11 & 0.00 & -0.03 & 0.04\end{array}$

$\begin{array}{llllll}23 & 36 & 0.22 & 0.00 & -0.08 & 0.04\end{array}$

$\begin{array}{llllll}24 & 37 & 0.24 & 0.00 & -0.05\end{array}$

$\begin{array}{rrrrr}25 & 38 & 0.25 & 0.00 & -0.01 \\ 26 & 39 & 0.24 & 0.00 & 0.02\end{array}$

$\begin{array}{lllll}27 & 40 & -0.31 & 0.00 & -0.12\end{array}$

$\begin{array}{llllllll}28 & 41 & -0.31 & 0.00 & -0.12 & 0.05 & -0.313\end{array}$

$\begin{array}{rrrrrrr}29 & 42 & -0.27 & 0.00 & -0.09 & 0.00 & -0.271\end{array}$

$\begin{array}{lllllll}30 & 43 & -0.27 & 0.00 & -0.05 & -0.05 & -0.271\end{array}$

$\begin{array}{lllllll}31 & 44 & -0.27 & 0.00 & -0.02 & -0.05 & -0.274\end{array}$

$\begin{array}{lllllll}32 & 45 & -0.27 & 0.00 & -0.02 & -0.05 & -0.274\end{array}$

$\begin{array}{lllllll}33 & 46 & -0.30 & 0.00 & 0.04 & -0.05 & -0.308\end{array}$

$\begin{array}{lllllll}34 & 47 & -0.36 & 0.00 & 0.06 & -0.05 & -0.369\end{array}$

$\begin{array}{llllllll}35 & 48 & -0.38 & 0.00 & 0.06 & -0.05 & -0.388\end{array}$

$\begin{array}{llllllll}36 & 49 & -0.38 & 0.00 & 0.03 & -0.05 & -0.386\end{array}$

$\begin{array}{lllllll}37 & 50 & 0.41 & 0.00 & 0.07 & -0.05 & 0.463\end{array}$

$\begin{array}{llllllll}38 & 51 & 0.41 & 0.00 & 0.08 & -0.06 & 0.464\end{array}$

$\begin{array}{llllllll}39 & 52 & 0.35 & 0.00 & 0.04 & -0.06 & 0.390\end{array}$

$\begin{array}{lllllll}40 & 53 & 0.35 & 0.00 & 0.04 & -0.06 & 0.390\end{array}$

$\begin{array}{lllllll}41 & 54 & 0.33 & 0.00 & 0.02 & -0.06 & 0.366\end{array}$

$\begin{array}{lllllll}42 & 55 & 0.34 & 0.00 & 0.05 & -0.04\end{array}$

0.378

$\begin{array}{lllllll}43 & 56 & 0.35 & 0.00 & 0.06 & 0.02 & 0.393\end{array}$

$\begin{array}{lllllll}44 & 57 & 0.35 & 0.00 & 0.07 & 0.06 & 0.397\end{array}$

$\begin{array}{lllllll}45 & 58 & 0.22 & 0.00 & -0.07 & 0.05 & 0.236\end{array}$

$\begin{array}{lllll}46 & 59 & 0.22 & 0.00 & -0.05\end{array}$

$0.06 \quad 0.236$

4760

$\begin{array}{llll}0.27 & 0.00 & -0.02\end{array}$

0.06

0.294

$48 \quad 6$

$\begin{array}{lll}0.30 & 0.00 & 0.02\end{array}$

0.07

0.332

4962

$\begin{array}{lll}0.28 & 0.00 & 0.04\end{array}$

0.07

0.312

$50 \quad 63$

\section{$Z=14$ (Si)}

$\begin{array}{rrrrr}8 & 22 & 0.00 & 0.01 & 0.02\end{array}$

$\begin{array}{llllll}9 & 23 & -0.23 & 0.00 & -0.12\end{array}$

$0.03 \quad 0.001-0$.

$0.03-0.232$

$0.03-0.292$

$\begin{array}{llllll}10 & 24 & -0.29 & 0.00 & -0.12\end{array}$

$0.03-0.350$

$\begin{array}{llllllll}12 & 26 & -0.37 & 0.00 & -0.12 & 0.03 & -0.370\end{array}$

$\begin{array}{llllllll}13 & 27 & -0.37 & 0.00 & -0.12 & -0.03 & -0.363\end{array}$

$\begin{array}{lllllll}14 & 28 & -0.37 & 0.00 & -0.12 & -0.03 & -0.363\end{array}$

$\begin{array}{lllllll}15 & 29 & -0.35 & 0.00 & -0.07 & -0.03 & -0.349\end{array}$

$\begin{array}{lllllll}16 & 30 & -0.23 & 0.00 & -0.02 & 0.03 & -0.236\end{array}$ $\begin{array}{lllllll}0.000 & 0.017 & -0.066 & -2.54 & -2.71 & 223.25 & 235.50\end{array}$

$\begin{array}{lllllll}0.000 & 0.034 & -0.059 & -2.97 & -2.95 & 217.85 & 248.97\end{array}$

$-0.78 \quad 236.57$

$-1.31 \quad 249.89$

$\begin{array}{lllllll}0.000 & -0.134 & -0.039 & -0.59 & 0.87 & 133.77 & 25.5\end{array}$

$\begin{array}{llll}0.000 & 0.083 & -0.015 & 1.38\end{array}$

$\begin{array}{llll}0.000 & 0.062 & -0.018 & 1.32\end{array}$

$\begin{array}{llll}0.000 & 0.020 & -0.032 & 1.97\end{array}$

$\begin{array}{llll}0.000 & 0.006 & -0.033 & 2.47\end{array}$

$\begin{array}{llll}0.000 & 0.193 & -0.027 & -0.29\end{array}$

$\begin{array}{llll}0.000 & -0.021 & 0.014 & 2.33\end{array}$

$\begin{array}{llll}0.000 & -0.045 & 0.019 & 1.86\end{array}$

$\begin{array}{llll}0.000 & -0.030 & 0.025 & 2.61\end{array}$

$\begin{array}{llll}0.000 & -0.030 & -0.033 & 2.27\end{array}$

$\begin{array}{llll}0.000 & -0.010 & -0.040 & 2.23\end{array}$

$\begin{array}{llll}0.000 & 0.001 & -0.039 & 1.52\end{array}$

$\begin{array}{llll}0.000 & 0.027 & 0.043 & 2.08\end{array}$

$\begin{array}{llll}0.000 & 0.043 & -0.036\end{array}$

1.87

0.000

$0.123-0.015$

0.59

$\begin{array}{llll}0.000 & 0.090 & -0.022 & 0.08\end{array}$

0.000

0.000

$\begin{array}{lll}0.044 & -0.034 & -0.10\end{array}$

0.000

$\begin{array}{lll}0.005 & -0.043 & -0.20\end{array}$

0.000

$\begin{array}{lll}0.165 & -0.077 & -2.10\end{array}$

0.000

$0.164-0.086-3.03$

$0.029-1.88$

$\begin{array}{llll}0.000 & 0.055 & 0.037 & -0.93\end{array}$

$\begin{array}{lllll}0.000 & 0.055 & 0.037 & -0.59\end{array}$

$\begin{array}{lll}0.000 & -0.005\end{array}$

$0.053-0.86$

$0.000-0.010$

$0.058-1.16$

$\begin{array}{lll}0.000 & -0.004\end{array}$

$0.058-1.12$

$\begin{array}{ll}0.000 & 0.027\end{array}$

$0.000-0.019$

$0.023-1.16$

$0.000-0.033$

$0.027-1.29$

$0.000-0.004$

$0.049-0.99$

$0.000-0.004$

$0.049-0.96$

$0.000 \quad 0.016$

$0.058-1.09$

$0.000-0.016$

$0.024-1.02$

$\begin{array}{lllll}0.000 & -0.014 & -0.037 & -1.53\end{array}$

0.000

0.000

0.000

0.000

$$
-0.0
$$

0.111

0.000

$$
0.063
$$

0.000

0.000

$\begin{array}{lll}3.39 & 149.89 & 17.50\end{array}$

$\begin{array}{lll}4.04 & 168.90 & 6.57\end{array}$

$\begin{array}{lll}4.86 & 183.18 & 0.37\end{array}$

$4.58 \quad 199.71$

$\begin{array}{llll}4.63 & 210.69 & -11.01 & -12.21\end{array}$

$\begin{array}{llll}4.16 & 223.56 & -15.81 & -17.20\end{array}$

$\begin{array}{llll}3.96 & 231.47 & -15.64 & -16.85\end{array}$

$\begin{array}{lllll}3.78 & 240.88 & -16.99 & -18.22\end{array}$

$\begin{array}{lllll}4.52 & 246.09 & -14.12 & -15.87\end{array}$

$\begin{array}{llll}3.06 & 255.07 & -15.03 & -14.95\end{array}$

$\begin{array}{llll}3.61 & 258.96 & -10.85 & -11.06\end{array}$

$3.20 \quad 265.42$

$\begin{array}{ll}3.55 & 268.23\end{array}$

$\begin{array}{ll}3.13 & 273.44\end{array}$

$-9.24$

$-3.98$

$\begin{array}{ll}3.07 & 275.54\end{array}$

$2.59 \quad 279.70$

$2.31 \quad 281.04$

$1.74 \quad 284.33$

$0.00 \quad 286.26$

$-1.05 \quad 289.18$

$0.22 \quad 287.35$

$0.38 \quad 288.31$

$0.28 \quad 287.16$

$0.76 \quad 287.12$

$0.40 \quad 285.64$

$0.26 \quad 285.62$

$0.11 \quad 283.38$

$0.74 \quad 282.04$

$1.28 \quad 278.62$

$1.11 \quad 277.60$

$\begin{array}{ll}0.77 \quad 274.62 \\ 0.56 & 273.19\end{array}$

$\begin{array}{ll}0.56 & 273.19\end{array}$

$0.06 \quad 269.96$

$0.72 \quad 267.26$

$0.22 \quad 263.67$

$-1.11$

\begin{abstract}
4.86
\end{abstract}
8.77

15.50

20.28

26.42

31.57

41.48

48.59

57.81

65.92

75.47

83.56

93.88

103.29

114.77

23.87

34.92

144.42

55.72

66.50

78.15

86.76

$\begin{array}{rrr}1.67 & 263.13 & 186.76 \\ -1.13 & 258.18 & 199.79 \\ -1.29 & 255.57 & 210.47\end{array}$

$\begin{array}{llllll}0.063 & -0.049 & -2.27 & -1.76 & 251.32 & 222.79\end{array}$

$\begin{array}{llllll}0.026 & -0.070 & -2.47 & -2.57 & 249.05 & 233.13\end{array}$

$\begin{array}{lllllll}0.000 & -0.003 & -0.077 & -2.84 & -3.41 & 244.88 & 245.37\end{array}$

$\begin{array}{lllllll}0.000 & -0.065 & -0.073 & -2.69 & -3.36 & 241.47 & 256.85\end{array}$ $\begin{array}{llll}0.000 & -0.013 & -0.042 & 1.55\end{array}$

$0.046-0.64$

\section{$-8.53$}

$-2.93$

$-0.13$

$\begin{array}{ll}5.78 & 0.215\end{array}$

$\begin{array}{ll}9.95 & 0.331\end{array}$

$\begin{array}{ll}16.05 & 0.731\end{array}$

$21.40 \quad 1.472$

$\begin{array}{ll}1.11 & 24.91\end{array}$

$3.36 \quad 16.94$

$3.99 \quad 6.27$

$4.74 \quad 0.25$

$\begin{array}{ll}4.50 & -8.00\end{array}$

$4.55-11.93$

$\begin{array}{ll}4.10 & -15.47\end{array}$

$3.91-15.23$

$3.76-16.52$

$4.51-13.66$

$3.08-14.57$

$3.64-10.44$

$\begin{array}{ll}3.25 & -8.89\end{array}$

$3.63-3.68$

$3.24-0.89$

$3.34 \quad 5.14$

$\begin{array}{ll}2.82 & 8.89\end{array}$

$2.48 \quad 15.45$

$\begin{array}{ll}1.98 & 20.19\end{array}$

$1.51 \quad 27.48$

$\begin{array}{ll}0.87 & 32.93\end{array}$

$0.98 \quad 41.57$

$0.97 \quad 48.41$

$0.77 \quad 57.43$

$1.31 \quad 65.51$

$\begin{array}{ll}1.16 & 75.20\end{array}$

$1.34 \quad 83.55$

$1.22 \quad 93.84$

$\begin{array}{ll}1.64 & 102.98\end{array}$

$1.79 \quad 114.05$

$1.92 \quad 123.44$

$1.74 \quad 134.63$

$\begin{array}{ll}1.60 & 144.22\end{array}$

$\begin{array}{ll}1.27 & 155.71\end{array}$

$1.17 \quad 165.77$

$\begin{array}{ll}0.68 & 177.49\end{array}$

$\begin{array}{ll}0.55 & 187.92\end{array}$

$0.18 \quad 200.12$

$0.19 \quad 211.06$

$-0.45 \quad 223.34$

$-0.42 \quad 234.64$

$-0.91 \quad 247.38$

$-0.75 \quad 259.14$

\begin{tabular}{rrrrrrrrrrr}
-0.013 & -0.023 & -0.029 & -1.57 & 0.18 & 133.93 & 32.68 & & & 0.22 & 31.34 \\
0.000 & 0.154 & -0.057 & 0.18 & 1.62 & 151.76 & 22.93 & & & 1.89 & 22.21 \\
0.000 & 0.163 & -0.065 & 0.44 & 1.98 & 173.33 & 9.43 & 10.76 & 0.019 & 2.22 & 9.01 \\
0.000 & 0.174 & -0.073 & 0.90 & 3.14 & 187.88 & 2.95 & 3.82 & 0.010 & 3.19 & 2.62 \\
0.000 & 0.178 & -0.076 & 0.07 & 3.35 & 206.11 & -7.21 & -7.14 & 0.003 & 3.42 & -7.27 \\
0.000 & 0.187 & -0.022 & -0.30 & 3.74 & 218.51 & -11.53 & -12.38 & 0.000 & 3.67 & -11.55 \\
0.000 & 0.187 & -0.022 & -1.52 & 3.06 & 234.72 & -19.68 & -21.49 & 0.000 & 3.08 & -19.46 \\
0.000 & 0.126 & -0.004 & -0.19 & 3.93 & 242.12 & -19.01 & -21.90 & 0.000 & 3.88 & -18.76 \\
0.000 & 0.040 & -0.034 & 1.00 & 3.14 & 254.09 & -22.90 & -24.43 & 0.000 & 3.14 & -22.53 \\
\hline
\end{tabular}




\section{$Z=14(\mathrm{Si})$}

$\begin{array}{llllllll}17 & 31 & -0.21 & 0.00 & 0.03 & -0.04 & -0.218\end{array}$ $\begin{array}{lllllll}18 & 32 & -0.12 & 0.00 & 0.03 & 0.04 & -0.124\end{array}$ $\begin{array}{lllllll}19 & 33 & -0.04 & 0.00 & 0.01 & 0.04 & -0.041\end{array}$ $\begin{array}{lllllll}20 & 34 & 0.00 & 0.00 & 0.00 & 0.04 & 0.000\end{array}$ $\begin{array}{lllllll}21 & 35 & -0.05 & 0.00 & -0.01 & 0.04 & -0.052\end{array}$ $\begin{array}{lllllll}22 & 36 & 0.01 & 0.00 & 0.00 & 0.04 & 0.011\end{array}$ $\begin{array}{lllllll}23 & 37 & 0.18 & 0.00 & -0.06 & 0.02 & 0.193\end{array}$ $\begin{array}{lllllll}24 & 38 & 0.21 & 0.00 & -0.04 & 0.04 & 0.226\end{array}$ $\begin{array}{lllllll}25 & 39 & 0.23 & 0.00 & 0.00 & 0.04 & 0.250\end{array}$ $\begin{array}{llllllll}26 & 40 & 0.23 & 0.00 & 0.04 & -0.04 & 0.252\end{array}$

$\begin{array}{llllllll}27 & 41 & -0.31 & 0.00 & -0.12 & -0.05 & -0.302\end{array}$ $\begin{array}{lllllll}28 & 42 & -0.31 & 0.00 & -0.12 & 0.05 & -0.313\end{array}$ $\begin{array}{lllllll}29 & 43 & -0.28 & 0.00 & -0.10 & -0.05 & -0.275\end{array}$

$\begin{array}{lllllll}30 & 44 & -0.28 & 0.00 & -0.09 & -0.05 & -0.277\end{array}$

$\begin{array}{llllllll}31 & 45 & -0.27 & 0.00 & -0.04 & -0.05 & -0.273\end{array}$

$\begin{array}{lllllll}32 & 46 & -0.27 & 0.00 & -0.01 & -0.05 & -0.275\end{array}$

$\begin{array}{lllllll}33 & 47 & -0.29 & 0.00 & 0.02 & -0.05 & -0.297\end{array}$

$\begin{array}{lllllll}34 & 48 & -0.36 & 0.00 & 0.05 & -0.05 & -0.368\end{array}$

$\begin{array}{llll}35 & 49 & -0.38 & 0.00\end{array}$

$0.04-0.05-0.387$

$\begin{array}{llll}36 & 50 & -0.44 & 0.00\end{array}$

$\begin{array}{llll}0.04 & -0.05 & -0.445\end{array}$

$\begin{array}{llll}37 & 51 & -0.47 & 0.00\end{array}$

$\begin{array}{lll}0.06 & -0.06 & -0.475\end{array}$

$\begin{array}{llll}38 & 52 & 0.41 & 0.00\end{array}$

$\begin{array}{lll}0.09 & -0.06 & 0.465\end{array}$

$\begin{array}{llll}39 & 53 & 0.41 & 0.00\end{array}$

$\begin{array}{llll}40 & 54 & 0.35 & 0.00\end{array}$

$0.09-0.06$

0.465

$\begin{array}{llll}41 & 55 & 0.35 & 0.00\end{array}$

$-0.06$

0.391

$\begin{array}{llll}42 & 56 & 0.35 & 0.00\end{array}$

$0.07-0.06$

0.391

$43 \quad 57$

$\begin{array}{ll}0.35 & 0.00\end{array}$

$\begin{array}{ll}0.08 & -0.04\end{array}$

0.393

4458

$\begin{array}{ll}0.33 & 0.00\end{array}$

$\begin{array}{lll}0.09 & -0.01\end{array}$

0.395

$\begin{array}{llllll}45 & 59 & 0.35 & 0.00 & 0.09 & 0.04\end{array}$

$46 \quad 60$

0.220 .00

0.06

0.371

0.399

$\begin{array}{llllll}47 & 61 & 0.26 & 0.00 & 0.00 & 0.07\end{array}$

0.285

$48 \quad 62$

$0.04 \quad 0.07$

0.301

4963

$0.27 \quad 0.00$

0.06

0.07

0.304

$50 \quad 64$

$\begin{array}{ll}-0.25 & 0.00\end{array}$

51

$\begin{array}{llll}52 & 66 & 0.30 & 0.00\end{array}$

$-0.256$

5367

$\begin{array}{ll}0.30 & 0.00\end{array}$

$0.12-0.01$

0.306

5468

0.339

0.339

0.351
$0.12 \quad 0.07$

$0.12-0.03$ $\begin{array}{lll}0.000 & -0.014 & 0.042\end{array}$

$\begin{array}{llll}0.000 & -0.030 & -0.033\end{array}$

$\begin{array}{llll}0.000 & -0.011 & -0.038\end{array}$

$\begin{array}{llll}0.000 & 0.000 & -0.039\end{array}$

1.57

1.59

1.67

0.000

$0.012-0.039$

0.82
1.66

$\begin{array}{llll}0.000 & 0.001 & -0.039\end{array}$

1.67

0.000

0.000

$0.089-0.004$

1.67
1.00

$\begin{array}{llll}0.000 & 0.027 & -0.037\end{array}$

0.37

$\begin{array}{llll}0.000 & -0.030 & 0.030 & -0.28\end{array}$

$\begin{array}{lllll}0.000 & 0.178 & 0.007 & -2.73\end{array}$

0.000$$
0.164
$$

0.000

0.149

$-0.0$

$\begin{array}{ll}0.000 & 0.137 \\ 0.000 & 0.077\end{array}$

$0.017-2.67$

$0.019-2.10$

0.000

0.043

0.032

0.000

0.015

$0.040-1.08$

$\begin{array}{llll}0.000 & 0.017 & 0.050 & -1.83\end{array}$

$0.000 \quad 0.035$

$0.046-1.93$

$0.000 \quad 0.026$

$0.061-1.74$

$0.000-0.045$

$0.022-1.36$

$0.000-0.045$

$0.022-1.60$

$\begin{array}{lll}0.000 & -0.029\end{array}$

$0.039-0.93$

$0.000-0.041$

$0.035-1.19$

$\begin{array}{lllll}0.000 & -0.050 & 0.011 & -1.21\end{array}$

$\begin{array}{lllll}0.000 & -0.056 & -0.021 & -1.58\end{array}$

0.000

0.000

0.000

0.000

$$
-0.04
$$$$
0.075
$$

0.000

0.000

$\begin{array}{lrlllll}0.000 & 0.153 & -0.097 & -3.20 & -6.45 & 282.66 & 222.95 \\ 0.000 & -0.113 & -0.025 & -3.42 & -2.86 & 273.84 & 239.85\end{array}$

$\begin{array}{lllllll}0.000 & -0.109 & -0.009 & -3.22 & -2.59 & 270.79 & 250.97\end{array}$

$\begin{array}{lllllll}0.000 & -0.109 & -0.009 & -3.52 & -2.92 & 265.64 & 264.19\end{array}$

$\begin{array}{lllllll}0.000 & -0.107 & -0.011 & -3.08 & -2.57 & 262.23 & 275.67\end{array}$

$3.23 \quad 294.93$

$2.46 \quad 300.95$

$1.94 \quad 307.05$

$0.07 \quad 309.42$

$-1.23 \quad 313.99$

$-0.16 \quad 312.62$

$0.05 \quad 314.96$

$0.46 \quad 315.16$

$0.40 \quad 313.58$

$0.32 \quad 314.70$

$0.44 \quad 312.38$

$0.76 \quad 312.51$

$0.49 \quad 310.06$

$\begin{array}{ll}1.38 & 309.08\end{array}$

$\begin{array}{ll}306.23 \\ .83 & 305.87\end{array}$

$0.62 \quad 302.48$

$0.85 \quad 301.22$

$0.40 \quad 297.68$

151.43

60.54

1.58
$0.047-1.27$

$\begin{array}{llll}0.000 & 0.000 & 0.054 & -1.80\end{array}$

$\begin{array}{llll}3.14 & 260.55 & -21.29 & -22.95\end{array}$

$\begin{array}{llll}270.27 & -22.95 & -24.08\end{array}$

$\begin{array}{lll}-19.67 & -20.49\end{array}$

$\begin{array}{lll}-19.63 & -19.96\end{array}$

$\begin{array}{llll}2.87 & 286.45 & -14.91 & -14.36\end{array}$

$\begin{array}{llll}2.59 & 293.16 & -13.54 & -12.48\end{array}$

$Z=15(P)$

$\begin{array}{llllllll}8 & 23 & -0.02 & 0.02 & 0.12 & -0.03 & -0.020 & -0 .\end{array}$

$\begin{array}{lllllll}9 & 24 & 0.10 & 0.00 & -0.11 & 0.03 & 0.108\end{array}$

$\begin{array}{lllllll}10 & 25 & 0.10 & 0.00 & 0.12 & 0.03 & 0.117 \\ 11 & 26 & 0.28 & 0.00 & 0.07 & 0.03 & 0.314\end{array}$

$\begin{array}{llllllll}11 & 26 & 0.28 & 0.00 & 0.07 & 0.03 & 0.314\end{array}$

$\begin{array}{lllllll}12 & 27 & 0.28 & 0.00 & 0.09 & 0.03 & 0.317\end{array}$

$\begin{array}{lllllll}13 & 28 & 0.20 & 0.00 & 0.04 & -0.03 & 0.218\end{array}$

$\begin{array}{lllllll}14 & 29 & -0.33 & 0.00 & -0.06 & -0.03 & -0.331\end{array}$

$\begin{array}{lllllll}15 & 30 & -0.12 & 0.00 & 0.01 & 0.03 & -0.125\end{array}$

$\begin{array}{llllllll}16 & 31 & -0.21 & 0.00 & 0.03 & -0.04 & -0.218\end{array}$

$\begin{array}{llllllll}17 & 32 & -0.19 & 0.00 & 0.05 & -0.01 & -0.198\end{array}$

$\begin{array}{lllllll}18 & 33 & -0.12 & 0.00 & 0.04 & 0.04 & -0.124\end{array}$

$\begin{array}{lllllll}19 & 34 & -0.06 & 0.00 & 0.01 & 0.00 & -0.063\end{array}$

$\begin{array}{lllllll}20 & 35 & 0.00 & 0.00 & 0.00 & 0.00 & 0.000\end{array}$

$\begin{array}{lllllll}21 & 36 & -0.06 & 0.00 & -0.01 & 0.01 & -0.063\end{array}$

$\begin{array}{lllll}22 & 37 & 0.11 & 0.00 & -0.01\end{array}$

$0.02 \quad 0.118$

$\begin{array}{llllll}23 & 38 & 0.18 & 0.00 & -0.02\end{array}$

$0.00 \quad 0.194$

2439

$\begin{array}{llll}0.21 & 0.00 & -0.01\end{array}$

0.03

0.227

0.229
$-0.025-0.139$

$\begin{array}{llll}0.000 & 0.141 & -0.013 & -0.45\end{array}$

$\begin{array}{llll}0.000 & -0.134 & -0.039 & 0.56\end{array}$

$\begin{array}{llll}0.000 & -0.068 & -0.054 & 0.94\end{array}$

$\begin{array}{llll}0.000 & -0.034 & 0.021 & 1.85\end{array}$

$\begin{array}{rrrr}0.000 & 0.110 & 0.002 & -0.11\end{array}$

$\begin{array}{llll}0.000 & -0.007 & -0.027 & 1.81\end{array}$

$\begin{array}{llll}0.000 & -0.014 & 0.042 & 1.39\end{array}$

$\begin{array}{llll}0.000 & -0.041 & 0.019 & 1.88\end{array}$

$\begin{array}{lll}0.000 & -0.041 & -0.032\end{array}$

$\begin{array}{lll}0.000 & -0.010 & 0.001\end{array}$

$\begin{array}{lll}0.000 & 0.000 & 0.000\end{array}$

$\begin{array}{llll}0.000 & 0.013 & -0.010\end{array}$

1.89

2.00

0.000

1.23

$\begin{array}{lll}0.000 & 0.038 & 0.006\end{array}$

$\begin{array}{llll}0.000 & 0.034 & -0.026\end{array}$

$\begin{array}{llll}0.000 & -0.004 & -0.013\end{array}$

1.77

1.47

0.67

0.37 $\begin{array}{llll}0.000 & -0.045 & -0.048 & 1.14\end{array}$
$-1.07 \quad 129.62$

$1.43 \quad 148.57$

\begin{tabular}{ll}
$1.45 \quad 171.08$ \\
\hline
\end{tabular}

$3.49 \quad 186.85$

3.41205 .96

$3.35 \quad 220.82$

$2.87 \quad 248.65-18.49-1$

$\begin{array}{lll}-18.25 & -20.20\end{array}$

$\begin{array}{lllll}2.70 & 261.53 & -23.06 & -24.44\end{array}$

$\begin{array}{lllll}3.71 & 268.81 & -22.26 & -24.31\end{array}$

$\begin{array}{llll}3.08 & 279.28 & -24.66 & -26.34\end{array}$

$\begin{array}{llll}3.27 & 285.73 & -23.04 & -24.56\end{array}$

$\begin{array}{llll}2.95 & 294.28 & -23.52 & -24.86\end{array}$

$\begin{array}{llll}3.34 & 299.10 & -20.27 & -20.25\end{array}$ $\begin{array}{lllll}3.32 & 305.95 & -19.05 & -18.99\end{array}$

$\begin{array}{lllll}3.56 & 309.68 & -14.70 & -14.76\end{array}$

$\begin{array}{llll}2.60 & 319.12 & -8.00 & -8.11\end{array}$ $\begin{array}{lllll}2.95 & 315.89 & -12.85 & -12.87\end{array}$

$\begin{array}{lll}0.000 & 3.14 & -20.89\end{array}$

$\begin{array}{lll}0.000 & 2.79 & -22.53\end{array}$

$\begin{array}{llll}0.016 & 2.91 & -19.27\end{array}$

$\begin{array}{lll}0.014 & 2.68 & -19.25\end{array}$

$\begin{array}{lll}0.038 & 2.92 & -14.57\end{array}$

$\begin{array}{lll}0.123 & 2.66 & -13.26\end{array}$

$\begin{array}{lll}0.169 & 3.36 & -6.99\end{array}$

$\begin{array}{lll}0.137 & 2.64 & -4.97\end{array}$

$2.35 \quad 1.33$

$2.14 \quad 4.89$

$\begin{array}{ll}1.03 & 11.25\end{array}$

$0.49 \quad 15.41$

$0.73 \quad 23.92$

$0.84 \quad 29.56$

$0.73 \quad 38.44$

$0.96 \quad 44.94$

$0.99 \quad 54.59$

$1.25 \quad 61.81$

$\begin{array}{ll}1.30 & 72.07\end{array}$

$1.75 \quad 80.09$

$1.99 \quad 91.08$

$2.22 \quad 99.44$

$\begin{array}{ll}1.87 & 110.32\end{array}$

$\begin{array}{ll}1.77 & 118.87\end{array}$

$1.55 \quad 130.33$

$\begin{array}{ll}1.43 & 139.33\end{array}$

$\begin{array}{ll}0.91 & 150.90\end{array}$

$0.95 \quad 160.50$

$\begin{array}{ll}0.31 & 172.34\end{array}$

$0.37 \quad 182.35$

$-0.20 \quad 194.61$

$-0.28 \quad 204.86$

$-0.80 \quad 217.50$

$-0.57 \quad 228.42$

$-1.24 \quad 241.20$

$\begin{array}{ll}-1.07 & 252.38\end{array}$ 


\begin{tabular}{|c|c|c|c|c|c|c|c|c|c|c|c|c|c|c|c|c|c|}
\hline \multicolumn{18}{|c|}{$Z=15(\mathrm{P})$} \\
\hline 26 & 41 & 0.22 & 0.00 & 0.05 & -0.03 & 0.241 & 0.000 & -0.043 & 0.018 & -0.19 & 1.94 & 324.30 & -5.11 & -5.28 & 0.216 & 2.05 & -5.01 \\
\hline 27 & 42 & 0.16 & 0.00 & 0.03 & -0.01 & 0.173 & 0.000 & -0.026 & 0.005 & 0.01 & 1.81 & 326.33 & 0.93 & 0.94 & 0.447 & 1.84 & 0.86 \\
\hline 28 & 43 & -0.27 & 0.00 & -0.09 & 0.00 & -0.271 & 0.000 & 0.129 & -0.027 & -2.18 & 0.88 & 330.82 & 4.51 & 5.77 & 0.969 & 1.41 & 4.85 \\
\hline 29 & 44 & -0.27 & 0.00 & -0.04 & -0.05 & -0.273 & 0.000 & 0.077 & 0.032 & -1.59 & 0.59 & 332.16 & 11.24 & & & 0.93 & 11.31 \\
\hline 30 & 45 & -0.23 & 0.00 & -0.02 & -0.05 & -0.235 & 0.000 & 0.047 & 0.040 & -0.71 & 0.72 & 334.73 & 16.74 & & & 1.06 & 16.71 \\
\hline 31 & 46 & -0.27 & 0.00 & 0.00 & -0.05 & -0.276 & 0.000 & 0.032 & 0.042 & -0.86 & 0.75 & 334.98 & 24.56 & & & 1.11 & 24.47 \\
\hline 32 & 47 & -0.27 & 0.00 & 0.02 & -0.05 & -0.277 & 0.000 & 0.010 & 0.048 & -0.59 & 0.88 & 336.80 & 30.82 & & & 1.34 & 30.75 \\
\hline 33 & 48 & -0.27 & 0.00 & 0.03 & -0.05 & -0.278 & 0.000 & -0.001 & 0.051 & -0.65 & 0.94 & 336.33 & 39.36 & & & 1.46 & 39.26 \\
\hline 34 & 49 & -0.29 & 0.00 & 0.04 & -0.05 & -0.299 & 0.000 & -0.007 & 0.053 & -0.67 & 0.97 & 337.55 & 46.21 & & & 1.63 & 46.18 \\
\hline 35 & 50 & -0.36 & 0.00 & 0.05 & -0.05 & -0.368 & 0.000 & 0.000 & 0.054 & -1.13 & 1.04 & 336.44 & 55.39 & & & 1.83 & 55.42 \\
\hline 36 & 51 & -0.36 & 0.00 & 0.04 & -0.06 & -0.368 & 0.000 & 0.012 & 0.059 & -0.77 & 1.29 & 336.83 & 63.08 & & & 2.31 & 63.28 \\
\hline 37 & 52 & -0.36 & 0.00 & 0.04 & -0.06 & -0.368 & 0.000 & 0.012 & 0.059 & -0.47 & 1.25 & 335.27 & 72.70 & & & 2.28 & 72.87 \\
\hline 38 & 53 & -0.21 & 0.00 & 0.10 & 0.06 & -0.213 & 0.000 & -0.096 & -0.030 & 0.81 & 1.49 & 335.11 & 80.94 & & & 2.55 & 81.10 \\
\hline 39 & 54 & 0.34 & 0.00 & 0.06 & -0.06 & 0.379 & 0.000 & -0.032 & 0.040 & -0.51 & 1.57 & 332.92 & 91.20 & & & 2.32 & 91.02 \\
\hline 40 & 55 & 0.34 & 0.00 & 0.06 & -0.06 & 0.379 & 0.000 & -0.032 & 0.040 & -0.54 & 1.39 & 332.66 & 99.53 & & & 2.21 & 99.40 \\
\hline 41 & 56 & 0.34 & 0.00 & 0.07 & -0.05 & 0.380 & 0.000 & -0.042 & 0.025 & -0.82 & 1.26 & 330.21 & 110.05 & & & 1.85 & 109.68 \\
\hline 42 & 57 & 0.31 & 0.00 & 0.05 & -0.04 & 0.344 & 0.000 & -0.024 & 0.025 & -0.39 & 1.71 & 328.86 & 119.47 & & & 2.13 & 118.94 \\
\hline 43 & 58 & 0.22 & 0.00 & -0.03 & -0.01 & 0.239 & 0.000 & 0.058 & 0.021 & -0.17 & 1.33 & 326.24 & 130.16 & & & 1.59 & 129.48 \\
\hline 44 & 59 & 0.22 & 0.00 & -0.02 & 0.01 & 0.238 & 0.000 & 0.046 & -0.002 & -0.45 & 1.28 & 324.95 & 139.52 & & & 1.41 & 138.74 \\
\hline 45 & 60 & 0.22 & 0.00 & -0.02 & 0.02 & 0.238 & 0.000 & 0.047 & -0.012 & -1.19 & 0.56 & 322.29 & 150.25 & & & 0.74 & 149.56 \\
\hline 46 & 61 & 0.22 & 0.00 & -0.01 & 0.03 & 0.238 & 0.000 & 0.036 & -0.025 & -1.42 & 0.22 & 320.89 & 159.72 & & & 0.53 & 159.21 \\
\hline 47 & 62 & 0.22 & 0.00 & 0.01 & 0.07 & 0.241 & 0.000 & 0.016 & -0.070 & -2.08 & -1.74 & 319.12 & 169.57 & & & -0.04 & 170.52 \\
\hline 48 & 63 & 0.22 & 0.00 & 0.04 & 0.02 & 0.242 & 0.000 & -0.025 & -0.028 & -2.04 & -0.44 & 315.73 & 181.03 & & & -0.09 & 180.71 \\
\hline 49 & 64 & 0.22 & 0.00 & 0.07 & 0.02 & 0.245 & 0.000 & -0.061 & -0.036 & -2.78 & -1.43 & 312.65 & 192.18 & & & -0.65 & 192.38 \\
\hline 50 & 65 & 0.26 & 0.00 & 0.11 & 0.01 & 0.295 & 0.000 & -0.100 & -0.040 & -3.19 & -2.20 & 310.99 & 201.91 & & & -0.70 & 202.94 \\
\hline 51 & 66 & 0.26 & 0.00 & 0.12 & -0.02 & 0.294 & 0.000 & -0.117 & -0.014 & -3.59 & -2.61 & 307.03 & 213.94 & & & -1.13 & 215.08 \\
\hline 52 & 67 & 0.27 & 0.00 & 0.12 & -0.03 & 0.304 & 0.000 & -0.117 & -0.006 & -3.28 & -2.44 & 304.13 & 224.92 & & & -0.90 & 226.24 \\
\hline 53 & 68 & 0.28 & 0.00 & 0.12 & -0.03 & 0.316 & 0.000 & -0.114 & -0.007 & -3.53 & -2.65 & 299.68 & 237.43 & & & -1.17 & 238.86 \\
\hline 54 & 69 & 0.30 & 0.00 & 0.12 & -0.03 & 0.339 & 0.000 & -0.109 & -0.009 & -3.11 & -2.26 & 296.28 & 248.91 & & & -0.84 & 250.45 \\
\hline 55 & 70 & 0.27 & 0.00 & 0.12 & -0.03 & 0.304 & 0.000 & -0.117 & -0.006 & -2.93 & -2.65 & 291.75 & 261.51 & & & -1.05 & 263.39 \\
\hline 56 & 71 & 0.27 & 0.00 & 0.12 & -0.03 & 0.304 & 0.000 & -0.117 & -0.006 & -2.27 & -2.29 & 288.10 & 273.23 & & & -0.63 & 275.37 \\
\hline 57 & 72 & -0.06 & 0.00 & 0.12 & 0.08 & -0.052 & 0.000 & -0.134 & -0.063 & -1.99 & -5.17 & 285.83 & 283.57 & & & -0.92 & 288.52 \\
\hline 58 & 73 & 0.06 & 0.00 & 0.12 & 0.08 & 0.078 & 0.000 & -0.132 & -0.081 & -1.63 & -5.93 & 283.05 & 294.42 & & & -0.82 & 300.45 \\
\hline \multicolumn{18}{|c|}{$Z=16(S)$} \\
\hline & 24 & 0.00 & 0.00 & 0.12 & -0.03 & 0.002 & 0.000 & -0.140 & 0.032 & -2.78 & -1.33 & 127.26 & 53.93 & & & -0.73 & 52.14 \\
\hline 9 & 25 & 0.10 & 0.00 & -0.10 & -0.03 & 0.114 & 0.000 & 0.131 & 0.049 & -0.69 & 1.00 & 146.91 & 42.36 & & & 1.38 & 40.81 \\
\hline 10 & 26 & 0.11 & 0.00 & 0.10 & 0.03 & 0.126 & 0.000 & -0.110 & -0.039 & 0.17 & 0.93 & 171.62 & 25.72 & & & 1.15 & 24.44 \\
\hline 11 & 27 & 0.28 & 0.00 & 0.1 & 0.03 & 0.321 & 0.000 & -0.103 & -0.063 & 0.4 & 2.71 & 188 & 17 & & & 2.83 & 16.19 \\
\hline 12 & 28 & 0.28 & 0.00 & 0.12 & 0.03 & 0.321 & 0.000 & -0.103 & -0.063 & 0.23 & 2.76 & 209.21 & 4.27 & 4.07 & 0.160 & 2.87 & 3.58 \\
\hline 13 & 29 & 0.21 & 0.00 & 0.07 & -0.03 & 0.231 & 0.000 & -0.069 & 0.014 & 1.25 & 3.16 & 224.15 & -2.60 & -3.16 & 0.050 & 3.14 & -3.15 \\
\hline 14 & 30 & -0.25 & 0.00 & 0.00 & -0.03 & -0.256 & 0.000 & & 0 & 0.60 & & & & 66 & 0.1 & 2.57 & 13.48 \\
\hline 15 & 31 & 0.19 & 0.00 & 0.07 & -0.04 & 0.208 & 0.000 & -0.073 & 0.02 & 1.2 & 3. & 255.01 & -17.32 & -19.05 & 0.002 & 3.11 & -17.46 \\
\hline 16 & 32 & 0.20 & 0.00 & 0.09 & -0.04 & 0.221 & 0.000 & -0.095 & 0.020 & 0.97 & 2.82 & 270.83 & -25.07 & -26.02 & 0.000 & 2.82 & -25.03 \\
\hline 17 & 33 & -0.20 & 0.00 & 0.09 & -0.04 & -0.209 & 0.000 & -0.082 & 0.056 & 1.41 & 3.37 & 279.06 & -25.22 & -26.59 & 0.000 & 3.36 & -25.08 \\
\hline 18 & 34 & -0.23 & 0.00 & 0.10 & 0.04 & -0.235 & 0.000 & -0.092 & -0.010 & 0.91 & 3.19 & 290.84 & -28.93 & -29.93 & 0.000 & 3.22 & -28.68 \\
\hline 19 & 35 & -0.09 & 0.00 & 0.03 & 0.04 & -0.093 & 0.000 & -0.032 & -0.035 & & 3.17 & 297.96 & -27.98 & -28.85 & 0.000 & 3.17 & -27.70 \\
\hline 20 & 36 & 0.00 & 0.00 & 0.00 & -0.03 & 0.000 & 0.000 & 0.000 & 0.030 & 1.27 & 2.86 & 308.18 & -30.13 & -30.66 & 0.000 & 2.86 & -29.82 \\
\hline 21 & 37 & -0.06 & 0.00 & 0.00 & 0.03 & -0.063 & 0.000 & & -0.029 & 2.10 & 3.31 & & -27.25 & -26.90 & 0.000 & 3.31 & -26.93 \\
\hline 22 & 38 & 0.11 & 0.00 & 0.01 & 0.02 & 0.118 & 0.000 & -0.006 & -0.021 & 1.81 & 3.31 & 321.79 & -27.60 & -26.86 & 0.007 & 3.32 & -27.29 \\
\hline 23 & 39 & 0.18 & 0.00 & 0.00 & -0.01 & 0.195 & 0.000 & 0.013 & 0.011 & 1.58 & 3.59 & 325.87 & -23.60 & -23.16 & 0.050 & 3.59 & -23.33 \\
\hline 24 & 40 & 0.21 & 0.00 & 0.02 & 0.00 & 0.229 & 0.000 & -0.005 & -0.004 & 0.6 & 2.96 & & -23.28 & -22.87 & 0.141 & 2.98 & -23.03 \\
\hline 25 & 41 & 0.23 & 0.00 & 0.04 & -0.02 & 0.252 & 0.000 & -0.028 & 0.011 & 0.07 & 2.60 & 337.20 & -18.80 & -19.02 & 0.118 & 2.64 & -18.58 \\
\hline 26 & 42 & 0.21 & 0.00 & 0.06 & -0.04 & 0.230 & 0.000 & -0.058 & 0.026 & -0.31 & 1.80 & 343.97 & -17.49 & -17.68 & 0.124 & 1.96 & -17.22 \\
\hline 27 & 43 & 0.18 & 0.00 & 0.04 & -0.02 & 0.196 & 0.000 & -0.036 & 0.012 & -0.23 & 1.36 & 346.63 & -12.08 & -11.97 & 0.202 & 1.43 & -11.97 \\
\hline 28 & 44 & -0.24 & 0.00 & -0.03 & 0.05 & -0.247 & 0.000 & 0.051 & -0.055 & -1.34 & 0.89 & 352.04 & -9.42 & -9.12 & 0.395 & 1.27 & -9.08 \\
\hline 29 & 45 & -0.20 & 0.00 & 0.01 & 0.05 & -0.206 & 0.000 & 0.001 & -0.045 & -0.77 & 0.80 & 353.47 & -2.77 & -3.25 & 1.742 & 1.04 & -2.65 \\
\hline 30 & 46 & -0.20 & 0.00 & 0.02 & -0.05 & -0.207 & 0.000 & -0.004 & 0.049 & -0.46 & 0.60 & 357.70 & 1.07 & & & 0.92 & 1.20 \\
\hline
\end{tabular}




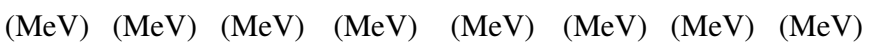

\begin{tabular}{|c|c|c|c|c|c|c|c|c|c|c|c|c|c|c|c|c|c|}
\hline \multicolumn{18}{|c|}{$Z=16(S)$} \\
\hline 31 & 47 & -0.23 & 0.00 & 0.04 & -0.05 & -0.238 & 0.000 & -0.021 & 0.054 & -0.53 & 0.75 & 358.07 & 8.76 & & & 1.18 & 8.92 \\
\hline 32 & 48 & -0.27 & 0.00 & 0.06 & -0.05 & -0.280 & 0.000 & -0.034 & 0.059 & -0.68 & 0.83 & 361.19 & 13.71 & & & 1.48 & 14.01 \\
\hline 33 & 49 & -0.27 & 0.00 & 0.07 & -0.05 & -0.280 & 0.000 & -0.045 & 0.062 & -0.84 & 0.62 & 361.23 & 21.75 & & & 1.38 & 22.08 \\
\hline 34 & 50 & -0.28 & 0.00 & 0.07 & -0.05 & -0.290 & 0.000 & -0.042 & 0.062 & -0.78 & 0.88 & 363.43 & 27.62 & & & 1.74 & 27.98 \\
\hline 35 & 51 & -0.27 & 0.00 & 0.07 & -0.06 & -0.280 & 0.000 & -0.044 & 0.071 & -0.36 & 1.14 & 362.34 & 36.78 & & & 2.23 & 37.30 \\
\hline 36 & 52 & -0.27 & 0.00 & 0.06 & -0.06 & -0.280 & 0.000 & -0.033 & 0.068 & -0.03 & 1.23 & 364.04 & 43.15 & & & 2.31 & 43.60 \\
\hline 37 & 53 & -0.21 & 0.00 & 0.10 & 0.06 & -0.213 & 0.000 & -0.096 & -0.030 & 0.49 & 1.58 & 362.27 & 52.99 & & & 2.49 & 53.21 \\
\hline 38 & 54 & -0.21 & 0.00 & 0.11 & 0.06 & -0.213 & 0.000 & -0.107 & -0.027 & 0.50 & 1.37 & 363.66 & 59.67 & & & 2.46 & 60.03 \\
\hline 39 & 55 & -0.22 & 0.00 & 0.11 & 0.06 & -0.223 & 0.000 & -0.105 & -0.026 & 0.45 & 1.36 & 361.74 & 69.67 & & & 2.46 & 70.01 \\
\hline 40 & 56 & -0.22 & 0.00 & 0.10 & 0.06 & -0.223 & 0.000 & -0.094 & -0.029 & 0.58 & 1.40 & 362.32 & 77.16 & & & 2.48 & 77.44 \\
\hline 41 & 57 & 0.33 & 0.00 & 0.06 & -0.05 & 0.367 & 0.000 & -0.033 & 0.030 & -0.52 & 1.67 & 359.61 & 87.94 & & & 2.25 & 87.69 \\
\hline 42 & 58 & 0.33 & 0.00 & 0.07 & -0.04 & 0.368 & 0.000 & -0.043 & 0.016 & -0.72 & 1.52 & 359.88 & 95.74 & & & 2.01 & 95.39 \\
\hline 43 & 59 & 0.26 & 0.00 & 0.02 & -0.01 & 0.285 & 0.000 & 0.004 & 0.007 & -0.37 & 1.52 & 357.01 & 106.68 & & & 1.59 & 105.92 \\
\hline 44 & 60 & 0.22 & 0.00 & 0.00 & 0.00 & 0.239 & 0.000 & 0.021 & 0.002 & -0.39 & 1.37 & 356.81 & 114.95 & & & 1.43 & 114.19 \\
\hline 45 & 61 & 0.22 & 0.00 & 0.00 & 0.00 & 0.239 & 0.000 & 0.021 & 0.002 & -1.11 & 0.84 & 354.07 & 125.76 & & & 0.88 & 125.01 \\
\hline 46 & 62 & 0.22 & 0.00 & 0.02 & 0.01 & 0.240 & 0.000 & -0.002 & -0.013 & -1.48 & 0.41 & 353.72 & 134.19 & & & 0.51 & 133.53 \\
\hline 47 & 63 & 0.22 & 0.00 & 0.05 & -0.01 & 0.241 & 0.000 & -0.041 & -0.002 & -2.17 & -0.25 & 350.74 & 145.24 & & & -0.04 & 144.74 \\
\hline 48 & 64 & 0.22 & 0.00 & 0.05 & -0.01 & 0.241 & 0.000 & -0.041 & -0.002 & -2.33 & -0.51 & 349.82 & 154.22 & & & -0.29 & 153.81 \\
\hline 49 & 65 & 0.22 & 0.00 & 0.08 & -0.02 & 0.243 & 0.000 & -0.078 & 0.001 & -3.11 & -1.54 & 346.85 & 165.26 & & & -0.89 & 165.33 \\
\hline 50 & 66 & 0.22 & 0.00 & 0.08 & 0.00 & 0.244 & 0.000 & -0.075 & -0.019 & -3.01 & -1.54 & 345.32 & 174.87 & & & -0.85 & 175.08 \\
\hline 51 & 67 & 0.23 & 0.00 & 0.11 & 0.00 & 0.259 & 0.000 & -0.108 & -0.027 & -3.52 & -2.47 & 341.94 & 186.32 & & & -1.12 & 187.29 \\
\hline 52 & 68 & 0.23 & 0.00 & 0.12 & 0.00 & 0.261 & 0.000 & -0.120 & -0.029 & -3.17 & -2.54 & 340.13 & 196.20 & & & -0.83 & 197.63 \\
\hline 53 & 69 & 0.23 & 0.00 & 0.12 & -0.01 & 0.260 & 0.000 & -0.122 & -0.020 & -3.28 & -2.74 & 335.73 & 208.67 & & & -1.11 & 210.15 \\
\hline 54 & 70 & -0.19 & 0.00 & 0.01 & -0.07 & -0.196 & 0.000 & 0.008 & 0.066 & -2.35 & -2.95 & 333.76 & 218.72 & & & -0.98 & 220.69 \\
\hline 55 & 71 & -0.19 & 0.00 & 0.02 & -0.08 & -0.197 & 0.000 & -0.003 & 0.078 & -2.54 & -3.95 & 329.88 & 230.66 & & & -1.23 & 233.54 \\
\hline 56 & 72 & -0.19 & 0.00 & 0.03 & -0.08 & -0.197 & 0.000 & -0.014 & 0.080 & -2.05 & -3.86 & 327.31 & 241.31 & & & -0.89 & 244.60 \\
\hline 57 & 73 & -0.19 & 0.00 & 0.03 & -0.08 & -0.197 & 0.000 & -0.014 & 0.080 & -2.00 & -4.22 & 322.55 & 254.14 & & & -1.20 & 257.67 \\
\hline 58 & 74 & -0.31 & 0.00 & 0.01 & -0.08 & -0.316 & 0.000 & 0.034 & 0.070 & -1.96 & -2.87 & 318.44 & 266.32 & & & -0.10 & 269.79 \\
\hline 59 & 75 & -0.25 & 0.00 & 0.02 & -0.08 & -0.257 & 0.000 & 0.009 & 0.076 & -1.78 & -3.99 & 314.20 & 278.64 & & & -0.99 & 282.54 \\
\hline 60 & 76 & -0.26 & 0.00 & 0.04 & -0.08 & -0.269 & 0.000 & -0.012 & 0.081 & -1.38 & -3.64 & 310.84 & 290.06 & & & -0.15 & 294.69 \\
\hline \multicolumn{18}{|c|}{$Z=17(\mathrm{Cl})$} \\
\hline 8 & 25 & -0.05 & 0.13 & 0.02 & 0.00 & -0.045 & -0.172 & -0.015 & 0.011 & -2.98 & -2.04 & 120.64 & 67.84 & & & -1.72 & 65.30 \\
\hline 9 & 26 & 0.11 & 0.00 & 0.02 & 0.03 & 0.119 & 0.000 & -0.017 & -0.032 & -0.88 & 0.99 & 141.61 & 54.94 & & & 1.05 & 52.63 \\
\hline 10 & 27 & 0.11 & 0.00 & 0.10 & 0.03 & 0.126 & 0.000 & -0.110 & -0.039 & -0.16 & 0.93 & 166.81 & 37.82 & & & 1.19 & 36.16 \\
\hline 11 & 28 & 0.11 & 0.00 & 0.04 & 0.03 & 0.120 & 0.000 & -0.041 & -0.034 & 1.39 & 2.26 & 185.82 & 26.88 & & & 2.29 & 25.40 \\
\hline 12 & 29 & -0.23 & 0.00 & 0.12 & -0.03 & -0.240 & 0.000 & -0.110 & 0.058 & 0.82 & 2.52 & 207.13 & 13.64 & & & 2.67 & 12.63 \\
\hline 13 & 30 & -0.23 & 0.00 & 0.09 & -0.03 & -0.240 & 0.000 & -0.077 & 0.049 & 1.04 & 2.92 & 223.97 & 4.87 & & & 2.92 & 4.03 \\
\hline 14 & 31 & -0.24 & 0.00 & 0.06 & -0.04 & -0.249 & 0.000 & -0.041 & 0.050 & 0.42 & 2.49 & 242.96 & -6.05 & -7.07 & 0.050 & 2.48 & -6.64 \\
\hline 15 & 32 & -0.21 & 0.00 & 0.07 & -0.04 & -0.219 & 0.000 & -0.058 & 0.052 & 1.02 & 3.01 & 257.05 & -12.07 & -13.33 & 0.007 & 2.97 & -12.47 \\
\hline 16 & 33 & -0.23 & 0.00 & 0.11 & 0.04 & -0.235 & 0.000 & -0.102 & -0.007 & 0.84 & 3.09 & 272.96 & -19.91 & -21.00 & 0.000 & 3.07 & -20.10 \\
\hline 17 & 34 & -0.23 & 0.00 & 0.12 & 0.04 & -0.234 & 0.000 & -0.113 & -0.004 & 1.03 & 3.57 & 283.94 & -22.81 & -24.44 & 0.000 & 3.52 & -23.77 \\
\hline 18 & 35 & -0.23 & 0.00 & 0.12 & 0.04 & -0.234 & 0.000 & -0.113 & -0.004 & 0.80 & 3.30 & 297.17 & -27.97 & -29.01 & 0.000 & 3.29 & -27.88 \\
\hline 19 & 36 & -0.11 & 0.00 & 0.04 & 0.03 & -0.114 & 0.000 & -0.042 & -0.023 & 1.90 & 3.49 & 305.74 & -28.47 & -29.52 & 0.000 & 3.48 & -28.31 \\
\hline 20 & 37 & 0.01 & 0.00 & 0.00 & 0.00 & 0.011 & 0.000 & 0.000 & 0.000 & 1.42 & 3.13 & 316.45 & -31.11 & -31.76 & 0.000 & 3.12 & -30.88 \\
\hline 21 & 38 & -0.07 & 0.00 & 0.00 & 0.01 & -0.073 & 0.000 & 0.002 & -0.010 & 2.14 & 3.54 & 323.26 & -29.85 & -29.80 & 0.000 & 3.53 & -29.58 \\
\hline 22 & 39 & 0.07 & 0.00 & 0.00 & 0.01 & 0.075 & 0.000 & 0.002 & -0.010 & 2.13 & 3.44 & 332.19 & -30.71 & -29.80 & 0.002 & 3.43 & -30.42 \\
\hline 23 & 40 & 0.14 & 0.00 & 0.00 & 0.00 & 0.150 & 0.000 & 0.008 & 0.001 & 2.17 & 4.16 & 337.33 & -27.78 & -27.56 & 0.032 & 4.15 & -27.50 \\
\hline 24 & 41 & 0.18 & 0.00 & 0.01 & 0.00 & 0.195 & 0.000 & 0.002 & -0.001 & 1.39 & 3.06 & 345.93 & -28.30 & -27.31 & 0.069 & 3.06 & -28.03 \\
\hline 25 & 42 & 0.19 & 0.00 & 0.03 & -0.01 & 0.207 & 0.000 & -0.022 & 0.004 & 0.95 & 2.88 & 350.77 & -25.08 & -24.91 & 0.144 & 2.89 & -24.84 \\
\hline 26 & 43 & 0.17 & 0.00 & 0.04 & -0.02 & 0.185 & 0.000 & -0.038 & 0.013 & 0.54 & 2.22 & 357.73 & -23.97 & -24.17 & 0.157 & 2.27 & -23.73 \\
\hline 27 & 44 & -0.17 & 0.00 & 0.03 & 0.05 & -0.175 & 0.000 & -0.025 & -0.041 & 0.20 & 2.00 & 361.57 & -19.73 & -20.23 & 0.108 & 2.12 & -19.47 \\
\hline 28 & 45 & -0.15 & 0.00 & 0.02 & 0.04 & -0.155 & 0.000 & -0.016 & -0.034 & -0.35 & 1.04 & 367.77 & -17.86 & -18.36 & 0.1 & 1.14 & -17.68 \\
\hline 29 & 46 & -0.17 & 0.00 & 0.03 & 0.03 & -0.176 & 0.000 & -0.024 & -0.023 & -0.41 & 0.93 & 370.54 & -12.56 & -14.71 & 0.717 & 1.00 & -12.47 \\
\hline 30 & 47 & -0.20 & 0.00 & 0.05 & 0.04 & -0.206 & 0.000 & -0.042 & -0.026 & -0.32 & 0.99 & 374.78 & -8.73 & & & 1.16 & -8.61 \\
\hline 31 & 48 & -0.23 & 0.00 & 0.05 & -0.05 & -0.239 & 0.000 & -0.032 & 0.056 & -0.37 & 1.12 & 376.45 & -2.33 & & & 1.51 & -2.07 \\
\hline 32 & 49 & -0.23 & 0.00 & 0.06 & -0.05 & -0.239 & 0.000 & -0.043 & 0.059 & -0.14 & 1.23 & 379.80 & 2.40 & & & 1.74 & 2.71 \\
\hline 33 & 50 & -0.27 & 0.00 & 0.08 & -0.05 & -0.280 & 0.000 & -0.056 & 0.065 & -0.68 & 1.19 & 380.87 & 9.39 & & & 1.89 & 9.83 \\
\hline
\end{tabular}




\begin{tabular}{|c|c|c|c|c|c|c|c|c|c|c|c|c|c|c|c|c|}
\hline \multicolumn{17}{|c|}{$Z=17(\mathrm{Cl})$} \\
\hline 3451 & -0.27 & 0.00 & 0.08 & -0.06 & -0.281 & 0.000 & -0.055 & 0.074 & -0.62 & 1.26 & 383.48 & 14.85 & & & 2.24 & 15.49 \\
\hline 3552 & -0.27 & 0.00 & 0.08 & -0.06 & -0.281 & 0.000 & -0.055 & 0.074 & -0.25 & 1.40 & 383.68 & 22.73 & & & 2.42 & 23.35 \\
\hline 3653 & -0.23 & 0.00 & 0.09 & 0.02 & -0.237 & 0.000 & -0.080 & 0.005 & 0.41 & 2.22 & 384.86 & 29.62 & & & 2.64 & 29.58 \\
\hline 3754 & -0.21 & 0.00 & 0.10 & 0.06 & -0.213 & 0.000 & -0.096 & -0.030 & 0.56 & 1.99 & 384.79 & 37.76 & & & 2.77 & 38.02 \\
\hline 3855 & -0.21 & 0.00 & 0.11 & 0.06 & -0.213 & 0.000 & -0.107 & -0.027 & 0.49 & 1.75 & 386.39 & 44.23 & & & 2.70 & 44.61 \\
\hline 3956 & -0.21 & 0.00 & 0.11 & 0.06 & -0.213 & 0.000 & -0.107 & -0.027 & 0.46 & 1.72 & 385.56 & 53.13 & & & 2.70 & 53.50 \\
\hline 4057 & -0.22 & 0.00 & 0.11 & 0.06 & -0.223 & 0.000 & -0.105 & -0.026 & 0.39 & 1.57 & 386.49 & 60.27 & & & 2.62 & 60.67 \\
\hline 4158 & -0.25 & 0.00 & 0.12 & 0.06 & -0.253 & 0.000 & -0.110 & -0.019 & 0.10 & 1.39 & 385.29 & 69.55 & & & 2.52 & 70.01 \\
\hline 4259 & -0.27 & 0.00 & 0.12 & 0.06 & -0.274 & 0.000 & -0.106 & -0.017 & -0.26 & 0.94 & 385.99 & 76.92 & & & 2.12 & 77.40 \\
\hline 4360 & -0.27 & 0.00 & 0.12 & 0.06 & -0.274 & 0.000 & -0.106 & -0.017 & -0.54 & 0.61 & 384.45 & 86.53 & & & 1.82 & 87.03 \\
\hline 4461 & 0.17 & 0.00 & -0.02 & 0.00 & 0.183 & 0.000 & 0.037 & 0.005 & 0.16 & 1.32 & 383.52 & 95.53 & & & 1.41 & 94.91 \\
\hline 4562 & 0.17 & 0.00 & -0.02 & 0.01 & 0.183 & 0.000 & 0.037 & -0.005 & -0.45 & 1.22 & 381.31 & 105.81 & & & 1.30 & 105.20 \\
\hline 4663 & 0.17 & 0.00 & 0.00 & 0.01 & 0.183 & 0.000 & 0.013 & -0.009 & -0.83 & 0.63 & 381.23 & 113.97 & & & 0.68 & 113.34 \\
\hline $47 \quad 64$ & 0.17 & 0.00 & 0.04 & 0.00 & 0.185 & 0.000 & -0.036 & -0.007 & -1.54 & -0.45 & 379.59 & 123.67 & & & -0.31 & 123.17 \\
\hline $48 \quad 65$ & 0.17 & 0.00 & 0.04 & -0.01 & 0.185 & 0.000 & -0.037 & 0.003 & -1.76 & -0.75 & 378.81 & 132.52 & & & -0.60 & 132.08 \\
\hline 4966 & 0.18 & 0.00 & 0.05 & -0.01 & 0.197 & 0.000 & -0.047 & 0.000 & -2.29 & -1.32 & 376.29 & 143.12 & & & -1.10 & 142.80 \\
\hline 5067 & -0.12 & 0.00 & -0.01 & 0.01 & -0.125 & 0.000 & 0.017 & -0.011 & -2.25 & -1.21 & 374.72 & 152.76 & & & -1.14 & 152.35 \\
\hline 5168 & -0.18 & 0.00 & -0.01 & 0.02 & -0.186 & 0.000 & 0.023 & -0.022 & -2.79 & -1.45 & 371.52 & 164.03 & & & -1.24 & 163.84 \\
\hline 5269 & -0.12 & 0.00 & -0.01 & 0.00 & -0.125 & 0.000 & 0.017 & -0.002 & -2.51 & -1.43 & 369.69 & 173.93 & & & -1.40 & 173.65 \\
\hline 5370 & -0.18 & 0.00 & 0.01 & -0.02 & -0.187 & 0.000 & 0.002 & 0.020 & -2.85 & -1.58 & 366.08 & 185.61 & & & -1.42 & 185.57 \\
\hline 5471 & -0.19 & 0.00 & 0.02 & -0.06 & -0.197 & 0.000 & -0.004 & 0.059 & -2.60 & -2.57 & 364.94 & 194.83 & & & -1.14 & 196.17 \\
\hline 5572 & -0.19 & 0.00 & 0.03 & -0.08 & -0.197 & 0.000 & -0.014 & 0.080 & -2.79 & -3.99 & 362.30 & 205.54 & & & -1.34 & 208.24 \\
\hline 5673 & -0.19 & 0.00 & 0.04 & -0.08 & -0.198 & 0.000 & -0.026 & 0.082 & -2.31 & -3.89 & 359.76 & 216.14 & & & -0.97 & 219.25 \\
\hline $57 \quad 74$ & -0.19 & 0.00 & 0.04 & -0.08 & -0.198 & 0.000 & -0.026 & 0.082 & -2.26 & -4.17 & 355.71 & 228.27 & & & -1.19 & 231.60 \\
\hline 5875 & -0.21 & 0.00 & 0.04 & -0.08 & -0.218 & 0.000 & -0.022 & 0.082 & -1.84 & -3.70 & 352.52 & 239.53 & & & -0.61 & 243.14 \\
\hline 5976 & -0.26 & 0.00 & 0.04 & -0.08 & -0.269 & 0.000 & -0.012 & 0.081 & -2.12 & -3.62 & 347.85 & 252.27 & & & -0.47 & 256.12 \\
\hline 6077 & -0.26 & 0.00 & 0.04 & -0.08 & -0.269 & 0.000 & -0.012 & 0.081 & -1.63 & -3.30 & 344.54 & 263.65 & & & -0.05 & 267.81 \\
\hline 6178 & -0.26 & 0.00 & 0.05 & -0.08 & -0.269 & 0.000 & -0.023 & 0.083 & -1.54 & -3.56 & 339.97 & 276.30 & & & -0.01 & 280.95 \\
\hline 6279 & -0.26 & 0.00 & 0.08 & -0.05 & -0.270 & 0.000 & -0.058 & 0.065 & -1.06 & -2.30 & 335.47 & 288.87 & & & 0.24 & 292.73 \\
\hline \multicolumn{17}{|c|}{$Z=18(\mathrm{Ar})$} \\
\hline 927 & 0.11 & 0.00 & 0.00 & -0.03 & 0.118 & 0.000 & 0.004 & 0.030 & -0.88 & 0.93 & 137.69 & 66.15 & & & 1.01 & 63.42 \\
\hline 1028 & 0.11 & 0.00 & 0.03 & 0.03 & 0.120 & 0.000 & -0.029 & -0.033 & -0.29 & 1.11 & 164.61 & 47.31 & & & 1.20 & 45.06 \\
\hline 1129 & -0.27 & 0.00 & 0.11 & -0.03 & -0.280 & 0.000 & -0.090 & 0.058 & 0.38 & 2.16 & 184.38 & 35.61 & & & 2.42 & 33.97 \\
\hline 1230 & -0.27 & 0.00 & 0.11 & -0.03 & -0.280 & 0.000 & -0.090 & 0.058 & 0.20 & 2.02 & 207.99 & 20.07 & & & 2.24 & 18.77 \\
\hline 1331 & -0.27 & 0.00 & 0.10 & -0.04 & -0.281 & 0.000 & -0.078 & 0.063 & 0.31 & 2.25 & 225.49 & 10.64 & & & 2.35 & 9.56 \\
\hline 1432 & -0.27 & 0.00 & 0.10 & -0.04 & -0.281 & 0.000 & -0.078 & 0.063 & -0.25 & 02 & 246.12 & 1 & 0 & & 2.12 & -2.69 \\
\hline 1533 & -0.20 & 0.00 & 0.09 & -0.04 & -0.209 & 0.000 & -0.082 & 0.056 & 0.62 & 2.47 & 260.75 & -8.48 & -9.38 & 0.000 & 2.49 & -9.08 \\
\hline 1634 & -0.22 & 0.00 & 0.12 & -0.04 & -0.230 & 0.000 & -0.111 & 0.066 & 0.42 & 2.74 & 278.25 & -17.91 & -18.38 & 0.000 & 2.80 & -18.25 \\
\hline $17 \quad 35$ & -0.23 & 0.00 & 0.12 & 0.04 & -0.234 & 0.000 & -0.113 & -0.004 & 0.57 & 3.28 & 290.52 & -22.11 & -23.05 & 0.001 & 3.26 & -22.34 \\
\hline 1836 & -0.25 & 0.00 & 0.12 & 0.04 & -0.255 & 0.000 & -0.109 & -0.002 & 0.21 & 3.22 & 306.06 & -29.57 & -30.23 & 0.000 & 3.24 & -29.62 \\
\hline 1937 & -0.07 & 0.00 & 0.02 & 0.03 & -0.073 & 0.000 & -0.022 & -0.027 & 1.50 & 2.88 & 315.61 & -31.06 & -30.95 & 00 & 2.87 & -31.01 \\
\hline 2038 & 0.00 & 0.00 & 0.00 & 0.00 & 0.000 & 0.000 & 0.000 & 0.000 & 0.78 & 2.68 & 327.77 & -35.14 & -34.72 & 0.000 & 2.67 & -35.00 \\
\hline 2139 & -0.04 & 0.00 & 0.00 & 0.02 & -0.042 & 0.000 & 0.000 & -0.019 & 1.63 & 3.01 & 335.08 & -34.38 & -33.24 & 0.005 & 3.00 & -34.17 \\
\hline 2240 & -0.03 & 0.00 & 0.00 & 0.04 & -0.031 & 0.000 & 0.000 & -0.039 & 1.60 & 2.79 & 345.67 & -36.90 & -35.04 & 0.000 & 2.80 & -36.63 \\
\hline 2341 & 0.07 & 0.00 & 0.00 & 0.02 & 0.075 & 0.000 & 0.003 & -0.020 & 2.10 & 3.33 & 351.38 & -34.54 & -33.07 & 0.000 & 3.33 & -34.27 \\
\hline $24 \quad 42$ & -0.03 & 0.00 & 0.00 & 0.02 & -0.032 & 0.000 & 0.000 & -0.020 & 1.88 & 2.45 & 361.23 & -36.31 & -34.42 & 0.006 & 2.46 & -36.03 \\
\hline 2543 & 0.11 & 0.00 & 0.01 & 0.01 & 0.118 & 0.000 & -0.007 & -0.011 & 1.43 & 2.88 & 365.83 & -32.85 & -32.01 & 0.005 & 2.88 & -32.57 \\
\hline 2644 & -0.14 & 0.00 & 0.04 & 0.05 & -0.144 & 0.000 & -0.039 & -0.040 & 0.56 & 2.02 & 374.41 & -33.35 & -32.67 & 0.002 & 2.12 & -33.01 \\
\hline $27 \quad 45$ & -0.15 & 0.00 & 0.04 & 0.05 & -0.154 & 0.000 & -0.038 & -0.040 & -0.06 & 1.38 & 378.97 & -29.85 & -29.77 & 0.001 & 1.49 & -29.52 \\
\hline 2846 & -0.13 & 0.00 & 0.02 & 0.03 & -0.135 & 0.000 & -0.017 & -0.025 & -0.67 & 0.71 & 386.25 & -29.05 & -29.72 & 0.041 & 0.76 & -28.83 \\
\hline 2947 & -0.14 & 0.00 & 0.03 & 0.02 & -0.146 & 0.000 & -0.027 & -0.014 & -0.63 & 0.84 & 389.07 & -23.80 & -25.91 & 0.100 & 0.88 & -23.64 \\
\hline 3048 & -0.20 & 0.00 & 0.06 & 0.05 & -0.205 & 0.000 & -0.054 & -0.032 & -0.64 & 1.19 & 394.33 & -20.99 & & & 1.41 & -20.70 \\
\hline 3149 & -0.21 & 0.00 & 0.08 & -0.05 & -0.220 & 0.000 & -0.069 & 0.064 & -0.58 & 1.26 & 396.32 & -14.91 & & & 1.76 & -14.41 \\
\hline 3250 & -0.23 & 0.00 & 0.07 & -0.05 & -0.240 & 0.000 & -0.054 & 0.062 & -0.39 & 1.57 & 400.72 & -11.24 & & & 2.10 & -10.77 \\
\hline 3351 & -0.27 & 0.00 & 0.09 & -0.06 & -0.281 & 0.000 & -0.066 & 0.077 & -0.94 & 1.39 & 402.17 & -4.61 & & & 2.26 & -3.87 \\
\hline 3452 & -0.27 & 0.00 & 0.09 & -0.06 & -0.281 & 0.000 & -0.066 & 0.077 & -0.87 & 1.39 & 406.06 & -0.43 & & & 2.38 & 0.37 \\
\hline 3553 & -0.27 & 0.00 & 0.10 & -0.02 & -0.280 & 0.000 & -0.079 & 0.045 & -0.42 & 2.02 & 405.99 & 7.71 & & & 2.62 & 8.06 \\
\hline
\end{tabular}




\begin{tabular}{|c|c|c|c|c|c|c|c|c|c|c|c|c|}
\hline \\
\hline 3654 & -0.27 & 0.00 & \multicolumn{9}{|c|}{$Z=18$ (Ar) } & \\
\hline $37 \quad 55$ & -0.21 & 0.00 & 0.11 & 0.06 & -0.213 & 0.000 & -0.107 & -0.027 & 0.28 & 2.06 & 408.99 & 20.85 \\
\hline 3856 & -0.21 & 0.00 & 0.12 & 0.06 & -0.212 & 0.000 & -0.118 & -0.024 & 0.15 & 1.85 & 411.68 & 26.23 \\
\hline 3957 & -0.21 & 0.00 & 0.11 & 0.06 & -0.213 & 0.000 & -0.107 & -0.027 & 0.18 & 1.82 & 411.02 & 34.97 \\
\hline $40 \quad 58$ & -0.22 & 0.00 & 0.12 & 0.06 & -0.222 & 0.000 & -0.116 & -0.023 & -0.03 & 1.59 & 413.11 & 40.95 \\
\hline 4159 & -0.25 & 0.00 & 0.12 & 0.06 & -0.253 & 0.000 & -0.110 & -0.019 & -0.31 & 1.52 & 411.94 & 50.19 \\
\hline 4260 & -0.25 & 0.00 & 0.12 & 0.06 & -0.253 & 0.000 & -0.110 & -0.019 & -0.40 & 1.18 & .57 & 56.63 \\
\hline 4361 & -0.27 & 0.00 & 0.12 & 0.07 & -0.273 & 0.000 & -0.107 & -0.026 & -0.79 & 0.58 & 412.44 & 65.83 \\
\hline 4462 & 0.12 & 0.00 & -0.02 & 0.00 & 0.128 & 0.000 & 0.030 & 0.003 & 0.35 & 1.57 & 412.21 & 74.13 \\
\hline $45 \quad 63$ & 0.16 & 0.00 & -0.02 & 0.01 & 0.172 & 0.000 & 0.036 & -0.005 & -0.38 & 1.04 & 410.56 & 83.85 \\
\hline 4664 & 0.1 & 0.00 & 0.0 & 0.01 & 0.172 & 0.000 & 0.012 & -0.009 & -0.80 & 0.70 & 19 & 91.29 \\
\hline $47 \quad 65$ & 0.16 & 0.00 & 0.02 & 0.00 & 0.173 & 0.000 & -0.013 & -0.003 & -1.54 & -0.02 & 409.30 & 101.25 \\
\hline 4866 & -0.12 & 0.00 & 0.02 & 0.02 & -0.125 & 0.000 & -0.018 & -0.016 & -1.63 & -0.57 & 70 & 08.93 \\
\hline 4967 & -0.12 & 0.00 & 0.02 & 0.02 & -0.125 & 0.000 & -0.018 & -0.016 & -2.46 & -1.37 & 407.50 & 119.19 \\
\hline $50 \quad 68$ & -0.01 & 0.00 & 0.00 & 0.00 & -0.011 & 0.000 & 0.000 & 0.000 & -2.53 & -1.87 & 407.44 & 127.33 \\
\hline 5169 & -0.12 & 0.00 & 0.02 & 0.01 & -0.125 & 0.000 & -0.017 & 0.012 & -3.37 & -2.19 & 404.40 & 138.44 \\
\hline 5270 & -0.12 & 0.00 & 0.03 & 0.00 & -0.125 & 0.000 & -0.029 & 0.004 & -3.14 & -2.04 & 403.31 & 147.60 \\
\hline 5371 & -0.16 & 0.00 & 0.04 & -0.03 & -0.167 & 0.000 & -0.034 & 0.035 & -3.32 & -2.33 & 399.91 & 159.07 \\
\hline 5472 & -0.12 & 0.00 & 0.02 & -0.02 & -0.125 & 0.000 & -0.016 & 0.022 & -2.86 & -1.93 & 398.22 & 168.83 \\
\hline 5573 & -0.16 & 0.00 & 0.05 & -0.06 & -0.168 & 0.000 & -0.044 & 0.065 & -3.02 & -3.31 & 395.60 & 179.52 \\
\hline 5674 & 0.00 & 0.00 & 0.01 & 0.06 & 0.001 & 0.000 & -0.011 & -0.058 & -2.03 & -2.51 & 393.17 & 190.02 \\
\hline $57 \quad 75$ & 0.01 & 0.00 & 0.00 & 0.08 & 0.012 & 0.000 & 0.001 & -0.078 & -2.13 & -3.38 & 389.76 & 201.51 \\
\hline 5876 & 0.01 & 0.00 & 0.00 & 0.08 & 0.012 & 0.000 & 0.001 & -0.078 & -1.64 & -2.99 & 387.43 & 211.91 \\
\hline 5977 & -0.26 & 0.00 & 0.05 & -0.08 & -0.269 & 0.000 & -0.023 & 0.083 & -2.29 & -3.57 & 383.46 & 223.95 \\
\hline 6078 & -0.26 & 0.00 & 0.06 & -0.07 & -0.270 & 0.000 & -0.034 & 0.077 & -1.83 & -2.88 & 380.55 & 234.93 \\
\hline 6179 & -0.26 & 0.00 & 0.08 & -0.05 & -0.270 & 0.000 & -0.058 & 0.065 & -1.77 & -2.31 & 375.18 & 248.37 \\
\hline 6280 & -0.26 & 0.00 & 0.09 & -0.03 & -0.270 & 0.000 & -0.070 & 0.051 & -1.35 & -1.50 & 371.88 & 259.74 \\
\hline 6381 & -0.25 & 0.00 & 0.12 & 0.00 & -0.258 & 0.000 & -0.107 & 0.033 & -1.48 & -1.48 & 366.82 & 272.88 \\
\hline 6482 & -0.25 & 0.00 & 0.12 & 0.00 & -0.258 & 0.000 & -0.107 & 0.033 & -1.18 & -1.27 & 363.87 & 283.89 \\
\hline 6583 & -0.26 & 0.00 & 0.10 & -0.01 & -0.269 & 0.000 & -0.083 & 0.036 & -1.19 & -0.92 & 358.26 & 297.57 \\
\hline
\end{tabular}

$2.72 \quad 13.02$

$2.86 \quad 21.27$

$\begin{array}{ll}2.82 & 26.78\end{array}$

$2.73 \quad 35.41$

$2.66 \quad 41.52$

$2.58 \quad 50.71$

$2.32 \quad 57.20$

$\begin{array}{ll}1.86 & 66.53\end{array}$

$\begin{array}{ll}1.64 & 73.60\end{array}$

$1.13 \quad 83.34$

$0.75 \quad 90.75$

$\begin{array}{ll}0.02 & 100.72\end{array}$

$\begin{array}{ll}-0.43 & 108.52\end{array}$

$-1.23 \quad 118.83$

$-1.87 \quad 126.88$

$\begin{array}{ll}-2.10 & 138.13\end{array}$

$\begin{array}{ll}-1.93 & 147.39\end{array}$

$\begin{array}{ll}-1.77 & 159.40\end{array}$

$\begin{array}{ll}-1.72 & 168.91\end{array}$

$\begin{array}{ll}-1.54 & 181.27\end{array}$

$-1.21 \quad 191.42$
-1.03

$\begin{array}{ll}-1.03 & 204.09\end{array}$

$-0.55 \quad 214.72$

$-0.34 \quad 227.71$

$0.05 \quad 238.56$

$0.05 \quad 251.62$

$0.31 \quad 262.64$

$0.45 \quad 276.10$

$0.71 \quad 287.40$

$0.60 \quad 300.86$

\section{$Z=19(\mathrm{~K})$}

$\begin{array}{lllll}0.07 & 0.00 & -0.01 & 0.03 & 0.075\end{array}$

$\begin{array}{lllllll}12 & 31 & 0.08 & 0.00 & 0.01 & 0.04 & 0.086\end{array}$

$\begin{array}{lllllll}13 & 32 & 0.08 & 0.00 & 0.01 & 0.04 & 0.086\end{array}$

$\begin{array}{lllllll}14 & 33 & 0.00 & 0.00 & 0.02 & 0.04 & 0.001\end{array}$

$\begin{array}{llllllll}15 & 34 & 0.02 & 0.00 & -0.02 & 0.04 & 0.021\end{array}$

$\begin{array}{llll}0.000 & 0.015 & -0.029 & -0.84\end{array}$

$\begin{array}{ll}0.85 & 158.29\end{array}$

60.91

$\begin{array}{lllll}0.000 & -0.006 & -0.031 & 0.38\end{array}$

$\begin{array}{ll}1.95 & 179.85\end{array}$

47.43

$\begin{array}{lll}0.000 & -0.008 & -0.040\end{array}$

0.45

$1.93 \quad 203.79$

31.56

$\begin{array}{llll}0.000 & -0.008 & -0.040 & 0.70\end{array}$

$\begin{array}{ll}2.43 & 222.81\end{array}$

20.61

$\begin{array}{llll}0.000 & -0.023 & -0.039\end{array}$

0.25

$1.72 \quad 244.38$

7.10

$\begin{array}{lllll}16 & 35 & -0.03 & 0.02\end{array}$

$\begin{array}{lllll}17 & 36 & -0.03 & 0.00\end{array}$

$\begin{array}{llll}0.00 & -0.04 & -0.031\end{array}$

$\begin{array}{lll}0.000 & 0.024 & -0.039\end{array}$

0.88

$\begin{array}{lll}2.45 & 260.47 & -0.91\end{array}$

$\begin{array}{llll}18 & 37 & -0.06 & 0.00\end{array}$

$\begin{array}{lll}0.01 & -0.04 & -0.032\end{array}$

$\begin{array}{rrr}-0.027 & 0.001 & 0.040\end{array}$

1.28

$\begin{array}{rrrr}0.000 & -0.011 & 0.040 & 1.66 \\ 0.000 & -0.022 & -0.037 & 1.42\end{array}$

$\begin{array}{lllll}19 & 38 & -0.04 & 0.00\end{array}$

$\begin{array}{lll}0.02 & 0.04 & -0.062\end{array}$

$\begin{array}{lll}0.000 & -0.011 & 0.001\end{array}$

1.17

$\begin{array}{llll}20 & 39 & -0.03 & 0.00\end{array}$

0.00

$0.01-0.032$

$\begin{array}{lll}0.000 & 0.000 & -0.010\end{array}$

0.40

$\begin{array}{lllll}22 & 41 & -0.03 & 0.00\end{array}$

$\begin{array}{llll}0.00 & 0.01 & -0.052\end{array}$

$\begin{array}{llll}0.00 & 0.01 & -0.032\end{array}$

$\begin{array}{lll}0.000 & 0.001 & -0.010\end{array}$

1.18

$\begin{array}{llll}0.000 & 0.000 & -0.010\end{array}$

1.03

$\begin{array}{lll}0.000 & -0.011 & 0.001\end{array}$

1.69

$\begin{array}{lllll}2.49 & 278.64 & -11.01 & -11.17\end{array}$

$\begin{array}{lllll}2.84 & 292.78 & -17.07 & -17.43\end{array}$

$\begin{array}{lllll}2.70 & 308.83 & -25.06 & -24.80\end{array}$

$\begin{array}{lllll}2.93 & 320.26 & -28.41 & -28.80\end{array}$

$\begin{array}{llll}2.50 & 333.85 & -33.93 & -33.81\end{array}$

$\begin{array}{lllll}2.99 & 342.54 & -34.55 & -33.53\end{array}$

$\begin{array}{lllll}2.75 & 353.54 & -37.48 & -35.56\end{array}$

$\begin{array}{lllll}3.08 & 360.93 & -36.80 & -35.02\end{array}$

$\begin{array}{llll}24 & 43 & -0.05 & 0.00\end{array}$

$\begin{array}{lll}0.01 & 0.00 & -0.053\end{array}$

$\begin{array}{lll}0.000 & 0.001 & -0.010\end{array}$

1.32

$\begin{array}{lllll}2.65 & 370.69 & -38.49 & -36.59\end{array}$

$\begin{array}{llll}0.000 & 0.001 & 0.000 & 1.21\end{array}$

$\begin{array}{lllll}2.50 & 377.28 & -37.01 & -35.81\end{array}$

$\begin{array}{lll}0.00 & 0.00 & -0.063\end{array}$

$\begin{array}{llll}26 & 45 & -0.05 & 0.00\end{array}$

$\begin{array}{llll}0.00 & 0.00 & -0.052\end{array}$

$\begin{array}{rrr}0.000 & 0.001 & 0.000\end{array}$

0.53

$\begin{array}{lllll}1.70 & 386.14 & -37.79 & -36.61\end{array}$

$\begin{array}{lllll}1.49 & 391.64 & -35.22 & -35.42\end{array}$

$\begin{array}{lllll}0.48 & 399.56 & -35.07 & -35.70\end{array}$

$\begin{array}{lllll}0.58 & 403.71 & -31.15 & -32.12\end{array}$

$\begin{array}{llll}0.000 & 0.001 & 0.000 & -0.59\end{array}$

$\begin{array}{llll}29 & 48 & -0.05 & 0.00\end{array}$

$\begin{array}{llll}0.00 & 0.00 & -0.042\end{array}$

$\begin{array}{llll}0.00 & 0.00 & -0.052\end{array}$

$\begin{array}{lllll}0.000 & -0.011 & 0.001 & 0.05\end{array}$

$\begin{array}{lllll}1.09 & 409.09 & -28.46 & -30.32\end{array}$

$\begin{array}{lll}0.000 & 0.001 & -0.010\end{array}$

0.68

$\begin{array}{llll}1.65 & 411.85 & -23.15 & -25.35\end{array}$

$\begin{array}{llll}0.000 & -0.009 & 0.010 & 1.25\end{array}$

$\begin{array}{llll}2.17 & 416.29 & -19.52\end{array}$

$\begin{array}{lll}0.01 & -0.01 & -0.084\end{array}$

$0.000-0.015$

$0.031 \quad 1.37$

$\begin{array}{lll}2.52 & 418.43 & -13.58\end{array}$

$\begin{array}{llll}0.000 & -0.061 & 0.078 & -0.26\end{array}$

$\begin{array}{lll}2.39 & 422.67 \quad-9.76\end{array}$

$\begin{array}{lllll}0.025 & 1.56 & 3.04 & 423.74 & -2.75\end{array}$
$0.92 \quad 58.25$

$1.99 \quad 45.18$

$2.00 \quad 29.74$

$2.47 \quad 19.13$

$1.74 \quad 5.95$

$2.46-1.79$

$2.50-11.65$

$2.83-17.51$

$2.70-25.31$

$2.91-29.31$

$2.49-33.92$

$2.98-34.44$

$2.74-37.30$

$3.07-36.58$

$2.65-38.23$

$2.49-36.74$

$1.70-37.53$

$1.49-34.97$

$0.47-34.85$

$\begin{array}{lll}0.57 & -30.97\end{array}$

$1.09-28.31$

$\begin{array}{lll}1.66 & -23.04\end{array}$

$2.18-19.46$

$2.62-13.49$

$3.22-8.99$

$3.17 \quad-2.74$ 


\section{$Z=19$ (K)}

$\begin{array}{llll}36 & 55 & -0.14 & 0.00\end{array}$ $\begin{array}{llll}37 & 56 & -0.13 & 0.00\end{array}$ $\begin{array}{lll}0.05 & 0.02 & -0.145\end{array}$ $\begin{array}{llll}38 & 57 & -0.13 & 0.00\end{array}$ $\begin{array}{lllll}39 & 58 & -0.07 & 0.00\end{array}$ $\begin{array}{llll}0.06 & 0.03 & -0.134\end{array}$ $\begin{array}{llll}0.07 & 0.06 & -0.132\end{array}$ $\begin{array}{llll}0.03 & 0.02 & -0.073\end{array}$ $\begin{array}{lll}0.000 & -0.050 & -0.010\end{array}$

1.67 $3.08 \quad 427.06$ $\begin{array}{llll}0.000 & -0.062 & -0.018\end{array}$ 1.64 $\begin{array}{llll}0.000 & -0.074 & -0.044\end{array}$ 1.37 $3.05 \quad 428.12$ 2.00 $\begin{array}{lll}0.000 & -0.033 & -0.016\end{array}$ 1.87 $2.44 \quad 431.39$ $\begin{array}{lllll}40 & 59 & -0.02 & 0.00\end{array}$ 0.00 $\begin{array}{lll}0.00 & -0.021\end{array}$

$\begin{array}{lll}0.000 & 0.000 & 0.000\end{array}$

1.56

$\begin{array}{ll}2.84 & 431.38 \\ 2.76 & 433.48\end{array}$

$\begin{array}{llll}41 & 60 & -0.07 & 0.07\end{array}$ $\begin{array}{llll}42 & 61 & -0.03 & 0.00\end{array}$ 0.00 $\begin{array}{lllll}0.00 & -0.071 & -0.092 & 0.004 & 0.003\end{array}$ 0.00 $\begin{array}{lll}0.00 & -0.032\end{array}$ 0.000

$0.000 \quad 0.000$

1.16

$\begin{array}{ll}2.51 & 433.54\end{array}$

$\begin{array}{llll}43 & 62 & -0.08 & 0.05\end{array}$

0.02

$0.00-0.083-0.1$

$\begin{array}{llll}44 & 63 & -0.09 & 0.00\end{array}$

$\begin{array}{llllll}45 & 64 & 0.12 & 0.00 & -0.02\end{array}$

$0.00-0.094$

$\begin{array}{ll}-0.019 & 0.003\end{array}$

1.17
0.81

$2.30 \quad 435.20$

$0.00 \quad 0.128$

0.000

$0.003-0.000$

0.53

$\begin{array}{ll}1.99 & 434.77\end{array}$

$\begin{array}{ll}1.71 & 435.97\end{array}$

$1.35 \quad 435.11$

$\begin{array}{llll}46 & 65 & 0.12 & 0.00\end{array}$

$\begin{array}{lll}0.00 & 0.01 & 0.129\end{array}$

$\begin{array}{lllll}0.000 & 0.007 & -0.010 & -0.64\end{array}$

$0.84 \quad 436.03$

$\begin{array}{lllll}47 & 66 & 0.12 & 0.00\end{array}$

$\begin{array}{lll}0.01 & 0.00 & 0.129\end{array}$

$\begin{array}{llll}48 & 67 & -0.10 & 0.00\end{array}$

$\begin{array}{lll}0.01 & 0.01 & -0.105\end{array}$

$\begin{array}{llll}49 & 68 & -0.09 & 0.00\end{array}$

$\begin{array}{lll}0.00 & 0.00 & -0.094\end{array}$

$\begin{array}{llll}50 & 69 & 0.00 & 0.00\end{array}$

$\begin{array}{lll}0.00 & 0.01 & 0.000\end{array}$

$\begin{array}{llll}51 & 70 & -0.10 & 0.00\end{array}$

$\begin{array}{lll}0.00 & 0.00 & -0.105\end{array}$

$\begin{array}{llll}52 & 71 & -0.10 & 0.00\end{array}$

$\begin{array}{lll}0.00 & 0.00 & -0.105\end{array}$

$\begin{array}{llll}53 & 72 & -0.10 & 0.00\end{array}$

$0.01-0.01-0.105$

$\begin{array}{llll}0.01 & -0.01 & -0.105\end{array}$

$\begin{array}{llll}54 & 73 & -0.10 & 0.00 \\ 55 & 74 & -0.10 & 0.00\end{array}$

$0.02-0.01-0.105$

$\begin{array}{llll}56 & 75 & 0.02 & 0.00\end{array}$

$\begin{array}{lll}0.01 & 0.04 & 0.022\end{array}$

$\begin{array}{llll}0.000 & -0.006 & -0.001 & -1.41\end{array}$

0.11435 .09

$\begin{array}{llllll}0.000 & -0.008 & -0.009 & -1.89 & -0.67 & 435.83\end{array}$

$\begin{array}{llllll}0.000 & 0.003 & -0.000 & -2.75 & -1.53 & 434.59\end{array}$

0.000

$0.000-0.010-3.29$

$\begin{array}{lllllll}0.000 & 0.004 & -0.000 & -3.65 & -2.39 & 432.52 & 117.61\end{array}$

$\begin{array}{lllllll}0.000 & 0.004 & -0.000 & -3.36 & -2.15 & 431.41 & 126.79\end{array}$

$\begin{array}{lllllll}0.000 & -0.007 & 0.011 & -3.39 & -2.26 & 428.69 & 137.58\end{array}$

$\begin{array}{lllllll}0.000 & -0.007 & 0.011 & -2.92 & -1.84 & 427.05 & 147.30\end{array}$

$\begin{array}{lllllll}0.000 & -0.019 & 0.012 & -2.89 & -1.89 & 423.91 & 158.50\end{array}$

$\begin{array}{llll}57 & 76 & 0.00 & 0.00\end{array}$

$\begin{array}{lll}0.00 & 0.08 & 0.001\end{array}$

0.000

$-0.0$

$-0.03$

0.000
0.000

$001-0.078$

$-2.24$

$-1.80 \quad 422.26$

168.22

$\begin{array}{llll}58 & 77 & 0.00 & 0.00\end{array}$

$0.00 \quad 0.08$

0.001

$\begin{array}{lllllll}59 & 78 & 0.04 & 0.00 & -0.02 & 0.08 & 0.043\end{array}$

$\begin{array}{lllllll}60 & 79 & 0.02 & 0.00 & 0.01 & 0.08 & 0.023\end{array}$

$\begin{array}{rrrr}61 & 80 & 0.06 & 0.00\end{array}$

$\begin{array}{lllll}62 & 81 & -0.06 & 0.00\end{array}$

$\begin{array}{lll}0.00 & 0.08 & 0.065\end{array}$

$\begin{array}{llll}63 & 82 & 0.05 & 0.00\end{array}$

$\begin{array}{llll}0.00 & 0.08 & -0.062\end{array}$

$\begin{array}{llll}64 & 83 & 0.05 & 0.00\end{array}$

$0.04-0.02$

0.054

$\begin{array}{llll}65 & 84 & 0.05 & 0.00\end{array}$

$0.04-0.02$

0.054

$0.04-0.02$

0.054

$\begin{array}{llll}66 & 85 & 0.05 & 0.00\end{array}$

$\begin{array}{llll}67 & 86 & 0.05 & 0.00\end{array}$

$\begin{array}{ll}0.03 & -0.01\end{array}$

0.054

0.000

0.001

0.000

0.000

$-0.010$

$0.000 \quad 0.001$

$0.000 \quad-0.047$

$0.000-0.047$

$0.000-0.047$

0.053

$0.000-0.035$

$0.000-0.035$

$0.00 \quad 0.00$

0.000

\section{$Z=20(\mathrm{Ca})$}

$\begin{array}{llllll}10 & 30 & 0.00 & 0.00 & 0.00 & -0.03\end{array}$

$\begin{array}{llll}11 & 31 & 0.00 & 0.00\end{array}$

$\begin{array}{llll}12 & 32 & 0.00 & 0.00\end{array}$

$0.00-0.03$

0.000

0.00

0.000

$\begin{array}{lll}-3.30 & 420.28 & 178.27\end{array}$

$\begin{array}{llll}0.00 & 0.00 & 0.00 & 0.04\end{array}$

0.000

0.000

$\begin{array}{lll}0.000 & 0.030 & -1.16\end{array}$

0.000

$\begin{array}{lll}0.000 & -0.039 & 0.08\end{array}$

$\begin{array}{lll}0.69 & 154.64 & 71.85\end{array}$

$\begin{array}{lll}1.71 & 176.70 & 57.87\end{array}$

$\begin{array}{lll}1.51 & 202.61 & 40.03\end{array}$

$\begin{array}{lllllllllll}13 & 33 & 0.03 & 0.00 & 0.01 & 0.04 & 0.033 & 0.000 & -0.011 & -0.040 & 0.09\end{array}$

$\begin{array}{llllll}14 & 34 & 0.00 & 0.00 & -0.0\end{array}$

$0.04 \quad 0.000$

0.000

$\begin{array}{lll}0.012 & -0.039 & -0.55\end{array}$

$1.30-245.36$

28.51

$\begin{array}{lllllll}15 & 35 & 0.00 & 0.00 & 0.00 & 0.04 & 0.000\end{array}$

$\begin{array}{llll}16 & 36 & 0.00 & 0.00\end{array}$

$\begin{array}{llll}17 & 37 & -0.02 & 0.00\end{array}$

0.00

$\begin{array}{lllll}18 & 38 & 0.00 & 0.00 & 0.00\end{array}$

$\begin{array}{lllll}19 & 39 & 0.01 & 0.00 & 0.00\end{array}$

$0.04 \quad 0.000$

0.000

$0.000-0.039$

0.10

$\begin{array}{ll}1.86 & 262.07\end{array}$

13.41

$0.04-0.021$

0.000

$0.000-0.039$

0.55

0.000

$\begin{array}{lll}0.000 & -0.039\end{array}$

0.93

$2.19 \quad 281.63-6.71$

$\begin{array}{llll}2.60 & 296.13 & -13.14 & -13.16\end{array}$

0.000

$0.000-0.039$

0.33

$\begin{array}{llll}2.55 & 313.72 & -22.66 & -22.06\end{array}$

$\begin{array}{lllll}2.57 & 326.55 & -27.42 & -27.27\end{array}$

$\begin{array}{llll}1.98 & 342.62 & -35.42 & -34.85\end{array}$

$\begin{array}{llll}0.000 & 0.000 & -0.010 & -0.52\end{array}$

$\begin{array}{lllll}21 & 41 & -0.02 & 0.00 & -0.01\end{array}$

$0.01 \quad 0.000$

$0.02-0.021$

0.000

$\begin{array}{lll}0.012 & -0.020 & 0.38\end{array}$

$\begin{array}{llll}2.55 & 351.62 & -36.34 & -35.14\end{array}$

$\begin{array}{lllll}22 & 42 & 0.00 & 0.00 & 0.00\end{array}$

$\begin{array}{ll}0.01 & 0.000\end{array}$

0.000

$0.000-0.010$

0.08

$\begin{array}{lllll}2.19 & 364.24 & -40.89 & -38.55\end{array}$

0.000

$0.000-0.020$

0.75

$\begin{array}{lllll}2.55 & 371.98 & -40.56 & -38.41\end{array}$

$\begin{array}{lllll}2.02 & 383.28 & -43.79 & -41.47\end{array}$

$\begin{array}{llll}0.000 & 0.000 & 0.000 & 0.28\end{array}$

$\begin{array}{llll}1.88 & 390.21 & -42.65 & -40.81\end{array}$

$\begin{array}{ll}0.00 & -0.011\end{array}$

$\begin{array}{llll}25 & 45 & -0.01 & 0.00\end{array}$

$\begin{array}{llll}26 & 46 & 0.00 & 0.00\end{array}$

0.00

$0.01 \quad 0.000$

0.000

$\begin{array}{llll}0.000 & -0.010 & -0.57\end{array}$

$\begin{array}{llll}27 & 47 & 0.03 & 0.00\end{array}$

$\begin{array}{lll}0.01 & 0.00 & 0.032\end{array}$

$\begin{array}{lllll}0.000 & -0.012 & -0.000 & -0.98\end{array}$

$\begin{array}{lllll}1.03 & 400.50 & -44.86 & -43.13\end{array}$

$\begin{array}{llll}0.76 & 406.38 & -42.67 & -42.34\end{array}$

$\begin{array}{llll}28 & 48 & 0.00 & 0.00\end{array}$

$0.00 \quad 0.000$

$\begin{array}{llll}0.000 & 0.000 & 0.000 & -1.94\end{array}$

$\begin{array}{llll}-0.11 & 415.48 & -43.71 & -44.21\end{array}$

$\begin{array}{lllll}0.000 & 0.000 & 0.000 & -1.54\end{array}$

$\begin{array}{lllll}0.16 & 419.77 & -39.92 & -41.29\end{array}$

$\begin{array}{llll}0.65 & 426.45 & -38.53 & -39.57\end{array}$

$\begin{array}{llll}0.000 & 0.000 & 0.000 & -0.88\end{array}$

$\begin{array}{lllll}1.34 & 429.35 & -33.36 & -35.86\end{array}$

$\begin{array}{llll}1.82 & 435.06 & -31.00 & -32.51\end{array}$

$\begin{array}{rrrrrr}0.00 & 0.000 & 0.000 & 0.000 & 0.000 & 0.56\end{array}$ $\begin{array}{lllllll}30 & 50 & 0.00 & 0.00 & 0.00 & 0.00 & 0.000 \\ 31 & 51 & 0.00 & 0.00 & 0.00 & 0.00 & 0.000 \\ 32 & 52 & 0.00 & 0.00 & 0.00 & 0.00 & 0.000\end{array}$ 


\begin{tabular}{|c|c|c|c|c|c|c|c|c|c|c|c|c|c|c|c|c|}
\hline \multicolumn{17}{|c|}{$Z=20(\mathrm{Ca})$} \\
\hline 3353 & 0.06 & 0.00 & 0.00 & 0.00 & 0.064 & 0.000 & 0.002 & 0.000 & 1.32 & 2.67 & 436.95 & -24.82 & & & 2.67 & -24.72 \\
\hline 3454 & -0.01 & 0.00 & 0.00 & 0.00 & -0.011 & 0.000 & 0.000 & 0.000 & 1.72 & 2.60 & 442.33 & -22.12 & & & 2.59 & -22.08 \\
\hline 3555 & -0.07 & 0.03 & 0.01 & 0.00 & -0.073 & -0.039 & -0.009 & 0.001 & 1.93 & 2.88 & 443.99 & -15.71 & & & 2.90 & -15.70 \\
\hline 3656 & -0.07 & 0.02 & 0.02 & 0.02 & -0.073 & -0.026 & -0.021 & -0.017 & 1.92 & 2.90 & 448.48 & -12.13 & & & 2.96 & -12.12 \\
\hline 3757 & -0.07 & 0.03 & 0.01 & 0.00 & -0.073 & -0.039 & -0.009 & 0.001 & 1.93 & 3.09 & 449.51 & -5.09 & & & 3.11 & -5.18 \\
\hline 3858 & 0.00 & 0.04 & 0.00 & 0.00 & 0.001 & -0.054 & 0.001 & 0.001 & 1.50 & 2.92 & 453.45 & -0.96 & & & 2.95 & -1.09 \\
\hline 3959 & 0.00 & 0.05 & 0.00 & 0.00 & 0.001 & -0.067 & 0.001 & 0.002 & 1.13 & 2.77 & 454.18 & 6.38 & & & 2.82 & 6.23 \\
\hline 4060 & 0.00 & 0.07 & 0.00 & 0.00 & 0.002 & -0.094 & 0.002 & 0.003 & 0.33 & 2.16 & 457.88 & 10.75 & & & 2.28 & 10.62 \\
\hline 4161 & -0.02 & 0.09 & 0.00 & 0.00 & -0.018 & -0.121 & 0.004 & 0.005 & 0.23 & 2.08 & 457.93 & 18.77 & & & 2.29 & 18.69 \\
\hline 4262 & 0.00 & 0.00 & 0.00 & 0.00 & 0.000 & 0.000 & 0.000 & 0.000 & 0.33 & 1.78 & 460.70 & 24.07 & & & 1.78 & 23.75 \\
\hline 4363 & 0.01 & 0.00 & 0.00 & 0.00 & 0.011 & 0.000 & 0.000 & 0.000 & 0.32 & 1.58 & 460.33 & 32.51 & & & 1.58 & 32.16 \\
\hline 4464 & 0.00 & 0.00 & 0.00 & 0.00 & 0.000 & 0.000 & 0.000 & 0.000 & -0.05 & 1.11 & 462.71 & 38.21 & & & 1.11 & 37.83 \\
\hline 4565 & 0.00 & 0.00 & 0.00 & 0.00 & 0.000 & 0.000 & 0.000 & 0.000 & -0.41 & 0.58 & 462.16 & 46.83 & & & 0.58 & 46.44 \\
\hline 4666 & 0.00 & 0.00 & 0.00 & 0.00 & 0.000 & 0.000 & 0.000 & 0.000 & -0.96 & 0.00 & 464.11 & 52.95 & & & -0.00 & 52.54 \\
\hline $47 \quad 67$ & 0.02 & 0.00 & -0.01 & 0.01 & 0.021 & 0.000 & 0.012 & -0.010 & -1.75 & -0.86 & 463.42 & 61.71 & & & -0.83 & 61.33 \\
\hline $48 \quad 68$ & 0.00 & 0.00 & 0.00 & 0.00 & 0.000 & 0.000 & 0.000 & 0.000 & -2.62 & -1.49 & 464.95 & 68.26 & & & -1.50 & 67.86 \\
\hline 4969 & 0.02 & 0.00 & 0.01 & 0.00 & 0.021 & 0.000 & -0.012 & -0.000 & -3.58 & -2.35 & 463.83 & 77.45 & & & -2.35 & 77.07 \\
\hline 5070 & 0.00 & 0.00 & 0.00 & 0.00 & 0.000 & 0.000 & 0.000 & 0.000 & -4.43 & -3.02 & 464.92 & 84.42 & & & -3.02 & 84.06 \\
\hline 5171 & -0.02 & 0.00 & 0.00 & 0.01 & -0.021 & 0.000 & 0.000 & -0.010 & -4.34 & -3.11 & 462.64 & 94.78 & & & -3.09 & 94.47 \\
\hline 5272 & 0.00 & 0.00 & 0.00 & 0.01 & 0.000 & 0.000 & 0.000 & -0.010 & -4.09 & -2.88 & 462.42 & 103.07 & & & -2.85 & 102.81 \\
\hline 5373 & 0.00 & 0.00 & 0.00 & 0.00 & 0.000 & 0.000 & 0.000 & 0.000 & -3.92 & -2.75 & 459.54 & 114.02 & & & -2.75 & 113.78 \\
\hline 5474 & 0.00 & 0.00 & 0.00 & 0.00 & 0.000 & 0.000 & 0.000 & 0.000 & -3.42 & -2.27 & 458.68 & 122.95 & & & -2.27 & 122.78 \\
\hline 5575 & 0.01 & 0.00 & 0.00 & 0.01 & 0.011 & 0.000 & 0.000 & -0.010 & -3.31 & -2.18 & 455.49 & 134.21 & & & -2.15 & 134.14 \\
\hline 5676 & 0.00 & 0.00 & 0.00 & 0.03 & 0.000 & 0.000 & 0.000 & -0.029 & -2.84 & -1.93 & 454.50 & 143.27 & & & -1.65 & 143.54 \\
\hline $57 \quad 77$ & 0.00 & 0.00 & 0.00 & 0.08 & 0.001 & 0.000 & 0.001 & -0.078 & -2.88 & -3.51 & 452.66 & 153.18 & & & -1.47 & 155.31 \\
\hline 5878 & 0.00 & 0.00 & 0.00 & 0.06 & 0.001 & 0.000 & 0.001 & -0.058 & -2.20 & -2.14 & 450.20 & 163.71 & & & -0.93 & 165.10 \\
\hline 5979 & 0.01 & 0.00 & 0.00 & 0.08 & 0.012 & 0.000 & 0.001 & -0.078 & -1.88 & -2.73 & 447.07 & 174.92 & & & -0.54 & 177.40 \\
\hline 6080 & 0.00 & 0.00 & 0.01 & 0.08 & 0.002 & 0.000 & -0.010 & -0.078 & -1.36 & -2.35 & 445.29 & 184.77 & & & -0.09 & 187.46 \\
\hline 6181 & 0.04 & 0.00 & 0.00 & 0.09 & 0.044 & 0.000 & 0.003 & -0.088 & -1.10 & -2.71 & 441.64 & 196.49 & & & 0.23 & 200.00 \\
\hline 6282 & 0.05 & 0.00 & 0.00 & 0.04 & 0.054 & 0.000 & 0.002 & -0.039 & -0.49 & 0.00 & 437.23 & 208.97 & & & 0.62 & 210.31 \\
\hline 6383 & 0.05 & 0.00 & 0.03 & -0.01 & 0.054 & 0.000 & -0.035 & 0.008 & -0.38 & 0.49 & 432.47 & 221.80 & & & 0.65 & 222.85 \\
\hline 6484 & 0.05 & 0.00 & 0.02 & 0.00 & 0.054 & 0.000 & -0.023 & -0.001 & -0.16 & 0.80 & 430.17 & 232.17 & & & 0.86 & 233.29 \\
\hline 6585 & 0.05 & 0.00 & 0.03 & -0.01 & 0.054 & 0.000 & -0.035 & 0.008 & -0.33 & 0.56 & 425.90 & 244.51 & & & 0.73 & 245.93 \\
\hline 6686 & 0.04 & 0.00 & 0.02 & -0.01 & 0.043 & 0.000 & -0.023 & 0.009 & -0.27 & 0.69 & 423.52 & 254.96 & & & 0.78 & 256.51 \\
\hline 6787 & 0.00 & 0.00 & 0.00 & 0.00 & 0.000 & 0.000 & 0.000 & 0.000 & -0.61 & 0.58 & 418.88 & 267.67 & & & 0.58 & 269.33 \\
\hline 6888 & 0.00 & 0.00 & 0.00 & 0.00 & 0.000 & 0.000 & 0.000 & 0.000 & -0.88 & 0.38 & 416.58 & 278.05 & & & 0.38 & 279.93 \\
\hline 6989 & -0.02 & 0.00 & 0.01 & 0.01 & -0.021 & 0.000 & -0.012 & -0.009 & -1.34 & -0.05 & 412.04 & 290.66 & & & 0.01 & 292.84 \\
\hline 7090 & 0.00 & 0.00 & 0.00 & 0.00 & 0.000 & 0.000 & 0.000 & 0.000 & -1.62 & -0.26 & 409.52 & 301.25 & & & -0.26 & 303.62 \\
\hline 7191 & -0.02 & 0.00 & -0.01 & 0.01 & -0.021 & 0.000 & 0.012 & -0.010 & -1.81 & -0.49 & 404.58 & 314.26 & & & -0.42 & 316.96 \\
\hline 7292 & 0.00 & 0.00 & 0.00 & 0.00 & 0.000 & 0.000 & 0.000 & 0.000 & -1.88 & -0.54 & 401.68 & 325.24 & & & -0.54 & 328.14 \\
\hline \multicolumn{17}{|c|}{$Z=21(\mathrm{Sc})$} \\
\hline 1132 & 0.40 & 0.00 & -0.07 & 0.04 & 0.440 & 0.000 & 0.167 & 0.013 & -1.67 & 2.09 & 169.89 & 71.97 & & & 2.20 & 69.07 \\
\hline 1233 & 0.11 & 0.00 & -0.07 & -0.04 & 0.122 & 0.000 & 0.093 & 0.054 & 0.06 & 0.87 & 197.22 & 52.70 & & & 1.20 & 50.47 \\
\hline $13 \quad 34$ & 0.11 & 0.00 & -0.06 & -0.04 & 0.121 & 0.000 & 0.079 & 0.052 & 0.25 & 1.71 & 218.02 & 39.97 & & & 1.90 & 38.00 \\
\hline 1435 & 0.08 & 0.00 & -0.05 & -0.04 & 0.088 & 0.000 & 0.064 & 0.047 & 0.03 & 1.62 & 241.12 & 24.95 & & & 1.75 & 23.28 \\
\hline 1536 & 0.08 & 0.00 & -0.02 & -0.04 & 0.086 & 0.000 & 0.026 & 0.043 & 0.76 & 2.29 & 259.35 & 14.79 & & & 2.33 & 13.37 \\
\hline 1637 & 0.08 & 0.00 & -0.01 & -0.04 & 0.086 & 0.000 & 0.014 & 0.041 & 1.26 & 2.70 & 279.25 & 2.96 & & & 2.72 & 1.83 \\
\hline 1738 & -0.06 & 0.00 & 0.00 & 0.04 & -0.063 & 0.000 & 0.001 & -0.039 & 1.73 & 2.90 & 295.55 & -5.27 & & & 2.91 & -6.15 \\
\hline 1839 & -0.06 & 0.00 & 0.00 & 0.04 & -0.063 & 0.000 & 0.001 & -0.039 & 1.48 & 2.87 & 313.54 & -15.19 & -14.17 & 0.024 & 2.87 & -15.84 \\
\hline 1940 & -0.04 & 0.00 & -0.01 & 0.03 & -0.042 & 0.000 & 0.012 & -0.030 & 1.23 & 2.98 & 327.81 & -21.38 & -20.52 & 0.003 & 2.98 & -21.85 \\
\hline 2041 & -0.02 & 0.00 & -0.01 & 0.03 & -0.021 & 0.000 & 0.012 & -0.030 & 0.49 & 2.58 & 344.09 & -29.59 & -28.64 & 0.000 & 2.57 & -29.88 \\
\hline 2142 & -0.06 & 0.00 & -0.01 & 0.03 & -0.063 & 0.000 & 0.013 & -0.030 & 1.18 & 3.09 & 355.38 & -32.81 & -32.12 & 0.000 & 3.08 & -33.68 \\
\hline 2243 & -0.04 & 0.00 & -0.02 & 0.02 & -0.042 & 0.000 & 0.024 & -0.020 & 0.96 & 2.75 & 369.08 & -38.44 & -36.19 & 0.002 & 2.74 & -38.47 \\
\hline 2344 & 0.05 & 0.00 & -0.02 & 0.00 & 0.053 & 0.000 & 0.025 & 0.001 & 1.44 & 2.91 & 378.44 & -39.73 & -37.82 & 0.002 & 2.90 & -39.67 \\
\hline $24 \quad 45$ & 0.04 & 0.00 & -0.01 & -0.03 & 0.043 & 0.000 & 0.013 & 0.031 & 1.10 & 2.27 & 390.21 & -43.43 & -41.07 & 0.001 & 2.27 & -43.28 \\
\hline 2546 & -0.05 & 0.00 & -0.01 & 0.01 & -0.052 & 0.000 & 0.013 & -0.010 & 0.97 & 2.40 & 398.24 & -43.39 & -41.76 & 0.001 & 2.39 & -43.19 \\
\hline 2647 & -0.02 & 0.00 & -0.01 & 0.00 & -0.021 & 0.000 & 0.012 & -0.000 & 0.14 & 1.45 & 408.97 & -46.04 & -44.33 & 0.002 & 1.44 & -45.81 \\
\hline
\end{tabular}




\section{$Z=21(\mathrm{Sc})$}

$\begin{array}{lllllll}27 & 48 & 0.06 & 0.00 & -0.01 & -0.01 & 0.064\end{array}$

$\begin{array}{lllllll}29 & 50 & -0.04 & 0.00 & -0.01 & 0.00 & -0.042\end{array}$

$\begin{array}{lllllll}30 & 51 & -0.04 & 0.00 & -0.01 & 0.00 & -0.042\end{array}$

$\begin{array}{rrrrrrr}31 & 52 & -0.04 & 0.00 & -0.01 & 0.00 & -0.042 \\ 32 & 53 & 0.06 & 0.00 & -0.02 & -0.01 & 0.064\end{array}$

$\begin{array}{lllllll}33 & 54 & 0.10 & 0.00 & 0.00 & -0.01 & 0.107\end{array}$

$\begin{array}{llllllll}34 & 55 & -0.10 & 0.00 & -0.01 & -0.02 & -0.104\end{array}$

$\begin{array}{lllllll}35 & 56 & -0.10 & 0.00 & 0.00 & -0.01 & -0.104\end{array}$

$\begin{array}{lllllll}36 & 57 & -0.10 & 0.00 & 0.01 & 0.00 & -0.105\end{array}$

$\begin{array}{llll}37 & 58 & -0.10 & 0.00\end{array}$

$\begin{array}{llll}38 & 59 & -0.07 & 0.03\end{array}$

$0.02 \quad 0.01-0.105$

0.01

$0.01-0.073$

0.00

$\begin{array}{lllll}40 & 61 & -0.02 & 0.08 & 0.00\end{array}$

$\begin{array}{lllll}41 & 62 & -0.04 & 0.09 & -0.01\end{array}$

$0.00-0.018-0.107$

$\begin{array}{llllll}42 & 63 & -0.02 & 0.00 & -0.01\end{array}$

$\begin{array}{ll}0.00 & -0.038 \\ 0.00 & -0.021\end{array}$

$\begin{array}{lllll}43 & 64 & 0.05 & 0.00 & -0.02\end{array}$

$0.00 \quad 0.053$

4465

$\begin{array}{lll}0.12 & 0.00 & -0.04\end{array}$

$-0.01$

0.129

4566

$\begin{array}{llll}0.12 & 0.00 & -0.03\end{array}$

0.00

0.128

$46 \quad 67$

$\begin{array}{lll}0.12 & 0.00 & -0.02\end{array}$

0.00

0.128

$\begin{array}{lllll}47 & 68 & 0.12 & 0.00 & -0.01\end{array}$

$0.00 \quad 0.128$

48

49

$\begin{array}{rrrrr}0.05 & 0.00 & 0.00 & -0.01 & 0.053\end{array}$

$\begin{array}{lllll}51 & 72 & -0.07 & 0.00 & -0.03\end{array}$

$0.00-0.032$

$0.01-0.073$

$\begin{array}{lllll}52 & 73 & -0.03 & 0.00 & 0.00\end{array}$

$0.01-0.032$

$\begin{array}{lllll}53 & 74 & -0.04 & 0.00 & 0.00\end{array}$

$\begin{array}{lllll}54 & 75 & -0.04 & 0.00 & -0.01\end{array}$

$\begin{array}{lllll}55 & 76 & -0.04 & 0.00 & -0.01\end{array}$

$\begin{array}{lllll}56 & 77 & -0.03 & 0.00 & -0.02\end{array}$

$0.01-0.042$

$0.01-0.042$

$0.01-0.042$

$0.01-0.032$

$\begin{array}{lllll}57 & 78 & -0.03 & 0.00 & -0.01\end{array}$

$\begin{array}{llllll}58 & 79 & 0.02 & 0.00 & -0.03\end{array}$

$\begin{array}{llllll}59 & 80 & 0.06 & 0.00 & -0.06\end{array}$

$\begin{array}{ll}-0.06 \quad 0.024 \\ 0.03 & 0.063\end{array}$

$0.03 \quad 0.063$

$\begin{array}{ll}0.04 & 0.063\end{array}$

$\begin{array}{rrrrr}60 & 81 & 0.06 & 0.00 & -0.06 \\ 61 & 82 & -0.06 & 0.00 & -0.04\end{array}$

$0.03-0.063$

$\begin{array}{lllllll}62 & 83 & -0.06 & 0.00 & -0.03 & 0.00 & -0.062\end{array}$

$\begin{array}{lllllll}63 & 84 & -0.06 & 0.00 & -0.03 & -0.02 & -0.062\end{array}$

$\begin{array}{lllllll}64 & 85 & -0.06 & 0.00 & 0.00 & 0.00 & -0.063\end{array}$

$\begin{array}{llllllll}65 & 86 & -0.06 & 0.00 & 0.01 & 0.01 & -0.063\end{array}$

$\begin{array}{lllll}66 & 87 & -0.06 & 0.00 & 0.03\end{array}$

$0.03-0.062$

$\begin{array}{lllll}67 & 88 & -0.06 & 0.00 & 0.03\end{array}$

$0.02-0.062$

$\begin{array}{lllll}68 & 89 & 0.02 & 0.00 & -0.01\end{array}$

$\begin{array}{ll}0.00 & 0.021\end{array}$

$0.01-0.042$

$\begin{array}{lllll}70 & 91 & -0.01 & 0.00 & -0.01\end{array}$

$0.00-0.010$

$\begin{array}{lllll}71 & 92 & -0.03 & 0.00 & -0.02\end{array}$

$0.01-0.032$

$\begin{array}{llllllll}72 & 93 & -0.02 & 0.00 & -0.01 & 0.00 & -0.021\end{array}$

$\begin{array}{lllllll}73 & 94 & 0.04 & 0.00 & -0.03 & -0.01 & 0.043\end{array}$

$\begin{array}{lllllll}74 & 95 & 0.08 & 0.00 & -0.07 & -0.04 & 0.090\end{array}$

0.000

0.000

0.000

0.000

0.000

0.000

0.000

0.000

0.000

0.000

0.000 $\begin{array}{lllllll}28 & 49 & -0.03 & 0.00 & -0.01 & 0.00 & -0.032\end{array}$

$0.05-0.031$
0.000

$\begin{array}{llll}0.000 & 0.012 & -0.000 & -1.07\end{array}$

$\begin{array}{lllll}0.000 & 0.012 & -0.001 & -0.62\end{array}$

$\begin{array}{llll}0.000 & 0.012 & -0.001 & -0.03\end{array}$

0.000

0.000

0.000

$\begin{array}{lll}0.012 & -0.001 & 0.79\end{array}$

$0.025 \quad 0.012$

0.004

0.010

1.41

$\begin{array}{lll}0.016 & 0.018 & 2.25\end{array}$

2.58
2.57

2.62

2.43

2.05

$0.002 \quad 0.002$

2.05
1.34

$0.016 \quad 0.005$

1.19

$0.012-0.000$

1.43

$\begin{array}{lll}0.025 & 0.001 & 1.25 \\ 0.055 & 0.017 & 0.37\end{array}$

$\begin{array}{llll}0.000 & 0.043 & 0.005 & -0.24\end{array}$

0.030

$0.003-0.75$

0.000

0.018

$0.002-1.53$

$\begin{array}{ll}0.002 & -1.53 \\ 0.000 & -1.81\end{array}$

0.001

0.010

$$
0.01
$$$$
0.03
$$

$-0.000$

$-2.85$

$$
0.000
$$

$$
0.012
$$

$\begin{array}{llll}0.010 & -2.99 & -1.98 & 485.07\end{array}$

$0.000-0.024-0.010-1.73-0.83-480.70-128$

$\begin{array}{lllllll}0.000 & 0.012 & -0.049 & -1.55 & -1.28 & 478.53 & 134.60\end{array}$

$\begin{array}{lllllll}0.000 & 0.038 & 0.062 & -1.07 & -1.19 & 477.43 & 143.77\end{array}$

$\begin{array}{lllllll}0.000 & 0.074 & -0.025 & -0.83 & -0.27 & 473.56 & 155.71\end{array}$

$\begin{array}{lllllll}0.000 & 0.074 & -0.035 & -0.36 & -0.05 & 472.00 & 165.35\end{array}$

$\begin{array}{lllllll}0.000 & 0.047 & -0.032 & 0.07 & 0.37 & 468.33 & 177.08\end{array}$

$\begin{array}{lllllll}0.000 & 0.037 & -0.002 & 0.49 & 1.10 & 465.96 & 187.53\end{array}$

$\begin{array}{lllllll}0.000 & 0.038 & 0.018 & 0.59 & 1.09 & 462.44 & 199.12\end{array}$

$\begin{array}{lllllll}0.000 & 0.001 & 0.000 & 0.81 & 1.42 & 460.15 & 209.48\end{array}$

$\begin{array}{llllllll}0.000 & -0.010 & -0.009 & 0.67 & 1.29 & 456.49 & 221.21\end{array}$

$\begin{array}{llllllll}0.000 & -0.034 & -0.026 & 0.56 & 0.92 & 454.64 & 231.13\end{array}$

$\begin{array}{llllllll}0.000 & -0.033 & -0.017 & 0.25 & 0.87 & 450.65 & 243.19\end{array}$

$\begin{array}{lllllll}0.000 & 0.012 & 0.000 & 0.26 & 1.31 & 447.73 & 254.18\end{array}$

$\begin{array}{llllllll}0.000 & -0.011 & -0.009 & -0.28 & 0.65 & 444.11 & 265.88\end{array}$

$\begin{array}{lllllll}0.000 & 0.012 & -0.000 & -0.50 & 0.68 & 441.37 & 276.69\end{array}$

$\begin{array}{llllllll}0.000 & 0.024 & -0.010 & -0.84 & 0.28 & 437.26 & 288.87\end{array}$

$\begin{array}{lllllll}0.000 & 0.012 & -0.000 & -0.91 & 0.26 & 434.33 & 299.87\end{array}$

$\begin{array}{lllllll}0.000 & 0.037 & 0.012 & -1.35 & -0.46 & 430.34 & 311.94\end{array}$

$\begin{array}{lllllll}0.000 & 0.090 & 0.051 & -1.85 & -2.27 & 428.99 & 321.36\end{array}$
$1.20-44.89$

$\begin{array}{lll}0.40 & -46.15\end{array}$

$\begin{array}{lll}0.73 & -43.59\end{array}$

$1.20-42.51$

$1.91-38.58$

$2.62-36.28$

$3.51-31.27$

$3.51-28.80$

$3.84-23.53$

$3.88-20.19$

$3.98-14.39$

$3.66-10.67$

$3.57-4.37$

$3.17-0.02$

$3.15 \quad 6.98$

$2.61 \quad 11.83$

$\begin{array}{ll}2.29 & 19.12\end{array}$

$2.16 \quad 24.98$

$\begin{array}{ll}1.53 & 32.52\end{array}$

$0.95 \quad 38.48$

$\begin{array}{ll}0.15 & 46.35\end{array}$

$-0.72 \quad 52.53$

$-1.78 \quad 60.62$

$-2.41 \quad 67.53$

$-2.33 \quad 77.21$

$-2.15 \quad 85.37$

$-1.95 \quad 95.58$

$\begin{array}{ll}-1.46 & 104.48\end{array}$

$-1.24 \quad 115.10$

$-0.75 \quad 124.39$

$-0.51 \quad 135.40$

$\begin{array}{ll}0.12 & 145.21\end{array}$

$0.41 \quad 156.61$

$\begin{array}{ll}0.87 & 166.60\end{array}$

$\begin{array}{ll}0.93 & 178.09\end{array}$

$\begin{array}{ll}1.22 & 188.24\end{array}$

$\begin{array}{ll}1.35 & 200.11\end{array}$

$\begin{array}{ll}1.42 & 210.36\end{array}$

$\begin{array}{ll}1.34 & 222.31\end{array}$

$1.35 \quad 232.79$

$1.12 \quad 244.86$

$\begin{array}{ll}1.33 & 255.82\end{array}$

$0.70 \quad 267.76$

$\begin{array}{ll}0.69 & 278.76\end{array}$

$\begin{array}{ll}0.39 & 291.27\end{array}$

$\begin{array}{ll}0.28 & 302.42\end{array}$

$-0.24 \quad 314.95$

$-0.36 \quad 326.34$

\section{$Z=22$ (Ti)}

$\begin{array}{llllll}12 & 34 & 0.23 & 0.02 & -0.12 & -0.04\end{array}$

$\begin{array}{lllllll}13 & 35 & 0.18 & 0.02 & -0.12 & -0.04\end{array}$

$0.261-0.029$

$\begin{array}{lll}0.181 & 0.092 & -1.36\end{array}$

$0.35 \quad 194.38$

62.83

$0.206-0.029$

$\begin{array}{lll}0.170 & 0.081 & -0.70\end{array}$

$1.15 \quad 215.61$

49.68

$\begin{array}{llllll}14 & 36 & 0.10 & 0.00 & -0.03 & 0.04\end{array}$

$0.106 \quad 0.000$

$\begin{array}{lll}0.042 & -0.036 & 0.14\end{array}$

$1.34 \quad 240.07$

33.28

$\begin{array}{rrrrrr}15 & 37 & 0.11 & 0.00 & -0.02 & 0.04\end{array}$

$\begin{array}{ll}0.117 & 0.000\end{array}$

$0.031-0.037$

0.67

22.79

$\begin{array}{llllll}16 & 38 & 0.11 & 0.00 & 0.00 & -0.04\end{array}$

$0.118 \quad 0.000$

$0.004 \quad 0.041$

1.08

2.39280 .22

9.28

$\begin{array}{lllllll}17 & 39 & 0.10 & 0.00 & 0.00 & 0.04 & 0.107\end{array}$

0.000

$0.006-0.040$

1.59

$\begin{array}{llr}3.11 & 296.39 & 1.18\end{array}$

$\begin{array}{lllllll}18 & 40 & -0.03 & 0.00 & 0.00 & 0.04 & -0.031\end{array}$

0.000

$\begin{array}{lll}0.000 & -0.039\end{array}$

1.54

$\begin{array}{llll}2.64 & 316.36 & -10.72\end{array}$

$\begin{array}{lllllll}0.000 & -0.049 & 1.27 & 2.84 & 330.94 & -17.23\end{array}$
$1.45 \quad 61.05$

$1.88 \quad 47.94$

$\begin{array}{ll}1.42 & 31.29\end{array}$

$2.12 \quad 21.11$

$2.43 \quad 7.90$

$3.13 \quad 0.06$

$2.66-11.58$

$2.85-17.87$ 


\begin{tabular}{|c|c|c|c|c|c|c|c|c|c|c|c|c|c|c|c|c|}
\hline \multicolumn{17}{|c|}{$Z=22(\mathrm{Ti})$} \\
\hline $20 \quad 42$ & 0.00 & 0.00 & 0.00 & 0.05 & 0.001 & 0.000 & 0.000 & -0.049 & 0.47 & 2.41 & 348.74 & -26.96 & -25.12 & 0.005 & 2.42 & -27.41 \\
\hline 2143 & -0.04 & 0.00 & -0.01 & 0.05 & -0.042 & 0.000 & 0.012 & -0.049 & 1.33 & 3.01 & 361.04 & -31.19 & -29.32 & 0.007 & 3.01 & -31.48 \\
\hline 2244 & 0.00 & 0.00 & 0.00 & 0.04 & 0.000 & 0.000 & 0.000 & -0.039 & 1.10 & 2.63 & 376.91 & -38.98 & -37.55 & 0.001 & 2.63 & -39.13 \\
\hline 2345 & 0.04 & 0.00 & 0.00 & 0.04 & 0.043 & 0.000 & 0.002 & -0.039 & 1.63 & 2.77 & 386.65 & -40.65 & -39.01 & 0.001 & 2.77 & -40.69 \\
\hline $24 \quad 46$ & 0.02 & 0.00 & 0.00 & 0.01 & 0.021 & 0.000 & 0.000 & -0.010 & 1.20 & 2.65 & 399.29 & -45.22 & -44.12 & 0.001 & 2.65 & -45.17 \\
\hline 2547 & 0.05 & 0.00 & -0.01 & 0.01 & 0.053 & 0.000 & 0.013 & -0.009 & 1.03 & 1.88 & 408.56 & -46.42 & -44.93 & 0.001 & 1.88 & -46.29 \\
\hline 2648 & 0.01 & 0.00 & 0.00 & 0.00 & 0.011 & 0.000 & 0.000 & 0.000 & 0.15 & 1.21 & 420.36 & -50.14 & -48.49 & 0.001 & 1.20 & -49.96 \\
\hline $27 \quad 49$ & 0.05 & 0.00 & 0.00 & 0.00 & 0.053 & 0.000 & 0.001 & 0.000 & -0.44 & 0.86 & 427.95 & -49.67 & -48.56 & 0.001 & 0.85 & -49.44 \\
\hline 2850 & 0.00 & 0.00 & 0.00 & 0.00 & 0.000 & 0.000 & 0.000 & 0.000 & -1.38 & 0.08 & 438.59 & -52.24 & -51.43 & 0.001 & 0.07 & -51.98 \\
\hline 2951 & 0.02 & 0.00 & 0.00 & 0.00 & 0.021 & 0.000 & 0.000 & 0.000 & -0.86 & 0.47 & 444.32 & -49.89 & -49.73 & 0.001 & 0.47 & -49.62 \\
\hline $30 \quad 52$ & 0.00 & 0.00 & 0.00 & 0.00 & 0.000 & 0.000 & 0.000 & 0.000 & -0.23 & 0.90 & 452.60 & -50.10 & -49.47 & 0.007 & 0.89 & -49.83 \\
\hline 3153 & 0.01 & 0.00 & 0.00 & 0.00 & 0.011 & 0.000 & 0.000 & 0.000 & 0.60 & 1.60 & 456.99 & -46.43 & -46.83 & 0.100 & 1.60 & -46.16 \\
\hline 3254 & -0.01 & 0.00 & 0.00 & 0.00 & -0.011 & 0.000 & 0.000 & 0.000 & 1.35 & 2.10 & 464.16 & -45.52 & -45.59 & 0.125 & 2.10 & -45.28 \\
\hline 3355 & 0.10 & 0.00 & 0.00 & 0.00 & 0.107 & 0.000 & 0.004 & 0.000 & 1.71 & 3.14 & 467.30 & -40.59 & -41.67 & 0.152 & 3.14 & -40.35 \\
\hline 3456 & 0.12 & 0.00 & 0.02 & -0.01 & 0.129 & 0.000 & -0.019 & 0.007 & 1.89 & 3.51 & 473.65 & -38.86 & -38.94 & 0.196 & 3.54 & -38.64 \\
\hline $35 \quad 57$ & 0.12 & 0.00 & 0.03 & -0.02 & 0.129 & 0.000 & -0.031 & 0.016 & 2.23 & 3.83 & 476.66 & -33.80 & -33.54 & 0.455 & 3.88 & -33.59 \\
\hline 3658 & -0.10 & 0.00 & 0.02 & 0.00 & -0.105 & 0.000 & -0.019 & 0.002 & 2.50 & 3.69 & 482.65 & -31.73 & & & 3.71 & -31.58 \\
\hline $37 \quad 59$ & -0.10 & 0.00 & 0.02 & 0.00 & -0.105 & 0.000 & -0.019 & 0.002 & 2.58 & 3.83 & 485.06 & -26.07 & & & 3.85 & -25.96 \\
\hline 3860 & -0.01 & 0.00 & 0.00 & 0.00 & -0.011 & 0.000 & 0.000 & 0.000 & 2.48 & 3.51 & 490.44 & -23.38 & & & 3.51 & -23.33 \\
\hline 3961 & 0.00 & 0.00 & 0.00 & 0.00 & 0.000 & 0.000 & 0.000 & 0.000 & 2.20 & 3.45 & 492.36 & -17.22 & & & 3.44 & -17.21 \\
\hline $40 \quad 62$ & 0.00 & 0.00 & 0.00 & 0.00 & 0.000 & 0.000 & 0.000 & 0.000 & 1.65 & 2.97 & 497.16 & -13.95 & & & 2.97 & -13.99 \\
\hline 4163 & -0.02 & 0.00 & -0.01 & 0.00 & -0.021 & 0.000 & 0.012 & -0.000 & 1.79 & 3.01 & 498.31 & -7.03 & & & 3.02 & -7.10 \\
\hline $42 \quad 64$ & 0.00 & 0.00 & 0.00 & 0.00 & 0.000 & 0.000 & 0.000 & 0.000 & 1.21 & 2.45 & 502.54 & -3.19 & & & 2.44 & -3.30 \\
\hline 4365 & 0.02 & 0.00 & 0.00 & 0.00 & 0.021 & 0.000 & 0.000 & 0.000 & 1.15 & 2.23 & 503.35 & 4.08 & & & 2.23 & 3.93 \\
\hline 4466 & 0.01 & 0.00 & 0.00 & 0.00 & 0.011 & 0.000 & 0.000 & 0.000 & 0.73 & 1.61 & 507.01 & 8.48 & & & 1.61 & 8.31 \\
\hline 4567 & 0.12 & 0.00 & -0.04 & 0.00 & 0.129 & 0.000 & 0.055 & 0.007 & -0.48 & 1.20 & 507.46 & 16.10 & & & 1.33 & 16.03 \\
\hline $46 \quad 68$ & 0.12 & 0.00 & -0.02 & 0.01 & 0.128 & 0.000 & 0.031 & -0.007 & -0.94 & 0.63 & 510.50 & 21.13 & & & 0.69 & 20.97 \\
\hline $47 \quad 69$ & 0.12 & 0.00 & -0.01 & 0.00 & 0.128 & 0.000 & 0.018 & 0.002 & -1.74 & -0.13 & 510.79 & 28.92 & & & -0.11 & 28.70 \\
\hline 4870 & 0.00 & 0.00 & 0.00 & 0.00 & 0.000 & 0.000 & 0.000 & 0.000 & -2.10 & -1.13 & 513.73 & 34.05 & & & -1.13 & 33.80 \\
\hline 4971 & 0.04 & 0.00 & 0.01 & 0.00 & 0.043 & 0.000 & -0.011 & -0.000 & -3.21 & -2.09 & 513.74 & 42.11 & & & -2.08 & 41.87 \\
\hline 5072 & 0.00 & 0.00 & 0.00 & 0.00 & 0.000 & 0.000 & 0.000 & 0.000 & -4.07 & -2.75 & 515.85 & 48.08 & & & -2.75 & 47.83 \\
\hline 5173 & -0.03 & 0.00 & -0.01 & -0.01 & -0.031 & 0.000 & 0.012 & 0.009 & -3.82 & -2.71 & 514.42 & 57.57 & & & -2.68 & 57.37 \\
\hline 5274 & 0.00 & 0.00 & 0.00 & 0.00 & 0.000 & 0.000 & 0.000 & 0.000 & -3.48 & -2.39 & 515.08 & 64.98 & & & -2.39 & 64.76 \\
\hline 5375 & 0.00 & 0.00 & 0.00 & 0.00 & 0.000 & 0.000 & 0.000 & 0.000 & -3.11 & -2.12 & 513.02 & 75.11 & & & -2.12 & 74.93 \\
\hline 5476 & 0.00 & 0.00 & 0.00 & 0.01 & 0.000 & 0.000 & 0.000 & -0.010 & -2.53 & -1.60 & 513.06 & 83.15 & & & -1.58 & 83.02 \\
\hline 5577 & 0.02 & 0.00 & 0.00 & 0.01 & 0.021 & 0.000 & 0.000 & -0.010 & -2.21 & -1.36 & 510.64 & 93.64 & & & -1.34 & 93.56 \\
\hline 5678 & 0.00 & 0.00 & 0.00 & 0.02 & 0.000 & 0.000 & 0.000 & -0.020 & -1.65 & -0.85 & 510.28 & 102.07 & & & -0.74 & 2.13 \\
\hline $57 \quad 79$ & 0.00 & 0.00 & 0.00 & 0.05 & 0.001 & 0.000 & 0.000 & -0.049 & -1.38 & -1.11 & 508.01 & 112.42 & & & -0.41 & 113.13 \\
\hline 5880 & 0.00 & 0.03 & 0.00 & 0.04 & 0.001 & -0.039 & 0.001 & -0.039 & -0.76 & -0.35 & 507.03 & 121.47 & & & 0.15 & 122.05 \\
\hline 5981 & 0.04 & 0.00 & -0.02 & 0.02 & 0.042 & 0.000 & 0.025 & -0.019 & -0.25 & 0.29 & 503.52 & 133.05 & & & 0.45 & 133.38 \\
\hline 6082 & 0.05 & 0.00 & -0.01 & 0.04 & 0.053 & 0.000 & 0.014 & -0.039 & 0.18 & 0.47 & 502.77 & 141.87 & & & 0.98 & 142.64 \\
\hline 6183 & 0.05 & 0.00 & -0.02 & 0.04 & 0.053 & 0.000 & 0.026 & -0.038 & 0.48 & 0.67 & 499.38 & 153.33 & & & 1.23 & 154.25 \\
\hline 6284 & 0.05 & 0.00 & -0.01 & 0.02 & 0.053 & 0.000 & 0.013 & -0.019 & 0.86 & 1.39 & 497.77 & 163.01 & & & 1.53 & 163.63 \\
\hline 6385 & 0.07 & 0.00 & 0.03 & -0.01 & 0.075 & 0.000 & -0.034 & 0.008 & 0.92 & 1.54 & 494.14 & 174.71 & & & 1.67 & 175.44 \\
\hline 6486 & 0.05 & 0.00 & 0.02 & 0.00 & 0.054 & 0.000 & -0.023 & -0.001 & 1.12 & 1.75 & 492.72 & 184.20 & & & 1.80 & 184.99 \\
\hline 6587 & 0.05 & 0.00 & 0.03 & -0.01 & 0.054 & 0.000 & -0.035 & 0.008 & 0.98 & 1.56 & 489.16 & 195.84 & & & 1.71 & .86 \\
\hline 6688 & 0.05 & 0.00 & 0.02 & -0.01 & 0.053 & 0.000 & -0.023 & 0.009 & 0.96 & 1.62 & 487.60 & 205.46 & & & 1.70 & 206.59 \\
\hline $67 \quad 89$ & 0.00 & 0.00 & 0.00 & 0.00 & 0.000 & 0.000 & 0.000 & 0.000 & 0.71 & 1.62 & 483.59 & 217.55 & & & 1.62 & 218.75 \\
\hline 6890 & 0.00 & 0.00 & 0.00 & 0.00 & 0.000 & 0.000 & 0.000 & 0.000 & 0.42 & 1.41 & 482.03 & 227.18 & & & 1.41 & 228.57 \\
\hline 6991 & -0.02 & 0.00 & 0.01 & 0.00 & -0.021 & 0.000 & -0.012 & 0.000 & -0.04 & 1.06 & 478.12 & 239.15 & & & 1.07 & 240.74 \\
\hline 7092 & 0.00 & 0.00 & 0.00 & 0.00 & 0.000 & 0.000 & 0.000 & 0.000 & -0.35 & 0.76 & 476.39 & 248.96 & & & 0.76 & 250.74 \\
\hline 7193 & -0.02 & 0.00 & -0.01 & 0.01 & -0.021 & 0.000 & 0.012 & -0.010 & -0.62 & 0.49 & 472.17 & 261.25 & & & 0.55 & 263.30 \\
\hline 7294 & 0.00 & 0.00 & 0.00 & 0.00 & 0.000 & 0.000 & 0.000 & 0.000 & -0.82 & 0.28 & 470.10 & 271.39 & & & 0.28 & 273.61 \\
\hline 7395 & -0.02 & 0.00 & 0.00 & -0.01 & -0.021 & 0.000 & 0.000 & 0.010 & -1.19 & -0.10 & 465.79 & 283.78 & & & -0.06 & 286.27 \\
\hline 7496 & 0.00 & 0.00 & 0.00 & 0.00 & 0.000 & 0.000 & 0.000 & 0.000 & -1.31 & -0.25 & 463.42 & 294.21 & & & -0.25 & 296.91 \\
\hline 7597 & 0.11 & 0.00 & -0.07 & -0.02 & 0.120 & 0.000 & 0.092 & 0.033 & -2.49 & -2.20 & 460.46 & 305.24 & & & -1.00 & 309.39 \\
\hline 7698 & 0.11 & 0.00 & -0.05 & 0.01 & 0.118 & 0.000 & 0.067 & -0.002 & -2.53 & -1.75 & 457.28 & 316.49 & & & -1.31 & 320.17 \\
\hline
\end{tabular}




\begin{tabular}{|c|c|c|c|c|c|c|c|c|c|c|c|c|c|c|c|c|}
\hline \multicolumn{17}{|c|}{$Z=23(V)$} \\
\hline 1336 & 0.24 & 0.00 & -0.10 & 0.04 & 9 & 0 & 4 & 04 & 8 & 0.64 & 4 & 62.44 & & & 0.93 & 59.97 \\
\hline 1437 & 0.18 & 0.00 & -0.09 & -0.04 & 0.201 & 0.000 & 0.129 & 0.069 & -0.75 & 1.02 & 234.78 & 45.87 & & & 1.47 & 43.94 \\
\hline 1538 & 0.18 & 0.00 & -0.05 & -0.04 & 0.197 & 0.000 & 0.075 & 0.056 & 0.08 & 2.01 & 254.64 & 34.08 & & & 2.15 & 32.21 \\
\hline 1740 & 0.17 & 0.00 & -0.01 & -0.04 & 0.184 & 0.000 & 0.023 & 0.044 & 1.39 & 2.89 & 294.46 & 10.40 & & & 2.90 & 9.04 \\
\hline 1841 & 0.11 & 0.00 & 0.00 & 0.05 & 0.118 & 0.000 & 0.008 & -0.049 & 1.72 & 3.23 & 313.99 & -1.06 & & & 3.26 & -2.13 \\
\hline 2144 & 0.07 & 0.00 & -0.02 & -0.02 & 0.075 & 0.000 & 0.026 & 0.022 & 1.75 & 3.08 & 362.45 & -25.31 & -2 & 0 & 3.07 & -25.78 \\
\hline 2245 & 0.03 & 0.00 & 0.00 & 0.04 & 0.032 & 0.000 & 0.001 & -0.039 & 1.60 & 2.71 & 378.67 & -33.45 & -31.88 & 0.017 & 2.71 & -33.75 \\
\hline 2346 & 0.18 & 0.00 & -0.08 & 0.01 & 0.195 & 0.000 & 0.115 & 0.012 & 0.22 & 3.26 & 390.07 & -36.79 & -37.07 & 01 & 3.24 & -37.61 \\
\hline $24 \quad 47$ & 0.19 & 0.00 & -0.07 & 0.02 & 0.205 & 0.000 & 0.104 & -0.001 & -0.38 & 2.58 & 404.27 & -42.92 & -42.00 & 0.001 & 2.59 & -42.95 \\
\hline 2548 & 0.19 & 0.00 & -0.05 & 0.01 & 0.205 & 0.000 & 0.078 & 0.004 & -0.34 & 2.38 & 414.30 & -44.88 & -44.47 & 0.003 & 2.37 & -44.84 \\
\hline 3053 & 0.11 & 0.00 & -0.02 & 0.01 & 0.117 & 0.000 & 0.030 & -0.007 & -0.06 & 1.43 & 461.77 & -51.98 & -51.85 & 0.003 & 1.43 & -51.70 \\
\hline 3154 & 0.11 & 0.00 & -0.02 & 0.01 & 0.117 & 0.000 & 0.030 & -0.007 & 0.74 & 2.20 & 467.30 & -49.44 & -49.89 & 0.015 & 2.21 & -49.15 \\
\hline 3255 & 0.16 & 0.00 & -0.01 & 0.01 & 0.172 & 0.000 & 0.024 & -0.007 & 0.90 & 3.05 & 474.39 & -48.46 & -49.15 & 0.100 & 3.06 & -48.17 \\
\hline 3356 & 0.16 & 0.00 & 0.01 & 0.00 & 0.173 & 0.000 & -0.001 & -0.001 & 1.50 & 3.69 & 479.08 & -45.08 & -46.08 & 0.204 & 3.70 & -44.81 \\
\hline 3457 & 0.16 & 0.00 & 0.03 & -0.02 & 0.173 & 0.000 & -0.027 & 0.015 & 1.79 & 3.89 & 485.85 & -43.78 & -4 & & 3.93 & -43.49 \\
\hline 3558 & 0.13 & 0.00 & 0.03 & -0.02 & 0.140 & 0.000 & -0.030 & 0.016 & 2.47 & 4.09 & 490.11 & -39.97 & -40.21 & 0.248 & 4.12 & -39.71 \\
\hline 3659 & 0.13 & 0.00 & 0.03 & -0.02 & 0.140 & 0.000 & -0.030 & 0.016 & 2.68 & 4.14 & 496 & -37.93 & -3 & 7 & 4.19 & -37.69 \\
\hline $37 \quad 60$ & -0.13 & 0.00 & 0.03 & 0.01 & -0.135 & 0.000 & -0.028 & -0.005 & 2.87 & 4.38 & 499.55 & -33.26 & & & 4.41 & -33.07 \\
\hline 3861 & -0.10 & 0.00 & 0.03 & 0.01 & -0.104 & 0.000 & -0.031 & -0.006 & 2.85 & 4.12 & 505.08 & -30.73 & & & 4.15 & -30.57 \\
\hline 3962 & 0.01 & 0.00 & 0.00 & 0.00 & 0.011 & 0.000 & 0.000 & 0.000 & 2.93 & 3.95 & & -25.73 & & & 3.94 & -25.64 \\
\hline $40 \quad 63$ & 0.01 & 0.00 & 0.00 & 0.00 & 0.011 & 0.000 & 0.000 & 0.000 & 2.43 & 3.51 & 513.12 & -22.63 & & & 3.50 & -22.58 \\
\hline 4871 & 0.12 & 0.00 & 0.01 & -0.01 & 0.129 & 0.000 & 0.007 & 0.009 & -1.94 & -0.43 & 534.07 & 21.00 & & & 0 & 20.86 \\
\hline 4972 & 0.05 & 0.00 & 0.01 & 0.00 & 0.053 & 0.000 & -0.011 & -0.001 & -2.67 & -1.71 & 30 & 27.84 & & & 70 & 27.67 \\
\hline 5073 & 0.01 & 0.00 & 0.00 & 0.01 & 0.011 & 0.000 & 0.000 & -0.010 & -3.49 & -2.33 & & 33.71 & & & & 33.54 \\
\hline 5174 & 0.03 & 0.00 & -0.02 & 0.00 & 0.032 & 0.000 & 0.024 & 0.001 & -3.18 & -2.28 & 536.93 & 42.35 & & & -2.25 & 42.20 \\
\hline 5275 & -0.01 & 0.00 & 0.01 & 0.00 & -0.010 & 0.000 & -0.012 & 0.000 & -2.78 & -1.86 & 537.61 & 49.75 & & & -1.85 & 49.58 \\
\hline 5376 & -0.01 & 0.00 & 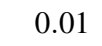 & 0.00 & -0.010 & 0.000 & 12 & 0 & -2.31 & -1.51 & & & & & & 58.96 \\
\hline 5477 & -0.01 & 0.00 & 0.01 & 0.00 & -0.010 & 0.000 & -0.012 & 0.000 & -1.68 & -0.94 & 41 & 67.09 & & & -0 & 66.96 \\
\hline 5578 & -0.02 & 0.00 & 0.01 & 0.00 & -0.021 & 0.000 & -0.012 & 0.000 & -1.27 & -0.53 & 534.65 & 76.92 & & & -0.52 & 76.83 \\
\hline 5679 & -0.01 & 0.00 & 0.01 & 0.00 & -0.010 & 0.000 & -0.012 & 0.000 & -0.66 & -0.03 & & 85.25 & & & -0.02 & 85.20 \\
\hline 5780 & 0.02 & 0.00 & 0.00 & 0.03 & 0.021 & 0.000 & 0.001 & -0.030 & -0.26 & 0.04 & 532.59 & 95.12 & & & 0.28 & 95.35 \\
\hline 5881 & & 0.00 & & & & & 26 & -0.027 & & 0.90 & & & & & & .50 \\
\hline 5982 & 0.27 & 0.00 & -0.07 & 0.00 & 0.295 & 0.000 & 0.121 & 0.031 & -1.90 & 0.95 & 529.46 & 114.40 & & & 1.66 & 115.24 \\
\hline 6083 & 0.27 & 0.00 & -0.07 & 0.00 & 0.295 & 0.000 & 0.121 & 0.031 & -1.63 & 1.12 & 528.79 & 123.14 & & & 1.88 & 124.10 \\
\hline 6184 & 0.28 & 0.00 & -0.06 & 0.00 & 0.306 & 0.000 & 0.110 & 0.028 & -1.67 & 1.30 & 526.19 & 133.80 & & & 1.88 & 134.67 \\
\hline 6285 & 0.28 & 0.00 & -0.06 & 0.01 & 0.305 & 0.000 & 0.111 & 0.018 & -1.27 & 1.53 & 525.12 & 142.95 & & & 2.07 & 143.87 \\
\hline 6386 & 0.27 & 0.00 & -0.06 & 0.0 & 94 & 0.000 & & 16 & -1 & 1.45 & 47 & 153.67 & & & 6 & 154.67 \\
\hline 6487 & 0.27 & 0.00 & -0.05 & 0.00 & 0.294 & 0.000 & 0.095 & 0.023 & -0.68 & 1.74 & 521.03 & 163.18 & & & 2.19 & 164.24 \\
\hline 6588 & 0.27 & 0.00 & -0.04 & -0.01 & 0.295 & 0.000 & 0.081 & 0.029 & -0.63 & 1.74 & 518.00 & 174.28 & & & 2.15 & 175.43 \\
\hline 6689 & 0.27 & 0.00 & -0.03 & -0.01 & 0.295 & 0.000 & 0.069 & 0.025 & -0.21 & 2.00 & 516.29 & 184.07 & & & 2.29 & 185.23 \\
\hline 6790 & 0.28 & 0.00 & -0.01 & -0.02 & 0.307 & 0.000 & 0.045 & 0.028 & -0.29 & 1.86 & 513.12 & 195.30 & & & 2.08 & 196.54 \\
\hline 6891 & 0.01 & 0.00 & 0.00 & 0.01 & 0.011 & 0.000 & 0.000 & 0.010 & 1.39 & 2.14 & 11 & 205.39 & & & 2.17 & 206.61 \\
\hline
\end{tabular}




\begin{tabular}{|c|c|c|c|c|c|c|c|c|c|c|c|c|c|c|c|c|c|}
\hline \multicolumn{18}{|c|}{$Z=23(\mathrm{~V})$} \\
\hline 70 & 93 & 0.00 & 0.00 & 0.00 & 0.00 & 0.000 & 0.000 & 0.000 & 0.000 & 0.61 & 1.51 & 506.17 & 226.47 & & & 1.51 & 228.01 \\
\hline 71 & 94 & -0.03 & 0.00 & -0.01 & 0.00 & -0.032 & 0.000 & 0.012 & -0.000 & 0.30 & 1.28 & 502.59 & 238.12 & & & 1.29 & 239.86 \\
\hline 72 & 95 & 0.01 & 0.00 & 0.00 & 0.01 & 0.011 & 0.000 & 0.000 & -0.010 & 0.03 & 0.94 & 500.67 & 248.11 & & & 0.98 & 250.08 \\
\hline 73 & 96 & 0.03 & 0.00 & -0.01 & 0.00 & 0.032 & 0.000 & 0.012 & 0.000 & -0.43 & 0.42 & 497.15 & 259.70 & & & 0.43 & 261.86 \\
\hline 74 & 97 & 0.11 & 0.00 & -0.06 & -0.02 & 0.120 & 0.000 & 0.079 & 0.031 & -1.26 & -0.82 & 495.90 & 269.03 & & & 0.06 & 272.28 \\
\hline 75 & 98 & 0.11 & 0.00 & -0.06 & 0.00 & 0.118 & 0.000 & 0.079 & 0.010 & -2.00 & -1.26 & 492.07 & 280.92 & & & -0.68 & 284.11 \\
\hline 76 & 99 & 0.11 & 0.00 & -0.05 & 0.00 & 0.118 & 0.000 & 0.066 & 0.008 & -2.24 & -1.41 & 489.50 & 291.57 & & & -0.99 & 294.84 \\
\hline 77 & 100 & 0.11 & 0.00 & -0.03 & 0.01 & 0.117 & 0.000 & 0.042 & -0.005 & -2.95 & -1.96 & 485.58 & 303.55 & & & -1.80 & 306.83 \\
\hline 78 & 101 & 0.11 & 0.00 & -0.01 & 0.02 & 0.118 & 0.000 & 0.018 & -0.018 & -3.20 & -2.27 & 482.96 & 314.25 & & & -2.10 & 317.81 \\
\hline \multicolumn{18}{|c|}{$Z=24(\mathrm{Cr})$} \\
\hline 14 & 38 & 0.24 & 0.00 & -0.06 & 0.04 & 0.259 & 0.000 & 0.103 & -0.019 & -1.19 & 0.56 & 232.19 & 55.75 & & & 0.74 & 53.27 \\
\hline 15 & 39 & 0.23 & 0.00 & -0.03 & 0.04 & 0.249 & 0.000 & 0.063 & -0.029 & -0.57 & 1.59 & 252.37 & 43.63 & & & 1.64 & 41.41 \\
\hline 16 & 40 & 0.23 & 0.00 & 0.01 & 0.04 & 0.251 & 0.000 & 0.015 & -0.040 & -0.25 & 1.83 & 275.93 & 28.15 & & & 1.89 & 26.27 \\
\hline 17 & 41 & 0.18 & 0.00 & 0.00 & 0.05 & 0.195 & 0.000 & 0.018 & -0.049 & 0.88 & 2.27 & 294.27 & 17.88 & & & 2.33 & 16.32 \\
\hline 18 & 42 & 0.11 & 0.00 & 0.00 & 0.05 & 0.118 & 0.000 & 0.008 & -0.049 & 1.42 & 2.63 & 315.26 & 4.96 & & & 2.70 & 3.69 \\
\hline 19 & 43 & 0.08 & 0.00 & 0.00 & 0.05 & 0.086 & 0.000 & 0.005 & -0.049 & 1.50 & 2.73 & 331.77 & -3.48 & & & 2.77 & -4.51 \\
\hline 20 & 44 & 0.00 & 0.00 & 0.00 & 0.05 & 0.001 & 0.000 & 0.000 & -0.049 & 0.87 & 2.28 & 351.40 & -15.04 & & & 2.31 & -15.85 \\
\hline 21 & 45 & 0.09 & 0.00 & -0.03 & -0.04 & 0.098 & 0.000 & 0.040 & 0.045 & 1.39 & 2.77 & 365.59 & -21.16 & -18.97 & 0.503 & 2.78 & -21.78 \\
\hline 22 & 46 & 0.15 & 0.00 & -0.05 & 0.00 & 0.161 & 0.000 & 0.071 & 0.011 & 0.66 & 2.69 & 382.90 & -30.40 & -29.47 & 0.020 & 2.71 & -30.85 \\
\hline 23 & 47 & 0.20 & 0.00 & -0.07 & 0.03 & 0.215 & 0.000 & 0.106 & -0.010 & -0.20 & 2.81 & 395.74 & -35.16 & -34.56 & 0.014 & 2.81 & -35.46 \\
\hline 24 & 48 & 0.21 & 0.00 & -0.05 & 0.03 & 0.226 & 0.000 & 0.083 & -0.015 & -0.79 & 2.11 & 411.93 & -43.29 & -42.82 & 0.007 & 2.13 & -43.43 \\
\hline 25 & 49 & 0.21 & 0.00 & -0.03 & 0.02 & 0.227 & 0.000 & 0.057 & -0.010 & -0.84 & 1.94 & 422.27 & -45.55 & -4 & 0.002 & 1.94 & -45.60 \\
\hline 26 & 50 & 0.18 & 0.00 & -0.02 & 0.00 & 0.194 & 0.000 & 0.038 & 0.006 & -0.92 & 1.19 & 435.77 & -50.98 & -50.26 & 0.001 & 1.20 & -50.93 \\
\hline 27 & 51 & 0.11 & 0.00 & -0.01 & 0.00 & 0.118 & 0.000 & 0.017 & 0.002 & -0.72 & 0.69 & 445.12 & -52.26 & -51.45 & 0.001 & 0.69 & -52.14 \\
\hline 28 & 52 & 0.00 & 0.00 & 0.00 & -0.02 & 0.000 & 0.000 & 0.000 & 0.020 & -1.27 & -0.17 & 457.40 & -56.47 & -55.42 & 0.001 & -0.17 & -56.29 \\
\hline 29 & 53 & 0.05 & 0.00 & 0.00 & 0.00 & 0.053 & 0.000 & 0.001 & 0.000 & -0.77 & 0.20 & 464.70 & -55.70 & -55.28 & 0.001 & 0.19 & -55.48 \\
\hline 30 & 54 & 0.15 & 0.00 & -0.03 & 0.03 & 0.161 & 0.000 & 0.048 & -0.024 & -0.86 & 1.21 & 473.90 & -56.83 & -56.93 & 0.001 & 1.25 & -56.54 \\
\hline 31 & 55 & 0.16 & 0.00 & -0.01 & 0.02 & 0.172 & 0.000 & 0.024 & -0.017 & -0.15 & 2.02 & 479.68 & -54.54 & -55.11 & 0.001 & 2.04 & -54.25 \\
\hline 32 & 56 & 0.17 & 0.00 & 0.00 & 0.01 & 0.183 & 0.000 & 0.013 & -0.009 & 0.27 & 2.17 & 488.65 & -55.43 & -55.28 & 0.002 & 2.19 & -55.12 \\
\hline 33 & 57 & 0.17 & 0.00 & 0.02 & 0.00 & 0.184 & 0.000 & -0.012 & -0.003 & 0.82 & 2.79 & 493.62 & -52.34 & -52.52 & 0.002 & 2.81 & -52.04 \\
\hline 34 & 58 & 0.17 & 0.00 & 0.04 & -0.02 & 0.185 & 0.000 & -0.038 & 0.013 & 1.09 & 3.11 & 501.42 & -52.06 & -51.83 & 0.203 & 3.16 & -51.74 \\
\hline 35 & 59 & 0.16 & 0.00 & 0.04 & -0.02 & 0.174 & 0.000 & -0.039 & 0.013 & 1.66 & 3.68 & 505.55 & -48.12 & -47.89 & 0. & 3.73 & -47.81 \\
\hline 36 & 60 & 0.16 & 0.00 & 0.03 & -0.02 & 0.173 & 0.000 & -0.027 & 0.015 & 1.97 & 3.70 & 512.72 & -47.21 & -46.50 & 0.213 & 3.76 & -46.92 \\
\hline 37 & 61 & -0.13 & 0.00 & 0.03 & 0.01 & -0.135 & 0.000 & -0.028 & -0.005 & 2.53 & 4.01 & 516.28 & -42.71 & -42.18 & 0.255 & 4.04 & -42.46 \\
\hline 38 & 62 & -0.10 & 0.00 & 0.03 & 0.01 & -0.104 & 0.000 & -0.031 & -0.006 & 2.53 & 3.79 & 522.85 & -41.20 & -40.42 & 0.337 & 3.83 & -40.98 \\
\hline 39 & 63 & 0.00 & 0.00 & 0.00 & 0.00 & 0.000 & 0.000 & 0.000 & 0.000 & 2.63 & 3.56 & 526.18 & -36.47 & & & 3.56 & -36.31 \\
\hline 40 & 64 & 0.00 & 0.00 & 0.00 & 0.00 & 0.000 & 0.000 & 0.000 & 0.000 & 2.14 & 3.18 & 532.14 & -34.35 & & & 3.18 & -34.23 \\
\hline 41 & 65 & -0.03 & 0.00 & -0.01 & 0.00 & -0.032 & 0.000 & 0.012 & -0.000 & 2.27 & 3.24 & 534.49 & -28.63 & & & 3.24 & -28.54 \\
\hline 42 & 66 & 0.00 & 0.00 & 0.00 & 0.00 & 0.000 & 0.000 & 0.000 & 0.000 & 1.74 & 2.69 & 539.89 & -25.96 & & & 2.68 & -25.91 \\
\hline 43 & 67 & 0.15 & 0.00 & -0.06 & -0.01 & 0.162 & 0.000 & 0.084 & 0.024 & 0.47 & 2.37 & 541.97 & -19.96 & & & 2.62 & -19.69 \\
\hline 44 & 68 & 0.16 & 0.00 & -0.04 & 0.00 & 0.172 & 0.000 & 0.060 & 0.009 & -0.08 & 1.87 & 546.66 & -16.58 & & & 2.00 & -16.46 \\
\hline 45 & 69 & 0.16 & 0.00 & -0.04 & 0.01 & 0.172 & 0.000 & 0.060 & -0.001 & -0 & 1.18 & 548.50 & -1 & & & 1.31 & -1 \\
\hline 46 & 70 & 0.16 & 0.00 & -0.02 & 0.01 & 0.172 & 0.000 & 0.036 & -0.005 & -1.36 & 0.59 & 552.67 & -6.46 & & & 0.66 & -6.45 \\
\hline 47 & 71 & 0.16 & 0.00 & 0.00 & 0.00 & 0.172 & 0.000 & 0.011 & 0.001 & -2.05 & 0.08 & 553.79 & 0.49 & & & 0.10 & 0.43 \\
\hline 48 & 72 & 0.12 & 0.00 & 0.01 & 0.00 & 0.129 & 0.000 & -0.006 & -0.001 & -2.30 & -0.78 & 557.65 & 4.71 & & & -0.76 & 4.62 \\
\hline 49 & 73 & 0.05 & 0.00 & 0.01 & 0.00 & 0.053 & 0.000 & -0.011 & -0.001 & -2.98 & -2.04 & 559.00 & 11.43 & & & -2.04 & 11.32 \\
\hline 50 & 74 & 0.00 & 0.00 & 0.00 & 0.00 & 0.000 & 0.000 & 0.000 & 0.000 & -3.81 & -2.64 & 562.06 & 16.44 & & & -2.64 & 16.31 \\
\hline 51 & 75 & 0.03 & 0.00 & -0.01 & 0.00 & 0.032 & 0.000 & 0.012 & 0.000 & -3.38 & -2.52 & 561.57 & 25.01 & & & -2.52 & 24.89 \\
\hline 52 & 76 & 0.00 & 0.00 & 0.00 & 0.00 & 0.000 & 0.000 & 0.000 & 0.000 & -2.96 & -2.08 & 563.09 & 31.56 & & & -2.08 & 31.43 \\
\hline 53 & 77 & 0.01 & 0.00 & 0.00 & 0.00 & 0.011 & 0.000 & 0.000 & 0.000 & -2.40 & -1.70 & 561.88 & 40.83 & & & -1.71 & 40.72 \\
\hline 54 & 78 & -0.01 & 0.00 & 0.00 & 0.00 & -0.011 & 0.000 & 0.000 & 0.000 & -1.72 & -1.05 & 562.73 & 48.05 & & & -1.05 & 47.95 \\
\hline 55 & 79 & 0.16 & 0.00 & -0.02 & 0.03 & 0.172 & 0.000 & 0.037 & -0.025 & -2.03 & -0.69 & & 57.73 & & & & 57.89 \\
\hline 56 & 80 & 0.17 & 0.00 & -0.01 & 0.03 & 0.183 & 0.000 & 0.026 & -0.027 & -1.50 & -0.10 & 561.60 & 65.33 & & & 0.14 & 65.52 \\
\hline 57 & 81 & 0.17 & 0.00 & 0.00 & 0.03 & 0.184 & 0.000 & 0.014 & -0.029 & -1.00 & 0.17 & 559.69 & 75.31 & & & 0.41 & 75.54 \\
\hline 58 & 82 & 0.17 & 0.00 & 0.00 & 0.03 & 0.184 & 0.000 & 0.014 & -0.029 & -0.53 & 0.67 & 559.86 & 83.21 & & & 0.92 & 83.51 \\
\hline 59 & 83 & 0.27 & 0.00 & -0.06 & 0.01 & 0.294 & 0.000 & 0.108 & 0.016 & -2.03 & 0.96 & 557.56 & 93.58 & & & 1.45 & 94.17 \\
\hline 60 & 84 & 0.27 & 0.00 & -0.06 & 0.01 & 0.294 & 0.000 & 0.108 & 0.016 & -1.79 & 1.14 & 557.66 & 101.56 & & & 1.67 & 102.24 \\
\hline
\end{tabular}




\begin{tabular}{|c|c|c|c|c|c|c|c|c|c|c|c|c|c|}
\hline \multicolumn{14}{|c|}{$Z=24(\mathrm{Cr})$} \\
\hline 61 & 85 & 0.28 & 0.00 & -0.05 & 0.01 & 0.305 & 0.000 & 0.098 & 0.014 & -1.87 & 1.15 & 555.31 & 111.98 \\
\hline 62 & 86 & 0.29 & 0.00 & -0.05 & 0.02 & 0.316 & 0.000 & 0.102 & 0.004 & -1.68 & 1.31 & 555.07 & 120.29 \\
\hline 63 & 87 & 0.28 & 0.00 & -0.04 & 0.00 & 0.306 & 0.000 & 0.085 & 0.020 & -1.38 & 1.44 & .27 & 131.16 \\
\hline 64 & 88 & 0.28 & 0.00 & -0.03 & 0.00 & 0.306 & 0.000 & 0.072 & 0.016 & & 1.74 & & 139.94 \\
\hline 65 & 89 & 0.28 & 0.00 & -0.03 & 0.01 & 0.305 & 0.000 & 0.073 & 0.005 & -0.99 & 1.69 & 64 & 150.93 \\
\hline 80 & 90 & 0.28 & 0.00 & -0.02 & 0.00 & 306 & 000 & 059 & 12 & - & .91 & 68 & 9.96 \\
\hline 67 & 91 & 0.28 & 0.00 & -0.01 & 0.00 & 0.306 & 0.000 & 0.047 & 0.008 & -( & 1.86 & & 171.24 \\
\hline 68 & 92 & 0.29 & 0.00 & 0.01 & -0.01 & 0.318 & 0.000 & 0.023 & 0.011 & - & 2.01 & 29 & 180.49 \\
\hline 69 & 93 & 0.27 & 0.00 & 0.02 & -0.02 & 0.296 & 0.000 & 0.005 & 0.017 & -0.56 & 1.74 & & 191.82 \\
\hline 70 & 94 & 0.00 & 0.00 & 0.00 & 0.00 & 0.000 & 0.000 & 0.000 & 0.000 & 0.91 & 1.67 & 538.79 & 201.13 \\
\hline 71 & 95 & 0.03 & 0.00 & -0.01 & 0.00 & 0.032 & 0.000 & 0.012 & -0.000 & 55 & 1.44 & 26 & 212.74 \\
\hline 72 & 96 & 0.00 & 0.00 & 0.00 & 0.00 & 0.000 & 0.000 & 0.000 & 0.000 & 0.27 & 1.08 & & 222.04 \\
\hline 73 & 97 & 0.03 & 0.00 & -0.01 & 0.00 & & 0.000 & 0. & 0.000 & -0.24 & 0.51 & & 233.56 \\
\hline 74 & 98 & 0.05 & 0.00 & -0.02 & 0.00 & 0.053 & 0.000 & 0.025 & 0.001 & -0.63 & 0.13 & 529.12 & 243.09 \\
\hline 75 & 99 & 0.05 & 0.00 & -0.02 & 0.00 & 0.053 & 0.000 & 0.025 & 0.001 & -1.33 & -0.50 & 525.52 & 254.77 \\
\hline 76 & 00 & 0.11 & 0.00 & -0.04 & 0.01 & 0.117 & 0.000 & 0.054 & -0.004 & -2.18 & -1.29 & 524.22 & 264.14 \\
\hline 77 & 101 & 0.11 & 0.00 & -0.03 & 0.02 & 0.117 & 0.000 & 0.042 & -0.016 & -3.04 & -2.14 & 520.62 & 275.8 \\
\hline 78 & 102 & 0.11 & 0.00 & -0.01 & 0.02 & 0.118 & 0.000 & 0.018 & -0.018 & -3.31 & -2.37 & 518.54 & 285.9 \\
\hline 79 & 103 & 0.09 & 0.00 & 0.01 & 0.00 & 0.096 & 0.000 & -0.009 & -0.001 & -4.15 & -3.08 & 514.58 & 297.9 \\
\hline
\end{tabular}

$\begin{array}{ll}1.53 & 112.59\end{array}$

$\begin{array}{ll}1.73 & 121.02\end{array}$

$\begin{array}{ll}1.76 & 131.88\end{array}$

$\begin{array}{ll}1.96 & 140.67\end{array}$

$1.85 \quad 151.71$

$2.03 \quad 160.82$

$\begin{array}{ll}1.88 & 172.13\end{array}$

$2.01 \quad 181.50$

$\begin{array}{ll}1.82 & 193.05\end{array}$

$1.67 \quad 202.46$

$\begin{array}{ll}1.45 & 214.25\end{array}$

$\begin{array}{ll}1.08 & 223.72\end{array}$

$0.52 \quad 235.45$

$0.19 \quad 245.23$

$-0.44 \quad 257.12$

$-1.02 \quad 266.92$

$-1.87 \quad 278.83$

$\begin{array}{ll}-2.20 & 289.14\end{array}$

$-3.08 \quad 301.24$

\section{$Z=25$ (Mn)}

\begin{tabular}{|c|c|c|c|c|c|c|c|c|c|c|c|c|c|c|c|c|c|}
\hline 15 & 40 & 0.23 & 0.00 & 0.01 & 0.04 & 0.251 & 0.000 & 0.015 & -0.040 & -0.93 & 1.00 & 247.29 & 56.01 & & & 1.05 & 53.52 \\
\hline 16 & 41 & 0.24 & 0.00 & 0.04 & 0.05 & 0.266 & 0.000 & -0.017 & -0.058 & -0.90 & 1.16 & 271.28 & 40.09 & & & 1.30 & 38.05 \\
\hline 17 & 42 & 0.21 & 0.00 & 0.03 & -0.05 & 0.229 & 0.000 & -0.022 & 0.044 & 0.24 & 1.79 & 290.87 & 28.57 & & & 1.84 & 26.76 \\
\hline 18 & 43 & 0.16 & 0.00 & 0.02 & -0.05 & 0.173 & 0.000 & -0.017 & 0.047 & 0.86 & 2.08 & 312.28 & 15.23 & & & 2.14 & 13.73 \\
\hline 19 & 44 & 0.10 & 0.00 & 0.00 & 0.05 & 0.108 & 0.000 & 0.007 & -0.049 & 1.16 & 2.53 & 329.86 & 5.72 & & & 2.57 & 4.48 \\
\hline 20 & 45 & 0.01 & 0.00 & 0.00 & 0.05 & 0.011 & 0.000 & 0.001 & -0.049 & 0.67 & 1.94 & 349.97 & -6.32 & & & 1.98 & -7.32 \\
\hline 21 & 46 & 0.11 & 0.00 & -0.03 & -0.02 & 0.118 & 0.000 & 0.041 & 0.025 & 1.05 & 2.61 & 365.35 & -13.62 & & & 2.61 & -14.45 \\
\hline 22 & 47 & 0.11 & 0.00 & -0.03 & -0.01 & 0.118 & 0.000 & 0.041 & 0.015 & 0.75 & 2.06 & 383.47 & -23.68 & & & 2.06 & -24.31 \\
\hline 23 & 48 & 0.20 & 0.00 & -0.04 & 0.01 & 0.216 & 0.000 & 0.067 & 0.002 & -0.12 & 2.54 & 397.28 & -29.41 & -29.32 & 0.112 & 2.52 & -29.89 \\
\hline 24 & 49 & 0.20 & 0.00 & -0.03 & 0.00 & 0.216 & 0.000 & 0.054 & 0.009 & -0.79 & 1.80 & 413.85 & -37.91 & -37.62 & 0.024 & 1.79 & -38.22 \\
\hline 25 & 50 & 0.21 & 0.00 & -0.01 & 0.00 & 0.228 & 0.000 & 0.031 & 0.004 & -1.08 & 1.56 & 426.17 & -42.16 & -42.63 & 0.001 & 1.54 & -42.96 \\
\hline 26 & 51 & 0.18 & 0.00 & 0.00 & 0.00 & 0.194 & 0.000 & 0.014 & 0.001 & -1.30 & 1.05 & 440.35 & -48.27 & -48.24 & 0.001 & 1.04 & -48.33 \\
\hline 27 & 52 & 0.14 & 0.00 & 0.00 & 0.00 & 0.150 & 0.000 & 0.008 & 0.001 & -1.26 & 0.53 & 450.97 & -50.82 & -5 & 02 & 0.52 & -50.80 \\
\hline 28 & 53 & -0.02 & 0.00 & 0.00 & -0.01 & -0.021 & 0.000 & 0.000 & 0.010 & -1.50 & -0.46 & 463.68 & -55.46 & -54.69 & 0.001 & -0.46 & -55.36 \\
\hline 9 & 54 & 0.11 & 0.00 & -0.01 & 0.01 & 0.118 & 0.000 & 0.018 & -0.008 & -1.35 & 0.11 & 472.00 & -55.71 & -55.56 & 0.001 & 0.11 & -55.54 \\
\hline 30 & 55 & 0.16 & 0.00 & -0.02 & 0.03 & 0.172 & 0.000 & 0.037 & -0.025 & -1.21 & 1.01 & .60 & -57.24 & -57.71 & & 1.02 & -57.01 \\
\hline 31 & 56 & 0.17 & 0.00 & -0.01 & 0.02 & 0.183 & 0.000 & 0.026 & -0.017 & -0.54 & 1.30 & 489.08 & -56.64 & -56 & 0.001 & 1.30 & -56.39 \\
\hline 32 & 57 & 0.18 & 0.00 & 0.01 & 0.01 & 0.195 & 0.000 & 0.002 & -0.011 & -0.11 & 1.98 & 497.78 & -57.27 & -5 & 0.002 & 1.99 & -56.99 \\
\hline 33 & 58 & 0.18 & 0.00 & 0.03 & -0.01 & 0.196 & 0.000 & -0.023 & 0.004 & 0.39 & 2.55 & 503.95 & -55.37 & -55.91 & 0.030 & 2.56 & -55.08 \\
\hline 4 & 59 & 0.18 & 0.00 & 0.04 & -0.02 & 0.196 & 0.000 & -0.036 & 0.012 & 0.69 & 2.89 & 511.97 & -55.32 & -55.48 & 0.030 & 2.93 & -55.00 \\
\hline 35 & 60 & 0.16 & 0.00 & 0.04 & -0.02 & 0.174 & 0.000 & -0.039 & 0.013 & 1.42 & 3.47 & 517.20 & -52.47 & -53.18 & 0.086 & 3.50 & -52.16 \\
\hline 36 & 61 & 0.16 & 0.00 & 0.04 & -0.02 & 0.174 & 0.000 & -0.039 & 0.013 & 1.73 & 3.53 & 524.56 & -51.77 & -5 & 0.2 & 3.58 & -51.45 \\
\hline 37 & 62 & -0.13 & 0.00 & 0.03 & 0.01 & -0.135 & 0.000 & -0.028 & -0.005 & 2.42 & 3.97 & 529.06 & -48.20 & -48.04 & 0.223 & 3.99 & -47.92 \\
\hline 38 & 63 & -0.10 & 0.00 & 0.03 & 0.01 & -0.104 & 0.000 & -0.031 & -0.006 & 2.42 & 3.68 & 535.92 & -46.99 & -46.35 & 0.258 & 3.70 & -46.73 \\
\hline 39 & 64 & -0.03 & 0.00 & 0.00 & 0.00 & -0.032 & 0.000 & 0.000 & 0.000 & 2.66 & 3.54 & 540.21 & -43.21 & -42.62 & 0.267 & 3.54 & -43.00 \\
\hline 40 & 65 & 0.00 & 0.00 & 0.00 & 0.00 & 0.000 & 0.000 & 0.000 & 0.000 & 2.18 & 3.15 & 546.37 & -41.29 & -40.67 & 0.537 & 3.15 & -41.12 \\
\hline 41 & 66 & -0.03 & 0.00 & -0.01 & 0.00 & -0.032 & 0.000 & 0.012 & -0.000 & 2.27 & 3.18 & 549.76 & -36.61 & & & 3.18 & -36.46 \\
\hline 42 & 67 & -0.01 & 0.00 & 0.00 & 0.00 & -0.011 & 0.000 & 0.000 & 0.000 & 1.76 & 2.65 & 555.33 & -34.11 & & & 2.65 & -33.99 \\
\hline 43 & 68 & 0.12 & 0.00 & -0.03 & -0.01 & 0.129 & 0.000 & 0.042 & 0.015 & 1.05 & 2.43 & 558.29 & -29.00 & & & 2.50 & -28.84 \\
\hline 44 & 69 & 0.16 & 0.00 & -0.03 & 0.01 & 0.172 & 0.000 & 0.048 & -0.003 & 0.05 & 1.96 & 563.13 & -25.76 & & & 2.03 & -25.63 \\
\hline 45 & 70 & 0.16 & 0.00 & -0.03 & 0.01 & 0.172 & 0.000 & 0.048 & -0.003 & -0.77 & 1.26 & 565.94 & -20.51 & & & 1.33 & -20.41 \\
\hline 46 & 71 & 0.16 & 0.00 & -0.01 & 0.01 & 0.172 & 0.000 & 0.024 & -0.007 & -1.32 & 0.83 & 570.10 & -16.59 & & & 0.87 & -16.55 \\
\hline 47 & 72 & 0.16 & 0.00 & 0.00 & 0.00 & 0.172 & 0.000 & 0.011 & 0.001 & -2.13 & 0.04 & 572.42 & -10.84 & & & 0.06 & -10.85 \\
\hline 48 & 73 & 0.12 & 0.00 & 0.01 & -0.01 & 0.129 & 0.000 & -0.007 & 0.009 & -2.40 & -0.85 & 576.47 & -6.82 & & & -0.83 & -6.83 \\
\hline 49 & 74 & 0.06 & 0.00 & 0.01 & 0.00 & 0.064 & 0.000 & -0.010 & -0.001 & -3.09 & -2.07 & 578.67 & -0.95 & & & -2.06 & -1.00 \\
\hline 50 & 75 & 0.00 & 0.00 & 0.00 & 0.00 & 0.000 & 0.000 & 0.000 & 0.000 & -3.97 & -2.79 & 582.00 & 3.79 & & & -2.79 & 3.72 \\
\hline 51 & 76 & 0.04 & 0.00 & -0.01 & 0.00 & 0.043 & 0.000 & 0.013 & 0.001 & -3.42 & -2.50 & 582.19 & 11.67 & & & -2.49 & 11.60 \\
\hline
\end{tabular}




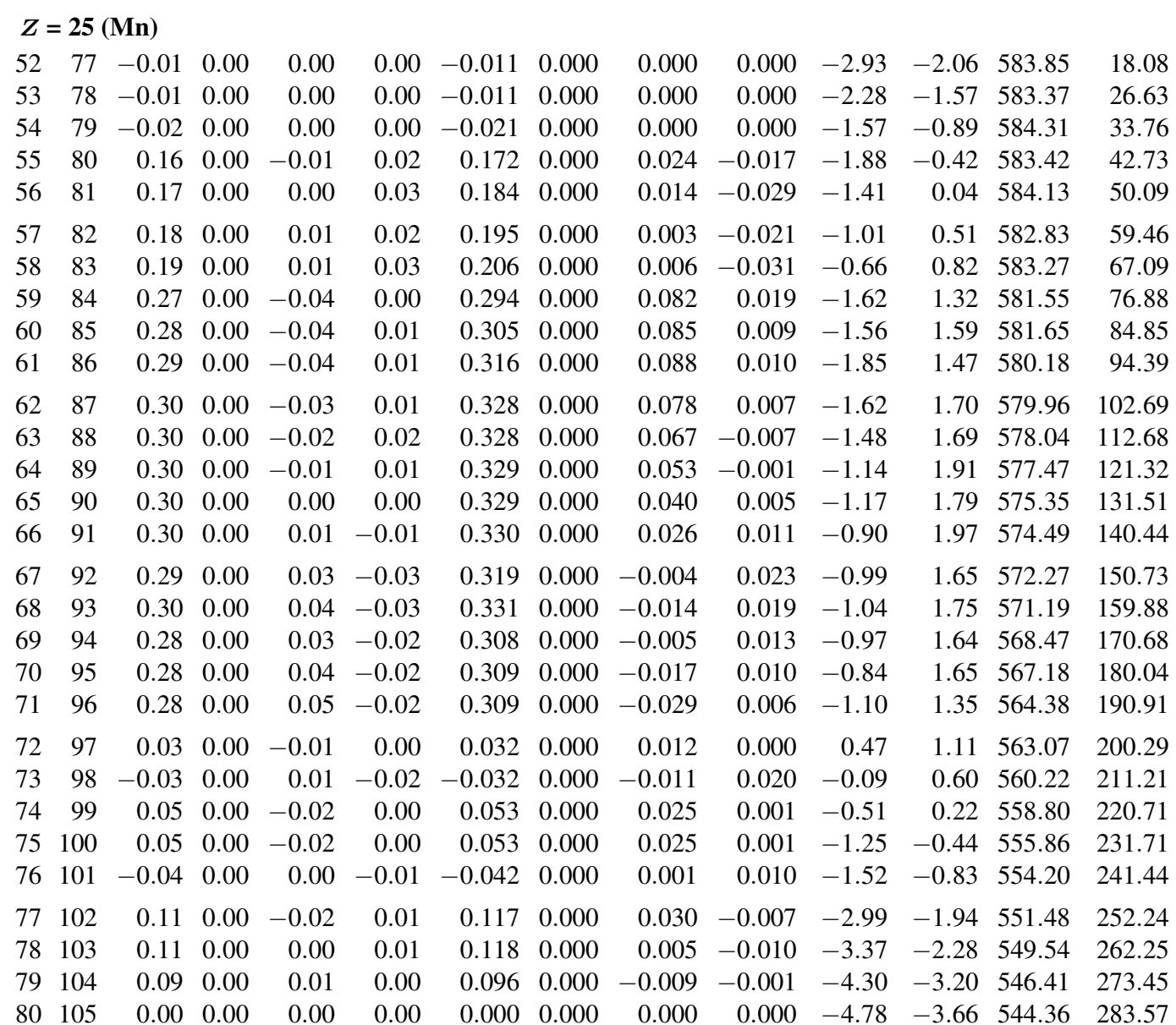

$-2.06 \quad 18.00$
-1.57

$-1.57 \quad 26.56$

$-0.89 \quad 33.69$

$\begin{array}{ll}-0.32 & 42.77\end{array}$

$\begin{array}{ll}0.26 & 50.27\end{array}$

$0.62 \quad 59.57$

$1.07 \quad 67.37$

$1.58 \quad 77.22$

$1.84 \quad 85.23$

$1.71 \quad 94.82$

$\begin{array}{ll}1.88 & 103.12\end{array}$

$\begin{array}{ll}1.82 & 113.14\end{array}$

$\begin{array}{ll}1.96 & 121.79\end{array}$

$1.77 \quad 132.01$

$1.97 \quad 141.06$

$\begin{array}{ll}1.85 & 151.67\end{array}$

$1.95 \quad 160.95$

$1.69 \quad 171.72$

$\begin{array}{ll}1.73 & 181.26\end{array}$

$1.46 \quad 192.31$

$1.12 \quad 201.76$

$0.77 \quad 213.00$

$0.28 \quad 222.58$

$-0.38 \quad 233.77$

$-0.79 \quad 243.70$

$\begin{array}{ll}-1.86 & 254.75\end{array}$

$-2.26 \quad 264.93$

$-3.20 \quad 276.34$

$-3.66 \quad 286.71$

\section{$Z=26(\mathrm{Fe})$}

$\begin{array}{llllllllllllll}16 & 42 & 0.26 & 0.00 & 0.09 & -0.05 & 0.289 & 0.000 & -0.086 & 0.024 & -1.59 & 0.53 & 269.07 & 49.58\end{array}$

$\begin{array}{llllllllllllll}17 & 43 & 0.20 & 0.00 & 0.06 & -0.05 & 0.219 & 0.000 & -0.060 & 0.036 & -0.24 & 1.09 & 289.07 & 37.66\end{array}$

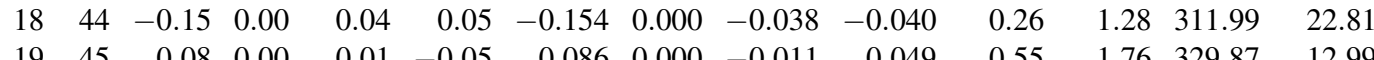

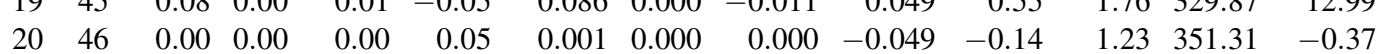

$\begin{array}{llllllllllllll}21 & 47 & 0.06 & 0.00 & -0.01 & -0.05 & 0.065 & 0.000 & 0.013 & 0.051 & 0.63 & 1.80 & 367.12 & -8.11\end{array}$

$\begin{array}{llllllllllllll}22 & 48 & 0.01 & 0.00 & 0.00 & 0.01 & 0.011 & 0.000 & 0.000 & -0.010 & 0.43 & 1.47 & 386.38 & -19.29\end{array}$

$\begin{array}{llllllllllllll}23 & 49 & 0.13 & 0.00 & -0.01 & -0.01 & 0.140 & 0.000 & 0.019 & 0.012 & 0.38 & 1.81 & 400.63 & -25.48\end{array}$

$\begin{array}{llllll}24 & 50 & 0.18 & 0.00 & -0.01 & -0.01\end{array}$

$\begin{array}{lllll}0.194 & 0.000 & 0.025 & 0.014 & -0.71\end{array}$

$\begin{array}{llll}1.30 & 418.28 & -35.05\end{array}$

$\begin{array}{llll}1.30 & 418.28 & -35.05 & -34.48 \\ 1.27 & 431.30 & -40.01 & -40.22\end{array}$

$\begin{array}{lll}0.195 & 0.000 & 0.001\end{array}$

$0.009-0.98$

$\begin{array}{rrrr}0.04 & 448.05 & -48.68 & -48.33 \\ -0.25 & 458.75 & -51.31 & -50.94\end{array}$

$\begin{array}{lll}0.118 & 0.000 & -0.007\end{array}$

$\begin{array}{ll}0.009 & -1.18\end{array}$

$\begin{array}{rrr}0.107 & 0.000 & -0.008 \\ 0.000 & 0.000 & 0.000\end{array}$

$0.009-1.64$

$\begin{array}{lllllll}0.000 & -2.31 & -1.17 & 472.62 & -57.11 & -56.25\end{array}$

$\begin{array}{llllll}28 & 54 & 0.00 & 0.00 & 0.00 & 0.00\end{array}$

$\begin{array}{llllll}29 & 55 & 0.02 & 0.00 & -0.01 & 0.00\end{array}$

$\begin{array}{lll}0.021 & 0.000 & 0.012\end{array}$

$\begin{array}{lllllll}0.000 & -1.70 & -0.74 & 481.36 & -57.78 & -57.48\end{array}$

$30 \quad 56$

$\begin{array}{lll}0.11 & 0.00 & -0.02\end{array}$

0.01

$0.117 \quad 0.000$

$\begin{array}{llll}0.030 & -0.007 & -1.35\end{array}$

$\begin{array}{llll}0.13 & 492.19 & -60.53 & -60.60\end{array}$

$\begin{array}{llll}1.15 & 499.21 & -59.48 & -60.18\end{array}$

$\begin{array}{llll}1.33 & 509.57 & -61.78 & -62.15\end{array}$

$\begin{array}{llll}1.95 & 515.95 & -60.08 & -60.66\end{array}$

$\begin{array}{llll}2.27 & 525.11 & -61.18 & -61.41\end{array}$

$0.185-0.000-0.036-0.007-0.14$

$0.04 \quad 0.00$

$\begin{array}{rrrrr}0.185 & 0.000 & -0.036 & -0.007 & -0.14 \\ 0.185 & 0.000 & -0.050 & 0.011 & 0.13\end{array}$

$\begin{array}{llll}2.78 & 530.65 & -58.64 & -58.92\end{array}$

$\begin{array}{llll}0.163 & 0.000 & -0.052\end{array}$

$0.012 \quad 0.80$

$\begin{array}{llll}2.85 & 539.10 & -59.02 & -58.90\end{array}$

$0.04-0.02$

$\begin{array}{lllll}0.152 & 0.000 & -0.041 & 0.014 & 1.28\end{array}$

$\begin{array}{llll}2.85 & 539.10 & -59.02 & -58.90 \\ 3.21 & 543.91 & -55.76 & -55.55\end{array}$

$\begin{array}{lllll}2.89 & 551.85 & -55.63 & -54.77\end{array}$

$\begin{array}{llll}2.87 & 556.23 & -51.94 & -50.88\end{array}$

$\begin{array}{lllll}2.46 & 563.44 & -51.07 & -49.57\end{array}$

$\begin{array}{lllll}2.54 & 566.98 & -46.54 & -45.69\end{array}$

$\begin{array}{lllll}2.02 & 573.53 & -45.02 & -43.13\end{array}$
$0.72 \quad 47.35$

$1.20 \quad 35.68$

$1.37 \quad 21.12$

$1.83 \quad 11.57$

$1.29-1.55$

$1.84-9.07$

$1.46-20.09$

$1.80-26.09$

$1.30-35.49$

$1.26-40.32$

$0.04-48.85$

$\begin{array}{rrr}0.007 & 0.04 & -48.85 \\ 0.002 & -0.25 & -51.38\end{array}$

$\begin{array}{llll}0.001 & -1.17 & -57.09\end{array}$

$\begin{array}{lll}0.001 & -0.74 & -57.69\end{array}$

$\begin{array}{llll}0.001 & 0.14 & -60.37\end{array}$

$\begin{array}{llll}0.001 & 1.15 & -59.27\end{array}$

$\begin{array}{lll}0.001 & 1.34 & -61.52\end{array}$

$\begin{array}{lll}0.001 & 1.97 & -59.79\end{array}$

$\begin{array}{lll}0.003 & 2.32 & -60.84\end{array}$

$\begin{array}{lll}0.020 & 2.83 & -58.30\end{array}$

$\begin{array}{lll}0.014 & 2.89 & -58.68\end{array}$

$\begin{array}{llll}0.168 & 3.21 & -55.47\end{array}$

$\begin{array}{lll}0.277 & 2.90 & -55.35\end{array}$

$\begin{array}{lll}0.243 & 2.87 & -51.69\end{array}$

$\begin{array}{llll}0.303 & 2.46 & -50.85\end{array}$

$\begin{array}{llll}0.416 & 2.54 & -46.34\end{array}$

$\begin{array}{lll}0.699 & 2.02 & -44.85\end{array}$ 


\begin{tabular}{|c|c|c|c|c|c|c|c|c|c|c|c|c|c|}
\hline \\
\hline \multicolumn{14}{|c|}{$\begin{array}{l}\boldsymbol{Z}=\mathbf{2 6}(\mathbf{F e}) \\
43 \quad 69 \quad 0.03\end{array}$} \\
\hline 44 & 70 & .12 & .00 & .02 & .00 & & 000 & & 03 & -0.02 & 45 & & -37.71 \\
\hline & 71 & 0.12 & 0.00 & -0.02 & 0.00 & & .000 & & 03 & -0.73 & 0.89 & & -32.49 \\
\hline 46 & 72 & .12 & 0.00 & 0.00 & 0.00 & & .000 & & 0.000 & -1 & 0.19 & & 9.79 \\
\hline 47 & 73 & .12 & 0.00 & 0.01 & 0.00 & & .000 & 0.006 & -0.001 & -2.28 & 0.65 & 11 & -24.25 \\
\hline 8 & 74 & 0.12 & 0.00 & 0.02 & -0.01 & 129 & 000 & 0.019 & .007 & -2.94 & -1.34 & 7.86 & -20.92 \\
\hline & 75 & 0.05 & .00 & 0.01 & 0.00 & & .000 & 11 & 01 & -3.74 & -2.67 & & -15.32 \\
\hline 50 & 76 & -0.01 & 0.00 & 0.00 & 0.00 & 1 & 000 & 00 & 00 & -4.62 & -3 & & 1.44 \\
\hline 1 & 77 & 0.04 & 0.00 & -0.01 & 0.01 & & 00 & & 9 & -4 & -2 & & 3.6 \\
\hline 52 & 78 & -0.01 & 0.00 & 0.00 & 0.00 & -0.011 & 0.000 & 00 & 0.000 & -3.49 & -2.53 & & 1.96 \\
\hline 53 & 79 & 0.01 & .00 & 0.00 & 0.00 & 11 & 000 & 00 & 00 & -2.75 & -1.97 & & .45 \\
\hline 54 & 80 & -0.01 & 0.00 & 0.00 & 0.00 & -0.011 & 000 & & & -1 & -1 & & .76 \\
\hline & 81 & 0.16 & 0.00 & -0.01 & 0.02 & & 00 & 24 & -0 & -2 & -0.32 & & 5.09 \\
\hline 6 & 82 & 0.17 & .00 & 0.00 & 0.03 & & 0 & & -0 & -1 & & & .61 \\
\hline 57 & 83 & 0.17 & 0.00 & 0.01 & 0.03 & & 0.000 & & -0 & -1.08 & & & 1.77 \\
\hline & 84 & & 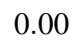 & 2 & 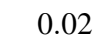 & & 00 & & 3 & -0 & & & .71 \\
\hline 59 & 85 & 0.27 & 0.00 & -0.04 & 0. & 4 & 000 & & 08 & -1 & 75 & & .75 \\
\hline & 86 & .29 & 0.00 & -0.03 & 0.02 & 16 & 00 & & 94 & -1 & 97 & & .9 \\
\hline & 87 & & .00 & -0.03 & 0. & & 00 & & -0 & -2 & & & 9 \\
\hline 02 & 88 & & 0.00 & -0.02 & 0.02 & & .000 & & -0 & -1.71 & & & 2.4 \\
\hline & 89 & & .00 & -0.01 & & & & & 20 & -1 & & & 17 \\
\hline 4 & 90 & & 0.00 & -0.01 & 0 . & & 00 & & -0 & -1 & & & .1 \\
\hline 6 & 91 & 0.30 & 0.00 & 0.01 & 0.01 & & 000 & & -0 & -1 & 72 & & \\
\hline 66 & 92 & & 0 & 0.0 & & & & & -0 & -1 & & & \\
\hline 67 & 93 & 0 & 0.00 & 0.0 & -0.01 & & .000 & 2 & 94 & -1 & 7 & & 0 \\
\hline 6 & 94 & 0 & .00 & 0.04 & -0.02 & & 000 & 12 & 99 & -1 & 70 & & .29 \\
\hline & 95 & & 0.0 & 0 & -0 . & & 00 & & & -1 & & & \\
\hline 70 & 96 & 0 & 0.00 & 0.06 & -0.02 & & 00 & -0 & 02 & -1 & 45 & & .54 \\
\hline 71 & 97 & & 0.0 & 0.06 & -0.02 & & 0.000 & -0 & & -1 & & & 7.3 \\
\hline 72 & 98 & 0.00 & 0.00 & 0.00 & 0.00 & 00 & 0.000 & 00 & & 0.37 & 7 & & .2 \\
\hline 1. & 99 & 0.02 & .00 & 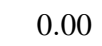 & 0 & & 00 & 0 & & -( & 8 & & 1 \\
\hline 4 & 00 & & 0.0 & & & & & & & & & & \\
\hline 75 & 101 & 0.05 & 0.00 & -0.01 & 0 & & 00 & & 01 & -1 & -0 & & 6.86 \\
\hline 76 & 102 & & 0.00 & -0.02 & 0. & & 0.000 & 0.030 & -0.007 & -2.14 & & & 215.7 \\
\hline 77 & 103 & 0 & 0.00 & -0.02 & 0.0 & & 0.000 & 0.030 & -0.007 & -3 & -2 & & 6.7 \\
\hline 78 & . & & 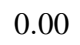 & 0 . & & & 00 & 1 & -0 & -3 & -2 & & $.1 J$ \\
\hline 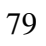 & 105 & & 0.0 & 0. & & & & & -0 & -4 & & & \\
\hline 80 & 106 & 0.00 & 0.00 & 0.00 & 0.00 & & 0.000 & 0.000 & 0.000 & -5.19 & -4.04 & 578.71 & 256.5 \\
\hline 81 & 107 & 0.01 & 0.00 & 0.01 & 0.00 & 0.011 & 0.000 & -0.012 & -0.000 & -6.50 & -5.21 & 575.64 & 267.6 \\
\hline
\end{tabular}

$\begin{array}{rr}1.82 & -39.93 \\ 1.49 & -37.55 \\ 0.92 & -32.36 \\ 0.20 & -29.70 \\ -0.64 & -24.19 \\ -1.30 & -20.86 \\ -2.66 & -15.31 \\ -3.37 & -11.46 \\ -2.97 & -3.62 \\ -2.53 & 1.92 \\ -1.97 & 10.41 \\ -1.27 & 16.73 \\ -0.22 & 26.17 \\ 0.33 & 32.80 \\ 0.70 & 42.00 \\ 1.12 & 48.96 \\ 1.99 & 59.06 \\ 2.20 & 66.24 \\ 1.69 & 75.35 \\ 1.82 & 82.85 \\ 1.67 & 92.69 \\ 1.86 & 100.62 \\ 1.73 & 110.83 \\ 1.86 & 119.07 \\ 1.77 & 129.63 \\ 1.80 & 138.12 \\ 1.55 & 148.85 \\ 1.64 & 157.71 \\ 1.34 & 168.67 \\ 1.06 & 177.49 \\ 0.58 & 188.55 \\ 0.14 & 197.50 \\ -0.54 & 208.63 \\ -1.07 & 217.77 \\ -1.96 & 228.95 \\ -2.36 & 238.49 \\ -3.44 & 249.72 \\ -4.04 & 259.30 \\ -5.19 & 270.70\end{array}$

$Z=27(\mathrm{Co})$

$\begin{array}{lllllllllll}17 & 44 & -0.23 & 0.00 & 0.01 & 0.05 & -0.237 & 0.000 & 0.006 & -0.045 & -0.88\end{array}$

$\begin{array}{lllllllllll}18 & 45 & -0.20 & 0.00 & 0.04 & 0.05 & -0.206 & 0.000 & -0.032 & -0.037 & -0.73\end{array}$

$\begin{array}{lll}0.66 & 284.06 & 49.96\end{array}$

$0.74 \quad 47.74$

$0.89 \quad 32.84$

$1.41 \quad 22.01$

$0.81 \quad 8.52$

$1.33-0.35$

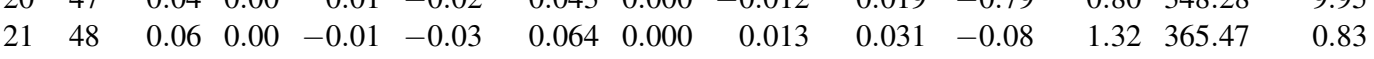

$\begin{array}{llllllllllllll}22 & 49 & 0.06 & 0.00 & 0.00 & -0.01 & 0.064 & 0.000 & 0.001 & 0.010 & -0.39 & 0.94 & 385.09 & -10.72\end{array}$

$\begin{array}{llllllllllllll}23 & 50 & 0.06 & 0.00 & 0.00 & 0.00 & 0.064 & 0.000 & 0.002 & 0.000 & 0.07 & 1.10 & 400.83 & -18.38\end{array}$

$\begin{array}{llllllllllllll}24 & 51 & 0.07 & 0.00 & -0.01 & 0.01 & 0.075 & 0.000 & 0.014 & -0.009 & -0.56 & 0.44 & 418.92 & -28.41\end{array}$

$\begin{array}{llllllllllllll}25 & 52 & 0.11 & 0.00 & 0.00 & 0.00 & 0.118 & 0.000 & 0.005 & 0.000 & -0.97 & 0.39 & 433.22 & -34.64\end{array}$

$\begin{array}{llllllllllllll}26 & 53 & 0.07 & 0.00 & -0.01 & 0.00 & 0.075 & 0.000 & 0.014 & 0.001 & -1.77 & -0.56 & 450.00 & -43.34\end{array}$

$0.93-11.69$

$1.09-19.16$

$0.44-29.00$

$0.38-35.08$

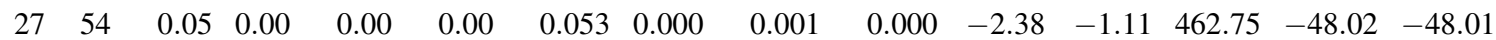

$\begin{array}{lllllllllllllll}28 & 55 & 0.04 & 0.00 & 0.01 & 0.00 & 0.043 & 0.000 & -0.011 & -0.000 & -3.12 & -1.73 & 477.17 & -54.37 & -54.03\end{array}$

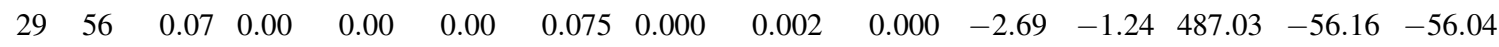

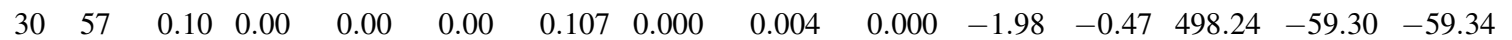

$\begin{array}{lllllllllllllll}31 & 58 & 0.09 & 0.00 & 0.00 & 0.00 & 0.096 & 0.000 & 0.003 & 0.000 & -1.04 & 0.26 & 506.70 & -59.69 & -59.85\end{array}$

$\begin{array}{llllll}0.000 & -1.04 & 0.26 & 506.70 & -59.69 & -59.85 \\ 0.000 & -0.46 & 1.01 & 516.75 & -61.67 & -62.23\end{array}$

$1.77 \quad 524.10-60.95-61.65$

$0.005-0.18$

$\begin{array}{rrrrrr}32 & 59 & 0.11 & 0.00 & 0.00 & 0.00 \\ 33 & 60 & 0.14 & 0.00 & 0.03 & -0.01\end{array}$ 


\begin{tabular}{|c|c|c|c|c|c|c|c|c|c|c|c|c|c|c|c|c|c|}
\hline \multicolumn{18}{|c|}{$Z=27($ Co $)$} \\
\hline 34 & 61 & 0.14 & 0.00 & 0.04 & -0.02 & 0.152 & 0.000 & -0.041 & 0.014 & 0.17 & 2.07 & 533.54 & -62.32 & -62.90 & 0.001 & 2.09 & -62.03 \\
\hline 35 & 62 & 0.12 & 0.00 & 0.04 & -0.01 & 0.130 & 0.000 & -0.043 & 0.005 & 0.74 & 2.32 & 540.43 & -61.13 & -61.43 & 0.020 & 2.33 & -60.83 \\
\hline 36 & 63 & 0.10 & 0.00 & 0.03 & -0.01 & 0.108 & 0.000 & -0.032 & 0.007 & 1.18 & 2.38 & 549.11 & -61.74 & -61.84 & 0.020 & 2.40 & -61.44 \\
\hline 37 & 64 & -0.10 & 0.00 & 0.02 & 0.01 & -0.105 & 0.000 & -0.019 & -0.007 & 1.41 & 2.60 & 555.12 & -59.68 & -59.79 & 0.020 & 2.60 & -59.39 \\
\hline 38 & 65 & 0.05 & 0.00 & 0.01 & 0.00 & 0.053 & 0.000 & -0.011 & -0.001 & 1.44 & 2.40 & 563.16 & -59.65 & -59.17 & 0.013 & 2.40 & -59.37 \\
\hline 39 & 66 & 0.03 & 0.00 & 0.01 & 0.00 & 0.032 & 0.000 & -0.012 & -0.000 & 1.33 & 2.46 & 568.49 & -56.90 & -56.11 & 0.252 & 2.46 & -56.63 \\
\hline 40 & 67 & 0.02 & 0.00 & 0.00 & 0.00 & 0.021 & 0.000 & 0.000 & 0.000 & 0.93 & 2.12 & 575.84 & -56.19 & -55.06 & 0.318 & 2.12 & -55.93 \\
\hline 41 & 68 & 0.03 & 0.00 & 0.01 & -0.01 & 0.032 & 0.000 & -0.012 & 0.009 & 1.07 & 2.18 & 580.39 & -52.67 & -51.35 & 0.318 & 2.18 & -52.42 \\
\hline 42 & 69 & 0.03 & 0.00 & 0.00 & 0.00 & 0.032 & 0.000 & 0.000 & 0.000 & 0.58 & 1.68 & 587.12 & -51.33 & -50.00 & 0.335 & 1.68 & -51.11 \\
\hline 43 & 70 & 0.04 & 0.00 & 0.00 & 0.00 & 0.043 & 0.000 & 0.001 & 0.000 & 0.45 & 1.46 & 591.23 & -47.37 & -45.64 & 0.838 & 1.46 & -47.17 \\
\hline 44 & 71 & 0.05 & 0.00 & 0.00 & 0.00 & 0.053 & 0.000 & 0.001 & 0.000 & -0.06 & 0.89 & 597.32 & -45.38 & -43.87 & 0.838 & 0.89 & -45.21 \\
\hline 45 & 72 & 0.12 & 0.00 & -0.01 & 0.00 & 0.128 & 0.000 & 0.018 & 0.002 & -1.00 & 0.64 & 600.79 & -40.78 & & & 0.65 & -40.62 \\
\hline 46 & 73 & 0.12 & 0.00 & 0.00 & 0.00 & 0.129 & 0.000 & 0.006 & 0.000 & -1.71 & -0.06 & 606.34 & -38.26 & & & -0.05 & -38.13 \\
\hline 47 & 74 & 0.12 & 0.00 & 0.01 & 0.00 & 0.129 & 0.000 & -0.006 & -0.001 & -2.62 & -0.92 & 609.80 & -33.65 & & & -0.91 & -33.54 \\
\hline 48 & 75 & 0.04 & 0.00 & 0.01 & 0.00 & 0.043 & 0.000 & -0.011 & -0.000 & -3.09 & -2.06 & 615.16 & -30.93 & & & -2.06 & -30.86 \\
\hline 49 & 76 & 0.04 & 0.00 & 0.01 & 0.00 & 0.043 & 0.000 & -0.011 & -0.000 & -4.32 & -3.09 & 618.22 & -25.92 & & & -3.09 & -25.86 \\
\hline 50 & 77 & 0.02 & 0.00 & 0.00 & 0.00 & 0.021 & 0.000 & 0.000 & 0.000 & -5.14 & -3.80 & 622.56 & -22.19 & & & -3.80 & -22.16 \\
\hline 51 & 78 & 0.04 & 0.00 & 0.01 & 0.00 & 0.043 & 0.000 & -0.011 & -0.000 & -4.51 & -3.33 & 623.58 & -15.14 & & & -3.32 & -15.12 \\
\hline 52 & 79 & 0.03 & 0.00 & 0.00 & 0.00 & 0.032 & 0.000 & 0.000 & 0.000 & -3.91 & -2.81 & 626.16 & -9.64 & & & -2.81 & -9.64 \\
\hline 53 & 80 & 0.04 & 0.00 & 0.01 & 0.00 & 0.043 & 0.000 & -0.011 & -0.000 & -3.17 & -2.19 & 626.52 & -1.94 & & & -2.18 & -1.93 \\
\hline 54 & 81 & 0.04 & 0.00 & 0.01 & 0.00 & 0.043 & 0.000 & -0.011 & -0.000 & -2.35 & -1.52 & 628.44 & 4.21 & & & -1.52 & 4.22 \\
\hline 55 & 82 & 0.04 & 0.00 & 0.01 & 0.00 & 0.043 & 0.000 & -0.011 & -0.000 & -1.60 & -0.97 & 628.40 & 12.32 & & & -0.97 & 12.33 \\
\hline 56 & 83 & 0.04 & 0.00 & 0.01 & 0.00 & 0.043 & 0.000 & -0.011 & -0.000 & -0.84 & -0.32 & 629.85 & 18.94 & & & -0.31 & 18.96 \\
\hline 57 & 84 & 0.12 & 0.00 & -0.01 & 0.02 & 0.128 & 0.000 & 0.019 & -0.018 & -0.58 & 0.48 & 629.13 & 27.74 & & & 0.56 & 27.85 \\
\hline 58 & 85 & 0.14 & 0.00 & 0.00 & 0.02 & 0.151 & 0.000 & 0.009 & -0.020 & -0.21 & 0.93 & 630.33 & 34.61 & & & 1.03 & 34.74 \\
\hline 59 & 86 & 0.14 & 0.00 & 0.01 & 0.02 & 0.151 & 0.000 & -0.002 & -0.021 & 0.28 & 1.30 & 629.62 & 43.39 & & & 1.40 & 43.56 \\
\hline 60 & 87 & 0.29 & 0.00 & -0.02 & 0.02 & 0.317 & 0.000 & 0.064 & -0.007 & -1.54 & 2.13 & 630.03 & 51.05 & & & 2. & 31 \\
\hline 61 & 88 & 0.30 & 0.00 & -0.02 & 0.02 & 0.328 & 0.000 & 0.067 & -0.007 & -1.87 & 2.13 & 629.29 & 59.86 & & & 2.26 & 60.13 \\
\hline 62 & 89 & 0.30 & 0.00 & -0.01 & 0.01 & 0.329 & 0.000 & 0.053 & -0.001 & -1.58 & 2.32 & 629.95 & 67.27 & & & 2.39 & 67.53 \\
\hline 63 & 90 & 0.31 & 0.00 & 0.00 & 0.02 & 0.340 & 0.000 & 0.045 & -0.014 & -1.76 & 1.73 & 629.43 & 75.86 & & & 1.81 & 76.18 \\
\hline 64 & 91 & 0.30 & 0.00 & 0.01 & 0.01 & 0.330 & 0.000 & 0.029 & -0.009 & -1.30 & 1.90 & 629.73 & 83.64 & & & 1.93 & 83.96 \\
\hline 65 & 92 & 0.30 & 0.00 & 0.02 & 0.00 & 0.330 & 0.000 & 0.015 & -0.002 & -1.39 & 1.81 & 628.38 & 93.06 & & & 1.78 & 93.40 \\
\hline 66 & 93 & 0.30 & 0.00 & 0.03 & 0.00 & 0.331 & 0.000 & 0.003 & -0.006 & -1.28 & 1.84 & 628.46 & 101.05 & & & 1.85 & 101.50 \\
\hline 67 & 94 & 0.30 & 0.00 & 0.04 & -0.01 & 0.332 & 0.000 & -0.011 & -0.000 & -1.54 & 1.62 & 626.90 & 110.68 & & & 1.64 & 111.22 \\
\hline 68 & 95 & 0.30 & 0.00 & 0.06 & -0.02 & 0.333 & 0.000 & -0.036 & 0.002 & -1.69 & 1.41 & 626.90 & 118.75 & & & 1.59 & 119.55 \\
\hline 69 & 96 & 0.31 & 0.00 & 0.07 & -0.02 & 0.346 & 0.000 & -0.046 & -0.002 & -2.10 & 1.17 & 625.05 & 128.67 & & & 1.38 & 129.61 \\
\hline 70 & 97 & 0.30 & 0.00 & 0.07 & -0.02 & 0.334 & 0.000 & -0.048 & -0.002 & -1.85 & 1.28 & 624.41 & 137.38 & & & 1.54 & 38.47 \\
\hline 71 & 98 & 0.30 & 0.00 & 0.07 & -0.02 & 0.334 & 0.000 & -0.048 & -0.002 & -2.10 & 1.00 & 622.32 & 147.55 & & & 1.23 & 148.74 \\
\hline 72 & 99 & 0.03 & 0.00 & 0.00 & 0.00 & 0.032 & 0.000 & 0.000 & 0.000 & 0.27 & 0.94 & 621.55 & 156.39 & & & 0.94 & 157.48 \\
\hline 73 & 100 & 0.04 & 0.00 & 0.00 & 0.00 & 0.043 & 0.000 & 0.001 & 0.000 & -0.31 & 0.35 & 619.48 & 166.53 & & & 0.35 & 167.76 \\
\hline 74 & 101 & 0.05 & 0.00 & -0.01 & 0.00 & 0.053 & 0.000 & 0.013 & 0.001 & -0.81 & -0.03 & 618.76 & 175.32 & & & -0.01 & 176.71 \\
\hline 75 & 102 & 0.05 & 0.00 & -0.01 & 0.00 & 0.053 & 0.000 & 0.013 & 0.001 & -1.58 & -0.72 & 616.53 & 185.62 & & & -0.71 & 187.17 \\
\hline 76 & 103 & 0.05 & 0.00 & 0.00 & 0.00 & 0.053 & 0.000 & 0.001 & 0.000 & -2.12 & -1.20 & 615.64 & 194.59 & & & -1.20 & 196.29 \\
\hline 77 & 104 & 0.05 & 0.00 & 0.00 & 0.00 & 0.053 & 0.000 & 0.001 & 0.000 & -3.12 & -2.12 & 613.39 & 204.91 & & & -2.12 & 206.79 \\
\hline 78 & 105 & 0.05 & 0.00 & 0.01 & 0.00 & 0.053 & 0.000 & -0.011 & -0.001 & -3.77 & -2.70 & 612.34 & 214.02 & & & -2.69 & 216.10 \\
\hline 79 & 106 & 0.05 & 0.00 & 0.01 & 0.00 & 0.053 & 0.000 & -0.011 & -0.001 & -4.98 & -3.83 & 610.06 & 224.37 & & & -3.82 & 226.65 \\
\hline 80 & 107 & 0.04 & 0.00 & 0.01 & 0.00 & 0.043 & 0.000 & -0.011 & -0.000 & -5.67 & -4.45 & 608.82 & 233.69 & & & -4.44 & 236.17 \\
\hline 81 & 108 & 0.02 & 0.00 & 0.0 & 0.00 & 0.021 & 0.000 & -0.012 & -0.000 & -7.03 & -5.69 & 606.43 & 244.15 & & & -5.68 & 246.85 \\
\hline 82 & 109 & 0.01 & 0.00 & 0.00 & 0.00 & 0.011 & 0.000 & 0.000 & 0.000 & -7.66 & -6.24 & 604.88 & 253.77 & & & -6.24 & 256.69 \\
\hline \multicolumn{18}{|c|}{$Z=28(\mathrm{Ni})$} \\
\hline 18 & 46 & -0.26 & 0.00 & 0.02 & 0.05 & -0.267 & 0.000 & -0.000 & -0.041 & -1.7 & 0.51 & 304.95 & 44.43 & & & 0.61 & 42.34 \\
\hline 19 & 47 & -0.05 & 0.00 & 0.00 & -0.04 & -0.052 & 0.000 & 0.002 & 0.039 & -0.74 & 0.13 & 325.34 & 32.10 & & & & 30.27 \\
\hline 20 & 48 & 0.00 & 0.00 & 0.00 & -0.01 & 0.000 & 0.000 & 0.000 & 0.010 & -1.61 & 0.01 & 348.01 & 17.51 & & & 0.01 & 15.88 \\
\hline 21 & 49 & -0.04 & 0.00 & -0.01 & 0.01 & -0.042 & 0.000 & 0.012 & -0.010 & -0.72 & 0.56 & 365.47 & 8.12 & & & 0.56 & 6.74 \\
\hline 22 & 50 & -0.01 & 0.00 & 0.00 & 0.00 & -0.011 & 0.000 & 0.000 & 0.000 & -1.12 & 0.28 & 386.29 & -4.63 & & & 0.27 & -5.78 \\
\hline 23 & 51 & 0.02 & 0.00 & 0.00 & 0.01 & 0 & 0.000 & 0.000 & -0.010 & -0.52 & 0.65 & 402.11 & -12.38 & & & 0.65 & -13.32 \\
\hline 24 & 52 & 0.01 & 0.00 & 0.00 & 0.00 & 0.011 & 0.000 & 0.000 & 0.000 & -1.09 & 0.03 & 421.44 & -23.64 & & & 0.03 & -24.38 \\
\hline
\end{tabular}




\begin{tabular}{|c|c|c|c|c|c|c|c|c|c|c|c|c|c|c|c|c|c|}
\hline \multicolumn{18}{|c|}{$Z=28(\mathrm{Ni})$} \\
\hline 25 & 53 & -0.02 & 0.00 & 0.00 & 0.00 & -0.021 & 0.000 & 0.000 & 0.000 & -1.28 & -0.20 & 436.22 & -30.35 & & & -0.20 & -30.92 \\
\hline 26 & 54 & 0.00 & 0.00 & 0.00 & 0.00 & 0.000 & 0.000 & 0.000 & 0.000 & -2.32 & -1.12 & 454.18 & -40.24 & -39.21 & 0.050 & -1.12 & -40.66 \\
\hline 27 & 55 & 0.03 & 0.00 & 0.00 & 0.00 & 0.032 & 0.000 & 0.000 & 0.000 & -2.98 & -1.55 & 467.68 & -45.66 & -45.34 & 0.011 & -1.56 & -45.95 \\
\hline 28 & 56 & 0.00 & 0.00 & 0.00 & 0.00 & 0.000 & 0.000 & 0.000 & 0.000 & -4.00 & -2.47 & 484.13 & -54.04 & -53.90 & 0.011 & -2.47 & -54.21 \\
\hline 29 & 57 & 0.02 & 0.00 & 0.00 & 0.00 & 0.021 & 0.000 & 0.000 & 0.000 & -3.24 & -1.85 & 494.14 & -55.98 & -56.08 & 0.002 & -1.86 & -56.05 \\
\hline 30 & 58 & 0.00 & 0.00 & 0.00 & 0.00 & 0.000 & 0.000 & 0.000 & 0.000 & -2.37 & -1.29 & 506.72 & -60.49 & -60.23 & 0.001 & -1.30 & -60.47 \\
\hline 31 & 59 & 0.00 & 0.00 & 0.00 & 0.00 & 0.000 & 0.000 & 0.000 & 0.000 & -1.44 & -0.49 & 515.37 & -61.07 & -61.16 & 0.001 & -0.50 & -60.98 \\
\hline 32 & 60 & 0.00 & 0.00 & 0.00 & 0.00 & 0.000 & 0.000 & 0.000 & 0.000 & -0.56 & 0.12 & 526.70 & -64.33 & -64.47 & 0.001 & 0.11 & -64.18 \\
\hline 33 & 61 & 0.10 & 0.00 & 0.01 & 0.00 & 0.107 & 0.000 & -0.008 & -0.001 & -0.14 & 1.20 & 533.98 & -63.54 & -64.22 & 0.001 & 1.20 & -6 \\
\hline 34 & 62 & 0.10 & 0.00 & 0.02 & -0.01 & 0.107 & 0.000 & -0.020 & 0.008 & 0.27 & 1.56 & 544.47 & -65.95 & -66.75 & 0.001 & 1.56 & -65.71 \\
\hline 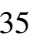 & 63 & 0.10 & 0.00 & 0.02 & -0.01 & 0.107 & 0.000 & -0.020 & 0.008 & 0.62 & 1.87 & 551.52 & -64.93 & -65.51 & 0.001 & 1.88 & -64.67 \\
\hline 36 & 64 & -0.09 & 0.00 & 0.01 & 0.00 & -0.094 & 0.000 & -0.008 & 0.001 & 0.77 & 1.71 & 561.51 & -66.85 & -67.10 & 01 & 1.71 & -6 \\
\hline 37 & 65 & -0.08 & 0.00 & 0.01 & 0.00 & -0.084 & 0.000 & -0.009 & 0.001 & 0.92 & 1.90 & & & & & 1.90 & \\
\hline 38 & 66 & 0.00 & 0.00 & 0.00 & 0.00 & 0.000 & 0.000 & 0.000 & 0.000 & 0.73 & 1.84 & 576.71 & -65.91 & -66.01 & 0.001 & 1.84 & -65.62 \\
\hline 39 & 67 & 0.00 & 0.00 & 0.00 & 0.00 & 0.000 & 0.000 & 0.000 & 0.000 & 0.51 & 1.78 & 582.37 & -63.49 & -63.74 & 0.003 & 1.78 & -63.21 \\
\hline 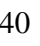 & 68 & 0.00 & 0.00 & 0.00 & 0.00 & 0.000 & 0.000 & 0.000 & 0.000 & 0.09 & 1.43 & .74 & -63.80 & -63.46 & 3 & 1.43 & 52 \\
\hline 1. & 69 & -0.02 & 0.00 & -0.01 & 0.00 & -0.021 & 0.000 & 0.012 & -0.000 & 0.28 & 1.51 & 595.47 & -60.45 & -59.98 & 04 & 1.51 & -60.19 \\
\hline 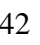 & 70 & 0.00 & 0.00 & 0.00 & 0.00 & 0.000 & 0.000 & 0.000 & 0.000 & -0.21 & 1.04 & & & & & 1.04 & \\
\hline 43 & 71 & 0.00 & 0.01 & 0.00 & 0.01 & 0.000 & -0.013 & 0.000 & -0.010 & -0.22 & 0.94 & & & & & 0.95 & \\
\hline 44 & 72 & 0.00 & 0.00 & 0.00 & 0.00 & 0.000 & 0.000 & 0.000 & 0.000 & -0.67 & 0.40 & 614.35 & -55.12 & -53.94 & & 0.40 & -54.91 \\
\hline 45 & 73 & 0.00 & 0.00 & 0.00 & 0.00 & 0.000 & 0.000 & 0.000 & 0.000 & -1.07 & -0.09 & 618.23 & -50.94 & & & -0.09 & -5 \\
\hline 40 & 74 & -0.01 & 0.00 & 0.00 & 0.00 & -0.011 & 0.000 & 0.000 & 0.000 & -1.77 & -0.80 & 624.72 & -49.35 & & & -0.80 & -49.19 \\
\hline 47 & 75 & 0.02 & 0.00 & 0.00 & 0.01 & 0.021 & 0.000 & 0.000 & -0.010 & -2.66 & -1.70 & 628.39 & & & & -1.69 & -44.80 \\
\hline 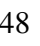 & 76 & 0.00 & 0.00 & 0.00 & 0.00 & 0.000 & 0.000 & 0.000 & 0.000 & -3.70 & -2.56 & 634.37 & -42 & & & -2.56 & -42.74 \\
\hline 49 & 77 & 0.03 & 0.00 & 0.01 & 0.00 & 0.032 & 0.000 & -0.012 & -0.000 & -4.89 & -3.56 & 637.56 & -37.97 & & & -3.56 & -37.87 \\
\hline 50 & 78 & 0.00 & 0.00 & 0.00 & 0.00 & 0.000 & 0.000 & 0.000 & 0.000 & -5.89 & -4.43 & 642.95 & -35.29 & & & -4.43 & -35.21 \\
\hline 51 & 79 & -0.02 & 0.00 & 0.00 & 0.00 & -0.021 & 0.000 & 0.000 & 0.000 & -5.06 & -3.84 & 643.99 & -28.26 & & & -3.84 & -28.20 \\
\hline 52 & 80 & 0.00 & 0.00 & 0.00 & 0.00 & 0.000 & 0.000 & 0.000 & 0.000 & -4.47 & -3.28 & .38 & .58 & & & -3.28 & -2 \\
\hline 53 & 81 & 0.00 & 0.00 & 0.00 & 0.00 & 0.000 & 0.000 & 0.000 & 0.000 & -3.57 & -2.52 & 647.74 & -15.87 & & & -2.52 & -15.83 \\
\hline 54 & 82 & 0.00 & 0.00 & 0.00 & 0.00 & 0.000 & 0.000 & 0.000 & 0.000 & -2.69 & -1.76 & 650.40 & -10.45 & & & -1.76 & .43 \\
\hline 55 & 83 & 0.01 & 0.00 & 0.00 & 0.00 & 0.011 & 0.000 & 0.000 & 0.000 & -1.85 & -1.09 & 650.37 & -2.35 & & & -1.09 & -2.33 \\
\hline 56 & 84 & 0.00 & 0.00 & 0.00 & 0.00 & 0.000 & 0.000 & 0.000 & 0.000 & -1.07 & -0.37 & 652.57 & 3.52 & & & -0.37 & 3.55 \\
\hline 57 & 85 & -0.12 & 0.00 & -0.03 & 0.01 & -0.124 & 0.000 & 0.040 & -0.014 & -0.70 & 0.44 & 651.94 & 12.22 & & & 0.55 & 12.36 \\
\hline 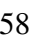 & 86 & 0.00 & 0.00 & 0.00 & 0.00 & 0.000 & 0.000 & 0.000 & 0.000 & 0.46 & 0.80 & 654.04 & 18.19 & & & 0.79 & 18.23 \\
\hline 59 & 87 & 0.27 & 0.00 & -0.02 & 0.03 & 0.294 & 0.000 & 0.060 & -0.019 & -1.38 & 1.80 & 652.79 & 27.50 & & & 2.03 & 27.80 \\
\hline 60 & 88 & 0.30 & 0.00 & -0.02 & 0.03 & 0.328 & 0.000 & 0.068 & -0.017 & -1.82 & 2.10 & 654.51 & 33.87 & & & 2.37 & 34.22 \\
\hline 61 & 89 & 0.30 & 0.00 & -0.02 & 0.03 & 0.328 & 0.000 & 68 & -0.017 & -1.98 & 2.00 & 3.97 & 42.47 & & & 2.24 & .83 \\
\hline 62 & 90 & 0.30 & 0.00 & -0.01 & 0.03 & 0.328 & 0.000 & 0.056 & -0.021 & -1.72 & 2.10 & .47 & 05 & & & 2.35 & 49.45 \\
\hline 63 & 91 & 0.31 & 0.00 & 0.00 & 0.03 & 0.340 & 0.000 & 0.047 & -0.024 & -1.93 & 1.56 & 654.99 & 57.60 & & & 1.79 & 58.02 \\
\hline 64 & 92 & 0.31 & 0.00 & 0.01 & 0.03 & 0.341 & 0.000 & 0.034 & -0.027 & -1.68 & 1.66 & 656.10 & 64.55 & & & 1.92 & 65.07 \\
\hline 65 & 93 & 31 & 0.00 & 0.02 & 0.02 & 0.342 & .000 & 0.021 & -0.022 & -1.73 & 1.65 & 654.73 & 99 & & & 1.79 & 4.43 \\
\hline 66 & 94 & 0.30 & 0.00 & 0.03 & 0.01 & 0.331 & .000 & & -0.016 & -1.42 & 1.76 & 5.47 & & & & 1.86 & 31.80 \\
\hline 67 & 95 & 0.30 & 0.00 & 0.05 & -0.01 & 0.333 & 0.000 & -0.023 & -0.004 & -1.72 & 1.56 & 653.96 & 90.91 & & & 1.65 & 91.45 \\
\hline 68 & 96 & 0.30 & 0.00 & 0.06 & -0.02 & 0.333 & 0.000 & -0.036 & 0.002 & -1.83 & 1.41 & 654.61 & 98.33 & & & 1.61 & 99.07 \\
\hline 69 & 97 & 0.31 & 0.00 & 0.07 & -0.02 & 0.346 & 0.000 & -0.046 & -0.002 & -2.30 & 1.13 & 652.86 & 108.15 & & & 1.37 & 109.01 \\
\hline 10 & 98 & 0.31 & 0.00 & م & -0.02 & 0.347 & .000 & -0.058 & -0.006 & -2.27 & 1.06 & 653.09 & .99 & & & 1.42 & 7.07 \\
\hline 71 & 99 & 0.30 & 0.00 & 0.07 & -0.01 & 0.335 & 0.000 & -0.047 & -0.011 & -2.28 & 0.87 & 650.96 & & & & 1.11 & 127.26 \\
\hline 72 & 100 & 0.00 & 0.00 & 0.00 & 0.00 & 0.000 & 0.000 & 0.000 & 0.000 & 0.15 & 0.87 & 650.81 & 134.42 & & & 0.87 & 135.35 \\
\hline 73 & 101 & 0.01 & 0.00 & م & 0.00 & 0.011 & 0.000 & 0.000 & 0.000 & -0.38 & 0.38 & 648.69 & 144.61 & & & 0.38 & 145.67 \\
\hline 74 & 102 & -0.01 & 0.00 & 0.00 & 0.00 & -0.011 & 0.000 & 0.000 & 0.000 & -0.89 & -0.08 & 648.70 & 152.67 & & & -0.08 & 153.87 \\
\hline 13 & 103 & 0.01 & 0.00 & 0 & 0.00 & 0.011 & .000 & 0.000 & 0.000 & -1.61 & -0.76 & 646.51 & 162.93 & & & .76 & 164.27 \\
\hline 76 & 104 & 0.01 & 0.00 & 0.00 & 0.00 & 0.011 & 0.000 & 0.000 & 0.000 & -2.20 & -1.28 & 646.30 & & & & -1.28 & 172.70 \\
\hline 77 & 105 & 0.05 & 0.00 & -0.01 & 0.00 & 0.053 & 0.000 & 0.013 & 0.001 & -3.27 & -2.23 & 644.12 & 181.46 & & & -2.22 & 183.12 \\
\hline 78 & 106 & 0.04 & 0.00 & 0.00 & 0.00 & & 0.000 & 0.001 & 0.000 & -3.92 & -2.84 & 643.73 & 189.92 & & & -2.84 & 191.74 \\
\hline 79 & 107 & -0.03 & 0.00 & 0.00 & -0.01 & -0.032 & 0.000 & 0.001 & 0.010 & -5.20 & -4.12 & 641.65 & 200.08 & & & -4.09 & 202.12 \\
\hline 80 & 108 & 0.00 & 0.00 & 0.00 & 0.00 & 0.000 & 0.000 & 0.000 & 0.000 & -6.02 & -4.80 & 641.07 & 208.73 & & & -4.80 & 210.91 \\
\hline 81 & 109 & 0.02 & 0.00 & 0.01 & -0.01 & 0.021 & 0.000 & -0.012 & 0.010 & -7.46 & -6.11 & 638.78 & 219.08 & & & -6.06 & 221.52 \\
\hline
\end{tabular}




\section{$Z=28(\mathrm{Ni})$} $\begin{array}{lllll}83 & 111 & -0.01 & 0.00 & -0.01\end{array}$

$\begin{array}{lllllllll}0.00 & 0.000 & 0.000 & 0.000 & 0.000 & -8.20 & -6.76 & 637.93 & 228.01\end{array}$

$\begin{array}{lllllllll}0.00 & -0.010 & 0.000 & 0.012 & -0.000 & -7.67 & -6.38 & 633.74 & 240.27\end{array}$

$-6.76 \quad 230.60$

$-6.37 \quad 243.10$

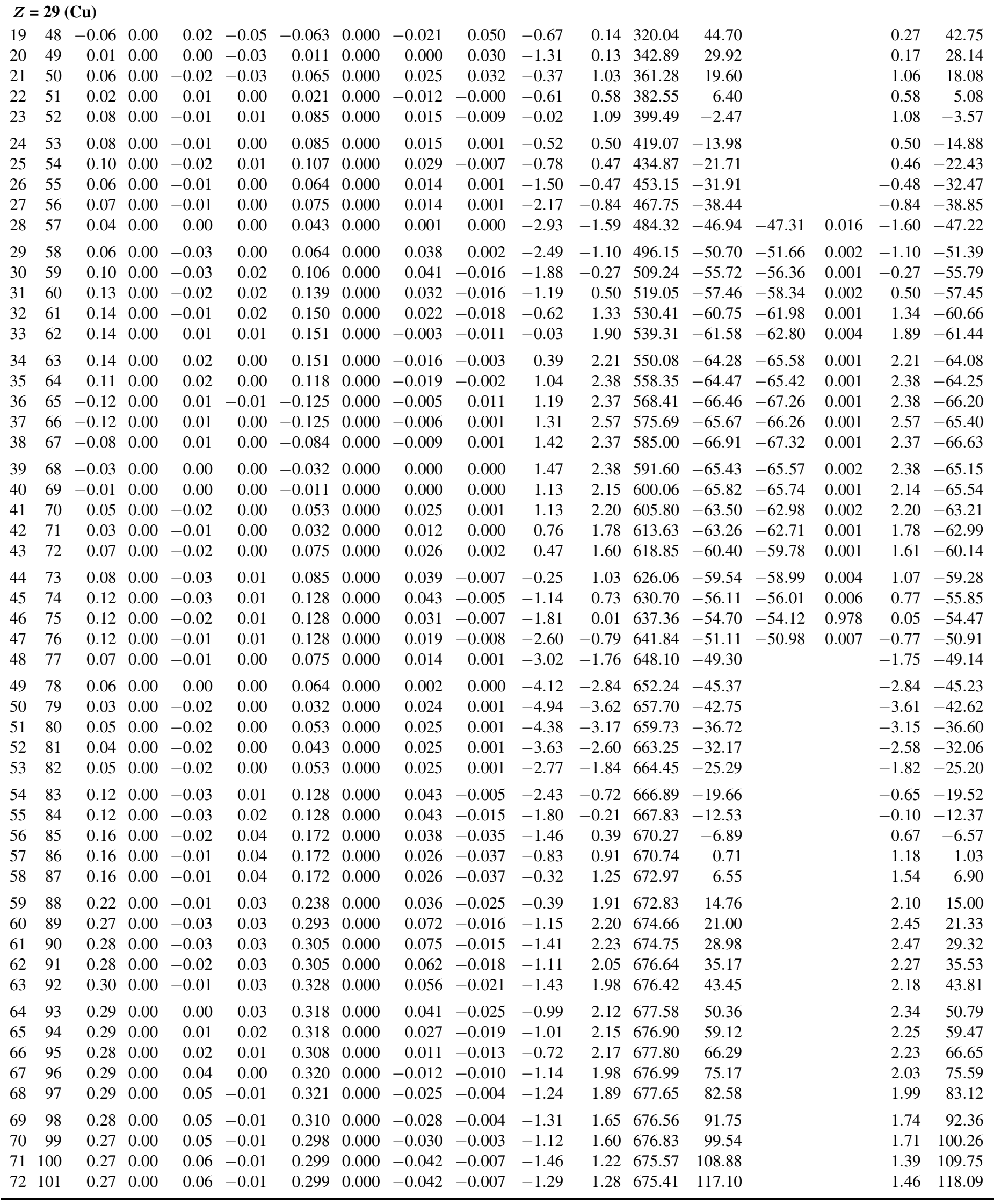




\begin{tabular}{rrrrrrrrrrrrrr}
\multicolumn{2}{c}{$\boldsymbol{Z}=\mathbf{2 9}(\mathbf{C u})$} \\
73 & 102 & 0.05 & 0.00 & -0.02 & 0.00 & 0.053 & 0.000 & 0.025 & 0.001 & 0.20 & 0.66 & 674.09 & 126.49 \\
74 & 103 & 0.05 & 0.00 & -0.02 & 0.00 & 0.053 & 0.000 & 0.025 & 0.001 & -0.40 & 0.31 & 674.05 & 134.61 \\
75 & 104 & 0.09 & 0.00 & -0.03 & 0.00 & 0.096 & 0.000 & 0.040 & 0.004 & -1.44 & -0.41 & 672.54 & 144.19 \\
76 & 105 & 0.11 & 0.00 & -0.02 & 0.02 & 0.117 & 0.000 & 0.030 & -0.017 & -2.21 & -1.11 & 672.56 & 152.24 \\
77 & 106 & 0.09 & 0.00 & -0.02 & 0.01 & 0.096 & 0.000 & 0.028 & -0.008 & -3.12 & -1.99 & 670.94 & 161.93 \\
78 & 107 & 0.06 & 0.00 & -0.01 & 0.01 & 0.064 & 0.000 & 0.014 & -0.009 & -3.63 & -2.59 & 670.58 & 170.36 \\
79 & 108 & 0.05 & 0.00 & -0.01 & 0.00 & 0.053 & 0.000 & 0.013 & 0.001 & -4.91 & -3.77 & 669.01 & 180.00 \\
80 & 109 & 0.04 & 0.00 & -0.01 & 0.00 & 0.043 & 0.000 & 0.013 & 0.001 & -5.67 & -4.47 & 668.50 & 188.59 \\
81 & 110 & 0.03 & 0.00 & 0.00 & 0.00 & 0.032 & 0.000 & 0.000 & 0.000 & -7.15 & -5.84 & 666.88 & 198.28 \\
82 & 111 & 0.01 & 0.00 & -0.01 & 0.00 & 0.011 & 0.000 & 0.012 & 0.000 & -7.88 & -6.49 & 666.06 & 207.17 \\
83 & 112 & 0.03 & 0.00 & -0.02 & 0.00 & 0.032 & 0.000 & 0.024 & 0.001 & -7.47 & -6.22 & 662.57 & 218.73
\end{tabular}

$\begin{array}{ll}0.70 & 127.45\end{array}$

$\begin{array}{ll}0.35 & 135.69\end{array}$

$-0.30 \quad 145.46$

$-0.95 \quad 153.70$

$-1.92 \quad 163.44$

$\begin{array}{ll}-2.54 & 172.00\end{array}$

$-3.76 \quad 181.77$

$-4.46 \quad 190.53$

$\begin{array}{ll}-5.84 & 200.39\end{array}$

\begin{tabular}{ll}
$-6.48 \quad 209.48$ \\
\hline
\end{tabular}

$-6.17 \quad 221.29$

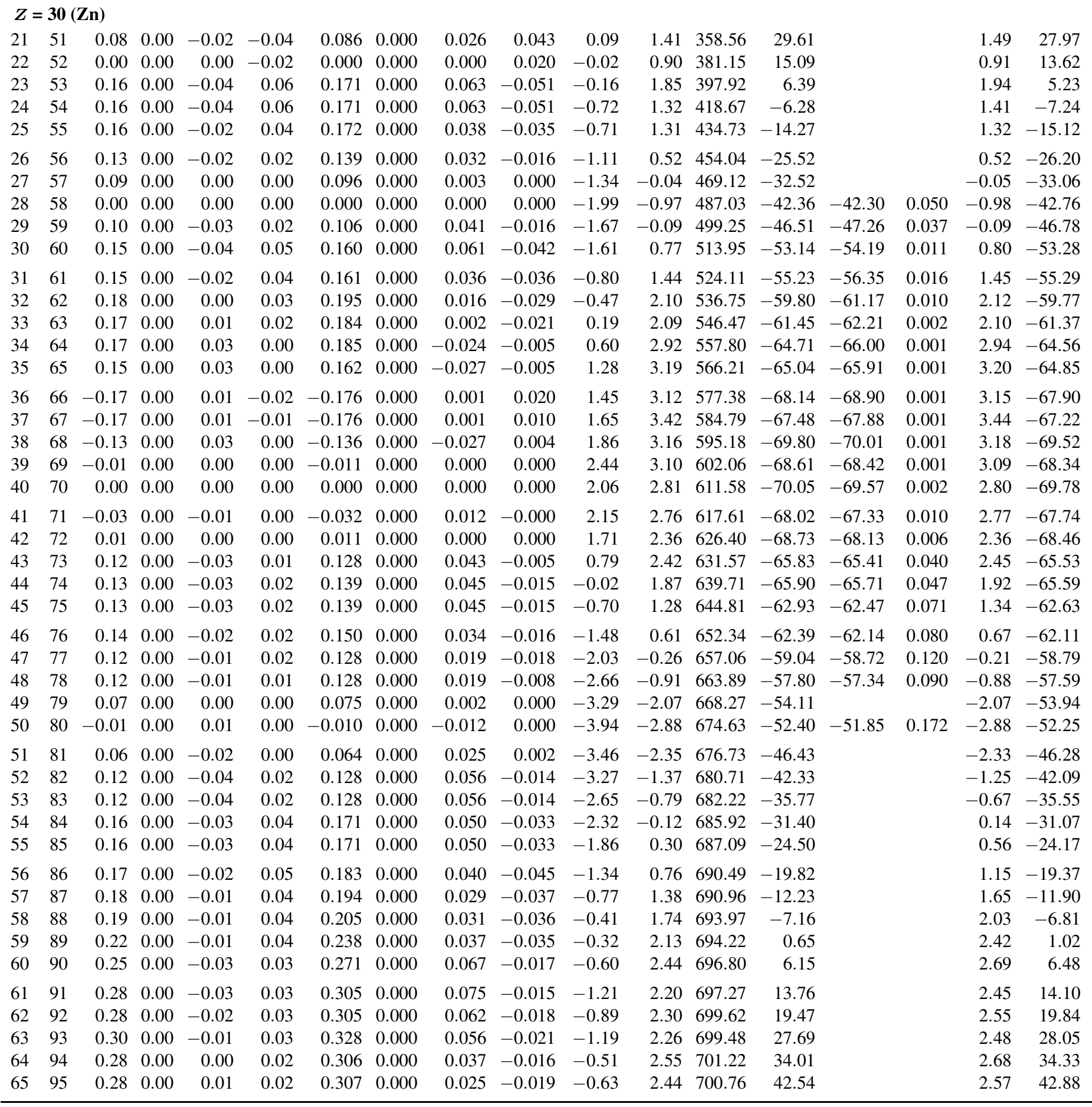




\section{$Z=\mathbf{3 0}(\mathrm{Zn})$}

$\begin{array}{rrrrrrrrrrrrrr}66 & 96 & 0.28 & 0.00 & 0.03 & 0.00 & 0.308 & 0.000 & -0.002 & -0.006 & -0.46 & 2.51 & 702.34 & 49.03 \\ 67 & 97 & 0.29 & 0.00 & 0.04 & 0.00 & 0.320 & 0.000 & -0.012 & -0.010 & -0.93 & 2.23 & 701.70 & 57.75 \\ 68 & 98 & 0.28 & 0.00 & 0.05 & -0.01 & 0.310 & 0.000 & -0.028 & -0.004 & -0.91 & 2.12 & 703.08 & 64.44 \\ 69 & 99 & 0.30 & 0.00 & 0.08 & -0.03 & 0.335 & 0.000 & -0.062 & 0.004 & -1.75 & 1.64 & 702.30 & 73.29 \\ 70 & 100 & 0.28 & 0.00 & 0.07 & -0.02 & 0.311 & 0.000 & -0.053 & -0.001 & -1.29 & 1.68 & 703.17 & 80.49 \\ 71 & 101 & 0.27 & 0.00 & 0.06 & -0.01 & 0.299 & 0.000 & -0.042 & -0.007 & -1.33 & 1.49 & 701.79 & 89.94 \\ 72 & 102 & 0.25 & 0.00 & 0.06 & -0.01 & 0.276 & 0.000 & -0.047 & -0.006 & -0.91 & 1.42 & 702.43 & 97.37 \\ 73 & 103 & 0.21 & 0.00 & 0.03 & 0.01 & 0.229 & 0.000 & -0.016 & -0.016 & -0.47 & 1.27 & 700.70 & 107.18 \\ 74 & 104 & 0.11 & 0.00 & -0.03 & 0.01 & 0.117 & 0.000 & 0.042 & -0.005 & -0.13 & 0.90 & 701.33 & 114.61 \\ 75 & 105 & 0.11 & 0.00 & -0.02 & 0.01 & 0.117 & 0.000 & 0.030 & -0.007 & -0.90 & 0.19 & 699.87 & 124.15 \\ 76 & 106 & 0.11 & 0.00 & -0.02 & 0.02 & 0.117 & 0.000 & 0.030 & -0.017 & -1.62 & -0.49 & 700.51 & 131.58 \\ 77 & 107 & 0.11 & 0.00 & -0.02 & 0.02 & 0.117 & 0.000 & 0.030 & -0.017 & -2.64 & -1.43 & 699.01 & 141.15 \\ 78 & 108 & 0.11 & 0.00 & -0.01 & 0.01 & 0.118 & 0.000 & 0.018 & -0.008 & -3.25 & -1.95 & 699.20 & 149.03 \\ 79 & 109 & 0.09 & 0.00 & 0.00 & 0.01 & 0.096 & 0.000 & 0.004 & -0.010 & -4.34 & -3.13 & 697.67 & 158.63 \\ 80 & 110 & 0.05 & 0.00 & 0.01 & 0.00 & 0.053 & 0.000 & -0.011 & -0.001 & -5.03 & -3.89 & 697.83 & 166.55 \\ 81 & 111 & 0.04 & 0.00 & 0.01 & 0.00 & 0.043 & 0.000 & -0.011 & -0.000 & -6.59 & -5.36 & 696.34 & 176.10 \\ 82 & 112 & 0.00 & 0.00 & 0.00 & 0.00 & 0.000 & 0.000 & 0.000 & 0.000 & -7.38 & -6.07 & 696.20 & 184.32 \\ 83 & 113 & 0.03 & 0.00 & -0.01 & 0.00 & 0.032 & 0.000 & 0.012 & 0.000 & -6.85 & -5.66 & 692.60 & 195.99\end{array}$

$2.57 \quad 49.36$

$2.30 \quad 58.14$

$2.25 \quad 64.95$

$2.03 \quad 74.12$

$1.98 \quad 81.30$

$1.67 \quad 90.73$

$\begin{array}{ll}1.65 & 98.29\end{array}$

$\begin{array}{ll}1.37 \quad 108.06 \\ 1.02 & 115.63\end{array}$

$\begin{array}{ll}1.02 & 115.63\end{array}$

$0.26 \quad 125.22$

$\begin{array}{ll}-0.33 & 132.87\end{array}$

$\begin{array}{ll}-1.27 & 142.57\end{array}$

$\begin{array}{ll}-1.91 & 150.47\end{array}$

$-3.10 \quad 160.20$

$\begin{array}{ll}-3.88 & 168.25\end{array}$

$\begin{array}{ll}-5.35 & 177.98\end{array}$

$-6.07 \quad 186.36$

$\begin{array}{ll}-5.65 & 198.22\end{array}$

\begin{tabular}{|c|c|c|c|c|c|c|c|c|c|c|c|c|c|c|c|c|c|}
\hline \multicolumn{18}{|c|}{$Z=31(\mathrm{Ga})$} \\
\hline 22 & 53 & 0.10 & 0.00 & -0.03 & 0.06 & 0.106 & 0.000 & 0.043 & -0.056 & 0.42 & 1.57 & 375.54 & 27.99 & & & 1.72 & 26.50 \\
\hline 23 & 54 & 0.17 & 0.00 & -0.02 & 0.05 & 0.183 & 0.000 & 0.040 & -0.045 & 0.38 & 1.96 & 394.08 & 17.52 & & & 2.01 & 16.16 \\
\hline 24 & 55 & 0.18 & 0.00 & -0.02 & 0.05 & 0.194 & 0.000 & 0.042 & -0.044 & -0.25 & 1.52 & 415.01 & 4.66 & & & 1.57 & 3.51 \\
\hline 25 & 56 & 0.18 & 0.00 & 0.00 & 0.03 & 0.195 & 0.000 & 0.016 & -0.029 & -0.27 & 1.55 & 432.21 & -4.47 & & & 1.53 & -5.48 \\
\hline 26 & 57 & 0.16 & 0.00 & 0.00 & 0.02 & 0.172 & 0.000 & 0.012 & -0.019 & -0.67 & 1.34 & 451.22 & -15.40 & & & 1.33 & -16.23 \\
\hline 27 & 58 & 0.13 & 0.00 & 0.00 & 0.01 & 0.140 & 0.000 & 0.008 & -0.010 & -0.73 & 0.97 & 467.25 & -23.36 & & & 0.95 & -24.03 \\
\hline 28 & 59 & 0.00 & 0.00 & 0.00 & 0.00 & 0.000 & 0.000 & 0.000 & 0.000 & -0.92 & -0.22 & 485.68 & -33.73 & & & -0.23 & -34.24 \\
\hline 29 & 60 & 0.13 & 0.00 & -0.02 & 0.02 & 0.139 & 0.000 & 0.032 & -0.016 & -0.94 & 0.92 & 498.77 & -38.74 & & & 0.92 & -39.13 \\
\hline 30 & 61 & 0.16 & 0.00 & -0.02 & 0.04 & 0.172 & 0.000 & 0.038 & -0.035 & -0.83 & 1.51 & 514.00 & -45.90 & -47.09 & 0.053 & 1.51 & -46.15 \\
\hline 31 & 62 & 0.18 & 0.00 & 0.00 & 0.03 & 0.195 & 0.000 & 0.016 & -0.029 & -0.39 & 1.72 & 526.22 & -50.05 & -52.00 & 0.028 & 1.71 & -50.71 \\
\hline 32 & 63 & 0.18 & 0.00 & 0.01 & 0.02 & 0.195 & 0.000 & 0.003 & -0.021 & 0.10 & 2.25 & 539.72 & -55.48 & -56.55 & 0.001 & 2.25 & -55.54 \\
\hline 33 & 64 & 0.18 & 0.00 & 0.04 & 0.01 & 0.197 & 0.000 & -0.034 & -0.017 & 0.45 & 2.67 & 550.08 & -57.77 & -58.83 & 02 & 2.67 & -57.77 \\
\hline 34 & 65 & 0.18 & 0.00 & 0.05 & -0.01 & 0.197 & 0.000 & -0.047 & 0.000 & 0.74 & 3.02 & 562.13 & -61.74 & -62.66 & 0.001 & 3.03 & -61.66 \\
\hline 35 & 66 & -0.25 & 0.00 & 0.00 & -0.06 & -0.256 & 0.000 & 0.029 & 0.052 & 0.53 & 3.27 & 571.60 & -63.14 & -63.72 & .003 & 3.29 & -62.99 \\
\hline 36 & 67 & -0.24 & 0.00 & 0.01 & -0.05 & -0.247 & 0.000 & 0.015 & 0.046 & 0.99 & 3.48 & 582.72 & -66.19 & -66.88 & 0.001 & 3.52 & -65.97 \\
\hline 37 & 68 & -0.20 & 0.00 & 0.02 & -0.02 & -0.207 & 0.000 & -0.006 & 0.022 & 1.85 & 3.78 & 591.15 & -66.55 & -67.09 & 0.002 & 3.79 & -66.33 \\
\hline 38 & 69 & -0.17 & 0.00 & 0.03 & 0.00 & -0.177 & 0.000 & -0.023 & 0.005 & 2.12 & 3.72 & 601.55 & -68.88 & -69.33 & & 3.73 & -68.63 \\
\hline 39 & 70 & -0.17 & 0.00 & 0.03 & 0.00 & -0.177 & 0.000 & -0.023 & 0.005 & 2.13 & 3.80 & 609.28 & -68.54 & -68.91 & 0.001 & 3.81 & -68.28 \\
\hline 40 & 71 & -0.20 & 0.00 & 0.03 & 0.00 & -0.207 & 0.000 & -0.018 & 0.006 & 1.63 & 3.36 & 619.14 & -70.33 & -70.14 & 0.001 & 3.39 & -70.04 \\
\hline 41 & 72 & -0.20 & 0.00 & 0.04 & 0.01 & -0.207 & 0.000 & -0.030 & -0.001 & 1.52 & 3.56 & 625.91 & -69.03 & -68.59 & 0.001 & 3.59 & -68.73 \\
\hline 42 & 73 & -0.20 & 0.00 & 0.04 & 0.01 & -0.207 & 0.000 & -0.030 & -0.001 & 1.15 & 3.19 & 86 & -69.90 & -69.70 & 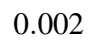 & 3.23 & -69.59 \\
\hline 43 & 74 & 0.13 & 0.00 & -0.02 & 0.01 & 0.139 & 0.000 & 0.032 & -0.006 & 1.51 & 3.24 & 640.98 & -67.95 & -68.05 & 44 & 3.25 & -67.67 \\
\hline 44 & 75 & 0.13 & 0.00 & -0.02 & 0.01 & 0.139 & 0.000 & 0.032 & -0.006 & 0.71 & 2.57 & 649.43 & -68.33 & -68.46 & 0.0 & 2.59 & -68.04 \\
\hline 45 & 76 & 0.16 & 0.00 & -0.02 & 0.02 & 0.172 & 0.000 & 0.036 & -0.015 & -0.30 & 2.11 & 655.32 & -66.15 & -66.30 & 0.002 & 2.15 & -65.86 \\
\hline 46 & 77 & 0.16 & 0.00 & 0.00 & 0.01 & 0.172 & 0.000 & 0.012 & -0.009 & -1.02 & 1.36 & 663.11 & -65.87 & -65.99 & 0.002 & 1.38 & -65.61 \\
\hline 47 & 78 & 0.14 & 0.00 & 0.00 & 0.01 & 50 & 0.000 & 0.009 & -0.009 & -1.56 & 7 & & -63.33 & & & 0.59 & -63.09 \\
\hline 48 & 79 & 0.12 & 0.00 & 0.01 & 0.00 & 0.129 & 0.000 & -0.006 & -0.001 & -2.09 & -0.30 & 675.86 & -62.47 & -62.51 & 0.098 & -0.29 & -62.26 \\
\hline 49 & 80 & 0.08 & 0.00 & 0.01 & 0.00 & 0.086 & 0.000 & -0.009 & -0.001 & -2.64 & -1.36 & 681.01 & -59.56 & -59.13 & 0.123 & -1.35 & -59.37 \\
\hline 50 & 81 & 0.03 & 0.00 & -0.01 & 0.00 & 0.032 & 0.000 & 0.012 & 0.000 & -3.10 & -2.13 & 687.48 & -57.96 & -57.98 & 0.192 & -2.13 & -57.78 \\
\hline 51 & 82 & -0.10 & 0.00 & 0.00 & -0.01 & -0.104 & 0.000 & 0.004 & 0.009 & -2.88 & -1.53 & 690.38 & -52.78 & & & -1.52 & -52.61 \\
\hline 52 & 83 & 0.12 & 0.00 & -0.02 & 0.01 & 0.128 & 0.000 & 0.031 & -0.007 & -2.42 & -0.63 & 694.56 & -48.89 & & & -0.59 & -48.72 \\
\hline 53 & 84 & 0.13 & 0.00 & -0.02 & 0.02 & 0.139 & 0.000 & 0.032 & -0.016 & -1.92 & -0.09 & 696.95 & -43.21 & & & -0.02 & -43.02 \\
\hline 54 & 85 & 0.16 & 0.00 & -0.02 & 0.03 & 0.172 & 0.000 & 0.037 & -0.025 & -1.63 & 0.64 & 700.74 & -38.93 & & & 0.77 & -38.69 \\
\hline 55 & 86 & 0.17 & 0.00 & -0.01 & 0.03 & 0.183 & 0.000 & 0.026 & -0.027 & -1.24 & 0.80 & 702.97 & -33.09 & & & 0.93 & -32.87 \\
\hline 56 & 87 & 0.18 & 0.00 & 0.00 & 0.04 & 0.195 & 0.000 & 0.017 & -0.039 & -0.81 & 1.12 & 706.64 & -28.68 & & & 1.35 & -28.37 \\
\hline 57 & 88 & 0.18 & 0.00 & 0.01 & 0.03 & 0.195 & 0.000 & 0.004 & -0.031 & -0.24 & 1.81 & 707.84 & -21.82 & & & 1.96 & -21.59 \\
\hline 58 & 89 & 0.19 & 0.00 & 0.01 & 0.03 & 0.206 & 0.000 & 0.006 & -0.031 & 0.12 & 2.17 & 710.95 & -16.86 & & & 2.34 & -16.61 \\
\hline 59 & 90 & 0.20 & 0.00 & 0.00 & 0.03 & 0.217 & 0.000 & 0.020 & -0.028 & 0.55 & 2.68 & 711.87 & -9.70 & & & 2.83 & -9.47 \\
\hline
\end{tabular}




\begin{tabular}{|c|c|c|c|c|c|c|c|c|c|c|c|c|c|c|c|c|c|}
\hline \multicolumn{18}{|c|}{$Z=31(\mathbf{G a})$} \\
\hline 60 & 91 & 0.28 & 0.00 & -0.05 & 0.02 & 0.304 & 0.000 & 0.099 & 0.003 & -1.08 & 2.84 & 714.71 & -4.47 & & & 3.12 & -4.11 \\
\hline 61 & 92 & 0.28 & 0.00 & -0.04 & 0.02 & 0.305 & 0.000 & 0.086 & -0.001 & -0.93 & 2.82 & 715.70 & 2.61 & & & 3.02 & 2.90 \\
\hline 62 & 93 & 0.29 & 0.00 & -0.04 & 0.02 & 0.316 & 0.000 & 0.089 & 0.000 & -0.90 & 3.02 & 718.06 & 8.32 & & & 3.25 & 8.66 \\
\hline 63 & 94 & 0.30 & 0.00 & -0.02 & 0.02 & 0.328 & 0.000 & 0.067 & -0.007 & -0.83 & 3.10 & 718.54 & 15.91 & & & 3.22 & 16.17 \\
\hline 64 & 95 & 0.30 & 0.00 & -0.01 & 0.02 & 0.328 & 0.000 & 0.054 & -0.011 & -0.61 & 3.12 & 720.65 & 21.88 & & & 3.24 & 22.16 \\
\hline 65 & 96 & 0.27 & 0.00 & 0.02 & 0.00 & 0.296 & 0.000 & 0.007 & -0.003 & -0.05 & 3.07 & 720.86 & 29.73 & & & 3.08 & 29.94 \\
\hline 66 & 97 & 0.27 & 0.00 & 0.03 & -0.01 & 0.297 & 0.000 & -0.006 & 0.003 & 0.01 & 3.00 & 722.65 & 36.01 & & & 3.06 & 36.29 \\
\hline 67 & 98 & 0.27 & 0.00 & 0.04 & -0.01 & 0.297 & 0.000 & -0.018 & 0.000 & -0.23 & 2.60 & 722.84 & 43.90 & & & 2.66 & 44.22 \\
\hline 68 & 99 & 0.27 & 0.00 & 0.05 & -0.02 & 0.298 & 0.000 & -0.032 & 0.007 & -0.39 & 2.52 & 724.27 & 50.54 & & & 2.67 & 51.00 \\
\hline 69 & 100 & 0.27 & 0.00 & 0.06 & -0.02 & 0.299 & 0.000 & -0.044 & 0.003 & -0.77 & 2.17 & 724.06 & 58.82 & & & 2.35 & 59.38 \\
\hline 70 & 101 & 0.27 & 0.00 & 0.07 & -0.02 & 0.299 & 0.000 & -0.056 & 0.000 & -0.89 & 2.03 & 725.18 & 65.77 & & & 2.31 & 66.48 \\
\hline 71 & 102 & 0.25 & 0.00 & 0.06 & -0.01 & 0.276 & 0.000 & -0.047 & -0.006 & -0.82 & 1.74 & 724.57 & 74.46 & & & 1.93 & 75.14 \\
\hline 72 & 103 & 0.24 & 0.00 & 0.06 & -0.01 & 0.265 & 0.000 & -0.049 & -0.005 & -0.63 & 1.57 & 725.39 & 81.71 & & & 1.78 & 82.50 \\
\hline 73 & 104 & 0.21 & 0.00 & 0.04 & 0.00 & 0.230 & 0.000 & -0.030 & -0.009 & -0.53 & 1.48 & 724.26 & 90.90 & & & 1.56 & 91.66 \\
\hline 74 & 105 & 0.18 & 0.00 & 0.02 & 0.01 & 0.195 & 0.000 & -0.010 & -0.013 & -0.50 & 1.20 & 724.86 & 98.38 & & & 1.26 & 99.20 \\
\hline 75 & 106 & 0.11 & 0.00 & -0.01 & 0.01 & 0.118 & 0.000 & 0.018 & -0.008 & -0.54 & 0.66 & 723.88 & 107.43 & & & 0.69 & 108.33 \\
\hline 76 & 107 & 0.11 & 0.00 & -0.01 & 0.01 & 0.118 & 0.000 & 0.018 & -0.008 & -1.13 & 0.11 & 724.43 & 114.94 & & & 0.15 & 115.95 \\
\hline 77 & 108 & 0.11 & 0.00 & -0.01 & 0.01 & 0.118 & 0.000 & 0.018 & -0.008 & -2.16 & -0.84 & 723.57 & 123.88 & & & -0.80 & 125.00 \\
\hline 78 & 109 & 0.11 & 0.00 & 0.00 & 0.01 & 0.118 & 0.000 & 0.005 & -0.010 & -2.91 & -1.55 & 724.01 & 131.51 & & & -1.52 & 132.75 \\
\hline 79 & 110 & 0.11 & 0.00 & 0.01 & 0.00 & 0.118 & 0.000 & -0.007 & -0.001 & -4.11 & -2.68 & 723.05 & 140.54 & & & -2.68 & 141.88 \\
\hline 80 & 111 & 0.06 & 0.00 & 0.02 & -0.01 & 0.064 & 0.000 & -0.023 & 0.009 & -4.70 & -3.57 & 723.39 & 148.28 & & & -3.50 & 149.83 \\
\hline 81 & 112 & 0.04 & 0.00 & 0.01 & 0.00 & 0.043 & 0.000 & -0.011 & -0.000 & -6.19 & -5.00 & 722.47 & 157.26 & & & -4.99 & 158.91 \\
\hline 82 & 113 & 0.00 & 0.00 & 0.00 & 0.00 & 0.000 & 0.000 & 0.000 & 0.000 & -6.97 & -5.73 & 722.38 & 165.43 & & & -5.73 & 167.22 \\
\hline 83 & 114 & 0.04 & 0.00 & -0.01 & -0.01 & 0.043 & 0.000 & 0.013 & 0.010 & -6.50 & -5.36 & 719.41 & 176.46 & & & -5.31 & 178.47 \\
\hline \multicolumn{18}{|c|}{$Z=32(\mathrm{Ge})$} \\
\hline 23 & 55 & 0.17 & 0.00 & -0.01 & 0.04 & 0.183 & 0.000 & 0.027 & -0.037 & 0.77 & 2.32 & 391.39 & 27.50 & & & 2.35 & 25.99 \\
\hline 24 & 56 & 0.18 & 0.00 & 0.00 & 0.03 & 0.195 & 0.000 & 0.016 & -0.029 & 0.20 & 1.94 & 413.43 & 13.53 & & & 1.96 & 12.21 \\
\hline 25 & 57 & 0.18 & 0.00 & 0.01 & 0.02 & 0.195 & 0.000 & 0.003 & -0.021 & 0.13 & 1.88 & 430.98 & 4.05 & & & 1.86 & 2.90 \\
\hline 26 & 58 & 0.16 & 0.00 & 0.02 & 0.00 & 0.173 & 0.000 & -0.013 & -0.003 & -0.25 & 1.35 & 451.47 & -8.37 & & & 1.34 & -9.32 \\
\hline 27 & 59 & 0.14 & 0.00 & 0.01 & 0.00 & 0.151 & 0.000 & -0.004 & -0.001 & -0.27 & 1.48 & 467.26 & -16.09 & & & 1.46 & -16.88 \\
\hline 28 & 60 & 0.07 & 0.00 & 0.00 & 0.00 & 0.075 & 0.000 & 0.002 & 0.000 & -0.29 & 0.66 & 486.45 & -27.20 & & & 0.66 & -27.83 \\
\hline 29 & 61 & 0.14 & 0.00 & -0.01 & 0.02 & 0.150 & 0.000 & 0.022 & -0.018 & -0.36 & 1.51 & 500.08 & -32.77 & & & 1.51 & -33.26 \\
\hline 30 & 62 & 0.17 & 0.00 & 0.00 & 0.03 & 0.184 & 0.000 & 0.014 & -0.029 & -0.20 & 2.14 & 516.37 & -40.99 & & & 2.15 & -41.34 \\
\hline 31 & 63 & 0.18 & 0.00 & 0.02 & 0.02 & 0.196 & 0.000 & -0.009 & -0.023 & 0.19 & 2.23 & 529.45 & -45.99 & & & 2.23 & -46.25 \\
\hline 32 & 64 & 0.19 & 0.00 & 0.04 & 0.00 & 0.207 & 0.000 & -0.033 & -0.008 & 0.46 & 2.65 & 544.61 & -53.08 & -54.35 & 0.032 & 2.66 & -53.23 \\
\hline 33 & 65 & 0.19 & 0.00 & 0.05 & -0.01 & 0.208 & 0.000 & -0.046 & -0.000 & 0.69 & 3.16 & 555.12 & -55.52 & -56.42 & 0.100 & 3.16 & -55.59 \\
\hline 34 & 66 & 0.19 & 0.00 & 0.06 & -0.02 & 0.208 & 0.000 & -0.059 & 0.007 & 0.89 & 3.38 & 568.35 & -60.68 & -61.62 & 0.030 & 3.41 & -60.65 \\
\hline 35 & 67 & -0.27 & 0.00 & 0.01 & -0.07 & -0.276 & 0.000 & 0.023 & 0.063 & 0.49 & 3.64 & 578.04 & -62.30 & -62.66 & 0. & 3.68 & -62.19 \\
\hline 36 & 68 & -0.27 & 0.00 & 0.01 & -0.07 & -0.276 & 0.000 & 0.023 & 0.063 & 0.71 & 3.65 & 590.39 & -66.58 & -66.98 & 0.0 & 3.74 & -66.37 \\
\hline 37 & 69 & -0.23 & 0.00 & 0.02 & -0.04 & -0.237 & 0.000 & 0.001 & 0.040 & 1.78 & 4.16 & 598.83 & -66.94 & -67.10 & 0.001 & 4.20 & -66.74 \\
\hline 38 & 70 & -0.21 & 0.00 & 0.03 & -0.02 & -0.218 & 0.000 & -0.015 & 0.024 & 2.09 & 4.20 & 610.13 & -70.18 & -70.56 & 0.00 & 4.24 & -69.94 \\
\hline 39 & 71 & -0.20 & 0.00 & 0.04 & 0.00 & -0.207 & 0.000 & -0.029 & 0.008 & 2.21 & 4. & 618 & -70.30 & -6 & 0. & 4.05 & -70.06 \\
\hline 40 & 72 & -0.21 & 0.00 & 0.04 & 0.00 & -0.217 & 0.000 & -0.028 & 0.008 & 1.84 & 3.81 & 628.96 & -72.86 & -72.59 & 0.002 & 3.85 & -72.57 \\
\hline 41 & 73 & -0.22 & 0.00 & 0.05 & 0.01 & -0.227 & 0.000 & -0.038 & 0.002 & 1.55 & 3.88 & 636.05 & -71.88 & -71.30 & 0.002 & 3.92 & -71.58 \\
\hline 42 & 74 & -0.23 & 0.00 & 0.05 & 0.01 & -0.237 & 0.000 & -0.036 & 0.002 & 1.02 & 3.49 & 645.96 & -73.72 & -73.42 & 0.002 & 3.55 & -73.40 \\
\hline 43 & 75 & 0.17 & 0.00 & 0.00 & 0.01 & 0.183 & 0.000 & 0.013 & -0.009 & 1.43 & 3.37 & 652.45 & -72.13 & -71.86 & 0.002 & 3.39 & -71.85 \\
\hline 44 & 76 & 0.15 & 0.00 & -0.01 & 0.01 & 0.161 & 0.000 & 0.022 & -0.007 & 1.07 & 3.11 & 661.42 & -73.04 & -73.21 & 0.002 & 3.13 & -72.75 \\
\hline 45 & 77 & 0.16 & 0.00 & 0.00 & 0.01 & 0.172 & 0.000 & 0.012 & -0.009 & 0.32 & 2.60 & 667.54 & -71.08 & -71.21 & 0.002 & 2.62 & -70.80 \\
\hline 46 & 78 & 0.16 & 0.00 & 0.01 & 0.01 & 0.173 & 0.000 & -0.001 & -0.011 & -0.57 & 1.79 & 676.30 & -71.77 & -71.86 & 0.0 & 1.82 & -71.49 \\
\hline 47 & 79 & 0.16 & 0.00 & 0.02 & 0.00 & 0.173 & 0.000 & -0.013 & -0.003 & -1.34 & 1.08 & 681.92 & -69.32 & -69.49 & 0.090 & 1.11 & -69.06 \\
\hline 48 & 80 & 0.13 & 0.00 & 0.02 & 0.00 & 0.140 & 0.000 & -0.017 & -0.003 & -1.72 & 0.22 & 690.01 & -69.34 & -69.51 & 0.028 & 0.25 & -69.10 \\
\hline 49 & 81 & 0.12 & 0.00 & 0.02 & 0.00 & 0.129 & 0.000 & -0.018 & -0.002 & -2.33 & -0.54 & 695.03 & -66.29 & & 0.120 & -0.52 & -66.06 \\
\hline 50 & 82 & 0.02 & 0.00 & 0.00 & 0.00 & 0.021 & 0.000 & 0.000 & 0.000 & -2.20 & -1.44 & 702.50 & -65.68 & -65.62 & 0.244 & -1.45 & -65.50 \\
\hline 51 & 83 & 0.12 & 0.00 & 0.00 & 0.00 & 0.129 & 0.000 & 0.006 & 0.000 & -2.39 & -0.67 & 705.36 & -60.48 & & & -0.65 & -60.30 \\
\hline 52 & 84 & 0.12 & 0.00 & -0.01 & 0.01 & 0.128 & 0.000 & 0.019 & -0.008 & -1.71 & -0.01 & 710.65 & -57.69 & & & 0.01 & -57.51 \\
\hline 53 & 85 & 0.16 & 0.00 & -0.01 & 0.02 & 0.172 & 0.000 & 0.024 & -0.017 & -1.52 & 0.76 & 712.94 & -51.91 & & & 0.82 & -51.71 \\
\hline 54 & 86 & 0.16 & 0.00 & -0.01 & 0.02 & 0.172 & 0.000 & 0.024 & -0.017 & -0.97 & 0.88 & 718.16 & -49.06 & & & 0.95 & -48.87 \\
\hline
\end{tabular}




\begin{tabular}{|c|c|c|c|c|c|c|c|c|c|c|c|c|c|}
\hline \\
\hline \multicolumn{14}{|c|}{$Z=32$} \\
\hline & 88 & & .00 & .01 & .0J & & .000 & & & & 1.72 & & \\
\hline 7 & 89 & & 0.00 & 0.02 & 02 & & .000 & 0.006 & & 0.03 & 2.16 & & \\
\hline & 90 & & .00 & 03 & & & .000 & -0.019 & & & 57 & & \\
\hline 59 & 91 & 0.22 & 0.00 & 0.01 & 0.02 & 40 & .000 & & 21 & 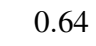 & 2.94 & & 0 \\
\hline & 92 & & ( & & 0 . & & & & & & & & \\
\hline & 93 & .30 & .00 & $T$ & 0.02 & & & & & & & & \\
\hline 2 & 94 & .30 & 0.00 & -0.03 & 0.02 & & .000 & & -0.003 & -0 & 3.36 & & -5.31 \\
\hline 3 & 95 & & 0.00 & -0.02 & 0.02 & & 0 & 7 & & & 39 & & 1 \\
\hline 64 & 96 & 0.27 & 0.00 & 0.01 & 0.00 & 0.296 & .000 & 0.020 & 0.000 & 0.34 & 3.49 & & 7.44 \\
\hline 65 & 97 & 0.27 & 0.00 & 0.0 & 01 & 6 & 0 & & & 0.14 & 1 & & .0 \\
\hline & 98 & & 00 & 08 & & & 00 & & & & & & .6 \\
\hline 67 & 99 & .27 & 0.00 & 0.05 & 2 & & 00 & - & & 9 & 1 & & 8.40 \\
\hline 8 & 100 & 0.27 & 0.00 & 0.06 & -0.02 & & 00 & -0 & & 39 & 57 & & 4.2 \\
\hline 69 & 101 & 027 & 0.00 & 0.07 & -0.02 & 99 & 00 & 0 & & 85 & 2.31 & & 12 \\
\hline U & 2 & 0.27 & 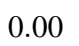 & 0.08 & -0.03 & & & & & & & & \\
\hline 71 & 103 & 0.26 & 00 & 0.0 & -0.02 & & 00 & -( & & & 77 & & 7.2 \\
\hline & 104 & & 0.00 & 0. & -0.02 & & 00 & -0 . & & & & & 3.9 \\
\hline & 105 & 0.21 & 0.00 & 0 & -0.01 & & 0 & -0 . & - & 3 & & & 73.1 \\
\hline 4 & 106 & مص م & 0.00 & 0.0 & -0.01 & 19 & .000 & -0.044 & -0 & & & & 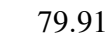 \\
\hline & & & & & & & & & & & & & \\
\hline 5 & 108 & 0.15 & 0.00 & 0.0 & 0 . & & 00 & -0 . & - & & & & 6.1 \\
\hline & 109 & & 0.00 & 0.0 & & & 00 & -0 . & -0 & & & & 105.0 \\
\hline 78 & 110 & 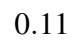 & 0.00 & 0.0 & 0. & & 00 & -0 . & -0 & - & 8 & & 120 \\
\hline 79 & 111 & 0.1 & 0.00 & 0.0 & 0.0 & 18 & .000 & -0.019 & -0.002 & -3 & -2.14 & .89 & 20. \\
\hline 80 & 112 & 0.0 & 0.0 & 0.0 & 0.0 & & 00 & -0 . & & - & -2 & & 128.2 \\
\hline 81 & 113 & & 0.0 & 0.0 & 0.0 & & .000 & -0.011 & -0 . & & -4.38 & & 137. \\
\hline - & 114 & 0.0 & 0.00 & & 0.0 & & 0.000 & & & & -5 & & 144.6 \\
\hline 83 & 115 & 0.05 & 0.00 & -0.01 & 0.00 & & 0.000 & 0.013 & & -5.89 & -4.72 & 747.47 & 155.7 \\
\hline & 16 & 0.06 & 0.00 & 0.01 & 0.00 & 0.064 & .000 & 0.014 & 0.001 & -5.03 & -3.89 & 746.15 & 16 \\
\hline
\end{tabular}

$\begin{array}{ll}1.31 & -42.98\end{array}$

$1.88-39.14$

$2.33-32.64$

$2.71-28.46$

$3.04-21.60$

$3.48-16.86$

$3.37-9.98$

$3.57-5.00$

$3.54 \quad 2.40$

$3.55 \quad 7.64$

$3.37 \quad 15.29$

$3.37 \quad 20.95$

$2.96 \quad 28.77$

$2.89 \quad 34.76$

$2.58 \quad 43.06$

$2.48 \quad 49.41$

$2.15 \quad 58.04$

$2.04 \quad 64.75$

$\begin{array}{ll}1.87 & 73.87\end{array}$

$1.55 \quad 80.73$

$0.99 \quad 89.79$

$0.71 \quad 97.02$

$-0.20 \quad 106.04$

$-0.95 \quad 113.12$

$\begin{array}{ll}-2.11 & 122.19\end{array}$

$-2.86129 .57$

$-4.37 \quad 138.57$

$-5.14 \quad 146.23$

$-4.71 \quad 157.44$

$-3.87 \quad 166.98$

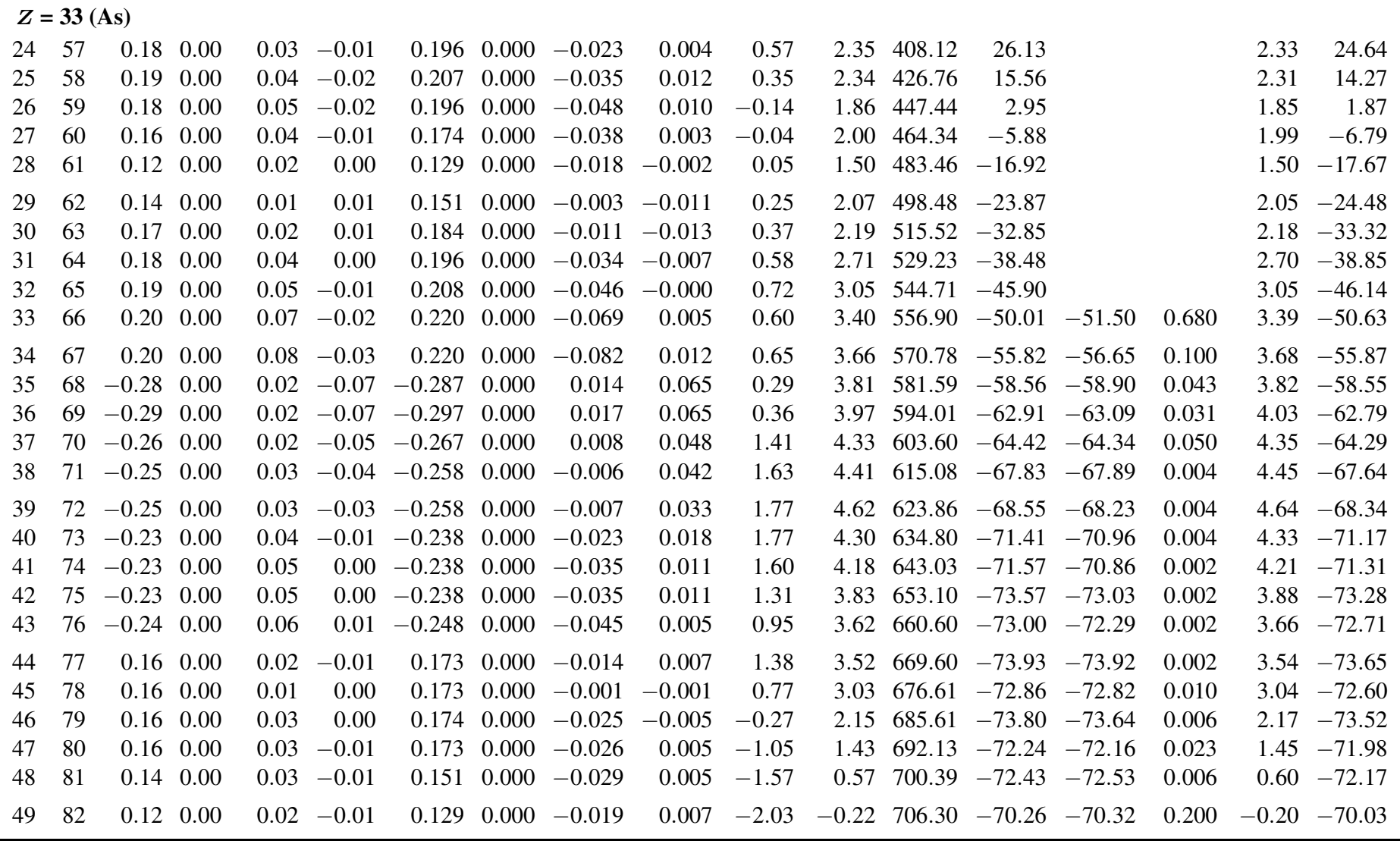




\begin{tabular}{|c|c|c|c|c|c|c|c|c|c|c|c|c|c|c|c|c|c|}
\hline \multicolumn{18}{|c|}{$Z=33(\mathrm{As})$} \\
\hline 50 & 83 & 0.07 & 0.00 & 0.00 & 0.00 & 0.075 & 0.000 & 0.002 & 0.000 & -2.00 & -0.82 & 713.62 & -69.52 & \multirow[t]{5}{*}{-69.88} & 0.220 & -0.82 & -69.32 \\
\hline 51 & 84 & 0.12 & 0.00 & 0.01 & -0.01 & 0.129 & 0.000 & -0.007 & 0.009 & -1.99 & -0.27 & 717.55 & -65.38 & & & -0.25 & -65.18 \\
\hline 52 & 85 & 0.12 & 0.00 & 0.01 & -0.01 & 0.129 & 0.000 & -0.007 & 0.009 & -1.30 & 0.37 & 723.00 & -62.76 & & & 0.39 & -62.57 \\
\hline 53 & 86 & 0.14 & 0.00 & 0.01 & 0.00 & 0.151 & 0.000 & -0.004 & -0.001 & -0.81 & 1.14 & 726.12 & -57.81 & & & 1.15 & -57.64 \\
\hline 54 & 87 & 0.16 & 0.00 & 0.01 & 0.01 & 0.173 & 0.000 & -0.001 & -0.011 & -0.48 & 1.74 & 731.00 & -54.61 & & & 1.78 & -54.44 \\
\hline 55 & 88 & 0.17 & 0.00 & 0.01 & 0.02 & 0.184 & 0.000 & 0.002 & -0.021 & -0.23 & 1.74 & 734.33 & -49.87 & & & 1.80 & -49.69 \\
\hline 56 & 89 & 0.19 & 0.00 & 0.03 & 0.02 & 0.207 & 0.000 & -0.019 & -0.025 & -0.03 & 2.25 & 738.74 & -46.22 & & & 2.36 & -45.99 \\
\hline 57 & 90 & 0.19 & 0.00 & 0.04 & 0.01 & 0.208 & 0.000 & -0.032 & -0.018 & 0.32 & 2.65 & 741.15 & -40.55 & & & 2.74 & -40.36 \\
\hline 58 & 91 & 0.19 & 0.00 & 0.04 & 0.01 & 0.208 & 0.000 & -0.032 & -0.018 & 0.76 & 2.94 & 745.25 & -36.58 & & & 3.04 & -36.38 \\
\hline 59 & 92 & 0.19 & 0.00 & 0.05 & 0.00 & 0.208 & 0.000 & -0.045 & -0.010 & 1.21 & 3.39 & 747.10 & -30.36 & & & 3.50 & -30.17 \\
\hline 60 & 93 & 0.29 & 0.00 & -0.05 & 0.01 & 0.316 & 0.000 & 0.101 & 0.015 & -0.88 & 3.52 & 750.86 & -26.05 & & & 3.77 & -25.71 \\
\hline 61 & 94 & 0.29 & 0.00 & -0.04 & 0.01 & 0.316 & 0.000 & 0.088 & 0.010 & -0.68 & 3.54 & 752.68 & -19.79 & & & 3.72 & -19.53 \\
\hline 62 & 95 & 0.30 & 0.00 & -0.03 & 0.01 & 0.328 & 0.000 & 0.078 & 0.007 & -0.49 & 3.66 & 755.97 & -15.01 & & & 3.83 & -14.76 \\
\hline 63 & 96 & 0.30 & 0.00 & -0.01 & 0.01 & 0.329 & 0.000 & 0.053 & -0.001 & -0.27 & 3.78 & 757.25 & -8.22 & & & 3.85 & -8.04 \\
\hline 64 & 97 & 0.27 & 0.00 & 0.02 & -0.01 & 0.296 & 0.000 & 0.006 & 0.007 & 0.55 & 3.84 & 760.15 & -3.05 & & & 3.90 & -2.88 \\
\hline 65 & 98 & 0.27 & 0.00 & 0.03 & -0.02 & 0.297 & 0.000 & -0.007 & 0.013 & 0.36 & 3.59 & 761.37 & 3.80 & & & 3.68 & 4.02 \\
\hline 66 & 99 & 0.27 & 0.00 & 0.05 & -0.02 & 0.298 & 0.000 & -0.032 & 0.007 & 0.34 & 3.53 & 763.97 & 9.27 & & & 3.68 & 9.57 \\
\hline 67 & 100 & 0.27 & 0.00 & 0.06 & -0.04 & 0.298 & 0.000 & -0.047 & 0.023 & -0.11 & 2.95 & 765.13 & 16.18 & & & 3.29 & 16.70 \\
\hline 68 & 101 & 0.27 & 0.00 & 0.07 & -0.03 & 0.299 & 0.000 & -0.057 & 0.010 & -0.42 & 2.85 & 767.38 & 22.01 & & & 3.15 & 22.53 \\
\hline 69 & 102 & 0.27 & 0.00 & 0.08 & -0.03 & 0.300 & 0.000 & -0.069 & 0.007 & -0.94 & 2.41 & 768.02 & 29.44 & & & 2.77 & 30.06 \\
\hline 70 & 103 & 0.25 & 0.00 & 0.07 & -0.02 & 0.277 & 0.000 & -0.060 & 0.001 & -0.54 & 2.37 & 769.81 & 35.72 & & & 2.65 & 36.30 \\
\hline 71 & 104 & 0.25 & 0.00 & 0.08 & -0.02 & 0.278 & 0.000 & -0.072 & -0.002 & -1.04 & 1.95 & 770.08 & 43.52 & & & 2.30 & 44.22 \\
\hline 72 & 105 & 0.23 & 0.00 & 0.07 & -0.02 & 0.254 & 0.000 & -0.064 & 0.002 & -0.59 & 1.93 & 771.48 & 50.19 & & & 2.24 & 50.90 \\
\hline 73 & 106 & 0.21 & 0.00 & 0.06 & -0.02 & 0.230 & 0.000 & -0.056 & 0.006 & -0.44 & 1.81 & 771.12 & 58.63 & & & 2.06 & 59.34 \\
\hline 74 & 107 & 0.20 & 0.00 & 0.06 & -0.01 & 0.220 & 0.000 & -0.056 & -0.003 & -0.56 & 1.55 & 772.43 & 65.39 & & & 1.78 & 66.17 \\
\hline 75 & 108 & 0.18 & 0.00 & 0.05 & -0.01 & 0.197 & 0.000 & -0.047 & 0.000 & -0.84 & 1.07 & 772.09 & 73.79 & & & 1.24 & 74.58 \\
\hline 76 & 109 & 0.15 & 0.00 & 0.04 & -0.01 & 0.163 & 0.000 & -0.039 & 0.003 & -0.76 & 0.80 & 773.08 & 80.88 & & & 0.93 & 81.71 \\
\hline 77 & 110 & 0.11 & 0.00 & 0.02 & 0.00 & 0.118 & 0.000 & -0.019 & -0.002 & -1.29 & 0.03 & 772.73 & 89.30 & & & 0.06 & 90.13 \\
\hline 78 & 111 & 0.11 & 0.00 & 0.03 & 0.00 & 0.119 & 0.000 & -0.031 & -0.004 & -2.18 & -0.78 & 773.96 & 96.14 & & & -0.71 & 97.12 \\
\hline 79 & 112 & 0.11 & 0.00 & 0.02 & 0.00 & 0.118 & 0.000 & -0.019 & -0.002 & -3.38 & -1.92 & 773.68 & 104.49 & & & -1.89 & 105.53 \\
\hline 80 & 113 & 0.09 & 0.00 & 0.03 & -0.01 & 0.097 & 0.000 & -0.033 & 0.007 & -4.09 & -2.78 & 774.65 & 111.59 & & & -2.68 & 112.81 \\
\hline 81 & 114 & 0.05 & 0.00 & 0.01 & 0.00 & 0.053 & 0.000 & -0.011 & -0.001 & -5.38 & -4.20 & 774.38 & 119.93 & & & -4.19 & 121.18 \\
\hline 82 & 115 & 0.01 & 0.00 & 0.00 & 0.00 & 0.011 & 0.000 & 0.000 & 0.000 & -6.26 & -5.03 & 775.05 & 127.34 & & & -5.03 & 128.71 \\
\hline 83 & 116 & 0.04 & 0.00 & -0.01 & 0.00 & 0.043 & 0.000 & 0.013 & 0.001 & -5.73 & -4.59 & 772.65 & 137.81 & & & -4.58 & 139.33 \\
\hline 84 & 117 & 0.08 & 0.00 & 0.00 & 0.00 & 0.085 & 0.000 & 0.003 & 0.000 & -4.87 & -3.64 & 771.26 & 147.27 & & & -3.64 & 148.93 \\
\hline 85 & 118 & 0.10 & 0.00 & 0.00 & 0.00 & 0.107 & 0.000 & 0.004 & 0.000 & -4.62 & -3.29 & 768.70 & 157.90 & & & -3.30 & 159.70 \\
\hline 86 & 119 & 0.11 & 0.00 & 0.00 & 0.00 & 0.118 & 0.000 & 0.005 & 0.000 & -3.79 & -2.44 & 767.15 & 167.52 & & & -2.45 & 169.48 \\
\hline \multicolumn{18}{|c|}{$Z=34(\mathrm{Se})$} \\
\hline 25 & 59 & 0.20 & 0.00 & 0.06 & -0.04 & 0.219 & 0.000 & -0.059 & 0.026 & 0.32 & 2.43 & 424.29 & 25.32 & & & 2.46 & 23.97 \\
\hline 26 & 60 & 0.19 & 0.00 & 0.07 & -0.04 & 0.208 & 0.000 & -0.073 & 0.025 & -0.19 & 1.99 & 446.05 & 11.62 & & & 2.05 & 10.49 \\
\hline 27 & 61 & 0.16 & 0.00 & 0.05 & -0.02 & 0.174 & 0.000 & -0.051 & 0.011 & 0.14 & 2.07 & 463.26 & 2.49 & & & 2.07 & 1.48 \\
\hline 28 & 62 & -0.20 & 0.00 & -0.02 & -0.06 & -0.204 & 0.000 & 0.043 & 0.051 & -0.39 & 1.54 & 483.51 & -9.69 & & & 1.60 & -10.48 \\
\hline 29 & 63 & -0.20 & 0.00 & -0.01 & -0.06 & -0.205 & 0.000 & 0.032 & 0.053 & 0.07 & 2.16 & 498.71 & -16.82 & & & 2.19 & -17.49 \\
\hline 30 & 64 & 0.18 & 0.00 & 0.04 & 0.00 & 0.196 & 0.000 & -0.034 & -0.007 & 0.61 & 2.60 & 516.52 & -26.55 & & & 2.60 & -27.12 \\
\hline 31 & 65 & 0.19 & 0.00 & 0.05 & -0.01 & 0.208 & 0.000 & -0.046 & -0.000 & 0.79 & 3.07 & 530.51 & -32.48 & & & 3.06 & -32.93 \\
\hline 32 & 66 & 0.20 & 0.00 & 0.07 & -0.02 & 0.220 & 0.000 & -0.069 & 0.005 & 0.72 & 3.37 & 547.09 & -40.98 & & & 3.39 & -41.29 \\
\hline 33 & 67 & 0.20 & 0.00 & 0.08 & -0.03 & 0.220 & 0.000 & -0.082 & 0.012 & 0.68 & 3.66 & 560.02 & -45.84 & & & 3.67 & -46.06 \\
\hline 34 & 68 & 0.21 & 0.00 & 0.10 & -0.04 & 0.233 & 0.000 & -0.106 & 0.016 & 0.38 & 3.85 & 575.44 & -53.19 & -54.22 & 0.033 & 3.90 & -53.28 \\
\hline 35 & 69 & -0.30 & 0.00 & 0.02 & -0.07 & -0.307 & 0.000 & 0.019 & 0.064 & 0.08 & 3.96 & 586.52 & -56.20 & -56.30 & 0.034 & 3.99 & -56.24 \\
\hline 36 & 70 & -0.30 & 0.00 & 0.02 & -0.07 & -0.307 & 0.000 & 0.019 & 0.064 & 0.27 & 4.06 & 600.01 & -61.61 & -62.05 & 0.062 & 4.13 & -61.54 \\
\hline 37 & 71 & -0.29 & 0.00 & 0.02 & -0.07 & -0.297 & 0.000 & 0.017 & 0.065 & 0.98 & 4.53 & 609.70 & -63.24 & -63.12 & 0.032 & 4.58 & -63.13 \\
\hline 38 & 72 & -0.29 & 0.00 & 0.03 & -0.06 & -0.298 & 0.000 & 0.005 & 0.059 & 1.07 & 4.55 & 622.22 & -67.69 & -67.89 & 0.012 & 4.63 & -67.50 \\
\hline 39 & 73 & -0.27 & 0.00 & 0.03 & -0.04 & -0.278 & 0.000 & -0.002 & 0.041 & 1.63 & 4.76 & 631.21 & -68.61 & -68.22 & 0.011 & 4.80 & -68.42 \\
\hline 40 & 74 & -0.23 & 0.00 & 0.04 & -0.01 & -0.238 & 0.000 & -0.023 & 0.018 & 2.00 & 4.56 & 642.98 & -72.31 & -72.21 & 0.002 & 4.60 & -72.09 \\
\hline 41 & 75 & -0.23 & 0.00 & 0.04 & -0.01 & -0.238 & 0.000 & -0.023 & 0.018 & 1.85 & 4.46 & 651.40 & -72.65 & -72.17 & 0.002 & 4.49 & -72.42 \\
\hline 42 & 76 & -0.24 & 0.00 & 0.05 & 0.00 & -0.248 & 0.000 & -0.033 & 0.011 & 1.36 & 4.10 & 662.42 & -75.60 & -75.25 & 0.002 & 4.15 & -75.33 \\
\hline 43 & 77 & -0.25 & 0.00 & 0.05 & 0.00 & -0.258 & 0.000 & -0.031 & 0.012 & 1.07 & 3.83 & 670.17 & -75.28 & -74.60 & 0.002 & 3.88 & -75.00 \\
\hline
\end{tabular}




\begin{tabular}{|c|c|c|c|c|c|c|c|c|c|c|c|c|c|c|c|c|c|}
\hline \multicolumn{18}{|c|}{$Z=34(\mathrm{Se})$} \\
\hline 44 & 78 & 0.16 & 0.00 & 0.03 & -0.01 & 0.173 & 0.000 & -0.026 & 0.005 & 1.71 & 3.75 & 680.06 & -77.10 & -77.03 & 0.002 & 3.78 & -76.83 \\
\hline 45 & 79 & 0.16 & 0.00 & 0.03 & -0.01 & 0.173 & 0.000 & -0.026 & 0.005 & 1.06 & 3.25 & 687.25 & -76.22 & -75.92 & 0.002 & 3.28 & -75.95 \\
\hline 46 & 80 & 0.16 & 0.00 & 0.04 & -0.01 & 0.174 & 0.000 & -0.038 & 0.003 & -0.01 & 2.39 & 697.14 & -78.03 & -77.76 & 0.002 & 2.43 & -77.75 \\
\hline 47 & 81 & 0.16 & 0.00 & 0.04 & -0.01 & 0.174 & 0.000 & -0.038 & 0.003 & -0.85 & 1.63 & 703.86 & -76.68 & -76.39 & 0.002 & 1.67 & -76.41 \\
\hline 48 & 82 & 0.14 & 0.00 & 0.05 & -0.02 & 0.152 & 0.000 & -0.053 & 0.012 & -1.49 & 0.75 & 713.01 & -77.76 & -77.59 & 0.002 & 0.82 & -77.46 \\
\hline 49 & 83 & 0.12 & 0.00 & 0.03 & -0.01 & 0.129 & 0.000 & -0.031 & 0.006 & -1.75 & 0.05 & 719.01 & -75.68 & -75.34 & 0.004 & 0.08 & -75.44 \\
\hline 50 & 84 & 0.07 & 0.00 & 0.01 & 0.00 & 0.075 & 0.000 & -0.010 & -0.001 & -1.72 & -0.57 & 727.19 & -75.80 & -75.95 & 0.015 & -0.56 & -75.59 \\
\hline 51 & 85 & 0.12 & 0.00 & 0.02 & -0.01 & 0.129 & 0.000 & -0.019 & 0.007 & -1.57 & 0.09 & 731.17 & -71.71 & -72.43 & 0.030 & 0.12 & -71.50 \\
\hline 52 & 86 & 0.12 & 0.00 & 0.02 & 0.00 & 0.129 & 0.000 & -0.018 & -0.002 & -0.86 & 0.74 & 737.44 & -69.91 & -70.54 & 0.016 & 0.76 & -69.71 \\
\hline 53 & 87 & 0.14 & 0.00 & 0.02 & 0.00 & 0.151 & 0.000 & -0.016 & -0.003 & -0.35 & 1.53 & 740.69 & -65.08 & -66.58 & 0.039 & 1.56 & -64.90 \\
\hline 54 & 88 & 0.16 & 0.00 & 0.02 & 0.00 & 0.173 & 0.000 & -0.013 & -0.003 & 0.00 & 2.16 & 746.36 & -62.69 & -63.88 & 0.049 & 2.19 & -62.51 \\
\hline 55 & 89 & 0.18 & 0.00 & 0.03 & 0.01 & 0.196 & 0.000 & -0.022 & -0.015 & 0.07 & 2.27 & 749.73 & -57.98 & & & 2.33 & -57.78 \\
\hline 56 & 90 & 0.19 & 0.00 & 0.05 & 0.01 & 0.208 & 0.000 & -0.044 & -0.020 & 0.21 & 2.62 & 755.09 & -55.27 & & & 2.75 & -55.02 \\
\hline 57 & 91 & 0.20 & 0.00 & 0.05 & 0.01 & 0.220 & 0.000 & -0.042 & -0.020 & 0.44 & 2.96 & 757.68 & -49.79 & & & 3.09 & -49.56 \\
\hline 58 & 92 & 0.20 & 0.00 & 0.05 & 0.00 & 0.219 & 0.000 & -0.043 & -0.010 & 0.88 & 3.38 & 762.43 & -46.47 & & & 3.49 & -46.25 \\
\hline 59 & 93 & -0.28 & 0.00 & -0.03 & -0.06 & -0.283 & 0.000 & 0.069 & 0.043 & -0.08 & 3.39 & 764.85 & -40.81 & & & 3.86 & -40.25 \\
\hline 60 & 94 & 0.31 & 0.00 & -0.04 & 0.01 & 0.339 & 0.000 & 0.094 & 0.013 & -1.11 & 3.74 & 769.15 & -37.04 & & & 3.98 & \\
\hline 61 & 95 & 0.30 & 0.00 & -0.03 & 0.00 & 0.328 & 0.000 & 0.077 & 0.018 & -0.74 & 3.65 & 771.19 & -31.02 & & & 3.83 & -30.75 \\
\hline 62 & 96 & 0.31 & 0.00 & -0.02 & 0.01 & 0.340 & 0.000 & 0.069 & 0.004 & -0.55 & 3.72 & 775.28 & -27.03 & & & 3.88 & -26.78 \\
\hline 63 & 97 & 0.31 & 0.00 & -0.01 & 0.01 & 0.340 & 0.000 & 0.056 & 0.000 & -0.50 & 3.90 & 776.60 & -20.28 & & & 4.01 & -20.08 \\
\hline 64 & 98 & 0.30 & 0.00 & 0.01 & -0.01 & 0.330 & 0.000 & 0.026 & 0.011 & -0.01 & 3.99 & 780.20 & -15.81 & & & 4.10 & -15.61 \\
\hline 65 & 99 & 0.29 & 0.00 & 0.03 & -0.02 & 0.319 & 0.000 & -0.002 & 0.013 & 0.05 & 3.75 & 781.52 & -9.06 & & & 3.86 & -8.84 \\
\hline 66 & 100 & 0.27 & 0.00 & 0.06 & -0.03 & 0.298 & 0.000 & -0.045 & 0.013 & 0.26 & 3.56 & 784.97 & -4.43 & & & 3.82 & -4.05 \\
\hline 67 & 101 & 0.27 & 0.00 & 0.07 & -0.04 & 0.299 & 0.000 & -0.059 & 0.019 & -0.28 & 3.01 & 786.18 & 2.42 & & & & 2.94 \\
\hline 68 & 102 & 0.27 & 0.00 & 0.08 & -0.04 & 0.300 & 0.000 & -0.071 & 0.016 & -0.69 & 2.71 & 789.32 & 7.36 & & & 3.18 & 7.99 \\
\hline 69 & 103 & 0.28 & 0.00 & 0.09 & -0.04 & 0.312 & 0.000 & -0.080 & 0.012 & -1.42 & 2.28 & 790.04 & 14.70 & & & 2.79 & 15.42 \\
\hline 70 & 104 & 0.27 & 0.00 & 0.09 & -0.04 & 0.301 & 0.000 & -0.083 & 0.013 & -1.31 & 2.17 & 792.59 & 20.22 & & & 2 & \\
\hline 71 & 105 & 0.25 & 0.00 & 0.09 & -0.03 & 0.278 & 0.000 & -0.085 & 0.005 & -1.32 & 1.88 & 792.81 & 28.08 & & & 2.38 & 8.86 \\
\hline 72 & 106 & 0.24 & 0.00 & 0.08 & -0.03 & 0.266 & 0.000 & -0.075 & 0.009 & -0.92 & 1.86 & 794.89 & 34.07 & & & 2.31 & 34.85 \\
\hline 73 & 107 & 0.22 & 0.00 & 0.09 & -0.03 & 0.244 & 0.000 & -0.091 & 0.008 & -0.95 & 1.37 & 794.97 & 42.06 & & & 1.94 & 43.02 \\
\hline 74 & 108 & 0.21 & 0.00 & 0.07 & -0.02 & 0.231 & 0.000 & -0.068 & 0.004 & -0.54 & 1.47 & 796.58 & 48.53 & & & 1.81 & 49.33 \\
\hline 75 & 109 & 0.19 & 0.00 & 0.07 & -0.02 & 0.209 & 0.000 & -0.071 & 0.005 & -0.87 & 1.21 & 796.09 & 57.08 & & & 1.57 & 57.97 \\
\hline 76 & 110 & 0.17 & 0.00 & 0.06 & -0.02 & 0.186 & 0.000 & -0.062 & 0.009 & -0.84 & 0.92 & 797.74 & 63.50 & & & 1.23 & 64.40 \\
\hline 77 & 111 & 0.15 & 0.00 & 0.05 & -0.01 & 0.163 & 0.000 & -0.051 & 0.002 & -1.38 & 0.43 & 797.17 & 72.14 & & & 0.62 & 73.01 \\
\hline 78 & 112 & 0.11 & 0.00 & 0.04 & 0.00 & 0.119 & 0.000 & -0.043 & -0.005 & -1.78 & -0.38 & 799.02 & 78.36 & & & -0.24 & 79.26 \\
\hline 79 & 113 & 0.11 & 0.00 & 0.03 & -0.01 & 0.119 & 0.000 & -0.032 & 0.006 & -2.97 & -1.52 & 798.81 & 86.64 & & & -1.43 & 87.60 \\
\hline 80 & 114 & 0.09 & 0.00 & 3 & -0.01 & 7 & 0.000 & -0.033 & 0.007 & -3.66 & -2.36 & 800.38 & 93.15 & & & 6 & 21 \\
\hline 81 & 115 & 0.05 & 0.00 & 0.01 & 0.00 & 0.053 & 0.000 & -0.011 & -0.001 & -5.02 & -3.84 & 800.22 & 101.38 & & & -3.83 & 102.46 \\
\hline 82 & 116 & 0.00 & 0.00 & 0.00 & 0.00 & 0.000 & 0.000 & 0.000 & 0.000 & -5.96 & -4.75 & 801.57 & 108.10 & & & -4.75 & 109.29 \\
\hline 83 & 117 & -0.03 & 0.00 & 0.00 & 0.00 & -0.032 & 0.000 & 0.000 & 0.000 & -5.34 & -4.30 & 799.22 & 118.53 & & & -4.30 & 119.84 \\
\hline 84 & 118 & 0.02 & 0.00 & 0.00 & 0.00 & 0.021 & 0.000 & 0.000 & 0.000 & -4.39 & -3.41 & 798.48 & 127.34 & & & -3.41 & 128.78 \\
\hline 85 & 119 & 0.09 & 0.00 & 0.00 & 0.00 & 0.096 & 0.000 & 0.003 & 0.000 & -4.09 & -2.86 & 795.76 & 138.12 & & & -2.86 & 139.71 \\
\hline 86 & 120 & 0.10 & 0.00 & 0.00 & 0.00 & 0.107 & 0.000 & 0.004 & 0.000 & -3.21 & -1.98 & 794.77 & 147.19 & & & -1.98 & 148.93 \\
\hline \multicolumn{18}{|c|}{$Z=35(\mathrm{Br})$} \\
\hline 26 & 61 & 0.17 & 0.00 & 0.06 & - & & 0.000 & - & 0.028 & 0. & 2.16 & 440.95 & 24 & & & 1 & 22.76 \\
\hline 27 & 62 & & 0.00 & & -0.03 & & 0.000 & -0.052 & 0.021 & & 2.34 & 459.15 & 13.89 & & & 2.34 & 12.77 \\
\hline 28 & 63 & -0.27 & 0.00 & -0.03 & -0.07 & -0.272 & 0.000 & 0.068 & 0.053 & -0.73 & 1.43 & 480.01 & 1.10 & & & 1.50 & 0.20 \\
\hline 29 & 64 & -0.26 & 0.00 & -0.01 & -0.07 & -0.264 & 0.000 & 0.044 & 0.058 & -0.10 & 2.28 & 496.05 & -6.87 & & & 2.30 & -7.66 \\
\hline 30 & 65 & -0.27 & 0.00 & -0.01 & -0.07 & -0.275 & 0.000 & 0.046 & 0.058 & 0.02 & 3.13 & 513.68 & -16.42 & & & 3.16 & -17.06 \\
\hline 31 & 66 & -0.28 & 0.00 & 0.01 & -0.07 & -0.286 & 0.000 & 0.025 & 0.062 & 0.24 & 3.17 & 529.15 & -23.82 & & & 3.17 & -24.36 \\
\hline 32 & 67 & -0.28 & 0.00 & 0.01 & -0.07 & -0.286 & 0.000 & & 0.062 & 0.7 & 3.45 & & & & & 3.47 & -32.98 \\
\hline 33 & 68 & -0.29 & 0.00 & 0.02 & -0.07 & -0.297 & 0.000 & 0.017 & 0.065 & 0.20 & 3.76 & 559.90 & -38.44 & & & 3.75 & -38.76 \\
\hline 34 & 69 & -0.30 & 0.00 & 0.02 & -0.07 & -0.307 & 0.000 & 0.019 & 0.064 & -0.02 & 3.81 & 575.69 & -46.15 & & & 3.83 & -46.35 \\
\hline 35 & 70 & -0.32 & 0.00 & 0.02 & -0.07 & -0.327 & 0.000 & 0.024 & 0.063 & -0.29 & 3.98 & 588.15 & -50.53 & & & 3.97 & -51.12 \\
\hline 36 & 71 & -0.35 & 0.00 & 0.03 & .08 & & 0.000 & 0.022 & 0.073 & -0 & 4.04 & 602.32 & & -5 & $\delta$ & 4.08 & -56.66 \\
\hline 37 & 72 & -0.35 & 0.0 & 0 & -1 & -0.357 & 0.000 & & & -0.35 & 4.47 & 613 & -59.27 & -59.01 & 0.060 & 4.49 & -59.26 \\
\hline 38 & 73 & 0.33 & 0.00 & 0.04 & -0.06 & 0.366 & 0.000 & -0.009 & 0.049 & 0.03 & 4.66 & 625.58 & -63.76 & -63.63 & 0.051 & 4.71 & -63.66 \\
\hline
\end{tabular}




\begin{tabular}{|c|c|c|c|c|c|c|c|c|c|c|c|c|c|c|c|c|c|}
\hline \multicolumn{18}{|c|}{$Z=35(\mathrm{Br})$} \\
\hline 39 & 74 & 0.36 & 0.00 & 0.04 & -0.04 & 0.401 & 0.000 & 0.002 & 0.029 & -0.38 & 4.85 & 635.55 & -65.66 & -65.31 & 0.015 & 4.86 & -65.56 \\
\hline 40 & 75 & -0.27 & 0.00 & 0.03 & -0.03 & -0.278 & 0.000 & -0.003 & 0.032 & 1.67 & 4.77 & 647.40 & -69.44 & -69.14 & 0.014 & 4.80 & -69.27 \\
\hline 41 & 76 & -0.27 & 0.00 & 0.04 & -0.02 & -0.278 & 0.000 & -0.015 & 0.027 & 1.56 & 4.76 & 656.66 & -70.62 & -70.29 & 0.009 & 4.78 & -70.44 \\
\hline 42 & 77 & -0.26 & 0.00 & 0.05 & -0.01 & -0.268 & 0.000 & -0.028 & 0.021 & 1.35 & 4.38 & 667.90 & -73.79 & -73.24 & 0.003 & 4.42 & -73.56 \\
\hline 43 & 78 & -0.27 & 0.00 & 0.05 & 0.00 & -0.278 & 0.000 & -0.027 & 0.012 & 1.02 & 4.14 & 676.53 & -74.35 & -73.45 & 0.004 & 4.16 & -74.12 \\
\hline 44 & 79 & -0.27 & 0.00 & 0.05 & 0.00 & -0.278 & 0.000 & -0.027 & 0.012 & 0.69 & 4.08 & 686.59 & -76.33 & -76.07 & 0.002 & 4.12 & -76.07 \\
\hline 45 & 80 & 0.13 & 0.00 & 0.02 & -0.01 & 0.140 & 0.000 & -0.018 & 0.007 & 1.93 & 3.45 & 694.80 & -76.48 & -75.89 & 0.002 & 3.46 & -76.25 \\
\hline 46 & 81 & 0.13 & 0.00 & 0.03 & -0.01 & 0.140 & 0.000 & -0.030 & 0.006 & 0.86 & 2.59 & 704.86 & -78.46 & -77.97 & 0.002 & 2.61 & -78.21 \\
\hline 47 & 82 & 0.13 & 0.00 & 0.03 & -0.01 & 0.140 & 0.000 & -0.030 & 0.006 & 0.09 & 1.91 & 712.38 & -77.91 & -77.50 & 0.002 & 1.93 & -77.67 \\
\hline 48 & 83 & 0.12 & 0.00 & 0.03 & -0.01 & 0.129 & 0.000 & -0.031 & 0.006 & -0.75 & 0.92 & 721.81 & -79.27 & -79.01 & 0.004 & 0.94 & -79.03 \\
\hline 49 & 84 & 0.09 & 0.00 & 0.02 & -0.01 & 0.097 & 0.000 & -0.021 & 0.008 & -1.16 & 0.13 & 728.74 & -78.13 & -77.80 & 0.015 & 0.14 & -77.91 \\
\hline 50 & 85 & -0.04 & 0.00 & 0.00 & 0.00 & -0.042 & 0.000 & 0.001 & 0.000 & -1.54 & -0.64 & 737.24 & -78.56 & -78.61 & 0.019 & -0.64 & -78.35 \\
\hline 51 & 86 & -0.09 & 0.00 & 0.00 & 0.00 & -0.094 & 0.000 & 0.003 & -0.000 & -1.15 & -0.08 & 742.16 & -75.40 & -75.64 & 0.011 & -0.07 & -75.21 \\
\hline 52 & 87 & -0.10 & 0.00 & 0.00 & 0.00 & -0.105 & 0.000 & 0.004 & -0.000 & -0.29 & 0.79 & 748.36 & -73.54 & -73.86 & 0.018 & 0.79 & -73.35 \\
\hline 53 & 88 & 0.12 & 0.00 & 0.02 & 0.00 & 0.129 & 0.000 & -0.018 & -0.002 & 0.30 & 1.75 & 752.25 & -69.36 & -70.73 & 0.038 & 1.77 & -69.17 \\
\hline 54 & 89 & 0.18 & 0.00 & 0.01 & 0.00 & 0.195 & 0.000 & 0.002 & -0.001 & 0.44 & 2.74 & 757.70 & -66.73 & -68.57 & 0.060 & 2.77 & -66.56 \\
\hline 55 & 90 & 0.19 & 0.00 & 0.02 & 0.00 & 0.206 & 0.000 & -0.009 & -0.003 & 0.60 & 2.78 & 761.93 & -62.89 & -64.62 & 0.077 & 2.81 & -62.73 \\
\hline 56 & 91 & 0.22 & 0.00 & 0.04 & 0.01 & 0.241 & 0.000 & -0.026 & -0.019 & 0.43 & 3.13 & 767.43 & -60.32 & -61.51 & 0.073 & 3.22 & -60.11 \\
\hline 57 & 92 & 0.22 & 0.00 & 0.04 & 0.00 & 0.241 & 0.000 & -0.028 & -0.009 & 0.80 & 3.49 & 770.79 & -55.61 & -56.58 & 0.050 & 3.55 & -55.44 \\
\hline 58 & 93 & 0.22 & 0.00 & 0.04 & 0.00 & 0.241 & 0.000 & -0.028 & -0.009 & 1.15 & 3.77 & 775.80 & -52.56 & & & 3.84 & -52.38 \\
\hline 59 & 94 & 0.29 & 0.00 & -0.03 & 0.00 & 0.317 & 0.000 & 0.075 & 0.017 & -0.51 & 3.84 & 778.92 & -47.60 & & & 3.97 & -47.38 \\
\hline 60 & 95 & 0.31 & 0.00 & -0.03 & 0.01 & 0.339 & 0.000 & 0.081 & 0.009 & -1.00 & 3.83 & 783.70 & -44.30 & & & 3.99 & -44.06 \\
\hline 61 & 96 & 0.31 & 0.00 & -0.02 & 0.00 & 0.340 & 0.000 & 0.068 & 0.014 & -0.92 & 3.75 & 786.48 & -39.02 & & & 3.86 & -38.83 \\
\hline 62 & 97 & 0.31 & 0.00 & -0.01 & 0.01 & 0.340 & 0.000 & 0.056 & 0.000 & -0.56 & 3.83 & 790.67 & -35.13 & & & 3.94 & -34.95 \\
\hline 63 & 98 & 0.31 & 0.00 & -0.01 & 0.00 & 0.340 & 0.000 & 0.055 & 0.010 & -0.60 & 3.96 & 792.78 & -29.17 & & & 4.04 & -29.01 \\
\hline 64 & 99 & 0.30 & 0.00 & 0.01 & -0.01 & 0.330 & 0.000 & 0.026 & 0.011 & -0.06 & 4.08 & 796.45 & -24.78 & & & 4.16 & -24.61 \\
\hline 65 & 100 & 0.29 & 0.00 & 0.03 & -0.02 & 0.319 & 0.000 & -0.002 & 0.013 & 0.03 & 3.89 & 798.44 & -18.69 & & & 3.97 & -18.51 \\
\hline 66 & 101 & 0.28 & 0.00 & 0.05 & -0.03 & 0.309 & 0.000 & -0.031 & 0.016 & 0.11 & 3.56 & 802.12 & -14.30 & & & 3.75 & -14.01 \\
\hline 67 & 102 & 0.28 & 0.00 & 0.06 & -0.03 & 0.310 & 0.000 & -0.043 & 0.013 & -0.36 & 3.25 & 803.80 & -7.91 & & & 3.46 & -7.59 \\
\hline 68 & 103 & 0.28 & 0.00 & 0.07 & -0.04 & 0.310 & 0.000 & -0.056 & 0.019 & -0.75 & 2.90 & 807.08 & -3.12 & & & 3.26 & -2.62 \\
\hline 69 & 104 & 0.29 & 0.00 & 0.09 & -0.04 & 0.324 & 0.000 & -0.078 & 0.011 & -1.62 & 2.46 & 808.50 & 3.53 & & & 2.90 & 4.14 \\
\hline 70 & 105 & 0.28 & 0.00 & 0.09 & -0.04 & 0.312 & 0.000 & -0.080 & 0.012 & -1.46 & 2.33 & 811.15 & 8. & & & 2 & 9.64 \\
\hline 71 & 106 & 0.27 & 0.00 & 0.09 & -0.04 & 0.301 & 0.000 & -0.083 & 0.013 & -1.56 & 2.02 & 812.07 & 16.11 & & & 2.51 & 16.83 \\
\hline 72 & 107 & 0.25 & 0.00 & 0.08 & -0.03 & 0.277 & 0.000 & -0.073 & 0.008 & -0.89 & 2.18 & 814.05 & 22.20 & & & 2.57 & 22.86 \\
\hline 73 & 108 & 0.25 & 0.00 & 0.08 & -0.04 & 0.277 & 0.000 & -0.075 & 0.018 & -0.90 & 1.86 & 814.61 & 29.71 & & & 2.34 & 30.51 \\
\hline 74 & 109 & 0.21 & 0.00 & 0.07 & -0.03 & 0.231 & 0.000 & -0.069 & 0.014 & -0.13 & 1.88 & 816.37 & 36.02 & & & 2.26 & 36.78 \\
\hline 75 & 110 & 0.20 & 0.00 & 0.06 & -0.03 & 0.219 & 0.000 & -0.058 & 0.017 & -0.51 & 1.60 & 816.56 & 43.91 & & & 1.92 & 44.66 \\
\hline 76 & 111 & -0.15 & 0.00 & 0.06 & 0.03 & -0.155 & 0.000 & -0.060 & -0.017 & -0.27 & 1.22 & 818.37 & 50.17 & & & 1.59 & 51.03 \\
\hline 77 & 112 & -0.15 & 0.00 & 0.06 & 0.03 & -0.155 & 0.000 & -0.060 & -0.017 & -1.14 & 0.44 & 818.72 & 57.88 & & & 0.81 & 58.81 \\
\hline 78 & 113 & 0.11 & 0.00 & 0.04 & 0.00 & 0.119 & 0.000 & -0.043 & -0.005 & -1.52 & -0.14 & 820.41 & 64.27 & & & -0.02 & 65.04 \\
\hline 79 & 114 & 0.09 & 0.00 & 0.02 & 0.00 & 0.097 & 0.000 & -0.021 & -0.002 & -2.54 & -1.30 & 820.83 & 71.92 & & & -1.27 & 72.68 \\
\hline 80 & 115 & 0.09 & 0.00 & 0.03 & -0.01 & 0.097 & 0.000 & -0.033 & 0.007 & -3.54 & -2.24 & 822.56 & 78.26 & & & - & 79.17 \\
\hline 81 & 116 & 0.04 & 0.00 & 0.01 & 0.00 & 0.043 & 0.000 & -0.011 & -0.000 & -5.01 & -3.83 & 823.13 & 85.76 & & & -3.83 & 86.69 \\
\hline 82 & 117 & 0.00 & 0.00 & 0.00 & 0.00 & 0.000 & 0.000 & 0.000 & 0.000 & -6.15 & -4.88 & 824.67 & 92.30 & & & -4.88 & 93.32 \\
\hline 83 & 118 & -0.03 & 0.00 & 0.00 & 0.00 & -0.032 & 0.000 & 0.000 & 0.000 & -5.41 & -4.32 & 822.79 & 102.24 & & & -4.32 & 103.38 \\
\hline 84 & 119 & 0.01 & 0.00 & 0.00 & 0.00 & 0.011 & 0.000 & 0.000 & 0.000 & -4.44 & -3.43 & 822.11 & 111.00 & & & -3.43 & 112.25 \\
\hline 85 & 120 & 0.06 & 0.00 & 0.00 & 0.00 & 0.064 & 0.000 & 0.002 & 0.000 & -3.87 & -2.81 & 819.91 & 121.26 & & & -2.82 & 122.65 \\
\hline 86 & 121 & 0.09 & 0.00 & 0.00 & 0.00 & 0.096 & 0.000 & 0.003 & 0.000 & -2.98 & -1.85 & 818.88 & 130.37 & & & -1.85 & 131.89 \\
\hline 87 & 122 & 0.10 & 0.00 & 0.02 & 0.00 & 0.107 & 0.000 & -0.020 & -0.002 & -2.54 & -1.40 & 816.59 & & & & & 142.42 \\
\hline 88 & 123 & 0.11 & 0.00 & 0.01 & 0.00 & 0.118 & 0.000 & -0.007 & -0.001 & -1.68 & -0.54 & 815.40 & 149.99 & & & -0.54 & 151.81 \\
\hline \multicolumn{18}{|c|}{$Z=36(\mathrm{Kr})$} \\
\hline 27 & 63 & -0.13 & 0.00 & 0.01 & -0.01 & -0.135 & 0.000 & -0.004 & 0.011 & 1.12 & 2.21 & 456.83 & 23.50 & & & 2.19 & 22.26 \\
\hline 28 & 64 & -0.13 & 0.00 & 0.00 & -0.01 & -0.135 & 0.000 & 0.007 & 0.009 & 0. & 1.57 & 478.49 & . & & & & 8.84 \\
\hline 29 & 65 & -0.14 & 0.00 & 0.01 & -0.01 & -0.146 & 0.000 & -0.003 & 0.011 & 1.2 & 2.34 & 494.84 & 1.63 & & & 2.33 & 0.71 \\
\hline 30 & 66 & -0.27 & 0.00 & -0.01 & -0.07 & -0.275 & 0.000 & 0.046 & 0.058 & 0.30 & 2.84 & 513.86 & -9.32 & & & 2.91 & -10.01 \\
\hline 31 & 67 & -0.27 & 0.00 & 0.00 & -0.07 & -0.275 & 0.000 & 0.034 & 0.060 & 0.66 & 3.26 & 529.18 & -16.57 & & & 3.29 & -17.17 \\
\hline 32 & 68 & -0.30 & 0.00 & 0.01 & -0.07 & -0.306 & 0.000 & 0.030 & 0.061 & 0.38 & 3.59 & 546.97 & -26.29 & & & 3.65 & -26.74 \\
\hline
\end{tabular}




\begin{tabular}{|c|c|c|c|c|c|c|c|c|c|c|c|c|c|c|c|c|c|}
\hline \multicolumn{18}{|c|}{$Z=36(\mathrm{Kr})$} \\
\hline 33 & 69 & -0.32 & 0.00 & 0.02 & -0.07 & -0.327 & 0.000 & 0.024 & 0.063 & 0.06 & 3.92 & 561.11 & -32.35 & & & 3.94 & -32.74 \\
\hline 34 & 70 & -0.32 & 0.00 & 0.02 & -0.07 & -0.327 & 0.000 & 0.024 & 0.063 & -0.10 & 3.89 & 577.98 & -41.15 & & & 3.94 & -41.40 \\
\hline 35 & 71 & -0.35 & 0.00 & 0.03 & -0.08 & -0.357 & 0.000 & 0.022 & 0.073 & -0.74 & 4.03 & 591.12 & -46.22 & -46.92 & 0.652 & 4.06 & -46.40 \\
\hline 36 & 72 & -0.36 & 0.00 & 0.03 & -0.08 & -0.366 & 0.000 & 0.025 & 0.073 & -0.91 & 4.05 & 606.72 & -53.76 & -53.94 & 0.008 & 4.13 & -53.81 \\
\hline 37 & 73 & -0.36 & 0.00 & 0.03 & -0.08 & -0.366 & 0.000 & 0.025 & 0.073 & -0.50 & 4.32 & 617.80 & -56.76 & -56.55 & 0.007 & 4.37 & -56.78 \\
\hline 38 & 74 & 0.36 & 0.00 & 0.04 & -0.05 & 0.401 & 0.000 & 0.001 & 0.039 & -0.92 & 4.35 & 631.49 & -62.38 & -62.33 & 0.002 & 4.41 & -62.32 \\
\hline 39 & 75 & 0.36 & 0.00 & 0.05 & -0.04 & 0.402 & 0.000 & -0.010 & 0.024 & -0.89 & 4.39 & 641.82 & & & 0.008 & 4.41 & \\
\hline 40 & 76 & 0.36 & 0.00 & 0.06 & -0.05 & 0.403 & 0.000 & -0.024 & 0.029 & -0.71 & 4.50 & 654.42 & -69.16 & & 0.004 & 4.58 & \\
\hline 41 & 77 & 0.32 & 0.00 & 0.05 & -0.04 & 0.355 & 0.000 & -0.022 & 0.025 & 0.39 & 4.55 & 663.81 & -70.48 & -70.17 & 0.002 & 4.59 & -70.32 \\
\hline 42 & 78 & -0.24 & 0.00 & 0.05 & 0.00 & -0.248 & 0.000 & -0.033 & 0.011 & 1.84 & 4.44 & 675.69 & -74.29 & -74.18 & 0.001 & 4.48 & -74.10 \\
\hline 43 & 79 & -0.25 & 0.00 & 0.06 & 0.01 & -0.258 & 0.000 & -0.043 & 0.006 & 1.46 & 4.22 & 684.49 & -75.02 & -74.44 & 0.004 & 4.25 & -74.81 \\
\hline 44 & 80 & -0.26 & 0.00 & 0.05 & 0.00 & -0.268 & 0.000 & -0.029 & 0.012 & 1.02 & 3.79 & 695.82 & -78.28 & & 0.001 & 3.84 & -78.03 \\
\hline 45 & 81 & 0.12 & 0.00 & 0.02 & -0.01 & & 0.000 & -0.019 & 0.007 & 2.2 & 3.41 & & & & 0.002 & 3.42 & \\
\hline 46 & 82 & 0.12 & 0.00 & 0.03 & -0.01 & 0.129 & 0.000 & -0.031 & 0.006 & 1.1 & 2.56 & & -81.21 & & 0.002 & 2.58 & \\
\hline 47 & 83 & 0.12 & 0.00 & 0.03 & -0.01 & 0.129 & 0.000 & -0.031 & 0.006 & 0.38 & 1.89 & 722.58 & -80.82 & -79.98 & 0.003 & 1.91 & -80.59 \\
\hline 48 & 84 & 0.08 & 0.00 & 0.02 & 0.00 & 0.086 & 0.000 & -0.021 & -0.002 & -0.27 & 0.80 & 732.97 & -83.14 & -8 & 0. & 0.81 & -82.92 \\
\hline 49 & 85 & 0.07 & 00 & 0.02 & 0.00 & & 0.000 & & -0.002 & & & & & & & & \\
\hline 50 & 86 & 0.00 & 0.00 & 0.00 & 0.00 & 0.000 & 0.000 & 0.000 & 0.000 & -1.76 & & & & & 0.000 & & \\
\hline 51 & 87 & -0.07 & 0.00 & 0.01 & 0.00 & -0.073 & 0.000 & -0.010 & 0.001 & -1.12 & -0.17 & 75 & & & 0.000 & -0.16 & -80.21 \\
\hline 52 & 88 & -0.10 & 0.00 & 0.00 & 0.00 & -0.105 & 0.000 & 0.004 & -0.000 & -0.28 & 0.82 & 761.35 & -79.24 & -79.69 & 0.013 & 0.83 & -79.05 \\
\hline 53 & 89 & 0.12 & 0.00 & 0.02 & 0.00 & 29 & 000 & -0 & -0 & 0.55 & 1.87 & 30 & -7 & -7 & 0.052 & 89 & -74.93 \\
\hline 54 & 90 & 0.16 & 0.00 & 0.01 & 0.00 & 0.173 & 0.000 & -0.001 & -0.001 & 0. & 2.79 & 771.64 & & -7 & 0. & 81 & -73.20 \\
\hline 55 & 91 & 0.19 & 0.00 & 0.02 & 0.01 & 0.2 & 0.000 & -0.008 & -0.013 & 0. & 2.97 & 775.85 & & -7 & & 2 & \\
\hline 56 & 92 & 0.22 & 0.00 & 0.04 & 0.01 & & 0.000 & -0.026 & -0.019 & & 3.34 & & & & & 4 & \\
\hline 57 & 93 & 0.22 & 0.00 & 0.05 & 0.00 & 0.242 & 0.000 & -0.040 & -0.011 & 0.99 & 3.66 & 785.66 & -63.19 & & & 3.76 & -62.98 \\
\hline 58 & 94 & 0.28 & 0.00 & 0.01 & -0.01 & 0.3 & .000 & 0.1 & 0.018 & 0 . & 3.99 & 791.39 & -60.85 & & & 4.12 & -60.62 \\
\hline 59 & 95 & 0.30 & 0.00 & -0.02 & 0.00 & & 000 & & & 0 & & & & & & & \\
\hline 60 & 96 & 0.31 & 0.00 & -0.02 & 0.00 & 0. & 0.000 & & 0.014 & -1.02 & 3.78 & 800.45 & -5 & & & 3 & -53.53 \\
\hline 61 & 97 & 0.31 & 0.00 & -0.01 & 0.00 & 0.340 & 0.000 & 0.055 & 0.010 & -0.96 & 3.65 & 803.40 & -48.64 & & & 3.76 & -48.46 \\
\hline 62 & 98 & 0.32 & 0.00 & 0.00 & 0.00 & 0.352 & 0.000 & 0.045 & 0.006 & -0.92 & 3.67 & 808.39 & -45.56 & & & 3.79 & -45.37 \\
\hline 63 & 99 & 0.31 & 00 & 0.01 & -0.01 & 0 . & 0.000 & 0.029 & 12 & -0.65 & 5 & 810.86 & -3 & & & 4 & \\
\hline 64 & 100 & 0.31 & 00 & 0.02 & -0.02 & 0 & 0.000 & 0.015 & 17 & & & 815.19 & & & & 88 & \\
\hline 65 & 101 & & & & 02 & & & & & -1 & 3 & & & & & 75 & \\
\hline 66 & 102 & 0.30 & ( & 0.05 & -0.03 & & 0.000 & -0. & 5 & -0 & 3. & 821.50 & -2 & & & 0 & \\
\hline 67 & 103 & 0.30 & 0.00 & 0.06 & -0.04 & 0.333 & 0.000 & -0.039 & 0.021 & -0.99 & 3.03 & 823.33 & -20.15 & & & 3.33 & -19.76 \\
\hline 68 & 104 & 0.29 & 00 & 0.07 & -0.04 & 0322 & 0.000 & -0.054 & 0.018 & -1.07 & 2.81 & 827.18 & -15.93 & & & 3.16 & -15.46 \\
\hline 69 & 105 & & & & 4 & & & & & & & & & & & & \\
\hline 70 & 106 & & & & 0.04 & & & & & & & & & & & & -3.87 \\
\hline 71 & 107 & 0.28 & 0.00 & 0.09 & -0.04 & & 0.000 & -0.080 & 0.012 & -1.73 & 2.12 & & 2.64 & & & 0 & 3.30 \\
\hline 72 & 108 & 0.27 & 0.00 & 0.08 & -0.04 & 0.300 & 0.000 & -0.071 & 0.016 & -1.13 & 2.27 & 835.49 & 8.05 & & & 2.73 & 8.72 \\
\hline 73 & 109 & 0.25 & 0.00 & 0.09 & -0.04 & 0.278 & 0.000 & -0.087 & 0.015 & -0.97 & 2.12 & 835.97 & 15.64 & & & 2.67 & 16.45 \\
\hline 74 & 110 & 0.21 & 00 & 0.07 & -0.03 & & 0.000 & -0.069 & & & 2.17 & 838.35 & 21.33 & & & 2.55 & 22.01 \\
\hline 75 & 111 & -0.15 & & & & & & & & & & & & & & & 29.79 \\
\hline 76 & 112 & -0.15 & & & 0 & -0 & n & -0 & -0 . & -0 & & & 34. & & & 1. & 14 \\
\hline 77 & 113 & -0.12 & 0.00 & 0.06 & 0.03 & -0.124 & 0.000 & -0.063 & -0.019 & -0.82 & 0.42 & 841.79 & 42.10 & & & 0.80 & 42.95 \\
\hline 78 & 14 & -0.12 & 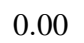 & 0.06 & 0.03 & 011 & 0.000 & -0.063 & -0.019 & -1.39 & 0.20 & 844.15 & 1701 & & & & 48 \\
\hline 79 & 115 & & & & 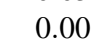 & & 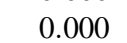 & 2 & 2 & & .02 & & & & & & \\
\hline 80 & 116 & 0.05 & & & 0.00 & 0.0 & 0.000 & -0.011 & -0.001 & -3.10 & -2.01 & 846.70 & 61.41 & & & -2.00 & 62.11 \\
\hline 81 & 117 & 0.04 & 0.00 & 0.01 & 0.00 & 0.043 & 0.000 & -0.011 & -0.000 & -4.91 & -3.71 & 847.42 & 68.75 & & & -3.70 & 69.54 \\
\hline 82 & 118 & 0.00 & 0.00 & 0.00 & 0.00 & 0.000 & 0.000 & 0.000 & 0.000 & -6.11 & -4.80 & 849.62 & 74.63 & & & -4.80 & 75.51 \\
\hline 83 & 119 & -0.03 & c & 0 & .00 & -0.032 & 00 & -0.0 & 00 & -5.32 & -4.19 & 847.74 & 84.58 & & & -4.18 & 85.56 \\
\hline 84 & 120 & 0.00 & 00 & 0 & 0.00 & 0.0 & 0.000 & 0.000 & & -4.35 & -3.34 & 847.70 & 92.69 & & & -3.34 & 93.78 \\
\hline 85 & 121 & 0.04 & 0.00 & 0.00 & 0.00 & 0.043 & 0.000 & 0.001 & 0.000 & -3.63 & -2.62 & 845.44 & 103.02 & & & -2.62 & 104.22 \\
\hline 86 & 122 & 0.06 & 0.00 & & 0.0 & & & & & & -1.61 & 844.95 & & & & & \\
\hline 87 & 123 & 0.08 & 0.05 & 0.00 & 0.00 & 0.086 & -0.068 & 0.004 & 0.002 & -2.34 & -1.12 & 842.67 & 121.94 & & & -1.03 & 123.48 \\
\hline 88 & 124 & 0.10 & 0.07 & 0.00 & 0.00 & 0.109 & -0.096 & 0.006 & 0.004 & -1.87 & -0.37 & 842.16 & 130.52 & & & -0.20 & 132.28 \\
\hline
\end{tabular}




\begin{tabular}{|c|c|c|c|c|c|c|c|c|c|c|c|c|c|c|c|c|c|}
\hline \multicolumn{18}{|c|}{$Z=37(\mathbf{R b})$} \\
\hline 29 & 66 & -0.13 & 0.00 & 0.02 & 0.00 & -0.136 & 0.000 & -0.016 & 0.003 & 1.44 & 2.53 & 490.71 & 13.05 & & & 2.51 & 12.04 \\
\hline 30 & 67 & 0.28 & 0.00 & 0.02 & -0.01 & 0.308 & 0.000 & 0.009 & 0.007 & 0.69 & 3.07 & 509.91 & 1.92 & & & 3.00 & 1.01 \\
\hline 31 & 68 & -0.27 & 0.00 & 0.01 & -0.05 & -0.277 & 0.000 & 0.021 & 0.045 & 1.21 & 3.70 & 526.04 & -6.14 & & & 3.68 & -6.87 \\
\hline 32 & 69 & -0.32 & 0.00 & 0.02 & -0.07 & -0.327 & 0.000 & 0.024 & 0.063 & 0.60 & 3.97 & 544.11 & -16.13 & & & 4.00 & -16.70 \\
\hline 33 & 70 & -0.34 & 0.00 & 0.04 & -0.07 & -0.348 & 0.000 & 0.008 & 0.069 & 0.16 & 4.15 & 559.39 & -23.34 & & & 4.14 & -23.84 \\
\hline 34 & 71 & -0.35 & 0.00 & 0.03 & -0.08 & -0.357 & 0.000 & 0.022 & 0.073 & -0.29 & 4.11 & 576.48 & -32.36 & & & 4.14 & -32.71 \\
\hline 35 & 72 & -0.36 & 0.00 & 0.03 & -0.08 & -0.366 & 0.000 & 0.025 & 0.073 & -0.59 & 4.27 & 590.57 & -38.38 & & & 4.26 & -38.68 \\
\hline 36 & 73 & -0.36 & 0.00 & 0.03 & -0.08 & -0.366 & 0.000 & 0.025 & 0.073 & -0.64 & 4.24 & 606.45 & -46.19 & & & 4.27 & -46.36 \\
\hline 37 & 74 & 0.33 & 0.00 & 0.04 & -0.06 & 0.366 & 0.000 & -0.009 & 0.049 & -0.55 & 4.22 & 619.19 & -50.86 & -51 & 0.004 & 4.20 & -51.42 \\
\hline 38 & 75 & 0.36 & 0.00 & 0.05 & -0.05 & 0.402 & 0.000 & -0.012 & 0.034 & -1.47 & 3.90 & 633.83 & -57.43 & -57.22 & 0.007 & 3.91 & -57.48 \\
\hline 39 & 76 & 0.36 & 0.00 & 0.06 & -0.05 & 0.403 & 0.000 & -0.024 & 0.029 & -1.58 & 3.90 & 645.13 & -60.65 & -60.48 & 0.002 & 3.89 & -60.68 \\
\hline 40 & 77 & 0.36 & 0.00 & 0.06 & -0.04 & 0.403 & 0.000 & -0.022 & 0.020 & -1.36 & 4.00 & 657.94 & -65.40 & -64.82 & 0.007 & 4.02 & -65.34 \\
\hline 41 & 78 & 0.33 & 0.00 & 0.05 & -0.03 & 0.367 & 0.000 & -0.018 & 0.015 & -0.34 & 4.23 & 668.07 & -67.45 & -66.94 & 0.007 & 4.22 & -67.38 \\
\hline 42 & 79 & 0.32 & 0.00 & 0.05 & -0.03 & 0.355 & 0.000 & -0.020 & 0.015 & 0.16 & 4.27 & 679.99 & -71.31 & -70.80 & 0.006 & 4.30 & -71.16 \\
\hline 43 & 80 & -0.24 & 0.00 & 0.06 & 0.02 & -0.247 & 0.000 & -0.045 & -0.004 & 1.75 & 4.28 & 689.46 & -72.70 & -72.17 & 0.007 & 4.29 & -72.55 \\
\hline 44 & 81 & -0.23 & 0.00 & 0.06 & 0.03 & -0.237 & 0.000 & -0.048 & -0.013 & 1.52 & 3.80 & 701.01 & -76.18 & -75.46 & 0.006 & 3.84 & -75.99 \\
\hline 45 & 82 & 0.08 & 0.00 & 0.01 & 0.00 & 0.086 & 0.000 & -0.009 & -0.001 & 2.73 & 3.26 & 710.20 & -77.30 & -7 & 0.003 & 3.26 & -77.12 \\
\hline 46 & 83 & 0.08 & 0.00 & 0.02 & 0.00 & 0.086 & 0.000 & -0.021 & -0.002 & 1.67 & 2.45 & 721.26 & -80.29 & -79.07 & 0.006 & 2.46 & -80.10 \\
\hline 47 & 84 & 0.09 & 0.00 & 0.01 & 0.00 & 0.096 & 0.000 & -0.009 & -0.001 & 0.89 & 1.83 & 729.76 & -80.71 & -79.75 & 0.003 & 1.83 & -80.51 \\
\hline 48 & 85 & 0.06 & 0.00 & 0.01 & 0.00 & 0.064 & 0.000 & -0.010 & -0.001 & -0.08 & 0.83 & 740.23 & -83.12 & -82.17 & 0.000 & 0.83 & -82.92 \\
\hline 49 & 86 & 0.07 & 0.00 & 0.02 & 0.00 & 0.075 & 0.000 & -0.022 & -0.002 & -1.09 & 0.06 & 748.14 & -82.95 & -82.75 & 0.000 & 0.07 & -82.75 \\
\hline 50 & 87 & -0.02 & 0.00 & 0.01 & 0.00 & -0.021 & 0.000 & -0.012 & 0.000 & -1.82 & -0.75 & 757.69 & -84.43 & -84.60 & 0.000 & -0.75 & -84.24 \\
\hline 51 & 88 & -0.07 & 0.00 & 0.00 & 0.00 & -0.073 & 0.000 & 0.002 & -0.000 & -1.15 & -0.14 & 763.54 & -82.21 & -82.61 & 0.000 & -0.14 & -82.02 \\
\hline 52 & 89 & -0.09 & 0.00 & 0.00 & 0.00 & -0.094 & 0.000 & 0.003 & -0.000 & -0.19 & 0.86 & 770.57 & -81.17 & -81.71 & 0.005 & 0.87 & -80.99 \\
\hline 53 & 90 & -0.10 & 0.00 & 0.01 & 0.00 & -0.105 & 0.000 & -0.008 & 0.001 & 0.81 & 1.97 & 775.28 & -77.81 & -79.36 & 0.007 & 1.97 & -77.63 \\
\hline 54 & 91 & -0.13 & 0.00 & 0.00 & 0.00 & -0.135 & 0.000 & 0.007 & -0.000 & 1.55 & 2.89 & 781.75 & -76.21 & -77.75 & 0.008 & 2.90 & -76.04 \\
\hline 55 & 92 & 0.22 & 0.00 & 0.02 & 0.01 & 0.240 & 0.000 & -0.002 & -0.013 & 0.77 & 3.30 & 786.53 & -72.92 & -74.77 & 0.006 & 3.34 & -72.74 \\
\hline 56 & 93 & 0.23 & 0.00 & 0.03 & 0.01 & 0.252 & 0.000 & -0.013 & -0.016 & 0.85 & 3.60 & 793.01 & -71.33 & -72.62 & 0.008 & 3.66 & -71.13 \\
\hline 57 & 94 & 0.26 & 0.00 & 0.01 & 0.00 & 0.284 & 0.000 & 0.017 & 0.000 & 0.57 & 3.86 & 797.37 & -67.62 & -68.55 & 0. & 3.90 & -67.46 \\
\hline 58 & 95 & 0.29 & 0.00 & -0.01 & -0.01 & 0.318 & 0.000 & 0.048 & 0.019 & -0.30 & 3.93 & 803.50 & -65.68 & -65.85 & 0.021 & 4.03 & -65.47 \\
\hline 59 & 96 & 0.30 & 0.00 & -0.01 & -0.01 & 0.329 & 0.000 & 0.051 & 0.019 & -0.88 & 3.69 & 807.82 & -61.92 & -61.22 & 0.029 & 3.78 & -61.73 \\
\hline 60 & 97 & 0.31 & 0.00 & -0.01 & 0.00 & 0.340 & 0.000 & 0.055 & 0.010 & -1.14 & 3.65 & .51 & -59.54 & -58.36 & 31 & 3.75 & -59.35 \\
\hline 61 & 98 & 0.31 & 0.00 & 0.00 & -0.01 & 0.341 & 0.000 & 0.041 & 0.016 & -1.21 & 3.43 & 817.29 & -55.25 & -54.22 & 0.050 & 3.51 & -55.10 \\
\hline 62 & 99 & 0.32 & 0.00 & 0.00 & 0.00 & 0.352 & 0.000 & 0.045 & 0.006 & -1.26 & 3.52 & 822.32 & -52.21 & -50.88 & 0.126 & 3.61 & -52.05 \\
\hline 63 & 100 & 0.32 & 0.00 & 0.01 & -0.01 & 0.353 & 0.000 & 0.032 & 0.012 & -1.25 & 3.45 & 825.47 & -47.28 & & & 3.52 & -47.15 \\
\hline 64 & 101 & 0.32 & 0.00 & 0.02 & -0.01 & 0.353 & 0.000 & 0.019 & 0.008 & -1.02 & 3.50 & 830.05 & -43.79 & -43.60 & 0.166 & 3.58 & -43.65 \\
\hline 65 & 102 & 0.31 & 0.00 & 0.03 & -0.02 & 0.342 & 0.000 & 0.003 & 0.013 & -0.95 & 3.30 & 832.86 & -38.53 & & & 3.39 & -38.39 \\
\hline 66 & 103 & 0.31 & 0.00 & 0.04 & -0.03 & 0.343 & 0.000 & -0.011 & 0.019 & -0.98 & 3.28 & 837.04 & -34.64 & & & 3.45 & -34.42 \\
\hline 67 & 104 & 0.31 & 0.00 & 0.06 & -0.03 & 0.344 & 0.000 & -0.035 & 0.011 & -1.44 & 2.99 & 839.51 & -29.04 & & & 3.16 & -28.80 \\
\hline 68 & 105 & 0.31 & 0.00 & 0.07 & -0.04 & 0.345 & 0.000 & -0.049 & 0.017 & -1.69 & 2.77 & 843.45 & -24.91 & & & 3.07 & -24.53 \\
\hline 69 & 106 & 0.30 & 0.00 & 0.08 & -0.04 & 0.334 & 0.000 & -0.064 & 0.014 & -2.02 & 2.41 & .56 & -18.95 & & & 2.74 & -18.53 \\
\hline 70 & 107 & 0.30 & 0.00 & 0.08 & -0.04 & 0.334 & 0.000 & -0.064 & 0.014 & -1.93 & 2.41 & 848.86 & -14.18 & & & 2.77 & -13.70 \\
\hline 71 & 108 & 0.29 & 0.00 & 0.08 & -0.04 & 0.323 & 0.000 & -0.066 & 0.015 & -1.83 & 2.24 & 850.38 & -7.62 & & & 2.61 & -7.13 \\
\hline 72 & 109 & 0.29 & 0.00 & 0.08 & -0.04 & 0.323 & 0.000 & -0.066 & 0.015 & -1.48 & 2.45 & 853.07 & -2.24 & & & 2.85 & -1.69 \\
\hline 73 & 110 & 0.29 & 0.00 & 0.07 & -0.04 & 0.322 & 0.000 & -0.054 & 0.018 & -1.22 & 2.56 & 853.94 & 4.95 & & & 2.89 & 5.48 \\
\hline 74 & 111 & 0.27 & 0.00 & 0.07 & -0.04 & 0.299 & 0.000 & -0.059 & 19 & -0.56 & 2.58 & & 10.54 & & & 2.97 & 11.15 \\
\hline 75 & 112 & -0.15 & 0.00 & 0.07 & 0.03 & -0.154 & 0.000 & -0.071 & -0.015 & 0.42 & 2.02 & 857.61 & 17.43 & & & 2.39 & 18.08 \\
\hline 76 & 113 & -0.12 & 0.00 & 0.06 & 0.03 & -0.124 & 0.000 & -0.063 & -0.019 & 0.17 & 1.47 & 860.32 & 22.79 & & & 1.81 & 23.46 \\
\hline 77 & 114 & -0.12 & 0.00 & 0.07 & 0.03 & -0.123 & 0.000 & -0.074 & -0.017 & -1.01 & 0.44 & 861.62 & 29.56 & & & 0.86 & 30.36 \\
\hline 78 & 115 & -0.12 & 0.00 & 0.06 & 0.02 & -0.124 & 0.000 & -0.063 & -0.010 & -1.46 & -0.09 & 863.96 & 35.29 & & & 0.19 & 36.02 \\
\hline 79 & 116 & 0.05 & 0.00 & 0.01 & 0.00 & 0.053 & 0.000 & -0.011 & -0.001 & -1.92 & -0.96 & 864.77 & 42.55 & & & -0.95 & 43.06 \\
\hline 80 & 117 & 0.04 & 0.00 & 0.01 & 0.00 & 0.043 & 0.000 & -0.011 & -0.000 & -3.08 & -2.00 & 867.29 & 48.11 & & & -2.00 & 48.69 \\
\hline 81 & 118 & 0.03 & 0.00 & 0.01 & 0.00 & 0.032 & 0.000 & -0.012 & -0.000 & -4.96 & -3.73 & 868.65 & 54.82 & & & -3.72 & 55.47 \\
\hline 82 & 119 & 0.01 & 0.00 & 0.00 & 0.00 & 0.011 & 0.000 & 0.000 & 0.000 & -6.20 & -4.83 & 870.91 & 60.63 & & & -4.83 & 61.36 \\
\hline 83 & 120 & -0.03 & 0.00 & 0.00 & 0.00 & -0.032 & 0.000 & 0.000 & 0.000 & -5.39 & -4.22 & 869.64 & 69.97 & & & -4.22 & 70.80 \\
\hline 84 & 121 & 0.01 & 0.00 & 0.00 & 0.00 & 0.011 & 0.000 & 0.000 & 0.000 & -4.39 & -3.30 & 869.57 & 78.11 & & & -3.30 & 79.04 \\
\hline 85 & 122 & 0.03 & 0.00 & 0.00 & 0.00 & 0.032 & 0.000 & 0.000 & 0.000 & -3.64 & -2.65 & 867.98 & 87.77 & & & -2.65 & 88.80 \\
\hline
\end{tabular}




\begin{tabular}{|c|c|c|c|c|c|c|c|c|c|c|c|c|c|c|c|c|c|}
\hline \multicolumn{18}{|c|}{$Z=37(\mathbf{R b})$} \\
\hline 86 & 123 & 0.03 & 0.00 & 0.00 & 0.00 & 0.032 & 0.000 & 0.000 & 0.000 & -2.46 & -1.59 & 867.49 & 96.33 & & & -1.60 & 97.48 \\
\hline 87 & 124 & 0.08 & 0.07 & 0.00 & 0.00 & 0.087 & -0.095 & 0.004 & 0.004 & -2.57 & -1.07 & 865.75 & 106.15 & & & -0.92 & 107.56 \\
\hline 88 & 125 & 0.09 & 0.08 & 0.00 & 0.00 & 0.099 & -0.109 & 0.006 & 0.005 & -1.91 & -0.27 & 865.23 & 114.73 & & & -0.06 & 116.32 \\
\hline 89 & 126 & 0.11 & 0.09 & -0.01 & 0.00 & 0.121 & -0.124 & 0.020 & 0.007 & -1.86 & 0.08 & 863.42 & 124.62 & & & 0.34 & 126.40 \\
\hline 90 & 127 & 0.11 & 0.09 & -0.02 & 0.00 & 0.121 & -0.124 & 0.032 & 0.009 & -1.12 & 0.73 & 862.78 & 133.33 & & & 1.04 & 135.30 \\
\hline 91 & 128 & 0.25 & 0.00 & -0.05 & -0.01 & 0.273 & 0.000 & 0.090 & 0.031 & -1.43 & 1.62 & 860.17 & 144.02 & & & 2.05 & 146.25 \\
\hline \multicolumn{18}{|c|}{$Z=38(\mathrm{Sr})$} \\
\hline 30 & 68 & 0.36 & 0.00 & 0.04 & -0.02 & 0.401 & 0.000 & 0.006 & 0.010 & -0.59 & 3.31 & 508.15 & 10.97 & & & 3.22 & 9.95 \\
\hline 31 & 69 & 0.27 & 0.00 & 0.03 & -0.01 & 0.297 & 0.000 & -0.006 & 0.003 & 1.16 & 3.61 & 524.81 & 2.38 & & & 3.55 & 1.52 \\
\hline 32 & 70 & 0.29 & 0.00 & 0.04 & -0.03 & 0.320 & 0.000 & -0.016 & 0.019 & 1.10 & 3.94 & 543.83 & -8.56 & & & 3.92 & -9.26 \\
\hline 33 & 71 & -0.29 & 0.00 & 0.02 & -0.07 & -0.297 & 0.000 & 0.017 & 0.065 & 1.17 & 4.16 & 559.28 & -15.94 & & & 4.20 & -16.47 \\
\hline 34 & 72 & -0.32 & 0.00 & 0.03 & -0.08 & -0.327 & 0.000 & 0.014 & 0.075 & 0.58 & 4.19 & 577.28 & -25.87 & & & 4.28 & -26.24 \\
\hline 35 & 73 & 0.36 & 0.00 & 0.03 & -0.03 & 0.400 & 0.000 & 0.016 & 0.024 & -0.29 & 4.51 & 591.42 & -31.94 & & & 4.47 & -32.34 \\
\hline 36 & 74 & 0.36 & 0.00 & 0.04 & -0.05 & 0.401 & 0.000 & 0.001 & 0.039 & -0.95 & 4.05 & 608.68 & -41.14 & & & 4.07 & -41.38 \\
\hline 37 & 75 & 0.36 & 0.00 & 0.05 & -0.05 & 0.402 & 0.000 & -0.012 & 0.034 & -1.41 & 3.83 & 622.23 & -46.61 & -46 & 0.220 & 3.82 & -46.82 \\
\hline 38 & 76 & 0.36 & .00 & 0.05 & -0.04 & 0.402 & 0.000 & -0.010 & 0.024 & -1.85 & 3.47 & 638.26 & -54.57 & & & 3.50 & -54.66 \\
\hline 39 & 77 & 0.36 & 0.00 & 0.06 & -0.04 & 0.403 & 0.000 & -0.022 & 0.020 & -1.99 & 3.40 & 649.82 & -58.06 & -57.80 & 0.009 & 3.40 & -58.12 \\
\hline 40 & 78 & 0.36 & 0.00 & 0.06 & -0.03 & 0.403 & 0.000 & -0.020 & 0.010 & -1.82 & 3.51 & .55 & -63.72 & -63.17 & .007 & 3.55 & -63.69 \\
\hline 41 & 79 & 0.36 & 0.00 & 0.06 & -0.03 & 0.403 & 0.000 & -0.020 & 0.010 & -1.47 & 3.81 & .80 & -65.89 & & & 3.83 & -65.84 \\
\hline 42 & 80 & 0.36 & 0.00 & 0.06 & -0.03 & 0.403 & 0.000 & -0.020 & 0.010 & -1.04 & 4.12 & 686.36 & -70.39 & -70.31 & 0.007 & 4.18 & -70.25 \\
\hline 43 & 81 & 0.36 & .00 & 0.06 & -0.02 & 0.403 & 0.000 & -0.019 & 0.001 & -0.69 & 4.40 & .74 & -71.69 & -7 & & 4.43 & -71.55 \\
\hline 44 & 82 & 0.00 & 0.00 & 0.00 & 0.00 & 0.000 & 0.000 & 0.000 & 0.000 & 3.05 & 3.76 & 708.35 & -76.23 & -76.01 & 0.006 & 3.76 & -76.10 \\
\hline 45 & 83 & 0.00 & 0.00 & 0.00 & 0.00 & 0.000 & 0.000 & 0.000 & 0.000 & 2.63 & 29 & 7.64 & -77.45 & -76.79 & .010 & 3.29 & -77.30 \\
\hline 46 & 84 & 0.01 & .00 & 0.00 & 0.00 & 0.011 & 0.000 & 0.000 & 0.000 & 1.57 & 2.31 & .73 & -81 & -8 & & 2.31 & -81.31 \\
\hline 47 & 85 & -0.05 & 0.00 & 0.01 & 0.00 & -0.053 & 0.000 & -0.011 & 0.001 & 0.73 & 1.57 & 738.52 & -82.18 & -81 & 3 & 1.57 & -82.01 \\
\hline 48 & 86 & 0.00 & .00 & 0.00 & 0.00 & 0.000 & 0.000 & 0.000 & 0.000 & -0.50 & 0.45 & 749.95 & -85.55 & -84.52 & 0.001 & 0.45 & -85.37 \\
\hline 49 & 87 & 0.04 & 0.00 & 0.01 & 0.00 & 0.043 & 0.000 & -0.011 & -0.000 & -1.33 & -0.24 & 757.95 & -85.48 & -84.88 & 0.001 & -0.24 & -85.29 \\
\hline 50 & 88 & 0.00 & 0.00 & 0 & 0.00 & 0 & 0 & 0 & 0 & -2.36 & & 4 & -8 & -8 & 1 & 1.16 & -87.71 \\
\hline 51 & 89 & -0.03 & 0.00 & 0.00 & 0.00 & -0.032 & 0.000 & 0.000 & 0.000 & -1.44 & -0.46 & 774.35 & -85.73 & -8 & & -0.46 & -85.55 \\
\hline 52 & 90 & 0.00 & 0.00 & 0.00 & 0.00 & 0.000 & 0.000 & 0.000 & 0.000 & -0.25 & 0.54 & 782.21 & -85.52 & -8 & & 0.54 & -85.35 \\
\hline 53 & 91 & 0.02 & 0.00 & 0.00 & 0.00 & 0.021 & 0.000 & 0.000 & 0.000 & 1.00 & 1.54 & .17 & -82.41 & -83.64 & 0.005 & 1.53 & -82.25 \\
\hline 54 & 92 & -0.13 & 0.00 & 0.01 & 0.00 & -0.135 & 0.000 & -0.005 & 0.001 & 1.40 & 2.70 & 794.20 & -81.36 & -82.87 & 0.003 & 2.72 & -81.20 \\
\hline 55 & 93 & 0.22 & 0.00 & 0 & 0 & & & & & 0.88 & & & -7 & & & 30 & \\
\hline 56 & 94 & 0.24 & 0.00 & 0.03 & 0.01 & 0.263 & 0.000 & -0.010 & -0.016 & 0.78 & 3.62 & 806.18 & -77.21 & -7 & & 3.70 & -77.00 \\
\hline 57 & 95 & 0.28 & 0.00 & 0.02 & 0.00 & 0.308 & 0.000 & 0.010 & -0.003 & 0.16 & 3.83 & 810.73 & -73.68 & & & 3.89 & -73.51 \\
\hline 58 & 96 & 0.31 & 0.00 & 0.00 & -0.01 & 0.341 & 0.000 & 0.041 & 0.016 & -0.78 & 3.84 & 817.68 & -72.56 & -72.94 & 0.027 & 3.96 & -72.34 \\
\hline 59 & 97 & 0.32 & 0.00 & 000 & -0.01 & 0.352 & $0 \Omega 00$ & & & & & & -68.91 & & & 3.73 & -68.71 \\
\hline 60 & 98 & 2 & 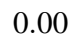 & 0.0 & 0.00 & & & & & -1.49 & & & -67.42 & & & 56 & -67.22 \\
\hline 61 & 99 & 0.32 & 0.00 & 0.00 & 0.00 & 0.352 & 0.000 & & 0.006 & -1.62 & 3.31 & 832.49 & -63.16 & -6 & & 3.40 & -63.00 \\
\hline 62 & 100 & 0.32 & 0.00 & 0.01 & 0.00 & 0.353 & 0.000 & 0.033 & 0.002 & -1.42 & 3.35 & 838.32 & -60.92 & -60.22 & & 3.45 & -60.75 \\
\hline 63 & 101 & 0.33 & 0.00 & 0.02 & 0.00 & 0.365 & 0.000 & 0.024 & -0.002 & -1.68 & 3.25 & 841.60 & -56.13 & -55.41 & 0.124 & 3.33 & -56.00 \\
\hline 64 & 102 & 0.32 & 0.00 & 0.02 & 0.00 & 0.353 & 0.000 & & -0.002 & -1.27 & 3.33 & 846.88 & -53.33 & & & .43 & -53.18 \\
\hline 65 & 103 & 0.32 & 00 & 4 & -0.01 & 5 & 00 & -0.005 & -0.000 & -1.41 & 3 & .79 & -48.18 & & & .22 & -48.04 \\
\hline 66 & 104 & 0.32 & 0.00 & 0.05 & -0.02 & 0.355 & 0.000 & -0.019 & 0.005 & -1.42 & 3.09 & 854.71 & -45.02 & & & 3.24 & -44.83 \\
\hline 67 & 105 & 0.32 & 0.00 & 0.06 & -0.03 & 0.356 & 0.000 & -0.032 & 0.011 & -1.86 & 2.79 & 857.27 & -39.51 & & & 2.98 & -39.28 \\
\hline 68 & 106 & 0.31 & 0.00 & 0.07 & -0.04 & 0.345 & 0.000 & -0.049 & 0.017 & -1.89 & 2.58 & 861.90 & -36.07 & & & 2.89 & -35.70 \\
\hline 69 & 107 & 0.31 & 0.00 & 0.08 & -0.04 & 0.346 & 0.000 & -0.061 & 0.013 & -2.41 & 22 & 864.10 & -30.20 & & & .56 & -29.81 \\
\hline 70 & 108 & 0.31 & 0.00 & 0.08 & -0.04 & 0.346 & 000 & -0.061 & 0.013 & -2.28 & 2.30 & 868.01 & -26.03 & & & 2.67 & -25.59 \\
\hline 71 & 109 & 0.31 & 0.00 & 0.08 & -0.04 & 0.346 & 0.000 & -0.061 & 0.013 & -2.27 & 2.29 & 869.46 & -19.42 & & & 2.64 & -18.97 \\
\hline 72 & 110 & 0.31 & 0.00 & 0.08 & -0.04 & 0.346 & 0.000 & -0.061 & 0.013 & -1.91 & 2.52 & 872.79 & -14.68 & & & 2.91 & -14.18 \\
\hline 73 & 111 & 0.31 & 0.00 & 0.07 & -0.03 & 0.345 & 0.000 & -0.047 & 0.007 & -1.61 & 2.44 & 873.93 & -7.75 & & & 2.69 & -7.36 \\
\hline 74 & 112 & -0.16 & 0.00 & 0.07 & 0.03 & -0.165 & 0.000 & -0.070 & -0.014 & 1.02 & 2.60 & 876.95 & -2.69 & & & 2.95 & -2.16 \\
\hline 75 & 113 & -0.15 & 0.00 & 0.08 & 0.04 & -0.154 & 0.000 & -0.082 & -0.022 & 0.09 & 1.76 & 878.49 & 3.84 & & & 2.28 & 4.57 \\
\hline 76 & 114 & -0.15 & 0.00 & 0.07 & 0.03 & -0.154 & 0.000 & -0.071 & -0.015 & -0.34 & 1.42 & 881.62 & 8.78 & & & 1.80 & 9.42 \\
\hline 77 & 115 & -0.12 & 0.00 & 0.07 & 0.03 & -0.123 & 0.000 & -0.074 & -0.017 & -1.19 & 0.33 & & 15.42 & & & 0.74 & 16.12 \\
\hline 78 & 116 & -0.12 & 0.00 & 0.06 & 0.03 & -0.124 & 0.000 & -0.063 & -0.019 & -1.66 & -0.27 & 886.09 & 20.45 & & & 0.08 & 21.15 \\
\hline 79 & 117 & -0.12 & 0.00 & 0.06 & 0.03 & -0.124 & 0.000 & -0.063 & -0.019 & -2.78 & -1.34 & 887.18 & 27.44 & & & -0.99 & 28.20 \\
\hline
\end{tabular}




\begin{tabular}{|c|c|c|c|c|c|c|c|c|c|c|c|c|}
\hline \\
\hline \multicolumn{13}{|c|}{ 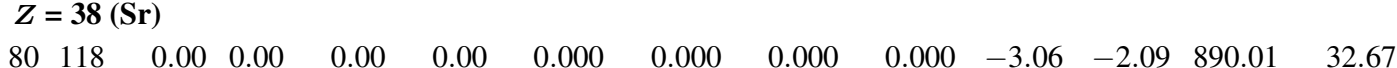 } \\
\hline $81 \quad 119$ & 0.02 & 0.00 & .01 & .00 & 021 & .000 & -0.012 & -0.000 & -5.01 & -3.78 & 891 & 39.35 \\
\hline $82 \quad 120$ & 0.00 & 0.00 & 0.00 & 0.00 & 0.000 & 0.000 & 0.000 & 0.000 & -6.34 & 97 & 85 & 44.47 \\
\hline $83 \quad 121$ & -0.02 & 0.00 & 0.00 & 0.00 & -0.021 & 0.000 & & 0.000 & & & & \\
\hline $84 \quad 122$ & 0.00 & 0.00 & 0.00 & 0.00 & 0.000 & 0.000 & 0.000 & 0.000 & - & -3.39 & & 61.34 \\
\hline 123 & 0.01 & חO & 0 & $0 \Omega$ & 1 & 0.000 & 0 & 00 & -3.64 & 66 & 1 & 3 \\
\hline $\begin{array}{ll}86 & 124\end{array}$ & 00 & 0.00 & 0.00 & 0.00 & 0.000 & .000 & 0.000 & 0.000 & -2.46 & -1.62 & & 79.00 \\
\hline $87 \quad 125$ & 0.05 & 0.07 & 0.00 & 0.00 & 0.055 & 0.095 & 0.003 & 0.003 & -2.39 & -0.99 & & 88.86 \\
\hline $88 \quad 126$ & 0.07 & 0.09 & -0.01 & 0.00 & 0.078 & -0.123 & 0.017 & 0.007 & -1.95 & -0.19 & 890.38 & 96.88 \\
\hline $89 \quad 127$ & 0.10 & 0.10 & -0.02 & 0.00 & 0.111 & -0.138 & 0.032 & 0.010 & -1.90 & 0.25 & 88 & 106.81 \\
\hline 90 & 0.10 & 0.10 & -0.02 & .01 & 1 & -0.137 & 032 & 0 & -1.02 & 0.96 & & \\
\hline $91 \quad 129$ & 0.24 & 0.00 & -0.05 & -0.01 & 0.261 & 0.000 & 0.087 & 0.030 & -1.07 & 2.03 & 885.63 & 125.84 \\
\hline $92 \quad 130$ & 0.26 & 0.00 & -0.05 & -0.01 & 0.284 & 0.000 & 0.092 & & -1.38 & 1.92 & & 133.48 \\
\hline $93 \quad 131$ & 0.27 & 0.00 & -0.05 & 0.00 & 0.294 & 0.000 & 0.095 & 0.023 & -1.82 & 1.70 & 884.36 & 143.26 \\
\hline
\end{tabular}

\section{$Z=39(\mathbf{Y})$}

$\begin{array}{lllll}31 & 70 & 0.36 & 0.00\end{array}$

$\begin{array}{llll}32 & 71 & 0.36 & 0.00\end{array}$

$\begin{array}{llll}33 & 72 & 0.36 & 0.00\end{array}$

$\begin{array}{llll}34 & 73 & 0.36 & 0.00\end{array}$

$\begin{array}{llll}35 & 74 & 0.36 & 0.00\end{array}$

$\begin{array}{llll}36 & 75 & 0.36 & 0.00\end{array}$

$\begin{array}{llll}37 & 76 & 0.36 & 0.00\end{array}$

$\begin{array}{llll}38 & 77 & 0.36 & 0.00\end{array}$

$\begin{array}{llll}39 & 78 & 0.36 & 0.00\end{array}$

$\begin{array}{llll}40 & 79 & 0.37 & 0.00\end{array}$

$\begin{array}{lllll}41 & 80 & 0.38 & 0.00\end{array}$

$\begin{array}{llll}42 & 81 & 0.36 & 0.00\end{array}$

$\begin{array}{lllll}43 & 82 & 0.36 & 0.00\end{array}$

$\begin{array}{llll}44 & 83 & 0.00 & 0.00\end{array}$

$\begin{array}{llll}45 & 84 & 0.00 & 0.00\end{array}$

$\begin{array}{rrrrr}46 & 85 & 0.00 & 0.00\end{array}$

$\begin{array}{llll}47 & 86 & -0.01 & 0.00\end{array}$

$\begin{array}{llll}48 & 87 & 0.00 & 0.00\end{array}$

$\begin{array}{llll}49 & 88 & 0.03 & 0.00\end{array}$

$\begin{array}{llll}50 & 89 & 0.00 & 0.00\end{array}$

$\begin{array}{llll}51 & 90 & -0.02 & 0.00\end{array}$

$\begin{array}{llll}52 & 91 & 0.00 & 0.00\end{array}$

$\begin{array}{llll}53 & 92 & 0.00 & 0.00\end{array}$

$\begin{array}{llll}54 & 93 & -0.14 & 0.00\end{array}$

$\begin{array}{llll}55 & 94 & 0.20 & 0.00\end{array}$

$\begin{array}{lllll}56 & 95 & 0.26 & 0.00\end{array}$

$\begin{array}{llll}57 & 96 & 0.28 & 0.00\end{array}$

$\begin{array}{llll}58 & 97 & 0.31 & 0.00\end{array}$

$\begin{array}{llll}59 & 98 & 0.32 & 0.00\end{array}$

$\begin{array}{llll}60 & 99 & 0.33 & 0.00\end{array}$

$\begin{array}{lllll}61 & 100 & 0.34 & 0.00\end{array}$

$\begin{array}{lllll}62 & 101 & 0.34 & 0.00\end{array}$

$\begin{array}{lllll}63 & 102 & 0.34 & 0.00\end{array}$

$\begin{array}{llll}64 & 103 & 0.33 & 0.00\end{array}$

$\begin{array}{llll}65 & 104 & 0.33 & 0.00\end{array}$

$66 \quad 105$

$67 \quad 106$

$\begin{array}{ll}0.32 & 0.00\end{array}$

$\begin{array}{ll}68 & 107\end{array}$

$0.32 \quad 0.00$

$69 \quad 108$

$\begin{array}{ll}0.32 & 0.00\end{array}$

70109

$\begin{array}{ll}0.32 & 0.00\end{array}$

0.320 .00

$\begin{array}{lllll}71 & 110 & 0.33 & 0.00\end{array}$

$72 \quad 111$ $\begin{array}{lll}0.03 & -0.01 & 0.400\end{array}$ $0.04-0.02$

$0.04-0.03$

$0.05-0.04$

$0.04-0.04$

$\begin{array}{ll}0.05 & -0.04\end{array}$

$0.06-0.04$

$0.07-0.03$

$\begin{array}{lll}0.07 & -0.03\end{array}$

$0.08-0.03$

$\begin{array}{lll}0.08 & -0.02\end{array}$

$\begin{array}{lll}0.07 & -0.02\end{array}$

$0.07-0.02$

$0.00 \quad 0.00$

$0.00 \quad 0.00$

$\begin{array}{lll}0.00 & 0.00 & 0.000\end{array}$

$\begin{array}{llll}0.00 & 0.00 & -0.011\end{array}$

$\begin{array}{lll}0.00 & 0.00 & 0.000\end{array}$

$\begin{array}{lll}0.01 & 0.00 & 0.032\end{array}$

0.00

0.0

$\begin{array}{lll}0.00 & 0.00 & -0.021\end{array}$

$\begin{array}{lll}0.00 & 0.00 & 0.000\end{array}$

$\begin{array}{lll}0.00 & 0.00 \quad 0.000\end{array}$

0.01

$0.00-0.146$

0.00

$\begin{array}{ll}0.01 & 0.217\end{array}$

0.03

0.02

0.01

0.01

0.286

0.01

0.00

$0.01 \quad 0.00$

0.364

$\begin{array}{lll}0.01 & 0.01 & 0.376\end{array}$

$\begin{array}{lll}0.02 & 0.01 & 0.376\end{array}$

$\begin{array}{lll}0.02 & 0.01 & 0.376\end{array}$

$\begin{array}{lll}0.03 & 0.00 & 0.366\end{array}$

$0.04-0.01$

0.366

$\begin{array}{lll}0.05 & -0.02\end{array}$

0.355

$\begin{array}{lll}0.06 & -0.02\end{array}$

$0.08-0.03$

$\begin{array}{lll}0.09 & -0.04\end{array}$

0.356

0.358

0.359

$0.09-0.04$

0.359

$\begin{array}{lll}0.08 & -0.03\end{array}$

$0.08-0.03$

0.370
$0.000 \quad 0.020$

$0.000 \quad 0.006$

$0.000 \quad 0.004$

$0.000-0.010$

$0.000 \quad 0.002$

$0.000-0.010$

$0.000-0.022$

$0.000-0.032$

$0.000-0.032$

$0.000-0.041$

$0.005-0.11$

$0.010-0.01$

$\begin{array}{ll}0.019 & 0.19\end{array}$

$0.024-0.12$

$0.029-0.37$

$0.024-0.98$

$0.020-1.48$

$0.006-2.05$

$0.006-2.26$

$0.001-2.45$

$\begin{array}{lllll}0.000 & -0.036 & -0.009 & -2.28\end{array}$

$\begin{array}{lllll}0.000 & -0.031 & -0.004 & -1.42\end{array}$

$\begin{array}{lllll}0.000 & -0.031 & -0.004 & -1.08\end{array}$

$\begin{array}{llll}0.000 & 0.000 & 0.000 & 2.92\end{array}$

$0.000 \quad 0.000$

0.000

$0.000 \quad 0.000$

$0.000 \quad 0.000$

0.000

2.51

$0.000 \quad 0.000$

0.000

1.45

$0.000-0.63$

$\begin{array}{rr}0.000 & -0.012 \\ 0.000 & 0.000\end{array}$

$0.000 \quad 0.000$

$-0.000-1.40$

0.000

$0.000-1.54$

$0.000 \quad 0.000$

$0.000 \quad 0.000$

$0.000-0.34$

$0.000-0.004$

0.000

$\begin{array}{lll}0.000 & 0.018 & -0.009\end{array}$

$\begin{array}{lll}0.000 & -0.006 & -0.016\end{array}$

$\begin{array}{llll}0.000 & 0.011 & -0.013\end{array}$

$0.013 \quad 0.15$

$\begin{array}{llll}0.000 & 0.030 & 0.002 & -0.68\end{array}$

$0.000 \quad 0.033$

$0.002-1.23$

$0.000 \quad 0.036$

$0.003-1.69$

$\begin{array}{lllll}0.000 & 0.041 & -0.006 & -2.11\end{array}$

0.000

0.000

$\begin{array}{llll}0.029 & -0.011 & -1.97\end{array}$

$\begin{array}{lllll}0.000 & 0.012 & -0.006 & -1.64\end{array}$

$\begin{array}{lllll}0.000 & -0.002 & -0.000 & -1.78\end{array}$

$0.000 \quad-0.019$

$0.005-1.57$

$0.000-0.031$

$0.001-2.00$

$0.003-2.35$

$0.000-0.070$

$0.009-2.95$

$0.009-2.84$

$\begin{array}{lll}3.47 & 520.89 & 13.59\end{array}$

$\begin{array}{lll}3.89 & 540.02 & 2.53\end{array}$

$\begin{array}{lll}4.41 & 556.15 & -5.53\end{array}$

$\begin{array}{lll}4.29 & 574.51 & -15.81\end{array}$

$\begin{array}{lll}4.38 & 589.83 & -23.06\end{array}$

$\begin{array}{llll}3.92 & 607.29 & -32.46\end{array}$

$\begin{array}{llll}3.70 & 621.78 & -38.87\end{array}$

$\begin{array}{llll}3.22 & 638.12 & -47.14\end{array}$

$\begin{array}{llll}3.16 & 650.99 & -51.94\end{array}$

$\begin{array}{lllll}3.23 & 665.33 & -58.21 & -58.36\end{array}$

$\begin{array}{lllll}3.62 & 676.38 & -61.19 & -61.22\end{array}$

$\begin{array}{lllll}3.84 & 689.23 & -65.96 & -66.02\end{array}$

$\begin{array}{lllll}4.12 & 699.48 & -68.14 & -68.19\end{array}$

$\begin{array}{lllll}3.67 & 712.08 & -72.67 & -72.33\end{array}$

$\begin{array}{lllll}3.21 & 722.23 & -74.75 & -74.16\end{array}$

$\begin{array}{lllll}2.21 & 734.51 & -78.96 & -77.84\end{array}$

$\begin{array}{lllll}1.53 & 744.08 & -80.46 & -79.28\end{array}$

$\begin{array}{lllll}0.35 & 755.76 & -84.06 & -83.02\end{array}$

$\begin{array}{rrrr}0.35 & 755.76 & -84.06 & -83.02 \\ -0.33 & 764.56 & -84.80 & -84.30\end{array}$

$\begin{array}{lllll}-1.27 & 775.24 & -87.40 & -87.70\end{array}$

$\begin{array}{llll}-0.55 & 781.93 & -86.03 & -86.49\end{array}$

$\begin{array}{lllll}0.47 & 789.93 & -85.96 & -86.35\end{array}$

$\begin{array}{lllll}1.48 & 795.68 & -83.63 & -84.81\end{array}$

$\begin{array}{lllll}2.77 & 802.72 & -82.60 & -84.22\end{array}$

$\begin{array}{lllll}3.37 & 808.24 & -80.05 & -82.35\end{array}$

$\begin{array}{lllll}3.64 & 815.66 & -79.40 & -81.21\end{array}$

$\begin{array}{lllll}3.84 & 820.99 & -76.65 & -78.35\end{array}$

$\begin{array}{lllll}3.84 & 828.08 & -75.68 & -76.26\end{array}$

$\begin{array}{lllll}3.63 & 833.24 & -72.77 & -72.47\end{array}$

$\begin{array}{llll}3.44 & 839.96 & -71.41 & -70.20\end{array}$

$\begin{array}{lllll}3.31 & 844.51 & -67.89 & -67.29\end{array}$

$\begin{array}{lllll}3.30 & 850.51 & -65.81 & -64.91\end{array}$

$\begin{array}{lllll}3.20 & 854.50 & -61.74 & -61.89\end{array}$

$\begin{array}{llll}3.25 & 859.92 & -59.09\end{array}$

$\begin{array}{lll}3.09 & 863.51 & -54.60\end{array}$

$\begin{array}{lll}3.04 & 868.54 & -51.56\end{array}$

$\begin{array}{llll}2.77 & 871.77 & -46.72\end{array}$

$\begin{array}{llll}2.58 & 876.48 & -43.36\end{array}$

$0.000-0.070$

$\begin{array}{lll}2.18 & 879.41 & -38.22\end{array}$

$\begin{array}{ll}0.000 & -0.054\end{array}$

$\begin{array}{ll}0.003 & -2.78\end{array}$

$\begin{array}{llll}2.22 & 883.44 & -34.17\end{array}$

$\begin{array}{ll}0.000 & -0.057\end{array}$
$0.003-2.22$ $\begin{array}{llll}2.38 & 885.39 & -28.06\end{array}$

$\begin{array}{llll}2.60 & 888.83 & -23.42\end{array}$

$\begin{array}{rr}-2.09 & 33.14 \\ -3.77 & 39.89 \\ -4.97 & 45.08 \\ -4.27 & 54.54 \\ -3.39 & 62.12 \\ -2.66 & 71.91 \\ -1.62 & 79.98 \\ -0.84 & 90.10 \\ 0.09 & 98.35 \\ 0.60 & 108.49 \\ 1.33 & 116.83 \\ 2.46 & 127.85 \\ 2.40 & 135.68 \\ 2.05 & 145.48\end{array}$

$3.31 \quad 12.56$

$3.78 \quad 1.68$

$\begin{array}{ll}4.28 & -6.28\end{array}$

$4.22-16.40$

$\begin{array}{ll}4.29 & -23.58\end{array}$

$3.88-32.83$

$3.63-39.20$

$3.19-47.35$

$3.11-52.49$

$3.22-58.27$

0.177

0.062

0.103

0.044

0.091

0.019

0.014

0.002

0.002

0.003

0.003

0.003

0.009

0.011

0.007

0.007

0.023

0.012

0.025

0.024

0.079

0.095

0.086

$3.59-61.24$

$3.85-65.92$

$\begin{array}{ll}4.11 & -68.09\end{array}$

$3.67-72.58$

$3.20-74.64$

$\begin{array}{ll}2.21 & -78.83\end{array}$

$\begin{array}{ll}1.53 & -80.31\end{array}$

$\begin{array}{lll}0.35 & -83.90\end{array}$

$-0.33-84.64$

$-1.27-87.24$

$-0.55-85.87$

$\begin{array}{lll}0.47 & -85.80\end{array}$

$\begin{array}{ll}1.48 & -83.48\end{array}$

$\begin{array}{lll}2.79 & -82.44\end{array}$

$\begin{array}{lll}3.39 & -79.89\end{array}$

$3.71-79.22$

$3.89-76.50$

$3.91-75.51$

$3.69-72.63$

$3.53-71.25$

$3.38-67.76$

$3.39-65.67$ 


\begin{tabular}{|c|c|c|c|c|c|c|c|c|c|c|c|c|c|c|c|c|c|}
\hline \multicolumn{18}{|c|}{$Z=39(Y)$} \\
\hline 73 & 112 & 0.32 & 0.00 & 0.07 & -0.03 & 0.357 & 0.000 & -0.045 & 0.007 & -1.93 & 2.73 & 890.41 & -16.93 & & & 2.94 & -16.63 \\
\hline 74 & 113 & -0.16 & 0.00 & 0.07 & 0.02 & -0.165 & 0.000 & -0.069 & -0.005 & 0.98 & 2.72 & 893.66 & -12.12 & & & 3.00 & -11.73 \\
\hline 75 & 114 & -0.16 & 0.00 & 0.07 & 0.03 & -0.165 & 0.000 & -0.070 & -0.014 & 0.14 & 2.12 & 895.62 & -6.00 & & & 2.44 & -5.53 \\
\hline 76 & 115 & -0.15 & 0.00 & 0.07 & 0.03 & -0.154 & 0.000 & -0.071 & -0.015 & -0.40 & 1.46 & 899.14 & -1.45 & & & 1.81 & -0.92 \\
\hline 77 & 116 & -0.15 & 0.00 & 0.07 & 0.04 & -0.154 & 0.000 & -0.071 & -0.024 & -1.45 & 0.41 & 901.17 & 4.59 & & & 0.85 & 5.25 \\
\hline 78 & 117 & -0.12 & 0.00 & 0.06 & 0.03 & -0.124 & 0.000 & -0.063 & -0.019 & -1.64 & -0.19 & 904.28 & 9.55 & & & 0.13 & 10.14 \\
\hline 79 & 118 & -0.12 & 0.00 & 0.05 & 0.03 & -0.124 & 0.000 & -0.052 & -0.021 & -2.67 & -1.25 & 905.98 & 15.93 & & & -0.98 & 16.51 \\
\hline 80 & 119 & 0.00 & 0.00 & 0.00 & 0.00 & 0.000 & 0.000 & 0.000 & 0.000 & -2.98 & -2.04 & 908.92 & 21.06 & & & -2.04 & 21.42 \\
\hline 81 & 120 & 0.02 & 0.00 & 0.01 & 0.00 & 0.021 & 0.000 & -0.012 & -0.000 & -4.95 & -3.72 & 910.92 & 27.13 & & & -3.72 & 27.57 \\
\hline 82 & 121 & 0.00 & 0.00 & 0.00 & 0.00 & 0.000 & 0.000 & 0.000 & 0.000 & -6.33 & -4.96 & 913.98 & 32.14 & & & -4.96 & 32.63 \\
\hline 83 & 122 & -0.01 & 0.00 & 0.00 & 0.00 & -0.011 & 0.000 & 0.000 & 0.000 & -5.41 & -4.22 & 913.23 & 40.96 & & & -4.22 & 41.53 \\
\hline 84 & 123 & 0.00 & 0.00 & 0.00 & 0.00 & 0.000 & 0.000 & 0.000 & 0.000 & -4.42 & -3.33 & 913.85 & 48.41 & & & -3.33 & 49.05 \\
\hline 85 & 124 & 0.01 & 0.00 & 0.00 & 0.00 & 0.011 & 0.000 & 0.000 & 0.000 & -3.53 & -2.54 & 912.75 & 57.58 & & & -2.54 & 58.31 \\
\hline 86 & 125 & 0.00 & 0.00 & 0.00 & 0.00 & 0.000 & 0.000 & 0.000 & 0.000 & -2.35 & -1.52 & 912.94 & 65.46 & & & -1.52 & 66.28 \\
\hline 87 & 126 & 0.03 & 0.07 & -0.01 & 0.00 & 0.034 & -0.095 & 0.014 & 0.004 & -2.27 & -0.92 & 911.75 & 74.72 & & & -0.77 & 75.79 \\
\hline 88 & 127 & 0.04 & 0.09 & -0.01 & 0.01 & 0.046 & -0.122 & 0.016 & -0.004 & -1.75 & -0.13 & 911.87 & 82.68 & & & 0.13 & 83.95 \\
\hline 89 & 128 & 0.04 & 0.11 & -0.01 & 0.01 & 0.048 & -0.149 & 0.017 & -0.001 & -1.74 & 0.28 & 910.60 & 92.02 & & & 0.64 & 93.51 \\
\hline 90 & 129 & 0.04 & 0.12 & -0.01 & 0.02 & 0.048 & -0.162 & 0.018 & -0.010 & -1.09 & 1.01 & 910.50 & 100.19 & & & 1.49 & 101.92 \\
\hline 91 & 130 & 0.09 & 0.13 & -0.03 & 0.01 & 0.103 & -0.180 & 0.046 & 0.006 & -1.42 & 1.37 & 909.01 & 109.75 & & & 1.93 & 11.68 \\
\hline 92 & 131 & 0.25 & 0.00 & -0.05 & 0.00 & 0.272 & 0.000 & 0.090 & 0.020 & -1.16 & 2.05 & 908.69 & 118.14 & & & 2.38 & 119.96 \\
\hline 93 & 132 & 0.26 & 0.00 & -0.05 & 0.00 & 0.283 & 0.000 & 0.092 & 0.022 & -1.68 & 1.75 & 907.61 & 127.29 & & & 2.06 & 129.24 \\
\hline 94 & 133 & 0.26 & 0.00 & -0.04 & 0.00 & 0.283 & 0.000 & 0.080 & 0.018 & -1.24 & 1.96 & 907.52 & 135.46 & & & 2.17 & 137.46 \\
\hline 95 & 134 & 0.27 & 0.00 & -0.04 & 0.01 & 0.294 & 0.000 & 0.083 & 0.008 & -1.57 & 1.76 & 906.09 & 144.95 & & & 1.92 & 147.05 \\
\hline \multicolumn{18}{|c|}{$(\mathbf{Z r})$} \\
\hline 32 & 72 & -0.24 & 0.00 & 0.04 & -0.01 & -0.248 & 0.000 & -0.022 & 0.018 & 1.70 & 3.57 & 538.66 & 11.18 & & & 3.55 & 10.36 \\
\hline 33 & 73 & -0.26 & 0.00 & 0.05 & -0.01 & -0.268 & 0.000 & -0.028 & 0.021 & 1.63 & 4.00 & 555.07 & 2.85 & & & 3.97 & 2.12 \\
\hline 34 & 74 & 0.36 & 0.00 & 0.06 & -0.03 & 0.403 & 0.000 & -0.020 & 0.010 & 0.15 & 4.25 & 574.01 & -8.03 & & & 4.19 & -8.67 \\
\hline 35 & 75 & 0.36 & 0.00 & 0.05 & -0.03 & 0.402 & 0.000 & -0.008 & 0.015 & -0.03 & 4.39 & 589.49 & -15.44 & & & 4.30 & -16.00 \\
\hline 36 & 76 & 0.36 & 0.00 & 0.07 & -0.03 & 0.404 & 0.000 & -0.032 & 0.006 & -0.74 & 3.95 & 607.88 & -25.75 & & & 3.92 & -26.17 \\
\hline 37 & 77 & 0.36 & 0.00 & 0.07 & -0.03 & 0.404 & 0.000 & -0.032 & 0.006 & -1.22 & 3.76 & 622.53 & -32.33 & & & 3.70 & -32.69 \\
\hline 38 & 78 & 0.37 & 0.00 & 0.08 & -0.03 & 0.417 & 0.000 & -0.041 & 0.001 & -2.05 & 3.32 & 639.75 & -41.48 & & & 3.31 & -41.72 \\
\hline 39 & 79 & 0.37 & 0.00 & 0.08 & -0.03 & 0.417 & 0.000 & -0.041 & 0.001 & -2.31 & 3.23 & 653.22 & -46.88 & & & 3.20 & -47.08 \\
\hline 40 & 80 & 0.38 & 0.00 & 0.09 & -0.02 & 0.430 & 0.000 & -0.047 & -0.014 & -2.57 & 3.29 & 668.85 & -54.44 & & & 3.31 & -54.53 \\
\hline 41 & 81 & 0.39 & 0.00 & 0.09 & -0.02 & 0.442 & 0.000 & -0.044 & -0.014 & -2.45 & 3.66 & 680.11 & -57.63 & -5 & 7 & 3.65 & -57.70 \\
\hline 42 & 82 & 0.39 & 0.00 & 0.09 & -0.01 & 43 & 0.000 & -0.042 & -0.023 & -2.08 & 3.93 & 693.79 & -63.23 & & & 3.98 & -63.20 \\
\hline 43 & 83 & -0.23 & 0.00 & 0.10 & 0.05 & -0.234 & 0.000 & -0.092 & -0.019 & 0.76 & 3.89 & 704.55 & -65.93 & -66.46 & 0.096 & 3.92 & -65.87 \\
\hline 44 & 84 & -0.23 & 0.00 & 0.09 & 0.05 & -0.235 & 0.000 & -0.081 & -0.022 & 0.49 & 3.39 & 718.07 & -71.37 & & & 3.44 & -71.26 \\
\hline 45 & 85 & -0.01 & 0.00 & 0.00 & 0.00 & -0.011 & 0.000 & & 0.000 & 2.32 & 2.98 & 728.34 & -73.58 & & & 2.98 & -73.49 \\
\hline 46 & 86 & 0.01 & 0.00 & 0.00 & 0.00 & 0.011 & 0.000 & 0.000 & 0.000 & 1.24 & 1.98 & 741.48 & -78.65 & -77.80 & 0. & 1.98 & -78.54 \\
\hline 47 & 87 & -0.01 & 0.00 & 0.0 & 0.00 & -0.011 & .000 & 00 & 0.000 & 0.53 & 1.27 & 751.25 & -80.34 & & & 1.27 & -80.22 \\
\hline 48 & 88 & -0.01 & 0.00 & 0.00 & 0.00 & -0.011 & 0.000 & 0.000 & 0.000 & -0.84 & 0.10 & 763.75 & -84.76 & -83.62 & 0.010 & 0.10 & -84.63 \\
\hline 49 & 89 & 0.03 & 0.00 & 0.01 & 0.00 & 0.032 & 0.000 & -0.012 & -0.000 & -1.80 & -0.69 & 772.83 & -85.78 & -84.87 & 0.004 & -0.69 & -85.64 \\
\hline 50 & 90 & 0.00 & 0.00 & 0.00 & 0.00 & 0.000 & 0.000 & 0.000 & 0.000 & -2.80 & -1.59 & 784.28 & -89.15 & -88.77 & 0.002 & -1.59 & -89.01 \\
\hline 51 & 91 & -0.02 & 0.00 & 0.00 & 0.00 & -0.021 & 0.000 & 0.000 & 0.000 & -1.84 & -0.84 & 791.11 & -87.92 & -87.89 & 0.002 & -0.85 & -87.77 \\
\hline 52 & 92 & 0.00 & 0.00 & 0.00 & 0.00 & 0.000 & .000 & & 0.000 & -0.69 & 0.12 & 799.96 & -88.69 & & & 0.12 & -88.55 \\
\hline 53 & 93 & 0.01 & 0.00 & 0.00 & 0.00 & 0.011 & 0.000 & 0.000 & 0.000 & 0.65 & 1.19 & 805.79 & -86.46 & -87.12 & 0.002 & 1.19 & -86.32 \\
\hline 54 & 94 & -0.15 & 0.00 & 0.01 & 0.01 & -0.156 & 0.000 & -0.003 & -0.008 & 0.95 & 2.62 & 813.49 & -86.08 & -87.27 & 0.002 & 2.64 & -85.93 \\
\hline 55 & 95 & -0.17 & 0.00 & 0.01 & 0.00 & -0.176 & 0.000 & -0.000 & 0.001 & 1.35 & 3.02 & 819.35 & -83.87 & -85.66 & 0.002 & 3.04 & -83.73 \\
\hline 56 & 96 & 0.22 & 0.00 & 0.01 & 0.02 & 0.240 & 0.000 & 0.011 & -0.021 & 1.13 & 3.38 & 827.47 & -83.91 & -85.44 & 0.003 & 3.44 & -83.74 \\
\hline 57 & 97 & 0.27 & 0.00 & 0.02 & 0.01 & 0.296 & 0.000 & 0.009 & -0.013 & 0.50 & 3.79 & 832.71 & -81.09 & -82.95 & 0.003 & 3.85 & -80.93 \\
\hline 58 & 98 & 0.31 & 0.00 & 0.02 & 0.00 & 0.342 & 0.000 & 0.018 & -0.002 & -0.37 & 3.94 & 840.41 & -80.72 & -81.29 & 0.020 & 4.03 & -80.54 \\
\hline 59 & 99 & 0.32 & 0.00 & 0.01 & 0.00 & 0.353 & 0.000 & 0.033 & 0.002 & -0.92 & 3.82 & 845.62 & -77.85 & -77.77 & 0.020 & 3.90 & -77.69 \\
\hline 60 & 100 & 0.33 & 0.00 & 0.01 & 0.01 & 0.364 & 0.000 & 0.038 & -0.007 & -1.39 & 3.62 & 853.08 & -77.24 & -76.60 & 0.036 & 3.74 & -77.06 \\
\hline 61 & 101 & 0.34 & 0.00 & 0.02 & 0.01 & 0.376 & 0.000 & 0.029 & -0.011 & -1.78 & 3.45 & 857.79 & -73.89 & -73.46 & 0.031 & 3.55 & -73.74 \\
\hline 62 & 102 & 0.34 & 0.00 & 0.02 & 0.01 & 0.376 & 0.000 & 0.029 & -0.011 & -1.73 & 3.44 & 864.51 & -72.53 & -71.74 & 0.051 & 3.57 & -72.37 \\
\hline 63 & 103 & 0.34 & 0.00 & 0.03 & 0.01 & 0.377 & 0.000 & 0.016 & -0.015 & -1.77 & 3.35 & 868.63 & -68.58 & -68.37 & 0.109 & 3.45 & -68.44 \\
\hline 64 & 104 & 0.34 & 0.00 & 0.04 & 0.00 & 0.378 & 0.000 & 0.003 & -0.010 & -1.64 & 3.38 & 874.78 & -66.66 & & & 3.51 & -66.51 \\
\hline
\end{tabular}




\begin{tabular}{|c|c|c|c|c|c|c|c|c|c|c|c|c|c|c|c|c|c|}
\hline \multicolumn{18}{|c|}{$Z=\mathbf{4 0}(\mathrm{Zr})$} \\
\hline 65 & 105 & 0.33 & 0.00 & 0.05 & -0.01 & 0.367 & 0.000 & -0.014 & -0.004 & -1.57 & 3.22 & 878.47 & -62.28 & & & 3.33 & -62.16 \\
\hline 66 & 106 & 0.33 & 0.00 & 0.06 & -0.01 & 0.368 & 0.000 & -0.026 & -0.008 & -1.62 & 3.21 & 884.16 & -59.90 & & & 3.37 & -59.73 \\
\hline 67 & 107 & 0.32 & 0.00 & 0.07 & -0.02 & 0.357 & 0.000 & -0.043 & -0.002 & -1.89 & 2.91 & 887.54 & -55.20 & & & 3.08 & -55.02 \\
\hline 68 & 108 & 0.32 & 0.00 & 0.08 & -0.03 & 0.358 & 0.000 & -0.057 & 0.003 & -2.13 & 2.78 & 892.86 & -52.46 & & & 3.04 & -52.19 \\
\hline 69 & 109 & 0.32 & 0.00 & 0.09 & -0.03 & 0.359 & 0.000 & -0.068 & -0.001 & -2.76 & 2.38 & 895.90 & -47.42 & & & 2.66 & -47.12 \\
\hline 70 & 110 & 0.32 & 0.00 & 0.09 & -0.03 & 0.359 & 0.000 & -0.068 & -0.001 & -2.65 & 2.43 & 900.59 & -44.04 & & & 2.75 & -43.70 \\
\hline 71 & 111 & 0.33 & 0.00 & 0.09 & -0.03 & 0.371 & 0.000 & -0.066 & -0.001 & -2.79 & 2.49 & 902.73 & -38.11 & & & 2.79 & -37.79 \\
\hline 72 & 112 & 0.32 & 0.00 & 0.09 & -0.03 & 0.359 & 0.000 & -0.068 & -0.001 & -2.18 & 2.77 & 906.78 & -34.08 & & & 3.10 & -33.71 \\
\hline 73 & 113 & 0.33 & 0.00 & 0.08 & -0.03 & 0.370 & 0.000 & -0.054 & 0.003 & -2.01 & 2.83 & 908.52 & -27.75 & & & 3.09 & -27.44 \\
\hline 74 & 114 & -0.18 & 0.00 & 0.07 & 0.03 & -0.185 & 0.000 & -0.067 & -0.013 & 0.52 & 2.47 & 912.78 & -23.94 & & & 2.78 & -23.57 \\
\hline 75 & 115 & -0.16 & 0.00 & 0.08 & 0.04 & -0.164 & 0.000 & -0.081 & -0.021 & -0.34 & 1.48 & 915.20 & -18.29 & & & 1.92 & -17.75 \\
\hline 76 & 116 & -0.16 & 0.00 & 0.08 & 0.04 & -0.164 & 0.000 & -0.081 & -0.021 & -0.95 & 1.17 & 919.01 & -14.03 & & & 1.65 & -13.43 \\
\hline 77 & 117 & -0.15 & 0.00 & 0.08 & 0.04 & -0.154 & 0.000 & -0.082 & -0.022 & -1.90 & 0.17 & 921.07 & -8.02 & & & 0.66 & -7.38 \\
\hline 78 & 118 & -0.15 & 0.00 & 0.07 & 0.03 & -0.154 & 0.000 & -0.071 & -0.015 & -2.27 & -0.31 & 924.68 & -3.56 & & & 0.05 & -3.01 \\
\hline 79 & 119 & -0.12 & 0.00 & 0.06 & 0.03 & -0.124 & 0.000 & -0.063 & -0.019 & -3.04 & -1.48 & 926.56 & 2.63 & & & -1.16 & 3.18 \\
\hline 80 & 120 & 0.00 & 0.00 & 0.00 & 0.00 & 0.000 & 0.000 & 0.000 & 0.000 & -3.18 & -2.22 & 930.08 & 7.19 & & & -2.22 & 7.47 \\
\hline 81 & 121 & 0.02 & 0.00 & 0.01 & 0.00 & 0.021 & 0.000 & -0.012 & -0.000 & -5.16 & -3.92 & 932.16 & 13.18 & & & -3.92 & 13.52 \\
\hline 82 & 122 & 0.00 & 0.00 & 0.00 & 0.00 & 0.000 & 0.000 & 0.000 & 0.000 & -6.57 & -5.19 & 935.85 & 17.56 & & & -5.19 & 17.95 \\
\hline 83 & 123 & -0.02 & 0.00 & 0.00 & 0.00 & -0.021 & 0.000 & 0.000 & 0.000 & -5.63 & -4.41 & 935.14 & 26.34 & & & -4.41 & 26.80 \\
\hline 84 & 124 & 0.00 & 0.00 & 0.00 & 0.00 & 0.000 & 0.000 & 0.000 & 0.000 & -4.62 & -3.51 & 936.34 & 33.21 & & & -3.51 & 33.74 \\
\hline 85 & 125 & 0.01 & 0.00 & 0.00 & 0.00 & 0.011 & 0.000 & 0.000 & 0.000 & -3.69 & -2.67 & 935.25 & 42.37 & & & -2.67 & 42.97 \\
\hline 86 & 126 & 0.00 & 0.00 & 0.00 & 0.00 & 0.000 & 0.000 & 0.000 & 0.000 & -2.50 & -1.64 & 936.01 & 49.69 & & & -1.64 & 50.37 \\
\hline 87 & 127 & 0.02 & 0.05 & 0.00 & 0.00 & 0.022 & -0.067 & 0.001 & 0.002 & -2.00 & -0.93 & 934.76 & 59.00 & & & -0.86 & 59.84 \\
\hline 88 & 128 & 0.03 & 0.09 & -0.01 & 0.01 & 0.035 & -0.122 & 0.015 & -0.004 & -1.78 & -0.15 & 935.46 & 66.37 & & & 0.10 & 67.49 \\
\hline 89 & 129 & 0.03 & 0.11 & -0.01 & 0.01 & 0.037 & -0.149 & 0.017 & -0.001 & -1.74 & 0.28 & 934.22 & 75.68 & & & 0.64 & 77.01 \\
\hline 90 & 130 & 0.03 & 0.12 & -0.01 & 0.02 & 0.038 & -0.162 & 0.018 & -0.010 & -1.10 & 1.01 & 934.69 & 83.29 & & & 1.49 & 84.84 \\
\hline 91 & 131 & 0.04 & 0.13 & -0.02 & 0.01 & 0.049 & -0.178 & 0.031 & 0.003 & -0.91 & 1.56 & 933.05 & 93.00 & & & 2.09 & 94.71 \\
\hline 92 & 132 & 0.24 & 0.00 & -0.06 & 0.00 & 0.261 & 0.000 & 0.101 & 0.023 & -1.08 & 2.14 & 933.39 & 100.73 & & & 2.59 & 102.48 \\
\hline 93 & 133 & 0.25 & 0.00 & -0.05 & 0.00 & 0.272 & 0.000 & 0.090 & 0.020 & -1.28 & 1.96 & 932.23 & 109.96 & & & 2.28 & 111.70 \\
\hline 94 & 134 & 0.26 & 0.00 & -0.04 & 0.00 & 0.283 & 0.000 & 0.080 & 0.018 & -1.02 & 2.18 & 932.66 & 117.60 & & & 2.42 & 119.40 \\
\hline 95 & 135 & 0.28 & 0.00 & -0.03 & 0.01 & 0.305 & 0.000 & 0.073 & 0.005 & -1.35 & 2.06 & 931.20 & 127.13 & & & 2.17 & 128.94 \\
\hline 96 & 136 & 0.28 & 0.00 & -0.02 & 0.01 & 0.305 & 0.000 & 0.060 & 0.002 & -1.03 & 2.23 & 931.44 & 134.97 & & & 2.28 & 136.88 \\
\hline 97 & 137 & 0.29 & 0.00 & -0.01 & 0.01 & 0.317 & 0.000 & 0.051 & -0.001 & -1.41 & 1.97 & 929.88 & 144.60 & & & 1.96 & 146.59 \\
\hline \multicolumn{18}{|c|}{$Z=41(\mathrm{Nb})$} \\
\hline 33 & 74 & -0.26 & 0.00 & 0.06 & 0.00 & -0.268 & 0.000 & -0.040 & 0.015 & 1.39 & 3.91 & 550.96 & 14.24 & & & 3.85 & 13.44 \\
\hline 34 & 75 & -0.26 & 0.00 & 0.06 & -0.01 & -0.269 & 0.000 & -0.039 & 0.024 & 1.53 & 4.21 & 570.05 & 3.22 & & & 4.18 & 2.56 \\
\hline 35 & 76 & 0.36 & 0.00 & 0.05 & -0.03 & 0.402 & 0.000 & -0.008 & 0.015 & 0.35 & 4.53 & 586.27 & -4.93 & & & 4.40 & -5.59 \\
\hline 36 & 77 & 0.38 & 0.00 & 0.06 & -0.02 & 0.426 & 0.000 & -0.012 & 0.001 & -0.58 & 4.20 & 604.75 & -15.33 & & & 4.10 & -15.88 \\
\hline 37 & 78 & 0.38 & 0.00 & 0.07 & -0.03 & 0.427 & 0.000 & -0.026 & 0.005 & -1.09 & 4.05 & 620.28 & -22.79 & & & 3.93 & -23.27 \\
\hline . & 79 & 0.38 & 0.00 & 0.07 & -0.02 & 0.428 & 0.000 & -0.024 & -0.004 & -1.78 & 3.56 & 637.73 & -32.17 & & & 3.49 & -32.53 \\
\hline 39 & 80 & 0.39 & 0.00 & 0.08 & -0.02 & 0.441 & 0.000 & -0.032 & -0.009 & -2.37 & 3.47 & 652.11 & -38.48 & & & 3.37 & -38.80 \\
\hline 40 & 81 & 0.40 & 0.00 & 0.09 & -0.01 & 0.455 & 0.000 & -0.038 & -0.023 & -2.71 & 3.48 & 667.97 & -46.27 & & & 3.44 & -46.47 \\
\hline 41 & 82 & 0.41 & 0.00 & 0.09 & 0.00 & 0.467 & 0.000 & -0.032 & -0.033 & -2.85 & 3.74 & 680.60 & -50.83 & & & 3.67 & -51.37 \\
\hline 42 & 83 & -0.23 & 0.00 & 0.10 & 0.05 & -0.234 & 0.000 & -0.092 & -0.019 & 0.58 & 3.78 & 695.06 & -57.21 & -58.96 & 0.315 & 3.80 & -57.25 \\
\hline 43 & 84 & -0.23 & 0.00 & 0.10 & 0.05 & -0.234 & 0.000 & -0.092 & -0.019 & 33 & 3.55 & 706.87 & -60.96 & & & 3.56 & -60.97 \\
\hline 44 & 85 & -0.23 & 0.00 & 0.10 & 0.05 & -0.234 & 0.000 & -0.092 & -0.019 & -0.08 & 3.07 & 720.54 & -66.56 & -67.15 & 0.224 & 3.10 & -66.50 \\
\hline 45 & 86 & -0.23 & 0.00 & 0.09 & 0.05 & -0.235 & 0.000 & -0.081 & -0.022 & -0.21 & 2.76 & 731.57 & -69.52 & -69.83 & 0.085 & 2.77 & -69.45 \\
\hline 46 & 87 & 0.01 & 0.01 & 0.00 & 0.00 & 0.011 & -0.013 & 0.000 & 0.000 & 1.22 & 1.91 & 744.73 & -74.60 & -74.18 & 0.061 & 1.91 & -74.52 \\
\hline 47 & 88 & 0.01 & 0.01 & 0.00 & 0.00 & 0.011 & -0.013 & 0.000 & 0.000 & 0.52 & 1.22 & 755.31 & -77.11 & -76.07 & 0.101 & 1.22 & -77.02 \\
\hline 48 & 89 & 0.01 & 0.01 & 0.00 & 0.00 & 0.011 & -0.013 & 0.000 & 0.000 & -0.86 & 0.05 & 767.97 & -81.70 & -80.65 & 0.027 & 0.05 & -81.59 \\
\hline 49 & 90 & 0.03 & 0.01 & 0.00 & 0.00 & 0.032 & -0.014 & 0.000 & 0.000 & -1.77 & -0.69 & 777.82 & -83.48 & -82.66 & 0.005 & -0.69 & -83.36 \\
\hline 50 & 91 & 0.01 & 0.01 & 0.00 & 0.00 & 0.011 & -0.013 & 0.000 & 0.000 & -2.87 & -1.66 & 789.50 & -87.09 & -86.63 & 0.004 & -1.66 & -86.96 \\
\hline 51 & 92 & 0.02 & 0.01 & -0.01 & 0.00 & 0.021 & -0.014 & 0.012 & 0.000 & -1.91 & -0.86 & 797.07 & -86.59 & -86.45 & 0.003 & -0.86 & -86.46 \\
\hline 52 & 93 & 0.01 & 0.01 & 0.00 & 0.00 & 0.011 & -0.013 & 0.000 & 0.000 & -0.73 & 0.06 & 806.13 & -87.57 & -87.21 & 0.002 & 0.06 & -87.44 \\
\hline 53 & 94 & 0.02 & 0.01 & 0.00 & 0.00 & 0.021 & -0.014 & 0.000 & 0.000 & 0.61 & 1.13 & 812.74 & -86.12 & -86.36 & 0.002 & 1.13 & -85.99 \\
\hline 54 & 95 & -0.17 & 0.00 & 0.02 & 0.01 & -0.176 & 0.000 & -0.012 & -0.006 & 0.51 & 2.29 & 820.86 & -86.16 & -86.78 & 0.002 & 2.31 & -86.02 \\
\hline 55 & 96 & 0.16 & 0.00 & -0.02 & 0.01 & 0.172 & 0.000 & 0.036 & -0.005 & 1.08 & 2.91 & 827.26 & -84.49 & -85.60 & 0.004 & 2.93 & -84.36 \\
\hline
\end{tabular}




\begin{tabular}{|c|c|c|c|c|c|c|c|c|c|c|c|c|c|c|c|c|c|}
\hline \multicolumn{18}{|c|}{$Z=41(\mathrm{Nb})$} \\
\hline 56 & 97 & 0.22 & 0.00 & 0.01 & 0.02 & 0.240 & 0.000 & 0.011 & -0.021 & 0.90 & 3.38 & 835.41 & -84.57 & -85.61 & 0.003 & 3.42 & -84.42 \\
\hline 57 & 98 & 0.24 & 0.00 & 0.01 & 0.02 & 0.262 & 0.000 & 0.015 & -0.020 & 0.95 & 3.70 & 841.51 & -82.59 & -83.53 & 0.006 & 3.74 & -82.46 \\
\hline 58 & 99 & 0.27 & 0.00 & 0.01 & 0.01 & 0.296 & 0.000 & 0.021 & -0.010 & 0.57 & 3.79 & 849.39 & -82.41 & -82.33 & 0.013 & 3.85 & -82.27 \\
\hline 59 & 100 & 0.37 & 0.00 & 0.03 & 0.01 & 0.412 & 0.000 & 0.027 & -0.014 & -1.92 & 4.14 & 854.87 & -79.82 & -79.94 & 0.026 & 4.22 & -79.67 \\
\hline 60 & 101 & 0.36 & 0.00 & 0.02 & 0.02 & 0.400 & 0.000 & 0.037 & -0.019 & -1.88 & 3.88 & 862.53 & -79.40 & -78.94 & 0.019 & 4.00 & -79.22 \\
\hline 61 & 102 & 0.36 & 0.00 & 0.02 & 0.02 & 0.400 & 0.000 & 0.037 & -0.019 & -2.12 & 3.65 & 868.02 & -76.83 & -76.35 & 0.041 & 3.74 & -76.69 \\
\hline 62 & 103 & 0.35 & 0.00 & 0.02 & 0.02 & 0.388 & 0.000 & 0.034 & -0.020 & -1.78 & 3.64 & 874.86 & -75.59 & -75.32 & 0.068 & 3.77 & -75.44 \\
\hline 63 & 104 & 0.35 & 0.00 & 0.03 & 0.02 & 0.389 & 0.000 & 0.022 & -0.024 & -1.84 & 3.54 & 879.70 & -72.36 & -72.22 & 0.105 & 3.64 & -72.24 \\
\hline 64 & 105 & 0.35 & 0.00 & 0.04 & 0.01 & 0.390 & 0.000 & 0.008 & -0.019 & -1.60 & 3.65 & 885.89 & -70.48 & -70.85 & 0.100 & 3.76 & -70.35 \\
\hline 65 & 106 & 0.34 & 0.00 & 0.05 & 0.00 & 0.379 & 0.000 & -0.009 & -0.014 & -1.57 & 3.65 & 890.12 & -66.64 & & & 3.73 & -66.56 \\
\hline 66 & 107 & 0.34 & 0.00 & 0.06 & -0.01 & 0.380 & 0.000 & -0.023 & -0.009 & -1.55 & 3.64 & 895.92 & -64.37 & & & 3.76 & -64.25 \\
\hline 67 & 108 & 0.30 & 0.00 & 0.06 & -0.02 & 0.333 & 0.000 & -0.036 & 0.002 & -0.95 & 3.32 & 900.01 & -60.38 & & & 3.43 & -60.28 \\
\hline 68 & 109 & 0.30 & .00 & 0.07 & -0.02 & 0.334 & 0.000 & -0.048 & -0.002 & -1.19 & 3.14 & 905.49 & -57.79 & & & 3.30 & -57.64 \\
\hline 69 & 110 & 0.30 & 0.00 & 0.08 & -0.03 & 0.335 & 0.000 & -0.062 & 0.004 & -1.82 & 2.76 & 909.17 & -53.40 & & & 2.97 & -53.21 \\
\hline 70 & 111 & 0.30 & 0.00 & 0.09 & -0.03 & 0.336 & 0.000 & -0.074 & 0.001 & -1.93 & 2.74 & 914.04 & -50.20 & & & 3.02 & -49.93 \\
\hline 71 & 112 & 0.29 & 0.00 & 0.08 & -0.03 & 0.323 & 0.000 & -0.064 & 0.005 & -1.54 & 2.73 & 916.92 & -45.01 & & & 2.96 & -44.78 \\
\hline 72 & 113 & 0.28 & 0.00 & 0.08 & -0.03 & 0.312 & 0.000 & -0.067 & 0.006 & -0.98 & 2.89 & 921.16 & -41.18 & & & 3.16 & -40.91 \\
\hline 73 & 114 & -0.21 & 0.00 & 0.07 & 0.02 & -0.217 & 0.000 & -0.062 & -0.002 & 0.38 & 2.78 & 923.73 & -35.67 & & & 2.99 & -35.46 \\
\hline 74 & 115 & -0.19 & 0.00 & 0.08 & 0.03 & -0.195 & 0.000 & -0.076 & -0.010 & 0.04 & 2.28 & 928.22 & -32.10 & & & 2.60 & -31.75 \\
\hline 75 & 116 & -0.18 & 0.00 & 0.08 & 0.04 & -0.184 & 0.000 & -0.078 & -0.020 & -0.60 & 1.45 & 931.11 & -26.91 & & & 1.84 & -26.48 \\
\hline 76 & 117 & -0.16 & 0.00 & 0.08 & 0.04 & -0.164 & 0.000 & -0.081 & -0.021 & -1.06 & 0.83 & 935.32 & -23.05 & & & 1.27 & -22.55 \\
\hline 77 & 118 & -0.16 & .00 & 0.07 & 0.04 & -0.164 & 0.000 & -0.070 & -0.023 & -1.93 & 0.22 & .61 & -17.28 & & & 0.60 & -16.80 \\
\hline 78 & 119 & -0.15 & .00 & 0.07 & 0.03 & -0.154 & 0.000 & -0.071 & -0.015 & -2.37 & -0.32 & 941.37 & -12.96 & & & 0.00 & -12.51 \\
\hline 79 & 120 & -0.15 & 0.00 & 0.07 & 0.03 & -0.154 & 0.000 & -0.071 & -0.015 & -3.42 & -1.36 & 943.73 & -7.25 & & & -1.03 & -6.76 \\
\hline 80 & 121 & 0.01 & 0.01 & 0.00 & 0.00 & 0.011 & -0.013 & 0.000 & 0.000 & -3.09 & -2.11 & 947.33 & -2.78 & & & -2.11 & -2.58 \\
\hline 81 & 122 & 0.02 & 0.01 & 0.01 & 0.00 & 0.021 & -0.013 & -0.012 & -0.000 & -5.09 & -3.87 & 950.08 & 2.55 & & & -3.86 & 2.80 \\
\hline 82 & 123 & 0.01 & .01 & -0.01 & 0.00 & 0.011 & -0.014 & 0.012 & 0.000 & -6.54 & -5.12 & 953.82 & 6.87 & & & -5.11 & 7.18 \\
\hline 83 & 124 & -0.02 & 0.00 & -0.01 & 0.00 & -0.021 & 0.000 & 0.012 & -0.000 & -5.48 & -4.29 & 953.64 & 15.12 & & & -4.28 & 15.48 \\
\hline 84 & 125 & 0.01 & 0.01 & 0.00 & 0.00 & 0.011 & -0.013 & & 0.000 & & -3.42 & & 21.90 & & & -3 . & 22.31 \\
\hline 85 & 126 & 0.01 & 0.01 & 0.00 & 0.00 & 0.011 & -0.013 & 0.000 & 0.000 & -3.58 & -2.58 & 954.45 & 30.47 & & & -2.58 & 30.95 \\
\hline 86 & 127 & 0.01 & 0.01 & 0.00 & 0.00 & 0.011 & -0.013 & 0.000 & 0.000 & -2.38 & -1.52 & 955.23 & 37.76 & & & -1.52 & 38.31 \\
\hline 87 & 128 & 0.03 & 0.05 & -0.01 & 0.00 & 0.033 & -0.068 & 13 & 0.002 & -1.88 & -0.81 & .55 & 46.50 & & & -0.74 & 47.20 \\
\hline 88 & 129 & 0.03 & 0.07 & -0.01 & 0.00 & 0.034 & -0.095 & 0.014 & 0.004 & -1.19 & 0.07 & 955.22 & 53.91 & & & 0.21 & 54.76 \\
\hline 89 & 130 & & .11 & -0.0 & 0.02 & & -0.147 & & -0.012 & -1 . & 0.53 & & & & & 0.91 & 63.87 \\
\hline 90 & 131 & 0.00 & 0.12 & -0.02 & 0.02 & 0.006 & -0.162 & 0.029 & -0.010 & -0.86 & 1.22 & 955.06 & 70.21 & & & 1.70 & 71.59 \\
\hline 91 & 132 & 0.21 & 0.00 & -0.05 & 0.00 & .227 & 0.000 & 0.081 & 0.016 & -0.73 & 1.86 & 953.90 & 79.43 & & & 2.12 & 80.70 \\
\hline 92 & 133 & 0.21 & .00 & -0.05 & 0.00 & 0.227 & 0.000 & 81 & 0.016 & -0.46 & 2.10 & 954.62 & 86.79 & & & 2.39 & 88.18 \\
\hline 93 & 134 & 0.24 & 0.00 & -0.05 & 0.00 & 0.261 & 0.000 & 0.088 & 0.019 & -1.17 & 1.98 & 953.96 & 95.52 & & & 2.26 & 97.03 \\
\hline 94 & 135 & 0.2 & 0.00 & -0.05 & 0.01 & & & & & & 2.11 & & & & & 38 & \\
\hline 95 & 136 & 0.26 & 0.00 & -0.04 & 0.01 & 0.282 & 0.000 & 0.080 & 0.007 & -1.19 & 1.95 & 953.63 & 111.99 & & & 2.12 & 113.64 \\
\hline 96 & 137 & 027 & 0.00 & -0.02 & 0.01 & & .000 & & & -0.86 & 2.28 & & & & & 2.32 & 121.61 \\
\hline 97 & 138 & 0.28 & 0.00 & -0.01 & 0.01 & 06 & .000 & 0.048 & -0.002 & -1.23 & 1.99 & .74 & 129.03 & & & 1.98 & 130.77 \\
\hline 98 & 139 & 0.29 & 0.00 & 0.00 & 0.01 & & 0.000 & 0.038 & -0.005 & -1.24 & 2.11 & 952.81 & 137.03 & & & 2.07 & 138.90 \\
\hline 99 & 140 & 0.29 & 0.00 & 0.01 & 0.01 & 0.318 & 0.000 & 0.026 & -0.009 & & 1.76 & 951.63 & 146.28 & & & 1.70 & 148.28 \\
\hline \multicolumn{18}{|c|}{$Z=42$ (Mo) } \\
\hline 35 & 77 & -0.27 & .00 & .06 & 0.01 & -0.278 & 000 & -0.037 & 0.024 & 1.44 & 4.17 & 584.96 & 3.68 & & & 4.13 & 3.06 \\
\hline 36 & 78 & 0.36 & 0.00 & 0.06 & -0.03 & 0.403 & 0.000 & -0.020 & 0.010 & 0.12 & 4.35 & 603.84 & -7.13 & & & 4.28 & -7.69 \\
\hline 37 & 79 & 0.36 & 0.00 & 0.06 & -0.03 & 0.403 & 0.000 & -0.020 & 0.010 & -0.30 & 4.09 & 619.66 & -14.89 & & & 4.00 & -15.39 \\
\hline 38 & 80 & 038 & 0.00 & 0.07 & -0.02 & 0.428 & 0.000 & -0.024 & -0.004 & -1.26 & 3.79 & 637.84 & -24.99 & & & 3.73 & -25.38 \\
\hline 39 & 81 & 0.39 & 0.00 & 0.07 & -0.01 & 0.440 & 0.000 & -0.018 & -0.014 & -1.74 & 3.76 & 652.34 & -31.42 & & & 3.68 & -31.76 \\
\hline 40 & 82 & 0.41 & 0.00 & 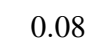 & 0.00 & 465 & .000 & -0.020 & -0.028 & -2.29 & 3.80 & 669.06 & -40.07 & & & 3.78 & -40.29 \\
\hline 41 & 83 & 0.43 & 0.00 & 0.09 & 0.01 & 0.492 & 0.000 & -0.021 & -0.042 & -2.91 & 3.96 & 682.34 & -45.28 & & & 3.91 & -45.46 \\
\hline 42 & 84 & -0.23 & 0.00 & 0.10 & 0.05 & -0.234 & 0.000 & -0.092 & -0.019 & 0.22 & 3.41 & 698.60 & -53.47 & & & 3.45 & -53.53 \\
\hline 43 & 85 & -0.23 & 0.00 & 01 & 0.05 & -0.234 & 0.000 & -0.092 & -0.019 & -0.04 & 3.13 & 710.64 & -57.44 & & & 3.15 & -57.47 \\
\hline 44 & 86 & -0.23 & 0.00 & 0.10 & 0.05 & -0.234 & 0.000 & -0.092 & -0.019 & -0.46 & 2.64 & 725.19 & -63.91 & -64.56 & 0.438 & 2.68 & -63.88 \\
\hline 45 & 87 & -0.23 & 0.00 & 0.1 & 0.05 & -0.234 & 0.000 & -0.092 & -0.019 & -0.75 & 2.32 & 736.39 & -67.04 & -67.69 & 0.223 & 2.35 & -66.99 \\
\hline 46 & 88 & 0.00 & 0.00 & 0.00 & 0.00 & 0.000 & 0.000 & 0.000 & 0.000 & 0.85 & 1.50 & 750.36 & -72.94 & -72.70 & 0.020 & 1.50 & -72.90 \\
\hline
\end{tabular}




\begin{tabular}{|c|c|c|c|c|c|c|c|c|c|c|c|c|c|c|c|c|c|}
\hline \multicolumn{18}{|c|}{$Z=42$ (Mo) } \\
\hline 47 & 89 & -0.02 & 0.00 & 0.00 & 0.00 & -0.021 & 0.000 & 0.000 & 0.000 & 0.12 & 0.80 & 761.12 & -75.63 & -75.00 & 0.015 & 0.80 & -75.56 \\
\hline 48 & 90 & 0.00 & 0.00 & 0.00 & 0.00 & 0.000 & 0.000 & 0.000 & 0.000 & -1.25 & -0.38 & 774.61 & -81.05 & -80.17 & 0.006 & -0.38 & -80.96 \\
\hline 49 & 91 & 0.03 & 0.00 & 0.01 & 0.00 & 0.032 & 0.000 & -0.012 & -0.000 & -2.19 & -1.13 & 784.63 & -83.00 & -82.20 & 0.011 & -1.13 & -82.91 \\
\hline 50 & 92 & 0.00 & 0.00 & 0.00 & 0.00 & 0.000 & 0.000 & 0.000 & 0.000 & -3.30 & -2.14 & 797.16 & -87.46 & -86.81 & 0.004 & -2.15 & -87.35 \\
\hline 51 & 93 & -0.01 & 0.00 & 0.00 & 0.00 & -0.011 & 0.000 & 0.000 & 0.000 & -2.39 & -1.44 & 804.99 & -87.22 & -86.80 & 0.004 & -1.45 & -87.11 \\
\hline 52 & 94 & 0.00 & 0.00 & 0.00 & 0.00 & 0.000 & 0.000 & 0.000 & 0.000 & -1.24 & -0.48 & 814.78 & -88.94 & -88.41 & 0.002 & -0.48 & -88.83 \\
\hline 53 & 95 & 0.00 & 0.00 & 0.00 & 0.00 & 0.000 & 0.000 & 0.000 & 0.000 & 0.10 & 0.55 & 821.59 & -87.68 & -87.71 & 0.002 & 0.55 & -87.57 \\
\hline 54 & 96 & 0.14 & 0.00 & -0.03 & 0.01 & 0.150 & 0.000 & 0.045 & -0.004 & 0.17 & 1.85 & 830.34 & -88.36 & -88.79 & 0.002 & 1.88 & -88.22 \\
\hline 55 & 97 & 0.16 & 0.00 & -0.02 & 0.02 & 0.172 & 0.000 & 0.036 & -0.015 & 0.60 & 2.39 & 836.98 & -86.92 & -87.54 & 0.002 & 2.42 & -86.79 \\
\hline 56 & 98 & 0.19 & 0.00 & 0.00 & 0.02 & 0.206 & 0.000 & 0.017 & -0.019 & 0.86 & 2.97 & 845.77 & -87.64 & -88.11 & 0.002 & 3.02 & -87.50 \\
\hline 57 & 99 & 0.22 & 0.00 & 0.00 & 0.02 & 0.239 & 0.000 & 0.023 & -0.018 & 0.85 & 3.48 & 851.81 & -85.61 & -85.97 & 0.002 & 3.53 & -85.48 \\
\hline 58 & 100 & 0.23 & 0.00 & 0.01 & 0.02 & 0.251 & 0.000 & 0.013 & -0.021 & 1.10 & 3.82 & 860.21 & -85.94 & -86.18 & 0.006 & 3.88 & -85.80 \\
\hline 59 & 101 & 0.27 & 0.00 & 0.00 & 0.01 & 0.295 & 0.000 & 0.033 & -0.006 & 0.55 & 3.93 & 866.05 & -83.71 & -83.51 & 0.006 & 3.99 & -83.59 \\
\hline 60 & 102 & 0.30 & 0.00 & 0.00 & 0.01 & 0.329 & 0.000 & 0.041 & -0.005 & -0.11 & 3.92 & 874.20 & -83.78 & -83.56 & 0.021 & 4.01 & -83.64 \\
\hline 61 & 103 & 0.34 & 0.00 & 0.01 & 0.02 & 0.376 & 0.000 & 0.042 & -0.016 & -1.19 & 3.79 & 879.72 & -81.23 & -80.85 & 0.061 & 3.89 & -81.09 \\
\hline 62 & 104 & 0.34 & 0.00 & 0.02 & 0.02 & 0.377 & 0.000 & 0.030 & -0.020 & -1.09 & 3.81 & 25 & -80.69 & -80.33 & 0.054 & 3.95 & -80.52 \\
\hline 63 & 105 & 0.35 & 0.00 & 0.03 & 0.02 & 0.389 & 0.000 & 0.022 & -0.024 & -1.40 & 3.94 & 891.97 & -77.35 & -77.34 & 0.071 & 4.07 & -77.20 \\
\hline 64 & 106 & 0.32 & 0.00 & 0.03 & 0.01 & 0.354 & 0.000 & 0.010 & -0.016 & -0.65 & 3.99 & 898.93 & -76.23 & -76.25 & 0.018 & 4.11 & -76.11 \\
\hline 65 & 107 & 0.31 & 0.00 & 0.04 & 0.00 & 0.343 & 0.000 & -0.006 & -0.010 & -0.63 & 3.84 & 903.43 & -72.66 & -72.94 & 0.162 & 3.92 & -72.58 \\
\hline 66 & 108 & 0.30 & 0.00 & 0.05 & -0.01 & 0.333 & 0.000 & -0.023 & -0.004 & -0.47 & 3.79 & 909.96 & -71.12 & & & 3.91 & -71.02 \\
\hline 67 & 109 & 0.30 & 0.00 & 0.06 & -0.02 & 3 & 0 & -0.036 & 02 & -0.65 & 3.59 & 33 & -67.11 & & & 72 & -67.01 \\
\hline 68 & 110 & 0.30 & 0.00 & 0.07 & -0.03 & 0.334 & 0.000 & -0.050 & 0.008 & -0.91 & 3.28 & 920.33 & -6 & & & 3.49 & -65.17 \\
\hline 69 & 111 & 0.30 & 0.00 & 0.08 & -0.03 & 0.335 & 0.000 & -0.062 & 0.004 & -1.50 & 3.01 & 924.00 & -60.94 & & & 3.23 & -60.76 \\
\hline 70 & 112 & 0.29 & 0.00 & 0.08 & -0.03 & 0.323 & 0.000 & -0.064 & 0.005 & -1.25 & 2.99 & 929.54 & -58.41 & & & 3.25 & -58.19 \\
\hline 71 & 113 & 0.28 & 0.00 & 0.08 & -0.03 & 0.312 & 0.000 & -0.067 & 0.006 & -1.14 & 2.87 & 932.62 & -53.42 & & & 3.12 & -53.21 \\
\hline 72 & 114 & 22 & 0.00 & 0 & 1 & i & 0 & 59 & 77 & & 34 & & & & & 05 & \\
\hline 73 & 115 & -0.22 & 0.00 & 0.08 & 0.02 & -0.227 & 0.000 & -0.071 & 0.001 & -0.35 & 2.25 & 940.85 & -45.51 & & & 2.51 & -45.28 \\
\hline 74 & 116 & -0.19 & 0.00 & 0.08 & 0.03 & -0.195 & 0.000 & -0.076 & -0.010 & -0.34 & 1.95 & 945.80 & -42.38 & & & 2.26 & -42.08 \\
\hline 75 & 117 & -0.18 & 0.00 & 0.08 & 0.04 & -0.184 & 0.000 & -0.078 & -0.020 & -0.97 & 1.06 & 948.83 & -37.35 & & & 1.44 & -36.97 \\
\hline 76 & 118 & -0.16 & 0.00 & 0.08 & 0.04 & -0.164 & 0.000 & -0.081 & -0.021 & -1.42 & 0.51 & 953.61 & -34.05 & & & 0.93 & -33.62 \\
\hline 77 & 119 & -0.16 & 0.00 & & & & & & & -2.45 & & & & & & 0.29 & \\
\hline 78 & 120 & -0.15 & 0.00 & 0.07 & 0.03 & -0.154 & 0.000 & -0.071 & -0.015 & -2.73 & -0.66 & 960.38 & -24.68 & & & -0.34 & -24.30 \\
\hline 79 & 121 & -0.12 & 0.00 & 0.06 & 0.03 & -0.124 & 0.000 & -0.063 & -0.019 & -3.43 & -1.79 & 962.91 & -19.14 & & & -1.50 & -18.76 \\
\hline 80 & 122 & 0.00 & 0.00 & -0.01 & 0.00 & 0.000 & 0.000 & 0.012 & 0.000 & -3.65 & -2.64 & 967.22 & -15.38 & & & -2.63 & -15.25 \\
\hline 81 & 123 & 0.02 & 0.00 & 0.01 & 0.00 & 0.021 & 0.000 & -0.012 & -0.000 & -5.61 & -4.36 & 970.00 & -10.09 & & & -4.36 & -9.92 \\
\hline 82 & 124 & 0.00 & 0.00 & 0.01 & 0.00 & & 0.000 & -0.012 & 0.000 & -7.06 & -5.67 & & -6.42 & & & -5.66 & -6.21 \\
\hline 83 & 125 & -0.01 & 0.00 & -0.01 & 0.01 & -0.011 & 0.000 & 0.012 & -0.010 & -6.05 & -4.83 & 974.28 & 1.77 & & & -4.80 & 2.05 \\
\hline 84 & 126 & 0.00 & 0.00 & 0.01 & 0.00 & 0.000 & 0.000 & -0.012 & 0.000 & -5.03 & -3.89 & 976.11 & 8.02 & & & -3.89 & 8.33 \\
\hline 85 & 127 & 0.00 & 0.00 & 0.01 & 0.00 & 0.000 & 0.000 & -0.012 & 0.000 & -4.06 & -3.05 & 975.66 & 16.54 & & & -3.04 & 16.91 \\
\hline 86 & 128 & 0.00 & 0.00 & 0.01 & 0.00 & 0.000 & 0.000 & -0.012 & 0.000 & -2.86 & -1.96 & 977.01 & 23.26 & & & -1.96 & 23.70 \\
\hline 87 & 129 & 0.00 & 0.00 & 0.01 & 0.00 & 00 & 0.000 & -0.012 & 0.000 & -1.93 & -1.16 & 976.30 & 32.04 & & & -1.15 & 32.54 \\
\hline 88 & 130 & 0.00 & 0.00 & 0.00 & 0.01 & 0.000 & 0.000 & 0.000 & -0.010 & -0.83 & -0.22 & 977.47 & 38.94 & & & -0.19 & 39.54 \\
\hline 89 & 131 & 0.02 & 0.09 & -0.01 & 0.02 & 0.025 & -0.121 & 0.015 & -0.014 & -1.22 & 0.29 & 976.77 & 47.71 & & & 0.57 & 48.65 \\
\hline 90 & 132 & 0.00 & 0.10 & -0.01 & 0.01 & 0.004 & -0.135 & 0.016 & -0.003 & -0.53 & 1.11 & 977.76 & 54.79 & & & 1.40 & 55.83 \\
\hline 91 & 133 & 0.20 & 0.00 & -0.06 & 0.00 & 0.216 & 0.000 & 0.092 & 0.018 & -0.94 & 1.70 & 976.70 & 63.93 & & & 2.07 & 65.14 \\
\hline 92 & 134 & 0.21 & 0.00 & -0.05 & 0.00 & 27 & 0.000 & 081 & 0.016 & -0.53 & 2.08 & 977.84 & 70.86 & & & 2.37 & 72.08 \\
\hline 93 & 135 & 0.21 & 0.00 & -0.05 & 0.00 & 0.227 & 0.000 & 0.081 & 0.016 & -0.59 & 2.01 & 977.16 & 79.60 & & & 2.29 & 80.93 \\
\hline 94 & 136 & 0.24 & 0.00 & -0.05 & 0.01 & 0.260 & 0.000 & 0.088 & 0.009 & -0.80 & 2.24 & 978.17 & 86.67 & & & 2.52 & 88.10 \\
\hline 95 & 137 & 0.25 & 0.00 & -0.04 & 0.01 & 0.271 & 0.000 & 0.078 & 0.006 & -0.91 & 2.22 & 977.19 & 95.72 & & & 2.40 & 97.18 \\
\hline 96 & 138 & 0.25 & 0.00 & -0.03 & 0.01 & 0.271 & 0.000 & 0.065 & 0.003 & -0.50 & 2.51 & 977.87 & 103.11 & & & 2.64 & 104.64 \\
\hline 97 & 139 & 0.27 & 0.00 & -0.02 & 0.02 & 94 & 00 & 59 & -0.009 & -0.98 & 2.24 & 39 & 16 & & & 2.35 & .80 \\
\hline 98 & 140 & 0.28 & 0.00 & -0.01 & 0.02 & 0.306 & 0.000 & 0.049 & -0.012 & -0.90 & 2.30 & 977.55 & 119.57 & & & 2.38 & 121.33 \\
\hline 99 & 141 & 0.28 & 0.00 & 0.00 & 0.02 & 0.306 & 0.000 & 0.037 & -0.016 & -1.21 & 1.91 & 976.45 & 128.75 & & & 1.97 & 130.63 \\
\hline 100 & 142 & 0.29 & 0.00 & 0.01 & 0.02 & 0.318 & 0.000 & 0.027 & -0.019 & -1.29 & 1.96 & 976.86 & 136.41 & & & 2.04 & 138.45 \\
\hline 101 & 143 & 0.29 & 0.00 & 0.02 & 0.02 & 0.319 & 0.000 & 0.015 & -0.022 & -1.61 & 1.63 & 975.48 & 145.86 & & & 1.72 & 148.07 \\
\hline 102 & 144 & 0.29 & 0.00 & 0.03 & 0.02 & 0.320 & 0.000 & 0.003 & -0.026 & -1.51 & 1.64 & 975.70 & 153.71 & & & 1.79 & 156.16 \\
\hline
\end{tabular}




\begin{tabular}{|c|c|c|c|c|c|c|c|c|c|c|c|c|c|c|c|c|c|}
\hline \multicolumn{18}{|c|}{$Z=43(\mathrm{Tc})$} \\
\hline 36 & 79 & -0.27 & 0.00 & 0.08 & 0.02 & -0.277 & 0.000 & -0.061 & 0.004 & 1.12 & 3.89 & 600.13 & 3.86 & & & 3.85 & 3.30 \\
\hline 37 & 80 & -0.24 & 0.00 & 0.08 & 0.03 & -0.247 & 0.000 & -0.068 & -0.007 & 1.64 & 4.18 & 616.30 & -4.24 & & & 4.14 & -4.72 \\
\hline 38 & 81 & 0.37 & 0.00 & 0.06 & -0.02 & 0.415 & 0.000 & -0.015 & 0.001 & -0.62 & 3.93 & 634.61 & -14.47 & & & 3.83 & -14.95 \\
\hline 39 & 82 & 0.38 & 0.00 & 0.07 & -0.01 & 0.428 & 0.000 & -0.022 & -0.014 & -1.10 & 3.92 & 649.96 & -21.76 & & & 3.79 & -22.19 \\
\hline 40 & 83 & -0.23 & 0.00 & 0.10 & 0.05 & -0.234 & 0.000 & -0.092 & -0.019 & 0.56 & 3.59 & 667.23 & -30.96 & & & 3.60 & -31.19 \\
\hline 41 & 84 & -0.23 & 0.00 & 0.10 & 0.05 & -0.234 & 0.000 & -0.092 & -0.019 & 0.28 & 3.51 & 681.62 & -37.27 & & & 3.50 & -37.47 \\
\hline 42 & 85 & -0.24 & 0.00 & 0.11 & 0.06 & -0.243 & 0.000 & -0.101 & -0.024 & -0.49 & 3.00 & 698.02 & -45.60 & & & 3.02 & -45.72 \\
\hline 43 & 86 & -0.24 & 0.00 & 0.12 & 0.06 & -0.243 & 0.000 & -0.112 & -0.021 & -0.95 & 2.73 & 711.27 & -50.78 & & & 2.73 & -51.21 \\
\hline 44 & 87 & -0.24 & 0.00 & 0.11 & 0.06 & -0.243 & 0.000 & -0.101 & -0.024 & -1.21 & 2.21 & 726.35 & -57.79 & & & 2.23 & -57.81 \\
\hline 45 & 88 & -0.24 & 0.00 & 0.11 & 0.05 & -0.244 & 0.000 & -0.101 & -0.015 & -1.50 & 2.11 & 738.17 & -61.54 & & & 2.11 & -61.55 \\
\hline 46 & 89 & 0.03 & 0.00 & 0.00 & 0.00 & 0.032 & 0.000 & 0.000 & 0.000 & 0.49 & 1.17 & 752.42 & -67.72 & & & 1.17 & -67.70 \\
\hline 47 & 90 & 0.05 & 0.00 & 0.00 & 0.00 & 0.053 & 0.000 & 0.001 & 0.000 & -0.28 & 0.53 & 763.95 & -71.17 & -71.21 & 0.242 & 0.53 & -71.13 \\
\hline 48 & 91 & 0.02 & 0.00 & 0.00 & 0.00 & 0.021 & 0.000 & 0.000 & 0.000 & -1.57 & -0.68 & 777.62 & -76.77 & -75.98 & 0.200 & -0.68 & -76.72 \\
\hline 49 & 92 & 0.04 & 0.00 & 0.00 & 0.00 & 0.043 & 0.000 & 0.001 & 0.000 & -2.51 & -1.43 & 788.45 & -79.53 & -78.93 & 0.026 & -1.43 & -79.46 \\
\hline 50 & 93 & 0.00 & 0.00 & 0.00 & 0.00 & 0.000 & 0.000 & 0.000 & 0.000 & -3.59 & -2.45 & 801.15 & -84.15 & -83.60 & 0.004 & -2.45 & -84.07 \\
\hline 51 & 94 & 0.02 & 0.00 & 0.00 & 0.00 & 0.021 & 0.000 & 0.000 & 0.000 & -2.59 & -1.62 & 809.64 & -84.58 & -84.15 & 0.004 & -1.62 & -84.49 \\
\hline 52 & 95 & 0.02 & 0.00 & 0.00 & 0.00 & 0.021 & 0.000 & 0.000 & 0.000 & -1.43 & -0.67 & 819.60 & -86.47 & -86.02 & 0.005 & -0.67 & -86.37 \\
\hline 53 & 96 & 0.12 & 0.00 & -0.03 & 0.00 & 0.128 & 0.000 & 0.043 & 0.005 & -0.91 & 0.89 & 826.66 & -85.45 & -85.82 & 0.005 & 0.90 & -85.35 \\
\hline 54 & 97 & 0.14 & 0.00 & -0.03 & 0.01 & 0.150 & 0.000 & 0.045 & -0.004 & -0.31 & 1.50 & 836.24 & -86.96 & -87.22 & 0.005 & 1.52 & -86.85 \\
\hline 55 & 98 & 0.16 & 0.00 & -0.01 & 0.02 & 0.172 & 0.000 & 0.024 & -0.017 & 0.21 & 2.03 & 843.65 & -86.30 & -86.43 & 0.004 & 2.04 & -86.20 \\
\hline 56 & 99 & 0.18 & 0.00 & -0.01 & 0.03 & 0.194 & 0.000 & 0.028 & -0.027 & 0.47 & 2.60 & 852.58 & -87.16 & -87.32 & 0.002 & 2.65 & -87.04 \\
\hline 57 & 100 & 0.19 & 0.00 & 0.01 & 0.02 & 0.206 & 0.000 & 0.005 & -0.021 & 0.88 & 3.19 & 859.29 & -85.80 & -86.02 & 0.002 & 3.21 & -85.71 \\
\hline 58 & 101 & 0.21 & 0.00 & 0.00 & 0.02 & 0.228 & 0.000 & 0.021 & -0.018 & 0.99 & 3.51 & 867.84 & -86.28 & -86.34 & 0.024 & 3.55 & -86.17 \\
\hline 59 & 102 & 0.25 & 0.00 & -0.01 & 0.02 & 0.272 & 0.000 & 0.041 & -0.014 & 0.51 & 3.80 & 874.24 & -84.61 & -84.57 & 0.009 & 3.84 & -84.52 \\
\hline 60 & 103 & 0.27 & 0.00 & -0.01 & 0.01 & 0.294 & 0.000 & 0.045 & -0.003 & 0.27 & 3.96 & 882.34 & -84.64 & -84.60 & 0.010 & 4.01 & -84.54 \\
\hline 61 & 104 & 0.28 & 0.00 & 0.00 & 0.01 & 0.306 & 0.000 & 0.036 & -0.006 & 0.13 & 4.01 & 8.40 & -82.62 & -82.49 & 946 & 4.05 & -82.56 \\
\hline 62 & 105 & 0.29 & 0.00 & 0.00 & 0.01 & 0.318 & 0.000 & 0.038 & -0.005 & -0.07 & 4.03 & 896.05 & -82.21 & -82.29 & 0.055 & 4.09 & -82.13 \\
\hline 63 & 106 & 0.29 & 0.00 & 0.01 & 0.01 & 0.318 & 0.000 & 0.026 & -0.009 & -0.16 & 3.94 & 901.71 & -79.79 & -79.78 & 0.013 & 3.99 & -79.74 \\
\hline 64 & 107 & 0.28 & 0.00 & 0.02 & 0.00 & 0.308 & 0.000 & 0.010 & -0.003 & 0.08 & 3.92 & 908.85 & -78.86 & -79.10 & 0.150 & 3.97 & -78.82 \\
\hline 65 & 108 & 0.28 & 0.00 & 0.03 & 0.00 & 0.308 & 0.000 & -0.002 & -0.006 & -0.13 & 3.73 & 914.09 & -76.03 & -75.95 & 0.126 & 3.77 & -76.01 \\
\hline 66 & 109 & 0.28 & 0.00 & 0.04 & -0.01 & 0.309 & 0.000 & -0.016 & -0.000 & -0.17 & 3.67 & 920.73 & -74.60 & -74.54 & 0.096 & 3.75 & -74.57 \\
\hline 67 & 110 & 0.28 & 0.00 & 0.05 & -0.02 & 0.309 & 0.000 & -0.029 & 0.006 & -0.29 & 3.58 & 925.38 & -71.17 & -70.96 & 0.077 & 3.66 & -71.14 \\
\hline 68 & 111 & 0.28 & 0.00 & 0.06 & -0.02 & 0.310 & 0.000 & -0.041 & 0.003 & -0.35 & 3.58 & 931.47 & -69.19 & -69.22 & 0.109 & 3.70 & -69.12 \\
\hline 69 & 112 & 0.28 & 0.00 & 0.07 & -0.03 & 0.311 & 0.000 & -0.055 & 0.009 & -0.92 & 3.20 & 935.93 & -65.58 & -66.00 & 0.124 & 3.36 & -65.48 \\
\hline 70 & 113 & -0.25 & 0.00 & 0.08 & 0.00 & -0.258 & 0.000 & -0.064 & 0.021 & -0.36 & 3.09 & 941.66 & -63.24 & & & 3.31 & -63.08 \\
\hline 71 & 114 & -0.25 & 0.00 & 0.08 & 0.01 & -0.258 & 0.000 & -0.064 & 0.012 & -0.58 & 2.74 & 945.63 & -59.14 & & & 2.93 & -59.01 \\
\hline 72 & 115 & -0.22 & 0.00 & 0.07 & 0.01 & -0.227 & 0.000 & -0.059 & 0.007 & -0.12 & 2.52 & 951.01 & -56.45 & & & 2.70 & -56.34 \\
\hline 73 & 116 & -0.22 & 0.00 & 0.08 & 0.02 & -0.227 & 0.000 & -0.071 & 0.001 & -0.74 & 2.03 & 954.69 & -52.06 & & & 2.26 & -51.90 \\
\hline 74 & 117 & -0.19 & 0.00 & 0.08 & 0.03 & -0.195 & 0.000 & -0.076 & -0.010 & -0.72 & 1.69 & 959.76 & -49.06 & & & 1.97 & -48.83 \\
\hline 75 & 118 & -0.18 & 0.00 & 0.08 & 0.03 & -0.185 & 0.000 & -0.078 & -0.011 & -1.34 & 1.09 & 963.15 & -44.37 & & & 1.38 & -44.13 \\
\hline 76 & 119 & -0.16 & 0.00 & 0.08 & 0.04 & -0.164 & 0.000 & -0.081 & -0.021 & -1.62 & 0.41 & 968.13 & -41.29 & & & 0.79 & -40.94 \\
\hline 77 & 120 & -0.16 & 0.00 & 0.08 & 0.04 & -0.164 & 0.000 & -0.081 & -0.021 & -2.67 & -0.60 & 971.54 & -36.62 & & & -0.22 & -36.26 \\
\hline 78 & 121 & -0.16 & 0.00 & 0.07 & 0.04 & -0.164 & 0.000 & -0.070 & -0.023 & -3.05 & -0.83 & 975.68 & -32.69 & & & -0.47 & -32.33 \\
\hline 79 & 122 & -0.15 & 0.00 & 0.07 & 0.03 & -0.154 & 0.000 & -0.071 & -0.015 & -3.99 & -1.83 & 978.70 & -27.64 & & & -1.53 & -27.32 \\
\hline 80 & 123 & 0.00 & 0.00 & 0.00 & 0.00 & 0.000 & 0.000 & 0.000 & 0.000 & -3.67 & -2.75 & 983.15 & -24.02 & & & -2.75 & -23.96 \\
\hline 81 & 124 & 0.02 & 0.00 & 0.01 & 0.00 & 0.021 & 0.000 & -0.012 & -0.000 & -5.71 & -4.49 & 986.55 & -19.35 & & & -4.48 & -19.25 \\
\hline 82 & 125 & 0.00 & 0.00 & 0.00 & 0.00 & 0.000 & 0.000 & 0.000 & 0.000 & -7.15 & -5.78 & 991.02 & -15.74 & & & -5.78 & -15.62 \\
\hline 83 & 126 & -0.02 & 0.00 & 0.00 & 0.00 & -0.021 & 0.000 & 0.000 & 0.000 & -6.13 & -4.94 & 991.49 & -8.15 & & & -4.94 & -7.98 \\
\hline 84 & 127 & 0.00 & 0.00 & 0.00 & 0.00 & 0.000 & 0.000 & 0.000 & 0.000 & -5.09 & -3.99 & 993.36 & -1.95 & & & -3.99 & -1.73 \\
\hline 85 & 128 & -0.01 & 0.01 & 0.01 & 0.00 & -0.010 & -0.013 & -0.012 & 0.000 & -4.10 & -3.11 & 993.47 & 6.02 & & & -3.10 & 6.29 \\
\hline 86 & 129 & 0.00 & 0.00 & 0.00 & 0.01 & 0.000 & 0.000 & 0.000 & -0.010 & -2.87 & -2.03 & 994.88 & 12.68 & & & -2.01 & 13.02 \\
\hline 87 & 130 & 0.00 & 0.01 & 0.00 & 0.00 & 0.000 & -0.013 & 0.000 & 0.000 & -1.90 & -1.18 & 994.71 & 20.92 & & & -1.18 & 21.31 \\
\hline 88 & 131 & 0.00 & 0.00 & 0.01 & -0.01 & 0.000 & 0.000 & -0.012 & 0.010 & -0.84 & -0.22 & 995.92 & 27.78 & & & -0.19 & 28.27 \\
\hline 89 & 132 & 0.03 & 0.08 & 0.00 & 0.01 & 0.034 & -0.108 & 0.003 & -0.006 & -0.90 & 0.44 & 995.63 & 36.14 & & & 0.60 & 36.83 \\
\hline 90 & 133 & 0.15 & 0.00 & -0.04 & 0.01 & 0.161 & 0.000 & 0.059 & -0.002 & -0.46 & 1.36 & 996.57 & 43.28 & & & 1.51 & 44.03 \\
\hline 91 & 134 & 0.18 & 0.00 & -0.05 & 0.01 & 0.194 & 0.000 & 0.076 & 0.003 & -0.61 & 1.56 & 996.45 & 51.47 & & & 1.78 & 52.37 \\
\hline 92 & 135 & 0.20 & 0.00 & -0.06 & 0.00 & 0.216 & 0.000 & 0.092 & 0.018 & -0.89 & 1.84 & 997.75 & 58.24 & & & 2.19 & 59.38 \\
\hline
\end{tabular}




\section{$Z=43(\mathrm{Tc})$}

\section{$\begin{array}{lllll}93 & 136 & 0.21 & 0.00 & -0.05\end{array}$}

$\begin{array}{lllll}94 & 137 & 0.23 & 0.00 & -0.05\end{array}$

$\begin{array}{lllll}95 & 138 & 0.25 & 0.00 & -0.04\end{array}$

$\begin{array}{lllll}96 & 139 & 0.25 & 0.00 & -0.03\end{array}$

$\begin{array}{llllll}97 & 140 & 0.26 & 0.00 & -0.02\end{array}$

$\begin{array}{llllll}98 & 141 & 0.27 & 0.00 & -0.01\end{array}$

$\begin{array}{lllll}99 & 142 & 0.28 & 0.00 & 0.00\end{array}$

$\begin{array}{lllll}100 & 143 & 0.28 & 0.00 & 0.01\end{array}$

$\begin{array}{lllll}101 & 144 & 0.29 & 0.00 & 0.02\end{array}$

$\begin{array}{lllll}102 & 145 & 0.28 & 0.00 & 0.02\end{array}$

$\begin{array}{llllll}103 & 146 & 0.29 & 0.00 & 0.03\end{array}$

$\begin{array}{lllll}104 & 147 & 0.28 & 0.00 & 0.04\end{array}$

\section{$Z=44$ (Ru)}

$\begin{array}{llllll}37 & 81 & -0.24 & 0.00 & 0.08\end{array}$

$\begin{array}{llllll}38 & 82 & -0.23 & 0.00 & 0.09\end{array}$

$\begin{array}{lllll}39 & 83 & -0.23 & 0.00 & 0.10\end{array}$

$\begin{array}{llllll}40 & 84 & -0.23 & 0.00 & 0.10\end{array}$

$\begin{array}{lllll}41 & 85 & -0.23 & 0.00 & 0.10\end{array}$

$\begin{array}{lllll}42 & 86 & -0.24 & 0.00\end{array}$

$\begin{array}{llll}43 & 87 & -0.24 & 0.00\end{array}$

$\begin{array}{llll}44 & 88 & -0.24 & 0.00\end{array}$

$\begin{array}{llll}45 & 89 & -0.24 & 0.00\end{array}$

$\begin{array}{llll}46 & 90 & 0.01 & 0.00\end{array}$

$\begin{array}{llllll}47 & 91 & -0.02 & 0.00 & 0.00\end{array}$

$\begin{array}{lllll}48 & 92 & 0.00 & 0.00 & 0.00\end{array}$

$\begin{array}{lllll}49 & 93 & 0.03 & 0.00 & 0.01\end{array}$

$\begin{array}{lllll}50 & 94 & 0.00 & 0.00 & 0.00\end{array}$

$\begin{array}{lllll}51 & 95 & -0.02 & 0.00 & 0.00\end{array}$

$\begin{array}{lllll}52 & 96 & 0.00 & 0.00 & 0.00\end{array}$

$\begin{array}{llllll}53 & 97 & 0.10 & 0.00 & -0.03\end{array}$

$\begin{array}{llllll}54 & 98 & 0.13 & 0.00 & -0.03\end{array}$

$\begin{array}{llllll}55 & 99 & 0.15 & 0.00 & -0.02\end{array}$

$\begin{array}{lllll}56 & 100 & 0.17 & 0.00 & -0.01\end{array}$

$\begin{array}{llllll}57 & 101 & 0.18 & 0.00 & 0.01\end{array}$

$\begin{array}{lllll}58 & 102 & 0.19 & 0.00 & 0.01\end{array}$

$\begin{array}{lllll}59 & 103 & 0.21 & 0.00 & 0.01\end{array}$

$\begin{array}{llllll}60 & 104 & 0.24 & 0.00 & -0.01\end{array}$

$\begin{array}{llllll}61 & 105 & 0.26 & 0.00 & -0.01\end{array}$

$\begin{array}{llllll}62 & 106 & 0.26 & 0.00 & 0.00\end{array}$

$\begin{array}{lllll}63 & 107 & 0.26 & 0.00\end{array}$

$\begin{array}{lllll}64 & 108 & 0.26 & 0.00\end{array}$

$\begin{array}{lllll}65 & 109 & 0.26 & 0.00\end{array}$

$\begin{array}{lllll}66 & 110 & -0.24 & 0.00\end{array}$

$\begin{array}{lllll}67 & 111 & -0.24 & 0.00\end{array}$

$\begin{array}{lllll}68 & 112 & -0.25 & 0.00\end{array}$

$\begin{array}{llll}69 & 113 & -0.25 & 0.00\end{array}$

$\begin{array}{lllll}70 & 114 & -0.25 & 0.00\end{array}$

$\begin{array}{llll}71 & 115 & -0.25 & 0.00\end{array}$

$\begin{array}{lllll}72 & 116 & -0.22 & 0.00\end{array}$

$\begin{array}{llll}73 & 117 & -0.22 & 0.00\end{array}$

$\begin{array}{llll}74 & 118 & -0.19 & 0.00\end{array}$

$\begin{array}{llll}75 & 119 & -0.18 & 0.00\end{array}$

$\begin{array}{llll}76 & 120 & -0.16 & 0.00\end{array}$

$\begin{array}{lllll}77 & 121 & -0.16 & 0.00\end{array}$

$\begin{array}{llll}78 & 122 & -0.12 & 0.00\end{array}$

$\begin{array}{llll}79 & 123 & -0.12 & 0.00\end{array}$

$\begin{array}{llll}80 & 124 & 0.00 & 0.00\end{array}$

0.03
0.06

0.06
0.07

0.08

0.06

0.06

$\begin{array}{rrrrrrrrr}0.00 & 0.227 & 0.000 & 0.081 & 0.016 & -0.85 & 1.84 & 997.55 & 66.51 \\ 0.01 & 0.249 & 0.000 & 0.086 & 0.008 & -0.80 & 2.10 & 998.57 & 73.56 \\ 0.02 & 0.271 & 0.000 & 0.079 & -0.004 & -1.08 & 2.15 & 998.05 & 82.15 \\ 0.01 & 0.271 & 0.000 & 0.065 & 0.003 & -0.69 & 2.37 & 998.86 & 89.42 \\ 0.02 & 0.283 & 0.000 & 0.056 & -0.010 & -0.97 & 2.17 & 998.33 & 98.01 \\ 0.02 & 0.294 & 0.000 & 0.046 & -0.013 & -0.86 & 2.25 & 999.00 & 105.41 \\ 0.02 & 0.306 & 0.000 & 0.037 & -0.016 & -1.31 & 1.89 & 998.40 & 114.09 \\ 0.02 & 0.307 & 0.000 & 0.025 & -0.019 & -1.18 & 1.92 & 998.87 & 121.69 \\ 0.02 & 0.319 & 0.000 & 0.015 & -0.022 & -1.66 & 1.62 & 997.97 & 130.66 \\ 0.02 & 0.308 & 0.000 & 0.013 & -0.023 & -1.34 & 1.70 & 998.15 & 138.55 \\ 0.02 & 0.320 & 0.000 & 0.003 & -0.026 & -1.80 & 1.40 & 997.01 & 147.76 \\ 0.02 & 0.310 & 0.000 & -0.011 & -0.029 & -1.44 & 1.46 & 996.98 & 155.86\end{array}$

$\begin{array}{llllll}0.03 & -0.247 & 0.000 & -0.068 & -0.007\end{array}$

1.35

$\begin{array}{llllll}0.04 & -0.235 & 0.000 & -0.081 & -0.013\end{array}$

1.15

3.69

614.9

0.88

$\begin{array}{llllll}0.05 & -0.234 & 0.000 & -0.092 & -0.019\end{array}$

0.36

3.57

3.56

$\begin{array}{llllll}0.05 & -0.234 & 0.000 & -0.092 & -0.019\end{array}$

0.08

3.04

$\begin{array}{lllllll}0.06 & -0.243 & 0.000 & -0.101 & -0.024 & -0.69\end{array}$

2.63

2.43

$\begin{array}{lllllll}0.06 & -0.243 & 0.000 & -0.101 & -0.024 & -0.98\end{array}$

$\begin{array}{lllllll}0.06 & -0.243 & 0.000 & -0.101 & -0.024 & -1.41\end{array}$

$\begin{array}{lllllll}0.06 & -0.243 & 0.000 & -0.101 & -0.024 & -1.72\end{array}$

2.13

1.87

$\begin{array}{lll}0.00 & 0.011 & 0.000\end{array}$

0.000

$0.000-0.27$

0.52

$\begin{array}{lll}0.00 & -0.021 & 0.000\end{array}$

0.000

$\begin{array}{llll}0.000 & -0.98 & -0.19\end{array}$

$\begin{array}{lllllll}0.00 & 0.000 & 0.000 & 0.000 & 0.000 & -2.37 & -1.41\end{array}$

$\begin{array}{lllllll}0.00 & 0.032 & 0.000 & -0.012 & -0.000 & -3.27 & -2.16\end{array}$

$\begin{array}{llll}0.00 & 0.000 & 0.000\end{array}$

0.000

$\begin{array}{lll}0.000 & -4.38 & -3.17\end{array}$

$\begin{array}{llll}0.00 & -0.021 & 0.000\end{array}$

0.000

$\begin{array}{llll}0.000 & -3.36 & -2.40\end{array}$

$\begin{array}{lll}0.00 & 0.000 & 0.000\end{array}$

$\begin{array}{llll}0.000 & 0.000 & -2.22 & -1.45\end{array}$

$\begin{array}{lll}0.00 & 0.107 & 0.000\end{array}$

0.041

$0.004-1.45$

$\begin{array}{lll}0.01 & 0.139 & 0.000\end{array}$

$0.044-0.005-0.89$

0.12

$\begin{array}{lll}0.02 & 0.161 & 0.000\end{array}$

$\begin{array}{llll}0.035 & -0.016 & -0.40\end{array}$

1.05

$\begin{array}{lll}0.03 & 0.183 & 0.000\end{array}$

$0.026-0.027$

0.00

1.72

$\begin{array}{llll}0.02 & 0.195 & 0.000\end{array}$

$0.003-0.021$

0.58

1.92

$\begin{array}{lll}0.02 & 0.206 & 0.000\end{array}$

$0.005-0.021$

0.86

2.63

0.02

$0.228 \quad 0.000$

$0.009-0.021$

0.86

3.13
3.45

$\begin{array}{lll}0.01 & 0.261 & 0.000\end{array}$

$0.038-0.004$

0.53

3.65

$\begin{array}{llll}0.01 & 0.283 & 0.000\end{array}$

$0.043-0.003$

$0.24 \quad 3.71$

$\begin{array}{llll}0.01 & 0.284 & 0.000\end{array}$

$\begin{array}{ll}0.031 & -0.007\end{array}$

0.42

3.83

$0.36 \quad 3.76$

0.37

3.73

0.00

$0.285 \quad 0.000$

$0.005-0.003$

0.21

3.63

$\begin{array}{lllll}0.01 & 0.285 & 0.000 & -0.008\end{array}$

0.014

0.42

3.53

$\begin{array}{lllll}0.00 & -0.248 & 0.000 & -0.044\end{array}$

$0.014 \quad 0.34$

3.34

$\begin{array}{lllll}0.00 & -0.258 & 0.000 & -0.053\end{array}$

$0.018-0.08$

3.29

$\begin{array}{lllll}0.00 & -0.258 & 0.000 & -0.064\end{array}$

$0.021-0.62$

2.92

$\begin{array}{lllll}0.01 & -0.258 & 0.000 & -0.064\end{array}$

$0.012-0.69$

2.72

$\begin{array}{lll}0.012 & -0.94 \quad 2.36\end{array}$

$\begin{array}{llllll}0.01 & -0.258 & 0.000 & -0.064\end{array}$

$0.007-0.51$

2.16

1.69

$\begin{array}{lllll}0.02 & -0.227 & 0.000 & -0.071\end{array}$

$0.001-1.13$

1.36

$\begin{array}{llllll}0.03 & -0.195 & 0.000 & -0.076 & -0.010 & -1.17 \\ 0.04 & -0.184 & 0.000 & -0.089 & -0.017 & -1.97\end{array}$

0.64

$\begin{array}{lllllll}0.04 & -0.164 & 0.000 & -0.081 & -0.021 & -2.02\end{array}$

0.13

$\begin{array}{lllllll}0.04 & -0.164 & 0.000 & -0.081 & -0.021 & -3.05 & -0.65\end{array}$

$$
0.03
$$

0.03

0.0

$756.85-64.86$
$633.97-6.54$

$649.51-14.01$

$667.67-24.10$

$682.32-30.68$

$699.47-39.76$

$713.16-45.38$

$729.21-53.36$

$741.35-57.43$

$768.60-68.53$

$783.10 \quad-74.97$

$\begin{array}{llll}794.09 & -77.88 & -77.27\end{array}$

$\begin{array}{llll}807.56 & -83.28 & -82.57\end{array}$

$\begin{array}{llll}816.27 & -83.92 & -83.45\end{array}$

$\begin{array}{lll}827.01 & -86.59 & -86.07\end{array}$

$\begin{array}{llll}834.20 & -85.70 & -86.11\end{array}$

$844.23-87.66-88.22$

$851.64-87.00 \quad-87.62$

$\begin{array}{llll}861.71 & -89.00 & -89.22\end{array}$

$868.43 \quad-87.65 \quad-87.95$

$\begin{array}{llll}877.54 & -88.69 & -89.10\end{array}$

$\begin{array}{lll}884.04 & -87.12 & -87.26\end{array}$

$\begin{array}{llll}892.82 & -87.83 & -88.09\end{array}$

$899.00-85.94 \quad-85.93$

$907.27-86.14 \quad-86.32$

$\begin{array}{llll}913.02 & -83.81 & -83.92\end{array}$

$\begin{array}{llll}920.87 & -83.59 & -83.67\end{array}$

$\begin{array}{llll}926.13 & -80.78 & -80.85\end{array}$

$\begin{array}{llll}933.52 & -80.10 & -79.98\end{array}$

$\begin{array}{lll}938.37 & -76.88 & -76.67\end{array}$

$\begin{array}{llll}945.19 & -75.62 & -75.48\end{array}$

$\begin{array}{llll}949.73 & -72.10 & -72.20\end{array}$

$\begin{array}{lll}956.22 & -70.51\end{array}$

$\begin{array}{llll}960.30 & -66.53 & -66.43\end{array}$

$966.32-64.47$

$970.08-60.16$

$975.77-57.78$

$979.37-53.31$

$984.83-50.69$

$988.08-45.87$

$993.28-43.00$

$996.50-38.15$

$1001.68-35.26$

$2.09 \quad 67.64$

$2.35 \quad 74.79$

$2.34 \quad 83.42$

$2.47 \quad 90.72$

$2.26 \quad 99.42$

$\begin{array}{ll}2.33 & 106.94\end{array}$

$1.94 \quad 115.72$

$1.99 \quad 123.48$

$1.69 \quad 132.60$

$1.79 \quad 140.67$

$\begin{array}{ll}1.51 & 150.06\end{array}$

$1.66 \quad 158.42$

$3.67 \quad 3.94$

$3.59-6.93$

$3.57-14.33$

$3.23-24.34$

$3.04-30.88$

$\begin{array}{ll}2.67 & -39.89\end{array}$

$2.44-45.48$

$2.17-53.39$

$\begin{array}{ll}1.89 & -57.45\end{array}$

$0.52-64.87$

$-0.19-68.52$

$\begin{array}{ll}-1.41 & -74.93\end{array}$

$\begin{array}{llll}0.085 & -2.16 & -77.83\end{array}$

$\begin{array}{llll}0.013 & -3.17 & -83.22\end{array}$

$\begin{array}{llll}0.012 & -2.40 & -83.85\end{array}$

$\begin{array}{llll}0.008 & -1.45 & -86.51\end{array}$

$\begin{array}{llll}0.008 & 0.13 & -85.61\end{array}$

$\begin{array}{llll}0.006 & 1.08 & -87.56\end{array}$

$\begin{array}{llll}0.002 & 1.74 & -86.91\end{array}$

$\begin{array}{llll}0.002 & 1.97 & -88.89\end{array}$

0.002

$2.66-87.56$

$3.17-88.59$

$3.49-87.04$

$3.71-87.74$

$3.76-85.87$

$\begin{array}{ll}3.89 & -86.07\end{array}$

$\begin{array}{ll}3.81 & -83.77\end{array}$

$3.80-83.56$

$3.69-80.76$

$3.65-80.03$

$\begin{array}{ll}3.45 & -76.82\end{array}$

$3.46-75.53$

$3.12-71.98$

$\begin{array}{ll}2.93 & -70.39\end{array}$

$2.56-66.41$

$2.34-64.38$

$1.91-60.03$

$1.64-57.59$

$1.02-53.01$

$0.49-50.40$ 


\begin{tabular}{|c|c|c|c|c|c|c|c|c|c|c|c|c|c|c|c|c|c|}
\hline \multicolumn{18}{|c|}{$Z=44(\mathrm{Ru})$} \\
\hline 81 & 125 & 0.02 & 0.00 & 0.01 & 0.00 & 0.021 & 0.000 & -0.012 & -0.000 & -6.48 & -5.22 & 1005.16 & -30.67 & & & -5.21 & -30.64 \\
\hline 82 & 126 & 0.00 & 0.00 & 0.00 & 0.00 & 0.000 & 0.000 & 0.000 & 0.000 & -7.98 & -6.57 & 1010.28 & -27.72 & & & -6.57 & -27.67 \\
\hline 83 & 127 & -0.01 & 0.00 & 0.00 & 0.00 & -0.011 & 0.000 & 0.000 & 0.000 & -6.89 & -5.65 & 1010.75 & -20.12 & & & -5.65 & -20.03 \\
\hline 84 & 128 & 0.00 & 0.00 & 0.00 & 0.00 & 0.000 & 0.000 & 0.000 & 0.000 & -5.86 & -4.71 & 1013.22 & -14.52 & & & -4.71 & -14.39 \\
\hline 85 & 129 & 0.01 & 0.00 & 0.00 & 0.00 & 0.011 & 0.000 & 0.000 & 0.000 & -4.77 & -3.73 & 1013.29 & -6.52 & & & -3.73 & -6.34 \\
\hline 86 & 130 & 0.00 & 0.00 & 0.00 & 0.00 & 0.000 & 0.000 & 0.000 & 0.000 & -3.54 & -2.64 & 1015.28 & -0.43 & & & -2.64 & -0.21 \\
\hline 87 & 131 & 0.00 & 0.00 & 0.00 & 0.00 & 0.000 & 0.000 & 0.000 & 0.000 & -2.55 & -1.78 & 1015.15 & 7.77 & & & -1.78 & 8.05 \\
\hline 88 & 132 & 0.00 & 0.00 & 0.00 & 0.00 & 0.000 & 0.000 & 0.000 & 0.000 & -1.42 & -0.76 & 1016.87 & 14.12 & & & -0.76 & 14.46 \\
\hline 89 & 133 & 0.11 & 0.03 & -0.03 & 0.00 & 0.118 & -0.041 & 0.042 & 0.005 & -1.33 & 0.17 & 1016.37 & 22.69 & & & 0.27 & 23.19 \\
\hline 90 & 134 & 0.14 & 0.02 & -0.04 & 0.01 & 0.150 & -0.028 & 0.058 & -0.002 & -0.83 & 0.97 & 1018.00 & 29.13 & & & 1.13 & 29.77 \\
\hline 91 & 135 & 0.15 & 0.00 & -0.04 & 0.01 & 0.161 & 0.000 & 0.059 & -0.002 & -0.40 & 1.44 & 1017.66 & 37.54 & & & 1.59 & 38.24 \\
\hline 92 & 136 & 0.19 & 0.00 & -0.05 & 0.01 & 0.205 & 0.000 & 0.078 & 0.004 & -0.59 & 1.78 & 1019.45 & 43.83 & & & 2.02 & 44.70 \\
\hline 93 & 137 & 0.21 & 0.00 & -0.05 & 0.01 & 0.227 & 0.000 & 0.082 & 0.006 & -0.93 & 1.81 & 1019.28 & 52.07 & & & 2.04 & 53.03 \\
\hline 94 & 138 & 0.21 & 0.00 & -0.05 & 0.01 & 0.227 & 0.000 & 0.082 & 0.006 & -0.61 & 2.08 & 1020.84 & 58.58 & & & 2.33 & 59.65 \\
\hline 95 & 139 & 0.23 & 0.00 & -0.04 & 0.01 & 0.249 & 0.000 & 0.073 & 0.005 & -0.72 & 2.24 & 1020.26 & 67.24 & & & 2.41 & 68.32 \\
\hline 96 & 140 & 0.24 & 0.00 & -0.04 & 0.02 & 0.260 & 0.000 & 0.076 & -0.005 & -0.72 & 2.29 & 1021.76 & 73.80 & & & 2.51 & 75.04 \\
\hline 97 & 141 & 0.25 & 0.00 & -0.03 & 0.02 & 0.271 & 0.000 & 0.066 & -0.007 & -0.93 & 2.16 & 1021.21 & 82.42 & & & 2.31 & 83.70 \\
\hline 98 & 142 & 0.25 & 0.00 & -0.02 & 0.02 & 0.271 & 0.000 & 0.054 & -0.010 & -0.55 & 2.36 & 1022.29 & 89.41 & & & 2.49 & 90.78 \\
\hline 99 & 143 & 0.26 & 0.00 & -0.01 & 0.02 & 0.283 & 0.000 & 0.044 & -0.013 & -0.90 & 2.05 & 1021.68 & 98.10 & & & 2.13 & 99.55 \\
\hline 100 & 144 & 0.27 & 0.00 & 0.00 & 0.02 & 0.295 & 0.000 & 0.034 & -0.016 & -0.89 & 2.11 & 1022.63 & 105.21 & & & 2.20 & 106.80 \\
\hline 101 & 145 & 0.28 & 0.00 & 0.01 & 0.03 & 0.307 & 0.000 & 0.026 & -0.029 & -1.41 & 1.65 & 92 & 113.99 & & & 1.88 & .85 \\
\hline 102 & 146 & 0.28 & 0.00 & 0.02 & 0.02 & 0.308 & 0.000 & 0.013 & -0.023 & -1.17 & 1.87 & 1022.48 & 121.50 & & & 1.98 & 123.40 \\
\hline 103 & 147 & 0.28 & 0.00 & 0.03 & 0.02 & 0.309 & 0.000 & 0.001 & -0.026 & -1.45 & 1.60 & 1021.34 & 130.71 & & & 1.75 & 132.79 \\
\hline 104 & 148 & 0.28 & 0.00 & 0.04 & 0.02 & 0.310 & 0.000 & -0.011 & -0.029 & -1.25 & 1.75 & 1021.73 & 138.40 & & & 1.98 & 140.71 \\
\hline 105 & 149 & 0.26 & 0.00 & 0.04 & 0.01 & 0.287 & 0.000 & -0.018 & -0.019 & -1.07 & 1.64 & 1020.22 & 147.99 & & & 1.74 & 150.33 \\
\hline 106 & 150 & 0.26 & 0.00 & 0.05 & 0.00 & 0.287 & 0.000 & -0.031 & -0.013 & -0.97 & 1.77 & 1020.38 & 155.89 & & & 1.88 & 158.41 \\
\hline \multicolumn{18}{|c|}{$Z=45(\mathrm{Rh})$} \\
\hline 38 & 83 & -0.23 & 0.00 & 0.09 & 0.04 & -0.235 & 0.000 & -0.081 & -0.013 & 0.69 & 3.18 & 630.02 & 4.70 & & & 3.18 & 4.28 \\
\hline 39 & 84 & -0.23 & 0.00 & 0.10 & 0.05 & -0.234 & 0.000 & -0.092 & -0.019 & 0.45 & 3.28 & 646.31 & -3.53 & & & 3.27 & -3.89 \\
\hline 40 & 85 & -0.23 & 0.00 & 0.10 & 0.05 & -0.234 & 0.000 & -0.092 & -0.019 & -0.08 & 2.74 & 664.83 & -13.97 & & & 2.75 & -14.26 \\
\hline 41 & 86 & -0.23 & 0.00 & 0.10 & 0.05 & -0.234 & 0.000 & -0.092 & -0.019 & -0.36 & 2.57 & 680.33 & -21.40 & & & 2.55 & -21.65 \\
\hline 42 & 87 & -0.24 & 0.00 & 0.11 & 0.06 & -0.243 & 0.000 & -0.101 & -0.024 & -1.14 & 2.20 & 697.61 & -30.61 & & & 2.21 & -30.79 \\
\hline 43 & 88 & -0.24 & 0.00 & 0.11 & 0.06 & -0.243 & 0.000 & -0.101 & -0.024 & -1.44 & 2.09 & 712.05 & -36.98 & & & 2.07 & -37.13 \\
\hline 44 & 89 & -0.24 & 0.00 & 0.11 & 0.06 & -0.243 & 0.000 & -0.101 & -0.024 & -1.87 & 1.70 & 728.35 & -45.21 & & & 1.71 & -45.30 \\
\hline 45 & 90 & 0.10 & 0.00 & -0.01 & 0.00 & 0.107 & 0.000 & 0.016 & 0.001 & -0.06 & 1.24 & 741.86 & -50.65 & & & 1.23 & -51.05 \\
\hline 46 & 91 & 0.07 & 0.00 & 0.00 & 0.00 & 0.075 & 0.000 & 0.002 & 0.000 & -1.00 & 0.02 & 757.71 & -58.43 & & & 0.02 & -58.47 \\
\hline 47 & 92 & 0.08 & 0.00 & 0.00 & 0.00 & 0.085 & 0.000 & 0.003 & 0.000 & -1.82 & -0.64 & 770.22 & -62.87 & & & -0.64 & -62.88 \\
\hline 48 & 93 & 0.00 & 0.00 & 0.00 & 0.01 & 0.000 & 0.000 & 0.000 & -0.010 & -2.96 & -2.02 & 785.04 & -69.61 & & & -2.02 & -69.60 \\
\hline 49 & 94 & 0.03 & 0.00 & 0.01 & 0.00 & 0.032 & 0.000 & -0.012 & -0.000 & -3.83 & -2.76 & 796.81 & -73.31 & & & -2.76 & -73.28 \\
\hline 50 & 95 & 0.00 & 0.00 & 0.00 & 0.00 & 0.000 & 0.000 & 0.000 & 0.000 & -4.95 & -3.78 & 810.44 & -78.87 & -78.34 & 0.150 & -3.78 & -78.83 \\
\hline 51 & 96 & 0.05 & 0.00 & -0.02 & 0.00 & 0.053 & 0.000 & 0.025 & 0.001 & -4.07 & -2.82 & 819.74 & -80.10 & -79.68 & 0.013 & -2.82 & -80.04 \\
\hline 52 & 97 & 0.04 & 0.00 & -0.01 & 0.00 & 0.043 & 0.000 & 0.013 & 0.001 & -2.85 & -1.91 & 830.67 & -82.96 & -82.59 & 0.036 & -1.91 & -82.90 \\
\hline 53 & 98 & 0.10 & 0.00 & -0.03 & 0.00 & 0.107 & 0.000 & 0.041 & 0.004 & -2.21 & -0.56 & 838.84 & -83.06 & -83.18 & 0.012 & -0.55 & -82.99 \\
\hline 54 & 99 & 0.13 & 0.00 & -0.03 & 0.01 & 0.139 & 0.000 & 0.044 & -0.005 & -1.60 & 0.42 & 848.97 & -85.11 & -85.57 & 0.007 & 0.44 & -85.04 \\
\hline 55 & 100 & 0.15 & 0.00 & -0.01 & 0.02 & 0.161 & 0.000 & 0.023 & -0.018 & -1.03 & 1.09 & 857.13 & -85.21 & -85.58 & 0.018 & 1.10 & -85.14 \\
\hline 56 & 101 & 0.17 & 0.00 & 0.00 & 0.03 & 0.184 & 0.000 & 0.014 & -0.029 & -0.65 & 1.25 & 867.38 & -87.38 & -87.41 & 0.017 & 1.28 & -87.29 \\
\hline 57 & 102 & 0.17 & 0.00 & 0.01 & 0.02 & 0.184 & 0.000 & 0.002 & -0.021 & 0.03 & 1.82 & 874.98 & -86.91 & -86.78 & 0.005 & 1.84 & -86.84 \\
\hline 58 & 103 & 0.18 & 0.00 & 0.01 & 0.02 & 0.195 & 0.000 & 0.003 & -0.021 & 0.43 & 2.33 & 884.21 & -88.07 & -88.02 & 0.003 & 2.35 & -88.00 \\
\hline 59 & 104 & 0.20 & 0.00 & 0.01 & 0.01 & 0.217 & 0.000 & 0.006 & -0.011 & 0.63 & 3.06 & 891.03 & -86.82 & -86.95 & 0.003 & 3.07 & -86.77 \\
\hline 60 & 105 & 0.20 & 0.00 & 0.01 & 0.01 & 0.217 & 0.000 & 0.006 & -0.011 & 0.88 & 3.28 & 899.92 & -87.64 & -87.85 & 0.004 & 3.30 & -87.60 \\
\hline 61 & 106 & 0.23 & 0.00 & -0.01 & 0.01 & 0.250 & 0.000 & 0.036 & -0.005 & 0.51 & 3.47 & 906.68 & -86.32 & -86.36 & 0.008 & 3.50 & -86.29 \\
\hline 62 & 107 & 0.23 & 0.00 & 0.00 & 0.00 & 0.250 & 0.000 & 0.023 & 0.002 & 0.63 & 3.49 & 915.17 & -86.74 & -86.86 & 0.012 & 3.53 & -86.72 \\
\hline 63 & 108 & 0.23 & 0.00 & 0.01 & 0.00 & 0.250 & 0.000 & 0.011 & -0.001 & 0.70 & 3.55 & 921.50 & -85.00 & -85.02 & 0.105 & 3.57 & -85.00 \\
\hline 64 & 109 & 0.24 & 0.00 & 0.02 & -0.01 & 0.262 & 0.000 & -0.001 & 0.006 & 0.48 & 3.46 & 929.53 & -84.96 & -85.01 & 0.012 & 3.50 & -84.96 \\
\hline 65 & 110 & -0.24 & 0.00 & 0.05 & 0.01 & -0.248 & 0.000 & -0.034 & 0.002 & 0.31 & 3.32 & 935.52 & -82.88 & -82.78 & 0.050 & 3.37 & -82.87 \\
\hline 66 & 111 & -0.24 & 0.00 & 0.06 & 0.01 & -0.248 & 0.000 & -0.045 & 0.005 & 0.21 & 3.29 & 942.94 & -82.23 & -82.36 & 0.030 & 3.38 & -82.21 \\
\hline 67 & 112 & -0.24 & 0.00 & 0.06 & 0.00 & -0.248 & 0.000 & -0.044 & 0.014 & 0.06 & 3.03 & 948.53 & -79.75 & -79.74 & 0.052 & 3.12 & -79.74 \\
\hline
\end{tabular}




\begin{tabular}{|c|c|c|c|c|c|c|c|c|c|c|c|c|c|c|c|c|c|}
\hline \multicolumn{18}{|c|}{$Z=45(\mathrm{Rh})$} \\
\hline 68 & 113 & -0.24 & 0.00 & 0.07 & 0.00 & -0.248 & 0.000 & -0.055 & 0.017 & -0.32 & 2.92 & 955.53 & -78.67 & -78.68 & 0.049 & 3.05 & -78.62 \\
\hline 69 & 114 & -0.25 & 0.00 & 0.07 & 0.00 & -0.258 & 0.000 & -0.053 & 0.018 & -0.83 & 2.62 & 960.67 & -75.74 & -75.63 & 0.113 & 2.75 & -75.71 \\
\hline 70 & 115 & -0.25 & 0.00 & 0.08 & 0.01 & -0.258 & 0.000 & -0.064 & 0.012 & -1.07 & 2.37 & 967.30 & -74.30 & -74.21 & 0.081 & 2.55 & -74.23 \\
\hline 71 & 116 & -0.25 & 0.00 & 0.08 & 0.01 & -0.258 & 0.000 & -0.064 & 0.012 & -1.32 & 2.03 & 972.02 & -70.95 & -70.74 & 0.138 & 2.20 & -70.89 \\
\hline 72 & 117 & -0.23 & 0.00 & 0.07 & 0.01 & -0.237 & 0.000 & -0.058 & 0.008 & -1.07 & 1.80 & 978.16 & -69.02 & & & 1.95 & -68.99 \\
\hline 73 & 118 & -0.22 & 0.00 & 0.08 & 0.02 & -0.227 & 0.000 & -0.071 & 0.001 & -1.53 & 1.38 & 982.52 & -65.31 & & & 1.57 & -65.23 \\
\hline 74 & 119 & -0.19 & 0.00 & 0.08 & 0.03 & -0.195 & 0.000 & -0.076 & -0.010 & -1.59 & 1.04 & 988.32 & -63.04 & & & 1.28 & -62.91 \\
\hline 75 & 120 & -0.18 & 0.00 & 0.08 & 0.03 & -0.185 & 0.000 & -0.078 & -0.011 & -2.16 & 0.43 & 992.43 & -59.08 & & & 0.67 & -58.95 \\
\hline 76 & 121 & -0.16 & 0.00 & 0.08 & 0.04 & -0.164 & 0.000 & -0.081 & -0.021 & -2.38 & -0.12 & 998.01 & -56.59 & & & 0.20 & -56.37 \\
\hline 77 & 122 & -0.16 & 0.00 & 0.08 & 0.04 & -0.164 & 0.000 & -0.081 & -0.021 & -3.38 & -0.92 & 1001.91 & -52.41 & & & -0.59 & -52.18 \\
\hline 78 & 123 & -0.12 & 0.00 & 0.06 & 0.03 & -0.124 & 0.000 & -0.063 & -0.019 & -3.35 & -1.67 & 1007.28 & -49.71 & & & -1.45 & -49.58 \\
\hline 79 & 124 & -0.03 & 0.00 & 0.00 & 0.00 & -0.032 & 0.000 & 0.000 & 0.000 & -3.54 & -2.76 & 1011.08 & -45.44 & & & -2.76 & -45.52 \\
\hline 80 & 125 & -0.02 & 0.00 & 0.00 & 0.00 & -0.021 & 0.000 & 0.000 & 0.000 & -4.96 & -4.00 & 1016.54 & -42.83 & & & -4.00 & -42.89 \\
\hline 81 & 126 & 0.02 & 0.00 & 0.01 & 0.00 & 0.021 & 0.000 & -0.012 & -0.000 & -6.82 & -5.57 & 1020.45 & -38.67 & & & -5.57 & -38.70 \\
\hline 82 & 127 & 0.00 & 0.00 & 0.00 & 0.00 & 0.000 & 0.000 & 0.000 & 0.000 & -8.36 & -6.96 & 1025.69 & -35.83 & & & -6.96 & -35.85 \\
\hline 83 & 128 & -0.01 & 0.00 & 0.00 & 0.00 & -0.011 & 0.000 & 0.000 & 0.000 & -7.24 & -6.00 & 1026.70 & -28.78 & & & -6.00 & -28.76 \\
\hline 84 & 129 & 0.00 & 0.00 & 0.00 & 0.00 & 0.000 & 0.000 & 0.000 & 0.000 & -6.20 & -5.05 & 1029.24 & -23.25 & & & -5.05 & -23.19 \\
\hline 85 & 130 & 0.01 & 0.00 & 0.00 & 0.00 & 0.011 & 0.000 & 0.000 & 0.000 & -5.07 & -4.04 & 1029.86 & -15.80 & & & -4.04 & -15.71 \\
\hline 86 & 131 & 0.00 & 0.00 & 0.00 & 0.00 & 0.000 & 0.000 & 0.000 & 0.000 & -3.84 & -2.94 & 1031.90 & -9.76 & & & -2.94 & -9.62 \\
\hline 87 & 132 & 0.00 & 0.00 & 0.00 & 0.00 & 0.000 & 0.000 & 0.000 & 0.000 & -2.82 & -2.06 & 1032.32 & -2.11 & & & -2.06 & -1.93 \\
\hline 88 & 133 & 0.00 & 0.00 & 0.00 & 0.00 & 0.000 & 0.000 & 0.000 & 0.000 & -1.69 & -1.04 & 1034.11 & 4.17 & & & -1.04 & 4.40 \\
\hline 89 & 134 & 0.11 & 0.02 & -0.03 & 0.00 & 0.118 & -0.028 & 0.042 & 0.005 & -1.60 & -0.11 & 1034.17 & 12.18 & & & -0.03 & 12.55 \\
\hline 90 & 135 & 0.14 & 0.00 & -0.04 & 0.01 & 0.150 & 0.000 & 0.058 & -0.002 & -1.17 & 0.50 & 1036.04 & 18.38 & & & 0.64 & 18.87 \\
\hline 91 & 136 & 0.15 & 0.00 & -0.02 & 0.01 & 0.161 & 0.000 & 0.034 & -0.006 & -0.49 & 1.23 & 1036.01 & 26.49 & & & 1.28 & 26.96 \\
\hline 92 & 137 & 0.15 & 0.00 & -0.03 & 0.01 & 0.161 & 0.000 & 0.047 & -0.004 & -0.03 & 1.70 & 1037.71 & 32.85 & & & 1.79 & 33.44 \\
\hline 93 & 138 & 0.21 & 0.00 & -0.05 & 0.01 & 0.227 & 0.000 & 0.082 & 0.006 & -1.20 & 1.65 & 1038.16 & 40.47 & & & 1.86 & 41.26 \\
\hline 94 & 139 & 0.21 & 0.00 & -0.05 & 0.01 & 0.227 & 0.000 & 0.082 & 0.006 & -0.88 & 1.86 & 1039.83 & 46.88 & & & 2.09 & 47.76 \\
\hline 95 & 140 & 0.21 & 0.00 & -0.04 & 0.01 & 0.227 & 0.000 & 0.069 & 0.003 & -0.64 & 1.93 & 1039.88 & 54.90 & & & 2.08 & 55.80 \\
\hline 96 & 141 & 0.23 & 0.00 & -0.04 & 0.02 & 0.249 & 0.000 & 0.074 & -0.006 & -0.68 & 2.19 & 1041.22 & 61.63 & & & 2.39 & 62.67 \\
\hline 97 & 142 & 0.25 & 0.00 & -0.03 & 0.02 & 0.271 & 0.000 & 0.066 & -0.007 & -1.05 & 2.09 & 1041.18 & 69.75 & & & 2.22 & 70.82 \\
\hline 98 & 143 & 0.26 & 0.00 & -0.02 & 0.02 & 0.283 & 0.000 & 0.056 & -0.010 & -0.92 & 2.25 & 1042.34 & 76.65 & & & 2.36 & 77.82 \\
\hline 99 & 144 & 0.26 & 0.00 & -0.01 & 0.02 & 0.283 & 0.000 & 0.044 & -0.013 & -1.10 & 1.94 & 1042.24 & 84.82 & & & 2.01 & 86.06 \\
\hline 100 & 145 & 0.27 & 0.00 & 0.00 & 0.02 & 0.295 & 0.000 & 0.034 & -0.016 & -1.07 & 2.03 & 1043.21 & 91.92 & & & 2.10 & 93.28 \\
\hline 101 & 146 & 0.27 & 0.00 & 0.01 & 0.02 & 0.296 & 0.000 & 0.022 & -0.019 & -1.30 & 1.74 & 1042.85 & 100.36 & & & 1.81 & 101.84 \\
\hline 102 & 147 & 0.27 & 0.00 & 0.02 & 0.02 & 0.296 & 0.000 & 0.010 & -0.023 & -1.16 & 1.80 & 1043.59 & 107.69 & & & 1.91 & 109.34 \\
\hline 103 & 148 & 0.28 & 0.00 & 0.03 & 0.02 & 0.309 & 0.000 & 0.001 & -0.026 & -1.59 & 1.58 & 104 & 116.42 & & & 1.70 & 118.23 \\
\hline 104 & 149 & 0.27 & 0.00 & 0.03 & 0.02 & 0.297 & 0.000 & -0.002 & -0.026 & -1.17 & 1.66 & 1043.40 & 124.02 & & & 1.81 & 125.99 \\
\hline 105 & 150 & 0.25 & 0.00 & 0.04 & 0.01 & 0.275 & 0.000 & -0.020 & -0.019 & -1.12 & 1.54 & 1042.40 & 133.09 & & & 1.63 & 135.16 \\
\hline 106 & 151 & 0.25 & 0.00 & 0.05 & 0.00 & 0.276 & 0.000 & -0.033 & -0.012 & -1.01 & 1.60 & 1042.67 & 140.90 & & & 1.70 & 143.14 \\
\hline 107 & 152 & 0.24 & 0.00 & 0.06 & -0.01 & 0.265 & 0.000 & -0.049 & -0.005 & -1.29 & 1.27 & 1041.65 & 149.98 & & & 1.43 & 152.45 \\
\hline 108 & 153 & 0.24 & 0.00 & 0.07 & -0.01 & 0.266 & 0.000 & -0.061 & -0.008 & -1.33 & 1.20 & 1041.82 & 157.88 & & & 1.48 & 160.63 \\
\hline \multicolumn{18}{|c|}{$Z=46(\mathrm{Pd})$} \\
\hline 40 & 86 & -0.23 & 0.00 & 0.09 & 0.05 & -0.235 & 0.000 & -0.081 & -0.022 & -0.10 & 2.39 & 663.95 & -5.80 & & & 2.41 & -6.09 \\
\hline 41 & 87 & -0.23 & 0.00 & 0.09 & 0.05 & -0.235 & 0.000 & -0.081 & -0.022 & -0.35 & 2.24 & 679.59 & -13.37 & & & 2.24 & -13.62 \\
\hline 42 & 88 & -0.23 & 0.00 & 0.10 & 0.05 & -0.234 & 0.000 & -0.092 & -0.019 & -1.00 & 1.89 & 697.69 & -23.40 & & & 1.91 & -23.58 \\
\hline 43 & 89 & -0.23 & 0.00 & 0.10 & 0.06 & -0.234 & 0.000 & -0.093 & -0.028 & -1.28 & 1.78 & 712.29 & -29.93 & & & 1.79 & -30.08 \\
\hline 44 & 90 & 0.00 & 0.00 & 0.00 & 0.00 & 0.000 & 0.000 & 0.000 & 0.000 & -0.12 & 0.63 & 730.18 & -39.75 & & & 0.63 & -39.87 \\
\hline 45 & 91 & -0.01 & 0.00 & 0.00 & 0.00 & -0.011 & 0.000 & 0.000 & 0.000 & -0.53 & 0.19 & 744.17 & -45.66 & & & 0.18 & -45.75 \\
\hline 46 & 92 & 0.00 & 0.00 & 0.00 & 0.00 & 0.000 & 0.000 & 0.000 & 0.000 & -1.72 & -0.90 & 761.03 & -54.45 & & & -0.90 & -54.51 \\
\hline 47 & 93 & -0.02 & 0.00 & 0.00 & 0.00 & -0.021 & 0.000 & 0.000 & 0.000 & -2.42 & -1.58 & 773.71 & -59.07 & & & -1.59 & -59.10 \\
\hline 48 & 94 & 0.01 & 0.00 & 0.00 & 0.00 & 0.011 & 0.000 & 0.000 & 0.000 & -3.84 & -2.84 & 789.20 & -66.49 & & & -2.84 & -66.49 \\
\hline 49 & 95 & 0.03 & 0.00 & 0.01 & 0.00 & 0.032 & 0.000 & -0.012 & -0.000 & -4.73 & -3.58 & 801.13 & -70.35 & & & -3.59 & -70.33 \\
\hline 50 & 96 & 0.00 & 0.00 & 0.00 & 0.00 & 0.000 & 0.000 & 0.000 & 0.000 & -5.87 & -4.63 & 815.59 & -76.73 & -76.23 & 0.151 & -4.64 & -76.70 \\
\hline 51 & 97 & -0.02 & 0.00 & 0.00 & 0.00 & -0.021 & 0.000 & 0.000 & 0.000 & -4.84 & -3.81 & 825.16 & -78.23 & -77.80 & 0.302 & -3.81 & -78.19 \\
\hline 52 & 98 & -0.01 & 0.00 & 0.00 & 0.00 & -0.011 & 0.000 & 0.000 & 0.000 & -3.69 & -2.83 & 836.80 & -81.79 & -81.30 & 0.021 & -2.83 & -81.75 \\
\hline 53 & 99 & 0.10 & 0.00 & -0.02 & 0.00 & 0.107 & 0.000 & 0.028 & 0.003 & -2.92 & -1.32 & 844.96 & -81.88 & -82.19 & 0.015 & -1.32 & -81.83 \\
\hline 54 & 100 & 0.12 & 0.00 & -0.02 & 0.01 & 0.128 & 0.000 & 0.031 & -0.007 & -2.15 & -0.35 & 855.85 & -84.71 & -85.23 & 0.011 & -0.34 & -84.64 \\
\hline
\end{tabular}




\begin{tabular}{|c|c|c|c|c|c|c|c|c|c|c|c|c|c|c|c|c|c|}
\hline \multicolumn{18}{|c|}{$Z=46(\mathrm{Pd})$} \\
\hline 55 & 101 & 0.14 & 0.00 & -0.01 & 0.02 & 0.150 & 0.000 & 0.022 & -0.018 & -1.67 & 0.34 & 864.12 & -84.91 & -85.43 & 0.018 & 0.35 & -84.85 \\
\hline 56 & 102 & 0.15 & 0.00 & 0.00 & 0.03 & 0.162 & 0.000 & 0.012 & -0.029 & -1.11 & 0.98 & 874.63 & -87.35 & -87.93 & 0.003 & 1.01 & -87.27 \\
\hline 57 & 103 & 0.16 & 0.00 & 0.01 & 0.02 & 0.173 & 0.000 & 0.000 & -0.021 & -0.54 & 1.60 & 882.32 & -86.97 & -87.48 & 0.003 & 1.62 & -86.91 \\
\hline 58 & 104 & 0.16 & 0.00 & 0.01 & 0.02 & 0.173 & 0.000 & 0.000 & -0.021 & 0.05 & 1.79 & 892.61 & -89.18 & -89.39 & 0.004 & 1.81 & -89.12 \\
\hline 59 & 105 & 0.16 & 0.00 & 0.03 & 0.00 & 0.174 & 0.000 & -0.025 & -0.005 & 0.63 & 2.34 & 899.73 & -88.23 & -88.41 & 0.004 & 2.36 & -88.19 \\
\hline 60 & 106 & 0.17 & 0.00 & 0.03 & 0.00 & 0.185 & 0.000 & -0.024 & -0.005 & 0.97 & 2.68 & 909.22 & -89.65 & -89.90 & 0.004 & 2.71 & -89.61 \\
\hline 61 & 107 & 0.18 & 0.00 & 0.02 & 0.00 & 0.195 & 0.000 & -0.010 & -0.003 & 1.22 & 3.24 & 915.74 & -88.09 & -88.37 & 0.004 & 3.26 & -88.08 \\
\hline 62 & 108 & 0.20 & 0.00 & 0.02 & -0.01 & 0.217 & 0.000 & -0.008 & 0.006 & 1.10 & 3.32 & 924.88 & -89.16 & -89.52 & 0.003 & 3.36 & -89.14 \\
\hline 63 & 109 & 0.22 & 0.00 & 0.01 & -0.01 & 0.239 & 0.000 & 0.008 & 0.009 & 0.84 & 3.37 & 931.33 & -87.54 & -87.61 & 0.003 & 3.41 & -87.54 \\
\hline 64 & 110 & 0.22 & 0.00 & 0.03 & -0.01 & 0.240 & 0.000 & -0.017 & 0.004 & 0.81 & 3.31 & 940.03 & -88.17 & -88.35 & 0.011 & 3.36 & -88.16 \\
\hline 65 & 111 & -0.24 & 0.00 & 0.05 & 0.01 & -0.248 & 0.000 & -0.034 & 0.002 & 0.36 & 3.31 & 945.99 & -86.07 & -86.00 & 0.011 & 3.37 & -86.06 \\
\hline 66 & 112 & -0.24 & 0.00 & 0.05 & 0.00 & -0.248 & 0.000 & -0.033 & 0.011 & 0.29 & 3.20 & 954.18 & -86.18 & -86.34 & 0.018 & 3.28 & -86.17 \\
\hline 67 & 113 & -0.24 & 0.00 & 0.06 & 0.00 & -0.248 & 0.000 & -0.044 & 0.014 & -0.12 & 2.97 & 959.85 & -83.78 & -83.69 & 0.036 & 3.07 & -83.77 \\
\hline 68 & 114 & -0.25 & 0.00 & 0.07 & 0.00 & -0.258 & 0.000 & -0.053 & 0.018 & -0.73 & 2.63 & 967.75 & -83.60 & -83.50 & 0.024 & 2.77 & -83.56 \\
\hline 69 & 115 & -0.25 & 0.00 & 0.07 & 0.00 & -0.258 & 0.000 & -0.053 & 0.018 & -1.10 & 2.37 & 972.95 & -80.74 & -80.40 & 0.061 & 2.51 & -80.71 \\
\hline 70 & 116 & -0.25 & 0.00 & 0.07 & 0.00 & -0.258 & 0.000 & -0.053 & 0.018 & -1.23 & 2.15 & 980.21 & -79.93 & -79.96 & 0.056 & 2.31 & -79.89 \\
\hline 71 & 117 & -0.25 & 0.00 & 0.07 & 0.00 & -0.258 & 0.000 & -0.053 & 0.018 & -1.46 & 1.84 & 985.01 & -76.65 & -76.53 & 0.059 & 1.99 & -76.63 \\
\hline 72 & 118 & -0.22 & 0.00 & 0.07 & 0.01 & -0.227 & 0.000 & -0.059 & 0.007 & -1.15 & 1.56 & 991.85 & -75.42 & -75.47 & 0.210 & 1.72 & -75.40 \\
\hline 73 & 119 & -0.20 & 0.00 & 0.07 & 0.02 & -0.206 & 0.000 & -0.063 & -0.003 & -1.33 & 1.21 & 996.23 & -71.73 & & & 1.36 & -71.72 \\
\hline 74 & 120 & -0.16 & 0.00 & 0.07 & 0.03 & -0.165 & 0.000 & -0.070 & -0.014 & -1.37 & 0.78 & 1002.75 & -70.18 & -70.15 & 0.124 & 0.99 & -70.12 \\
\hline 75 & 121 & -0.16 & 0.00 & 0.07 & 0.03 & -0.165 & 0.000 & -0.070 & -0.014 & -2.06 & 0.10 & 1007.03 & -66.39 & & & 0.30 & -66.33 \\
\hline 76 & 122 & -0.12 & 0.00 & 0.06 & 0.03 & -0.124 & 0.000 & -0.063 & -0.019 & -2.25 & -0.66 & 1013.46 & -64.74 & & & -0.47 & -64.69 \\
\hline 77 & 123 & -0.12 & 0.00 & 0.06 & 0.03 & -0.124 & 0.000 & -0.063 & -0.019 & -3.28 & -1.56 & 1017.54 & -60.76 & & & -1.37 & -60.70 \\
\hline 78 & 124 & 0.02 & 0.00 & 0.01 & 0.00 & 0.021 & 0.000 & -0.012 & -0.000 & -3.12 & -2.30 & 1023.51 & -58.65 & & & -2.29 & -58.78 \\
\hline 79 & 125 & 0.01 & 0.00 & 0.00 & 0.01 & 0.011 & 0.000 & 0.000 & -0.010 & -4.52 & -3.58 & 1027.58 & -54.65 & & & -3.57 & -54.76 \\
\hline 80 & 126 & 0.00 & 0.00 & 0.00 & 0.00 & 0.000 & 0.000 & 0.000 & 0.000 & -6.11 & -5.03 & 1033.86 & -52.86 & & & -5.03 & -52.97 \\
\hline 81 & 127 & 0.02 & 0.00 & 0.01 & 0.00 & 0.021 & 0.000 & -0.012 & -0.000 & -7.72 & -6.42 & 1037.67 & -48.60 & & & -6.42 & -48.69 \\
\hline 82 & 128 & 0.00 & 0.00 & 0.00 & 0.00 & 0.000 & 0.000 & 0.000 & 0.000 & -9.09 & -7.66 & 1043.35 & -46.21 & & & -7.66 & -46.28 \\
\hline 83 & 129 & -0.01 & 0.00 & 0.00 & 0.00 & -0.011 & 0.000 & 0.000 & 0.000 & -8.17 & -6.89 & 1044.63 & -39.42 & & & -6.89 & -39.47 \\
\hline 84 & 130 & 0.00 & 0.00 & 0.00 & 0.00 & 0.000 & 0.000 & 0.000 & 0.000 & -7.12 & -5.92 & 1047.73 & -34.45 & & & -5.92 & -34.47 \\
\hline 85 & 131 & 0.00 & 0.00 & 0.00 & 0.00 & 0.000 & 0.000 & 0.000 & 0.000 & -5.95 & -4.88 & 1048.40 & -27.04 & & & -4.88 & -27.03 \\
\hline 86 & 132 & 0.00 & 0.00 & 0.00 & 0.00 & 0.000 & & 0.000 & & -4.70 & -3.74 & 1050.97 & -21.55 & & & -3.74 & -21.50 \\
\hline 87 & 133 & 0.00 & 0.00 & 0.00 & 0.00 & 0.000 & 0.000 & 0.000 & 0.000 & -3.66 & -2.82 & 1051.43 & -13.93 & & & -2.82 & -13.84 \\
\hline 88 & 134 & 0.03 & 0.02 & 0.00 & 0.00 & 0.032 & -0.027 & 0.001 & 0.000 & -2.60 & -1.75 & 1053.73 & -8.16 & & & -1.74 & -8.01 \\
\hline 89 & 135 & 0.11 & 0.00 & -0.03 & 0.00 & 0.118 & 0.000 & 0.042 & 0.005 & -2.19 & -0.72 & 1053.75 & -0.11 & & & -0.65 & 0.14 \\
\hline 90 & 136 & 0.11 & 0.00 & -0.03 & 0.00 & 0.118 & 0.000 & 0.042 & 0.005 & -1.44 & -0.04 & 1056.12 & 5.59 & & & 0.04 & 5.91 \\
\hline 91 & 137 & 0.14 & 0.01 & -0.02 & 0.01 & 0.150 & -0.014 & 0.033 & -0.006 & -0.91 & 0.71 & 1056.12 & 13.67 & & & 0.77 & 14.03 \\
\hline 92 & 138 & 0.15 & 0.00 & -0.03 & 0.01 & 0.161 & 0.000 & 0.047 & -0.004 & -0.52 & 1.24 & 1058.32 & 19.54 & & & 1.34 & 20.00 \\
\hline 93 & 139 & 0.20 & 0.00 & -0.06 & 0.01 & 0.216 & 0.000 & 0.093 & 0.008 & -1.53 & 1.65 & 1058.36 & 27.56 & & & 1.94 & 28.30 \\
\hline 94 & 140 & 0.20 & 0.00 & -0.05 & 0.01 & 0.216 & 0.000 & 0.080 & 0.005 & -0.89 & 1.74 & 1060.69 & 33.30 & & & 1.97 & 34.05 \\
\hline 95 & 141 & 0.21 & 0.00 & -0.05 & 0.01 & 0.227 & 0.000 & 0.082 & 0.006 & -1.07 & 1.76 & 1060.85 & 41.22 & & & 1.99 & 42.05 \\
\hline 96 & 142 & 0.23 & 0.00 & -0.04 & 0.02 & 0.249 & 0.000 & 0.074 & -0.006 & -0.83 & 2.11 & 1062.63 & 47.51 & & & 2.32 & 48.40 \\
\hline 97 & 143 & 0.24 & 0.00 & -0.03 & 0.02 & 0.260 & 0.000 & 0.064 & -0.008 & -0.96 & 2.03 & 1062.60 & 55.61 & & & 2.18 & 56.54 \\
\hline 98 & 144 & 0.25 & 0.00 & -0.02 & 0.02 & 0.271 & 0.000 & 0.054 & -0.010 & -0.81 & 2.20 & 1064.29 & 62.00 & & & 2.33 & 63.00 \\
\hline 99 & 145 & 0.25 & 0.00 & -0.02 & 0.03 & 0.271 & 0.000 & 0.055 & -0.021 & -1.12 & 1.79 & 1064.33 & 70.02 & & & 2.01 & 71.22 \\
\hline 100 & 146 & 0.26 & 0.00 & -0.01 & 0.03 & 0.283 & 0.000 & 0.045 & -0.023 & -1.03 & 1.93 & 1065.78 & 76.65 & & & 2.15 & 77.96 \\
\hline 101 & 147 & 0.26 & 0.00 & 0.00 & 0.02 & 0.284 & 0.000 & 0.032 & -0.016 & -1.13 & 1.84 & 1065.25 & 85.25 & & & 1.92 & 86.53 \\
\hline 102 & 148 & 0.26 & 0.00 & 0.01 & 0.02 & 0.284 & 0.000 & 0.020 & -0.020 & -0.94 & 1.92 & 1066.49 & 92.08 & & & 2.03 & 93.51 \\
\hline 103 & 149 & 0.26 & 0.00 & 0.02 & 0.02 & 0.285 & 0.000 & 0.007 & -0.023 & -1.15 & 1.68 & 1065.88 & 100.76 & & & 1.79 & 102.33 \\
\hline 104 & 150 & 0.26 & 0.00 & 0.03 & 0.01 & 0.286 & 0.000 & -0.006 & -0.016 & -0.88 & 1.86 & 1066.76 & 107.95 & & & 1.93 & 109.61 \\
\hline 105 & 151 & 0.25 & 0.00 & 0.04 & 0.01 & 0.275 & 0.000 & -0.020 & -0.019 & -0.99 & 1.66 & 1065.87 & 116.91 & & & 1.77 & 118.75 \\
\hline 106 & 152 & 0.24 & 0.00 & 0.05 & 0.00 & 0.264 & 0.000 & -0.036 & -0.012 & -0.80 & 1.75 & 1066.60 & 124.25 & & & 1.88 & 126.26 \\
\hline 107 & 153 & 0.23 & 0.00 & 0.05 & -0.01 & 0.253 & 0.000 & -0.039 & -0.002 & -0.91 & 1.48 & 1065.56 & 133.36 & & & 1.59 & 135.51 \\
\hline 108 & 154 & 0.22 & 0.00 & 0.06 & -0.01 & 0.242 & 0.000 & -0.053 & -0.004 & -0.82 & 1.42 & 1066.21 & 140.79 & & & 1.64 & 143.20 \\
\hline 109 & 155 & 0.21 & 0.00 & 0.07 & -0.02 & 0.231 & 0.000 & -0.068 & 0.004 & -1.14 & 1.03 & 1065.07 & 150.00 & & & 1.39 & 152.72 \\
\hline 110 & 156 & 0.21 & 0.00 & 0.07 & -0.02 & 0.231 & 0.000 & -0.068 & 0.004 & -1.05 & 0.92 & 1065.54 & 157.60 & & & 1.30 & 160.51 \\
\hline
\end{tabular}




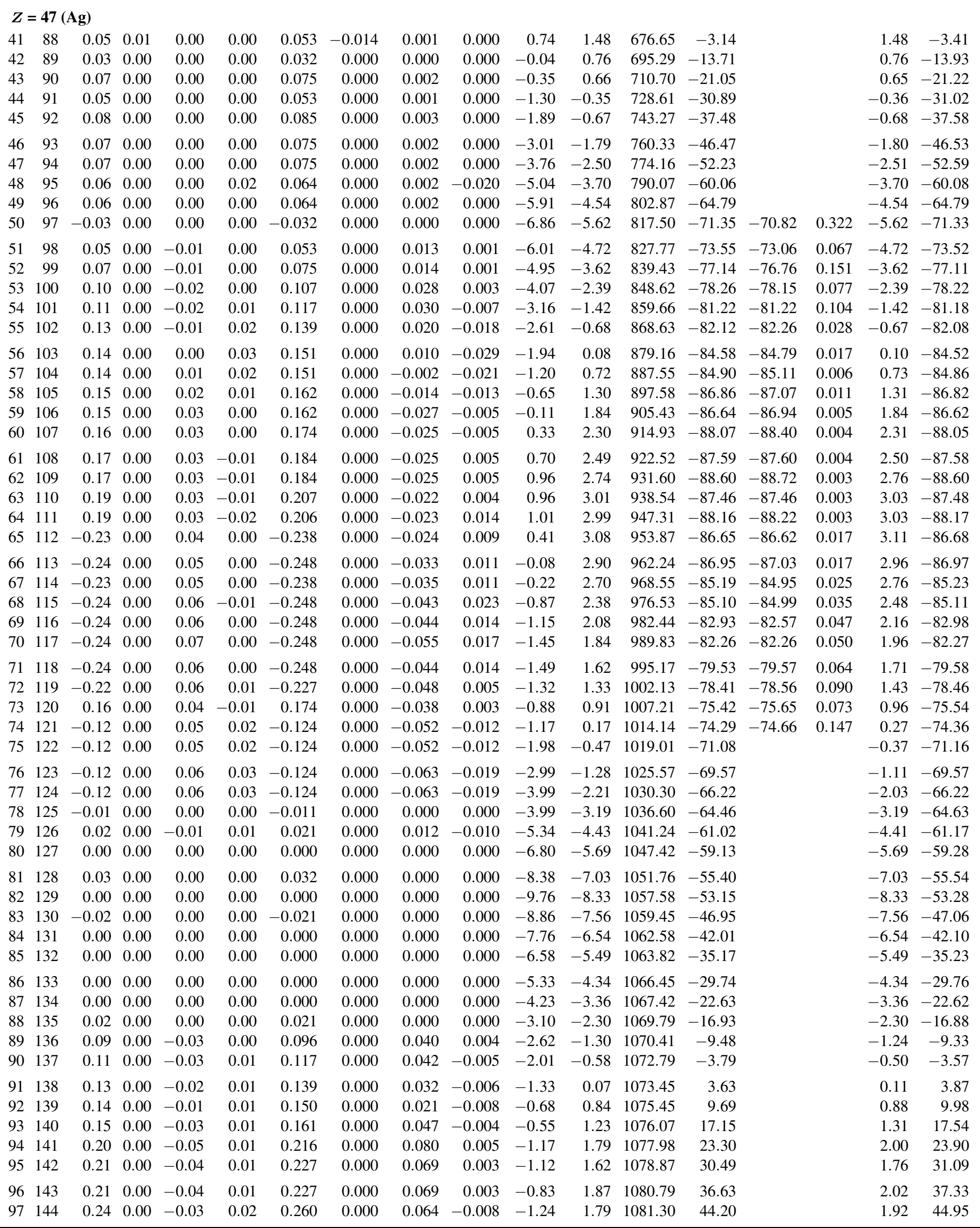




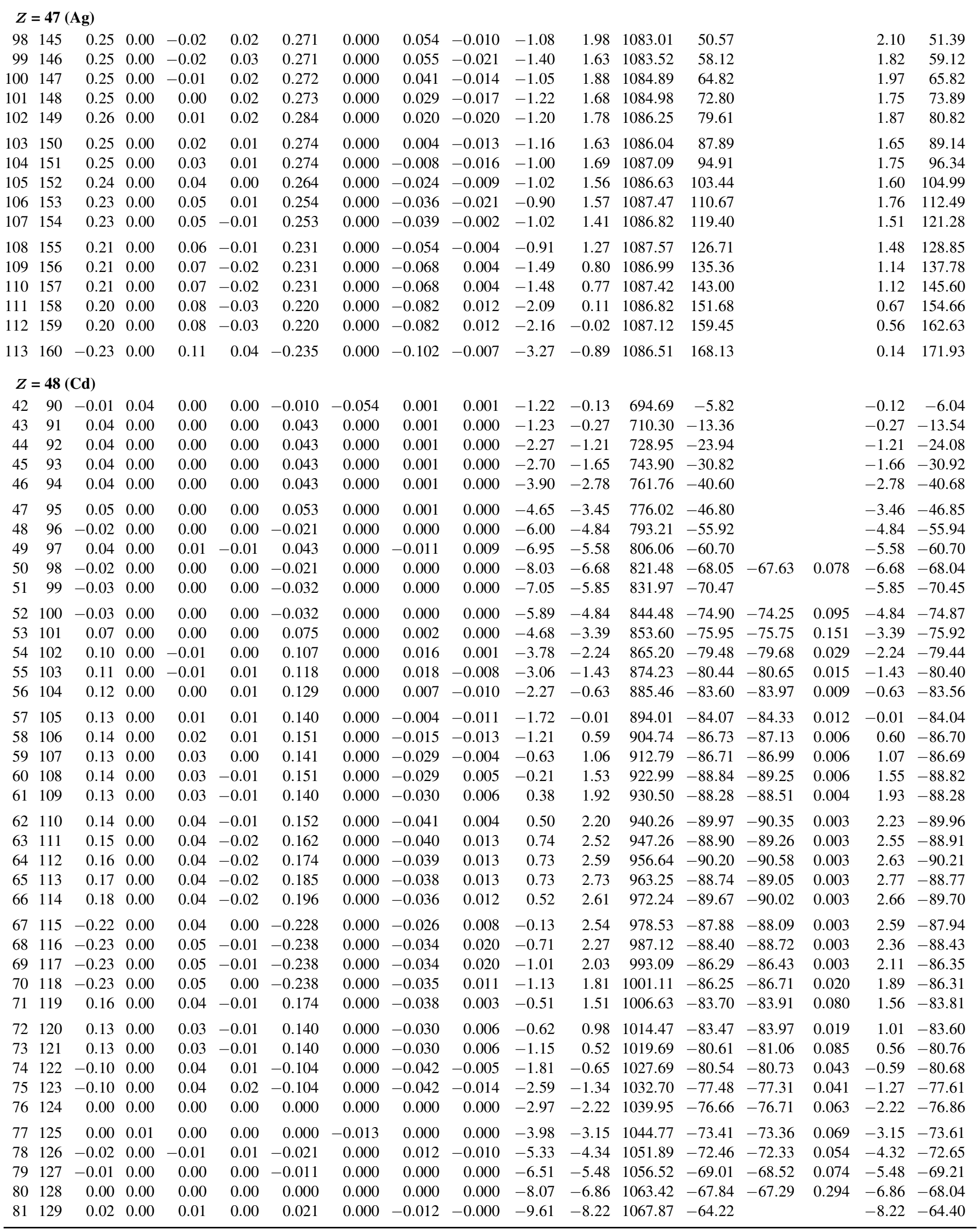




\begin{tabular}{|c|c|c|c|c|c|c|c|c|c|c|c|c|c|c|c|c|c|}
\hline \multicolumn{18}{|c|}{$Z=48(\mathrm{Cd})$} \\
\hline 82 & 130 & 0.00 & 0.00 & 0.00 & 0.00 & 0.000 & 0.000 & 0.000 & 0.000 & -11.02 & -9.52 & 1074.28 & -62.56 & \multirow[t]{5}{*}{-61.57} & \multirow[t]{2}{*}{0.283} & -9.52 & -62.74 \\
\hline 83 & 131 & -0.01 & 0.00 & 0.00 & 0.00 & -0.011 & 0.000 & 0.000 & 0.000 & -10.04 & -8.67 & 1076.15 & -56.36 & & & -8.67 & -56.52 \\
\hline 84 & 132 & 0.00 & 0.00 & 0.00 & 0.00 & 0.000 & 0.000 & 0.000 & 0.000 & -8.98 & -7.67 & 1079.88 & -52.02 & & & -7.67 & -52.16 \\
\hline 85 & 133 & 0.01 & 0.00 & 0.00 & 0.00 & 0.011 & 0.000 & 0.000 & 0.000 & -7.73 & -6.54 & 1081.10 & -45.17 & & & -6.54 & -45.29 \\
\hline 86 & 134 & 0.00 & 0.00 & 0.00 & 0.00 & 0.000 & 0.000 & 0.000 & 0.000 & -6.47 & -5.39 & 1084.31 & -40.31 & & & -5.39 & -40.40 \\
\hline 87 & 135 & 0.00 & 0.00 & 0.00 & 0.00 & 0.000 & 0.000 & 0.000 & 0.000 & -5.36 & -4.38 & 1085.32 & -33.25 & & & -4.39 & -33.31 \\
\hline 88 & 136 & 0.00 & 0.00 & 0.00 & 0.00 & 0.000 & 0.000 & 0.000 & 0.000 & -4.20 & -3.31 & 1088.26 & -28.11 & & & -3.31 & -28.14 \\
\hline 89 & 137 & 0.01 & 0.00 & 0.00 & 0.00 & 0.011 & 0.000 & 0.000 & 0.000 & -3.11 & -2.34 & 1088.96 & -20.74 & & & -2.34 & -20.74 \\
\hline 90 & 138 & 0.09 & 0.00 & -0.03 & 0.00 & 0.096 & 0.000 & 0.040 & 0.004 & -2.56 & -1.30 & 1091.59 & -15.30 & & & -1.24 & -15.19 \\
\hline 91 & 139 & 0.11 & 0.00 & -0.02 & 0.00 & 0.118 & 0.000 & 0.029 & 0.003 & -1.87 & -0.58 & 1092.22 & -7.86 & & & -0.54 & -7.73 \\
\hline 92 & 140 & 0.11 & 0.00 & -0.02 & 0.01 & 0.117 & 0.000 & 0.030 & -0.007 & -1.17 & 0.02 & 1094.96 & -2.53 & & & 0.07 & -2.33 \\
\hline 93 & 141 & 0.14 & 0.00 & -0.02 & 0.01 & 0.150 & 0.000 & 0.033 & -0.006 & -0.70 & 0.82 & 1095.22 & 5.28 & & & 0.87 & 5.54 \\
\hline 94 & 142 & 0.15 & 0.00 & -0.02 & 0.01 & 0.161 & 0.000 & 0.034 & -0.006 & -0.32 & 1.30 & 1097.76 & 10.82 & & & 1.36 & 11.14 \\
\hline 95 & 143 & 0.21 & 0.00 & -0.05 & 0.01 & 0.227 & 0.000 & 0.082 & 0.006 & -1.62 & 1.35 & 1098.47 & 18.17 & & & 1.56 & 18.72 \\
\hline 96 & 144 & 0.21 & 0.00 & -0.04 & 0.01 & 0.227 & 0.000 & 0.069 & 0.003 & -1.07 & 1.67 & 1100.86 & 23.85 & & & 1.84 & 24.42 \\
\hline 97 & 145 & 0.23 & 0.00 & -0.04 & 0.02 & 0.249 & 0.000 & 0.074 & -0.006 & -1.45 & 1.60 & 1101.41 & 31.38 & & & 1.79 & 32.04 \\
\hline 98 & 146 & 0.24 & 0.00 & -0.03 & 0.02 & 0.260 & 0.000 & 0.064 & -0.008 & -1.23 & 1.83 & 1103.61 & 37.25 & & & 1.99 & 37.97 \\
\hline 99 & 147 & 0.25 & 0.00 & -0.02 & 0.03 & 0.271 & 0.000 & 0.055 & -0.021 & -1.55 & 1.54 & 1104.10 & 44.83 & & & 1.75 & 45.68 \\
\hline 100 & 148 & 0.25 & 0.00 & -0.01 & 0.02 & 0.272 & 0.000 & 0.041 & -0.014 & -1.18 & 1.81 & 1105.97 & 51.03 & & & 1.92 & 51.88 \\
\hline 101 & 149 & 0.25 & 0.00 & 0.00 & 0.02 & 0.273 & 0.000 & 0.029 & -0.017 & -1.32 & 1.63 & 1106.10 & 58.97 & & & 1.71 & 59.90 \\
\hline 102 & 150 & 0.25 & 0.00 & 0.01 & 0.02 & 0.273 & 0.000 & 0.017 & -0.020 & -1.12 & 1.75 & 1107.85 & 65.29 & & & 1.85 & 66.34 \\
\hline 103 & 151 & 0.25 & 0.00 & 0.01 & 0.02 & 0.273 & 0.000 & 0.017 & -0.020 & -1.30 & 1.54 & 1107.74 & 73.48 & & & 1.64 & 74.62 \\
\hline 104 & 152 & 0.25 & 0.00 & 0.02 & 0.01 & 0.274 & 0.000 & 0.004 & -0.013 & -1.02 & 1.74 & 1109.15 & 80.14 & & & 1.78 & 81.35 \\
\hline 105 & 153 & 0.24 & 0.00 & 0.03 & 0.00 & 0.263 & 0.000 & -0.012 & -0.006 & -1.00 & 1.55 & 1108.79 & 88.57 & & & 1.57 & 89.88 \\
\hline 106 & 154 & 0.23 & 0.00 & 0.04 & 0.00 & 0.252 & 0.000 & -0.026 & -0.009 & -0.75 & 1.70 & 1109.99 & 95.44 & & & 1.77 & 96.92 \\
\hline 107 & 155 & 0.21 & 0.00 & 0.05 & -0.01 & 0.230 & 0.000 & -0.043 & -0.001 & -0.81 & 1.36 & 1109.54 & 103.96 & & & 1.49 & 105.64 \\
\hline 108 & 156 & 0.21 & 0.00 & 0.05 & -0.01 & 0.230 & 0.000 & -0.043 & -0.001 & -0.80 & 1.42 & 1110.59 & 110.98 & & & 1.57 & 112.81 \\
\hline 109 & 157 & 0.20 & 0.00 & 0.06 & -0.01 & 0.220 & 0.000 & -0.056 & -0.003 & -1.18 & 1.01 & 1109.99 & 119.66 & & & 1.23 & 121.71 \\
\hline 110 & 158 & 0.20 & 0.00 & 0.07 & -0.02 & 0.220 & 0.000 & -0.069 & 0.005 & -1.41 & 0.78 & 1111.09 & 126.63 & & & 1.15 & 128.98 \\
\hline 111 & 159 & 0.19 & 0.00 & 0.07 & -0.02 & 0.209 & 0.000 & -0.071 & 0.005 & -1.78 & 0.39 & 1110.25 & 135.54 & & & 0.76 & 138.06 \\
\hline 112 & 160 & 0.18 & 0.00 & 0.07 & -0.03 & 0.197 & 0.000 & -0.073 & 0.016 & -1.87 & 0.06 & 1111.22 & 142.63 & & & 0.57 & 145.45 \\
\hline 113 & 161 & 0.18 & 0.00 & 0.08 & -0.03 & 0.198 & 0.000 & -0.085 & 0.014 & -2.61 & -0.36 & 1110.19 & 151.74 & & & 0.26 & 154.83 \\
\hline 114 & 162 & 0.15 & 0.00 & 0.07 & -0.02 & 0.164 & 0.000 & -0.076 & 0.008 & -2.29 & -0.46 & 1110.72 & 159.28 & & & 0.00 & 162.39 \\
\hline 115 & 163 & 0.15 & 0.00 & 0.07 & -0.03 & 0.164 & 0.000 & -0.077 & 0.018 & -2.99 & -1.19 & 1109.80 & 168.27 & & & -0.62 & 171.68 \\
\hline \multicolumn{18}{|c|}{$Z=49($ In) } \\
\hline 43 & 92 & 0.04 & 0.00 & 0.01 & 0.00 & 0.043 & 0.000 & -0.011 & -0.000 & -3.03 & -1.81 & 707.92 & -3.69 & & & -1.81 & -3.86 \\
\hline 44 & 93 & 0.03 & 0.00 & 0.01 & 0.00 & 0.032 & 0.000 & -0.012 & -0.000 & -4.07 & -2.81 & 726.78 & -14.48 & & & -2.81 & -14.62 \\
\hline 45 & 94 & 0.04 & 0.00 & 0.01 & 0.00 & 0.043 & 0.000 & -0.011 & -0.000 & -4.51 & -3.24 & 742.51 & -22.14 & & & -3.24 & -22.24 \\
\hline 46 & 95 & 0.03 & 0.00 & 0.01 & 0.00 & 0.032 & 0.000 & -0.012 & -0.000 & -5.72 & -4.42 & 760.57 & -32.13 & & & -4.42 & -32.20 \\
\hline 47 & 96 & 0.04 & 0.00 & 0.00 & 0.00 & 0.043 & 0.000 & 0.001 & 0.000 & -6.44 & -5.09 & 775.62 & -39.11 & & & -5.10 & -39.16 \\
\hline 48 & 97 & 0.03 & 0.00 & 0.01 & 0.00 & 0.032 & 0.000 & -0.012 & -0.000 & -7.86 & -6.43 & 792.91 & -48.33 & & & -6.43 & -48.35 \\
\hline 49 & 98 & 0.04 & 0.00 & 0.01 & 0.00 & 0.043 & 0.000 & -0.011 & -0.000 & -8.79 & -7.24 & 806.92 & -54.26 & & & -7.25 & -54.58 \\
\hline 50 & 99 & 0.02 & 0.00 & 0.00 & 0.00 & 0.021 & 0.000 & 0.000 & 0.000 & -9.85 & -8.28 & 822.73 & -62.01 & & & -8.28 & -62.00 \\
\hline 51 & 100 & 0.03 & 0.00 & 0.00 & 0.00 & 0.032 & 0.000 & 0.000 & 0.000 & -8.88 & -7.42 & 833.94 & -65.14 & -64.17 & 0.249 & -7.42 & -65.12 \\
\hline 52 & 101 & 0.03 & 0.00 & 0.01 & 0.00 & 0.032 & 0.000 & -0.012 & -0.000 & -7.77 & -6.41 & 846.59 & -69.73 & & & -6.41 & -69.70 \\
\hline 53 & 102 & 0.04 & 0.00 & 0.01 & 0.00 & 0.043 & 0.000 & -0.011 & -0.000 & -6.41 & -5.16 & 856.65 & -71.71 & -70.71 & 0.112 & -5.16 & -71.68 \\
\hline 54 & 103 & 0.05 & 0.00 & 0.00 & -0.01 & 0.053 & 0.000 & 0.001 & 0.010 & -5.17 & -4.00 & 868.38 & -75.37 & -74.60 & 0.025 & -4.01 & -75.34 \\
\hline 55 & 104 & 0.08 & 0.00 & -0.01 & 0.00 & 0.085 & 0.000 & 0.015 & 0.001 & -4.29 & -2.95 & 877.91 & -76.83 & -76.11 & 0.085 & -2.95 & -76.80 \\
\hline 56 & 105 & 0.08 & 0.00 & -0.01 & 0.01 & 0.085 & 0.000 & 0.015 & -0.009 & -3.33 & -2.09 & 889.20 & -80.04 & -79.48 & 0.017 & -2.08 & -80.01 \\
\hline 57 & 106 & 0.10 & 0.00 & 0.00 & 0.01 & 0.107 & 0.000 & 0.005 & -0.010 & -2.71 & -1.33 & 898.34 & -81.11 & -80.61 & 0.012 & -1.33 & -81.09 \\
\hline 58 & 107 & 0.11 & 0.00 & 0.01 & 0.01 & 0.118 & 0.000 & -0.007 & -0.011 & -2.05 & -0.63 & 909.09 & -83.79 & -83.56 & 0.011 & -0.62 & -83.77 \\
\hline 59 & 108 & 0.10 & 0.00 & 0.02 & 0.00 & 0.107 & 0.000 & -0.020 & -0.002 & -1.44 & -0.13 & 917.83 & -84.46 & -84.12 & 0.010 & -0.13 & -84.45 \\
\hline 60 & 109 & 0.10 & 0.00 & 0.02 & -0.01 & 0.107 & 0.000 & -0.020 & 0.008 & -0.77 & 0.45 & 928.03 & -86.60 & -86.49 & 0.006 & 0.46 & -86.60 \\
\hline 61 & 110 & 0.09 & 0.00 & 0.02 & 0.00 & 0.097 & 0.000 & -0.021 & -0.002 & -0.11 & 0.94 & 936.15 & -86.64 & -86.47 & 0.012 & 0.94 & -86.66 \\
\hline 62 & 111 & 0.10 & 0.00 & 0.03 & -0.01 & 0.108 & 0.000 & -0.032 & 0.007 & 0.10 & 1.29 & 945.96 & -88.38 & -88.40 & 0.005 & 1.30 & -88.40 \\
\hline 63 & 112 & 0.10 & 0.00 & 0.03 & -0.01 & 0.108 & 0.000 & -0.032 & 0.007 & 0.54 & 1.65 & 953.61 & -87.96 & -88.00 & 0.005 & 1.66 & -88.00 \\
\hline 64 & 113 & 0.08 & 0.00 & 0.02 & -0.01 & 0.086 & 0.000 & -0.022 & 0.008 & 0.87 & 1.66 & 963.16 & -89.43 & -89.37 & 0.003 & 1.67 & -89.48 \\
\hline
\end{tabular}




\section{$Z=49$ (In)}

\section{$\begin{array}{llll}65 & 114 & 0.08 & 0.00\end{array}$}

$\begin{array}{llll}66 & 115 & -0.11 & 0.00\end{array}$

$\begin{array}{lllll}67 & 116 & -0.14 & 0.00\end{array}$

$\begin{array}{lllll}68 & 117 & -0.12 & 0.01\end{array}$

$\begin{array}{llll}69 & 118 & -0.12 & 0.00\end{array}$

$\begin{array}{lllll}70 & 119 & -0.12 & 0.00\end{array}$

$\begin{array}{lllll}71 & 120 & -0.10 & 0.00\end{array}$

$\begin{array}{llll}72 & 121 & -0.10 & 0.00\end{array}$

$\begin{array}{llll}73 & 122 & -0.09 & 0.00\end{array}$

$\begin{array}{lllll}74 & 123 & -0.09 & 0.00\end{array}$

$\begin{array}{lllll}75 & 124 & -0.09 & 0.00\end{array}$

$\begin{array}{lllll}76 & 125 & 0.04 & 0.00\end{array}$

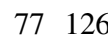

$\begin{array}{ll}78 & 127\end{array}$

$\begin{array}{ll}79 & 128\end{array}$

$0.04 \quad 0.00$

$0.01 \quad 0.00$

$0.03 \quad 0.00$

$\begin{array}{ll}80 & 129\end{array}$

$81 \quad 130$

$82 \quad 131$

$83 \quad 132$

84133

$\begin{array}{ll}85 & 134\end{array}$

$86 \quad 135$

$87 \quad 136$

$\begin{array}{lll}88 & 137\end{array}$

89138

$90 \quad 139$

$91 \quad 140$

$\begin{array}{ll}92 & 141\end{array}$

$93 \quad 142$

$94 \quad 143$

$95 \quad 144$

$96 \quad 145$

$97 \quad 146$

$\begin{array}{lll}98 & 147\end{array}$

$99 \quad 148$

100149

$101 \quad 150$

$102 \quad 151$

103152

104153

105154

$106 \quad 155$

$107 \quad 156$

$108 \quad 157$

$109 \quad 158$

110159

$111 \quad 160$

112161

113162

114163

115164

116165

$117 \quad 166$

$0.02 \quad 0.00$

$\begin{array}{ll}0.01 & 0.00\end{array}$

$\begin{array}{ll}0.02 & 0.00\end{array}$

$\begin{array}{ll}0.01 & 0.00\end{array}$

$0.03 \quad 0.00$

$\begin{array}{ll}0.02 & 0.00\end{array}$

$\begin{array}{ll}0.02 & 0.00\end{array}$

$\begin{array}{ll}0.03 & 0.00\end{array}$

$0.03 \quad 0.00$

$\begin{array}{ll}0.25 & 0.00\end{array}$

0.250 .00

$\begin{array}{ll}0.23 & 0.00\end{array}$

$\begin{array}{ll}0.23 & 0.00\end{array}$

$0.23 \quad 0.00$

0.210 .00

$0.21 \quad 0.00$

$0.20 \quad 0.00$

$\begin{array}{ll}0.15 & 0.00\end{array}$

$\begin{array}{ll}0.15 & 0.00\end{array}$

0.150 .00

$\begin{array}{ll}0.15 & 0.00\end{array}$

0.110 .00

0.110 .00 $\begin{array}{lll}0.02 & -0.01 \quad 0.086\end{array}$

$\begin{array}{lll}0.00 & -0.01 & -0.115\end{array}$

$\begin{array}{llll}0.01 & -0.01 & -0.146\end{array}$

$0.01-0.01-0.125$

$\begin{array}{llll}0.02 & 0.00 & -0.125\end{array}$

$\begin{array}{llll}0.02 & 0.00 & -0.125\end{array}$

$\begin{array}{llll}0.02 & 0.00 & -0.105\end{array}$

$\begin{array}{llll}0.03 & 0.01 & -0.104\end{array}$

$\begin{array}{llll}0.03 & 0.01 & -0.094\end{array}$

$\begin{array}{llll}0.03 & 0.01 & -0.094\end{array}$

$\begin{array}{llll}0.04 & 0.02 & -0.094\end{array}$

$\begin{array}{lll}0.01 & 0.00 & 0.043\end{array}$

$\begin{array}{lll}0.01 & 0.00 & 0.043\end{array}$

$\begin{array}{lll}0.01 & 0.00 & 0.011\end{array}$

$\begin{array}{lll}0.00 & 0.00 & 0.032\end{array}$

$\begin{array}{lll}0.01 & -0.01 & 0.011\end{array}$

$0.01 \quad 0.00$

0.021

0.00

0.00

0.011

0.00

0.00

0.021

0.01

0.00

0.011

0.01

0.00

0.032

0.01

0.021

$0.01-0.01$

$\begin{array}{lll}0.04 & 0.00 & 0.00\end{array}$

$\begin{array}{llll}0.09 & 0.00 & -0.02\end{array}$

0.00

0.00

0.00

$\begin{array}{llll}0.10 & 0.00 & -0.02 \\ 0.11 & 0.00 & -0.01\end{array}$

0.01

0.01

$\begin{array}{llll}0.11 & 0.00 & -0.01\end{array}$

$\begin{array}{llll}0.20 & 0.00 & -0.05\end{array}$

0.0

$\begin{array}{llll}0.20 & 0.00 & -0.04\end{array}$

0.0

$\begin{array}{llll}0.23 & 0.00 & -0.04\end{array}$

0.02

0.02

$\begin{array}{lll}0.23 & 0.00 & -0.03 \\ 0.24 & 0.00 & -0.02\end{array}$

0.02

$\begin{array}{lll}0.25 & 0.00 & -0.01\end{array}$

0.02

$\begin{array}{lll}0.25 & 0.00 & 0.00\end{array}$

0.02

0.01

0.02

0.01

0.02

0.01

0.03

0.00

$0.04 \quad 0.00$

$0.05-0.01$

$0.06-0.02$

$0.06-0.02$

$\begin{array}{lll}0.06 & -0.02\end{array}$

$\begin{array}{lll}0.05 & -0.01\end{array}$

$\begin{array}{lll}0.06 & -0.02\end{array}$

$\begin{array}{lll}0.06 & -0.02\end{array}$

$\begin{array}{lll}0.07 & -0.03\end{array}$ $\begin{array}{ll}0.05 & -0.02\end{array}$

$0.05-0.02$
0.096

0.107

0.118

0.260

0.272

0.274

0.252

0.230

0.219

0.163

0.163

0.164
0.119
$0.000-0.022$

$0.000 \quad 0.005$

$0.000-0.003$

$-0.013-0.005$

$0.000 \quad-0.017$

$\begin{array}{ll}0.000 & -0.017\end{array}$

$0.000-0.019$

$\begin{array}{llll}0.000 & -0.031 & -0.006\end{array}$

$\begin{array}{llll}0.000 & -0.032 & -0.006\end{array}$

$\begin{array}{llll}0.000 & -0.032 & -0.006\end{array}$

$\begin{array}{llll}0.000 & -0.043 & -0.014\end{array}$

$\begin{array}{llll}0.000 & -0.011 & -0.000\end{array}$

$0.000-0.011-0.000$

$0.000-0.012-0.000$

$\begin{array}{lll}0.000 & 0.000 & 0.000\end{array}$

$0.000-0.012-0$.

$0.000 \quad 0.000$

$0.000 \quad 0.000$

$0.010-9.11$

$0.000 \quad 0.000$

$0.000-11.70$

$0.000-10.81$

$\begin{array}{rr}0.000 & -10.81 \\ 0.000 & -9.70\end{array}$

$\begin{array}{llll}0.000 & -0.012 & -0.000 & -8.51\end{array}$

$\begin{array}{llll}0.000 & -0.012 & -0.000 & -7.20\end{array}$

$\begin{array}{llll}0.000 & 0.000 & -0.010 & -6.09\end{array}$

$\begin{array}{llll}0.000 & -0.012 & -0.000 & -4.95\end{array}$

$\begin{array}{llll}0.000 & -0.012 & 0.009 & -3.85\end{array}$

$0.000 \quad 0.001$

0.000

0.028

$0.000-2.85$

0.000

0.028

0.002

$-2.32$

0.000

$\begin{array}{lll}0.018 & -0.008 & -1.02\end{array}$

0.000

$0.018-0$.

0.008

$-0.47$

$\begin{array}{llll}0.000 & 0.080 & 0.005 & -1.74\end{array}$

$\begin{array}{lllll}0.000 & 0.067 & 0.002 & -1.18\end{array}$

$\begin{array}{llll}0.000 & 0.074 & -0.006 & -1.72\end{array}$

$\begin{array}{lllll}0.000 & 0.062 & -0.009 & -1.29\end{array}$

0.000

-1.29
-1.52

0.000

$\begin{array}{lll}0.041 & -0.014 & -1.38\end{array}$

$\begin{array}{lllll}0.000 & 0.029 & -0.017 & -1.51\end{array}$

$\begin{array}{llll}0.000 & 0.017 & -0.020 & -1.32\end{array}$

0.000

$0.004-0.013-1.45$

0.000

$0.004-0.013$

$-1.25$

$\begin{array}{lllll}0.000 & -0.014 & -0.006 & -1.12\end{array}$

$\begin{array}{llll}0.000 & -0.026 & -0.009 & -1.03\end{array}$

$\begin{array}{llll}0.000 & -0.039 & -0.002 & -1.29\end{array}$

$\begin{array}{llll}0.000 & -0.043 & -0.001 & -1.04\end{array}$

$0.000-0.056$

$0.006-1.52$

$0.000-0.057$

$0.007-1.37$

$0.000-0.060$

$0.008-1.62$

$0.000-0.051$

$0.002-1.38$

$0.000-0.064$

$0.000-0.064$

$0.010-2.11$

-2.11
-2.43

$0.000-0.077$

0.018

$0.000-0.056$

$0.000-0.056$ $\begin{array}{llllll}0.018 & -3.32 & -1.45 & 1131.88 & 153.48 \\ 0.014 & -3.20 & -1.88 & 1132.54 & 160.89\end{array}$

$\begin{array}{lllll}0.014 & -4.05 & -2.68 & 1131.95 & 169.55\end{array}$

$\begin{array}{lllllll}1.80 & 970.45 & -88.65 & -88.57 & 0.003 & 1.81 & -88.72\end{array}$

$\begin{array}{llllll}1.83 & 979.40 & -89.54 & -89.54 & 0.004\end{array}$

$\begin{array}{lllll}1.87 & 986.25 & -88.31 & -88.25 & 0.004\end{array}$

$\begin{array}{lllll}1.50 & 995.05 & -89.04 & -88.94 & 0.006\end{array}$

$\begin{array}{lllll}1.32 & 1001.61 & -87.53 & -87.23 & 0.008\end{array}$

$\begin{array}{lllll}0.86 & 1009.97 & -87.82 & -87.70 & 0.008\end{array}$

$\begin{array}{lllll}0.46 & 1016.24 & -86.02 & -85.74 & 0.040\end{array}$

$\begin{array}{lllll}-0.17 & 1024.28 & -85.99 & -85.84 & 0.027\end{array}$

$\begin{array}{lllll}-0.68 & 1030.18 & -83.82 & -83.58 & 0.050\end{array}$

$\begin{array}{llll}-1.48 & 1037.90 & -83.47 & -83.43\end{array}$

0.024

0.049

0.030

0.040

0.040

0.049

0.043

0.039

$0.028-10$

0.062

$\begin{array}{llll}-9.35 & 1089.58 & -62.50 & -62.42\end{array}$

$\begin{array}{lll}-8.34 & 1093.37 & -58.22\end{array}$

$\begin{array}{lll}-7.21 & 1095.17 & -51.95\end{array}$

$\begin{array}{lll}-6.01 & 1098.41 & -47.11\end{array}$

$\begin{array}{llll}-5.03 & 1100.01 & -40.64\end{array}$ 


\section{$Z=50$ (Sn)}

$\begin{array}{llll}47 & 97 & -0.01 & 0.00\end{array}$

$\begin{array}{llll}48 & 98 & 0.00 & 0.00\end{array}$

$\begin{array}{llll}49 & 99 & 0.02 & 0.00\end{array}$

$\begin{array}{rrrr}50 & 100 & 0.00 & 0.00\end{array}$

$\begin{array}{llll}51 & 101 & -0.01 & 0.00\end{array}$

$\begin{array}{llll}52 & 102 & 0.00 & 0.00\end{array}$

$\begin{array}{llll}53 & 103 & 0.00 & 0.00\end{array}$

$\begin{array}{llll}54 & 104 & 0.00 & 0.00\end{array}$

$\begin{array}{llll}55 & 105 & 0.00 & 0.00\end{array}$

$\begin{array}{llll}56 & 106 & 0.00 & 0.00\end{array}$

$\begin{array}{lllll}57 & 107 & 0.00 & 0.00\end{array}$

$\begin{array}{lllll}58 & 108 & 0.06 & 0.00\end{array}$

$\begin{array}{llll}59 & 109 & 0.08 & 0.00\end{array}$

$\begin{array}{llll}60 & 110 & 0.00 & 0.00\end{array}$

$\begin{array}{llll}61 & 111 & -0.01 & 0.00\end{array}$

$\begin{array}{lllll}62 & 112 & 0.00 & 0.00\end{array}$

$\begin{array}{llll}63 & 113 & 0.03 & 0.00\end{array}$

$\begin{array}{llll}64 & 114 & 0.00 & 0.00\end{array}$

$\begin{array}{llll}65 & 115 & 0.02 & 0.00\end{array}$

$\begin{array}{llll}66 & 116 & 0.00 & 0.00\end{array}$

$\begin{array}{lllll}67 & 117 & -0.07 & 0.00\end{array}$

$\begin{array}{llll}68 & 118 & -0.09 & 0.00\end{array}$

$\begin{array}{llll}69 & 119 & -0.10 & 0.00\end{array}$

$\begin{array}{llll}70 & 120 & -0.08 & 0.00\end{array}$

$\begin{array}{lllll}71 & 121 & -0.09 & 0.00\end{array}$

$\begin{array}{ll}72 & 122\end{array}$

$\begin{array}{ll}73 & 123\end{array}$

$\begin{array}{lll}74 & 124\end{array}$

$\begin{array}{ll}75 & 125\end{array}$

$0.00 \quad 0.00$

$0.00 \quad 0.00$

$\begin{array}{ll}0.00 & 0.00\end{array}$

$76 \quad 126$

$\begin{array}{ll}0.00 & 0.00\end{array}$

$0.00 \quad 0.00$

$\begin{array}{llll}77 & 127 & -0.01 & 0.00\end{array}$

$\begin{array}{llll}78 & 128 & 0.00 & 0.00\end{array}$

$\begin{array}{llll}79 & 129 & -0.01 & 0.00\end{array}$

$\begin{array}{llll}80 & 130 & 0.01 & 0.00\end{array}$

$\begin{array}{llll}81 & 131 & 0.01 & 0.00\end{array}$

$\begin{array}{lllll}82 & 132 & 0.00 & 0.00\end{array}$

$\begin{array}{llll}83 & 133 & -0.01 & 0.00\end{array}$

$\begin{array}{llll}84 & 134 & 0.00 & 0.00\end{array}$

$\begin{array}{lllll}85 & 135 & 0.00 & 0.00\end{array}$

$\begin{array}{llll}86 & 136 & 0.00 & 0.00\end{array}$

$\begin{array}{lllll}87 & 137 & 0.00 & 0.00\end{array}$

$\begin{array}{lllll}88 & 138 & 0.00 & 0.00\end{array}$

$\begin{array}{lllll}89 & 139 & 0.00 & 0.00\end{array}$

$\begin{array}{llll}90 & 140 & 0.00 & 0.00\end{array}$

$\begin{array}{lllll}91 & 141 & 0.00 & 0.00\end{array}$

$92 \quad 142$

$93 \quad 143$

$94 \quad 144$

$95 \quad 145$

96146

$\begin{array}{ll}97 & 147\end{array}$

$98 \quad 148$

$99 \quad 149$

$100 \quad 150$

101151

$0.00 \quad 0.00$

$0.09-0.00-0.02 \quad 0.00$

$\begin{array}{lllll}0.20 & 0.00 & -0.05 & 0.01\end{array}$

$\begin{array}{lllll}0.21 & 0.00 & -0.05 & 0.02\end{array}$

$\begin{array}{llll}0.21 & 0.00 & -0.04 & 0.02\end{array}$

$\begin{array}{lllll}0.23 & 0.00 & -0.03 & 0.02\end{array}$

$\begin{array}{lllll}0.24 & 0.00 & -0.02 & 0.02\end{array}$

$\begin{array}{lllll}0.24 & 0.00 & -0.01 & 0.02\end{array}$

$102 \quad 152$

$\begin{array}{lllll}0.24 & 0.00 & -0.01 & 0.02\end{array}$

103153

$\begin{array}{ll}0.00 & 0.00 \\ 0.00 & 0.00\end{array}$

$\begin{array}{llll}0.24 & 0.00 & 0.00 & 0.02\end{array}$ $\begin{array}{llll}0.00 & 0.00 & -0.011 & 0.000\end{array}$

$\begin{array}{llll}0.00 & 0.000 & 0.000\end{array}$

$0.021-0.000$

$0.000 \quad 0.000$

$\begin{array}{lllll}0.00 & -0.011 & 0.000\end{array}$

$0.000 \quad 0.000$

$0.000 \quad 0.000$

$\begin{array}{ll}0.000 & 0.000\end{array}$

$0.000 \quad 0.000$

$\begin{array}{ll}0.000 & 0.000\end{array}$

$\begin{array}{ll}0.000 & 0.000\end{array}$

$\begin{array}{ll}0.064 & 0.000\end{array}$

$0.086 \quad 0.000$

$0.000 \quad 0.000$

0.000

$0.000 \quad 0.000$

$0.032 \quad 0.000-0$.

$\begin{array}{ll}0.000 & 0.000\end{array}$

$0.021 \quad 0.000$

$0.000 \quad 0.000$

0.000

0.000

$\begin{array}{lll}0.000 & 0.000 & -9.06\end{array}$

0.000

$0.000-9.84$

0.000

$0.000-11.12$

0.000

$0.000-10.04$

0.000

$0.000-8.96$

0.000

0.000

0.000

0.000

0.000

0.000

0.000

0.002

0.000

$-0.009$

0.000

$0.000 \quad 0.000$

$0.012-0.000$

$-7.51$

$-6.20$

$-5.04$

$0.000 \quad 0.000$

$-3.96$

$-3.00$

$-2.21$

$-1.70$

$-0.90$

$-0.47$

$-0.19$

0.18

0.26

0.52

0.37

$-0.012-0.000$

$\begin{array}{lr}0.000 & 0.000\end{array}$

$\begin{array}{rrr}0.002 & -0.000 & 0.31 \\ 0.003 & -0.000 & -0.15\end{array}$

$-0.008$

0.001

$-0.37$

$0.001-0.74$

$-1.12$

0.001

$-1.62$

0.000

0.000

$\begin{array}{lll}0.000 \quad 0.000 & 0.000\end{array}$

0.000

$0.000-2.10$

0.000

0.000

$-3.20$

$0.000 \quad 0.000$

0.000

$-3.88$

0.000

0.000

0.00

0.000

$0.000-6.05$

6.05

$0.000-7.5$

$\begin{array}{lll}0.000 & 0.000 & -8.61 \\ 0.000 & 0.000 & -10.24\end{array}$

$0.011 \quad 0.000$

0.000$$
-0.000
$$

0.000
0.000

0.000

$$
\begin{aligned}
& 0.000 \\
& 0.000
\end{aligned}
$$

$-11.40$

0.000$$
0.000
$$$$
-12.82
$$

.0000 .000

$0.000 \quad 0.000$

0.000

0.000

$-11.8$

1.82
0.73

$-9.42$

0.000

0.000

$\begin{array}{lll}0.000 & 0.000\end{array}$

0.000

0.000

$0.000 \quad 0.000$

0.000

0.000

$0.000 \quad 0.000$

0.000

0.000

$\begin{array}{lll}0.000 & 0.000\end{array}$

$0.000 \quad 0.000$

$\begin{array}{ll}0.075 & 0.000\end{array}$

0.000

0.000

$-8.13$

$\begin{array}{ll}0.096 & 0.000\end{array}$

$\begin{array}{ll}0.216 & 0.000\end{array}$

0.026

0.000

$\begin{array}{ll}0.028 & 0.002\end{array}$

$0.080 \quad 0.00$

$0.226 \quad 0.000$

$0.082-0.00$

$\begin{array}{ll}0.226 & 0.000\end{array}$

$\begin{array}{ll}0.249 & 0.000\end{array}$

$0.260 \quad 0.000$

$\begin{array}{lll}0.070 & -0.007 & -1.68\end{array}$

$\begin{array}{lll}0.062 & -0.009 & -1.53\end{array}$

$\begin{array}{llll}0.051 & -0.011 & -1.72\end{array}$

$\begin{array}{ll}0.261 \quad 0.000 \\ 0.261 & 0.000\end{array}$

$\begin{array}{llll}0.039 & -0.014 & -1.40\end{array}$

$0.261 \quad 0.000$
0.000

$\begin{array}{lll}0.039 & -0.014 & -1.59\end{array}$

$\begin{array}{ll}0.261 & 0.000\end{array}$

$\begin{array}{llll}0.027 & -0.017 & -1.34\end{array}$

$\begin{array}{lll}0.017 & -0.020 & -1.66\end{array}$

-6.16
-7.53

$776.03-32.23$

$794.13-42.26$

$\begin{array}{lll}-8.24 & 808.48 & -48.53\end{array}$

$-9.44$

$\begin{array}{llll}825.52 & -57.50 & -56.78\end{array}$

$836.79-60.71$

$-7.53$

$-6.24$

$\begin{array}{lll}850.23 & -66.07 & -64.93\end{array}$

$860.39-68.16$

$872.84-72.54 \quad-71.59$

$\begin{array}{llll}882.51 & -74.14 & -73.26\end{array}$

$\begin{array}{llll}894.41 & -77.97 & -77.43\end{array}$

$\begin{array}{llll}903.54 & -79.03 & -78.58\end{array}$

$\begin{array}{llll}914.78 & -82.20 & -82.04\end{array}$

$\begin{array}{llll}923.54 & -82.88 & -82.64\end{array}$

$-0.18$

0.26

$\begin{array}{llll}934.59 & -85.87 & -85.84\end{array}$

$\begin{array}{llll}942.89 & -86.09 & -85.94\end{array}$

$\begin{array}{lllll}0.51 & 953.49 & -88.62 & -88.66\end{array}$

0.87

0.89

1.12

$\begin{array}{llll}961.25 & -88.31 & -88.33\end{array}$

$\begin{array}{lll}971.47 & -90.45 & -90.56\end{array}$

$\begin{array}{lll}988.60 & -91.45 & -91.53\end{array}$

$\begin{array}{llll}1.09 & 995.47 & -90.24 & -90.40\end{array}$

$\begin{array}{llll}0.81 & 1004.84 & -91.54 & -91.66\end{array}$

$\begin{array}{llll}0.64 & 1011.48 & -90.12 & -90.07\end{array}$

$\begin{array}{llll}0.14 & 1020.54 & -91.10 & -91.11\end{array}$

$\begin{array}{llll}-0.14 & 1026.80 & -89.29 & -89.20\end{array}$

$\begin{array}{lllll}-0.93 & 1035.63 & -90.05 & -89.95\end{array}$

$\begin{array}{lllll}-1.39 & 1041.59 & -87.93 & -87.82\end{array}$

$\begin{array}{lllll}-2.35 & 1050.10 & -88.37 & -88.24\end{array}$

$\begin{array}{lllll}-3.01 & 1055.79 & -85.99 & -85.90\end{array}$

$\begin{array}{llll}-4.15 & 1064.02 & -86.15 & -86.02\end{array}$

$\begin{array}{lllll}-5.02 & 1069.47 & -83.53 & -83.50\end{array}$

$\begin{array}{lllll}-6.34 & 1077.42 & -83.41 & -83.33\end{array}$

$\begin{array}{lllll}-7.39 & 1082.66 & -80.57 & -80.59\end{array}$

$\begin{array}{lllll}-8.84 & 1090.31 & -80.15 & -80.14\end{array}$

$\begin{array}{lllll}-9.92 & 1095.15 & -76.92 & -77.31\end{array}$

$\begin{array}{lllll}-11.22 & 1102.23 & -75.94 & -76.55\end{array}$

$\begin{array}{llll}-10.33 & 1104.73 & -70.36 & -70.95\end{array}$

$\begin{array}{lllll}-9.31 & 1109.09 & -66.65 & -66.80\end{array}$

$\begin{array}{llll}-8.12 & 1110.91 & -60.40\end{array}$

$\begin{array}{llll}-6.91 & 1114.71 & -56.13\end{array}$ 


\begin{tabular}{|c|c|c|c|c|c|c|c|c|c|c|c|c|c|c|c|c|c|}
\hline \multicolumn{18}{|c|}{$Z=50(\mathrm{Sn})$} \\
\hline 104 & 154 & 0.24 & 0.00 & 0.02 & 0.01 & 0.262 & 0.000 & 0.002 & -0.013 & -1.23 & 1.49 & 1148.88 & 54.99 & & & 1.54 & 55.84 \\
\hline 105 & 155 & 0.24 & 0.00 & 0.02 & 0.01 & 0.262 & 0.000 & 0.002 & -0.013 & -1.36 & 1.38 & 1148.97 & 62.97 & & & 1.42 & 63.91 \\
\hline 106 & 156 & 0.23 & 0.00 & 0.03 & 0.00 & 0.252 & 0.000 & -0.014 & -0.006 & -0.99 & 1.53 & 1150.71 & 69.30 & & & 1.57 & 70.34 \\
\hline 107 & 157 & 0.23 & 0.00 & 0.04 & 0.00 & 0.252 & 0.000 & -0.026 & -0.009 & -1.19 & 1.35 & 1150.62 & 77.46 & & & 1.42 & 78.64 \\
\hline 108 & 158 & 0.21 & 0.00 & 0.04 & -0.01 & 0.229 & 0.000 & -0.031 & 0.001 & -0.85 & 1.36 & 1152.25 & 83.90 & & & 1.45 & 85.23 \\
\hline 109 & 159 & 0.21 & 0.00 & 0.05 & -0.01 & 0.230 & 0.000 & -0.043 & -0.001 & -1.24 & 1.03 & 1152.08 & 92.14 & & & 1.16 & 93.64 \\
\hline 110 & 160 & 0.18 & 0.00 & 0.04 & -0.01 & 0.196 & 0.000 & -0.035 & 0.002 & -0.71 & 1.05 & 1153.45 & 98.84 & & & 1.15 & 100.44 \\
\hline 111 & 161 & 0.18 & 0.00 & 0.05 & -0.02 & 0.196 & 0.000 & -0.048 & 0.010 & -1.29 & 0.74 & 1153.04 & 107.33 & & & 0.94 & 109.17 \\
\hline 112 & 162 & 0.15 & 0.00 & 0.04 & -0.01 & 0.163 & 0.000 & -0.039 & 0.003 & -1.09 & 0.52 & 1154.41 & 114.03 & & & 0.63 & 115.92 \\
\hline 113 & 163 & 0.15 & 0.00 & 0.05 & -0.01 & 0.163 & 0.000 & -0.051 & 0.002 & -1.76 & -0.05 & 1154.02 & 122.49 & & & 0.13 & 124.59 \\
\hline 114 & 164 & 0.11 & 0.00 & 0.03 & 0.00 & 0.119 & 0.000 & -0.031 & -0.004 & -1.73 & -0.56 & 1155.46 & 129.11 & & & -0.50 & 131.27 \\
\hline 115 & 165 & 0.11 & 0.00 & 0.04 & -0.01 & 0.119 & 0.000 & -0.043 & 0.005 & -2.54 & -1.28 & 1155.02 & 137.63 & & & -1.14 & 140.02 \\
\hline 116 & 166 & 0.10 & 0.00 & 0.04 & -0.01 & 0.108 & 0.000 & -0.044 & 0.005 & -3.05 & -1.84 & 1156.29 & 144.44 & & & -1.69 & 147.00 \\
\hline 117 & 167 & 0.10 & 0.00 & 0.04 & -0.01 & 0.108 & 0.000 & -0.044 & 0.005 & -3.92 & -2.66 & 1155.73 & 153.06 & & & -2.51 & 155.80 \\
\hline 118 & 168 & 0.10 & 0.00 & 0.04 & -0.02 & 0.108 & 0.000 & -0.045 & 0.015 & -4.46 & -3.22 & 1156.79 & 160.08 & & & -2.99 & 163.08 \\
\hline 119 & 169 & 0.08 & 0.00 & 0.03 & -0.01 & 0.086 & 0.000 & -0.034 & 0.007 & -5.15 & -4.01 & 1156.01 & 168.93 & & & -3.91 & 171.99 \\
\hline \multicolumn{18}{|c|}{$Z=51(\mathrm{Sb})$} \\
\hline 46 & 97 & -0.02 & 0.00 & 0.00 & 0.00 & -0.021 & ل & 000 & 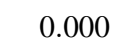 & - & -4.10 & & -12 & & & -4.10 & -1 \\
\hline 47 & 98 & 0.02 & 0.00 & -0.01 & 0.00 & 0.021 & 0.000 & 0.012 & 00 & -5 & -4.76 & 771.40 & -2 & & & -4.77 & \\
\hline 48 & 99 & 0.01 & 0.00 & -0.01 & 0.00 & 0.011 & 0.000 & 0.012 & 0.000 & -7.36 & -6.06 & 789.57 & -30.41 & & & -6.06 & -30.42 \\
\hline 49 & 100 & 0.03 & 0.00 & -0.01 & 0.00 & 0.032 & 0.000 & 0.012 & 0.000 & -8.19 & -6.78 & 804.68 & -37.45 & & & -6.78 & -37.44 \\
\hline 50 & 101 & 0.01 & 0.00 & -0.01 & 0.00 & 0.011 & 0.000 & 0.012 & 0.000 & -9.37 & -7.90 & 821.78 & -46.48 & & & -7.90 & -46.46 \\
\hline 51 & 102 & 0.03 & 0.00 & -0.02 & -0.01 & 0.032 & 0.000 & 0.024 & 0.011 & -8.41 & -6.98 & 834.12 & -50.75 & & & -6.98 & -51.01 \\
\hline 52 & 103 & & 00 & -0.01 & -0.01 & 0.021 & 0. & & & -7 & -6.03 & & -5 & & & - & -5 \\
\hline 53 & 104 & 0.07 & 0.00 & -0.04 & 0.00 & 0.075 & 0.000 & 0.0 & 0.004 & -6.25 & -4.61 & & -59.25 & & & -4.60 & -59.21 \\
\hline 54 & 105 & 0.08 & 0.00 & -0.04 & -0.01 & 0.086 & 0.000 & 0.051 & 0.015 & -5.20 & -3.51 & 871.44 & -63.85 & -6382 & 0105 & -3.50 & -63.80 \\
\hline 55 & 106 & 0.10 & 0.00 & -0.04 & 0.00 & 0.107 & 0.000 & 0.053 & 0.006 & -4.40 & -2.57 & 881.94 & -66.28 & & & -2.57 & -66.24 \\
\hline 56 & 107 & 0.11 & 0.00 & -0.03 & 0.01 & 0.117 & 0.0 & 0.042 & -0.005 & -3 & -1 & 98 & -70 & & & - & \\
\hline 57 & 108 & & & -0.03 & 0.01 & & & 42 & -0 & & -1 & & -7 & & & & -72.27 \\
\hline 58 & 109 & & 00 & -0.02 & 0.01 & 0.128 & 0.000 & 0.031 & -0.007 & -1.87 & -0.32 & & -75.85 & -76.26 & 0.019 & -0.32 & -75.83 \\
\hline 59 & 110 & 1 & 0.00 & -0.01 & 0 & 0 . & 08 & 0. & 0. & -1 & 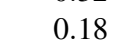 & & -7 & & & 0.17 & -7 \\
\hline 60 & 111 & 0.11 & 0.00 & 0.00 & 0.00 & 0.118 & 0.000 & 0.005 & 0.000 & -0.46 & 0.68 & 936.40 & -80.39 & -80.89 & 0.028 & 0.68 & -80.39 \\
\hline 61 & 112 & 0.11 & 0 & 0.00 & 0.00 & 118 & 000 & 0005 & 0.000 & 0.05 & 1.11 & 945.39 & -81.31 & -81.60 & 001 & 1.11 & -81.32 \\
\hline 62 & 113 & & & & -0 & & 0 & & & & & & & & & 6 & - \\
\hline 63 & 114 & & & -0.01 & & & & & -0 & & & & & & & 1.90 & - \\
\hline 64 & 115 & & & -0.01 & -0.01 & & & & & & & & & & & 89 & -86.50 \\
\hline 65 & 116 & -0.13 & 0.00 & -0.01 & -0.01 & -0.135 & 0.000 & 0.019 & 0.008 & 0.46 & 1.94 & 982.91 & -86.54 & -86.82 & 0.006 & 1.95 & -86.61 \\
\hline 66 & 117 & -0.14 & 00 & -0.01 & -0.01 & - & 0 & 0. & 0. & & 1.79 & 2 & -8 & & 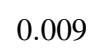 & 0 & $-\varepsilon$ \\
\hline 67 & 118 & & & & & & & & & & 1 & & & & & 73 & -88.15 \\
\hline 68 & 119 & -0.14 & & 0.00 & 0.01 & -0.146 & 0.000 & 0.008 & 0.009 & & 1.44 & & & & & 1.45 & -89.57 \\
\hline 69 & 120 & 014 & & 0.00 & .01 & - & 0.0 & & & & 2 & & & & & 1.33 & -88.76 \\
\hline 70 & 121 & -0.12 & 0.01 & 0.01 & & -0.125 & -0.013 & -0.006 & & & & & -89.75 & -89.60 & 0.002 & 0.79 & -89.91 \\
\hline 71 & 122 & -0.12 & 00 & 0.02 & 0.00 & -0.125 & 0.000 & -0.017 & 0.003 & -0.86 & 0.46 & & -88.61 & & & 0.46 & -88.79 \\
\hline 72 & 123 & 1 & & & & & & & & & & & & & & -0.18 & \\
\hline 73 & 124 & 0.10 & & & 1 & 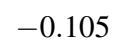 & 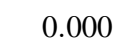 & 19 & -0 & -1 & & & & & & & $-\varepsilon$ \\
\hline 74 & 125 & -0.10 & & & & & & & & & & & & & & & -88.48 \\
\hline 75 & 126 & -0.10 & 0.00 & 0.03 & 0.01 & -0.104 & 0.000 & -0.031 & -0.006 & -3 & -2.16 & 1063.60 & -86.51 & -86.40 & 0.032 & -2.14 & -86.73 \\
\hline 76 & 127 & & & 0 & & & & & & & & & & & 0.005 & & -8 \\
\hline 77 & 128 & + & & & & & & & & & & & & & & & \\
\hline 78 & 129 & -0.03 & ( & 0. & 0 & & & & & & & & -84.67 & -84.63 & & -5.43 & -84.94 \\
\hline 79 & 130 & -0.03 & 0.00 & & & 0.0 & 0 & & & -7.54 & & 1091.79 & -82.42 & -82.29 & & -6.48 & -82.71 \\
\hline 80 & 131 & -0.02 & 0.00 & 0.00 & 0.00 & -0.021 & 0.000 & 0.000 & 0.000 & -9.17 & -7.96 & & -82.11 & -81.99 & 0.021 & -7.96 & -82.40 \\
\hline 81 & 132 & 0. & 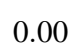 & 0.00 & 0.01 & 0.032 & 0.000 & 0.000 & 0.010 & -10.33 & -8.90 & 1104.86 & -79.34 & -79.67 & 0.014 & -8.90 & -79.63 \\
\hline 82 & 133 & -0.01 & 0.00 & & & & & & & & & & & & & & \\
\hline 83 & 134 & 000 & 0.00 & & 0. & & & & 0 & & & & & & & & \\
\hline 84 & 135 & -0.02 & & & 0. & & & & & & -8.31 & 11 & -69.82 & -69.71 & 0.103 & -8.31 & -70.10 \\
\hline 85 & 136 & 0.02 & 0.00 & -0.01 & 0.00 & 0.021 & 0.000 & 0.012 & 0.000 & -8.33 & -7.13 & 1121.96 & -64.16 & & & -7.12 & -64.43 \\
\hline
\end{tabular}




\begin{tabular}{|c|c|c|c|c|c|c|c|c|c|c|c|c|c|c|c|c|c|}
\hline \\
\hline \multicolumn{7}{|c|}{$\begin{array}{llllll}0.012 & -0.000 & -7.06 & -5.97 & 1125.88 & -60.01\end{array}$} & 0.000 & 0.012 & -0.000 & -7.06 & -5.97 & & & & & & \\
\hline 87 & 138 & -0.03 & 0.00 & -0.01 & 0.00 & -0.032 & 0.000 & 0.012 & -0.000 & -5.92 & -4.91 & 1128.03 & -54.09 & & & -4.91 & -54.33 \\
\hline 88 & 139 & 0.05 & 0.00 & -0.03 & -0.02 & 0.054 & 0.000 & 0.037 & 0.022 & -5.15 & -3.94 & 1131.77 & -49.76 & & & -3.84 & -49.88 \\
\hline 89 & 140 & 0.06 & 0.00 & -0.03 & -0.02 & 0.065 & 0.000 & 0.038 & 0.023 & -4.19 & -3.03 & 1133.72 & -43.64 & & & -2.93 & -43.74 \\
\hline 90 & 141 & 0.09 & 0.00 & -0.04 & -0.01 & 0.097 & 0.000 & 0.052 & 0.015 & -3.61 & -2.13 & 1137.17 & -39.02 & & & -2.03 & -39.09 \\
\hline 91 & 142 & 0.10 & 0.00 & -0.04 & 0.00 & 0.107 & 0.000 & 0.053 & 0.006 & -2.86 & -1.40 & 1138.96 & -32.74 & & & -1.31 & -32.80 \\
\hline 92 & 143 & 0.11 & 0.00 & -0.04 & 0.00 & 0.118 & 0.000 & 0.054 & 0.006 & -2.23 & -0.72 & 1142.29 & -27.99 & & & -0.62 & -28.02 \\
\hline 93 & 144 & 0.13 & 0.00 & -0.04 & 0.00 & 0.140 & 0.000 & 0.056 & 0.007 & -1.74 & -0.00 & 1143.77 & -21.40 & & & 0.09 & -21.39 \\
\hline 94 & 145 & 0.15 & 0.00 & -0.05 & 0.00 & 0.161 & 0.000 & 0.071 & 0.011 & -1.71 & 0.52 & 1146.92 & -16.48 & & & 0.69 & -16.35 \\
\hline 95 & 146 & 0.20 & 0.00 & -0.05 & 0.01 & 0.216 & 0.000 & 0.080 & 0.005 & -2.30 & 0.86 & 1148.48 & -9.96 & & & 1.02 & -9.79 \\
\hline 96 & 147 & 0.20 & 0.00 & -0.05 & 0.01 & 0.216 & 0.000 & 0.080 & 0.005 & -1.97 & 1.14 & 1151.55 & -4.97 & & & 1.32 & -4.72 \\
\hline 97 & 148 & 0.21 & 0.00 & -0.04 & 0.02 & 0.226 & 0.000 & 0.070 & -0.007 & -1.92 & 0.95 & 1153.32 & 1.34 & & & 1.11 & 1.60 \\
\hline 98 & 149 & 0.21 & 0.00 & -0.04 & 0.02 & 0.226 & 0.000 & 0.070 & -0.007 & -1.63 & 1.19 & 1156.13 & 6.59 & & & 1.36 & 6.93 \\
\hline 99 & 150 & 0.23 & 0.00 & -0.02 & 0.02 & 0.249 & 0.000 & 0.049 & -0.012 & -1.67 & 1.24 & 1157.37 & 13.43 & & & 1.34 & 13.76 \\
\hline 100 & 151 & 0.21 & 0.00 & -0.02 & 0.02 & 0.227 & 0.000 & 0.045 & -0.013 & -1.12 & 1.39 & 1159.98 & 18.89 & & & 1.50 & 19.30 \\
\hline 101 & 152 & 0.23 & 0.00 & -0.01 & 0.02 & 0.250 & 0.000 & 0.037 & -0.015 & -1.52 & 1.31 & 1161.07 & 25.87 & & & 1.39 & 26.33 \\
\hline 102 & 153 & 0.23 & 0.00 & 0.00 & 0.02 & 0.250 & 0.000 & 0.025 & -0.018 & -1.25 & 1.47 & 1163.38 & 31.63 & & & 1.55 & 32.17 \\
\hline 103 & 154 & 0.24 & 0.00 & 0.01 & 0.02 & 0.262 & 0.000 & 0.015 & -0.020 & -1.57 & 1.28 & 1164.31 & 38.77 & & & 1.36 & 39.40 \\
\hline 104 & 155 & 0.23 & 0.00 & 0.01 & 0.01 & 0.250 & 0.000 & 0.012 & -0.011 & -1.14 & 1.49 & 1166.29 & 44.86 & & & 1.52 & 45.52 \\
\hline 105 & 156 & 0.24 & 0.00 & 0.03 & 0.01 & 0.263 & 0.000 & -0.010 & -0.016 & -1.53 & 1.28 & 1166.98 & 52.24 & & & 1.34 & 53.02 \\
\hline 106 & 157 & 0.23 & 0.00 & 0.03 & 0.00 & 0.252 & 0.000 & -0.014 & -0.006 & -1.10 & 1.45 & 1168.74 & 58.55 & & & 1.48 & 59.41 \\
\hline 107 & 158 & 0.23 & 0.00 & 0.04 & 0.00 & 0.252 & 0.000 & -0.026 & -0.009 & -1.33 & 1.28 & 1169.14 & 66.23 & & & 1.33 & 67.21 \\
\hline 108 & 159 & 0.21 & 0.00 & 0.04 & -0.01 & 0.229 & 0.000 & -0.031 & 0.001 & -0.98 & 1.28 & 1170.81 & 72.63 & & & 1.36 & 73.75 \\
\hline 109 & 160 & 0.21 & 0.00 & 0.04 & -0.01 & 0.229 & 0.000 & -0.031 & 0.001 & -1.22 & 1.03 & 1171.04 & 80.47 & & & 1.10 & 81.69 \\
\hline 110 & 161 & 0.18 & 0.00 & 0.04 & -0.01 & 0.196 & 0.000 & -0.035 & 0.002 & -0.80 & 1.00 & 1172.50 & 8708 & & & 1.09 & 88.45 \\
\hline 111 & 162 & 0.18 & 0.00 & 0.05 & -0.02 & 0.196 & 0.000 & -0.048 & 0.010 & -1.34 & 0.52 & 1172.73 & 94.93 & & & 0.71 & 96.52 \\
\hline 112 & 163 & 0.15 & 0.00 & 0.03 & -0.01 & 0.162 & 0.000 & -0.027 & 0.005 & -0.99 & 0.64 & 1173.80 & 101.93 & & & 0.70 & 103.53 \\
\hline 113 & 164 & 0.15 & 0.00 & 0.04 & -0.01 & 0.163 & 0.000 & -0.039 & 0.003 & -1.62 & 0.09 & 1173.86 & 109.93 & & & 0.19 & 111.72 \\
\hline 114 & 165 & -0.14 & 0.00 & 0.02 & -0.01 & -0.146 & 0.000 & -0.015 & 0.012 & -1.79 & -0.46 & 1175.37 & 116.50 & & & -0.40 & 118.39 \\
\hline 115 & 166 & 0.11 & 0.00 & 0.02 & 0.00 & 0.118 & 0.000 & -0.019 & -0.002 & -2.23 & -1.04 & 1175.26 & 124.68 & & & -1.02 & 126.68 \\
\hline 116 & 167 & 0.11 & 0.00 & 0.03 & -0.01 & 0.119 & 0.000 & -0.032 & 0.006 & -2.85 & -1.59 & 1176.53 & 131.48 & & & -1.51 & 133.70 \\
\hline 117 & 168 & 0.10 & 0.00 & 0.04 & -0.01 & 0.108 & 0.000 & -0.044 & 0.005 & -3.75 & -2.47 & 1176.50 & 139.58 & & & -2.33 & 142.02 \\
\hline 118 & 169 & 0.10 & 0.00 & 0.04 & -0.02 & 0.108 & 0.000 & -0.045 & 0.015 & -4.31 & -3.05 & 1177.59 & 146.56 & & & -2.83 & 149.25 \\
\hline 119 & 170 & 0.10 & 0.00 & 0.04 & -0.02 & 0.108 & 0.000 & -0.045 & 0.015 & -5.37 & -4.30 & 1177.73 & 154.49 & & & -4.08 & 157.36 \\
\hline 120 & 171 & 0.08 & 0.00 & 0.02 & -0.01 & 0.086 & 0.000 & -0.022 & 0.008 & -5.46 & -4.45 & 1178.19 & 162.11 & & & -4.40 & 165.00 \\
\hline 121 & 172 & -0.06 & 0.00 & 0.01 & 0.00 & -0.063 & 0.000 & -0.010 & 0.001 & -6.49 & -5.70 & 1178.12 & 170.25 & & & -5.70 & 173.27 \\
\hline \multicolumn{18}{|c|}{$Z=52(\mathrm{Te})$} \\
\hline 47 & 99 & -0.01 & 0.00 & 0.00 & 0.00 & -0.011 & 0.000 & 0.000 & 0.000 & -4.73 & -3.71 & 768.49 & -10.12 & & & -3.71 & -10.11 \\
\hline 48 & 100 & 0.00 & 0.00 & 0.00 & 0.00 & 0.000 & 0.000 & 0.000 & 0.000 & -6.13 & -4.98 & 787.40 & -20.95 & & & -4.98 & -20.93 \\
\hline 49 & 101 & 0.02 & 0.00 & 0.01 & 0.00 & 0.021 & 0.000 & -0.012 & -0.000 & -6.92 & -5.66 & 802.61 & -28.09 & & & -5.66 & -28.06 \\
\hline 50 & 102 & 0.00 & 0.00 & 0.00 & 0.00 & 0.000 & 0.000 & 0.000 & 0.000 & -8.09 & -6.75 & 820.43 & -37.84 & & & -6.75 & -37.80 \\
\hline 51 & 103 & -0.02 & 0.00 & 0.00 & 0.00 & -0.021 & 0.000 & 0.000 & 0.000 & -6.99 & -5.82 & 833.20 & -42.54 & & & -5.83 & -42.49 \\
\hline 52 & 104 & 0.00 & 0.00 & 0.00 & 0.00 & 0.000 & 0.000 & 0.000 & 0.000 & -5.87 & -4.85 & 848.09 & -49.36 & & & -4.85 & -49.31 \\
\hline 53 & 105 & 0.08 & 0.00 & -0.04 & -0.01 & 0.086 & 0.000 & 0.051 & 0.015 & -5.16 & -3.48 & 859.04 & -52.23 & & & -3.47 & -52.17 \\
\hline 54 & 106 & 0.11 & 0.00 & -0.05 & -0.01 & 0.119 & 0.000 & 0.066 & 0.018 & -4.54 & -2.37 & 872.41 & -57.53 & -58.21 & 0.132 & -2.35 & -57.46 \\
\hline 55 & 107 & 0.12 & 0.00 & -0.05 & 0.01 & 0.128 & 0.000 & 0.068 & -0.002 & -3.78 & -1.58 & 883.19 & -60.24 & & & -1.57 & -60.18 \\
\hline 56 & 108 & 0.13 & 0.00 & -0.04 & 0.01 & 0.139 & 0.000 & 0.056 & -0.003 & -2.84 & -0.77 & 896.11 & -65.09 & -65.72 & 0.104 & -0.76 & -65.03 \\
\hline 57 & 109 & 0.13 & 0.00 & -0.03 & 0.01 & 0.139 & 0.000 & 0.044 & -0.005 & -1.91 & -0.06 & 906.26 & -67.17 & -67 & 0.063 & -0.06 & -67.13 \\
\hline 58 & 110 & 0.14 & 0.00 & -0.03 & 0.01 & 0.150 & 0.000 & 0.045 & -0.004 & -1.36 & 0.52 & 918.68 & -71.52 & -72.28 & 0.053 & 0.53 & -71.48 \\
\hline 59 & 111 & 0.16 & 0.00 & -0.03 & 0.01 & 0.172 & 0.000 & 0.048 & -0.003 & -0.94 & 1.14 & 928.25 & -73.02 & -73.49 & 0.071 & 1.14 & -72.99 \\
\hline 60 & 112 & 0.17 & 0.00 & -0.04 & 0.01 & 0.183 & 0.000 & 0.062 & -0.000 & -0.71 & 1.58 & 940.13 & -76.82 & -77.30 & 0.170 & 1.60 & -76.79 \\
\hline 61 & 113 & 0.18 & 0.00 & -0.04 & 0.00 & 0.194 & 0.000 & 0.063 & 0.011 & -0.50 & 1.85 & 949.39 & -78.02 & -78.35 & 0.028 & 1.87 & -78.00 \\
\hline 62 & 114 & 0.18 & 0.00 & -0.03 & 0.00 & 0.194 & 0.000 & 0.051 & 0.008 & -0.01 & 2.03 & 960.87 & -81.42 & -81.89 & 0.028 & 2.05 & -81.42 \\
\hline 63 & 115 & 0.19 & 0.00 & -0.03 & 0.00 & 0.205 & 0.000 & 0.052 & 0.009 & 0.03 & 2.27 & 969.55 & -82.03 & -82.06 & 0.028 & 2.29 & -82.05 \\
\hline 64 & 116 & 0.20 & 0.00 & -0.02 & 0.00 & 0.216 & 0.000 & 0.042 & 0.007 & 0.19 & 2.43 & 980.42 & -84.83 & -85.27 & 0.028 & 2.45 & -84.86 \\
\hline 65 & 117 & 0.20 & 0.00 & -0.01 & -0.01 & 0.216 & 0.000 & 0.029 & 0.014 & 0.32 & 2.50 & 988.68 & -85.01 & -85.10 & 0.013 & 2.52 & -85.07 \\
\hline 66 & 118 & -0.16 & 0.00 & -0.01 & -0.02 & -0.165 & 0.000 & 0.023 & 0.016 & 0.70 & 2.56 & 999.04 & -87.31 & -87.72 & 0.015 & 2.59 & -87.38 \\
\hline
\end{tabular}




\begin{tabular}{|c|c|c|c|c|c|c|c|c|c|c|c|c|c|c|c|c|c|}
\hline \multicolumn{18}{|c|}{$Z=52(\mathrm{Te})$} \\
\hline & 119 & -0.17 & 0.00 & 0.00 & -0.02 & -0.176 & 0.000 & 0.012 & 0.018 & 0.44 & 2.30 & 1007.07 & -87.27 & -87.18 & 0.008 & 2.32 & -87.36 \\
\hline 68 & 120 & -0.17 & 0.00 & 0.00 & -0.02 & -0.176 & 0.000 & 0.012 & 0.018 & 0.20 & 2.18 & 1017.05 & -89.18 & -89.40 & 0.010 & 2.20 & -89.28 \\
\hline 69 & 121 & -0.17 & 0.00 & 0.00 & -0.02 & -0.176 & 0.000 & 0.012 & 0.018 & 0.05 & 1.85 & 1024.60 & -88.65 & -88.55 & 0.026 & 1.88 & -88.78 \\
\hline 70 & 122 & -0.16 & 0.00 & 0.01 & -0.01 & -0.166 & 0.000 & -0.001 & 0.010 & -0.11 & 1.65 & 1034.11 & -90.10 & -90.31 & 0.001 & 1.67 & -90.24 \\
\hline 71 & 123 & -0.14 & 0.00 & 0.01 & -0.01 & -0.146 & 0.000 & -0.003 & 0.011 & -0.14 & 1.36 & 1041.12 & -89.03 & -89.17 & 0.001 & 1.37 & -89.20 \\
\hline 72 & 124 & -0.12 & 0.01 & 0.01 & 0.00 & -0.125 & -0.013 & -0.006 & 0.001 & -0.54 & 0.74 & 1050.51 & -90.35 & -90.53 & 0.001 & 0.75 & -90.55 \\
\hline 73 & 125 & -0.12 & 0.00 & 0.02 & 0.00 & -0.125 & 0.000 & -0.017 & 0.003 & -1.05 & 0.26 & 1057.21 & -88.98 & -89.02 & 0.001 & 0.27 & -89.19 \\
\hline 74 & 126 & -0.10 & 0.00 & 0.02 & 0.00 & -0.105 & 0.000 & -0.019 & 0.002 & -1.74 & -0.57 & 1066.31 & -90.01 & -90.07 & 0.001 & -0.56 & -90.24 \\
\hline 75 & 127 & -0.10 & 0.00 & 0.02 & 0.01 & -0.105 & 0.000 & -0.019 & -0.007 & -2.43 & -1.21 & 1072.70 & -88.33 & -88.28 & 0.002 & -1.20 & -88.57 \\
\hline 76 & 128 & -0.09 & 0.00 & 0.02 & 0.01 & -0.094 & 0.000 & -0.020 & -0.007 & -3.37 & -2.17 & 1081.45 & -89.01 & -88.99 & 0.002 & -2.15 & -89.26 \\
\hline 77 & 129 & -0.09 & 0.00 & 0.02 & 0.01 & -0.094 & 0.000 & -0.020 & -0.007 & -4.24 & -3.01 & 1087.58 & -87.06 & -87.00 & 0.002 & -2.99 & -87.33 \\
\hline 78 & 130 & 0.00 & 0.00 & 0.00 & 0.00 & 0.000 & 0.000 & 0.000 & 0.000 & -5.39 & -4.50 & 1096.40 & -87.81 & -87.35 & 0.002 & -4.50 & -88.11 \\
\hline 79 & 131 & 0.01 & 0.00 & 0.00 & 0.01 & 0.011 & 0.000 & 0.000 & -0.010 & -6.45 & -5.44 & 1102.20 & -85.54 & -85.21 & 0.002 & -5.44 & -85.84 \\
\hline 80 & 132 & 0.00 & 0.00 & 0.00 & 0.00 & 0.000 & 0.000 & 0.000 & 0.000 & -8.19 & -7.04 & 1110.68 & -85.95 & -85.18 & 0.007 & -7.04 & -86.26 \\
\hline 81 & 133 & 0.02 & 0.00 & 0.01 & 0.00 & 0.021 & 0.000 & -0.012 & -0.000 & -9.26 & -7.95 & 1116.03 & -83.22 & -82.94 & 0.024 & -7.94 & -83.54 \\
\hline 82 & 134 & 0.00 & 0.00 & 0.00 & 0.00 & 0.000 & 0.000 & 0.000 & 0.000 & -10.64 & -9.23 & 1123.77 & -82.90 & -82.56 & 0.011 & -9.23 & -83.22 \\
\hline 83 & 135 & -0.01 & 0.00 & 0.00 & 0.00 & -0.011 & 0.000 & 0.000 & 0.000 & -9.71 & -8.42 & 1127.00 & -78.06 & -77.83 & 0.090 & -8.42 & -78.38 \\
\hline 84 & 136 & 0.00 & 0.00 & 0.00 & 0.00 & 0.000 & 0.000 & 0.000 & 0.000 & -8.61 & -7.40 & .02 & -75.00 & -7 & 0.045 & -7.40 & -75.32 \\
\hline 85 & 137 & 0.01 & 0.00 & 0.00 & 0.00 & 0.011 & 0.000 & 0.000 & 0.000 & -7.26 & -6.18 & 4.46 & -69.37 & -69.56 & 0.122 & -6.18 & -69.68 \\
\hline 86 & 138 & 0.00 & 0.00 & 0.00 & 0.00 & 0.000 & 0.000 & 0.000 & 0.000 & -5.96 & -5.00 & 1138.92 & -65.76 & & & -5.00 & -66.07 \\
\hline 87 & 139 & 0.08 & 0.00 & -0.05 & -0.02 & 0.087 & 0.000 & 0.064 & 0.026 & -5.82 & -3.91 & 1141.12 & -59.89 & & & -3.74 & -60.02 \\
\hline 88 & 140 & 0.09 & 0.00 & -0.05 & -0.01 & 0.097 & 0.000 & 0.064 & 0.017 & -4.80 & -2.95 & 1145.43 & -56.13 & & & -2.81 & -56.27 \\
\hline 89 & 141 & 0.11 & 0.00 & -0.05 & 0.00 & 0.118 & 0.000 & 0.066 & 0.008 & -4.13 & -2.15 & 1147.56 & -50.19 & & & -2.03 & -50.33 \\
\hline 90 & 142 & 0.11 & 0.00 & -0.05 & 0.00 & 0.118 & 0.000 & 0.066 & 0.008 & -3.37 & -1.49 & 1151.81 & -46.36 & & & -1.35 & -46.47 \\
\hline 91 & 143 & 0.14 & 0.00 & -0.05 & 0.01 & 0.150 & 0000 & 0.070 & -0.000 & -3.03 & -0.79 & 1153.70 & -40.19 & & & -0.65 & -40 \\
\hline 92 & 144 & 0.15 & 0.00 & -0.06 & 0.01 & 0.161 & 0.000 & 0.084 & 0.003 & -2.82 & -0.24 & 1157.70 & -36.12 & & & -0.03 & -36.10 \\
\hline 93 & 145 & 0.18 & 0.00 & -0.07 & 0.01 & 0.194 & 0.000 & 0.102 & 0.009 & -3.32 & 0.12 & 1159.61 & -29.95 & & & 0.40 & -29.83 \\
\hline 94 & 146 & 0.18 & 0.00 & -0.06 & 0.01 & 0.194 & 0.000 & 0.089 & 0.006 & -2.52 & 0.56 & 3.38 & -25.65 & & & 0.80 & -25.53 \\
\hline 95 & 147 & 0.19 & 0.00 & -0.06 & 0.01 & 0.205 & 0.000 & 0.091 & 0.007 & -2.58 & 0.49 & 1165.39 & -19.59 & & & 0.72 & -19.44 \\
\hline 96 & 148 & 0.20 & 0.00 & -0.05 & 0.02 & 0.215 & 0.000 & 0.080 & -0.005 & -2.09 & 0.80 & 1168.97 & -15.10 & & & 1.01 & -14.92 \\
\hline 97 & 149 & 0.21 & 0.00 & -0.05 & 0.02 & 0.226 & 0.000 & 0.082 & -0.004 & -2.26 & 0.84 & 1170.57 & -8.62 & & & 1.05 & -8.41 \\
\hline 98 & 150 & 0.21 & 0.00 & -0.04 & 0.02 & 0.226 & 0.000 & 0.070 & -0.007 & -1.73 & 1.12 & .86 & -3.84 & & & 1.30 & -3.61 \\
\hline 99 & 151 & 0.21 & 0.00 & -0.03 & 0.02 & 0.227 & 0.000 & 0.057 & -0.010 & -1.59 & 1.11 & 1175.21 & 2.88 & & & 1.24 & 3.12 \\
\hline 100 & 152 & 0.21 & 0.00 & -0.03 & 0.02 & 0.227 & 0.000 & 0.057 & -0.010 & -1.33 & 1.34 & 1178.26 & 7.90 & & & 1.48 & 8.22 \\
\hline 101 & 153 & 0.23 & 0.00 & -0.01 & 0.02 & 0.250 & 0.000 & 0.037 & -0.015 & -1.56 & 1.30 & 1179.35 & 14.87 & & & 1.39 & 15.22 \\
\hline 102 & 154 & 0.24 & 0.00 & 0.00 & 0.03 & 0.261 & 0.000 & 0.028 & -0.027 & -1.58 & 1.35 & 1182.29 & 20.02 & & & 1.55 & 20.53 \\
\hline 103 & 155 & 0.24 & 0.00 & 0.01 & 0.02 & 0.262 & 0.000 & 0.015 & -0.020 & -1.64 & 1.25 & 3.17 & 27.20 & & & 1.34 & 27.69 \\
\hline 104 & 156 & 0.24 & 0.00 & 0.02 & 0.02 & 0.263 & 0.000 & 0.003 & -0.023 & -1.46 & 1.38 & .73 & 32.71 & & & 1.51 & 33.32 \\
\hline 105 & 157 & 0.23 & 0.00 & 0.02 & 0.01 & 0.251 & 0.000 & -0.001 & -0.013 & -1.35 & 1.29 & 1186.35 & 40.17 & & & 1.34 & 40.78 \\
\hline 106 & 158 & 0.23 & 0.00 & 0.03 & 0.01 & 0.252 & 0.000 & -0.013 & -0.016 & -1.18 & 1.41 & 1188.66 & 45.93 & & & 1.49 & 46.67 \\
\hline 107 & 159 & 0.23 & 0.00 & 0.04 & 0.00 & 0.252 & 0.000 & -0.026 & -0.009 & -1.34 & 1.31 & 1189.02 & 53.63 & & & 1.38 & 54.46 \\
\hline 108 & 160 & 0.21 & 0.00 & 0.03 & 0.00 & 0.229 & 0.000 & -0.018 & -0.006 & -0.85 & 1.36 & 1191.13 & 59.60 & & & 1.40 & 60.50 \\
\hline 109 & 161 & 0.21 & 0.00 & 0.04 & 0.00 & 0.230 & 0.000 & -0.030 & -0.009 & -1.16 & 1.12 & 1191.39 & 67.41 & & & 1.20 & 68.45 \\
\hline 110 & 162 & 0.20 & 0.00 & 0.04 & -0.01 & 0.218 & 0.000 & -0.032 & 0.002 & -0.90 & 1.17 & 1193.25 & 73.62 & & & 1.26 & 74.79 \\
\hline 111 & 163 & 0.18 & 0.00 & 0.04 & -0.01 & 0.196 & 0.000 & -0.035 & 0.002 & -0.99 & 0.86 & 1193.34 & 81.60 & & & 0.95 & 82.89 \\
\hline 12 & 164 & 0.17 & 0.00 & 0.04 & -0.01 & 0.185 & 0.000 & -0.037 & 0.003 & -0.99 & 0.94 & 1194.92 & 88.10 & & & 1.04 & 89.52 \\
\hline 113 & 165 & 0.15 & 0.00 & 0.03 & -0.01 & 0.162 & 0.000 & -0.027 & 0.005 & -1.20 & 0.49 & 1194.92 & 96.16 & & & 0.55 & 97.67 \\
\hline 114 & 166 & 0.15 & 0.00 & 0.04 & -0.01 & 0.163 & 0.000 & -0.039 & 0.003 & -1.65 & -0.00 & 1196.84 & 102.31 & & & 0.11 & 104.01 \\
\hline 115 & 167 & -0.15 & 0.00 & 0.03 & 0.00 & -0.156 & 0.000 & -0.025 & 0.005 & -2.20 & -0.65 & 1196.82 & 110.41 & & & -0.60 & 112.19 \\
\hline 116 & 168 & 0.11 & 0.00 & 0.03 & -0.01 & 0.119 & 0.000 & -0.032 & 0.006 & -2.40 & -1.13 & 1198.49 & 116.81 & & & -1.04 & 118.77 \\
\hline 17 & 169 & 0.11 & 0.00 & 0.04 & -0.01 & 0.119 & 0.000 & -0.043 & 0.005 & -3.37 & -1.98 & 1198.46 & 124.91 & & & -1.85 & 127.07 \\
\hline 118 & 170 & 0.11 & 0.00 & 0.04 & -0.02 & 0.119 & 0.000 & -0.044 & 0.015 & -3.87 & -2.51 & 1199.96 & 131.48 & & & -2.30 & 133.88 \\
\hline 119 & 171 & 0.10 & 0.00 & 0.04 & -0.02 & 0.108 & 0.000 & -0.045 & 0.015 & -4.69 & -3.37 & 1199.72 & 139.79 & & & -3.15 & 142.36 \\
\hline 120 & 172 & -0.12 & 0.00 & 0.01 & 0.00 & -0.125 & 0.000 & -0.006 & 0.001 & -5.02 & -3.82 & 1200.93 & 146.65 & & & -3.82 & 149.18 \\
\hline 121 & 173 & -0.07 & 0.00 & 0.01 & 0.00 & -0.073 & 0.000 & -0.010 & 0.001 & -5.81 & -4.99 & 1200.81 & 154.85 & & & -4.98 & 157.56 \\
\hline 122 & 174 & -0.06 & 0.00 & 0.02 & 0.01 & -0.063 & 0.000 & -0.022 & -0.008 & -6.72 & -5.87 & 1202.23 & 161.50 & & & -5.81 & 164.45 \\
\hline 123 & 175 & -0.06 & 0.00 & 0.02 & 0.01 & -0.063 & 0.000 & -0.022 & -0.008 & -8.08 & -7.11 & 1201.99 & 169.81 & & & -7.05 & 172.95 \\
\hline
\end{tabular}


$Z=52(\mathrm{Te})$

$124 \quad 176-0.04$

\section{$Z=\mathbf{5 3}(\mathbf{I})$}

$\begin{array}{lllll}48 & 101 & 0.01 & 0.00 & 0.00\end{array}$

$\begin{array}{llllllllllllll}49 & 102 & 0.03 & 0.00 & 0.01 & 0.00 & 0.032 & 0.000 & -0.012 & -0.000 & -5.38 & -4.31 & 797.77 & -15.96\end{array}$

$\begin{array}{llllllllllllll}50 & 103 & 0.00 & 0.00 & 0.00 & 0.00 & 0.000 & 0.000 & 0.000 & 0.000 & -6.45 & -5.33 & 815.65 & -25.77\end{array}$

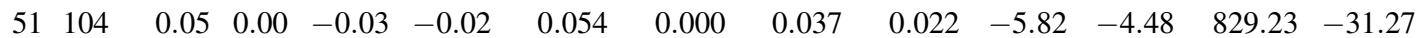

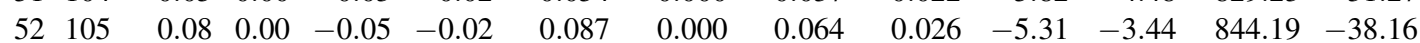

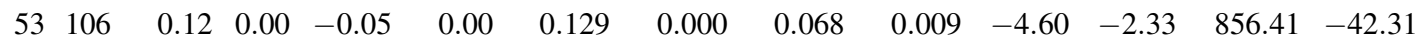

54107

$\begin{array}{ll}856.41 & -42.31 \\ 870.57 & -48.40\end{array}$

55108

$\begin{array}{lll}0.14 & 0.05 & -0.05\end{array}$

0.02

$0.129-0.041$

$0.068-0.001-3.94-1.59$

$882.12-51.88$

$\begin{array}{llllll}56 & 109 & 0.15 & 0.06 & -0.04\end{array}$

$\begin{array}{llll}0.02 & 0.162 & -0.082\end{array}$

$\begin{array}{llll}0.071 & -0.009 & -3.72 & -0.86\end{array}$

$\begin{array}{llll}0.060 & -0.009 & -3.11 & -0.18\end{array}$

$\begin{array}{llll}895.29 & -56.98 & -57.61\end{array}$

$\begin{array}{lll}0.02 & 0.173 & -0.082\end{array}$

$\begin{array}{llll}0.049 & -0.011 & -2.37\end{array}$

$\begin{array}{lll}0.52 & 906.16 & -59.78\end{array}$

$58 \quad 111$

$\begin{array}{lll}0.16 & 0.06 & -0.03\end{array}$

0.02

$0.173-0.082$

$\begin{array}{llllll}59 & 112 & 0.17 & 0.04 & -0.03\end{array}$

$\begin{array}{llll}0.02 & 0.183 & -0.055\end{array}$

$\begin{array}{llll}0.049 & -0.011 & -1.79\end{array}$

1.01

$60 \quad 113$

$\begin{array}{llll}0.19 & 0.02 & -0.04\end{array}$

0.02

$\begin{array}{lllll}61 & 114 & 0.20 & 0.02 & -0.04 \\ 62 & 115 & 0.20 & 0.00 & -0.03\end{array}$

$\begin{array}{llll}0.01 & 0.216 & -0.028\end{array}$

$\begin{array}{llll}0.051 & -0.011 & -1.03\end{array}$

1.22

$918.79-64.34$

0.01

$\begin{array}{lll}0.066 & -0.009 & -0.79\end{array}$

1.64
2.00

$929.46-66.94$

$63 \quad 116$

$\begin{array}{lll}0.21 & 0.00 & -0.02\end{array}$

$\begin{array}{lll}0.01 & 0.227 & 0.000\end{array}$

$\begin{array}{lll}0.067 & 0.002 & -0.63\end{array}$

2.26

$\begin{array}{ll}941.48 & -70.89 \\ 951.34 & -72.67\end{array}$

$\begin{array}{lll}64 & 117\end{array}$

$\begin{array}{lll}0.21 & 0.00 & -0.01\end{array}$

0.00

0.000

$0.044-0.003$

0.02

2.42

$962.85 \quad-76.11 \quad-76.34$

0.00

0.239

0.000

$\begin{array}{ll}0.021 & 0.002\end{array}$

0.24

2.54

$\begin{array}{llll}972.28 & -77.48 & -77.49\end{array}$

$66 \quad 119$

$\begin{array}{ll}0.22 & 0.00\end{array}$

0.01

$\begin{array}{ll}0.00 & 0.239\end{array}$

0.000

$0.009-0.001$

0.16
0.23

$\begin{array}{llll}2.60 & 992.25 & -81.29 & -80.97\end{array}$

$\begin{array}{lllll}2.61 & 1002.77 & -83.75 & -83.77\end{array}$

$0.009-0.001$

0.10

$\begin{array}{lllll}2.61 & 1011.19 & -84.10 & -83.79\end{array}$

$\begin{array}{llll}68 & 121 & -0.18 & 0.00\end{array}$

$\begin{array}{lll}0.00 & -0.02 & -0.186\end{array}$

0.000

$\begin{array}{ll}0.014 & 0.018\end{array}$

0.48

$\begin{array}{lllll}2.44 & 1021.32 & -86.15 & -86.29\end{array}$

0.000

0.016

0.027

0.11

$\begin{array}{lllll}2.18 & 1029.46 & -86.22 & -86.08\end{array}$

$\begin{array}{llll}70 & 123 & -0.18 & 0.00\end{array}$

$\begin{array}{llll}0.01 & -0.02 & -0.187\end{array}$

0.000

0.012

$0.001-0.03$

$\begin{array}{lllll}1.94 & 1039.10 & -87.79 & -87.94\end{array}$

$\begin{array}{llll}71 & 124 & 0.17 & 0.00\end{array}$

$\begin{array}{lll}0.00 & 0.00 & 0.183\end{array}$

$\begin{array}{ll}0.000 & 0.012 \\ 0.000 & -0.001\end{array}$

$0.010-0.34$

$\begin{array}{lllll}1.68 & 1046.70 & -87.33 & -87.36\end{array}$

$\begin{array}{rrrr}72 & 125 & -0.16 & 0.00 \\ 73 & 126 & 0.15 & 0.00\end{array}$

$\begin{array}{lll}0.01 & -0.01 & -0.166\end{array}$

$\begin{array}{lllll}0.000 & 0.009 & 0.001 & -0.76\end{array}$

$\begin{array}{lllll}1.26 & 1056.00 & -88.55 & -88.84\end{array}$

$\begin{array}{llll}74 & 127 & -0.12 & 0.00\end{array}$

0.02

$0.00 \quad 0.162$

$\begin{array}{llll}0.000 & -0.018 & -0.007 & -1.11\end{array}$

$\begin{array}{lllll}1.14 & 1062.97 & -87.45 & -87.91\end{array}$

$\begin{array}{llll}75 & 128 & -0.12 & 0.00\end{array}$

$\begin{array}{lll}0.02 & 0.01 & -0.125\end{array}$

$\begin{array}{llll}76 & 129 & -0.11 & 0.00\end{array}$

$\begin{array}{lll}0.02 & 0.01 & -0.115\end{array}$

$\begin{array}{llllllll}0.000 & -0.018 & -0.007 & -1.78 & -0.17 & 1079.01 & -87.35 & -87.74\end{array}$

$\begin{array}{lllll}77 & 130 & -0.12 & 0.00\end{array}$

$\begin{array}{llll}0.03 & 0.02 & -0.125\end{array}$

$\begin{array}{llllllll}0.000 & -0.019 & -0.007 & -2.56 & -1.24 & 1087.96 & -88.22 & -88.50\end{array}$

$\begin{array}{lllll}78 & 131 & 0.03 & 0.00 & 0.00\end{array}$

$0.01 \quad 0.032$

0.000

7913

$\begin{array}{ll}0.03 & 0.00\end{array}$

0.00

$0.01 \quad 0.032$

0.000

$$
-0.02
$$

$0.029-0.0$

$\begin{array}{lllllll}0.015 & -3.64 & -2.05 & 1094.67 & -86.87 & -86.93\end{array}$

$80 \quad 133$

$0.02 \quad 0.00$

0.00

$0.01 \quad 0.021$

0.000

81134

$\begin{array}{lll}0.03 & 0.00 & 0.01\end{array}$

$0.00 \quad 0.032$

0.000

$0.000-0.1$

$\begin{array}{lllllll}0.010 & -4.04 & -3.29 & 1103.32 & -87.44 & -87.44 \\ -0.010 & -5.21 & -4.37 & 1109.86 & -85.91 & -85.70\end{array}$

$\begin{array}{lllllll}.010 & -6.81 & -5.78 & 1118.24 & -86.22 & -85.89\end{array}$

82135

$\begin{array}{lll}0.00 & 0.00 & 0.00\end{array}$

0.00

0.000

0.000

$-0.012-0$.

$\begin{array}{lllllll}-0.000 & -7.95 & -6.73 & 1124.22 & -84.13 & -84.07\end{array}$

83136

$\begin{array}{lll}0.03 & 0.00 & -0.01\end{array}$

$0.00 \quad 0.032$

0.000

0.012

$0.000-9.27$

$85 \quad 138$

$\begin{array}{lll}0.01 & 0.00 & 0.00\end{array}$

$0.00 \quad 0.011$

0.000

0.000

$\begin{array}{lllllll}0.000 & -8.37 & -7.12 & 1135.76 & -79.53 & -79.50\end{array}$

86139

$\begin{array}{lll}0.09 & 0.00 & -0.05\end{array}$

0.01

0.032

0.000

0.001

$\begin{array}{lllllll}0.000 & -7.22 & -6.13 & 1140.89 & -76.58 & -76.50\end{array}$

$87 \quad 140$

$\begin{array}{llll}0.10 & 0.00 & -0.05\end{array}$

0.00

0.097

0.000

0.064

8814

$\begin{array}{lll}0.11 & 0.00 & -0.05\end{array}$

0.00

0.107

0.065

$\begin{array}{lllllll}0.017 & -5.71 & -3.70 & 1148.42 & -67.97 & -68.84\end{array}$

$89 \quad 142$

$\begin{array}{llll}0.13 & 0.07 & -0.05\end{array}$

0.01

$\begin{array}{ll}0.118 & 0.000\end{array}$

0.066

$\begin{array}{llllll}0.007 & -4.98 & -2.95 & 1151.51 & -62.99\end{array}$

$90 \quad 143$

$\begin{array}{lll}0.14 & 0.06 & -0.05\end{array}$

0.01

$0.141-0.097$

0.070

$\begin{array}{llllll}0.008 & -4.19 & -2.12 & 1156.02 & -59.43\end{array}$

$\begin{array}{llllll}91 & 144 & 0.14 & 0.07 & -0.05\end{array}$

$0.151-0.083$
-0.03

0.071

$\begin{array}{llllll}0.003 & -4.62 & -1.46 & 1158.85 & -54.19\end{array}$

$\begin{array}{llllll}92 & 145 & 0.16 & 0.00 & -0.06\end{array}$

$0.152-0.097$

0.072

$\begin{array}{llllll}0.003 & -3.86 & -0.85 & 1163.21 & -50.48\end{array}$

$\begin{array}{lll}0.01 & 0.172 & 0.000\end{array}$

0.086

$\begin{array}{lllll}0.004 & -3.59 & -0.40 & 1165.90 & -45.10\end{array}$

$\begin{array}{lllll}93 & 146 & 0.18 & 0.00 & -0.06\end{array}$

$0.01 \quad 0.194$

0.000

0.089

$\begin{array}{llllll}0.004 & -2.67 & -0.05 & 1170.17 & -41.29\end{array}$

$\begin{array}{llllll}94 & 147 & 0.18 & 0.00 & -0.06\end{array}$

$0.01 \quad 0.194$

0.000

0.08

$\begin{array}{llllll}0.006 & -2.81 & 0.42 & 1172.50 & -35.55\end{array}$

$\begin{array}{lll}0.02 & 0.215 & 0.000\end{array}$

$\begin{array}{lll}0.52 & 1176.67 & -31.65\end{array}$

$\begin{array}{lllll}95 & 148 & 0.20 & 0.00 & -0.05 \\ 96 & 149 & 0.20 & 0.00 & -0.05\end{array}$

$0.02 \quad 0.215$

0.000

$0.080-0.005 \quad-2.04$

$\begin{array}{lll}0.64 & 1179.02 & -25.93\end{array}$

97150

$\begin{array}{llll}0.21 & 0.00 & -0.04\end{array}$

0.02

0.226

0.000

$\begin{array}{llll}0.070 & -0.007 & -1.99\end{array}$

$\begin{array}{lll}0.90 & 1182.71 & -21.55\end{array}$

$\begin{array}{llll}0.96 & 1184.81 & -15.58\end{array}$

$\begin{array}{lllll}98 & 151 & 0.21 & 0.00 & -0.04\end{array}$

$\begin{array}{lll}0.03 & 0.226 & 0.000\end{array}$

$\begin{array}{lll}0.071 & -0.018 & -1.78\end{array}$

$\begin{array}{lll}1.11 & 1188.30 & -10.99\end{array}$

$99 \quad 152$

$0.03 \quad 0.226$

0.000

$\begin{array}{llll}0.058 & -0.020 & -1.67\end{array}$

$\begin{array}{lll}1.04 & 1190.22 & -4.84\end{array}$

100153

$\begin{array}{llll}0.21 & 0.00 & -0.03\end{array}$

$0.02 \quad 0.227$

0.000

$\begin{array}{llll}0.045 & -0.013 & -1.21\end{array}$

$\begin{array}{lll}1.34 & 1193.24 & 0.20\end{array}$

$\begin{array}{lll}0.038 & -0.025 & -1.71\end{array}$

$\begin{array}{ll}1.19 & 1194.97\end{array}$

6.55

$102 \quad 155$

$\begin{array}{llr}0.24 & 0.00 & 0.00\end{array}$

$0.03 \quad 0.261$

0.000

$\begin{array}{lll}0.028 & -0.027 & -1.65\end{array}$

$\begin{array}{lll}1.31 & 1197.87 & 11.72\end{array}$

$\begin{array}{ll}-3.66 & -8.05\end{array}$

$-4.31-15.90$

$-5.33-25.70$

$-4.47-31.20$

$-3.43-38.07$

$-2.34-42.52$

$-1.58-48.32$

$-0.86-51.81$

$\begin{array}{lll}0.104 & -0.17 & -56.91\end{array}$

$0.51-59.73$

$1.02-64.29$

$1.22-66.91$

$1.65-70.85$

$2.01-72.66$

$2.28-76.11$

$\begin{array}{llll}0.097 & 2.42 & -77.50\end{array}$

$\begin{array}{lll}0.028 & 2.55 & -80.46\end{array}$

$\begin{array}{lll}0.020 & 2.60 & -81.36\end{array}$

$\begin{array}{lll}0.028 & 2.62 & -83.82\end{array}$

$\begin{array}{llll}0.018 & 2.61 & -84.20\end{array}$

$\begin{array}{lll}0.010 & 2.46 & -86.26\end{array}$

$\begin{array}{lll}0.005 & 2.21 & -86.33\end{array}$

$\begin{array}{llll}0.004 & 1.97 & -87.93\end{array}$ 


\begin{tabular}{|c|c|c|c|c|c|c|c|c|c|c|c|c|c|c|c|c|c|}
\hline \multicolumn{18}{|c|}{$Z=53(\mathrm{I})$} \\
\hline 103 & 156 & 0.24 & 0.00 & 0.01 & 0.03 & 0.262 & 0.000 & 0.016 & -0.030 & -1.85 & 1.10 & 1199.38 & 18.29 & & & 1.28 & 18.73 \\
\hline 104 & 157 & 0.24 & 0.00 & 0.02 & 0.02 & 0.263 & 0.000 & 0.003 & -0.023 & -1.55 & 1.33 & 1201.89 & 23.85 & & & 1.44 & 24.30 \\
\hline 105 & 158 & 0.23 & 0.00 & 0.02 & 0.02 & 0.251 & 0.000 & 0.001 & -0.023 & -1.55 & 1.15 & 1203.08 & 30.72 & & & 1.25 & 31.24 \\
\hline 106 & 159 & 0.23 & 0.00 & 0.03 & 0.01 & 0.252 & 0.000 & -0.013 & -0.016 & -1.30 & 1.33 & 1205.37 & 36.51 & & & 1.40 & 37.08 \\
\hline 107 & 160 & 0.23 & 0.00 & 0.04 & 0.00 & 0.252 & 0.000 & -0.026 & -0.009 & -1.47 & 1.23 & 1206.22 & 43.72 & & & 1.29 & 44.37 \\
\hline 108 & 161 & 0.21 & 0.00 & 0.03 & 0.00 & 0.229 & 0.000 & -0.018 & -0.006 & -0.98 & 1.28 & 1208.38 & 49.64 & & & 1.31 & 50.36 \\
\hline 109 & 162 & 0.21 & 0.00 & 0.04 & 0.00 & 0.230 & 0.000 & -0.030 & -0.009 & -1.29 & 1.05 & 1209.11 & 56.98 & & & 1.12 & 57.83 \\
\hline 110 & 163 & 0.20 & 0.00 & 0.04 & 0.00 & 0.219 & 0.000 & -0.031 & -0.008 & -1.02 & 1.11 & 1210.99 & 63.17 & & & 1.19 & 64.13 \\
\hline 111 & 164 & 0.20 & 0.00 & 0.05 & -0.01 & 0.219 & 0.000 & -0.044 & -0.001 & -1.45 & 0.76 & 1211.60 & 70.63 & & & 0.88 & 71.74 \\
\hline 112 & 165 & 0.18 & 0.00 & 0.04 & -0.01 & 0.196 & 0.000 & -0.035 & 0.002 & -1.11 & 0.73 & 1213.32 & 76.98 & & & 0.82 & 78.18 \\
\hline 113 & 166 & 0.17 & 0.00 & 0.04 & -0.01 & 0.185 & 0.000 & -0.037 & 0.003 & -1.40 & 0.32 & 1213.76 & 84.61 & & & 0.41 & 85.93 \\
\hline 114 & 167 & 0.15 & 0.00 & 0.04 & -0.01 & 0.163 & 0.000 & -0.039 & 0.003 & -1.48 & 0.32 & 1215.22 & 91.23 & & & 0.42 & 92.69 \\
\hline 115 & 168 & 0.15 & 0.00 & 0.04 & -0.01 & 0.163 & 0.000 & -0.039 & 0.003 & -2.16 & -0.43 & 1215.76 & 98.76 & & & -0.33 & 100.35 \\
\hline 116 & 169 & 0.13 & 0.00 & 0.03 & -0.01 & 0.140 & 0.000 & -0.030 & 0.006 & -2.25 & -0.79 & 1217.34 & 105.25 & & & -0.72 & 106.95 \\
\hline 117 & 170 & 0.11 & 0.00 & 0.03 & -0.01 & 0.119 & 0.000 & -0.032 & 0.006 & -2.94 & -1.59 & 1217.71 & 112.95 & & & -1.51 & 114.80 \\
\hline 118 & 171 & 0.11 & 00 & 0.04 & -0.01 & 0.119 & 000 & -0.043 & 0.005 & -3.53 & -2.10 & 1219.23 & 119.50 & & & -1.97 & 121.56 \\
\hline 119 & 172 & 0.10 & 0.00 & 0.04 & -0.01 & 0.108 & 0.000 & -0.044 & 0.005 & -4.30 & -2.92 & 1219.41 & 127.40 & & & -2.79 & 129.61 \\
\hline 120 & 173 & -0.12 & 0.00 & 0.01 & -0.01 & -0.125 & 0.000 & -0.005 & 0.011 & -4.66 & -3.40 & 1220.66 & 134.21 & & & -3.36 & 136.49 \\
\hline 121 & 174 & -0.12 & 0.00 & 0.01 & 0.00 & -0.125 & 0.000 & -0.006 & 0.001 & -5.69 & -4.45 & 1220.86 & 142.08 & & & -4.45 & 144.49 \\
\hline 122 & 175 & -0.06 & 0.00 & 0.02 & 0.00 & -0.063 & 0.000 & -0.022 & 0.002 & -6.11 & -5.31 & 1222.30 & 148.72 & & & -5.28 & 151.34 \\
\hline 123 & 176 & -0.06 & 0.00 & 0.02 & 0.00 & -0.063 & 000 & -0.022 & 002 & -7.49 & -6.56 & .50 & 156.59 & & & -6.53 & .39 \\
\hline 124 & 177 & -0.05 & 0.00 & 0.02 & 0.01 & -0.052 & 0.000 & -0.022 & -0.008 & -8.33 & -7.34 & 1223.64 & 163.52 & & & -7.28 & 166.53 \\
\hline 125 & 178 & 0.01 & 0.00 & 0.00 & 0.00 & 0.011 & 0.000 & 0.000 & 0.000 & -9.78 & -8.62 & 1223.68 & 171.55 & & & -8.62 & 174.69 \\
\hline 126 & 179 & 0.00 & 0.00 & 0.00 & 0.00 & 0.000 & 0.000 & 0.000 & 0.000 & -10.56 & -9.35 & 1224.57 & 178.73 & & & -9.35 & 182.07 \\
\hline \multicolumn{18}{|c|}{$Z=54(\mathrm{Xe})$} \\
\hline 49 & 103 & 0.05 & 0.01 & -0.01 & 0.00 & 0.053 & 14 & 0.013 & 0.001 & -4.18 & -3.14 & 79 & -5.38 & & & -3 & -5.29 \\
\hline 50 & 104 & 0.00 & 0.00 & 0.00 & 0.00 & 0.000 & 0.000 & 0.000 & 0.000 & -5.16 & -4.15 & 09 & -15.92 & & & -4.15 & -15.82 \\
\hline 51 & 105 & 0.07 & 0.00 & -0.04 & -0.02 & 0.076 & 0.000 & 0.051 & 0.024 & -4.73 & -3.19 & 826.70 & -21.45 & & & -3.18 & -21.34 \\
\hline 52 & 106 & 0.12 & 0.00 & -0.05 & 0.00 & 0.129 & 0.000 & 0.068 & 0.009 & -4.42 & -2.27 & 842.50 & -29.19 & & & -2.26 & -29.07 \\
\hline 53 & 107 & 0.13 & 0.03 & -0.05 & 0.01 & 0.140 & -0.041 & 0.069 & -0.000 & -3.88 & -1.47 & 855.44 & -34.05 & & & -1.46 & -33.94 \\
\hline 54 & 108 & 0.15 & 0.05 & -0.05 & 0.02 & 2 & 0.069 & 0.073 & -0.008 & & & & -41.15 & & & & \\
\hline 55 & 109 & 0.15 & 0.07 & -0.04 & 0.02 & 0.162 & -0.096 & 0.061 & -0.008 & -3.32 & -0.21 & .49 & -44.96 & & & -0.20 & -44.86 \\
\hline 56 & 110 & 0.16 & 0.07 & -0.04 & 0.03 & 0.173 & -0.095 & 0.063 & -0.018 & -2.85 & 0.37 & 896.47 & -50.87 & -51.90 & . & 0.40 & -50.76 \\
\hline 57 & 111 & 0.17 & 0.08 & -0.03 & 0.03 & 0.185 & -0.109 & 0.052 & -0.018 & -2.39 & 0.58 & 907.95 & -54.28 & & & 0.59 & -54.19 \\
\hline 58 & 112 & 0.18 & 0.07 & -0.03 & 0.03 & 0.195 & -0.095 & 0.054 & -0.019 & -1.77 & & & -59.46 & -59.97 & . & & -59.36 \\
\hline 59 & 113 & 0 & 6 & -0 . & & & & & & & & & & & & 56 & \\
\hline 60 & 114 & 0.22 & 0.01 & -0.05 & 0.02 & 0.237 & -0.014 & 0.084 & -0.003 & & 2.06 & 944.41 & -66.53 & -67.09 & & 2.09 & -66.46 \\
\hline 61 & 115 & 0.22 & 0.00 & -0.05 & 0.02 & 0.237 & 0.000 & 0.084 & -0.003 & -0.85 & 2.29 & 954.53 & -68.57 & -68.66 & & 2.31 & -68.53 \\
\hline 62 & 116 & 0.22 & 0.00 & -0.03 & 0.02 & 0.238 & 0.000 & 0.059 & -0.009 & -0.17 & 2.49 & 966.77 & -72.74 & -73.05 & 0.013 & 2.52 & -72.71 \\
\hline 63 & 117 & 0.22 & 0.00 & -0.02 & 0.01 & 0.238 & 0.000 & 0.046 & -0.002 & 0.12 & 2.70 & 976.27 & -74.18 & -74.18 & 0.010 & 2.70 & -74.18 \\
\hline 64 & 118 & 0.22 & 0.00 & -0.01 & 0.0 & & & & & 6 & 1 & 97 & -7 & & & 83 & \\
\hline 65 & 119 & 0.23 & 0.00 & 0.00 & 0.01 & & 0.000 & 0.024 & -0.008 & 0.23 & 2.80 & 997.08 & -78.84 & -78.79 & & 2.81 & -78.89 \\
\hline 66 & 120 & 0.23 & 0.00 & 0.01 & 0.00 & 0.250 & 0.000 & 0.011 & -0.001 & 0.28 & 2.78 & 1008.30 & -81.99 & & & 2.80 & -82.04 \\
\hline 67 & 121 & 0.23 & 0.00 & 0.02 & 0.00 & 0.251 & 0.000 & -0.002 & -0.004 & 0.12 & 2.80 & 1016.81 & -82.43 & -82.47 & 0.011 & 2.81 & -82.51 \\
\hline 68 & 122 & 0.23 & 0.00 & 0.03 & 0.00 & 0.252 & 0.000 & -0.014 & -0.006 & 0.01 & 2.73 & 1027.49 & -85.03 & -85.36 & 0.011 & 2.75 & -85.12 \\
\hline 69 & 123 & 0.23 & 0.00 & 0.03 & 0.00 & 52 & 00 & -0.014 & -0.006 & -0.23 & 59 & 60 & -85.07 & -8 & 10 & 2.61 & -85.19 \\
\hline 70 & 124 & 0.21 & 0.00 & 0.03 & 0.00 & 0.229 & 0.000 & -0.018 & -0.006 & -0.01 & 2.47 & 1045.77 & -87.17 & -87.66 & & 2.50 & -87.30 \\
\hline 71 & 125 & 0.20 & 0.00 & 0.02 & 0.00 & 0.217 & 0.000 & -0.007 & -0.004 & -0.11 & 2.11 & 1053.58 & -86.91 & -87.19 & 0.002 & 2.12 & -87.07 \\
\hline 72 & 126 & 0.18 & 0.00 & 0.01 & 0.00 & 0.195 & 0.000 & 0.002 & -0.001 & -0.11 & 1.65 & 1063.54 & -88.80 & -89.17 & 0.006 & 1.67 & -88.98 \\
\hline 73 & 127 & 0.16 & 0.00 & 0.00 & 0.01 & 0.172 & 0.000 & 0.012 & -0.009 & -0.32 & 1.42 & 1070.71 & -87.90 & -88.32 & 0.004 & 1.43 & -88.11 \\
\hline 74 & 128 & 0.16 & 0.00 & 0.01 & 0.00 & 3 & 00 & -0.001 & -0.001 & -0.79 & 0.96 & 16 & -89.28 & -89.86 & 0.0 & 0.98 & -89.50 \\
\hline 75 & 129 & 0.15 & 0.00 & 0.01 & 0.00 & 0.162 & 0.000 & -0.003 & -0.001 & -1.32 & 0.38 & 1087.19 & -88.24 & -88.70 & 0.001 & 0.39 & -88.49 \\
\hline 76 & 130 & -0.12 & 0.00 & 0.02 & 0.01 & -0.125 & 0.000 & -0.018 & -0.007 & -1.69 & -0.09 & 1096.16 & -89.14 & -89.88 & 0.001 & -0.08 & -89.40 \\
\hline 77 & 131 & -0.12 & 0.00 & 0.03 & 0.01 & -0.125 & 0.000 & -0.029 & -0.005 & -2.59 & -1.08 & 1103.14 & -88.04 & -88.42 & 0.001 & -1.06 & -88.32 \\
\hline 78 & 132 & -0.12 & 0.00 & 0.03 & 0.01 & -0.125 & 0.000 & -0.029 & -0.005 & -3.54 & -2.00 & 1112.07 & -88.90 & -89.28 & 0.001 & -1.97 & -89.19 \\
\hline 79 & 133 & 0.05 & 0.00 & -0.01 & 0.00 & & 0.000 & 0.013 & 0.001 & -4.04 & -3.24 & 111 & -87.61 & -87.64 & 0.002 & -3.23 & -87.94 \\
\hline 80 & 134 & 0.00 & 0.00 & 0.00 & 0.00 & 0.000 & 0.000 & 0.000 & 0.000 & -5.59 & -4.69 & 1127.86 & -88.55 & -88.12 & 0.001 & -4.69 & -88.89 \\
\hline
\end{tabular}




\begin{tabular}{|c|c|c|c|c|c|c|c|c|c|c|c|c|c|c|c|c|}
\hline \multicolumn{17}{|c|}{$Z=54(\mathrm{Xe})$} \\
\hline $81 \quad 135$ & 0.03 & 0.00 & 0.01 & 0.00 & 0.032 & 0.000 & -0.012 & -0.000 & -6.74 & -5.60 & 1133.89 & -86.51 & -86.42 & 0.005 & -5.60 & -86.86 \\
\hline $82 \quad 136$ & 0.00 & 0.00 & 0.00 & 0.00 & 0.000 & 0.000 & 0.000 & 0.000 & -8.12 & -6.91 & 1142.31 & -86.86 & -86.43 & 0.007 & -6.91 & -87.22 \\
\hline $83 \quad 137$ & -0.02 & 0.00 & -0.01 & 0.00 & -0.021 & 0.000 & 0.012 & -0.000 & -7.20 & -6.09 & 1146.20 & -82.68 & -82.38 & 0.007 & -6.09 & -83.04 \\
\hline $84 \quad 138$ & 0.00 & 0.00 & 0.00 & 0.00 & 0.000 & 0.000 & 0.000 & 0.000 & -5.99 & -5.01 & 1151.81 & -80.21 & -80.15 & 0.043 & -5.01 & -80.59 \\
\hline $85 \quad 139$ & 0.08 & 0.00 & -0.04 & 0.00 & 0.086 & 0.000 & 0.051 & 0.005 & -5.38 & -3.70 & 1154.81 & -75.14 & -75.64 & 0.021 & -3.64 & -75.46 \\
\hline $86 \quad 140$ & 0.09 & 0.00 & -0.05 & -0.01 & 0.097 & 0.000 & 0.064 & 0.017 & -4.80 & -2.84 & 1160.24 & -72.50 & -72.99 & 0.061 & -2.73 & -72.76 \\
\hline $87 \quad 141$ & 0.12 & 0.04 & -0.05 & 0.00 & 0.129 & -0.056 & 0.068 & 0.010 & -4.74 & -2.17 & 1163.49 & -67.68 & -68.33 & 0.091 & -2.05 & -67.93 \\
\hline $88 \quad 142$ & 0.13 & 0.06 & -0.06 & 0.01 & 0.141 & -0.083 & 0.083 & 0.004 & -4.73 & -1.50 & 1168.72 & -64.84 & -65.47 & 0.101 & -1.30 & -65.01 \\
\hline $89 \quad 143$ & 0.14 & 0.08 & -0.05 & 0.01 & 0.152 & -0.111 & 0.072 & 0.005 & -4.62 & -1.03 & 1171.81 & -59.85 & & & -0.84 & -60.02 \\
\hline $90 \quad 144$ & 0.15 & 0.07 & -0.05 & 0.02 & 0.162 & -0.096 & 0.073 & -0.006 & -3.84 & -0.46 & 1176.76 & -56.74 & & & -0.25 & -56.88 \\
\hline $91 \quad 145$ & 0.16 & 0.08 & -0.05 & 0.02 & 0.174 & -0.110 & 0.075 & -0.004 & -3.83 & -0.28 & 1179.78 & -51.68 & & & -0.05 & -51.79 \\
\hline $92 \quad 146$ & 0.18 & 0.01 & -0.07 & 0.01 & 0.194 & -0.014 & 0.102 & 0.009 & -3.14 & 0.22 & 1184.44 & -48.28 & & & 0.48 & -48.33 \\
\hline $\begin{array}{ll}93 & 147\end{array}$ & 0.20 & 0.00 & -0.07 & 0.01 & 0.216 & 0.000 & 0.105 & 0.011 & -3.47 & 0.28 & 1187.25 & -43.01 & & & 0.54 & -43.05 \\
\hline $94 \quad 148$ & 0.20 & 0.00 & -0.07 & 0.01 & 0.216 & 0.000 & 0.105 & 0.011 & -3.06 & 0.60 & 1191.74 & -39.43 & & & 0.88 & -39.42 \\
\hline $95 \quad 149$ & 0.20 & 0.01 & -0.06 & 0.02 & 0.215 & -0.014 & 0.093 & -0.002 & -2.65 & 0.62 & 1194.26 & -33.88 & & & 0.85 & -33.89 \\
\hline $96 \quad 150$ & 0.21 & 0.00 & -0.05 & 0.02 & 0.226 & 0.000 & 0.082 & -0.004 & -2.22 & 0.94 & 1198.41 & -29.96 & & & 1.14 & -29.97 \\
\hline $97 \quad 151$ & 0.21 & 0.00 & -0.05 & 0.02 & 0.226 & 0.000 & 0.082 & -0.004 & -2.24 & 0.92 & 1200.64 & -24.12 & & & 1.11 & -24.10 \\
\hline $98 \quad 152$ & 0.21 & 0.00 & -0.04 & 0.03 & 0.226 & 0.000 & 0.071 & -0.018 & -1.78 & 1.11 & 1204.61 & -20.01 & & & 1.33 & -19.92 \\
\hline $99 \quad 153$ & 0.23 & 0.00 & -0.03 & 0.03 & 0.249 & 0.000 & 0.062 & -0.019 & -2.05 & 1.08 & 1206.55 & -13.89 & & & 1.27 & -13.78 \\
\hline $100 \quad 154$ & 0.23 & 0.00 & -0.02 & 0.03 & 0.249 & 0.000 & 0.050 & -0.022 & -1.68 & 1.28 & 1210.19 & -9.45 & & & 1.47 & -9.30 \\
\hline $101 \quad 155$ & 0.24 & 0.00 & -0.01 & 0.04 & 0.261 & 0.000 & 0.041 & -0.034 & -2.09 & 1.06 & 1212.04 & -3.23 & & & 1.34 & -2.92 \\
\hline 102156 & 0.24 & 0.00 & 0.00 & 0.03 & 0.261 & 0.000 & 0.028 & -0.027 & -1.71 & 1.29 & 1215.34 & 1.54 & & & 1.48 & 1.81 \\
\hline 103157 & 0.24 & 0.00 & 0.01 & 0.03 & 0.262 & 0.000 & 0.016 & -0.030 & -1.91 & 1.07 & 1216.90 & 8.06 & & & 1.26 & 8.39 \\
\hline $104 \quad 158$ & 0.24 & 0.00 & 0.02 & 0.03 & 0.263 & 0.000 & 0.004 & -0.033 & -1.73 & 1.20 & 1220.01 & 13.02 & & & 1.44 & 13.46 \\
\hline 105159 & 0.23 & 0.00 & 0.02 & 0.02 & 0.251 & 0.000 & 0.001 & -0.023 & -1.59 & 1.14 & 1221.13 & 19.96 & & & 1.25 & 20.35 \\
\hline 106160 & 0.23 & 0.00 & 0.03 & 0.02 & 0.252 & 0.000 & -0.011 & -0.026 & -1.42 & 1.26 & 1223.97 & 25.19 & & & 1.42 & 25.71 \\
\hline $107 \quad 161$ & 0.23 & 0.00 & 0.04 & 0.01 & 0.253 & 0.000 & -0.024 & -0.019 & -1.57 & 1.18 & 1224.85 & 32.38 & & & 1.29 & 32.93 \\
\hline $108 \quad 162$ & 0.21 & 0.00 & 0.03 & 0.01 & 0.229 & 0.000 & -0.016 & -0.016 & -1.01 & 1.30 & 1227.42 & 37.89 & & & 1.38 & 38.49 \\
\hline 109163 & 0.21 & 0.00 & 0.04 & 0.00 & 0.230 & 0.000 & -0.030 & -0.009 & -1.26 & 1.12 & 1228.14 & 45.24 & & & 1.20 & 45.93 \\
\hline 110164 & 0.21 & 0.00 & 0.05 & 0.00 & 0.230 & 0.000 & -0.041 & -0.011 & -1.24 & 1.17 & 1230.52 & 50.93 & & & 1.30 & 51.78 \\
\hline $111 \quad 165$ & 0.20 & 0.00 & 0.05 & 0.00 & 0.219 & 0.000 & -0.043 & -0.010 & -1.38 & 0.89 & 1231.09 & 58.43 & & & 1.02 & 59.37 \\
\hline 112166 & 0.18 & 0.00 & 0.04 & 0.00 & 0.196 & 0.000 & -0.034 & -0.007 & -0.93 & 0.98 & 1233.18 & 64.42 & & & 1.07 & 65.43 \\
\hline 113167 & 0.18 & 0.00 & 0.05 & -0.01 & 0.197 & 0.000 & -0.047 & 0.000 & -1.44 & 0.55 & 1233.66 & 72.00 & & & 0.69 & 73.18 \\
\hline 114168 & 0.15 & 0.00 & 0.03 & 0.00 & 0.162 & 0.000 & -0.027 & -0.005 & -0.99 & 0.48 & 1235.66 & 78.08 & & & 0.53 & 79.28 \\
\hline 115169 & 0.15 & 0.00 & 0.04 & -0.01 & 0.163 & 0.000 & -0.039 & 0.003 & -1.73 & 0.12 & 1235.85 & 85.96 & & & 0.22 & 87.34 \\
\hline 116170 & 0.15 & 0.00 & 0.04 & -0.01 & 0.163 & 0.000 & -0.039 & 0.003 & -1.98 & -0.15 & 1237.80 & 92.07 & & & -0.05 & 93.59 \\
\hline $117 \quad 171$ & 0.13 & 0.00 & 0.04 & -0.01 & 0.141 & 0.000 & -0.042 & 0.004 & -2.51 & -0.87 & 1238.13 & 99.82 & & & -0.76 & 101.48 \\
\hline $118 \quad 172$ & 0.11 & 0.00 & 0.04 & -0.02 & 0.119 & 0.000 & -0.044 & 0.015 & -2.95 & -1.55 & 1240.26 & 105.76 & & & -1.36 & 107.64 \\
\hline 119173 & 0.11 & 0.00 & 0.04 & -0.02 & 0.119 & 0.000 & -0.044 & 0.015 & -3.76 & -2.32 & 1240.41 & 113.68 & & & -2.12 & 115.70 \\
\hline $120 \quad 174$ & -0.12 & 0.00 & 0.01 & -0.01 & -0.125 & 0.000 & -0.005 & 0.011 & -3.90 & -2.69 & 1242.02 & 120.15 & & & -2.65 & 122.16 \\
\hline $121 \quad 175$ & -0.12 & 0.00 & 0.01 & 0.00 & -0.125 & 0.000 & -0.006 & 0.001 & -4.96 & -3.67 & 1242.17 & 128.06 & & & -3.67 & 130.20 \\
\hline $122 \quad 176$ & -0.06 & 0.00 & 0.02 & 0.00 & -0.063 & 0.000 & -0.022 & 0.002 & -5.24 & -4.49 & 1244.01 & 134.29 & & & -4.46 & 136.62 \\
\hline $123 \quad 177$ & -0.06 & 0.00 & 0.02 & 0.00 & -0.063 & 0.000 & -0.022 & 0.002 & -6.64 & -5.74 & 1244.23 & 142.14 & & & -5.71 & 144.64 \\
\hline $124 \quad 178$ & -0.06 & 0.00 & 0.02 & 0.01 & -0.063 & 0.000 & -0.022 & -0.008 & -7.47 & -6.49 & 1245.78 & 148.67 & & & -6.43 & 151.37 \\
\hline 125179 & 0.00 & 0.00 & 0.00 & 0.00 & 0.000 & 0.000 & 0.000 & 0.000 & -8.88 & -7.78 & 1245.85 & 156.66 & & & -7.78 & 159.49 \\
\hline 126180 & 0.00 & 0.00 & 0.00 & 0.00 & 0.000 & 00 & 0.000 & 0.000 & -9.65 & -8.48 & 1247.15 & $16 ?$ & & & -8.48 & 166.45 \\
\hline $127 \quad 181$ & -0.01 & 0.00 & 0.00 & 0.00 & -0.011 & 0.000 & 0.000 & 0.000 & -8.77 & -7.68 & 1244.94 & 173.72 & & & -7.68 & 176.93 \\
\hline $128 \quad 182$ & 0.00 & 0.00 & 0.00 & 0.00 & 0.000 & 0.000 & 0.000 & 0.000 & -7.67 & -6.64 & 1244.31 & 182.42 & & & -6.64 & 185.83 \\
\hline \multicolumn{17}{|c|}{$Z=55(\mathrm{Cs})$} \\
\hline $51 \quad 106$ & 0.09 & 0.00 & -0.04 & 0.00 & 0.096 & 0.000 & 0.052 & 0.005 & -3.68 & -2.16 & 821.91 & -9.38 & & & -2.17 & -9.23 \\
\hline 52107 & 0.12 & 0.04 & -0.05 & 0.01 & 0.129 & -0.056 & 0.068 & -0.000 & -3.79 & -1.51 & 838.11 & -17.51 & & & -1.51 & -17.36 \\
\hline $\begin{array}{ll}53 & 108\end{array}$ & 0.15 & 0.07 & -0.05 & 0.02 & 0.162 & -0.096 & 0.073 & -0.006 & -4.02 & -0.81 & 851.86 & -23.19 & & & -0.82 & -23.06 \\
\hline $54 \quad 109$ & 0.16 & 0.08 & -0.05 & 0.03 & 0.173 & -0.109 & 0.075 & -0.014 & -3.92 & -0.35 & 867.42 & -30.68 & & & -0.34 & -30.54 \\
\hline $55 \quad 110$ & 0.16 & 0.09 & -0.04 & 0.03 & 0.174 & -0.123 & 0.064 & -0.015 & -3.66 & -0.39 & 880.86 & -36.04 & & & -0.40 & -36.20 \\
\hline $56 \quad 111$ & 0.17 & 0.09 & -0.04 & 0.03 & 0.185 & -0.123 & 0.065 & -0.014 & -3.29 & 0.14 & 895.28 & -42.40 & & & 0.15 & -42.27 \\
\hline $57 \quad 112$ & 0.18 & 0.09 & -0.03 & 0.03 & 0.196 & -0.122 & 0.054 & -0.016 & -2.67 & 0.76 & 907.05 & -46.09 & & & 0.75 & -45.99 \\
\hline $58 \quad 113$ & 0.19 & 0.09 & -0.03 & 0.03 & 0.207 & -0.122 & 0.056 & -0.016 & -2.32 & 1.23 & 920.53 & -51.49 & -51.70 & 0.104 & 1.24 & -51.39 \\
\hline
\end{tabular}




\begin{tabular}{|c|c|c|c|c|c|c|c|c|c|c|c|c|c|c|c|c|c|}
\hline \multicolumn{18}{|c|}{$Z=55(\mathrm{Cs})$} \\
\hline 59 & 114 & 0.21 & 0.07 & -0.04 & 0.03 & 0.228 & -0.095 & 0.072 & -0.014 & -1.83 & 1.72 & 931.72 & -54.62 & & & 1.71 & -54.55 \\
\hline 60 & 115 & 0.24 & 0.00 & -0.06 & 0.01 & 0.260 & 0.000 & 0.101 & 0.013 & -1.82 & 2.06 & 944.62 & -59.45 & & & 2.07 & -59.37 \\
\hline 61 & 116 & 0.24 & 0.00 & -0.06 & 0.01 & 0.260 & 0.000 & 0.101 & 0.013 & -1.64 & 2.25 & 955.44 & -62.20 & & & 2.25 & -62.15 \\
\hline 62 & 117 & 0.24 & 0.00 & -0.04 & 0.01 & 0.260 & 0.000 & 0.076 & 0.005 & -0.80 & 2.46 & 967.79 & -66.48 & -66.44 & 0.062 & 2.47 & -66.44 \\
\hline 63 & 118 & 0.24 & 0.01 & -0.03 & 0.02 & 0.260 & -0.013 & 0.064 & -0.008 & -0.47 & 2.64 & 977.99 & -68.60 & -68.41 & 0.013 & 2.64 & -68.60 \\
\hline 64 & 119 & 0.24 & 0.00 & -0.01 & 0.01 & 0.261 & 0.000 & 0.038 & -0.004 & -0.03 & 2.86 & 989.69 & -72.23 & -72.31 & 0.014 & 2.87 & -72.24 \\
\hline 65 & 120 & 0.23 & 0.00 & 0.00 & 0.01 & 0.250 & 0.000 & 0.024 & -0.008 & 0.19 & 2.84 & 999.48 & -73.95 & -73.89 & 0.010 & 2.83 & -73.99 \\
\hline 66 & 121 & 0.23 & 0.00 & 0.01 & 0.01 & 0.250 & 0.000 & 0.012 & -0.011 & 0.19 & 2.74 & 1010.88 & -77.28 & -77.10 & 0.014 & 2.74 & -77.33 \\
\hline 67 & 122 & 0.24 & 0.00 & 0.02 & 0.01 & 0.262 & 0.000 & 0.002 & -0.013 & -0.15 & 2.70 & 1020.09 & -78.41 & -78.14 & 0.032 & 2.70 & -78.50 \\
\hline 68 & 123 & 0.23 & 0.00 & 0.03 & 0.01 & 0.252 & 0.000 & -0.013 & -0.016 & -0.09 & 2.70 & 1030.80 & -81.06 & -81.04 & 0.012 & 2.72 & -81.14 \\
\hline 69 & 124 & 0.23 & 0.00 & 0.03 & 0.00 & 0.252 & 0.000 & -0.014 & -0.006 & -0.29 & 2.52 & 1039.59 & -81.78 & -81.73 & 0.008 & 2.52 & -81.90 \\
\hline 70 & 125 & 0.22 & 0.00 & 0.03 & 0.00 & 0.240 & 0.000 & -0.016 & -0.006 & -0.16 & 2.49 & 1049.78 & -83.89 & -84.09 & 0.008 & 2.50 & -84.02 \\
\hline 71 & 126 & 0.22 & 0.00 & 0.04 & 0.00 & 0.241 & 0.000 & -0.028 & -0.009 & -0.51 & 2.23 & 1058.11 & -84.15 & -84.35 & 0.012 & 2.24 & -84.31 \\
\hline 72 & 127 & 0.19 & 0.00 & 0.02 & 0.01 & 0.206 & 0.000 & -0.008 & -0.013 & -0.15 & 1.91 & 1068.03 & -86.00 & -86.24 & 0.006 & 1.93 & -86.18 \\
\hline 73 & 128 & 0.19 & 0.00 & 0.02 & 0.01 & 0.206 & 0.000 & -0.008 & -0.013 & -0.56 & 1.50 & 1076.00 & -85.90 & -85.93 & 0.005 & 1.51 & -86.11 \\
\hline 74 & 129 & 0.17 & 0.00 & 0.02 & 0.01 & 0.184 & 0.000 & -0.011 & -0.013 & -0.66 & 1.25 & 1085.33 & -87.16 & -87.50 & 0.005 & 1.27 & -87.38 \\
\hline 75 & 130 & 0.16 & 0.00 & 0.01 & 0.01 & 0.173 & 0.000 & -0.001 & -0.011 & -1.09 & 0.77 & 1092.89 & -86.64 & -8 & 0.008 & 0.77 & -8 \\
\hline 76 & 131 & 0.14 & 0.00 & 0.01 & 0.01 & 0.151 & 0.000 & -0.003 & -0.011 & -1.36 & 0.44 & 1101.80 & -87.48 & -88.06 & 0.005 & 0.45 & -87.75 \\
\hline 77 & 132 & 0.13 & 0.00 & 0.01 & 0.00 & 0.140 & 0.000 & -0.005 & -0.001 & -1.82 & -0.27 & 1109.10 & -86.72 & -87.16 & 0.002 & -0.27 & -87.01 \\
\hline 78 & 133 & -0.12 & 0.00 & 0.03 & 0.01 & -0.125 & 0.000 & -0.029 & -0.005 & -2.76 & -1.27 & 1118.20 & -87.74 & -88.07 & 0.000 & -1.25 & -88.04 \\
\hline 79 & 134 & -0.12 & 0.00 & 0.03 & 0.01 & -0.125 & 0.000 & -0.029 & -0.005 & -3.69 & -2.27 & 1125.33 & -86.81 & -86.89 & 0.000 & -2.25 & -87.12 \\
\hline 80 & 135 & 0.00 & 0.00 & 0.00 & 0.00 & 0.000 & 0.000 & 0.000 & 0.000 & -4.49 & -3.68 & & -87.79 & $-\varepsilon$ & 0.001 & -3.68 & 14 \\
\hline 81 & 136 & 0.05 & 0.00 & 0.00 & 0.00 & 0.053 & 0.000 & 0.001 & 0.000 & -5.75 & -4.60 & 1141.01 & -86.34 & -86.34 & 0.002 & -4.60 & -86.70 \\
\hline 82 & 137 & 0.00 & 0.00 & 0.00 & 0.00 & 0.000 & 0.000 & 0.000 & 0.000 & -7.06 & -5.91 & 1149.51 & -86.77 & -86.55 & 0.000 & -5.91 & -87.15 \\
\hline 83 & 138 & -0.02 & 0.00 & 0.00 & 0.00 & -0.021 & 0.000 & 0.000 & 0.000 & -6.12 & -5.10 & 1153.99 & -83.17 & -82.89 & 0.009 & -5.10 & -83.56 \\
\hline 84 & 139 & -0.01 & 0.00 & 0.00 & 0.00 & -0.011 & 0.000 & 0.000 & 0.000 & -4.94 & -4.03 & 1159.69 & -80.81 & -80.70 & 0.003 & -4.03 & -81.20 \\
\hline 85 & 140 & 0.09 & 0.03 & -0.04 & -0.01 & 0.097 & -0.042 & 0.052 & 0.016 & -4.84 & -2.86 & 1163.39 & -76.44 & -77.05 & 0.008 & -2.79 & -76.76 \\
\hline 86 & 141 & 0.11 & 0.04 & -0.05 & 0.00 & 0.119 & -0.056 & 0.067 & 0.009 & -4.52 & -2.06 & 1168.96 & -73.94 & -74.48 & 0.011 & -1.96 & -74.23 \\
\hline 87 & 142 & 0.13 & 0.07 & -0.05 & 0.01 & 0.141 & -0.097 & 0.070 & 0.003 & -4.97 & -1.68 & 1173.06 & -69.96 & -70.51 & 0.011 & -1.54 & -70.23 \\
\hline 88 & 143 & 0.14 & 0.08 & -0.06 & 0.01 & 0.153 & -0.112 & 0.084 & 0.007 & -5.09 & -1.13 & 1178.48 & -67.32 & -67.67 & 0.024 & -0.92 & -67.51 \\
\hline 89 & 144 & 0.15 & 0.09 & -0.05 & 0.02 & 0.163 & -0.124 & 0.074 & -0.004 & -4.83 & -0.77 & 1182.24 & -62.99 & -63.27 & 0.026 & -0.58 & -63.20 \\
\hline 90 & 145 & 0.15 & 0.08 & -0.05 & 0.02 & 0.163 & -0.110 & 0.073 & -0.005 & -3.91 & -0.22 & 1187.28 & -59.97 & -6 & 0.011 & -0.03 & -60.16 \\
\hline 91 & 146 & 0.16 & 0.09 & -0.05 & 0.02 & 0.174 & -0.124 & 0.075 & -0.003 & -4.03 & -0.14 & 1190.94 & -55.56 & -55.62 & 0.071 & 0.07 & -55.73 \\
\hline 92 & 147 & 0.19 & 0.04 & -0.07 & 0.01 & 0.206 & -0.055 & 0.104 & 0.011 & -3.48 & 0.41 & 1195.62 & -52.17 & -52.02 & 0.053 & 0.66 & -52.28 \\
\hline 93 & 148 & 0.20 & 0.00 & -0.07 & 0.01 & 0.216 & 0.000 & 0.105 & 0.011 & -3.40 & 0.40 & 1199.02 & -47.50 & -47.30 & 0.576 & 0.63 & -47.62 \\
\hline 94 & 149 & 0.21 & 0.00 & -0.07 & 0.01 & 0.227 & 0.000 & 0.107 & 0.012 & -3.22 & 0.74 & 1203.56 & -43.97 & & & 0.99 & -44.04 \\
\hline 95 & 150 & 0.22 & 0.02 & -0.06 & 0.02 & 0.237 & -0.027 & 0.097 & 0.000 & -3.10 & 0.68 & 1206.69 & -39.02 & & & 0.89 & -39.11 \\
\hline 96 & 151 & 0.21 & 0.00 & -0.05 & 0.02 & 0.226 & 0.000 & 0.082 & -0.004 & -2.19 & 1.02 & 1210.88 & -35.14 & & & 1.20 & -35.24 \\
\hline 97 & 152 & 0.23 & 0.00 & -0.05 & 0.03 & 0.248 & 0.000 & 0.087 & -0.013 & -2.68 & 0.87 & 1213.78 & -29.97 & & & 1.08 & -30.00 \\
\hline 98 & 153 & 0.23 & 0.00 & -0.03 & 0.03 & 0.249 & 0.000 & 0.062 & -0.019 & -1.97 & 1.13 & 1217.71 & -25.83 & & & 1.31 & -25.87 \\
\hline 99 & 154 & 0.24 & 0.00 & -0.02 & 0.03 & 0.260 & 0.000 & 0.052 & -0.021 & -2.18 & 1.06 & 1220.21 & -20.26 & & & 1.21 & -20.28 \\
\hline 100 & 155 & 0.24 & 0.00 & -0.01 & 0.03 & 0.261 & 0.000 & 0.040 & -0.024 & -1.85 & 1.26 & 1223.91 & -15.89 & & & 1.42 & -15.85 \\
\hline 101 & 156 & 0.24 & 0.00 & -0.01 & 0.04 & 0.261 & 0.000 & 0.041 & -0.034 & -2.21 & 0.96 & 1226.33 & -10.24 & & & 1.22 & -10.06 \\
\hline 102 & 157 & 0.24 & 0.00 & 0.00 & 0.04 & 0.262 & 0.000 & 0.029 & -0.037 & -1.98 & 1.09 & 1229.80 & -5.63 & & & 1.37 & -5.37 \\
\hline 103 & 158 & 0.24 & 0.00 & 0.01 & 0.03 & 0.262 & 0.000 & 0.016 & -0.030 & -2.03 & 1.01 & 1231.72 & 0.52 & & & 1.18 & 0.72 \\
\hline 104 & 159 & 0.24 & 0.00 & 0.02 & 0.03 & 0.263 & 0.000 & 0.004 & -0.033 & -1.85 & 1.11 & 1234.90 & 5.41 & & & 1.32 & 5.71 \\
\hline 105 & 160 & 0.24 & 0.00 & 0.03 & 0.02 & 0.263 & 0.000 & -0.009 & -0.026 & -1.93 & 1.06 & 1236.51 & 11.87 & & & 1.19 & 12.15 \\
\hline 106 & 161 & 0.23 & 0.00 & 0.03 & 0.02 & 0.252 & 0.000 & -0.011 & -0.026 & -1.54 & 1.20 & 1239.37 & 17.08 & & & 1.35 & 17.44 \\
\hline 107 & 162 & 0.23 & 0.00 & 0.04 & 0.01 & 0.253 & 0.000 & -0.024 & -0.019 & -1.68 & 1.11 & 1240.75 & 23.77 & & & 1.21 & 24.17 \\
\hline 108 & 163 & 0.21 & 0.00 & 0.04 & 0.01 & 0.230 & 0.000 & -0.028 & -0.018 & -1.21 & 1.22 & 1243.38 & 29.22 & & & 1.33 & 29.71 \\
\hline 109 & 164 & 0.21 & 0.00 & 0.04 & 0.01 & 0.230 & 0.000 & -0.028 & -0.018 & -1.40 & 1.05 & 1244.57 & 36.10 & & & 1.16 & 36.66 \\
\hline 110 & 165 & 0.21 & 0.00 & 0.05 & 0.00 & 0.230 & 0.000 & -0.041 & -0.011 & -1.32 & 1.15 & 1246.94 & 41.80 & & & 1.27 & 42.47 \\
\hline 111 & 166 & 0.20 & 0.00 & 0.05 & 0.00 & 0.219 & 0.000 & -0.043 & -0.010 & -1.43 & 0.89 & 1247.97 & 48.84 & & & 1.01 & 49.60 \\
\hline 112 & 167 & 0.19 & 0.00 & 0.05 & 0.00 & 0.208 & 0.000 & -0.045 & -0.010 & -1.20 & 0.93 & 1250.14 & 54.74 & & & 1.06 & 55.61 \\
\hline 113 & 168 & 0.18 & 0.00 & 0.05 & -0.01 & 0.197 & 0.000 & -0.047 & 0.000 & -1.41 & 0.65 & 1250.95 & 62.00 & & & 0.77 & 62.98 \\
\hline 114 & 169 & 0.18 & 0.00 & 0.05 & -0.01 & 0.197 & 0.000 & -0.047 & 0.000 & -1.45 & 0.55 & 1253.01 & 68.02 & & & 0.68 & 69.11 \\
\hline 115 & 170 & 0.15 & 0.00 & 0.05 & -0.01 & 0.163 & 0.000 & -0.051 & 0.002 & -1.75 & 0.23 & 1253.61 & 75.49 & & & 0.38 & 76.70 \\
\hline
\end{tabular}




\section{$Z=55(\mathrm{Cs})$}

$\begin{array}{rrrrrrrrrrrrrr}116 & 171 & 0.15 & 0.00 & 0.05 & -0.01 & 0.163 & 0.000 & -0.051 & 0.002 & -1.99 & -0.04 & 1255.60 & 81.57 \\ 117 & 172 & 0.13 & 0.00 & 0.04 & -0.02 & 0.140 & 0.000 & -0.042 & 0.014 & -2.32 & -0.67 & 1256.30 & 88.94 \\ 118 & 173 & 0.11 & 0.00 & 0.04 & -0.02 & 0.119 & 0.000 & -0.044 & 0.015 & -2.64 & -1.22 & 1258.32 & 94.98 \\ 119 & 174 & 0.11 & 0.00 & 0.04 & -0.02 & 0.119 & 0.000 & -0.044 & 0.015 & -3.43 & -1.98 & 1258.92 & 102.46 \\ 120 & 175 & 0.10 & 0.00 & 0.03 & -0.01 & 0.108 & 0.000 & -0.032 & 0.007 & -3.48 & -2.19 & 1260.39 & 109.06 \\ 121 & 176 & -0.12 & 0.00 & 0.01 & 0.00 & -0.125 & 0.000 & -0.006 & 0.001 & -4.51 & -3.22 & 1261.05 & 116.48 \\ 122 & 177 & -0.07 & 0.00 & 0.02 & 0.01 & -0.073 & 0.000 & -0.021 & -0.008 & -4.80 & -4.02 & 1262.88 & 122.71 \\ 123 & 178 & -0.07 & 0.00 & 0.02 & 0.00 & -0.073 & 0.000 & -0.021 & 0.002 & -6.15 & -5.23 & 1263.52 & 130.15 \\ 124 & 179 & -0.06 & 0.00 & 0.02 & 0.01 & -0.063 & 0.000 & -0.022 & -0.008 & -6.96 & -5.99 & 1265.10 & 136.64 \\ 125 & 180 & -0.01 & 0.00 & 0.00 & 0.00 & -0.011 & 0.000 & 0.000 & 0.000 & -8.34 & -7.27 & 1265.60 & 144.21 \\ 126 & 181 & 0.00 & 0.00 & 0.00 & 0.00 & 0.000 & 0.000 & 0.000 & 0.000 & -9.08 & -7.92 & 1266.86 & 151.02 \\ 127 & 182 & -0.01 & 0.00 & 0.00 & 0.00 & -0.011 & 0.000 & 0.000 & 0.000 & -8.25 & -7.17 & 1265.13 & 160.82 \\ 128 & 183 & -0.01 & 0.00 & 0.00 & 0.00 & -0.011 & 0.000 & 0.000 & 0.000 & -7.14 & -6.14 & 1264.53 & 169.50 \\ 129 & 184 & 0.02 & 0.00 & 0.00 & 0.00 & 0.021 & 0.000 & 0.000 & 0.000 & -6.17 & -5.20 & 1262.42 & 179.68 \\ 130 & 185 & -0.01 & 0.00 & 0.00 & 0.00 & -0.011 & 0.000 & 0.000 & 0.000 & -4.94 & -4.11 & 1261.56 & 188.61\end{array}$

$0.12 \quad 82.92$

$-0.51 \quad 90.42$

$-1.04 \quad 96.61$

$-1.80 \quad 104.22$

$-2.11 \quad 110.87$

$-3.22 \quad 118.35$

$\begin{array}{ll}-3.97 & 124.79\end{array}$

$-5.20 \quad 132.37$

$-5.93 \quad 139.05$

$-7.27 \quad 146.74$

$\begin{array}{ll}-7.92 & 153.72\end{array}$

$-7.17 \quad 163.70$

$-6.14 \quad 172.57$

$-5.20 \quad 182.95$

$-4.11 \quad 192.08$

\begin{tabular}{|c|c|c|c|c|c|c|c|c|c|c|c|c|c|c|c|c|c|}
\hline \multicolumn{18}{|c|}{$Z=56(\mathrm{Ba})$} \\
\hline 52 & 108 & 0.13 & 0.05 & -0.05 & 0.01 & 0.140 & -0.069 & 0.070 & 0.001 & -3.12 & -0.72 & 835.66 & -7.76 & & & -0.71 & -7.55 \\
\hline 53 & 109 & 0.15 & 0.08 & -0.05 & 0.02 & 0.163 & -0.110 & 0.073 & -0.005 & -3.47 & -0.17 & 849.69 & -13.73 & & & -0.17 & -13.53 \\
\hline 54 & 110 & 0.17 & 0.09 & -0.04 & 0.03 & 0.185 & -0.123 & 0.065 & -0.014 & -3.42 & -0.28 & 866.53 & -22.49 & & & -0.27 & -22.29 \\
\hline 55 & 111 & 0.17 & 0.10 & -0.04 & 0.03 & 0.186 & -0.136 & 0.066 & -0.013 & -3.43 & 0.13 & 879.90 & -27.80 & & & 0.13 & -27.62 \\
\hline 56 & 112 & 0.18 & 0.10 & -0.03 & 0.04 & 0.197 & -0.135 & 0.056 & -0.025 & -3.03 & 0.59 & 895.36 & -35.19 & & & 0.62 & -35.00 \\
\hline 57 & 113 & 0.19 & 0.10 & -0.03 & 0.04 & 0.208 & -0.135 & 0.057 & -0.024 & -2.63 & 1.09 & 907.37 & -39.13 & & & 1.09 & -38.97 \\
\hline 58 & 114 & 0.20 & 0.09 & -0.03 & 0.04 & 0.218 & -0.121 & 0.059 & -0.025 & -2.10 & 1.52 & 921.58 & -45.26 & 4 & 0.139 & 1.54 & -45.10 \\
\hline 59 & 115 & 0.24 & 0.00 & -0.07 & 0.01 & 0.261 & 0.000 & 0.114 & 0.016 & -2.25 & 1.88 & 933.01 & -48.62 & & & 1.90 & -48.49 \\
\hline 60 & 116 & 0.26 & 0.00 & -0.06 & 0.02 & 0.282 & 0.000 & 0.106 & 0.005 & -2.20 & 2.01 & 946.80 & -54.34 & & & 2.04 & -54.21 \\
\hline 61 & 117 & 0.26 & 0.00 & -0.05 & 0.02 & 0.282 & 0.000 & 0.094 & 0.001 & -1.75 & 2.17 & 957.77 & -57.24 & & & 2.18 & -57.15 \\
\hline 62 & 118 & 0.27 & 0.00 & -0.04 & 0.02 & 0.293 & 0.000 & 0.084 & -0.002 & -1.45 & 2.32 & 970.86 & -62.25 & & & 2.34 & -62.17 \\
\hline 63 & 119 & 0.26 & 0.00 & -0.03 & 0.01 & 0.283 & 0.000 & 0.068 & 0.004 & -0.89 & 2.57 & 981.09 & -64.41 & -6 & 0.200 & 2.58 & -64.37 \\
\hline 64 & 120 & 0.26 & 0.00 & -0.01 & 0.02 & 0.283 & 0.000 & 0.044 & -0.013 & -0.39 & 2.85 & 993.39 & -68.64 & -68 & 0.300 & 2.87 & -68.61 \\
\hline 65 & 121 & 0.26 & 0.00 & 0.00 & 0.02 & 0.284 & 0.000 & 0.032 & -0.016 & -0.35 & 2.87 & 1003.24 & -70.43 & -70.74 & 0.142 & 2.87 & -70.43 \\
\hline 66 & 122 & 0.25 & 0.00 & 0.01 & 0.01 & 0.273 & 0.000 & 0.016 & -0.010 & -0.10 & 2.78 & 1015.29 & -74.40 & -74.61 & 0.028 & 2.79 & -74.42 \\
\hline 67 & 123 & 0.25 & 0.00 & 0.02 & 0.01 & 0.274 & 0.000 & 0.004 & -0.013 & -0.29 & 2.71 & 1024.64 & -75.68 & -75 & 0.012 & 2.71 & -75.73 \\
\hline 68 & 124 & 0.24 & 0.00 & 0.03 & 0.01 & 0.263 & 0.000 & -0.010 & -0.016 & -0.21 & 2.73 & 1035.97 & -78.94 & -79.09 & 0.012 & 2.75 & -79.00 \\
\hline 69 & 125 & 0.24 & 0.00 & 0.04 & 0.01 & 0.264 & 0.000 & -0.022 & -0.019 & -0.55 & 2.55 & 1044.87 & -79.77 & -79.67 & 0.011 & 2.56 & -79.86 \\
\hline 70 & 126 & 0.24 & 0.00 & 0.05 & 0.00 & 0.264 & 0.000 & -0.036 & -0.012 & -0.62 & 2.55 & 1055.64 & -82.47 & -82.67 & 0.012 & 2.59 & -82.56 \\
\hline 71 & 127 & 0.23 & 0.00 & 0.04 & 0.01 & 0.253 & 0.000 & -0.024 & -0.019 & -0.57 & 2.35 & 1064.02 & -82.77 & -82.82 & 0.011 & 2.38 & -82.90 \\
\hline 72 & 128 & 0.21 & 0.00 & 0.03 & 0.01 & 0.229 & 0.000 & -0.016 & -0.016 & -0.21 & 2.22 & 1074.38 & -85.07 & -85.40 & 0.010 & 2.25 & -85.21 \\
\hline 73 & 129 & 0.20 & 0.00 & 0.03 & 0.01 & 0.218 & 0.000 & -0.018 & -0.016 & -0.43 & 1.85 & 41 & -85.02 & -8 & 1 & 1.87 & -85.20 \\
\hline 74 & 130 & 0.18 & 0.00 & 0.02 & 0.01 & 0.195 & 0.000 & -0.010 & -0.013 & -0.36 & 1.50 & 1092.45 & -86.99 & -87.26 & 0.003 & 1.53 & -87.19 \\
\hline 75 & 131 & 0.17 & 0.00 & 0.02 & 0.01 & 0.184 & 0.000 & -0.011 & -0.013 & -0.74 & 1.20 & 1099.91 & -86.38 & -86.68 & 0.003 & 1.22 & -86.61 \\
\hline 76 & 132 & 0.15 & 0.00 & 0.02 & 0.01 & 0.162 & 0.000 & -0.014 & -0.013 & -0.90 & 0.99 & 1109.31 & -87.71 & -88.43 & 0.001 & 1.01 & -87.96 \\
\hline 77 & 133 & 0.14 & 0.00 & 0.02 & 0.00 & 0.151 & 0.000 & -0.016 & -0.003 & -1.41 & 0.38 & 1116.60 & -86.93 & -87.55 & 0.001 & 0.39 & -87.21 \\
\hline 78 & 134 & -0.12 & 0.00 & 0.03 & 0.01 & -0.125 & 0.000 & -0.029 & -0.005 & -1.95 & -0.40 & .09 & -88.34 & -8 & 00 & -0.38 & -88.63 \\
\hline 79 & 135 & -0.12 & 0.00 & 0.03 & 0.01 & -0.125 & 0.000 & -0.029 & -0.005 & -2.83 & -1.32 & 1133.23 & -87.41 & -87.85 & 0.000 & -1.30 & -87.72 \\
\hline 80 & 136 & 0.02 & 0.00 & 0.00 & 0.00 & 0.021 & 0.000 & 0.000 & 0.000 & -3.57 & -2.75 & 1142.89 & -89.00 & -8 & 0.000 & -2.75 & -89.36 \\
\hline 81 & 137 & 0.05 & 0.00 & 0.00 & 0.00 & 0.053 & 0.000 & 0.001 & 0.000 & -4.83 & -3.74 & 1149.66 & -87.70 & -87.72 & 0.000 & -3.74 & -88.07 \\
\hline 82 & 138 & 0.00 & 0.00 & 0.00 & 0.00 & 0.000 & 0.000 & 0.000 & 0.000 & -6.12 & -5.02 & 1158.72 & -88.69 & -8 & 0.000 & -5.02 & -89.07 \\
\hline 83 & 139 & -0.03 & 0.00 & 0.00 & 0.00 & -0.032 & 0.000 & 0.000 & 0.000 & -5.23 & -4.24 & 30 & -85.20 & -84 & 0. & -4.24 & -85.60 \\
\hline 84 & 140 & 0.00 & 0.00 & 0.00 & 0.00 & 0.000 & 0.000 & 0.000 & 0.000 & -4.05 & -3.21 & 1169.61 & -83.44 & -83.27 & 0.008 & -3.21 & -83.85 \\
\hline 85 & 141 & 0.09 & 0.05 & -0.04 & 0.00 & 0.097 & -0.069 & 0.053 & 0.007 & -4.20 & -2.05 & 1173.41 & -79.16 & -79.73 & 0.008 & -1.98 & -79.50 \\
\hline 86 & 142 & 0.12 & 0.06 & -0.05 & 0.01 & 0.130 & -0.083 & 0.069 & 0.001 & -4.20 & -1.33 & 1179.62 & -77.30 & -77.82 & 0.006 & -1.20 & -77.60 \\
\hline 87 & 143 & 0.14 & 0.08 & -0.05 & 0.01 & 0.152 & -0.111 & 0.072 & 0.005 & -4.76 & -1.07 & 1183.92 & -73.53 & -73.94 & 0.013 & -0.92 & -73.80 \\
\hline 88 & 144 & 0.15 & 0.09 & -0.05 & 0.02 & 0.163 & -0.124 & 0.074 & -0.004 & -4.66 & -0.60 & 1189.97 & -71.52 & -71.77 & 0.013 & -0.40 & -71.74 \\
\hline 89 & 145 & 0.16 & 0.10 & -0.05 & 0.02 & 0.175 & -0.138 & 0.076 & -0.001 & -4.88 & -0.32 & 1193.87 & -67.34 & -67.42 & 0.071 & -0.10 & -67.55 \\
\hline 90 & 146 & 0.16 & 0.09 & -0.05 & 0.02 & 0.174 & -0.124 & 0.075 & -0.003 & -3.98 & -0.11 & 1199.80 & -65.20 & -65.00 & 0.072 & 0.12 & -65.40 \\
\hline 91 & 147 & 0.17 & 0.09 & -0.05 & 0.03 & 0.185 & -0.123 & 0.077 & -0.012 & -3.87 & 0.18 & 1203.33 & -60.66 & & & 0.42 & -60.83 \\
\hline 92 & 148 & 0.21 & 0.00 & -0.08 & 0.01 & 0.228 & 0.000 & 0.120 & 0.016 & -3.92 & 0.54 & 1208.73 & -57.99 & -58.01 & 0.084 & 0.85 & -58.09 \\
\hline
\end{tabular}




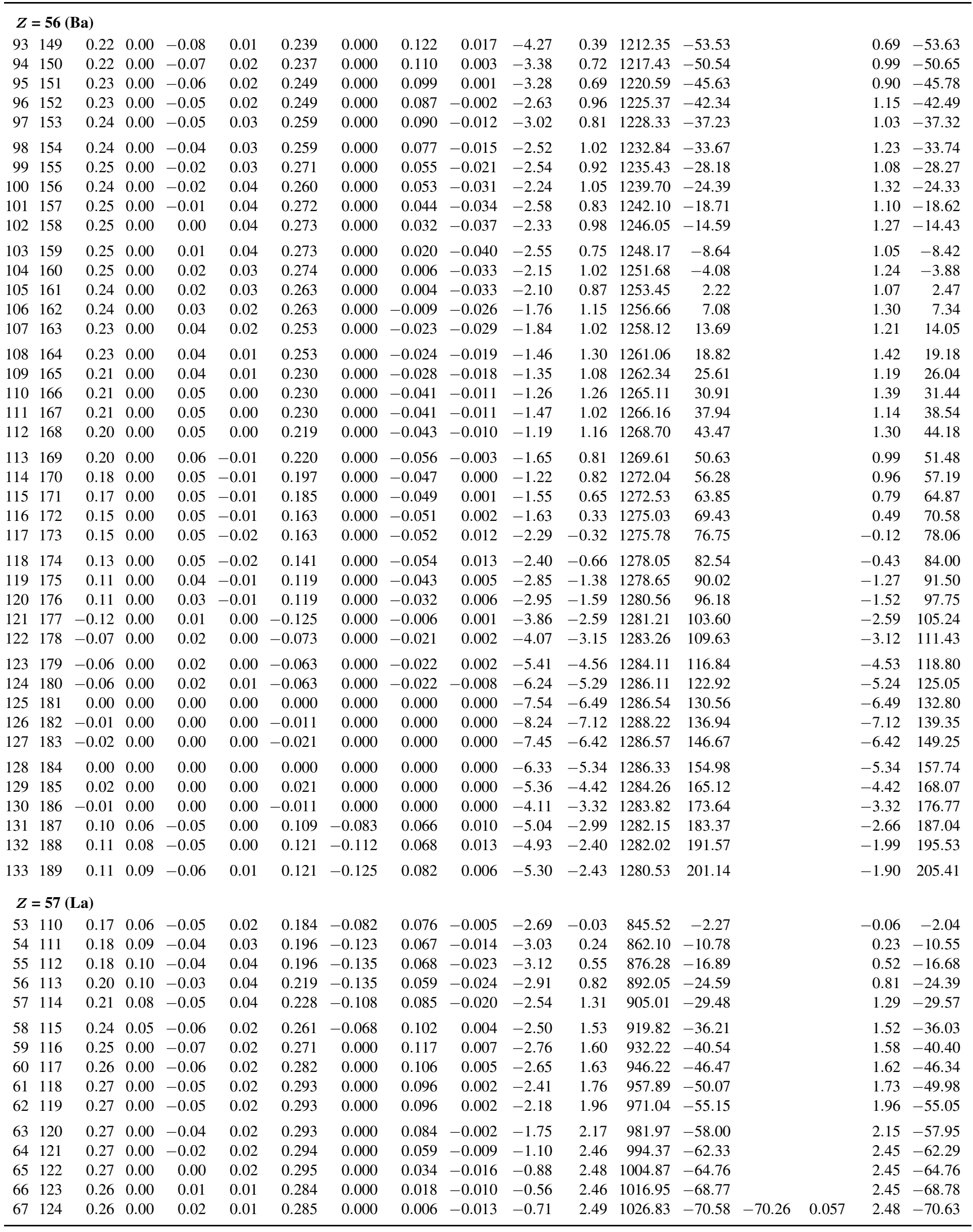




\begin{tabular}{|c|c|c|c|c|c|c|c|c|c|c|c|c|c|c|c|c|c|}
\hline \multicolumn{18}{|c|}{$Z=57($ La $)$} \\
\hline 68 & 125 & 0.26 & 0.00 & 0.03 & 0.01 & 0.286 & 0.000 & -0.006 & -0.016 & -0.69 & 2.53 & 1038.25 & -73.93 & -73.76 & 0.026 & 2.54 & -73.98 \\
\hline 69 & 126 & 0.26 & 0.00 & 0.04 & 0.00 & 0.286 & 0.000 & -0.019 & -0.010 & -0.92 & 2.43 & 1047.70 & -75.31 & -74.97 & 0.091 & 2.42 & -75.40 \\
\hline 70 & 127 & 0.25 & 0.00 & 0.04 & 0.00 & 0.275 & 0.000 & -0.022 & -0.009 & -0.65 & 2.50 & 1058.52 & -78.05 & -77.90 & 0.026 & 2.51 & -78.15 \\
\hline 71 & 128 & 0.24 & 0.00 & 0.04 & 0.00 & 0.264 & 0.000 & -0.024 & -0.009 & -0.62 & 2.36 & 1067.44 & -78.91 & -78.63 & 0.054 & 2.36 & -79.04 \\
\hline 72 & 129 & 0.22 & 0.00 & 0.03 & 0.01 & 0.241 & 0.000 & -0.014 & -0.016 & -0.19 & 2.36 & 1077.78 & -81.17 & -81.33 & 0.021 & 2.37 & -81.31 \\
\hline 73 & 130 & 0.21 & 0.00 & 0.02 & 0.01 & 0.229 & 0.000 & -0.004 & -0.013 & -0.25 & 2.09 & 1086.31 & -81.64 & -81.63 & 0.026 & 2.09 & -81.82 \\
\hline 74 & 131 & 0.19 & 0.00 & 0.02 & 0.01 & 0.206 & 0.000 & -0.008 & -0.013 & -0.11 & 1.93 & 1096.27 & -83.52 & -83.77 & 0.028 & 1.94 & -83.72 \\
\hline 75 & 132 & 0.17 & 0.00 & 0.03 & 0.00 & 0.185 & 0.000 & -0.024 & -0.005 & -0.37 & 1.67 & 1104.29 & -83.47 & -83.74 & 0.039 & 1.67 & -83.70 \\
\hline 76 & 133 & 0.16 & 0.00 & 0.03 & 0.00 & 0.174 & 0.000 & -0.025 & -0.005 & -0.67 & 1.25 & 1113.99 & -85.10 & -85.49 & 0.028 & 1.26 & -85.35 \\
\hline 77 & 134 & 0.14 & 0.00 & 0.02 & 0.00 & 0.151 & 0.000 & -0.016 & -0.003 & -0.94 & 0.83 & 1121.69 & -84.73 & -85.22 & 0.020 & 0.83 & -85.01 \\
\hline 78 & 135 & 0.12 & 0.00 & 0.02 & 0.00 & 0.129 & 0.000 & -0.018 & -0.002 & -1.30 & 0.15 & 1131.16 & -86.12 & -86.65 & 0.010 & 0.16 & -86.42 \\
\hline 79 & 136 & 0.10 & 0.00 & 0.01 & 0.00 & 0.107 & 0.000 & -0.008 & -0.001 & -1.97 & -0.66 & 1138.78 & -85.68 & -86.04 & 0.053 & -0.66 & -86.00 \\
\hline 80 & 137 & 0.05 & 0.00 & 0.00 & 0.00 & 0.053 & 0.000 & 0.001 & 0.000 & -2.84 & -1.88 & 1148.32 & -87.15 & -87.10 & 0.013 & -1.88 & -87.50 \\
\hline 81 & 138 & 0.05 & 0.00 & 0.01 & 0.00 & 0.053 & 0.000 & -0.011 & -0.001 & -4.06 & -3.00 & 1155.79 & -86.55 & -86.53 & 0.004 & -2.99 & -86.91 \\
\hline 82 & 139 & 0.00 & 0.00 & 0.00 & 0.00 & 0.000 & 0.000 & 0.000 & 0.000 & -5.29 & -4.22 & 1164.88 & -87.56 & -87.23 & 0.002 & -4.22 & -87.95 \\
\hline 83 & 140 & 0.05 & 0.00 & -0.01 & 0.00 & 0.053 & 0.000 & 0.013 & 0.001 & -4.49 & -3.40 & 1169.99 & -84.60 & -84.32 & 0.002 & -3.40 & -85.00 \\
\hline 84 & 141 & 0.05 & 0.00 & 0.00 & 0.00 & 0.053 & 0.000 & 0.001 & 0.000 & -3.32 & -2.35 & 1176.37 & -82.90 & -82.94 & 0.005 & -2.35 & -83.32 \\
\hline 85 & 142 & 0.10 & 0.06 & -0.03 & 0.00 & 0.108 & -0.083 & 0.042 & 0.007 & -3.62 & -1.37 & 1180.90 & -79.36 & -80.04 & 0.006 & -1.31 & -79.74 \\
\hline 86 & 143 & 0.12 & 0.08 & -0.04 & 0.00 & 0.131 & -0.111 & 0.057 & 0.012 & -3.97 & -0.77 & 1187.31 & -77.71 & -78.19 & 0.015 & -0.66 & -78.03 \\
\hline 87 & 144 & 0.14 & 0.09 & -0.05 & 0.01 & 0.153 & -0.125 & 0.073 & 0.006 & -4.61 & -0.60 & 1192.25 & -74.57 & -74.89 & 0.049 & -0.45 & -74.87 \\
\hline 88 & 145 & 0.16 & 0.09 & -0.05 & 0.02 & 0.174 & -0.124 & 0.075 & -0.003 & -4.42 & -0.19 & .45 & -72.70 & -72.99 & .090 & -0.02 & -72.97 \\
\hline 89 & 146 & 0.16 & 0.10 & -0.04 & 0.02 & 0.175 & -0.137 & 0.064 & -0.004 & -4.28 & -0.23 & 1203.21 & -69.39 & -69.12 & 0.071 & -0.07 & -69.68 \\
\hline 90 & 147 & 0.17 & 0.09 & -0.05 & 0.02 & 0.185 & -0.124 & 0.077 & -0.002 & -3.84 & 0.27 & 1208.92 & -67.03 & -66.85 & 0.048 & 0.46 & -67.29 \\
\hline 91 & 148 & 0.21 & 0.02 & -0.08 & 0.01 & 0.228 & -0.028 & 0.120 & 0.016 & -4.27 & 0.39 & 1213.16 & -63.19 & -63.13 & 0.059 & 0.63 & -63.40 \\
\hline 92 & 149 & 0.22 & 0.00 & -0.08 & 0.01 & 0.239 & 0.000 & 0.122 & 0.017 & -4.30 & 0.47 & 1218.91 & -60.88 & & & 0.74 & -61.06 \\
\hline 93 & 150 & 0.22 & 0.00 & -0.08 & 0.01 & 239 & 0.000 & 122 & 0.017 & -4.46 & 0.31 & 07 & -56.97 & & & 0.57 & -57.14 \\
\hline 94 & 151 & 0.23 & 0.00 & -0.07 & 0.02 & 0.249 & 0.000 & 0.112 & 0.004 & -3.83 & 0.62 & 1228.23 & -54.05 & & & 0.87 & -54.24 \\
\hline 95 & 152 & 0.24 & 0.00 & -0.06 & 0.02 & 0.260 & 0.000 & 0.101 & 0.002 & -3.75 & 0.56 & 1231.95 & -49.70 & & & 0.74 & -49.93 \\
\hline 96 & 153 & 0.24 & 0.00 & -0.06 & 0.03 & 0.259 & 0.000 & 0.102 & -0.008 & -3.45 & 0.77 & 1236.86 & -46.54 & & & 1.01 & -46.70 \\
\hline 97 & 154 & 0.24 & 0.00 & -0.05 & 0.03 & 0.259 & 0.000 & 0.090 & -0.012 & -3.31 & 0.61 & 1240.34 & -41.95 & & & 0.80 & -42.13 \\
\hline 98 & 155 & 0.25 & 0.00 & -0.04 & 0.03 & 0.271 & 0.000 & 0.079 & -0.014 & -3.00 & 0.84 & 1244.90 & -38.44 & & & 1.02 & -38.60 \\
\hline 99 & 156 & 0.25 & 0.00 & -0.03 & 0.03 & 0.271 & 0.000 & 0.067 & -0.017 & -2.96 & 0.73 & 1248.00 & -33.47 & & & 0.88 & -33.64 \\
\hline 100 & 157 & 0.25 & 0.00 & -0.02 & 0.04 & 0.271 & 0.000 & 0.056 & -0.031 & -2.70 & 0.83 & 1252.36 & -29.76 & & & 1.08 & -29.80 \\
\hline 101 & 158 & 0.25 & 0.00 & -0.01 & 0.04 & 0.272 & 0.000 & 0.044 & -0.034 & -2.83 & 0.65 & 1255.23 & -24.55 & & & 0.88 & -24.58 \\
\hline 102 & 159 & 0.25 & 0.00 & 0.00 & 0.04 & 0.273 & 0.000 & 0.032 & -0.037 & -2.56 & 0.81 & 1259.21 & -20.47 & & & 1.07 & -20.42 \\
\hline 103 & 160 & 0.25 & 0.00 & 0.01 & 0.04 & 0.273 & 0.000 & 0.020 & -0.040 & -2.76 & 0.60 & 1261.81 & -14.99 & & & 0.87 & -14.89 \\
\hline 104 & 161 & 0.25 & 0.00 & 0.01 & 0.03 & 0.273 & 0.000 & 0.018 & -0.030 & -2.30 & 0.93 & 1265.33 & -10.44 & & & 1.10 & -10.39 \\
\hline 105 & 162 & 0.25 & 0.00 & 0.02 & 0.03 & 0.274 & 0.000 & 0.006 & -0.033 & -2.41 & 0.79 & 1267.56 & -4.60 & & & 0.98 & -4.49 \\
\hline 106 & 163 & 0.24 & 0.00 & 0.03 & 0.02 & 0.263 & 0.000 & -0.009 & -0.026 & -1.84 & 1.10 & 1270.80 & 0.23 & & & 1.23 & 0.35 \\
\hline 107 & 164 & 0.24 & 0.00 & 0.04 & 0.01 & 0.264 & 0.000 & -0.022 & -0.019 & -1.92 & 1.04 & 1272.68 & 6.42 & & & 1.12 & 6.56 \\
\hline 108 & 165 & 0.23 & 0.00 & 0.04 & 0.01 & 0.253 & 0.000 & -0.024 & -0.019 & -1.44 & 1.33 & 1275.65 & 11.53 & & & 1.44 & 11.74 \\
\hline 109 & 166 & 0.23 & 0.00 & 0.05 & 0.00 & 0.253 & 0.000 & -0.038 & -0.012 & -1.60 & 1.22 & 1277.31 & 17.94 & & & 1.32 & 18.22 \\
\hline 110 & 167 & 0.21 & 0.00 & 0.05 & 0.00 & 0.230 & 0.000 & -0.041 & -0.011 & -1.11 & 1.35 & 1280.16 & 23.15 & & & 1.47 & 23.52 \\
\hline 111 & 168 & 0.21 & 0.00 & 0.06 & -0.01 & 0.231 & 0.000 & -0.054 & -0.004 & -1.53 & 1.16 & 1281.64 & 29.75 & & & 1.31 & 30.23 \\
\hline 112 & 169 & 0.20 & 0.00 & 0.06 & -0.01 & 0.220 & 0.000 & -0.056 & -0.003 & -1.24 & 1.26 & 1284.27 & 35.19 & & & 1.43 & 35.78 \\
\hline 113 & 170 & 0.20 & 0.00 & 0.07 & -0.02 & 0.220 & 0.000 & -0.069 & 0.005 & -1.80 & 0.86 & 1285.70 & 41.83 & & & 1.11 & 42.60 \\
\hline 114 & 171 & 0.18 & 0.00 & 0.06 & -0.01 & 0.197 & 0.000 & -0.059 & -0.002 & -1.28 & 0.98 & 1288.04 & 47.56 & & & 1.17 & 48.35 \\
\hline 115 & 172 & 0.17 & 0.00 & 0.06 & -0.02 & 0.186 & 0.000 & -0.062 & 0.009 & -1.65 & 0.71 & 1289.10 & 54.57 & & & 0.93 & 55.50 \\
\hline 116 & 173 & 0.15 & 0.00 & 0.05 & -0.01 & 0.163 & 0.000 & -0.051 & 0.002 & -1.46 & 0.53 & 1291.49 & 60.25 & & & 0.67 & 61.20 \\
\hline 117 & 174 & 0.15 & 0.00 & 0.05 & -0.02 & 0.163 & 0.000 & -0.052 & 0.012 & -2.14 & -0.14 & 1292.72 & 67.10 & & & 0.04 & 68.20 \\
\hline 118 & 175 & 0.14 & 0.00 & 0.05 & -0.02 & 0.152 & 0.000 & -0.053 & 0.012 & -2.28 & -0.42 & 1294.97 & 72.92 & & & -0.22 & 74.15 \\
\hline 119 & 176 & 0.11 & 0.00 & 0.04 & -0.01 & 0.119 & 0.000 & -0.043 & 0.005 & -2.55 & -1.08 & 1295.94 & 80.01 & & & -0.97 & 81.27 \\
\hline 120 & 177 & 0.11 & 0.00 & 0.04 & -0.02 & 0.119 & 0.000 & -0.044 & 0.015 & -2.81 & -1.38 & 1297.99 & 86.04 & & & -1.21 & 87.49 \\
\hline 121 & 178 & 0.10 & 0.00 & 0.03 & -0.01 & 0.108 & 0.000 & -0.032 & 0.007 & -3.46 & -2.26 & 1298.97 & 93.14 & & & -2.19 & 94.61 \\
\hline 122 & 179 & -0.07 & 0.00 & 0.02 & 0.00 & -0.073 & 0.000 & -0.021 & 0.002 & -3.69 & -2.72 & 1300.93 & 99.24 & & & -2.69 & 100.81 \\
\hline 123 & 180 & -0.06 & 0.00 & 0.02 & 0.00 & -0.063 & 0.000 & -0.022 & 0.002 & -4.98 & -4.15 & 1302.24 & 106.00 & & & -4.12 & 107.71 \\
\hline 124 & 181 & -0.06 & 0.00 & 0.02 & 0.01 & -0.063 & 0.000 & -0.022 & -0.008 & -5.81 & -4.87 & 1304.26 & 112.06 & & & -4.82 & 113.94 \\
\hline
\end{tabular}




\begin{tabular}{|c|c|c|c|c|c|c|c|c|c|c|c|c|c|c|c|c|c|}
\hline \multicolumn{18}{|c|}{$Z=57($ La $)$} \\
\hline 125 & 182 & 0.01 & 0.00 & 0.00 & 0.00 & 0.011 & 0.000 & 0.000 & 0.000 & -7.09 & -6.02 & 1305.08 & 119.31 & & & -6.02 & 121.28 \\
\hline 126 & 183 & 0.01 & 0.00 & 0.00 & 0.00 & 0.011 & 0.000 & 0.000 & 0.000 & -7.76 & -6.62 & 1306.76 & 125.70 & & & -6.62 & 127.84 \\
\hline 127 & 184 & -0.02 & 0.00 & 0.00 & 0.00 & -0.021 & 0.000 & 0.000 & 0.000 & -6.98 & -5.96 & 1305.57 & 134.96 & & & -5.96 & 137.26 \\
\hline 128 & 185 & 0.01 & 0.00 & 0.00 & 0.00 & 0.011 & 0.000 & 0.000 & 0.000 & -5.86 & -4.86 & 1305.34 & 143.26 & & & -4.86 & 145.73 \\
\hline 129 & 186 & 0.02 & 0.00 & 0.00 & 0.00 & 0.021 & 0.000 & 0.000 & 0.000 & -4.89 & -3.96 & 1303.71 & 152.96 & & & -3.96 & 155.60 \\
\hline 130 & 187 & 0.01 & 0.00 & 0.00 & 0.00 & 0.011 & 0.000 & 0.000 & 0.000 & -3.64 & -2.82 & 1303.25 & 161.50 & & & -2.83 & 164.32 \\
\hline 131 & 188 & 0.10 & 0.07 & -0.04 & 0.00 & 0.109 & -0.097 & 0.054 & 0.009 & -4.69 & -2.56 & 1302.06 & 170.75 & & & -2.30 & 174.02 \\
\hline 132 & 189 & 0.11 & 0.08 & -0.05 & 0.00 & 0.121 & -0.112 & 0.068 & 0.013 & -4.59 & -2.02 & 1302.00 & 178.89 & & & -1.63 & 182.48 \\
\hline 133 & 190 & 0.11 & 0.09 & -0.05 & 0.00 & 0.121 & -0.126 & 0.069 & 0.014 & -4.79 & -1.98 & 1300.85 & 188.10 & & & -1.56 & 191.93 \\
\hline 134 & 191 & 0.13 & 0.10 & -0.05 & 0.01 & 0.143 & -0.139 & 0.072 & 0.007 & -4.69 & -1.49 & 1300.65 & 196.38 & & & -1.04 & 200.43 \\
\hline 135 & 192 & 0.14 & 0.10 & -0.06 & 0.02 & 0.153 & -0.138 & 0.085 & -0.001 & -5.01 & -1.61 & 1299.48 & 205.61 & & & -1.05 & 209.98 \\
\hline \multicolumn{18}{|c|}{$Z=58(\mathrm{Ce})$} \\
\hline 55 & 113 & 0.20 & 0.08 & -0.04 & 0.04 & 0.217 & -0.108 & 0.071 & -0.024 & -2.41 & 0.98 & 874.02 & -7.33 & & & 0.96 & -7.06 \\
\hline 56 & 114 & 0.21 & 0.08 & -0.03 & 0.04 & 0.228 & -0.107 & 0.060 & -0.026 & -2.13 & 1.21 & 890.51 & -15.76 & & & 1.21 & -15.49 \\
\hline 57 & 115 & 0.23 & 0.06 & -0.05 & 0.03 & 0.249 & -0.082 & 0.088 & -0.010 & -2.16 & 1.47 & 904.09 & -21.27 & & & 1.45 & -21.04 \\
\hline 58 & 116 & 0.26 & 0.00 & -0.06 & 0.02 & 0.282 & 0.000 & 0.106 & 0.005 & -2.54 & 1.50 & 920.01 & -29.11 & & & 1.50 & -28.89 \\
\hline 59 & 117 & 0.26 & 0.00 & -0.06 & 0.02 & 0.282 & 0.000 & 0.106 & 0.005 & -2.77 & 1.44 & 932.66 & -33.69 & & & 1.42 & -33.51 \\
\hline 60 & 118 & 0.28 & 0.00 & -0.05 & 0.03 & 0.304 & 0.000 & 0.100 & -0.007 & -2.99 & 1.34 & 947.47 & -40.43 & & & 1.34 & -40.24 \\
\hline 61 & 119 & 0.28 & 0.00 & -0.05 & 0.03 & 0.304 & 0.000 & 0.100 & -0.007 & -2.93 & 1.45 & 959.27 & -44.16 & & & 1.43 & -44.01 \\
\hline 62 & 120 & 0.28 & 0.00 & -0.04 & 0.03 & 0.304 & 0.000 & 0.087 & -0.011 & -2.48 & 1.62 & 973.10 & -49.92 & & & 1.63 & -49.78 \\
\hline 63 & 121 & 0.28 & 0.00 & -0.03 & 0.03 & 0.305 & 0.000 & 0.075 & -0.015 & -2.13 & 1.79 & 984.19 & -52.94 & & & 1.78 & -52.84 \\
\hline 64 & 122 & 0.28 & 0.00 & -0.02 & 0.02 & 0.305 & 0.000 & 0.061 & -0.008 & -1.64 & 2.00 & 997.32 & -58.00 & & & 2.00 & -57.91 \\
\hline 65 & 123 & 0.28 & 0.00 & -0.01 & 0.02 & 0.306 & 0.000 & 0.049 & -0.012 & -1.49 & 2.08 & 1007.87 & -60.47 & & & 2.06 & -60.43 \\
\hline 66 & 124 & 0.27 & 0.00 & 0.00 & 0.01 & 0.295 & 0.000 & 0.033 & -0.006 & -1.04 & 2.21 & 1020.44 & -64.97 & & & 2.22 & -64.93 \\
\hline 67 & 125 & 0.27 & 0.00 & 0.01 & 0.00 & 0.296 & 0.000 & 0.020 & 0.000 & -1.03 & 2.24 & 1030.43 & -66.89 & & & 2.23 & -66.90 \\
\hline 68 & 126 & 0.27 & 0.00 & 0.03 & 0.00 & 0.297 & 0.000 & -0.005 & -0.007 & -0.93 & 2.35 & 1042.42 & -70.81 & -70.82 & 0.028 & 2.35 & -70.83 \\
\hline 69 & 127 & 0.27 & 0.00 & 0.03 & 0.00 & 0.297 & 0.000 & -0.005 & -0.007 & -1.06 & 2.32 & 1051.90 & -72.22 & -71.98 & 0.058 & 2.31 & -72.28 \\
\hline 70 & 128 & 0.26 & 0.00 & 0.04 & 0.00 & 0.286 & 0.000 & -0.019 & -0.010 & -0.81 & 2.46 & 1063.26 & -75.51 & -75.53 & 0.028 & 2.48 & -75.57 \\
\hline 71 & 129 & 0.25 & 0.00 & 0.04 & 0.00 & 0.275 & 0.000 & -0.022 & -0.009 & -0.70 & 2.41 & 1072.21 & -76.38 & -76.29 & 0.028 & 2.42 & -76.49 \\
\hline 72 & 130 & 0.23 & 0.00 & 0.03 & 0.01 & 0.252 & 0.000 & -0.013 & -0.016 & -0.17 & 2.49 & 1083.07 & -79.17 & -79.42 & 0.028 & 2.52 & -79.29 \\
\hline 73 & 131 & 0.21 & 0.00 & 0.03 & 0.01 & 0.229 & 0.000 & -0.016 & -0.016 & -0.10 & 2.28 & 1091.65 & -79.68 & -79.71 & 0.034 & 2.29 & -79.83 \\
\hline 74 & 132 & 0.19 & 0.00 & 0.03 & 0.01 & 0.207 & 0.000 & -0.020 & -0.015 & 0.09 & 2.11 & 1102.22 & -82.18 & -82.47 & 0.021 & 2.13 & -82.35 \\
\hline 75 & 133 & 0.18 & 0.00 & 0.03 & 0.00 & 0.196 & 0.000 & -0.023 & -0.005 & -0.14 & 1.89 & 1110.30 & -82.19 & -82.42 & 0.016 & 1.90 & -82.39 \\
\hline 76 & 134 & 0.17 & 0.00 & 0.03 & 0.00 & 0.185 & 0.000 & -0.024 & -0.005 & -0.38 & 1.61 & 1120.45 & -84.27 & -84.84 & 0.020 & 1.63 & -84.50 \\
\hline 77 & 135 & 0.15 & 0.00 & 0.03 & 0.00 & 0.162 & 0.000 & -0.027 & -0.005 & -0.70 & 1.10 & 1128.33 & -84.08 & -84.62 & 0.011 & 1.12 & -84.33 \\
\hline 78 & 136 & 0.12 & 0.00 & 0.02 & 0.00 & 0.129 & 0.000 & -0.018 & -0.002 & -0.79 & 0.68 & 1138.13 & -85.81 & -86.47 & 0.013 & 0.69 & -86.10 \\
\hline 79 & 137 & 0.12 & 0.00 & 0.02 & 0.00 & 0.129 & 0.000 & -0.018 & -0.002 & -1.58 & -0.13 & 1145.84 & -85.45 & -85.88 & 0.013 & -0.12 & -85.76 \\
\hline 80 & 138 & 0.06 & 0.00 & 0.01 & 0.00 & 0.064 & 0.000 & -0.010 & -0.001 & -2.24 & -1.25 & .87 & -87.40 & & 010 & -1.25 & -87.74 \\
\hline 81 & 139 & 0.05 & 0.00 & 0.01 & -0.01 & 0.053 & 0.000 & -0.011 & 0.009 & -3.43 & -2.40 & 1163.46 & -86.92 & -86.95 & 0.007 & -2.39 & -87.28 \\
\hline 82 & 140 & 0.00 & 0.00 & 0.00 & 0.00 & 0.000 & 0.000 & 0.000 & 0.000 & -4.65 & -3.62 & 1173.12 & -88.51 & -88.08 & 0.002 & -3.62 & -88.89 \\
\hline 83 & 141 & -0.03 & 0.00 & 0.00 & 0.00 & -0.032 & 0.000 & 0.000 & 0.000 & -3.74 & -2.84 & 1178.35 & -85.67 & -85.44 & 0.002 & -2.84 & -86.07 \\
\hline 84 & 142 & 0.01 & 0.00 & 0.00 & 0.00 & 0.011 & 0.000 & 0.000 & 0.000 & -2.57 & -1.79 & 1185.30 & -84.55 & -84.54 & 0.003 & -1.79 & -84.96 \\
\hline 85 & 143 & 0.12 & 0.06 & -0.03 & 0.00 & 0.130 & -0.083 & 0.044 & 0.008 & -3.22 & -0.79 & .88 & -81.06 & 61 & 0.003 & -0.73 & -81.43 \\
\hline 86 & 144 & 0.13 & 0.07 & -0.04 & 0.01 & 0.141 & -0.097 & 0.058 & 0.001 & -3.16 & -0.22 & 1196.88 & -79.99 & -80.44 & 0.003 & -0.12 & -80.33 \\
\hline 87 & 145 & 0.15 & 0.08 & -0.04 & 0.01 & 0.163 & -0.110 & 0.061 & 0.003 & -3.57 & -0.04 & 1201.90 & -76.93 & -77.10 & 0.041 & 0.07 & -77.27 \\
\hline 88 & 146 & 0.16 & 0.09 & -0.05 & 0.02 & 0.174 & -0.124 & 0.075 & -0.003 & -3.88 & 0.04 & 1208.96 & -75.93 & -75.68 & 0.066 & 0.22 & -76.21 \\
\hline 89 & 147 & 0.17 & 0.08 & -0.05 & 0.02 & 0.185 & -0.110 & 0.077 & -0.003 & -3.49 & 0.33 & 1213.47 & -72.37 & -72.03 & 0.031 & 0.50 & -72.68 \\
\hline 90 & 148 & 0.19 & 0.07 & -0.05 & 0.02 & 0.206 & -0.096 & 0.080 & -0.002 & -3.14 & 0.74 & 1219.82 & -70.64 & -70.39 & 0.029 & 0.92 & -70.94 \\
\hline 91 & 149 & 0.21 & 0.00 & -0.08 & 0.01 & 0.228 & 0.000 & 0.120 & 0.016 & -4.11 & 0.46 & 1224.53 & -67.28 & -66.69 & 0.097 & 0.70 & -67.51 \\
\hline 92 & 150 & 0.22 & 0.00 & -0.08 & 0.01 & 0.239 & 0.000 & 0.122 & 0.017 & -4.33 & 0.49 & 1230.88 & -65.55 & -64.82 & 0.048 & 0.77 & -65.75 \\
\hline 93 & 151 & 0.23 & 0.00 & -0.07 & 0.02 & 0.249 & 0.000 & 0.112 & 0.004 & -4.25 & 0.31 & 1235.12 & -61.73 & -61.50 & 0.103 & 0.53 & -61.97 \\
\hline 94 & 152 & 0.24 & 0.00 & -0.07 & 0.03 & 0.259 & 0.000 & 0.115 & -0.005 & -4.22 & 0.48 & 1240.95 & -59.49 & & & 0.75 & -59.68 \\
\hline 95 & 153 & 0.24 & 0.00 & -0.06 & 0.03 & 0.259 & 0.000 & 0.102 & -0.008 & -4.00 & 0.34 & 1244.80 & -55.27 & & & 0.57 & -55.50 \\
\hline 96 & 154 & 0.25 & 0.00 & -0.05 & 0.03 & 0.270 & 0.000 & 0.092 & -0.010 & -3.63 & 0.53 & 1250.27 & -52.66 & & & 0.74 & -52.89 \\
\hline 97 & 155 & 0.25 & 0.00 & -0.05 & 0.03 & 0.270 & 0.000 & 0.092 & -0.010 & -3.85 & 0.33 & 1253.85 & -48.17 & & & 0.53 & -48.39 \\
\hline 98 & 156 & 0.25 & 0.00 & -0.04 & 0.03 & 0.271 & 0.000 & 0.079 & -0.014 & -3.33 & 0.54 & 1258.94 & -45.19 & & & 0.73 & -45.40 \\
\hline 99 & 157 & 0.26 & 0.00 & -0.03 & 0.04 & 0.282 & 0.000 & 0.071 & -0.026 & -3.64 & 0.37 & 1262.17 & -40.35 & & & 0.60 & -40.49 \\
\hline
\end{tabular}




\begin{tabular}{|c|c|c|c|c|c|c|c|c|c|c|c|c|c|c|c|c|c|}
\hline \multicolumn{18}{|c|}{$Z=58(\mathrm{Ce})$} \\
\hline 100 & 158 & 0.26 & 0.00 & -0.02 & 0.04 & 0.282 & 0.000 & 0.058 & -0.030 & -3.24 & 0.56 & 1266.94 & -37.05 & & & 0.81 & -37.15 \\
\hline 101 & 159 & 0.26 & 0.00 & -0.01 & 0.04 & 0.283 & 0.000 & 0.046 & -0.033 & -3.36 & 0.38 & 1269.86 & -31.90 & & & 0.62 & -31.99 \\
\hline 102 & 160 & 0.26 & 0.00 & 0.00 & 0.04 & 0.284 & 0.000 & 0.034 & -0.036 & -3.02 & 0.60 & 1274.30 & -28.26 & & & 0.87 & -28.28 \\
\hline 103 & 161 & 0.26 & 0.00 & 0.01 & 0.04 & 0.285 & 0.000 & 0.022 & -0.039 & -3.19 & 0.39 & 1276.94 & -22.84 & & & 0.67 & -22.82 \\
\hline 104 & 162 & 0.25 & 0.00 & 0.01 & 0.03 & 0.273 & 0.000 & 0.018 & -0.030 & -2.48 & 0.78 & 1280.90 & -18.72 & & & 0.96 & -18.75 \\
\hline 105 & 163 & 0.25 & 0.00 & 0.02 & 0.03 & 0.274 & 0.000 & 0.006 & -0.033 & -2.58 & 0.66 & 1283.16 & -12.91 & & & 0.85 & -12.89 \\
\hline 106 & 164 & 0.25 & 0.00 & 0.03 & 0.02 & 0.275 & 0.000 & -0.007 & -0.026 & -2.14 & 1.00 & 1286.86 & -8.54 & & & 1.14 & -8.51 \\
\hline 107 & 165 & 0.24 & 0.00 & 0.04 & 0.01 & 0.264 & 0.000 & -0.022 & -0.019 & -2.04 & 0.95 & 1288.78 & -2.38 & & & 1.05 & -2.34 \\
\hline 108 & 166 & 0.24 & 0.00 & 0.04 & 0.01 & 0.264 & 0.000 & -0.022 & -0.019 & -1.69 & 1.21 & 1292.26 & 2.20 & & & 1.33 & 2.32 \\
\hline 109 & 167 & 0.23 & 0.00 & 0.05 & 0.01 & 0.254 & 0.000 & -0.036 & -0.021 & -1.76 & 1.13 & 1293.93 & 8.60 & & & 1.28 & 8.81 \\
\hline 110 & 168 & 0.21 & 0.00 & 0.05 & 0.00 & 0.230 & 0.000 & -0.041 & -0.011 & -1.13 & 1.41 & 1297.12 & 13.48 & & & 1.53 & 13.74 \\
\hline 111 & 169 & 0.21 & 0.00 & 0.06 & 0.00 & 0.231 & 0.000 & -0.053 & -0.013 & -1.55 & 1.12 & 1298.73 & 19.94 & & & 1.29 & 20.31 \\
\hline 112 & 170 & 0.21 & 0.00 & 0.06 & -0.01 & 0.231 & 0.000 & -0.054 & -0.004 & -1.31 & 1.35 & 1301.71 & 25.04 & & & 1.52 & 25.49 \\
\hline 113 & 171 & 0.20 & 0.00 & 0.07 & -0.02 & 0.220 & 0.000 & -0.069 & 0.005 & -1.71 & 0.99 & 1303.14 & 31.68 & & & 1.24 & 32.30 \\
\hline 114 & 172 & 0.19 & 0.00 & 0.07 & -0.02 & 0.209 & 0.000 & -0.071 & 0.005 & -1.48 & 1.10 & 1305.96 & 36.93 & & & 1.38 & 37.66 \\
\hline 115 & 173 & 0.18 & 0.00 & 0.06 & -0.02 & 0.197 & 0.000 & -0.060 & 0.008 & -1.57 & 0.70 & 1307.18 & 43.78 & & & 0.92 & 44.54 \\
\hline 116 & 174 & 0.15 & 0.00 & 0.06 & -0.02 & 0.163 & 0.000 & -0.064 & 0.010 & -1.39 & 0.74 & 1309.81 & 49.22 & & & 0.99 & 50.10 \\
\hline 117 & 175 & 0.15 & 0.00 & 0.06 & -0.02 & 0.163 & 0.000 & -0.064 & 0.010 & -2.07 & 0.08 & 1311.06 & 56.05 & & & 0.33 & 57.03 \\
\hline 118 & 176 & 0.14 & 0.00 & 0.05 & -0.02 & 0.152 & 0.000 & -0.053 & 0.012 & -1.95 & -0.09 & 1313.66 & 61.51 & & & 0.11 & 62.56 \\
\hline 119 & 177 & 0.13 & 0.00 & 0.05 & -0.02 & 0.141 & 0.000 & -0.054 & 0.013 & -2.35 & -0.59 & 1314.51 & 68.74 & & & -0.38 & 69.90 \\
\hline 120 & 178 & 0.11 & 0.00 & 0.04 & -0.02 & 0.119 & 0.000 & -0.044 & 0.015 & -2.33 & -0.92 & 1317.03 & 74.29 & & & -0.74 & 75.54 \\
\hline 121 & 179 & 0.10 & 0.00 & 0.03 & -0.01 & 0.108 & 0.000 & -0.032 & 0.007 & -2.90 & -1.68 & 1317.92 & 81.47 & & & -1.61 & 82.73 \\
\hline 122 & 180 & -0.07 & 0.00 & 0.02 & 0.00 & -0.073 & 0.000 & -0.021 & 0.002 & -3.11 & -2.17 & 1320.36 & 87.10 & & & -2.14 & 88.44 \\
\hline 123 & 181 & -0.06 & 0.00 & 0.02 & 0.00 & -0.063 & 0.000 & -0.022 & 0.002 & -4.41 & -3.59 & 1321.69 & 93.84 & & & -3.56 & 95.32 \\
\hline 124 & 182 & -0.06 & 0.00 & 0.02 & 0.01 & -0.063 & 0.000 & -0.022 & -0.008 & -5.24 & -4.30 & 1324.14 & 99.46 & & & -4.25 & 101.10 \\
\hline 125 & 183 & 0.00 & 0.00 & 0.00 & 0.00 & 0.000 & 0.000 & 0.000 & 0.000 & -6.50 & -5.47 & 1325.01 & 106.67 & & & -5.47 & 108.40 \\
\hline 126 & 184 & 0.00 & 0.00 & 0.00 & 0.00 & 0.000 & 0.000 & 0.000 & 0.000 & -7.20 & -6.09 & 1327.13 & 112.61 & & & -6.09 & 114.49 \\
\hline 127 & 185 & -0.01 & 0.00 & 0.00 & 0.00 & -0.011 & 0.000 & 0.000 & 0.000 & -6.37 & -5.34 & 1325.88 & 121.94 & & & -5.34 & 123.97 \\
\hline 128 & 186 & 0.00 & 0.00 & 0.00 & 0.00 & 0.000 & 0.000 & 0.000 & 0.000 & -5.27 & -4.30 & 1326.14 & 129.75 & & & -4.30 & 131.94 \\
\hline 129 & 187 & 0.01 & 0.00 & 0.00 & 0.00 & 0.011 & 0.000 & 0.000 & 0.000 & -4.25 & -3.36 & 1324.49 & 139.47 & & & -3.36 & 141.83 \\
\hline 130 & 188 & 0.00 & 0.00 & 0.00 & 0.00 & 0.000 & 0.000 & 0.000 & 0.000 & -3.02 & -2.24 & 1324.47 & 147.57 & & & -2.24 & 150.09 \\
\hline 131 & 189 & 0.10 & 0.08 & -0.04 & 0.00 & 0.110 & -0.111 & 0.055 & 0.011 & -4.43 & -2.04 & 1323.37 & 156.74 & & & -1.74 & 159.74 \\
\hline 132 & 190 & 0.11 & 0.08 & -0.04 & 0.00 & 0.120 & -0.111 & 0.056 & 0.011 & -3.82 & -1.41 & 1323.63 & 164.54 & & & -1.11 & 167.74 \\
\hline 133 & 191 & 0.12 & 0.09 & -0.05 & 0.01 & 0.131 & -0.125 & 0.070 & 0.005 & -4.31 & -1.43 & 1322.56 & 173.68 & & & -1.03 & 177.16 \\
\hline 134 & 192 & 0.13 & 0.10 & -0.05 & 0.01 & 0.143 & -0.139 & 0.072 & 0.007 & -4.22 & -0.99 & 1322.83 & 181.49 & & & -0.54 & 185.21 \\
\hline 135 & 193 & 0.14 & 0.10 & -0.05 & 0.02 & 0.153 & -0.138 & 0.073 & -0.003 & -4.27 & -1.02 & 1321.59 & 190.80 & & & -0.55 & 194.74 \\
\hline 136 & 194 & 0.15 & 0.10 & -0.05 & 0.02 & 0.164 & -0.138 & 0.074 & -0.002 & -3.93 & -0.70 & 1321.78 & 198.68 & & & -0.22 & 202.84 \\
\hline 137 & 195 & 0.18 & 0.00 & -0.09 & 0.00 & 0.196 & 0.000 & 0.127 & 0.025 & -4.46 & -1.15 & 1320.79 & 207.75 & & & -0.36 & 212.42 \\
\hline \multicolumn{18}{|c|}{$Z=59(\operatorname{Pr})$} \\
\hline 56 & 115 & 0.24 & 0.00 & -0.06 & 0.02 & 0.260 & 0.000 & 0.101 & 0.002 & -2.36 & 1.11 & 886.44 & -4.40 & & & 1.07 & -4.10 \\
\hline 57 & 116 & 0.26 & 0.00 & -0.06 & 0.02 & 0.282 & 0.000 & 0.106 & 0.005 & -2.81 & 1.18 & 900.88 & -10.77 & & & 1.12 & -10.52 \\
\hline 58 & 117 & 0.27 & 0.00 & -0.05 & 0.03 & 0.293 & 0.000 & 0.097 & -0.008 & -2.97 & 0.99 & 917.14 & -18.96 & & & 0.94 & -18.71 \\
\hline 59 & 118 & 0.27 & 0.00 & -0.05 & 0.03 & 0.293 & 0.000 & 0.097 & -0.008 & -3.24 & 0.87 & 930.78 & -24.52 & & & 0.81 & -24.57 \\
\hline 60 & 119 & 0.28 & 0.00 & -0.05 & 0.03 & 0.304 & 0.000 & 0.100 & -0.007 & -3.60 & 0.78 & 945.94 & -31.61 & & & 0.74 & -31.41 \\
\hline 61 & 120 & 0.29 & 0.00 & -0.04 & 0.04 & 0.315 & 0.000 & 0.091 & -0.020 & -3.58 & 0.85 & 958.43 & -36.03 & & & 0.79 & -35.88 \\
\hline 62 & 121 & 0.29 & 0.00 & -0.03 & 0.03 & 0.316 & 0.000 & 0.078 & -0.014 & -3.12 & 0.97 & 972.43 & -41.96 & & & 0.94 & -41.81 \\
\hline 63 & 122 & 0.29 & 0.00 & -0.03 & 0.03 & 0.316 & 0.000 & 0.078 & -0.014 & -3.01 & 1.10 & 984.21 & -45.67 & & & 1.04 & -45.56 \\
\hline 64 & 123 & 0.29 & 0.00 & -0.02 & 0.03 & 0.316 & 0.000 & 0.065 & -0.018 & -2.55 & 1.42 & 997.33 & -50.72 & & & 1.40 & -50.61 \\
\hline 65 & 124 & 0.29 & 0.00 & -0.01 & 0.02 & 0.317 & 0.000 & 0.052 & -0.011 & -2.29 & 1.45 & 1008.57 & -53.88 & & & 1.41 & -53.83 \\
\hline 66 & 125 & 0.29 & 0.00 & 0.00 & 0.01 & 0.318 & 0.000 & 0.038 & -0.005 & -1.95 & 1.72 & 1021.11 & -58.36 & & & 1.69 & -58.31 \\
\hline 67 & 126 & 0.28 & 0.00 & 0.01 & 0.01 & 0.307 & 0.000 & 0.023 & -0.009 & -1.71 & 1.80 & 1031.69 & -60.86 & & & 1.76 & -60.85 \\
\hline 68 & 127 & 0.28 & 0.00 & 0.01 & 0.01 & 0.307 & 0.000 & 0.023 & -0.009 & -1.53 & 1.98 & 1043.70 & -64.80 & & & 1.97 & -64.80 \\
\hline 69 & 128 & 0.27 & 0.00 & 0.02 & 0.00 & 0.296 & 0.000 & 0.007 & -0.003 & -1.36 & 1.99 & 1053.76 & -66.79 & -66.33 & 0.030 & 1.97 & -66.84 \\
\hline 70 & 129 & 0.27 & 0.00 & 0.03 & 0.00 & 0.297 & 0.000 & -0.005 & -0.007 & -1.18 & 2.19 & 1065.18 & -70.14 & -69.77 & 0.030 & 2.18 & -70.19 \\
\hline 71 & 130 & 0.26 & 0.00 & 0.03 & 0.00 & 0.285 & 0.000 & -0.007 & -0.007 & -0.95 & 2.23 & 1074.64 & -71.53 & -71.18 & 0.064 & 2.22 & -71.62 \\
\hline 72 & 131 & 0.24 & 0.00 & 0.03 & 0.00 & 0.263 & 0.000 & -0.012 & -0.006 & -0.33 & 2.45 & 1085.47 & -74.28 & -74.28 & 0.052 & 2.45 & -74.39 \\
\hline 73 & 132 & 0.22 & 0.00 & 0.02 & 0.01 & 0.240 & 0.000 & -0.002 & -0.013 & -0.11 & 2.32 & 1094.57 & -75.32 & -75.21 & 0.057 & 2.31 & -75.46 \\
\hline
\end{tabular}




\begin{tabular}{|c|c|c|c|c|c|c|c|c|c|c|c|c|c|c|c|c|c|}
\hline \multicolumn{18}{|c|}{$Z=59(\operatorname{Pr})$} \\
\hline 74 & 133 & 0.21 & 0.00 & 0.02 & 0.01 & 0.229 & 0.000 & -0.004 & -0.013 & 0.08 & 2.26 & 1105.12 & -77.80 & -77.94 & 0.012 & 2.27 & -77.96 \\
\hline 75 & 134 & 0.19 & 0.00 & 0.03 & 0.00 & 0.207 & 0.000 & -0.021 & -0.006 & -0.13 & 2.12 & 1113.72 & -78.32 & -78.51 & 0.035 & 2.12 & -78.51 \\
\hline 76 & 135 & 0.17 & 0.00 & 0.04 & -0.01 & 0.185 & 0.000 & -0.037 & 0.003 & -0.21 & 1.87 & 1123.94 & -80.47 & -80.94 & 0.012 & 1.89 & -80.68 \\
\hline 77 & 136 & 0.15 & 0.00 & 0.04 & -0.01 & 0.163 & 0.000 & -0.039 & 0.003 & -0.55 & 1.37 & 1132.40 & -80.86 & -81.33 & 0.012 & 1.38 & -81.10 \\
\hline 78 & 137 & 0.13 & 0.00 & 0.03 & -0.01 & 0.140 & 0.000 & -0.030 & 0.006 & -0.61 & 1.03 & 1142.21 & -82.60 & -83.18 & 0.012 & 1.04 & -82.86 \\
\hline 79 & 138 & 0.12 & 0.00 & 0.03 & -0.01 & 0.129 & 0.000 & -0.031 & 0.006 & -1.20 & 0.29 & 1150.44 & -82.75 & -83.13 & 0.014 & 0.30 & -83.04 \\
\hline 80 & 139 & 0.07 & 0.00 & 0.01 & 0.00 & 0.075 & 0.000 & -0.010 & -0.001 & -1.70 & -0.69 & 1160.40 & -84.65 & -84.82 & 0.008 & -0.69 & -84.98 \\
\hline 81 & 140 & 0.06 & 0.00 & 0.01 & 0.00 & 0.064 & 0.000 & -0.010 & -0.001 & -2.84 & -1.80 & 1168.53 & -84.71 & -84.69 & 0.006 & -1.80 & -85.06 \\
\hline 82 & 141 & 0.00 & 0.00 & 0.00 & 0.00 & 0.000 & 0.000 & 0.000 & 0.000 & -4.02 & -3.02 & 1178.27 & -86.38 & -86.02 & 0.002 & -3.02 & -86.75 \\
\hline 83 & 142 & -0.03 & 0.00 & 0.00 & 0.00 & -0.032 & 0.000 & 0.000 & 0.000 & -3.12 & -2.24 & 1184.07 & -84.11 & -83.79 & 0.002 & -2.24 & -84.50 \\
\hline 84 & 143 & 0.03 & 0.00 & 0.00 & 0.00 & 0.032 & 0.000 & 0.000 & 0.000 & -1.98 & -1.18 & 1191.09 & -83.05 & -83.07 & 0.003 & -1.18 & -83.46 \\
\hline 85 & 144 & 0.12 & 0.07 & -0.03 & 0.00 & 0.130 & -0.097 & 0.044 & 0.009 & -2.97 & -0.34 & 1196.40 & -80.29 & -80.76 & 0.003 & -0.28 & -80.66 \\
\hline 86 & 145 & 0.13 & 0.07 & -0.04 & 0.00 & 0.141 & -0.097 & 0.058 & 0.011 & -2.71 & 0.23 & 1203.47 & -79.29 & -79.63 & 0.007 & 0.31 & -79.65 \\
\hline 87 & 146 & 0.15 & 0.08 & -0.04 & 0.01 & 0.163 & -0.110 & 0.061 & 0.003 & -3.11 & 0.19 & 1209.25 & -76.99 & -76.71 & 0.062 & 0.29 & -77.36 \\
\hline 88 & 147 & 0.17 & 0.07 & -0.05 & 0.02 & 0.184 & -0.096 & 0.076 & -0.004 & -3.03 & 0.50 & 1216.17 & -75.85 & -75.46 & 0.023 & 0.63 & -76.19 \\
\hline 89 & 148 & 0.19 & 0.05 & -0.06 & 0.02 & 0.205 & -0.069 & 0.092 & -0.002 & -3.13 & 0.63 & 1221.39 & -72.99 & -72.53 & 0.026 & 0.77 & -73.34 \\
\hline 90 & 149 & 0.21 & 0.00 & -0.07 & 0.02 & 0.227 & 0.000 & 0.108 & 0.002 & -3.39 & 0.75 & 1228.10 & -71.63 & -71.06 & 0.082 & 0.92 & -71.94 \\
\hline 91 & 150 & 0.22 & 0.00 & -0.08 & 0.02 & 0.238 & 0.000 & 0.123 & 0.006 & -4.44 & 0.37 & 1233.44 & -68.90 & -68.30 & 0.026 & 0.58 & -69.18 \\
\hline 92 & 151 & 0.23 & 0.00 & -0.07 & 0.02 & 0.249 & 0.000 & 0.112 & 0.004 & -4.26 & 0.37 & 1239.88 & -67.27 & -66.77 & 0.023 & 0.56 & -67.57 \\
\hline 93 & 152 & 0.24 & 0.00 & -0.07 & 0.02 & 0.260 & 0.000 & 0.114 & 0.006 & -4.74 & 0.15 & 1244.70 & -64.01 & -63.81 & 0.122 & 0.34 & -64.32 \\
\hline 94 & 153 & 0.24 & 0.00 & -0.07 & 0.03 & 0.259 & 0.000 & 0.115 & -0.005 & -4.54 & 0.25 & 1250.66 & -61.91 & -61.63 & 0.104 & 0.49 & -62.17 \\
\hline 95 & 154 & 0.25 & 0.00 & -0.06 & 0.03 & 0.270 & 0.000 & 0.105 & -0.007 & -4.56 & 0.03 & 1255.13 & -58.30 & -58.20 & 0.152 & 0.22 & -58.60 \\
\hline 96 & 155 & 0.25 & 0.00 & -0.05 & 0.03 & 0.270 & 0.000 & 0.092 & -0.010 & -3.99 & 0.26 & 1260.60 & -55.70 & & & 0.45 & -56.00 \\
\hline 97 & 156 & 0.26 & 0.00 & -0.04 & 0.03 & 0.282 & 0.000 & 0.082 & -0.013 & -4.15 & 0.08 & 1264.68 & -51.71 & & & 0.23 & -52.03 \\
\hline 98 & 157 & 0.26 & 0.00 & -0.03 & 0.03 & 0.282 & 0.000 & 0.069 & -0.016 & -3.70 & 0.27 & 1269.85 & -48.81 & & & 0.42 & -49.12 \\
\hline 99 & 158 & 0.26 & 0.00 & -0.03 & 0.04 & 0.282 & 0.000 & 0.071 & -0.026 & -4.03 & 0.06 & 1273.63 & -44.52 & & & 0.26 & -44.76 \\
\hline 100 & 159 & 0.26 & 0.00 & -0.02 & 0.04 & 0.282 & 0.000 & 0.058 & -0.030 & -3.58 & 0.30 & 1278.42 & -41.24 & & & 0.52 & -41.43 \\
\hline 101 & 160 & 0.27 & 0.00 & -0.01 & 0.04 & 0.294 & 0.000 & 0.049 & -0.032 & -3.94 & 0.04 & 1281.92 & -36.67 & & & 0.25 & -36.85 \\
\hline 102 & 161 & 0.27 & 0.00 & 0.00 & 0.04 & 0.295 & 0.000 & 0.037 & -0.036 & -3.58 & 0.28 & 1286.39 & -33.06 & & & 0.52 & -33.19 \\
\hline 103 & 162 & 0.27 & 0.00 & 0.01 & 0.04 & 0.296 & 0.000 & 0.025 & -0.039 & -3.70 & 0.14 & 1289.46 & -28.07 & & & 0.38 & -28.15 \\
\hline 104 & 163 & 0.26 & 0.00 & 0.01 & 0.03 & 0.285 & 0.000 & 0.021 & -0.030 & -2.95 & 0.52 & 1293.48 & -24.01 & & & 0.68 & -24.14 \\
\hline 105 & 164 & 0.26 & 0.00 & 0.02 & 0.03 & 0.285 & 0.000 & 0.009 & -0.033 & -2.97 & 0.50 & 1296.13 & -18.59 & & & 0.67 & -18.67 \\
\hline 106 & 165 & 0.25 & 0.00 & 0.02 & 0.02 & 0.274 & 0.000 & 0.005 & -0.023 & -2.26 & 0.88 & 1299.84 & -14.23 & & & 0.98 & -14.34 \\
\hline 107 & 166 & 0.25 & 0.00 & 0.04 & 0.01 & 0.275 & 0.000 & -0.020 & -0.019 & -2.35 & 0.84 & 1302.23 & -8.55 & & & 0.92 & -8.63 \\
\hline 108 & 167 & 0.24 & 0.00 & 0.04 & 0.01 & 0.264 & 0.000 & -0.022 & -0.019 & -1.79 & 1.15 & 1305.72 & -3.96 & & & 1.25 & -3.97 \\
\hline 109 & 168 & 0.24 & 0.00 & 0.05 & 0.00 & 0.264 & 0.000 & -0.036 & -0.012 & -1.88 & 1.10 & 1307.84 & 1.98 & & & 1.18 & 2.02 \\
\hline 110 & 169 & 0.23 & 0.00 & 0.05 & 0.00 & 0.253 & 0.000 & -0.038 & -0.012 & -1.38 & 1.43 & 1311.02 & 6.88 & & & 1.53 & 6.99 \\
\hline 111 & 170 & 0.21 & 0.00 & 0.06 & -0.01 & 0.231 & 0.000 & -0.054 & -0.004 & -1.43 & 1.23 & 1313.02 & 12.95 & & & 1.37 & 13.17 \\
\hline 112 & 171 & 0.21 & 0.00 & 0.06 & -0.01 & 0.231 & 0.000 & -0.054 & -0.004 & -1.22 & 1.43 & 1316.05 & 17.99 & & & 1.59 & 18.29 \\
\hline 113 & 172 & 0.21 & 0.00 & 0.07 & -0.02 & 0.231 & 0.000 & -0.068 & 0.004 & -1.71 & 1.12 & 1317.91 & 24.20 & & & 1.34 & 24.65 \\
\hline 114 & 173 & 0.19 & 0.00 & 0.07 & -0.02 & 0.209 & 0.000 & -0.071 & 0.005 & -1.33 & 1.28 & 1320.71 & 29.47 & & & 1.54 & 30.02 \\
\hline 115 & 174 & 0.18 & 0.00 & 0.07 & -0.03 & 0.197 & 0.000 & -0.073 & 0.016 & -1.74 & 0.75 & 1322.53 & 35.72 & & & 1.08 & 36.44 \\
\hline 116 & 175 & 0.15 & 0.00 & 0.06 & -0.02 & 0.163 & 0.000 & -0.064 & 0.010 & -1.20 & 0.95 & 1325.04 & 41.28 & & & 1.18 & 41.99 \\
\hline 117 & 176 & 0.15 & 0.00 & 0.06 & -0.03 & 0.163 & 0.000 & -0.065 & 0.020 & -1.98 & 0.20 & 1326.83 & 47.56 & & & 0.51 & 48.44 \\
\hline 118 & 177 & 0.14 & 0.00 & 0.06 & -0.02 & 0.152 & 0.000 & -0.065 & 0.011 & -1.98 & 0.07 & 1329.43 & 53.03 & & & 0.31 & 53.95 \\
\hline 119 & 178 & 0.13 & 0.00 & 0.05 & -0.02 & 0.141 & 0.000 & -0.054 & 0.013 & -2.10 & -0.33 & 1330.63 & 59.90 & & & -0.14 & 60.87 \\
\hline 120 & 179 & 0.11 & 0.00 & 0.04 & -0.02 & 0.119 & 0.000 & -0.044 & 0.015 & -2.00 & -0.60 & 1333.12 & 65.49 & & & -0.44 & 66.53 \\
\hline 121 & 180 & -0.12 & 0.00 & 0.01 & 0.00 & -0.125 & 0.000 & -0.006 & 0.001 & -2.51 & -1.27 & 1334.36 & 72.32 & & & -1.26 & 73.31 \\
\hline 122 & 181 & -0.07 & 0.00 & 0.01 & 0.00 & -0.073 & 0.000 & -0.010 & 0.001 & -2.69 & -1.80 & 1336.88 & 77.87 & & & -1.79 & 78.99 \\
\hline 123 & 182 & -0.06 & 0.00 & 0.02 & 0.00 & -0.063 & 0.000 & -0.022 & 0.002 & -4.06 & -3.24 & 1338.68 & 84.15 & & & -3.22 & 85.41 \\
\hline 124 & 183 & -0.06 & 0.00 & 0.02 & 0.01 & -0.063 & 0.000 & -0.022 & -0.008 & -4.87 & -3.95 & 1341.14 & 89.76 & & & -3.90 & 91.17 \\
\hline 125 & 184 & -0.02 & 0.00 & 0.00 & 0.00 & -0.021 & 0.000 & 0.000 & 0.000 & -6.08 & -5.08 & 1342.40 & 96.56 & & & -5.08 & 98.06 \\
\hline 126 & 185 & -0.01 & 0.00 & 0.00 & 0.00 & -0.011 & 0.000 & 0.000 & 0.000 & -6.72 & -5.63 & 1344.49 & 102.54 & & & -5.63 & 104.18 \\
\hline 127 & 186 & -0.02 & 0.00 & 0.00 & 0.00 & -0.021 & 0.000 & 0.000 & 0.000 & -5.92 & -4.93 & 1343.71 & 111.40 & & & -4.93 & 113.18 \\
\hline 128 & 187 & -0.01 & 0.00 & 0.00 & 0.00 & -0.011 & 0.000 & 0.000 & 0.000 & -4.79 & -3.85 & 1343.95 & 119.22 & & & -3.85 & 121.16 \\
\hline 129 & 188 & 0.02 & 0.00 & 0.00 & 0.00 & 0.021 & 0.000 & 0.000 & 0.000 & -3.80 & -2.96 & 1342.78 & 128.46 & & & -2.96 & 130.55 \\
\hline 130 & 189 & 0.07 & 0.07 & -0.03 & 0.00 & 0.077 & -0.097 & 0.040 & 0.007 & -3.87 & -2.02 & 1342.96 & 136.36 & & & -1.83 & 138.80 \\
\hline
\end{tabular}




\section{$Z=59(P r)$}

$\begin{array}{lllll}131 & 190 & 0.09 & 0.09 & -0.03\end{array}$ $\begin{array}{lllll}132 & 191 & 0.10 & 0.09 & -0.04\end{array}$ $\begin{array}{llllll}133 & 192 & 0.11 & 0.10 & -0.05\end{array}$ $\begin{array}{lllll}134 & 193 & 0.13 & 0.10 & -0.05\end{array}$ $\begin{array}{lllll}135 & 194 & 0.14 & 0.10 & -0.05\end{array}$

$\begin{array}{llllll}136 & 195 & 0.17 & 0.00 & -0.09\end{array}$ $\begin{array}{lllll}137 & 196 & 0.18 & 0.00 & -0.09\end{array}$ $\begin{array}{llllll}138 & 197 & 0.19 & 0.00 & -0.09\end{array}$ $\begin{array}{lllll}139 & 198 & 0.21 & 0.00 & -0.08\end{array}$

\section{$\boldsymbol{Z}=\mathbf{6 0}(\mathrm{Nd})$}

$\begin{array}{lllll}58 & 118 & 0.28 & 0.00 & -0.05\end{array}$ $\begin{array}{lllll}59 & 119 & 0.28 & 0.00 & -0.05\end{array}$ $\begin{array}{lllll}60 & 120 & 0.30 & 0.00 & -0.04\end{array}$ $\begin{array}{llllll}61 & 121 & 0.30 & 0.00 & -0.04\end{array}$ $\begin{array}{llllll}62 & 122 & 0.30 & 0.00 & -0.03\end{array}$

$\begin{array}{llllll}63 & 123 & 0.30 & 0.00 & -0.03\end{array}$ $\begin{array}{llllll}64 & 124 & 0.30 & 0.00 & -0.02\end{array}$ $\begin{array}{lllll}65 & 125 & 0.30 & 0.00 & -0.01\end{array}$ $\begin{array}{lllll}66 & 126 & 0.29 & 0.00 & 0.00\end{array}$ $\begin{array}{lllll}67 & 127 & 0.29 & 0.00 & 0.01\end{array}$

$\begin{array}{lllll}68 & 128 & 0.29 & 0.00 & 0.02\end{array}$

$\begin{array}{llllll}69 & 129 & 0.29 & 0.00 & 0.02\end{array}$

$\begin{array}{lllll}70 & 130 & 0.29 & 0.00 & 0.03\end{array}$

$\begin{array}{lllll}71 & 131 & 0.30 & 0.00 & 0.02\end{array}$

$\begin{array}{lllll}72 & 132 & 0.26 & 0.00 & 0.02\end{array}$

$\begin{array}{lllll}73 & 133 & 0.23 & 0.00 & 0.02\end{array}$

$\begin{array}{llllll}74 & 134 & 0.21 & 0.00 & 0.03\end{array}$

$\begin{array}{lllll}75 & 135 & 0.20 & 0.00 & 0.03\end{array}$

$\begin{array}{llll}76 & 136 & 0.17 & 0.00\end{array}$

$\begin{array}{llll}77 & 137 & 0.16 & 0.00\end{array}$

$\begin{array}{llll}78 & 138 & 0.13 & 0.00\end{array}$

$\begin{array}{llll}79 & 139 & 0.12 & 0.00\end{array}$

$\begin{array}{llll}80 & 140 & 0.04 & 0.00\end{array}$

$\begin{array}{llll}81 & 141 & 0.05 & 0.00\end{array}$

0.04

$0.04-0$.

0.03

0.03

0.00 $\begin{array}{lllll}82 & 142 & 0.00 & 0.00 & 0.00\end{array}$

$\begin{array}{lllll}83 & 143 & 0.03 & 0.00 & -0.01\end{array}$ $\begin{array}{lllll}84 & 144 & 0.00 & 0.00 & 0.00\end{array}$ $\begin{array}{llllll}85 & 145 & 0.10 & 0.07 & -0.02\end{array}$ $\begin{array}{llllll}86 & 146 & 0.14 & 0.06 & -0.04\end{array}$ $\begin{array}{llllll}87 & 147 & 0.16 & 0.06 & -0.04\end{array}$ $\begin{array}{llllll}88 & 148 & 0.18 & 0.06 & -0.05\end{array}$ $\begin{array}{llllll}89 & 149 & 0.20 & 0.03 & -0.06\end{array}$ $\begin{array}{llllll}90 & 150 & 0.22 & 0.00 & -0.07\end{array}$ $\begin{array}{lllll}91 & 151 & 0.23 & 0.00 & -0.07\end{array}$ $\begin{array}{llllll}92 & 152 & 0.24 & 0.00 & -0.07\end{array}$

$\begin{array}{lllll}93 & 153 & 0.24 & 0.00 & -0.07\end{array}$ $\begin{array}{llllll}94 & 154 & 0.25 & 0.00 & -0.06\end{array}$ $\begin{array}{lllll}95 & 155 & 0.25 & 0.00 & -0.06\end{array}$ $\begin{array}{llllll}96 & 156 & 0.26 & 0.00 & -0.05\end{array}$ $\begin{array}{lllll}97 & 157 & 0.26 & 0.00 & -0.05\end{array}$ $\begin{array}{lllll}98 & 158 & 0.27 & 0.00 & -0.03\end{array}$ $\begin{array}{lllll}99 & 159 & 0.27 & 0.00 & -0.02\end{array}$ $\begin{array}{lllll}100 & 160 & 0.27 & 0.00 & -0.02\end{array}$ $\begin{array}{lllll}101 & 161 & 0.27 & 0.00 & -0.01\end{array}$ $\begin{array}{lllll}102 & 162 & 0.28 & 0.00 & 0.00\end{array}$ $\begin{array}{llllll}103 & 163 & 0.27 & 0.00 & 0.01\end{array}$ $\begin{array}{lllll}104 & 164 & 0.27 & 0.00 & 0.01\end{array}$ $\begin{array}{llll}0.00 & 0.110 & -0.125\end{array}$ $\begin{array}{lll}0.01 & 0.121 & -0.139\end{array}$ $\begin{array}{lll}0.01 & 0.143 & -0.139\end{array}$ $\begin{array}{llll}0.02 & 0.153 & -0.138\end{array}$

$-0.01 \quad 0.186$ $0.00 \quad 0.196$ $\begin{array}{ll}0.00 & 0.207\end{array}$ $\begin{array}{ll}0.01 & 0.228\end{array}$

0.000

0.000

0.000

0.000 $\begin{array}{llll}0.00 & 0.099 & -0.125\end{array}$

$\begin{array}{lrrrrr}0.043 & 0.010 & -4.25 & -1.76 & 1342.22 & 145.17 \\ 0.056 & 0.012 & -3.83 & -1.18 & 1342.56 & 152.91 \\ 0.069 & 0.005 & -4.29 & -1.15 & 1341.86 & 161.68 \\ 0.072 & 0.007 & -3.97 & -0.68 & 1342.10 & 169.50 \\ 0.073 & -0.003 & -4.01 & -0.76 & 1341.33 & 178.35 \\ 0.126 & 0.035 & -4.05 & -0.69 & 1341.78 & 185.97 \\ 0.127 & 0.025 & -4.44 & -0.98 & 1341.03 & 194.79 \\ 0.129 & 0.027 & -4.46 & -0.95 & 1341.34 & 202.55 \\ 0.120 & 0.016 & -4.57 & -1.12 & 1340.30 & 211.66\end{array}$

$\begin{array}{llllll}0.043 & 0.010 & -4.25 & -1.76 & 1342.22 & 145.17\end{array}$

$\begin{array}{llllll}0.069 & 0.005 & -4.29 & -1.15 & 1341.86 & 161.68\end{array}$

$\begin{array}{llllll}0.072 & 0.007 & -3.97 & -0.68 & 1342.10 & 169.50\end{array}$

$\begin{array}{llllll}0.120 & 0.016 & -4.57 & -1.12 & 1340.30 & 211.66\end{array}$

$\begin{array}{ll}0.03 & 0.304\end{array}$ $\begin{array}{ll}0.03 & 0.304\end{array}$

$0.04 \quad 0.326$

$0.04 \quad 0.326$

$\begin{array}{ll}0.04 & 0.327\end{array}$

$\begin{array}{ll}0.04 & 0.327\end{array}$

$\begin{array}{ll}0.03 & 0.328\end{array}$

$\begin{array}{ll}0.03 & 0.328\end{array}$

$\begin{array}{ll}0.02 & 0.318\end{array}$

0.010 .318

$\begin{array}{ll}0.01 & 0.319\end{array}$

$\begin{array}{ll}0.00 & 0.319\end{array}$

$\begin{array}{ll}0.01 & 0.320\end{array}$

$\begin{array}{ll}0.00 & 0.330\end{array}$

$0.00 \quad 0.285$

$\begin{array}{ll}0.01 & 0.251\end{array}$

$\begin{array}{ll}0.00 & 0.229\end{array}$

$0.00 \quad 0.218$

$-0.01 \quad 0.185$

$-0.01 \quad 0.174$

$-0.01 \quad 0.140$

$\begin{array}{ll}-0.01 & 0.129\end{array}$

$\begin{array}{ll}0.00 & 0.043\end{array}$

$\begin{array}{ll}0.00 & 0.053\end{array}$

$0.00 \quad 0.000$

$\begin{array}{ll}-0.01 & 0.032\end{array}$

$0.00 \quad 0.000$

$\begin{array}{ll}0.00 & 0.109\end{array}$

$\begin{array}{llll}0.00 & 0.152 & -0.083\end{array}$

$\begin{array}{llll}0.01 & 0.173 & -0.083\end{array}$

$\begin{array}{llll}0.02 & 0.194 & -0.082\end{array}$

$\begin{array}{llll}0.02 & 0.216 & -0.041\end{array}$

$\begin{array}{ll}0.02 & 0.237\end{array}$

$\begin{array}{ll}0.02 & 0.249\end{array}$

$0.02 \quad 0.260$

$\begin{array}{ll}0.03 & 0.259\end{array}$

$\begin{array}{ll}0.03 & 0.270\end{array}$

$\begin{array}{ll}0.03 & 0.270\end{array}$

$\begin{array}{lll}0.04 & 0.281\end{array}$

$0.04 \quad 0.281$

0.000

0.000

$\begin{array}{lll}0.100 & -0.007 & -3.36\end{array}$

$\begin{array}{lllll}0.000 & 0.100 & -0.007 & -3.69\end{array}$

$\begin{array}{lll}0.094 & -0.019 & -4.16\end{array}$

$\begin{array}{lllll}0.000 & 0.094 & -0.019 & -4.23\end{array}$

$\begin{array}{lllll}0.000 & 0.082 & -0.023 & -3.85\end{array}$

$\begin{array}{lllll}0.000 & 0.082 & -0.023 & -3.69\end{array}$

0.000

$\begin{array}{llll}0.068 & -0.017 & -3.13\end{array}$

$\begin{array}{lllll}0.000 & 0.056 & -0.021 & -2.89\end{array}$

$\begin{array}{lllll}0.000 & 0.039 & -0.015 & -2.31\end{array}$

$\begin{array}{lll}0.026 & -0.009 & -2.15\end{array}$

$\begin{array}{lll}0.014 & -0.013 & -1.94\end{array}$

$\begin{array}{llll}0.012 & -0.003 & -1.91\end{array}$

$\begin{array}{llll}0.002 & -0.016 & -1.70\end{array}$

$\begin{array}{lllll}0.000 & 0.015 & -0.002 & -1.71\end{array}$

$\begin{array}{lllll}0.000 & 0.005 & -0.003 & -0.64\end{array}$

$\begin{array}{lllll}0.000 & -0.001 & -0.013 & -0.16\end{array}$

$\begin{array}{llll}0.000 & -0.018 & -0.006 & 0.21\end{array}$

$\begin{array}{lllll}0.000 & -0.019 & -0.006 & -0.06\end{array}$

$\begin{array}{lllll}0.000 & -0.037 & 0.003 & -0.03\end{array}$

$\begin{array}{lllll}0.000 & -0.038 & 0.003 & -0.40\end{array}$

$\begin{array}{lllll}0.000 & -0.030 & 0.006 & -0.30\end{array}$

$\begin{array}{lllll}0.000 & -0.031 & 0.006 & -0.86\end{array}$

$\begin{array}{lllll}0.000 & 0.001 & 0.000 & -1.14\end{array}$

$\begin{array}{llll}0.000 & -0.011 & -0.001 & -2.39\end{array}$

0.000

0.000

$$
0.000-3.67
$$

0.012

$0.010-2.74$

\section{$\begin{array}{llll}0.000 & 0.000 & -1.55\end{array}$}

$\begin{array}{lll}0.030 & 0.006 & -2.08\end{array}$

$\begin{array}{lll}0.058 & 0.011 & -2.04\end{array}$

$\begin{array}{llll}0.062 & 0.002 & -2.18\end{array}$

$\begin{array}{llll}0.078 & -0.004 & -2.46\end{array}$

$\begin{array}{lll}0.093 & -0.002 & -2.72\end{array}$

$\begin{array}{lll}0.110 & 0.003 & -3.49\end{array}$

$\begin{array}{lll}0.112 & 0.004 & -4.17\end{array}$

0.000

$\begin{array}{lll}0.114 & 0.006 & -4.55\end{array}$

$\begin{array}{lllll}0.000 & 0.115 & -0.005 & -4.82\end{array}$

$\begin{array}{lllll}0.000 & 0.105 & -0.007 & -4.55\end{array}$

0.000

$\begin{array}{lll}0.105 & -0.007 & -4.76\end{array}$

$\begin{array}{lll}0.75 & 915.92 & -10.44\end{array}$

$\begin{array}{llll}0.58 & 929.97 & -16.43\end{array}$

$\begin{array}{lll}0.31 & 946.23 & -24.61\end{array}$

$\begin{array}{llll}0.31 & 958.90 & -29.22\end{array}$

$\begin{array}{lll}0.48 & 973.51 & -35.74\end{array}$

$\begin{array}{llll}0.67 & 985.33 & -39.50\end{array}$

$\begin{array}{lll}0.94 & 999.15 & -45.25\end{array}$

$\begin{array}{llll}1.07 & 1010.39 & -48.42\end{array}$

$\begin{array}{lll}1.30 & 1023.60 & -53.56\end{array}$

$\begin{array}{lll}1.43 & 1034.24 & -56.12\end{array}$

$\begin{array}{lll}1.65 & 1046.84 & -60.65\end{array}$

$\begin{array}{lll}1.74 & 1056.93 & -62.67\end{array}$

$\begin{array}{lllll}2.00 & 1068.89 & -66.56 & -66.60\end{array}$

$\begin{array}{lllll}2.17 & 1078.33 & -67.93 & -67.77\end{array}$

$\begin{array}{lllll}2.49 & 1089.66 & -71.19 & -71.43\end{array}$

$\begin{array}{lllll}2.37 & 1098.85 & -72.30 & -72.33\end{array}$

$\begin{array}{lllll}2.32 & 1110.00 & -75.38 & -75.65\end{array}$

$\begin{array}{lllll}2.21 & 1118.66 & -75.97 & -76.21\end{array}$

$\begin{array}{lllll}2.06 & 1129.37 & -78.61 & -79.20\end{array}$

$\begin{array}{lllll}1.59 & 1137.89 & -79.06 & -79.58\end{array}$

$\begin{array}{lllll}1.28 & 1148.26 & -81.35 & -82.02\end{array}$

$\begin{array}{lllll}0.69 & 1156.41 & -81.44 & -81.99\end{array}$

$\begin{array}{lllll}-0.39 & 1167.07 & -84.02 & -84.25\end{array}$

$\begin{array}{lllll}-1.45 & 1175.23 & -84.11 & -84.20\end{array}$

$\begin{array}{lllll}-2.68 & 1185.55 & -86.36 & -85.96\end{array}$

$\begin{array}{lllll}-1.83 & 1191.36 & -84.10 & -84.01\end{array}$

$\begin{array}{lllll}-0.84 & 1199.01 & -83.68 & -83.75\end{array}$

$\begin{array}{lllll}0.11 & 1204.29 & -80.89 & -81.44\end{array}$

$\begin{array}{lllll}0.69 & 1211.91 & -80.44 & -80.93\end{array}$

$\begin{array}{lllll}0.70 & 1217.72 & -78.18 & -78.15\end{array}$

$\begin{array}{lllll}0.93 & 1225.26 & -77.65 & -77.41\end{array}$

$\begin{array}{lllll}0.92 & 1230.69 & -75.01 & -74.38\end{array}$

$\begin{array}{lllll}0.83 & 1238.15 & -74.39 & -73.69\end{array}$

$\begin{array}{llll}0.46 & 1243.55 & -71.73 & -70.95\end{array}$

$\begin{array}{llll}0.32 & 1250.67 & -70.77 & -70.16\end{array}$

$\begin{array}{llll}0.01 & 1255.65 & -67.68 & -67.35\end{array}$

$\begin{array}{llll}0.09 & 1262.16 & -66.12 & -65.69\end{array}$

$-0.12 \quad 1266.68-62.56$

0.000

$\begin{array}{llllllll}0.095 & -0.020 & -4.55 & -0.01 & 1272.80 & -60.61 & -60.53\end{array}$

$\begin{array}{lllllll}0.095 & -0.020 & -4.79 & -0.23 & 1276.98 & -56.72\end{array}$

$\begin{array}{ll}0.04 & 0.293\end{array}$

$0.04 \quad 0.294$

$\begin{array}{ll}0.04 & 0.294\end{array}$

$\begin{array}{ll}0.04 & 0.294\end{array}$

0.000

$\begin{array}{lllllll}0.073 & -0.026 & -4.34 & -0.11 & 1282.73 & -54.40\end{array}$

$\begin{array}{lllllll}0.000 & 0.061 & -0.029 & -4.40 & -0.28 & 1286.54 & -50.14\end{array}$

$\begin{array}{lllllll}0.000 & 0.061 & -0.029 & -4.17 & -0.07 & 1291.86 & -47.39\end{array}$

0.040 .306

0.000

$\begin{array}{lllllll}0.049 & -0.032 & -4.30 & -0.29 & 1295.38 & -42.84\end{array}$

$0.04 \quad 0.296$

0.000

$\begin{array}{llllll}0.039 & -0.035 & -4.14 & -0.04 & 1300.34 & -39.73\end{array}$

0.000

$\begin{array}{llllll}0.025 & -0.039 & -4.02 & -0.16 & 1303.45 & -34.77\end{array}$

$\begin{array}{llllll}0.023 & -0.029 & -3.40 & 0.28 & 1307.89 & -31.13\end{array}$

$-1.50 \quad 147.84$

$-0.85 \quad 155.82$

$-0.74 \quad 164.86$

$-0.26 \quad 172.88$

$\begin{array}{ll}-0.32 & 181.93\end{array}$

$0.17 \quad 190.16$

$-0.23 \quad 199.07$

$-0.18 \quad 207.06$

$-0.57 \quad 216.16$

$$
\begin{array}{lrr} 
& 0.72 & -10.11 \\
& 0.53 & -16.14 \\
& 0.28 & -24.33 \\
& 0.26 & -28.98 \\
& 0.46 & -35.51 \\
& 0.63 & -39.32 \\
& 0.93 & -45.08 \\
& 1.04 & -48.29 \\
& 1.29 & -53.45 \\
& 1.40 & -56.06 \\
0.028 & 2.00 & -66.56 \\
0.028 & 2.16 & -67.98 \\
0.024 & 2.50 & -71.25 \\
0.047 & 2.37 & -72.40 \\
0.012 & 2.33 & -75.50 \\
0.019 & 2.21 & -76.13 \\
0.012 & 2.08 & -78.79 \\
0.011 & 1.61 & -79.27 \\
0.012 & 1.30 & -81.59 \\
0.026 & 0.70 & -81.71 \\
0.028 & -0.39 & -84.33 \\
0.004 & -1.45 & -84.45 \\
0.002 & -2.68 & -86.72 \\
0.002 & -1.82 & -84.48 \\
0.002 & -0.84 & -84.09 \\
0.002 & 0.16 & -81.27 \\
0.002 & 0.78 & -80.80 \\
0.002 & 0.78 & -78.56 \\
0.003 & 1.07 & -77.99 \\
0.003 & 1.05 & -75.36 \\
0.003 & 1.02 & -74.71 \\
0.003 & 0.64 & -72.05 \\
0.025 & 0.54 & -71.07 \\
0.027 & 0.23 & -67.98 \\
& 0.31 & -66.42 \\
& 0.09 & -62.88 \\
& 0.24 & -60.88 \\
& 0.00 & -56.99 \\
& 0.12 & -54.67
\end{array}
$$$$
\begin{array}{llll}
0.024 & 2.50 & -71.25
\end{array}
$$$$
\begin{array}{lll}
0.012 & 2.33 & -75.50
\end{array}
$$$$
\begin{array}{llll}
0.012 & 2.08 & -78.79
\end{array}
$$$$
\begin{array}{llll}
0.012 & 1.30 & -81.59
\end{array}
$$$$
\begin{array}{lll}
0.028 & -0.39 & -84.33
\end{array}
$$$$
\begin{array}{llll}
0.002 & -2.68 & -86.72
\end{array}
$$$$
\begin{array}{llll}
0.002 & -0.84 & -84.09
\end{array}
$$$$
\begin{array}{lll}
0.002 & 0.78 & -80.80
\end{array}
$$$$
\begin{array}{lll}
0.003 & 1.07 & -77.99
\end{array}
$$$$
\begin{array}{llll}
0.003 & 1.02 & -74.71
\end{array}
$$$$
0.025
$$

0.114 


\begin{tabular}{|c|c|c|c|c|c|c|c|c|c|c|c|c|c|c|c|c|c|}
\hline \multicolumn{18}{|c|}{$Z=60(\mathrm{Nd})$} \\
\hline 105 & 165 & 0.26 & 0.00 & 0.02 & 0.03 & 0.285 & 0.000 & 0.009 & -0.033 & -3.19 & 0.30 & 1310.56 & -25.74 & & & 0.48 & -25.89 \\
\hline 106 & 166 & 0.26 & 0.00 & 0.02 & 0.02 & 0.285 & 0.000 & 0.007 & -0.023 & -2.60 & 0.74 & 1314.70 & -21.80 & & & 0.85 & -21.98 \\
\hline 107 & 167 & 0.25 & 0.00 & 0.04 & 0.01 & 0.275 & 0.000 & -0.020 & -0.019 & -2.52 & 0.68 & 1317.16 & -16.19 & & & 0.78 & -16.34 \\
\hline 108 & 168 & 0.25 & 0.00 & 0.04 & 0.01 & 0.275 & 0.000 & -0.020 & -0.019 & -2.11 & 1.01 & 1321.10 & -12.06 & & & 1.12 & -12.15 \\
\hline 109 & 169 & 0.24 & 0.00 & 0.05 & 0.00 & 0.264 & 0.000 & -0.036 & -0.012 & -2.03 & 0.98 & 1323.26 & -6.14 & & & 1.07 & -6.20 \\
\hline 110 & 170 & 0.23 & 0.00 & 0.05 & 0.00 & 0.253 & 0.000 & -0.038 & -0.012 & -1.48 & 1.31 & 1326.91 & -1.73 & & & 1.42 & -1.71 \\
\hline 111 & 171 & 0.23 & 0.00 & 0.06 & -0.01 & 0.254 & 0.000 & -0.051 & -0.005 & -1.74 & 1.22 & 1328.85 & 4.41 & & & 1.36 & 4.51 \\
\hline 112 & 172 & 0.21 & 0.00 & 0.07 & -0.02 & 0.231 & 0.000 & -0.068 & 0.004 & -1.52 & 1.37 & 1332.41 & 8.92 & & & 1.61 & 9.19 \\
\hline 113 & 173 & 0.21 & 0.00 & 0.07 & -0.02 & 0.231 & 0.000 & -0.068 & 0.004 & -1.79 & 1.09 & 1334.27 & 15.13 & & & 1.32 & 15.46 \\
\hline 114 & 174 & 0.20 & 0.00 & 0.07 & -0.02 & 0.220 & 0.000 & -0.069 & 0.005 & -1.48 & 1.22 & 1337.57 & 19.90 & & & 1.47 & 20.32 \\
\hline 115 & 175 & 0.18 & 0.00 & 0.07 & -0.03 & 0.197 & 0.000 & -0.073 & 0.016 & -1.70 & 0.83 & 1339.29 & 26.25 & & & 1.15 & 26.82 \\
\hline 116 & 176 & 0.17 & 0.00 & 0.07 & -0.03 & 0.186 & 0.000 & -0.075 & 0.017 & -1.56 & 1.01 & 1342.28 & 31.33 & & & 1.36 & 32.01 \\
\hline 117 & 177 & 0.15 & 0.00 & 0.07 & -0.03 & 0.164 & 0.000 & -0.077 & 0.018 & -2.07 & 0.33 & 1344.04 & 37.65 & & & 0.70 & 38.43 \\
\hline 118 & 178 & 0.15 & 0.00 & 0.06 & -0.02 & 0.163 & 0.000 & -0.064 & 0.010 & -1.87 & 0.27 & 1347.01 & 42.74 & & & 0.51 & 43.49 \\
\hline 119 & 179 & 0.14 & 0.00 & 0.05 & -0.02 & 0.152 & 0.000 & -0.053 & 0.012 & -1.89 & -0.05 & 1348.17 & 49.66 & & & 0.14 & 50.44 \\
\hline 120 & 180 & 0.11 & 0.00 & 0.05 & -0.02 & 0.119 & 0.000 & -0.056 & 0.014 & -1.80 & -0.28 & 1351.07 & 54.83 & & & -0.07 & 55.74 \\
\hline 121 & 181 & -0.12 & 0.00 & 0.01 & 0.00 & -0.125 & 0.000 & -0.006 & 0.001 & -2.10 & -0.99 & 1352.39 & 61.58 & & & -0.98 & 62.40 \\
\hline 122 & 182 & -0.08 & 0.00 & 0.01 & 0.00 & -0.084 & 0.000 & -0.009 & 0.001 & -2.31 & -1.40 & 1355.22 & 66.82 & & & -1.39 & 67.74 \\
\hline 123 & 183 & -0.06 & 0.00 & 0.02 & 0.00 & -0.063 & 0.000 & -0.022 & 0.002 & -3.61 & -2.81 & 1357.02 & 73.09 & & & -2.79 & 74.15 \\
\hline 124 & 184 & -0.06 & 0.00 & 0.03 & 0.01 & -0.063 & 0.000 & -0.033 & -0.007 & -4.52 & -3.53 & 1359.94 & 78.24 & & & -3.45 & 79.48 \\
\hline 125 & 185 & 0.00 & 0.00 & 0.00 & 0.00 & 0.000 & 0.000 & 0.000 & 0.000 & -5.64 & -4.63 & 1361.20 & 85.06 & & & -4.63 & 86.34 \\
\hline 126 & 186 & 0.00 & 0.00 & 0.00 & 0.00 & 0.000 & 0.000 & 0.000 & 0.000 & -6.29 & -5.21 & 1363.75 & 90.58 & & & -5.21 & 91.99 \\
\hline 127 & 187 & -0.01 & 0.00 & 0.00 & 0.00 & -0.011 & 0.000 & 0.000 & 0.000 & -5.44 & -4.44 & 1362.93 & 99.47 & & & -4.44 & 101.02 \\
\hline 128 & 188 & 0.00 & 0.00 & 0.00 & 0.00 & 0.000 & 0.000 & 0.000 & 0.000 & -4.34 & -3.40 & 1363.63 & 106.83 & & & -3.40 & 108.52 \\
\hline 129 & 189 & 0.01 & 0.00 & 0.00 & 0.00 & 0.011 & 0.000 & 0.000 & 0.000 & -3.30 & -2.43 & 1362.41 & 116.12 & & & -2.43 & 117.96 \\
\hline 130 & 190 & 0.07 & 0.07 & -0.03 & 0.00 & 0.077 & -0.097 & 0.040 & 0.007 & -3.44 & -1.59 & 1363.10 & 123.50 & & & -1.39 & 125.68 \\
\hline 131 & 191 & 0.09 & 0.09 & -0.03 & 0.00 & 0.099 & -0.125 & 0.043 & 0.010 & -3.83 & -1.32 & 1362.39 & 132.30 & & & -1.06 & 134.70 \\
\hline 132 & 192 & 0.10 & 0.09 & -0.03 & 0.00 & 0.110 & -0.125 & 0.043 & 0.010 & -3.19 & -0.66 & 1363.06 & 139.69 & & & -0.39 & 142.27 \\
\hline 133 & 193 & 0.11 & 0.09 & -0.05 & 0.00 & 0.121 & -0.126 & 0.069 & 0.014 & -3.53 & -0.62 & 1362.36 & 148.46 & & & -0.22 & 151.34 \\
\hline 134 & 194 & 0.13 & 0.10 & -0.05 & 0.01 & 0.143 & -0.139 & 0.072 & 0.007 & -3.42 & -0.12 & 1362.99 & 155.90 & & & 0.31 & 158.98 \\
\hline 135 & 195 & 0.14 & 0.10 & -0.05 & 0.02 & 0.153 & -0.138 & 0.073 & -0.003 & -3.44 & -0.16 & 1362.20 & 164.77 & & & 0.28 & 168.04 \\
\hline 136 & 196 & 0.18 & 0.00 & -0.08 & -0.01 & 0.196 & 0.000 & 0.114 & 0.033 & -3.34 & -0.11 & 1363.09 & 171.95 & & & 0.59 & 175.67 \\
\hline 137 & 197 & 0.19 & 0.00 & -0.08 & 0.00 & 0.206 & 0.000 & 0.116 & 0.023 & -3.77 & -0.42 & 1362.36 & 180.74 & & & 0.18 & 184.54 \\
\hline 138 & 198 & 0.20 & 0.00 & -0.08 & 0.01 & 0.216 & 0.000 & 0.118 & 0.014 & -3.72 & -0.37 & 1363.06 & 188.12 & & & 0.20 & 192.09 \\
\hline 139 & 199 & 0.21 & 0.00 & -0.08 & 0.01 & 0.228 & 0.000 & 0.120 & 0.016 & -4.32 & -0.80 & 1362.30 & 196.96 & & & -0.25 & 201.12 \\
\hline 140 & 200 & 0.21 & 0.00 & -0.08 & 0.02 & 0.227 & 0.000 & 0.120 & 0.005 & -4.23 & -0.83 & 1362.89 & 204.43 & & & -0.25 & 208.83 \\
\hline 141 & 201 & 0.22 & 0.00 & -0.07 & 0.02 & 0.237 & 0.000 & 0.110 & 0.003 & -4.49 & -1.22 & 1361.91 & 213.49 & & & -0.79 & 217.96 \\
\hline \multicolumn{18}{|c|}{$Z=61(\mathrm{Pm})$} \\
\hline 59 & 120 & 0.29 & 0.00 & -0.04 & 0.04 & 0.315 & 0.000 & 0.091 & -0.020 & -3.94 & 0.21 & 926.56 & -5.73 & & & 0.11 & -5.41 \\
\hline 60 & 121 & 0.30 & 0.00 & -0.04 & 0.05 & 0.326 & 0.000 & 0.095 & -0.029 & -4.59 & -0.10 & 942.96 & -14.06 & & & -0.16 & -13.73 \\
\hline 61 & 122 & 0.30 & 0.00 & -0.04 & 0.05 & 0.326 & 0.000 & 0.095 & -0.029 & -4.69 & -0.11 & 956.55 & -19.57 & & & -0.19 & -19.55 \\
\hline 62 & 123 & 0.31 & 0.00 & -0.03 & 0.05 & 0.338 & 0.000 & 0.086 & -0.031 & -4.57 & 0.01 & 971.56 & -26.51 & & & -0.05 & -26.25 \\
\hline 63 & 124 & 0.31 & 0.00 & -0.02 & 0.05 & 0.339 & 0.000 & 0.074 & -0.035 & -4.26 & 0.20 & 984.02 & -30.90 & & & 0.12 & -30.69 \\
\hline 64 & 125 & 0.31 & 0.00 & -0.01 & 0.04 & 0.340 & 0.000 & 0.060 & -0.030 & -3.70 & 0.47 & 997.94 & -36.75 & & & 0.42 & -36.55 \\
\hline 65 & 126 & 0.31 & 0.00 & 0.00 & 0.04 & 0.341 & 0.000 & 0.048 & -0.033 & -3.51 & 0.62 & 1009.80 & -40.54 & & & 0.55 & -40.39 \\
\hline 66 & 127 & 0.30 & 0.00 & 0.00 & 0.03 & 0.329 & 0.000 & 0.044 & -0.024 & -2.98 & 0.85 & 1023.12 & -45.78 & & & 0.81 & -45.64 \\
\hline 67 & 128 & 0.30 & 0.00 & 0.01 & 0.02 & 0.330 & 0.000 & 0.030 & -0.018 & -2.75 & 1.03 & 1034.33 & -48.92 & & & 0.97 & -48.83 \\
\hline 68 & 129 & 0.30 & 0.00 & 0.02 & 0.01 & 0.330 & 0.000 & 0.016 & -0.012 & -2.48 & 1.25 & 1047.03 & -53.55 & & & 1.22 & -53.47 \\
\hline 69 & 130 & 0.29 & 0.00 & 0.02 & 0.01 & 0.319 & 0.000 & 0.014 & -0.013 & -2.30 & 1.36 & 1057.71 & -56.16 & & & 1.31 & -56.13 \\
\hline 70 & 131 & 0.29 & 0.00 & 0.03 & 0.01 & 0.320 & 0.000 & 0.002 & -0.016 & -2.06 & 1.61 & 1069.78 & -60.16 & & & 1.59 & -60.14 \\
\hline 71 & 132 & 0.31 & 0.00 & 0.03 & 0.00 & 0.343 & 0.000 & 0.006 & -0.006 & -2.29 & 1.75 & 1079.86 & -62.17 & & & 1.71 & -62.20 \\
\hline 72 & 133 & 0.31 & 0.00 & 0.02 & 0.01 & 0.342 & 0.000 & 0.019 & -0.012 & -1.96 & 2.05 & 1091.30 & -65.54 & -65.41 & 0.050 & 2.04 & -65.58 \\
\hline 73 & 134 & 0.30 & 0.00 & 0.01 & 0.01 & 0.330 & 0.000 & 0.029 & -0.009 & -1.60 & 2.26 & 1100.77 & -66.94 & -66.74 & 0.058 & 2.23 & -67.03 \\
\hline 74 & 135 & 0.21 & 0.00 & 0.03 & 0.00 & 0.229 & 0.000 & -0.018 & -0.006 & 0.14 & 2.42 & 1111.80 & -69.90 & -69.98 & 0.059 & 2.42 & -69.99 \\
\hline 75 & 136 & 0.21 & 0.00 & 0.03 & -0.01 & 0.229 & 0.000 & -0.019 & 0.004 & -0.17 & 2.22 & 1121.14 & -71.16 & -71.20 & 0.078 & 2.21 & -71.30 \\
\hline 76 & 137 & 0.19 & 0.00 & 0.04 & -0.01 & 0.207 & 0.000 & -0.034 & 0.002 & -0.22 & 2.08 & 1131.94 & -73.89 & -74.07 & 0.013 & 2.09 & -74.05 \\
\hline 77 & 138 & 0.17 & 0.00 & 0.04 & -0.01 & 0.185 & 0.000 & -0.037 & 0.003 & -0.37 & 1.71 & 1140.94 & -74.83 & -74.94 & 0.027 & 1.71 & -75.01 \\
\hline
\end{tabular}




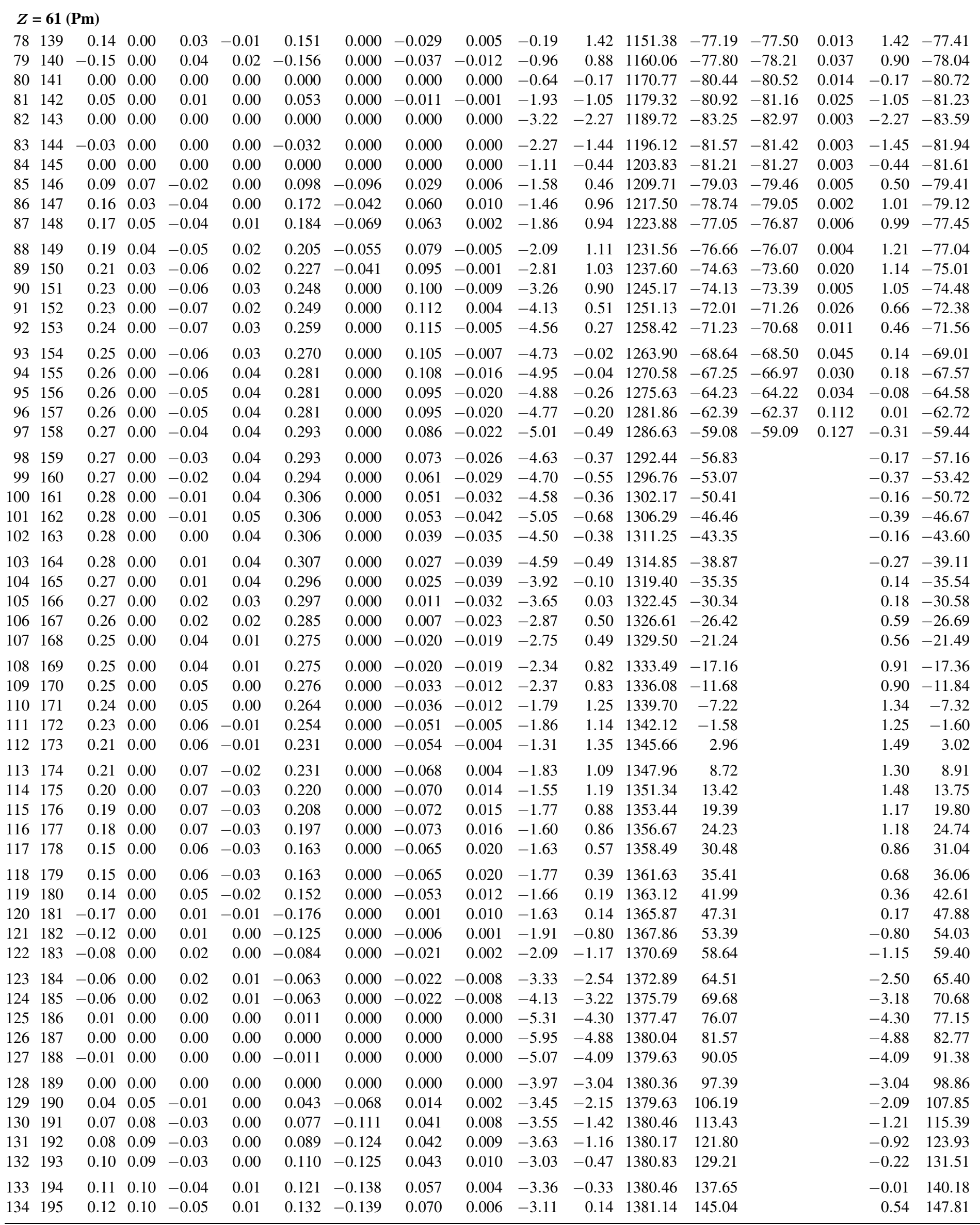




\section{$Z=61(\mathrm{Pm})$}

$\begin{array}{lllll}135 & 196 & 0.13 & 0.10 & -0.05\end{array}$ $\begin{array}{llllll}136 & 197 & 0.18 & 0.00 & -0.08\end{array}$ $\begin{array}{llllll}137 & 198 & 0.19 & 0.00 & -0.08\end{array}$ $\begin{array}{llllll}138 & 199 & 0.20 & 0.00 & -0.08\end{array}$ $\begin{array}{lllll}139 & 200 & 0.21 & 0.00 & -0.07\end{array}$

0.01

$0.01=0.196$

$\begin{array}{ll}0.196 & 0.000\end{array}$

$\begin{array}{lll}0.00 & 0.217 & 0.000\end{array}$

$\begin{array}{lll}0.01 & 0.227 & 0.000\end{array}$

140201

141202

142203

143204

$\begin{array}{llll}0.21 & 0.00 & -0.07\end{array}$

$\begin{array}{llll}0.22 & 0.00 & -0.07\end{array}$

$\begin{array}{llll}0.23 & 0.00 & -0.06\end{array}$

$\begin{array}{llll}0.23 & 0.00 & -0.06\end{array}$

144205

$\begin{array}{llll}0.24 & 0.00 & -0.05\end{array}$ $\begin{array}{lll}0.01 & 0.227 & 0.000\end{array}$

$\begin{array}{lll}0.02 & 0.237 & 0.000\end{array}$

$\begin{array}{lll}0.03 & 0.248 & 0.000\end{array}$

$\begin{array}{lll}0.04 & 0.248 & 0.000\end{array}$

$0.04 \quad 0.259$

0.000

\subsection{2}

0.114

0.116

0.118

0.107

0.110

0.100 $\begin{array}{lllll}0.007 & -3.15 & 0.17 & 1380.68 & 153.58\end{array}$ $\begin{array}{lllll}0.033 & -3.27 & 0.06 & 1381.74 & 160.59\end{array}$ $\begin{array}{llllll}0.023 & -3.70 & -0.25 & 1381.44 & 168.96\end{array}$ $\begin{array}{lllll}0.025 & -3.79 & -0.27 & 1382.22 & 176.25\end{array}$ $\begin{array}{llllll}0.012 & -3.86 & -0.54 & 1381.69 & 184.85\end{array}$ $\begin{array}{llllll}0.012 & -3.85 & -0.63 & 1382.36 & 192.25\end{array}$ $\begin{array}{lllll}0.003 & -4.47 & -1.13 & 1381.88 & 200.80\end{array}$ $\begin{array}{llllll}0.090 & -0.022 & -4.87 & -1.81 & 1382.03 & 224.87\end{array}$
$0.56 \quad 156.50$

$0.72 \quad 163.96$

$\begin{array}{ll}0.30 & 172.41\end{array}$

$0.31 \quad 179.91$

$-0.15 \quad 188.51$

$-0.23 \quad 196.13$

$-0.72 \quad 204.89$

$\begin{array}{ll}-0.81 & 212.69\end{array}$

$\begin{array}{ll}-1.33 & 221.61\end{array}$

$-1.32 \quad 229.68$

\section{$Z=62(\mathrm{Sm})$}

$\begin{array}{lllll}61 & 123 & 0.31 & 0.00 & -0.03\end{array}$

$\begin{array}{lllll}62 & 124 & 0.32 & 0.00 & -0.02\end{array}$

$\begin{array}{llllll}63 & 125 & 0.31 & 0.00 & -0.02\end{array}$

0.06

0.000

$\begin{array}{lllllll}0.087 & -0.041 & -4.61 & -0.09 & 955.12 & -10.85\end{array}$

$0.06 \quad 0.350$

0.000

$\begin{array}{lll}0.078 & -0.045 & -4.56\end{array}$

0.01

$971.03-18.69$

$\begin{array}{lllll}64 & 126 & 0.31 & 0.00 & -0.01\end{array}$

0.05

0.339

0.000

$0.074-0.035-4.07$

$\begin{array}{lll}0.16 & 983.64 & -23.23\end{array}$

$\begin{array}{ll}65 & 127\end{array}$

$\begin{array}{lll}0.31 & 0.00 & 0.00\end{array}$

0.04

0.341

0.000

$0.048-0.033-3.36$

$\begin{array}{lll}0.46 & 998.18 & -29.69\end{array}$

66128

$\begin{array}{ll}0.31 & 0.00\end{array}$

0.01

0.03

0.341

0.000

$\begin{array}{llll}0.034 & -0.027 & -2.97\end{array}$

$\begin{array}{lll}0.60 & 1010.14 & -33.59\end{array}$

$\begin{array}{ll}67 & 129\end{array}$

$0.30 \quad 0.00$

0.02
0.02

0.02
0.02

$\begin{array}{ll}68 & 130 \\ 69 & 131\end{array}$

$\begin{array}{ll}0.30 & 0.00\end{array}$

0.331

0.000

$\begin{array}{llll}0.018 & -0.022 & -2.64\end{array}$

$\begin{array}{llll}0.83 & 1024.09 & -39.46\end{array}$

$\begin{array}{llll}0.98 & 1035.42 & -42.73\end{array}$

0.000

$\begin{array}{llll}0.018 & -0.022 & -2.48\end{array}$

$\begin{array}{lll}1.19 & 1048.76 & -47.99\end{array}$

$\begin{array}{ll}70 & 132\end{array}$

$\begin{array}{lll}0.29 & 0.00 & 0.03\end{array}$

$0.01 \quad 0.320$

0.000

$0.002-0.016-2.32$

$\begin{array}{lll}1.25 & 1059.58 & -50.75\end{array}$

$71 \quad 133$

$\begin{array}{lll}0.31 & 0.00 & 0.03\end{array}$

0.320

$\begin{array}{llll}0.000 & -0.012 & -0.010 & -2.09\end{array}$

$\begin{array}{lll}1.51 & 1072.26 & -55.35\end{array}$

$\begin{array}{ll}72 & 134\end{array}$

$\begin{array}{lll}0.31 & 0.00 & 0.03\end{array}$

$0.01-0.343$

$\begin{array}{lllll}0.000 & 0.007 & -0.016 & -2.30\end{array}$

$\begin{array}{lll}1.66 & 1082.43 & -57.45\end{array}$

$\begin{array}{lll}73 & 135\end{array}$

$\begin{array}{lll}0.30 & 0.00 & 0.02\end{array}$

0.01

0.343

0.000

$0.006-0.006-1.88$

$\begin{array}{llll}1.99 & 1094.45 & -61.40\end{array}$

$\begin{array}{ll}74 & 136\end{array}$

$0.22 \quad 0.00$

0.04

0.00

0.241

$\begin{array}{lllll}0.000 & 0.016 & -0.012 & -1.51\end{array}$

$\begin{array}{llll}2.21 & 1104.00 & -62.88\end{array}$

$\begin{array}{lllll}0.000 & -0.028 & -0.009 & -0.13\end{array}$

$\begin{array}{ll}76 & 138\end{array}$

$0.20 \quad 0.00$

$0.04-0.01$

0.218

$\begin{array}{llll}0.000 & -0.032 & 0.002 & -0.20\end{array}$

$\begin{array}{llll}333 & 1115.66 & -66.46 & -66.81\end{array}$

$\begin{array}{lllll}2.15 & 1125.08 & -67.81 & -68.03\end{array}$

$\begin{array}{lll}0.000 & -0.032\end{array}$

$\begin{array}{ll}0.002 & -0.32\end{array}$

$\begin{array}{lllll}2.02 & 1136.45 & -71.11 & -71.50\end{array}$

$\begin{array}{ll}0.000 & -0.037\end{array}$

$0.003-0.34$

$\begin{array}{lllll}1.73 & 1145.47 & -72.06 & -72.38\end{array}$

$\begin{array}{llll}0.000 & -0.025 & -0.014 & -0.32\end{array}$

$\begin{array}{lllll}1.43 & 1156.50 & -75.02 & -75.46\end{array}$

$\begin{array}{lllll}0.000 & -0.037 & -0.012 & -0.85\end{array}$

$\begin{array}{lllll}1.00 & 1165.16 & -75.61 & -75.94\end{array}$

$\begin{array}{llll}79 & 141 & -0.15 & 0.00\end{array}$

$\begin{array}{llll}0.04 & 0.02 & -0.156\end{array}$

$80 \quad 14$

$0.00 \quad 0.00$

0.00

$0.00 \quad 0.000$

0.000

$\begin{array}{llll}0.000 & 0.000 & -0.49\end{array}$

$\begin{array}{lllll}-0.04 & 1176.42 & -78.80 & -78.99\end{array}$

$\begin{array}{lllll}81 & 143 & 0.04 & 0.00 & 0.01\end{array}$

$0.00 \quad 0.043$

$\begin{array}{lllll}82 & 144 & 0.00 & 0.00 & 0.00\end{array}$

$0.00 \quad 0.000$

0.000

$-0.011-0.1$

$0.000-0.49$

0.000

0.000

$0.000-3.06$

$\begin{array}{llll}-0.88 & 1185.02 & -79.33 & -79.52\end{array}$

$0.00-0.032$

0.000

0.012

$\begin{array}{lllll}84 & 146 & 0.00 & 0.00 & 0.00\end{array}$

$0.00 \quad 0.000$

$\begin{array}{ll}85 & 147\end{array}$

$\begin{array}{llll}0.13 & 0.00 & -0.03\end{array}$

$-0.01$

0.140

0.000

0.043

$-0.000$

$.000-2.11-1$

$\begin{array}{llll}2.12 & 1196.01 & -82.24 & -81.97\end{array}$

$\begin{array}{llllll}86 & 148 & 0.16 & 0.00 & -0.04\end{array}$

$\begin{array}{lll}0.00 & 0.172 & 0.000\end{array}$

0.060

$0.016-0.93$

$-0.27$

$\begin{array}{llll}0.27 & 1210.74 & -80.83 & -81.00\end{array}$

$\begin{array}{lllll}87 & 149 & 0.17 & 0.03 & -0.04\end{array}$

$\begin{array}{llll}0.01 & 0.183 & -0.041\end{array}$

0.062

$0.009-0.98$

$\begin{array}{llll}1216.59 & -78.62 & -79.27\end{array}$

0.02

$0.205-0.055$

$\begin{array}{llll}0.066 & -0.008 & -1.50\end{array}$

$\begin{array}{lllll}1.09 & 1225.07 & -79.02 & -79.34\end{array}$

$\begin{array}{llll}1.22 & 1231.38 & -77.26 & -77.14\end{array}$

$\begin{array}{lllll}1.39 & 1239.61 & -77.42 & -77.06\end{array}$

$\begin{array}{lll}0.082 & -0.004 & -2.10\end{array}$

$\begin{array}{lllll}1.30 & 1245.73 & -75.47 & -74.58\end{array}$

$\begin{array}{llll}0.097 & -0.000 & -2.69\end{array}$

$\begin{array}{lllll}1.20 & 1253.82 & -75.48 & -74.77\end{array}$

$\begin{array}{lll}0.102 & -0.008 & -3.60\end{array}$

$\begin{array}{llll}0.80 & 1259.85 & -73.44 & -72.57\end{array}$

$0.03 \quad 0.259$

0.000

$\begin{array}{lll}0.102 & -0.008 & -3.60 \\ 0.105 & -0.007 & -4.04\end{array}$

$\begin{array}{lllll}0.57 & 1267.66 & -73.19 & -72.46\end{array}$

$0.03 \quad 0.270$

0.000

$\begin{array}{lll}0.105 & -0.007 & -4.38\end{array}$

$\begin{array}{lllll}0.25 & 1273.24 & -70.69 & -70.20\end{array}$

$0.03 \quad 0.282$

0.000

$0.094-0.009-4.26$

$\begin{array}{llll}0.24 & 1280.44 & -69.82 & -69.37\end{array}$

$\begin{array}{llllllll}0.095 & -0.020 & -4.63 & -0.07 & 1285.64 & -66.95 & -66.73\end{array}$

$\begin{array}{lllll}95 & 157 & 0.26 & 0.00 & -0.05\end{array}$

0.04

0.281

0.000

$\begin{array}{llllllll}0.086 & -0.022 & -4.52 & -0.04 & 1292.43 & -65.66 & -65.21\end{array}$

$\begin{array}{lllll}96 & 158 & 0.27 & 0.00 & -0.04\end{array}$

$\begin{array}{lllll}97 & 159 & 0.27 & 0.00 & -0.03\end{array}$

$0.04 \quad 0.293$

0.000

$\begin{array}{lll}0.04 & 0.293 & 0.000\end{array}$

$\begin{array}{llllllll}0.073 & -0.026 & -4.61 & -0.36 & 1297.28 & -62.45 & -62.21\end{array}$

0.000

$\begin{array}{lllllll}0.073 & -0.026 & -4.52 & -0.31 & 1303.68 & -60.78\end{array}$

$0.04-0.293$

0.000

$\begin{array}{lllllll}0.061 & -0.029 & -4.63 & -0.52 & 1308.08 & -57.10\end{array}$

99161

$\begin{array}{lll}0.27 & 0.00 & -0.02\end{array}$

$0.04 \quad 0.294$

0.000

$\begin{array}{lllllll}0.051 & -0.032 & -4.58 & -0.41 & 1314.07 & -55.03\end{array}$

101163

$\begin{array}{lll}0.28 & 0.00 & 0.00\end{array}$

$0.04 \quad 0.306$

0.000

$\begin{array}{llllll}0.039 & -0.035 & -4.79 & -0.66 & 1318.19 & -51.07\end{array}$

10216

103165

$0.28 \quad 0.00$

0.01

0.04

0.307

0.000

$\begin{array}{llllll}0.027 & -0.039 & -4.55 & -0.48 & 1323.77 & -48.58\end{array}$

$0.28 \quad 0.00$

0.01

$0.04 \quad 0.307$

0.000

$\begin{array}{llllll}0.027 & -0.039 & -4.68 & -0.60 & 1327.44 & -44.17\end{array}$

$\begin{array}{lllllll}0.000 & 0.011 & -0.032 & -3.87 & -0.17 & 1332.43 & -41.10\end{array}$

$\begin{array}{lllllll}0.000 & -0.002 & -0.026 & -3.74 & -0.12 & 1335.62 & -36.21\end{array}$

$0.02 \quad 0.297$

$-0.16-10.48$

$-0.03-18.33$

$\begin{array}{lllllll}0.000 & -0.004 & -0.026 & -3.14 & 0.21 & 1340.40 & -32.92\end{array}$ 


\begin{tabular}{|c|c|c|c|c|c|c|c|c|c|c|c|c|c|c|c|c|}
\hline \multicolumn{17}{|c|}{$Z=62(\mathrm{Sm})$} \\
\hline $107 \quad 169$ & 0.25 & 0.00 & 0.04 & 0.01 & 0.275 & 0.000 & -0.020 & -0.019 & -2.99 & 0.25 & 1343.29 & -27.75 & & & 0.34 & -28.05 \\
\hline 108170 & 0.25 & 0.00 & 0.05 & 0.00 & 0.276 & 0.000 & -0.033 & -0.012 & -2.66 & 0.60 & 1347.76 & -24.14 & & & 0.70 & -24.39 \\
\hline 109171 & 0.25 & 0.00 & 0.06 & 0.00 & 0.277 & 0.000 & -0.045 & -0.015 & -2.83 & 0.56 & 1350.44 & -18.75 & & & 0.69 & -18.94 \\
\hline 110172 & 0.24 & 0.00 & 0.06 & -0.01 & 0.265 & 0.000 & -0.049 & -0.005 & -2.21 & 0.95 & 1354.56 & -14.79 & & & 1.08 & -14.94 \\
\hline 111173 & 0.24 & 0.00 & 0.07 & -0.02 & 0.265 & 0.000 & -0.062 & 0.002 & -2.47 & 0.89 & 1356.98 & -9.15 & & & 1.07 & -9.19 \\
\hline 112174 & 0.23 & 0.00 & 0.07 & -0.02 & 0.254 & 0.000 & -0.064 & 0.002 & -1.99 & 1.16 & 1360.92 & -5.02 & & & 1.37 & -4.99 \\
\hline 113175 & 0.21 & 0.00 & 0.07 & -0.02 & 0.231 & 0.000 & -0.068 & 0.004 & -2.00 & 0.95 & 1363.22 & 0.75 & & & 1.16 & 0.84 \\
\hline 114176 & 0.21 & 0.00 & 0.07 & -0.03 & 0.231 & 0.000 & -0.069 & 0.014 & -1.81 & 1.09 & 1367.03 & 5.02 & & & 1.37 & 5.24 \\
\hline 115177 & 0.20 & 0.00 & 0.07 & -0.03 & 0.220 & 0.000 & -0.070 & 0.014 & -1.98 & 0.78 & 1369.17 & 10.95 & & & 1.06 & 11.23 \\
\hline 116178 & 0.18 & 0.00 & 0.07 & -0.03 & 0.197 & 0.000 & -0.073 & 0.016 & -1.66 & 0.85 & 1372.76 & 15.43 & & & 1.17 & 15.81 \\
\hline $117 \quad 179$ & 0.15 & 0.00 & 0.07 & -0.03 & 0.164 & 0.000 & -0.077 & 0.018 & -1.87 & 0.57 & 1374.62 & 21.64 & & & 0.90 & 22.12 \\
\hline 118180 & 0.15 & 0.00 & 0.06 & -0.03 & 0.163 & 0.000 & -0.065 & 0.020 & -1.74 & 0.43 & 1378.16 & 26.17 & & & 0.73 & 26.68 \\
\hline 119181 & 0.15 & 0.00 & 0.06 & -0.03 & 0.163 & 0.000 & -0.065 & 0.020 & -1.95 & 0.19 & 1379.73 & 32.68 & & & 0.48 & 33.27 \\
\hline 120182 & -0.17 & 0.00 & 0.02 & -0.01 & -0.177 & 0.000 & -0.011 & 0.012 & -1.62 & 0.12 & 1382.95 & 37.52 & & & 0.17 & 37.96 \\
\hline $121 \quad 183$ & -0.14 & 0.00 & 0.01 & 0.00 & -0.146 & 0.000 & -0.004 & 0.001 & -1.91 & -0.54 & 1384.70 & 43.85 & & & -0.54 & 44.33 \\
\hline $122 \quad 184$ & -0.12 & 0.00 & 0.01 & 0.00 & -0.125 & 0.000 & -0.006 & 0.001 & -2.11 & -1.03 & 1388.09 & 48.53 & & & -1.02 & 49.11 \\
\hline 123185 & -0.06 & 0.00 & 0.02 & 0.00 & -0.063 & 0.000 & -0.022 & 0.002 & -3.07 & -2.29 & 1390.20 & 54.48 & & & -2.27 & 55.18 \\
\hline $124 \quad 186$ & -0.06 & 0.00 & 0.02 & 0.01 & -0.063 & 0.000 & -0.022 & -0.008 & -3.86 & -2.96 & 1393.54 & 59.22 & & & -2.92 & 60.04 \\
\hline 125187 & 0.00 & 0.00 & 0.00 & 0.00 & 0.000 & 0.000 & 0.000 & 0.000 & -4.99 & -4.01 & 1395.21 & 65.62 & & & -4.01 & 66.50 \\
\hline 126188 & 0.00 & 0.00 & 0.00 & 0.00 & 0.000 & 0.000 & 0.000 & 0.000 & -5.62 & -4.57 & 1398.20 & 70.70 & & & -4.57 & 71.70 \\
\hline $127 \quad 189$ & -0.01 & 0.00 & 0.00 & 0.00 & -0.011 & 0.000 & 0.000 & 0.000 & -4.69 & -3.72 & 1397.77 & 79.21 & & & -3.73 & 80.33 \\
\hline $128 \quad 190$ & 0.00 & 0.00 & 0.00 & 0.00 & 0.000 & 0.000 & 0.000 & 0.000 & -3.58 & -2.67 & 1398.92 & 86.12 & & & -2.68 & 87.37 \\
\hline 129191 & 0.04 & 0.06 & -0.01 & 0.00 & 0.044 & -0.082 & 0.014 & 0.003 & -3.31 & -1.84 & 1398.28 & 94.83 & & & -1.76 & 96.30 \\
\hline 130192 & 0.07 & 0.08 & -0.02 & 0.00 & 0.077 & -0.110 & 0.028 & 0.007 & -3.08 & -1.04 & 1399.47 & 101.72 & & & -0.87 & 103.40 \\
\hline $131 \quad 193$ & 0.08 & 0.09 & -0.03 & 0.00 & 0.089 & -0.124 & 0.042 & 0.009 & -3.29 & -0.81 & 1399.22 & 110.04 & & & -0.56 & 111.94 \\
\hline 132194 & 0.09 & 0.10 & -0.03 & 0.01 & 0.100 & -0.138 & 0.043 & 0.001 & -2.88 & -0.12 & 1400.30 & 117.03 & & & 0.17 & 119.11 \\
\hline 133195 & 0.10 & 0.09 & -0.04 & 0.00 & 0.110 & -0.125 & 0.056 & 0.012 & -2.62 & 0.08 & 1399.89 & 125.51 & & & 0.38 & 127.76 \\
\hline 134196 & 0.12 & 0.10 & -0.04 & 0.01 & 0.132 & -0.138 & 0.058 & 0.004 & -2.42 & 0.65 & 1400.89 & 132.59 & & & 0.99 & 135.03 \\
\hline 135197 & 0.17 & 0.00 & -0.07 & -0.01 & 0.185 & 0.000 & 0.100 & 0.029 & -2.28 & 0.59 & 1400.53 & 141.01 & & & 1.09 & 143.77 \\
\hline 136198 & 0.18 & 0.00 & -0.08 & -0.01 & 0.196 & 0.000 & 0.114 & 0.033 & -2.87 & 0.50 & 1401.99 & 147.62 & & & 1.16 & 150.71 \\
\hline 137199 & 0.19 & 0.00 & -0.08 & 0.00 & 0.206 & 0.000 & 0.116 & 0.023 & -3.30 & 0.18 & 1401.71 & 155.98 & & & 0.73 & 159.14 \\
\hline $138 \quad 200$ & 0.20 & 0.00 & -0.07 & 0.00 & 0.217 & 0.000 & 0.105 & 0.022 & -2.96 & 0.26 & 1402.80 & 162.96 & & & 0.71 & 166.19 \\
\hline 139201 & 0.21 & 0.00 & -0.07 & 0.01 & 0.227 & 0.000 & 0.107 & 0.012 & -3.49 & -0.16 & 1402.44 & 171.39 & & & 0.23 & 174.76 \\
\hline 140202 & 0.21 & 0.00 & -0.07 & 0.02 & 0.227 & 0.000 & 0.108 & 0.002 & -3.44 & -0.28 & 1403.53 & 178.37 & & & 0.16 & 181.96 \\
\hline 141203 & 0.22 & 0.00 & -0.06 & 0.02 & 0.237 & 0.000 & 0.097 & -0.000 & -3.79 & -0.70 & 1402.99 & 186.99 & & & -0.38 & 190.65 \\
\hline $142 \quad 204$ & 0.23 & 0.00 & -0.06 & 0.03 & 0.248 & 0.000 & 0.100 & -0.009 & -4.09 & -0.92 & 1404.00 & 194.04 & & & -0.50 & 198.01 \\
\hline 143205 & 0.23 & 0.00 & -0.05 & 0.03 & 0.248 & 0.000 & 0.087 & -0.013 & -4.34 & -1.34 & 1403.29 & 202.83 & & & -1.01 & 206.91 \\
\hline 144206 & 0.24 & 0.00 & -0.05 & 0.04 & 0.259 & 0.000 & 0.090 & -0.022 & -4.62 & -1.55 & 1404.10 & 210.08 & & & -1.05 & 214.55 \\
\hline 145207 & 0.24 & 0.00 & -0.04 & 0.04 & 0.259 & 0.000 & 0.078 & -0.025 & -4.90 & -1.98 & 1403.23 & 219.02 & & & -1.54 & 223.65 \\
\hline 146208 & 0.24 & 0.00 & -0.04 & 0.05 & 0.259 & 0.000 & 0.079 & -0.035 & -5.03 & -2.27 & 1403.95 & 226.38 & & & -1.56 & 231.49 \\
\hline \multicolumn{17}{|c|}{$Z=63(\mathrm{Eu})$} \\
\hline $62 \quad 125$ & 0.31 & 0.00 & -0.02 & 0.05 & 0.339 & 0.000 & 0.074 & -0.035 & -4.08 & -0.05 & 967.11 & -7.49 & & & -0.13 & -7.08 \\
\hline $63 \quad 126$ & 0.31 & 0.00 & -0.01 & 0.05 & 0.340 & 0.000 & 0.062 & -0.039 & -3.89 & 0.12 & 980.58 & -12.88 & & & 0.02 & -12.77 \\
\hline $64 \quad 127$ & 0.31 & 0.00 & 0.00 & 0.04 & 0.341 & 0.000 & 0.048 & -0.033 & -3.43 & 0.33 & 995.55 & -19.78 & & & 0.25 & -19.45 \\
\hline $65 \quad 128$ & 0.30 & 0.00 & 0.01 & 0.03 & 0.330 & 0.000 & 0.032 & -0.028 & -3.07 & 0.59 & 1008.03 & -24.18 & & & 0.49 & -23.91 \\
\hline $66 \quad 129$ & 0.30 & 0.00 & 0.02 & 0.02 & 0.331 & 0.000 & 0.018 & -0.022 & -2.81 & 0.75 & 1022.14 & -30.23 & & & 0.68 & -29.98 \\
\hline $67 \quad 130$ & 0.30 & 0.00 & 0.03 & 0.01 & 0.331 & 0.000 & 0.004 & -0.016 & -2.78 & 0.68 & 1034.31 & -34.33 & & & 0.60 & -34.13 \\
\hline $68 \quad 131$ & 0.29 & 0.00 & 0.03 & 0.01 & 0.320 & 0.000 & 0.002 & -0.016 & -2.55 & 0.85 & 1047.79 & -39.74 & & & 0.79 & -39.55 \\
\hline $69 \quad 132$ & 0.29 & 0.00 & 0.04 & 0.00 & 0.320 & 0.000 & -0.012 & -0.010 & -2.64 & 0.89 & 1059.25 & -43.12 & & & 0.81 & -42.99 \\
\hline $70 \quad 133$ & 0.29 & 0.00 & 0.05 & 0.00 & 0.321 & 0.000 & -0.024 & -0.013 & -2.55 & 1.10 & 1072.07 & -47.87 & & & 1.06 & -47.75 \\
\hline $71 \quad 134$ & 0.30 & 0.00 & 0.04 & 0.00 & 0.332 & 0.000 & -0.009 & -0.010 & -2.42 & 1.33 & 1082.76 & -50.49 & & & 1.26 & -50.43 \\
\hline $72 \quad 135$ & 0.30 & 0.00 & 0.04 & 0.00 & 0.332 & 0.000 & -0.009 & -0.010 & -2.00 & 1.69 & 1094.85 & -54.51 & & & 1.65 & -54.46 \\
\hline $73 \quad 136$ & 0.29 & 0.00 & 0.03 & 0.00 & 0.320 & 0.000 & 0.000 & -0.006 & -1.54 & 1.80 & 1105.09 & -56.68 & & & 1.76 & -56.68 \\
\hline $74 \quad 137$ & 0.23 & 0.00 & 0.03 & 0.00 & 0.252 & 0.000 & -0.014 & -0.006 & -0.41 & 1.89 & 1116.89 & -60.41 & & & 1.87 & -60.41 \\
\hline $\begin{array}{ll}75 & 138\end{array}$ & 0.21 & 0.00 & 0.04 & -0.01 & 0.229 & 0.000 & -0.031 & 0.001 & -0.54 & 1.92 & 1126.68 & -62.12 & -61.75 & 0.028 & 1.90 & -62.17 \\
\hline $76 \quad 139$ & 0.20 & 0.00 & 0.04 & -0.01 & 0.218 & 0.000 & -0.032 & 0.002 & -0.43 & 1.89 & 1138.04 & -65.42 & -65.40 & 0.013 & 1.89 & -65.49 \\
\hline $77 \quad 140$ & 0.18 & 0.00 & 0.04 & -0.02 & 0.196 & 0.000 & -0.036 & 0.012 & -0.56 & 1.61 & 1147.64 & -66.94 & -66.99 & 0.052 & 1.60 & -67.05 \\
\hline
\end{tabular}




\begin{tabular}{|c|c|c|c|c|c|c|c|c|c|c|c|c|c|c|c|c|c|}
\hline \multicolumn{18}{|c|}{$Z=63(\mathrm{Eu})$} \\
\hline 78 & 141 & -0.17 & 0.00 & 0.03 & 0.02 & -0.176 & 0.000 & -0.024 & -0.013 & -0.39 & 1.43 & 1158.63 & -69.86 & -69.93 & 0.013 & 1.43 & -70.00 \\
\hline 79 & 142 & -0.16 & 0.00 & 0.03 & 0.02 & -0.166 & 0.000 & -0.025 & -0.014 & -0.72 & 0.97 & 1167.89 & -71.05 & -71.32 & 0.031 & 0.97 & -71.22 \\
\hline 80 & 143 & -0.13 & 0.00 & 0.02 & 0.01 & -0.135 & 0.000 & -0.017 & -0.007 & -0.88 & 0.45 & 1178.72 & -73.81 & -74.24 & 0.011 & 0.45 & -74.02 \\
\hline 81 & 144 & -0.06 & 0.00 & 0.01 & 0.00 & -0.063 & 0.000 & -0.010 & 0.001 & -1.47 & -0.77 & 1188.26 & -75.28 & -75.62 & 0.011 & -0.77 & -75.53 \\
\hline 82 & 145 & 0.01 & 0.00 & 0.00 & 0.00 & 0.011 & 0.000 & 0.000 & 0.000 & -2.72 & -1.82 & 1199.14 & -78.09 & -78.00 & 0.004 & -1.82 & -78.37 \\
\hline 83 & 146 & -0.03 & 0.00 & 0.00 & 0.00 & -0.032 & 0.000 & 0.000 & 0.000 & -1.77 & -1.00 & 1206.18 & -77.06 & -77.12 & 0.006 & -1.00 & -77.37 \\
\hline 84 & 147 & 0.04 & 0.05 & -0.01 & 0.00 & 0.043 & -0.068 & 0.014 & 0.002 & -1.14 & 0.00 & 1214.54 & -77.34 & -77.55 & 0.003 & 0.02 & -77.67 \\
\hline 85 & 148 & 0.14 & 0.00 & -0.03 & -0.01 & 0.151 & 0.000 & 0.045 & 0.016 & -0.88 & 0.91 & 1221.05 & -75.78 & -76.30 & 0.010 & 0.93 & -76.13 \\
\hline 86 & 149 & 0.17 & 0.00 & -0.03 & 0.00 & 0.183 & 0.000 & 0.049 & 0.008 & -0.80 & 1.20 & 1229.67 & -76.33 & -76.45 & 0.004 & 1.22 & -76.70 \\
\hline 87 & 150 & 0.18 & 0.00 & -0.03 & 0.01 & 0.194 & 0.000 & 0.051 & -0.002 & -0.88 & 1.34 & 1236.52 & -75.11 & -74.80 & 0.006 & 1.36 & -75.51 \\
\hline 88 & 151 & 0.20 & 0.01 & -0.04 & 0.02 & 0.215 & -0.014 & 0.068 & -0.008 & -1.32 & 1.45 & 1244.88 & -75.40 & -74.66 & 0.002 & 1.50 & -75.79 \\
\hline 89 & 152 & 0.21 & 0.00 & -0.04 & 0.02 & 0.226 & 0.000 & 0.070 & -0.007 & -1.67 & 1.37 & 1251.53 & -73.97 & -72.89 & 0.002 & 1.42 & -74.39 \\
\hline 90 & 153 & 0.23 & 0.00 & -0.05 & 0.02 & 0.249 & 0.000 & 0.087 & -0.002 & -2.47 & 1.26 & 1259.69 & -74.07 & -73.37 & 0.002 & 1.35 & -74.47 \\
\hline 91 & 154 & 0.23 & 0.00 & -0.05 & 0.02 & 0.249 & 0.000 & 0.087 & -0.002 & -2.90 & 0.91 & 1266.21 & -72.51 & -71.74 & 0.002 & 0.99 & -72.94 \\
\hline 92 & 155 & 0.25 & 0.00 & -0.05 & 0.03 & 0.270 & 0.000 & 0.092 & -0.010 & -3.55 & 0.69 & 1274.08 & -72.31 & -71.82 & 0.002 & 0.81 & -72.72 \\
\hline 93 & 156 & 0.25 & 0.00 & -0.05 & 0.03 & 0.270 & 0.000 & 0.092 & -0.010 & -3.88 & 0.39 & 1280.16 & -70.32 & -70.09 & 0.006 & 0.50 & -70.75 \\
\hline 94 & 157 & 0.26 & 0.00 & -0.04 & 0.03 & 0.282 & 0.000 & 0.082 & -0.013 & -3.84 & 0.35 & 1287.46 & -69.55 & -69.47 & 0.005 & 0.47 & -69.98 \\
\hline 95 & 158 & 0.26 & 0.00 & -0.04 & 0.03 & 0.282 & 0.000 & 0.082 & -0.013 & -4.16 & 0.05 & 1293.17 & -67.19 & -67.21 & 0.077 & 0.15 & -67.64 \\
\hline 96 & 159 & 0.26 & 0.00 & -0.03 & 0.03 & 0.282 & 0.000 & 0.069 & -0.016 & -3.89 & 0.06 & 1300.03 & -65.98 & -66.05 & 0.007 & 0.17 & -66.44 \\
\hline 97 & 160 & 0.27 & 0.00 & -0.03 & 0.04 & 0.293 & 0.000 & 0.073 & -0.026 & -4.58 & -0.36 & 1305.51 & -63.38 & & & -0.22 & -63.81 \\
\hline 98 & 161 & 0.27 & 0.00 & -0.02 & 0.04 & 0.294 & 0.000 & 0.061 & -0.029 & -4.41 & -0.37 & 1312.02 & -61.83 & & & -0.20 & -62.24 \\
\hline 99 & 162 & 0.27 & 0.00 & -0.01 & 0.04 & 0.294 & 0.000 & 0.049 & -0.032 & -4.59 & -0.60 & 1316.96 & -58.69 & & & -0.45 & -59.11 \\
\hline 100 & 163 & 0.28 & 0.00 & 0.00 & 0.04 & 0.306 & 0.000 & 0.039 & -0.035 & -4.65 & -0.56 & 1323.07 & -56.73 & & & -0.37 & -57.12 \\
\hline 101 & 164 & 0.28 & 0.00 & 0.01 & 0.04 & 0.307 & 0.000 & 0.027 & -0.039 & -4.92 & -0.81 & 1327.69 & -53.28 & & & -0.63 & -53.67 \\
\hline 102 & 165 & 0.28 & 0.00 & 0.01 & 0.04 & 0.307 & 0.000 & 0.027 & -0.039 & -4.75 & -0.67 & 1333.37 & -50.89 & & & -0.47 & -51.24 \\
\hline 103 & 166 & 0.28 & 0.00 & 0.02 & 0.04 & 0.308 & 0.000 & 0.015 & -0.042 & -4.94 & -0.83 & 1337.56 & -47.01 & & & -0.62 & -47.34 \\
\hline 104 & 167 & 0.27 & 0.00 & 0.02 & 0.03 & 0.297 & 0.000 & 0.011 & -0.032 & -4.16 & -0.44 & 1342.66 & -44.04 & & & -0.29 & -44.41 \\
\hline 105 & 168 & 0.27 & 0.00 & 0.03 & 0.02 & 0.297 & 0.000 & -0.002 & -0.026 & -4.06 & -0.42 & 1346.36 & -39.67 & & & -0.33 & -40.08 \\
\hline 106 & 169 & 0.26 & 0.00 & 0.03 & 0.02 & 0.286 & 0.000 & -0.004 & -0.026 & -3.47 & -0.11 & 1351.21 & -36.45 & & & -0.00 & -36.82 \\
\hline 107 & 170 & 0.26 & 0.00 & 0.04 & 0.01 & 0.287 & 0.000 & -0.018 & -0.019 & -3.49 & -0.06 & 1354.58 & -31.75 & & & 0.00 & -32.13 \\
\hline 108 & 171 & 0.25 & 0.00 & 0.05 & 0.00 & 0.276 & 0.000 & -0.033 & -0.012 & -3.01 & 0.27 & 1359.10 & -28.20 & & & 0.35 & -28.54 \\
\hline 109 & 172 & 0.25 & 0.00 & 0.06 & 0.00 & 0.277 & 0.000 & -0.045 & -0.015 & -3.17 & 0.28 & 1362.22 & -23.24 & & & 0.38 & -23.52 \\
\hline 110 & 173 & 0.24 & 0.00 & 0.06 & -0.01 & 0.265 & 0.000 & -0.049 & -0.005 & -2.52 & 0.68 & 1366.38 & -19.33 & & & 0.79 & -19.56 \\
\hline 111 & 174 & 0.24 & 0.00 & 0.07 & -0.02 & 0.265 & 0.000 & -0.062 & 0.002 & -2.77 & 0.64 & 1369.24 & -14.12 & & & 0.80 & -14.27 \\
\hline 112 & 175 & 0.23 & 0.00 & 0.07 & -0.02 & 0.254 & 0.000 & -0.064 & 0.002 & -2.25 & 0.95 & 1373.19 & -10.00 & & & 1.14 & -10.08 \\
\hline 113 & 176 & 0.21 & 0.00 & 0.07 & -0.02 & 0.231 & 0.000 & -0.068 & 0.004 & -2.17 & 0.83 & 1375.87 & -4.61 & & & 1.02 & -4.64 \\
\hline 114 & 177 & 0.21 & 0.00 & 0.07 & -0.03 & 0.231 & 0.000 & -0.069 & 0.014 & -1.97 & 0.98 & 1379.71 & -0.37 & & & 1.24 & -0.28 \\
\hline 115 & 178 & 0.20 & 0.00 & 0.07 & -0.03 & 0.220 & 0.000 & -0.070 & 0.014 & -2.11 & 0.70 & 1382.28 & 5.13 & & & 0.95 & 5.28 \\
\hline 116 & 179 & 0.18 & 0.00 & 0.07 & -0.03 & 0.197 & 0.000 & -0.073 & 0.016 & -1.74 & 0.82 & 1385.87 & 9.61 & & & 1.11 & 9.86 \\
\hline 117 & 180 & 0.17 & 0.00 & 0.07 & -0.03 & 0.186 & 0.000 & -0.075 & 0.017 & -2.04 & 0.62 & 1388.09 & 15.46 & & & 0.91 & 15.77 \\
\hline 118 & 181 & 0.15 & 0.00 & 0.06 & -0.02 & 0.163 & 0.000 & -0.064 & 0.010 & -1.62 & 0.55 & 1391.61 & 20.01 & & & 0.75 & 20.31 \\
\hline 119 & 182 & 0.15 & 0.00 & 0.05 & -0.02 & 0.163 & 0.000 & -0.052 & 0.012 & -1.58 & 0.18 & 1393.75 & 25.94 & & & 0.33 & 26.26 \\
\hline 120 & 183 & -0.17 & 0.00 & 0.02 & -0.01 & -0.177 & 0.000 & -0.011 & 0.012 & -1.67 & 0.10 & 1397.02 & 30.74 & & & 0.14 & 31.04 \\
\hline 121 & 184 & -0.14 & 0.00 & 0.02 & 0.00 & -0.146 & 0.000 & -0.015 & 0.003 & -1.93 & -0.53 & 1399.18 & 36.66 & & & -0.51 & 37.01 \\
\hline 122 & 185 & -0.12 & 0.00 & 0.01 & 0.00 & -0.125 & 0.000 & -0.006 & 0.001 & -2.04 & -0.95 & 1402.54 & 41.37 & & & -0.94 & 41.80 \\
\hline 123 & 186 & -0.08 & 0.00 & 0.02 & 0.00 & -0.084 & 0.000 & -0.021 & 0.002 & -2.96 & -2.11 & 1404.99 & 46.99 & & & -2.09 & 47.52 \\
\hline 124 & 187 & -0.06 & 0.00 & 0.02 & 0.01 & -0.063 & 0.000 & -0.022 & -0.008 & -3.62 & -2.74 & 1408.32 & 51.73 & & & -2.70 & 52.38 \\
\hline 125 & 188 & 0.00 & 0.00 & 0.00 & 0.00 & 0.000 & 0.000 & 0.000 & 0.000 & -4.74 & -3.79 & 1410.43 & 57.69 & & & -3.79 & 58.41 \\
\hline 126 & 189 & 0.00 & 0.00 & 0.00 & 0.00 & 0.000 & 0.000 & 0.000 & 0.000 & -5.36 & -4.33 & 1413.43 & 62.77 & & & -4.33 & 63.59 \\
\hline 127 & 190 & -0.01 & 0.00 & 0.00 & 0.00 & -0.011 & 0.000 & 0.000 & 0.000 & -4.42 & -3.48 & 1413.41 & 70.86 & & & -3.48 & 71.79 \\
\hline 128 & 191 & 0.00 & 0.00 & 0.00 & 0.00 & 0.000 & 0.000 & 0.000 & 0.000 & -3.31 & -2.42 & 1414.59 & 77.75 & & & -2.43 & 78.79 \\
\hline 129 & 192 & 0.04 & 0.07 & -0.02 & 0.00 & 0.045 & -0.096 & 0.026 & 0.004 & -3.50 & -1.75 & 1414.53 & 85.88 & & & -1.62 & 87.17 \\
\hline 130 & 193 & 0.06 & 0.09 & -0.02 & 0.00 & 0.067 & -0.124 & 0.029 & 0.007 & -3.22 & -0.91 & 1415.70 & 92.77 & & & -0.71 & 94.27 \\
\hline 131 & 194 & 0.07 & 0.10 & -0.03 & 0.01 & 0.078 & -0.137 & 0.042 & 0.000 & -3.45 & -0.71 & 1415.91 & 100.64 & & & -0.46 & 102.32 \\
\hline 132 & 195 & 0.08 & 0.10 & -0.03 & 0.01 & 0.089 & -0.138 & 0.043 & 0.001 & -2.72 & 0.01 & 1416.98 & 107.64 & & & 0.28 & 109.47 \\
\hline 133 & 196 & 0.10 & 0.10 & -0.04 & 0.01 & 0.110 & -0.138 & 0.056 & 0.003 & -2.75 & 0.26 & 1416.93 & 115.76 & & & 0.57 & 117.77 \\
\hline 134 & 197 & 0.11 & 0.10 & -0.05 & 0.01 & 0.121 & -0.139 & 0.069 & 0.005 & -2.42 & 0.81 & 1417.97 & 122.79 & & & 1.18 & 125.02 \\
\hline
\end{tabular}




\section{$Z=63(\mathrm{Eu})$}

$\begin{array}{rrrrrrrrrrrrrr}135 & 198 & 0.17 & 0.00 & -0.07 & -0.01 & 0.185 & 0.000 & 0.100 & 0.029 & -2.16 & 0.79 & 1418.00 & 130.84 \\ 136 & 199 & 0.17 & 0.00 & -0.08 & -0.01 & 0.185 & 0.000 & 0.113 & 0.032 & -2.58 & 0.71 & 1419.46 & 137.44 \\ 137 & 200 & 0.19 & 0.00 & -0.07 & 0.00 & 0.206 & 0.000 & 0.103 & 0.020 & -2.73 & 0.47 & 1419.50 & 145.48 \\ 138 & 201 & 0.20 & 0.00 & -0.07 & 0.00 & 0.217 & 0.000 & 0.105 & 0.022 & -2.86 & 0.41 & 1420.75 & 152.30 \\ 139 & 202 & 0.21 & 0.00 & -0.06 & 0.01 & 0.227 & 0.000 & 0.094 & 0.009 & -3.01 & 0.09 & 1420.68 & 160.43 \\ 140 & 203 & 0.22 & 0.00 & -0.05 & 0.01 & 0.238 & 0.000 & 0.084 & 0.007 & -2.91 & 0.00 & 1421.77 & 167.42 \\ 141 & 204 & 0.22 & 0.01 & -0.05 & 0.02 & 0.237 & -0.014 & 0.084 & -0.003 & -3.45 & -0.54 & 1421.74 & 175.52 \\ 142 & 205 & 0.23 & 0.00 & -0.05 & 0.03 & 0.248 & 0.000 & 0.087 & -0.013 & -3.76 & -0.74 & 1422.76 & 182.58 \\ 143 & 206 & 0.23 & 0.00 & -0.05 & 0.03 & 0.248 & 0.000 & 0.087 & -0.013 & -4.33 & -1.27 & 1422.54 & 190.86 \\ 144 & 207 & 0.24 & 0.00 & -0.04 & 0.04 & 0.259 & 0.000 & 0.078 & -0.025 & -4.39 & -1.45 & 1423.33 & 198.14 \\ 145 & 208 & 0.24 & 0.00 & -0.04 & 0.04 & 0.259 & 0.000 & 0.078 & -0.025 & -4.93 & -1.96 & 1422.93 & 206.61 \\ 146 & 209 & 0.24 & 0.00 & -0.04 & 0.05 & 0.259 & 0.000 & 0.079 & -0.035 & -5.08 & -2.25 & 1423.67 & 213.95 \\ 147 & 210 & 0.25 & 0.00 & -0.03 & 0.05 & 0.271 & 0.000 & 0.069 & -0.037 & -5.54 & -2.69 & 1423.03 & 222.66 \\ 148 & 211 & 0.25 & 0.00 & -0.02 & 0.05 & 0.271 & 0.000 & 0.057 & -0.040 & -5.28 & -2.61 & 1423.22 & 230.54\end{array}$

$\begin{array}{ll}1.25 & 133.31\end{array}$

$\begin{array}{ll}1.31 & 140.22\end{array}$

$\begin{array}{ll}0.87 & 148.21\end{array}$

$\begin{array}{ll}0.83 & 155.22\end{array}$

$0.36 \quad 163.38$

$\begin{array}{ll}0.19 & 170.47\end{array}$

$-0.32 \quad 178.77$

$-0.43 \quad 186.12$

$-0.97 \quad 194.59$

$-1.02 \quad 202.19$

$-1.55 \quad 210.86$

$-1.58 \quad 218.66$

$-2.07 \quad 227.55$

$-1.96 \quad 235.68$

\begin{tabular}{|c|c|c|c|c|c|c|c|c|c|c|c|c|c|c|c|c|c|}
\hline \multicolumn{18}{|c|}{$Z=64(\mathrm{Gd})$} \\
\hline 64 & 128 & 0.31 & 0.00 & 0.01 & 0.03 & 0.341 & 0.000 & 0.034 & -0.027 & -2.88 & 0.69 & 994.32 & -11.26 & & & 0.61 & -10.84 \\
\hline 65 & 129 & 0.30 & 0.00 & 0.02 & 0.02 & 0.331 & 0.000 & 0.018 & -0.022 & -2.65 & 0.83 & 1007.01 & -15.88 & & & 0.74 & -15.52 \\
\hline 66 & 130 & 0.30 & 0.00 & 0.03 & 0.01 & 0.331 & 0.000 & 0.004 & -0.016 & -2.55 & 0.92 & 1021.83 & -22.62 & & & 0.85 & -22.28 \\
\hline 67 & 131 & 0.29 & 0.00 & 0.03 & 0.01 & 0.320 & 0.000 & 0.002 & -0.016 & -2.53 & 0.66 & 1034.29 & -27.01 & & & 0.59 & -26.72 \\
\hline 68 & 132 & 0.29 & 0.00 & 0.04 & 0.00 & 0.320 & 0.000 & -0.012 & -0.010 & -2.54 & 0.76 & 1048.45 & -33.10 & & & 0.70 & -32.83 \\
\hline 69 & 133 & 0.29 & 0.00 & 0.05 & 0.00 & 0.321 & 0.000 & -0.024 & -0.013 & -2.79 & 0.82 & 1059.98 & -36.57 & & & 0.75 & -36.35 \\
\hline 70 & 134 & 0.29 & 0.00 & 0.06 & -0.01 & 0.322 & 0.000 & -0.037 & -0.007 & -2.76 & 0.98 & 1073.46 & -41.98 & & & 0.94 & -41.78 \\
\hline 71 & 135 & 0.27 & 0.00 & 0.05 & 0.00 & 0.299 & 0.000 & -0.029 & -0.013 & -2.16 & 1.17 & 1084.29 & -44.73 & & & 1.12 & -44.58 \\
\hline 72 & 136 & 0.27 & 0.00 & 0.05 & -0.01 & 0.298 & 0.000 & -0.030 & -0.003 & -1.72 & 1.50 & 1096.99 & -49.36 & & & 1.48 & -49.23 \\
\hline 73 & 137 & 0.24 & 0.00 & 0.05 & -0.01 & 0.264 & 0.000 & -0.037 & -0.002 & -1.16 & 1.58 & 1107.37 & -51.67 & & & 1.55 & -51.58 \\
\hline 74 & 138 & 0.23 & 0.00 & 0.04 & -0.01 & 0.252 & 0.000 & -0.027 & 0.001 & -0.67 & 1.72 & 1119.70 & -55.93 & & & 1.71 & -55.87 \\
\hline 75 & 139 & 0.21 & 0.00 & 0.05 & -0.01 & 0.230 & 0.000 & -0.043 & -0.001 & -0.81 & 1.75 & 1129.58 & -57.74 & & & 1.74 & -57.72 \\
\hline 76 & 140 & 0.20 & 0.00 & 0.04 & -0.01 & 0.218 & 0.000 & -0.032 & 0.002 & -0.54 & 1.71 & 1141.54 & -61.63 & -6 & 0.028 & 1.71 & -61.64 \\
\hline 77 & 141 & 0.19 & 0.00 & 0.04 & -0.01 & 0.207 & 0.000 & -0.034 & 0.002 & -0.72 & 1.47 & 1151.18 & -63.19 & -63.22 & 0.020 & 1.46 & -63.25 \\
\hline 78 & 142 & -0.17 & 0.00 & 0.03 & 0.01 & -0.176 & 0.000 & -0.023 & -0.004 & -0.40 & 1.32 & 1162.72 & -66.66 & -66.96 & 0.028 & 1.32 & -6 \\
\hline 79 & 143 & -0.16 & 0.00 & 0.03 & 0.02 & -0.166 & 0.000 & -0.025 & -0.014 & -0.81 & 0.83 & 1172.10 & -67.97 & -68.23 & 0.200 & 0.83 & -68.09 \\
\hline 80 & 144 & -0.14 & 0.00 & 0.02 & 0.01 & -0.146 & 0.000 & -0.015 & -0.007 & -1.15 & 0.30 & 1183.51 & -71.31 & -71.76 & .028 & 0.31 & -71.47 \\
\hline 81 & 145 & -0.06 & 0.00 & 0.01 & 0.00 & -0.063 & 0.000 & -0.010 & 0.001 & -1.53 & -0.83 & 1193.05 & -72.78 & -72.93 & 0.019 & -0.84 & -72.99 \\
\hline 82 & 146 & 0.00 & 0.00 & 0.00 & 0.00 & 0.000 & 0.000 & 0.000 & 0.000 & -2.80 & -1.91 & 1204.52 & -76.18 & -76.09 & 0.005 & -1.91 & -76.42 \\
\hline 83 & 147 & -0.03 & 0.00 & 0.00 & 0.00 & -0.032 & 0.000 & 0.000 & 0.000 & -1.81 & -1.05 & 1211.60 & -75.19 & -75.36 & 0.003 & -1.05 & -75.46 \\
\hline 84 & 148 & 0.00 & 0.00 & 0.00 & 0.00 & 0.000 & 0.000 & 0.000 & 0.000 & -0.63 & -0.06 & 1220.53 & -76.04 & -76.28 & 0.003 & -0.06 & -76.35 \\
\hline 85 & 149 & 0.13 & 0.00 & -0.03 & -0.01 & 0.140 & 0.000 & 0.043 & 0.016 & -0.67 & 1.00 & 1226.96 & -74.40 & -75.13 & 0.004 & 1.02 & -7 \\
\hline 86 & 150 & 0.16 & 0.00 & -0.03 & -0.01 & 0.172 & 0.000 & 0.047 & 0.017 & -0.60 & 1.44 & 1235.97 & -75.34 & -75.77 & 0.006 & 1.48 & -75.67 \\
\hline 87 & 151 & 0.18 & 0.00 & -0.03 & 0.01 & 0.194 & 0.000 & 0.051 & -0.002 & -0.73 & 1.44 & 1243.03 & -74.33 & -74.19 & 0.004 & 1.47 & -74.70 \\
\hline 88 & 152 & 0.19 & 0.00 & -0.03 & 0.01 & 0.205 & 0.000 & 0.053 & -0.001 & -0.69 & 1.64 & 1251.85 & -75.08 & -74.71 & 0.003 & 1.68 & -75.46 \\
\hline 89 & 153 & 0.21 & 0.00 & -0.04 & 0.02 & 0.226 & 0.000 & 0.070 & -0.007 & -1.38 & 1.58 & 1258.55 & -73.71 & -72.89 & 0.003 & 1.64 & -74.10 \\
\hline 90 & 154 & 0.22 & 0.00 & -0.04 & 0.02 & 0.237 & 0.000 & 0.072 & -0.007 & -1.65 & 1.52 & 1267.20 & -74.29 & -73.71 & 0.003 & 1.59 & -74.69 \\
\hline 91 & 155 & 0.23 & 0.00 & -0.05 & 0.02 & 0.249 & 0.000 & 0.087 & -0.002 & -2.49 & 1.23 & 1273.73 & -72.75 & -72.08 & 0.003 & 1.32 & -73.15 \\
\hline 92 & 156 & 0.24 & 0.00 & -0.05 & 0.02 & 0.260 & 0.000 & 0.089 & -0.001 & -2.88 & 1.04 & 1282.10 & -73.04 & -72.54 & 0.003 & 1.15 & -73.45 \\
\hline 93 & 157 & 0.25 & 0.00 & -0.04 & 0.02 & 0.271 & 0.000 & 0.079 & -0.004 & -3.13 & 0.74 & 1288.25 & -71.13 & -70.83 & 0.003 & 0.82 & -71.57 \\
\hline 94 & 158 & 0.26 & 0.00 & -0.04 & 0.03 & 0.282 & 0.000 & 0.082 & -0.013 & -3.43 & 0.65 & 1296.12 & -70.92 & -70.70 & 0.003 & 0.78 & -71.34 \\
\hline 95 & 159 & 0.26 & 0.00 & -0.03 & 0.03 & 0.282 & 0.000 & 0.069 & -0.016 & -3.54 & 0.35 & 1301.89 & -68.62 & -68.57 & 0.003 & 0.46 & -69.08 \\
\hline 96 & 160 & 0.26 & 0.00 & -0.03 & 0.03 & 0.282 & 0.000 & 0.069 & -0.016 & -3.57 & 0.30 & 1309.34 & -68.00 & -67.95 & 0.003 & 0.42 & -68.44 \\
\hline 97 & 161 & 0.27 & 0.00 & -0.02 & 0.03 & 0.294 & 0.000 & 0.060 & -0.019 & -3.96 & -0.10 & 1314.85 & -65.44 & -65.51 & 0.003 & 0.00 & -65.92 \\
\hline 98 & 162 & 0.27 & 0.00 & -0.01 & 0.03 & 0.294 & 0.000 & 0.047 & -0.023 & -3.89 & -0.14 & 1321.91 & -64.43 & -64.29 & 0.005 & -0.02 & -64.89 \\
\hline 99 & 163 & 0.27 & 0.00 & -0.01 & 0.04 & 0.294 & 0.000 & 0.049 & -0.032 & -4.38 & -0.47 & 1327.00 & -61.45 & & & -0.31 & -61.87 \\
\hline 100 & 164 & 0.27 & 0.00 & 0.00 & 0.04 & 0.295 & 0.000 & 0.037 & -0.036 & -4.28 & -0.44 & 1333.64 & -60.01 & & & -0.26 & -60.42 \\
\hline 101 & 165 & 0.28 & 0.00 & 0.01 & 0.04 & 0.307 & 0.000 & 0.027 & -0.039 & -4.80 & -0.73 & 1338.35 & -56.65 & & & -0.55 & -57.05 \\
\hline 102 & 166 & 0.28 & 0.00 & 0.02 & 0.04 & 0.308 & 0.000 & 0.015 & -0.042 & -4.72 & -0.66 & 1344.59 & -54.82 & & & -0.43 & -55.17 \\
\hline 103 & 167 & 0.28 & 0.00 & 0.03 & 0.03 & 0.309 & 0.000 & 0.002 & -0.036 & -4.77 & -0.79 & 1348.81 & -50.97 & & & -0.63 & -51.39 \\
\hline 104 & 168 & 0.27 & 0.00 & 0.03 & 0.03 & 0.298 & 0.000 & -0.001 & -0.036 & -4.28 & -0.55 & 1354.55 & -48.64 & & & -0.37 & -49.02 \\
\hline 105 & 169 & 0.27 & 0.00 & 0.04 & 0.02 & 0.298 & 0.000 & -0.014 & -0.029 & -4.30 & -0.60 & 1358.38 & -44.40 & & & -0.48 & -44.81 \\
\hline
\end{tabular}

\section{$Z=64(G d)$}




\begin{tabular}{|c|c|c|c|c|c|c|c|c|c|c|c|c|c|c|c|}
\hline \multicolumn{16}{|c|}{$Z=64(\mathrm{Gd})$} \\
\hline 106170 & 0.26 & 0.00 & 0.04 & 0.01 & 0.287 & 0.000 & -0.018 & -0.019 & -3.67 & -0.30 & 1363.73 & -41.68 & & -0.21 & -42.11 \\
\hline $107 \quad 171$ & 0.26 & 0.00 & 0.05 & 0.01 & 0.287 & 0.000 & -0.030 & -0.022 & -3.95 & -0.39 & 1367.29 & -37.16 & & -0.28 & -37.56 \\
\hline $108 \quad 172$ & 0.25 & 0.00 & 0.05 & 0.00 & 0.276 & 0.000 & -0.033 & -0.012 & -3.33 & -0.04 & 1372.27 & -34.07 & & 0.06 & -34.46 \\
\hline 109173 & 0.25 & 0.00 & 0.06 & 0.00 & 0.277 & 0.000 & -0.045 & -0.015 & -3.54 & -0.10 & 1375.50 & -29.23 & & 0.02 & -29.56 \\
\hline 110174 & 0.25 & 0.00 & 0.07 & -0.01 & 0.277 & 0.000 & -0.059 & -0.009 & -3.28 & 0.29 & 1380.14 & -25.81 & & 0.45 & -26.06 \\
\hline 111175 & 0.24 & 0.00 & 0.07 & -0.02 & 0.265 & 0.000 & -0.062 & 0.002 & -3.14 & 0.29 & 1383.02 & -20.61 & & 0.46 & -20.82 \\
\hline 112176 & 0.23 & 0.00 & 0.08 & -0.03 & 0.254 & 0.000 & -0.077 & 0.010 & -2.94 & 0.56 & 1387.48 & -17.00 & & 0.84 & -17.06 \\
\hline 113177 & 0.21 & 0.00 & 0.07 & -0.02 & 0.231 & 0.000 & -0.068 & 0.004 & -2.47 & 0.55 & 1390.10 & -11.55 & & 0.74 & -11.65 \\
\hline $114 \quad 178$ & 0.21 & 0.00 & 0.07 & -0.03 & 0.231 & 0.000 & -0.069 & 0.014 & -2.26 & 0.72 & 1394.37 & -7.74 & & 0.98 & -7.74 \\
\hline 115179 & 0.20 & 0.00 & 0.08 & -0.03 & 0.220 & 0.000 & -0.082 & 0.012 & -2.68 & 0.41 & 1397.01 & -2.32 & & 0.71 & -2.21 \\
\hline 116180 & 0.19 & 0.00 & 0.07 & -0.03 & 0.208 & 0.000 & -0.072 & 0.015 & -2.04 & 0.66 & 1400.93 & 1.84 & & 0.94 & 1.97 \\
\hline $117 \quad 181$ & 0.17 & 0.00 & 0.07 & -0.03 & 0.186 & 0.000 & -0.075 & 0.017 & -2.18 & 0.29 & 1403.37 & 7.47 & & 0.58 & 7.68 \\
\hline 118182 & 0.15 & 0.00 & 0.06 & -0.02 & 0.163 & 0.000 & -0.064 & 0.010 & -1.69 & 0.49 & 1407.06 & 11.85 & & 0.69 & 12.03 \\
\hline 119183 & -0.18 & 0.00 & 0.03 & -0.01 & -0.187 & 0.000 & -0.021 & 0.014 & -1.63 & 0.24 & 1409.12 & 17.86 & & 0.31 & 17.98 \\
\hline $120 \quad 184$ & -0.17 & 0.00 & 0.02 & -0.01 & -0.177 & 0.000 & -0.011 & 0.012 & -1.81 & -0.01 & 1413.01 & 22.05 & & 0.04 & 22.22 \\
\hline $121 \quad 185$ & -0.15 & 0.00 & 0.02 & 0.00 & -0.156 & 0.000 & -0.014 & 0.003 & -2.10 & -0.55 & 1415.11 & 28.01 & & -0.53 & 28.23 \\
\hline 122186 & -0.12 & 0.00 & 0.01 & 0.00 & -0.125 & 0.000 & -0.006 & 0.001 & -2.04 & -0.95 & 1418.89 & 32.31 & & -0.94 & 32.60 \\
\hline 123187 & -0.08 & 0.00 & 0.02 & 0.00 & -0.084 & 0.000 & -0.021 & 0.002 & -2.90 & -2.07 & 1421.33 & 37.93 & & -2.05 & 38.32 \\
\hline $124 \quad 188$ & -0.06 & 0.00 & 0.02 & 0.01 & -0.063 & 0.000 & -0.022 & -0.008 & -3.52 & -2.65 & 1425.05 & 42.29 & & -2.61 & 42.78 \\
\hline 125189 & 0.00 & 0.00 & 0.00 & 0.00 & 0.000 & 0.000 & 0.000 & 0.000 & -4.54 & -3.61 & 1427.10 & 48.31 & & -3.61 & 48.86 \\
\hline 126190 & 0.00 & 0.00 & 0.00 & 0.00 & 0.000 & 0.000 & 0.000 & 0.000 & -5.14 & -4.12 & 1430.51 & 52.97 & & -4.12 & 53.62 \\
\hline $127 \quad 191$ & -0.02 & 0.00 & -0.01 & 0.00 & -0.021 & 0.000 & 0.012 & -0.000 & -4.22 & -3.29 & 1430.54 & 61.01 & & -3.29 & 61.77 \\
\hline $128 \quad 192$ & 0.00 & 0.00 & 0.00 & 0.00 & 0.000 & 0.000 & 0.000 & 0.000 & -3.07 & -2.20 & 1432.11 & 67.51 & & -2.20 & 68.37 \\
\hline 129193 & 0.04 & 0.07 & -0.02 & 0.00 & 0.045 & -0.096 & 0.026 & 0.004 & -3.24 & -1.49 & 1432.04 & 75.65 & & -1.36 & 76.76 \\
\hline 130194 & 0.05 & 0.08 & -0.02 & 0.00 & 0.056 & -0.110 & 0.027 & 0.006 & -2.59 & -0.61 & 1433.60 & 82.16 & & -0.45 & 83.42 \\
\hline $131 \quad 195$ & 0.07 & 0.10 & -0.02 & 0.01 & 0.079 & -0.137 & 0.030 & -0.001 & -3.00 & -0.37 & 1433.79 & 90.04 & & -0.14 & 91.49 \\
\hline 132196 & 0.08 & 0.10 & -0.03 & 0.01 & 0.089 & -0.138 & 0.043 & 0.001 & -2.41 & 0.34 & 1435.30 & 96.61 & & 0.61 & 98.23 \\
\hline 133197 & 0.10 & 0.09 & -0.04 & 0.00 & 0.110 & -0.125 & 0.056 & 0.012 & -2.17 & 0.59 & 1435.27 & 104.71 & & 0.87 & 106.47 \\
\hline 134198 & 0.12 & 0.09 & -0.05 & 0.01 & 0.131 & -0.125 & 0.070 & 0.005 & -1.84 & 1.17 & 1436.69 & 111.36 & & 1.51 & 113.32 \\
\hline 135199 & 0.16 & 0.00 & -0.07 & -0.01 & 0.174 & 0.000 & 0.098 & 0.027 & -1.71 & 1.18 & 1436.70 & 119.42 & & 1.64 & 121.64 \\
\hline 136200 & 0.17 & 0.00 & -0.07 & -0.01 & 0.185 & 0.000 & 0.100 & 0.029 & -1.81 & 1.13 & 1438.55 & 125.64 & & 1.61 & 128.04 \\
\hline 137201 & 0.18 & 0.00 & -0.07 & -0.01 & 0.196 & 0.000 & 0.101 & 0.030 & -2.36 & 0.76 & 1438.74 & 133.52 & & 1.25 & 136.08 \\
\hline $138 \quad 202$ & 0.19 & 0.00 & -0.07 & 0.00 & 0.206 & 0.000 & 0.103 & 0.020 & -2.32 & 0.79 & 1440.31 & 140.03 & & 1.21 & 142.68 \\
\hline 139203 & 0.21 & 0.00 & -0.06 & 0.01 & 0.227 & 0.000 & 0.094 & 0.009 & -2.60 & 0.48 & 1440.25 & 148.16 & & 0.76 & 150.83 \\
\hline $140 \quad 204$ & 0.22 & 0.00 & -0.05 & 0.01 & 0.238 & 0.000 & 0.084 & 0.007 & -2.53 & 0.37 & 1441.75 & 154.73 & & 0.58 & 157.50 \\
\hline 141205 & 0.22 & 0.00 & -0.05 & 0.02 & 0.237 & 0.000 & 0.084 & -0.003 & -3.06 & -0.17 & 1441.74 & 162.81 & & 0.06 & 165.78 \\
\hline $142 \quad 206$ & 0.22 & 0.00 & -0.05 & 0.02 & 0.237 & 0.000 & 0.084 & -0.003 & -3.17 & -0.29 & 1443.07 & 169.55 & & -0.05 & 172.72 \\
\hline 143207 & 0.23 & 0.00 & -0.04 & 0.03 & 0.248 & 0.000 & 0.075 & -0.016 & -3.69 & -0.85 & 1442.90 & 177.79 & & -0.59 & 181.16 \\
\hline 144208 & 0.24 & 0.00 & -0.04 & 0.04 & 0.259 & 0.000 & 0.078 & -0.025 & -4.02 & -1.08 & 1444.15 & 184.62 & & -0.65 & 188.35 \\
\hline 145209 & 0.24 & 0.00 & -0.03 & 0.04 & 0.260 & 0.000 & 0.066 & -0.028 & -4.39 & -1.61 & 1443.77 & 193.06 & & -1.22 & 196.96 \\
\hline 146210 & 0.24 & 0.00 & -0.03 & 0.04 & 0.260 & 0.000 & 0.066 & -0.028 & -4.42 & -1.67 & 1444.67 & 200.24 & & -1.26 & 204.35 \\
\hline 147211 & 0.25 & 0.00 & -0.02 & 0.04 & 0.271 & 0.000 & 0.056 & -0.031 & -4.89 & -2.06 & 1443.98 & 208.99 & & -1.69 & 213.28 \\
\hline 148212 & 0.25 & 0.00 & -0.02 & 0.04 & 0.271 & 0.000 & 0.056 & -0.031 & -4.80 & -2.06 & 1444.64 & 216.41 & & -1.67 & 220.92 \\
\hline 149213 & 0.25 & 0.00 & -0.01 & 0.04 & 0.272 & 0.000 & 0.044 & -0.034 & -5.07 & -2.42 & 1443.75 & 225.37 & & -2.03 & 230.10 \\
\hline $150 \quad 214$ & 0.25 & 0.00 & 0.00 & 0.04 & 0.273 & 0.000 & 0.032 & -0.037 & -4.83 & -2.30 & 1444.12 & 233.07 & & -1.88 & 238.05 \\
\hline \multicolumn{16}{|c|}{$Z=65(\mathrm{~Tb})$} \\
\hline $65 \quad 130$ & 0.30 & 0.00 & 0.03 & 0.02 & 0.331 & 0.000 & 0.006 & -0.026 & -2.93 & 0.65 & 1003.79 & -5.37 & & 0.52 & -5.17 \\
\hline 66131 & 0.29 & 0.00 & 0.03 & 0.01 & 0.320 & 0.000 & 0.002 & -0.016 & -2.84 & 0.45 & 1019.22 & -12.73 & & 0.35 & -12.31 \\
\hline $67 \quad 132$ & 0.29 & 0.00 & 0.04 & 0.01 & 0.321 & 0.000 & -0.010 & -0.020 & -3.02 & 0.40 & 1032.08 & -17.52 & & 0.29 & -17.17 \\
\hline $68 \quad 133$ & 0.29 & 0.00 & 0.05 & 0.00 & 0.321 & 0.000 & -0.024 & -0.013 & -3.08 & 0.28 & 1046.56 & -23.93 & & 0.19 & -23.59 \\
\hline $69 \quad 134$ & 0.29 & 0.00 & 0.06 & -0.01 & 0.322 & 0.000 & -0.037 & -0.007 & -3.37 & 0.23 & 1058.81 & -28.10 & & 0.13 & -27.83 \\
\hline $70 \quad 135$ & 0.29 & 0.00 & 0.06 & -0.01 & 0.322 & 0.000 & -0.037 & -0.007 & -3.27 & 0.43 & 1072.34 & -33.56 & & 0.36 & -33.31 \\
\hline $71 \quad 136$ & 0.28 & 0.00 & 0.06 & -0.01 & 0.310 & 0.000 & -0.040 & -0.007 & -2.92 & 0.65 & 1083.73 & -36.88 & & 0.57 & -36.68 \\
\hline $\begin{array}{ll}72 & 137\end{array}$ & 0.27 & 0.00 & 0.06 & -0.01 & 0.299 & 0.000 & -0.042 & -0.007 & -2.37 & 0.99 & 1096.53 & -41.61 & & 0.94 & -41.43 \\
\hline $\begin{array}{ll}73 & 138\end{array}$ & 0.25 & 0.00 & 0.05 & -0.01 & 0.275 & 0.000 & -0.035 & -0.003 & -1.72 & 1.17 & 1107.39 & -44.40 & & 1.12 & -44.27 \\
\hline 74139 & 0.23 & 0.00 & 0.05 & -0.02 & 0.252 & 0.000 & -0.040 & 0.008 & -1.24 & 1.31 & 1119.81 & -48.75 & & 1.28 & -48.63 \\
\hline $75 \quad 140$ & 0.22 & 0.00 & 0.05 & -0.01 & 0.241 & 0.000 & -0.041 & -0.002 & -1.28 & 1.35 & 1130.26 & $-51.13-50.48$ & 0.800 & 1.32 & -51.06 \\
\hline
\end{tabular}




\begin{tabular}{|c|c|c|c|c|c|c|c|c|c|c|c|c|c|c|c|c|c|}
\hline \multicolumn{18}{|c|}{$Z=65(\mathrm{~Tb})$} \\
\hline 76 & 141 & 0.21 & 0.00 & 0.05 & -0.02 & 0.230 & 0.000 & -0.044 & 0.009 & -1.14 & 1.43 & 1142.19 & -54.99 & -54.54 & 0.105 & 1.42 & -54.95 \\
\hline 77 & 142 & 0.19 & 0.00 & 0.05 & -0.02 & 0.207 & 0.000 & -0.047 & 0.010 & -1.10 & 1.26 & 1152.34 & -57.06 & & & 1.24 & -57.06 \\
\hline 78 & 143 & -0.17 & 0.00 & 0.03 & 0.01 & -0.176 & 0.000 & -0.023 & -0.004 & -0.47 & 1.26 & 1163.80 & -60.46 & -60.43 & 0.060 & 1.26 & -60.50 \\
\hline 79 & 144 & -0.16 & 0.00 & 0.03 & 0.01 & -0.166 & 0.000 & -0.024 & -0.004 & -0.86 & 0.75 & 1173.77 & -62.36 & -62.37 & 0.028 & 0.74 & -62.44 \\
\hline 80 & 145 & -0.15 & 0.00 & 0.03 & 0.01 & -0.156 & 0.000 & -0.026 & -0.005 & -1.37 & 0.28 & 1185.21 & -65.73 & -65.88 & 0.057 & 0.28 & -65.84 \\
\hline 81 & 146 & -0.06 & 0.00 & 0.00 & 0.00 & -0.063 & 0.000 & 0.001 & 0.000 & -1.41 & -0.75 & 1195.21 & -67.65 & -67.77 & 0.045 & -0.75 & -67.80 \\
\hline 82 & 147 & -0.02 & 0.00 & 0.00 & 0.00 & -0.021 & 0.000 & 0.000 & 0.000 & -2.53 & -1.70 & 1206.63 & -71.00 & -70.75 & 0.012 & -1.70 & -71.19 \\
\hline 83 & 148 & -0.04 & 0.00 & -0.01 & 0.00 & -0.042 & 0.000 & 0.012 & -0.001 & -1.69 & -0.93 & 1214.36 & -70.65 & -70.54 & 0.014 & -0.93 & -70.88 \\
\hline 84 & 149 & -0.05 & 0.00 & -0.01 & 0.00 & -0.052 & 0.000 & 0.013 & -0.001 & -0.55 & 0.14 & 1223.28 & -71.51 & -71.50 & 0.004 & 0.14 & -71.77 \\
\hline 85 & 150 & 0.14 & 0.00 & -0.03 & -0.01 & 0.151 & 0.000 & 0.045 & 0.016 & -0.77 & 1.04 & 1230.43 & -70.58 & -71.11 & 0.008 & 1.05 & -70.86 \\
\hline 86 & 151 & 0.16 & 0.00 & -0.03 & 0.00 & 0.172 & 0.000 & 0.048 & 0.007 & -0.57 & 1.43 & 1239.57 & -71.65 & -71.63 & 0.005 & 1.45 & -71.96 \\
\hline 87 & 152 & 0.18 & 0.00 & -0.02 & 0.00 & 0.194 & 0.000 & 0.038 & 0.006 & -0.57 & 1.44 & 1247.16 & -71.17 & -70.72 & 0.040 & 1.45 & -71.53 \\
\hline 88 & 153 & 0.20 & 0.00 & -0.02 & 0.02 & 0.216 & 0.000 & 0.043 & -0.013 & -0.75 & 1.58 & .11 & -72.05 & -71.32 & 0.004 & 1.61 & -72.41 \\
\hline 89 & 154 & 0.21 & 0.00 & -0.03 & 0.02 & 0.227 & 0.000 & 0.057 & -0.010 & -1.21 & 1.53 & 1263.34 & -71.21 & -70.16 & 0.045 & 1.56 & -71.60 \\
\hline 90 & 155 & 0.22 & 0.00 & -0.03 & 0.01 & 0.238 & 0.000 & 0.059 & 0.001 & -1.42 & 1.49 & 1272.04 & -71.84 & -71.25 & 0.012 & 1.52 & -72.25 \\
\hline 91 & 156 & 0.23 & 0.00 & -0.04 & 0.02 & 0.249 & 0.000 & 0.074 & -0.006 & -2.17 & 1.24 & 1279.06 & -70.79 & -70.10 & 0.004 & 1.29 & -71.21 \\
\hline 92 & 157 & 0.24 & 0.00 & -0.04 & 0.02 & 0.260 & 0.000 & 0.076 & -0.005 & -2.53 & 1.06 & 1287.48 & -71.14 & -70.77 & 0.003 & 1.13 & -71.57 \\
\hline 93 & 158 & 0.25 & 0.00 & -0.04 & 0.02 & 0.271 & 0.000 & 0.079 & -0.004 & -3.05 & 0.77 & .15 & -69.73 & -69.48 & 0.003 & 0.83 & .19 \\
\hline 94 & 159 & 0.25 & 0.00 & -0.03 & 0.02 & 0.271 & 0.000 & 0.066 & -0.007 & -2.90 & 0.68 & 2.08 & -69.59 & -69.54 & 0.003 & 0.75 & .06 \\
\hline 95 & 160 & 0.26 & 0.00 & -0.02 & 0.02 & 0.283 & 0.000 & 0.056 & -0.010 & -3.22 & 0.40 & 1308.35 & -67.80 & -67.84 & 0.003 & 0.44 & -68.31 \\
\hline 96 & 161 & 0.26 & 0.00 & -0.02 & 0.02 & 0.283 & 0.000 & 0.056 & -0.010 & -3.31 & 0.30 & .92 & -67.29 & -67.47 & 0.003 & 0.35 & -67.79 \\
\hline 97 & 162 & 0.26 & 0.00 & -0.02 & 0.03 & 0.283 & 0.000 & 0.057 & -0.020 & -3.76 & -0.11 & 1321.94 & -65.24 & -65.68 & 0.036 & -0.03 & -65.74 \\
\hline 98 & 163 & 0.27 & 0.00 & 0.00 & 0.03 & 0.295 & 0.000 & 0.035 & -0.026 & -3.88 & -0.24 & 9.15 & -64.38 & -64.60 & 0.005 & -0.14 & 88 \\
\hline 99 & 164 & 0.27 & 0.00 & 0.00 & 0.03 & 0.295 & 0.000 & 0.035 & -0.026 & -4.26 & -0.56 & 4.74 & -61.90 & -62.08 & 0.100 & -0.48 & -62.42 \\
\hline 100 & 165 & 0.27 & 0.00 & 0.01 & 0.03 & 0.296 & 0.000 & 0.023 & -0.029 & -4.23 & -0.54 & 13 & -60.53 & & & -0.43 & 02 \\
\hline 101 & 166 & 0.27 & 0.00 & 0.02 & 0.03 & 0.297 & 0.000 & 0.011 & -0.032 & -4.60 & -0.85 & 5.67 & -57.68 & -57.76 & 0.100 & -0.74 & -58.18 \\
\hline 102 & 167 & 0.27 & 0.00 & 0.02 & 0.03 & 0.297 & 0.000 & 0.011 & -0.032 & -4.52 & -0.79 & 1352.98 & -55.93 & & & -0.67 & -56.40 \\
\hline 103 & 168 & 0.27 & 0.00 & 0.03 & 0.03 & 0.298 & 0.000 & -0.001 & -0.036 & -4.86 & -1.04 & 1357.82 & -52.69 & & & -0.91 & 15 \\
\hline 104 & 169 & 0.27 & 0.00 & 0.03 & 0.03 & 0.298 & 0.000 & -0.001 & -0.036 & -4.59 & -0.84 & 1363.65 & -50.45 & & & -0.69 & -50.89 \\
\hline 105 & 170 & 0.27 & 0.00 & 0.04 & 0.02 & 0.298 & 0.000 & -0.014 & -0.029 & -4.67 & -0.97 & 1368.04 & -46.77 & & & -0.87 & -47.25 \\
\hline 106 & 171 & 0.26 & 0.00 & 0.04 & 0.01 & 0.287 & 0.000 & -0.018 & -0.019 & -4.07 & -0.61 & 1373.39 & -44.05 & & & -0.54 & -44.54 \\
\hline 107 & 172 & 0.26 & 0.00 & 0.05 & 0.01 & 0.287 & 0.000 & -0.030 & -0.022 & -4.39 & -0.79 & 1377.52 & -40.10 & & & -0.71 & -40.56 \\
\hline 108 & 173 & 0.25 & 0.00 & 0.06 & 0.00 & 0.277 & 0.000 & -0.045 & -0.015 & -3.98 & -0.47 & 1382.58 & -37.10 & & & -0.37 & -37.52 \\
\hline 109 & 174 & 0.25 & 0.00 & 0.06 & 0.00 & 0.277 & 0.000 & -0.045 & -0.015 & -3.97 & -0.50 & 1386.25 & -32.69 & & & -0.40 & -33.10 \\
\hline 110 & 175 & 0.25 & 0.00 & 0.07 & -0.01 & 0.277 & 0.000 & -0.059 & -0.009 & -3.69 & -0.08 & 1390.92 & -29.29 & & & 0.05 & -29.63 \\
\hline 111 & 176 & 0.24 & 0.00 & 0.07 & -0.01 & 0.266 & 0.000 & -0.061 & -0.008 & -3.53 & -0.11 & 1394.29 & -24.59 & & & 0.02 & -24.90 \\
\hline 112 & 177 & 0.24 & 0.00 & 0.08 & -0.03 & 0.266 & 0.000 & -0.075 & 0.009 & -3.41 & 0.28 & 1398.69 & -20.92 & & & 0.51 & -21.09 \\
\hline 113 & 178 & 0.23 & 0.00 & 0.08 & -0.03 & 0.254 & 0.000 & -0.077 & 0.010 & -3.38 & 0.18 & 140 & -10 & & & 0.42 & 13 \\
\hline 114 & 179 & 0.21 & 0.00 & 0.07 & -0.03 & 0.231 & 0.000 & -0.069 & 0.014 & -2.56 & 0.47 & 1406.05 & -12.14 & & & 0.69 & -12.24 \\
\hline 115 & 180 & 0.20 & 0.00 & 0.08 & -0.03 & 0.220 & 0.000 & -0.082 & 0.012 & -2.95 & 0.20 & 1409.11 & -7.12 & & & 0.47 & -7.13 \\
\hline 116 & 181 & 0.19 & 0.00 & 0.08 & -0.03 & 0.209 & 0.000 & -0.084 & 0.013 & -2.59 & 0.43 & 1413.08 & -3.03 & & & 0.73 & -2.96 \\
\hline 117 & 182 & 0.18 & 0.00 & 0.07 & -0.03 & 0.197 & 0.000 & -0.073 & 0.016 & -2.48 & 0.14 & 1415.89 & 2.23 & & & 0.40 & 2.31 \\
\hline 118 & 183 & 0.15 & 0.00 & 0.06 & -0.02 & 0.163 & 0.000 & -0.064 & 0.010 & -1.80 & 0.40 & 1419.57 & 6.63 & & & 0.58 & 6.69 \\
\hline 119 & 184 & -0.18 & 0.00 & 0.03 & -0.01 & -0.187 & 0.000 & -0.021 & 0.014 & -1.82 & 0.09 & 1422.14 & 12.13 & & & 0.15 & 12.13 \\
\hline 120 & 185 & -0.17 & 0.00 & 0.02 & -0.01 & -0.177 & 0.000 & -0.011 & 0.012 & -1.98 & -0.15 & 1426.05 & 16.29 & & & -0.10 & 16.34 \\
\hline 121 & 186 & -0.15 & 0.00 & 0.02 & -0.01 & -0.156 & 0.000 & -0.014 & 0.012 & -2.27 & -0.67 & 1428.58 & 21.83 & & & -0.63 & 21.95 \\
\hline 122 & 187 & -0.12 & 0.00 & 0.01 & 0.00 & -0.125 & 0.000 & -0.006 & 0.001 & -2.09 & -0.98 & 1432.30 & 26.18 & & & -0.97 & 26.34 \\
\hline 123 & 188 & -0.08 & 0.00 & 0.02 & 0.00 & -0.084 & 0.000 & -0.021 & 0.002 & -2.89 & -2.07 & 1435.16 & 31.40 & & & -2.05 & 31.65 \\
\hline 124 & 189 & -0.06 & 0.00 & 0.02 & 0.01 & -0.063 & 0.000 & -0.022 & -0.008 & -3.47 & -2.63 & 1438.88 & 35.75 & & & -2.59 & 36.10 \\
\hline 125 & 190 & 0.01 & 0.01 & 0.01 & 0.00 & 0.011 & -0.013 & -0.012 & 0.000 & -4.40 & -3.44 & 1441.22 & 41.48 & & & -3.43 & 41.89 \\
\hline 126 & 191 & 0.00 & 0.01 & 0.00 & 0.01 & 0.000 & -0.013 & 0.000 & -0.010 & -4.95 & -3.94 & 1444.64 & 46.13 & & & -3.92 & 46.64 \\
\hline 127 & 192 & -0.02 & 0.00 & -0.01 & 0.00 & -0.021 & 0.000 & 0.012 & -0.000 & -4.04 & -3.13 & 1445.12 & 53.72 & & & -3.12 & 54.31 \\
\hline 128 & 193 & 0.01 & 0.02 & 0.00 & 0.00 & 0.011 & -0.027 & 0.000 & 0.000 & -2.95 & -2.02 & 1446.70 & 60.21 & & & -2.01 & 60.91 \\
\hline 129 & 194 & 0.03 & 0.05 & -0.01 & 0.01 & 0.033 & -0.067 & 0.013 & -0.008 & -2.49 & -1.25 & 1447.00 & 67.98 & & & -1.18 & 68.85 \\
\hline 130 & 195 & 0.07 & 0.08 & -0.03 & 0.00 & 0.077 & -0.111 & 0.041 & 0.008 & -2.63 & -0.43 & 1448.64 & 74.41 & & & -0.25 & 75.51 \\
\hline 131 & 196 & 0.07 & 0.10 & -0.03 & 0.01 & 0.078 & -0.137 & 0.042 & 0.000 & -2.96 & -0.17 & 1449.24 & 81.89 & & & 0.06 & 83.15 \\
\hline 132 & 197 & 0.08 & 0.10 & -0.03 & 0.01 & 0.089 & -0.138 & 0.043 & 0.001 & -2.25 & 0.53 & 1450.77 & 88.42 & & & 0.78 & 89.82 \\
\hline
\end{tabular}




\section{$Z=65(\mathrm{~Tb})$}

$\begin{array}{lllll}133 & 198 & 0.11 & 0.08 & -0.04\end{array}$ 134199

135200

$\begin{array}{llll}0.13 & 0.08 & -0.05\end{array}$

0.00

$0.160 .00-0.07$

$0.120-0.111$

0.056

$\begin{array}{ll}0.011-1.81 \\ 0.004 & -1.55\end{array}$

$\begin{array}{lll}0.75 & 1451.20 & 96.07\end{array}$

$\begin{array}{llllll}0.071 & 0.004 & -1.55 & 1.32 & 1452.65 & 102.69\end{array}$

0.098

$\begin{array}{ll}0.027 & -1.72\end{array}$

$\begin{array}{ll}1.09 & 1453.31 \\ 1\end{array}$

110.10

$\begin{array}{lllll}0.17 & 0.00 & -0.07 & -0.01\end{array}$

0.185

0.000

0.100

$\begin{array}{lll}0.029 & -1.79\end{array}$

$\begin{array}{lll}1.16 & 1455.06 & 116.42\end{array}$

137202

$\begin{array}{lll}0.18 & 0.00 & -0.07\end{array}$

0.00

0.195

0.000

0.101

$\begin{array}{ll}0.019 & -2.17\end{array}$

$\begin{array}{lll}0.92 & 1455.53 & 124.02\end{array}$

138203

$\begin{array}{llll}0.19 & 0.00 & -0.06\end{array}$

0.00

0.206

0.000

0.090

$\begin{array}{ll}0.017 & -1.87\end{array}$

$\begin{array}{llll}0.97 & 1457.09 & 130.53\end{array}$

140205

$0.00-0.06$

0.216

0.000

0.093

$\begin{array}{ll}0.008 & -2.36\end{array}$

$\begin{array}{lll}0.60 & 1457.50 & 138.19\end{array}$

$0.00-0.05$

$$
0.02
$$$$
142 \quad 207
$$

143208

$\begin{array}{ll}0.02 & 0.237\end{array}$

0.000

0.084

$\begin{array}{ll}0.007 & -2.45\end{array}$

$\begin{array}{lll}0.46 & 1459.05 & 144.72\end{array}$

0.000

$0.072-0.6$

$-0.007-2.98$

152.41

$\begin{array}{lll}0.03 & 0.248 & 0.000\end{array}$

$\begin{array}{lllllll}0.075 & -0.016 & -3.63 & -0.76 & 1461.00 & 166.98\end{array}$

144209

$0.00-0.04$

0.03

145210

$\begin{array}{lll}0.23 & 0.00 & -0.04\end{array}$

0.04

0.248

0.000

$\begin{array}{llllll}0.075 & -0.016 & -3.69 & -0.84 & 1462.11 & 173.94\end{array}$

$\begin{array}{lll}0.24 & 0.00 & -0.03 \\ 0.24 & 0.00 & -0.02\end{array}$

0.04

0.260

0.000

\section{$147 \quad 212$}

$\begin{array}{llll}0.24 & 0.00 & -0.02\end{array}$

0.04

0.260

0.000

$\begin{array}{lllll}0.066 & -0.028 & -4.34 & -1.53 & 1462.29\end{array}$

181.84

$\begin{array}{llllll}0.053 & -0.031 & -4.24 & -1.52 & 1463.13 & 189.07\end{array}$

148213

$\begin{array}{llll}0.24 & 0.00 & -0.01\end{array}$

0.04

0.261

0.000

$\begin{array}{llllll}0.041 & -0.034 & -4.60 & -2.05 & 1463.63 & 204.71\end{array}$

$150 \quad 215$

$\begin{array}{llll}0.24 & 0.00 & -0.01\end{array}$

0.04

0.261

0.000

0.000

$\begin{array}{llllll}0.029 & -0.037 & -4.80 & -2.36 & 1463.57 & 220.91\end{array}$

$\begin{array}{lll}0.25 & 0.00 & 0.01\end{array}$

0.04

0.273

0.000

$\begin{array}{lllllll}0.020 & -0.040 & -5.27 & -2.72 & 1462.91 & 229.64\end{array}$

$\begin{array}{lll}152 & 217\end{array}$

0.250 .00

0.02

0.03

0.274

$\begin{array}{lll}153 \quad 218 & 0.25\end{array}$

\section{$Z=66(\mathrm{Dy})$}

0.000

$\begin{array}{lllllll}0.006 & -0.033 & -4.89 & -2.37 & 1462.89 & 237.74\end{array}$

$\begin{array}{llllllll}0.000 & -0.005 & -0.036 & -5.18 & -2.63 & 1461.96 & 246.74\end{array}$

$\begin{array}{lllllll}67 & 133 & 0.29 & 0.00 & 0.05 & 0.00 & 0.321\end{array}$

$\begin{array}{lllllll}68 & 134 & 0.29 & 0.00 & 0.06 & -0.01 & 0.322\end{array}$

$\begin{array}{lllllll}69 & 135 & 0.29 & 0.00 & 0.07 & -0.01 & 0.323\end{array}$

$\begin{array}{llllllll}70 & 136 & 0.28 & 0.00 & 0.07 & -0.01 & 0.311\end{array}$

$\begin{array}{lllllll}71 & 137 & 0.27 & 0.00 & 0.07 & -0.01 & 0.300\end{array}$

$\begin{array}{lllllll}72 & 138 & 0.27 & 0.00 & 0.07 & -0.02 & 0.299\end{array}$

$\begin{array}{lllllll}73 & 139 & 0.25 & 0.00 & 0.06 & -0.01 & 0.276\end{array}$

$\begin{array}{lllllll}74 & 140 & 0.23 & 0.00 & 0.05 & -0.01 & 0.253\end{array}$

$\begin{array}{lllllll}75 & 141 & 0.22 & 0.00 & 0.05 & -0.01 & 0.241\end{array}$

$\begin{array}{lllllll}76 & 142 & 0.21 & 0.00 & 0.05 & -0.02 & 0.230\end{array}$

$\begin{array}{llllllll}77 & 143 & 0.19 & 0.00 & 0.05 & -0.01 & 0.208\end{array}$

$\begin{array}{lllllll}78 & 144 & -0.17 & 0.00 & 0.04 & 0.01 & -0.176\end{array}$

$\begin{array}{lllllll}79 & 145 & -0.17 & 0.00 & 0.04 & 0.01 & -0.176\end{array}$

$\begin{array}{lllllll}80 & 146 & -0.15 & 0.00 & 0.03 & 0.01 & -0.156\end{array}$

$\begin{array}{lllllll}81 & 147 & -0.07 & 0.00 & 0.01 & 0.00 & -0.073\end{array}$

$\begin{array}{lllll}82 & 148 & 0.01 & 0.00 & 0.00\end{array}$

$\begin{array}{lllll}83 & 149 & -0.04 & 0.00 & -0.01\end{array}$

84150

$\begin{array}{ll}0.00 & 0.011\end{array}$

$0.00-0.042$

$\begin{array}{lllll}0.00 & 0.00 & 0.00 & 0.00 & 0.000\end{array}$

$\begin{array}{lllllll}85 & 151 & 0.11 & 0.00 & -0.03 & -0.01 & 0.118\end{array}$

$86 \quad 15$

$\begin{array}{llllll}87 & 153 & 0.17 & 0.00 & -0.02\end{array}$

$88 \quad 154$

$89 \quad 155$

$90 \quad 156$

$\begin{array}{lll}0.19 & 0.00 & -0.02\end{array}$

0.00

0.172

$\begin{array}{llll}0.000 & -0.024 & -0.013 & -3.10 \\ 0.000 & -0.037 & -0.007 & -3.33\end{array}$

$\begin{array}{llll}0.000 & -0.037 & -0.007 & -3.33\end{array}$

$\begin{array}{llll}0.000 & -0.049 & -0.011 & -3.76\end{array}$

$\begin{array}{llll}0.000 & -0.052 & -0.010 & -3.61\end{array}$

$\begin{array}{llll}0.000 & -0.054 & -0.010 & -3.28\end{array}$

$\begin{array}{lllll}0.000 & -0.056 & 0.000 & -2.87\end{array}$

$\begin{array}{lllll}0.000 & -0.047 & -0.006 & -2.24\end{array}$

$\begin{array}{llll}0.000 & -0.039 & -0.002 & -1.52\end{array}$

$\begin{array}{lllll}0.000 & -0.041 & -0.002 & -1.49\end{array}$

$\begin{array}{llll}0.000 & -0.044 & 0.009 & -1.30\end{array}$

$\begin{array}{lllll}0.000 & -0.046 & -0.000 & -1.18\end{array}$

$\begin{array}{llll}0.000 & -0.034 & -0.002 & -0.65\end{array}$

$\begin{array}{lllll}0.000 & -0.034 & -0.002 & -1.33\end{array}$

$\begin{array}{llll}0.000 & -0.026 & -0.005 & -1.65\end{array}$

$\begin{array}{lllll}0.000 & -0.010 & 0.001 & -1.79\end{array}$

0.000

0.000

0.000

0.000

0.000

0.000

0.000

$0.012-0.0$

0.000

0.041

$-0.001-1.97$

$\begin{array}{lll}0.23 & 1031.14 & -9.29\end{array}$

$\begin{array}{lll}0.16 & 1046.17 & -16.25\end{array}$

$\begin{array}{llll}0.03 & 1058.60 & -20.61\end{array}$

$\begin{array}{llll}0.09 & 1072.87 & -26.81\end{array}$

$\begin{array}{llll}0.29 & 1084.37 & -30.23\end{array}$

$\begin{array}{llll}0.62 & 1097.77 & -35.56\end{array}$

$\begin{array}{llll}0.78 & 1108.74 & -38.47\end{array}$

$\begin{array}{llll}1.03 & 1121.64 & -43.29\end{array}$

$\begin{array}{lll}1.04 & 1132.22 & -45.80\end{array}$

$\begin{array}{lll}1.19 & 1144.66-50.16\end{array}$

$\begin{array}{lll}1.07 & 1154.82 & -52.26\end{array}$

$\begin{array}{lllll}1.12 & 1166.82 & -56.18 & -56.58\end{array}$

$\begin{array}{lllll}0.41 & 1177.07 & -58.37 & -58.29\end{array}$

$\begin{array}{llll}-0.02 & 1189.04 & -62.26 & -62.55\end{array}$

$\begin{array}{llll}-1.05 & 1199.12 & -64.27 & -64.19\end{array}$

$0.00 \quad 0.183$

$\begin{array}{ll}0.01 & 0.205\end{array}$

$0.01 \quad 0.227$

0.000

0.035

$0.000-0.76$

$\begin{array}{llll}-2.02 & 1211.12 & -68.20 & -67.86\end{array}$

0.000

$\begin{array}{llll}0.037 & 0.005 & -0.40\end{array}$

$\begin{array}{llll}0.041 & -0.004 & -0.45\end{array}$

0.000

$\begin{array}{lll}0.044 & -0.003 & -0.80\end{array}$

$0.01 \quad 0.238$

0.000

$\begin{array}{lll}0.046 & -0.002 & -0.99\end{array}$

$\begin{array}{llll}0.061 & 0.001 & -1.60\end{array}$

$0.01 \quad 0.260$

$\begin{array}{llll}0.063 & 0.002 & -1.92\end{array}$

$\begin{array}{lllll}0.000 & 0.063 & 0.002 & -2.21\end{array}$

$0.01 \quad 0.260$

$\begin{array}{ll}0.01 & 0.272\end{array}$

$0.02 \quad 0.271$

$0.02 \quad 0.283$

0.000

$\begin{array}{llll}0.053 & -0.001 & -2.30\end{array}$

0.000

$\begin{array}{lll}0.054 & -0.010 & -2.68\end{array}$

$96 \quad 162$

$\begin{array}{llll}0.25 & 0.00 & -0.02\end{array}$

0.02

0.283

$\begin{array}{ll}97 & 163\end{array}$

$\begin{array}{lll}0.26 & 0.00 & -0.01\end{array}$

$98 \quad 164$

$99 \quad 165$

$\begin{array}{lll}0.27 & 0.00 & 0.01\end{array}$

$0.02 \quad 0.296$

0.000

$\begin{array}{lll}0.044 & -0.013 & -2.88\end{array}$

0.000

$\begin{array}{lll}0.044 & -0.013 & -3.24\end{array}$

$\begin{array}{llll}-1.19 & 1218.87 & -67.88 & -67.71\end{array}$

$\begin{array}{lllll}0.90 & 1235.43 & -68.30 & -68.76\end{array}$

$\begin{array}{lllll}1.42 & 1244.99 & -69.79 & -70.12\end{array}$

$\begin{array}{llll}1.45 & 1252.65 & -69.37 & -69.15\end{array}$

$\begin{array}{lllll}1.69 & 1262.03 & -70.68 & -70.40\end{array}$

$\begin{array}{lllll}1.67 & 1269.30 & -69.89 & -69.16\end{array}$

$\begin{array}{lllll}1.66 & 1278.50 & -71.01 & -70.53\end{array}$

$\begin{array}{lllll}1.49 & 1285.52 & -69.96 & -69.43\end{array}$

$\begin{array}{lllll}1.33 & 1294.44 & -70.81 & -70.41\end{array}$

$\begin{array}{lllll}1.09 & 1301.13 & -69.42 & -69.17\end{array}$

$\begin{array}{lllll}0.99 & 1309.59 & -69.81 & -69.68\end{array}$

$\begin{array}{lllll}0.67 & 1315.97 & -68.13 & -68.06\end{array}$

$\begin{array}{lllll}0.53 & 1324.08 & -68.16 & -68.19\end{array}$

0.000

$\begin{array}{lllllll}-0.019 & -3.47 & -0.00 & 1337.89 & -65.83 & -65.97\end{array}$

0.000

$\begin{array}{llllllll}0.023 & -0.029 & -4.03 & -0.40 & 1343.61 & -63.48 & -63.62\end{array}$

$\begin{array}{llllllll}0.011 & -0.032 & -4.11 & -0.46 & 1350.90 & -62.69 & -62.59\end{array}$

$\begin{array}{llllllll}0.011 & -0.032 & -4.49 & -0.79 & 1356.21 & -59.93 & -59.94\end{array}$

$0.97 \quad 97.57$

$\begin{array}{ll}1.61 & 104.39\end{array}$

$1.52 \quad 112.07$

1.61118 .56

$1.29 \quad 126.22$

$\begin{array}{ll}1.26 & 132.80\end{array}$

$\begin{array}{ll}0.85 & 140.59\end{array}$

$0.65 \quad 147.21$

$0.14 \quad 155.08$

$0.02 \quad 161.99$

$-0.52 \quad 170.03$

$-0.58 \quad 177.20$

$-1.16 \quad 185.39$

$-1.15 \quad 192.82$

$-1.69201 .24$

$-1.67208 .87$

$-2.06 \quad 217.62$

$-1.95 \quad 225.52$

$-2.30 \quad 234.49$

$-2.13 \quad 242.63$ 


\begin{tabular}{|c|c|c|c|c|c|c|c|c|c|c|c|c|c|c|c|c|c|}
\hline \multicolumn{18}{|c|}{$Z=66(D y)$} \\
\hline 102 & 168 & 0.27 & 0.00 & 0.03 & 0.03 & 0.298 & 0.000 & -0.001 & -0.036 & -4.55 & -0.79 & 1363.07 & -58.72 & -58.56 & 0.140 & -0.64 & -59.19 \\
\hline 103 & 169 & 0.27 & 0.00 & 0.03 & 0.03 & 0.298 & 0.000 & -0.001 & -0.036 & -4.83 & -1.06 & 1367.98 & -55.56 & -55.60 & 0.301 & -0.92 & -56.04 \\
\hline 104 & 170 & 0.27 & 0.00 & 0.04 & 0.02 & 0.298 & 0.000 & -0.014 & -0.029 & -4.59 & -0.90 & 1374.34 & -53.86 & & & -0.78 & -54.34 \\
\hline 105 & 171 & 0.27 & 0.00 & 0.05 & 0.01 & 0.299 & 0.000 & -0.027 & -0.023 & -4.80 & -1.00 & 1378.76 & -50.20 & & & -0.91 & -50.71 \\
\hline 106 & 172 & 0.26 & 0.00 & 0.05 & 0.01 & 0.287 & 0.000 & -0.030 & -0.022 & -4.45 & -0.87 & 1384.81 & -48.18 & & & -0.76 & -48.66 \\
\hline 107 & 173 & 0.26 & 0.00 & 0.06 & 0.00 & 0.288 & 0.000 & -0.043 & -0.016 & -4.76 & -1.06 & 1389.01 & -44.31 & & & -0.96 & -44.79 \\
\hline 108 & 174 & 0.25 & 0.00 & 0.06 & 0.00 & 0.277 & 0.000 & -0.045 & -0.015 & -4.32 & -0.82 & 1394.64 & -41.86 & & & -0.71 & -42.31 \\
\hline 109 & 175 & 0.25 & 0.00 & 0.07 & -0.01 & 0.277 & 0.000 & -0.059 & -0.009 & -4.57 & -0.90 & 1398.41 & -37.56 & & & -0.77 & -37.98 \\
\hline 110 & 176 & 0.25 & 0.00 & 0.07 & -0.01 & 0.277 & 0.000 & -0.059 & -0.009 & -4.12 & -0.50 & 1403.56 & -34.64 & & & -0.36 & -35.02 \\
\hline 111 & 177 & 0.24 & 0.00 & 0.08 & -0.02 & 0.266 & 0.000 & -0.074 & -0.001 & -4.29 & -0.55 & 1407.00 & -30.01 & & & -0.36 & -30.32 \\
\hline 112 & 178 & 0.24 & 0.00 & 0.08 & -0.03 & 0.266 & 0.000 & -0.075 & 0.009 & -3.88 & -0.12 & 1411.81 & -26.75 & & & 0.13 & -26.98 \\
\hline 113 & 179 & 0.23 & 0.00 & 0.09 & -0.03 & 0.255 & 0.000 & -0.089 & 0.007 & -4.20 & -0.27 & 1415.07 & -21.94 & & & 0.02 & -22.09 \\
\hline 114 & 180 & 0.22 & 0.00 & 0.09 & -0.04 & 0.243 & 0.000 & -0.092 & 0.018 & -3.81 & 0.03 & 1419.73 & -18.53 & & & 0.41 & -18.54 \\
\hline 115 & 181 & 0.20 & 0.00 & 0.08 & -0.03 & 0.220 & 0.000 & -0.082 & 0.012 & -3.33 & -0.08 & 1422.68 & -13.40 & & & 0.19 & -13.48 \\
\hline 116 & 182 & 0.20 & 0.00 & 0.08 & -0.04 & 0.220 & 0.000 & -0.083 & 0.022 & -3.18 & 0.03 & 1427.23 & -9.89 & & & 0.39 & -9.83 \\
\hline 117 & 183 & 0.18 & 0.00 & 0.07 & -0.03 & 0.197 & 0.000 & -0.073 & 0.016 & -2.82 & -0.14 & 1429.95 & -4.54 & & & 0.12 & -4.55 \\
\hline 118 & 184 & 0.15 & 0.00 & 0.06 & -0.02 & 0.163 & 0.000 & -0.064 & 0.010 & -2.04 & 0.17 & 1434.03 & -0.54 & & & 0.36 & -0.57 \\
\hline 119 & 185 & -0.18 & 0.00 & 0.03 & -0.01 & -0.187 & 0.000 & -0.021 & 0.014 & -2.11 & -0.18 & 1436.69 & 4.87 & & & -0.12 & 4.78 \\
\hline 120 & 186 & -0.17 & 0.00 & 0.02 & -0.01 & -0.177 & 0.000 & -0.011 & 0.012 & -2.25 & -0.40 & 1441.02 & 8.61 & & & -0.35 & 8.56 \\
\hline 121 & 187 & -0.15 & 0.00 & 0.02 & -0.01 & -0.156 & 0.000 & -0.014 & 0.012 & -2.49 & -0.89 & 1443.56 & 14.14 & & & -0.85 & 14.15 \\
\hline 122 & 188 & -0.12 & 0.00 & 0.01 & 0.00 & -0.125 & 0.000 & -0.006 & 0.001 & -2.26 & -1.14 & 1447.67 & 18.10 & & & -1.14 & 18.15 \\
\hline 123 & 189 & -0.09 & 0.00 & 0.02 & 0.01 & -0.094 & 0.000 & -0.020 & -0.007 & -3.10 & -2.20 & 1450.53 & 23.32 & & & -2.17 & 23.46 \\
\hline 124 & 190 & -0.06 & 0.00 & 0.02 & 0.01 & -0.063 & 0.000 & -0.022 & -0.008 & -3.55 & -2.70 & 1454.63 & 27.29 & & & -2.66 & 27.51 \\
\hline 125 & 191 & -0.01 & 0.00 & 0.00 & 0.00 & -0.011 & 0.000 & 0.000 & 0.000 & -4.43 & -3.54 & 1457.02 & 32.97 & & & -3.54 & 33.23 \\
\hline 126 & 192 & 0.00 & 0.00 & 0.00 & 0.00 & 0.000 & 0.000 & 0.000 & 0.000 & -4.98 & -4.00 & 1460.84 & 37.22 & & & -4.00 & 37.57 \\
\hline 127 & 193 & -0.02 & 0.00 & -0.01 & 0.00 & -0.021 & 0.000 & 0.012 & -0.000 & -4.05 & -3.15 & 1461.32 & 44.81 & & & -3.15 & 45.26 \\
\hline 128 & 194 & 0.00 & 0.00 & 0.00 & 0.00 & 0.000 & 0.000 & 0.000 & 0.000 & -2.88 & -2.05 & 1463.33 & 50.87 & & & -2.05 & 51.41 \\
\hline 129 & 195 & 0.03 & 0.06 & -0.01 & 0.00 & 0.033 & -0.082 & 0.014 & 0.003 & -2.64 & -1.20 & 1463.58 & 58.69 & & & -1.13 & 59.40 \\
\hline 130 & 196 & 0.05 & 0.08 & -0.02 & 0.00 & 0.056 & -0.110 & 0.027 & 0.006 & -2.28 & -0.29 & 1465.56 & 64.79 & & & -0.14 & 65.68 \\
\hline 131 & 197 & 0.07 & 0.09 & -0.02 & 0.01 & 0.078 & -0.123 & 0.029 & -0.002 & -2.30 & 0.03 & 1466.11 & 72.30 & & & 0.21 & 73.33 \\
\hline 132 & 198 & 0.08 & 0.10 & -0.02 & 0.01 & 0.089 & -0.137 & 0.030 & -0.001 & -1.88 & 0.77 & 1468.03 & 78.46 & & & 0.99 & 79.64 \\
\hline 133 & 199 & 0.11 & 0.08 & -0.04 & 0.00 & 0.120 & -0.111 & 0.056 & 0.011 & -1.62 & 0.96 & 1468.50 & 86.05 & & & 1.19 & 87.36 \\
\hline 134 & 200 & 0.11 & 0.08 & -0.04 & 0.00 & 0.120 & -0.111 & 0.056 & 0.011 & -0.95 & 1.56 & 1470.34 & 92.29 & & & 1.80 & 93.74 \\
\hline 135 & 201 & 0.15 & 0.00 & -0.07 & -0.01 & 0.163 & 0.000 & 0.097 & 0.026 & -1.32 & 1.50 & 1470.86 & 99.84 & & & 1.92 & 101.59 \\
\hline 136 & 202 & 0.17 & 0.00 & -0.07 & -0.01 & 0.185 & 0.000 & 0.100 & 0.029 & -1.51 & 1.44 & 1473.14 & 105.63 & & & 1.89 & 107.55 \\
\hline 137 & 203 & 0.18 & 0.00 & -0.07 & 0.00 & 0.195 & 0.000 & 0.101 & 0.019 & -1.86 & 1.21 & 1473.63 & 113.21 & & & 1.58 & 115.19 \\
\hline 138 & 204 & 0.19 & 0.00 & -0.06 & 0.00 & 0.206 & 0.000 & 0.090 & 0.017 & -1.55 & 1.28 & 1475.57 & 119.34 & & & 1.57 & 121.38 \\
\hline 139 & 205 & 0.19 & 0.00 & -0.06 & 0.00 & 0.206 & 0.000 & 0.090 & 0.017 & -1.93 & 0.91 & 1476.00 & 126.98 & & & 1.19 & 129.17 \\
\hline 140 & 206 & 0.22 & 0.00 & -0.04 & 0.01 & 0.238 & 0.000 & 0.071 & 0.004 & -1.75 & 0.89 & 1477.83 & 133.23 & & & 1.02 & 135.4 \\
\hline 141 & 207 & 0.22 & 0.00 & -0.05 & 0.02 & 0.237 & 0.000 & 0.084 & -0.003 & -2.57 & 0.31 & 1478.27 & 140.85 & & & 0.53 & 143.28 \\
\hline 142 & 208 & 0.22 & 0.00 & -0.04 & 0.02 & 0.237 & 0.000 & 0.072 & -0.007 & -2.47 & 0.21 & 1479.99 & 147.21 & & & 0.38 & 149.74 \\
\hline 143 & 209 & 0.23 & 0.00 & -0.04 & 0.03 & 0.248 & 0.000 & 0.075 & -0.016 & -3.22 & -0.37 & 1480.26 & 155.01 & & & -0.12 & 157.80 \\
\hline 144 & 210 & 0.23 & 0.00 & -0.03 & 0.03 & 0.249 & 0.000 & 0.062 & -0.019 & -3.09 & -0.40 & 1481.71 & 161.63 & & & -0.18 & 164.57 \\
\hline 145 & 211 & 0.24 & 0.00 & -0.02 & 0.03 & 0.260 & 0.000 & 0.052 & -0.021 & -3.62 & -0.88 & 1481.69 & 169.73 & & & -0.69 & 172.81 \\
\hline 146 & 212 & 0.24 & 0.00 & -0.02 & 0.04 & 0.260 & 0.000 & 0.053 & -0.031 & -3.85 & -1.15 & 1483.19 & 176.29 & & & -0.77 & 179.7 \\
\hline 147 & 213 & 0.24 & 0.00 & -0.02 & 0.04 & 0.260 & 0.000 & 0.053 & -0.031 & -4.36 & -1.68 & 1483.04 & 184.51 & & & -1.31 & 188.15 \\
\hline 148 & 214 & 0.24 & 0.00 & -0.01 & 0.04 & 0.261 & 0.000 & 0.041 & -0.034 & -4.25 & -1.70 & 1484.12 & 191.51 & & & -1.31 & 195.36 \\
\hline 149 & 215 & 0.24 & 0.00 & 0.00 & 0.04 & 0.262 & 0.000 & 0.029 & -0.037 & -4.64 & -2.14 & 1483.70 & 200.00 & & & -1.74 & 204.05 \\
\hline 150 & 216 & 0.24 & 0.00 & 0.01 & 0.03 & 0.262 & 0.000 & 0.016 & -0.030 & -4.36 & -1.91 & 1484.35 & 207.42 & & & -1.68 & 211.51 \\
\hline 151 & 217 & 0.25 & 0.00 & 0.02 & 0.03 & 0.274 & 0.000 & 0.006 & -0.033 & -4.91 & -2.32 & 1483.75 & 216.09 & & & -2.08 & 220.4 \\
\hline 152 & 218 & 0.25 & 0.00 & 0.03 & 0.03 & 0.275 & 0.000 & -0.005 & -0.036 & -4.81 & -2.23 & 1484.36 & 223.55 & & & -1.92 & 228.16 \\
\hline 153 & 219 & 0.25 & 0.00 & 0.03 & 0.03 & 0.275 & 0.000 & -0.005 & -0.036 & -5.05 & -2.49 & 1483.44 & 232.54 & & & -2.18 & 237.3 \\
\hline 154 & 220 & 0.25 & 0.00 & 0.04 & 0.03 & 0.276 & 0.000 & -0.017 & -0.039 & -4.91 & -2.36 & 1483.84 & 240.21 & & & -1.96 & 245.3 \\
\hline 155 & 221 & 0.24 & 0.00 & 0.04 & 0.03 & 0.265 & 0.000 & -0.020 & -0.038 & -4.99 & -2.58 & 1482.72 & 249.41 & & & -2.18 & 254.7 \\
\hline
\end{tabular}

$Z=67($ Ho)

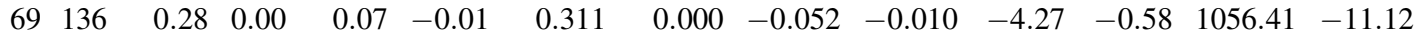

$-0.70-10.67$

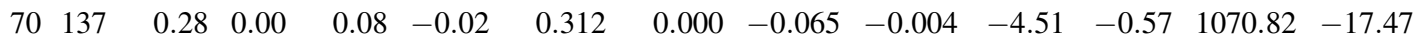

$-0.66-17.04$ 


\begin{tabular}{|c|c|c|c|c|c|c|c|c|c|c|c|c|c|c|c|c|c|}
\hline \multicolumn{18}{|c|}{$Z=67(\mathrm{Ho})$} \\
\hline 71 & 138 & 0.27 & 0.00 & 0.07 & -0.01 & 0.300 & 0.000 & -0.054 & -0.010 & -3.94 & -0.35 & 1082.89 & -21.47 & & & -0.44 & -21.10 \\
\hline 72 & 139 & 0.27 & 0.00 & 0.07 & -0.01 & 0.300 & 0.000 & -0.054 & -0.010 & -3.53 & 0.00 & 1096.36 & -26.87 & & & -0.07 & -26.52 \\
\hline 73 & 140 & 0.26 & 0.00 & 0.08 & -0.02 & 0.289 & 0.000 & -0.070 & -0.002 & -3.43 & 0.21 & 1107.87 & -30.30 & & & 0.13 & -30.02 \\
\hline 74 & 141 & 0.24 & 0.00 & 0.07 & -0.02 & 0.265 & 0.000 & -0.062 & 0.002 & -2.58 & 0.44 & 1120.88 & -35.24 & & & 0.40 & -34.97 \\
\hline 75 & 142 & 0.22 & 0.00 & 0.06 & -0.01 & 0.242 & 0.000 & -0.053 & -0.004 & -2.19 & 0.48 & 1131.99 & -38.28 & & & 0.44 & -38.07 \\
\hline 76 & 143 & 0.21 & 0.00 & 0.06 & -0.02 & 0.230 & 0.000 & -0.056 & 0.006 & -1.91 & 0.74 & 1144.41 & -42.63 & & & 0.71 & -42.44 \\
\hline 77 & 144 & 0.20 & 0.00 & 0.05 & -0.01 & 0.219 & 0.000 & -0.044 & -0.001 & -1.58 & 0.81 & 1154.96 & -45.11 & & & 0.78 & -44.98 \\
\hline 78 & 145 & -0.18 & 0.00 & 0.04 & 0.01 & -0.187 & 0.000 & -0.033 & -0.002 & -0.98 & 0.87 & 1167.04 & -49.12 & & & 0.85 & -49.02 \\
\hline 79 & 146 & -0.17 & 0.00 & 0.04 & 0.01 & -0.176 & 0.000 & -0.034 & -0.002 & -1.56 & 0.20 & 1177.81 & -51.82 & & & 0.18 & -51.77 \\
\hline 80 & 147 & -0.16 & 0.00 & 0.03 & 0.01 & -0.166 & 0.000 & -0.024 & -0.004 & -1.90 & -0.19 & 1189.82 & -55.75 & -55.84 & 0.028 & -0.20 & -55.74 \\
\hline 81 & 148 & -0.08 & 0.00 & 0.00 & 0.00 & -0.084 & 0.000 & 0.002 & -0.000 & -1.78 & -1.01 & 1200.24 & -58.10 & -58.01 & 0.129 & -1.01 & -58.13 \\
\hline 82 & 149 & -0.02 & 0.00 & 0.00 & 0.00 & -0.021 & 0.000 & 0.000 & 0.000 & -2.62 & -1.83 & 1212.18 & -61.97 & -61.69 & 0.018 & -1.83 & -62.04 \\
\hline 83 & 150 & -0.06 & 0.00 & -0.01 & 0.00 & -0.063 & 0.000 & 0.013 & -0.001 & -1.88 & -1.11 & 1220.58 & -62.31 & -61.95 & 0.014 & -1.12 & -62.42 \\
\hline 84 & 151 & -0.07 & 0.00 & -0.01 & 0.00 & -0.073 & 0.000 & 0.014 & -0.001 & -0.84 & -0.09 & 1230.19 & -63.84 & -63.63 & 0.012 & -0.10 & -63.99 \\
\hline 85 & 152 & 0.13 & 0.00 & -0.03 & -0.01 & 0.140 & 0.000 & 0.043 & 0.016 & -0.83 & 0.89 & 1237.87 & -63.45 & -63.61 & 0.014 & 0.90 & -63.63 \\
\hline 86 & 153 & 0.15 & 0.00 & -0.03 & 0.00 & 0.161 & 0.000 & 0.046 & 0.007 & -0.52 & 1.37 & 1247.55 & -65.05 & -65.02 & 0.006 & 1.38 & -65.28 \\
\hline 87 & 154 & 0.18 & 0.00 & -0.01 & 0.01 & 0.194 & 0.000 & 0.027 & -0.007 & -0.45 & 1.35 & 1255.78 & -65.21 & -64.64 & 0.008 & 1.35 & -65.49 \\
\hline 88 & 155 & 0.20 & 0.00 & -0.01 & 0.01 & 0.216 & 0.000 & 0.030 & -0.006 & -0.56 & 1.59 & 1265.24 & -66.61 & -66.04 & 0.018 & 1.59 & -66.91 \\
\hline 89 & 156 & 0.21 & 0.00 & -0.01 & 0.01 & 0.227 & 0.000 & 0.032 & -0.006 & -0.74 & 1.59 & 1273.02 & -66.31 & -6 & 0. & 1.59 & -66.65 \\
\hline 90 & 157 & 0.22 & 0.00 & -0.01 & 0.01 & 0.238 & 0.000 & 0.034 & -0.005 & -0.90 & 1.59 & 1282.28 & -67.50 & -66.83 & 0.024 & 1.60 & -67.87 \\
\hline 91 & 158 & 0.23 & 0.00 & -0.02 & 0.01 & 249 & 0.000 & 0.048 & -0.002 & -1.39 & 1.48 & .76 & -66.91 & -66 & 7 & 1.49 & -67.31 \\
\hline 92 & 159 & 0.24 & 0.00 & -0.02 & 0.01 & 0.261 & 0.000 & 0.051 & -0.001 & -1.67 & 1.39 & 1298.68 & -67.76 & -67.34 & 0.004 & 1.42 & -68.17 \\
\hline 93 & 160 & 0.24 & 0.00 & -0.02 & 0.01 & 0.261 & 0.000 & 0.051 & -0.001 & -1.96 & 1.11 & 1305.93 & -66.93 & & 0.015 & 1.13 & -67.38 \\
\hline 94 & 161 & 0.25 & 0.00 & -0.01 & 0.01 & 0.272 & 0.000 & 0.041 & -0.004 & -2.12 & 1.00 & 1314.48 & -67.41 & & 0.003 & 1.02 & -67.88 \\
\hline 95 & 162 & & 0.00 & -0.01 & 0.01 & 0.272 & 0.000 & 0.041 & -0.004 & -2.48 & & & -66.22 & -66.05 & & 0.69 & -66.72 \\
\hline 96 & 163 & 0.26 & 0.00 & 0.00 & 0.02 & 284 & 0.000 & 032 & -0.016 & -2.82 & 0.48 & .59 & -66.39 & & 003 & 0.52 & -66.88 \\
\hline 97 & 164 & 0.26 & 0.00 & 0.01 & 0.02 & 0.284 & 0.000 & 0.020 & -0.020 & -3.17 & 0.14 & 1336.13 & -64.85 & & 03 & 0.17 & -65.37 \\
\hline 98 & 165 & 0.26 & 0.00 & 0.01 & 0.02 & 0.284 & & 0.020 & & & -0.13 & & & & & & \\
\hline 99 & 166 & 0.27 & 0.00 & 0.02 & 0.02 & 0.296 & 0.000 & 0.010 & -0.023 & -4.02 & -0.50 & 1350.25 & -62.83 & -63 & 0.003 & -0.46 & -63.37 \\
\hline 100 & 167 & 0.27 & 0.00 & 0.02 & 0.03 & 0.297 & 0.000 & 0.011 & -0.032 & -4.28 & -0.63 & 1357.67 & -62.17 & -62.29 & 0 & -0.54 & -62.67 \\
\hline 101 & 168 & 0.27 & 0.00 & 0.03 & 0.03 & 0.298 & 0.000 & -0.001 & -0.036 & -4.77 & -0.98 & 1363.48 & -59.92 & -60.07 & 0.030 & -0.88 & -60.43 \\
\hline 102 & 169 & 0.27 & 0.00 & 0.04 & 0.03 & 0.299 & 0.000 & -0.013 & -0.039 & -4.92 & -1.04 & 1370.47 & -58.83 & & & -0.90 & -59.31 \\
\hline 103 & 170 & 0.27 & 0.00 & 0.04 & 0.02 & 0.298 & 0.000 & -0.014 & -0.029 & -5.06 & -1.30 & & -56 & & & & -56.70 \\
\hline 104 & 171 & 0.27 & 0.00 & 0.05 & 0.02 & 0.299 & 0.000 & -0.026 & -0.032 & -5.09 & -1.22 & 1382.36 & -54.58 & & 0.600 & -1.11 & -55.08 \\
\hline 105 & 172 & 0.26 & 0.00 & 0.05 & 0.01 & 0.287 & 0.000 & -0.030 & -0.022 & -5.04 & -1.46 & 1387.39 & -51.54 & & & -1.39 & -52.09 \\
\hline 106 & 173 & 0.26 & 0.00 & 0.06 & 0.01 & 0.289 & 0.000 & -0.042 & -0.025 & -5.11 & -1.29 & 1393.47 & -49.55 & & & -1.18 & -50.05 \\
\hline 107 & 174 & 0.26 & 0.00 & 0.06 & 0.00 & 0.288 & 0.000 & -0.043 & -0.016 & -5.25 & -1.51 & 1398.16 & -46.17 & & & -1.43 & -46.70 \\
\hline 108 & 175 & 0.25 & 0.00 & 0.07 & 0.00 & 0.278 & 0.000 & -0.057 & -0.018 & -5.11 & -1.33 & 1403.91 & -43.84 & & & -1.21 & -44.31 \\
\hline 109 & 176 & 0.25 & 0.00 & 0.07 & -0.01 & 0.277 & 0.000 & -0.059 & -0.009 & -5.08 & -1.35 & 1408.09 & -39.96 & & & -1.25 & -40.43 \\
\hline 110 & 177 & 0.25 & 0.00 & 0.08 & -0.01 & 0.278 & 0.000 & -0.071 & -0.011 & -4.96 & -1.02 & 1413.36 & -37.15 & & & -0.86 & -37.55 \\
\hline 111 & 178 & 0.24 & 0.00 & 0.08 & -0.02 & 0.266 & 0.000 & -0.074 & -0.001 & -4.83 & -1.03 & 1417.24 & -32.96 & & & -0.87 & -33.34 \\
\hline 112 & 179 & 0.24 & 0.00 & 0.09 & -0.03 & 0.267 & 0.000 & -0.087 & 0.006 & -4.78 & -0.65 & 1422.14 & -29.79 & & & -0.39 & -30.05 \\
\hline 113 & 180 & 0.23 & 0.00 & 0.09 & -0.03 & 0.255 & 0.000 & -0.089 & 0.007 & -4.74 & -0.74 & 1425.81 & -25.39 & & & -0.49 & -25.62 \\
\hline 114 & 181 & 0.22 & 0.00 & 0.09 & -0.04 & 0.243 & 0.000 & -0.092 & 0.018 & -4.32 & -0.46 & 1430.53 & -22.04 & & & -0.12 & -22.15 \\
\hline 115 & 182 & 0.21 & 0.00 & 0.09 & -0.04 & 0.232 & 0.000 & -0.094 & 0.019 & -4.35 & -0.56 & 1433.92 & -17.35 & & & -0.22 & -17.43 \\
\hline 116 & 183 & 0.20 & 0.00 & 0.08 & -0.04 & 0.220 & 0.000 & -0.083 & 0.022 & -3.61 & -0.33 & 1438.39 & -13.76 & & & 0.00 & -13.81 \\
\hline 117 & 184 & 0.18 & 0.00 & 0.07 & -0.03 & 0.197 & 0.000 & -0.073 & 0.016 & -3.18 & -0.45 & 1441.52 & -8.82 & & & -0.22 & -8.93 \\
\hline 118 & 185 & 0.15 & 0.00 & 0.06 & -0.02 & 0.163 & 0.000 & -0.064 & 0.010 & -2.33 & -0.08 & 1445.58 & -4.80 & & & 0.09 & -4.93 \\
\hline 119 & 186 & -0.19 & 0.00 & 0.04 & -0.01 & -0.197 & 0.000 & -0.030 & 0.017 & -2.65 & -0.45 & 1448.70 & 0.15 & & & -0.37 & -0.01 \\
\hline 120 & 187 & -0.18 & 0.00 & 0.03 & -0.01 & -0.187 & 0.000 & -0.021 & 0.014 & -2.74 & -0.68 & 1453.08 & 3.84 & & & -0.61 & 3.71 \\
\hline 121 & 188 & -0.15 & 0.00 & 0.02 & -0.01 & -0.156 & 0.000 & -0.014 & 0.012 & -2.72 & -1.10 & 1455.99 & 9.00 & & & -1.06 & 8.90 \\
\hline 122 & 189 & -0.13 & 0.00 & 0.02 & 0.00 & -0.136 & 0.000 & -0.016 & 0.003 & -2.57 & -1.29 & 1460.07 & 12.99 & & & -1.27 & 12.94 \\
\hline 123 & 190 & -0.09 & 0.00 & 0.02 & 0.00 & -0.094 & 0.000 & -0.020 & 0.002 & -3.23 & -2.36 & 1463.37 & 17.76 & & & -2.34 & 17.77 \\
\hline 124 & 191 & -0.07 & 0.00 & 0.02 & 0.01 & -0.073 & 0.000 & -0.021 & -0.008 & -3.69 & -2.82 & 1467.48 & 21.72 & & & -2.79 & 21.82 \\
\hline 125 & 192 & -0.01 & 0.00 & 0.00 & 0.00 & -0.011 & 0.000 & 0.000 & 0.000 & -4.44 & -3.55 & 1470.20 & 27.08 & & & -3.56 & 27.22 \\
\hline 126 & 193 & 0.00 & 0.00 & 0.00 & 0.00 & 0.000 & 0.000 & 0.000 & 0.000 & -4.95 & -3.99 & 1474.02 & 31.32 & & & -3.99 & 31.54 \\
\hline 127 & 194 & -0.02 & 0.00 & 0.00 & 0.00 & -0.021 & 0.000 & 0.000 & 0.000 & -4.02 & -3.16 & 1474.95 & 38.47 & & & -3.16 & 38.78 \\
\hline
\end{tabular}




\section{$Z=67(\mathrm{Ho})$}

\begin{tabular}{|c|c|c|c|c|c|c|c|c|c|c|c|c|}
\hline $28 \quad 195$ & -0.01 & 0.00 & 0.00 & 0.00 & -0.011 & 0.000 & 0.000 & 0.000 & -2.86 & -2.06 & 1476.99 & 44.49 \\
\hline 99196 & 0.04 & 0.05 & -0.01 & 0.00 & 0.043 & -0.068 & 0.014 & 0.002 & -2.38 & -1.12 & 1477.58 & 51.98 \\
\hline 197 & 0.07 & 0.06 & -0.02 & 0.00 & 0.076 & -0.082 & 0.027 & 0.004 & -1.72 & -0.17 & 1479.54 & 58.10 \\
\hline 198 & 0.07 & 0.09 & -0.02 & 0.01 & 0.078 & -0.123 & 0.029 & -0.002 & -2.13 & 0.21 & 1480.46 & 65.24 \\
\hline 199 & 0.11 & 0.05 & -0.04 & -0.01 & 0.119 & -0.070 & 0.055 & 0.019 & -1.09 & 0.83 & 1482.52 & 1.26 \\
\hline 200 & 0.11 & 0.07 & -0.04 & 0.00 & 0.120 & -0.097 & 0.056 & 0.010 & -1.30 & 1.03 & 40 & $10.4 J$ \\
\hline 201 & 0.13 & 0.05 & -0.05 & 0.00 & 0.141 & -0.069 & 0.069 & 0.011 & -0.67 & 1.62 & 85.27 & 1.65 \\
\hline 202 & 0.15 & 0.00 & -0.07 & -0.01 & 0.163 & 0.000 & 0.097 & 0.026 & -1.39 & 1.50 & 1486.26 & 91.73 \\
\hline 203 & 0.17 & 0.00 & -0.06 & -0.01 & 0.184 & 0.000 & 0.087 & 0.026 & -1.11 & 1.54 & 1488.47 & 97.59 \\
\hline 204 & 0.17 & 0.00 & -0.07 & 0.00 & 0.184 & 0.000 & 0.100 & 0.018 & -1.73 & 1.22 & 1489.44 & 04.69 \\
\hline 205 & 0.18 & 0.00 & -0.06 & 0.00 & 0.194 & 0.000 & 0.089 & 0.016 & -1.39 & 1.36 & 1491.34 & 10.86 \\
\hline 206 & 0.19 & 0.00 & -0.06 & 0.01 & 0.205 & 0.000 & 0.091 & 07 & -1.86 & 0.94 & .22 & 3.06 \\
\hline 207 & 0.20 & 0.00 & -0.05 & 0.01 & 0.216 & 0.000 & 0.080 & 0.005 & -1.69 & 0.98 & 1494.01 & 124.33 \\
\hline 208 & 0.22 & 0.00 & -0.04 & 0.02 & 0.237 & 0.000 & 0.072 & -0.007 & -2.20 & 0.47 & 1494.79 & 131.63 \\
\hline 209 & 0.22 & 0.00 & -0.04 & 0.02 & 0.237 & 0.000 & 0.072 & -0.007 & -2.38 & 0.32 & 1496.57 & 137.92 \\
\hline 210 & 0.22 & 0.00 & -0.04 & 0.03 & 0.237 & 0.000 & 0.073 & -0.017 & -2.97 & -0.23 & 1497.19 & 145.37 \\
\hline 211 & 0.23 & 0.00 & -0.03 & 0.03 & 0.249 & 0.000 & 0.062 & -0.019 & -2.98 & -0.28 & 1498.68 & 151.95 \\
\hline 212 & 0.24 & 0.00 & -0.02 & 0.03 & 0.260 & 0.000 & 0.052 & -0.021 & -3.52 & -0.75 & 1499.04 & 159.66 \\
\hline 213 & 0.24 & 0.00 & -0.01 & 0.03 & 0.261 & 0.000 & 0.040 & -0.024 & -3.49 & -0.85 & 1500.39 & 166.38 \\
\hline $7 \quad 214$ & 0.24 & 0.00 & -0.01 & 0.03 & 0.261 & 0.000 & 0.040 & -0.024 & -4.03 & -1.39 & 1500.64 & 174.20 \\
\hline 215 & 0.24 & 0.00 & 0.00 & 0.05 & 0.201 & & 0.028 & -0.027 & -4.00 & -1.46 & & 01.14 \\
\hline 216 & 0.24 & 0.00 & 0.00 & 0.03 & 0.261 & 0.000 & 0.028 & -0.027 & -4.47 & -1.91 & 1501.76 & 189.23 \\
\hline 217 & 0.24 & 0.00 & 0.01 & 0.03 & 0.262 & 0.000 & 0.016 & -0.030 & -4.43 & -1.95 & 1502.68 & 196.38 \\
\hline 218 & 0.24 & 0.00 & 0.02 & 0.03 & 0.263 & 0.000 & 0.004 & -0.033 & -4.90 & -2.40 & 1502.50 & 204.63 \\
\hline 219 & 0.25 & 0.00 & 0.03 & 0.03 & 0.275 & 0.000 & -0.005 & -0.036 & -4.98 & -2.35 & 1503.16 & 212.04 \\
\hline 220 & 0.25 & 0.00 & 0.04 & 0.0 & 0.276 & 0.000 & -0.017 & -0.039 & -5.36 & -2.68 & 1502.69 & 220.59 \\
\hline 221 & 0.24 & 0.00 & 0.04 & 0.03 & 0.265 & 0.000 & -0.020 & -0.038 & -5.01 & -2.52 & 1503.06 & 228.28 \\
\hline 222 & 0.24 & 0.00 & 0.05 & 0.02 & 0.265 & 0.000 & -0.033 & -0.031 & -5.21 & -2.61 & 1502.18 & 237.23 \\
\hline 223 & 0.24 & 0.00 & 0.05 & 0.02 & 0.265 & 0.000 & -0.033 & -0.031 & -4.99 & -2.44 & 1502.39 & 245.09 \\
\hline 224 & 0.24 & 0.00 & 0.06 & 0.02 & 0.266 & 0.000 & -0.045 & -0.034 & -5.46 & -2.79 & 1501.61 & 253.94 \\
\hline
\end{tabular}

$\begin{array}{rr}-2.06 & 44.89 \\ -1.08 & 52.51 \\ -0.08 & 58.77 \\ 0.37 & 66.09 \\ 1.02 & 72.24 \\ 1.22 & 79.53 \\ 1.84 & 85.88 \\ 1.89 & 93.25 \\ 1.86 & 99.17 \\ 1.56 & 106.42 \\ 1.63 & 112.66 \\ 1.18 & 119.96 \\ 1.16 & 126.32 \\ 0.61 & 133.73 \\ 0.48 & 140.19 \\ 0.01 & 147.88 \\ -0.07 & 154.60 \\ -0.58 & 162.44 \\ -0.68 & 169.34 \\ -1.23 & 177.33 \\ -1.28 & 184.48 \\ -1.74 & 192.75 \\ -1.74 & 200.12 \\ -2.17 & 208.61 \\ -2.06 & 216.28 \\ -2.33 & 225.10 \\ -2.14 & 233.04 \\ -2.35 & 242.09 \\ -2.17 & 250.19 \\ -2.43 & 259.37\end{array}$

\section{$Z=68(\mathrm{Er})$}

$\begin{array}{lllll}70 & 138 & 0.28 & 0.00 & 0\end{array}$

$\begin{array}{llll}71 & 139 & 0.28 & 0.00\end{array}$

$0.09-0.02$

0.313

$$
0.000
$$

$0.09-0.03$

0.312

$\begin{array}{lllllll}0.000 & -0.077 & -0.007 & -4.96 & -0.83 & 1070.22 & -9.58\end{array}$

$\begin{array}{llll}72 & 140 & 0.27 & 0.00\end{array}$

$0.09-0.02$

0.301

$\begin{array}{llll}73 & 141 & 0.25 & 0.00\end{array}$

$0.08-0.02$

0.278

$0.000-0.079$

0.002

$-4.77$

$\begin{array}{llll}-0.62 & 1082.39 & -13.68\end{array}$

$\begin{array}{lllllll}0.000 & -0.079 & -0.006 & -4.25 & -0.27 & 1096.45 & -19.66\end{array}$

$\begin{array}{llll}74 & 142 & 0.24 & 0.00\end{array}$

$\begin{array}{ll}0.08 & -0.02\end{array}$

0.266

$0.000-0.072-$

$0.000-0.074-0.0$

$-0.002-3.55-0$.

$\begin{array}{llll}0.04 & 1108.03 & -23.17\end{array}$

$\begin{array}{llll}75 & 143 & 0.23 & 0.00\end{array}$

$\begin{array}{llll}76 & 144 & 0.21 & 0.00\end{array}$

$0.07-0.02$

0.254

$0.000-0.064$

$0.002-2.73$

$0.28 \quad 1121.53-28.60$

$\begin{array}{llll}77 & 145 & 0.20 & 0.00\end{array}$

$0.06-0.02$

0.219

$0.000-0.068$

$0.004-2.36$

$\begin{array}{lll}0.22 & 1132.83 & -31.83\end{array}$

$0.000-0.057$

$0.007-1.93$

$\begin{array}{lll}0.36 & 1145.94 & -36.87\end{array}$

$\begin{array}{llll}0.51 & 1156.50 & -39.36\end{array}$

$\begin{array}{lllllll}0.000 & -0.022 & -0.004 & -1.24 & 0.42 & 1169.29 & -44.08\end{array}$

$\begin{array}{llll}78 & 146 & -0.18 & 0.00 \\ 79 & 147 & -0.18 & 0.00\end{array}$

$\begin{array}{llll}0.03 & 0.01 & -0.187\end{array}$

$\begin{array}{llll}0.04 & 0.01 & -0.187\end{array}$

$0.000-0.033-0.002-1.98$

$\begin{array}{rrr}0.23 & 1180.13 & -46.85\end{array}$

$\begin{array}{lllll}80 & 148 & -0.14 & 0.00\end{array}$

$\begin{array}{llllll}81 & 149 & -0.09 & 0.00 & 0.01\end{array}$

$\begin{array}{lllll}82 & 150 & 0.01 & 0.00 & 0.00\end{array}$

$\begin{array}{lllll}83 & 151 & -0.06 & 0.00 & -0.01\end{array}$

$0.00-0.146$

$0.00-0.094$

$0.00 \quad 0.011$

$0.000-0.026$

$0.004-2.08$

$\begin{array}{llll}-0.60 & 1192.67 & -51.32\end{array}$

$0.00-0.063$

$0.000-0.008$

$\begin{array}{lllllll}0.001 & -2.16 & -1.31 & 1203.07 & -53.64 & -53.74\end{array}$

0.000

0.000

$\begin{array}{lllllll}0.000 & -2.92 & -2.11 & 1215.53 & -58.03 & -57.83\end{array}$

0.000

$\begin{array}{llllllll}0.013 & -0.001 & -2.17 & -1.41 & 1224.04 & -58.47 & -58.27\end{array}$

$0.00-0.084$

$85 \quad 15$

$86 \quad 154$

$\begin{array}{llll}-0.08 & 0.00 & -0.01\end{array}$

$-0.0$

0.129

0.000

0.014

0.000

0.042

$0.00 \quad 0.150$

0.000

0.045

0.001

$-1.18-0$.

0.000

$\begin{array}{lllll}0.39 & 1234.18 & -60.54 & -60.50\end{array}$

$0.01 \quad 0.172$

$88 \quad 156$

$\begin{array}{lll}0.16 & 0.00 & -0.02\end{array}$

$0.01 \quad 0.205$

0.000

$\begin{array}{lll}0.036 & -0.005 & -0.35\end{array}$

$\begin{array}{lllll}0.64 & 1241.90 & -60.19 & -60.49\end{array}$

$\begin{array}{lllll}1.20 & 1252.03 & -62.25 & -62.61\end{array}$

$\begin{array}{lllll}1.51 & 1260.01 & -62.15 & -62.22\end{array}$

$\begin{array}{lllll}1.60 & 1270.16 & -64.23 & -64.21\end{array}$

$\begin{array}{lllll}1.70 & 1277.91 & -63.92 & -63.42\end{array}$

$\begin{array}{lllll}1.82 & 1287.58 & -65.52 & -65.30\end{array}$

$\begin{array}{lllll}1.74 & 1295.10 & -64.96 & -64.57\end{array}$

$\begin{array}{lllll}1.68 & 1304.52 & -66.31 & -66.06\end{array}$

$\begin{array}{lllll}1.43 & 1311.80 & -65.51 & -65.21\end{array}$

$\begin{array}{lllll}1.30 & 1320.88 & -66.52 & -66.34\end{array}$

$\begin{array}{llll}0.97 & 1327.84 & -65.42 & -65.17\end{array}$ $\begin{array}{ll}-0.91 & -9.03\end{array}$

$\begin{array}{ll}-0.71 & -13.19\end{array}$

$-0.32-19.20$

$\begin{array}{ll}-0.10 & -22.77\end{array}$

$0.24-28.22$

$0.17-31.52$

$0.34-36.58$

$0.48-39.13$

$0.40-43.89$

$-0.25-46.72$

$-0.61-51.22$

$\begin{array}{lll}0.028 & -1.32 & -53.60\end{array}$

$\begin{array}{lll}0.017 & -2.11 & -58.03\end{array}$

$\begin{array}{llll}0.016 & -1.41 & -58.51\end{array}$

$\begin{array}{llll}0.011 & -0.39 & -60.63\end{array}$

$\begin{array}{lll}0.009 & 0.65 & -60.31\end{array}$

0.005

$1.21-62.41$

$1.52-62.36$

$1.61-64.47$

$1.70-64.20$

$1.83-65.83$

$1.75-65.31$

$1.71-66.68$

$1.45-65.92$

$1.33-66.95$

$0.99-65.88$ 


\begin{tabular}{|c|c|c|c|c|c|c|c|c|c|c|c|c|c|c|c|c|}
\hline \multicolumn{17}{|c|}{$Z=68(\mathrm{Er})$} \\
\hline 96164 & 0.26 & 0.00 & 0.01 & 0.01 & 0.284 & 0.000 & 0.018 & -0.010 & -2.36 & 0.77 & 1336.59 & -66.09 & -65.95 & 0.003 & 0.80 & -66.56 \\
\hline $97 \quad 165$ & 0.26 & 0.00 & 0.01 & 0.01 & 0.284 & 0.000 & 0.018 & -0.010 & -2.77 & 0.44 & 1343.18 & -64.61 & -64.53 & 0.003 & 0.46 & -65.12 \\
\hline $98 \quad 166$ & 0.26 & 0.00 & 0.02 & 0.02 & 0.285 & 0.000 & 0.007 & -0.023 & -3.14 & 0.09 & 1351.68 & -65.04 & -64.93 & 0.003 & 0.15 & -65.53 \\
\hline $99 \quad 167$ & 0.27 & 0.00 & 0.03 & 0.02 & 0.297 & 0.000 & -0.002 & -0.026 & -3.82 & -0.29 & 1357.95 & -63.24 & -63.30 & 0.003 & -0.23 & -63.75 \\
\hline 100168 & 0.27 & 0.00 & 0.03 & 0.02 & 0.297 & 0.000 & -0.002 & -0.026 & -3.99 & -0.42 & 1365.87 & -63.08 & -63.00 & 0.003 & -0.35 & -63.59 \\
\hline 101169 & 0.27 & 0.00 & 0.04 & 0.02 & 0.298 & 0.000 & -0.014 & -0.029 & -4.54 & -0.82 & 1371.80 & -60.94 & -60.93 & 0.003 & -0.75 & -61.46 \\
\hline 102170 & 0.27 & 0.00 & 0.04 & 0.02 & 0.298 & 0.000 & -0.014 & -0.029 & -4.61 & -0.90 & 1379.29 & -60.37 & -60.12 & 0.003 & -0.81 & -60.88 \\
\hline 103171 & 0.27 & 0.00 & 0.05 & 0.02 & 0.299 & 0.000 & -0.026 & -0.032 & -5.16 & -1.27 & 1384.85 & -57.85 & -57.72 & 0.003 & -1.16 & -58.36 \\
\hline $104 \quad 172$ & 0.27 & 0.00 & 0.06 & 0.01 & 0.300 & 0.000 & -0.039 & -0.026 & -5.11 & -1.19 & 1391.84 & -56.77 & -56.49 & 0.005 & -1.08 & -57.28 \\
\hline 105173 & 0.26 & 0.00 & 0.06 & 0.01 & 0.289 & 0.000 & -0.042 & -0.025 & -5.31 & -1.46 & 1396.96 & -53.82 & & & -1.36 & -54.34 \\
\hline 106174 & 0.26 & 0.00 & 0.06 & 0.00 & 0.288 & 0.000 & -0.043 & -0.016 & -5.11 & -1.39 & 1403.61 & -52.40 & & & -1.29 & -52.93 \\
\hline $107 \quad 175$ & 0.26 & 0.00 & 0.07 & 0.00 & 0.289 & 0.000 & -0.055 & -0.019 & -5.69 & -1.73 & 1408.48 & -49.20 & & & -1.62 & -49.71 \\
\hline 108176 & 0.25 & 0.00 & 0.07 & 0.00 & 0.278 & 0.000 & -0.057 & -0.018 & -5.34 & -1.55 & 1414.69 & -47.34 & & & -1.42 & -47.82 \\
\hline 109177 & 0.25 & 0.00 & 0.08 & -0.01 & 0.278 & 0.000 & -0.071 & -0.011 & -5.68 & -1.68 & 1419.04 & -43.61 & & & -1.53 & -44.07 \\
\hline 110178 & 0.25 & 0.00 & 0.08 & -0.02 & 0.278 & 0.000 & -0.072 & -0.002 & -5.25 & -1.32 & 1424.75 & -41.25 & & & -1.15 & -41.67 \\
\hline 111179 & 0.24 & 0.00 & 0.09 & -0.02 & 0.267 & 0.000 & -0.086 & -0.004 & -5.53 & -1.41 & 1428.75 & -37.19 & & & -1.20 & -37.55 \\
\hline 112180 & 0.24 & 0.00 & 0.09 & -0.03 & 0.267 & 0.000 & -0.087 & 0.006 & -5.18 & -1.03 & 1434.13 & -34.49 & & & -0.77 & -34.78 \\
\hline 113181 & 0.23 & 0.00 & 0.09 & -0.03 & 0.255 & 0.000 & -0.089 & 0.007 & -5.14 & -1.12 & 1437.84 & -30.13 & & & -0.86 & -30.41 \\
\hline 114182 & 0.22 & 0.00 & 0.09 & -0.04 & 0.243 & 0.000 & -0.092 & 0.018 & -4.74 & -0.85 & 1443.02 & -27.24 & & & -0.50 & -27.41 \\
\hline 115183 & 0.21 & 0.00 & 0.09 & -0.04 & 0.232 & 0.000 & -0.094 & 0.019 & -4.77 & -0.95 & 1446.46 & -22.61 & & & -0.61 & -22.74 \\
\hline 116184 & 0.20 & 0.00 & 0.09 & -0.04 & 0.221 & 0.000 & -0.095 & 0.020 & -4.35 & -0.73 & 1451.40 & -19.48 & & & -0.35 & -19.54 \\
\hline 117185 & 0.18 & 0.00 & 0.08 & -0.03 & 0.198 & 0.000 & -0.085 & 0.014 & -3.87 & -0.82 & 1454.54 & -14.55 & & & -0.54 & -14.68 \\
\hline 118186 & 0.15 & 0.00 & 0.07 & -0.03 & 0.164 & 0.000 & -0.077 & 0.018 & -3.08 & -0.52 & 1459.13 & -11.06 & & & -0.26 & -11.16 \\
\hline 119187 & -0.19 & 0.00 & 0.04 & -0.01 & -0.197 & 0.000 & -0.030 & 0.017 & -3.04 & -0.82 & 1462.21 & -6.07 & & & -0.73 & -6.30 \\
\hline 120188 & -0.18 & 0.00 & 0.03 & -0.01 & -0.187 & 0.000 & -0.021 & 0.014 & -3.11 & -1.04 & 1467.04 & -2.83 & & & -0.98 & -3.03 \\
\hline $121 \quad 189$ & -0.15 & 0.00 & 0.02 & -0.01 & -0.156 & 0.000 & -0.014 & 0.012 & -3.07 & -1.44 & 1469.96 & 2.32 & & & -1.41 & 2.14 \\
\hline 122190 & -0.13 & 0.00 & 0.02 & 0.00 & -0.136 & 0.000 & -0.016 & 0.003 & -2.90 & -1.62 & 1474.48 & 5.88 & & & -1.60 & 5.73 \\
\hline 123191 & -0.09 & 0.00 & 0.02 & 0.01 & -0.094 & 0.000 & -0.020 & -0.007 & -3.58 & -2.69 & 1477.81 & 10.61 & & & -2.66 & 10.54 \\
\hline 124192 & -0.07 & 0.00 & 0.03 & 0.01 & -0.073 & 0.000 & -0.033 & -0.007 & -4.09 & -3.12 & 1482.32 & 14.17 & & & -3.07 & 14.19 \\
\hline 125193 & 0.00 & 0.00 & 0.00 & 0.00 & 0.000 & 0.000 & 0.000 & 0.000 & -4.79 & -3.89 & 1485.11 & 19.45 & & & -3.89 & 19.48 \\
\hline 126194 & 0.00 & 0.00 & 0.00 & 0.00 & 0.000 & 0.000 & 0.000 & 0.000 & -5.22 & -4.25 & 1489.29 & 23.35 & & & -4.25 & 23.45 \\
\hline $127 \quad 195$ & -0.02 & 0.00 & -0.01 & 0.00 & -0.021 & 0.000 & 0.012 & -0.000 & -4.27 & -3.38 & 1490.21 & 30.50 & & & -3.38 & 30.68 \\
\hline 128196 & 0.00 & 0.00 & 0.00 & 0.00 & 0.000 & 0.000 & 0.000 & 0.000 & -3.10 & -2.28 & 1492.68 & 36.10 & & & -2.28 & 36.36 \\
\hline 129197 & 0.03 & 0.04 & -0.01 & 0.00 & 0.032 & -0.054 & 0.013 & 0.002 & -2.35 & -1.26 & 1493.21 & 43.64 & & & -1.23 & 44.01 \\
\hline 130198 & 0.06 & 0.05 & -0.02 & 0.00 & 0.065 & -0.069 & 0.027 & 0.003 & -1.56 & -0.22 & 1495.50 & 49.42 & & & -0.15 & 49.92 \\
\hline 131199 & 0.07 & 0.08 & -0.02 & 0.00 & 0.077 & -0.110 & 0.028 & 0.007 & -1.88 & 0.20 & 1496.41 & 56.58 & & & 0.34 & 57.25 \\
\hline $132 \quad 200$ & 0.11 & 0.04 & -0.04 & -0.01 & 0.119 & -0.056 & 0.054 & 0.018 & -0.90 & 0.86 & 1498.85 & 62.21 & & & 1.03 & 63.02 \\
\hline 133201 & 0.11 & 0.06 & -0.04 & 0.00 & 0.119 & -0.083 & 0.055 & 0.009 & -1.02 & 1.09 & 1499.73 & 69.41 & & & 1.25 & 70.31 \\
\hline 134202 & 0.11 & 0.06 & -0.04 & 0.00 & 0.119 & -0.083 & 0.055 & 0.009 & -0.37 & 1.69 & 1502.01 & 75.20 & & & 1.86 & 76.22 \\
\hline 135203 & 0.15 & 0.00 & -0.06 & -0.01 & 0.162 & 0.000 & 0.084 & 0.024 & -0.86 & 1.68 & 1502.91 & 82.37 & & & 1.98 & 83.63 \\
\hline 136204 & 0.16 & 0.00 & -0.06 & -0.01 & 0.173 & 0.000 & 0.085 & 0.025 & -0.80 & 1.68 & 1505.56 & 87.78 & & & 1.99 & 89.18 \\
\hline $137 \quad 205$ & 0.17 & 0.00 & -0.06 & -0.01 & 0.184 & 0.000 & 0.087 & 0.026 & -1.25 & 1.43 & 1506.49 & 94.93 & & & 1.75 & 96.44 \\
\hline 138206 & 0.18 & 0.00 & -0.06 & 0.00 & 0.194 & 0.000 & 0.089 & 0.016 & -1.15 & 1.58 & 1508.79 & 100.70 & & & 1.85 & 102.30 \\
\hline 139207 & 0.19 & 0.00 & -0.05 & 0.00 & 0.205 & 0.000 & 0.078 & 0.014 & -1.29 & 1.26 & 1509.59 & 107.97 & & & 1.45 & 109.62 \\
\hline $140 \quad 208$ & 0.20 & 0.00 & -0.05 & 0.01 & 0.216 & 0.000 & 0.080 & 0.005 & -1.37 & 1.27 & 1511.80 & 113.83 & & & 1.46 & 115.61 \\
\hline 141209 & 0.20 & 0.00 & -0.05 & 0.01 & 0.216 & 0.000 & 0.080 & 0.005 & -1.80 & 0.81 & 2.56 & 121.15 & & & 0.99 & 123.06 \\
\hline $142 \quad 210$ & 0.22 & 0.00 & -0.04 & 0.02 & 0.237 & 0.000 & 0.072 & -0.007 & -1.99 & 0.68 & 1514.71 & 127.07 & & & 0.84 & 129.11 \\
\hline 143211 & 0.22 & 0.00 & -0.03 & 0.02 & 0.238 & 0.000 & 0.059 & -0.009 & -2.30 & 0.25 & 1515.23 & 134.62 & & & 0.36 & 136.76 \\
\hline 144212 & 0.22 & 0.00 & -0.03 & 0.03 & 0.237 & 0.000 & 0.060 & -0.020 & -2.43 & 0.10 & 1517.21 & 140.71 & & & 0.33 & 143.12 \\
\hline 145213 & 0.23 & 0.00 & -0.02 & 0.03 & 0.249 & 0.000 & 0.050 & -0.022 & -2.97 & -0.38 & 1517.60 & 148.39 & & & -0.20 & 150.93 \\
\hline 146214 & 0.23 & 0.00 & -0.02 & 0.03 & 0.249 & 0.000 & 0.050 & -0.022 & -3.03 & -0.44 & 1519.30 & 154.76 & & & -0.25 & 157.48 \\
\hline $147 \quad 215$ & 0.24 & 0.00 & -0.01 & 0.03 & 0.261 & 0.000 & 0.040 & -0.024 & -3.60 & -0.95 & 1519.53 & 162.61 & & & -0.77 & 165.47 \\
\hline $148 \quad 216$ & 0.24 & 0.00 & 0.00 & 0.03 & 0.261 & 0.000 & 0.028 & -0.027 & -3.61 & -1.09 & 1521.12 & 169.08 & & & -0.90 & 172.14 \\
\hline 149217 & 0.24 & 0.00 & 0.01 & 0.03 & 0.262 & 0.000 & 0.016 & -0.030 & -4.12 & -1.60 & 1521.17 & 177.10 & & & -1.39 & 180.36 \\
\hline $150 \quad 218$ & 0.24 & 0.00 & 0.02 & 0.03 & 0.263 & 0.000 & 0.004 & -0.033 & -4.19 & -1.70 & 1522.54 & 183.81 & & & -1.44 & 187.30 \\
\hline 151219 & 0.24 & 0.00 & 0.03 & 0.03 & 0.264 & 0.000 & -0.008 & -0.036 & -4.72 & -2.16 & 1522.38 & 192.04 & & & -1.87 & 195.76 \\
\hline $152 \quad 220$ & 0.24 & 0.00 & 0.03 & 0.03 & 0.264 & 0.000 & -0.008 & -0.036 & -4.63 & -2.12 & 1523.42 & 199.07 & & & -1.80 & 203.01 \\
\hline
\end{tabular}




\section{$Z=68(\mathrm{Er})$}

$153 \quad 221$

$\begin{array}{llll}154 & 222 & 0.24 & 0.00\end{array}$

$\begin{array}{llll}155 & 223 & 0.24 & 0.00\end{array}$

0.04

0.03

$\begin{array}{llllllll}0.265 & 0.000 & -0.020 & -0.038 & -5.04 & -2.48 & 1522.99 & 207.58\end{array}$

$\begin{array}{llllllll}0.265 & 0.000 & -0.020 & -0.038 & -4.83 & -2.29 & 1523.72 & 214.92\end{array}$

$\begin{array}{lll}0.24 & 0.00\end{array}$

0.06

0.02

$\begin{array}{llllllll}0.265 & 0.000 & -0.033 & -0.031 & -5.09 & -2.46 & 1522.93 & 223.77\end{array}$

$156 \quad 22$

$0.24 \quad 0.00$

0.06

0.02

$\begin{array}{llllllll}0.266 & 0.000 & -0.045 & -0.034 & -5.09 & -2.39 & 1523.61 & 231.17\end{array}$

158226

$0.24 \quad 0.00$

0.07

0.01

$\begin{array}{llllllll}0.266 & 0.000 & -0.046 & -0.024 & -5.25 & -2.55 & 1522.64 & 240.21\end{array}$

159227

0.07 $\begin{array}{llllllll}0.267 & 0.000 & -0.058 & -0.027 & -5.37 & -2.55 & 1523.22 & 247.70\end{array}$

$\begin{array}{llllllll}0.255 & 0.000 & -0.061 & -0.017 & -5.60 & -2.84 & 1522.24 & 256.75\end{array}$ $\begin{array}{ll}-2.11 \quad 211.77 \\ -1.91 & 219.34\end{array}$

$-1.91 \quad 219.34$
-2.11

$\begin{array}{ll}-2.19 & 228.29\end{array}$

$\begin{array}{ll}-2.02 & 236.02\end{array}$

$\begin{array}{ll}-2.33 & 245.12\end{array}$

$\begin{array}{ll}-2.21 & 252.96\end{array}$

$-2.59 \quad 262.16$

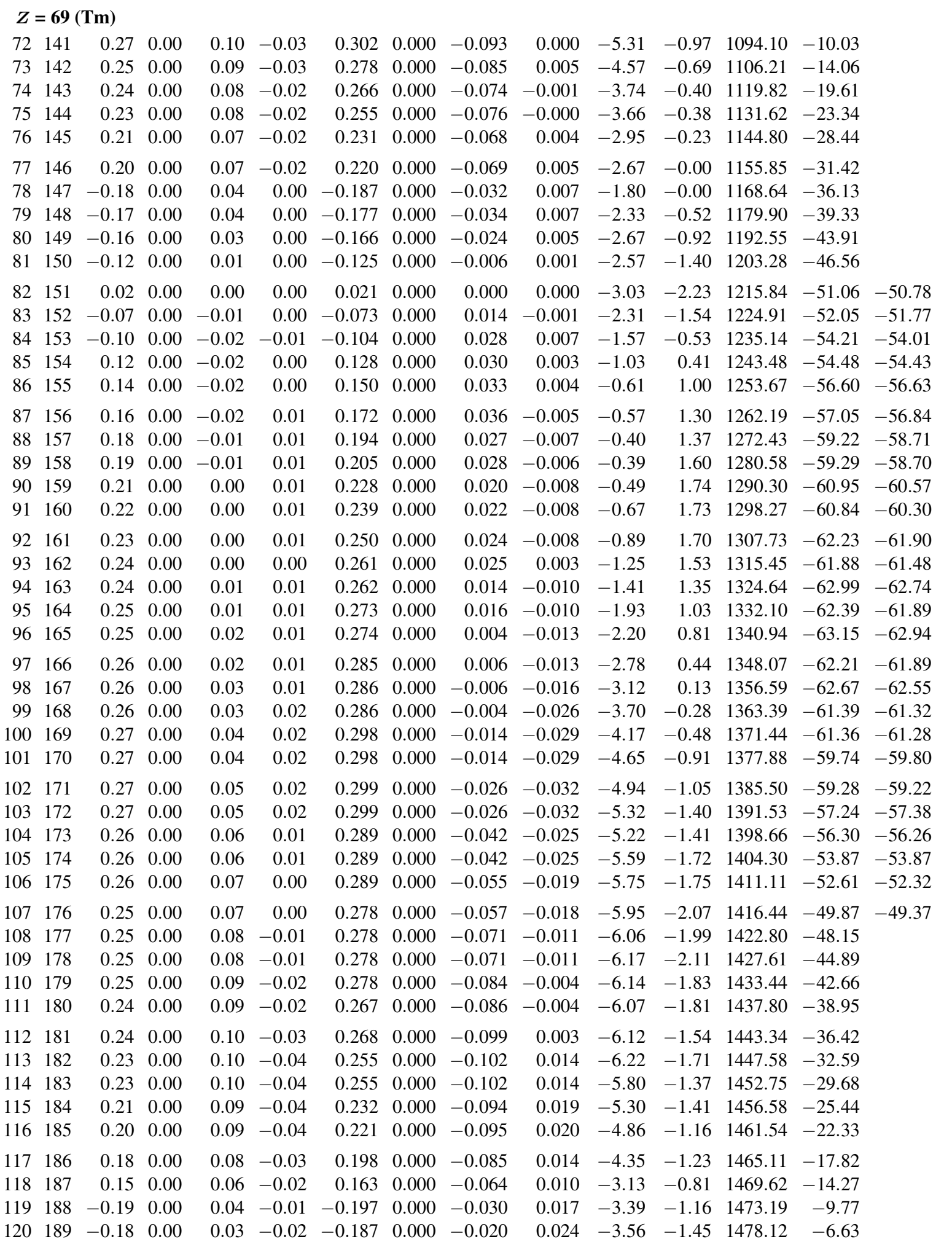

\section{$Z=69(\mathrm{Tm})$}

$\begin{array}{llll}74 & 143 & 0.24 & 0.00\end{array}$

$\begin{array}{lllll}76 & 145 & 0.21 & 0.00\end{array}$

$\begin{array}{llll}77 & 146 & 0.20 & 0.00\end{array}$

$\begin{array}{llll}79 & 148 & -0.17 & 0.00\end{array}$

$\begin{array}{lllll}80 & 149 & -0.16 & 0.00\end{array}$

$\begin{array}{lllll}81 & 150 & -0.12 & 0.00\end{array}$

$\begin{array}{llllll}85 & 154 & 0.12 & 0.00 & -0.02\end{array}$

$\begin{array}{llllll}86 & 155 & 0.14 & 0.00 & -0.02\end{array}$

$87 \quad 156$

$89 \quad 158$

0.01

1.60

$\begin{array}{llll}1.74 & 1290.30 & -60.95 & -60.57\end{array}$

$\begin{array}{lllll}1.73 & 1298.27 & -60.84 & -60.30\end{array}$

.0 .02
.48

0.026

0.006

0.028

0.003

0.012

0.003

0.003

0.002

0.002

$-1.33-57.77$

$\begin{array}{lll}0.005 & -1.32 & -56.83\end{array}$

$\begin{array}{llll}0.045 & -1.64 & -54.42\end{array}$

$\begin{array}{llll}0.050 & -1.66 & -53.14\end{array}$

$\begin{array}{llll}0.100 & -1.98 & -50.40\end{array}$

$\begin{array}{ll}-1.86 & -48.65\end{array}$

$\begin{array}{ll}-1.99 & -45.39\end{array}$

$\begin{array}{ll}-1.64 & -43.09\end{array}$

$\begin{array}{ll}-1.63 & -39.37\end{array}$

$\begin{array}{ll}-1.28 & -36.74\end{array}$

$\begin{array}{ll}-1.39 & -32.84\end{array}$

$\begin{array}{ll}-1.02 & -29.88\end{array}$

$-1.11-25.66$

$-0.82-22.48$

$\begin{array}{ll}-0.98 & -18.04\end{array}$

$\begin{array}{ll}-0.67 & -14.54\end{array}$

$-1.08-10.08$

$-1.34 \quad-6.86$ 


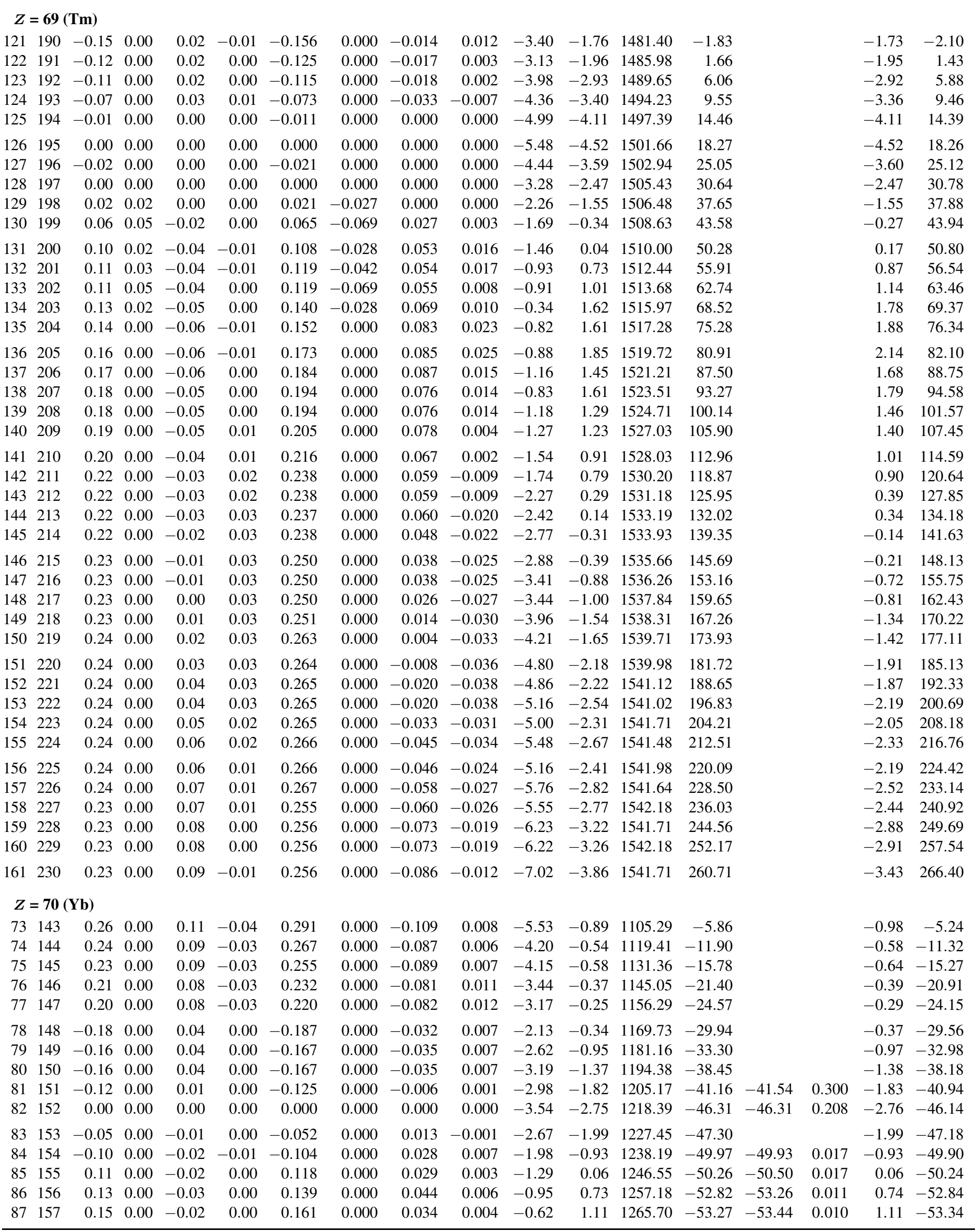




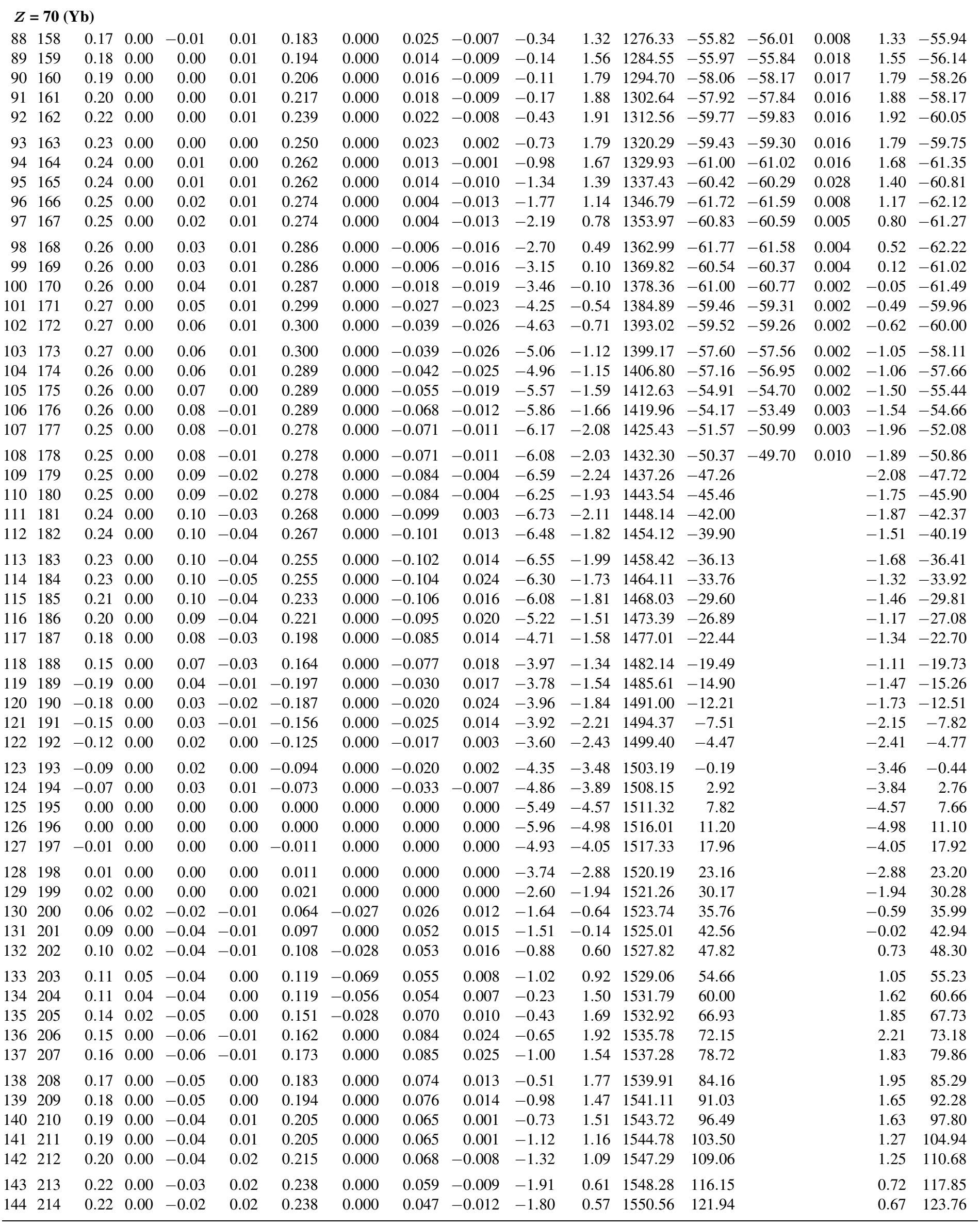




\section{$Z=70(Y b)$}

$\begin{array}{lllll}145 & 215 & 0.22 & 0.00 & -0.02\end{array}$

\section{$\begin{array}{llllll}146 & 216 & 0.23 & 0.00 & -0.01\end{array}$}

$\begin{array}{lllll}147 & 217 & 0.23 & 0.00 & -0.01\end{array}$

\section{$\begin{array}{lll}0.03 & 0.250 & 0.000\end{array}$}

$148 \quad 218$

$\begin{array}{lll}0.23 & 0.00 & 0.00\end{array}$

0.03

149219

$0.23 \quad 0.00$

0.01

0.03

150220

$\begin{array}{ll}0.23 & 0.00\end{array}$

0.02

0.03

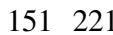

$0.24 \quad 0.00$

0.03

0.02

\section{3}

\section{4}

\section{5}

156226

$\begin{array}{lll}157 & 227\end{array}$

158228

\section{9}

$0.24 \quad 0.00$

0.05$$
\begin{array}{llllllll}
0.264 & 0.000 & -0.021 & -0.029 & -4.38 & -1.76 & 1560.06 & 177.01
\end{array}
$$

$\begin{array}{llllllll}0.264 & 0.000 & -0.021 & -0.029 & -4.38 & -1.76 & 1560.06 & 177.01 \\ 0.265 & 0.000 & -0.033 & -0.031 & -4.86 & -2.13 & 1560.02 & 185.12\end{array}$

$\begin{array}{llllllll}0.266 & 0.000 & -0.046 & -0.024 & -5.07 & -2.27 & 1560.88 & 200.40\end{array}$ $\begin{array}{llllllll}0.254 & 0.000 & -0.048 & -0.024 & -4.82 & -2.14 & 1561.88 & 207.48\end{array}$ $\begin{array}{lllllllllllll}0.23 & 0.00 & 0.07 & 0.01 & 0.255 & 0.000 & -0.060 & -0.026 & -5.48 & -2.62 & 1561.61 & 215.81 \\ 0.23 & 0.00 & 0.07 & 0.00 & 0.255 & 0.000 & -0.061 & -0.017 & -5.32 & -2.49 & 1562.44 & 223.05\end{array}$ $\begin{array}{llllllllllll}0.23 & 0.00 & 0.08 & 0.00 & 0.256 & 0.000 & -0.073 & -0.019 & -6.16 & -3.14 & 1562.18 & 231.38\end{array}$ $\begin{array}{llll}0.23 & 0.00 & 0.09 & -0.01\end{array}$ $\begin{array}{llllll}161 & 231 & 0.22 & 0.00 & 0.09 & -0.01\end{array}$

$\begin{array}{llllll}162 & 232 & 0.22 & 0.00 & 0.09 & -0.02\end{array}$ $\begin{array}{llllll}163 & 233 & 0.22 & 0.00 & 0.10 & -0.02\end{array}$ $\begin{array}{llllllll}0.244 & 0.000 & -0.090 & -0.002 & -6.97 & -3.93 & 1563.32 & 254.46\end{array}$ $\begin{array}{llllllll}0.245 & 0.000 & -0.101 & -0.004 & -7.43 & -4.16 & 1562.34 & 263.51\end{array}$ $\begin{array}{llllll}164 & 234 & 0.22 & 0.00 & 0.10 & -0.03\end{array}$

$\begin{array}{llllllll}0.245 & 0.000 & -0.103 & 0.006 & -7.05 & -3.88 & 1562.52 & 271.40\end{array}$ $\begin{array}{llllllll}0.256 & 0.000 & -0.086 & -0.012 & -6.50 & -3.31 & 1563.14 & 238.50 \\ 0.245 & 0.000 & -0.088 & -0.011 & -6.97 & -3.85 & 1562.61 & 247.10\end{array}$ $\begin{array}{llllllll}0.256 & 0.000 & -0.086 & -0.012 & -6.50 & -3.31 & 1563.14 & 238.50 \\ 0.245 & 0.000 & -0.088 & -0.011 & -6.97 & -3.85 & 1562.61 & 247.10\end{array}$ $\begin{array}{lllllllll}0.03 & 0.238 & 0.000 & 0.048 & -0.022 & -2.42 & 0.02 & 1551.42 & 129.15\end{array}$

$0.250 \quad 0.000$

$0.038-0.025-2.50$

$0.03 \quad 1553.5$

135.13

$0.250 \quad 0.000$

$0.038-0.025$

$0.026-0.027$

0.2510 .000

$\begin{array}{llllll}0.014 & -0.030 & -3.62 & -1.17 & 1556.57 & 156.28\end{array}$

$0.252 \quad 0.000$

$0.002-0.033$$$
\begin{array}{llllllll}
0.263 & 0.000 & -0.009 & -0.026 & -4.29 & -1.71 & 1558.54 & 170.46
\end{array}
$$

$0.20 \quad 131.21$

$0.16 \quad 137.34$

$-0.35 \quad 144.93$

$-0.46 \quad 151.21$

$-0.96 \quad 158.99$ $\begin{array}{llllllll}0.244 & 0.000 & -0.104 & 0.015 & -4.92 & -1.01 & 1128.98 & -6.11\end{array}$

\begin{tabular}{|c|c|c|c|c|c|c|c|c|c|c|c|c|c|c|c|c|c|}
\hline 75 & 146 & 0.22 & 0.00 & 0.10 & -0.04 & 0.244 & 0.000 & -0.104 & 0.015 & -4.92 & -1.01 & 1128.98 & -6.11 & & & -1.09 & -5.49 \\
\hline 76 & 147 & 0.21 & 0.00 & 0.09 & -0.03 & .232 & 0.000 & -0.093 & 0.009 & -4.23 & -0.88 & 1142.83 & -11.90 & & & -0.93 & \\
\hline 77 & 148 & 0.19 & 0.00 & 0.08 & -0.03 & 0.209 & 0.000 & -0.084 & 0.013 & -3.64 & -0.82 & 1154.69 & -15.68 & & & 87 & \\
\hline 8 & 149 & -0.18 & 0.00 & 0.05 & 0.00 & -0.187 & 0.000 & -0.043 & 0.010 & -2.84 & -0.92 & 1168.21 & -21 & & & & \\
\hline 79 & 150 & -0.17 & 0.00 & 0.05 & 0.01 & -0.176 & 0.000 & -0.045 & 0.000 & -3.38 & -1.48 & 1180.16 & -25.01 & & & .52 & \\
\hline 80 & 151 & -0.16 & 00 & 0.04 & 0.00 & -0.167 & 00 & -0.035 & 07 & -3.68 & -1.82 & 39 & -30.16 & & & 84 & -2 \\
\hline & 152 & -0.10 & 0.00 & 0.01 & 0.00 & -0.105 & 0.000 & -0.008 & 001 & -3.29 & -2.37 & & -3 & & & & \\
\hline & 153 & -0.02 & 0.00 & 0.00 & 0.00 & -0.021 & 0.000 & 0.000 & 0.000 & & -3.14 & & -38.58 & 1 & 209 & .14 & \\
\hline & 154 & -0.08 & 0.00 & -0.01 & 0.00 & -0.084 & 0.000 & 0.014 & -0.001 & -3.21 & -2.40 & 1227.57 & -40.14 & & & 41 & \\
\hline 4 & 155 & 0.09 & 0.00 & 0.01 & 0.00 & 0.096 & 0.000 & 0.015 & 0.001 & - & -1.16 & 20 & -4 & - & 0 & 17 & \\
\hline & 156 & 0.11 & 0.00 & -0.02 & 0.00 & 0.118 & 0.000 & 0.029 & 0.003 & -1.76 & -0.36 & 1247.30 & -43 & -43 & 074 & -0.37 & -43.61 \\
\hline & 157 & 0.13 & 0.00 & -0.02 & 0.00 & 0.139 & 00 & & & & 0.35 & & & & & 34 & \\
\hline & 158 & 0.15 & 0.00 & -0.01 & 0.00 & 0.161 & 0.000 & 0.022 & 0.002 & -0.92 & 0.72 & 7.02 & -47.30 & -4 & 15 & 0.70 & .31 \\
\hline & 159 & 0.16 & 0.00 & -0.01 & 0.01 & 0.172 & 0.000 & 0.024 & -0.007 & & 1.14 & & & & & .14 & \\
\hline 89 & 160 & 0.17 & 0.00 & 0.00 & 0.01 & 0.183 & 0.000 & 0.013 & -0.009 & -0.38 & 1.25 & 12 & -50.51 & -5 & 57 & 1.24 & -50.62 \\
\hline 0 & 161 & 0.19 & 0.00 & 0.00 & 0.01 & 0.206 & 0.000 & 0.016 & -0.009 & -0.38 & 1.52 & .57 & -52.63 & -52 & .028 & 1.51 & -52.77 \\
\hline 1 & 162 & 0.19 & 0.00 & 0.00 & 0.01 & 0.206 & 00 & 16 & -0.009 & & 1.63 & & & & & 1.62 & \\
\hline 2 & 163 & 0.21 & 0.00 & 0.01 & 0.01 & 0.228 & 0.000 & 0.008 & -0.011 & -0.41 & 1.75 & .90 & -5 & -5 & 28 & 1.74 & .05 \\
\hline & 164 & 0.22 & 0.00 & 0.01 & 0.00 & 0.239 & 0.000 & 0.009 & -0.001 & -0.60 & 1.68 & & -5 & & & 67 & \\
\hline 94 & 165 & 0.22 & 0.00 & 0.01 & 0.01 & 0.239 & 0.000 & 0.010 & -0.011 & -0.72 & 1.64 & 72 & -5 & -5 & 0.027 & 1.64 & \\
\hline 05 & 166 & 0.23 & 0.00 & 0.01 & 0.00 & 250 & 00 & 011 & -0.001 & -1.09 & 1.42 & 66 & -5 & -5 & 0 & 1.41 & - \\
\hline 96 & 167 & 0.24 & 0.00 & 0.02 & 0.01 & 0.262 & 0.000 & 002 & -0.013 & -1.49 & 1.21 & .06 & -5 & -5 & & 1.21 & .06 \\
\hline 97 & 168 & 0.24 & 0.00 & 0.02 & 0.01 & 0.262 & 0.000 & 002 & -0.013 & -1.86 & 0.89 & 69 & -57.26 & -5 & & 0.89 & 67 \\
\hline 98 & 169 & 0.25 & 0.00 & 0.04 & 0.00 & 0.275 & 0.000 & -0.022 & -0.009 & -2.37 & 0.71 & 66 & -5 & -5 & 95 & 0.72 & .58 \\
\hline 90 & 170 & 0.26 & 0.00 & 00 & 0.0 & 0286 & 0.000 & 19 & -0 & -2 & 034 & & -5 & & & 0.33 & \\
\hline & 171 & & 0.00 & & & & & & 22 & & & & & & & 2 & \\
\hline 11 & 172 & 0.26 & 0.00 & 0.05 & 0.01 & 0.287 & 0.000 & -0.030 & -0.022 & -3.89 & -0.31 & 60 & -56.88 & -56 & 03 & .28 & -57.37 \\
\hline & 173 & 0.26 & 0.00 & 0.06 & 0.01 & 0.289 & 0.000 & -0.042 & -0.025 & -4.31 & -0.53 & & -57.05 & -5 & 2 & 47 & -57.53 \\
\hline 103 & 174 & 0.26 & 0.00 & 0.06 & 0.01 & 0.289 & 0.000 & -0.042 & -0.025 & -4.77 & -0.96 & .48 & -55.62 & -55.58 & 0.002 & .91 & -56.13 \\
\hline 104 & 175 & 0.26 & 0.00 & 0.07 & 0.00 & 0.289 & 0.000 & -0.055 & -0.019 & -5.06 & -1.11 & 30 & -5 & -5 & 0. & .04 & 5.87 \\
\hline 25 & 176 & 0.25 & 0.00 & 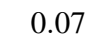 & & 78 & 0 & -( & -0.018 & -5 & -1.56 & & -5 & & 02 & .50 & .15 \\
\hline 06 & 177 & 0.25 & 0.00 & 0.08 & -0.01 & 0.278 & 0.000 & -0.071 & -0.011 & -5.83 & -1.76 & & -53.06 & -52 & 0.002 & 67 & -53.56 \\
\hline 7 & 178 & 0.25 & 0.00 & 0.08 & -0.01 & 0.278 & 0.000 & -0.071 & -0.011 & & -2.21 & & -50.96 & -5 & 0.0 & -2.12 & -51.49 \\
\hline 108 & 179 & 0.25 & 0.00 & 0.09 & -0.02 & 0.278 & 0.000 & -0.084 & -0.004 & -6.62 & -2.25 & 1439.12 & -49.90 & -49.06 & 0.005 & -2.11 & -50.38 \\
\hline 109 & 180 & 0.24 & 0.00 & 0.09 & -0.02 & 0.267 & 0.000 & -0.086 & -0.004 & -6.69 & -2.43 & 1.51 & -47.22 & -46.69 & 0.071 & -2.29 & -47.70 \\
\hline 10 & 181 & 0.24 & 0.00 & 0.10 & -0.03 & 0.268 & 0.000 & -0.099 & 0.003 & -6.90 & -2.28 & 1451.00 & -45.64 & & & -2.07 & -46.05 \\
\hline
\end{tabular}
$\begin{array}{lll}0.232 & 0.000 & -0.093\end{array}$ 


\section{$Z=71(\mathrm{Lu})$}

\begin{tabular}{|c|c|c|c|c|c|c|c|c|c|c|c|c|c|c|c|}
\hline 111 & 182 & 0.24 & 0.00 & 0.10 & -0.03 & 0.268 & 0.000 & -0.099 & 0.003 & -7.05 & -2.35 & 1455.95 & -42.52 & -2.14 & -42.94 \\
\hline 112 & 183 & 0.23 & 0.00 & 0.10 & -0.04 & 0.255 & 0.000 & -0.102 & 0.014 & -6.76 & -2.17 & 1462.10 & -40.60 & -1.90 & -40.94 \\
\hline 113 & 184 & 0.23 & 0.00 & 0.11 & -0.05 & 0.256 & 0.000 & -0.116 & 0.021 & -7.57 & -2.46 & 1466.98 & -37.40 & -2.10 & -37.64 \\
\hline 114 & 185 & 0.22 & 0.00 & 0.11 & -0.05 & 0.244 & 0.000 & -0.117 & 0.023 & -7.18 & -2.17 & 1472.69 & -35.04 & -1.76 & -35.23 \\
\hline 115 & 186 & 0.21 & 0.00 & 0.10 & -0.05 & 0.232 & 0.000 & -0.107 & 0.026 & -6.78 & -2.31 & 1477.12 & -31.40 & -1.94 & -31.60 \\
\hline 116 & 187 & 0.20 & 0.00 & 0.10 & -0.04 & 0.221 & 0.000 & -0.107 & 0.018 & -6.19 & -2.01 & 1482.53 & -28.74 & -1.67 & -28.95 \\
\hline 117 & 188 & 0.18 & 0.00 & 0.09 & -0.04 & 0.198 & 0.000 & -0.098 & 0.022 & -5.81 & -2.21 & 1486.72 & -24.86 & -1.90 & -25.09 \\
\hline 118 & 189 & 0.15 & 0.00 & 0.07 & -0.03 & 0.164 & 0.000 & -0.077 & 0.018 & -4.55 & -1.86 & 1491.78 & -21.85 & -1.65 & -22.15 \\
\hline 19 & 190 & -0.18 & 0.00 & 0.04 & -0.01 & -0.187 & 0.000 & -0.032 & 0.017 & -4.05 & -1.93 & 1495.57 & -17.56 & -1.87 & -17.98 \\
\hline 120 & 191 & -0.17 & 0.00 & 0.03 & -0.02 & -0.177 & 0.000 & -0.022 & 0.024 & -4.26 & -2.25 & 1501.01 & -14.94 & -2.15 & -15.30 \\
\hline 121 & 192 & -0.15 & 0.00 & 0.03 & -0.01 & -0.156 & 0.000 & -0.025 & 0.014 & -4.33 & -2.62 & 1504.82 & -10.68 & -2.57 & -11.05 \\
\hline 122 & 193 & -0.12 & 0.00 & 0.02 & 0.00 & -0.125 & 0.000 & -0.017 & 0.003 & -4.04 & -2.86 & 1509.92 & -7.70 & -2.85 & -8.07 \\
\hline 123 & 194 & -0.09 & 0.00 & 0.03 & 0.01 & -0.094 & 0.000 & -0.032 & -0.006 & -4.86 & -3.88 & 1514.11 & -3.82 & -3.84 & -4.12 \\
\hline 124 & 195 & -0.07 & 0.00 & 0.03 & 0.01 & -0.073 & 0.000 & -0.033 & -0.007 & -5.25 & -4.28 & 1519.10 & -0.74 & -4.24 & -0.99 \\
\hline 125 & 196 & -0.03 & 0.00 & 0.00 & 0.00 & -0.032 & 0.000 & 0.000 & 0.000 & -5.79 & -4.92 & 1522.66 & 3.77 & -4.92 & 3.53 \\
\hline 126 & 197 & 0.00 & 0.00 & 0.00 & 0.00 & 0.000 & 0.000 & 0.000 & 0.000 & -6.21 & -5.24 & 1527.30 & 7.21 & -5.24 & 7.02 \\
\hline 127 & 198 & -0.02 & 0.00 & -0.01 & 0.00 & -0.021 & 0.000 & 0.012 & -0.000 & -5.32 & -4.42 & 1529.15 & 13.43 & -4.42 & 13.31 \\
\hline 128 & 199 & 0.00 & 0.00 & 0.00 & 0.00 & 0.000 & 0.000 & 0.000 & 0.000 & -4.14 & -3.31 & 1532.11 & 18.54 & -3.31 & 18.48 \\
\hline 129 & 200 & 0.01 & 0.00 & -0.01 & 0.00 & 0.011 & 0.000 & 0.012 & 0.000 & -2.96 & -2.18 & 1533.40 & 25.31 & -2.18 & 25.32 \\
\hline 130 & 201 & 0.06 & 0.00 & -0.02 & 0.00 & 0.064 & 0.000 & 0.025 & 0.002 & -1.88 & -0.95 & 1535.98 & 30.80 & -0.93 & 30.90 \\
\hline 131 & 202 & 0.09 & 0.00 & -0.03 & -0.01 & 0.096 & 0.000 & 0.040 & 0.014 & -1.63 & -0.43 & 1537.65 & 37.21 & -0.36 & 37.43 \\
\hline 132 & 203 & 0.10 & 0.00 & -0.04 & -0.01 & 0.107 & 0.000 & 0.053 & 0.016 & -1.13 & 0.31 & 1540.50 & 42.43 & 0.42 & 42.78 \\
\hline 133 & 204 & 0.11 & 0.00 & -0.04 & -0.01 & 0.118 & 0.000 & 0.054 & 0.016 & -0.87 & 0.65 & 1542.11 & 48.89 & 0.77 & 49.32 \\
\hline 134 & 205 & 0.11 & 0.04 & -0.04 & 0.00 & 0.119 & -0.056 & 0.054 & 0.007 & -0.53 & 1.24 & 1544.87 & 54.20 & 1.35 & 54.72 \\
\hline 135 & 206 & 0.14 & 0.01 & -0.04 & 0.00 & 0.150 & -0.014 & 0.057 & 0.008 & -0.32 & 1.50 & 1546.34 & 60.81 & 1.60 & 61.39 \\
\hline 136 & 207 & 0.15 & 0.00 & -0.05 & 0.00 & 0.161 & 0.000 & 0.071 & 0.011 & -0.37 & 1.83 & 1549.13 & 66.09 & 1.99 & 66.83 \\
\hline 137 & 208 & 0.16 & 0.00 & -0.05 & -0.01 & 0.173 & 0.000 & 0.072 & 0.022 & -0.78 & 1.41 & 1551.06 & 72.22 & 1.61 & 73.11 \\
\hline 138 & 209 & 0.17 & 0.00 & -0.05 & 0.00 & 0.183 & 0.000 & 0.074 & 0.013 & -0.64 & 1.62 & 1553.74 & 77.61 & 1.79 & 78.57 \\
\hline 139 & 210 & 0.17 & 0.00 & -0.05 & 0.00 & 0.183 & 0.000 & 0.074 & 0.013 & -0.93 & 1.39 & 1555.28 & 84.15 & 1.55 & 85.21 \\
\hline 140 & 211 & 0.19 & 0.00 & -0.04 & 0.01 & 0.205 & 0.000 & 0.065 & 0.001 & -0.81 & 1.50 & 1557.85 & 89.65 & 1.61 & 90.78 \\
\hline 141 & 212 & 0.19 & 0.00 & -0.04 & 0.01 & 0.205 & 0.000 & 0.065 & 0.001 & -1.21 & 1.10 & 1559.35 & 96.22 & 1.20 & 97.46 \\
\hline 142 & 213 & 0.20 & 0.00 & -0.03 & 0.01 & 0.216 & 0.000 & 0.055 & -0.001 & -1.14 & 1.14 & 1561.77 & 101.88 & 1.21 & 103.20 \\
\hline 143 & 214 & 0.22 & 0.00 & -0.03 & 0.02 & 0.238 & 0.000 & 0.059 & -0.009 & -1.90 & 0.69 & 1563.12 & 108.59 & 0.78 & 110.08 \\
\hline 144 & 215 & 0.22 & 0.00 & -0.02 & 0.02 & 0.238 & 0.000 & 0.047 & -0.012 & -1.82 & 0.58 & 1565.49 & 114.30 & 0.66 & 115.90 \\
\hline 145 & 216 & 0.22 & 0.00 & -0.02 & 0.02 & 0.238 & 0.000 & 0.047 & -0.012 & -2.32 & 0.14 & 1566.63 & 121.22 & 0.20 & 122.95 \\
\hline 146 & 217 & 0.22 & 0.00 & -0.01 & 0.03 & 0.238 & 0.000 & 0.036 & -0.025 & -2.40 & -0.04 & 1568.87 & 127.06 & 0.13 & 129.03 \\
\hline 147 & 218 & 0.22 & 0.00 & -0.01 & 0.03 & 0.238 & 0.000 & 0.036 & -0.025 & -2.91 & -0.52 & 1569.86 & 134.14 & -0.36 & 136.26 \\
\hline 148 & 219 & 0.22 & 0.00 & 0.00 & 0.03 & 0.239 & 0.000 & 0.024 & -0.028 & -2.96 & -0.63 & 1571.84 & 140.23 & -0.45 & 142.52 \\
\hline 149 & 220 & 0.23 & 0.00 & 0.01 & 0.03 & 0.251 & 0.000 & 0.014 & -0.030 & -3.65 & -1.18 & 1572.70 & 147.44 & -0.99 & 149.88 \\
\hline 150 & 221 & 0.23 & 0.00 & 0.02 & 0.02 & 0.251 & 0.000 & 0.001 & -0.023 & -3.64 & -1.21 & 1574.41 & 153.80 & -1.12 & 156.31 \\
\hline 151 & 222 & 0.23 & 0.00 & 0.03 & 0.02 & 0.252 & 0.000 & -0.011 & -0.026 & -4.24 & -1.73 & 1575.07 & 161.21 & -1.61 & 163.92 \\
\hline 152 & 223 & 0.23 & 0.00 & 0.04 & 0.02 & 0.253 & 0.000 & -0.023 & -0.029 & -4.32 & -1.77 & 1576.60 & 167.75 & -1.58 & 170.70 \\
\hline 153 & 224 & 0.23 & 0.00 & 0.04 & 0.02 & 0.253 & 0.000 & -0.023 & -0.029 & -4.63 & -2.08 & 1576.88 & 175.54 & -1.91 & 178.65 \\
\hline 154 & 225 & 0.23 & 0.00 & 0.05 & 0.02 & 0.254 & 0.000 & -0.035 & -0.031 & -4.70 & -2.09 & 1578.20 & 182.30 & -1.83 & 185.67 \\
\hline 155 & 226 & 0.23 & 0.00 & 0.06 & 0.01 & 0.254 & 0.000 & -0.048 & -0.024 & -5.09 & -2.33 & 1578.23 & 190.34 & -2.12 & 193.85 \\
\hline 156 & 227 & 0.23 & 0.00 & 0.06 & 0.01 & 0.254 & 0.000 & -0.048 & -0.024 & -4.96 & -2.25 & 1579.29 & 197.35 & -2.03 & 201.07 \\
\hline 157 & 228 & 0.23 & 0.00 & 0.07 & 0.01 & 0.255 & 0.000 & -0.060 & -0.026 & -5.63 & -2.73 & 1579.40 & 205.32 & -2.44 & 209.31 \\
\hline 158 & 229 & 0.22 & 0.00 & 0.07 & 0.00 & 0.243 & 0.000 & -0.063 & -0.016 & -5.42 & -2.67 & 1580.29 & 212.49 & -2.42 & 216.63 \\
\hline 159 & 230 & 0.22 & 0.00 & 0.08 & 0.00 & 0.244 & 0.000 & -0.075 & -0.019 & -6.30 & -3.33 & 1580.41 & 220.44 & -2.99 & 224.88 \\
\hline 160 & 231 & 0.22 & 0.00 & 0.08 & -0.01 & 0.244 & 0.000 & -0.076 & -0.009 & -6.37 & -3.45 & 1581.33 & 227.60 & -3.13 & 232.24 \\
\hline 161 & 232 & 0.22 & 0.00 & 0.09 & -0.01 & 0.245 & 0.000 & -0.088 & -0.011 & -7.29 & -4.11 & 1581.29 & 235.71 & -3.68 & 240.67 \\
\hline 162 & 233 & 0.22 & 0.00 & 0.10 & -0.02 & 0.245 & 0.000 & -0.101 & -0.004 & -7.73 & -4.34 & 1582.14 & 242.93 & -3.74 & 248.27 \\
\hline 163 & 234 & 0.22 & 0.00 & 0.10 & -0.03 & 0.245 & 0.000 & -0.103 & 0.006 & -7.87 & -4.54 & 1581.48 & 251.66 & -3.88 & 257.29 \\
\hline 164 & 235 & 0.22 & 0.00 & 0.10 & -0.03 & 0.245 & 0.000 & -0.103 & 0.006 & -7.45 & -4.19 & 1581.61 & 259.60 & -3.51 & 265.49 \\
\hline 165 & 236 & 0.21 & 0.00 & 0.10 & -0.03 & 0.233 & 0.000 & -0.105 & 0.007 & -7.39 & -4.26 & 1580.67 & 268.62 & -3.56 & 274.77 \\
\hline 166 & 237 & 0.20 & 0.00 & 0.10 & -0.04 & 0.221 & 0.000 & -0.107 & 0.018 & -6.93 & -4.03 & 1580.75 & 276.60 & -3.13 & 283.19 \\
\hline
\end{tabular}




\begin{tabular}{|c|c|c|c|c|c|c|c|c|c|c|c|c|c|c|c|c|}
\hline \multicolumn{17}{|c|}{$Z=72(\mathrm{Hf})$} \\
\hline $\begin{array}{ll}77 & 149\end{array}$ & 0.18 & 0.00 & 0.08 & -0.03 & 0.198 & 0.000 & -0.085 & 0.014 & -3.66 & -0.92 & 1153.97 & -7.67 & & & -0.96 & -7.00 \\
\hline $78 \quad 150$ & -0.17 & 0.00 & 0.05 & 0.01 & -0.176 & 0.000 & -0.045 & 0.000 & -3.05 & -1.26 & 1168.29 & -13.92 & & & -1.29 & -13.31 \\
\hline $79 \quad 151$ & -0.16 & 0.00 & 0.05 & 0.01 & -0.166 & 0.000 & -0.047 & -0.000 & -3.62 & -1.63 & 1180.13 & -17.69 & & & -1.66 & -17.14 \\
\hline $80 \quad 152$ & -0.15 & 0.00 & 0.04 & 0.01 & -0.156 & 0.000 & -0.037 & -0.003 & -3.97 & -2.26 & 1194.19 & -23.67 & & & -2.28 & -23.19 \\
\hline $81 \quad 153$ & -0.09 & 0.00 & 0.02 & 0.00 & -0.094 & 0.000 & -0.020 & 0.002 & -3.85 & -2.94 & 1205.82 & -27.24 & & & -2.95 & -26.80 \\
\hline $82 \quad 154$ & 0.02 & 0.00 & 0.00 & 0.00 & 0.021 & 0.000 & 0.000 & 0.000 & -4.53 & -3.66 & 1219.45 & -32.79 & & & -3.66 & -32.41 \\
\hline $83 \quad 155$ & -0.06 & 0.00 & 0.00 & 0.00 & -0.063 & 0.000 & 0.001 & 0.000 & -3.64 & -2.97 & 1229.20 & -34.48 & & & -2.97 & -34.16 \\
\hline $84 \quad 156$ & -0.08 & 0.00 & -0.01 & 0.00 & -0.084 & 0.000 & 0.014 & -0.001 & -2.75 & -1.99 & 1240.63 & -37.83 & -37.85 & 0.208 & -1.99 & -37.57 \\
\hline $85 \quad 157$ & 0.11 & 0.00 & -0.02 & 0.00 & 0.118 & 0.000 & 0.029 & 0.003 & -2.20 & -0.78 & 1249.39 & -38.52 & & & -0.79 & -38.31 \\
\hline 86158 & 0.12 & 0.00 & -0.02 & 0.00 & 0.128 & 0.000 & 0.030 & 0.003 & -1.48 & -0.01 & 1260.53 & -41.59 & -42.10 & 0.018 & -0.01 & -41.44 \\
\hline $87 \quad 159$ & 0.14 & 0.00 & -0.01 & 0.00 & 0.150 & 0.000 & 0.020 & 0.002 & -1.14 & 0.41 & 1269.61 & -42.60 & -42.85 & 0.017 & 0.40 & -42.51 \\
\hline $88 \quad 160$ & 0.15 & 0.00 & -0.01 & 0.01 & 0.161 & 0.000 & 0.022 & -0.007 & -0.73 & 0.91 & 1280.55 & -45.47 & -45.94 & 0.012 & 0.90 & -45.42 \\
\hline $89 \quad 161$ & 0.16 & 0.00 & 0.00 & 0.01 & 0.172 & 0.000 & 0.012 & -0.009 & -0.43 & 1.03 & 1289.47 & -46.32 & -46.32 & 0.023 & 1.02 & -46.33 \\
\hline $90 \quad 162$ & 0.17 & 0.00 & 0.00 & 0.01 & 0.183 & 0.000 & 0.013 & -0.009 & -0.24 & 1.38 & 1300.10 & -48.88 & -49.17 & 0.010 & 1.38 & -48.93 \\
\hline $91 \quad 163$ & 0.18 & 0.00 & 0.00 & 0.01 & 0.194 & 0.000 & 0.014 & -0.009 & -0.15 & 1.60 & 1308.50 & -49.21 & -49.29 & 0.028 & 1.59 & -49.31 \\
\hline $92 \quad 164$ & 0.19 & 0.00 & 0.00 & 0.01 & 0.206 & 0.000 & 0.016 & -0.009 & -0.08 & 1.76 & 1318.87 & -51.50 & -51.82 & 0.020 & 1.76 & -51.65 \\
\hline $\begin{array}{ll}93 & 165\end{array}$ & 0.20 & 0.00 & 0.00 & 0.01 & 0.217 & 0.000 & 0.018 & -0.009 & -0.20 & 1.79 & 1327.03 & -51.59 & -51.64 & 0.028 & 1.79 & -51.78 \\
\hline $94 \quad 166$ & 0.21 & 0.00 & 0.01 & 0.01 & 0.228 & 0.000 & 0.008 & -0.011 & -0.34 & 1.80 & 1337.12 & -53.61 & -53.86 & 0.028 & 1.81 & -53.84 \\
\hline $\begin{array}{ll}95 & 167\end{array}$ & 0.22 & 0.00 & 0.01 & 0.00 & 0.239 & 0.000 & 0.009 & -0.001 & -0.62 & 1.64 & 1345.06 & -53.48 & -53.47 & 0.028 & 1.64 & -53.76 \\
\hline 96168 & 0.23 & 0.00 & 0.02 & 0.00 & 0.251 & 0.000 & -0.002 & -0.004 & -0.94 & 1.52 & 1354.87 & -55.22 & -55.36 & 0.028 & 1.52 & -55.53 \\
\hline $97 \quad 169$ & 0.24 & 0.00 & 0.02 & 0.00 & 0.262 & 0.000 & 0.001 & -0.004 & -1.40 & 1.23 & 1362.55 & -54.83 & -54.72 & 0.028 & 1.22 & -55.18 \\
\hline $98 \quad 170$ & 0.25 & 0.00 & 0.03 & 0.00 & 0.274 & 0.000 & -0.009 & -0.006 & -1.82 & 1.02 & .04 & -56.24 & -5 & 0.028 & 1.04 & 6.61 \\
\hline $99 \quad 171$ & 0.25 & 0.00 & 0.03 & 0.01 & 0.274 & 0.000 & -0.008 & -0.016 & -2.28 & 0.71 & 1379.35 & -55.49 & -55.43 & 0.029 & 0.72 & -55.89 \\
\hline $100 \quad 172$ & 0.25 & 0.00 & 0.04 & 0.01 & 0.275 & 0.000 & -0.020 & -0.019 & -2.60 & 0.51 & 1388.44 & -56.50 & -56.40 & 0.024 & 0.55 & -56.92 \\
\hline $101 \quad 173$ & 0.26 & 0.00 & 0.05 & 0.00 & 0.287 & 0.000 & -0.031 & -0.013 & -3.25 & 0.13 & 1395.46 & -55.45 & -55.41 & 0.028 & 0.15 & -55.91 \\
\hline $102 \quad 174$ & 0.26 & 0.00 & 0.06 & 0.00 & 0.288 & 0.000 & -0.043 & -0.016 & -3.64 & -0.06 & 1404.16 & -56.08 & -55.85 & 0.003 & -0.01 & -56.53 \\
\hline $103 \quad 175$ & 0.26 & 0.00 & 0.06 & 0.00 & 0.288 & 0.000 & -0.043 & -0.016 & -4.11 & -0.50 & 1410.87 & -54.72 & -54.48 & 0.003 & -0.46 & -55.20 \\
\hline 104176 & 0.25 & 0.00 & 0.07 & 0.00 & 0.278 & 0.000 & -0.057 & -0.018 & -4.43 & -0.71 & 1419.23 & -55.01 & -54.58 & 0.002 & -0.63 & -55.47 \\
\hline $105 \quad 177$ & 0.25 & 0.00 & 0.07 & -0.01 & 0.277 & 0.000 & -0.059 & -0.009 & -4.89 & -1.19 & 1425.63 & -53.34 & -52.89 & 0.002 & -1.13 & -53.84 \\
\hline 106178 & 0.25 & 0.00 & 0.08 & -0.01 & 0.278 & 0.000 & -0.071 & -0.011 & -5.39 & -1.42 & 1433.65 & -53.28 & -52.44 & 0.002 & -1.32 & -53.76 \\
\hline $107 \quad 179$ & 0.24 & 0.00 & 0.08 & -0.01 & 0.267 & 0.000 & -0.073 & -0.010 & -5.77 & -1.89 & 1439.71 & -51.27 & -50.47 & 0.002 & -1.79 & -51.77 \\
\hline $108 \quad 180$ & 0.24 & 0.00 & 0.09 & -0.02 & 0.267 & 0.000 & -0.086 & -0.004 & -6.16 & -1.92 & 7.18 & -50.67 & .79 & 0.002 & -1.77 & -51.12 \\
\hline 109181 & 0.24 & 0.00 & 0.09 & -0.02 & 0.267 & 0.000 & -0.086 & -0.004 & -6.39 & -2.16 & 1452.68 & -48.10 & -47.41 & 0.002 & -2.02 & -48.56 \\
\hline 110182 & 0.24 & 0.00 & 0.10 & -0.03 & 0.268 & 0.000 & -0.099 & 0.003 & -6.64 & -2.03 & 1459.65 & -47.00 & -46.06 & 0.006 & -1.81 & -47.40 \\
\hline 111183 & 0.23 & 0.00 & 0.10 & -0.03 & 0.256 & 0.000 & -0.101 & 0.004 & -6.77 & -2.23 & 1464.80 & -44.07 & -43.29 & 0.030 & -2.02 & -44.48 \\
\hline 112184 & 0.23 & 0.00 & 0.11 & -0.04 & 0.256 & 0.000 & -0.114 & 0.012 & -7.10 & -2.14 & 1471.48 & -42.69 & -41.50 & 0.040 & -1.82 & -42.99 \\
\hline 113185 & 0.22 & 0.00 & 0.11 & -0.05 & 0.244 & 0.000 & -0.117 & 0.023 & -7.43 & -2.40 & 1476.38 & -39.51 & & & -2.03 & -39.75 \\
\hline 114186 & 0.21 & 0.00 & 0.11 & -0.05 & 0.233 & 0.000 & -0.119 & 0.024 & -7.12 & -2.23 & 1482.67 & -37.73 & & & -1.82 & -37.92 \\
\hline $115 \quad 187$ & 0.20 & 0.00 & 0.10 & -0.05 & 0.221 & 0.000 & -0.109 & 0.027 & -6.81 & -2.45 & 1487.22 & -34.22 & & & -2.08 & -34.44 \\
\hline 116188 & 0.19 & 0.00 & 0.10 & -0.04 & 0.210 & 0.000 & -0.109 & 0.019 & -6.31 & -2.24 & 1493.17 & -32.09 & & & -1.89 & -32.32 \\
\hline $117 \quad 189$ & 0.18 & 0.00 & 0.09 & -0.04 & 0.198 & 0.000 & -0.098 & 0.022 & -6.13 & -2.54 & 1497.51 & -28.36 & & & -2.23 & -28.61 \\
\hline $118 \quad 190$ & 0.15 & 0.00 & 0.07 & -0.03 & 0.164 & 0.000 & -0.077 & 0.018 & -5.07 & -2.35 & 1503.18 & -25.96 & & & -2.14 & -26.29 \\
\hline 119191 & 0.14 & 0.00 & 0.06 & -0.02 & 0.152 & 0.000 & -0.065 & 0.011 & -4.65 & -2.36 & 1506.95 & -21.65 & & & -2.23 & -22.04 \\
\hline $120 \quad 192$ & -0.17 & 0.00 & 0.03 & -0.02 & -0.177 & 0.000 & -0.022 & 0.024 & -4.66 & -2.61 & 1512.76 & -19.40 & & & -2.51 & -19.80 \\
\hline $121 \quad 193$ & -0.14 & 0.00 & 0.02 & -0.01 & -0.146 & 0.000 & -0.015 & 0.012 & -4.60 & -3.10 & 1516.74 & -15.31 & & & -3.07 & -15.74 \\
\hline $122 \quad 194$ & -0.11 & 0.00 & 0.02 & 0.00 & -0.115 & 0.000 & -0.018 & 0.002 & -4.53 & -3.47 & 1522.40 & -12.89 & & & -3.46 & -13.31 \\
\hline $123 \quad 195$ & -0.09 & 0.00 & 0.03 & 0.00 & -0.094 & 0.000 & -0.031 & 0.003 & -5.43 & -4.45 & 1526.59 & -9.01 & & & -4.41 & -9.37 \\
\hline $124 \quad 196$ & -0.07 & 0.00 & 0.03 & 0.01 & -0.073 & 0.000 & -0.033 & -0.007 & -5.89 & -4.90 & 1532.06 & -6.41 & & & -4.85 & -6.71 \\
\hline $125 \quad 197$ & -0.01 & 0.00 & 0.00 & 0.00 & -0.011 & 0.000 & 0.000 & 0.000 & -6.41 & -5.49 & 1535.60 & -1.88 & & & -5.49 & -2.19 \\
\hline 126198 & 0.00 & 0.00 & 0.00 & 0.00 & 0.000 & 0.000 & 0.000 & 0.000 & -6.84 & -5.83 & 1540.70 & 1.09 & & & -5.83 & 0.84 \\
\hline $127 \quad 199$ & -0.02 & 0.00 & 0.00 & 0.00 & -0.021 & 0.000 & 0.000 & 0.000 & -5.93 & -5.03 & 1542.61 & 7.26 & & & -5.04 & 7.05 \\
\hline $128 \quad 200$ & 0.00 & 0.00 & 0.00 & 0.00 & 0.000 & 0.000 & 0.000 & 0.000 & -4.80 & -3.93 & 1545.99 & 11.95 & & & -3.93 & 11.80 \\
\hline 129201 & 0.01 & 0.00 & 0.00 & 0.00 & 0.011 & 0.000 & 0.000 & 0.000 & -3.61 & -2.91 & 1547.43 & 18.57 & & & -2.91 & 18.49 \\
\hline $130 \quad 202$ & 0.01 & 0.00 & 0.00 & 0.00 & 0.011 & 0.000 & 0.000 & 0.000 & -2.29 & -1.60 & 1550.35 & 23.72 & & & -1.60 & 23.70 \\
\hline 131203 & 0.08 & 0.00 & -0.03 & -0.01 & 0.086 & 0.000 & 0.039 & 0.014 & -1.93 & -0.76 & 1551.74 & 30.41 & & & -0.69 & 30.53 \\
\hline $132 \quad 204$ & 0.10 & 0.01 & -0.03 & -0.01 & 0.107 & 0.014 & 0.041 & 0.014 & -1.22 & 0.05 & 1554.92 & 35.30 & & & 0.13 & 35.50 \\
\hline 133205 & 0.11 & 0.04 & -0.03 & -0.01 & 0.119 & -0.056 & 0.042 & 0.016 & -1.21 & 0.45 & 1556.51 & 41.78 & & & 0.55 & 42.08 \\
\hline
\end{tabular}




\section{$Z=72$ (Hf)}

134206

$\begin{array}{lll}0.11 & 0.02 & -0.04\end{array}$ $\begin{array}{ll}135 & 207\end{array}$

136208 $\begin{array}{llll}0.13 & 0.01 & -0.04\end{array}$ $\begin{array}{lll}0.14 & 0.00 & -0.04\end{array}$

0.00

0.054

$\begin{array}{llll}0.007 & -0.50 & 1.04 & 1559.68\end{array}$ 0.056 $\begin{array}{ll}0.008 & -0.30\end{array}$ $\begin{array}{ll}1.39 & 1561.08\end{array}$

137209

$\begin{array}{llll}0.15 & 0.00 & -0.05\end{array}$

$-0.01$

$\begin{array}{ll}0.150 & 0.000\end{array}$

0.057

$\begin{array}{ll}0.008 & 0.01\end{array}$

$1.80 \quad 1564.20$

$\begin{array}{ll}1.73 \quad 1565.81 \\ 1.76 & 1569.07\end{array}$

$\begin{array}{ll}0.162 & 0.000\end{array}$

0.071

$\begin{array}{ll}0.021 & -0.58\end{array}$

$\begin{array}{ll}1.76 & 1569.07\end{array}$

139211

$\begin{array}{llll}0.17 & 0.00 & -0.04\end{array}$

$140 \quad 212$

$\begin{array}{ll}141 & 213\end{array}$

$\begin{array}{ll}142 & 214\end{array}$

$143 \quad 215$

$144 \quad 216$

$145 \quad 217$

$146 \quad 218$

$\begin{array}{lll}147 & 219\end{array}$

$148 \quad 220$

$\begin{array}{llll}0.18 & 0.00 & -0.04\end{array}$

$\begin{array}{lll}0.19 & 0.00 & -0.04\end{array}$

$\begin{array}{llll}0.19 & 0.00 & -0.03\end{array}$

$\begin{array}{llll}0.20 & 0.00 & -0.03\end{array}$

0.00

0.172

0.000

0.060

0.00

0.000

0.062

$0.009-0.11$

$\begin{array}{ll}1.56 & 1570.61\end{array}$

0.000

0.063

$0.010-0.50$

$1.63 \quad 1573.61$

$\begin{array}{ll}0.01 \quad 0.205 \\ 0.01 & 0.205\end{array}$

0.000

0.065

$\begin{array}{ll}1.33 & 1575.04\end{array}$

$\begin{array}{ll}0.01 & 0.205\end{array}$

0.000

$\begin{array}{ll}1.34 & 1577.88\end{array}$

$\begin{array}{lll}0.96 & 1579.18\end{array}$

$\begin{array}{llll}0.22 & 0.00 & -0.02\end{array}$

0.02

0.238

0.000

$\begin{array}{llll}0.056 & -0.011 & -1.31\end{array}$

$\begin{array}{ll}0.90 & 1581.90\end{array}$

$\begin{array}{llll}0.22 & 0.00 & -0.02\end{array}$

0.02

$\begin{array}{llll}0.22 & 0.00 & -0.01\end{array}$

0.02

0.238

0.000

$-0.012-1.49$

$0.42 \quad 1583.09$

$\begin{array}{rr}0.37 & 1585.60 \\ -0.21 & 1586.70\end{array}$

0.000

$0.035-0.015-1.94$

$\begin{array}{lll}0.036 & -0.025 & -2.57\end{array}$

$\begin{array}{lll}0.22 & 0.00 & 0.00\end{array}$

0.02

0.000

0.023

$0.025-2.57$

$0.22 \quad 1588.96$
-0.75

$\begin{array}{lllllll}0.000 & 0.011 & -0.021 & -3.04 & -0.75 & 1589.83 & 137.60\end{array}$

$\begin{array}{lllllll}0.000 & -0.001 & -0.023 & -3.19 & -0.91 & 1592.05 & 143.46\end{array}$

$\begin{array}{llllllll}0.000 & -0.011 & -0.026 & -3.90 & -1.42 & 1592.71 & 150.86\end{array}$

$\begin{array}{lllllll}0.000 & -0.013 & -0.026 & -3.73 & -1.41 & 1594.57 & 157.08\end{array}$

$\begin{array}{lllllll}0.000 & -0.025 & -0.028 & -4.18 & -1.77 & 1594.91 & 164.81\end{array}$

153225

$0.23 \quad 0.00$

0.03

0.02

0.252

0.241

$\begin{array}{ll}0.22 & 0.00\end{array}$

0.04

0.02

0.242

0.000

$\begin{array}{lllll}-0.031 & -4.27 & -1.76 & 1596.58\end{array}$

171.21

155227

0.220 .00

0.05

0.02

0.243

$\begin{array}{lll}0.22 & 0.00 & 0.06\end{array}$

0.01

0.243

$\begin{array}{llllll}0.000 & -0.038 & -0.021 & -4.46 & -1.99 & 1596.61\end{array}$

179.25

$157 \quad 229$

$\begin{array}{ll}0.22 & 0.00\end{array}$

0.07

0.00

0.243

$\begin{array}{lllllll}0.000 & -0.063 & -0.016 & -5.19 & -2.43 & 1598.20 & 193.81\end{array}$

$\begin{array}{llllllll}0.000 & -0.063 & -0.016 & -5.21 & -2.47 & 1599.56 & 200.52\end{array}$

159231

$160 \quad 232$

$0.22 \quad 0.00$

$0.08-0.01$

0.244

$\begin{array}{lll}0.22 & 0.00\end{array}$

$0.09-0.01$

0.245

$\begin{array}{llllllll}0.000 & -0.076 & -0.009 & -6.07 & -3.12 & 1599.68 & 208.47\end{array}$

$\begin{array}{lllllll}0.000 & -0.088 & -0.011 & -6.56 & -3.36 & 1601.07 & 215.15\end{array}$

$\begin{array}{lllllll}0.000 & -0.088 & -0.011 & -7.16 & -3.95 & 1600.97 & 223.32\end{array}$

$162 \quad 234$

$\begin{array}{ll}0.22 & 0.00 \\ 0.22 & 0.00\end{array}$

$0.09-0.01$

0.245

$\begin{array}{lllllll}0.000 & -0.101 & -0.004 & -7.64 & -4.22 & 1602.23 & 230.12\end{array}$

163235

$0.21 \quad 0.00$

$0.10-0.02$

0.234

$0.000-0.103-0.0$

0.233

$0.000-0.105$

$0.000-0.106$

$0.000-0.107$

$\begin{array}{lllll}-7.39 & -4.18 & 1602.17 & 246.34\end{array}$

165237

$\begin{array}{ll}0.20 & 0.00\end{array}$

$0.10-0.03$

0.221

$0.000-0.120$

$\begin{array}{llll}-7.39 & -4.32 & 1601.30 & 255.27\end{array}$

0.222

$0.000-0.110$

$\begin{array}{llllll}0.018 & -7.02 & -4.05 & 1601.70 & 262.95\end{array}$

$\begin{array}{llllll}0.025 & -7.69 & -4.52 & 1601.02 & 271.70\end{array}$

$\begin{array}{lllll}0.028 & -6.82 & -4.14 & 1601.14 & 279.64\end{array}$
$1.14 \quad 47.06$

$1.49 \quad 53.81$

$1.90 \quad 58.87$

$1.92 \quad 65.50$

$\begin{array}{ll}1.87 & 70.33\end{array}$

$1.66 \quad 76.96$

$1.75 \quad 82.16$

$1.43 \quad 88.90$

$1.42 \quad 94.21$

\begin{tabular}{ll}
$1.07 \quad 101.14$ \\
\hline
\end{tabular}

$0.99 \quad 106.60$

$0.50 \quad 113.59$

$\begin{array}{ll}0.44 & 119.29\end{array}$

$\begin{array}{ll}-0.03 & 126.50\end{array}$

$\begin{array}{ll}-0.15 & 132.35\end{array}$

$-0.68 \quad 139.71$

$\begin{array}{ll}-0.81 & 145.74\end{array}$

$\begin{array}{ll}-1.29 & 153.34\end{array}$

$\begin{array}{ll}-1.26 & 159.73\end{array}$

$\begin{array}{ll}-1.58 & 167.67\end{array}$

$\begin{array}{ll}-1.49 & 174.32\end{array}$

$\begin{array}{ll}-1.84 & 182.42\end{array}$

$\begin{array}{ll}-1.77 & 189.24\end{array}$

$\begin{array}{ll}-2.19 & 197.44\end{array}$

$-2.21 \quad 204.36$

$\begin{array}{ll}-2.80 & 212.57\end{array}$

$\begin{array}{ll}-2.91 \quad 219.58 \\ -3.52 & 227.95\end{array}$

$\begin{array}{ll}-3.52 & 227.95\end{array}$

$\begin{array}{ll}-3.63 & 235.13\end{array}$

$\begin{array}{ll}-3.79 & 244.11\end{array}$

$\begin{array}{ll}-3.47 \quad 251.89 \\ -3.59 & 261.08\end{array}$

$\begin{array}{ll}-3.59 & 261.08\end{array}$

$\begin{array}{ll}-3.16 & 269.15\end{array}$

$\begin{array}{ll}-3.30 & 278.47\end{array}$

$-2.98 \quad 286.60$

\section{$Z=73$ (Ta)}

$\begin{array}{llllll}78 & 151 & -0.17 & 0.00 & 0.05\end{array}$

$\begin{array}{lllll}79 & 152 & -0.16 & 0.00\end{array}$

$\begin{array}{lllll}80 & 153 & -0.15 & 0.00\end{array}$

$\begin{array}{lllll}81 & 154 & -0.08 & 0.00\end{array}$

$\begin{array}{llll}82 & 155 & 0.02 & 0.00\end{array}$

0.05

$0.01-0.176$

$0.000-0.045$

$0.000-0.047-0.10$

$0.000-0.036$

$\begin{array}{lllll}0.000 & -3.71 & -1.89 & 1165.84 & -4.19\end{array}$

$\begin{array}{lll}0.000 & -0.009\end{array}$

$\begin{array}{llll}0.04 & 0.00 & -0.156\end{array}$

$\begin{array}{llll}0.01 & 0.00 & -0.084\end{array}$

$0.000 \quad 0.000$

$\begin{array}{ll}0.00 & 0.021\end{array}$

0.000

0.002

0.000

0.000

$\begin{array}{llllll}0.003 & -0.010 & -3.52 & -2.42 & 1239.85 & -29.77\end{array}$

$\begin{array}{rrrrrrr}83 & 156 & -0.07 & 0.00 & 0.00 & 0.00 & -0.073 \\ 84 & 157 & 0.08 & 0.00 & 0.00 & 0.01 & 0.085\end{array}$

$\begin{array}{lllllll}85 & 158 & 0.10 & 0.00 & -0.01 & -0.01 & 0.107\end{array}$

$\begin{array}{llllll}0.012 & -2.76 & -1.47 & 1249.39 & -31.24\end{array}$

$\begin{array}{lllll}86 & 159 & 0.12 & 0.00 & -0.01\end{array}$

$0.00 \quad 0.128$

0.000

0.018

$\begin{array}{lllllll}0.002 & -2.01 & -0.62 & 1260.53 & -34.30 & -34.45\end{array}$

0.000

0.020

$\begin{array}{lllllll}0.002 & -1.70 & -0.12 & 1270.05 & -35.75 & -35.88\end{array}$

$\begin{array}{llll}0.022 & -0.007 & -1.25\end{array}$

$\begin{array}{lll}0.42 & 1281.03 & -38.65\end{array}$

$\begin{array}{llll}0.000 & 0.012 & -0.009 & -0.95\end{array}$

$\begin{array}{lllll}0.000 & 0.001 & -0.011 & -0.71\end{array}$

$\begin{array}{llll}0.79 & 1290.23 & -39.78 & -39.78\end{array}$

$\begin{array}{llll}0.89 & 1301.17 & -42.65 & -42.54\end{array}$

$\begin{array}{lllll}1.19 & 1309.99 & -43.41 & -43.28\end{array}$

$\begin{array}{llll}1.41 & 1320.38 & -45.72 & -45.85\end{array}$

$\begin{array}{lllll}1.44 & 1329.05 & -46.32 & -46.10\end{array}$

$\begin{array}{llll}1.51 & 1339.15 & -48.35 & -48.35\end{array}$

$\begin{array}{llll}1.47 & 1347.47 & -48.60 & -48.39\end{array}$

$\begin{array}{llll}1.34 & 1357.35 & -50.41 & -50.29\end{array}$

$\begin{array}{llll}1.15 & 1365.43 & -50.42 & -50.14\end{array}$

$\begin{array}{lllll}0.000 & -0.004 & -0.004 & -1.16\end{array}$

$\begin{array}{llllllllll}0.000 & -0.014 & -0.006 & -1.52 & 1.00 & 1374.92 & -51.83 & -51.72 & 0.028\end{array}$

$-1.93-3.45$

$-2.37-7.90$

$-2.95-14.00$

$-3.69-18.22$

$-4.29-23.81$

$-3.68-26.17$

$\begin{array}{ll}-2.43 & -29.39\end{array}$

$\begin{array}{ll}-1.48 & -30.92\end{array}$

$\begin{array}{llll}0.021 & -0.63 & -34.05\end{array}$

$\begin{array}{llll}0.089 & -0.13 & -35.56\end{array}$

$\begin{array}{ll}0.41 & -38.52\end{array}$

$\begin{array}{ll}0.77 & -39.71\end{array}$

$\begin{array}{lll}0.88 & -42.63\end{array}$

$1.18-43.44$

$1.40-45.80$

$1.42-46.45$

$1.50-48.52$

$1.45-48.82$

$1.33-50.66$ 


\begin{tabular}{|c|c|c|c|c|c|c|c|c|c|c|c|c|c|c|c|c|}
\hline \multicolumn{17}{|c|}{$Z=73$ (Ta) } \\
\hline $99 \quad 172$ & 0.24 & 0.00 & 0.03 & 0.01 & 0.263 & 0.000 & -0.010 & -0.016 & -2.03 & 0.76 & 1382.65 & -51.50 & -51.33 & 0.028 & 0.76 & -51.86 \\
\hline 100173 & 0.24 & 0.00 & 0.04 & 0.01 & 0.264 & 0.000 & -0.022 & -0.019 & -2.33 & 0.59 & 1391.78 & -52.55 & -52.40 & 0.028 & 0.61 & -52.93 \\
\hline $101 \quad 174$ & 0.24 & 0.00 & 0.04 & 0.01 & 0.264 & 0.000 & -0.022 & -0.019 & -2.73 & 0.22 & 1399.27 & -51.97 & -51.74 & 0.028 & 0.22 & -52.39 \\
\hline 102175 & 0.24 & 0.00 & 0.05 & 0.00 & 0.264 & 0.000 & -0.036 & -0.012 & -3.03 & 0.01 & 1408.05 & -52.68 & -52.41 & 0.028 & 0.03 & -53.11 \\
\hline 103176 & 0.24 & 0.00 & 0.06 & 0.00 & 0.265 & 0.000 & -0.047 & -0.015 & -3.71 & -0.42 & 1415.23 & -51.79 & -51.37 & 0.031 & -0.40 & -52.25 \\
\hline 104177 & 0.24 & 0.00 & 0.06 & 0.00 & 0.265 & 0.000 & -0.047 & -0.015 & -3.93 & -0.63 & 1423.65 & -52.13 & -51.72 & 0.004 & -0.60 & -52.60 \\
\hline 105178 & 0.24 & 0.00 & 0.07 & -0.01 & 0.266 & 0.000 & -0.061 & -0.008 & -4.68 & -1.12 & 1430.53 & -50.95 & -50.51 & 0.015 & -1.08 & -51.43 \\
\hline 106179 & 0.23 & 0.00 & 0.07 & -0.01 & 0.254 & 0.000 & -0.063 & -0.007 & -4.82 & -1.40 & 1438.66 & -51.00 & -50.37 & 0.002 & -1.34 & -51.49 \\
\hline $107 \quad 180$ & 0.23 & 0.00 & 0.08 & -0.01 & 0.255 & 0.000 & -0.075 & -0.010 & -5.64 & -1.87 & 1445.18 & -49.46 & -48.94 & 0.002 & -1.80 & -49.95 \\
\hline 108181 & 0.23 & 0.00 & 0.08 & -0.02 & 0.255 & 0.000 & -0.076 & -0.000 & -5.70 & -1.95 & 1452.76 & -48.96 & -48.44 & 0.002 & -1.86 & -49.45 \\
\hline $109 \quad 182$ & 0.23 & 0.00 & 0.09 & -0.02 & 0.256 & 0.000 & -0.088 & -0.003 & -6.34 & -2.26 & 1458.79 & -46.92 & -46.43 & 0.002 & -2.14 & -47.40 \\
\hline 110183 & 0.22 & 0.00 & 0.09 & -0.03 & 0.244 & 0.000 & -0.091 & 0.008 & -6.14 & -2.17 & 1465.86 & -45.92 & -45.30 & 0.002 & -2.02 & -46.37 \\
\hline 111184 & 0.22 & 0.00 & 0.10 & -0.03 & 0.245 & 0.000 & -0.103 & 0.006 & -6.83 & -2.40 & 1471.49 & -43.48 & -42.84 & 0.026 & -2.22 & -43.90 \\
\hline 112185 & 0.22 & 0.00 & 0.10 & -0.04 & 0.244 & 0.000 & -0.104 & 0.015 & -6.78 & -2.33 & 1478.25 & -42.17 & -41.40 & 0.014 & -2.09 & -42.54 \\
\hline 113186 & 0.21 & 0.00 & 0.10 & -0.04 & 0.233 & 0.000 & -0.106 & 0.016 & -7.03 & -2.60 & 1483.61 & -39.46 & -38.61 & 0.060 & -2.36 & -39.83 \\
\hline 114187 & 0.20 & 0.00 & 0.10 & -0.04 & 0.221 & 0.000 & -0.107 & 0.018 & -6.82 & -2.54 & 1490.06 & -37.83 & & & -2.26 & -38.16 \\
\hline 115188 & 0.20 & 0.00 & 0.10 & -0.05 & 0.221 & 0.000 & -0.109 & 0.027 & -7.31 & -2.93 & 1495.24 & -34.94 & & & -2.61 & -35.21 \\
\hline 116189 & 0.18 & 0.00 & 0.09 & -0.04 & 0.198 & 0.000 & -0.098 & 0.022 & -6.39 & -2.76 & 1501.27 & -32.90 & & & -2.48 & -33.21 \\
\hline 117190 & 0.17 & 0.00 & 0.08 & -0.04 & 0.186 & 0.000 & -0.087 & 0.025 & -6.30 & -2.91 & 1505.91 & -29.47 & & & -2.67 & -29.80 \\
\hline 118191 & 0.15 & 0.00 & 0.07 & -0.03 & 0.164 & 0.000 & -0.077 & 0.018 & -5.82 & -3.02 & 1511.92 & -27.41 & & & -2.84 & -27.79 \\
\hline 119192 & 0.14 & 0.00 & 0.06 & -0.02 & 0.152 & 0.000 & -0.065 & 0.011 & -5.40 & -3.05 & 1516.15 & -23.56 & & & -2.93 & -24.00 \\
\hline 120193 & -0.17 & 0.00 & 0.03 & -0.02 & -0.177 & 0.000 & -0.022 & 0.024 & -5.15 & -3.09 & 1521.80 & -21.14 & & & -3.00 & -21.58 \\
\hline $121 \quad 194$ & -0.14 & 0.00 & 0.02 & -0.01 & -0.146 & 0.000 & -0.015 & 0.012 & -5.13 & -3.62 & 1526.25 & -17.53 & & & -3.59 & -18.00 \\
\hline 122195 & -0.11 & 0.00 & 0.02 & 0.00 & -0.115 & 0.000 & -0.018 & 0.002 & -5.10 & -4.03 & 1531.99 & -15.19 & & & -4.01 & -15.65 \\
\hline 123196 & -0.09 & 0.00 & 0.03 & 0.01 & -0.094 & 0.000 & -0.032 & -0.006 & -5.99 & -4.99 & 1536.60 & -11.73 & & & -4.95 & -12.13 \\
\hline 124197 & -0.07 & 0.00 & 0.03 & 0.01 & -0.073 & 0.000 & -0.033 & -0.007 & -6.44 & -5.43 & 1542.10 & -9.16 & & & -5.39 & -9.52 \\
\hline 125198 & -0.02 & 0.00 & 0.00 & 0.00 & -0.021 & 0.000 & 0.000 & 0.000 & -6.90 & -5.98 & 1546.03 & -5.02 & & & -5.99 & -5.39 \\
\hline 126199 & -0.01 & 0.00 & 0.00 & 0.00 & -0.011 & 0.000 & 0.000 & 0.000 & -7.28 & -6.29 & 1551.13 & -2.05 & & & -6.30 & -2.37 \\
\hline 127200 & -0.02 & 0.00 & 0.00 & 0.00 & -0.021 & 0.000 & 0.000 & 0.000 & -6.39 & -5.50 & 1553.46 & 3.69 & & & -5.50 & 3.42 \\
\hline $128 \quad 201$ & -0.01 & 0.00 & 0.00 & 0.00 & -0.011 & 0.000 & 0.000 & 0.000 & -5.24 & -4.40 & 1556.89 & 8.34 & & & -4.40 & 8.12 \\
\hline 129202 & 0.03 & 0.00 & -0.01 & 0.00 & 0.032 & 0.000 & 0.012 & 0.000 & -4.15 & -3.41 & 1558.78 & 14.52 & & & -3.41 & 14.36 \\
\hline 130203 & -0.02 & 0.00 & 0.00 & 0.00 & -0.021 & 0.000 & 0.000 & 0.000 & -2.80 & -2.18 & 1561.82 & 19.55 & & & -2.18 & 19.44 \\
\hline 131204 & 0.08 & 0.00 & -0.03 & -0.01 & 0.086 & 0.000 & 0.039 & 0.014 & -2.43 & -1.24 & 1563.51 & 25.93 & & & -1.17 & 25.95 \\
\hline 132205 & 0.09 & 0.00 & -0.03 & -0.01 & 0.096 & 0.000 & 0.040 & 0.014 & -1.61 & -0.40 & 1566.70 & 30.81 & & & -0.33 & 30.91 \\
\hline 133206 & 0.11 & 0.00 & -0.03 & -0.01 & 0.118 & 0.000 & 0.041 & 0.015 & -1.32 & 0.03 & 1568.67 & 36.91 & & & 0.10 & 37.08 \\
\hline 134207 & 0.11 & 0.00 & -0.03 & -0.01 & 0.118 & 0.000 & 0.041 & 0.015 & -0.72 & 0.61 & 1571.87 & 41.78 & & & 0.69 & 42.03 \\
\hline 135208 & 0.11 & 0.03 & -0.03 & 0.00 & 0.118 & -0.041 & 0.042 & 0.005 & -0.52 & 0.95 & 1573.70 & 48.02 & & & 1.01 & 48.33 \\
\hline 136209 & 0.14 & 0.00 & -0.03 & 0.00 & 0.150 & 0.000 & 0.045 & 0.006 & -0.11 & 1.49 & 1576.71 & 53.08 & & & 1.54 & 53.47 \\
\hline $137 \quad 210$ & 0.15 & 0.00 & -0.04 & 0.00 & 0.161 & 0.000 & 0.059 & 0.009 & -0.47 & 1.51 & 1578.63 & 59.23 & & & 1.60 & 59.74 \\
\hline 138211 & 0.16 & 0.00 & -0.04 & 0.00 & 0.172 & 0.000 & 0.060 & 0.009 & -0.36 & 1.76 & 1581.71 & 64.22 & & & 1.86 & 64.83 \\
\hline $139 \quad 212$ & 0.17 & 0.00 & -0.04 & 0.00 & 0.183 & 0.000 & 0.062 & 0.010 & -0.70 & 1.37 & 1583.83 & 70.18 & & & 1.46 & 70.88 \\
\hline $140 \quad 213$ & 0.17 & 0.00 & -0.03 & 0.00 & 0.183 & 0.000 & 0.049 & 0.008 & -0.37 & 1.50 & 1586.80 & 75.28 & & & 1.56 & 76.04 \\
\hline 141214 & 0.18 & 0.00 & -0.03 & 0.01 & 0.194 & 0.000 & 0.051 & -0.002 & -0.79 & 1.22 & 1588.59 & 81.56 & & & 1.28 & 82.42 \\
\hline $142 \quad 215$ & 0.19 & 0.00 & -0.03 & 0.01 & 0.205 & 0.000 & 0.053 & -0.001 & -0.91 & 1.20 & 1591.50 & 86.72 & & & 1.26 & 87.71 \\
\hline 143216 & 0.20 & 0.00 & -0.03 & 0.01 & 0.216 & 0.000 & 0.055 & -0.001 & -1.35 & 0.93 & 1593.08 & 93.21 & & & 0.98 & 94.30 \\
\hline $144 \quad 217$ & 0.22 & 0.00 & -0.02 & 0.01 & 0.238 & 0.000 & 0.046 & -0.002 & -1.46 & 0.92 & 1595.76 & 98.60 & & & 0.95 & 99.79 \\
\hline 145218 & 0.22 & 0.00 & -0.02 & 0.02 & 0.238 & 0.000 & 0.047 & -0.012 & -2.01 & 0.42 & 1597.37 & 105.07 & & & 0.49 & 106.41 \\
\hline 146219 & 0.22 & 0.00 & -0.01 & 0.02 & 0.238 & 0.000 & 0.035 & -0.015 & -1.92 & 0.38 & 1599.88 & 110.63 & & & 0.44 & 112.10 \\
\hline $147 \quad 220$ & 0.22 & 0.00 & 0.00 & 0.02 & 0.239 & 0.000 & 0.023 & -0.018 & -2.36 & -0.09 & 1601.26 & 117.32 & & & -0.03 & 118.91 \\
\hline $148 \quad 221$ & 0.22 & 0.00 & 0.01 & 0.02 & 0.240 & 0.000 & 0.011 & -0.021 & -2.50 & -0.25 & 1603.69 & 122.96 & & & -0.18 & 124.70 \\
\hline $149 \quad 222$ & 0.22 & 0.00 & 0.01 & 0.02 & 0.240 & 0.000 & 0.011 & -0.021 & -3.07 & -0.78 & 1604.93 & 129.79 & & & -0.71 & 131.66 \\
\hline $150 \quad 223$ & 0.22 & 0.00 & 0.02 & 0.02 & 0.240 & 0.000 & -0.001 & -0.023 & -3.25 & -0.96 & 1607.20 & 135.60 & & & -0.87 & 137.65 \\
\hline $151 \quad 224$ & 0.22 & 0.00 & 0.03 & 0.02 & 0.241 & 0.000 & -0.013 & -0.026 & -3.86 & -1.49 & 1608.26 & 142.61 & & & -1.37 & 144.84 \\
\hline $152 \quad 225$ & 0.22 & 0.00 & 0.03 & 0.02 & 0.241 & 0.000 & -0.013 & -0.026 & -3.82 & -1.49 & 1610.14 & 148.80 & & & -1.36 & 151.20 \\
\hline 153226 & 0.22 & 0.00 & 0.04 & 0.02 & 0.242 & 0.000 & -0.025 & -0.028 & -4.27 & -1.86 & 1610.85 & 156.15 & & & -1.68 & 158.75 \\
\hline $154 \quad 227$ & 0.22 & 0.00 & 0.05 & 0.01 & 0.242 & 0.000 & -0.038 & -0.021 & -4.24 & -1.76 & 1612.45 & 162.62 & & & -1.62 & 165.36 \\
\hline $155 \quad 228$ & 0.22 & 0.00 & 0.05 & 0.01 & 0.242 & 0.000 & -0.038 & -0.021 & -4.58 & -2.10 & 1612.97 & 170.18 & & & -1.97 & 173.07 \\
\hline
\end{tabular}




\section{$Z=73$ (Ta)}

$\begin{array}{llllllllllllll}156 & 229 & 0.22 & 0.00 & 0.06 & 0.01 & 0.243 & 0.000 & -0.050 & -0.023 & -4.72 & -2.12 & 1614.50 & 176.72 \\ 157 & 230 & 0.22 & 0.00 & 0.07 & 0.00 & 0.243 & 0.000 & -0.063 & -0.016 & -5.33 & -2.56 & 1614.94 & 184.35 \\ 158 & 231 & 0.21 & 0.00 & 0.07 & 0.00 & 0.232 & 0.000 & -0.065 & -0.015 & -5.26 & -2.57 & 1616.29 & 191.07 \\ 159 & 232 & 0.21 & 0.00 & 0.07 & 0.00 & 0.232 & 0.000 & -0.065 & -0.015 & -5.88 & -3.15 & 1616.70 & 198.73 \\ 160 & 233 & 0.21 & 0.00 & 0.08 & -0.01 & 0.233 & 0.000 & -0.078 & -0.008 & -6.34 & -3.43 & 1618.15 & 205.35 \\ 161 & 234 & 0.21 & 0.00 & 0.09 & -0.01 & 0.234 & 0.000 & -0.090 & -0.010 & -7.30 & -4.13 & 1618.52 & 213.06 \\ 162 & 235 & 0.21 & 0.00 & 0.09 & -0.02 & 0.233 & 0.000 & -0.091 & -0.001 & -7.45 & -4.34 & 1619.73 & 219.92 \\ 163 & 236 & 0.20 & 0.00 & 0.09 & -0.02 & 0.222 & 0.000 & -0.093 & 0.000 & -7.59 & -4.61 & 1619.49 & 228.23 \\ 164 & 237 & 0.20 & 0.00 & 0.10 & -0.03 & 0.222 & 0.000 & -0.106 & 0.008 & -7.70 & -4.51 & 1620.24 & 235.55 \\ 165 & 238 & 0.19 & 0.00 & 0.09 & -0.03 & 0.210 & 0.000 & -0.096 & 0.011 & -7.33 & -4.51 & 1619.58 & 244.28 \\ 166 & 239 & 0.19 & 0.00 & 0.09 & -0.03 & 0.210 & 0.000 & -0.096 & 0.011 & -6.89 & -4.16 & 1619.91 & 252.03 \\ 167 & 240 & 0.18 & 0.00 & 0.10 & -0.04 & 0.199 & 0.000 & -0.110 & 0.020 & -7.47 & -4.61 & 1619.55 & 260.45 \\ 168 & 241 & 0.18 & 0.00 & 0.10 & -0.04 & 0.199 & 0.000 & -0.110 & 0.020 & -7.13 & -4.38 & 1619.83 & 268.24 \\ 169 & 242 & 0.17 & 0.00 & 0.10 & -0.04 & 0.187 & 0.000 & -0.111 & 0.021 & -7.28 & -4.41 & 1618.91 & 277.24 \\ 170 & 243 & 0.17 & 0.00 & 0.10 & -0.05 & 0.187 & 0.000 & -0.112 & 0.031 & -7.16 & -4.41 & 1619.27 & 284.95\end{array}$

$\begin{array}{ll}-1.90 & 179.87\end{array}$

$-2.34 \quad 187.69$

$-2.33 \quad 194.61$

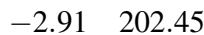

$-3.11 \quad 209.36$

$\begin{array}{ll}-3.71 & 217.36\end{array}$

$\begin{array}{ll}-3.88 & 224.47\end{array}$

$\begin{array}{ll}-4.13 & 232.99\end{array}$

$-3.82 \quad 240.76$

$-3.92 \quad 249.60$

$\begin{array}{ll}-3.55 & 257.59\end{array}$

$-3.71 \quad 266.54$
-3.46

$\begin{array}{ll}-3.46 & 274.59\end{array}$

$\begin{array}{ll}-3.46 \quad 283.85 \\ -3.21 & 292.05\end{array}$

$-3.21 \quad 292.05$

\begin{tabular}{|c|c|c|c|c|c|c|c|c|c|c|c|c|c|c|c|c|c|}
\hline \multicolumn{18}{|c|}{$Z=74(W)$} \\
\hline 80 & 154 & -0.13 & 0.00 & 0.04 & 0.01 & -0.135 & .000 & -0.039 & -0.003 & -5.01 & -3.50 & 1192.35 & -7.26 & & & -3.52 & -6.50 \\
\hline 81 & 155 & -0.08 & 0.00 & 0.02 & 0.00 & -0.084 & 0.000 & -0.021 & 0.002 & -5.15 & -4.37 & 1204.78 & -11 & & & & -10 \\
\hline 82 & 156 & -0.03 & 0.00 & 0.00 & 0.00 & -0.032 & 0.000 & 0.000 & 0.000 & -5.87 & -5.07 & 1219.01 & -17.78 & & & .07 & -17.15 \\
\hline 83 & 157 & -0.06 & 0.00 & 0.00 & 0.00 & -0.063 & 0.000 & 0.001 & 0.000 & & -4.34 & & -20.04 & & & & \\
\hline 84 & 158 & 0.08 & 0.00 & 0.00 & 0.00 & 0.085 & 0.000 & 0.003 & 0.000 & -4.15 & -3.03 & 1241.05 & -23.67 & & & .04 & -23.17 \\
\hline 85 & 159 & 0.10 & .00 & 0.00 & 0.01 & 0.107 & 0.000 & 0.004 & 0.010 & -3.26 & -2.01 & .59 & -25.15 & & & -2.01 & -24.72 \\
\hline 86 & 160 & 0.12 & 0.00 & -0.01 & 0.00 & 0.128 & 0.000 & 0.018 & 0.002 & -2.47 & -1.07 & .17 & -2 & & 9 & 1.08 & -2 \\
\hline 87 & 161 & 0.13 & 0.00 & 0.01 & 0.00 & 0.139 & 0.000 & 0.019 & 0.002 & & .52 & & -3 & & & & \\
\hline 88 & 162 & 0.14 & 0.00 & 0.01 & 0.00 & 0.150 & 0.000 & 0.020 & 0.002 & & 0.05 & & & & & 0.04 & \\
\hline 89 & 163 & 0.15 & 0.00 & 0.01 & 0.00 & 0.162 & 0.000 & -0.003 & -0.001 & & 0.48 & & & & & 0.46 & \\
\hline 90 & 164 & 0.16 & 00 & 0.01 & 0.00 & 173 & 00 & .001 & 0.001 & - & 86 & 57 & -3 & - & 2 & .85 & - \\
\hline 91 & 165 & 0.16 & .00 & 0.01 & 0.01 & 0.173 & 000 & -0.001 & -0.011 & -0.56 & 0.94 & 69 & -38.81 & -3 & & 0.93 & - \\
\hline 92 & 166 & 0.17 & 0.00 & 0.01 & 0.01 & & 00 & & -0 . & & 1.22 & & & & & 1.21 & \\
\hline 93 & 167 & 0.18 & .00 & 0.01 & 0.00 & 95 & 0.000 & 0.002 & -0.001 & & 1.37 & .14 & -42 & -4 & 19 & 1.36 & -4 \\
\hline 94 & 168 & 0.19 & 0.00 & 0.02 & 0.00 & 6 & 0.000 & -0.009 & -0.003 & & 1.44 & & -4 & -4 & & .44 & \\
\hline 95 & 169 & 0.20 & 0.00 & 0.02 & 0.00 & 0.217 & 000 & -0.007 & -0.004 & - & 1.46 & .07 & -4 & -6 & 3 & .45 & - \\
\hline 96 & 170 & 0.20 & 0.00 & 0.02 & 0.00 & 0.217 & 0.000 & -0.007 & -0.004 & -0 & 1.44 & & -4 & -4 & & 1.44 & - \\
\hline 97 & 171 & 0.21 & 0.00 & 0.02 & 0.00 & 29 & 0.000 & 05 & -0 . & & 1.32 & & -4 & & & 1.31 & - \\
\hline 98 & 172 & 0.22 & 0.00 & 0.02 & 0.01 & 0.240 & 0.000 & -0.002 & -0.013 & & 1.20 & .36 & -48.99 & -4 & 88 & 1.21 & -49.23 \\
\hline 99 & 173 & 0.22 & 0.00 & 0.03 & 0.01 & 0.241 & 0.000 & -0.014 & -0.016 & -1 & 1.00 & 12 & -48.68 & -4 & 8 & 1.00 & -48.97 \\
\hline 00 & 174 & 0.22 & 0.00 & 0.03 & 0.01 & 0.241 & .000 & -0.014 & -0.016 & -1.54 & 0.87 & 5.69 & -50.17 & -5 & 028 & 0.89 & -50.49 \\
\hline 01 & 175 & 0.23 & 0.00 & 0.04 & 0.01 & 3 & 000 & -0.024 & -0.019 & -2 & 0.56 & & 60 & -2 & & 0.57 & - \\
\hline 102 & 176 & 0.23 & 0.00 & 0.05 & 0.00 & & 0.000 & -0.038 & -0.012 & & .36 & & 78 & & & 0.38 & -51.15 \\
\hline 03 & 177 & 0.23 & 0.00 & 0.05 & 0.00 & 3 & 0.000 & -0.038 & -0.012 & -2 & -0.04 & .65 & -49.92 & -4 & 28 & .02 & -50.32 \\
\hline 104 & 178 & 0.23 & 0.00 & 0.06 & 0.00 & 0.254 & 0.000 & -0.049 & -0.014 & -3.37 & -0.31 & .60 & -50.80 & -50.42 & 0.015 & -0.27 & -51.21 \\
\hline 105 & 179 & 0.23 & 0.00 & 0.06 & .00 & 254 & 000 & -0.049 & -0.014 & -3.86 & -0.77 & 51 & -4 & -4 & 16 & .73 & -50.09 \\
\hline 106 & 180 & 0.22 & 0.00 & 0.07 & -0.01 & & 000 & 65 & -0.007 & & 11 & & -5 & & & 5 & - \\
\hline 107 & 181 & 0.22 & 0.00 & 0.07 & 0.01 & & 0.000 & -0.065 & -0.007 & & 56 & & .72 & -2 & & & -49.19 \\
\hline 108 & 182 & 0.21 & 0.00 & 0.07 & -0.01 & & 0.000 & -0.066 & -0.006 & & & & -48.73 & & 1 & -1.60 & -49.20 \\
\hline 109 & 183 & 0.22 & 0.00 & 0.08 & -0.02 & 3 & 0.000 & -0.078 & 0.001 & -5 & -1.89 & 80 & -46.65 & -4 & 01 & -1.79 & -47.11 \\
\hline 10 & 184 & 21 & 0.00 & 0.09 & -0.03 & 32 & 00 & 93 & 09 & - & 38 & 42 & - & & & 72 & - \\
\hline 111 & 185 & 0.20 & 0.00 & 0.09 & -0.03 & 221 & 0.000 & -0.094 & 0.010 & - & -2.20 & 19 & -43.89 & -4 & 01 & -2.04 & -44.31 \\
\hline 112 & 186 & 0.20 & 0.00 & 0.09 & -0.04 & 0.221 & 0.000 & -0.095 & 0.020 & & -2.21 & & -43.12 & -4 & 02 & -1.99 & -43.49 \\
\hline 13 & 187 & 0.20 & 0.00 & 0.10 & -0.04 & & 0.000 & -0.107 & 0.018 & & -2 & & -40.59 & -3 & 02 & -2.37 & -40.94 \\
\hline 114 & 188 & 0.19 & 0.00 & 0.10 & -0.04 & 0 & 0.000 & -0.109 & 0.019 & -6 & -2.55 & 93 & -39.41 & -3 & 03 & 27 & -39.73 \\
\hline 10 & 89 & & 0 & & -0.05 & & & & & & & & & & & & \\
\hline 116 & 190 & 0.17 & 0.00 & 0.09 & -0.04 & 87 & 0.000 & -0.099 & 0.023 & -6.54 & -2.78 & .64 & -34.99 & -3 & 0.165 & -2.50 & -35.30 \\
\hline 117 & 191 & 0.15 & 0.00 & 0.07 & -0.03 & 0.164 & 0.000 & -0.077 & 0.018 & & -3.28 & 5.68 & -31.95 & & & -3.12 & -32.37 \\
\hline 118 & 192 & 0.15 & 0.00 & 0.07 & -0.03 & & 0.000 & -0.077 & 0.018 & & & & -30.43 & & & -3.30 & -30.82 \\
\hline 119 & 193 & 0.14 & 0.00 & 0.07 & -0.03 & 0.153 & 0.000 & -0.078 & 0.019 & -6.34 & -3.62 & 1526.61 & -26.74 & & & -3.44 & -27.12 \\
\hline 20 & 194 & -0.15 & 0.00 & 0.02 & -0.02 & -0.156 & 0.000 & -0.013 & 0.022 & -5.26 & -3.55 & 1532.59 & -24.65 & & & -3.49 & -25.13 \\
\hline
\end{tabular}


$Z=74(W)$

$\begin{array}{llll}121 & 195 & -0.12 & 0.00\end{array}$

$\begin{array}{llll}122 & 196 & -0.11 & 0.00\end{array}$

$\begin{array}{llll}123 & 197 & -0.08 & 0.00\end{array}$

$\begin{array}{llll}124 & 198 & -0.06 & 0.00\end{array}$

$\begin{array}{lll}0.02 & -0.01 & -0.125\end{array}$

$\begin{array}{llll}0.02 & 0.00 & -0.115\end{array}$

$\begin{array}{llll}0.03 & 0.01 & -0.084\end{array}$

$\begin{array}{llll}0.03 & 0.01 & -0.063\end{array}$

$\begin{array}{llll}125 & 199 & 0.00 & 0.00\end{array}$

0.00

$0.00 \quad 0.000$

$\begin{array}{lllll}126 & 200 & 0.00 & 0.00 & 0.00\end{array}$

$\begin{array}{lllll}127 & 201 & -0.02 & 0.00 & -0.01\end{array}$

$\begin{array}{lllll}128 & 202 & 0.00 & 0.00 & 0.00\end{array}$

$0.00 \quad 0.000$

$0.00-0.021$

$0.00 \quad 0.000$

$\begin{array}{lllll}129 & 203 & -0.01 & 0.00 & 0.00\end{array}$

$0.00-0.011$

0.00

$0.00 \quad 0.000$

131205

132206

$0.00 \quad 0.00$

0.00

0.021

$\begin{array}{lllll}0.07 & 0.00 & -0.02 & -0.01\end{array}$

0.075

$\begin{array}{lllll}0.10 & 0.00 & -0.03 & -0.01\end{array}$

0.107

134208

$\begin{array}{lllll}0.11 & 0.00 & -0.03 & -0.01\end{array}$

$\begin{array}{lllll}0.11 & 0.04 & -0.03 & 0.00\end{array}$

0.118

135209

136210

$137 \quad 211$

$\begin{array}{lll}0.11 & 0.01 & -0.03\end{array}$

0.00

0.118

$\begin{array}{llll}0.14 & 0.00 & -0.04\end{array}$

$\begin{array}{lll}0.15 & 0.00 & -0.03\end{array}$

$-0.01$

0.118

\subsection{5}

0.00

0.161

139213

140214

$\begin{array}{llll}0.16 & 0.00 & -0.04\end{array}$

0.00

0.172

$\begin{array}{llll}0.17 & 0.00 & -0.03\end{array}$

0.00

0.183

141215

142216

$\begin{array}{ll}143 & 217\end{array}$

144218

145219

$\begin{array}{llll}0.18 & 0.00 & -0.03\end{array}$

$\begin{array}{llll}0.18 & 0.00 & -0.03\end{array}$

0.00

0.194

$\begin{array}{llll}0.19 & 0.00 & -0.03\end{array}$

0.194

$\begin{array}{llll}0.20 & 0.00 & -0.02\end{array}$

0.0

$\begin{array}{llll}0.22 & 0.00 & -0.02\end{array}$

0.02

0.205

$\begin{array}{llll}0.22 & 0.00 & -0.01\end{array}$

0.02

$\begin{array}{ll}147 & 221\end{array}$

$\begin{array}{llll}0.22 & 0.00 & -0.01\end{array}$

0.02

$\begin{array}{lll}0.22 & 0.00 & 0.00\end{array}$

0.02

$\begin{array}{ll}0.22 & 0.00\end{array}$

0.01

0.02

$150 \quad 224$

$0.22 \quad 0.00$

0.02

0.02

151225

$0.22 \quad 0.00$

0.02

0.02

0.238

0.238

0.238

0.239

0.240

0.240

$152 \quad 226$

$\begin{array}{lll}153 & 227\end{array}$

154228

$155 \quad 229$

0.04

0.02

0.240

$0.22 \quad 0.00$

$0.22 \quad 0.00$

0.05

0.01

0.241

0.241

$0.22 \quad 0.00$

0.05

0.01

0.242

156230

$0.22 \quad 0.00$
0.21

0.06

0.00

0.242

0.243

$157 \quad 23$

$\begin{array}{ll}0.21 & 0.00\end{array}$

0.06

0.00

0.231

159233

0.00

160234

$0.20 \quad 0.00$

$0.08-0.01$

0.232

0.221

0.221

$0.20 \quad 0.00$

$\begin{array}{lll}0.08 & -0.01\end{array}$

0.221

$162 \quad 236$

$0.20 \quad 0.00$

$\begin{array}{lll}0.09 & -0.02\end{array}$

0.222

$0.19 \quad 0.00$

$\begin{array}{ll}0.09 & -0.02\end{array}$

0.210

164238

$\begin{array}{ll}0.19 & 0.00\end{array}$

$\begin{array}{ll}0.09 & -0.02\end{array}$

0.210

165239

$\begin{array}{ll}0.19 & 0.00\end{array}$

$\begin{array}{lll}0.09 & -0.03\end{array}$

0.210

166240

$\begin{array}{ll}0.18 & 0.00\end{array}$

$\begin{array}{lll}0.09 & -0.03\end{array}$

0.199

$\begin{array}{ll}167 & 241\end{array}$

$0.18 \quad 0.00$

$\begin{array}{lll}0.10 & -0.04\end{array}$

0.199

0.187

169243

$\begin{array}{ll}0.17 & 0.00\end{array}$

$\begin{array}{lll}0.09 & -0.04\end{array}$

0.187

$170 \quad 244$

$\begin{array}{ll}0.17 & 0.00\end{array}$

$\begin{array}{lll}0.10 & -0.05\end{array}$

0.187

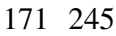

$0.14 \quad 0.00$

$0.09-0.04$

0.153

$172 \quad 246$

$\begin{array}{ll}0.14 & 0.00\end{array}$

$\begin{array}{lll}0.09 & -0.04\end{array}$

0.153

$173 \quad 247$ $\begin{array}{lllllll}0.000 & -0.017 & 0.012 & -5.49 & -4.25 & 1537.25 & -21.24\end{array}$

$\begin{array}{lllllll}0.000 & -0.018 & 0.002 & -5.71 & -4.64 & 1543.40 & -19.32\end{array}$

$\begin{array}{llllllll}0.000 & -0.032 & -0.007 & -6.62 & -5.64 & 1548.10 & -15.94\end{array}$

$\begin{array}{lllllll}0.000 & -0.033 & -0.007 & -7.12 & -6.11 & 1554.05 & -13.82\end{array}$

$\begin{array}{lllllll}0.000 & 0.000 & 0.000 & -7.73 & -6.75 & 1558.11 & -9.81\end{array}$

$\begin{array}{lllllll}0.000 & 0.000 & 0.000 & -8.12 & -7.08 & 1563.65 & -7.28\end{array}$

$\begin{array}{lllllll}0.000 & 0.012 & -0.000 & -7.20 & -6.22 & 1565.96 & -1.52\end{array}$

$\begin{array}{lllllll}0.000 & 0.000 & 0.000 & -6.06 & -5.15 & 1569.83 & 2.68\end{array}$

$\begin{array}{lllllll}0.000 & 0.000 & 0.000 & -4.86 & -4.11 & 1571.72 & 8.87\end{array}$

$\begin{array}{lllllll}0.000 & 0.000 & 0.000 & -3.59 & -2.86 & 1575.15 & 13.51\end{array}$

$\begin{array}{lllllll}0.000 & 0.000 & 0.000 & -2.50 & -1.80 & 1576.77 & 19.96\end{array}$

$\begin{array}{lllllll}0.000 & 0.026 & 0.012 & -1.80 & -0.83 & 1580.23 & 24.57\end{array}$

$\begin{array}{lllllll}0.000 & 0.041 & 0.014 & -1.61 & -0.33 & 1582.16 & 30.71\end{array}$

$\begin{array}{lllllll}0.000 & 0.041 & 0.015 & -1.03 & 0.31 & 1585.71 & 35.23\end{array}$

$-0.055$

0.042

$0.006-0.96$

$0.65 \quad 1587.57$

0.042

$\begin{array}{llll}0.005 & -0.04 & 1.24 & 1590.94\end{array}$

0.000

0.057

$0.018-0.52$

$1.38 \quad 1592.78$

$0.000 \quad 0.046$

$0.007-0.02$

$\begin{array}{ll}1.71 & 1596.17\end{array}$

0.000

0.060

$0.009-0.53$

$1.61 \quad 1598.03$

$1.56 \quad 1601.57$

0.000

0.051

$0.008-0.28$

$\begin{array}{ll}1.33 & 1603.34\end{array}$

0.000

$1.39 \quad 1606.56$

0.000

$\begin{array}{lll}0.051 & -0.002 & -0.60\end{array}$

$\begin{array}{ll}1.09 & 1608.19\end{array}$

0.000

$0.01-1$.

$1.13 \quad 1611.21$

$0.66 \quad 1612.82$

0.000

$\begin{array}{llll}0.047 & -0.012 & -1.73\end{array}$

$\begin{array}{ll}0.62 & 1615.71\end{array}$

0.000

$\begin{array}{lll}0.035 & -0.015 & -1.65\end{array}$

$\begin{array}{ll}0.16 & 1617.10\end{array}$

$\begin{array}{llllll}0.000 & 0.023 & -0.018 & -2.19 & 0.03 & 1619.88\end{array}$

$\begin{array}{llllll}0.000 & 0.011 & -0.021 & -2.72 & -0.48 & 1621.13\end{array}$

$\begin{array}{llllll}0.000 & -0.001 & -0.023 & -2.90 & -0.66 & 1623.76\end{array}$

$\begin{array}{llllll}0.000 & -0.001 & -0.023 & -3.39 & -1.13 & 1624.78\end{array}$

$\begin{array}{llllll}0.000 & -0.013 & -0.026 & -3.45 & -1.16 & 1627.07\end{array}$

$\begin{array}{llllll}0.000 & -0.026 & -0.019 & -3.76 & -1.43 & 1627.70\end{array}$

$\begin{array}{llllll}0.000 & -0.038 & -0.021 & -3.87 & -1.44 & 1629.79\end{array}$

$\begin{array}{lllllll}0.000 & -0.038 & -0.021 & -4.20 & -1.76 & 1630.29\end{array}$

$\begin{array}{llllll}0.000 & -0.052 & -0.014 & -4.25 & -1.71 & 1632.13\end{array}$

$\begin{array}{llllll}0.000 & -0.053 & -0.013 & -4.60 & -2.11 & 1632.54\end{array}$

$\begin{array}{lllllll}0.000 & -0.065 & -0.015 & -4.96 & -2.30 & 1634.43\end{array}$

$\begin{array}{llllll}0.000 & -0.067 & -0.015 & -5.52 & -2.92 & 1634.90\end{array}$

$\begin{array}{llllll}0.000 & -0.080 & -0.007 & -6.04 & -3.25 & 1636.76\end{array}$

$\begin{array}{llllll}0.000 & -0.080 & -0.007 & -6.66 & -3.86 & 1637.04\end{array}$

$0.000-0.093$

$0.000-0.094$

$0.000-0.094$

$0.000-0.096$

$\begin{array}{llll}0.000 & -7.24 & -4.22 & 1638.76\end{array}$

$\begin{array}{llll}0.001 & -7.40 & -4.48 & 1638.54\end{array}$

$\begin{array}{lllll}0.001 & -7.11 & -4.21 & 1639.45 & 223.63\end{array}$

$0.000-0.097$

$0.000-0.110$

$\begin{array}{lllll}0.011 & -7.29 & -4.47 & 1639.06 & 232.09\end{array}$

$\begin{array}{lll}0.000 & -0.099\end{array}$

$\begin{array}{lllll}0.012 & -6.84 & -4.17 & 1639.79 & 239.43\end{array}$

$0.000-0.112$

$0.000-0.112$

$0.000-0.102$

$0.000-0.102$

$0.000-0.091$ $\begin{array}{llllll}0.023 & -6.79 & -4.12 & 1639.81 & 255.55\end{array}$

$\begin{array}{llllll}0.031 & -7.62 & -4.71 & 1639.45 & 263.99\end{array}$

$\begin{array}{llllll}0.031 & -7.31 & -4.49 & 1639.93 & 271.58\end{array}$

$\begin{array}{lllll}0.026 & -7.03 & -4.68 & 1639.03 & 280.55\end{array}$

$\begin{array}{llllll}0.026 & -7.03 & -4.72 & 1639.62 & 288.02\end{array}$

$\begin{array}{lllll}0.028 & -7.09 & -5.10 & 1638.76 & 296.96\end{array}$

$-4.22-21.74$

$-4.62-19.80$

$-5.61-16.38$

$-6.07-14.22$

$-6.75-10.22$

$-7.08-7.65$

$-6.22-1.85$

$\begin{array}{ll}-5.15 & 2.40\end{array}$ 


\begin{tabular}{|c|c|c|c|c|c|c|c|c|c|c|c|c|c|c|c|c|c|}
\hline \multicolumn{18}{|c|}{$Z=75(\mathrm{Re})$} \\
\hline 84 & 159 & 0.06 & 0.00 & 0.00 & 0.01 & 0.064 & 0.000 & 0.002 & -0.010 & -5.08 & -4.04 & 1239.87 & -15.20 & & & -4.05 & -14.57 \\
\hline 85 & 160 & 0.10 & 0.00 & 0.01 & -0.01 & 0.107 & 0.000 & -0.008 & 0.009 & -4.15 & -2.85 & 1249.78 & -17.04 & & & -2.87 & -16.49 \\
\hline 86 & 161 & 0.11 & 0.00 & 0.00 & -0.01 & 0.118 & 0.000 & 0.005 & 0.010 & -3.23 & -1.90 & 1261.41 & -20.60 & -20.88 & 0.209 & -1.91 & -20.11 \\
\hline 87 & 162 & 0.12 & 0.00 & 0.00 & -0.01 & 0.129 & 0.000 & 0.006 & 0.010 & -2.67 & -1.26 & 1271.38 & -22.51 & & & -1.27 & -22.08 \\
\hline 88 & 163 & 0.14 & 0.00 & 0.00 & 0.00 & 0.150 & 0.000 & 0.008 & 0.001 & -2.14 & -0.61 & 1282.84 & -25.89 & -26.01 & 0.020 & -0.63 & -25.54 \\
\hline 89 & 164 & 0.14 & 0.00 & 0.01 & 0.00 & 0.151 & 0.000 & -0.004 & -0.001 & -1.69 & -0.15 & 1292.53 & -27.51 & & & -0.17 & -27.22 \\
\hline 90 & 165 & 0.15 & 0.00 & 0.01 & 0.00 & 0.162 & 0.000 & -0.003 & -0.001 & -1.33 & 0.29 & 1303.73 & -30.64 & -30.66 & 0.028 & 0.27 & -30.40 \\
\hline 91 & 166 & 0.16 & 0.00 & 0.02 & 0.00 & 0.173 & 0.000 & -0.013 & -0.003 & -1.14 & 0.63 & 1313.09 & -31.93 & & & 0.60 & -31.76 \\
\hline 92 & 167 & 0.16 & 0.00 & 0.02 & 0.00 & 0.173 & 0.000 & -0.013 & -0.003 & -0.81 & 0.67 & 1324.23 & -35.00 & & & 0.65 & -34.88 \\
\hline 93 & 168 & 0.17 & 0.00 & 0.02 & 0.00 & 0.184 & 0.000 & -0.012 & -0.003 & -0.70 & 0.87 & 1333.30 & -35.99 & -35.79 & 0.031 & 0.85 & -35.94 \\
\hline 94 & 169 & 0.18 & 0.00 & 0.03 & 0.00 & 0.196 & 0.000 & -0.023 & -0.005 & -0.71 & 1.05 & 1343.86 & -38.49 & -38.39 & 0.028 & 1.03 & -38.47 \\
\hline 95 & 170 & 0.19 & 0.00 & 0.02 & 0.00 & 0.206 & 0.000 & -0.009 & -0.003 & -0.68 & 1.05 & 1352.71 & -39.26 & -38.92 & 0.026 & 1.03 & -39.31 \\
\hline 96 & 171 & 0.19 & 0.00 & 0.02 & 0.00 & 0.206 & 0.000 & -0.009 & -0.003 & -0.66 & 1.11 & 1362.96 & -41.44 & -41.25 & 0.028 & 1.10 & -41.53 \\
\hline 97 & 172 & 0.20 & 0.00 & 0.02 & 0.00 & 0.217 & 0.000 & -0.007 & -0.004 & -0.88 & 1.04 & 1371.47 & -41.88 & -41.52 & 0.054 & 1.02 & -42.02 \\
\hline 98 & 173 & 0.21 & 0.00 & 0.03 & 0.00 & 0.229 & 0.000 & -0.018 & -0.006 & -1.14 & 1.00 & 1381.42 & -43.76 & -43.55 & 0.028 & 0.98 & -43.94 \\
\hline 99 & 174 & 0.21 & 0.00 & 0.03 & 0.00 & 0.229 & 0.000 & -0.018 & -0.006 & -1.33 & 0.83 & 1389.63 & -43.90 & -43.67 & 0.028 & 0.82 & -44.12 \\
\hline 100 & 175 & 0.21 & 0.00 & 0.03 & 0.00 & 0.229 & 0.000 & -0.018 & -0.006 & -1.48 & 0.71 & 1399.25 & -45.44 & -4 & 0.028 & 0.71 & -2 \\
\hline 101 & 176 & 0.21 & 0.00 & 0.04 & 0.00 & 0.230 & 0.000 & -0.030 & -0.009 & -1.92 & 0.41 & 1407.22 & -45.34 & -45.06 & 0.028 & 0.40 & -45.64 \\
\hline 102 & 177 & 0.21 & 0.00 & 0.04 & 0.00 & 0.230 & 0.000 & -0.030 & -0.009 & -2.13 & 0.23 & 1416.51 & -46.56 & -46.27 & 0.028 & 0.23 & -46.89 \\
\hline 103 & 178 & 0.22 & 0.00 & 0.05 & 0.00 & 0.242 & 0.000 & -0.040 & -0.011 & -2.83 & -0.17 & 1424.20 & -46.18 & -45.65 & 0.028 & -0.17 & -46.54 \\
\hline 104 & 179 & 0.21 & 0.00 & 0.06 & -0.01 & 0.231 & 0.000 & -0.054 & -0.004 & -3.25 & -0.46 & 1433.23 & -47.14 & -46.59 & 0.024 & -0.44 & -47.51 \\
\hline 105 & 180 & 0.21 & 0.00 & 0.06 & -0.01 & 0.231 & 0.000 & -0.054 & -0.004 & -3.71 & -0.94 & & -46.47 & -4 & 0.021 & -0.92 & - \\
\hline 106 & 181 & 0.21 & 0.00 & 0.06 & -0.01 & 0.231 & 0.000 & -0.054 & -0.004 & -4.04 & -1.20 & 1449.27 & -47.04 & -46.51 & 0.013 & -1.17 & -47.46 \\
\hline 107 & 182 & 0.21 & 0.00 & 0.07 & -0.01 & 0.232 & 0.000 & -0.066 & -0.006 & -4.84 & -1.70 & 1456.35 & -46.05 & -45.45 & 0.102 & -1.66 & -46.49 \\
\hline 108 & 183 & 0.21 & 0.00 & 0.07 & -0.01 & 0.232 & 0.000 & -0.066 & -0.006 & -4.95 & -1.84 & 1464.51 & -46.13 & -45.81 & 0.008 & -1.78 & -46.58 \\
\hline 109 & 184 & 0.20 & 0.00 & 0.08 & -0.02 & 0.221 & 0.000 & -0.081 & 0.002 & -5.48 & -2.13 & 1471.04 & -44.59 & -44.23 & 0.004 & -2.06 & -45.04 \\
\hline 110 & 185 & 0.20 & 0.00 & 0.08 & -0.02 & 0.221 & 0.000 & -0.081 & 0.002 & -5.45 & -2.15 & 1478.73 & -44.21 & -43.82 & 0.001 & -2.06 & -44.65 \\
\hline 111 & 186 & 0.20 & 0.00 & 0.09 & -0.03 & 0.221 & 0.000 & -0.094 & 0.010 & -6.20 & -2.47 & 1484.96 & -42.38 & -41.93 & 0.001 & -2.34 & -42.80 \\
\hline 112 & 187 & 0.20 & 0.00 & 0.09 & -0.04 & 0.221 & 0.000 & -0.095 & 0.020 & -6.26 & -2.50 & 1492.33 & -41.67 & -41.22 & 0.001 & -2.31 & -42.05 \\
\hline 113 & 188 & 0.19 & 0.00 & 0.09 & -0.04 & 0.209 & 0.000 & -0.097 & 0.021 & -6.62 & -2.84 & 1498.26 & -39.53 & -39.02 & 0.001 & -2.65 & -39.91 \\
\hline 114 & 189 & 0.18 & 0.00 & 0.09 & -0.04 & 0.198 & 0.000 & -0.098 & 0.022 & -6.55 & -2.89 & 1505.33 & -38.52 & -37.98 & 0.008 & -2.68 & -38.89 \\
\hline 115 & 190 & 0.18 & 0.00 & 0.09 & -0.04 & 0.198 & 0.000 & -0.098 & 0.022 & -7.04 & -3.38 & 1511.10 & -36.22 & -35.57 & 0.149 & -3.17 & -36.59 \\
\hline 116 & 191 & 0.15 & 0.00 & 0.07 & -0.03 & 0.164 & 0.000 & -0.077 & 0.018 & -6.13 & -3.27 & 1517.68 & -34.73 & -34.35 & 0.010 & -3.12 & -35.17 \\
\hline 117 & 192 & 0.15 & 0.00 & 0.07 & -0.03 & 0.164 & 0.000 & -0.077 & 0.018 & -6.82 & -3.93 & 1523.32 & -32.30 & & & -3.78 & -32.74 \\
\hline 118 & 193 & 0.14 & 0.00 & 0.07 & -0.03 & 0.153 & 0.000 & -0.078 & 0.019 & -6.95 & -4.14 & 1529.93 & -30.84 & & & -3.98 & -31.25 \\
\hline 119 & 194 & 0.14 & 0.00 & 0.07 & -0.03 & 0.153 & 0.000 & -0.078 & 0.019 & -7.09 & -4.31 & 1534.78 & -27.62 & & & -4.15 & -28.02 \\
\hline 120 & 195 & 0.11 & 0.00 & 0.05 & -0.02 & 0.119 & 0.000 & -0.056 & 0.014 & -6.05 & -4.15 & 1540.71 & -25.48 & & & -4.06 & -25.95 \\
\hline 121 & 196 & -0.12 & 0.00 & 0.02 & -0.01 & -0.125 & 0.000 & -0.017 & 0.012 & -6.18 & -4.92 & 1545.88 & -22.57 & & & -4.90 & -23.09 \\
\hline 122 & 197 & -0.10 & 0.00 & 0.02 & 0.00 & -0.105 & 0.000 & -0.019 & 0.002 & -6.37 & -5.37 & 1552.14 & -20.77 & & & -5.36 & -21.27 \\
\hline 123 & 198 & -0.08 & 0.00 & 0.03 & 0.01 & -0.084 & 0.000 & -0.032 & -0.007 & -7.35 & -6.35 & 1557.23 & -17.79 & & & -6.32 & -18.25 \\
\hline 124 & 199 & -0.07 & 0.00 & 0.03 & 0.01 & -0.073 & 0.000 & -0.033 & -0.007 & -7.89 & -6.83 & 1563.24 & -15.73 & & & -6.80 & -16.16 \\
\hline 125 & 200 & 0.02 & 0.00 & 0.00 & 0.00 & 0.021 & 0.000 & 0.000 & 0.000 & -8.29 & -7.25 & 1567.51 & -11.92 & & & -7.25 & -12.36 \\
\hline 126 & 201 & 0.01 & 0.00 & 0.00 & 0.00 & 0.011 & 0.000 & 0.000 & 0.000 & -8.62 & -7.55 & 1573.06 & -9.40 & & & -7.55 & -9.81 \\
\hline 127 & 202 & -0.02 & 0.00 & 0.00 & 0.00 & -0.021 & 0.000 & 0.000 & 0.000 & -7.71 & -6.77 & 1575.86 & -4.14 & & & -6.77 & -4.51 \\
\hline 128 & 203 & 0.02 & 0.00 & 0.00 & 0.00 & 0.021 & 0.000 & 0.000 & 0.000 & -6.59 & -5.61 & 1579.69 & 0.11 & & & -5.61 & -0.22 \\
\hline 129 & 204 & 0.03 & 0.00 & 0.00 & 0.00 & 0.032 & 0.000 & 0.000 & 0.000 & -5.47 & -4.58 & 1581.99 & 5.88 & & & -4.59 & 5.59 \\
\hline 130 & 205 & 0.03 & 0.00 & 0.00 & 0.00 & 0.032 & 0.000 & 0.000 & 0.000 & -4.20 & -3.35 & 1585.49 & 10.46 & & & -3.35 & 10.22 \\
\hline 131 & 206 & 0.06 & 0.00 & -0.01 & 0.00 & 0.064 & 0.000 & 0.014 & 0.001 & -3.36 & -2.45 & 1587.67 & 16.35 & & & -2.45 & 16.16 \\
\hline 132 & 207 & 0.07 & 0.00 & -0.02 & -0.01 & 0.075 & 0.000 & 0.026 & 0.012 & -2.47 & -1.48 & 1591.17 & 20.92 & & & -1.44 & 20.82 \\
\hline 133 & 208 & 0.09 & 0.00 & -0.03 & -0.01 & 0.096 & 0.000 & 0.040 & 0.014 & -2.12 & -0.89 & 1593.42 & 26.74 & & & -0.83 & 26.73 \\
\hline 134 & 209 & 0.10 & 0.02 & -0.03 & -0.01 & 0.107 & -0.028 & 0.041 & 0.015 & -1.57 & -0.21 & 1596.97 & 31.26 & & & -0.14 & 31.32 \\
\hline 135 & 210 & 0.11 & 0.03 & -0.03 & 0.00 & 0.118 & -0.041 & 0.042 & 0.005 & -1.34 & 0.16 & 1599.20 & 37.10 & & & 0.22 & 37.21 \\
\hline 136 & 211 & 0.11 & 0.00 & -0.03 & 0.00 & 0.118 & 0.000 & 0.042 & 0.005 & -0.56 & 0.73 & 1602.61 & 41.76 & & & 0.78 & 41.93 \\
\hline 137 & 212 & 0.13 & 0.00 & -0.03 & 0.00 & 0.139 & 0.000 & 0.044 & 0.006 & -0.51 & 1.01 & 1604.72 & 47.73 & & & 1.05 & 47.97 \\
\hline 138 & 213 & 0.14 & 0.00 & -0.03 & 0.00 & 0.150 & 0.000 & 0.045 & 0.006 & -0.30 & 1.33 & 1608.15 & 52.37 & & & 1.38 & 52.69 \\
\hline 139 & 214 & 0.15 & 0.00 & -0.04 & 0.00 & 0.161 & 0.000 & 0.059 & 0.009 & -0.70 & 1.31 & 1610.31 & 58.27 & & & 1.40 & 58.72 \\
\hline 140 & 215 & 0.16 & 0.00 & -0.03 & 0.00 & 0.172 & 0.000 & 0.048 & 0.007 & -0.43 & 1.49 & 1613.66 & 62.99 & & & 1.54 & 63.50 \\
\hline
\end{tabular}




\section{$Z=75(\operatorname{Re})$}

141216

$\begin{array}{llllll}142 & 217 & 0.18 & 0.00 & -0.03\end{array}$

$\begin{array}{lllllllll}0.00 & 0.183 & 0.000 & 0.049 & 0.008 & -0.77 & 1.12 & 1615.97 & 68.76\end{array}$

$\begin{array}{lllllllll}0.01 & 0.194 & 0.000 & 0.051 & -0.002 & -0.79 & 1.20 & 1619.19 & 73.61\end{array}$

$\begin{array}{lll}0.19 & 0.00 & -0.03\end{array}$

$\begin{array}{lll}0.01 & 0.205 & 0.000\end{array}$

$\begin{array}{lll}0.053 & -0.001 & -1.19\end{array}$

0.961621 .15

79.72

144219

$\begin{array}{lll}0.19 & 0.00 & -0.02\end{array}$

$\begin{array}{lll}0.01 & 0.205 & 0.000\end{array}$

$\begin{array}{lll}0.041 & -0.004 & -0.98\end{array}$

$\begin{array}{ll}0.98 & 1624.22\end{array}$

84.72

145220

$\begin{array}{llll}0.20 & 0.00 & -0.02\end{array}$

$0.216 \quad 0.000$

$\begin{array}{lll}0.043 & -0.003 & -1.47\end{array}$

$\begin{array}{ll}0.65 & 1626.06\end{array}$

90.95

14622

$\begin{array}{llll}0.22 & 0.00 & -0.01\end{array}$

$\begin{array}{llll}0.01 & 0.238 & 0.000\end{array}$

$\begin{array}{llll}0.034 & -0.005 & -1.64\end{array}$

$\begin{array}{ll}0.61 & 1628.98\end{array}$

96.10

222

$\begin{array}{llll}0.22 & 0.00 & -0.01\end{array}$

$\begin{array}{lll}0.02 & 0.238 & 0.000\end{array}$

$\begin{array}{lll}0.035 & -0.015 & -2.20\end{array}$

$\begin{array}{lll}0.09 & 1630.82\end{array}$

102.34

149224

$\begin{array}{lll}0.22 & 0.00 & 0.00\end{array}$

$0.239 \quad 0.000$

$\begin{array}{lllll}0.023 & -0.018 & -2.24 & -0.02 & 1633.60\end{array}$

107.63

$150 \quad 225$

$0.22 \quad 0.00$

0.02

0.01

0.2390 .000

$\begin{array}{lllll}0.010 & -0.011 & -2.65 & -0.46 & 1635.15\end{array}$

114.15

151226

$\begin{array}{lll}0.22 & 0.00 & 0.02\end{array}$

0.02

0.2400 .000

$\begin{array}{lllll}-0.002 & -0.013 & -2.77 & -0.59 & 1637.76\end{array}$

119.62

$152 \quad 227$

$\begin{array}{ll}0.22 & 0.00\end{array}$

0.03

0.02

126.22

$153 \quad 228$

$\begin{array}{ll}0.22 & 0.00\end{array}$

0.04

0.01

$\begin{array}{lll}0.241 & 0.000 & -0 \\ 0.241 & 0.000 & -0\end{array}$

0.013

$-0.026$

$-3.44$

$-1.17 \quad 1641.54$

131.98

154229

$\begin{array}{lll}0.22 & 0.00 & 0.04\end{array}$

$\begin{array}{llllllll}0.241 & 0.000 & -0.026 & -0.019 & -3.63 & -1.36 & 1644.55 & 145.10\end{array}$

155230

$0.22 \quad 0.00$

0.01

152.29

156231

$\begin{array}{ll}0.20 & 0.00\end{array}$

$0.05 \quad 0.01$

$\begin{array}{llllllll}0.220 & 0.000 & -0.042 & -0.020 & -3.94 & -1.72 & 1647.36 & 158.43\end{array}$

157232

$0.20 \quad 0.00$

0.00

$$
\begin{array}{llllllll}
0.220 & 0.000 & -0.055 & -0.013 & -4.58 & -2.20 & 1648.23 & 165.64
\end{array}
$$

159234

$\begin{array}{ll}0.20 & 0.00 \\ 0.20 & 0.00\end{array}$

$\begin{array}{ll}0.06 \quad 0.00 \\ 0.07 & -0.01\end{array}$

$\begin{array}{llllllll}0.220 & 0.000 & -0.055 & -0.013 & -4.67 & -2.32 & 1650.07 & 171.87\end{array}$

$\begin{array}{llllllll}0.220 & 0.000 & -0.068 & -0.005 & -5.57 & -3.01 & 1650.95 & 179.06\end{array}$

160235

$\begin{array}{ll}0.20 & 0.00 \\ 0.19 & 0.00\end{array}$

$0.07-0.01$

161236

$\begin{array}{lll}0.19 & 0.00\end{array}$

$0.08-0.01$

162237

$\begin{array}{lll}0.19 & 0.00\end{array}$

$0.08-0.01$

$0.19 \quad 0.00$

$0.09-0.02$

164239

$\begin{array}{ll}0.19 & 0.00\end{array}$

$0.09-0.03$

$\begin{array}{llllllll}0.210 & 0.000 & -0.082 & -0.006 & -6.89 & -4.19 & 1655.08 & 199.14\end{array}$

$\begin{array}{llllllll}0.210 & 0.000 & -0.094 & 0.001 & -7.58 & -4.57 & 1655.33 & 206.97\end{array}$

$\begin{array}{llllll}165 & 240 & 0.18 & 0.00 & 0.09 & -0.03\end{array}$

$\begin{array}{lll}0.210 & 0.000 & -0.096\end{array}$

$\begin{array}{llllll}0.011 & -7.36 & -4.44 & 1656.40 & 213.97\end{array}$

$\begin{array}{llll}0.199 & 0.000 & -0.097\end{array}$

$\begin{array}{llllll}0.012 & -7.47 & -4.67 & 1656.33 & 222.11\end{array}$

166241

$\begin{array}{ll}0.17 & 0.00\end{array}$

$0.09-0.03$

$\begin{array}{llll}0.187 & 0.000 & -0.098\end{array}$

$\begin{array}{llllll}0.013 & -7.07 & -4.43 & 1657.13 & 229.39\end{array}$

167242

$\begin{array}{lll}0.17 & 0.00\end{array}$

$0.09-0.04$

$\begin{array}{llll}0.187 & 0.000 & -0.099\end{array}$

$\begin{array}{llllll}0.023 & -7.41 & -4.60 & 1656.85 & 237.74\end{array}$

$\begin{array}{lll}0.187 & 0.000 & -0.099\end{array}$

$\begin{array}{lllll}0.023 & -7.12 & -4.38 & 1657.50 & 245.15\end{array}$

169244

$\begin{array}{lll}0.17 & 0.00 \\ 0.16 & 0.00\end{array}$

$0.09-0.04$

$\begin{array}{llll}0.176 & 0.000 & -0.101\end{array}$

$\begin{array}{llllll}0.024 & -7.32 & -4.68 & 1657.20 & 253.53\end{array}$

170245

$\begin{array}{ll}0.15 & 0.00\end{array}$

$0.09-0.04$

$\begin{array}{lll}0.164 & 0.000 & -0.101\end{array}$

$\begin{array}{llllll}0.025 & -7.11 & -4.59 & 1657.82 & 260.97\end{array}$

$\begin{array}{lll}0.153 & 0.000 & -0.091\end{array}$

$\begin{array}{llllll}0.027 & -7.21 & -5.03 & 1657.50 & 269.36\end{array}$

$172 \quad 247$

$\begin{array}{ll}0.14 & 0.00\end{array}$

$0.08-0.04$

$\begin{array}{llll}0.153 & 0.000 & -0.091\end{array}$

$\begin{array}{llllll}0.027 & -7.20 & -5.07 & 1658.10 & 276.84\end{array}$

$\begin{array}{llll}0.142 & 0.000 & -0.091\end{array}$

$\begin{array}{lllll}0.028 & -7.68 & -5.60 & 1657.73 & 285.28\end{array}$

$\begin{array}{lll}0.130 & 0.000 & -0.080\end{array}$

$\begin{array}{lllll}0.030 & -7.34 & -5.60 & 1658.14 & 292.94\end{array}$

174249

$\begin{array}{lll}0.13 & 0.00 \\ 0.12 & 0.00 & (1400\end{array}$

$0.08-0.04$

$\begin{array}{ll}0.12 & 0.00 \\ 0.12 & 0.00\end{array}$

$0.07-0.04$

$\begin{array}{llll}0.130 & 0.000 & -0.080\end{array}$

$\begin{array}{llllll}0.030 & -7.66 & -5.93 & 1657.43 & 301.73\end{array}$

\section{$Z=76(O s)$}

$\begin{array}{llll}83 & 159 & -0.05 & 0.00\end{array}$

$\begin{array}{lllll}84 & 160 & 0.06 & 0.00\end{array}$

$\begin{array}{llll}85 & 161 & 0.08 & 0.00\end{array}$

$\begin{array}{llll}86 & 162 & 0.10 & 0.00\end{array}$

$\begin{array}{llll}87 & 163 & 0.11 & 0.00\end{array}$

88164

89165

$\begin{array}{ll}0.12 & 0.00\end{array}$

0.140 .00

$\begin{array}{llll}91 & 167 & 0.15 & 0.00\end{array}$

$\begin{array}{llll}92 & 168 & 0.16 & 0.00\end{array}$

$\begin{array}{llllllll}0.064 & 0.000 & -0.010 & -0.001 & -5.96 & -4.87 & 1240.30 & -8.34\end{array}$

$\begin{array}{llllllllll}0.03 & 0.00 & 0.086 & 0.000 & -0.033 & -0.003 & -4.90 & -3.61 & 1250.21 & -10.19\end{array}$

$0.01-0.01$

$\begin{array}{lll}0.107 & 0.000 & -0.008\end{array}$

$\begin{array}{lllll}0.009 & -3.75 & -2.50 & 1262.21 & -14.11\end{array}$

$0.01-0.01$

$\begin{array}{lll}0.118 & 0.000 & -0.007\end{array}$

$0.009-3.08-1.76 \quad 1272.16-15.99$

$0.00 \quad 0.00$

$\begin{array}{lll}0.129 & 0.000 \quad 0.006\end{array}$

$0.000-2.38$

$0.01 \quad 0.00$$$
\begin{array}{llllllll}
0.140 & 0.000 & -0.005 & -0.001 & -1.93 & -0.51 & 1293.75 & -21.44
\end{array}
$$

$$
\begin{array}{llllllllll}
0.151 & 0.000 & -0.004 & -0.001 & -1.51 & -0.03 & 1305.41 & -25.03 & -25.44
\end{array}
$$

0.02

$\begin{array}{lllll}0.162 & 0.000 & -0.015 & -0.003 & -1.25\end{array}$

$\begin{array}{llll}0.37 & 1314.79 & -26.33 & -26.50\end{array}$

$\begin{array}{lllll}0.73 & 1326.11 & -29.59 & -29.99\end{array}$

$\begin{array}{llllll}0.173 & 0.000 & -0.013 & -0.003 & -0.96\end{array}$

$\begin{array}{lllll}0.67 & 1335.51 & -30.92 & -30.72\end{array}$

$\begin{array}{lllll}0.90 & 1346.52 & -33.85 & -33.93\end{array}$

$\begin{array}{lllll}1.06 & 1355.28 & -34.54 & -34.29\end{array}$

$\begin{array}{lllll}1.14 & 1366.01 & -37.20 & -37.24\end{array}$

$\begin{array}{lllll}1.16 & 1374.50 & -37.62 & -37.44\end{array}$

$\begin{array}{lllll}1.11 & 1384.94 & -39.99 & -40.00\end{array}$

$\begin{array}{llll}1.01 & 1393.14 & -40.12 & -40.10\end{array}$

$\begin{array}{llll}0.90 & 1403.24 & -42.15 & -42.10\end{array}$

$\begin{array}{lllll}0.68 & 1411.18 & -42.01 & -41.95\end{array}$

$\begin{array}{lllll}0.48 & 1420.97 & -43.74 & -43.55\end{array}$

$\begin{array}{llllll}0.16 & 1428.65 & -43.34 & -43.02 & 0.018\end{array}$
$99 \quad 175$

100176

101177

102178

$0.20 \quad 0.00$

$0.20 \quad 0.00$

0.03

0.04
0.00

$\begin{array}{llllll}0.219 & 0.000 & -0.031 & -0.008 & -1.63\end{array}$

$\begin{array}{llllll}0.219 & 0.000 & -0.031 & -0.008 & -1.99\end{array}$ $\begin{array}{ll}1.16 & 69.35\end{array}$

$\begin{array}{ll}1.26 & 74.31\end{array}$

$\begin{array}{ll}1.01 & 80.51\end{array}$

$1.01 \quad 85.60$

$0.68 \quad 91.92$

$0.62 \quad 97.17$

$0.14 \quad 103.57$

$\begin{array}{ll}0.04 & 108.99\end{array}$

$-0.46 \quad 115.57$

$-0.57 \quad 121.19$

$\begin{array}{ll}-1.05 & 127.99\end{array}$

$\begin{array}{ll}-1.04 & 133.94\end{array}$

$-1.35 \quad 141.10$
-1.28

$-1.28 \quad 147.31$

$\begin{array}{ll}-1.56 & 154.70\end{array}$

$-1.57 \quad 161.02$

$\begin{array}{ll}-2.05 & 168.40\end{array}$

$-2.16 \quad 174.81$

$\begin{array}{ll}-2.79 & 182.22\end{array}$

$\begin{array}{ll}-3.02 & 188.70\end{array}$

$\begin{array}{ll}-3.64 & 196.30\end{array}$

$\begin{array}{ll}-3.86 & 202.97\end{array}$

$-4.11 \quad 211.12$

$-3.88 \quad 218.42$

$-4.09 \quad 226.77$

$\begin{array}{ll}-3.81 & 234.29\end{array}$

$\begin{array}{ll}-3.84 & 243.00\end{array}$

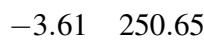

$\begin{array}{ll}-3.88 & 259.28\end{array}$

$\begin{array}{ll}-3.75 & 266.99\end{array}$

$-4.27 \quad 275.53$

$-4.29 \quad 283.26$

$\begin{array}{ll}-4.80 & 291.97\end{array}$

$-4.86 \quad 299.82$

$-5.19308 .86$ $\begin{array}{ll}-6.01 & -3.09\end{array}$

$-4.87 \quad-7.57$

$\begin{array}{ll}-3.62 & -9.48\end{array}$

$\begin{array}{ll}-2.51 & -13.48\end{array}$

$-1.78-15.44$

0.209

$-1.05-19.33$

$\begin{array}{ll}-0.52 & -21.02\end{array}$

$\begin{array}{llll}0.018 & -0.04 & -24.67\end{array}$

$\begin{array}{llll}0.073 & 0.35 & -26.04\end{array}$

$\begin{array}{lll}0.012 & 0.71 & -29.36\end{array}$

0.025

0.011

$\begin{array}{ll}0.66 & -30.74\end{array}$

$\begin{array}{ll}0.89 & -33.73\end{array}$

$1.04-34.48$

$1.13-37.18$

$1.14-37.66$

$1.10 \quad-40.07$

$1.00-40.25$

$\begin{array}{lll}0.90 & -42.31\end{array}$

$0.68-42.23$

$0.49-43.98$

$0.16-43.63$ 


\section{$Z=76(O s)$}

\begin{tabular}{|c|c|c|c|c|c|c|c|c|c|c|c|c|c|c|c|c|c|}
\hline 104 & 180 & 0.20 & 0.00 & 0.05 & 0.00 & 0.219 & 0.000 & -0.043 & -0.010 & -2.50 & -0.15 & 1438.17 & -44.79 & -44.36 & 0.020 & -0.13 & -45.10 \\
\hline 105 & 181 & 0.21 & 0.00 & 0.05 & 0.00 & 0.230 & 0.000 & -0.041 & -0.011 & -3.03 & -0.54 & 1445.54 & -44.09 & -43.55 & 0.032 & -0.52 & -44.44 \\
\hline 106 & 182 & 0.20 & 0.00 & 0.06 & -0.01 & 0.220 & 0.000 & -0.056 & -0.003 & -3.50 & -0.90 & 1454.74 & -45.22 & -44.61 & 0.022 & -0.86 & -45.58 \\
\hline 107 & 183 & 0.20 & 0.00 & 0.06 & -0.01 & 0.220 & 0.000 & -0.056 & -0.003 & -3.97 & -1.34 & 1461.82 & -44.23 & -43.66 & 0.050 & -1.31 & -44.62 \\
\hline 108 & 184 & 0.20 & 0.00 & 0.07 & -0.01 & 0.220 & 0.000 & -0.068 & -0.005 & -4.40 & -1.50 & 1470.47 & -44.81 & -44.26 & 0.001 & -1.45 & -45.19 \\
\hline 109 & 185 & 0.20 & 0.00 & 0.07 & -0.02 & 0.220 & 0.000 & -0.069 & 0.005 & -4.68 & -1.78 & 1477.04 & -43.30 & -42.81 & 0.001 & -1.72 & -43.71 \\
\hline 110 & 186 & 0.19 & 0.00 & 0.08 & -0.02 & 0.209 & 0.000 & -0.083 & 0.003 & -4.92 & -1.82 & 1485.21 & -43.40 & -43.00 & 0.001 & -1.72 & -43.80 \\
\hline 11 & 187 & 0.19 & 0.00 & 0.08 & -0.03 & 0.209 & 0.000 & -0.084 & 0.013 & -5.31 & -2.07 & 1491.42 & -41.55 & -41.22 & 0.001 & -1.96 & -41.94 \\
\hline 12 & 188 & 0.18 & 0.00 & 0.08 & -0.03 & 0.198 & 0.000 & -0.085 & 0.014 & -5.22 & -2.11 & 1499.26 & -41.31 & -41.14 & 0.001 & -1.98 & -41.70 \\
\hline 113 & 189 & 0.18 & 0.00 & 0.08 & -0.03 & 0.198 & 0.000 & -0.085 & 0.014 & -5.67 & -2.54 & 1505.33 & -39.31 & -38.99 & 0.001 & -2.41 & -39.72 \\
\hline 114 & 190 & 0.17 & 0.00 & 0.08 & -0.03 & 0.187 & 0.000 & -0.086 & 0.015 & -5.71 & -2.41 & 1512.67 & -38.58 & -38.71 & 0.001 & -2.26 & -38.98 \\
\hline 15 & 191 & 0.15 & 0.00 & 0.07 & -0.03 & 0.164 & 0.000 & -0.077 & 0.018 & -5.79 & -2.94 & 1518.52 & -36.36 & -36.39 & 0.001 & -2.81 & -36.79 \\
\hline 116 & 192 & 0.15 & 0.00 & 0.07 & -0.03 & 0.164 & 0.000 & -0.077 & 0.018 & -6.24 & -3.40 & 1526.12 & -35.89 & -35.88 & 0.003 & -3.25 & -36.31 \\
\hline 117 & 193 & 0.15 & 0.00 & 0.07 & -0.03 & 0.164 & 0.000 & -0.077 & 0.018 & -6.92 & -4.05 & 1531.80 & -33.49 & -33.39 & 0.003 & -3.91 & -33.92 \\
\hline 118 & 194 & 0.14 & 0.00 & 0.07 & -0.03 & 0.153 & 0.000 & -0.078 & 0.019 & -7.17 & -4.38 & 1538.96 & -32.58 & -32.43 & 0.003 & -4.22 & -32.99 \\
\hline 19 & 195 & 0.11 & 0.00 & 0.06 & -0.02 & 0.119 & 0.000 & -0.068 & 0.013 & -6.87 & -4.66 & 1543.97 & -29.53 & -29.69 & 0.500 & -4.55 & -29.98 \\
\hline 120 & 196 & 0.11 & 0.00 & 0.05 & -0.02 & 0.119 & 0.000 & -0.056 & 0.014 & -6.64 & -4.72 & 1550.57 & -28.05 & -28.28 & 0.040 & -4.63 & -28.51 \\
\hline 121 & 197 & -0.11 & 0.00 & 0.02 & -0.01 & -0.115 & 0.000 & -0.018 & 0.012 & -6.74 & -5.59 & 1555.88 & -25.29 & & & -5.57 & -25.81 \\
\hline 122 & 198 & -0.09 & 0.00 & 0.02 & 0.00 & -0.094 & 0.000 & -0.020 & 0.002 & -7.06 & -6.12 & 1562.65 & -23.99 & & & -6.11 & -24.50 \\
\hline 123 & 199 & -0.07 & 0.00 & 0.02 & 0.00 & -0.073 & 0.000 & -0.021 & 0.002 & -7.93 & -7.04 & 1567.73 & -20.99 & & & -7.03 & -21.49 \\
\hline 124 & 200 & -0.06 & 0.00 & 0.03 & 0.01 & -0.063 & 0.000 & -0.033 & -0.007 & -8.65 & -7.57 & 1574.20 & -19.40 & & & -7.53 & -19.85 \\
\hline 125 & 201 & 0.00 & 0.00 & 0.00 & 0.00 & 0.000 & 0.000 & 0.000 & 0.000 & -9.26 & -8.22 & 1578.74 & -15.87 & & & -8.22 & -16.33 \\
\hline 126 & 202 & 0.00 & 0.00 & 0.00 & 0.00 & 0.000 & 0.000 & 0.000 & 0.000 & -9.62 & -8.52 & 1584.73 & -13.78 & & & -8.53 & -14.22 \\
\hline 127 & 203 & -0.02 & 0.00 & 0.00 & 0.00 & -0.021 & 0.000 & 0.000 & 0.000 & -8.65 & -7.66 & 1587.49 & -8.47 & & & -7.66 & -8.88 \\
\hline 128 & 204 & 0.00 & 0.00 & 0.00 & 0.00 & 0.000 & 0.000 & 0.000 & 0.000 & -7.55 & -6.57 & 1591.80 & -4.71 & & & -6.57 & -5.08 \\
\hline 129 & 205 & -0.01 & 0.00 & 0.00 & 0.00 & -0.011 & 0.000 & 0.000 & 0.000 & -6.31 & -5.50 & 1594.11 & 1.06 & & & -5.51 & 0.72 \\
\hline 130 & 206 & 0.00 & 0.00 & 0.00 & 0.00 & 0.000 & 0.000 & 0.000 & 0.000 & -5.04 & -4.24 & 1597.98 & 5.25 & & & -4.24 & 4.96 \\
\hline 131 & 207 & 0.02 & 0.00 & 0.00 & 0.00 & 0.021 & 0.000 & 0.000 & 0.000 & -3.96 & -3.16 & 1600.02 & 11.29 & & & -3.16 & 11.04 \\
\hline 132 & 208 & 0.05 & 0.00 & -0.01 & 0.00 & 0.053 & 0.000 & 0.013 & 0.001 & -2.92 & -2.07 & 1603.81 & 15.56 & & & -2.07 & 15.37 \\
\hline 133 & 209 & 0.08 & 0.00 & -0.02 & -0.01 & 0.086 & 0.000 & 0.027 & 0.012 & -2.41 & -1.37 & 1605.99 & 21.46 & & & -1.34 & 21.35 \\
\hline 134 & 210 & 0.09 & 0.02 & -0.02 & -0.01 & 0.096 & -0.028 & 0.027 & 0.013 & -1.79 & -0.64 & 1609.89 & 25.62 & & & -0.60 & 25.58 \\
\hline 35 & 211 & 0.11 & 0.04 & -0.02 & 0.00 & 0.118 & -0.055 & 0.030 & 0.004 & -1.71 & -0.21 & 1612.10 & 31.49 & & & -0.17 & 31.51 \\
\hline 136 & 212 & 0.11 & 0.03 & -0.02 & 0.00 & 0.118 & -0.041 & 0.030 & 0.004 & -0.94 & 0.39 & 1615.89 & 35.77 & & & 0.42 & 35.85 \\
\hline 137 & 213 & 0.11 & 0.00 & -0.03 & 0.00 & 0.118 & 0.000 & 0.042 & 0.005 & -0.58 & 0.68 & 1618.00 & 41.73 & & & 0.73 & 41.89 \\
\hline 138 & 214 & 0.13 & 0.00 & -0.02 & 0.00 & 0.139 & 0.000 & 0.032 & 0.004 & -0.16 & 1.15 & 1621.68 & 46.12 & & & 1.18 & 46.33 \\
\hline 139 & 215 & 0.14 & 0.00 & -0.03 & 0.00 & 0.150 & 0.000 & 0.045 & 0.006 & -0.40 & 1.22 & 1623.79 & 52.08 & & & 1.27 & 52.39 \\
\hline 140 & 216 & 0.15 & 0.00 & -0.03 & 0.00 & 0.161 & 0.000 & 0.046 & 0.007 & -0.28 & 1.46 & 1627.47 & 56.47 & & & 1.52 & 56.87 \\
\hline 141 & 217 & 0.16 & 0.00 & -0.03 & 0.00 & 0.172 & 0.000 & 0.048 & 0.007 & -0.57 & 1.16 & 1629.74 & 62.28 & & & 1.22 & 62.76 \\
\hline 142 & 218 & 0.17 & 0.00 & -0.03 & 0.00 & 0.183 & 0.000 & 0.049 & 0.008 & -0.56 & 1.27 & 1633.32 & 66.77 & & & 1.34 & 67.35 \\
\hline 143 & 219 & 0.18 & 0.00 & -0.03 & 0.01 & 0.194 & 0.000 & 0.051 & -0.002 & -0.90 & 1.07 & 1635.27 & 72.89 & & & 1.13 & 73.56 \\
\hline 144 & 220 & 0.19 & 0.00 & -0.03 & 0.01 & 0.205 & 0.000 & 0.053 & -0.001 & -0.96 & 1.12 & 1638.70 & 77.53 & & & 1.18 & 78.30 \\
\hline 145 & 221 & 0.20 & 0.00 & -0.02 & 0.01 & 0.216 & 0.000 & 0.043 & -0.003 & -1.26 & 0.82 & 1640.54 & 83.77 & & & 0.86 & 84.61 \\
\hline 146 & 222 & 0.22 & 0.00 & -0.01 & 0.01 & 0.238 & 0.000 & 0.034 & -0.005 & -1.37 & 0.81 & 1643.81 & 88.56 & & & 0.83 & 89.50 \\
\hline 147 & 223 & 0.22 & 0.00 & -0.01 & 0.02 & 0.238 & 0.000 & 0.035 & -0.015 & -1.92 & 0.32 & 1645.64 & 94.81 & & & 0.38 & 95.90 \\
\hline 148 & 224 & 0.22 & 0.00 & 0.00 & 0.01 & 0.239 & 0.000 & 0.022 & -0.008 & -1.85 & 0.28 & 1648.72 & 99.79 & & & 0.30 & 100.94 \\
\hline 149 & 225 & 0.22 & 0.00 & 0.01 & 0.01 & 0.239 & 0.000 & 0.010 & -0.011 & -2.33 & -0.19 & 1650.33 & 106.25 & & & -0.18 & 107.52 \\
\hline 150 & 226 & 0.22 & 0.00 & 0.01 & 0.01 & 0.239 & 0.000 & 0.010 & -0.011 & -2.41 & -0.30 & 1653.29 & 111.37 & & & -0.28 & 112.76 \\
\hline 151 & 227 & 0.22 & 0.00 & 0.02 & 0.01 & 0.240 & 0.000 & -0.002 & -0.013 & -2.92 & -0.77 & 1654.71 & 118.02 & & & -0.76 & 119.54 \\
\hline 152 & 228 & 0.22 & 0.00 & 0.03 & 0.01 & 0.241 & 0.000 & -0.014 & -0.016 & -2.93 & -0.75 & 1657.34 & 123.46 & & & -0.70 & 125.16 \\
\hline 153 & 229 & 0.22 & 0.00 & 0.03 & 0.01 & 0.241 & 0.000 & -0.014 & -0.016 & -3.19 & -1.02 & 1658.37 & 130.50 & & & -0.98 & 132.33 \\
\hline 154 & 230 & 0.22 & 0.00 & 0.04 & 0.01 & 0.241 & 0.000 & -0.026 & -0.019 & -3.20 & -0.98 & 1660.78 & 136.16 & & & -0.89 & 138.18 \\
\hline 155 & 231 & 0.20 & 0.00 & 0.04 & 0.01 & 0.219 & 0.000 & -0.030 & -0.018 & -3.29 & -1.25 & 1661.63 & 143.39 & & & -1.16 & 145.55 \\
\hline 156 & 232 & 0.20 & 0.00 & 0.05 & 0.00 & 0.219 & 0.000 & -0.043 & -0.010 & -3.39 & -1.27 & 1663.91 & 149.18 & & & -1.16 & 151.50 \\
\hline 157 & 233 & 0.19 & 0.00 & 0.05 & 0.00 & 0.208 & 0.000 & -0.045 & -0.010 & -3.76 & -1.71 & 1664.75 & 156.41 & & & -1.61 & 158.89 \\
\hline 158 & 234 & 0.19 & 0.00 & 0.06 & 0.00 & 0.209 & 0.000 & -0.057 & -0.012 & -4.12 & -1.92 & 1667.04 & 162.20 & & & -1.74 & 164.90 \\
\hline 159 & 235 & 0.19 & 0.00 & 0.06 & -0.01 & 0.208 & 0.000 & -0.058 & -0.002 & -4.69 & -2.48 & 1667.82 & 169.49 & & & -2.32 & 172.35 \\
\hline 160 & 236 & 0.19 & 0.00 & 0.07 & -0.01 & 0.209 & 0.000 & -0.070 & -0.004 & -5.25 & -2.82 & 1670.06 & 175.32 & & & -2.58 & 178.43 \\
\hline
\end{tabular}




\section{$Z=76(O s)$}

$161 \quad 237$

$\begin{array}{llll}162 & 238 & 0.19 & 0.00\end{array}$

$\begin{array}{llll}164 & 240 & 0.18 & 0.00\end{array}$

$\begin{array}{llll}165 & 241 & 0.17 & 0.00\end{array}$

$\begin{array}{llll}166 & 242 & 0.17 & 0.00\end{array}$

$\begin{array}{llll}167 & 243 & 0.17 & 0.00\end{array}$

$\begin{array}{llll}168 & 244 & 0.15 & 0.00\end{array}$

$\begin{array}{llll}169 & 245 & 0.15 & 0.00\end{array}$

$\begin{array}{llll}170 & 246 & 0.14 & 0.00\end{array}$

$\begin{array}{llll}171 & 247 & 0.14 & 0.00\end{array}$

$\begin{array}{llll}172 & 248 & 0.12 & 0.00\end{array}$

$\begin{array}{llll}173 & 249 & 0.12 & 0.00\end{array}$

$\begin{array}{llll}174 & 250 & 0.12 & 0.00\end{array}$

$\begin{array}{llll}175 & 251 & -0.12 & 0.00\end{array}$

$\begin{array}{llll}176 & 252 & -0.12 & 0.00\end{array}$

$\begin{array}{llll}177 & 253 & -0.11 & 0.00\end{array}$ $\begin{array}{llll}163 & 239 & 0.18 & 0.00\end{array}$
$0.08-0.01$

$0.08-0.02$

$\begin{array}{lll}0.08 & -0.02\end{array}$

$0.08-0.03$

$0.09-0.03$

$0.09-0.04$

$0.08-0.03$

$0.08-0.03$

$\begin{array}{lll}0.08 & -0.04\end{array}$

$\begin{array}{lll}0.08 & -0.04\end{array}$

$0.07-0.03$

$0.07-0.03$

$\begin{array}{ll}0.07 & -0.04\end{array}$

$0.02-0.01$

$\begin{array}{llllll}0.03 & 0.00 & -0.125 & 0.000 & -0.029\end{array}$

$\begin{array}{llllll}0.04 & 0.00 & -0.115 & 0.000 & -0.041\end{array}$

$\begin{array}{llll}0.198 & 0.000 & -0.084\end{array}$

$\begin{array}{llll}0.198 & 0.000 & -0.084\end{array}$

$\begin{array}{llll}0.187 & 0.000 & -0.086\end{array}$

$\begin{array}{llll}0.187 & 0.000 & -0.098\end{array}$

$\begin{array}{llll}0.187 & 0.000 & -0.099\end{array}$

$\begin{array}{llll}0.164 & 0.000 & -0.089\end{array}$

$\begin{array}{llll}0.164 & 0.000 & -0.089\end{array}$

$\begin{array}{llll}0.153 & 0.000 & -0.091\end{array}$

$\begin{array}{llll}0.130 & 0.000 & -0.080\end{array}$

$\begin{array}{lll}0.130 & 0.000 & -0.080\end{array}$

$\begin{array}{llll}0.130 & 0.000 & -0.080\end{array}$
$0.08-0.01$ $\begin{array}{llllllll}0.210 & 0.000 & -0.082 & -0.006 & -6.18 & -3.49 & 1670.77 & 182.67\end{array}$

$\begin{array}{llllllll}0.210 & 0.000 & -0.082 & -0.006 & -6.39 & -3.66 & 1672.67 & 188.84\end{array}$

$\begin{array}{lllll}0.004 & -6.63 & -4.01 & 1672.89 & 196.69\end{array}$

$\begin{array}{lllll}0.004 & -6.38 & -3.83 & 1674.27 & 203.39\end{array}$

$\begin{array}{lllll}0.015 & -6.59 & -4.16 & 1674.32 & 211.41\end{array}$

$\begin{array}{lllll}0.013 & -6.68 & -3.83 & 1675.37 & 218.43\end{array}$

$\begin{array}{lllll}0.023 & -7.01 & -4.19 & 1675.29 & 226.58\end{array}$

$\begin{array}{lllll}0.017 & -6.19 & -3.87 & 1676.19 & 233.76\end{array}$

$\begin{array}{lllll}0.017 & -6.52 & -4.20 & 1675.92 & 242.09\end{array}$

$\begin{array}{lllll}0.027 & -6.56 & -4.36 & 1677.14 & 248.95\end{array}$

$\begin{array}{lllll}0.027 & -7.07 & -4.87 & 1676.91 & 257.25\end{array}$

$\begin{array}{llllll}0.021 & -6.61 & -4.78 & 1677.71 & 264.52\end{array}$

$\begin{array}{llllll}0.021 & -7.20 & -5.37 & 1677.40 & 272.90\end{array}$

$\begin{array}{llllll}0.030 & -7.41 & -5.63 & 1678.42 & 279.95\end{array}$

$\begin{array}{lllll}0.012 & -6.47 & -5.49 & 1677.23 & 289.21\end{array}$

$\begin{array}{llllll}0.004 & -6.75 & -5.72 & 1678.06 & 296.45\end{array}$

$\begin{array}{llllll}0.005 & -7.56 & -6.51 & 1677.66 & 304.92\end{array}$ $\begin{array}{llll}0.153 & 0.000 & -0.091\end{array}$
$-3.16 \quad 186.04$

$-3.32 \quad 192.41$

$-3.63200 .48$

$\begin{array}{ll}-3.44 & 207.38\end{array}$

$\begin{array}{ll}-3.67 & 215.69\end{array}$

$-3.22 \quad 223.03$

$\begin{array}{ll}-3.44 & 231.52\end{array}$

$-3.31 \quad 238.71$

$-3.65247 .26$

$-3.61 \quad 254.53$

$-4.12 \quad 263.06$

$\begin{array}{ll}-4.25 & 270.33\end{array}$

$-4.84 \quad 278.95$

$-4.90 \quad 286.44$

$-5.44 \quad 295.26$

$-5.67 \quad 302.75$

$-6.39 \quad 311.54$

\section{$Z=77$ (Ir)}

$\begin{array}{llllllllllllll}85 & 162 & -0.07 & 0.00 & 0.00 & 0.00 & -0.073 & 0.000 & 0.002 & -0.000 & -5.85 & -5.05 & 1249.03 & -1.72\end{array}$

$\begin{array}{lllllllllllllll}86 & 163 & -0.08 & 0.00 & -0.01 & 0.00 & -0.084 & 0.000 & 0.014 & -0.001 & -4.76 & -3.92 & 1261.07 & -5.69\end{array}$

$\begin{array}{llllllllllllll}87 & 164 & 0.10 & 0.00 & 0.00 & -0.01 & 0.107 & 0.000 & 0.004 & 0.010 & -3.92 & -2.68 & 1271.04 & -7.59\end{array}$

$\begin{array}{llllllllllllll}88 & 165 & 0.11 & 0.00 & 0.00 & -0.01 & 0.118 & 0.000 & 0.005 & 0.010 & -3.17 & -1.90 & 1282.95 & -11.42\end{array}$

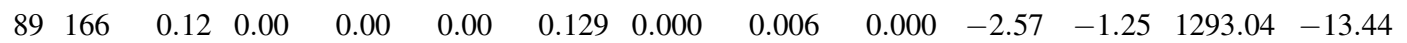

$\begin{array}{lllllllllllllll}90 & 167 & 0.13 & 0.00 & 0.00 & 0.00 & 0.140 & 0.000 & 0.007 & 0.000 & -2.04 & -0.68 & 1304.68 & -17.01\end{array}$

$\begin{array}{llll}92 & 169 & 0.14 & 0.00\end{array}$

$\begin{array}{llll}93 & 170 & 0.15 & 0.00\end{array}$

$\begin{array}{ll}0.01 & 0.00\end{array}$

$\begin{array}{ll}0.140 & 0.000\end{array}$

$\begin{array}{lll}-0.22 & 1314.50 & -18.76\end{array}$

$\begin{array}{llll}94 & 171 & 0.15 & 0.00\end{array}$

$\begin{array}{ll}0.01 & 0.01\end{array}$

$\begin{array}{llllll}0.151 & 0.000 & -0.003 & -0.011 & -1.23\end{array}$

$\begin{array}{llll}0.23 & 1325.80 & -21.99\end{array}$

$\begin{array}{ll}0.01 & 0.00 \\ 0.02 & 0.00\end{array}$

$\begin{array}{llllll}0.162 & 0.000 & -0.003 & -0.001 & -0.88\end{array}$

$\begin{array}{llll}0.61 & 1335.26 & -23.38\end{array}$

$\begin{array}{llll}95 & 172 & 0.16 & 0.00\end{array}$

$\begin{array}{llll}96 & 173 & 0.16 & 0.00\end{array}$

$0.02 \quad 0.00$

$\begin{array}{llllll}0.162 & 0.000 & -0.015 & -0.003 & -0.65\end{array}$

$\begin{array}{lllll}0.88 & 1346.30 & -26.34 & -26.43\end{array}$

$\begin{array}{llllll}0.173 & 0.000 & -0.013 & -0.003 & -0.52\end{array}$

$\begin{array}{lll}1.10 & 1355.49 & -27.46\end{array}$

$\begin{array}{llll}97 & 174 & 0.17 & 0.00\end{array}$

$0.02 \quad 0.00$

$\begin{array}{llllll}0.173 & 0.000 & -0.013 & -0.003 & -0.36\end{array}$

$\begin{array}{llll}98 & 175 & 0.18 & 0.00\end{array}$

$0.01 \quad 0.00$

$\begin{array}{lllll}0.184 & 0.000 & 0.000 & -0.001 & -0.33\end{array}$

$\begin{array}{llll}1.23 & 1366.23 & -30.14 & -30.27\end{array}$

$\begin{array}{lllll}0.98 & 1375.47 & -31.31 & -30.87\end{array}$

$\begin{array}{lllll}1.03 & 1385.88 & -33.63 & -33.43\end{array}$

$\begin{array}{lllll}0.98 & 1394.52 & -34.21 & -33.86\end{array}$

$\begin{array}{llllll}0.239 & 0.000 & 0.009 & -0.001 & -0.93\end{array}$

$\begin{array}{lllll}0.96 & 1404.59 & -36.21 & -36.05\end{array}$

$\begin{array}{lllll}0.81 & 1412.94 & -36.49 & -36.25\end{array}$

$\begin{array}{lllll}0.60 & 1422.80 & -38.28 & -38.08\end{array}$

$\begin{array}{lllll}0.36 & 1430.87 & -38.27 & -37.98\end{array}$

$103 \quad 180$

$0.22 \quad 0.00$

$0.02 \quad 0.00$

$\begin{array}{lllll}0.240 & 0.000 & -0.004 & -0.004 & -0.99\end{array}$

$101 \quad 178$

$\begin{array}{ll}0.19 & 0.00\end{array}$

0.03

0.00

$\begin{array}{lllll}0.240 & 0.000 & -0.004 & -0.004 & -1.23\end{array}$

0.00

$\begin{array}{llllll}0.240 & 0.000 & -0.016 & -0.006 & -1.78\end{array}$

$\begin{array}{lllll}0.03 & 1440.47 & -39.80 & -39.47\end{array}$

$\begin{array}{llll}105 & 182 & 0.22 & 0.00\end{array}$

$\begin{array}{llll}106 & 183 & 0.19 & 0.00\end{array}$

$0.05-0.01$

$\begin{array}{llllll}0.207 & 0.000 & -0.033 & -0.008 & -1.91\end{array}$

$\begin{array}{lllll}-0.27 & 1448.23 & -39.49 & -39.05\end{array}$

$0.05-0.01$

$\begin{array}{llllll}0.241 & 0.000 & -0.041 & -0.002 & -2.73\end{array}$

$\begin{array}{llllll}0.208 & 0.000 & -0.046 & -0.000 & -2.84\end{array}$

$\begin{array}{lllll}-0.68 & 1457.53 & -40.72 & -40.20\end{array}$

$\begin{array}{lllllllll}0.208 & 0.000 & -0.058 & -0.002 & -3.53 & -1.10 & 1465.06 & -40.17 & -39.61\end{array}$

$0.06-0.01$

$0.208 \quad 0.000-0.058-0.1$

$\begin{array}{llll}108 & 185 & 0.19 & 0.00\end{array}$

$\begin{array}{llll}109 & 186 & 0.19 & 0.00\end{array}$

$0.06-0.01$

$0.209 \quad 0.000-0.071$

$\begin{array}{rrrrrr}-0.002 & -3.67 & -1.26 & 1473.75 & -40.80 & -40.34 \\ 0.005 & -4.24 & -1.48 & 1480.72 & -39.70 & -39.17\end{array}$

$\begin{array}{llll}0.198 & 0.000 & -0.072\end{array}$

0.006

$\begin{array}{llll}110 & 187 & 0.18 & 0.00 \\ 111 & 188 & 0.18 & 0.00\end{array}$

$0.07-0.02$

$\begin{array}{lll}0.198 & 0.000 & -0.072\end{array}$

$0.07-0.02$

$\begin{array}{lll}0.186 & 0.000 & -0.074\end{array}$

$0.07-0.02$

$\begin{array}{llll}0.163 & 0.000 & -0.064\end{array}$

$\begin{array}{llll}113 & 190 & 0.15 & 0.00\end{array}$

$\begin{array}{llll}114 & 191 & 0.15 & 0.00\end{array}$

$0.07-0.03$

$\begin{array}{llll}0.164 & 0.000 & -0.077\end{array}$

$\begin{array}{lllllll}0.006 & -4.54 & -1.94 & 1495.79 & -38.62 & -38.33\end{array}$

$\begin{array}{lllllll}0.007 & -4.53 & -1.69 & 1503.38 & -38.14 & -38.45\end{array}$

$\begin{array}{lllllll}0.010 & -4.57 & -2.14 & 1509.93 & -36.62 & -36.75\end{array}$

$\begin{array}{lllllll}0.018 & -5.32 & -2.52 & 1517.82 & -36.44 & -36.71\end{array}$

$0.07-0.03$

$\begin{array}{lll}0.164 & 0.000 & -0.077\end{array}$

$\begin{array}{lllllll}0.018 & -5.99 & -3.15 & 1524.23 & -34.78 & -34.83\end{array}$

$0.06-0.02$

$\begin{array}{lll}0.141 & 0.000 & -0.066\end{array}$

$\begin{array}{lllllll}0.011 & -5.94 & -3.60 & 1531.87 & -34.34 & -34.53\end{array}$

$\begin{array}{lllllll}0.019 & -7.12 & -4.31 & 1538.04 & -32.45 & -32.53\end{array}$

$\begin{array}{ll}0.07 & -0.03 \\ 0.06 & -0.03\end{array}$

$\begin{array}{llll}0.153 & 0.000 & -0.078\end{array}$

$\begin{array}{ll}117 & 194 \\ 118 & 195\end{array}$

$0.14 \quad 0.00$

$\begin{array}{llll}119 & 196 & 0.11 & 0.00\end{array}$

$0.05-0.02$

$\begin{array}{lll}0.141 & 0.000 & -0.067\end{array}$

$\begin{array}{lllllll}0.021 & -7.21 & -4.76 & 1545.38 & -31.71 & -31.69\end{array}$

$\begin{array}{lllllll}0.014 & -7.23 & -5.26 & 1551.04 & -29.30 & -29.44\end{array}$

$\begin{array}{llllll}0.02 & -0.01 & -0.125 & 0.000 & -0.017\end{array}$

$\begin{array}{lllllll}0.012 & -6.89 & -5.59 & 1557.95 & -28.14 & -28.27\end{array}$

$\begin{array}{llllll}0.002 & -7.59 & -6.43 & 1563.66 & -25.78\end{array}$

$\begin{array}{lllllll}0.002 & -7.93 & -7.01 & 1570.52 & -24.57 & -24.40\end{array}$

$\begin{array}{llllll}0.02 & 0.00 & -0.084 & 0.000 & -0.021\end{array}$

$\begin{array}{lllll}0.002 & -8.85 & -7.90 & 1576.00 & -21.98\end{array}$

$-5.06-0.86$

$-3.93-4.91$

$-2.70-6.89$

$-1.91-10.80$

$-1.27-12.89$

$\begin{array}{llll}122 & 199 & -0.08 & 0.00\end{array}$

$\begin{array}{llllll}0.02 & 0.00 & -0.073 & 0.000 & -0.021\end{array}$
$-0.70-16.53$

$-0.24-18.34$

$0.22-21.63$

$0.59-23.09$

$0.86-26.11$

$1.07-27.30$

$1.21-30.02$

$0.96-31.25$

$1.02-33.63$

$0.95-34.27$

$\begin{array}{lll}0.020 & 0.94 & -36.30\end{array}$

$\begin{array}{lll}0.020 & 0.78 & -36.64\end{array}$

$\begin{array}{lll}0.011 & 0.59 & -38.45\end{array}$

$\begin{array}{llll}0.022 & 0.34 & -38.50\end{array}$

$\begin{array}{llll}0.021 & -0.28 & -39.78\end{array}$

$0.025-0.66-41.03$

$\begin{array}{lll}0.028 & -1.09 & -40.51\end{array}$

$\begin{array}{lll}0.028 & -1.24 & -41.16\end{array}$

$\begin{array}{llll}0.017 & -1.44 & -40.07\end{array}$

$\begin{array}{llll}0.006 & -1.53 & -40.31\end{array}$

$\begin{array}{llll}0.007 & -1.89 & -39.03\end{array}$

$0.013-1.62-38.55$

$\begin{array}{llll}0.002 & -2.09 & -37.06\end{array}$

$\begin{array}{llll}0.002 & -2.41 & -36.84\end{array}$

$\begin{array}{llll}0.002 & -3.04 & -35.19\end{array}$

$\begin{array}{llll}0.002 & -3.52 & -34.80\end{array}$

$\begin{array}{llll}0.002 & -4.18 & -32.86\end{array}$

$\begin{array}{llll}0.002 & -4.64 & -32.13\end{array}$

$\begin{array}{llll}0.038 & -5.19 & -29.77\end{array}$

$\begin{array}{lll}0.020 & -5.57 & -28.66\end{array}$ $-6.42-26.31$

$\begin{array}{ll}-7.00 & -25.09\end{array}$

$\begin{array}{ll}-7.89 & -22.48\end{array}$ $\begin{array}{llll}0.026 & 0.03 & -40.05\end{array}$ 
$Z=77$ (Ir)

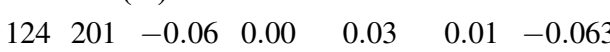

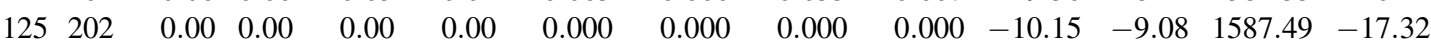

$\begin{array}{llllllllllllll}126 & 203 & 0.00 & 0.00 & 0.00 & 0.00 & 0.000 & 0.000 & 0.000 & 0.000 & -10.49 & -9.38 & 1593.50 & -15.26\end{array}$

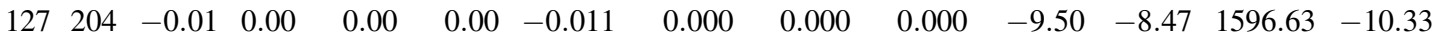

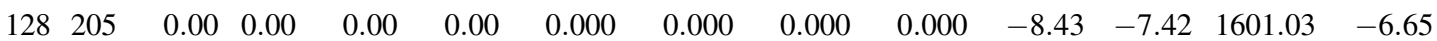

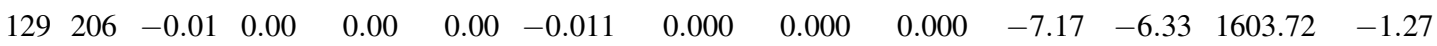

$\begin{array}{llllllllllllll}130 & 207 & 0.00 & 0.00 & 0.00 & 0.00 & 0.000 & 0.000 & 0.000 & 0.000 & -5.92 & -5.08 & 1607.65 & 2.87\end{array}$

$\begin{array}{llllllllllllll}131 & 208 & 0.01 & 0.00 & 0.00 & 0.00 & 0.011 & 0.000 & 0.000 & 0.000 & -4.78 & -3.99 & 1610.09 & 8.50\end{array}$

$\begin{array}{llllllllllllll}132 & 209 & -0.04 & 0.00 & 0.00 & 0.00 & -0.042 & 0.000 & 0.001 & 0.000 & -3.70 & -3.09 & 1614.11 & 12.56\end{array}$

$\begin{array}{llllllllllllll}133 & 210 & -0.06 & 0.00 & -0.01 & 0.00 & -0.063 & 0.000 & 0.013 & -0.001 & -2.86 & -2.23 & 1616.53 & 18.20\end{array}$

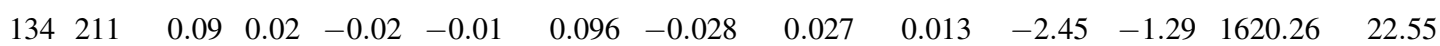

$\begin{array}{llllllllllllll}135 & 212 & 0.10 & 0.03 & -0.02 & -0.01 & 0.108 & -0.042 & 0.029 & 0.014 & -2.16 & -0.83 & 1622.84 & 28.04\end{array}$

$\begin{array}{llllllllllllll}136 & 213 & 0.11 & 0.03 & -0.02 & 0.00 & 0.118 & -0.041 & 0.030 & 0.004 & -1.53 & -0.19 & 1626.62 & 32.33\end{array}$

$\begin{array}{llllllllllllll}137 & 214 & 0.11 & 0.01 & -0.02 & 0.00 & 0.118 & -0.014 & 0.029 & 0.003 & -1.02 & 0.14 & 1629.10 & 37.92\end{array}$

$\begin{array}{llllllllllllll}138 & 215 & 0.11 & 0.00 & -0.02 & 0.00 & 0.118 & 0.000 & 0.029 & 0.003 & -0.50 & 0.60 & 1632.82 & 42.28\end{array}$

$\begin{array}{llllllllllllll}139 & 216 & 0.13 & 0.00 & -0.03 & 0.00 & 0.139 & 0.000 & 0.044 & 0.006 & -0.70 & 0.80 & 1635.20 & 47.97\end{array}$

$\begin{array}{llllllllllllll}140 & 217 & 0.14 & 0.00 & -0.03 & 0.00 & 0.150 & 0.000 & 0.045 & 0.006 & -0.52 & 1.09 & 1638.86 & 52.38\end{array}$

$\begin{array}{llllllllllllll}141 & 218 & 0.16 & 0.00 & -0.03 & 0.00 & 0.172 & 0.000 & 0.048 & 0.007 & -0.82 & 1.07 & 1641.23 & 58.08\end{array}$

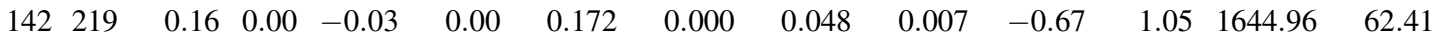

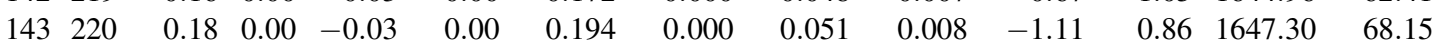

$\begin{array}{llllllllllllll}144 & 221 & 0.19 & 0.00 & -0.03 & 0.01 & 0.205 & 0.000 & 0.053 & -0.001 & -1.12 & 0.94 & 1650.72 & 72.80\end{array}$

$\begin{array}{llllllllllllll}145 & 222 & 0.19 & 0.00 & -0.02 & 0.01 & 0.205 & 0.000 & 0.041 & -0.004 & -1.26 & 0.67 & 1652.91 & 78.68\end{array}$

$\begin{array}{llllllllllllll}146 & 223 & 0.19 & 0.00 & -0.01 & 0.01 & 0.205 & 0.000 & 0.028 & -0.006 & -1.09 & 0.72 & 1656.15 & 83.52\end{array}$

$\begin{array}{llllllllllllll}147 & 224 & 0.22 & 0.00 & -0.01 & 0.01 & 0.238 & 0.000 & 0.034 & -0.005 & -1.88 & 0.31 & 1658.28 & 89.46\end{array}$

$\begin{array}{llllllllllllll}148 & 225 & 0.22 & 0.00 & 0.00 & 0.01 & 0.239 & 0.000 & 0.022 & -0.008 & -1.87 & 0.23 & 1661.43 & 94.38\end{array}$

$\begin{array}{llllllllllllll}149 & 226 & 0.22 & 0.00 & 0.00 & 0.01 & 0.239 & 0.000 & 0.022 & -0.008 & -2.38 & -0.23 & 1663.41 & 100.47\end{array}$

150227

151228

152229

$0.22 \quad 0.00$

0.01

$0.01-0.239$

0.000

0.220 .00

0.02

$0.01 \quad 0.240$

$0.000-0.002-0.013$

$\begin{array}{lll}-2.92 & -0.79 & 1668.16\end{array}$

$\begin{array}{lllllll}0.000 & -0.002 & -0.013 & -2.82 & -0.73 & 1670.77 & 117.32\end{array}$

153230

0.220 .00

0.03

$0.01 \quad 0.240$

$0.000-0.014-0.016$

$-3.17$

$\begin{array}{lllllll}0.000 & -0.014 & -0.016 & -2.98 & -0.89 & 1674.53 & 129.70\end{array}$

155232

$0.22 \quad 0.00$

0.03

$0.01 \quad 0.241$

156233

157234

$\begin{array}{ll}0.20 & 0.00 \\ 0.20 & 0.00\end{array}$

$\begin{array}{lll}0.04 & 0.00 & 0.219\end{array}$

$\begin{array}{lllllll}0.000 & -0.028 & -0.009 & -3.30 & -1.12 & 1675.69 & 136.61\end{array}$

$\begin{array}{lllllll}0.000 & -0.031 & -0.008 & -3.04 & -1.10 & 1677.96 & 142.42\end{array}$

$\begin{array}{lllllll}0.000 & -0.043 & -0.010 & -3.66 & -1.56 & 1679.18 & 149.26\end{array}$

$\begin{array}{lllllll}0.000 & -0.045 & -0.010 & -3.63 & -1.63 & 1681.35 & 155.17\end{array}$

\section{6}

\section{7}

\section{8}

162239

163240

0.190 .00

0.05
0.05

$0.00 \quad 0.219$

$\begin{array}{ll}0.19 & 0.00\end{array}$

$0.06-0.01$

0.208

0.000

0.208

$\begin{array}{ll}0.18 & 0.00\end{array}$

$0.06-0.01$

$\begin{array}{lll}0.000 & -0.058 & -0.002\end{array}$

$\begin{array}{lll}-4.42 & -2.19 & 1682.49\end{array}$

162.10

$\begin{array}{llll}0.000 & -0.059 & -0.002\end{array}$

$\begin{array}{llll}0.000 & -0.071 & -0.004\end{array}$

$\begin{array}{llll}-4.57 & -2.43 & 1684.64 & 168.02\end{array}$

$\begin{array}{lll}0.18 & 0.00 \\ 0.18 & 0.00\end{array}$

$0.07-0.01$

$\begin{array}{llll}0.000 & -0.071 & -0.004\end{array}$

$\begin{array}{llll}-5.44 & -3.07 & 1685.68\end{array}$

175.05

$0.000-0.084$

0.004

$\begin{array}{lll}-5.64 & -3.26 & 1687.61\end{array}$

181.20

16424

$0.18 \quad 0.00$

$\begin{array}{ll}0.08 & -0.02\end{array}$

0.198

$0.000-0.085$

$0.000-0.085$

0.005

$\begin{array}{lll}-6.33 & -3.71 & 1688.30\end{array}$

188.57

165242

$\begin{array}{ll}0.17 & 0.00\end{array}$

$0.08-0.02$

0.187

$0.000-0.088$

0.005

$\begin{array}{lll}-6.10 & -3.63 & 1689.78\end{array}$

195.16

166243

$0.16 \quad 0.00$

$\begin{array}{lll}0.08 & -0.03\end{array}$

0.175

$0.000-0.089$

0.016

$-6.08$

168245

$0.15 \quad 0.00$

$0.08-0.03$

0.164

$0.000-0.089$

0.017

$\begin{array}{lll}-6.34 & -3.94 & 1691.46\end{array}$

203.15

169246

$0.14 \quad 0.00$

$0.07-0.03$

0.153

$\begin{array}{ll}0.000 & -0.078\end{array}$

\subsection{1}

$-6.17$

$\begin{array}{lll}0.000 & -0.067\end{array}$

0.012

$\begin{array}{llll}-6.15 & -4.13 & 1692.62 & 232.68\end{array}$

$\begin{array}{ll}171 \quad 248 \\ 172 & 249\end{array}$

$\begin{array}{ll}0.12 & 0.00\end{array}$

$0.07-0.03$

0.130

$0.000-0.080$

0.021

$-6.76$

$0.000-0.080$

0.021

$\begin{array}{lll}0.07 & -0.03 & 0.130\end{array}$

$0.000-0.080$

0.02

$\begin{array}{llll}-6.93 & -5.06 & 1695.13 & 254.39\end{array}$

173250

$\begin{array}{ll}0.12 & 0.00 \\ 0.12 & 0.00\end{array}$

$\begin{array}{lll}0.07 & -0.03 & 0.130\end{array}$

$\begin{array}{lll}0.000 & -0.017\end{array}$

0.01

$-7.5$

$0.000-0.017$

0.012

$-6.43$

$\begin{array}{rrr}0.02 & -0.01 & -0.125 \\ 0.03 & 0.00 & -0.115\end{array}$

-7.16
-7.36

$\begin{array}{lll}-6.39 & 1696.55 & 285.25\end{array}$ $\begin{array}{lll}0.000 & -0.030\end{array}$

0.004

$\begin{array}{lll}0.000 & -0.031\end{array}$

0.004

$0.000-0.042$

0.005

$\begin{array}{llll}0.04 & 0.00 & -0.105\end{array}$

$0.000-0.031$

$-8$

\begin{tabular}{|c|c|}
\hline-8.41 & -20.90 \\
\hline-9.08 & -17.80 \\
\hline-9.38 & -15.72 \\
\hline-8.47 & -10.76 \\
\hline-7.42 & -7.05 \\
\hline-6.33 & -1.64 \\
\hline-5.08 & 2.54 \\
\hline-4.00 & 8.21 \\
\hline-3.09 & 12.31 \\
\hline-2.23 & 18.01 \\
\hline-1.25 & 22.44 \\
\hline-0.78 & 28.00 \\
\hline-0.16 & 32.33 \\
\hline 0.15 & 37.97 \\
\hline 0.62 & 42.39 \\
\hline 0.84 & 48.17 \\
\hline 1.14 & 52.67 \\
\hline 1.12 & 58.45 \\
\hline 1.11 & 62.87 \\
\hline 0.90 & 68.69 \\
\hline 1.00 & 73.44 \\
\hline 0.69 & 79.38 \\
\hline 0.74 & 84.31 \\
\hline 0.31 & 90.34 \\
\hline 0.24 & 95.37 \\
\hline-0.24 & 101.56 \\
\hline-0.34 & 106.78 \\
\hline-0.78 & 113.21 \\
\hline-0.71 & 118.80 \\
\hline-1.00 & 125.59 \\
\hline-0.85 & 131.47 \\
\hline-1.09 & 138.50 \\
\hline-1.05 & 144.48 \\
\hline-1.48 & 151.51 \\
\hline-1.54 & 157.58 \\
\hline-2.05 & 164.72 \\
\hline-2.26 & 170.82 \\
\hline-2.84 & 178.08 \\
\hline-3.02 & 184.40 \\
\hline-3.36 & 192.07 \\
\hline-3.25 & 198.87 \\
\hline-3.27 & 207.04 \\
\hline-3.10 & 214.08 \\
\hline-3.42 & 222.11 \\
\hline-3.30 & 229.29 \\
\hline-3.67 & 237.43 \\
\hline-3.79 & 244.53 \\
\hline-4.37 & 252.64 \\
\hline-4.55 & 259.85 \\
\hline-5.14 & 268.12 \\
\hline-5.38 & 275.43 \\
\hline-6.11 & 283.71 \\
\hline-6.34 & 291.18 \\
\hline-7.10 & 299.60 \\
\hline-7.23 & 307.32 \\
\hline-7.59 & 316.28 \\
\hline
\end{tabular}

$\begin{array}{llllllll}179 & 256 & -0.09 & 0.00 & 0.03 & 0.00 & -0.094\end{array}$ 


\begin{tabular}{|c|c|c|c|c|c|c|c|c|c|c|c|c|c|c|c|c|c|}
\hline \multicolumn{18}{|c|}{$Z=78(\mathrm{Pt})$} \\
\hline 87 & 165 & -0.09 & 0.00 & 0.01 & 0.00 & -0.094 & 0.000 & -0.008 & 0.001 & -4.50 & -3.57 & 1271.23 & -0.49 & & & -3.58 & 0.37 \\
\hline 88 & 166 & -0.10 & 0.00 & 0.00 & 0.00 & -0.105 & 0.000 & 0.004 & -0.000 & -3.57 & -2.63 & 1283.50 & -4.68 & & & -2.64 & -3.90 \\
\hline 89 & 167 & 0.10 & 0.00 & 0.00 & 0.00 & 0.107 & 0.000 & 0.004 & 0.000 & -2.70 & -1.60 & 1293.27 & -6.38 & & & -1.61 & -5.68 \\
\hline 90 & 168 & 0.11 & 0.00 & 0.00 & 0.00 & 0.118 & 0.000 & 0.005 & 0.000 & -2.09 & -0.95 & 1305.34 & -10.38 & -11.04 & 0.209 & -0.96 & -9.75 \\
\hline 91 & 169 & 0.12 & 0.00 & 0.00 & 0.01 & 0.129 & 0.000 & 0.007 & -0.010 & -1.64 & -0.42 & 1315.16 & -12.13 & & & -0.43 & -11.57 \\
\hline 92 & 170 & 0.12 & 0.00 & 0.01 & 0.00 & 0.129 & 0.000 & -0.006 & -0.001 & -1.07 & 0.10 & 1326.89 & -15.79 & -16.31 & 0.019 & 0.09 & -15.30 \\
\hline 93 & 171 & 0.13 & 0.00 & 0.00 & 0.00 & 0.140 & 0.000 & 0.007 & 0.000 & -0.67 & 0.54 & 1336.36 & -17.19 & -17.47 & 0.088 & 0.52 & -16.76 \\
\hline 94 & 172 & 0.13 & 0.00 & 0.01 & 0.00 & 0.140 & 0.000 & -0.005 & -0.001 & -0.31 & 0.88 & 1347.82 & -20.57 & -21.10 & 0.013 & 0.87 & -20.21 \\
\hline 95 & 173 & 0.14 & 0.00 & 0.01 & 0.00 & 0.151 & 0.000 & -0.004 & -0.001 & -0.14 & 1.15 & 1357.03 & -21.72 & -21.94 & 0.056 & 1.13 & -21.42 \\
\hline 96 & 174 & 0.15 & 0.00 & 0.01 & 0.00 & 0.162 & 0.000 & -0.003 & -0.001 & 0.01 & 1.34 & 1368.20 & -24.81 & -25.32 & 0.012 & 1.33 & -24.57 \\
\hline 97 & 175 & 0.16 & 0.00 & 0.01 & 0.00 & 0.173 & 0.000 & -0.001 & -0.001 & 0.10 & 1.50 & 1377.10 & -25.64 & -25.69 & 0.019 & 1.48 & -25.47 \\
\hline 98 & 176 & 0.22 & 0.00 & 0.00 & 0.00 & 0.239 & 0.000 & 0.021 & 0.002 & -0.46 & 1.39 & 1388.15 & -28.62 & -28.93 & 0.014 & 1.37 & -28.50 \\
\hline 99 & 177 & 0.23 & 0.00 & 0.00 & 0.00 & 0.250 & 0.000 & 0.023 & 0.002 & -0.73 & 1.29 & 1396.90 & -29.30 & -29.37 & 0.015 & 1.27 & -29.25 \\
\hline 100 & 178 & 0.23 & 0.00 & 0.01 & 0.00 & 0.250 & 0.000 & 0.011 & -0.001 & -0.71 & 1.27 & 1407.46 & -31.79 & -32.00 & 0.011 & 1.25 & -31.77 \\
\hline 101 & 179 & 0.23 & 0.00 & 0.01 & 0.00 & 0.250 & 0.000 & 0.011 & -0.001 & -0.94 & 1.07 & 1415.92 & -32.17 & -32.26 & 0.009 & 1.05 & -32.22 \\
\hline 102 & 180 & 0.23 & 0.00 & 0.02 & 0.00 & 0.251 & 0.000 & -0.002 & -0.004 & -0.98 & 1.03 & 1426.08 & -34.27 & -34.44 & 0.011 & 1.02 & -34.35 \\
\hline 103 & 181 & 0.22 & 0.00 & 0.03 & 0.00 & 0.240 & 0.000 & -0.016 & -0.006 & -1.27 & 0.75 & 1434.25 & -34.36 & -3 & 0.015 & 0.74 & -34.49 \\
\hline 104 & 182 & 0.20 & 0.00 & 0.04 & 0.00 & 0.219 & 0.000 & -0.031 & -0.008 & -1.39 & 0.52 & 1444.23 & -36.27 & -36.17 & 0.016 & 0.52 & -36.43 \\
\hline 105 & 183 & 0.21 & 0.00 & 0.04 & 0.00 & 0.230 & 0.000 & -0.030 & -0.009 & -1.89 & 0.19 & 1452.07 & -36.04 & -35.77 & 0.016 & 0.18 & -36.25 \\
\hline 106 & 184 & 0.20 & 0.00 & 0.05 & -0.01 & 0.219 & 0.000 & -0.044 & -0.001 & -2.28 & -0.14 & 1461.76 & -37.66 & -37.33 & 0.018 & -0.12 & -37.89 \\
\hline 107 & 185 & 0.20 & 0.00 & 0.05 & -0.01 & 0.219 & 0.000 & -0.044 & -0.001 & -2.70 & -0.54 & 1469.32 & -37.15 & -36.68 & 0.041 & -0.54 & -37.42 \\
\hline 108 & 186 & 0.19 & 0.00 & 0.06 & -0.01 & 0.208 & 0.000 & -0.058 & -0.002 & -2.96 & -0.67 & 1478.45 & -38.21 & -3 & 0.022 & -0.64 & -38.49 \\
\hline 109 & 187 & 0.19 & 0.00 & 0.06 & -0.01 & 0.208 & 0.000 & -0.058 & -0.002 & -3.18 & -0.85 & 1485.43 & -37.12 & -36.71 & 0.028 & -0.82 & -37.44 \\
\hline 110 & 188 & 0.17 & 0.00 & 0.06 & -0.01 & 0.186 & 0.000 & -0.061 & -0.001 & -3.09 & -0.92 & 1494.15 & -37.77 & -37.82 & 0.005 & -0.88 & -38.10 \\
\hline 111 & 189 & 0.16 & 0.00 & 0.06 & -0.01 & 0.175 & 0.000 & -0.062 & -0.001 & -3.34 & -1.21 & 1500.91 & -36.45 & -36.48 & 0.011 & -1.17 & -36.81 \\
\hline 112 & 190 & 0.15 & 0.00 & 0.05 & -0.01 & 0.163 & 0.000 & -0.051 & 0.002 & -3.37 & -1.30 & 1509.30 & -36.78 & -37.32 & 0.006 & -1.27 & -37.17 \\
\hline 113 & 191 & 0.14 & 0.00 & 0.05 & -0.01 & 0.152 & 0.000 & -0.052 & 0.002 & -3.89 & -1.85 & 1515.99 & -35.39 & -35.70 & 0.004 & -1.81 & -35.80 \\
\hline 114 & 192 & 0.13 & 0.00 & 0.05 & -0.01 & 0.141 & 0.000 & -0.054 & 0.003 & -4.31 & -2.34 & 1524.44 & -35.77 & -36.29 & 0.002 & -2.30 & -36.20 \\
\hline 115 & 193 & 0.13 & 0.00 & 0.05 & -0.01 & 0.141 & 0.000 & -0.054 & 0.003 & -4.93 & -2.93 & 1530.86 & -34.12 & -34.48 & 0.002 & -2.89 & -34.56 \\
\hline 116 & 194 & 0.12 & 0.00 & 0.05 & -0.02 & 0.130 & 0.000 & -0.055 & 0.013 & -5.43 & -3.46 & 1539.03 & -34.22 & -34.76 & 0.001 & -3.40 & -34.65 \\
\hline 117 & 195 & 0.12 & 0.00 & 0.05 & -0.02 & 0.130 & 0.000 & -0.055 & 0.013 & -6.10 & -4.10 & 1545.18 & -32.30 & -32.80 & 0.001 & -4.04 & -32.74 \\
\hline 118 & 196 & 0.11 & 0.00 & 0.05 & -0.02 & 0.119 & 0.000 & -0.056 & 0.014 & -6.63 & -4.69 & 1553.09 & -32.14 & -32.65 & 0.001 & -4.62 & -32.58 \\
\hline 119 & 197 & -0.12 & 0.00 & 0.02 & -0.01 & -0.125 & 0.000 & -0.017 & 0.012 & -6.86 & -5.56 & 1559.17 & -30.14 & -30.42 & 0.001 & -5.54 & -30.64 \\
\hline 120 & 198 & -0.11 & 0.00 & 0.02 & -0.01 & -0.115 & 0.000 & -0.018 & 0.012 & -7.46 & -6.25 & 1566.87 & -29.77 & -29.91 & 0.003 & -6.23 & -30.27 \\
\hline 121 & 199 & -0.11 & 0.00 & 0.02 & 0.00 & -0.115 & 0.000 & -0.018 & 0.002 & -8.28 & -7.09 & 1572.62 & -27.46 & -27.39 & 0.003 & -7.08 & -27.97 \\
\hline 122 & 200 & -0.08 & 0.00 & 0.03 & 0.01 & -0.084 & 0.000 & -0.032 & -0.007 & -8.85 & -7.75 & 1580.00 & -26.76 & -26.60 & 0.020 & -7.72 & -27.25 \\
\hline 123 & 201 & -0.07 & 0.00 & 0.03 & 0.01 & -0.073 & 0.000 & -0.033 & -0.007 & -9.77 & -8.64 & 1585.53 & -24.21 & -23.74 & 0.050 & -8.61 & -24.69 \\
\hline 124 & 202 & -0.06 & 0.00 & 0.03 & 0.01 & -0.063 & 0.000 & -0.033 & -0.007 & -10.34 & -9.16 & 1592.46 & -23.08 & & & -9.13 & -23.54 \\
\hline 125 & 203 & 0.00 & 0.00 & 0.00 & 0.00 & 0.000 & 0.000 & 0.000 & 0.000 & -10.90 & -9.77 & 1597.43 & -19.98 & & & -9.77 & -20.46 \\
\hline 126 & 204 & 0.00 & 0.00 & 0.00 & 0.00 & 0.000 & 0.000 & 0.000 & 0.000 & -11.23 & -10.06 & 1603.85 & -18.33 & & & -10.06 & -18.79 \\
\hline 127 & 205 & -0.02 & 0.00 & 0.00 & 0.00 & -0.021 & 0.000 & 0.000 & 0.000 & -10.23 & -9.16 & 1607.04 & -13.44 & & & -9.16 & -13.88 \\
\hline 128 & 206 & 0.00 & 0.00 & 0.00 & 0.00 & 0.000 & 0.000 & 0.000 & 0.000 & -9.15 & -8.08 & 1611.82 & -10.15 & & & -8.09 & -10.57 \\
\hline 129 & 207 & 0.01 & 0.00 & 0.00 & 0.00 & 0.011 & 0.000 & 0.000 & 0.000 & -7.88 & -6.98 & 1614.54 & -4.80 & & & -6.98 & -5.19 \\
\hline 130 & 208 & 0.00 & 0.00 & 0.00 & 0.00 & 0.000 & 0.000 & 0.000 & 0.000 & -6.63 & -5.72 & 1618.87 & -1.06 & & & -5.72 & -1.42 \\
\hline 131 & 209 & 0.01 & 0.00 & 0.00 & 0.00 & 0.011 & 0.000 & 0.000 & 0.000 & -5.48 & -4.63 & 1621.34 & 4.54 & & & -4.63 & 4.21 \\
\hline 132 & 210 & -0.04 & 0.00 & 0.00 & 0.00 & -0.042 & 0.000 & 0.001 & 0.000 & -4.41 & -3.72 & 1625.76 & 8.19 & & & -3.72 & 7.91 \\
\hline 133 & 211 & -0.05 & 0.00 & 0.00 & 0.00 & -0.052 & 0.000 & 0.001 & 0.000 & -3.51 & -2.85 & 1628.21 & 13.81 & & & -2.85 & 13.57 \\
\hline 134 & 212 & -0.06 & 0.00 & 0.00 & 0.00 & -0.063 & 0.000 & 0.001 & 0.000 & -2.61 & -1.98 & 1632.41 & 17.69 & & & -1.98 & 17.49 \\
\hline 135 & 213 & 0.09 & 0.04 & -0.02 & 0.00 & 0.097 & -0.055 & 0.028 & 0.004 & -2.59 & -1.22 & 1634.73 & 23.44 & & & -1.18 & 23.33 \\
\hline 136 & 214 & 0.09 & 0.03 & -0.02 & 0.00 & 0.096 & -0.041 & 0.028 & 0.003 & -1.73 & -0.55 & 1638.88 & 27.35 & & & -0.52 & 27.29 \\
\hline 137 & 215 & 0.11 & 0.03 & -0.03 & 0.00 & 0.118 & -0.041 & 0.042 & 0.005 & -1.60 & -0.13 & 1641.30 & 33.01 & & & -0.08 & 33.02 \\
\hline 138 & 216 & 0.11 & 0.00 & -0.02 & 0.00 & 0.118 & 0.000 & 0.029 & 0.003 & -0.72 & 0.37 & 1645.38 & 37.01 & & & 0.40 & 37.05 \\
\hline 139 & 217 & 0.11 & 0.00 & -0.03 & 0.00 & 0.118 & 0.000 & 0.042 & 0.005 & -0.59 & 0.64 & 1647.71 & 42.74 & & & 0.68 & 42.87 \\
\hline 140 & 218 & 0.13 & 0.00 & -0.03 & 0.00 & 0.139 & 0.000 & 0.044 & 0.006 & -0.42 & 1.03 & 1651.67 & 46.85 & & & 1.08 & 47.06 \\
\hline 141 & 219 & 0.16 & 0.00 & -0.04 & 0.00 & 0.172 & 0.000 & 0.060 & 0.009 & -1.01 & 1.08 & 1654.01 & 52.59 & & & 1.16 & 52.90 \\
\hline 142 & 220 & 0.17 & 0.00 & -0.04 & 0.00 & 0.183 & 0.000 & 0.062 & 0.010 & -0.94 & 1.09 & 1658.11 & 56.56 & & & 1.19 & 56.96 \\
\hline 143 & 221 & 0.18 & 0.00 & -0.04 & 0.00 & 0.194 & 0.000 & 0.063 & 0.011 & -1.24 & 0.94 & 1660.42 & 62.32 & & & 1.03 & 62.79 \\
\hline
\end{tabular}




\section{$Z=78(\mathrm{Pt})$}

144222

$\begin{array}{llllll}145 & 223 & 0.19 & 0.00 & -0.03\end{array}$

$\begin{array}{lllll}146 & 224 & 0.20 & 0.00 & -0.02\end{array}$

$\begin{array}{lll}0.01 & 0.205 & 0.000\end{array}$

$\begin{array}{lll}0.01 & 0.205 & 0.000\end{array}$

$\begin{array}{lll}0.053 & -0.001 & -0.96\end{array}$

$\begin{array}{ll}1.05 & 1664.20\end{array}$

$\begin{array}{ll}0.79 & 1666.41\end{array}$

$\begin{array}{ll}0.87 & 1670.00\end{array}$

$0.043-0.003-1.12$

$\begin{array}{ll}0.44 & 1672.17\end{array}$

$\begin{array}{lll}0.02 & 0.238 & 0.000\end{array}$

$\begin{array}{llll}0.047 & -0.012 & -1.84\end{array}$

$0.39 \quad 1675.68$

$148 \quad 226$

$\begin{array}{llll}0.22 & 0.00 & -0.01\end{array}$

0.02

$0.238 \quad 0.000$

$\begin{array}{lll}0.035 & -0.015 & -1.75\end{array}$

$\begin{array}{lllll}0.022 & -0.008 & -2.09 & -0.02 & 1677.62 \\ 0.010 & -0.011 & -2.10 & -0.08 & 1680.93\end{array}$

149227

$\begin{array}{lll}0.22 & 0.00 & 0.00\end{array}$

0.01

$\begin{array}{ll}0.239 & 0.000\end{array}$

151229

$0.22 \quad 0.00$

0.01

0.01

$0.239 \quad 0.000$

$\begin{array}{llllll}0.010 & -0.011 & -2.10 & -0.08 & 1680.93 \\ 0.010 & -0.011 & -2.54 & -0.50 & 1682.69\end{array}$

$152 \quad 230$

0.02

0.01

0.2390 .000

0.02

0.01

$0.240-0.000$

$\begin{array}{lllll}-0.002 & -0.013 & -2.46 & -0.43 & 1685.67\end{array}$

0.01

$\begin{array}{lllllll}0.241 & 0.000 & -0.014 & -0.016 & -2.56 & -0.53 & 1689.76\end{array}$

154232

0.220 .00

0.04

0.00$$
\begin{array}{lllllll}
0.241 & 0.000 & -0.028 & -0.009 & -2.83 & -0.75 & 1690.93 \\
0.207 & 0.000 & -0.033 & -0.008 & -2.44 & -0.66 & 1693.49 \\
0.207 & 0.000 & -0.033 & -0.008 & -2.85 & -1.07 & 1694.68
\end{array}
$$$$
\begin{array}{llllllll}
0.207 & 0.000 & -0.033 & -0.008 & -2.85 & -1.07 & 1694.68
\end{array}
$$$$
\begin{array}{lllllll}
0.208 & 0.000 & -0.045 & -0.010 & -3.12 & -1.17 & 1697.24
\end{array}
$$

$\begin{array}{lllllll}0.208 & 0.000 & -0.058 & -0.002 & -3.89 & -1.72 & 1698.38\end{array}$

$\begin{array}{llll}159 & 237 & 0.19 & 0.00\end{array}$

$\begin{array}{llll}160 & 238 & 0.18 & 0.00\end{array}$

$\begin{array}{llll}161 & 239 & 0.19 & 0.00\end{array}$

$\begin{array}{llll}162 & 240 & 0.18 & 0.00\end{array}$

$\begin{array}{llll}163 & 241 & 0.17 & 0.00\end{array}$

$\begin{array}{llll}164 & 242 & 0.17 & 0.00\end{array}$

$\begin{array}{llll}165 & 243 & 0.16 & 0.00\end{array}$

$\begin{array}{llll}166 & 244 & 0.15 & 0.00\end{array}$

$\begin{array}{llll}167 & 245 & 0.15 & 0.00\end{array}$

$\begin{array}{llll}168 & 246 & 0.13 & 0.00\end{array}$

$0.06-0.01$$$
\begin{array}{lllllll}
0.197 & 0.000 & -0.059 & -0.002 & -4.01 & -1.93 & 1700.86
\end{array}
$$

$0.07-0.01$

$\begin{array}{lllllll}0.209 & 0.000 & -0.070 & -0.004 & -4.94 & -2.54 & 1701.90\end{array}$

$\begin{array}{lllllll}0.198 & 0.000 & -0.071 & -0.004 & -5.05 & -2.71 & 1704.17\end{array}$

$\begin{array}{ll}0.07 & -0.01 \\ 0.07 & -0.01\end{array}$

$\begin{array}{llllllll}0.187 & 0.000 & -0.072 & -0.003 & -5.29 & -3.05 & 1704.75 & 179.42\end{array}$

$0.07-0.02$

$\begin{array}{lll}0.186 & 0.000 & -0.074\end{array}$$$
\begin{array}{lllll}
0.007 & -5.13 & -2.96 & 1706.57 & 185.66
\end{array}
$$

$0.07-0.02$

$\begin{array}{lll}0.175 & 0.000 & -0.075\end{array}$

$\begin{array}{lllll}0.008 & -5.27 & -3.03 & 1706.72 & 193.59\end{array}$

$0.08-0.02$

$\begin{array}{lll}0.165 & 0.000 & -0.088\end{array}$

$\begin{array}{lllll}0.007 & -5.35 & -2.96 & 1708.40 & 199.98\end{array}$

$0.06-0.02$

$0.000-0.089$

$\begin{array}{lllll}0.017 & -5.75 & -3.37 & 1708.73 & 207.72\end{array}$

$\begin{array}{lllll}0.011 & -4.80 & -3.09 & 1710.02 & 214.50\end{array}$

$\begin{array}{llll}169 & 247 & 0.12 & 0.00\end{array}$

$\begin{array}{llll}170 & 248 & -0.13 & 0.00\end{array}$

$\begin{array}{llll}171 & 249 & -0.13 & 0.00\end{array}$

$\begin{array}{llll}172 & 250 & -0.13 & 0.00\end{array}$

$\begin{array}{llll}173 & 251 & -0.12 & 0.00\end{array}$

$\begin{array}{llll}174 & 252 & -0.12 & 0.00\end{array}$

$\begin{array}{llll}175 & 253 & -0.12 & 0.00\end{array}$

$\begin{array}{llll}176 & 254 & -0.10 & 0.00\end{array}$

$\begin{array}{llll}177 & 255 & -0.10 & 0.00\end{array}$

$\begin{array}{llll}178 & 256 & -0.10 & 0.00\end{array}$

$\begin{array}{llll}179 & 257 & -0.09 & 0.00\end{array}$

$\begin{array}{llll}180 & 258 & -0.07 & 0.00\end{array}$

$\begin{array}{llll}181 & 259 & -0.07 & 0.00\end{array}$

$\begin{array}{llllll}0.06 & -0.02 & 0.130 & 0.000 & -0.067\end{array}$

$\begin{array}{llllll}0.01 & -0.01 & -0.135 & 0.000 & -0.004\end{array}$

$\begin{array}{llllll}0.012 & -5.30 & -3.64 & 1710.33 & 222.26\end{array}$

$\begin{array}{llllll}0.01 & -0.01 & -0.135 & 0.000 & -0.004\end{array}$

$\begin{array}{llllll}0.011 & -4.77 & -3.67 & 1711.78 & 228.89\end{array}$

$\begin{array}{llllll}0.01 & -0.01 & -0.135 & 0.000 & -0.004\end{array}$

$\begin{array}{lllll}0.011 & -5.50 & -4.39 & 1712.09 & 236.64\end{array}$

$\begin{array}{llllll}0.02 & -0.01 & -0.125 & 0.000 & -0.017\end{array}$

$\begin{array}{lllll}0.011 & -5.80 & -4.70 & 1713.66 & 243.15\end{array}$

$\begin{array}{llllll}0.02 & -0.01 & -0.125 & 0.000 & -0.017\end{array}$

$\begin{array}{llllll}0.012 & -6.54 & -5.50 & 1713.90 & 250.98\end{array}$

$\begin{array}{llllll}0.03 & 0.00 & -0.125 & 0.000 & -0.029\end{array}$

$\begin{array}{lllll}0.012 & -6.77 & -5.76 & 1715.25 & 257.70\end{array}$

$\begin{array}{llllll}0.03 & 0.00 & -0.105 & 0.000 & -0.031\end{array}$

$\begin{array}{lllll}0.004 & -7.55 & -6.47 & 1715.27 & 265.75\end{array}$

0.04

$\begin{array}{lllll}0.00 & -0.105 & 0.000 & -0.042\end{array}$

$\begin{array}{llllll}0.004 & -7.76 & -6.84 & 1716.57 & 272.52\end{array}$

0.04

$\begin{array}{lllll}0.00 & -0.105 & 0.000 & -0.042\end{array}$

$\begin{array}{llllll}0.005 & -8.68 & -7.62 & 1716.50 & 280.66\end{array}$

0.04

$\begin{array}{lllll}0.00 & -0.094 & 0.000 & -0.043\end{array}$

$\begin{array}{llllll}0.005 & -8.83 & -7.77 & 1717.44 & 287.80\end{array}$

0.04

$\begin{array}{lllll}0.01 & -0.073 & 0.000 & -0.044\end{array}$

$\begin{array}{lllll}0.004 & -9.14 & -8.11 & 1716.79 & 296.51\end{array}$

0.03

0.01

0.00

$-0.073$

0.000

\section{$Z=79$ (Au)}

$\begin{array}{llll}88 & 167 & -0.08 & 0.00\end{array}$

$\begin{array}{lllll}89 & 168 & -0.10 & 0.00\end{array}$

$\begin{array}{llll}90 & 169 & -0.10 & 0.00\end{array}$

$\begin{array}{llll}91 & 170 & -0.10 & 0.00\end{array}$

$\begin{array}{llll}92 & 171 & -0.11 & 0.00\end{array}$

$\begin{array}{llll}93 & 172 & 0.11 & 0.00\end{array}$

$\begin{array}{llll}94 & 173 & 0.12 & 0.00\end{array}$

$\begin{array}{llll}95 & 174 & 0.12 & 0.00\end{array}$

$\begin{array}{llll}96 & 175 & 0.12 & 0.00\end{array}$

$\begin{array}{llll}97 & 176 & 0.13 & 0.00\end{array}$

$\begin{array}{llll}98 & 177 & 0.13 & 0.00\end{array}$

$\begin{array}{llll}99 & 178 & 0.22 & 0.00\end{array}$

$\begin{array}{llll}100 & 179 & 0.22 & 0.00\end{array}$

0.01

$\begin{array}{lllll}0.00 & -0.084 & 0.000 & -0.009\end{array}$

$\begin{array}{llllll}0.01 & 0.00 & -0.105 & 0.000 & -0.008\end{array}$

$\begin{array}{llllll}0.01 & 0.00 & -0.105 & 0.000 & -0.008\end{array}$

$\begin{array}{llllll}0.01 & 0.00 & -0.105 & 0.000 & -0.008\end{array}$

$\begin{array}{llllll}0.02 & 0.00 & -0.115 & 0.000 & -0.018\end{array}$

$-0.006$

0.01

0.00

$\begin{array}{llll}0.118 & 0.000 & -0.007\end{array}$

0.000

$\begin{array}{llll}-9.11 & -8.11 & 1717.43 & 303.95\end{array}$

$0.01 \quad 0.00$

$\begin{array}{llll}0.129 & 0.000 & -0.006 & -0.001\end{array}$

$\begin{array}{llll}0.001 & -4.81 & -3.89 & 1281.85\end{array}$

$\begin{array}{llll}0.001 & -3.98 & -2.95 & 1292.22\end{array}$

4.26
1.96

$\begin{array}{lllll}0.001 & -3.14 & -2.15 & 1304.21 & -1.96\end{array}$

$\begin{array}{lllll}0.001 & -2.32 & -1.27 & 1314.18 & -3.86\end{array}$

$\begin{array}{llll}0.002 & -1.79 & -0.70 & 1325.93\end{array}$

$-7.54$

$0.129-0.000-0.006-0.001-0.54$

$\begin{array}{lll}-0.22 & 1335.84 & -9.38\end{array}$

$\begin{array}{lll}0.18 & 1347.31 & -12.78 \\ 0.54 & 1356.93 & -14.32\end{array}$

$\begin{array}{llllll}0.129 & 0.000 & -0.006 & -0.001 & -0.23\end{array}$

$\begin{array}{llll}0.54 & 1356.93 & -14.32\end{array}$

$0.01 \quad 0.00$

$\begin{array}{llllll}0.140 & 0.000 & -0.005 & -0.001 & -0.12\end{array}$

$\begin{array}{lll}0.81 & 1368.08 & -17.41 \\ 1.02 & 1377.42 & -18.67\end{array}$

0.01

0.00

$\begin{array}{llllll}0.140 & 0.000 & -0.005 & -0.001 & 0.03\end{array}$

$\begin{array}{llll}1.15 & 1388.29 & -21.47 & -21.55\end{array}$

$\begin{array}{llll}1.24 & 1397.34 & -22.45 & -22.33\end{array}$

$\begin{array}{llll}1.25 & 1407.92 & -24.96 & -24.95\end{array}$

$\begin{array}{llll}1.25 & 1416.66 & -25.63 & -25.60\end{array}$

$\begin{array}{llll}1.17 & 1426.93 & -27.82 & -27.87\end{array}$

$\begin{array}{llllllll}0.02 & 0.00 & 0.262 & 0.000 & 0.001 & -0.004 & -0.89\end{array}$

$\begin{array}{llll}102 & 181 & 0.24 & 0.00\end{array}$

0.02

0.2620 .000

$\begin{array}{lll}0.001 & -0.004 & -0.97\end{array}$

$\begin{array}{llll}0.89 & 1435.56 & -28.38 & -28.30\end{array}$

$\begin{array}{lllll}0.263 & 0.000 & -0.012 & -0.006 & -1.22\end{array}$

$\begin{array}{llll}103 & 182 & 0.24 & 0.00\end{array}$ $\begin{array}{ll}1.12 & 67.15\end{array}$

$0.84 \quad 73.09$

$0.91 \quad 77.64$

$0.51 \quad 83.68$

$0.46 \quad 88.34$

$-0.01 \quad 94.51$

$\begin{array}{ll}-0.06 & 99.39\end{array}$

$-0.49 \quad 105.81$

$-0.41 \quad 111.04$

$-0.64 \quad 117.86$

$-0.48 \quad 123.37$

$-0.71 \quad 130.38$

$-0.60 \quad 136.05$

$\begin{array}{ll}-1.02 & 143.07\end{array}$

$-1.06 \quad 148.78$

$-1.57 \quad 155.90$

$-1.76 \quad 161.66$

$\begin{array}{ll}-2.32 & 168.92\end{array}$

$-2.47 \quad 174.90$

$-2.80 \quad 182.57$

$\begin{array}{ll}-2.66 & 189.03\end{array}$

$-2.72 \quad 197.14$

$-2.55 \quad 203.82$

$-2.86 \quad 211.85$

$\begin{array}{ll}-2.81 & 218.59\end{array}$

$-3.36 \quad 226.55$

$\begin{array}{ll}-3.65 & 233.12\end{array}$

$-4.37 \quad 241.08$

$\begin{array}{ll}-4.68 & 247.80\end{array}$

$-5.45 \quad 255.87$

$-5.71 \quad 262.81$
-6.43

$\begin{array}{ll}-6.43 & 271.09\end{array}$

$-6.78 \quad 278.10$

$\begin{array}{ll}-7.51 \quad 286.53 \\ -7.65 & 293.91\end{array}$

$\begin{array}{ll}-7.65 & 293.91\end{array}$

$\begin{array}{ll}-8.00 \quad 302.87 \\ -7.96 & 310.59\end{array}$

$\begin{array}{ll}-7.96 & 310.59\end{array}$

$-8.47 \quad 319.54$

$-8.31 \quad 327.53$

$0.03-0.01$

$\begin{array}{llllll}0.218 & 0.000 & -0.020 & 0.004 & -0.99\end{array}$

$-3.90 \quad 5.20$
-2.96

$-2.96 \quad 2.82$

$-2.16-1.18$

$-1.29-3.15$

$-0.71-6.90$

$-0.23 \quad-8.82$

$\begin{array}{lll}0.17 & -12.29\end{array}$

$\begin{array}{lll}0.52 & -13.90\end{array}$

$\begin{array}{lll}0.80 & -17.04\end{array}$

$\begin{array}{lll}1.00 & -18.37\end{array}$

$1.14-21.23$ 


\section{$Z=79$ (Au)}

\begin{tabular}{|c|c|c|c|c|c|c|c|c|c|c|c|c|c|c|c|c|}
\hline 105184 & 0.23 & 0.00 & 0.04 & 0.00 & 0.252 & 0.000 & -0.026 & -0.009 & -1.75 & 0.39 & 1453.84 & -30.52 & -30.32 & 0.022 & 0.37 & -30.66 \\
\hline $106 \quad 185$ & 0.20 & 0.00 & 0.04 & -0.01 & 0.218 & 0.000 & -0.032 & 0.002 & -1.72 & 0.20 & 1463.45 & -32.06 & -31.87 & 0.026 & 0.20 & -32.22 \\
\hline 7186 & 0.20 & 00 & 0.04 & -0.01 & 0.218 & 0.000 & -0.032 & 0.002 & -2.08 & -0.19 & 1471.46 & -32.00 & -31.72 & 0.021 & -0.20 & -32.21 \\
\hline 187 & -0.15 & 0.00 & 0.02 & 0.00 & -0.156 & 0.000 & -0.014 & 0.003 & -1.59 & -0.22 & 1480.54 & -33.01 & -33.01 & 0.025 & -0.22 & -33.25 \\
\hline 9188 & 0.15 & 0.00 & 0.01 & 0.00 & -0.156 & 0.000 & -0.003 & 0.001 & -1.92 & -0.47 & 488.06 & -32.46 & -32.30 & 0.020 & -0.48 & -32.74 \\
\hline 189 & -0.15 & 0.00 & 0.02 & 0.00 & -0.156 & 0.000 & -0.014 & 0.003 & -2.35 & -0.81 & 497.10 & -33.43 & -33.58 & 0.020 & -0.82 & -33.74 \\
\hline 1190 & -0.14 & 0.00 & 0.02 & 0.00 & -0.146 & 0.000 & -0.015 & 0.003 & -2.78 & -1.27 & 1504.48 & -32.74 & -32.88 & 0.016 & -1.28 & -33.08 \\
\hline 191 & -0.14 & 0.00 & 0.02 & 0.00 & -0.146 & 0.000 & -0.015 & 0.003 & -3.26 & -1.82 & 1513.38 & -33.56 & -33.81 & 0.037 & -1.82 & -33.92 \\
\hline 192 & -0.14 & 0.00 & 0.03 & 0.00 & -0.146 & 0.000 & -0.026 & 0.004 & -3.97 & -2.39 & 1520.54 & -32.66 & -32.78 & 0.016 & -2.39 & -33.04 \\
\hline 4193 & -0.12 & 0.00 & 0.02 & 0.00 & -0.125 & 0.000 & -0.017 & 0.003 & -4.30 & -2.93 & 1529.10 & -33.14 & -33.39 & 0.011 & -2.93 & -33.55 \\
\hline 194 & -0.12 & 0.00 & 0.03 & 0.00 & -0.125 & 0.000 & -0.029 & 0.004 & -5.04 & -3.54 & 1535.97 & -31.94 & -32.26 & 0.010 & -3.54 & -32.37 \\
\hline 6195 & -0.12 & 0.00 & 0.03 & 0.00 & -0.125 & 0.000 & -0.029 & 0.004 & -5.55 & -4.04 & 1544.16 & -32.06 & -32.57 & 0.001 & -4.03 & -32.49 \\
\hline 7196 & -0.12 & 0.00 & 0.03 & 0.00 & -0.125 & 0.000 & -0.029 & 0.004 & -6.30 & -4.93 & 1551.00 & -30.83 & .14 & 0.003 & -4.93 & -31.28 \\
\hline $8 \quad 197$ & -0.12 & 0.00 & 0.02 & -0.01 & -0.125 & 0.000 & -0.017 & 0.012 & -6.94 & -5.63 & 9.07 & -30.83 & & 0.001 & -5.62 & -31.29 \\
\hline 9198 & -0.11 & 0.00 & 0.02 & 0.00 & -0.115 & 0.000 & -0.018 & 0.002 & -7.61 & -6.41 & 1565.49 & -29.17 & -29.58 & 0.001 & -6.41 & -29.66 \\
\hline 199 & -0.11 & 0.00 & 0.02 & -0.01 & -0.115 & 0.000 & -0.018 & 0.012 & -8.33 & -7.09 & 73.23 & -28.84 & -29.09 & 0.001 & -7.07 & -29.31 \\
\hline 1200 & -0.09 & 0.00 & 0.02 & 0.00 & -0.094 & 0.000 & -0.020 & 0.002 & -9.07 & -8.02 & 9.51 & -27.05 & -2 & 0.050 & -8.01 & -27.53 \\
\hline 2201 & -0.08 & 0.00 & 0.03 & 0.01 & -0.084 & 0.000 & -0.032 & -0.007 & -9.78 & -8.64 & 1586.88 & -26.35 & -26.40 & 0.003 & -8.61 & -26.82 \\
\hline 3202 & -0.07 & 0.00 & 0.03 & 0.01 & -0.073 & 0.000 & -0 & -0.007 & 67 & -9.49 & & -24.19 & & & -9.47 & -24.66 \\
\hline 4203 & -0.06 & 0.00 & 0.03 & 0.01 & -0.063 & 0.000 & -0.033 & -0.007 & -11.26 & -10.03 & 1599.79 & -23.12 & -23.14 & 0.003 & -10.00 & -23.58 \\
\hline 204 & 0.00 & 0.00 & 0.00 & 0.00 & 0.000 & 000 & 0.000 & 0.000 & -11.88 & -10.70 & 25 & -20.50 & & & -10.71 & -20.98 \\
\hline 205 & 0.00 & 0.00 & 0.00 & 0.00 & 0.000 & 0.000 & 0.000 & 0.000 & -12.19 & -10.98 & 1.70 & -18.88 & & & -10.98 & -19.34 \\
\hline 7206 & -0.02 & 0.00 & 0.00 & 0.00 & -0.021 & & 0.000 & 0.000 & -11.19 & -10.07 & 5.30 & -14.41 & & & -10.07 & -14.85 \\
\hline 207 & 0.00 & 0.00 & 0.00 & 0.00 & 0.000 & 0.000 & 0.000 & 0.000 & -10.10 & -8.99 & & -11 & & & -8.99 & -11.58 \\
\hline 208 & 0.01 & 0.00 & 0.00 & 0.00 & 0.011 & 0.000 & 0.000 & & & -7.76 & & -6.09 & & & -7.76 & -6.49 \\
\hline 209 & 0.01 & 0.00 & 0.00 & 0.00 & .011 & 00 & 0.000 & 00 & 59 & -6.59 & .58 & -2.48 & & & -6.59 & -2.85 \\
\hline 210 & 0.01 & 0.00 & 0.00 & 0.00 & 0.011 & 00 & 0.000 & & & -5.51 & & 2.70 & & & -5.51 & 2.35 \\
\hline 211 & 0.02 & 0.00 & & 0.00 & & & & & & -4.45 & & & & & & 16 \\
\hline 12 & 0.04 & 0.00 & 0.00 & 0.00 & 0.043 & 00 & 0.001 & 0.000 & -4 & -3.69 & .74 & 11.57 & & & -3.70 & 11.30 \\
\hline 213 & -0.05 & 0.00 & 0.00 & 0.00 & -0.052 & 0.000 & 0.001 & 0.000 & -3.45 & -2.79 & 1641.94 & 1541 & & & -2.79 & 21 \\
\hline 214 & 0.07 & 0.04 & 0.02 & 0.00 & 0.075 & 0.055 & 0.027 & 0.003 & -3.24 & -1.95 & & 20.87 & & & -1.92 & 20.71 \\
\hline 15 & 0.08 & 0.04 & 0.02 & 0.00 & 0.086 & 55 & 0.027 & & & -1.25 & & & & & -1.22 & .68 \\
\hline 216 & 0.09 & 0.04 & -0.02 & 0.00 & & -0.055 & & & & -0.74 & & & & & -0.71 & 08 \\
\hline 17 & 0.10 & 0.03 & -0.02 & 0.00 & 0.107 & -0.041 & 0.029 & 0.004 & & -0.19 & .53 & & & & -0.17 & 34.13 \\
\hline 218 & 0.11 & 0.00 & -0.03 & 0.00 & 0.118 & 0.000 & 0.042 & 0.005 & -1.07 & 0.16 & 3.18 & 39.57 & & & 0.20 & 39.62 \\
\hline 219 & 0.11 & 0.00 & -0.03 & 0.00 & 0.118 & 0.000 & 0.042 & 0.005 & -0.63 & 0.57 & 1662.14 & 43.67 & & & 0.61 & 43.80 \\
\hline 220 & 0.15 & 0.00 & -0.05 & 0.00 & 51 & & 71 & & & 0.77 & & & & & 0.88 & 49.43 \\
\hline 221 & 0.16 & 0.00 & -0.04 & 0.00 & 172 & 00 & 60 & & & 1.01 & 62 & & & & 1.09 & 64 \\
\hline 222 & 0.17 & 0.00 & -0.04 & 0.00 & & & 0.062 & & -1.30 & 0.73 & 1671.45 & & & & 0.80 & 58.95 \\
\hline 14223 & 0.18 & 0.00 & -0.04 & 0.01 & 0.194 & 0.000 & 0.064 & 0.001 & -1.23 & 0.88 & 1675.22 & 62.88 & & & 0.96 & 63.34 \\
\hline 224 & 0.19 & 0.00 & -0.03 & 0.01 & 205 & 000 & 053 & -0.001 & -1.38 & 0.63 & 7.80 & 68.37 & & & 0.68 & 68.88 \\
\hline 225 & 19 & 0.00 & -0.03 & 0.01 & & & & -( & & 0.73 & & & & & 0.79 & 73.45 \\
\hline 7226 & 0.20 & 0.00 & -0.02 & 0.01 & 216 & 00 & 0.043 & -0.003 & 54 & 0.44 & 81 & 78.51 & & & 0.46 & 79.16 \\
\hline 227 & 0.22 & 0.00 & -0.01 & 0.02 & & & 0.035 & -0.015 & -1.74 & 0.37 & 7.36 & 83.03 & & & 0.43 & 83.82 \\
\hline $49 \quad 228$ & 0.22 & 0.00 & 0.00 & 0.02 & 0.239 & 0.000 & 0.023 & -0.018 & -2.15 & -0.07 & 1689.70 & 88.75 & & & -0.02 & 89.63 \\
\hline 229 & 0.22 & 0.00 & 0.00 & 0.02 & 239 & 00 & 023 & -0.018 & -2.18 & -0.13 & .03 & .49 & & & -0.07 & 4.48 \\
\hline 230 & 0.22 & 0.00 & 0.01 & 0.02 & & & & -0.0 & & -0.55 & & & & & -0.50 & 0.52 \\
\hline 2231 & 0.22 & 0.00 & 0.02 & 0.01 & 0.240 & 0.000 & -0.002 & -0.013 & -2.40 & -0.41 & 1698.09 & 104.57 & & & -0.39 & 105.74 \\
\hline 232 & 0.22 & 0.00 & 0.02 & 0.01 & & & .002 & -0.013 & & -0.63 & 9.83 & 10.91 & & & -0.62 & 12.19 \\
\hline 154233 & 0.22 & 0.00 & 0.03 & 0.01 & 0.241 & 0.000 & -0.014 & -0.016 & -2.47 & -0.47 & 1702.54 & 116.28 & & & -0.43 & 117.71 \\
\hline 234 & 0.22 & 0.00 & 0 & 0.01 & $T 1$ & 00 & -0.014 & -0.016 & -2.65 & -0.67 & 4.06 & 122.83 & & & -0 & 24.37 \\
\hline 6235 & 0.19 & 0.00 & 0.03 & 0.00 & & & -0.021 & -0.006 & & -0.50 & & 128.40 & & & -0.48 & 130.06 \\
\hline $77 \quad 236$ & 0.19 & 0.00 & 0.04 & 0.00 & 0.207 & 0.000 & -0.033 & -0.008 & -2.63 & -0.90 & 1708.10 & 134.93 & & & -0.86 & 136.74 \\
\hline 237 & 0.18 & 0.00 & 0.04 & 0.00 & 0.196 & 0.000 & -0.034 & -0.007 & -2.59 & -0.95 & 1710.63 & 140.47 & & & -0.90 & 142.43 \\
\hline $59 \quad 238$ & 0.19 & 0.00 & 0.05 & -0.01 & 0.208 & 0.000 & -0.046 & -0.000 & -3.39 & -1.50 & 1712.12 & 147.04 & & & -1.41 & 149.18 \\
\hline 239 & 0.19 & 0.00 & 0.06 & 0.01 & & & -0.058 & -0.002 & -3.80 & -1.68 & .60 & 152.64 & & & -1.53 & 154.99 \\
\hline 240 & 0.18 & 0.00 & 0.06 & -0.01 & 0.197 & 0.000 & -0.059 & -0.002 & -4.24 & -2.20 & 1715.90 & 159.42 & & & -2.05 & 161.92 \\
\hline
\end{tabular}


$Z=79$ (Au)

$\begin{array}{llllll}162 & 241 & 0.19 & 0.00 & 0.07 & -0.01\end{array}$

$\begin{array}{lllllll}163 & 242 & 0.18 & 0.00 & 0.07 & -0.02\end{array}$

$\begin{array}{lllllll}164 & 243 & 0.17 & 0.00 & 0.07 & -0.02\end{array}$

$\begin{array}{lllllll}165 & 244 & 0.16 & 0.00 & 0.07 & -0.02\end{array}$

$\begin{array}{llll}0.209 & 0.000 & -0.070 & -0.004\end{array}$

$\begin{array}{llll}0.198 & 0.000 & -0.072 & 0.006\end{array}$

$\begin{array}{lll}0.186 & 0.000 & -0.074\end{array}$

$\begin{array}{llll}0.175 & 0.000 & -0.075\end{array}$

0.007

$\begin{array}{llll}-4.77 & -2.40 & 1718.21 & 165.18\end{array}$

$\begin{array}{llll}-5.03 & -2.76 & 1719.16 & 172.29\end{array}$

$\begin{array}{llll}0.141 & 0.000 & -0.054\end{array}$

0.008

$\begin{array}{lll}-4.75 & -2.63 & 1720.97\end{array}$

178.55

$\begin{array}{llllll}166 & 245 & 0.13 & 0.00 & 0.05 & -0.01\end{array}$

$\begin{array}{lll}0.130 & 0.000 & -0.054\end{array}$

$\begin{array}{lllllll}167 & 246 & 0.12 & 0.00 & 0.05 & -0.01\end{array}$

0.003

$\begin{array}{llll}-4.91 & -2.68 & 1721.45 & 186.15 \\ -3.92 & -2.41 & 1722.93 & 192.73\end{array}$

$\begin{array}{lllll}0.003 & -4.41 & -2.90 & 1723.69 & 200.05\end{array}$

$\begin{array}{lllllllll}168 & 247 & -0.13 & 0.00 & 0.01 & -0.01 & -0.135 & 0.000 & -0.004\end{array}$

$\begin{array}{lllllllll}169 & 248 & -0.13 & 0.00 & 0.01 & -0.01 & -0.135 & 0.000 & -0.004\end{array}$

$\begin{array}{lllllllll}170 & 249 & -0.13 & 0.00 & 0.01 & -0.01 & -0.135 & 0.000 & -0.004\end{array}$

$\begin{array}{llllllllll}171 & 250 & -0.13 & 0.00 & 0.01 & -0.01 & -0.135 & 0.000 & -0.004\end{array}$

0.011

$\begin{array}{llll}-4.29 & -3.16 & 1725.54 & 206.27\end{array}$

$\begin{array}{lllll}0.011 & -4.98 & -3.88 & 1726.36 & 213.52\end{array}$

$\begin{array}{lllll}0.011 & -5.38 & -4.29 & 1728.19 & 219.77\end{array}$

$\begin{array}{llllllllll}172 & 251 & -0.12 & 0.00 & 0.01 & -0.02 & -0.125 & 0.000 & -0.005\end{array}$

$\begin{array}{lllllllll}173 & 252 & -0.12 & 0.00 & 0.02 & -0.01 & -0.125 & 0.000 & -0.017\end{array}$

$\begin{array}{lllllllll}174 & 253 & -0.12 & 0.00 & 0.02 & -0.01 & -0.125 & 0.000 & -0.017\end{array}$

0.011

$\begin{array}{llll}-6.10 & -4.99 & 1728.83 & 227.20\end{array}$

$\begin{array}{lllll}0.020 & -6.42 & -5.41 & 1730.51 & 233.59\end{array}$

$\begin{array}{lllll}0.012 & -7.14 & -6.09 & 1730.98 & 241.19\end{array}$

$\begin{array}{lllll}0.012 & -7.36 & -6.33 & 1732.32 & 247.92\end{array}$

$\begin{array}{lllllllll}175 & 254 & -0.10 & 0.00 & 0.02 & -0.01 & -0.105 & 0.000 & -0.019\end{array}$

$\begin{array}{lllll}176 & 255 & -0.10 & 0.00 & 0.03\end{array}$

$\begin{array}{lllll}0.00 & -0.105 & 0.000 & -0.031\end{array}$

0.012

$\begin{array}{llll}-7.36 & -6.33 & 1732.32 & 247.92 \\ -8.01 & -7.14 & 1732.76 & 255.55\end{array}$

0.004

$\begin{array}{lllll}256 & -0.10 & 0.00 & 0.04\end{array}$ $\begin{array}{lllll}178 & 257 & -0.10 & 0.00 & 0.04\end{array}$ $\begin{array}{lllll}179 & 258 & -0.08 & 0.00 & 0.03\end{array}$ $\begin{array}{lllll}180 & 259 & -0.07 & 0.00 & 0.04\end{array}$ $\begin{array}{lllll}181 & 260 & -0.07 & 0.00 & 0.03\end{array}$

$\begin{array}{lllll}182 & 261 & 0.00 & 0.00 & 0.00\end{array}$ $\begin{array}{lllll}183 & 262 & -0.01 & 0.00 & 0.00\end{array}$ $\begin{array}{lllll}184 & 263 & 0.00 & 0.00 & 0.00\end{array}$

$\begin{array}{lllll}0.00 & -0.105 & 0.000 & -0.042\end{array}$

$\begin{array}{llll}0.00 & -0.105 & 0.000 & -0.042\end{array}$

$\begin{array}{lllll}0.00 & -0.084 & 0.000 & -0.032\end{array}$

0.005

$\begin{array}{llllll}0.01 & -0.073 & 0.000 & -0.044 & -0.006\end{array}$

$\begin{array}{lllll}0.01 & -0.073 & 0.000 & -0.033\end{array}$

$\begin{array}{lll}0.00 & 0.000 & 0.000\end{array}$

0.000

$\begin{array}{llll}0.00 & -0.011 & 0.000\end{array}$

0.000

$\begin{array}{llll}0.00 & 0.000 & 0.000 & 0.000\end{array}$

$$
-0.007-10
$$

$\begin{array}{llll}-9.30 & -8.21 & 1734.26 & 270.19\end{array}$

$\begin{array}{llll}-9.43 & -8.34 & 1735.18 & 277.34\end{array}$

$\begin{array}{llll}-9.60 & -8.67 & 1734.85 & 285.74\end{array}$

$\begin{array}{llll}-9.76 & -8.72 & 1735.55 & 293.12\end{array}$

$\begin{array}{llllll}0.000 & -9.97 & -9.02 & 1735.54 & 309.27\end{array}$

$\begin{array}{llllll}0.000 & -10.72 & -9.73 & 1735.31 & 317.57\end{array}$

$\begin{array}{lllll}0.000 & -10.73 & -9.73 & 1735.66 & 325.29\end{array}$
$-2.19 \quad 167.90$

$\begin{array}{ll}-2.50 & 175.22\end{array}$

$-2.36 \quad 181.67$

$-2.39 \quad 189.44$

$-2.27 \quad 196.05$

$-2.76 \quad 203.55$

$-3.14 \quad 209.84$

$\begin{array}{ll}-3.87 & 217.27\end{array}$

$-4.27 \quad 223.71$

$-4.97 \quad 231.34$

$-5.30 \quad 238.02$

$\begin{array}{ll}-6.05 & 245.77\end{array}$

$-6.29 \quad 252.71$

$\begin{array}{ll}-7.09 & 260.57\end{array}$

$\begin{array}{ll}-7.39 & 267.61\end{array}$

$\begin{array}{ll}-8.10 & 275.71\end{array}$

$\begin{array}{ll}-8.23 & 283.10\end{array}$

$-8.61 \quad 291.68$

$-8.58 \quad 299.38$

$-9.08 \quad 308.01$

$-9.02 \quad 315.89$
$-9.73 \quad 324.45$

$-9.73 \quad 324.45$

$-9.73 \quad 332.43$

\section{$Z=80$ (Hg)}

$\begin{array}{lllll}90 & 170 & -0.09 & 0.00 & 0.02\end{array}$ $\begin{array}{lllll}91 & 171 & -0.10 & 0.00 & 0.02\end{array}$ $\begin{array}{lllll}92 & 172 & -0.10 & 0.00 & 0.02\end{array}$ $\begin{array}{lllll}93 & 173 & -0.10 & 0.00 & 0.02\end{array}$ $\begin{array}{lllll}94 & 174 & -0.10 & 0.00 & 0.02\end{array}$ $\begin{array}{lllll}95 & 175 & -0.11 & 0.00 & 0.03\end{array}$ $\begin{array}{lllll}96 & 176 & -0.11 & 0.00 & 0.03\end{array}$ $\begin{array}{llllll}97 & 177 & -0.11 & 0.00 & 0.02\end{array}$ $\begin{array}{lllll}98 & 178 & -0.12 & 0.00 & 0.02\end{array}$ $\begin{array}{lllll}99 & 179 & -0.13 & 0.00 & 0.02\end{array}$

$\begin{array}{lllll}100 & 180 & -0.13 & 0.00 & 0.01\end{array}$ $\begin{array}{lllll}101 & 181 & -0.14 & 0.00 & 0.01\end{array}$ $\begin{array}{lllll}102 & 182 & -0.14 & 0.00 & 0.01\end{array}$ $\begin{array}{lllll}103 & 183 & -0.14 & 0.00 & 0.01\end{array}$ $\begin{array}{lllll}104 & 184 & -0.14 & 0.00 & 0.01\end{array}$ $\begin{array}{lllll}105 & 185 & -0.14 & 0.00 & 0.01\end{array}$ $\begin{array}{lllll}106 & 186 & -0.14 & 0.00 & 0.01\end{array}$ $\begin{array}{lllll}107 & 187 & -0.14 & 0.00 & 0.02\end{array}$ $\begin{array}{lllll}108 & 188 & -0.14 & 0.00 & 0.02\end{array}$ $\begin{array}{lllll}109 & 189 & -0.14 & 0.00 & 0.02\end{array}$

$\begin{array}{lllll}110 & 190 & -0.12 & 0.00 & 0.02\end{array}$ $\begin{array}{lllll}111 & 191 & -0.12 & 0.00 & 0.03\end{array}$ $\begin{array}{lllll}112 & 192 & -0.12 & 0.00 & 0.03\end{array}$ $\begin{array}{lllll}113 & 193 & -0.12 & 0.00 & 0.03\end{array}$ $\begin{array}{lllll}114 & 194 & -0.12 & 0.00 & 0.03\end{array}$ $\begin{array}{lllll}115 & 195 & -0.12 & 0.00 & 0.03\end{array}$ $\begin{array}{lllll}116 & 196 & -0.11 & 0.00 & 0.03\end{array}$ $\begin{array}{lllll}117 & 197 & -0.12 & 0.00 & 0.03\end{array}$ $\begin{array}{lllll}118 & 198 & -0.11 & 0.00 & 0.02\end{array}$ $\begin{array}{lllll}119 & 199 & -0.11 & 0.00 & 0.03\end{array}$ $\begin{array}{lllll}120 & 200 & -0.09 & 0.00 & 0.02\end{array}$ $\begin{array}{lllll}121 & 201 & -0.08 & 0.00 & 0.02\end{array}$ $\begin{array}{lllll}122 & 202 & -0.07 & 0.00 & 0.03\end{array}$ $\begin{array}{lllll}0.00 & -0.094 & 0.000 & -0.020 & 0.002\end{array}$

$\begin{array}{llllll}0.01 & -0.105 & 0.000 & -0.019 & -0.007\end{array}$

$\begin{array}{llllll}0.00 & -0.105 & 0.000 & -0.019 & 0.002\end{array}$

$\begin{array}{lllll}0.00 & -0.105 & 0.000 & -0.019\end{array}$

$\begin{array}{lllll}0.00 & -0.105 & 0.000 & -0.019\end{array}$

0.002

0.002

$\begin{array}{lllll}0.00 & -0.115 & 0.000 & -0.030 & 0.004\end{array}$

$\begin{array}{llllll}0.00 & -0.115 & 0.000 & -0.030 & 0.004\end{array}$

$\begin{array}{llllll}0.01 & -0.115 & 0.000 & -0.019 & -0.007\end{array}$

$\begin{array}{llllll}0.00 & -0.125 & 0.000 & -0.017 & 0.003\end{array}$

$\begin{array}{llllll}0.01 & -0.135 & 0.000 & -0.017 & -0.007\end{array}$

$\begin{array}{llllll}0.01 & -0.135 & 0.000 & -0.005 & -0.008\end{array}$

$\begin{array}{llllll}0.01 & -0.146 & 0.000 & -0.004 & -0.008\end{array}$

$\begin{array}{llllll}0.00 & -0.146 & 0.000 & -0.004 & 0.001\end{array}$

$\begin{array}{llllll}0.01 & -0.146 & 0.000 & -0.004 & -0.008\end{array}$

$\begin{array}{llllll}0.00 & -0.146 & 0.000 & -0.004 & 0.001\end{array}$

$\begin{array}{lllll}0.00 & -0.146 & 0.000 & -0.004\end{array}$

0.001

$\begin{array}{lllll}0.00 & -0.146 & 0.000 & -0.004\end{array}$

0.001

$\begin{array}{lllll}0.00 & -0.146 & 0.000 & -0.015\end{array}$

$\begin{array}{lllll}0.00 & -0.146 & 0.000 & -0.015\end{array}$

$\begin{array}{lllll}0.00 & -0.146 & 0.000 & -0.015\end{array}$

0.003

0.003

0.003

$\begin{array}{llll}0.00 & -0.125 & 0.000 & -0.017\end{array}$

$\begin{array}{lllll}0.00 & -0.125 & 0.000 & -0.029\end{array}$

$\begin{array}{lllll}0.00 & -0.125 & 0.000 & -0.029\end{array}$

$\begin{array}{lllll}0.00 & -0.125 & 0.000 & -0.029\end{array}$

$\begin{array}{lllll}0.00 & -0.125 & 0.000 & -0.029\end{array}$

0.003

0.004

0.004

0.004

$\begin{array}{lllll}0.00 & -0.125 & 0.000 & -0.029\end{array}$

$\begin{array}{lllll}0.00 & -0.115 & 0.000 & -0.030\end{array}$

$\begin{array}{lllll}0.00 & -0.125 & 0.000 & -0.029\end{array}$

$\begin{array}{lllll}0.00 & -0.115 & 0.000 & -0.018\end{array}$

$\begin{array}{lllll}0.00 & -0.115 & 0.000 & -0.030\end{array}$

0.004

0.004

0.004

0.004

0.002

$\begin{array}{lllll}0.00 & -0.094 & 0.000 & -0.020\end{array}$

0.004

$\begin{array}{lllll}0.00 & -0.084 & 0.000 & -0.021\end{array}$

0.002

$\begin{array}{lllll}0.01 & -0.073 & 0.000 & -0.033\end{array}$

0.002 $\begin{array}{lll}-3.71 & -2.67 & 1304.17\end{array}$

$\begin{array}{lll}-2.97 & -1.88 & 1314.29\end{array}$

5.37

$\begin{array}{llll}-1.59 & -0.55 & 1336.25 & -2.50\end{array}$

$\begin{array}{lll}-1.10 & -0.08 & 1348.14\end{array}$

$-6.32$

$-0.77$

$0.41 \quad 1357.69$

$-7.79$

$-0.42$

$13.28-11.32-11.78$

$\begin{array}{lllll}0.09 & 1.16 & 1389.95 & -15.84 & -16.32\end{array}$

$\begin{array}{lllll}0.15 & 1.29 & 1399.01 & -16.83 & -16.92\end{array}$

0.23

$\begin{array}{lllll}1.32 & 1410.05 & -19.80 & -20.25\end{array}$

0.15

$\begin{array}{llll}1.33 & 1418.84 & -20.52 & -20.66\end{array}$

$\begin{array}{llllll}0.00 & 1.16 & 1429.67 & -23.28 & -23.58\end{array}$

$\begin{array}{llllll}-0.11 & 1.08 & 1438.17 & -23.70 & -23.80\end{array}$

$\begin{array}{llllll}-0.39 & 0.78 & 1448.73 & -26.20 & -26.35\end{array}$

$-0.62$

$\begin{array}{llll}0.56 & 1456.98 & -26.38 & -26.18\end{array}$

$\begin{array}{llllll}-0.93 & 0.26 & 1467.18 & -28.50 & -28.54\end{array}$

$\begin{array}{lllll}-1.24 & -0.00 & 1475.11 & -28.36 & -28.12\end{array}$

$\begin{array}{llllll}-1.62 & -0.38 & 1485.00 & -30.18 & -30.20\end{array}$

$\begin{array}{llllll}-1.94 & -0.70 & 1492.64 & -29.75 & -29.63\end{array}$

$\begin{array}{llllll}-2.25 & -1.18 & 1502.28 & -31.31 & -31.37\end{array}$

$\begin{array}{llllll}-2.79 & -1.58 & 1509.65 & -30.62 & -30.59\end{array}$

$\begin{array}{llllll}-3.35 & -2.12 & 1518.99 & -31.89 & -32.01\end{array}$

$\begin{array}{llllll}-3.89 & -2.64 & 1526.15 & -30.98 & -31.05\end{array}$

$\begin{array}{llllll}-4.52 & -3.24 & 1535.21 & -31.97 & -32.19\end{array}$

$\begin{array}{llllll}-5.18 & -3.88 & 1542.16 & -30.84 & -31.00\end{array}$

$\begin{array}{llllll}-5.82 & -4.58 & 1550.99 & -31.60 & -31.83\end{array}$

$\begin{array}{llllll}-6.64 & -5.28 & 1557.69 & -30.23 & -30.54\end{array}$

$\begin{array}{llllll}-7.20 & -6.01 & 1566.23 & -30.70 & -30.95\end{array}$

$\begin{array}{llllll}-8.09 & -6.77 & 1572.68 & -29.08 & -29.55\end{array}$

$\begin{array}{llllll}-8.56 & -7.51 & 1580.91 & -29.23 & -29.50\end{array}$

$\begin{array}{llllll}-9.50 & -8.48 & 1587.27 & -27.52 & -27.66\end{array}$

$\begin{array}{rrrrr}-9.50 & -8.48 & 1587.27 & -27.52 & -27.66 \\ -10.31 & -9.14 & 1595.12 & -27.31 & -27.35\end{array}$ $\begin{array}{rrr} & -2.68 & 6.32 \\ & -1.89 & 4.19 \\ 0.209 & -1.22 & 0.04 \\ & -0.56 & -1.78 \\ 0.020 & -0.09 & -5.67 \\ 0.101 & 0.39 & -7.22\end{array}$

$\begin{array}{lll}0.014 & 0.71 & -10.81\end{array}$

$\begin{array}{lll}0.075 & 0.96 & -12.16\end{array}$

$\begin{array}{lll}0.013 & 1.15 & -15.46\end{array}$

$\begin{array}{lll}0.027 & 1.28 & -16.52\end{array}$

$\begin{array}{lll}0.014 & 1.31 & -19.54\end{array}$

$\begin{array}{lll}0.015 & 1.31 & -20.33\end{array}$

$\begin{array}{lll}0.010 & 1.15 & -23.14\end{array}$

$\begin{array}{lll}0.008 & 1.06 & -23.62\end{array}$

$\begin{array}{lll}0.010 & 0.77 & -26.16\end{array}$

$\begin{array}{lll}0.016 & 0.55 & -26.40\end{array}$

$\begin{array}{lll}0.011 & 0.25 & -28.56\end{array}$

$\begin{array}{llll}0.014 & -0.01 & -28.47\end{array}$

$0.012-0.38-30.33$

$\begin{array}{lll}0.033 & -0.70 & -29.93\end{array}$

$\begin{array}{lll}0.016 & -1.18 & -31.53\end{array}$

$\begin{array}{llll}0.023 & -1.58 & -30.87\end{array}$

$\begin{array}{lll}0.016 & -2.12 & -32.17\end{array}$

$\begin{array}{llll}0.015 & -2.64 & -31.29\end{array}$

$0.013-3.23-32.30$

$\begin{array}{lll}0.023 & -3.87 & -31.20\end{array}$

$\begin{array}{lll}0.003 & -4.57 & -31.98\end{array}$

$\begin{array}{llll}0.003 & -5.27 & -30.63\end{array}$

$\begin{array}{llll}0.000 & -6.01 & -31.12\end{array}$

$\begin{array}{llll}0.000 & -6.76 & -29.50\end{array}$

$\begin{array}{lll}0.000 & -7.50 & -29.67\end{array}$

$\begin{array}{llll}0.001 & -8.47 & -27.97\end{array}$

$\begin{array}{llll}0.001 & -9.12 & -27.74\end{array}$ 


\section{$Z=80(\mathbf{H g})$}

$\begin{array}{llll}123 & 203 & -0.07 & 0.00\end{array}$ $\begin{array}{llll}124 & 204 & -0.05 & 0.00\end{array}$ $\begin{array}{llll}125 & 205 & 0.00 & 0.00\end{array}$ $\begin{array}{llll}126 & 206 & 0.00 & 0.00\end{array}$ $\begin{array}{llll}127 & 207 & -0.01 & 0.00\end{array}$ $\begin{array}{llll}128 & 208 & 0.00 & 0.00\end{array}$ $\begin{array}{llll}129 & 209 & 0.01 & 0.00\end{array}$ $\begin{array}{llll}130 & 210 & 0.00 & 0.00\end{array}$ $\begin{array}{llll}131 & 211 & 0.00 & 0.00\end{array}$ $\begin{array}{lllll}132 & 212 & 0.00 & 0.00 & 0.00\end{array}$ $\begin{array}{lllll}133 & 213 & 0.00 & 0.00 & 0.00\end{array}$ $\begin{array}{lllll}134 & 214 & -0.04 & 0.00 & 0.00\end{array}$ $\begin{array}{lllll}135 & 215 & 0.05 & 0.06 & -0.01\end{array}$ $\begin{array}{lllll}136 & 216 & 0.06 & 0.05 & -0.01\end{array}$ $\begin{array}{llllll}137 & 217 & 0.07 & 0.06 & -0.02\end{array}$

$\begin{array}{lllll}138 & 218 & 0.08 & 0.05 & -0.02\end{array}$ $\begin{array}{lllll}139 & 219 & 0.09 & 0.05 & -0.02\end{array}$ $\begin{array}{llllll}140 & 220 & 0.10 & 0.03 & -0.03\end{array}$ $\begin{array}{llllll}141 & 221 & 0.15 & 0.00 & -0.05\end{array}$ $\begin{array}{llllll}142 & 222 & 0.16 & 0.00 & -0.05\end{array}$

$\begin{array}{llllll}143 & 223 & 0.17 & 0.00 & -0.04\end{array}$ $\begin{array}{llllll}144 & 224 & 0.18 & 0.00 & -0.04\end{array}$ $\begin{array}{llllll}145 & 225 & 0.19 & 0.00 & -0.04\end{array}$ $\begin{array}{llllll}146 & 226 & 0.19 & 0.00 & -0.03\end{array}$ $\begin{array}{lllll}147 & 227 & 0.20 & 0.00 & -0.03\end{array}$ $148 \quad 228$ 149229 $\begin{array}{llll}0.22 & 0.00 & -0.01\end{array}$ 150230 $\begin{array}{lll}0.22 & 0.00 & -0.01\end{array}$ $\begin{array}{lll}0.22 & 0.00 & 0.00\end{array}$ $\begin{array}{lllll}151 & 231 & 0.22 & 0.00 & 0.01\end{array}$ $\begin{array}{lllll}152 & 232 & 0.22 & 0.00 & 0.01\end{array}$

$\begin{array}{lllll}153 & 233 & 0.22 & 0.00 & 0.02\end{array}$ $\begin{array}{lllll}154 & 234 & 0.22 & 0.00 & 0.02\end{array}$ 155235 156236 $\begin{array}{lll}0.22 & 0.00\end{array}$ 0.03 $0.20 \quad 0.00$ 0.03

$\begin{array}{ll}158 & 238\end{array}$ $0.19 \quad 0.00$ 0.04

159239 $0.19 \quad 0.00$ 0.04 160240 $\begin{array}{ll}0.19 & 0.00\end{array}$ $\begin{array}{ll}161 & 241\end{array}$ $0.18 \quad 0.00$ $162 \quad 242$ $\begin{array}{ll}0.19 & 0.00\end{array}$ 0.05 $0.00 \quad 0.208$ $\begin{array}{lll}0.05 & -0.01 & 0.197\end{array}$ $\begin{array}{lll}0.06 & -0.01 \quad 0.208\end{array}$ $\begin{array}{llll}0.06 & -0.01 & 0.197\end{array}$ 163243 $0.18 \quad 0.00$

164244 $\begin{array}{ll}0.17 & 0.00\end{array}$ $\begin{array}{lll}0.16 & 0.00 \\ 0.16 & 0.00\end{array}$ $\begin{array}{llll}166 & 246 & -0.13 & 0.00\end{array}$ $\begin{array}{llll}167 & 247 & -0.13 & 0.00\end{array}$

$\begin{array}{llll}168 & 248 & -0.13 & 0.00\end{array}$ $\begin{array}{llll}169 & 249 & -0.13 & 0.00\end{array}$ $\begin{array}{llll}170 & 250 & -0.13 & 0.00\end{array}$ $\begin{array}{llll}171 & 251 & -0.13 & 0.00\end{array}$ $\begin{array}{llll}172 & 252 & -0.12 & 0.00\end{array}$ $\begin{array}{lll}0.07 & -0.01 \quad 0.187\end{array}$ $\begin{array}{lll}0.06 & -0.01 \quad 0.175\end{array}$ $\begin{array}{llll}0.06 & -0.02 & 0.175\end{array}$ $\begin{array}{llll}0.02 & 0.00 & -0.136\end{array}$ $\begin{array}{llll}0.02 & 0.00 & -0.136\end{array}$

$\begin{array}{lll}0.01 & 0.00 & -0.135\end{array}$ $\begin{array}{llll}0.01 & 0.00 & -0.135\end{array}$ $\begin{array}{llll}0.01 & -0.01 & -0.135\end{array}$ $\begin{array}{llll}0.01 & -0.01 & -0.135\end{array}$ $\begin{array}{lll}0.02 & -0.01 & -0.125\end{array}$

$\begin{array}{llll}173 & 253 & -0.12 & 0.00\end{array}$ $\begin{array}{llll}174 & 254 & -0.10 & 0.00\end{array}$ $\begin{array}{llll}175 & 255 & -0.10 & 0.00\end{array}$ $\begin{array}{llll}176 & 256 & -0.10 & 0.00\end{array}$ $\begin{array}{llll}177 & 257 & -0.10 & 0.00\end{array}$

$\begin{array}{lll}0.02 & 0.00 & -0.125\end{array}$

$\begin{array}{llll}0.02 & 0.00 & -0.105\end{array}$

$\begin{array}{llll}0.03 & 0.00 & -0.105\end{array}$

$0.03 \quad 0.00-0.105$

$\begin{array}{llll}0.04 & 0.00 & -0.105\end{array}$

$\begin{array}{lll}0.04 & 0.00 & -0.105\end{array}$

$\begin{array}{llll}178 & 258 & -0.10 & 0.00\end{array}$ $\begin{array}{llll}179 & 259 & -0.08 & 0.00\end{array}$
$0.03 \quad 0.00 \quad-0.084$ $\begin{array}{lllllllllll}0.000 & -0.033 & -0.007 & -11.24 & -10.01 & 1601.09 & -25.20 & -25.27 & 0.002 & -9.98 & -25.64\end{array}$

$\begin{array}{lllllllllll}0.000 & -0.022 & -0.008 & -11.69 & -10.51 & 1608.48 & -24.52 & -24.69 & 0.000 & -10.49 & -24.96\end{array}$

$\begin{array}{llllllllllll}0.000 & 0.000 & 0.000 & -12.56 & -11.33 & 1614.12 & -22.09 & -22.29 & 0.004 & -11.33 & -22.54\end{array}$

$\begin{array}{lllllllllll}0.000 & 0.000 & 0.000 & -12.87 & -11.60 & 1620.99 & -20.88 & -20.95 & 0.020 & -11.60 & -21.33\end{array}$

$\begin{array}{llllllllllll}0.000 & 0.000 & 0.000 & -11.85 & -10.66 & 1624.59 & -16.41 & -16.22 & 0.150 & -10.66 & -16.85\end{array}$

$\begin{array}{lllllll}0.000 & 0.000 & 0.000 & -10.80 & -9.63 & 1629.88 & -13.63\end{array}$

$\begin{array}{lllllll}0.000 & 0.000 & 0.000 & -9.48 & -8.37 & 1632.89 & -8.57\end{array}$

$\begin{array}{lllllll}0.000 & 0.000 & 0.000 & -8.27 & -7.24 & 1637.79 & -5.40\end{array}$

$\begin{array}{lllllll}0.000 & 0.000 & 0.000 & -7.09 & -6.14 & 1640.71 & -0.25\end{array}$

$\begin{array}{lllllll}0.000 & 0.000 & 0.000 & -5.97 & -5.07 & 1645.41 & 3.12\end{array}$

$\begin{array}{lllllll}0.000 & 0.000 & 0.000 & -4.90 & -4.08 & 1648.18 & 8.42\end{array}$

$\begin{array}{lllll}0.000 & -3.97 & -3.24 & 1652.85 & 11.82 \\ 0.003 & -3.88 & -2.32 & 1655.44 & 17.30\end{array}$

$\begin{array}{llllll}0.014 & 0.003 & -3.88 & -2.32 & 1655.44 & 17.30 \\ 0.014 & 0.002 & -2.90 & -1.58 & 1659.96 & 20.85\end{array}$

$\begin{array}{llllll}0.027 & 0.004 & -2.66 & -1.05 & 1662.70 & 26.19\end{array}$

$-9.63-14.05$

$-8.37-8.97$

$-7.24-5.79$

$-6.14-0.60$

$-5.07 \quad 2.79$

$-4.08 \quad 8.12$

$-3.24 \quad 11.56$

$-2.28 \quad 17.12$

$-1.55 \quad 20.71$

$-1.00 \quad 26.10$

$-0.39 \quad 29.82$

$0.06 \quad 35.37$

$0.59 \quad 39.26$

$0.87 \quad 44.87$

$0.96 \quad 48.56$

$0.84 \quad 54.00$

$1.03 \quad 58.03$

$0.82 \quad 63.61$

$\begin{array}{ll}0.92 & 67.79\end{array}$

$0.63 \quad 73.51$

$0.60 \quad 77.78$

$\begin{array}{ll}0.17 & 83.57\end{array}$

$0.14 \quad 88.07$

$-0.24 \quad 94.12$

$-0.1198 .99$

$-0.30 \quad 105.44$

$\begin{array}{ll}-0.09 & 110.60\end{array}$

$-0.27 \quad 117.27$

$-0.10 \quad 122.60$

$-0.42 \quad 129.32$

$-0.46 \quad 134.64$

$\begin{array}{ll}-0.91 & 141.42\end{array}$

$-1.00 \quad 146.90$

$-1.50 \quad 153.82$

$-1.60 \quad 159.48$

$-1.92 \quad 166.76$

$-1.83 \quad 172.80$

$-1.88 \quad 180.54$

$-2.26 \quad 186.29$

$-2.90 \quad 193.61$

$-3.29199 .54$

$-4.00 \quad 206.97$

$-4.36 \quad 213.10$

$\begin{array}{ll}-5.05 & 220.72\end{array}$

$-5.40 \quad 227.04$

$\begin{array}{ll}-6.14 & 234.77\end{array}$

$-6.42 \quad 241.33$

$\begin{array}{ll}-7.20 & 249.19\end{array}$

$-7.52 \quad 255.88$

$-8.23 \quad 263.96$

$-8.34 \quad 271.02$ $-8.74 \quad 279.57$ 


\section{$Z=80(\mathbf{H g})$}

$\begin{array}{llll}180 & 260 & -0.07 & 0.00\end{array}$ $\begin{array}{llll}181 & 261 & 0.00 & 0.00\end{array}$ $\begin{array}{llll}182 & 262 & 0.00 & 0.00\end{array}$ $\begin{array}{lll}0.04 & 0.01 & -0.073\end{array}$ $\begin{array}{lll}0.00 & 0.00 & 0.000\end{array}$ $\begin{array}{lll}0.00 & 0.00 & 0.000\end{array}$ $\begin{array}{llll}183 & 263 & 0.00 & 0.00\end{array}$ $\begin{array}{lll}0.00 & 0.00 & 0.000\end{array}$ $\begin{array}{llll}184 & 264 & 0.00 & 0.00\end{array}$ $\begin{array}{lll}0.00 & 0.00 & 0.000\end{array}$

$\begin{array}{llll}185 & 265 & -0.01 & 0.00\end{array}$ $\begin{array}{lll}0.00 & 0.00 & -0.011\end{array}$ $\begin{array}{llll}186 & 266 & 0.00 & 0.00\end{array}$ $\begin{array}{lllllll}0.000 & -0.044 & -0.006 & -9.94 & -8.85 & 1754.94 & 281.02\end{array}$ $\begin{array}{lllllll}0.000 & 0.000 & 0.000 & -10.04 & -9.09 & 1754.37 & 289.65\end{array}$ $\begin{array}{lllllll}0.000 & 0.000 & 0.000 & -10.36 & -9.38 & 1755.49 & 296.61\end{array}$ $\begin{array}{lllllll}0.000 & 0.000 & 0.000 & -11.07 & -10.05 & 1755.21 & 304.95\end{array}$ $\begin{array}{lllllll}0.000 & 0.000 & 0.000 & -11.06 & -10.03 & 1755.88 & 312.36\end{array}$ $\begin{array}{lllllll}0.000 & 0.000 & 0.000 & -10.18 & -9.19 & 1753.96 & 322.35\end{array}$ $\begin{array}{lllllll}0.000 & 0.000 & 0.000 & -9.39 & -8.42 & 1753.73 & 330.65\end{array}$
$-8.72 \quad 286.93$

$-9.09 \quad 295.66$

$-9.38 \quad 302.86$

$-10.05 \quad 311.45$

$-10.03 \quad 319.11$

$-9.19 \quad 329.36$

$-8.42 \quad 337.93$

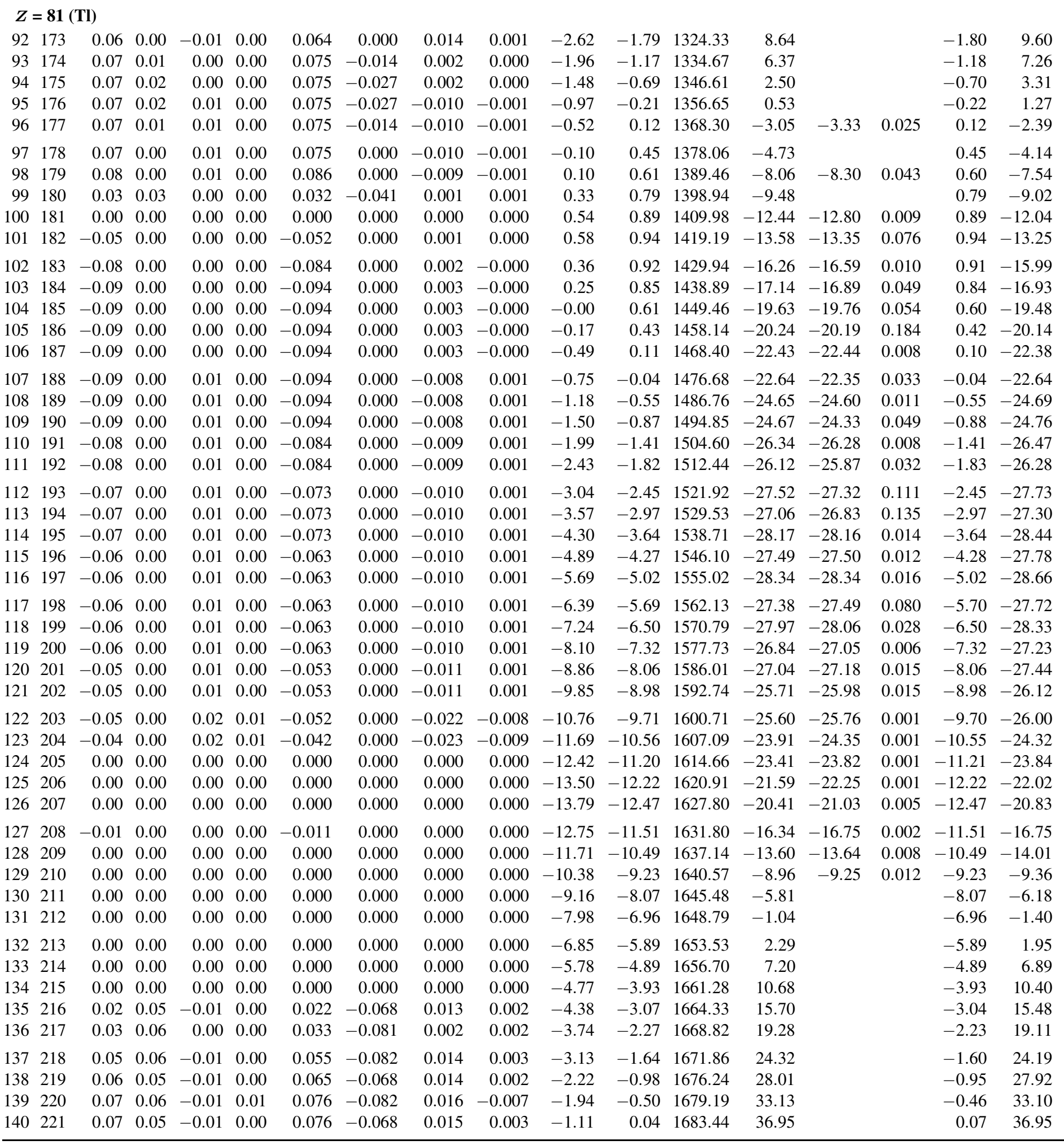




\begin{tabular}{|c|c|c|c|c|c|c|c|c|c|c|c|c|c|c|c|c|}
\hline \multicolumn{17}{|c|}{$Z=81(\mathrm{Tl})$} \\
\hline 141222 & 0.15 & 0.00 & -0.06 & 0.00 & 0.162 & 0.000 & 0.084 & 0.013 & -2.07 & 0.47 & 1686.21 & 42.25 & & & 0.61 & 42.42 \\
\hline 142223 & 0.16 & 0.00 & -0.05 & 0.00 & 0.172 & 0.000 & 0.073 & 0.012 & -1.51 & 0.76 & 1690.48 & 46.05 & & & 0.87 & 46.26 \\
\hline 143224 & 0.16 & 0.00 & -0.05 & 0.00 & 0.172 & 0.000 & 0.073 & 0.012 & -1.60 & 0.53 & 1693.68 & 50.93 & & & 0.64 & 51.19 \\
\hline 144225 & 0.17 & 0.00 & -0.05 & 0.01 & 0.183 & 0.000 & 0.075 & 0.002 & -1.41 & 0.74 & 1697.80 & 54.88 & & & 0.85 & 55.21 \\
\hline 145226 & 0.17 & 0.00 & -0.04 & 0.01 & 0.183 & 0.000 & 0.062 & -0.000 & -1.28 & 0.61 & 1700.67 & 60.08 & & & 0.69 & 60.44 \\
\hline 146227 & 0.19 & 0.00 & -0.03 & 0.01 & 0.205 & 0.000 & 0.053 & -0.001 & -1.09 & 0.80 & 1704.59 & 64.23 & & & 0.85 & 64.65 \\
\hline 147228 & 0.20 & 0.00 & -0.03 & 0.02 & 0.216 & 0.000 & 0.056 & -0.011 & -1.60 & 0.47 & 1707.44 & 69.46 & & & 0.55 & 69.97 \\
\hline $148 \quad 229$ & 0.20 & 0.00 & -0.02 & 0.02 & 0.216 & 0.000 & 0.043 & -0.013 & -1.39 & 0.51 & 1711.29 & 73.68 & & & 0.58 & 74.27 \\
\hline 149230 & 0.22 & 0.00 & -0.01 & 0.02 & 0.238 & 0.000 & 0.035 & -0.015 & -1.97 & 0.09 & 1714.02 & 79.01 & & & 0.13 & 79.67 \\
\hline $150 \quad 231$ & 0.22 & 0.00 & 0.00 & 0.02 & 0.239 & 0.000 & 0.023 & -0.018 & -1.88 & 0.08 & 1717.70 & 83.40 & & & 0.13 & 84.16 \\
\hline 151232 & 0.22 & 0.00 & 0.00 & 0.02 & 0.239 & 0.000 & 0.023 & -0.018 & -2.27 & -0.30 & 1720.19 & 88.99 & & & -0.25 & 89.83 \\
\hline $152 \quad 233$ & 0.22 & 0.00 & 0.01 & 0.02 & 0.240 & 0.000 & 0.011 & -0.021 & -2.09 & -0.17 & 1723.51 & 93.73 & & & -0.10 & 94.69 \\
\hline $153 \quad 234$ & 0.22 & 0.00 & 0.02 & 0.01 & 0.240 & 0.000 & -0.002 & -0.013 & -2.13 & -0.26 & 1725.52 & 99.80 & & & -0.25 & 100.81 \\
\hline 154235 & 0.22 & 0.00 & 0.02 & 0.01 & 0.240 & 0.000 & -0.002 & -0.013 & -1.87 & -0.06 & 1728.57 & 104.82 & & & -0.04 & 105.94 \\
\hline 155236 & 0.22 & 0.00 & 0.03 & 0.01 & 0.241 & 0.000 & -0.014 & -0.016 & -2.08 & -0.22 & 1730.44 & 111.03 & & & -0.19 & 112.27 \\
\hline 156237 & 0.19 & 0.00 & 0.02 & 0.01 & 0.206 & 0.000 & -0.008 & -0.013 & -1.48 & -0.05 & 1733.32 & 116.21 & & & -0.03 & 117.56 \\
\hline 157238 & 0.19 & 0.00 & 0.03 & 0.00 & 0.207 & 0.000 & -0.021 & -0.006 & -1.84 & -0.37 & 1735.17 & 122.44 & & & -0.37 & 123.90 \\
\hline $158 \quad 239$ & 0.18 & 0.00 & 0.04 & 0.00 & 0.196 & 0.000 & -0.034 & -0.007 & -1.88 & -0.30 & 1737.95 & 127.72 & & & -0.25 & 129.35 \\
\hline 159240 & 0.19 & 0.00 & 0.04 & 0.00 & 0.207 & 0.000 & -0.033 & -0.008 & -2.41 & -0.81 & 1739.79 & 133.96 & & & -0.77 & 135.70 \\
\hline 160241 & 0.18 & 0.00 & 0.05 & 0.00 & 0.197 & 0.000 & -0.046 & -0.009 & -2.67 & -0.98 & 1742.63 & 139.19 & & & -0.89 & 141.12 \\
\hline 161242 & 0.18 & 0.00 & 0.05 & -0.01 & 0.197 & 0.000 & -0.047 & 0.000 & -3.14 & -1.40 & 1744.20 & 145.69 & & & -1.31 & 147.75 \\
\hline 162243 & 0.18 & 0.00 & 0.06 & -0.01 & 0.197 & 0.000 & -0.059 & -0.002 & -3.52 & -1.56 & 1746.84 & 151.12 & & & -1.41 & 153.38 \\
\hline 163244 & 0.17 & 0.00 & 0.06 & -0.01 & 0.186 & 0.000 & -0.061 & -0.001 & -3.75 & -1.88 & 1748.13 & 157.90 & & & -1.73 & 160.31 \\
\hline 164245 & 0.16 & 0.00 & 0.06 & -0.01 & 0.175 & 0.000 & -0.062 & -0.001 & -3.51 & -1.61 & 1750.16 & 163.95 & & & -1.45 & 166.52 \\
\hline 165246 & -0.13 & 0.00 & 0.01 & 0.00 & -0.135 & 0.000 & -0.005 & 0.001 & -3.10 & -2.05 & 1751.40 & 170.78 & & & -2.06 & 173.34 \\
\hline 166247 & -0.13 & 0.00 & 0.01 & 0.00 & -0.135 & 0.000 & -0.005 & 0.001 & -3.46 & -2.48 & 1753.94 & 176.31 & & & -2.48 & 179.02 \\
\hline 167248 & -0.13 & 0.00 & 0.01 & 0.00 & -0.135 & 0.000 & -0.005 & 0.001 & -4.11 & -3.08 & 1755.17 & 183.15 & & & -3.09 & 186.03 \\
\hline 168249 & -0.13 & 0.00 & 0.01 & 0.00 & -0.135 & 0.000 & -0.005 & 0.001 & -4.53 & -3.50 & 1757.53 & 188.86 & & & -3.50 & 191.91 \\
\hline 169250 & -0.13 & 0.00 & 0.01 & 0.00 & -0.135 & 0.000 & -0.005 & 0.001 & -5.25 & -4.20 & 1758.69 & 195.77 & & & -4.21 & 198.99 \\
\hline $170 \quad 251$ & -0.13 & 0.00 & 0.01 & -0.01 & -0.135 & 0.000 & -0.004 & 0.011 & -5.66 & -4.59 & 1760.86 & 201.67 & & & -4.58 & 205.10 \\
\hline 171252 & -0.13 & 0.00 & 0.01 & -0.01 & -0.135 & 0.000 & -0.004 & 0.011 & -6.37 & -5.29 & 1761.85 & 208.76 & & & -5.27 & 212.36 \\
\hline $172 \quad 253$ & -0.13 & 0.00 & 0.01 & -0.01 & -0.135 & 0.000 & -0.004 & 0.011 & -6.63 & -5.56 & 1763.72 & 214.95 & & & -5.54 & 218.74 \\
\hline $173 \quad 254$ & -0.12 & 0.00 & 0.02 & 0.00 & -0.125 & 0.000 & -0.017 & 0.003 & -7.30 & -6.27 & 1764.57 & 222.18 & & & -6.26 & 226.15 \\
\hline $174 \quad 255$ & -0.12 & 0.00 & 0.02 & 0.00 & -0.125 & 0.000 & -0.017 & 0.003 & -7.50 & -6.49 & 1766.23 & 228.58 & & & -6.48 & 232.76 \\
\hline 175256 & -0.10 & 0.00 & 0.02 & 0.00 & -0.105 & 0.000 & -0.019 & 0.002 & -8.12 & -7.24 & 1766.97 & 235.92 & & & -7.23 & 240.30 \\
\hline 176257 & -0.10 & 0.00 & 0.03 & 0.00 & -0.105 & 0.000 & -0.031 & 0.004 & -8.51 & -7.54 & 1768.56 & 242.40 & & & -7.49 & 247.02 \\
\hline $177 \quad 258$ & -0.10 & 0.00 & 0.03 & 0.00 & -0.105 & 0.000 & -0.031 & 0.004 & -9.23 & -8.25 & 1769.08 & 249.95 & & & -8.20 & 254.77 \\
\hline $178 \quad 259$ & -0.08 & 0.00 & 0.03 & 0.00 & -0.084 & 0.000 & -0.032 & 0.003 & -9.32 & -8.37 & 1770.34 & 256.77 & & & -8.31 & 261.82 \\
\hline 179260 & -0.07 & 0.01 & 0.03 & 0.01 & -0.073 & -0.013 & -0.033 & -0.007 & -9.90 & -8.92 & 1770.57 & 264.61 & & & -8.85 & 269.90 \\
\hline 180261 & 0.00 & 0.00 & 0.00 & 0.00 & 0.000 & 0.000 & 0.000 & 0.000 & -9.80 & -8.88 & 1771.50 & 271.75 & & & -8.88 & 277.19 \\
\hline 181262 & 0.00 & 0.00 & 0.00 & 0.00 & 0.000 & 0.000 & 0.000 & 0.000 & -10.64 & -9.67 & 1771.82 & 279.50 & & & -9.67 & 285.17 \\
\hline $182 \quad 263$ & 0.00 & 0.00 & 0.00 & 0.00 & 0.000 & 0.000 & 0.000 & 0.000 & -10.93 & -9.93 & 1772.91 & 286.48 & & & -9.93 & 292.38 \\
\hline 183264 & 0.00 & 0.00 & 0.00 & 0.00 & 0.000 & 0.000 & 0.000 & 0.000 & -11.61 & -10.57 & 1772.93 & 294.52 & & & -10.57 & 300.67 \\
\hline 184265 & 0.00 & 0.00 & 0.00 & 0.00 & 0.000 & 0.000 & 0.000 & 0.000 & -11.58 & -10.53 & 1773.58 & 301.95 & & & -10.53 & 308.34 \\
\hline 185266 & -0.01 & 0.00 & 0.00 & 0.00 & -0.011 & 0.000 & 0.000 & 0.000 & -10.67 & -9.66 & 1771.96 & 311.64 & & & -9.67 & 318.27 \\
\hline 186267 & 0.00 & 0.00 & 0.00 & 0.00 & 0.000 & 0.000 & 0.000 & 0.000 & -9.89 & -8.90 & 1771.74 & 319.93 & & & -8.90 & 326.82 \\
\hline 187268 & 0.01 & 0.03 & 0.00 & 0.00 & 0.011 & -0.040 & 0.000 & 0.001 & -8.92 & -7.84 & 1769.78 & 329.96 & & & -7.82 & 337.12 \\
\hline $188 \quad 269$ & 0.00 & 0.00 & 0.00 & 0.00 & 0.000 & 0.000 & 0.000 & 0.000 & -7.77 & -6.87 & 1769.21 & 338.60 & & & -6.87 & 346.01 \\
\hline \multicolumn{17}{|c|}{$Z=82(\mathrm{~Pb})$} \\
\hline $93 \quad 175$ & 0.00 & 0.00 & 0.00 & 0.00 & 0.000 & 0.000 & 0.000 & 0.000 & -2.13 & -1.38 & 1334.01 & 14.32 & & & -1.38 & 15.39 \\
\hline $94 \quad 176$ & 0.00 & 0.00 & 0.00 & 0.00 & 0.000 & 0.000 & 0.000 & 0.000 & -1.59 & -0.87 & 1346.42 & 9.98 & & & -0.87 & 10.97 \\
\hline 95177 & 0.00 & 0.02 & 0.00 & 0.00 & 0.000 & -0.027 & 0.000 & 0.000 & -1.09 & -0.34 & 1356.47 & 8.00 & & & -0.34 & 8.91 \\
\hline $96 \quad 178$ & 0.01 & 0.02 & 0.00 & 0.00 & 0.011 & -0.027 & 0.000 & 0.000 & -0.76 & -0.03 & 1368.62 & 3.92 & 3.57 & 0.024 & -0.03 & 4.75 \\
\hline $97 \quad 179$ & 0.01 & 0.04 & 0.00 & 0.00 & 0.011 & -0.054 & 0.001 & 0.001 & -0.58 & 0.35 & 1378.40 & 2.22 & & & 0.34 & 2.97 \\
\hline $98 \quad 180$ & 0.00 & 0.03 & 0.00 & 0.00 & 0.000 & -0.040 & 0.000 & 0.001 & -0.32 & 0.44 & 1390.33 & -1.64 & -1.94 & 0.021 & 0.44 & -0.96 \\
\hline $99 \quad 181$ & 0.02 & 0.03 & 0.00 & 0.00 & 0.022 & -0.040 & 0.001 & 0.001 & -0.02 & 0.72 & 1399.78 & -3.03 & -3.14 & 0.090 & 0.72 & -2.42 \\
\hline $100 \quad 182$ & 0.01 & 0.02 & 0.00 & 0.00 & 0.011 & -0.027 & 0.000 & 0.000 & 0.05 & 0.68 & 1411.43 & -6.60 & -6.83 & 0.014 & 0.68 & -6.06 \\
\hline
\end{tabular}




\section{$Z=82(\mathrm{~Pb})$}

$\begin{array}{llllllll}101 & 183 & -0.01 & 0.04 & -0.01 & 0.00 & -0.010 & -0.054\end{array}$

$\begin{array}{llllllll}102 & 184 & 0.00 & 0.02 & -0.01 & 0.00 & 0.000 & -0.027\end{array}$

$\begin{array}{llllllll}103 & 185 & -0.01 & 0.01 & 0.00 & 0.00 & -0.010 & -0.013\end{array}$

$\begin{array}{llllllll}104 & 186 & 0.00 & 0.00 & 0.00 & 0.00 & 0.000 & 0.000\end{array}$

$0.013-0.001$

105187

$0.01 \quad 0.00$

$0.00 \quad 0.00$

0.011

0.000

106188

107189

$0.00 \quad 0.00$

$0.00 \quad 0.00$

$0.000 \quad 0.000$

$108 \quad 190$

$0.00 \quad 0.00$

$0.00 \quad 0.00$

0.000

109191

$0.00 \quad 0.00$

$0.00 \quad 0.00$
0.00

0.000

0.000

$0.00 \quad 0.00$
0.00

0.000

0.000

110192

$0.00 \quad 0.00$

$0.00 \quad 0.00$

0.000

0.000

111193

$0.02 \quad 0.00$

$0.00 \quad 0.00$

0.021

0.000

112194

113195

114196

$\begin{array}{ll}0.00 & 0.00\end{array}$

$\begin{array}{ll}0.03 & 0.00\end{array}$

$\begin{array}{ll}0.00 & 0.00\end{array}$

0.000

0.000

$\begin{array}{ll}0.00 & 0.00\end{array}$

0.032

0.000

$\begin{array}{ll}0.00 & 0.00\end{array}$

$\begin{array}{ll}0.00 & 0.00\end{array}$

0.000

0.000

115197

$0.01 \quad 0.00$

$0.00 \quad 0.00$

0.011

0.000

116198

$117 \quad 199$

118200

119201

$\begin{array}{ll}0.00 & 0.00\end{array}$

$0.00 \quad 0.00$
0.00

0.000

$\begin{array}{ll}0.01 & 0.00\end{array}$

$\begin{array}{ll}0.00 & 0.00\end{array}$

0.011

0.000

0.000

$\begin{array}{ll}0.00 & 0.00\end{array}$

$0.00 \quad 0.00$

$0.00 \quad 0.00$
0.00

0.000

0.000

120202

$\begin{array}{ll}0.00 & 0.00\end{array}$

$0.00 \quad 0.00$

0.000

0.000

0.000

121203

$0.00 \quad 0.00$
0.00

$0.00 \quad 0.00$

0.000

$122 \quad 204$

$0.00 \quad 0.00$

0.000

0.000

$0.012 \quad 0.000$

0.01

$\begin{array}{lllll}0.87 & 1420.58 & -7.68 & -7.57 & 0.028\end{array}$

$\begin{array}{lll}0.000 & 0.000 \quad 0.28\end{array}$

$\begin{array}{lll}0.000 & 0.000 & 0.09\end{array}$

0.000

0.000

$\begin{array}{lll}0.000 & 0.000 & -0.30\end{array}$

0.000

$0.000-0.53$

$0.000-0.95$

0.000

$0.000-1.28$

$0.000-1.87$

0.000

$0.000-2.26$

0.000

$0.000 \quad-2.99$

$0.000-3.48$

0.000

0.000

-3.48
-4.28

0.000

$0.000-4.88$

0.000

$0.000 \quad-5.69$

0.000

0.000

0.000

0.000

$-6.3$

0.000

0.000

$-8.04$

-8.04
-8.91

0.000

$0.000 \quad-9.82$

$0.000 \quad 0.000$

$0.000-10.74$

$\begin{array}{llll}0.01 & 0.00 & -0.010\end{array}$

$0.000-0.012$

124206

125207

$0.00 \quad 0.00$

$\begin{array}{lll}0.00 & 0.00 & 0.000\end{array}$

$\begin{array}{lll}0.00 & 0.00 & 0.000\end{array}$

$0.000 \quad 0.000$

$\begin{array}{lllll}-11.73 & -10.57 & 1613.90 & -23.43 & -23.77\end{array}$

$\begin{array}{lllll}126 & 208 & 0.00 & 0.00\end{array}$

$\begin{array}{llll}127 & 209 & -0.01 & 0.00\end{array}$

$\begin{array}{lll}0.00 & 0.00 & 0.000\end{array}$

0.000

0.000

$\begin{array}{llllll}0.000 & -13.66 & -12.34 & 1628.30 & -21.69 & -22.45\end{array}$

$\begin{array}{llll}0.00 & 0.00 & -0.011\end{array}$

$0.000 \quad 0.000$

$\begin{array}{lllllll}0.000 & -13.93 & -12.58 & 1635.60 & -20.92 & -21.75\end{array}$

$\begin{array}{lllllllll}0.000 & 0.000 & 0.000 & -12.88 & -11.60 & 1639.62 & -16.87 & -17.61\end{array}$

128210

12921

130212

$0.00 \quad 0.00$

$0.00 \quad 0.00$

0.000

0.000

0.000

0.000

$0.00 \quad 0.00$

0.000

0.000

0.000

$\begin{array}{llllll}0.000 & -10.51 & -9.33 & 1648.84 & -9.94 & -10.49\end{array}$

$\begin{array}{llllll}0.000 & -9.30 & -8.18 & 1654.18 & -7.21 & -7.55\end{array}$

0.000

$132 \quad 214$

133215

134216

$0.00 \quad 0.00$

$0.000 \quad 0.000$

0.000 .00

$0.000 \quad 0.000$

$0.000-0.027$

0.000

$\begin{array}{llll}0.000 & -8.10 & -7.04 & 1657.50\end{array}$

$\begin{array}{lllll}0.000 & -6.98 & -5.98 & 1662.65\end{array}$

$-2.46$

5.33

$\begin{array}{lllll}0.000 & 0.000 & -5.98 & -4.97 & 1665.85\end{array}$

8.41

$\begin{array}{ll}0.011 & -0.054 \\ 0.023 & -0.094\end{array}$

$-5.22$

$-4.01 \quad 1670.84$

8.41
13.29

$0.012-0.081$

0.002

$-0.006$

$0.034-0.095$

137219

138220

$\begin{array}{llll}0.01 & 0.06 & 0.00 & 0.00\end{array}$

$\begin{array}{lllll}0.03 & 0.07 & -0.01 & 0.00\end{array}$

$\begin{array}{llll}0.01 & 0.07 & -0.01 & 0.00\end{array}$

$0.013-0.095$

0.014

$\begin{array}{lllll}0.002 & -3.91 & -2.44 & 1678.91\end{array}$

16.49

$0.034-0.094$

$0.014-0.003$

139221

$\begin{array}{lllll}0.03 & 0.07 & -0.01 & 0.01\end{array}$

140222

$\begin{array}{llll}0.01 & 0.07 & 0.00 & 0.01\end{array}$

$0.013-0.094$

$-2.73$

1.74

21.57

$\begin{array}{llll}0.002 & -0.007 & -1.44\end{array}$

$-0.50 \quad 1689.56$

30.05

$0.162 \quad 0.000$

0.173

0.000

$\begin{array}{lll}0.084 & 0.013 & -2.01\end{array}$

$\begin{array}{ll}0.50 & 1696.97\end{array}$

33.48

$142 \quad 224$

$\begin{array}{llll}0.16 & 0.00 & -0.06 & 0.00\end{array}$

0.172

$\begin{array}{lllll}0.16 & 0.00 & -0.05 & 0.00\end{array}$

0.183

0.000

$\begin{array}{lll}0.085 & 0.014 & -1.82\end{array}$

$\begin{array}{ll}0.80 & 1701.62\end{array}$

38.78

$\begin{array}{ll}0.75 & 1704.67\end{array}$

42.20

$\begin{array}{lll}0.79 & 1709.33 & 50.63\end{array}$

$\begin{array}{lllllll}145 & 227 & 0.18 & 0.00 & -0.04 & 0.01\end{array}$

0.194

0.000

$\begin{array}{lll}0.075 & 0.002 & -1.34\end{array}$

55.83

$\begin{array}{lll}0.81 & 1716.57 & 59.54\end{array}$

$\begin{array}{lll}0.55 & 1719.39 & 64.79\end{array}$

$\begin{array}{lll}0.62 & 1723.58 & 68.67\end{array}$

$\begin{array}{llll}0.22 & 1726.32 & 74.00\end{array}$

$\begin{array}{lll}0.25 & 1730.34 \quad 78.06\end{array}$

$\begin{array}{lllll}0.238 & 0.000 & 0.035 & -0.015 & -1.78\end{array}$

0.000

$\begin{array}{lll}0.035 & -0.015 & -1.73\end{array}$

$0.239 \quad 0.000$

$\begin{array}{lll}0.023 & -0.018 & -2.06\end{array}$

$-0.14 \quad 1732.86$

0.000

$0.011-0.021-1.85$

83.61

0.240

$\begin{array}{llll}0.000 & 0.011 & -0.021 & -1.96\end{array}$

$\begin{array}{llllll}0.240 & 0.000 & -0.002 & -0.013 & -1.56\end{array}$

$-0.09 \quad 1738.57$

0.201741 .90

$\begin{array}{lll}0.35 & 1746.90 & 109.92\end{array}$

$\begin{array}{lll}0.09 & 1748.70 & 116.19\end{array}$

$0.87-7.20$

$0.73-10.52$

$0.74-11.44$

$0.55-14.42$

$0.45-15.06$

$0.16-17.74$

$-0.09-18.15$

$-0.47-20.54$

$-0.79-20.66$

$-1.32-22.83$ 


\section{$Z=82(\mathrm{~Pb})$}

\begin{tabular}{|c|c|c|c|c|c|c|c|c|c|c|c|c|}
\hline 240 & 0.18 & 00 & 0.03 & 00 & & & & & & & & \\
\hline 9241 & 0.19 & 00 & 0.04 & .00 & & 0.000 & -0.033 & -0.008 & & & & \\
\hline $0 \quad 242$ & 18 & 00 & .04 & .00 & & .000 & & & & & & \\
\hline 43 & 18 & 00 & 05 & .01 & & & & & & & & \\
\hline 44 & 18 & 00 & .06 & 0.01 & & & -0 & & & & & 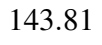 \\
\hline 15 & 17 & 00 & 06 & 0.01 & 6 & 00 & -0.061 & 01 & -3.19 & -1.37 & & 58 \\
\hline 16 & 0.00 & 00 & & 0.00 & & & & & & & & \\
\hline 17 & -0.01 & 0.00 & .00 & 0.00 & -0.011 & 00 & 000 & & & -1.93 & & 2.70 \\
\hline 18 & 0.00 & 00 & 0.00 & 0.00 & & & & & & & & .02 \\
\hline 49 & 0.02 & 0.00 & 001 & 0.00 & & & & & & & & 75.01 \\
\hline 0 & 02 & 00 & 0 & 00 & 1 & 0 & 0 & 0 & $(2$ & -2 & & .46 \\
\hline 51 & & 00 & 1 & 0.00 & & & & & & -3.78 & & 27 \\
\hline 52 & 0.15 & 00 & 1 & -0.01 & -0 . & 0 & & & & -4 & & \\
\hline 53 & 0.13 & 00 & 01 & -0.01 & -0 . & & & & & -4 & & \\
\hline & & 00 & 01 & 001 & -0 . & & & & & & & \\
\hline 255 & 2 & 0.00 & & 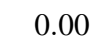 & & & & & & & & \\
\hline 56 & -0.01 & 0.00 & 00 & 0.00 & -0 . & & & & & & & \\
\hline 57 & -0.12 & 0.00 & 0 & .00 & -0 . & & & & & & & \\
\hline$=0$ & 0.0 & 00 & & 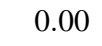 & & & & & & & & \\
\hline 259 & 0.0 & 0.00 & 0.00 & 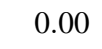 & & & & & & & & \\
\hline 60 & 0 & 00 & & & & & & & & & & \\
\hline 61 & 0.00 & 00 & & 0 & & & & & & & & \\
\hline 62 & 00 & 00 & 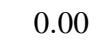 & 0.00 & & & & & & -8 & & \\
\hline 0 & & & & & & & & & & & & \\
\hline 264 & 0.00 & 0.00 & .0 & 0.1 & 0. & 0 & 0 & 00 & & -9.76 & & 215.55 \\
\hline 65 & 0.00 & 0.00 & 00 & 0.00 & 0.000 & 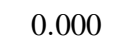 & .000 & 0 & -1 & 7 & & 40 \\
\hline$G$ & & 00 & & 00 & & & & & & & & \\
\hline 67 & -0.01 & 0.00 & . & .00 & -0.011 & 0.000 & 0 & 00 & -10.42 & -9.42 & 65 & 300.24 \\
\hline 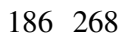 & & 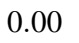 & & & & & & & & & & 08.19 \\
\hline 9 & 0.01 & 0.04 & 00 & 0.00 & 0.0 & 0.0 & & & & -7.64 & & 18 \\
\hline 70 & & & & & & & & & & & & \\
\hline & & & & & & & & & & & & \\
\hline 212 & 0.00 & 0.09 & & 0.00 & & -0.123 & & & & -5.61 & & 344.15 \\
\hline 273 & 0.03 & 0.09 & -0.01 & 0.00 & 0.035 & -0.123 & 0.016 & 0.006 & -7.42 & -5.36 & 1785.69 & 353.63 \\
\hline
\end{tabular}

$\begin{array}{ll}0.16 & 122.54\end{array}$

$-0.28 \quad 128.94$

$-0.37 \quad 134.02$

$-0.82 \quad 140.61$

$-0.90 \quad 145.90$

$\begin{array}{ll}-1.22 & 152.81\end{array}$

$\begin{array}{ll}-1.36 & 158.22\end{array}$

$\begin{array}{ll}-1.93 & 165.07\end{array}$

$-2.21 \quad 170.54$

$\begin{array}{ll}-2.65 & 177.69\end{array}$

$-2.98 \quad 183.30$

$\begin{array}{ll}-3.79 & 190.26\end{array}$

$\begin{array}{ll}-4.13 & 196.04\end{array}$

$\begin{array}{ll}-4.83 & 203.28\end{array}$

$-5.09209 .33$

$-5.72 \quad 216.80$

$\begin{array}{ll}-5.91 & 223.09\end{array}$

$-6.68 \quad 230.60$

$\begin{array}{ll}-6.93 & 237.00\end{array}$

$\begin{array}{ll}-7.58 & 244.79\end{array}$

$\begin{array}{ll}-7.71 & 251.47\end{array}$

$-8.45 \quad 259.33$

$\begin{array}{ll}-8.76 \quad 266.00 \\ -9.52 & 273.99\end{array}$

$\begin{array}{ll}-9.52 & 273.99\end{array}$

$-9.76 \quad 280.90$

$-10.37 \quad 289.20$

$-10.31 \quad 296.57$

$-9.42 \quad 306.51$

$-8.67 \quad 314.71$

$-7.61 \quad 324.98$

$-6.65 \quad 333.55$

$-6.14 \quad 343.41$

$-5.44 \quad 351.87$

$-5.19361 .61$

\begin{tabular}{|c|c|c|c|c|c|c|c|c|c|c|c|c|c|c|c|c|}
\hline \multicolumn{17}{|c|}{$Z=83(\mathrm{Bi})$} \\
\hline $95 \quad 178$ & 0.07 & 0.07 & -0.01 & 0.00 & 0.077 & -0.096 & 0.016 & 0.004 & -1.23 & 0.28 & 1353.29 & 18.48 & & & 0.26 & 19.55 \\
\hline $96 \quad 179$ & 0.07 & 0.07 & -0.01 & 0.00 & 0.077 & -0.096 & 0.016 & 0.004 & -0.73 & 0.72 & 1365.37 & 14.46 & & & 0.71 & 15.46 \\
\hline $97 \quad 180$ & 0.05 & 0.07 & -0.01 & 0.00 & 0.055 & -0.095 & 0.015 & 0.004 & -0.30 & 1.09 & 1375.62 & 12.28 & & & 1.08 & 13.19 \\
\hline $98 \quad 181$ & 0.05 & 0.07 & -0.01 & 0.00 & 0.055 & -0.095 & 0.015 & 0.004 & 0.03 & 1.38 & 1387.43 & 8.55 & & & 1.38 & 9.39 \\
\hline $99 \quad 182$ & 0.27 & 0.00 & -0.01 & 0.01 & 0.294 & 0.000 & 0.045 & -0.003 & -0.77 & 1.35 & 1397.67 & 6.38 & & & 1.27 & 7.07 \\
\hline $100 \quad 183$ & 0.28 & 0.00 & 0.00 & 0.01 & 0.306 & 0.000 & 0.036 & -0.006 & -0.79 & 1.40 & 1409.28 & 2.84 & & & 1.33 & 3.46 \\
\hline $101 \quad 184$ & 0.28 & 0.00 & 0.01 & 0.01 & 0.307 & 0.000 & 0.023 & -0.009 & -0.92 & 1.30 & 1419.19 & 1.00 & & & 1.21 & 1.54 \\
\hline 102185 & 0.28 & 0.00 & 0.01 & 0.01 & 0.307 & 0.000 & 0.023 & -0.009 & -0.81 & 1.44 & 1430.29 & -2.04 & & & 1.37 & -1.54 \\
\hline 103186 & 0.27 & 0.00 & 0.02 & 0.01 & 0.296 & 0.000 & 0.009 & -0.013 & -0.85 & 1.28 & 1439.86 & -3.53 & -3.17 & 0.077 & 1.20 & -3.11 \\
\hline $104 \quad 187$ & 0.27 & 0.00 & 0.03 & 0.00 & 0.297 & 0.000 & -0.005 & -0.007 & -0.68 & 1.45 & 1450.53 & -6.13 & -6.37 & 0.015 & 1.39 & -5.76 \\
\hline $105 \quad 188$ & -0.19 & 0.00 & 0.00 & 0.00 & -0.196 & 0.000 & 0.014 & -0.001 & -0.27 & 1.19 & 1459.82 & -7.35 & -7.20 & 0.050 & 1.15 & -7.02 \\
\hline 106189 & -0.19 & 0.00 & 0.00 & 0.00 & -0.196 & 0.000 & 0.014 & -0.001 & -0.58 & 0.93 & 1470.54 & -9.99 & -10.06 & 0.054 & 0.90 & -9.71 \\
\hline $107 \quad 190$ & -0.19 & 0.00 & 0.00 & 0.00 & -0.196 & 0.000 & 0.014 & -0.001 & -0.88 & 0.66 & 1479.45 & -10.84 & -10.90 & 0.185 & 0.62 & -10.62 \\
\hline 108191 & -0.19 & 0.00 & 0.00 & 0.00 & -0.196 & 0.000 & 0.014 & -0.001 & -1.13 & 0.40 & 1489.79 & -13.11 & -13.24 & 0.007 & 0.37 & -12.94 \\
\hline 109192 & 0.08 & 0.00 & -0.01 & 0.00 & 0.085 & 0.000 & 0.015 & 0.001 & -0.66 & 0.05 & 1498.42 & -13.66 & -13.55 & 0.033 & 0.04 & -13.52 \\
\hline 110193 & 0.07 & 0.00 & -0.01 & 0.00 & 0.075 & 0.000 & 0.014 & 0.001 & -1.14 & -0.41 & 1508.59 & -15.76 & -15 & 0 & -0.41 & -15.67 \\
\hline $111 \quad 194$ & 0.06 & 0.00 & -0.01 & 0.00 & 0.064 & 0.000 & 0.014 & 0.001 & -1.56 & -0.82 & 1516.93 & -16.02 & -15.99 & 0.049 & -0.82 & -15.98 \\
\hline 112195 & -0.05 & 0.00 & -0.01 & 0.00 & -0.052 & 0.000 & 0.013 & -0.001 & -2.17 & -1.77 & 1527.23 & -18.26 & -18.02 & 0.006 & -1.77 & -18.26 \\
\hline 113196 & 0.05 & 0.00 & -0.01 & 0.00 & 0.053 & 0.000 & 0.013 & 0.001 & -2.68 & -1.89 & 1534.93 & -17.88 & -18.01 & 0.024 & -1.89 & -17.93 \\
\hline 114197 & -0.05 & 0.00 & -0.01 & 0.00 & -0.052 & 0.000 & 0.013 & -0.001 & -3.45 & -2.94 & 1544.98 & -19.87 & -19.69 & 0.008 & -2.94 & -19.95 \\
\hline 115198 & -0.05 & 0.00 & -0.01 & 0.00 & -0.052 & 0.000 & 0.013 & -0.001 & -4.08 & -3.54 & 1552.83 & -19.65 & -19.37 & 0.028 & -3.55 & -19.76 \\
\hline 116199 & -0.05 & 0.00 & -0.01 & 0.00 & -0.052 & 0.000 & 0.013 & -0.001 & -4.88 & -4.26 & 1562.21 & -20.96 & -20.80 & 0.012 & -4.26 & -21.11 \\
\hline
\end{tabular}




\section{$Z=83(\mathrm{Bi})$}

$\begin{array}{lllll}117 & 200 & -0.04 & 0.00 & -0.01\end{array}$ $\begin{array}{llllll}118 & 201 & -0.05 & 0.00 & -0.01\end{array}$ $\begin{array}{lllll}119 & 202 & -0.05 & 0.00 & 0.00\end{array}$ $\begin{array}{lllll}120 & 203 & -0.04 & 0.00 & 0.00\end{array}$ $\begin{array}{lllll}121 & 204 & -0.05 & 0.00 & 0.00\end{array}$

$\begin{array}{llllll}122 & 205 & -0.04 & 0.00 & 0.00\end{array}$ $\begin{array}{lllll}123 & 206 & -0.03 & 0.00 & 0.00\end{array}$ $\begin{array}{lllll}124 & 207 & -0.02 & 0.00 & 0.00\end{array}$ $\begin{array}{lllll}125 & 208 & -0.01 & 0.00 & 0.00\end{array}$ $\begin{array}{lllll}126 & 209 & -0.01 & 0.00 & 0.00\end{array}$ $\begin{array}{llllll}127 & 210 & -0.02 & 0.00 & -0.01\end{array}$ $\begin{array}{llllll}128 & 211 & -0.01 & 0.00 & -0.01\end{array}$ $\begin{array}{lllll}129 & 212 & -0.01 & 0.00 & 0.00\end{array}$ $\begin{array}{lllll}130 & 213 & -0.01 & 0.00 & -0.01\end{array}$ $\begin{array}{llllll}131 & 214 & -0.01 & 0.00 & -0.01\end{array}$ $\begin{array}{lllll}132 & 215 & -0.02 & 0.00 & -0.01\end{array}$ $\begin{array}{lllll}133 & 216 & 0.04 & 0.09 & -0.02\end{array}$ $\begin{array}{llllll}134 & 217 & 0.04 & 0.08 & -0.02\end{array}$ $\begin{array}{llllll}135 & 218 & 0.05 & 0.08 & -0.02\end{array}$ $\begin{array}{lllll}136 & 219 & 0.05 & 0.08 & -0.02\end{array}$

$\begin{array}{lllll}137 & 220 & 0.07 & 0.08 & -0.03\end{array}$ $\begin{array}{llllll}138 & 221 & 0.08 & 0.08 & -0.03\end{array}$ $\begin{array}{llllll}139 & 222 & 0.09 & 0.07 & -0.03\end{array}$ $\begin{array}{llllll}140 & 223 & 0.13 & 0.00 & -0.06\end{array}$ $\begin{array}{llllll}141 & 224 & 0.14 & 0.00 & -0.06\end{array}$

$\begin{array}{llllll}142 & 225 & 0.15 & 0.00 & -0.06\end{array}$ $\begin{array}{lllll}144 & 227 & 0.17 & 0.00 & -0.05\end{array}$ $\begin{array}{llllll}145 & 228 & 0.18 & 0.00 & -0.05\end{array}$ $\begin{array}{llllll}146 & 229 & 0.19 & 0.00 & -0.04\end{array}$

$\begin{array}{llllll}147 & 230 & 0.20 & 0.01 & -0.03\end{array}$ $\begin{array}{llllll}148 & 231 & 0.22 & 0.00 & -0.02\end{array}$ $\begin{array}{llllll}149 & 232 & 0.22 & 0.00 & -0.02\end{array}$ $\begin{array}{llllll}150 & 233 & 0.22 & 0.00 & -0.01\end{array}$ $\begin{array}{lllll}151 & 234 & 0.22 & 0.00 & 0.00\end{array}$

$\begin{array}{llllll}152 & 235 & 0.22 & 0.00 & 0.00\end{array}$

$\begin{array}{lllll}153 & 236 & 0.22 & 0.00 & 0.01\end{array}$ $\begin{array}{lllll}154 & 237 & 0.22 & 0.00 & 0.01\end{array}$ $\begin{array}{lllll}155 & 238 & 0.22 & 0.00 & 0.02\end{array}$ $\begin{array}{lllll}156 & 239 & 0.18 & 0.00 & 0.01\end{array}$

$157 \quad 240$ $\begin{array}{lll}0.18 & 0.00 & 0.02\end{array}$

$158 \quad 241$ $\begin{array}{lll}0.18 & 0.00 & 0.03\end{array}$ 159242 $\begin{array}{lll}0.17 & 0.00 & 0.03\end{array}$ 160243 $\begin{array}{ll}0.17 & 0.00\end{array}$ 0.04

$0.00-0.042$ $0.00-0.052$ $0.00-0.052$ $0.00-0.042$ $0.00-0.052$

$\begin{array}{lll}0.00 & -0.042\end{array}$

$0.00-0.032$

$0.00-0.021$

$0.00-0.011$

$0.00-0.011$

$0.00-0.010$

$0.00-0.011$

$0.00-0.010$

$0.00 \quad-0.021$

$0.046-0.123$

$\begin{array}{lll}0.00 & 0.056 & -0.110\end{array}$

0.00

$0.056-0.110$

$\begin{array}{llll}0.00 & 0.077 & -0.111\end{array}$

$\begin{array}{llll}0.00 & 0.088 & -0.111\end{array}$

0.00

$0.098-0.097$

$-0.01$

0.141

$0.00 \quad 0.15$

$0.00 \quad 0.162$

$0.01 \quad 0.172$

$0.01 \quad 0.183$

$0.02 \quad 0.193$

0.02

0.02

0.02

0.204

0.000

0.000

0.02

0.216

0.238

0.238

0.02

0.238

0.02

0.239

0.000

0.000

0.000

0.000

0.000

0.02

0.239

$0.02 \quad 0.240$

$0.02 \quad 0.240$

$-0.013$

0.000

0.000

0.000

0.000

0.000

0.000

0.000

$0.01 \quad 0.240$

$0.01 \quad 0.195$

0.000

0.000

$0.00 \quad 0.195$

$0.00 \quad 0.196$

$0.00 \quad 0.185$

$0.00 \quad 0.185$

161244

0.180 .00

$\begin{array}{lll}0.05 & -0.01\end{array}$

0.197

0.000
0.000

$\begin{array}{lllll}0.000 & -0.023 & -0.005 & -1.28\end{array}$

$\begin{array}{llll}0.000 & -0.024 & -0.005 & -1.54\end{array}$

$\begin{array}{lllll}0.000 & -0.036 & -0.007 & -1.75\end{array}$

$\begin{array}{lllll}0.000 & -0.047 & 0.000 & -2.46\end{array}$

$162 \quad 245$

$0.17 \quad 0.00$

$\begin{array}{lll}0.05 & -0.01 \quad 0.185\end{array}$

$0.000-0.049$

$0.000-0.049$

$0.001-2.45$

0.000

$\begin{array}{lllll}164 & 247 & 0.16 & 0.00 & 0.05\end{array}$

$\begin{array}{llllll}165 & 248 & -0.03 & 0.00 & -0.01\end{array}$

$-0.01 \quad 0.174$

$0.00-0.032$

$0.00-0.032$

0.000

$-0.050$

$0.001-2.76$

0.001

$-2.54$

0.000

$0.012-0.000$

$-1.99$

0.000

$0.012-0.000$

$-2.28$

$0.00-0.032$

$0.00-0.104$

$0.00-0.104$

0.000

$0.012-0.000$

$-2.89$

0.000

$0.015-0.00$

$-3.62$

0.000

$0.015-0.001$

0.000

0.018

0.000

0.000

$\begin{array}{llllllll}170 & 253 & -0.10 & 0.00 & -0.01 & 0.00 & -0.104\end{array}$

0.018

$-0.001$

$-4.37$

$-4.72$

$\begin{array}{lllllll}172 & 255 & -0.12 & 0.00 & -0.01 & -0.01 & -0.125\end{array}$

0.004 $\begin{array}{lllll}143 & 226 & 0.16 & 0.00 & -0.06\end{array}$

$0.00-0.021$

$0.00-0.010$

$\begin{array}{lllllll}-4.92 & 1569.79 & -20.47 & -20.37 & 0.024 & -4.92 & -20.65\end{array}$ $\begin{array}{lllllll}-5.70 & 1578.91 & -21.51 & -21.42 & 0.015 & -5.70 & -21.72\end{array}$ $\begin{array}{lllllll}-6.54 & 1586.35 & -20.88 & -20.73 & 0.020 & -6.54 & -21.12\end{array}$ $\begin{array}{lllllll}-7.24 & 1595.07 & -21.52 & -21.54 & 0.022 & -7.25 & -21.79\end{array}$ $\begin{array}{lllllll}-8.18 & 1602.30 & -20.68 & -20.67 & 0.026 & -8.19 & -20.97\end{array}$

$\begin{array}{llll}-8.85 & 1610.67 & -20.98 & -21.06\end{array}$

$0.007-8.85-21.28$

$0.008-9.65-20.02$

$0.002-10.43-20.13$

$0.002-11.33-18.67$

$0.001-11.56-17.93$

$0.001-10.69-14.41$

$0.005-9.62-12.08$

$0.002-8.46-7.98$

$0.005 \quad-7.24 \quad-5.22$

$0.011-6.12-0.88$

$\begin{array}{llllllll}171 & 254 & -0.12 & 0.00 & -0.01 & -0.01 & -0.125\end{array}$

$\begin{array}{rr}-9.62 & 1649.84 \\ -8.46 & 1653.82\end{array}$

$11.73-11$
-7.64

$-4.88-5.23$

$-0.56-1.20$

2.24

1.65

0.015

$-5.14$

6.61

9.55

13.96

17.08

$-2.701680 .65$

5.87

21.52

24.79

29.50

$\begin{array}{lll}0.25 & 1702.06 & 32.91 \\ 0.31 & 1705.61 & 37.43\end{array}$

$0.31 \quad 1705.61$

40.85

$0.64 \quad 1713.64$

45.54

$0.66 \quad 1718.37$

48.88

$\begin{array}{ll}0.52 & 1721.66\end{array}$

53.66

$\begin{array}{ll}0.70 & 1725.99\end{array}$

57.40

$\begin{array}{ll}0.50 & 1729.13\end{array}$

62.34

$\begin{array}{lll}0.53 & 1733.38 & 66.16\end{array}$

$\begin{array}{lll}0.15 & 1736.48 & 71.13\end{array}$

$0.24 \quad 1740.46$

75.23

$-0.11 \quad 1743.31$

80.44

$\begin{array}{ll}0.06 & 1747.00\end{array}$

84.83

$-0.03 \quad 1749.39$

90.51

$\begin{array}{lll}0.25 & 1752.74 & 95.22\end{array}$

$\begin{array}{lll}0.23 & 1754.87 & 101.17\end{array}$

$\begin{array}{lll}0.49 & 1758.05 & 106.06\end{array}$

$\begin{array}{lll}0.21 & 1760.22 & 111.96\end{array}$

$\begin{array}{lll}0.21 & 1763.47 & 116.79\end{array}$

$\begin{array}{lll}-0.13 & 1765.51 & 122.81\end{array}$

$\begin{array}{lll}-0.29 & 1768.72 & 127.68\end{array}$

$\begin{array}{lll}-0.74 & 1770.70 & 133.77\end{array}$

$\begin{array}{lll}-0.83 & 1773.63 & 138.91\end{array}$

$\begin{array}{lll}-1.15 & 1775.30 & 145.31\end{array}$

$\begin{array}{lll}-0.99 & 1777.80 & 150.88\end{array}$

$\begin{array}{lll}-1.46 & 1779.42 & 157.33\end{array}$

$\begin{array}{lll}-1.88 & 1782.33 & 162.49\end{array}$

$\begin{array}{lll}-2.36 & 1783.80 & 169.10\end{array}$

$\begin{array}{lll}-2.79 & 1786.53 & 174.44\end{array}$

$\begin{array}{lll}-3.51 & 1788.06 & 180.97\end{array}$

$\begin{array}{lll}-3.85 & 1790.54 & 186.57\end{array}$

193.28
199.10

205.99

$-4.17 \quad 6.39$

$-3.33 \quad 9.34$

$\begin{array}{ll}-2.64 & 13.77\end{array}$

$\begin{array}{ll}-1.86 & 16.92\end{array}$

$\begin{array}{ll}-1.39 & 21.40\end{array}$

$-0.71 \quad 24.72$

$-0.24 \quad 29.45$

$0.41 \quad 32.98$

$0.44 \quad 37.51$

$\begin{array}{ll}0.78 & 40.99\end{array}$

$0.77 \quad 45.73$

$0.76 \quad 49.10$

$0.64 \quad 53.96$

$0.80 \quad 57.74$

$0.57 \quad 62.71$

$\begin{array}{ll}0.59 & 66.59\end{array}$

$0.20 \quad 71.63$

$0.29 \quad 75.80$

$-0.06 \quad 81.09$

$0.11 \quad 85.57$

$0.02 \quad 91.35$

$0.31 \quad 96.16$

$0.24 \quad 102.16$ 


\section{$Z=83(\mathrm{Bi})$}

$\begin{array}{lllll}174 & 257 & -0.05 & 0.00 & -0.01\end{array}$ $\begin{array}{lllll}175 & 258 & -0.10 & 0.00 & 0.01\end{array}$ $\begin{array}{llll}176 & 259 & -0.08 & 0.00\end{array}$ $\begin{array}{llll}177 & 260 & -0.05 & 0.00\end{array}$ $0.00-0.105$ $\begin{array}{lllllll}0.000 & 0.013 & -0.001 & -6.34 & -5.76 & 1797.33 & 212.07\end{array}$ $\begin{array}{lllllll}0.000 & -0.008 & 0.001 & -7.25 & -6.46 & 1798.35 & 219.11\end{array}$ $\begin{array}{llll}178 & 261 & -0.03 & 0.00\end{array}$ $\begin{array}{lll}0.00 & 0.00 & -0.052\end{array}$ $0.000-0.009$ $\begin{array}{lllll}0.010 & -7.45 & -6.72 & 1800.24 & 225.29\end{array}$ $\begin{array}{lllllll}0.000 & 0.001 & 0.000 & -7.97 & -7.32 & 1801.01 & 232.60\end{array}$ 0.00 $0.000 \quad 0.000$ $\begin{array}{lllll}0.000 & -8.13 & -7.49 & 1802.65 & 239.03\end{array}$ $\begin{array}{lllll}179 & 262 & -0.03 & 0.00 & 0.00\end{array}$ $0.00-0.032$

$0.000 \quad 0.000$ $\begin{array}{lllll}0.000 & -8.87 & -8.03 & 1803.20 & 246.55\end{array}$ $\begin{array}{lllll}180 & 263 & -0.02 & 0.00 & 0.00\end{array}$

$0.00-0.032$ 0.000 0.000

$0.00-0.011$ 0.000 $\begin{array}{llllll}0.000 & -9.11 & -8.23 & 1804.71 & 253.11\end{array}$ $\begin{array}{lll}264 & -0.01 & 0.00\end{array}$ $0.00-0.011$ 0.000 0.000 $\begin{array}{lllll}0.000 & -9.88 & -8.94 & 1805.28 & 260.61\end{array}$ $\begin{array}{lllll}182 & 265 & -0.01 & 0.00 & 0.00\end{array}$ $0.00-0.011$ 0.000

0.000 $\begin{array}{llllll}0.000 & -10.10 & -9.13 & 1806.64 & 267.33\end{array}$ $\begin{array}{llllll}183 & 266 & -0.01 & 0.00 & 0.00\end{array}$ $0.00 \quad 0.000$ $0.000-0.012$ $\begin{array}{lllll}185 & 268 & -0.01 & 0.00 & -0.01\end{array}$ $\begin{array}{lllll}186 & 269 & -0.01 & 0.00 & 0.00\end{array}$ $\begin{array}{lllll}187 & 270 & 0.01 & 0.03 & -0.01\end{array}$ $0.00-0.010$ 0.000 $0.000-10.74$

$0.00-0.011$

0.000

$\begin{array}{lllll}188 & 271 & 0.00 & 0.05 & -0.01\end{array}$

$\begin{array}{lllll}189 & 272 & 0.03 & 0.08 & -0.01\end{array}$

$\begin{array}{lllll}190 & 273 & 0.04 & 0.09 & -0.02\end{array}$

$\begin{array}{lllll}191 & 274 & 0.04 & 0.10 & -0.02\end{array}$

$\begin{array}{llllll}192 & 275 & 0.04 & 0.11 & -0.01\end{array}$

$\begin{array}{lllll}193 & 276 & 0.04 & 0.11 & -0.01\end{array}$
$-0.01 \quad 0.011-0.041$

$\begin{array}{lll}0.00 & 0.001 & -0.068\end{array}$

$\begin{array}{lll}0.00 & 0.035 & -0.109\end{array}$

$\begin{array}{lll}0.00 & 0.046 & -0.123\end{array}$

$\begin{array}{lll}0.00 & 0.047 & -0.137\end{array}$

$\begin{array}{lll}0.01 & 0.048 & -0.149\end{array}$

$\begin{array}{lll}0.01 & 0.048 & -0.149\end{array}$
0.012

0.012

0.013

0.015

0.028

$\begin{array}{llllll}0.028 & 0.008 & -7.40 & -4.96 & 1802.82 & 343.79\end{array}$

$\begin{array}{llllll}0.017 & -0.001 & -6.97 & -4.38 & 1802.68 & 352.00\end{array}$

$\begin{array}{llllll}0.017 & -0.001 & -6.70 & -4.14 & 1801.47 & 361.28\end{array}$ $\begin{array}{ll}-5.75 & 215.72\end{array}$

$\begin{array}{ll}-6.47 & 222.94\end{array}$

$\begin{array}{ll}-6.69 & 229.35\end{array}$

$\begin{array}{ll}-7.32 & 236.82\end{array}$

$\begin{array}{ll}-7.49 & 243.44\end{array}$

$\begin{array}{ll}-8.03 & 251.17\end{array}$

$-8.23 \quad 257.94$

$-8.94 \quad 265.65$

$-9.13 \quad 272.59$

$-9.74 \quad 280.55$

$-9.61 \quad 287.97$

$-8.80 \quad 297.51$

$-8.01 \quad 305.74$

$-6.95 \quad 315.68$

$-6.00 \quad 324.23$

$-5.54 \quad 333.72$

$-4.90 \quad 342.10$

$-4.75 \quad 351.43$

$\begin{array}{ll}-4.15 & 359.92\end{array}$

$-3.91 \quad 369.47$

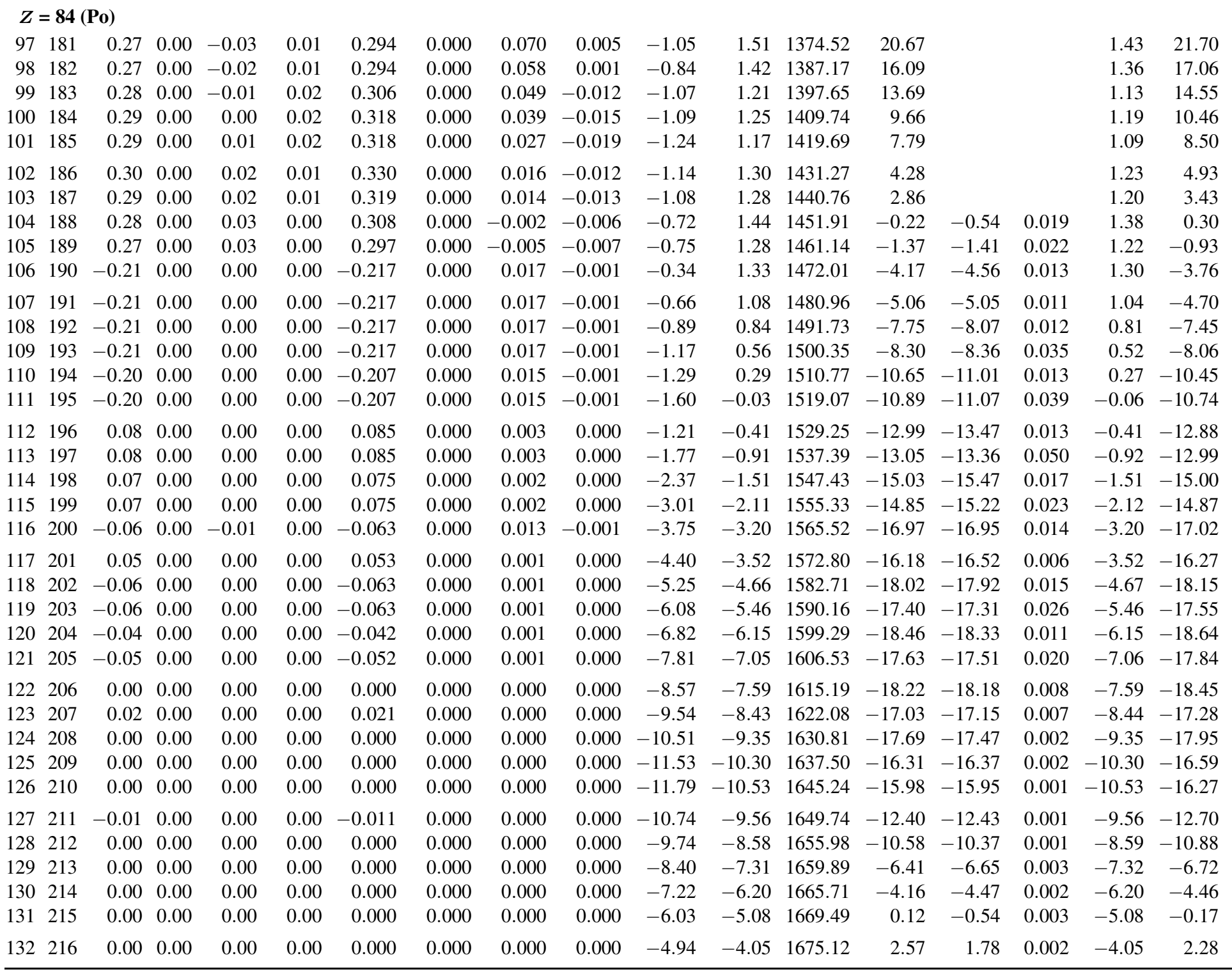




\section{$Z=84$ (Po)}

$\begin{array}{lllll}133 & 217 & 0.04 & 0.09 & -0.02\end{array}$

$\begin{array}{llllll}134 & 218 & 0.05 & 0.09 & -0.02\end{array}$

$\begin{array}{lllll}135 & 219 & 0.08 & 0.08 & -0.04\end{array}$

$\begin{array}{llll}0.00 & 0.046 & -0.123 & 0.028\end{array}$

$\begin{array}{lllll}0.00 & 0.056 & -0.123 & 0.028\end{array}$

$\begin{array}{llll}0.007 & -6.05 & -3.44 & 1679.15\end{array}$

6.61

$\begin{array}{llll}5.90 & 0.007 & -3.37 & 6.41\end{array}$

136220

$\begin{array}{lll}0.09 & 0.09 & -0.04\end{array}$

$\begin{array}{lll}0.00 & 0.088 & -0.111\end{array}$

0.054

$\begin{array}{llll}0.007 & -5.19 & -2.61 & 1684.71\end{array}$

9.12

$\begin{array}{llll}0.009 & -4.78 & -2.09 & 1688.57\end{array}$

13.33

$\begin{array}{llll}0.00 & 0.099 & -0.125\end{array}$

0.055

$\begin{array}{llll}0.011 & -4.45 & -1.39 & 1694.00\end{array}$

15.97

$137 \quad 221$

$\begin{array}{lll}0.09 & 0.09 & -0.04\end{array}$

0.00

$0.099-0.125$

0.055

$\begin{array}{llll}0.011 & -4.03 & -1.00 & 1697.74\end{array}$

20.30

138222

$\begin{array}{llll}0.10 & 0.08 & -0.04 & 0.00\end{array}$

$\begin{array}{ll}0.110 & -0.111\end{array}$

139223

$\begin{array}{lllll}0.13 & 0.00 & -0.07 & -0.01\end{array}$

$\begin{array}{ll}0.141 & 0.000\end{array}$

0.055

$\begin{array}{llll}0.011 & -3.02 & -0.33 & 1702.93\end{array}$

23.18

$140 \quad 224$

$\begin{array}{llll}0.13 & 0.00 & -0.07 & -0.01\end{array}$

$\begin{array}{ll}0.141 & 0.000\end{array}$

0.094

$\begin{array}{llll}0.024 & -2.87 & 0.02 & 1706.46\end{array}$

$0.024-2.46$

$\begin{array}{ll}0.40 & 1711.69\end{array}$

27.73

141225

$\begin{array}{llll}0.14 & 0.00 & -0.07\end{array}$

0.00

0.151

0.000

0.095

$0.015-2.44$

$\begin{array}{lll}0.00 & 0.162 & 0.000\end{array}$

0.084

$0.013-1.76$

$\begin{array}{lll}0.78 & 1720.33\end{array}$

35.02

$143 \quad 227$

$\begin{array}{llll}0.17 & 0.00 & -0.06\end{array}$

$\begin{array}{ll}0.01 & 0.183\end{array}$

0.000

$144 \quad 228$

$\begin{array}{llll}0.18 & 0.00 & -0.05\end{array}$

0.01

$\begin{array}{llll}0.19 & 0.00 & -0.05\end{array}$

0.02

0.194

0.000

0.087

$\begin{array}{ll}0.005 & -1.98\end{array}$

$\begin{array}{lll}0.77 & 1723.74\end{array}$

0.000

$\begin{array}{ll}0.76 & 1728.88\end{array}$

$\begin{array}{lll}0.58 & 1732.24\end{array}$

$\begin{array}{llll}0.079 & -0.006 & -1.82\end{array}$

$\begin{array}{lll}0.79 & 1736.93 \\ 0.39 & 1740.29 & -\end{array}$

$\begin{array}{ll}0.39 & 1740.29\end{array}$

0.000

$-0.009-1.3$

$\begin{array}{ll}0.48 & 1744.87\end{array}$

$\begin{array}{llll}0.060 & -0.020 & -1.79\end{array}$

$\begin{array}{lll}0.12 & 1747.97\end{array}$

$\begin{array}{ll}0.28 & 1752.25\end{array}$

$0.035-0.015-1.65$

$\begin{array}{ll}0.12 & 1755.17\end{array}$

$\begin{array}{ll}0.12 & 1759.16\end{array}$

$\begin{array}{llll}0.023 & -0.018 & -1.71\end{array}$

$\begin{array}{ll}0.12 & 1759.16 \\ 0.06 & 1761.55\end{array}$

$\begin{array}{lll}153 & 237\end{array}$

154238

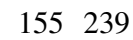

$\begin{array}{lll}156 & 240\end{array}$

$157 \quad 241$

$\begin{array}{lll}0.22 & 0.00 & 0.00\end{array}$

0.03
0.02

0.238

0.000

$\begin{array}{lll}0.22 & 0.00 & 0.01 \\ 0.22 & 0.00 & 0.01\end{array}$

0.02

0.240

0.000

$\begin{array}{lll}0.011 & -0.021 & -1.75\end{array}$

$\begin{array}{lll}0.34 & 1765.28 & 89.97\end{array}$

$\begin{array}{lll}0.22 & 0.00 & 0.02\end{array}$

$\begin{array}{ll}0.01 & 0.240\end{array}$

$\begin{array}{lllll}0.000 & -0.002 & -0.013 & -1.32\end{array}$

$\begin{array}{lll}0.41 & 1767.32 & 96.00\end{array}$

$\begin{array}{lllll}0.000 & 0.004 & -0.011 & -0.73\end{array}$

$\begin{array}{lll}0.66 & 1770.88 & 100.52\end{array}$

$\begin{array}{lll}0.19 & 0.00 & 0.02\end{array}$

0.01

0.206

$\begin{array}{lllll}0.000 & -0.008 & -0.013 & -1.08\end{array}$

$\begin{array}{lll}0.46 & 1773.00 & 106.47\end{array}$

\section{$158 \quad 242$}

159243

$\begin{array}{lll}0.18 & 0.00 & 0.02\end{array}$

$0.00 \quad 0.195$

$\begin{array}{lllll}0.000 & -0.010 & -0.003 & -0.83\end{array}$

$\begin{array}{lll}0.58 & 1776.48 & 111.06\end{array}$

160244

$\begin{array}{ll}0.18 & 0.00\end{array}$

0.03

0.00

0.196

$\begin{array}{lllll}0.000 & -0.023 & -0.005 & -1.27\end{array}$

$\begin{array}{lll}0.17 & 1778.62 & 116.99\end{array}$

161245

$\begin{array}{ll}0.18 & 0.00 \\ 0.18 & 0.00\end{array}$

0.00

0.196

$\begin{array}{lllll}0.000 & -0.034 & -0.007 & -1.46\end{array}$

$\begin{array}{lll}0.01 & 1782.18 & 121.50\end{array}$

162246

$0.18 \quad 0.00$

$0.05-0.01$

0.197

$\begin{array}{lll}0.000 & -0.047\end{array}$

$\begin{array}{lllll}0.000 & -2.09 & -0.38 & 1784.11 & 127.64\end{array}$

$\begin{array}{lll}0.000 & -0.047\end{array}$

$\begin{array}{lllll}0.000 & -2.13 & -0.48 & 1787.42 & 132.40\end{array}$

$\begin{array}{ll}163 & 247 \\ 164 & 248\end{array}$

165249

$\begin{array}{ll}0.17 & 0.00\end{array}$

$0.05-0.01$

0.185

$\begin{array}{lll}0.000 & -0.049\end{array}$

$\begin{array}{llllll}0.001 & -2.33 & -0.75 & 1789.04 & 138.85\end{array}$

$0.000-0.050$

$\begin{array}{llllll}0.001 & -2.10 & -0.55 & 1791.86 & 144.11\end{array}$

166250

$\begin{array}{lll}0.08 & 0.00 & 0.00\end{array}$

0.00

0.085

0.000

0.003

$\begin{array}{llllll}0.000 & -1.65 & -1.01 & 1793.50 & 150.54\end{array}$

$0.00 \quad 0.075$

0.000

0.014

$\begin{array}{lllll}0.001 & -1.91 & -1.28 & 1796.61 & 155.50\end{array}$

$\begin{array}{lllll}167 & 251 & -0.10 & 0.00 & -0.01 \\ 168 & 252 & -0.10 & 0.00 & -0.01\end{array}$

$0.00-0.104$

0.000

0.015

$\begin{array}{lllll}-0.001 & -2.70 & -1.92 & 1798.25 & 161.94\end{array}$

$0.00-0.104$

$\begin{array}{llllllll}170 & 254 & -0.10 & 0.00 & -0.01 & -0.01 & -0.104\end{array}$

0.000

0.015

$-0.001$

0.000

0.01$$
\begin{array}{rr}
-0.001 \\
0 & 0.008 \\
.016 & 0.008
\end{array}
$$

$\begin{array}{lllllll}171 & 255 & -0.10 & 0.00 & -0.01 & -0.01 & -0.104\end{array}$

0.000

0.016

$\begin{array}{lllllll}172 & 256 & -0.10 & 0.00 & -0.01 & -0.01 & -0.104\end{array}$

$\begin{array}{ll}0.000 & 0.016 \\ 0.000 & 0.016\end{array}$

$0.000 \quad 0.004$

$0.000-0.008$

$\begin{array}{llll}174 & 258 & -0.10 & 0.00\end{array}$

$0.00-0.01-0.104$

$0.00-0.105$

$\begin{array}{llll}175 & 259 & -0.10 & 0.00\end{array}$

$\begin{array}{lll}0.01 & 0.00 & -0.105 \\ 0.00 & 0.00 & -0.052\end{array}$

$\begin{array}{ll}0.000 & 0.001\end{array}$

$\begin{array}{llll}177 & 261 & -0.08 & 0.00\end{array}$

$\begin{array}{lll}0.01 & 0.00 & -0.084\end{array}$

$0.000-0.009$

$0.00-0.032$

$0.000 \quad 0.000$

$0.000 \quad 0.000$

$0.000 \quad 0.000$

0.000

$0.00 \quad 0.011$

0.000

0.000

$\begin{array}{llllll}0.008 & -5.67 & -4.87 & 1810.60 & 198.01\end{array}$

$\begin{array}{llllll}0.009 & -5.82 & -5.07 & 1812.94 & 203.75\end{array}$

$\begin{array}{llllll}0.001 & -6.48 & -5.73 & 1813.92 & 210.83\end{array}$

$\begin{array}{llllll}0.000 & -6.53 & -5.96 & 1816.12 & 216.71\end{array}$

$\begin{array}{lllll}0.001 & -7.28 & -6.58 & 1816.92 & 223.98\end{array}$

$\begin{array}{lllll}0.000 & -7.32 & -6.72 & 1818.86 & 230.11\end{array}$

$\begin{array}{lllll}0.000 & -8.02 & -7.15 & 1819.30 & 237.74\end{array}$

$\begin{array}{llllll}0.000 & -8.29 & -7.39 & 1821.19 & 243.92\end{array}$

181265

$0.00 \quad 0.00$

0.00

0.00

0.000

0.000

$\begin{array}{llllll}0.000 & -9.10 & -8.17 & 1821.84 & 251.34\end{array}$

$\begin{array}{lllll}0.000 & -9.30 & -8.34 & 1823.50 & 257.75\end{array}$

183267

$\begin{array}{llll}0.00 & 0.00 & 0.00\end{array}$

$\begin{array}{ll}0.00 & 0.000\end{array}$

$0.000 \quad 0.000$

$\begin{array}{lllll}0.000 & -9.94 & -8.95 & 1823.82 & 265.50\end{array}$

$\begin{array}{lllll}184 & 268 & 0.00 & 0.00 & 0.00\end{array}$

$0.00 \quad 0.000$
0.00

0.000

0.000

$\begin{array}{lllll}0.000 & -9.84 & -8.85 & 1825.06 & 272.33\end{array}$

$\begin{array}{lllll}185 & 269 & -0.01 & 0.00 & 0.00\end{array}$

$\begin{array}{lll}0.00 & -0.011 & 0.000\end{array}$

0.000

$\begin{array}{lllll}0.000 & -8.91 & -7.96 & 1823.75 & 281.72\end{array}$

$\begin{array}{llllllll}186 & 270 & 0.00 & 0.00 & 0.00 & 0.00 & 0.000 & 0.000\end{array}$

0.000

$\begin{array}{llllll}0.000 & -8.16 & -7.23 & 1824.20 & 289.34\end{array}$

$187 \quad 271$

0.00

0.00

$0.011-0.054$

0.001

$\begin{array}{llllll}0.001 & -7.37 & -6.23 & 1822.64 & 298.97\end{array}$

$\begin{array}{lllll}188 & 272 & 0.01 & 0.06 & 0.00\end{array}$

$\begin{array}{lll}0.00 & 0.012 & -0.081\end{array}$

0.002

$\begin{array}{llllll}0.002 & -6.75 & -5.34 & 1822.80 & 306.89\end{array}$

$\begin{array}{llllll}0.005 & -6.89 & -5.05 & 1821.80 & 315.96\end{array}$

$\begin{array}{rrrr}8.36 & 0.002 & -2.53 & 8.94\end{array}$

$\begin{array}{ll}-1.99 & 13.19\end{array}$

$-1.27 \quad 15.88$

$\begin{array}{ll}-0.89 & 20.23\end{array}$

$\begin{array}{ll}-0.21 & 23.14\end{array}$

$0.21 \quad 27.78$

$\begin{array}{ll}0.61 & 30.67\end{array}$

$0.61 \quad 35.14$

$0.93 \quad 38.20$

$0.91 \quad 42.89$

$0.87 \quad 45.85$

$0.71 \quad 50.63$

$0.90 \quad 54.05$

$0.52 \quad 58.84$

$\begin{array}{ll}0.62 & 62.42\end{array}$

$0.24 \quad 67.44$

$0.34 \quad 71.24$

$0.01 \quad 76.52$

$0.19 \quad 80.63$

$\begin{array}{ll}0.12 & 86.39\end{array}$

$0.40 \quad 90.83$

$0.43 \quad 96.90$

$0.68 \quad 101.51$

$0.48 \quad 107.56$

$\begin{array}{ll}0.59 & 112.24\end{array}$

$0.19 \quad 118.30$

$\begin{array}{ll}0.07 & 122.95\end{array}$ 


\section{$Z=84($ Po $)$} $\begin{array}{llllll}191 & 275 & 0.04 & 0.10 & -0.01\end{array}$ $\begin{array}{lllll}192 & 276 & 0.04 & 0.11 & -0.01\end{array}$ $\begin{array}{lllll}193 & 277 & 0.04 & 0.12 & -0.01\end{array}$ $\begin{array}{lllll}194 & 278 & 0.04 & 0.12 & -0.01\end{array}$ $\begin{array}{lllll}195 & 279 & 0.03 & 0.12 & -0.01\end{array}$

\section{$Z=85$ (At)}

$\begin{array}{lllll}99 & 184 & 0.29 & 0.00 & -0.01\end{array}$ $\begin{array}{lll}0.29 & 0.00 & 0.00\end{array}$ $\begin{array}{lllll}101 & 186 & 0.30 & 0.00 & 0.01\end{array}$ $\begin{array}{lllll}102 & 187 & 0.30 & 0.00 & 0.01\end{array}$ $\begin{array}{lllll}103 & 188 & 0.32 & 0.00 & 0.01\end{array}$

$\begin{array}{lllll}104 & 189 & 0.33 & 0.00 & 0.0\end{array}$ $\begin{array}{lllll}105 & 190 & 0.33 & 0.00 & 0.01\end{array}$ $\begin{array}{lllll}106 & 191 & -0.22 & 0.00 & 0.00\end{array}$ $\begin{array}{lllll}107 & 192 & -0.22 & 0.00 & 0.00\end{array}$ $\begin{array}{lllll}108 & 193 & -0.22 & 0.00 & 0.00\end{array}$

$\begin{array}{lllll}109 & 194 & -0.22 & 0.00 & 0.00\end{array}$ $\begin{array}{lllll}110 & 195 & -0.21 & 0.00 & 0.00\end{array}$ $\begin{array}{llll}111 & 196 & -0.21 & 0.00\end{array}$ $\begin{array}{llll}112 & 197 & -0.20 & 0.00\end{array}$

0.00 $\begin{array}{llll}113 & 198 & -0.20 & 0.00\end{array}$

0.00

$\begin{array}{lllll}114 & 199 & 0.09 & 0.00 & 0.00\end{array}$

$\begin{array}{lllll}115 & 200 & 0.09 & 0.00 & 0.00\end{array}$ $\begin{array}{lllll}116 & 201 & 0.08 & 0.00 & 0.01\end{array}$ $\begin{array}{rrrrr}117 & 202 & 0.08 & 0.00 & 0.01\end{array}$ $\begin{array}{lllll}118 & 203 & -0.08 & 0.00 & -0.01\end{array}$

$\begin{array}{lllll}119 & 204 & -0.08 & 0.00 & 0.00\end{array}$ $\begin{array}{lllll}120 & 205 & -0.07 & 0.00 & 0.00\end{array}$ $\begin{array}{lllll}121 & 206 & -0.07 & 0.00 & 0.00\end{array}$ $\begin{array}{lllll}122 & 207 & -0.05 & 0.00 & 0.01\end{array}$ $\begin{array}{lllll}123 & 208 & -0.04 & 0.00 & 0.02\end{array}$

$\begin{array}{lllll}124 & 209 & 0.01 & 0.00 & 0.00\end{array}$ $\begin{array}{lllll}125 & 210 & 0.01 & 0.00 & 0.00\end{array}$ $\begin{array}{lllll}126 & 211 & 0.00 & 0.00 & 0.00\end{array}$ $\begin{array}{llllll}127 & 212 & 0.01 & 0.00 & -0.01\end{array}$ $\begin{array}{lllll}128 & 213 & -0.01 & 0.00 & 0.00\end{array}$

$\begin{array}{lllll}129 & 214 & -0.01 & 0.00 & 0.00\end{array}$

\section{5}

131216

$132 \quad 217$

133218

13421

$135 \quad 220$

136221

$\begin{array}{ll}137 & 222\end{array}$

138223

139224

$140 \quad 225$

141226

$\begin{array}{ll}142 & 227\end{array}$

143228

144229

145230

146231

$147 \quad 232$

148233

$\begin{array}{llll}0.05 & 0.09 & -0.03\end{array}$

$\begin{array}{lll}0.06 & 0.10 & -0.03\end{array}$

$\begin{array}{lll}0.10 & 0.09 & -0.05\end{array}$

$\begin{array}{lll}0.10 & 0.09 & -0.05\end{array}$

\begin{abstract}
$\begin{array}{lll}0.00 & 0.035 & -0.123\end{array}$
$\begin{array}{lll}0.01 & 0.048 & -0.149\end{array}$

$\begin{array}{llll}0.01 & 0.049 & -0.163\end{array}$

$\begin{array}{lll}0.02 & 0.048 & -0.162\end{array}$

$\begin{array}{lll}0.02 & 0.038 & -0.162\end{array}$
\end{abstract}

$\begin{array}{llllll}0.016 & 0.006 & -6.53 & -4.46 & 1822.11 & 323.72\end{array}$

$\begin{array}{llllll}0.016 & -0.003 & -6.66 & -4.35 & 1821.15 & 332.74\end{array}$

$\begin{array}{lllllll}0.017 & -0.001 & -6.42 & -3.84 & 1821.40 & 340.57\end{array}$

$\begin{array}{llllll}0.018 & 0.000 & -6.55 & -3.65 & 1820.23 & 349.80\end{array}$

$\begin{array}{llllll}0.018 & -0.010 & -5.86 & -3.07 & 1820.27 & 357.84\end{array}$

$\begin{array}{llllll}0.018 & -0.010 & -5.45 & -2.72 & 1818.82 & 367.36\end{array}$ $\begin{array}{ll}-4.30 & 330.69\end{array}$

$-4.16 \quad 340.00$

$-3.60 \quad 348.12$

$-3.38 \quad 357.65$

$-2.73 \quad 366.01$

$-2.39 \quad 375.80$

$\begin{array}{lllllllll}0.01 & 0.317 & 0.000 & 0.051 & -0.001 & -1.38 & 0.88 & 1395.58 & 23.04\end{array}$ $\begin{array}{lllllllll}0.01 & 0.318 & 0.000 & 0.038 & -0.005 & -1.27 & 0.94 & 1407.72 & 18.97\end{array}$ $\begin{array}{lllllllll}0.02 & 0.330 & 0.000 & 0.030 & -0.018 & -1.56 & 0.88 & 1418.10 & 16.66\end{array}$ $\begin{array}{lllllllll}0.02 & 0.330 & 0.000 & 0.030 & -0.018 & -1.45 & 1.03 & 1429.73 & 13.10\end{array}$ $\begin{array}{lllllllll}0.01 & 0.353 & 0.000 & 0.034 & -0.008 & -1.64 & 1.05 & 1439.64 & 11.27\end{array}$

$\begin{array}{lllllllll}0.01 & 0.364 & 0.000 & 0.038 & -0.007 & -1.55 & 1.24 & 1450.81 & 8.17\end{array}$ $\begin{array}{lllllllll}0.00 & 0.364 & 0.000 & 0.036 & 0.003 & -1.50 & 1.30 & 1460.28 & 6.77\end{array}$ $\begin{array}{lllllllll}0.00 & -0.227 & 0.000 & 0.019 & -0.002 & -0.49 & 1.32 & 1471.24 & 3.88\end{array}$ $\begin{array}{lllllllll}0.00 & -0.227 & 0.000 & 0.019 & -0.002 & -0.82 & 1.03 & 1480.68 & 2.52\end{array}$ $\begin{array}{llllll}0.00 & -0.227 & 0.000 & 0.019 & -0.002 & -1.02\end{array}$ $0.83 \quad 1491.47$ $-0.20$

$0.56 \quad 1500.52$

$\begin{array}{llllll}0.00 & -0.227 & 0.000 & 0.019 & -0.002 & -1.31\end{array}$

$0.35 \quad 1510.95$

$-1.19$

$\begin{array}{rr}0.04 & 1519.68 \\ -0.22 & 1529.78\end{array}$

$\begin{array}{ll}0.00 & -0.217\end{array}$

0.000

$\begin{array}{llll}0.017 & -0.001 & -1.38\end{array}$

$\begin{array}{lll}0.00 & -0.207\end{array}$

0.000

$0.017-0.001$

$-1.68$

$-3.54$

$-4.20$

$\begin{array}{ll}0.00 & -0.207\end{array}$

0.000

$0.015-0.001$

$\begin{array}{lll}-1.80 & -0.22 & 1529.78 \\ -2.19 & -0.62 & 1538.26\end{array}$

-6.23
-6.64

$0.00 \quad 0.096$

0.000

$0.015-0.001$

$\begin{array}{lll}-1.72 & -0.88 & 1548.02\end{array}$

$-8.33$

$0.00 \quad 0.096$

0.000

$0.003 \quad 0.000$

$\begin{array}{lll}-2.33 & -1.36 & 1556.23\end{array}$

$-8.47$

$0.00 \quad 0.086$

$\begin{array}{lll}0.000 & -0.009 & -0.00\end{array}$

$-2.98$

$\begin{array}{llll}0.000 & -0.009 & -0.001\end{array}$

0.000

$0.014 \quad 0.009$

$-4.52$

$\begin{array}{ll}0.00 & -0.084\end{array}$

0.000

$0.002-0.000$
$0.002-0.000$

$-5.29$

$\begin{array}{lll}0.00 & -0.073\end{array}$

0.000

$0.002-0.000$

$\begin{array}{ll}0.00 & -0.053\end{array}$

0.000

$0.002-0.000$

$-5.99$

$0.000-0.023$

0.001

$-7.6$

$\begin{array}{ll}0.00 & -0.042\end{array}$

$0.000 \quad 0.000$

0.000

$-9.26$

$0.00 \quad 0.011$

0.000

0.000

$$
0.000
$$

$0.00 \quad 0.01$

0.000
0.000

0.012

0.000

$\begin{array}{lllllll}0.000 & -10.45 & -9.27 & 1648.03 & -11.48 & -11.65\end{array}$

$0.00-0.011$

0.000

0.000

0.000

$\begin{array}{lll}-9.50 & -8.42 & 1653.06\end{array}$

$\begin{array}{lll}-0.01 & -0.010 & 0.000\end{array}$

0.000

0.010

$-8.44$

$-7.38$

$\begin{array}{lll}0.00 & 0.011 & 0.000\end{array}$

0.000

0.000

$-7.24$

$0.00-0.068-0.138$

0.040

0.008

$-7.14$

$-6$

$6.30 \quad 1663.79$

0.041

0.01

$-6.73$

0.055

0.012

$\begin{array}{lll}-6.59 & -3.48 & 1679.95 \\ -3.01 & 1684.52\end{array}$

0.067

0.023

$\begin{array}{lll}-5.95 & -2.33 & 1690.26\end{array}$

$\begin{array}{llll}0.00 & 0.110 & -0.125\end{array}$

$0.100-0.127$

0.068

0.014

$\begin{array}{lll}-5.51 & -1.99 & 1694.70\end{array}$

$\begin{array}{lll}0.00 & 0.110 & -0.125\end{array}$

0.068

$\begin{array}{lllll}0.014 & -4.81 & -1.34 & 1700.21\end{array}$

$\begin{array}{llll}0.014 & -4.39 & -0.95 & 1704.34\end{array}$

0.068

$\begin{array}{lllll}0.003 & -3.33 & -0.29 & 1709.58\end{array}$

\subsection{4}

0.025

$\begin{array}{llll}-3.35 & -0.06 & 1713.63\end{array}$

$0.152-0.028$
$0.162-0.028$

$\begin{array}{lllll}0.14 & 0.02 & -0.07 & -0.01\end{array}$

$\begin{array}{lll}0.162 & -0.028\end{array}$

0.095

0.026

$-2.78$

$\begin{array}{ll}0.35 & 1718.86\end{array}$

$\begin{array}{ll}0.34 & 1722.91\end{array}$

$0.68 \quad 1727.96$

$0.006-2.40$

$0.44 \quad 1732.00$

$\begin{array}{ll}0.58 & 1737.02\end{array}$

$0.41 \quad 1740.75$

$0.52 \quad 1745.56$

$0.20 \quad 1749.22$

$\begin{array}{ll}0.32 & 1753.79\end{array}$
$-8.44$

$-6.59$

$-3.03$

$-0.74$

2.87

5.03

8.53

10.86

14.49

17.05

20.99

23.82

27.85

30.68

34.71

37.72

41.76

44.81

49.15

52.41

56.83

60.33
$-8.62$

$-6.58$

$-1.25$

2.26

4.40

8.10

10.40
14.35 $\begin{array}{lr}0.77 & 24.05 \\ 0.84 & 19.92 \\ 0.77 & 17.51 \\ 0.94 & 13.90 \\ 0.93 & 11.96 \\ 1.14 & 8.80 \\ 1.19 & 7.32 \\ 1.27 & 4.43 \\ 0.98 & 3.00 \\ 0.79 & 0.22\end{array}$

$\begin{array}{ll}0.51 & -0.83 \\ 0.31 & -3.22\end{array}$

$-0.01 \quad-3.95$

$\begin{array}{lll}0.051 & -0.25 & -6.02\end{array}$

$\begin{array}{lll}0.049 & -0.65 & -6.48\end{array}$

$\begin{array}{lll}0.050 & -0.89 & -8.19\end{array}$

$\begin{array}{lll}0.024 & -1.37 & -8.37\end{array}$

$\begin{array}{llll}0.008 & -2.27 & -10.39\end{array}$

$\begin{array}{llll}0.028 & -2.64 & -10.13\end{array}$

$\begin{array}{lll}0.012 & -3.89 & -12.17\end{array}$

$\begin{array}{lll}0.024 & -4.67 & -11.99\end{array}$

$\begin{array}{llll}0.015 & -5.34 & -13.11\end{array}$

$\begin{array}{lll}0.020 & -6.23 & -12.72\end{array}$ 


\section{$Z=85$ (At)}

\begin{tabular}{|c|c|c|c|c|c|c|c|c|c|c|c|c|}
\hline 34 & 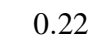 & 00 & -0.02 & .03 & & & 8 & & -2 & & & \\
\hline 35 & 0.22 & 00 & -0.02 & .03 & & 000 & 0.048 & -0.022 & -2.03 & 0.10 & & 266 \\
\hline 36 & 22 & 00 & -0.01 & .03 & & & 36 & -0.025 & -2.24 & & & \\
\hline 37 & 22 & 00 & 0.00 & .02 & & & 23 & & -1.75 & 0.07 & & .62 \\
\hline 38 & .22 & 00 & 0.00 & .02 & .239 & & .023 & & -1.80 & 0.03 & & \\
\hline 239 & 0.22 & .00 & 0.01 & 0.02 & .240 & 00 & 011 & & & & & \\
\hline 40 & 22 & 00 & 0.01 & .02 & & & & & & & & \\
\hline 41 & 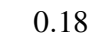 & 0 & 0.01 & .01 & & & & & & & & \\
\hline 42 & 18 & 00 & 0.01 & .01 & 5 & & 0.002 & & & 52 & & \\
\hline 43 & .17 & .00 & 0.02 & .00 & .184 & 00 & -0.012 & & -0.73 & 0.61 & & 076 \\
\hline 244 & 17 & 00 & 0.03 & 00 & 185 & 00 & 0.024 & -0.005 & -1.11 & 33 & .54 & 1336 \\
\hline 45 & 17 & 0 & 0.03 & 00 & & & -0.024 & & & 2 & & \\
\hline 246 & 0.17 & 00 & 0.04 & 0.00 & 5 & & -0.0 & -( & - & 28 & & \\
\hline 247 & 0.18 & 00 & 0.05 & 0.01 & 7 & & & & & & & \\
\hline 48 & 0.17 & 0.00 & 0.05 & 01 & 85 & & -0 & & -2.21 & 62 & & \\
\hline 249 & 16 & 00 & 05 & -0.01 & 4 & 0 & 0 & 1 & 8 & 12 & & 30.60 \\
\hline 50 & 0.12 & 00 & 01 & 0.00 & & & -0.006 & & & & & \\
\hline 251 & 0.08 & 00 & 00 & 0.00 & 5 & & & & 74 & - & & \\
\hline 52 & -0.13 & 00 & & 00 & & & & & & & & \\
\hline 53 & 12 & 0 & 00 & 0.00 & & & & & & & & \\
\hline 54 & .13 & 0.00 & 0 & & 5 & & & & 1 & $J$ & & \\
\hline 55 & .10 & 00 & 0.01 & & -0 . & & & & & -2 & & \\
\hline 256 & -0.13 & 0 & 0.00 & 0.01 & -0 . & & & & 72 & -3 & & \\
\hline 57 & 0.13 & 0 & 00 & & 5 & & & & & & & \\
\hline & & 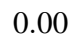 & & & & & & & & & & \\
\hline 59 & $\Omega_{1}$ & .00 & 00 & & & & & & & & & \\
\hline 60 & 0.10 & .00 & 01 & & -0 & & & & & & & \\
\hline 261 & -0.09 & .00 & 01 & -0.01 & -0.0 & & & & & & & \\
\hline 62 & 0.09 & 0 & & 0.0 & -0 . & & & & & & & \\
\hline 63 & -0.08 & 0 & 0.02 & -0.01 & -0.084 & 0 & -0.020 & 1 & 4 & -6 & & 2 \\
\hline 64 & 0.05 & OQ & 01 & 0 & -0.053 & & - & & -7.44 & & & \\
\hline 65 & 0.03 & 0 & & & & & & & & & & \\
\hline 266 & 0.0 & 0 & & & & & & & & & & \\
\hline 67 & 0.0 & & & & & & & & & & & \\
\hline 268 & 0.01 & 0.0 & 0.00 & 0 & 0.0 & 0. & 00 & 0 & -9.08 & -8.10 & & 25 \\
\hline 69 & 0.00 & 0 & $0 \Omega$ & 0.00 & 0 & 000 & 0 & 0 & $-\varepsilon$ & -8.00 & & 16 \\
\hline 70 & -0.0 & & & & & & & & & & & \\
\hline 71 & & J & & & & & & & & & & \\
\hline 72 & 0.01 & . & & & & & & & & & & 29 \\
\hline 273 & 0.02 & 0.06 & -0.01 & 0.00 & 0.023 & 1 & 13 & 3 & -5.95 & -4.55 & & 299.00 \\
\hline 74 & 0.04 & 0.09 & ר & (1) 00 & 6 & 0123 & 8 & 7 & - & - & 42 & 307.62 \\
\hline 275 & & & & & & & & & & & & \\
\hline 276 & 8 & 11 & - & & & r & & -0 . & & -3 & & \\
\hline 277 & & 0.1 & & & & & & & & & & 331.71 \\
\hline 278 & 0.0 & 0.1 & -0.01 & 0.0 & & -0 . & & -0 . & -6.10 & -3.22 & & \\
\hline (1) & & & & & & & & c & & & & \\
\hline 200 & & & & & & & & & & & & \\
\hline 281 & 0.1 & 0.12 & -0.05 & 000 & & -0 . & & & 26 & -1.63 & & 366.1 \\
\hline 282 & 0.10 & 0.11 & -0.05 & 0.00 & 0.112 & -0.154 & 0.069 & 0.017 & -4.74 & -1.53 & 4.42 & 375.1 \\
\hline
\end{tabular}

$0.11 \quad 65.40$

$0.22 \quad 69.18$

$-0.11 \quad 74.10$

$0.11 \quad 78.22$

$0.01 \quad 83.57$

$0.40 \quad 88.08$

$0.47 \quad 93.83$

$\begin{array}{ll}0.80 & 98.49\end{array}$

$\begin{array}{ll}0.52 & 104.09\end{array}$

$0.61 \quad 108.73$

$0.34 \quad 114.55$

$0.14 \quad 119.10$

$-0.24 \quad 125.01$

$-0.21 \quad 130.00$

$-0.54 \quad 136.15$

$-0.32 \quad 141.52$

$-0.80 \quad 147.72$

$-1.07 \quad 152.81$

$-1.70 \quad 159.04$

$-2.04 \quad 164.23$

$\begin{array}{ll}-2.74 & 170.58\end{array}$

$\begin{array}{ll}-2.96 & 176.09\end{array}$

$-3.66 \quad 182.62$

$-3.87 \quad 188.32$

$-4.53 \quad 195.06$

$-4.73 \quad 200.96$

$-5.40 \quad 207.86$

\begin{tabular}{ll}
$-5.61 \quad 213.92$ \\
\hline
\end{tabular}

$\begin{array}{ll}-6.21 \quad 221.07 \\ -6.32 & 227.40\end{array}$

$-6.32 \quad 227.40$

$-6.70 \quad 234.93$

$-6.77 \quad 241.47$

$-7.45 \quad 248.86$

$-7.54 \quad 255.55$

$-8.11 \quad 263.22$

$-8.00 \quad 270.26$

$-7.14 \quad 279.51$

$\begin{array}{ll}-6.40 \quad 287.36 \\ -5.37 & 296.92\end{array}$

$-5.37 \quad 296.92$

$-4.48 \quad 305.07$

$-4.23 \quad 314.01$

$-3.69321 .96$

$\begin{array}{ll}-3.65 & 330.84\end{array}$

\begin{tabular}{ll}
$-3.11 \quad 338.94$ \\
\hline
\end{tabular}

$-2.92 \quad 348.11$

$\begin{array}{ll}-2.45 & 356.29\end{array}$

$-1.84 \quad 366.04$

$-1.23 \quad 374.49$

$-1.18 \quad 383.82$

\section{$Z=86(\mathrm{Rn})$}

\begin{tabular}{|c|c|c|c|c|c|c|c|c|c|c|c|c|c|c|}
\hline 100186 & 0.30 & 0.00 & 0.00 & 0.02 & 0.329 & 0.000 & 0.042 & -0.014 & -1.65 & 0.69 & 1407.37 & 26.61 & 0.60 & 27.76 \\
\hline $101 \quad 187$ & 0.30 & 0.00 & 0.00 & 0.02 & 0.329 & 0.000 & 0.042 & -0.014 & -1.82 & 0.61 & 1417.83 & 24.22 & 0.50 & 25.26 \\
\hline 102188 & 0.30 & 0.00 & 0.01 & 0.02 & 0.330 & 0.000 & 0.030 & -0.018 & -1.64 & 0.77 & 1429.91 & 20.21 & 0.69 & 21.20 \\
\hline 103189 & 0.32 & 0.00 & 0.01 & 0.01 & 0.353 & 0.000 & 0.034 & -0.008 & -1.75 & 0.80 & 1439.86 & 18.33 & 0.69 & 19.21 \\
\hline 104190 & 0.33 & 0.00 & 0.01 & 0.01 & 0.364 & 0.000 & 0.038 & -0.007 & -1.63 & 1.02 & 1451.47 & 14.80 & 0.93 & 15.62 \\
\hline 5191 & 0.28 & 0.00 & 0.03 & 0.00 & 0.308 & 0.000 & -0.002 & -0.006 & -1.01 & 1.15 & 1460.93 & 13.41 & 1.07 & 14.16 \\
\hline $06 \quad 192$ & 0.33 & 0.00 & 0.01 & 0.00 & 0.364 & 0.000 & 0.036 & 0.003 & -1.26 & 1.35 & 1472.16 & 10.25 & 1.27 & 10.93 \\
\hline
\end{tabular}




\section{$Z=86(\mathrm{Rn})$} $\begin{array}{llll}108 & 194 & -0.23 & 0.00\end{array}$ $\begin{array}{llll}109 & 195 & -0.23 & 0.00\end{array}$ $\begin{array}{llll}110 & 196 & -0.22 & 0.00\end{array}$ $\begin{array}{lll}0.00 & -0.237\end{array}$ $\begin{array}{llll}0.000 & -0.009 & -0.006 & -0.56\end{array}$ $\begin{array}{llll}111 & 197 & -0.22 & 0.00\end{array}$

0.00 $0.00 \quad-0.227$ $\begin{array}{lllll}0.000 & 0.020 & -0.002 & -0.70\end{array}$ $\begin{array}{lllll}0.000 & 0.020 & -0.002 & -0.98\end{array}$ $\begin{array}{ll}1.32 & 1481.40\end{array}$ $\begin{array}{ll}1.21 & 1492.55\end{array}$ $0.93 \quad 1501.66$ $\begin{array}{llll}0.000 & 0.019 & -0.002 & -1.03\end{array}$ $0.76 \quad 1512.49$ $\begin{array}{lll}0.00 & -0.227\end{array}$ 0.000 $\begin{array}{lll}0.019 & -0.002 & -1.32\end{array}$ $0.48 \quad 1521.25$

9.08 6.01 4.97 2.20 2.20
1.52 $0.27 \quad 1531.75$ $-0.91$

$\begin{array}{llll}112 & 198 & -0.22 & 0.00\end{array}$

$\begin{array}{llll}113 & 199 & -0.21 & 0.00\end{array}$

$\begin{array}{lll}0.00 & -0.227\end{array}$

0.000

$\begin{array}{lll}0.019 & -0.002 & -1.51\end{array}$

$\begin{array}{lll}0.00 & -0.217\end{array}$

0.000

$0.017-0.001-1.75$

$\begin{array}{rr}0.27 & 1531.75 \\ -0.12 & 1540.26\end{array}$

$\begin{array}{lllll}114 & 200 & -0.20 & 0.00 & 0.01\end{array}$

$0.00-0.207$

0.000

0.004

\subsection{1}

0.000
0.000

0.004

$\begin{array}{lllll}0.001 & -2.40 & -0.85 & 1558.71\end{array}$

$\begin{array}{llllllll}116 & 202 & -0.11 & 0.00 & -0.01 & -0.01 & -0.115\end{array}$

0.000

0.017

$\begin{array}{llll}0.008 & -2.24 & -1.43 & 1568.87\end{array}$

$\begin{array}{llllllll}117 & 203 & -0.11 & 0.00 & -0.01 & -0.01 & -0.115\end{array}$

$\begin{array}{lllllll}118 & 204 & -0.11 & 0.00 & -0.01 & -0.01 & -0.115\end{array}$

$\begin{array}{lllllll}119 & 205 & -0.10 & 0.00 & -0.01 & -0.01 & -0.104\end{array}$

0.000

0.017

$\begin{array}{llll}0.008 & -2.92 & -2.06 & 1576.95\end{array}$

0.000

0.017

$$
0.008
$$

$\begin{array}{llllllll}120 & 206 & -0.09 & 0.00 & -0.01 & -0.01 & -0.094\end{array}$

0.000

0.016

$$
0.000
$$$$
\begin{array}{lllllll}
0.008 & -4.43 & -3.58 & 1594.83 & -7.50 & -7.71
\end{array}
$$

$\begin{array}{llllllll}122 & 208 & -0.06 & 0.00 & 0.01 & 0.00 & -0.063\end{array}$

$\begin{array}{llllllll}123 & 209 & -0.06 & 0.00 & 0.01 & 0.00 & -0.063\end{array}$

$\begin{array}{lllllll}124 & 210 & 0.00 & 0.00 & 0.00 & 0.00 & 0.000\end{array}$

$\begin{array}{lllllll}125 & 211 & 0.00 & 0.00 & 0.00 & 0.00 & 0.000\end{array}$

$\begin{array}{lllllll}126 & 212 & 0.00 & 0.00 & 0.00 & 0.00 & 0.000\end{array}$

$\begin{array}{llllll}127 & 213 & -0.01 & 0.00 & -0.0\end{array}$

$\begin{array}{lllll}128 & 214 & 0.00 & 0.00 & 0.00\end{array}$

$\begin{array}{lllll}129 & 215 & -0.01 & 0.00 & 0.00\end{array}$

$\begin{array}{lllll}130 & 216 & 0.00 & 0.00 & 0.00\end{array}$

$0.00-0.010$

$0.000-0.010$

$$
0.001
$$

$\begin{array}{lllllll}0.001 & -6.48 & -5.76 & 1621.33 & -9.78 & -9.65\end{array}$

$\begin{array}{lllllll}0.001 & -7.35 & -6.55 & 1628.63 & -9.00 & -8.93\end{array}$

$0.000 \quad 0.000$

$\begin{array}{lllllll}0.000 & -8.04 & -7.01 & 1637.37 & -9.67 & -9.60\end{array}$

$\begin{array}{llllll}0.000 & -9.05 & -7.94 & 1644.50 & -8.74 & -8.76\end{array}$

0.000

0.000

$\begin{array}{lllllll}0.000 & -9.30 & -8.16 & 1652.69 & -8.85 & -8.66\end{array}$

0.000

$0.00 \quad 0.000$
0.00

0.000

0.012
0.000

$\begin{array}{ll}0.00 & -0.011\end{array}$

0.000

$\begin{array}{lll}0.00 & 0.000 & 0.000\end{array}$

$\begin{array}{lll}0.00 & 0.068 & -0.138\end{array}$

0.000$$
\begin{array}{r}
-0.000 \\
0.000
\end{array}
$$$$
-5.74-5.70
$$

$\begin{array}{llllll}0.041 & 0.010 & -6.69 & -3.44 & 1679.92\end{array}$

$132 \quad 218$

133219

$0.07 \quad 0.10-0.04$

0.00

$0.079-0.139$

134220

$\begin{array}{lll}0.09 & 0.09 & -0.05\end{array}$

$-0.01$

$0.100-0.127$

0.054

$\begin{array}{lllll}0.012 & -6.21 & -2.73 & 1686.31\end{array}$

0.067

$\begin{array}{lllll}0.023 & -6.10 & -2.46 & 1691.12\end{array}$

$\begin{array}{llll}0.00 & 0.110 & -0.125\end{array}$

0.068

$\begin{array}{llll}0.014 & -5.32 & -1.82 & 1697.30\end{array}$

$\begin{array}{lll}0.10 & 0.09 & -0.05 \\ 0.10 & 0.09 & -0.05\end{array}$

$\begin{array}{lll}0.00 & 0.110 & -0.125\end{array}$

136222

$\begin{array}{lll}0.10 & 0.09 & -0.05\end{array}$

0.00

$0.110-0.125$

0.068

$\begin{array}{llll}0.014 & -5.05 & -1.56 & 1701.86\end{array}$

$\begin{array}{llll}0.014 & -4.37 & -0.93 & 1707.78\end{array}$

$\begin{array}{llll}0.00 & 0.143 & -0.126\end{array}$

0.084

$\begin{array}{llll}0.018 & -4.74 & -0.61 & 1712.02\end{array}$

$\begin{array}{lllll}0.017 & -3.79 & -0.06 & 1717.76\end{array}$

$\begin{array}{lll}0.00 & 0.142 & -0.112\end{array}$

0.083

$0.018-3.49$

$0.12 \quad 1721.89$

$\begin{array}{llll}0.00 & 0.163 & -0.070\end{array}$

0.097

$0.017-2.92$

$\begin{array}{ll}0.47 & 1727.57\end{array}$

0.097
0.098

$\begin{array}{ll}0.017 & -2.85\end{array}$

$0.45 \quad 1731.66$

$\begin{array}{ll}0.007 & -2.53\end{array}$

$\begin{array}{ll}0.46 \quad 1737.43 \\ 0.41 & 1741.31\end{array}$

0.100

$0.006-2.32$

$0.41 \quad 1741.31$

$\begin{array}{llll}0.091 & -0.004 & -2.22\end{array}$

$\begin{array}{ll}0.54 & 1746.71\end{array}$

$\begin{array}{lll}0.02 & 0.204 & 0.000\end{array}$

$\begin{array}{lll}0.02 & 0.215 & 0.000\end{array}$

$\begin{array}{llll}0.080 & -0.005 & -2.20\end{array}$

$0.29 \quad 1750.56$

$\begin{array}{llll}0.083 & -0.014 & -2.31\end{array}$

$0.39 \quad 1755.76$

$\begin{array}{llll}0.03 & 0.226 & -0.027\end{array}$

$\begin{array}{llll}0.073 & -0.017 & -2.43\end{array}$

$\begin{array}{ll}0.07 & 1759.44\end{array}$

$\begin{array}{lll}0.03 & 0.237 & 0.000\end{array}$

$\begin{array}{llll}0.060 & -0.020 & -2.04\end{array}$

$0.24 \quad 1764.34$

$\begin{array}{ll}0.03 & 0.237\end{array}$

0.000

$0.060-0.020-2.40$

$-0.09 \quad 1767.81$

$\begin{array}{ll}0.03 & 0.238\end{array}$

0.000

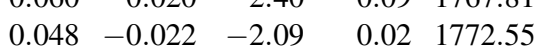

0.000

$\begin{array}{lll}0.036 & -0.025 & -2.27\end{array}$

$-0.25 \quad 1775.74$

$0.03 \quad 0.238$

0.000

$\begin{array}{llllll}0.036 & -0.025 & -2.00 & -0.03 & 1780.14\end{array}$

0.000

$\begin{array}{lll}0.023 & -0.018 & -1.77\end{array}$

$0.02 \quad 1782.81$

$\begin{array}{lll}0.023 & -0.018-1.36\end{array}$

$\begin{array}{ll}0.42 & 1786.81\end{array}$

0.000

$\begin{array}{lll}0.011 & -0.021 & -1.31\end{array}$

$0.47 \quad 1789.26$

$\begin{array}{lll}0.006 & -0.011 & -0.64\end{array}$

$0.87 \quad 1793.06$

$\begin{array}{lllll}0.000 & 0.004 & -0.011 & -0.81\end{array}$

$\begin{array}{lll}0.64 & 1795.59 & 98.46\end{array}$

$157 \quad 243$

$158 \quad 244$

$\begin{array}{ll}0.19 & 0.00\end{array}$

0.01

0.01

0.206

0.000

$\begin{array}{lll}0.004 & -0.011 & -0.81 \\ -0.010 & -0.003 & -0.62\end{array}$

$\begin{array}{rrr}0.64 & 1795.59 & 98.46 \\ 0.76 & 1799.45 & 102.67\end{array}$

159245

$0.18 \quad 0.00$

0.03

$0.00 \quad 0.195$

$\begin{array}{lllll}0.000 & -0.023 & -0.005 & -1.01\end{array}$

$\begin{array}{lll}0.49 & 1801.83 & 108.36\end{array}$

160246

$0.18 \quad 0.00$

0.03

$0.00-0.196$

$\begin{array}{lllll}0.000 & -0.023 & -0.005 & -0.94\end{array}$

$\begin{array}{lll}0.47 & 1805.63 & 112.63\end{array}$

$\begin{array}{llllllll}0.000 & -0.034 & -0.007 & -1.51 & -0.00 & 1808.01 & 118.32\end{array}$

162248 $\begin{array}{ll}0.18 & 0.00\end{array}$

$0.04 \quad 0.00$

0.196

$0.000-0.047$

$\begin{array}{lllll}0.000 & -1.71 & -0.03 & 1811.62 & 122.79\end{array}$

$\begin{array}{llllll}0.001 & -1.93 & -0.32 & 1813.64 & 128.83\end{array}$

\section{$-4.32$}

$1.09 \quad 0.26$

4.28

5.96
9.22

9.22
11.11

14.62

16.77

22.93

26.88

29.26

33.25

39.74

42.41

46.63

53.89

57.06

61.67

69.88

78.95

83.03

88.64

163249 $\begin{array}{ll}0.18 \quad 0.00 \\ 0.17 & 0.000\end{array}$

$0.05-0.01$
0.185 


\section{$Z=86(\mathrm{Rn})$}

$\begin{array}{lllllll}164 & 250 & 0.16 & 0.00 & 0.04 & -0.01 & 0.174\end{array}$

$\begin{array}{llllllllllllll}165 & 251 & 0.16 & 0.00 & 0.04 & -0.01 & 0.174 & 0.000 & -0.038 & 0.003 & -1.77 & -0.35 & 1818.60 & 140.02\end{array}$

$0.000-0.005-0.011-2.05-1.17 \quad 1823.98-150.78$

$\begin{array}{llllllllllllll}168 & 254 & -0.13 & 0.00 & -0.01 & 0.00 & -0.135 & 0.000 & 0.018 & -0.002 & -2.39 & -1.44 & 1827.26 & 155.57\end{array}$

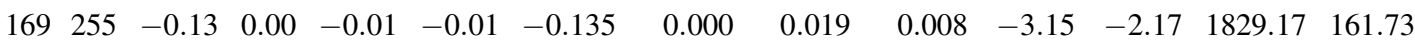

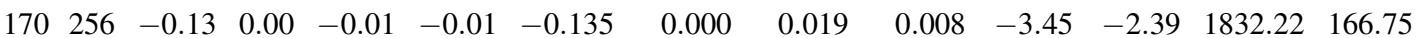

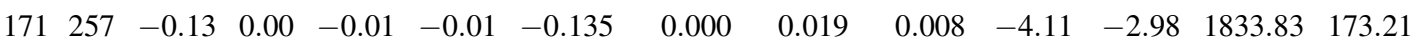

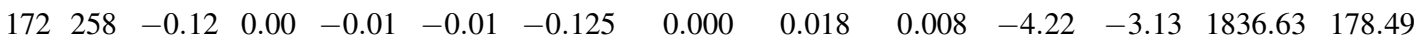

$\begin{array}{llllllllllllll}173 & 259 & -0.10 & 0.00 & -0.01 & -0.01 & -0.104 & 0.000 & 0.016 & 0.008 & -4.64 & -3.89 & 1838.24 & 184.95\end{array}$

$\begin{array}{llllllllllllll}174 & 260 & -0.10 & 0.00 & 0.00 & -0.01 & -0.104 & 0.000 & 0.004 & 0.009 & -4.76 & -4.07 & 1840.89 & 190.37\end{array}$

$\begin{array}{lllllll}175 & 261 & -0.10 & 0.00 & 0.00 & -0.01 & -0.104\end{array}$

$0.000 \quad 0.004$

$\begin{array}{lllll}0.009 & -5.41 & -4.72 & 1842.22 & 197.11\end{array}$

$\begin{array}{llll}176 & 262 & -0.09 & 0.00\end{array}$

$\begin{array}{lll}0.01 & -0.01 & -0.094\end{array}$

$0.000-0.008$

$\begin{array}{llll}177 & 263 & -0.09 & 0.00\end{array}$

$\begin{array}{lll}0.01 & -0.01 & -0.094\end{array}$

$\begin{array}{llllll}0.010 & -5.55 & -4.92 & 1844.73 & 202.67\end{array}$

$\begin{array}{llll}178 & 264 & -0.08 & 0.00\end{array}$

$\begin{array}{lll}0.01 & -0.01 & -0.084\end{array}$

$0.000-0.009$

$\begin{array}{lllll}0.010 & -6.15 & -5.50 & 1845.83 & 209.64\end{array}$

$\begin{array}{lllll}179 & 265 & -0.07 & 0.00\end{array}$

$\begin{array}{llll}180 & 266 & -0.04 & 0.00\end{array}$

$\begin{array}{lll}0.01 & 0.00 & -0.073\end{array}$

$0.000-0.010$

$\begin{array}{lllll}0.010 & -6.24 & -5.56 & 1848.03 & 215.51\end{array}$

$\begin{array}{llll}181 & 267 & 0.00 & 0.00\end{array}$

182268

$0.00 \quad 0.00$

$0.00-0.042$

$0.000-0.011$

$\begin{array}{lllll}0.001 & -6.66 & -6.00 & 1848.83 & 222.78\end{array}$

$\begin{array}{llllll}0.001 & -6.65 & -5.90 & 1850.71 & 228.98\end{array}$

$\begin{array}{ll}0.00 & 0.00\end{array}$

0.00

$0.00 \quad 0.000$

$0.000 \quad 0.000$

$\begin{array}{llllll}0.000 & -7.28 & -6.41 & 1851.43 & 236.33\end{array}$

183269

$\begin{array}{rrrrrrr}184 & 270 & 0.00 & 0.00 & 0.00 & 0.00 & 0.000\end{array}$

$\begin{array}{lll}0.00 & 0.00 & 0.000\end{array}$

0.000

0.000

$\begin{array}{llllll}0.000 & -7.45 & -6.55 & 1853.39 & 242.44\end{array}$

$\begin{array}{llllll}0.000 & -8.05 & -7.12 & 1854.02 & 249.89\end{array}$

$\begin{array}{llll}185 & 271 & -0.01 & 0.00\end{array}$

0.00

$0.00-0.011$

0.000

0.000

$\begin{array}{lllll}186 & 272 & 0.00 & 0.00 & 0.00\end{array}$

$\begin{array}{lll}0.00 & 0.000 & 0.000\end{array}$

0.000

$\begin{array}{lllll}0.000 & -7.91 & -6.99 & 1855.55 & 256.43\end{array}$

$187 \quad 273$

$\begin{array}{lll}0.01 & 0.05 & 0.00\end{array}$

$\begin{array}{lll}0.00 & 0.012 & -0.067\end{array}$

0.000

$\begin{array}{lllll}0.000 & -6.99 & -6.12 & 1854.58 & 265.47\end{array}$

188274

$\begin{array}{lll}0.02 & 0.07 & -0.01\end{array}$

0.00

$0.023-0.095$

0.001

$\begin{array}{llllll}0.000 & -6.26 & -5.39 & 1855.37 & 272.75\end{array}$

189275

190276

$\begin{array}{lll}0.04 & 0.09 & -0.02\end{array}$

0.00

$0.046-0.123$

0.014

0.002

$\begin{array}{llll}4 & -3.67 & 1854.77 & 289.49\end{array}$

191277

$\begin{array}{llll}0.04 & 0.10 & -0.02\end{array}$

$\begin{array}{llll}0.00 & 0.047 & -0.137\end{array}$

0.028

$\begin{array}{llllll}0.007 & -5.71 & -3.56 & 1854.27 & 298.06\end{array}$

$\begin{array}{llll}0.01 & 0.058 & -0.150\end{array}$

0.030

$\begin{array}{llllll}0.008 & -5.50 & -3.09 & 1855.01 & 305.39\end{array}$

192278

$\begin{array}{lll}0.05 & 0.11 & -0.02 \\ 0.05 & 0.12 & -0.02\end{array}$

$\begin{array}{lll}0.02 & 0.059 & -0.163\end{array}$

$\begin{array}{lllllll}0.031 & -0.009 & -5.67 & -2.87 & 1855.34 & 321.20\end{array}$

193279

$\begin{array}{lll}0.05 & 0.12 & -0.01\end{array}$

0.02

$0.059-0.162$

0.018

$\begin{array}{lllllll}0.019 & -0.008 & -5.10 & -2.08 & 1854.83 & 337.86\end{array}$

195281

$\begin{array}{lll}0.05 & 0.13 & -0.01\end{array}$

$\begin{array}{lll}0.02 & 0.060 & -0.176\end{array}$

0.070$$
0.019
$$

$\begin{array}{llllll}0.019 & -5.32 & -1.76 & 1853.71 & 347.04\end{array}$

19628

$\begin{array}{llll}0.10 & 0.12 & -0.05 & 0.00\end{array}$

$0.113-0.168$

0.070

$\begin{array}{llllll}0.019 & -4.83 & -1.33 & 1854.08 & 354.75\end{array}$

197283

$\begin{array}{lllll}0.11 & 0.07 & -0.06 & -0.01\end{array}$

$0.121-0.099$

0.080

$\begin{array}{llllll}0.024 & -3.34 & -0.98 & 1852.80 & 364.10\end{array}$

198284

$\begin{array}{llll}0.10 & 0.11 & -0.05 & 0.00\end{array}$

$0.112-0.154$

0.069

$\begin{array}{llllll}0.017 & -3.77 & -0.67 & 1853.15 & 371.82\end{array}$

$\begin{array}{llllll}199 & 285 & 0.11 & 0.02 & -0.08 & -0.02\end{array}$

$\begin{array}{ll}0.121 & -0.029\end{array}$

0.105

$\begin{array}{lllll}0.035 & -3.20 & -0.89 & 1852.32 & 380.72\end{array}$

200286

$\begin{array}{lllll}0.028 & -2.93 & -0.38 & 1852.34 & 388.77\end{array}$
$0.000-0.008$

$0.000 \quad 0.000$ $\begin{array}{llll}0.14 & 0.00 & -0.08 & -0.01\end{array}$
$0.153 \quad 0.000$
0.108
$-0.10 \quad 135.35$

$-0.30 \quad 141.81$

$-0.49 \quad 146.61$

$-1.15 \quad 152.79$

$-1.44 \quad 157.69$

$-2.15 \quad 164.00$

$-2.37 \quad 169.17$

$\begin{array}{ll}-2.97 & 175.77\end{array}$

$-3.11 \quad 181.20$

$-3.87 \quad 187.82$

$\begin{array}{ll}-4.05 & 193.39\end{array}$

$-4.70 \quad 200.29$

$\begin{array}{ll}-4.89 & 206.03\end{array}$

$\begin{array}{ll}-5.48 & 213.17\end{array}$

$\begin{array}{ll}-5.53 & 219.22\end{array}$

$-6.00 \quad 226.64$

$-5.89233 .03$

$\begin{array}{ll}-6.41 & 240.57\end{array}$

$-6.56246 .86$

$-7.13 \quad 254.51$

$-6.99261 .26$

$-6.12 \quad 270.51$

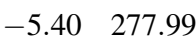

$-4.42 \quad 287.50$

$-3.58 \quad 295.27$

$-3.39 \quad 304.14$

$-2.88 \quad 311.74$

$-2.89 \quad 320.56$

$-2.54 \quad 328.14$

$\begin{array}{ll}-2.36 & 337.29\end{array}$

$-1.73 \quad 345.31$

$-1.36 \quad 354.80$

$-0.92 \quad 362.78$

$-0.57 \quad 372.39$

$-0.29 \quad 380.35$

$-0.18 \quad 389.85$

$0.20 \quad 398.05$

\section{$Z=87$ (Fr)}

\begin{tabular}{|c|c|c|c|c|c|c|c|c|c|c|c|c|c|c|c|c|}
\hline 102189 & 0.31 & 0.00 & 0.01 & 0.02 & 0.341 & 0.000 & 0.033 & -0.018 & -2.04 & 0.40 & 1427.59 & 29.83 & & & 0.27 & 30.97 \\
\hline 103190 & 0.32 & 0.00 & 0.00 & 0.02 & 0.352 & 0.000 & 0.048 & -0.013 & -2.22 & 0.40 & 1438.02 & 27.46 & & & 0.26 & 28.50 \\
\hline 4191 & 0.33 & 0.00 & 0.00 & 0.02 & 0.363 & 0.000 & 0.051 & -0.013 & -2.09 & 0.61 & 1449.70 & 23.86 & & & 0.49 & 24.83 \\
\hline $5 \quad 192$ & 0.33 & 0.00 & 0.01 & 0.01 & 0.364 & 0.000 & 0.038 & -0.007 & -1.88 & 0.71 & 1459.64 & 21.99 & & & 0.57 & 2.87 \\
\hline 6193 & 0.33 & 0.00 & 0.01 & 0.00 & 0.364 & 0.000 & 0.036 & 0.003 & -1.57 & 0.95 & 1470.88 & 18.82 & & & 0.84 & 19.65 \\
\hline $7 \quad 194$ & 0.27 & 0.00 & 0.03 & 0.01 & 0.297 & 0.000 & -0.006 & 0.003 & -0.85 & 1.10 & 1480.39 & 17.38 & & & 1.00 & 18.15 \\
\hline $8 \quad 195$ & 0.32 & 0.00 & 0.02 & -0.01 & 0.353 & 0.000 & 0.019 & 0.008 & -1.04 & 1.30 & 1491.28 & 14.56 & & & 1.20 & 15.26 \\
\hline 99196 & -0.24 & 0.00 & 0.00 & -0.01 & -0.247 & 0.000 & 0.023 & 0.007 & -0.93 & 1.07 & 1500.80 & 13.12 & & & 1.00 & 13.77 \\
\hline $\begin{array}{ll}0 & 197\end{array}$ & -0.24 & 0.00 & 0.00 & -0.01 & -0.247 & 0.000 & 0.023 & 0.007 & -1.08 & 0.91 & 1511.67 & 10.32 & & & 0.86 & 10.92 \\
\hline 1198 & -0.23 & 0.00 & $0 \Omega 0$ & 0.00 & -0.237 & 0.000 & 0. & -0.002 & -1.21 & 0.66 & 83 & 9.23 & & & 0.60 & 9.76 \\
\hline 199 & -0.23 & 0.00 & 0.00 & 0.00 & -0.237 & .000 & 0.020 & -0.002 & -1.38 & 49 & & 6.78 & & & 0.44 & 7.26 \\
\hline 200 & -0.22 & 0.00 & 0.00 & 0.00 & -0.227 & 0.000 & 0.019 & -0.002 & -1.58 & 0.16 & 23 & 5.96 & & & 0.11 & 6.39 \\
\hline 201 & -0.21 & 0.00 & 0.01 & 0.00 & -0.217 & 0.000 & 0.006 & 0.001 & -1.68 & -0.07 & 1550.45 & 3.82 & 3.60 & 0.071 & -0.10 & 4.20 \\
\hline 202 & -0.21 & 0.00 & 0.01 & 0.00 & -0.217 & 0.000 & 0.006 & 0.001 & -2.15 & -0.52 & 1559.12 & 3.22 & 3.14 & & -0.57 & 3.54 \\
\hline 6203 & -0.20 & 0.00 & 0.02 & 0.00 & -0.207 & 0.000 & -0.007 & 0.003 & -2.47 & -0.90 & 1569.13 & 1.28 & 0.86 & 0.016 & -0.93 & 1.56 \\
\hline 204 & -0.19 & 0.00 & 0.02 & 0.00 & -0.197 & .000 & -0.009 & 0.003 & -2.99 & -1.33 & 1577.44 & 1.04 & 0.61 & 25 & -1.36 & 1.27 \\
\hline 205 & -0.12 & 0.00 & -0.01 & -0.01 & -0.125 & 0.000 & 0.018 & 0.008 & -3.25 & -2.23 & 1587.64 & -1.08 & & 0.008 & -2.24 & -0.87 \\
\hline 206 & -0.12 & 0.00 & -0.01 & -0.01 & -0.125 & 0.000 & 0.018 & 0.008 & -3.97 & -2.91 & 1595.87 & -1.24 & -1.24 & 0.028 & -2.92 & -1.08 \\
\hline 20207 & -0.11 & 0.00 & -0.01 & -0.01 & -0.115 & 0.000 & 0.017 & 0.008 & -4.53 & -3.57 & 1605.48 & -2.79 & -2.84 & 0.051 & -3.57 & -2.66 \\
\hline
\end{tabular}




\section{$Z=87(\mathrm{Fr})$}

$\begin{array}{lllllll}121 & 208 & -0.09 & 0.00 & 0.00 & 0.00 & -0.094\end{array}$

$\begin{array}{lllllll}122 & 209 & -0.08 & 0.00 & 0.00 & 0.00 & -0.084\end{array}$

$\begin{array}{llllllll}123 & 210 & -0.06 & 0.00 & 0.01 & 0.00 & -0.063\end{array}$

$\begin{array}{lllllllllll}0.000 & 0.003 & -0.000 & -5.10 & -4.40 & 1613.55 & -2.78 & -2.66 & 0.047 & -4.41 & -2.69\end{array}$

$\begin{array}{lllll}124 & 211 & 0.01 & 0.00 & 0.0\end{array}$

$\begin{array}{lllll}125 & 212 & 0.00 & 0.00 & 0.00\end{array}$

$\begin{array}{lllll}126 & 213 & 0.00 & 0.00 & 0.00\end{array}$

$\begin{array}{lllll}127 & 214 & -0.01 & 0.00 & -0.01\end{array}$

$\begin{array}{lllll}128 & 215 & 0.00 & 0.00 & 0.00\end{array}$

$\begin{array}{lllll}129 & 216 & 0.01 & 0.00 & 0.00\end{array}$

130217

$\begin{array}{lll}0.07 & 0.09 & -0.04\end{array}$

131218

$\begin{array}{lll}0.08 & 0.10 & -0.04\end{array}$

132219

$\begin{array}{llll}0.09 & 0.10 & -0.05\end{array}$

$\begin{array}{lll}0.10 & 0.09 & -0.06\end{array}$

$0.00 \quad 0.011$

0.000

0.002

$0.000-0.010$

$0.000 \quad 0.000$

$0.00 \quad 0.000$

0.000

$0.00 \quad 0.000$

$0.00-0.010$

$0.00 \quad 0.000$

0.000

0.000

0.000

$\begin{array}{llllll}-0.000 & -5.60 & -4.93 & 1622.71 & -3.87 & -3.77\end{array}$

$\begin{array}{llllll}0.001 & -6.39 & -5.69 & 1630.40 & -3.48 & -3.35\end{array}$

$\begin{array}{lllllll}0.000 & -6.97 & -6.06 & 1639.09 & -4.11 & -4.16\end{array}$

$\begin{array}{lllllll}0.000 & -7.95 & -6.90 & 1646.55 & -3.49 & -3.54\end{array}$

0.000

0.000

$\begin{array}{llll}0.000 & -8.20 & -7.12 & 1654.78\end{array}$

0.00
-0.01

$0.079-0.126$

0.012

\subsection{0}

$\begin{array}{llll}-0.000 & -7.19 & -6.17 & 1660.15\end{array}$

$-3.65-3.55$

$-0.95-0.96$

$0.34 \quad 0.32$

$3.48 \quad 2.98$

$\begin{array}{llll}0.000 & -4.93 & -4.13 & 1671.86\end{array}$

$\begin{array}{llllll}0.054 & 0.021 & -6.58 & -3.34 & 1678.50\end{array}$

4.91

4.32

$\begin{array}{llll}0.00 & 0.090 & -0.139\end{array}$

0.055

$\begin{array}{llll}0.012 & -6.65 & -3.07 & 1683.97\end{array}$

7.51

9.02

0.00
-0.01

$0.100-0.140$

0.068

$\begin{array}{lllll}0.014 & -6.40 & -2.49 & 1690.53\end{array}$

$0.111-0.127$

0.081

$\begin{array}{llll}0.026 & -6.50 & -2.36 & 1695.89\end{array}$

$134 \quad 221$

$\begin{array}{lll}0.10 & 0.10 & -0.06\end{array}$

0.00

$0.111-0.140$

0.081

$\begin{array}{llll}0.017 & -6.12 & -1.80 & 1702.18\end{array}$

11.74

135222

$\begin{array}{llll}0.12 & 0.10 & -0.06\end{array}$

$0.122-0.141$

0.083

$\begin{array}{lllll}0.018 & -6.01 & -1.59 & 1707.19\end{array}$

137224

$\begin{array}{llll}0.13 & 0.10 & -0.06\end{array}$

0.01

$0.132-0.139$

0.084

$\begin{array}{llll}0.008 & -5.34 & -1.03 & 1713.22\end{array}$

138225

$\begin{array}{lll}0.14 & 0.09 & -0.06\end{array}$

139226

$\begin{array}{lll}0.15 & 0.06 & -0.07\end{array}$

0.01

$0.153-0.125$

0.085

$\begin{array}{lllll}0.009 & -5.26 & -0.84 & 1717.98\end{array}$

0.00

$0.163-0.084$

0.097

$\begin{array}{lllll}0.008 & -4.38 & -0.26 & 1723.73\end{array}$

0.097

$\begin{array}{llll}0.019 & -4.01 & -0.13 & 1728.29\end{array}$

$\begin{array}{llll}0.018 & -3.38 & 0.25 & 1733.98\end{array}$

0.098

$\begin{array}{llll}0.018 & -3.22 & -0.05 & 1738.73\end{array}$

$\begin{array}{llll}0.00 & 0.173 & -0.042\end{array}$

141228

$\begin{array}{lll}0.16 & 0.03 & -0.07\end{array}$

142229

$\begin{array}{lll}0.18 & 0.00 & -0.07\end{array}$

0.0

0.194

0.000

$144 \quad 231$

$\begin{array}{lll}0.00 & -0.07\end{array}$

145232

$\begin{array}{llll}0.20 & 0.00 & -0.06\end{array}$

0.02

0.03

0.205

0.000

0.102

$0.009-2.92$

$\begin{array}{ll}0.05 & 1738.73 \\ 0.21 & 1744.29\end{array}$

$\begin{array}{lll}0.104 & -0.001 & -3.16\end{array}$

$0.08 \quad 1748.63$

0.093

0.000

$\begin{array}{lllll}0.095 & -0.012 & -3.08 & -0.06 & 1758.31\end{array}$

13.52

16.58

18.62

21.93

24.25

27.76

30.14

33.47

$\begin{array}{llllll}146 & 233 & 0.21 & 0.02 & -0.05\end{array}$

$\begin{array}{llll}0.03 & 0.226 & -0.027\end{array}$

$\begin{array}{lllll}0.083 & -0.014 & -2.62 & 0.13 & 1763.45\end{array}$

$\begin{array}{lllll}0.073 & -0.017 & -2.71 & -0.16 & 1767.48\end{array}$

$\begin{array}{lllll}0.073 & -0.017 & -2.54 & -0.02 & 1772.44\end{array}$

$\begin{array}{lll}0.03 & 0.237 & 0.000\end{array}$

0.000

$\begin{array}{lllll}0.060 & -0.020 & -2.65 & -0.32 & 1776.25\end{array}$

0.000

$0.048-0$.

$-0.022$

.

$-0.17 \quad 1780.97$

$\begin{array}{llllll}0.038 & -0.025 & -2.57 & -0.44 & 1784.53\end{array}$

0.000

$\begin{array}{lllll}0.036 & -0.025 & -2.17 & -0.19 & 1788.93\end{array}$

$\begin{array}{llllll}0.023 & -0.018 & -1.90 & -0.11 & 1791.93\end{array}$

$\begin{array}{ll}0.30 & 1795.94\end{array}$

$\begin{array}{lll}0.023 & -0.018 & -1.48\end{array}$

$\begin{array}{ll}0.30 & 1795.94 \\ 0.46 & 1798.66\end{array}$

$\begin{array}{lll}0.011 & -0.021 & -1.39\end{array}$

$\begin{array}{lll}0.80 & 1802.53 & 90.73 \\ 0.58 & 1805.42 & 95.92\end{array}$

35.98
39.7

42.41

46.17

49.11

53.14

56.26

60.52

63.87

68.38

153240

$\begin{array}{lll}0.22 & 0.00 & 0.00\end{array}$

0.02

0.239

0.000

$\begin{array}{lll}0.006 & -0.011 & -0.74\end{array}$

$157 \quad 244$

$0.20 \quad 0.00$

0.01

0.01
0.01

0.217

0.000

$\begin{array}{llll}0.004 & -0.011 & -0.87\end{array}$

$\begin{array}{lll}0.77 & 1809.23 & 100.18\end{array}$

0.000

$0.002-0.001 \quad-0.58$

$\begin{array}{llll}0.48 & 1811.99 & 105.49\end{array}$

$\begin{array}{lll}0.52 & 1815.74 & 109.81\end{array}$

160247

$\begin{array}{lll}0.18 & 0.01 & 0.02\end{array}$

0.00

0.195

$\begin{array}{llll}0.014 & -0.010 & -0.003 & -0.92\end{array}$

$\begin{array}{lllll}0.000 & -0.023 & -0.005 & -0.89\end{array}$

$\begin{array}{lll}0.09 & 1818.45 & 115.18\end{array}$

161248

$162 \quad 249$

$0.18 \quad 0.00$

0.04

0.00

0.196

$\begin{array}{llll}0.000 & -0.034 & -0.007 & -1.44\end{array}$

$\begin{array}{rrrrrrrr}0.000 & -0.046 & -0.009 & -1.64 & 0.09 & 1822.04 & 119.66 \\ 0.000 & -0.049 & 0.001 & -1.85 & -0.20 & 1824.41 & 125.36\end{array}$

$\begin{array}{lllllll}0.000 & -0.037 & -0.007 & -1.36 & -0.04 & 1827.65 & 130.18\end{array}$

16425

$\begin{array}{ll}0.17 & 0.00\end{array}$

$\begin{array}{ll}0.05 & -0.01\end{array}$

0.185

$\begin{array}{ll}0.16 & 0.00\end{array}$

$0.04 \quad 0.00$

0.174

$0.000-0.038$

$\begin{array}{llllll}0.003 & -1.57 & -0.19 & 1829.69 & 136.22\end{array}$

$\begin{array}{lllllll}166 & 253 & 0.12 & 0.00 & 0.01 & 0.01 & 0.129\end{array}$

$\begin{array}{lllllll}0.000 & -0.005 & -0.011 & -1.17 & -0.26 & 1832.98 & 140.99\end{array}$

$\begin{array}{lllllll}167 & 254 & 0.12 & 0.00 & 0.01 & 0.01 & 0.129\end{array}$

$0.000-0.005$

$\begin{array}{lllll}0.011 & -1.76 & -0.87 & 1835.30 & 146.75\end{array}$

$0.000-0.000$

$\begin{array}{lllll}0.001 & -2.37 & -1.16 & 1838.61 & 151.51\end{array}$

$0.000-0.000$

$\begin{array}{lllll}0.001 & -3.11 & -1.86 & 1840.85 & 157.35\end{array}$

$\begin{array}{llllllll}169 & 256 & -0.17 & 0.00 & 0.01 & 0.00 & -0.176\end{array}$

0.000

0.019

$\begin{array}{lllll}0.008 & -3.14 & -2.09 & 1843.92 & 162.35\end{array}$

$0.000 \quad 0.019$

$\begin{array}{lllll}0.008 & -3.79 & -2.68 & 1845.86 & 168.48\end{array}$

$\begin{array}{lllllll}171 & 258 & -0.13 & 0.00 & -0.01 & -0.01 & -0.135\end{array}$

0.000

0.018

$\begin{array}{llllll}0.008 & -3.90 & -2.95 & 1848.80 & 173.60\end{array}$

$0.000 \quad 0.018$

$\begin{array}{lllll}0.008 & -4.44 & -3.50 & 1850.53 & 179.95\end{array}$

$\begin{array}{llllllll}173 & 260 & -0.12 & 0.00 & -0.01 & -0.01 & -0.125\end{array}$

$0.000 \quad 0.016$

$\begin{array}{llllll}0.008 & -4.36 & -3.66 & 1853.18 & 185.37\end{array}$

$-0.01-0.01-0.104$

$0.000 \quad 0.004$

$\begin{array}{lllll}0.009 & -4.94 & -4.28 & 1854.82 & 191.80\end{array}$

$\begin{array}{llllllll}176 & 263 & -0.10 & 0.00 & 0.01 & -0.01 & -0.105\end{array}$

$0.000-0.007$

$\begin{array}{llllll}0.011 & -5.11 & -4.44 & 1857.30 & 197.39\end{array}$

$0.000-0.020$

$\begin{array}{llllll}0.002 & -5.71 & -5.05 & 1858.75 & 204.01\end{array}$

$\begin{array}{lll}0.015 & -4.93 & -3.82\end{array}$

$\begin{array}{lll}0.022 & -5.69 & -3.46\end{array}$

$\begin{array}{lll}0.021 & -6.06 & -4.11\end{array}$

$\begin{array}{lll}0.026 & -6.90 & -3.52\end{array}$

$\begin{array}{lll}0.008 & -7.12 & -3.70\end{array}$

$\begin{array}{lll}0.009 & -6.17 & -1.02\end{array}$

$\begin{array}{lll}0.007 & -5.24 & 0.25\end{array}$

$\begin{array}{lll}0.014 & -4.13 \quad 3.37\end{array}$

$0.007-3.26 \quad 4.88$

$\begin{array}{llll}7.06 & 0.005 & -2.99 & 7.46\end{array}$

$\begin{array}{llll}8.62 & 0.007 & -2.37 & 9.00\end{array}$

$\begin{array}{llll}11.48 & 0.004 & -2.23 & 11.74\end{array}$

$\begin{array}{llll}13.28 & 0.005 & -1.65 & 13.52\end{array}$

$\begin{array}{llll}16.35 & 0.021 & -1.45 & 16.58\end{array}$

$\begin{array}{llll}18.38 & 0.002 & -0.88 & 18.65\end{array}$

$\begin{array}{lllll}21.66 & 0.050 & -0.70 & 21.96\end{array}$

$\begin{array}{llll}23.81 & 0.030 & -0.11 & 24.30\end{array}$

$\begin{array}{llll}27.37 & 0.100 & 0.02 & 27.82\end{array}$

$\begin{array}{llll}29.66 & 0.100 & 0.41 & 30.24\end{array}$

$0.36 \quad 36.11$

$0.23 \quad 39.87$

$\begin{array}{ll}0.41 & 42.59\end{array}$

$0.09 \quad 46.41$

$0.28 \quad 49.38$

$-0.05 \quad 53.43$

$0.11 \quad 56.60$

$-0.21 \quad 60.89$

$-0.06 \quad 64.30$

$-0.34 \quad 68.85$

$-0.09 \quad 72.60$

$-0.07 \quad 77.67$

$0.35 \quad 81.81$

$0.50 \quad 87.23$

$\begin{array}{ll}0.80 & 91.47\end{array}$ 


\section{$Z=87(\mathrm{Fr})$} $\begin{array}{lllll}179 & 266 & -0.07 & 0.00\end{array}$ $\begin{array}{lllll}180 & 267 & -0.04 & 0.00\end{array}$ $\begin{array}{lllll}181 & 268 & 0.00 & 0.00 & 0.0\end{array}$ $\begin{array}{llll}182 & 269 & 0.00 & 0.00\end{array}$

$\begin{array}{lllll}183 & 270 & 0.00 & 0.00 & 0.0 \\ 184 & 271 & 0.00 & 0.00 & 0.00\end{array}$ $\begin{array}{rrrr}184 & 271 & 0.00 & 0.00 \\ 185 & 272 & -0.01 & 0.00\end{array}$ $\begin{array}{lllll}186 & 273 & 0.00 & 0.00 & 0.0\end{array}$ $\begin{array}{lllll}187 & 274 & 0.01 & 0.05 & 0.00\end{array}$ $\begin{array}{lllll}188 & 275 & 0.03 & 0.07 & -0.01\end{array}$ $\begin{array}{llllll}189 & 276 & 0.05 & 0.09 & -0.02\end{array}$ $\begin{array}{llllll}190 & 277 & 0.05 & 0.11 & -0.02\end{array}$ $\begin{array}{lllll}191 & 278 & 0.06 & 0.12 & -0.02\end{array}$ $\begin{array}{llllll}192 & 279 & 0.06 & 0.12 & -0.02\end{array}$

$\begin{array}{llllll}193 & 280 & 0.06 & 0.13 & -0.01\end{array}$ $\begin{array}{lllll}194 & 281 & 0.05 & 0.13 & -0.01\end{array}$ $\begin{array}{llllll}195 & 282 & 0.10 & 0.11 & -0.06\end{array}$ $\begin{array}{llllll}196 & 283 & 0.10 & 0.11 & -0.06\end{array}$ $\begin{array}{lllll}197 & 284 & 0.12 & 0.11 & -0.06\end{array}$

\section{$0.00-0.084$} $0.000-0.021$

$\begin{array}{lllll}0.002 & -5.78 & -5.09 & 1860.95 & 209.88\end{array}$ $0.00-0.073$ $\begin{array}{ll}0.01 & -0.042\end{array}$ $0.000-0.021$ $\begin{array}{llllll}0.002 & -6.23 & -5.55 & 1862.09 & 216.81\end{array}$ $0.00 \quad 0.000$ $0.000-0.023$ $\begin{array}{ll}0.00 & 0.000 \\ 0.00 & 0.000\end{array}$ 0.000 0.000 $\begin{array}{llllll}0.009 & -6.14 & -5.40 & 1863.93 & 223.04\end{array}$ $0.00 \quad 0.000$ 0.000

0.000

$\begin{array}{ll}0.00 & 0.000\end{array}$

0.000

$0.00-0.011$

0.000

0.000

$0.00 \quad 0.000$

$\begin{array}{lll}0.00 & 0.012 & -0.067\end{array}$

0.000

$\begin{array}{llll}0.00 & 0.034 & -0.095\end{array}$

$\begin{array}{llll}0.00 & 0.056 & -0.123\end{array}$

$\begin{array}{llll}0.01 & 0.058 & -0.150\end{array}$

$\begin{array}{lll}0.01 & 0.069 & -0.164\end{array}$

0.02

$0.069-0.163$

$\begin{array}{llll}0.02 & 0.070 & -0.176\end{array}$

$\begin{array}{lll}0.02 & 0.060 & -0.176\end{array}$

$\begin{array}{llll}0.00 & 0.112 & -0.154\end{array}$

0.00

$0.112-0.154$

0.01

$0.133-0.154$

$198 \quad 285$

$\begin{array}{lll}0.12 & 0.11 & -0.06\end{array}$

0.01

$\begin{array}{ll}0.133 & -0.154\end{array}$

199286

$\begin{array}{lllll}0.13 & 0.03 & -0.08 & -0.01\end{array}$

$0.142-0.042$

200287

$\begin{array}{lllll}0.14 & 0.00 & -0.08 & -0.01\end{array}$

$0.153 \quad 0.000$

201288

$\begin{array}{llll}0.15 & 0.00 & -0.08\end{array}$

202289

$\begin{array}{llll}0.15 & 0.00 & -0.08\end{array}$
0.00

0.00
$0.163 \quad 0.000$

0.000

\subsection{0}

0.001

0.014

0.028

$\begin{array}{llllll}0.030 & -0.000 & -5.40 & -2.66 & 1869.95 & 297.74\end{array}$

$\begin{array}{lllllll}0.031 & 0.002 & -5.86 & -2.91 & 1869.99 & 305.77\end{array}$ $\begin{array}{lllllll}0.031 & -0.009 & -5.34 & -2.49 & 1870.65 & 313.19\end{array}$ $\begin{array}{llllll}0.020 & -0.008 & -5.47 & -2.34 & 1870.15 & 321.76\end{array}$ $\begin{array}{llllll}0.019 & -0.008 & -4.79 & -1.72 & 1870.47 & 329.51\end{array}$ $\begin{array}{llllll}0.082 & 0.019 & -5.22 & -1.72 & 1869.98 & 338.07\end{array}$ $\begin{array}{llllll}0.082 & 0.019 & -4.75 & -1.30 & 1870.36 & 345.76\end{array}$ $\begin{array}{llllll}0.084 & 0.010 & -4.57 & -1.09 & 1869.53 & 354.67\end{array}$ $\begin{array}{llllll}0.084 & 0.010 & -4.18 & -0.72 & 1869.82 & 362.45\end{array}$ $\begin{array}{llllll}0.107 & 0.027 & -3.28 & -0.58 & 1868.93 & 371.40\end{array}$ $\begin{array}{lllllll}0.108 & 0.028 & -3.06 & -0.44 & 1869.32 & 379.09\end{array}$ $\begin{array}{lllllll}0.109 & 0.018 & -3.21 & -0.58 & 1868.57 & 387.90\end{array}$ $\begin{array}{llllll}0.109 & 0.018 & -3.09 & -0.48 & 1868.88 & 395.67\end{array}$ $\begin{array}{ll}-5.08 & 213.37\end{array}$

$\begin{array}{ll}-5.53 & 220.47\end{array}$

$\begin{array}{ll}-5.35 & 226.90\end{array}$

$\begin{array}{ll}-5.80 & 234.18\end{array}$

$-5.93 \quad 240.48$

$-6.47 \quad 247.82$

$-6.32 \quad 254.56$

$\begin{array}{ll}-5.44 & 263.49\end{array}$

$-4.74 \quad 270.95$

$-3.73 \quad 280.16$

$-2.95 \quad 287.86$

$\begin{array}{ll}-2.89 & 296.27\end{array}$

$-2.44 \quad 303.80$

$\begin{array}{ll}-2.67 & 312.08\end{array}$

$-2.19 \quad 319.78$

$\begin{array}{ll}-2.03 & 328.59\end{array}$

$-1.40 \quad 336.60$

$-1.31 \quad 345.49$

$-0.88 \quad 353.45$

$-0.71 \quad 362.56$

$-0.33 \quad 370.61$

$\begin{array}{ll}-0.03 & 379.99\end{array}$

$0.11 \quad 387.95$

$-0.11 \quad 396.95$

$-0.01 \quad 405.01$

\section{$Z=88$ (Ra)}

\begin{tabular}{|c|c|c|c|c|c|c|c|c|c|c|c|c|c|c|c|c|}
\hline $04 \quad 192$ & 0.33 & 0.00 & 0.00 & 0.02 & 0.363 & 0.000 & 0.051 & -0.013 & -2.02 & 0.52 & 1449.33 & 31.52 & & & 0.41 & 32.70 \\
\hline $05 \quad 193$ & 0.33 & 0.00 & 0.01 & 0.01 & 0.364 & 0.000 & 0.038 & -0.007 & -1.78 & 0.65 & 1459.30 & 29.62 & & & 0.51 & 30.70 \\
\hline $6 \quad 194$ & 0.33 & 0.00 & 0.01 & 0.01 & 0.364 & 0.000 & .038 & -0.007 & -1.48 & 0.91 & 1470.97 & 26.02 & & & 0.80 & 7.04 \\
\hline $77 \quad 195$ & 0.26 & 0.00 & 0.02 & 0.00 & 0.285 & 0.000 & 0.005 & -0.003 & -0.66 & 1.14 & 1480.45 & 24.61 & & & 06 & 5.58 \\
\hline $8 \quad 196$ & 0.32 & 0.00 & 0.02 & -0.01 & 0.353 & 0.000 & 0.019 & 0.008 & -0.92 & 1.29 & 1491.84 & 21.29 & & & 1.20 & 2.17 \\
\hline 9197 & 0.31 & 0.00 & 0.02 & -0.01 & 0.342 & 00 & 6 & 0.008 & -0.81 & 1.50 & 96 & & & & & 1.04 \\
\hline $\begin{array}{ll}0 & 198\end{array}$ & -0.24 & 0.00 & 0.00 & -0.01 & -0.247 & 0.000 & 0.023 & 0.007 & -0.52 & 1.45 & 1512.17 & 7.10 & & & .40 & 7.88 \\
\hline 1199 & 0.24 & 0.00 & 0.00 & 0.00 & -0.247 & 0.000 & 0.022 & -0.002 & -0.79 & 1.21 & 1521.38 & 15.96 & & & 1.15 & 6.66 \\
\hline 2200 & -0.23 & 0.00 & 0.00 & 0.00 & -0.237 & 0.000 & 0.020 & -0.002 & -0.82 & 1.04 & .33 & 3.08 & & & .99 & 3.73 \\
\hline 3201 & -0.23 & 0.00 & 0.01 & 0.00 & -0.237 & 0.000 & 0.009 & 0.001 & -1.15 & 0.75 & 1541.24 & 12.25 & & & 0.70 & 2.83 \\
\hline $4 \quad 202$ & -0.22 & 0.00 & 0.01 & 0.00 & -0.227 & 0.000 & 0.007 & 0.001 & -1.23 & 0.53 & 15 & 9.68 & & & 0.49 & 0.21 \\
\hline 5203 & -0.21 & 0.00 & 0.01 & 0.00 & -0.217 & 00 & 0.006 & 01 & -1.59 & 0.12 & & 9.07 & & & 0.07 & 9.55 \\
\hline 6204 & -0.20 & 0.00 & 0.02 & 0.00 & -0.207 & 0.000 & -0.007 & 0.003 & -1.88 & -0.30 & .04 & 6.66 & 6. & 0. & -0.33 & 7.09 \\
\hline 7205 & -0.19 & 0.00 & 0.02 & 0.00 & -0.197 & 0.000 & -0.009 & 0.003 & -2.39 & .86 & & 6.25 & & & 89 & 6.62 \\
\hline 8206 & -0.12 & 0.00 & -0.01 & -0.01 & -0.125 & 0.000 & 0.018 & 0.008 & -2.49 & -1.50 & 89 & 3.96 & 7 & & -1.51 & 4.30 \\
\hline 07 & -0 & 0 & .01 & -0.01 & .125 & 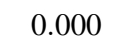 & 0.0 & 0.008 & -3.21 & 8 & & 3.75 & & & & 05 \\
\hline 208 & -0.12 & 0.00 & 0.01 & -0.01 & -0.125 & 0.000 & 0.018 & 08 & -3.79 & -2.75 & & 1.87 & & & -2.75 & 2.13 \\
\hline 209 & -0.10 & 0.00 & 0.00 & -0.01 & -0.104 & 0.000 & 0.004 & 0.009 & -4.35 & -3.56 & & 1.85 & 1. & & -3.57 & 2.06 \\
\hline 210 & -0.08 & 0.00 & 0.01 & 0.00 & -0.084 & 0.000 & -0.009 & 0.001 & -4.75 & -4.10 & 80 & 0.33 & 0.46 & & -4.10 & 0.50 \\
\hline 3211 & -0.07 & 0.00 & $0 \Omega 1$ & 0.00 & -0.073 & 0.000 & -0.010 & & -5.54 & -4.84 & & 0.70 & & & & 083 \\
\hline 12 & -0.05 & 0.00 & 0.01 & 0.00 & -0. & & -0.0 & & & -5.25 & & & & & & -0.28 \\
\hline 213 & 0.00 & 0.00 & 0.00 & 0.00 & 0.000 & 0.000 & 0.000 & 0.000 & -6.94 & -5.94 & & 0.34 & & & & 0.41 \\
\hline 214 & 0.00 & 0.00 & 0.00 & 0.00 & 0.000 & 0.000 & 0.000 & 0.000 & -7.18 & -6.14 & 64 & -0.22 & & & -6.15 & -0.18 \\
\hline 215 & -0.02 & 0.00 & -0.01 & 0.00 & -0.021 & 0.000 & 0.012 & -0.000 & -6.20 & -5.24 & 10 & 2.39 & 2.53 & 08 & -5.24 & 2.41 \\
\hline 216 & 0.00 & 0.00 & 0.00 & 0.00 & 0.000 & 0.000 & 0.000 & 00 & -5.20 & -4.27 & 16 & 3.30 & 9 & & -4.28 & 3.30 \\
\hline 217 & 0.05 & 0.08 & -0.03 & 0.00 & 0.056 & 0 & 9 & 07 & -5.66 & -3.23 & 9 & 6.34 & 9 & & -3 & 6.36 \\
\hline $0 \quad 218$ & 0.07 & 0.09 & -0.04 & 0.00 & 0.078 & -0.125 & 0.054 & 0.010 & -5.67 & -2.61 & & 7.21 & & & & 7.24 \\
\hline 219 & 0.08 & 0.10 & -0.04 & 0.00 & 0.090 & -0.139 & 0.055 & 0.012 & -5.96 & -2.42 & & 9.67 & & & -2.34 & 9.70 \\
\hline 220 & 0.10 & 0.09 & -0.06 & -0.01 & 0.111 & -0.127 & 0.081 & 0.026 & -6.02 & -1.93 & & 10.70 & 10.27 & & -1.78 & 10.78 \\
\hline 221 & 0.10 & 0.10 & -0.06 & 0.00 & 0.111 & -0.140 & 0.081 & 0.017 & -6.19 & -1.86 & & 13.32 & 12.96 & 0.005 & -1.72 & 13.39 \\
\hline 222 & 0.11 & 0.10 & -0.06 & 0.00 & 0.122 & -0.141 & 0.082 & 0.018 & -5.75 & -1.36 & 1708.36 & 14.63 & 14.32 & 0.005 & -1.21 & 14.71 \\
\hline
\end{tabular}




\section{$Z=88$ (Ra)}

$\begin{array}{lllll}135 & 223 & 0.12 & 0.10 & -0.06\end{array}$

$\begin{array}{llllll}136 & 224 & 0.13 & 0.10 & -0.06\end{array}$

$\begin{array}{lllll}137 & 225 & 0.14 & 0.10 & -0.06\end{array}$

$\begin{array}{lllll}138 & 226 & 0.15 & 0.08 & -0.07\end{array}$

$\begin{array}{lll}0.01 & 0.132 & -0.139\end{array}$

0.083

$\begin{array}{llll}0.008 & -5.58 & -1.26 & 1713.50\end{array}$

$\begin{array}{lll}0.01 & 0.143 & -0.139\end{array}$

$\begin{array}{llll}0.01 & 0.154 & -0.139\end{array}$

0.084

$\begin{array}{llll}0.009 & -5.16 & -0.77 & 1720.00\end{array}$

0.085

$\begin{array}{llll}0.01 & 0.164 & -0.112\end{array}$

0.098

$\begin{array}{llll}0.010 & -5.13 & -0.63 & 1724.85\end{array}$

$\begin{array}{lllll}0.010 & -4.46 & -0.15 & 1731.08\end{array}$

0.099

139227

$\begin{array}{lll}0.16 & 0.07 & -0.07\end{array}$

$\begin{array}{lll}141 & 229\end{array}$

$142 \quad 230$

$\begin{array}{lll}0.16 & 0.06 & -0.07\end{array}$

0.01

$0.174-0.097$

$\begin{array}{llll}0.01 & 0.174 & -0.083\end{array}$

0.099

$\begin{array}{lll}0.17 & 0.02 & -0.08\end{array}$

$\begin{array}{lll}0.18 & 0.00 & -0.07\end{array}$

0.01

$0.184-0.028$

$\begin{array}{lll}0.01 & 0.194 & 0.000\end{array}$

$\begin{array}{lll}0.02 & 0.205 & 0.000\end{array}$

0.113

$\begin{array}{llll}0.010 & -4.17 & -0.03 & 1735.70\end{array}$

0.102

$\begin{array}{lllll}0.009 & -3.57 & 0.07 & 1742.06\end{array}$

$\begin{array}{lllll}0.011 & -3.72 & -0.07 & 1746.67\end{array}$

$\begin{array}{llll}0.009 & -3.07 & 0.10 & 1752.71\end{array}$

$143 \quad 231$

$\begin{array}{llll}0.19 & 0.00 & -0.07\end{array}$

145233

$\begin{array}{lll}0.20 & 0.00 & -0.06\end{array}$

0.02

0.215

0.000

0.104

$-0.001$

0.0

$\begin{array}{lll}0.22 & 0.00 & -0.05\end{array}$

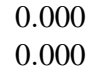

$\begin{array}{lll}0.03 & 0.237 & 0.000 \\ 0.03 & 0.237 & 0.000\end{array}$

$\begin{array}{llllll}0.095 & -0.012 & -3.31 & -0.25 & 1767.24\end{array}$

$\begin{array}{lllll}0.085 & -0.014 & -2.89 & -0.06 & 1772.77\end{array}$

$\begin{array}{lllll}0.073 & -0.017 & -2.90 & -0.35 & 1776.83\end{array}$

0.000

$\begin{array}{lllll}0.073 & -0.017 & -2.73 & -0.21 & 1782.16\end{array}$

$\begin{array}{lll}0.03 & 0.237 & 0.000\end{array}$

$\begin{array}{lllll}0.060 & -0.020 & -2.82 & -0.49 & 1785.98\end{array}$

149237

$\begin{array}{llll}0.22 & 0.00 & -0.04\end{array}$

0.03

$0.249 \quad 0.000$

$\begin{array}{lllll}0.050 & -0.022 & -2.56 & -0.33 & 1791.06\end{array}$

0.03

0.250

0.000$$
\begin{array}{lllll}
0.038 & -0.025 & -2.72 & -0.59 & 1794.65
\end{array}
$$

$\begin{array}{lllll}0.038 & -0.025 & -2.42 & -0.34 & 1799.41\end{array}$

$\begin{array}{lll}0.03 & 0.238 & 0.000\end{array}$

$\begin{array}{lllll}0.036 & -0.025 & -2.24 & -0.27 & 1802.45\end{array}$

$\begin{array}{llll}0.023 & -0.018 & -1.56\end{array}$

$\begin{array}{ll}0.19 & 1806.78\end{array}$

$\begin{array}{lll}0.011 & -0.021 & -1.44\end{array}$

$\begin{array}{lll}0.33 & 1809.53\end{array}$

$0.75 \quad 1813.68$

17.56

19.13

22.35

24.19

27.64

29.36

32.81
34.85

34.85
38.45

40.75

44.53

47.08

51.08

53.83

58.08

61.06

65.55

$153 \quad 24$

155243

$\begin{array}{lll}0.22 & 0.00 & 0.01\end{array}$

0.02

0.240

0.000

$\begin{array}{lll}0.010 & -0.011 & -0.93\end{array}$

$0.56 \quad 1816.56$

158246

$\begin{array}{lll}0.20 & 0.00 & 0.01\end{array}$

0.01

0.217

0.000

$\begin{array}{llll}0.006 & -0.011 & -0.96\end{array}$

$\begin{array}{lll}0.57 & 1823.41 \quad 101.36\end{array}$

$\begin{array}{lllll}159 & 247 & 0.18 & 0.00 & 0.02\end{array}$

$0.00 \quad 0.195$

$0.000-0.010-0.003-0.81$

$\begin{array}{llll}0.57 & 1827.56 & 105.28\end{array}$

161249

$\begin{array}{lll}0.19 & 0.00 & 0.03\end{array}$

0.00

0.207

$\begin{array}{lllll}0.000 & -0.021 & -0.006 & -0.85\end{array}$

$\begin{array}{lll}0.22 & 1830.19 & 110.72\end{array}$

$162 \quad 250$

$0.18 \quad 0.00$

0.04
0.05

0.00
0.00

0.196

$\begin{array}{lllll}0.000 & -0.034 & -0.007 & -1.31\end{array}$

$\begin{array}{lll}0.25 & 1834.12 & 114.86\end{array}$

$\begin{array}{rrrr}0.000 & -0.045 & -0.010 & -1.55 \\ 0.000 & -0.047 & 0.000 & -1.70\end{array}$

$\begin{array}{lll}0.00 & 1836.47 & 120.59\end{array}$

164252

$0.18 \quad 0.00$

$0.05-0.01$

$\begin{array}{llll}0.000 & -0.037 & -0.007 & -1.15\end{array}$

$\begin{array}{lll}0.18 & 1840.05 & 125.08\end{array}$

165253

$\begin{array}{ll}0.16 & 0.00\end{array}$

$0.04-0.01$

0.174

$\begin{array}{lllll}0.000 & -0.038 & 0.003 & -1.35\end{array}$

$\begin{array}{lll}0.06 & 1842.07 & 131.12\end{array}$

166254

0.120 .00

$0.000-0.005$

$-0.011-0.76$

$\begin{array}{lll}0.14 & 1845.56 & 135.71\end{array}$

$\begin{array}{ll}167 & 255 \\ 168 & 256\end{array}$

0.120 .00

0.01

0.01

0.129

0.000

$\begin{array}{rrrrrrrr}168 & 256 & 0.12 & 0.00 & 0.01 & 0.01 & 0.129\end{array}$

0.000

$-0.005$

$-0.120 .0$

$\begin{array}{lllll}-0.011 & -1.52 & -0.66 & 1851.45 & 145.96\end{array}$

$\begin{array}{lllll}0.008 & -2.35 & -1.41 & 1853.74 & 151.74\end{array}$

$\begin{array}{lllllll}170 & 258 & -0.13 & 0.00 & -0.01 & -0.01 & -0.135\end{array}$

0.000

0.019

$\begin{array}{lllll}0.008 & -2.62 & -1.60 & 1857.12 & 156.43\end{array}$

$\begin{array}{lllllll}171 & 259 & -0.13 & 0.00 & -0.01 & -0.01 & -0.135\end{array}$

$0.000 \quad 0.019$

$\begin{array}{lllll}0.008 & -3.26 & -2.16 & 1859.05 & 162.57\end{array}$

$\begin{array}{lllllll}172 & 260 & -0.17 & 0.00 & 0.01 & -0.01 & -0.176\end{array}$

$\begin{array}{ll}0.000 \quad 0.001 \\ 0.000 & 0.018\end{array}$

$\begin{array}{lllll}0.010 & -3.75 & -2.35 & 1862.24 & 167.45\end{array}$

$0.000 \quad 0.018$

$\begin{array}{llllll}0.008 & -3.81 & -2.89 & 1863.98 & 173.79\end{array}$

$\begin{array}{llll}174 & 262-0.10 & 0.00-0.00-0.01-0.104\end{array}$

$0.000 \quad 0.004$

$\begin{array}{llllll}0.009 & -3.68 & -3.03 & 1866.95 & 178.89\end{array}$

$\begin{array}{llll}175 & 263 & -0.10 & 0.00\end{array}$

$\begin{array}{llll}0.00 & -0.01 & -0.104\end{array}$

$\begin{array}{ll}0.000 \quad 0.004 \\ 0.000 & -0.007\end{array}$

$0.000-0.007$

$\begin{array}{llllll}0.009 & -4.30 & -3.66 & 1868.61 & 185.30\end{array}$

$\begin{array}{llll}176 & 264 & -0.10 & 0.00\end{array}$

$0.01-0.01-0.105$

$0.000-0.019$

$\begin{array}{llllll}0.011 & -4.46 & -3.83 & 1871.43 & 190.55\end{array}$

$\begin{array}{llll}177 & 265 & -0.10 & 0.00\end{array}$

$\begin{array}{llll}0.02 & 0.00 & -0.105\end{array}$

$0.000-0.020$

$\begin{array}{llllll}0.002 & -5.05 & -4.37 & 1872.84 & 197.22\end{array}$

$\begin{array}{llll}179 & 267 & -0.07 & 0.00\end{array}$

$-0.01-0.09$

$0.000-0.021$

$\begin{array}{llllll}0.012 & -5.10 & -4.44 & 1875.38 & 202.74\end{array}$

$\begin{array}{lllll}0.002 & -5.44 & -4.79 & 1876.43 & 209.76\end{array}$

$\begin{array}{llll}180 & 268 & -0.04 & 0.00\end{array}$

$\begin{array}{llll}181 & 269 & 0.00 & 0.00\end{array}$

$\begin{array}{llll}0.01 & 0.00 & -0.042\end{array}$

$0.000-0.011$

$\begin{array}{llllll}0.001 & -5.21 & -4.51 & 1878.48 & 215.79\end{array}$

$182 \quad 270$

$0.00 \quad 0.00$

$0.000 \quad 0.000$

$\begin{array}{llllll}0.000 & -5.78 & -4.98 & 1879.48 & 222.85\end{array}$

$\begin{array}{lll}183 & 271\end{array}$

$0.00 \quad 0.00$

0.00

$0.00 \quad 0.000$

0.000

0.000

$\begin{array}{llllll}0.000 & -5.91 & -5.08 & 1881.74 & 228.67\end{array}$

184272

$\begin{array}{lll}0.00 & 0.00 & 0.00\end{array}$

0.00

0.000

0.000

0.000

$\begin{array}{llllll}0.000 & -6.47 & -5.61 & 1882.66 & 235.82\end{array}$

$\begin{array}{llllll}0.000 & -6.30 & -5.44 & 1884.48 & 242.07\end{array}$

$\begin{array}{lllll}185 & 273 & -0.01 & 0.00 & 0.00\end{array}$

$\begin{array}{lllll}186 & 274 & 0.00 & 0.00 & 0.00\end{array}$

$\begin{array}{lll}0.00 & -0.011 & 0.000\end{array}$

0.000

$\begin{array}{lllll}0.000 & -5.36 & -4.55 & 1883.83 & 250.79\end{array}$

\section{5}

$188 \quad 276$

$\begin{array}{lll}0.02 & 0.06 & -0.01\end{array}$

$\begin{array}{lll}0.00 & 0.000 & 0.000\end{array}$

0.000

$\begin{array}{llllll}0.000 & -4.66 & -3.86 & 1884.98 & 257.72\end{array}$

$\begin{array}{llll}0.00 & 0.023 & -0.081\end{array}$

0.013

$\begin{array}{llllll}0.003 & -4.34 & -3.00 & 1884.19 & 266.57\end{array}$ 18927

$\begin{array}{llll}0.03 & 0.08 & -0.01\end{array}$

$\begin{array}{llll}0.00 & 0.035 & -0.109\end{array}$

0.015

$\begin{array}{llllll}0.005 & -4.07 & -2.31 & 1885.20 & 273.64\end{array}$

$\begin{array}{llllll}190 & 278 & 0.06 & 0.11 & -0.03\end{array}$

$\begin{array}{lll}0.00 & 0.057 & -0.137\end{array}$

0.029

$\begin{array}{lllll}0.009 & -4.83 & -2.38 & 1885.20 & 281.71\end{array}$

$\begin{array}{lllll}191 & 279 & 0.06 & 0.12 & -0.02\end{array}$ $\begin{array}{lll}0.01 & 0.069 & -0.151\end{array}$

$\begin{array}{llll}0.02 & 0.069 & -0.163\end{array}$ $\begin{array}{lllllll}0.042 & 0.001 & -4.92 & -2.20 & 1886.56 & 288.42\end{array}$

$\begin{array}{llllll}0.031 & -0.009 & -5.22 & -2.32 & 1886.48 & 296.58\end{array}$

$\begin{array}{llll}17.24 & 0.003 & -1.12 & 17.62\end{array}$ $\begin{array}{llll}18.83 & 0.002 & -0.61 & 19.21\end{array}$

$\begin{array}{llll}21.99 & 0.003 & -0.48 & 22.43\end{array}$

$\begin{array}{llll}23.67 & 0.002 & 0.03 & 24.31\end{array}$

$\begin{array}{llll}27.18 & 0.002 & 0.13 & 27.75\end{array}$

$\begin{array}{llll}28.94 & 0.002 & 0.23 & 29.49\end{array}$

$\begin{array}{llll}32.56 & 0.019 & 0.11 & 32.97\end{array}$

$\begin{array}{llll}34.52 & 0.012 & 0.25 & 35.01\end{array}$

$0.02 \quad 38.64$

0.1940 .95

$-0.08 \quad 44.78$

$0.09 \quad 47.35$

$-0.23 \quad 51.37$

$-0.07 \quad 54.17$

$-0.38 \quad 58.44$

$-0.22 \quad 61.48$

$-0.49 \quad 66.01$

$-0.23 \quad 69.39$

$-0.17 \quad 74.48$

$0.24 \quad 78.23$

$0.38 \quad 83.61$

$0.77 \quad 87.57$ 


\section{$Z=88$ (Ra)}

$\begin{array}{llll}192 & 280 & 0.06 & 0.12\end{array}$ $\begin{array}{lll}0.06 & 0.13 & -0.02\end{array}$ $\begin{array}{llllll}194 & 282 & 0.10 & 0.12 & -0.05\end{array}$ $\begin{array}{llllll}195 & 283 & 0.10 & 0.11 & -0.06\end{array}$ $\begin{array}{lllll}196 & 284 & 0.10 & 0.11 & -0.06\end{array}$

$\begin{array}{llllll}197 & 285 & 0.12 & 0.11 & -0.06\end{array}$ 198286 199287 200288 $\begin{array}{lll}0.13 & 0.11 & -0.06\end{array}$ $\begin{array}{lllll}0.38 & 0.00 & 0.11 & -0.03\end{array}$ 201289 $\begin{array}{llll}0.14 & 0.01 & -0.08\end{array}$ 202290 $\begin{array}{lll}0.15 & 0.01 & -0.08\end{array}$ 203291 $\begin{array}{lll}0.15 & 0.00 & -0.08\end{array}$ $\begin{array}{lllll}204 & 292 & 0.16 & 0.02 & -0.07\end{array}$ $\begin{array}{lll}0.02 & 0.069 & -0.163\end{array}$ $\begin{array}{llll}0.02 & 0.070 & -0.177\end{array}$ $\begin{array}{llll}0.00 & 0.113 & -0.168\end{array}$ $\begin{array}{lll}0.00 & 0.112 & -0.154\end{array}$ $\begin{array}{llll}0.00 & 0.112 & -0.154\end{array}$ $\begin{array}{lll}0.01 & 0.133 & -0.154\end{array}$ $\begin{array}{llll}0.01 & 0.144 & -0.153\end{array}$

\section{$-0.01$} $\begin{array}{ll}0.433 & 0.000\end{array}$ $0.153-0.014$ 0.00 $0.163-0.014$ $\begin{array}{lll}0.00 & 0.163 & 0.000\end{array}$ $\begin{array}{llll}0.01 & 0.173 & -0.014\end{array}$ $\begin{array}{lll}0.01 & 0.172 & -0.028\end{array}$ $\begin{array}{llllll}0.031 & -0.009 & -4.72 & -1.87 & 1887.41 & 303.71\end{array}$ $\begin{array}{llllll}0.032 & -0.007 & -4.99 & -1.75 & 1886.95 & 312.25\end{array}$ $\begin{array}{lllllll}0.070 & 0.019 & -4.85 & -1.19 & 1887.64 & 319.63\end{array}$ $\begin{array}{llllll}0.082 & 0.019 & -4.74 & -1.23 & 1887.19 & 328.14\end{array}$ $\begin{array}{llllll}0.082 & 0.019 & -4.28 & -0.79 & 1887.85 & 335.55\end{array}$ $\begin{array}{lllllll}0.084 & 0.010 & -4.24 & -0.75 & 1887.20 & 344.28\end{array}$ $\begin{array}{llllll}0.084 & 0.010 & -3.93 & -0.43 & 1887.85 & 351.70\end{array}$ $\begin{array}{lllllll}-0.073 & -0.014 & -3.98 & 0.03 & 1886.64 & 360.98\end{array}$ $\begin{array}{lllllll}0.108 & 0.028 & -2.84 & -0.17 & 1887.67 & 368.03\end{array}$ $\begin{array}{llllll}0.109 & 0.018 & -3.06 & -0.37 & 1886.99 & 376.77\end{array}$ $\begin{array}{lllllll}0.109 & 0.018 & -2.91 & -0.28 & 1887.60 & 384.24\end{array}$ $\begin{array}{llllll}0.111 & 0.009 & -3.23 & -0.72 & 1887.04 & 392.87\end{array}$ $\begin{array}{lllllll}0.098 & 0.007 & -2.72 & -0.54 & 1887.42 & 400.56\end{array}$ $\begin{array}{ll}-1.56 & 310.01\end{array}$

$\begin{array}{ll}-1.42 & 318.79\end{array}$

$-0.80 \quad 326.46$

$-0.81 \quad 335.24$

$-0.35 \quad 342.91$

$-0.36 \quad 351.84$

$-0.04 \quad 359.52$

$0.18 \quad 368.81$

$0.39 \quad 376.52$

$0.10 \quad 385.44$

$\begin{array}{ll}0.20 & 393.19\end{array}$

$-0.28 \quad 402.06$

$-0.19 \quad 409.93$

\section{$Z=89$ (Ac)}

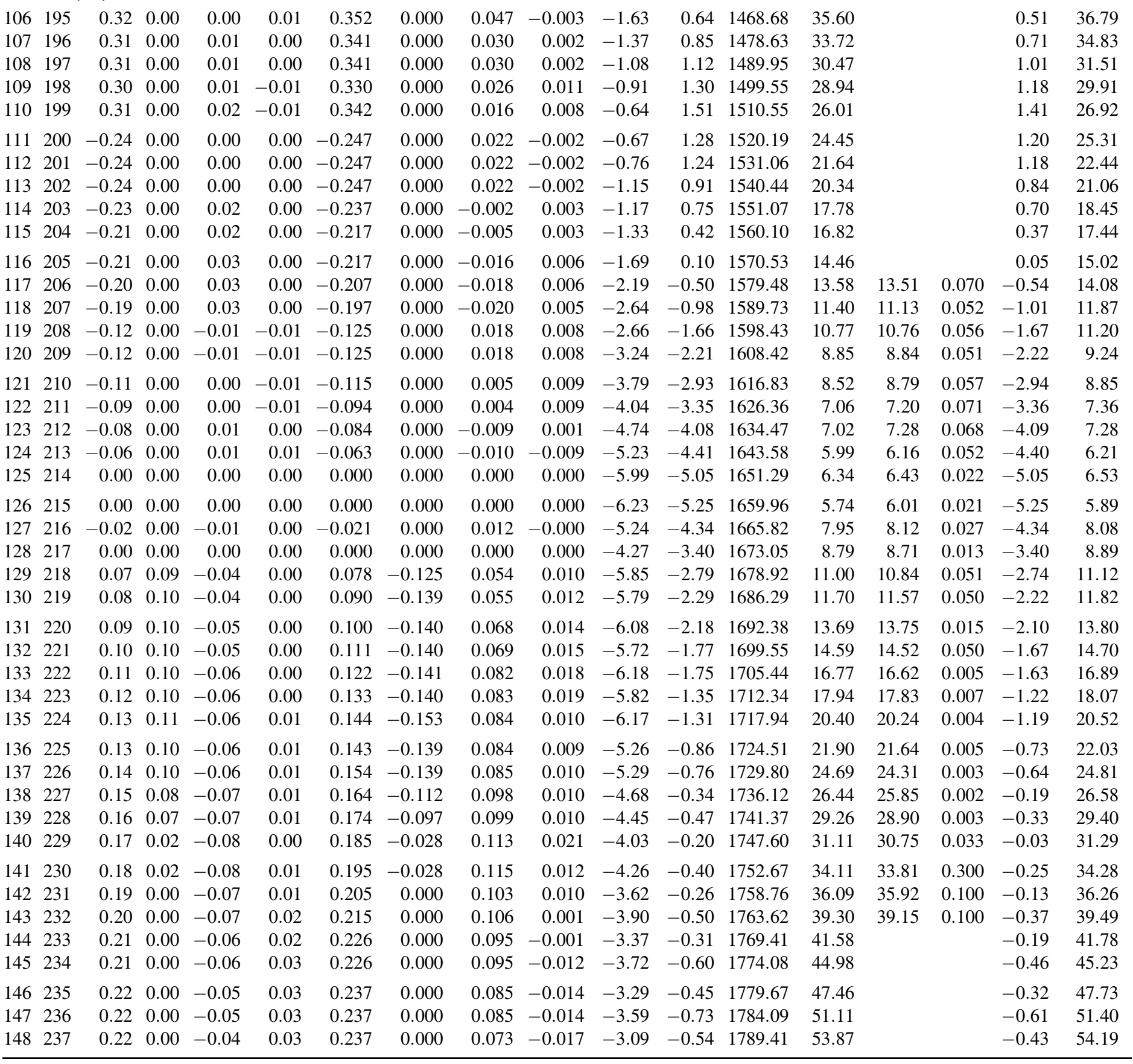




\section{$Z=89$ (Ac)}

\begin{tabular}{|c|c|c|c|c|c|c|c|c|c|c|c|c|c|c|}
\hline 149238 & 0.23 & 0.00 & -0.03 & 0.03 & 0.249 & 0.000 & 0.062 & -0.019 & -3.27 & -0.82 & 1793.60 & 57.75 & -0.73 & 58.09 \\
\hline $150 \quad 239$ & 0.23 & 0.00 & -0.02 & 0.03 & 0.249 & 0.000 & 0.050 & -0.022 & -2.88 & -0.63 & 1798.68 & 60.74 & -0.54 & 61.13 \\
\hline 151240 & 0.23 & 0.00 & -0.02 & 0.03 & 0.249 & 0.000 & 0.050 & -0.022 & -3.15 & -0.90 & 1802.63 & 64.86 & -0.81 & 65.29 \\
\hline $52 \quad 241$ & 0.23 & 0.00 & -0.01 & 0.03 & 0.250 & 0.000 & 0.038 & -0.025 & -2.71 & -0.62 & 1807.39 & 68.17 & -0.52 & 68.66 \\
\hline $153 \quad 242$ & 0.22 & 0.00 & -0.01 & 0.03 & 0.238 & 0.000 & 0.036 & -0.025 & -2.50 & -0.54 & 1810.79 & 72.84 & -0.45 & 73.38 \\
\hline 4243 & 0.22 & 0.00 & 0.00 & 0.02 & 0.239 & 0.000 & 0.023 & -0.018 & -1.79 & -0.06 & 1815.12 & 76.58 & -0.02 & 77.13 \\
\hline $5 \quad 244$ & 0.22 & 0.00 & 0.00 & 0.02 & 0.239 & 0.000 & 0.023 & -0.018 & -1.65 & 0.15 & 1818.18 & 81.60 & 0.18 & 82.20 \\
\hline $6 \quad 245$ & 0.22 & 0.00 & 0.01 & 0.01 & 0.239 & 0.000 & 0.010 & -0.011 & -1.09 & 0.59 & 1822.33 & 85.51 & 0.59 & 86.15 \\
\hline $7 \quad 246$ & 0.20 & 0.00 & 0.01 & 0.01 & 0.217 & 0.000 & 0.006 & -0.011 & -1.13 & 0.40 & 1825.56 & 90.35 & 0.40 & 91.06 \\
\hline $58 \quad 247$ & 0.19 & 0.00 & 0.01 & 0.01 & 0.206 & 0.000 & 0.004 & -0.011 & -0.82 & 0.62 & 1829.73 & 94.26 & 0.63 & 95.05 \\
\hline 59248 & 0.19 & 0.00 & 0.02 & 0.00 & 0.206 & 0.000 & -0.009 & -0.003 & -0.98 & 0.49 & 1832.71 & 99.35 & 0.48 & 100.20 \\
\hline 160249 & 0.19 & 0.00 & 0.03 & 0.00 & 0.207 & 0.000 & -0.021 & -0.006 & -0.90 & 0.61 & 1836.77 & 103.36 & 0.62 & 104.31 \\
\hline 161250 & 0.19 & 0.00 & 0.04 & 0.00 & 0.207 & 0.000 & -0.033 & -0.008 & -1.41 & 0.17 & 1839.85 & 108.35 & 0.19 & 109.40 \\
\hline 62251 & 0.19 & 0.00 & 0.04 & 0.00 & 0.207 & 0.000 & -0.033 & -0.008 & -1.34 & 0.27 & 1843.72 & 112.55 & 0.31 & 113.70 \\
\hline 163252 & 0.18 & 0.00 & 0.05 & -0.01 & 0.197 & 0.000 & -0.047 & 0.000 & -1.67 & 0.05 & 1846.39 & 117.95 & 0.11 & 119.22 \\
\hline 64253 & 0.16 & 0.01 & 0.04 & 0.00 & 0.174 & -0.014 & -0.037 & -0.007 & -1.08 & 0.27 & 1849.95 & 122.47 & 0.31 & 123.82 \\
\hline $165 \quad 254$ & 0.16 & 0.01 & 0.04 & 0.00 & 0.174 & -0.014 & -0.037 & -0.007 & -1.30 & 0.13 & 1852.34 & 128.15 & 0.17 & 129.59 \\
\hline 166255 & 0.12 & 0.01 & 0.00 & 0.01 & 0.129 & -0.014 & 0.007 & -0.010 & -0.58 & 0.35 & 1855.70 & 132.85 & 0.36 & 134.38 \\
\hline $67 \quad 256$ & 0.12 & 0.01 & 0.00 & 0.01 & 0.129 & -0.014 & 0.007 & -0.010 & -1.12 & -0.23 & 1858.35 & 138.27 & -0.22 & 139.90 \\
\hline $168 \quad 257$ & -0.18 & 0.00 & 0.01 & 0.00 & -0.186 & 0.000 & 0.001 & 0.001 & -1.70 & -0.39 & 1861.90 & 142.80 & -0.40 & 144.52 \\
\hline 169258 & -0.17 & 0.00 & 0.01 & 0.00 & -0.176 & 0.000 & -0.000 & 0.001 & -2.37 & -1.20 & 1864.59 & 148.18 & -1.21 & 150.01 \\
\hline $170 \quad 259$ & -0.17 & 0.00 & 0.01 & -0.01 & -0.176 & 0.000 & 0.001 & 0.010 & -2.67 & -1.39 & 1867.99 & 152.85 & -1.38 & 154.83 \\
\hline 171260 & -0.17 & 0.00 & 0.01 & -0.01 & -0.176 & 0.000 & 0.001 & 0.010 & -3.34 & -1.99 & 1870.29 & 158.62 & -1.98 & 160.72 \\
\hline $172 \quad 261$ & -0.17 & 0.00 & 0.01 & -0.01 & -0.176 & 0.000 & 0.001 & 0.010 & -3.44 & -2.05 & 1873.38 & 163.60 & -2.04 & 165.84 \\
\hline $173 \quad 262$ & -0.12 & 0.00 & -0.01 & -0.01 & -0.125 & 0.000 & 0.018 & 0.008 & -3.46 & -2.55 & 1875.41 & 169.65 & -2.54 & 172.02 \\
\hline 174263 & -0.12 & 0.00 & -0.01 & -0.01 & -0.125 & 0.000 & 0.018 & 0.008 & -3.44 & -2.58 & 1878.28 & 174.85 & -2.56 & 177.36 \\
\hline $175 \quad 264$ & -0.10 & 0.00 & 0.00 & -0.01 & -0.104 & 0.000 & 0.004 & 0.009 & -3.88 & -3.27 & 1880.33 & 180.86 & -3.26 & 183.51 \\
\hline 176265 & -0.10 & 0.00 & 0.01 & 0.00 & -0.105 & 0.000 & -0.008 & 0.001 & -4.00 & -3.41 & 1883.14 & 186.13 & -3.41 & 188.91 \\
\hline 177266 & -0.10 & 0.00 & 0.02 & 0.00 & -0.105 & 0.000 & -0.019 & 0.002 & -4.61 & -3.96 & 1884.88 & 192.46 & -3.95 & 195.40 \\
\hline $178 \quad 267$ & -0.09 & 0.00 & 0.02 & -0.01 & -0.094 & 0.000 & -0.020 & 0.012 & -4.60 & -3.97 & 1887.39 & 198.03 & -3.93 & 201.16 \\
\hline 179268 & -0.08 & 0.00 & 0.02 & 0.00 & -0.084 & 0.000 & -0.021 & 0.002 & -4.97 & -4.32 & 1888.76 & 204.72 & -4.30 & 207.98 \\
\hline 180269 & -0.04 & 0.00 & 0.01 & 0.00 & -0.042 & 0.000 & -0.011 & 0.001 & -4.65 & -3.98 & 1890.75 & 210.80 & -3.97 & 214.22 \\
\hline 181270 & 0.01 & 0.00 & 0.00 & 0.00 & 0.011 & 0.000 & 0.000 & 0.000 & -5.23 & -4.43 & 1892.07 & 217.55 & -4.43 & 221.13 \\
\hline $182 \quad 271$ & 0.01 & 0.00 & 0.00 & 0.00 & 0.011 & 0.000 & 0.000 & 0.000 & -5.33 & -4.51 & 1894.32 & 223.38 & -4.51 & 227.13 \\
\hline 183272 & 0.01 & 0.00 & 0.00 & 0.00 & 0.011 & 0.000 & 0.000 & 0.000 & -5.87 & -5.02 & 1895.54 & 230.23 & -5.03 & 234.15 \\
\hline 184273 & 0.00 & 0.00 & 0.00 & 0.00 & 0.000 & 0.000 & 0.000 & 0.000 & -5.68 & -4.85 & 1897.37 & 236.47 & -4.85 & 240.57 \\
\hline 185274 & -0.01 & 0.00 & 0.00 & 0.00 & -0.011 & 0.000 & 0.000 & 0.000 & -4.76 & -3.98 & 1897.06 & 244.85 & -3.98 & 249.14 \\
\hline 186275 & 0.00 & 0.00 & 0.00 & 0.00 & 0.000 & 0.000 & 0.000 & 0.000 & -4.04 & -3.28 & 1898.20 & 251.78 & -3.28 & 256.26 \\
\hline 187276 & 0.01 & 0.05 & 0.00 & 0.00 & 0.012 & -0.067 & 0.001 & 0.002 & -3.51 & -2.40 & 1897.73 & 260.33 & -2.37 & 265.04 \\
\hline 188277 & 0.04 & 0.09 & -0.02 & 0.00 & 0.046 & -0.123 & 0.028 & 0.007 & -4.03 & -1.90 & 1898.92 & 267.21 & -1.75 & 272.23 \\
\hline 189278 & 0.06 & 0.10 & -0.03 & 0.00 & 0.068 & -0.138 & 0.041 & 0.010 & -4.66 & -2.18 & 1899.45 & 274.75 & -1.98 & 280.02 \\
\hline $190 \quad 279$ & 0.06 & 0.11 & -0.03 & 0.01 & 0.069 & -0.151 & 0.042 & 0.001 & -4.63 & -1.89 & 1900.70 & 281.57 & -1.65 & 287.08 \\
\hline 191280 & 0.06 & 0.12 & -0.02 & 0.02 & 0.069 & -0.163 & 0.031 & -0.009 & -4.92 & -2.00 & 1900.92 & 289.42 & -1.73 & 295.18 \\
\hline 192281 & 0.06 & 0.12 & -0.02 & 0.02 & 0.069 & -0.163 & 0.031 & -0.009 & -4.43 & -1.55 & 1901.87 & 296.54 & -1.27 & 302.53 \\
\hline $193 \quad 282$ & 0.06 & 0.13 & -0.02 & 0.02 & 0.070 & -0.177 & 0.032 & -0.007 & -4.70 & -1.43 & 1901.71 & 304.77 & -1.12 & 311.00 \\
\hline 194283 & 0.10 & 0.12 & -0.05 & 0.01 & 0.112 & -0.167 & 0.070 & 0.008 & -4.63 & -1.04 & 1902.58 & 311.98 & -0.70 & 318.47 \\
\hline 195284 & 0.10 & 0.12 & -0.05 & 0.01 & 0.112 & -0.167 & 0.070 & 0.008 & -4.60 & -1.01 & 1902.38 & 320.25 & -0.69 & 326.96 \\
\hline 196285 & 0.38 & 0.00 & 0.10 & -0.02 & 0.432 & 0.000 & -0.059 & -0.018 & -4.01 & -0.39 & 1902.86 & 327.84 & -0.34 & 334.50 \\
\hline 197286 & 0.37 & 0.00 & 0.10 & -0.02 & 0.420 & 0.000 & -0.063 & -0.018 & -4.08 & -0.51 & 1902.67 & 336.10 & -0.46 & 342.99 \\
\hline 198287 & 0.37 & 0.00 & 0.10 & -0.02 & 0.420 & 0.000 & -0.063 & -0.018 & -3.77 & -0.28 & 1903.40 & 343.44 & -0.20 & 350.60 \\
\hline 199288 & 0.38 & 0.00 & 0.11 & -0.03 & 0.433 & 0.000 & -0.073 & -0.014 & -4.22 & -0.18 & 1902.86 & 352.05 & -0.06 & 359.51 \\
\hline $200 \quad 289$ & 0.38 & 0.00 & 0.12 & -0.03 & 0.434 & 0.000 & -0.085 & -0.018 & -4.32 & -0.05 & 1903.57 & 359.41 & 0.18 & 367.24 \\
\hline 201290 & 0.38 & 0.00 & 0.12 & -0.03 & 0.434 & 0.000 & -0.085 & -0.018 & -4.62 & -0.35 & 1903.29 & 367.77 & -0.14 & 375.82 \\
\hline $202 \quad 291$ & 0.16 & 0.01 & -0.08 & 0.01 & 0.173 & -0.014 & 0.111 & 0.009 & -3.12 & -0.67 & 1904.31 & 374.82 & -0.25 & 383.35 \\
\hline 203292 & 0.16 & 0.01 & -0.08 & 0.01 & 0.173 & -0.014 & 0.111 & 0.009 & -3.48 & -1.06 & 1903.99 & 383.20 & -0.64 & 391.99 \\
\hline 293 & 0.17 & 0.01 & -0.07 & 0.01 & 0.183 & -0.014 & 0.100 & 0.008 & -3.02 & -0.75 & 1904.24 & 391.02 & -0.43 & 399.98 \\
\hline $05 \quad 294$ & 0.18 & 0.01 & -0.07 & 0.02 & 0.193 & -0.014 & 0.102 & -0.002 & -3.45 & -1.16 & 1903.82 & 399.51 & -0.82 & 408.77 \\
\hline
\end{tabular}


$Z=89$ (Ac)

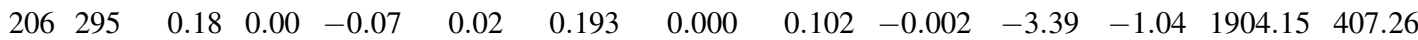

$-0.69416 .81$

$$
Z=90 \text { (Th) }
$$

\begin{tabular}{|c|c|c|c|c|c|c|c|c|c|c|c|c|c|c|c|c|}
\hline 108198 & 0.26 & 0.00 & 0.02 & 0.00 & 0.285 & 0.000 & 0.005 & -0.003 & -0.79 & 0.94 & 1489.80 & 37.92 & & & 0.86 & 39.20 \\
\hline 109199 & 0.30 & 0.00 & 0.01 & 0.00 & 0.330 & 0.000 & 0.027 & 0.001 & -0.92 & 1.15 & 1499.41 & 36.37 & & & 1.04 & 37.54 \\
\hline 0200 & 0.31 & 0.00 & 0.02 & -0.01 & 0.342 & 0.000 & 0.016 & 0.008 & -0.56 & 1.52 & 1510.69 & 33.16 & & & 1.42 & 34.26 \\
\hline 1201 & 0.17 & 0.00 & -0.01 & 0.01 & 0.183 & 0.000 & 0.025 & -0.007 & 0.31 & 1.47 & 1520.21 & 31.72 & & & 1.43 & 32.81 \\
\hline 2202 & 0.16 & 0.00 & -0.02 & 0.01 & 0.172 & 0.000 & 0.036 & -0.005 & 0.28 & 1.52 & 1531.42 & 28.57 & & & 1.50 & 29.60 \\
\hline 3203 & 0.15 & 0.00 & -0.01 & 0.01 & 0.161 & 0.000 & 0.022 & -0.007 & 0.05 & 1.19 & 1540.85 & 27.21 & & & 1.16 & 28.17 \\
\hline 4204 & -0.23 & 0.00 & 0.02 & 0.00 & -0.237 & 0.000 & -0.002 & 0.003 & -0.80 & 1.08 & 1551.86 & 24.27 & & & 1.03 & 25.13 \\
\hline 5205 & 0.14 & 0.00 & 0.00 & 0.00 & 0.150 & 0.000 & 0.008 & 0.001 & -0.32 & 0.73 & 1560.96 & 23.25 & & & 0.71 & 24.07 \\
\hline 6206 & -0.21 & 0.00 & 0.03 & 0.00 & -0.217 & 0.000 & -0.016 & 0.006 & -1.20 & 0.57 & 1571.66 & 20.62 & & & 0.53 & 21.35 \\
\hline 7207 & -0.20 & 0.00 & 0.04 & 0.00 & -0.207 & 0.000 & -0.029 & 0.008 & -1.80 & -0.01 & 1580.65 & 19.70 & & & -0.05 & 20.38 \\
\hline 8208 & -0.19 & 0.00 & 0.03 & 0.00 & -0.197 & 0.000 & -0.020 & 0.005 & -2.08 & -0.39 & 1591.22 & 17.20 & & & -0.42 & 17.82 \\
\hline 9209 & -0.14 & 0.00 & 0.00 & -0.01 & -0.146 & 0.000 & 0.008 & 0.009 & -2.24 & -1.10 & 1600.00 & 16.50 & 50 & .100 & -1.12 & 17.08 \\
\hline 210 & -0.13 & 0.00 & 0.00 & -0.01 & -0.135 & 0.000 & 0.007 & 0.009 & -2.67 & -1.62 & 1610.37 & 14.19 & 14.04 & .025 & -1.63 & 14.72 \\
\hline 1211 & -0.11 & 0.00 & 0.00 & -0.01 & -0.115 & 0.000 & 0.005 & 0.009 & -3.15 & -2.31 & 1618.80 & 13.83 & & 0.075 & -2.32 & 14.32 \\
\hline 2212 & -0.09 & 0.00 & 0.01 & 0.00 & -0.094 & 0.000 & -0.008 & 0.001 & -3.37 & -2.73 & 1628.74 & 11.96 & 12.09 & 0.018 & -2.74 & 12.40 \\
\hline 3213 & -0.08 & 0.00 & 0.01 & 0.00 & -0.084 & 0.000 & -0.009 & 0.001 & -4.06 & -3.43 & 5.87 & 11.91 & 12 & 071 & -3.44 & 12.30 \\
\hline 4214 & -0.06 & 0.00 & 0.02 & 0.01 & -0.063 & 0.000 & -0.022 & -0.008 & -4.58 & -3.75 & 1646.39 & 10.47 & 10.71 & 0.017 & -3.74 & 10.82 \\
\hline 215 & 0.00 & 0.00 & 0.00 & 0.00 & 0.000 & 0.000 & 0.000 & 0.000 & -5.20 & -4.29 & 1654.05 & 10.88 & 10.93 & 27 & -4.29 & 11.19 \\
\hline 6216 & 0.00 & 0.00 & 0.00 & 0.00 & 0.000 & 0.000 & 0.000 & 0.000 & -5.43 & -4.48 & 1663.12 & 9.88 & 10.30 & 0.013 & -4.48 & 10.15 \\
\hline 7217 & -0.02 & 0.00 & -0.01 & 0.00 & -0.021 & 0.000 & 0.012 & -0.000 & -4.45 & -3.58 & 1669.03 & 12.03 & 12.22 & 0.021 & -3.58 & 12.28 \\
\hline 8218 & 0.00 & 0.00 & 0.00 & 0.00 & 0.000 & 0.000 & 0.000 & 0.000 & -3.47 & -2.64 & 67 & 12.47 & 12 & 13 & -2.64 & 12.68 \\
\hline 9219 & 0.07 & 0.09 & -0.03 & 0.00 & 0.078 & -0.124 & 0.041 & 0.009 & -4.93 & -2.17 & 1682.71 & 14.50 & 14 & 0.051 & -2.12 & 14.72 \\
\hline $0 \quad 220$ & 0.08 & 0.10 & -0.04 & 0.00 & 0.090 & -0.139 & 0.055 & 0.012 & -5.12 & -1.68 & 1690.50 & 14.78 & 67 & 22 & -1.61 & 15.01 \\
\hline 221 & 0.10 & 0.09 & -0.05 & 0.00 & 0.110 & -0.125 & 0.068 & 0.014 & -5.09 & -1.61 & 1696.66 & 16.69 & 16.94 & 0.009 & -1.54 & 16.90 \\
\hline 2222 & 0.10 & 0.10 & -0.05 & 0.00 & 0.111 & -0.140 & 0.069 & 0.015 & -5.11 & -1.22 & 1704.25 & 17.17 & 17.20 & 0 & -1.11 & 17.39 \\
\hline 3223 & 0.12 & 0.10 & -0.06 & 0.00 & 0.133 & -0.140 & 0.083 & 0.019 & -5.69 & -1.25 & .23 & 19.26 & 19.39 & 0.009 & -1.13 & 19.47 \\
\hline $4 \quad 224$ & 0.13 & 0.11 & -0.06 & 0.01 & 0.144 & -0.153 & 0.084 & 0.010 & -5.69 & -0.91 & 1717.59 & 19.97 & 20.00 & 0.011 & -0.77 & 20.20 \\
\hline $5 \quad 225$ & 0.13 & 0.10 & -0.06 & 0.01 & 0.143 & -0.139 & 0.084 & 0.009 & -5.31 & -0.93 & .29 & 22.34 & 22.31 & 05 & -0.81 & 22.54 \\
\hline 6226 & 0.14 & 0.10 & -0.06 & 0.01 & 0.154 & -0.139 & 0.085 & 0.010 & -4.98 & -0.52 & 1730.30 & 23.41 & 23.20 & 0.005 & -0.38 & 23.62 \\
\hline $137 \quad 227$ & 0.15 & 0.10 & -0.06 & 0.02 & 0.164 & -0.138 & 0.087 & 0.000 & -5.01 & -0.51 & 1735.70 & 26.08 & 25.81 & 0.003 & -0.38 & 26.28 \\
\hline $8 \quad 228$ & 0.16 & 0.08 & -0.07 & 0.01 & 0.174 & -0.111 & 0.100 & 0.011 & -4.52 & -0.23 & 1742.56 & 27.29 & 26.77 & 0.002 & -0.07 & 27.51 \\
\hline 39229 & 0.17 & 0.00 & -0.08 & 0.00 & 0.184 & 0.000 & 0.113 & 0.021 & -4.18 & -0.37 & 7.86 & 30.06 & 29.59 & 0.003 & -0.22 & 30.28 \\
\hline $0 \quad 230$ & 0.18 & 0.00 & -0.08 & 0.00 & 0.195 & 0.000 & 0.114 & 0.022 & -4.18 & -0.27 & .63 & 31.36 & 30.86 & 0.002 & -0.09 & 31.60 \\
\hline 11231 & 0.18 & 0.00 & -0.08 & 0.01 & 0.195 & 0.000 & 0.115 & 0.012 & -4.34 & -0.53 & 1759.79 & 34.27 & 33.82 & 0.002 & -0.38 & 34.50 \\
\hline $142 \quad 232$ & 0.19 & 0.00 & -0.07 & 0.01 & 0.205 & 0.000 & 0.103 & 0.010 & -3.84 & -0.50 & 1766.38 & 35.75 & 35.45 & 0.002 & -0.36 & 35.98 \\
\hline $143 \quad 233$ & 0.20 & 0.00 & -0.07 & 0.02 & 0.215 & 0.000 & 0.106 & 0.001 & -4.16 & -0.73 & 1771.26 & 38.95 & 38.73 & 0.002 & -0.59 & 39.19 \\
\hline $44 \quad 234$ & 0.21 & 0.00 & -0.06 & 0.02 & 0.226 & 0.000 & 0.095 & -0.001 & -3.63 & -0.53 & 1777.43 & 40.85 & 40.61 & 0.004 & -0.41 & 41.09 \\
\hline $145 \quad 235$ & 0.21 & 0.00 & -0.06 & 0.03 & 0.226 & 0.000 & 0.095 & -0.012 & -3.98 & -0.83 & 1782.14 & 44.21 & 44.26 & 0.050 & -0.69 & 44.50 \\
\hline $46 \quad 236$ & 0.22 & 0.00 & -0.05 & 0.03 & 0.237 & 0.000 & 0.085 & -0.014 & -3.53 & -0.63 & 1788.06 & 46.36 & & & -0.50 & 46.67 \\
\hline $147 \quad 237$ & 0.22 & 0.00 & -0.05 & 0.03 & 0.237 & 0.000 & 0.085 & -0.014 & -3.82 & -0.95 & 1792.55 & 49.94 & & & -0.83 & 50.26 \\
\hline $48 \quad 238$ & 0.22 & 0.00 & -0.04 & 0.03 & 37 & 0.000 & 0.073 & -0.017 & -3.31 & -0.75 & .22 & 52.34 & & & -0.63 & 52.69 \\
\hline 49239 & 0.22 & 0.00 & -0.04 & 0.03 & 0.237 & 0.000 & 0.073 & -0.017 & -3.59 & -1.02 & 2.44 & 56.20 & & & -0 & 56.58 \\
\hline $50 \quad 240$ & 0.22 & 0.00 & -0.03 & 0.03 & 0.237 & 0.000 & 0.060 & -0.020 & -3.15 & -0.84 & 1807.89 & 58.81 & & & -0.73 & 59.23 \\
\hline 51241 & 0.23 & 0.00 & -0.02 & 0.03 & 0.249 & 0.000 & 0.050 & -0.022 & -3.35 & -1.10 & 1811.87 & 62.91 & & & -1.01 & 63.35 \\
\hline $152 \quad 242$ & 0.23 & 0.00 & -0.01 & 0.03 & 0.250 & 0.000 & 0.038 & -0.025 & -2.91 & -0.82 & 1817.00 & 65.85 & & & -0.72 & 66.35 \\
\hline 53243 & 0.22 & 0.00 & -0.01 & 0.03 & 0.250 & 0.000 & 0.036 & -0.025 & -2.69 & -0.73 & 1820.41 & 70.51 & & & -0.63 & 71.05 \\
\hline 4244 & 0.22 & 0.00 & -0.01 & 0.03 & & 0.000 & 0.036 & -0.025 & -2.20 & -0.26 & 1825.11 & 73.88 & & & -0.15 & 74.48 \\
\hline $55 \quad 245$ & 0.22 & 0.00 & 0.00 & 0.02 & 0.239 & 0.000 & 0.023 & -0.018 & -1.81 & -0.05 & 1828.19 & 78.88 & & & -0.01 & 79.47 \\
\hline 246 & 0.22 & 0.00 & 0.00 & 0.02 & 0.239 & 0.000 & 0.023 & -0.018 & -1.33 & 0.40 & 1832.70 & 82.43 & & & 0.45 & 83.10 \\
\hline $157 \quad 247$ & 0.21 & 0.00 & 0.01 & 0.01 & 0.228 & 0.000 & 0.008 & -0.011 & -1.27 & 0.33 & 1835.84 & 87.37 & & & 0.34 & 88.05 \\
\hline 58248 & 0.20 & 0.00 & 0.01 & 0.01 & 0.217 & 0.000 & .006 & -0.011 & -0.96 & 0.53 & 1840.38 & 90.89 & & & 0.55 & 91.65 \\
\hline 159249 & 0.20 & 0.00 & 0.02 & 0.01 & 0.218 & 0.000 & -0.006 & -0.013 & -1.18 & 0.38 & 1843.40 & 95.94 & & & 0.39 & 96.77 \\
\hline $160 \quad 250$ & 0.20 & 0.00 & 0.03 & 0.00 & 0.218 & 0.000 & -0.019 & -0.006 & -1.02 & 0.57 & 1847.74 & 99.67 & & & 0.58 & 100.58 \\
\hline 251 & 0.19 & 0.00 & 0.04 & 0.00 & 0.207 & 0.000 & -0.033 & -0.008 & -1.43 & 0.12 & 1850.85 & 104.64 & & & 0.16 & 105.64 \\
\hline $62 \quad 252$ & 0.19 & 0.00 & 0.04 & 0.00 & 0.207 & 0.000 & -0.033 & -0.008 & -1.35 & 0.18 & 1855.12 & 108.44 & & & 0.22 & 109.53 \\
\hline
\end{tabular}




\section{$Z=90(\mathbf{T h})$}

\begin{tabular}{|c|c|c|c|c|c|c|c|c|c|c|c|c|}
\hline 33 & 18 & 00 & 4 & 00 & & & & & & & & \\
\hline 54 & 0.17 & 00 & 0.04 & .00 & & & -0.036 & -0.007 & & & & \\
\hline $5=$ & 16 & 00 & & & & & & & & & & \\
\hline 56 & & 00 & & & & & & & & & & \\
\hline 57 & 12 & 00 & 01 & & & & & & & & & \\
\hline 58 & 2 & 00 & 0 & 1 & & 00 & & & & & & \\
\hline 9 & .17 & 00 & & & & & & & & & & \\
\hline 50 & & 00 & .00 & 0.01 & & & & & & & & \\
\hline 61 & & 00 & .00 & 01 & & & & & & & & \\
\hline 62 & 13 & 00 & 0.01 & .01 & 35 & & 19 & & & & & 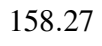 \\
\hline 63 & .12 & 00 & .01 & .01 & 25 & 0 & 8 & & -2.98 & -2.09 & & $642 \theta$ \\
\hline & & 00 & 0.01 & 0.01 & 25 & & & & & & & \\
\hline 55 & & 00 & .00 & 0.01 & -0 . & & & & & -2 & & \\
\hline 66 & & 00 & 0.01 & 001 & -0 . & & & & & & & \\
\hline 67 & & 00 & 002 & $0 \Omega 0$ & & & & & & & & \\
\hline 58 & 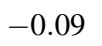 & .00 & 0.02 & $x$ & 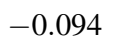 & & & & & & & \\
\hline 59 & .08 & UU & & 0.00 & 4 & & & & & & & \\
\hline 70 & . .05 & 00 & & 0. & 3 & & & & & & & \\
\hline 71 & & & & & & & & & & & & \\
\hline 72 & 0.00 & .00 & & & & & & & & & & \\
\hline ( & & & & & & & & & & & & \\
\hline 74 & & & & & & & & & & & & \\
\hline 275 & -0.01 & 0.00 & & & & & & & & & & \\
\hline 76 & & & & & & & & & & & & \\
\hline 77 & & 07 & 0.01 & & & -0.095 & & & & & & \\
\hline 78 & & 9 & & & & & & & & & & \\
\hline 79 & & 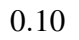 & & & & & & & & & & \\
\hline 80 & & 1 & & & & & & & & & & \\
\hline 31 & & & & & & & & & & & & \\
\hline 282 & 0 & 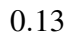 & -0.02 & 0.02 & 0 & 7 & 2 & & 8 & & & 28 \\
\hline 33 & & 13 & 0 & & & & & & & & & .0 \\
\hline 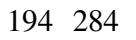 & & & & & & & & & & & & \\
\hline 285 & & 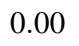 & & & & & & & & & & \\
\hline 86 & & & & & & & & & & & & \\
\hline 287 & 0.37 & 0.00 & 0. & 2 & & & & & & & & \\
\hline 88 & & 00 & .10 & 07 & & & & & & & & 43 \\
\hline 89 & & & & & & & & & & & & \\
\hline 90 & & & & & & & & & & & & \\
\hline 91 & & . & & -0 & & & & & & & & \\
\hline 292 & U & 0.00 & & 0. & & & & & & & & \\
\hline 293 & & 00 & & 0.01 & & & & & & & & .75 \\
\hline 294 & & & & & & & & & & & & \\
\hline 295 & & $x^{\circ}$ & & & & & & & & & & \\
\hline 90 & & & & & & & & & & & & .9 \\
\hline 07 & & 000 & & & & & & & & & & 046 \\
\hline & & & & & & & & & & & & \\
\hline 99 & .19 & 0.00 & 0.05 & .03 & .204 & 0.000 & .079 & -0 . & .47 & -1.61 & .90 & \\
\hline
\end{tabular}

$0.14 \quad 115.14$

$0.41 \quad 119.44$

$\begin{array}{ll}0.14 & 125.07\end{array}$

$\begin{array}{ll}0.58 & 129.75\end{array}$

$0.15 \quad 135.41$

$-0.10 \quad 139.60$

$-0.78 \quad 145.20$

$-0.97 \quad 149.65$

$-1.53 \quad 155.56$

$\begin{array}{ll}-1.54 & 160.39\end{array}$

$-2.07 \quad 166.51$

$-2.08 \quad 171.52$

$-2.72 \quad 177.71$

$\begin{array}{ll}-2.87 & 182.77\end{array}$

$-3.40 \quad 189.25$

$-3.37 \quad 194.67$

$-3.66 \quad 201.56$

$\begin{array}{ll}-3.39 & 207.41\end{array}$

$-3.79 \quad 214.36$

$-3.86 \quad 220.04$

$\begin{array}{ll}-4.34 & 227.08\end{array}$

$\begin{array}{ll}-4.14 & 233.19\end{array}$

$-3.24 \quad 241.77$

$-2.57 \quad 248.53$

$\begin{array}{ll}-1.76 & 257.19\end{array}$

$\begin{array}{ll}-1.18 \quad 264.02 \\ -1.42 & 271.78\end{array}$

$-1.42 \quad 271.78$

$-1.11 \quad 278.51$
-1.23

$-1.23 \quad 286.55$

$-0.76 \quad 293.58$

$\begin{array}{ll}-0.65 & 302.01\end{array}$

$-0.22 \quad 309.17$

$-0.44 \quad 317.41$

$-0.19 \quad 324.54$

$-0.33 \quad 333.01$

$-0.06 \quad 340.31$

$-0.01 \quad 349.11$

$\begin{array}{lll}0.42 & 356.72\end{array}$

$-0.08 \quad 365.12$

$\begin{array}{ll}-0.13 & 372.39\end{array}$

$-0.37 \quad 381.18$

$-0.33 \quad 388.69$

$-0.75 \quad 397.43$

$-0.72405 .07$

$-0.99 \quad 414.11$

$-0.95 \quad 421.89$

$-1.32430 .96$

\section{$Z=91(\mathrm{~Pa})$}

\begin{tabular}{rrrrrrrrrrrrrrrr}
109 & 200 & 0.30 & 0.00 & 0.02 & 0.00 & 0.330 & 0.000 & 0.015 & -0.002 & -1.16 & 0.76 & 1497.32 & 45.75 & \\
110 & 201 & 0.30 & 0.00 & 0.02 & -0.01 & 0.330 & 0.000 & 0.014 & 0.007 & -0.79 & 1.06 & 1508.72 & 42.42 & 0.62 & 47.10 \\
111 & 202 & 0.18 & 0.00 & -0.01 & 0.01 & 0.194 & 0.000 & 0.027 & -0.007 & 0.04 & 1.22 & 1518.46 & 40.75 & 0.94 & 43.71 \\
112 & 203 & 0.17 & 0.00 & -0.02 & 0.01 & 0.183 & 0.000 & 0.037 & -0.005 & 0.13 & 1.41 & 1529.59 & 37.70 & \\
113 & 204 & 0.16 & 0.00 & -0.02 & 0.01 & 0.172 & 0.000 & 0.036 & -0.005 & -0.11 & 1.18 & 1539.35 & 36.01 & \\
114 & 205 & 0.15 & 0.00 & -0.01 & 0.00 & 0.161 & 0.000 & 0.022 & 0.002 & -0.04 & 1.09 & 1550.39 & 33.03 & \\
115 & 206 & 0.15 & 0.00 & 0.00 & 0.00 & 0.162 & 0.000 & 0.009 & 0.001 & -0.35 & 0.78 & 1559.88 & 31.62 & 42.03 \\
116 & 207 & 0.14 & 0.02 & 0.01 & 0.00 & 0.151 & -0.027 & -0.004 & -0.001 & -0.46 & 0.65 & 1570.60 & 28.97 & 38.91 \\
117 & 208 & -0.21 & 0.00 & 0.05 & 0.01 & -0.217 & 0.000 & -0.039 & 0.002 & -1.78 & 0.25 & 1579.83 & 27.81 & 37.14 \\
\hline
\end{tabular}




\section{$Z=91$ (Pa)}

\begin{tabular}{|c|c|c|c|c|c|c|c|c|c|c|c|c|c|c|c|c|}
\hline $18 \quad 209$ & -0.20 & 0.00 & 0.04 & 0.00 & -0.207 & 0.000 & -0.029 & 0.008 & -1.96 & -0.20 & 1590.52 & 25.19 & & & -0.24 & \\
\hline 19210 & -0.15 & 0.00 & 0.00 & -0.01 & -0.156 & 0.000 & 0.009 & 0.009 & -1.92 & -0.70 & 1599.51 & 24.27 & & & -0.73 & 25.00 \\
\hline 211 & -0.14 & 0.00 & 0.00 & -0.02 & -0.146 & 0.000 & 0.009 & 0.019 & -2.41 & -1.20 & 1609.91 & 21.95 & & & & 22.64 \\
\hline 212 & -0.12 & 0.00 & 0.00 & -0.02 & -0.125 & 0.000 & 0.007 & 0.019 & -2.77 & -1.76 & 1618.63 & 21.30 & .61 & 0.075 & -1.76 & 1.94 \\
\hline 2213 & -0.10 & 0.00 & 0.01 & 0.00 & -0.105 & 0.000 & -0.008 & 0.001 & -2.84 & -2.15 & 1628.59 & 19.41 & 19.66 & 0.071 & -2.16 & 9.99 \\
\hline 214 & -0.09 & 0.00 & 0.01 & 0.00 & -0.094 & 0.000 & -0.008 & 0.001 & -3.44 & -2.82 & 1637.09 & 18.98 & 19.49 & .076 & -2.83 & 9.51 \\
\hline 15 & -0.06 & 0.00 & 0.02 & 0.01 & -0.063 & 0.000 & -0.022 & -0.008 & -3.82 & -3.03 & 1646.54 & 17.60 & & 87 & -3.02 & 3.09 \\
\hline 216 & 0.00 & 0.00 & 0.00 & 0.00 & 0.000 & 0.000 & 0.000 & 0.000 & -4.39 & -3.55 & 1654.59 & 17.62 & 17.80 & .070 & -3.55 & 8.07 \\
\hline 217 & 0.00 & 0.00 & 0.00 & 0.00 & 0.000 & 0.000 & 0.000 & 0.000 & -4.62 & -3.73 & 1663.70 & 16.58 & 17.07 & 052 & -3.73 & 16.99 \\
\hline 7218 & -0.02 & 0.00 & -0.01 & 0.00 & -0.021 & 0.000 & 0.012 & -0.000 & -3.65 & -2.84 & 1670.03 & 18.33 & 18.67 & 0.025 & -2.84 & 18.69 \\
\hline 219 & 0.05 & 0.07 & -0.02 & 0.00 & 0.055 & -0.096 & 0.027 & 0.005 & -3.85 & -1.99 & 7.79 & 18.64 & 18.52 & 0.054 & -1.97 & 18.99 \\
\hline 220 & 0.08 & 0.09 & -0.04 & 0.00 & 0.089 & -0.125 & 0.054 & 0.011 & -4.86 & -1.83 & .56 & 19.94 & 20.38 & 0.057 & -1.79 & 20.28 \\
\hline 221 & 0.09 & 0.10 & -0.04 & 0.00 & 0.100 & -0.139 & 0.056 & 0.013 & -4.89 & -1.41 & & 20.12 & 20.38 & 0.052 & -1.35 & 20.45 \\
\hline 222 & 0.10 & 0.09 & -0.05 & 0.00 & 0.110 & -0.125 & 0.068 & 0.014 & -4.86 & -1.40 & 1699.07 & 21.57 & & & -1.34 & 21.87 \\
\hline 2223 & 0.11 & 0.10 & -0.05 & 0.00 & 0.122 & -0.140 & 0.069 & 0.016 & -4.98 & -1.05 & 1706.74 & 21.97 & 22.32 & 0.071 & -0.97 & 22.27 \\
\hline 224 & 0.12 & 10 & -0.06 & 0.01 & 0.132 & -0.139 & 0.083 & 0.008 & -5.45 & -1.15 & 20 & 23.58 & 87 & 016 & -1.07 & 23.87 \\
\hline 225 & 0.13 & 0.11 & -0.06 & 0.01 & 0.144 & -0.153 & 0.084 & 0.010 & -5.65 & -0.88 & 1720.66 & 24.20 & & & -0.76 & 24.49 \\
\hline 226 & 0.14 & 0.11 & -0.06 & 0.02 & 0.154 & -0.152 & 0.086 & 0.001 & -5.72 & -0.92 & 1726.77 & 26.15 & & & & 26.43 \\
\hline 227 & 0.14 & 0.10 & -0.06 & 0.02 & 0.153 & -0.138 & 0.085 & -0.001 & -4.95 & -0.57 & & 27.12 & & & & 27.40 \\
\hline $7 \quad 228$ & 0.15 & 0.10 & -0.06 & 0.02 & 0.164 & -0.138 & 0.087 & 0.000 & -5.11 & -0.66 & & 29.30 & 2 & & & 29.55 \\
\hline 229 & 0.17 & 00 & -0.09 & 0.00 & 85 & 0 & 26 & 24 & -4.90 & -0.48 & & 38 & 0 & & 31 & .68 \\
\hline 230 & 0.17 & 0.00 & -0.09 & 0.00 & 0.185 & 0.000 & 0.126 & 0.024 & -5.18 & -0.73 & & 32.66 & 32.17 & 0.003 & 57 & 32.95 \\
\hline 231 & 0.18 & .00 & -0.08 & 0.01 & 0.195 & 0.000 & 0.115 & 0.012 & -4.43 & -0.61 & & 33.94 & & & & 34.21 \\
\hline 32 & 0.19 & 00 & -0.08 & 0.01 & 0.206 & 0.000 & 0.116 & 0.013 & & -0.89 & & 36.44 & & & & 36.71 \\
\hline 33 & 0.19 & 0.00 & -0.08 & 0.02 & & & & & & -0.90 & & & & & & 38.15 \\
\hline 234 & 0.20 & 00 & -0.07 & 0 & & 00 & 06 & 01 & -4.58 & -1.12 & & & & & & 40.94 \\
\hline 35 & 0.21 & 0.00 & -0.07 & 0.03 & 0.226 & 0.000 & 0.108 & -0.009 & -4.60 & -1.02 & & 42.45 & & & & 42.77 \\
\hline 236 & 0.21 & 0.00 & -0.06 & 0.03 & 0.226 & 0.000 & 0.095 & -0.012 & -4.43 & -1.24 & & 45.51 & & & & 45.81 \\
\hline 37 & 0.22 & 0.00 & -0.05 & 0.03 & 0.237 & 0.000 & 0.085 & -0.014 & -3.98 & -1.11 & & 47.57 & & & -1.00 & 47.89 \\
\hline 38 & 0 & 0.00 & -0.05 & 0.03 & 0 & 0 & 35 & -0 & - & -1.37 & 1 & 50 & 50.77 & & - & 51.17 \\
\hline 239 & 0.22 & 0.00 & -0.04 & 0.03 & 237 & 0.000 & .073 & -0.017 & -3.74 & -1.16 & .64 & 53.21 & & & -1.05 & 53.57 \\
\hline 240 & 0.22 & 00 & -0.04 & 0.03 & & 00 & 0.073 & -0.017 & & -1.42 & & 56.71 & & & & 57.09 \\
\hline 0241 & 0.22 & 0.00 & -0.03 & 0.03 & 37 & 0.000 & 0.060 & -0.020 & -3.56 & -1.23 & .69 & 59.30 & & & & 59.72 \\
\hline 242 & 0.23 & 0.00 & -0.02 & 0.03 & 0.249 & 0.000 & 0.050 & -0.022 & -3.73 & -1.47 & 9.02 & 63.05 & & & & 63.48 \\
\hline 2243 & 0.23 & 0.00 & -0.01 & 0.03 & 0.250 & 0.000 & 0.038 & -0.025 & -3.28 & -1.17 & 16 & 65.98 & & & 09 & 66.46 \\
\hline 244 & 0.22 & 0.00 & -0.01 & 0.03 & 0.238 & 0.000 & 0.036 & -0.025 & -3.05 & -1.07 & 7.92 & 70.29 & & & 99 & 70.81 \\
\hline 45 & 0.22 & 00 & -0.01 & 0 & & & 36 & -0.025 & & -0.65 & & 73 & & & & 74.16 \\
\hline 5246 & 0.22 & 0.00 & 0.00 & 0.02 & & 0.000 & 0.023 & -0.018 & & -0.35 & & 78.30 & & & .33 & 78.87 \\
\hline 6247 & 0.22 & 0.00 & 0.00 & 0.02 & 39 & 0.000 & 0.023 & -0.018 & -1.66 & 0.09 & & 81.83 & & & 0.13 & 82.46 \\
\hline $7 \quad 248$ & 0.21 & 0.00 & 0.01 & 0.01 & 0.228 & 0.000 & 0.008 & -0.011 & -1.51 & 0.08 & 1844.04 & 86.46 & & & 0.07 & 87.11 \\
\hline 249 & 0.20 & 0.00 & 0.01 & 0.01 & 217 & 0.000 & 006 & -0.011 & -1.18 & 0.32 & 56 & 90.00 & & & 0.32 & 90.72 \\
\hline 250 & 0.20 & 0.00 & 0.02 & 0.00 & & & .007 & -0.004 & & 0.17 & & 94.71 & & & 0.16 & 95.47 \\
\hline 51 & 0.20 & 0.00 & 0.03 & 0.00 & 18 & 0.000 & -0.019 & -0.006 & 20 & 0.37 & 29 & 98.42 & & & 0.38 & 99.28 \\
\hline 1252 & 0.20 & 0.00 & 0.04 & 0.00 & 0.219 & 0.000 & -0.031 & -0.008 & -1.67 & -0.02 & .70 & 103.08 & & & -0.00 & 104.03 \\
\hline $62 \quad 253$ & 0.20 & 0.00 & 0.04 & 0.00 & 0.219 & 0.000 & -0.031 & -0.008 & -1.54 & 0.08 & 1863.95 & 106.90 & & & 0.10 & 107.93 \\
\hline 54 & 0.18 & 0.00 & 00 & 0.00 & 96 & 0.000 & -0.034 & -0.007 & -1.55 & 0.02 & 82 & 112.10 & & & 0.04 & 113.21 \\
\hline $4 \quad 255$ & 0.17 & 0.00 & 0.04 & 0.00 & & 0.000 & -0.036 & -0.007 & & 0.32 & & 116.33 & & & 0.35 & 117.53 \\
\hline $52 \quad 256$ & 0.16 & 0.00 & 0.03 & 0.00 & 0.174 & 0.000 & -0.025 & -0.005 & -1.05 & 0.10 & 1873.50 & 121.56 & & & .12 & 122.83 \\
\hline $66 \quad 257$ & 0.16 & 0.00 & 0.0 & 0.0 & & 0.000 & -0.025 & -0.005 & & 0.41 & & 125.99 & & & 42 & 127.36 \\
\hline $57 \quad 258$ & 0.13 & 0.00 & 0.0 & 0.01 & 0.140 & 0.000 & -0.004 & -0.011 & -0.71 & 0.24 & 187 & 131.46 & & & .25 & 132.92 \\
\hline 59 & 0.12 & 0.00 & 0.00 & 0.01 & & 00 & 0.007 & -0.010 & & .07 & & 135.61 & & & .08 & 137.17 \\
\hline 59260 & -0.17 & 0.00 & 0.01 & 0.00 & -0.176 & 0.000 & -0.000 & 0.001 & & -0.51 & 5.50 & 140.85 & & & & 142.49 \\
\hline $70 \quad 261$ & -0.17 & 0.00 & 0.01 & -0.01 & -0.176 & 0.000 & 0.001 & 0.010 & -1.97 & -0.77 & 1890.31 & 145.11 & & & -0.76 & 146.88 \\
\hline 262 & -0.17 & 0.00 & 0.01 & -0.01 & -0.176 & 0.000 & 0.001 & 0.010 & & -1.34 & 2.94 & 150.55 & & & & 152.43 \\
\hline $72 \quad 263$ & -0.13 & 0.00 & -0.01 & -0.01 & -0.135 & 0.000 & 0.019 & 0.008 & -2.35 & -1.30 & 1896.28 & 155.28 & & & -1.29 & 157.29 \\
\hline 64 & -0.13 & 0.00 & 0.00 & -0.01 & -0.135 & 0.000 & 0.007 & 0.009 & -2.69 & -1.75 & 62 & 161.02 & & & & 163.13 \\
\hline 265 & -0.12 & 0.00 & -0.01 & -0.01 & -0.125 & 0.000 & 0.018 & 0.008 & -2.64 & -1.80 & 1901.85 & 165.85 & & & -1.78 & 168.10 \\
\hline
\end{tabular}




\section{$Z=91(\mathrm{~Pa})$}

$\begin{array}{llll}175 & 266 & -0.10 & 0.00\end{array}$ $\begin{array}{llll}176 & 267 & -0.10 & 0.00\end{array}$ $\begin{array}{llll}177 & 268 & -0.10 & 0.00\end{array}$

$\begin{array}{lll}0.00 & -0.01 & -0.104\end{array}$

$\begin{array}{llll}0.01 & 0.00 & -0.105\end{array}$

$\begin{array}{llll}0.02 & 0.00 & -0.105\end{array}$

$\begin{array}{llll}0.02 & -0.01 & -0.094\end{array}$

$\begin{array}{llll}178 & 269 & -0.09 & 0.00\end{array}$

$\begin{array}{llll}179 & 270 & -0.08 & 0.00\end{array}$

$\begin{array}{llll}180 & 271 & -0.04 & 0.00\end{array}$

$\begin{array}{llll}0.02 & 0.00 & -0.084\end{array}$

$\begin{array}{llll}0.01 & 0.00 & -0.042\end{array}$

$\begin{array}{llll}181 & 272 & -0.03 & 0.00\end{array}$

$\begin{array}{llll}182 & 273 & 0.01 & 0.00\end{array}$

$\begin{array}{llll}183 & 274 & 0.01 & 0.00\end{array}$

$\begin{array}{lllll}184 & 275 & 0.00 & 0.00\end{array}$

$\begin{array}{llllll}185 & 276 & -0.01 & 0.00 & 0.0\end{array}$

$\begin{array}{lllll}186 & 277 & 0.00 & 0.00 & 0.00\end{array}$

$\begin{array}{lllll}187 & 278 & 0.03 & 0.08 & -0.01\end{array}$

$\begin{array}{llllll}188 & 279 & 0.05 & 0.09 & -0.02\end{array}$

189280

$\begin{array}{lll}0.06 & 0.10 & -0.03\end{array}$

190281

191282

192283

193284

194285

195286

196287

$197 \quad 288$

198289

199290

$\begin{array}{lll}0.06 & 0.11 & -0.03\end{array}$

$\begin{array}{lll}0.06 & 0.12 & -0.02\end{array}$

$0.00-0.032$

$\begin{array}{lll}0.00 & 0.00 & 0.011\end{array}$

$\begin{array}{lll}0.00 & 0.00 \quad 0.011\end{array}$

0.00

$0.00 \quad 0.000$

$\begin{array}{ll}0.000 \quad 0.004 \\ 0.000 & -0.008\end{array}$

$\begin{array}{lllll}0.009 & -2.99 & -2.42 & 1904.18 & 171.59\end{array}$

$0.000-0.008$ $\begin{array}{llllll}0.001 & -3.04 & -2.49 & 1907.27 & 176.58\end{array}$ $0.000-0.019$ $\begin{array}{lllll}0.002 & -3.68 & -3.08 & 1909.39 & 182.52\end{array}$ $0.000-0.020$ $\begin{array}{lllll}0.012 & -3.62 & -3.04 & 1912.20 & 187.79\end{array}$ $0.000-0.021$ $\begin{array}{lllll}0.002 & -3.85 & -3.24 & 1913.76 & 194.30\end{array}$

$0.000-0.011$ $\begin{array}{lllll}0.001 & -3.52 & -2.88 & 1916.08 & 200.06\end{array}$ $0.000-0.011$ $0.000 \quad 0.000$ $\begin{array}{lllll}0.000 & -4.08 & -3.37 & 1917.76 & 206.44\end{array}$ $\begin{array}{llllll}0.000 & -4.07 & -3.32 & 1920.22 & 212.06\end{array}$ $0.000 \quad 0.000$ $\begin{array}{lllll}0.000 & -4.58 & -3.80 & 1921.73 & 218.62\end{array}$ $0.000 \quad 0.000$ $\begin{array}{lllll}0.000 & -4.34 & -3.58 & 1923.85 & 224.57\end{array}$ $\begin{array}{llllllllll}0.00 & -0.011 & 0.000 & 0.000 & 0.000 & -3.41 & -2.70 & 1923.85 & 232.64\end{array}$ $\begin{array}{llllllllll}0.00 & 0.000 & 0.000 & 0.000 & 0.000 & -2.71 & -2.02 & 1925.35 & 239.21\end{array}$ $\begin{array}{lllllllll}0.00 & 0.035 & -0.109 & 0.015 & 0.005 & -3.22 & -1.47 & 1925.54 & 247.10\end{array}$ $\begin{array}{llllllllll}0.00 & 0.056 & -0.123 & 0.028 & 0.007 & -3.14 & -1.02 & 1927.10 & 253.60\end{array}$ $\begin{array}{lllllllll}0.00 & 0.068 & -0.138 & 0.041 & 0.010 & -3.85 & -1.36 & 1928.02 & 260.76\end{array}$ $\begin{array}{lllllllll}0.01 & 0.069 & -0.151 & 0.042 & 0.001 & -3.86 & -1.11 & 1929.63 & 267.22\end{array}$ $\begin{array}{lllllllll}0.02 & 0.069 & -0.163 & 0.031 & -0.009 & -4.21 & -1.25 & 1930.21 & 274.71\end{array}$ $\begin{array}{llllllllllll}0.06 & 0.13 & -0.02 & 0.02 & 0.070 & -0.177 & 0.032 & -0.007 & -4.17 & -0.85 & 1931.51 & 281.48\end{array}$ $\begin{array}{llllllllllll}0.37 & 0.00 & 0.09 & -0.01 & 0.419 & 0.000 & -0.049 & -0.022 & -4.00 & -0.61 & 1931.56 & 289.50\end{array}$ $\begin{array}{llllllllllll}0.37 & 0.00 & 0.09 & -0.01 & 0.419 & 0.000 & -0.049 & -0.022 & -3.80 & -0.48 & 1932.99 & 296.14\end{array}$ $\begin{array}{llllllllllll}0.37 & 0.00 & 0.09 & -0.01 & 0.419 & 0.000 & -0.049 & -0.022 & -3.99 & -0.68 & 1933.33 & 303.88\end{array}$ $\begin{array}{lllllllllllll}0.37 & 0.00 & 0.10 & -0.02 & 0.420 & 0.000 & -0.063 & -0.018 & -4.02 & -0.51 & 1934.58 & 310.69\end{array}$ $\begin{array}{lllllllllllll}0.37 & 0.00 & 0.10 & -0.02 & 0.420 & 0.000 & -0.063 & -0.018 & -4.10 & -0.60 & 1934.68 & 318.67\end{array}$ $\begin{array}{lllllllllllll}0.37 & 0.00 & 0.10 & -0.02 & 0.420 & 0.000 & -0.063 & -0.018 & -3.78 & -0.24 & 1935.59 & 325.83\end{array}$ $\begin{array}{lllllllllllll}0.38 & 0.00 & 0.12 & -0.03 & 0.434 & 0.000 & -0.085 & -0.018 & -4.67 & -0.42 & 1935.63 & 333.86\end{array}$ 200291 201292 202293 203294 204295 $0.38 \quad 0.00$ $0.12-0.03$ $\begin{array}{llll}0.28 & 0.00 & 0.02 & 0.02\end{array}$

0.434 $\begin{array}{lllllll}0.000 & -0.085 & -0.018 & -4.42 & -0.35 & 1936.70 & 340.86\end{array}$ $\begin{array}{lll}0.28 & 0.00 & 0.02\end{array}$ $\begin{array}{lll}0.17 & 0.00 & -0.08\end{array}$ $\begin{array}{lll}0.18 & 0.01 & -0.07\end{array}$

0.02 0.308 0.308

0.000

$\begin{array}{llllll}0.013 & -0.023 & -2.13 & -0.35 & 1936.43 & 349.20\end{array}$

$\begin{array}{lllllllll}0.01 & 0.184 & 0.000 & 0.113 & 0.010 & -3.68 & -0.95 & 1937.62 & 364.15\end{array}$

205296

206297

$\begin{array}{lll}0.18 & 0.01 & -0.07\end{array}$

0.02

$0.193-0.014$

$0.102-0$.

$-0.002$

3.68

$\begin{array}{llllll}0.102 & -0.002 & -3.66 & -1.30 & 1938.31 & 379.61\end{array}$

$\begin{array}{lll}0.02 & 0.193 & -0.014\end{array}$

$\begin{array}{llllll}0.102 & -0.002 & -3.61 & -1.29 & 1939.03 & 386.96\end{array}$

207298

$0.19-0.01-0.06$

$\begin{array}{lll}0.02 & 0.193 & 0.000\end{array}$$$
\begin{array}{lllllll}
0.091 & -0.014 & -3.79 & -1.58 & 1938.66 & 395.40
\end{array}
$$

208299

$\begin{array}{lll}0.19 & 0.00 & -0.06\end{array}$

0.03

$0.204-0.014$

$\begin{array}{lllllll}0.091 & -0.014 & -3.71 & -1.56 & 1939.24 & 402.89\end{array}$

209300

$\begin{array}{lll}0.19 & 0.00 & -0.05\end{array}$

0.03

0.204

0.000

$\begin{array}{lllllll}0.079 & -0.016 & -3.75 & -1.85 & 1938.75 & 411.46\end{array}$

$\begin{array}{lllll}210 & 301 & 0.20 & 0.00 & -0.05\end{array}$

$0.04 \quad 0.215$

211302

$\begin{array}{lll}0.20 & 0.00 & -0.05 \\ 0.20 & 0.00 & -0.04\end{array}$

0.0

\section{$Z=92(\mathrm{U})$}

$\begin{array}{lllll}112 & 204 & 0.17 & 0.00 & -0.02\end{array}$

0.000

$\begin{array}{llllll}0.082 & -0.026 & -3.85 & -2.01 & 1939.38 & 418.89\end{array}$

0.000
$-2.41 \quad 173.97$

$\begin{array}{ll}-2.50 & 179.07\end{array}$

$-3.07 \quad 185.17$

$-3.01 \quad 190.60$

$-3.22197 .24$

$-2.88 \quad 203.13$

$-3.37 \quad 209.66$

$-3.33 \quad 215.43$

$\begin{array}{ll}-3.80 & 222.15\end{array}$

$-3.58 \quad 228.27$

$-2.70 \quad 236.51$

$\begin{array}{ll}-2.02 & 243.25\end{array}$

$-1.38 \quad 251.42$

$-0.88 \quad 258.15$

$-1.18 \quad 265.54$

$\begin{array}{ll}-0.89 & 272.23\end{array}$

$-1.00 \quad 279.95$

$\begin{array}{ll}-0.56 \quad 286.95 \\ -0.61 & 294.90\end{array}$

$-0.61 \quad 294.90$

$-0.46 \quad 301.76$

$\begin{array}{ll}-0.68 & 309.69\end{array}$

$-0.43 \quad 316.80$

$-0.55 \quad 324.98$

$-0.16 \quad 332.38$

$-0.22 \quad 340.77$

$\begin{array}{ll}-0.12 & 348.04\end{array}$

$-0.36 \quad 356.38$

$-0.23 \quad 363.83$

$-0.56 \quad 372.22$

$-0.56 \quad 379.68$

$-0.98 \quad 388.12$

$-0.95 \quad 395.75$

$-1.24 \quad 404.46$

$-1.21 \quad 412.23$

$-1.57 \quad 421.00$

$-1.55 \quad 428.89$

$-1.71438 .00$ $\begin{array}{lllll}111 & 203 & 0.19 & 0.00 & -0.01\end{array}$

113205

114206

$\begin{array}{lll}0.17 & 0.00 & -0.01\end{array}$

$\begin{array}{llll}0.16 & 0.00 & -0.01\end{array}$

$0.01 \quad 0.183$

$0.01 \quad 0.183$

$0.01 \quad 0.172$

115207

$\begin{array}{lll}0.15 & 0.00 & 0.00\end{array}$

0.00

0.162

0.000

0.000

0.028

0.000

0.037

0.000

0.025

0.000

0.009

$0.000-0.003$

$\begin{array}{llll}116 & 208 & 0.15 & 0.00\end{array}$

$\begin{array}{ll}0.01-0.01 \\ 0.02 & -0.01\end{array}$

0.162

$\begin{array}{rr}0.000 & -0.003 \\ -0.027 & -0.016\end{array}$

$\begin{array}{rrrrr}0.05 & 0.00 & -0.207 & 0.000 & -0.040\end{array}$

$\begin{array}{llll}118 & 210 & -0.20 & 0.00\end{array}$

$\begin{array}{llll}0.01 & -0.01 & -0.156\end{array}$

$0.000-0.002$

$0.000-0.003$

$\begin{array}{llll}120 & 212 & -0.14 & 0.00\end{array}$

$\begin{array}{llll}0.01 & -0.01 & -0.146\end{array}$

$0.000-0.005$

$\begin{array}{ll}0.000 & -0.007\end{array}$

$0.000-0.020$

$\begin{array}{llll}122 & 214 & -0.11 & 0.00\end{array}$

$0.01-0.00-0.115$

0.02

$0.00-0.094$

$0.01-0.073$

$0.000-0.021$

$0.000 \quad 0.000$

$\begin{array}{llll}125 & 217 & 0.00 & 0.00\end{array}$

0.00

$0.00 \quad 0.000$

0.000

0.000

$\begin{array}{llll}0.00 & 0.022 & -0.054 & 0.013\end{array}$

$\begin{array}{lll}0.00 & 0.056 & -0.110\end{array}$

0.027

0.042
$-0.006$

$-0.005$

$-0.16$

$\begin{array}{ll}1.06 & 1517.95\end{array}$

$\begin{array}{ll}1.38 & 1529.38\end{array}$

0.20

$\begin{array}{ll}1.24 & 1539.11\end{array}$

$\begin{array}{ll}1.24 & 1539.11 \\ 1.25 & 1550.48\end{array}$

$-0.007$

0.06

$0.94 \quad 1560.00$

$0.009-0.23$

$\begin{array}{ll}0.87 & 1571.09\end{array}$

$0.007-0.59$

$\begin{array}{ll}0.55 & 1580.29\end{array}$

$\begin{array}{llll}0.010 & -1.71 & 0.15 & 1591.36\end{array}$

$\begin{array}{llll}0.011 & -1.52 & -0.42 & 1600.46\end{array}$

$\begin{array}{lllll}0.011 & -1.91 & -0.79 & 1611.16\end{array}$

$\begin{array}{lllll}0.011 & -2.24 & -1.34 & 1619.91\end{array}$

$\begin{array}{lllll}0.001 & -2.41 & -1.67 & 1630.22\end{array}$

$\begin{array}{llll}0.002 & -3.01 & -2.35 & 1638.78\end{array}$

$\begin{array}{llll}-0.008 & -3.35 & -2.60 & 1648.69\end{array}$

$\begin{array}{lllll}0.000 & -3.82 & -3.01 & 1656.66\end{array}$

$\begin{array}{lllll}0.000 & -4.04 & -3.18 & 1666.17\end{array}$

$\begin{array}{llll}0.001 & -3.41 & -2.29 & 1672.54\end{array}$

$\begin{array}{llll}0.006 & -3.63 & -1.51 & 1680.79\end{array}$

$\begin{array}{lllll}0.009 & -4.02 & -1.31 & 1687.55\end{array}$
48.55

45.19

43.54

40.24

38.78

35.76

34.64

31.65

30.61

27.99

27.31

25.06

24.58

22.74

22.84

21.40

23.10

22.93

24.24 


\section{$Z=92(\mathrm{U})$}

\section{2}

$\begin{array}{llll}0.10 & 0.10 & -0.04\end{array}$

$\begin{array}{llllll}132 & 224 & 0.12 & 0.10 & -0.05\end{array}$

$\begin{array}{llllll}133 & 225 & 0.13 & 0.10 & -0.06\end{array}$

$\begin{array}{lllll}134 & 226 & 0.13 & 0.10 & -0.06\end{array}$

$135 \quad 227$

$\begin{array}{lll}0.14 & 0.10 & -0.06\end{array}$

136228

$\begin{array}{ll}137 & 229\end{array}$

$\begin{array}{llll}0.17 & 0.00 & -0.09\end{array}$

$\begin{array}{lll}0.17 & 0.00 & -0.09\end{array}$

$\begin{array}{llll}0.17 & 0.00 & -0.09\end{array}$

139231

$\begin{array}{llll}0.18 & 0.00 & -0.08\end{array}$

$140 \quad 232$

$\begin{array}{lll}0.19 & 0.00 & -0.08\end{array}$

141233

$\begin{array}{llll}0.19 & 0.00 & -0.08\end{array}$

$142 \quad 234$

$\begin{array}{llll}0.20 & 0.00 & -0.07\end{array}$

$\begin{array}{lll}0.20 & 0.00 & -0.07\end{array}$

144236

$\begin{array}{llll}0.21 & 0.00 & -0.07\end{array}$

$\begin{array}{ll}145 & 237\end{array}$

$\begin{array}{lll}0.21 & 0.00 & -0.06\end{array}$

146238

$\begin{array}{llll}0.22 & 0.00 & -0.06\end{array}$

$147 \quad 239$

$\begin{array}{llll}0.22 & 0.00 & -0.05\end{array}$

$\begin{array}{llll}0.22 & 0.00 & -0.05\end{array}$

$\begin{array}{ll}148 & 240 \\ 149 & 241\end{array}$

$\begin{array}{llll}0.22 & 0.00 & -0.04\end{array}$

$150 \quad 242$

$\begin{array}{llll}0.22 & 0.00 & -0.03\end{array}$

151243

$\begin{array}{llll}0.23 & 0.00 & -0.02\end{array}$

$152 \quad 244$

$\begin{array}{llll}0.23 & 0.00 & -0.01\end{array}$

153245

$\begin{array}{llll}0.22 & 0.00 & -0.01\end{array}$

154246

$\begin{array}{llll}0.22 & 0.00 & -0.01\end{array}$

155247

$\begin{array}{ll}0.22 & 0.00\end{array}$

0.00

$\begin{array}{ll}0.22 & 0.00\end{array}$

0.00

157249

$0.22 \quad 0.00$

0.01

$\begin{array}{ll}0.21 & 0.00\end{array}$

0.01

159251

$0.20 \quad 0.00$

0.02

$160 \quad 252$

$\begin{array}{ll}0.20 & 0.00\end{array}$

0.03

$\begin{array}{ll}161 & 253\end{array}$

$0.20 \quad 0.00$

0.04

$\begin{array}{ll}0.20 & 0.00\end{array}$

0.04

163255

$\begin{array}{ll}0.19 & 0.00\end{array}$

0.04

164256

$0.18 \quad 0.00$

0.04

$\begin{array}{ll}0.16 & 0.00\end{array}$

0.03

166258

$0.16 \quad 0.00$

0.03

$167 \quad 259$

$0.16 \quad 0.00$

0.02

$0.12 \quad 0.00$

0.01

0.120 .00

0.00

$\begin{array}{lll}0.01 & 0.100 & -0.138\end{array}$

$\begin{array}{llll}0.01 & 0.110 & -0.138\end{array}$

$\begin{array}{llll}0.01 & 0.132 & -0.139\end{array}$

0.056

$\begin{array}{lllll}0.002 & -4.18 & -0.87 & 1695.82 & 24.03\end{array}$

$\begin{array}{llllll}0.056 & 0.003 & -4.25 & -0.87 & 1702.49 & 25.44\end{array}$

$\begin{array}{lll}0.01 & 0.143 & -0.139\end{array}$

0.070

$\begin{array}{llll}0.006 & -4.33 & -0.52 & 1710.56\end{array}$

0.084

$\begin{array}{lllll}0.009 & -4.93 & -0.63 & 1717.06\end{array}$

$\begin{array}{llll}0.01 & 0.143 & -0.139\end{array}$

0.084

$\begin{array}{llll}0.009 & -4.67 & -0.38 & 1724.94\end{array}$

0.02

$0.153-0.138$

0.00

$0.186 \quad 0.000$

$\begin{array}{lllll}0.085 & -0.001 & -4.81 & -0.48 & 1731.15\end{array}$

0.126

$\begin{array}{llll}0.035 & -4.90 & -0.25 & 1738.76\end{array}$

0.00

$0.185 \quad 0.000$

0.126

$\begin{array}{llll}0.024 & -5.00 & -0.54 & 1744.90\end{array}$

$\begin{array}{lll}0.00 & 0.195 \quad 0.000\end{array}$

0.126

$\begin{array}{llll}0.024 & -4.95 & -0.50 & 1752.42\end{array}$

0.114

$\begin{array}{lllll}0.022 & -4.77 & -0.78 & 1758.28\end{array}$

$\begin{array}{lll}0.01 & 0.206 & 0.000\end{array}$

0.116

$\begin{array}{lllll}0.013 & -4.74 & -0.78 & 1765.57\end{array}$

$\begin{array}{lll}0.01 & 0.206 & 0.000\end{array}$

$\begin{array}{lll}0.02 & 0.215 & 0.000\end{array}$

0.116

$\begin{array}{lllll}0.013 & -5.09 & -1.10 & 1771.21\end{array}$

0.106

$\begin{array}{llll}0.001 & -4.58 & -1.12 & 1778.27\end{array}$

$\begin{array}{lll}0.02 & 0.215 & 0.000\end{array}$

0.106

$\begin{array}{llll}0.001 & -4.84 & -1.37 & 1783.57\end{array}$

0.000

0.108

$\begin{array}{llll}-0.009 & -4.83 & -1.24 & 1790.23\end{array}$

$\begin{array}{lll}0.03 & 0.226 & 0.000\end{array}$

$\begin{array}{lll}0.04 & 0.236 & 0.000\end{array}$

$\begin{array}{lllll}0.095 & -0.012 & -4.65 & -1.45 & 1795.25\end{array}$

$\begin{array}{lll}0.04 & 0.237 & 0.000\end{array}$

$\begin{array}{lllll}0.098 & -0.021 & -4.70 & -1.31 & 1801.64\end{array}$

$\begin{array}{lllll}0.086 & -0.024 & -4.65 & -1.64 & 1806.54\end{array}$

$\begin{array}{lll}0.04 & 0.237 & 0.000 \\ 0.04 & 0.237 & 0.000\end{array}$

$\begin{array}{lllll}0.086 & -0.024 & -4.42 & -1.44 & 1812.62\end{array}$

$\begin{array}{lllll}0.073 & -0.027 & -4.42 & -1.70 & 1817.23\end{array}$

$\begin{array}{lll}0.03 & 0.237 & 0.000\end{array}$

$\begin{array}{lllll}0.060 & -0.020 & -3.77 & -1.45 & 1823.01\end{array}$

$\begin{array}{lll}0.03 & 0.249 & 0.000\end{array}$

$\begin{array}{lllll}0.050 & -0.022 & -3.95 & -1.70 & 1827.38\end{array}$

$0.03 \quad 0.250$

0.000

$\begin{array}{lllll}0.038 & -0.025 & -3.52 & -1.43 & 1832.90\end{array}$

$0.03 \quad 0.238$

$0.03 \quad 0.238$

0.000

$\begin{array}{lllll}0.036 & -0.025 & -3.30 & -1.32 & 1836.68\end{array}$

$\begin{array}{lllll}0.036 & -0.025 & -2.79 & -0.89 & 1841.82\end{array}$

0.000

$\begin{array}{lllll}0.023 & -0.018 & -2.36 & -0.65 & 1845.26\end{array}$

0.000

$\begin{array}{lllll}0.023 & -0.018 & -1.89 & -0.19 & 1850.14\end{array}$

$\begin{array}{lllll}0.010 & -0.011 & -1.75 & -0.15 & 1853.55\end{array}$

0.000

$\begin{array}{lllll}0.008 & -0.011 & -1.40 & 0.13 & 1858.39\end{array}$

$\begin{array}{lllllll}0.000 & -0.006 & -0.013 & -1.52 & 0.00 & 1861.77\end{array}$

$0.00 \quad 0.218$

$\begin{array}{lllllll}0.000 & -0.019 & -0.006 & -1.40 & 0.03 & 1866.65 & 95.34\end{array}$

$0.00 \quad 0.219$

$\begin{array}{lllllll}0.000 & -0.031 & -0.008 & -1.85 & -0.22 & 1869.94 & 100.12\end{array}$

0.00

0.219

$\begin{array}{lllllll}0.000 & -0.031 & -0.008 & -1.72 & -0.09 & 1874.51 & 103.62\end{array}$

$\begin{array}{lllllll}0.000 & -0.033 & -0.008 & -1.72 & -0.18 & 1877.44 & 108.77\end{array}$

$\begin{array}{lllllll}0.000 & -0.034 & -0.007 & -1.26 & 0.25 & 1881.50 & 112.78\end{array}$

$0.00 \quad 0.196$

$\begin{array}{llll}0.000 & -0.025 & -0.005 & -1.05\end{array}$

$\begin{array}{lll}0.13 & 1884.27 & 118.08\end{array}$

$0.00 \quad 0.174$

$\begin{array}{llll}0.000 & -0.025 & -0.005 & -0.79\end{array}$

$\begin{array}{lll}0.41 & 1888.28 & 122.14\end{array}$

$0.00 \quad 0.173$

$\begin{array}{llll}0.000 & -0.013 & -0.003 & -0.84\end{array}$

$\begin{array}{lll}0.22 & 1890.92 & 127.57\end{array}$

169261

$0.01 \quad 0.129$

$\begin{array}{lllllll}0.000 & -0.005 & -0.011 & -0.60 & 0.22 & 1895.01 & 131.55\end{array}$

$\begin{array}{lllllll}0.000 & 0.007 & -0.010 & -1.07 & -0.26 & 1897.76 & 136.88\end{array}$

$\begin{array}{lllllll}170 & 262 & -0.17 & 0.00 & 0.01 & -0.01 & -0.176\end{array}$

0.000

0.001

$\begin{array}{lllll}0.010 & -1.61 & -0.46 & 1901.86 & 140.85\end{array}$

$\begin{array}{llll}171 & 263 & -0.17 & 0.00\end{array}$

$\begin{array}{llll}0.01 & -0.01 & -0.176\end{array}$

0.000

0.001

$\begin{array}{llllll}0.010 & -2.26 & -1.01 & 1904.49 & 146.29\end{array}$

$\begin{array}{llllllll}172 & 264 & -0.17 & 0.00 & 0.01 & -0.01 & -0.176\end{array}$

0.000

0.001

$\begin{array}{lllll}0.010 & -2.33 & -1.04 & 1908.23 & 150.62\end{array}$

$\begin{array}{lllllll}173 & 265 & -0.12 & 0.00 & -0.01 & -0.01 & -0.125\end{array}$

$0.000 \quad 0.018$

$\begin{array}{lllll}0.008 & -2.36 & -1.49 & 1910.58 & 156.34\end{array}$

$0.000 \quad 0.018$

$\begin{array}{lllll}0.008 & -2.29 & -1.46 & 1914.08 & 160.91\end{array}$

$\begin{array}{llll}175 & 267 & -0.10 & 0.00\end{array}$

$\begin{array}{llll}176 & 268 & -0.10 & 0.00\end{array}$

$0.00-0.01-0.104$

$0.000 \quad 0.004$

$\begin{array}{llllll}0.009 & -2.61 & -2.04 & 1916.38 & 166.68\end{array}$

$0.000-0.008$

$\begin{array}{lllll}0.001 & -2.69 & -2.16 & 1919.85 & 171.29\end{array}$

$\begin{array}{llll}177 & 269 & -0.10 & 0.00\end{array}$

$0.00-0.105$

$0.000-0.019$

$\begin{array}{lllll}0.002 & -3.27 & -2.68 & 1921.92 & 177.29\end{array}$

$\begin{array}{llll}0.02 & 0.00 & -0.105\end{array}$

$0.000-0.020$

$\begin{array}{lllll}0.012 & -3.21 & -2.63 & 1925.04 & 182.24\end{array}$

$\begin{array}{llll}178 & 270 & -0.09 & 0.00 \\ 179 & 271 & -0.08 & 0.00\end{array}$

$\begin{array}{llll}0.02 & -0.01 & -0.094\end{array}$

$0.000-0.021$

$\begin{array}{llllll}0.002 & -3.41 & -2.81 & 1926.60 & 188.75\end{array}$

$\begin{array}{llll}180 & 272 & -0.04 & 0.00\end{array}$

$\begin{array}{llll}0.01 & 0.00 & -0.042\end{array}$

$0.000-0.011$

$\begin{array}{lllll}0.001 & -3.06 & -2.44 & 1929.23 & 194.20\end{array}$

$\begin{array}{llll}181 & 273 & 0.00 & 0.00\end{array}$

$\begin{array}{llll}182 & 274 & 0.00 & 0.00\end{array}$

$\begin{array}{lll}0.00 & 0.00 & 0.000\end{array}$

$0.000 \quad 0.000$

$\begin{array}{llllll}0.000 & -3.54 & -2.84 & 1930.84 & 200.65\end{array}$

$\begin{array}{llll}183 & 275 & 0.00 & 0.00\end{array}$

$\begin{array}{lll}0.00 & 0.00 & 0.000\end{array}$

$0.000 \quad 0.000$

$\begin{array}{llllll}0.000 & -3.59 & -2.87 & 1933.70 & 205.87\end{array}$

$\begin{array}{llll}184 & 276 & 0.00 & 0.00\end{array}$

0.00

$0.00 \quad 0.000$

0.000

0.000

$\begin{array}{lllll}0.000 & -4.06 & -3.32 & 1935.19 & 212.44\end{array}$

$\begin{array}{llll}185 & 277 & -0.01 & 0.00\end{array}$

$\begin{array}{llll}186 & 278 & 0.00 & 0.0\end{array}$ $\begin{array}{llll}0.00 & 0.00 & -0.011 & 0.000\end{array}$

$\begin{array}{llll}0.00 & 0.00 & 0.000 & -0.013\end{array}$
0.000

$\begin{array}{lllll}0.000 & -3.83 & -3.09 & 1937.62 & 218.08\end{array}$

$\begin{array}{lllllll}0.000 & 0.000 & -2.85 & -2.16 & 1937.59 & 226.19\end{array}$

$\begin{array}{lllllll}0.000 & 0.000 & -2.19 & -1.51 & 1939.44 & 232.41\end{array}$

$\begin{array}{lll}-0.81 & 24.49\end{array}$

$\begin{array}{llll}25.84 & 0.071 & -0.81 & 25.85\end{array}$

$\begin{array}{llll}25.71 & 0.025 & -0.43 & 25.86\end{array}$

$\begin{array}{llll}27.38 & 0.012 & -0.54 & 27.41\end{array}$

$\begin{array}{llll}27.33 & 0.013 & -0.27 & 27.60\end{array}$

$\begin{array}{llll}29.02 & 0.017 & -0.37 & 29.44\end{array}$

$\begin{array}{llll}29.23 & 0.015 & -0.06 & 29.96\end{array}$

$\begin{array}{llll}31.21 & 0.006 & -0.39 & 31.85\end{array}$

$\begin{array}{llll}31.61 & 0.005 & -0.33 & 32.40\end{array}$

$\begin{array}{llll}33.81 & 0.003 & -0.64 & 34.58\end{array}$

$\begin{array}{lllll}34.61 & 0.002 & -0.63 & 35.35\end{array}$

$\begin{array}{llll}36.92 & 0.003 & -0.96 & 37.78\end{array}$

$\begin{array}{llll}38.15 & 0.002 & -1.00 & 38.79\end{array}$

$\begin{array}{llll}40.92 & 0.002 & -1.25 & 41.55\end{array}$

$\begin{array}{lllll}42.45 & 0.002 & -1.08 & 43.02\end{array}$

$\begin{array}{lllll}45.39 & 0.002 & -1.33 & 46.04\end{array}$

$\begin{array}{llll}47.31 & 0.002 & -1.13 & 47.80\end{array}$

$\begin{array}{llll}50.57 & 0.002 & -1.48 & 50.96\end{array}$

$\begin{array}{llll}52.72 & 0.005 & -1.26 & 52.99\end{array}$

$-1.55 \quad 56.46$

$-1.35 \quad 58.72$

$\begin{array}{ll}-1.62 & 62.44\end{array}$

$-1.34 \quad 65.03$

$-1.23 \quad 69.36$

$-0.79 \quad 72.35$

$-0.62 \quad 76.96$

$-0.14 \quad 80.21$

$-0.15 \quad 84.88$

$0.14 \quad 88.17$

$0.01 \quad 92.93$

$0.04 \quad 96.18$

$-0.20 \quad 101.05$

$-0.06 \quad 104.62$ 


\section{$Z=92(\mathrm{U})$}

$\begin{array}{lllll}187 & 279 & 0.03 & 0.08 & -0.01\end{array}$ $\begin{array}{lllll}188 & 280 & 0.05 & 0.09 & -0.02\end{array}$ $\begin{array}{llllll}189 & 281 & 0.06 & 0.10 & -0.03\end{array}$

$\begin{array}{lllllllll}0.00 & 0.035 & -0.109 & 0.015 & 0.005 & -2.77 & -1.04 & 1939.71 & 240.21\end{array}$ $\begin{array}{lllllllll}0.00 & 0.056 & -0.123 & 0.028 & 0.007 & -2.71 & -0.74 & 1941.74 & 246.25\end{array}$ $\begin{array}{lllll}190 & 282 & 0.06 & 0.11 & -0.02\end{array}$ $\begin{array}{lllll}191 & 283 & 0.06 & 0.12 & -0.02\end{array}$

$\begin{array}{llll}0.01 & 0.068 & -0.137\end{array}$

$0.01 \quad 0.068-0.150$

$\begin{array}{llllll}0.041 & -0.000 & -3.34 & -0.94 & 1942.53 & 253.54\end{array}$ $\begin{array}{llllll}0.030 & 0.000 & -3.25 & -0.66 & 1944.43 & 259.71\end{array}$

192284 193285

194286

195287

196288

$\begin{array}{lll}0.38 & 0.00 & 0.09\end{array}$

0.02

$0.069-0.163$

$\begin{array}{llllll}0.031 & -0.009 & -3.79 & -0.82 & 1945.02 & 267.18\end{array}$

$\begin{array}{llll}0.37 & 0.00 & 0.09 & 0.00\end{array}$

$\begin{array}{llll}0.37 & 0.00 & 0.09 & -0.01\end{array}$

0.431

0.000

$\begin{array}{lllllll}-0.043 & -0.032 & -3.68 & -0.26 & 1946.49 & 273.79\end{array}$

0.419

$\begin{array}{lllllll}0.000 & -0.047 & -0.032 & -3.88 & -0.51 & 1947.03 & 281.33\end{array}$

$\begin{array}{llll}0.37 & 0.00 & 0.10 & -0.01\end{array}$

$\begin{array}{ll}0.37 & 0.00\end{array}$

$0.10-0.01$

$\begin{array}{lllllll}0.000 & -0.049 & -0.022 & -3.51 & -0.27 & 1948.67 & 287.75\end{array}$

0.420

197289

198290

$\begin{array}{lllll}0.37 & 0.00 & 0.10 & -0.02\end{array}$

0.420

$0.000-0.060-0.027$

199291

200292

201293

$\begin{array}{ll}0.37 & 0.00\end{array}$

$\begin{array}{ll}0.11 & -0.02\end{array}$

$0.37 \quad 0.00$
0.27

$\begin{array}{ll}0.11 & -0.02\end{array}$

$\begin{array}{ll}0.27 & 0.00\end{array}$

$\begin{array}{ll}0.01 & 0.02\end{array}$

$0.27 \quad 0.00$

0.01

0.02

0.421

$\begin{array}{lllllll}0.000 & -0.063 & -0.018 & -3.80 & -0.24 & 1950.51 & 310.12\end{array}$

0.421$$
0.000
$$

$\begin{array}{lllllll}0.000 & -0.075 & -0.022 & -3.95 & -0.17 & 1952.02 & 316.69\end{array}$

$\begin{array}{llllllll}0.296 & 0.000 & 0.022 & -0.019 & -1.79 & -0.09 & 1953.25 & 331.60\end{array}$

20229

203295

204296

205297

206298

$\begin{array}{lll}0.27 & 0.00 & 0.01\end{array}$

0.02

0.296

0.000

0.022

0.000

$\begin{array}{lllllll}0.022 & -0.019 & -1.92 & -0.31 & 1954.50 & 346.49\end{array}$ $\begin{array}{lll}0.19 & 0.00 & -0.07\end{array}$

0.02

0.000$$
0.1
$$

$\begin{array}{lll}0.18 & 0.00-0.07\end{array}$

0.02

0.205

0.000

$\begin{array}{llllll}0.104 & -0.001 & -3.35 & -0.92 & 1955.87 & 361.26\end{array}$

$\begin{array}{lll}0.18 & 0.00 & -0.07 \\ 0.19 & 0.00 & -0.07\end{array}$

0.03

0.204

0.000

$\begin{array}{llllll}0.102 & -0.002 & -3.64 & -1.27 & 1955.69 & 369.52\end{array}$

207299

$\begin{array}{lll}0.19 & 0.00 & -0.06\end{array}$

0.03

0.204

0.000

$\begin{array}{llllll}0.104 & -0.011 & -3.76 & -1.34 & 1956.80 & 376.48\end{array}$

208300

$\begin{array}{lll}0.19 & 0.00 & -0.06\end{array}$

0.03

0.204

0.000

$\begin{array}{lllllll}0.091 & -0.014 & -3.82 & -1.68 & 1956.47 & 384.88\end{array}$

209301

$\begin{array}{lll}0.20 & 0.00 & -0.05\end{array}$

0.04

0.215

0.000

$\begin{array}{lllllll}0.091 & -0.014 & -3.76 & -1.58 & 1957.27 & 392.15\end{array}$

210302

$\begin{array}{lll}0.20 & 0.00 & -0.05\end{array}$

0.04

211303

$\begin{array}{lll}0.20 & 0.00 & -0.04\end{array}$

0.04

0.215

0.000

$\begin{array}{lllllll}0.082 & -0.026 & -4.05 & -2.15 & 1957.05 & 400.44\end{array}$

$\begin{array}{lllllll}0.082 & -0.026 & -3.92 & -2.05 & 1957.72 & 407.84\end{array}$

$0.04 \quad 0.215$

0.000

$\begin{array}{llllll}0.069 & -0.028 & -3.88 & -2.18 & 1956.94 & 416.69\end{array}$

213305

0.04
0.000

0.000 $\begin{array}{lllllll}0.069 & -0.028 & -3.61 & -1.96 & 1957.37 & 424.34\end{array}$

$\begin{array}{llllll}0.057 & -0.031 & -3.57 & -2.08 & 1956.46 & 433.32\end{array}$
$-0.95 \quad 244.34$

$-0.60 \quad 250.60$

$-0.75 \quad 258.11$

$-0.46 \quad 264.48$

$\begin{array}{ll}-0.56 & 272.20\end{array}$

$-0.15 \quad 278.85$

$-0.41 \quad 286.57$

$-0.22 \quad 293.16$

$-0.44 \quad 301.07$

$-0.20 \quad 307.86$

$-0.16 \quad 316.19$

$\begin{array}{ll}0.03 & 323.08\end{array}$

$-0.24 \quad 331.24$

$-0.09 \quad 338.25$

$-0.41 \quad 346.50$

$-0.31 \quad 353.61$

$-0.54 \quad 362.09$

$\begin{array}{ll}-0.59 \quad 369.20 \\ -0.94 & 377.70\end{array}$

$-0.94 \quad 377.70$

$-0.92 \quad 385.01$

$\begin{array}{ll}-1.34 & 393.59\end{array}$

$-1.22 \quad 401.14$

$-1.70 \quad 409.78$

$-1.59 \quad 417.47$

$-1.76 \quad 426.55$

$\begin{array}{rr}-1.53 & 434.49\end{array}$

$-1.68 \quad 443.73$

\section{$Z=93(\mathbf{N p})$}

$\begin{array}{lllll}113 & 206 & 0.17 & 0.00 & -0.01\end{array}$

$\begin{array}{lllll}114 & 207 & 0.17 & 0.00 & -0.01\end{array}$

$\begin{array}{lllllllll}0.01 & 0.183 & 0.000 & 0.025 & -0.007 & -0.07 & 1.10 & 1536.87 & 53.06\end{array}$ 115208

116209

$\begin{array}{rrr}0.17 & 0.00 & -0.01 \\ 0.16 & 0.00 & 0.00\end{array}$

$0.01 \quad 0.183$

53.06

$0.00 \quad 0.172$

0.000

$\begin{array}{lll}0.025 & -0.007 & -0.04\end{array}$

$1.14 \quad 1548.26$

$\begin{array}{lllll}0.000 & 0.011 & 0.001 & -0.24\end{array}$

$\begin{array}{ll}0.90 & 1558.16\end{array}$

47.92

$117 \quad 210$

0.150 .00

$0.00 \quad 0.00$

0.162

$\begin{array}{ll}0.000 & 0.009\end{array}$

$0.001-0.20$

$\begin{array}{ll}0.87 & 1569.26\end{array}$

$0.000-0.003$

$0.009-0.50$

$\begin{array}{ll}0.60 & 1578.83\end{array}$

44.89

118211

$\begin{array}{ll}0.14 & 0.00\end{array}$

$\begin{array}{llll}0.02 & -0.01 \quad 0.151\end{array}$

$0.000-0.016$

$\begin{array}{lllll}0.007 & -0.66 & 0.30 & 1589.84\end{array}$

$0.000-0.011$

$\begin{array}{llll}0.012 & -1.43 & -0.18 & 1599.27\end{array}$

43.39

$\begin{array}{llll}119 & 212 & -0.17 & 0.00\end{array}$

$\begin{array}{llll}0.02 & -0.01 & -0.177\end{array}$

$0.000-0.002$

$\begin{array}{llll}0.020 & -1.76 & -0.47 & 1609.92\end{array}$

$\begin{array}{llll}121 & 213 & -0.15 & 0.00 \\ 121 & 214 & -0.13 & 0.00\end{array}$

$0.01-0.02-0.156$

$0.000-0.004$

$\begin{array}{llll}0.011 & -1.94 & -0.94 & 1619.02\end{array}$

$0.000-0.007$

$\begin{array}{llll}0.001 & -2.01 & -1.28 & 1629.38\end{array}$

39.09

$\begin{array}{llll}122 & 215 & -0.11 & 0.00\end{array}$

$\begin{array}{lll}0.01 & 0.00 & -0.115\end{array}$

$0.000-0.020$

$\begin{array}{lllll}0.002 & -2.54 & -1.90 & 1638.30\end{array}$

$\begin{array}{lll}123 & 216 & 217\end{array}$

$\begin{array}{lll}0.09 & 0.00 \quad 0.0\end{array}$

$0.00-0.094$

$0.000-0.021$

$\begin{array}{llll}-0.008 & -2.78 & -2.06 & 1648.16\end{array}$

125218

$0.00-0.01-0.00$

$0.01-0.073$

0.000

$\begin{array}{lllll}0.000 & -3.14 & -2.36 & 1656.43\end{array}$

$\begin{array}{llll}0.00 & 0.000 & -0.013\end{array}$

0.000

$\begin{array}{llll}0.000 & -3.35 & -2.53 & 1665.98\end{array}$

$\begin{array}{ll}126 & 219 \\ 127 & 220\end{array}$

$\begin{array}{lll}0.00 & 0.01 & 0.00\end{array}$

0.00

$0.000-0.013$

0.014

$\begin{array}{llll}0.003 & -3.28 & -1.83 & 1672.95\end{array}$

0.00

$0.033-0.082$

0.027

$\begin{array}{llll}0.006 & -3.24 & -1.17 & 1681.35\end{array}$

$\begin{array}{ll}128 & 221 \\ 129 & 222\end{array}$

$\begin{array}{llll}0.05 & 0.08 & -0.02\end{array}$

0.00

$0.056-0.110$

0.042

$\begin{array}{lllll}0.009 & -3.68 & -1.02 & 1688.56\end{array}$

$\begin{array}{lll}0.01 & 0.100 & -0.138\end{array}$

0.043

$\begin{array}{llll}0.001 & -3.63 & -0.61 & 1696.91\end{array}$

$\begin{array}{ll}131 & 224\end{array}$

$\begin{array}{lll}0.09 & 0.10 & -0.03\end{array}$

0.01

$0.112-0.152$

0.045

$\begin{array}{llll}0.003 & -4.10 & -0.57 & 1703.94\end{array}$

$\begin{array}{llll}0.01 & 0.132 & -0.139\end{array}$

0.070

$\begin{array}{llll}0.006 & -4.00 & -0.22 & 1712.05\end{array}$

$\begin{array}{llllll}0.084 & 0.007 & -4.23 & -0.38 & 1718.99\end{array}$

$\begin{array}{lll}0.01 & 0.142 & -0.125\end{array}$

$\begin{array}{llllll}0.085 & -0.001 & -4.45 & -0.19 & 1726.97\end{array}$

36.51

133226

$\begin{array}{lll}0.13 & 0.09 & -0.06\end{array}$

0.02

$0.153-0.138$

0.085

0.000

$\begin{array}{lll}0.14 & 0.10 & -0.06 \\ 0.15 & 0.10 & -0.06\end{array}$

0.02

$0.164-0.138$

$\begin{array}{ll}136 & 229\end{array}$

$\begin{array}{llll}0.17 & 0.00 & -0.09\end{array}$

$\begin{array}{lll}0.00 & 0.185 & 0.000\end{array}$

0.126

$\begin{array}{llll}-4.73 & -0.35 & 1733.63\end{array}$

$137 \quad 230$

$\begin{array}{llll}0.17 & 0.00 & -0.09\end{array}$

$\begin{array}{lll}0.00 & 0.185 & 0.000\end{array}$

0.126

$\begin{array}{llll}0.024 & -4.86 & -0.42 & 1741.58\end{array}$

$\begin{array}{lll}0.00 & 0.196 & 0.000\end{array}$ 


\section{$Z=93$ (Np)}

142235

$\begin{array}{llll}0.20 & 0.00 & -0.08\end{array}$

$\begin{array}{llllll}144 & 237 & 0.21 & 0.00 & -0.07\end{array}$

$\begin{array}{llllll}145 & 238 & 0.21 & 0.00 & -0.06\end{array}$

$\begin{array}{lllll}146 & 239 & 0.22 & 0.00 & -0.06\end{array}$

$\begin{array}{llllll}147 & 240 & 0.22 & 0.00 & -0.05\end{array}$

$\begin{array}{llllll}148 & 241 & 0.22 & 0.00 & -0.05\end{array}$

$\begin{array}{llllll}149 & 242 & 0.22 & 0.00 & -0.04\end{array}$

$\begin{array}{llllll}150 & 243 & 0.22 & 0.00 & -0.03\end{array}$

$\begin{array}{llllll}151 & 244 & 0.23 & 0.00 & -0.02\end{array}$

$\begin{array}{llllll}152 & 245 & 0.23 & 0.00 & -0.01\end{array}$

$\begin{array}{llllll}153 & 246 & 0.23 & 0.00 & -0.01\end{array}$

$154 \quad 247$

155248

$\begin{array}{llll}0.22 & 0.00 & -0.01\end{array}$

$\begin{array}{lll}0.22 & 0.00 & 0.00\end{array}$

$\begin{array}{lll}0.22 & 0.00 & 0.00\end{array}$

$0.02 \quad 0.216$

0.000

$\begin{array}{lllll}0.119 & 0.004 & -5.56 & -1.59 & 1782.64\end{array}$

$0.03 \quad 0.226$

$0.03 \quad 0.226$

0.000

$0.03 \quad 0.226$

$0.04 \quad 0.236$

0.000

$\begin{array}{lllll}0.108 & -0.009 & -5.44 & -1.81 & 1788.30\end{array}$

0.000

$\begin{array}{llllll}0.108 & -0.009 & -5.31 & -1.71 & 1795.01\end{array}$

$\begin{array}{lllll}0.095 & -0.012 & -5.14 & -1.92 & 1800.41\end{array}$

$\begin{array}{llllll}0.098 & -0.021 & -5.20 & -1.79 & 1806.84\end{array}$

$\begin{array}{ll}0.04 & 0.237\end{array}$

$0.04 \quad 0.237$

$0.04 \quad 0.237$

0.000

$\begin{array}{llllll}0.086 & -0.024 & -5.12 & -2.09 & 1812.09\end{array}$

0.000

$\begin{array}{lllll}0.086 & -0.024 & -4.89 & -1.89 & 1818.20\end{array}$

0.000

$\begin{array}{llllll}0.073 & -0.027 & -4.89 & -2.15 & 1823.17\end{array}$

$0.03 \quad 0.237$

0.000

$\begin{array}{llllll}0.060 & -0.020 & -4.24 & -1.90 & 1828.99\end{array}$

0.000

$\begin{array}{llllll}0.051 & -0.032 & -4.64 & -2.21 & 1833.77\end{array}$

$0.03 \quad 0.250$

0.000

$\begin{array}{llllll}0.038 & -0.025 & -3.98 & -1.87 & 1839.26\end{array}$

$0.03 \quad 0.250$

$\begin{array}{ll}0.03 & 0.238\end{array}$

$0.02 \quad 0.239$

0.000

$\begin{array}{llllll}0.038 & -0.025 & -3.84 & -1.76 & 1843.41\end{array}$

0.000

$\begin{array}{llllll}0.036 & -0.025 & -3.23 & -1.31 & 1848.54\end{array}$

$\begin{array}{llllll}0.023 & -0.018 & -2.76 & -1.08 & 1852.35\end{array}$

0.000

0.023

$0.02 \quad 0.240$

0.000

$\begin{array}{lllll}0.011 & -0.021 & -2.24 & -0.52 & 1860.98\end{array}$

0.000

$\begin{array}{lllll}0.008 & -0.011 & -1.72 & -0.18 & 1865.78\end{array}$

$\begin{array}{llllll}0.000 & -0.004 & -0.013 & -1.89 & -0.25 & 1869.45 \\ 0.000 & -0.018 & -0.006 & -1.76 & -0.15 & 1874.28\end{array}$

$\begin{array}{lllllll}0.000 & -0.030 & -0.009 & -2.18 & -0.47 & 1877.99 & 99.37\end{array}$

$\begin{array}{ll}162 & 255 \\ 163 & 256\end{array}$

\section{7}

\section{8}

$0.20 \quad 0.00$

0.04

$0.00 \quad 0.219$

$\begin{array}{lllllll}0.000 & -0.031 & -0.008 & -1.96 & -0.32 & 1882.57 & 102.86\end{array}$

$\begin{array}{lllllll}0.000 & -0.033 & -0.008 & -1.94 & -0.39 & 1885.82 & 107.68\end{array}$

$\begin{array}{llllllll}0.000 & -0.034 & -0.007 & -1.44 & 0.09 & 1889.86 & 111.71\end{array}$

$\begin{array}{lllllll}0.000 & -0.024 & -0.005 & -1.26 & -0.00 & 1892.94 & 116.70\end{array}$

$\begin{array}{lllllll}0.000 & -0.013 & -0.003 & -0.79 & 0.22 & 1897.04 & 120.68\end{array}$

$\begin{array}{llllllllllllll}167 & 260 & 0.16 & 0.00 & 0.02 & 0.00 & 0.173 & 0.000 & -0.013 & -0.003 & -0.97 & 0.11 & 1899.94 & 125.85\end{array}$

$\begin{array}{lllllllllllllll}168 & 261 & 0.13 & 0.00 & 0.01 & 0.01 & 0.140 & 0.000 & -0.004 & -0.011 & -0.65 & 0.21 & 1903.95 & 129.91\end{array}$

$\begin{array}{llllllllllllll}169 & 262 & 0.12 & 0.00 & 0.00 & 0.01 & 0.129 & 0.000 & 0.007 & -0.010 & -1.03 & -0.23 & 1907.00 & 134.93\end{array}$

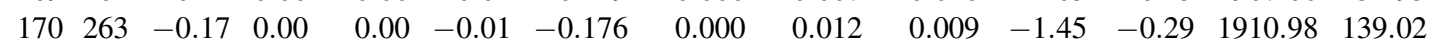

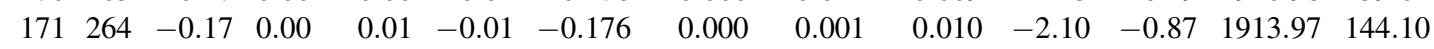

$\begin{array}{llllllllllllll}172 & 265 & -0.17 & 0.00 & 0.01 & -0.01 & -0.176 & 0.000 & 0.001 & 0.010 & -2.16 & -0.89 & 1917.72 & 148.42\end{array}$

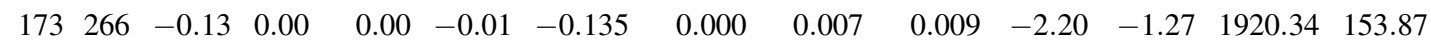

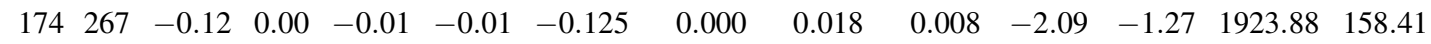

$\begin{array}{llllllllllllll}175 & 268 & -0.11 & 0.00 & 0.00 & -0.01 & -0.115 & 0.000 & 0.005 & 0.009 & -2.43 & -1.78 & 1926.44 & 163.91\end{array}$

$\begin{array}{llllllllllllll}176 & 269 & -0.10 & 0.00 & 0.01 & -0.01 & -0.105 & 0.000 & -0.007 & 0.011 & -2.48 & -1.94 & 1929.97 & 168.46\end{array}$

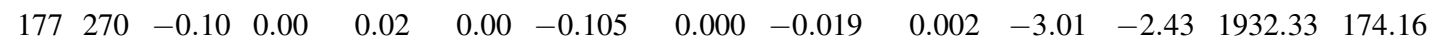

$\begin{array}{llllllllllllll}178 & 271 & -0.10 & 0.00 & 0.02 & -0.01 & -0.105 & 0.000 & -0.019 & 0.012 & -2.97 & -2.38 & 1935.47 & 179.10\end{array}$

$\begin{array}{llllllllllllll}179 & 272 & -0.08 & 0.00 & 0.02 & 0.00 & -0.084 & 0.000 & -0.021 & 0.002 & -3.07 & -2.49 & 1937.29 & 185.35\end{array}$

$\begin{array}{llllllllllllll}180 & 273 & -0.07 & 0.00 & 0.02 & 0.00 & -0.073 & 0.000 & -0.021 & 0.002 & -2.80 & -2.22 & 1940.03 & 190.68\end{array}$

$\begin{array}{llllllllllllll}181 & 274 & -0.04 & 0.00 & 0.01 & 0.00 & -0.042 & 0.000 & -0.011 & 0.001 & -3.10 & -2.46 & 1941.81 & 196.98\end{array}$

$\begin{array}{llllllllllllll}182 & 275 & 0.00 & 0.01 & 0.00 & 0.00 & 0.000 & -0.013 & 0.000 & 0.000 & -3.01 & -2.32 & 1944.50 & 202.35\end{array}$

$\begin{array}{llllllllllllll}183 & 276 & 0.00 & 0.01 & 0.00 & 0.00 & 0.000 & -0.013 & 0.000 & 0.000 & -3.47 & -2.75 & 1946.31 & 208.62\end{array}$

$\begin{array}{llllllllllllll}184 & 277 & 0.00 & 0.01 & 0.00 & 0.00 & 0.000 & -0.013 & 0.000 & 0.000 & -3.22 & -2.51 & 1948.74 & 214.26\end{array}$

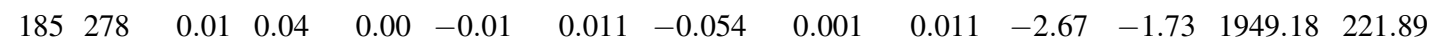

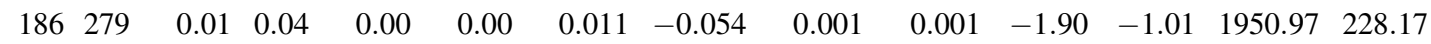

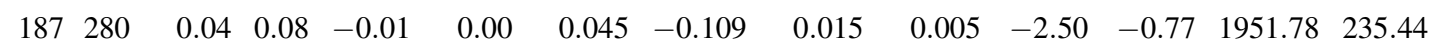

$\begin{array}{llllllllllllll}188 & 281 & 0.05 & 0.09 & -0.02 & 0.00 & 0.056 & -0.123 & 0.028 & 0.007 & -2.51 & -0.53 & 1953.88 & 241.40\end{array}$

$\begin{array}{llllllllllllll}189 & 282 & 0.06 & 0.11 & -0.02 & 0.01 & 0.068 & -0.150 & 0.030 & 0.000 & -3.41 & -0.77 & 1955.02 & 248.33\end{array}$

$\begin{array}{llllllllllllll}190 & 283 & 0.38 & 0.00 & 0.09 & 0.00 & 0.431 & 0.000 & -0.043 & -0.032 & -3.77 & -0.33 & 1956.77 & 254.65\end{array}$

$\begin{array}{llllllllllllll}191 & 284 & 0.38 & 0.00 & 0.09 & 0.00 & 0.431 & 0.000 & -0.043 & -0.032 & -4.01 & -0.53 & 1957.72 & 261.78\end{array}$

$\begin{array}{lllllllllllllll}192 & 285 & 0.37 & 0.00 & 0.09 & 0.00 & 0.419 & 0.000 & -0.047 & -0.032 & -3.79 & -0.43 & 1959.65 & 267.92\end{array}$

$\begin{array}{llllllllllllll}193 & 286 & 0.37 & 0.00 & 0.09 & 0.00 & 0.419 & 0.000 & -0.047 & -0.032 & -4.03 & -0.67 & 1960.49 & 275.15\end{array}$

$\begin{array}{llllllllllllll}194 & 287 & 0.37 & 0.00 & 0.09 & -0.01 & 0.419 & 0.000 & -0.049 & -0.022 & -3.64 & -0.42 & 1962.12 & 281.58\end{array}$

$\begin{array}{llllllllllllll}195 & 288 & 0.37 & 0.00 & 0.10 & -0.01 & 0.420 & 0.000 & -0.060 & -0.027 & -4.24 & -0.71 & 1962.87 & 288.91\end{array}$

$\begin{array}{llllllllllllll}196 & 289 & 0.37 & 0.00 & 0.10 & -0.01 & 0.420 & 0.000 & -0.060 & -0.027 & -3.94 & -0.33 & 1964.22 & 295.63\end{array}$

$\begin{array}{llllllllllllll}197 & 290 & 0.37 & 0.00 & 0.10 & -0.01 & 0.420 & 0.000 & -0.060 & -0.027 & -4.07 & -0.49 & 1964.69 & 303.24\end{array}$

$\begin{array}{llllllllllllll}198 & 291 & 0.36 & 0.00 & 0.10 & -0.01 & 0.408 & 0.000 & -0.064 & -0.026 & -3.94 & -0.47 & 1966.26 & 309.74\end{array}$ $\begin{array}{llll}41.04 & 0.002 & -1.46 & 41.79\end{array}$ $\begin{array}{llll}43.38 & 0.050 & -1.70 & 44.18\end{array}$

$\begin{array}{llll}44.87 & 0.002 & -1.58 & 45.57\end{array}$

$\begin{array}{llll}47.46 & 0.002 & -1.82 & 48.21\end{array}$

$\begin{array}{llll}49.31 & 0.002 & -1.63 & 49.92\end{array}$

$\begin{array}{llll}52.31 & 0.015 & -1.96 & 52.73\end{array}$

$\begin{array}{lllll}54.26 & 0.071 & -1.74 & 54.73\end{array}$

$\begin{array}{lllll}57.42 & 0.200 & -2.02 & 57.83\end{array}$

$\begin{array}{ll}-1.83 & 60.06\end{array}$

$-2.08 \quad 63.42$

$-1.80 \quad 65.97$

$-1.70 \quad 69.93$

$-1.23 \quad 72.91$

$-1.06 \quad 77.16$

$-0.59 \quad 80.38$

$-0.50 \quad 84.77$

$-0.18 \quad 88.07$

$-0.25 \quad 92.52$

$-0.15 \quad 95.82$

$\begin{array}{ll}-0.46 & 100.25\end{array}$

$-0.30 \quad 103.82$

$-0.37 \quad 108.71$

$0.11 \quad 112.82$

$0.00 \quad 117.87$

$0.21 \quad 121.92$

$\begin{array}{ll}0.10 & 127.17\end{array}$

$0.22 \quad 131.34$

$\begin{array}{ll}-0.23 & 136.45\end{array}$

$-0.29 \quad 140.63$

$-0.87 \quad 145.81$

$\begin{array}{ll}-0.88 & 150.24\end{array}$

$-1.27 \quad 155.80$

$-1.25160 .45$

$-1.77 \quad 166.07$

$-1.93 \quad 170.74$

$-2.42 \quad 176.56$

$-2.35 \quad 181.65$

$-2.48 \quad 188.01$

$-2.20 \quad 193.48$

$-2.46199 .90$

$\begin{array}{ll}-2.32 \quad 205.42 \\ -2.75 & 211.83\end{array}$

$-2.75 \quad 211.83$

$-2.51 \quad 217.62$

$-1.69 \quad 225.46$

$-0.99 \quad 231.88$

$-0.68 \quad 239.37$

$-0.40 \quad 245.54$

$-0.60 \quad 252.70$

$-0.25 \quad 259.10$
-0.48

$-0.48 \quad 266.37$

$\begin{array}{ll}-0.33 & 272.74\end{array}$

$-0.60 \quad 280.14$

$-0.39 \quad 286.73$

$-0.63 \quad 294.31$

$-0.23 \quad 301.26$

$\begin{array}{ll}-0.41 \quad 309.04 \\ -0.35 & 315.80\end{array}$

$-0.35 \quad 315.80$ 


\section{$Z=93$ (Np)}

\begin{tabular}{|c|c|c|c|c|c|c|c|c|c|c|c|c|c|c|c|c|c|}
\hline 199 & 292 & 0.27 & 0.00 & 0.00 & 0.02 & 0.295 & 0.000 & 0.034 & -0.016 & -2.27 & -0.50 & 1966.46 & 317.61 & & & -0.53 & 323.73 \\
\hline 200 & 293 & 0.27 & 0.00 & 0.01 & 0.02 & 0.296 & 0.000 & 0.022 & -0.019 & -2.17 & -0.49 & 1967.89 & 324.25 & & & -0.49 & 330.62 \\
\hline 201 & 294 & 0.27 & 0.00 & 0.01 & 0.02 & 0.296 & 0.000 & 0.022 & -0.019 & -2.44 & -0.75 & 1968.19 & 332.02 & & & -0.77 & 338.60 \\
\hline 202 & 295 & 0.27 & 0.00 & 0.01 & 0.02 & 0.296 & 0.000 & 0.022 & -0.019 & -2.31 & -0.71 & 1969.44 & 338.84 & & & -0.72 & 345.65 \\
\hline 203 & 296 & 0.19 & 0.00 & -0.07 & 0.02 & 0.205 & 0.000 & 0.104 & -0.001 & -3.70 & -1.14 & 1969.78 & 346.57 & & & -0.85 & 353.92 \\
\hline 204 & 297 & 0.19 & 0.00 & -0.07 & 0.02 & 0.205 & 0.000 & 0.104 & -0.001 & -3.68 & -1.22 & 1971.02 & 353.40 & & & -0.92 & 361.00 \\
\hline 205 & 298 & 0.20 & 0.00 & -0.06 & 0.03 & 0.215 & 0.000 & 0.093 & -0.013 & -3.79 & -1.56 & 1971.12 & 361.37 & & & -1.25 & 369.21 \\
\hline 206 & 299 & 0.19 & 0.00 & -0.06 & 0.03 & 0.204 & 0.000 & 0.091 & -0.014 & -3.73 & -1.58 & 1972.18 & 368.38 & & & -1.25 & 376.50 \\
\hline 207 & 300 & 0.19 & 0.00 & -0.06 & 0.03 & 0.204 & 0.000 & 0.091 & -0.014 & -4.16 & -2.00 & 1972.23 & 376.41 & & & -1.67 & 384.77 \\
\hline 208 & 301 & 0.20 & 0.00 & -0.05 & 0.03 & 0.215 & 0.000 & 0.081 & -0.016 & -3.86 & -1.93 & 1973.06 & 383.64 & & & -1.67 & 392.20 \\
\hline 209 & 302 & 0.20 & 0.00 & -0.05 & 0.04 & 0.215 & 0.000 & 0.082 & -0.026 & -4.42 & -2.46 & 1973.10 & 391.68 & & & -2.04 & 400.65 \\
\hline 210 & 303 & 0.20 & 0.00 & -0.05 & 0.04 & 0.215 & 0.000 & 0.082 & -0.026 & -4.29 & -2.37 & 1973.78 & 399.07 & & & -1.93 & 408.32 \\
\hline 211 & 304 & 0.20 & 0.00 & -0.04 & 0.04 & 0.215 & 0.000 & 0.069 & -0.028 & -4.26 & -2.54 & 1973.33 & 407.59 & & & -2.15 & 417.07 \\
\hline 212 & 305 & 0.20 & 0.00 & -0.04 & 0.04 & 0.215 & 0.000 & 0.069 & -0.028 & -3.98 & -2.29 & 1973.72 & 415.27 & & & -1.88 & 425.04 \\
\hline 213 & 306 & 0.20 & 0.00 & -0.03 & 0.04 & 0.215 & 0.000 & 0.057 & -0.031 & -3.93 & -2.39 & 1973.09 & 423.98 & & & -2.02 & 433.99 \\
\hline 214 & 307 & 0.20 & 0.00 & -0.03 & 0.04 & 0.215 & 0.000 & 0.057 & -0.031 & -3.64 & -2.14 & 1973.35 & 431.78 & & & -1.76 & 442.09 \\
\hline 215 & 308 & 0.20 & 0.00 & -0.02 & 0.04 & 0.216 & 0.000 & 0.045 & -0.034 & -3.73 & -2.35 & 1972.70 & 440.51 & & & -1.98 & 451.09 \\
\hline \multicolumn{18}{|c|}{$Z=94(\mathrm{Pu})$} \\
\hline 115 & 209 & 0.17 & 0.00 & 0.00 & 0.00 & 0.183 & 0.000 & 0.012 & 0.001 & -0.22 & 0.75 & 1557.73 & 55.63 & & & 0.70 & 57.23 \\
\hline 116 & 210 & 0.16 & 0.00 & 0.01 & 0.00 & 0.173 & 0.000 & -0.001 & -0.001 & -0.14 & 0.90 & 1569.07 & 52.36 & & & 0.87 & 53.89 \\
\hline 117 & 211 & 0.15 & 0.00 & 0.01 & 0.00 & 0.162 & 0.000 & -0.003 & -0.001 & -0.49 & 0.56 & 1578.75 & 50.75 & & & 0.53 & 52.20 \\
\hline 118 & 212 & 0.15 & 0.00 & 0.02 & -0.01 & 0.162 & 0.000 & -0.015 & 0.007 & -0.67 & 0.44 & 1590.01 & 47.57 & & & 0.42 & 48.95 \\
\hline 119 & 213 & 0.13 & 0.01 & 0.02 & -0.01 & 0.140 & -0.014 & -0.018 & 0.007 & -0.92 & 0.16 & 1599.30 & 46.35 & & & 0.13 & 47.65 \\
\hline 120 & 214 & -0.15 & 0.00 & 0.02 & -0.01 & -0.156 & 0.000 & -0.014 & 0.012 & -1.45 & -0.23 & 1610.47 & 43.25 & & & -0.26 & 44.48 \\
\hline 121 & 215 & -0.13 & 0.00 & 0.01 & -0.01 & -0.135 & 0.000 & -0.004 & 0.011 & -1.65 & -0.69 & 1619.59 & 42.21 & & & -0.70 & 43.37 \\
\hline 122 & 216 & -0.11 & 0.00 & 0.01 & 0.00 & -0.115 & 0.000 & -0.007 & 0.001 & -1.70 & -1.00 & 1630.34 & 39.52 & & & -1.01 & 40.62 \\
\hline 123 & 217 & -0.09 & 0.00 & 0.02 & 0.01 & -0.094 & 0.000 & -0.020 & -0.007 & -2.24 & -1.60 & 1639.28 & 38.66 & & & -1.61 & 39.70 \\
\hline 124 & 218 & -0.07 & 0.00 & 0.02 & 0.01 & -0.073 & 0.000 & -0.021 & -0.008 & -2.47 & -1.76 & 1649.55 & 36.46 & & & -1.76 & 37.44 \\
\hline 125 & 219 & 0.00 & 0.00 & 0.00 & 0.00 & 0.000 & 0.000 & 0.000 & 0.000 & -2.81 & -2.07 & 1657.88 & 36.19 & & & -2.08 & 37.13 \\
\hline 126 & 220 & 0.00 & 0.00 & 0.00 & 0.00 & 0.000 & 0.000 & 0.000 & 0.000 & -3.00 & -2.22 & 1667.81 & 34.33 & & & -2.23 & 35.21 \\
\hline 127 & 221 & 0.03 & 0.06 & -0.01 & 0.00 & 0.033 & -0.082 & 0.014 & 0.003 & -2.94 & -1.52 & 1674.83 & 35.40 & & & -1.52 & 36.22 \\
\hline 128 & 222 & 0.05 & 0.08 & -0.02 & 0.00 & 0.056 & -0.110 & 0.027 & 0.006 & -2.87 & -0.84 & 1683.60 & 34.69 & & & -0.82 & 35.48 \\
\hline 129 & 223 & 0.07 & 0.09 & -0.02 & 0.00 & 0.078 & -0.124 & 0.029 & 0.008 & -2.99 & -0.60 & 1690.78 & 35.58 & & & -0.59 & 36.33 \\
\hline 130 & 224 & 0.09 & 0.10 & -0.03 & 0.01 & 0.100 & -0.138 & 0.043 & 0.001 & -3.13 & -0.18 & 1699.51 & 34.92 & & & -0.14 & 35.65 \\
\hline 131 & 225 & 0.10 & 0.11 & -0.03 & 0.01 & 0.112 & -0.152 & 0.045 & 0.003 & -3.57 & -0.11 & 1706.55 & 35.95 & & & -0.07 & 36.63 \\
\hline 132 & 226 & 0.12 & 0.10 & -0.05 & 0.01 & 0.132 & -0.139 & 0.070 & 0.006 & -3.49 & 0.21 & 1715.08 & 35.49 & & & 0.28 & 36.17 \\
\hline 133 & 227 & 0.13 & 0.10 & -0.05 & 0.01 & 0.143 & -0.139 & 0.072 & 0.007 & -3.75 & 0.03 & 1722.08 & 36.56 & & & 0.10 & 37.20 \\
\hline 134 & 228 & 0.14 & 0.10 & -0.05 & 0.01 & 0.154 & -0.139 & 0.073 & 0.008 & -3.62 & 0.27 & 1730.41 & 36.31 & 36.09 & 0.032 & 0.35 & 36.94 \\
\hline 135 & 229 & 0.18 & 0.00 & -0.08 & 0.00 & 0.195 & 0.000 & 0.114 & 0.022 & -3.86 & 0.01 & .21 & 37.58 & 37.40 & 0.051 & 0.10 & 38.19 \\
\hline 136 & 230 & 0.18 & 0.00 & -0.08 & 0.00 & 0.195 & 0.000 & 0.114 & 0.022 & -4.03 & -0.13 & 1745.62 & 37.24 & 36.93 & 0.015 & -0.02 & 37.84 \\
\hline 137 & 231 & 0.18 & 0.00 & -0.08 & 0.00 & 0.195 & 0.000 & 0.114 & 0.022 & -4.43 & -0.50 & 1752.25 & 38.69 & 38.28 & 0.026 & -0.39 & 39.25 \\
\hline 138 & 232 & 0.19 & 0.00 & -0.08 & 0.01 & 0.206 & 0.000 & 0.116 & 0.013 & -4.46 & -0.56 & 1760.30 & 38.71 & 38.37 & 0.018 & -0.44 & 39.26 \\
\hline 139 & 233 & 0.19 & 0.00 & -0.08 & 0.01 & 0.206 & 0.000 & 0.116 & 0.013 & -4.90 & -0.96 & 1766.70 & 40.38 & 40.05 & 0.050 & -0.86 & 40.90 \\
\hline 140 & 234 & 0.20 & 0.00 & -0.08 & 0.02 & 0.216 & 0.000 & 0.119 & 0.004 & -5.01 & -1.12 & .57 & 40.58 & 40 & 0.007 & -1.00 & 41.11 \\
\hline 141 & 235 & 0.20 & 0.00 & -0.07 & 0.02 & 0.215 & 0.000 & 0.106 & 0.001 & -4.89 & -1.46 & 1780.64 & 42.58 & 42.18 & 0.021 & -1.37 & 43.06 \\
\hline 142 & 236 & 0.20 & 0.00 & -0.07 & 0.02 & 0.215 & 0.000 & 0.106 & 0.001 & -4.97 & -1.54 & 1788.18 & 43.12 & 42.90 & 0.002 & -1.44 & 43.61 \\
\hline 143 & 237 & 0.21 & 0.00 & -0.07 & 0.03 & 0.226 & 0.000 & 0.108 & -0.009 & -5.43 & -1.85 & 1793.95 & 45.41 & 45.09 & 0.002 & -1.73 & 45.91 \\
\hline 144 & 238 & 0.21 & 0.00 & -0.06 & 0.03 & 0.226 & 0.000 & 0.095 & -0.012 & -4.90 & -1.75 & 1801.04 & 46.39 & 46.17 & 0.002 & -1.63 & 46.89 \\
\hline 145 & 239 & 0.22 & 0.00 & -0.06 & 0.04 & 0.236 & 0.000 & 0.098 & -0.021 & -5.44 & -2.06 & 1806.57 & 48.93 & 48.59 & 0.002 & -1.91 & 49.46 \\
\hline 146 & 240 & 0.22 & 0.00 & -0.05 & 0.04 & 0.237 & 0.000 & 0.086 & -0.024 & -4.95 & -1.97 & 1813.42 & 50.15 & 50.13 & 0.002 & -1.83 & 50.69 \\
\hline 147 & 241 & 0.22 & 0.00 & -0.05 & 0.04 & 0.237 & 0.000 & 0.086 & -0.024 & -5.26 & -2.26 & 1818.69 & 52.96 & 52.96 & 0.002 & -2.12 & 53.50 \\
\hline 148 & 242 & 0.22 & 0.00 & -0.04 & 0.04 & 0.237 & 0.000 & 0.073 & -0.027 & -4.78 & -2.07 & 1825.17 & 54.54 & 54.72 & 0.002 & -1.93 & 55.10 \\
\hline 149 & 243 & 0.22 & 0.00 & -0.04 & 0.04 & 0.237 & 0.000 & 0.073 & -0.027 & -5.07 & -2.35 & 1830.20 & 57.59 & 57.76 & 0.003 & -2.22 & 58.15 \\
\hline 150 & 244 & 0.22 & 0.00 & -0.03 & 0.04 & 0.237 & 0.000 & 0.061 & -0.030 & -4.67 & -2.19 & 1836.47 & 59.39 & 59.81 & 0.005 & -2.05 & 59.98 \\
\hline 151 & 245 & 0.23 & 0.00 & -0.02 & 0.04 & 0.249 & 0.000 & 0.051 & -0.032 & -4.88 & -2.47 & 1841.26 & 62.67 & 63.11 & 0.014 & -2.34 & 63.27 \\
\hline 152 & 246 & 0.23 & 0.00 & -0.01 & 0.03 & 0.250 & 0.000 & 0.038 & -0.025 & -4.23 & -2.15 & 1847.13 & 64.87 & 65.39 & 0.015 & -2.07 & 65.44 \\
\hline 153 & 247 & 0.22 & 0.00 & -0.01 & 0.03 & 0.238 & 0.000 & 0.036 & -0.025 & -4.02 & -2.04 & 1851.30 & 68.78 & & & -1.97 & 69.37 \\
\hline
\end{tabular}

\begin{tabular}{|c|c|c|c|c|c|c|c|c|c|c|c|c|c|c|c|c|c|}
\hline 199 & 292 & 0.27 & 0.00 & 0.00 & 0.02 & 0.295 & 0.000 & 0.034 & -0.016 & -2.27 & -0.50 & 1966.46 & 317.61 & & & -0.53 & 323.73 \\
\hline 200 & 293 & 0.27 & 0.00 & 0.01 & 0.02 & 0.296 & 0.000 & 0.022 & -0.019 & -2.17 & -0.49 & 1967.89 & 324.25 & & & -0.49 & 330.62 \\
\hline 201 & 294 & 0.27 & 0.00 & 0.01 & 0.02 & 0.296 & 0.000 & 0.022 & -0.019 & -2.44 & -0.75 & 1968.19 & 332.02 & & & -0.77 & 338.60 \\
\hline 202 & 295 & 0.27 & 0.00 & 0.01 & 0.02 & 0.296 & 0.000 & 0.022 & -0.019 & -2.31 & -0.71 & 1969.44 & 338.84 & & & -0.72 & 345.65 \\
\hline 203 & 296 & 0.19 & 0.00 & -0.07 & 0.02 & 0.205 & 0.000 & 0.104 & -0.001 & -3.70 & -1.14 & 1969.78 & 346.57 & & & -0.85 & 353.92 \\
\hline 204 & 297 & 0.19 & 0.00 & -0.07 & 0.02 & 0.205 & 0.000 & 0.104 & -0.001 & -3.68 & -1.22 & 1971.02 & 353.40 & & & -0.92 & 361.00 \\
\hline 205 & 298 & 0.20 & 0.00 & -0.06 & 0.03 & 0.215 & 0.000 & 0.093 & -0.013 & -3.79 & -1.56 & 1971.12 & 361.37 & & & -1.25 & 369.21 \\
\hline 206 & 299 & 0.19 & 0.00 & -0.06 & 0.03 & 0.204 & 0.000 & 0.091 & -0.014 & -3.73 & -1.58 & 1972.18 & 368.38 & & & -1.25 & 376.50 \\
\hline 207 & 300 & 0.19 & 0.00 & -0.06 & 0.03 & 0.204 & 0.000 & 0.091 & -0.014 & -4.16 & -2.00 & 1972.23 & 376.41 & & & -1.67 & 384.77 \\
\hline 208 & 301 & 0.20 & 0.00 & -0.05 & 0.03 & 0.215 & 0.000 & 0.081 & -0.016 & -3.86 & -1.93 & 1973.06 & 383.64 & & & -1.67 & 392.20 \\
\hline 209 & 302 & 0.20 & 0.00 & -0.05 & 0.04 & 0.215 & 0.000 & 0.082 & -0.026 & -4.42 & -2.46 & 1973.10 & 391.68 & & & -2.04 & 400.65 \\
\hline 210 & 303 & 0.20 & 0.00 & -0.05 & 0.04 & 0.215 & 0.000 & 0.082 & -0.026 & -4.29 & -2.37 & 1973.78 & 399.07 & & & -1.93 & 408.32 \\
\hline 211 & 304 & 0.20 & 0.00 & -0.04 & 0.04 & 0.215 & 0.000 & 0.069 & -0.028 & -4.26 & -2.54 & 1973.33 & 407.59 & & & -2.15 & 417.07 \\
\hline 212 & 305 & 0.20 & 0.00 & -0.04 & 0.04 & 0.215 & 0.000 & 0.069 & -0.028 & -3.98 & -2.29 & 1973.72 & 415.27 & & & -1.88 & 425.04 \\
\hline 213 & 306 & 0.20 & 0.00 & -0.03 & 0.04 & 0.215 & 0.000 & 0.057 & -0.031 & -3.93 & -2.39 & 1973.09 & 423.98 & & & -2.02 & 433.99 \\
\hline 214 & 307 & 0.20 & 0.00 & -0.03 & 0.04 & 0.215 & 0.000 & 0.057 & -0.031 & -3.64 & -2.14 & 1973.35 & 431.78 & & & -1.76 & 442.09 \\
\hline 215 & 308 & 0.20 & 0.00 & -0.02 & 0.04 & 0.216 & 0.000 & 0.045 & -0.034 & -3.73 & -2.35 & 1972.70 & 440.51 & & & -1.98 & 451.09 \\
\hline \multicolumn{18}{|c|}{$Z=94(\mathrm{Pu})$} \\
\hline 115 & 209 & 0.17 & 0.00 & 0.00 & 0.00 & 0.183 & 0.000 & 0.012 & 0.001 & -0.22 & 0.75 & 1557.73 & 55.63 & & & 0.70 & 57.23 \\
\hline 116 & 210 & 0.16 & 0.00 & 0.01 & 0.00 & 0.173 & 0.000 & -0.001 & -0.001 & -0.14 & 0.90 & 1569.07 & 52.36 & & & 0.87 & 53.89 \\
\hline 117 & 211 & 0.15 & 0.00 & 0.01 & 0.00 & 0.162 & 0.000 & -0.003 & -0.001 & -0.49 & 0.56 & 1578.75 & 50.75 & & & 0.53 & 52.20 \\
\hline 118 & 212 & 0.15 & 0.00 & 0.02 & -0.01 & 0.162 & 0.000 & -0.015 & 0.007 & -0.67 & 0.44 & 1590.01 & 47.57 & & & 0.42 & 48.95 \\
\hline 119 & 213 & 0.13 & 0.01 & 0.02 & -0.01 & 0.140 & -0.014 & -0.018 & 0.007 & -0.92 & 0.16 & 1599.30 & 46.35 & & & 0.13 & 47.65 \\
\hline 120 & 214 & -0.15 & 0.00 & 0.02 & -0.01 & -0.156 & 0.000 & -0.014 & 0.012 & -1.45 & -0.23 & 1610.47 & 43.25 & & & -0.26 & 44.48 \\
\hline 121 & 215 & -0.13 & 0.00 & 0.01 & -0.01 & -0.135 & 0.000 & -0.004 & 0.011 & -1.65 & -0.69 & 1619.59 & 42.21 & & & -0.70 & 43.37 \\
\hline 122 & 216 & -0.11 & 0.00 & 0.01 & 0.00 & -0.115 & 0.000 & -0.007 & 0.001 & -1.70 & -1.00 & 1630.34 & 39.52 & & & -1.01 & 40.62 \\
\hline 123 & 217 & -0.09 & 0.00 & 0.02 & 0.01 & -0.094 & 0.000 & -0.020 & -0.007 & -2.24 & -1.60 & 1639.28 & 38.66 & & & -1.61 & 39.70 \\
\hline 124 & 218 & -0.07 & 0.00 & 0.02 & 0.01 & -0.073 & 0.000 & -0.021 & -0.008 & -2.47 & -1.76 & 1649.55 & 36.46 & & & -1.76 & 37.44 \\
\hline 125 & 219 & 0.00 & 0.00 & 0.00 & 0.00 & 0.000 & 0.000 & 0.000 & 0.000 & -2.81 & -2.07 & 1657.88 & 36.19 & & & -2.08 & 37.13 \\
\hline 126 & 220 & 0.00 & 0.00 & 0.00 & 0.00 & 0.000 & 0.000 & 0.000 & 0.000 & -3.00 & -2.22 & 1667.81 & 34.33 & & & -2.23 & 35.21 \\
\hline 127 & 221 & 0.03 & 0.06 & -0.01 & 0.00 & 0.033 & -0.082 & 0.014 & 0.003 & -2.94 & -1.52 & 1674.83 & 35.40 & & & -1.52 & 36.22 \\
\hline 128 & 222 & 0.05 & 0.08 & -0.02 & 0.00 & 0.056 & -0.110 & 0.027 & 0.006 & -2.87 & -0.84 & 1683.60 & 34.69 & & & -0.82 & 35.48 \\
\hline 129 & 223 & 0.07 & 0.09 & -0.02 & 0.00 & 0.078 & -0.124 & 0.029 & 0.008 & -2.99 & -0.60 & 1690.78 & 35.58 & & & -0.59 & 36.33 \\
\hline 130 & 224 & 0.09 & 0.10 & -0.03 & 0.01 & 0.100 & -0.138 & 0.043 & 0.001 & -3.13 & -0.18 & 1699.51 & 34.92 & & & -0.14 & 35.65 \\
\hline 131 & 225 & 0.10 & 0.11 & -0.03 & 0.01 & 0.112 & -0.152 & 0.045 & 0.003 & -3.57 & -0.11 & 1706.55 & 35.95 & & & -0.07 & 36.63 \\
\hline 132 & 226 & 0.12 & 0.10 & -0.05 & 0.01 & 0.132 & -0.139 & 0.070 & 0.006 & -3.49 & 0.21 & 1715.08 & 35.49 & & & 0.28 & 36.17 \\
\hline 133 & 227 & 0.13 & 0.10 & -0.05 & 0.01 & 0.143 & -0.139 & 0.072 & 0.007 & -3.75 & 0.03 & 1722.08 & 36.56 & & & 0.10 & 37.20 \\
\hline 134 & 228 & 0.14 & 0.10 & -0.05 & 0.01 & 0.154 & -0.139 & 0.073 & 0.008 & -3.62 & 0.27 & 1730.41 & 36.31 & 36.09 & 0.032 & 0.35 & 36.94 \\
\hline 135 & 229 & 0.18 & 0.00 & -0.08 & 0.00 & 0.195 & 0.000 & 0.114 & 0.022 & -3.86 & 0.01 & .21 & 37.58 & 37.40 & 0.051 & 0.10 & 38.19 \\
\hline 136 & 230 & 0.18 & 0.00 & -0.08 & 0.00 & 0.195 & 0.000 & 0.114 & 0.022 & -4.03 & -0.13 & 1745.62 & 37.24 & 36.93 & 0.015 & -0.02 & 37.84 \\
\hline 137 & 231 & 0.18 & 0.00 & -0.08 & 0.00 & 0.195 & 0.000 & 0.114 & 0.022 & -4.43 & -0.50 & 1752.25 & 38.69 & 38.28 & 0.026 & -0.39 & 39.25 \\
\hline 138 & 232 & 0.19 & 0.00 & -0.08 & 0.01 & 0.206 & 0.000 & 0.116 & 0.013 & -4.46 & -0.56 & 1760.30 & 38.71 & 38.37 & 0.018 & -0.44 & 39.26 \\
\hline 139 & 233 & 0.19 & 0.00 & -0.08 & 0.01 & 0.206 & 0.000 & 0.116 & 0.013 & -4.90 & -0.96 & 1766.70 & 40.38 & 40.05 & 0.050 & -0.86 & 40.90 \\
\hline 140 & 234 & 0.20 & 0.00 & -0.08 & 0.02 & 0.216 & 0.000 & 0.119 & 0.004 & -5.01 & -1.12 & .57 & 40.58 & 40 & 0.007 & -1.00 & 41.11 \\
\hline 141 & 235 & 0.20 & 0.00 & -0.07 & 0.02 & 0.215 & 0.000 & 0.106 & 0.001 & -4.89 & -1.46 & 1780.64 & 42.58 & 42.18 & 0.021 & -1.37 & 43.06 \\
\hline 142 & 236 & 0.20 & 0.00 & -0.07 & 0.02 & 0.215 & 0.000 & 0.106 & 0.001 & -4.97 & -1.54 & 1788.18 & 43.12 & 42.90 & 0.002 & -1.44 & 43.61 \\
\hline 143 & 237 & 0.21 & 0.00 & -0.07 & 0.03 & 0.226 & 0.000 & 0.108 & -0.009 & -5.43 & -1.85 & 1793.95 & 45.41 & 45.09 & 0.002 & -1.73 & 45.91 \\
\hline 144 & 238 & 0.21 & 0.00 & -0.06 & 0.03 & 0.226 & 0.000 & 0.095 & -0.012 & -4.90 & -1.75 & 1801.04 & 46.39 & 46.17 & 0.002 & -1.63 & 46.89 \\
\hline 145 & 239 & 0.22 & 0.00 & -0.06 & 0.04 & 0.236 & 0.000 & 0.098 & -0.021 & -5.44 & -2.06 & 1806.57 & 48.93 & 48.59 & 0.002 & -1.91 & 49.46 \\
\hline 146 & 240 & 0.22 & 0.00 & -0.05 & 0.04 & 0.237 & 0.000 & 0.086 & -0.024 & -4.95 & -1.97 & 1813.42 & 50.15 & 50.13 & 0.002 & -1.83 & 50.69 \\
\hline 147 & 241 & 0.22 & 0.00 & -0.05 & 0.04 & 0.237 & 0.000 & 0.086 & -0.024 & -5.26 & -2.26 & 1818.69 & 52.96 & 52.96 & 0.002 & -2.12 & 53.50 \\
\hline 148 & 242 & 0.22 & 0.00 & -0.04 & 0.04 & 0.237 & 0.000 & 0.073 & -0.027 & -4.78 & -2.07 & 1825.17 & 54.54 & 54.72 & 0.002 & -1.93 & 55.10 \\
\hline 149 & 243 & 0.22 & 0.00 & -0.04 & 0.04 & 0.237 & 0.000 & 0.073 & -0.027 & -5.07 & -2.35 & 1830.20 & 57.59 & 57.76 & 0.003 & -2.22 & 58.15 \\
\hline 150 & 244 & 0.22 & 0.00 & -0.03 & 0.04 & 0.237 & 0.000 & 0.061 & -0.030 & -4.67 & -2.19 & 1836.47 & 59.39 & 59.81 & 0.005 & -2.05 & 59.98 \\
\hline 151 & 245 & 0.23 & 0.00 & -0.02 & 0.04 & 0.249 & 0.000 & 0.051 & -0.032 & -4.88 & -2.47 & 1841.26 & 62.67 & 63.11 & 0.014 & -2.34 & 63.27 \\
\hline 152 & 246 & 0.23 & 0.00 & -0.01 & 0.03 & 0.250 & 0.000 & 0.038 & -0.025 & -4.23 & -2.15 & 1847.13 & 64.87 & 65.39 & 0.015 & -2.07 & 65.44 \\
\hline 153 & 247 & 0.22 & 0.00 & -0.01 & 0.03 & 0.238 & 0.000 & 0.036 & -0.025 & -4.02 & -2.04 & 1851.30 & 68.78 & & & -1.97 & 69.37 \\
\hline
\end{tabular}

$\begin{array}{ll}-0.53 & 323.73\end{array}$

$-0.49 \quad 330.62$

$-0.77 \quad 338.60$

$-0.72 \quad 345.65$

$-0.85 \quad 353.92$

$-0.92 \quad 361.00$

$-1.25 \quad 369.21$

$-1.25 \quad 376.50$

$-1.67384 .77$

$-1.67 \quad 392.20$

$-2.04 \quad 400.65$

$-1.93 \quad 408.32$

$-2.15 \quad 417.07$

$-1.88 \quad 425.04$

$\begin{array}{ll}-2.02 & 433.99\end{array}$

$-1.76442 .09$

$-1.98 \quad 451.09$

\section{$Z=94$ (Pu)}

\section{2}

\section{2}

\section{2}




\begin{tabular}{|c|c|c|c|c|c|c|c|c|c|c|c|c|c|c|}
\hline \multicolumn{15}{|c|}{$Z=94(\mathrm{Pu})$} \\
\hline 154248 & 0.22 & 0.00 & -0.01 & 0.03 & 0.238 & 0.000 & 0.036 & -0.025 & -3.51 & -1.59 & 1856.81 & 71.34 & -1.50 & 71.98 \\
\hline 155249 & 0.22 & 0.00 & 0.00 & 0.03 & 0.239 & 0.000 & 0.024 & -0.028 & -3.24 & -1.42 & 1860.69 & 75.52 & -1.34 & 76.19 \\
\hline 156250 & 0.22 & 0.00 & 0.00 & 0.02 & 0.239 & 0.000 & 0.023 & -0.018 & -2.58 & -0.96 & 1865.97 & 78.32 & -0.92 & 78.99 \\
\hline $157 \quad 251$ & 0.22 & 0.00 & 0.01 & 0.02 & 0.240 & 0.000 & 0.011 & -0.021 & -2.54 & -0.87 & 1869.72 & 82.64 & -0.84 & 83.35 \\
\hline $158 \quad 252$ & 0.21 & 0.00 & 0.01 & 0.01 & 0.228 & 0.000 & 0.008 & -0.011 & -1.98 & -0.49 & 1874.84 & 85.59 & -0.48 & 86.32 \\
\hline 159253 & 0.21 & 0.00 & 0.02 & 0.01 & 0.229 & 0.000 & -0.004 & -0.013 & -2.20 & -0.69 & 1878.67 & 89.84 & -0.69 & 90.61 \\
\hline $160 \quad 254$ & 0.21 & 0.00 & 0.03 & 0.01 & 0.229 & 0.000 & -0.016 & -0.016 & -2.13 & -0.54 & 1883.80 & 92.77 & -0.51 & 93.61 \\
\hline 161255 & 0.21 & 0.00 & 0.04 & 0.00 & 0.230 & 0.000 & -0.030 & -0.009 & -2.47 & -0.76 & 1887.43 & 97.21 & -0.74 & 98.10 \\
\hline $162 \quad 256$ & 0.20 & 0.00 & 0.04 & 0.00 & 0.219 & 0.000 & -0.031 & -0.008 & -2.25 & -0.60 & 1892.35 & 100.36 & -0.58 & 101.33 \\
\hline 163257 & 0.20 & 0.00 & 0.04 & 0.00 & 0.219 & 0.000 & -0.031 & -0.008 & -2.26 & -0.63 & 1895.59 & 105.20 & -0.61 & 106.22 \\
\hline 164258 & 0.19 & 0.00 & 0.04 & 0.00 & 0.207 & 0.000 & -0.033 & -0.008 & -1.72 & -0.21 & 1900.03 & 108.83 & -0.18 & 109.93 \\
\hline $165 \quad 259$ & 0.17 & 0.00 & 0.03 & 0.00 & 0.185 & 0.000 & -0.024 & -0.005 & -1.41 & -0.15 & 1902.98 & 113.95 & -0.14 & 115.10 \\
\hline 166260 & 0.16 & 0.00 & 0.03 & 0.00 & 0.174 & 0.000 & -0.025 & -0.005 & -1.02 & 0.11 & 1907.38 & 117.62 & 0.13 & 118.85 \\
\hline 167261 & 0.16 & 0.00 & 0.02 & 0.00 & 0.173 & 0.000 & -0.013 & -0.003 & -1.08 & -0.00 & 1910.31 & 122.77 & -0.01 & 124.06 \\
\hline 168262 & 0.14 & 0.00 & 0.01 & 0.01 & 0.151 & 0.000 & -0.003 & -0.011 & -0.69 & 0.21 & 1914.55 & 126.60 & 0.22 & 127.98 \\
\hline 169263 & 0.12 & 0.00 & 0.00 & 0.01 & 0.129 & 0.000 & 0.007 & -0.010 & -0.97 & -0.18 & 1917.57 & 131.65 & -0.17 & 133.12 \\
\hline $170 \quad 264$ & 0.12 & 0.00 & 0.01 & 0.01 & 0.129 & 0.000 & -0.005 & -0.011 & -0.97 & -0.17 & 1921.81 & 135.47 & -0.16 & 137.04 \\
\hline 171265 & -0.17 & 0.00 & 0.01 & -0.01 & -0.176 & 0.000 & 0.001 & 0.010 & -1.85 & -0.67 & 1924.74 & 140.62 & -0.66 & 142.27 \\
\hline 172266 & -0.17 & 0.00 & 0.01 & -0.01 & -0.176 & 0.000 & 0.001 & 0.010 & -1.91 & -0.68 & 1928.82 & 144.61 & -0.67 & 146.36 \\
\hline 173267 & -0.13 & 0.00 & 0.00 & -0.01 & -0.135 & 0.000 & 0.007 & 0.009 & -1.97 & -1.07 & 1931.46 & 150.04 & -1.06 & 151.89 \\
\hline 174268 & -0.12 & 0.00 & -0.01 & -0.01 & -0.125 & 0.000 & 0.018 & 0.008 & -1.86 & -1.05 & 1935.32 & 154.25 & -1.03 & 156.22 \\
\hline $175 \quad 269$ & -0.11 & 0.00 & 0.00 & -0.01 & -0.115 & 0.000 & 0.005 & 0.009 & -2.19 & -1.56 & 1937.90 & 159.74 & -1.55 & 161.81 \\
\hline 176270 & -0.10 & 0.00 & 0.01 & -0.01 & -0.105 & 0.000 & -0.007 & 0.011 & -2.25 & -1.72 & 1941.76 & 163.96 & -1.70 & 166.15 \\
\hline $177 \quad 271$ & -0.10 & 0.00 & 0.02 & 0.00 & -0.105 & 0.000 & -0.019 & 0.002 & -2.78 & -2.22 & 1944.15 & 169.63 & -2.21 & 171.93 \\
\hline $178 \quad 272$ & -0.10 & 0.00 & 0.02 & -0.01 & -0.105 & 0.000 & -0.019 & 0.012 & -2.74 & -2.17 & 1947.61 & 174.24 & -2.13 & 176.69 \\
\hline 179273 & -0.08 & 0.00 & 0.02 & -0.01 & -0.084 & 0.000 & -0.020 & 0.011 & -2.84 & -2.24 & 1949.41 & 180.52 & -2.20 & 183.09 \\
\hline $180 \quad 274$ & -0.05 & 0.00 & 0.01 & 0.00 & -0.053 & 0.000 & -0.011 & 0.001 & -2.36 & -1.80 & 1952.30 & 185.70 & -1.80 & 188.36 \\
\hline 181275 & -0.03 & 0.00 & 0.01 & 0.00 & -0.032 & 0.000 & -0.011 & 0.000 & -2.81 & -2.16 & 1954.22 & 191.85 & -2.16 & 194.65 \\
\hline 182276 & 0.00 & 0.00 & 0.00 & 0.00 & 0.000 & 0.000 & 0.000 & 0.000 & -2.76 & -2.10 & 1957.32 & 196.82 & -2.10 & 199.76 \\
\hline $183 \quad 277$ & 0.00 & 0.00 & 0.00 & 0.00 & 0.000 & 0.000 & 0.000 & 0.000 & -3.20 & -2.52 & 1959.12 & 203.09 & -2.52 & 206.17 \\
\hline 184278 & 0.00 & 0.00 & 0.00 & 0.00 & 0.000 & 0.000 & 0.000 & 0.000 & -2.93 & -2.25 & 1961.85 & 208.43 & -2.25 & 211.65 \\
\hline $185 \quad 279$ & 0.01 & 0.04 & 0.00 & 0.00 & 0.011 & -0.054 & 0.001 & 0.001 & -2.33 & -1.42 & 1962.24 & 216.12 & -1.40 & 219.51 \\
\hline 186280 & 0.00 & 0.00 & 0.00 & 0.00 & 0.000 & 0.000 & 0.000 & 0.000 & -1.31 & -0.70 & 1964.35 & 222.07 & -0.71 & 225.60 \\
\hline $187 \quad 281$ & 0.03 & 0.08 & -0.01 & 0.00 & 0.035 & -0.109 & 0.015 & 0.005 & -2.20 & -0.49 & 1965.21 & 229.29 & -0.40 & 233.06 \\
\hline $188 \quad 282$ & 0.05 & 0.09 & -0.02 & 0.00 & 0.056 & -0.123 & 0.028 & 0.007 & -2.19 & -0.23 & 1967.61 & 234.96 & -0.10 & 238.93 \\
\hline 189283 & 0.06 & 0.11 & -0.02 & 0.01 & 0.068 & -0.150 & 0.030 & 0.000 & -3.08 & -0.46 & 1968.74 & 241.90 & -0.28 & 246.09 \\
\hline $190 \quad 284$ & 0.37 & 0.00 & 0.08 & 0.01 & 0.419 & 0.000 & -0.033 & -0.036 & -3.23 & -0.09 & 1970.88 & 247.83 & 0.05 & 252.16 \\
\hline 191285 & 0.37 & 0.00 & 0.08 & 0.01 & 0.419 & 0.000 & -0.033 & -0.036 & -3.46 & -0.33 & 1971.88 & 254.91 & -0.21 & 259.39 \\
\hline 192286 & 0.37 & 0.00 & 0.09 & 0.00 & 0.419 & 0.000 & -0.047 & -0.032 & -3.43 & -0.15 & 1974.04 & 260.81 & -0.03 & 265.47 \\
\hline 193287 & 0.37 & 0.00 & 0.09 & 0.00 & 0.419 & 0.000 & -0.047 & -0.032 & -3.66 & -0.39 & 1974.89 & 268.04 & -0.30 & 272.85 \\
\hline 194288 & 0.36 & 0.00 & 0.09 & 0.00 & 0.408 & 0.000 & -0.050 & -0.031 & -3.38 & -0.20 & 1976.89 & 274.11 & -0.06 & 279.15 \\
\hline $195 \quad 289$ & 0.36 & 0.00 & 0.09 & -0.01 & 0.407 & 0.000 & -0.052 & -0.022 & -3.46 & -0.22 & 1977.38 & 281.69 & -0.17 & 286.84 \\
\hline 196290 & 0.36 & 0.00 & 0.09 & -0.01 & 0.407 & 0.000 & -0.052 & -0.022 & -3.33 & -0.16 & 1979.35 & 287.79 & -0.09 & 293.15 \\
\hline 197291 & 0.36 & 0.00 & 0.10 & -0.01 & 0.408 & 0.000 & -0.064 & -0.026 & -4.04 & -0.57 & 1980.07 & 295.14 & -0.44 & 300.76 \\
\hline 198292 & 0.24 & 0.00 & -0.03 & 0.02 & 0.260 & 0.000 & 0.064 & -0.008 & -2.25 & -0.39 & 1981.79 & 301.49 & -0.32 & 307.25 \\
\hline 199293 & 0.26 & 0.00 & -0.01 & 0.02 & 0.283 & 0.000 & 0.044 & -0.013 & -2.32 & -0.61 & 1982.18 & 309.17 & -0.61 & 315.07 \\
\hline $200 \quad 294$ & 0.27 & 0.00 & 0.01 & 0.02 & 0.296 & 0.000 & 0.022 & -0.019 & -2.17 & -0.52 & 1983.85 & 315.58 & -0.51 & 321.70 \\
\hline 201295 & 0.27 & 0.00 & 0.01 & 0.02 & 0.296 & 0.000 & 0.022 & -0.019 & -2.45 & -0.80 & 1984.16 & 323.34 & -0.80 & 329.67 \\
\hline $202 \quad 296$ & 0.27 & 0.00 & 0.01 & 0.03 & 0.296 & 0.000 & 0.023 & -0.029 & -2.55 & -0.89 & 1985.86 & 329.71 & -0.75 & 336.41 \\
\hline $203 \quad 297$ & 0.27 & 0.00 & 0.01 & 0.03 & 0.296 & 0.000 & 0.023 & -0.029 & -2.81 & -1.14 & 1986.00 & 337.64 & -1.01 & 344.55 \\
\hline 204298 & 0.20 & 0.00 & -0.06 & 0.03 & 0.215 & 0.000 & 0.093 & -0.013 & -3.36 & -1.15 & 1987.48 & 344.23 & -0.83 & 351.56 \\
\hline $205 \quad 299$ & 0.20 & 0.00 & -0.06 & 0.03 & 0.215 & 0.000 & 0.093 & -0.013 & -3.77 & -1.53 & 1987.62 & 352.16 & -1.22 & 359.71 \\
\hline 206300 & 0.20 & 0.00 & -0.05 & 0.03 & 0.215 & 0.000 & 0.081 & -0.016 & -3.45 & -1.49 & 1988.92 & 358.94 & -1.22 & 366.69 \\
\hline 207301 & 0.20 & 0.00 & -0.05 & 0.03 & 0.215 & 0.000 & 0.081 & -0.016 & -3.86 & -1.89 & 1988.95 & 366.97 & -1.63 & 374.96 \\
\hline 208302 & 0.20 & 0.00 & -0.05 & 0.03 & 0.215 & 0.000 & 0.081 & -0.016 & -3.87 & -1.94 & 1990.19 & 373.80 & -1.67 & 382.05 \\
\hline 209303 & 0.20 & 0.00 & -0.05 & 0.04 & 0.215 & 0.000 & 0.082 & -0.026 & -4.44 & -2.47 & 1990.23 & 381.83 & -2.05 & 390.48 \\
\hline 210304 & 0.20 & 0.00 & -0.04 & 0.04 & 0.215 & 0.000 & 0.069 & -0.028 & -4.09 & -2.36 & 1991.18 & 388.96 & -1.96 & 397.84 \\
\hline
\end{tabular}




\section{$Z=94(\mathrm{Pu})$}

\section{5} 212306 213307 214308 215309

216310

$217 \quad 311$

$218 \quad 312$ $\begin{array}{lll}0.20 & 0.00 & -0.04\end{array}$ $\begin{array}{llll}0.20 & 0.00 & -0.03\end{array}$ $\begin{array}{llll}0.20 & 0.00 & -0.03\end{array}$ $\begin{array}{llll}0.20 & 0.00 & -0.03\end{array}$ $\begin{array}{llll}0.20 & 0.00 & -0.02\end{array}$ $\begin{array}{lll}0.20 & 0.00 & -0.01\end{array}$ $\begin{array}{llll}0.20 & 0.00 & -0.01\end{array}$ $\begin{array}{lll}0.20 & 0.00 & 0.00\end{array}$
$0.04 \quad 0.215$ $0.04 \quad 0.215$ $0.04 \quad 0.215$ $0.04 \quad 0.215$ $0.04 \quad 0.216$

$0.04 \quad 0.216$

$0.04 \quad 0.216$ $0.04 \quad 0.217$ $\begin{array}{lllllll}0.000 & 0.069 & -0.028 & -4.32 & -2.58 & 1990.79 & 397.42\end{array}$ $\begin{array}{lllllll}0.000 & 0.057 & -0.031 & -3.88 & -2.34 & 1991.47 & 404.81\end{array}$ $\begin{array}{lllllll}0.000 & 0.057 & -0.031 & -4.05 & -2.51 & 1990.91 & 413.44\end{array}$ $\begin{array}{lllllll}0.000 & 0.057 & -0.031 & -3.77 & -2.27 & 1991.47 & 420.96\end{array}$ $\begin{array}{lllllll}0.000 & 0.045 & -0.034 & -3.87 & -2.49 & 1990.83 & 429.67\end{array}$ $\begin{array}{lllllll}0.000 & 0.033 & -0.036 & -3.57 & -2.31 & 1991.33 & 437.24\end{array}$ $\begin{array}{lllllll}0.000 & 0.033 & -0.036 & -3.88 & -2.64 & 1990.68 & 445.96\end{array}$ $\begin{array}{lllllll}0.000 & 0.021 & -0.038 & -3.72 & -2.61 & 1991.20 & 453.51\end{array}$
$-2.19 \quad 406.56$

$-1.95 \quad 414.20$

$-2.13 \quad 423.10$

$-1.87 \quad 430.90$

$-2.11439 .87$

$-1.92 \quad 447.74$

$-2.26 \quad 456.74$

$-2.20 \quad 464.61$

\section{$Z=95$ (Am)}

\begin{tabular}{|c|c|c|c|c|c|c|c|c|c|c|c|c|c|c|c|c|}
\hline $17 \quad 212$ & 0.16 & 0.00 & 0.01 & 0.00 & 0.173 & 0.000 & -0.001 & -0.001 & -0.68 & 0.40 & 1576.65 & 60.15 & & & 0.36 & 61.80 \\
\hline $18 \quad 213$ & 0.15 & 0.00 & 0.02 & -0.01 & 0.162 & 0.000 & -0.015 & 0.007 & -0.79 & 0.32 & 1587.91 & 56.96 & & & 0.28 & 58.54 \\
\hline 9214 & 0.14 & 0.00 & 0.02 & -0.01 & 0.151 & 0.000 & -0.016 & 0.007 & -0.98 & 0.10 & 1597.54 & 55.40 & & & 0.07 & 56.90 \\
\hline 0215 & -0.15 & 0.00 & 0.02 & -0.01 & -0.156 & 0.000 & -0.014 & 0.012 & -1.19 & 0.01 & 1608.47 & 52.54 & & & -0.03 & 53.97 \\
\hline 12216 & -0.14 & 0.00 & 0.02 & -0.01 & -0.146 & 0.000 & -0.015 & 0.012 & -1.44 & -0.34 & 1617.90 & 51.19 & & & -0.37 & 52.54 \\
\hline 217 & -0.11 & 0.00 & 0.02 & 0.00 & -0.115 & 0.000 & -0.018 & 0.002 & -1.43 & -0.69 & 1628.73 & 48.42 & & & -0.71 & 49.71 \\
\hline 3218 & -0.10 & 0.00 & 0.02 & 0.00 & -0.105 & 0.000 & -0.019 & 0.002 & -1.93 & -1.29 & 1638.08 & 47.14 & & & -1.31 & 48.37 \\
\hline $4 \quad 219$ & -0.08 & 0.00 & 0.02 & 0.01 & -0.084 & 0.000 & -0.021 & -0.008 & -2.11 & -1.46 & 1648.40 & 44.89 & & & -1.47 & 46.06 \\
\hline 5220 & 0.01 & 0.03 & 0.00 & 0.00 & 0.011 & -0.040 & 0.000 & 0.001 & -2.47 & -1.62 & 1656.99 & 44.38 & & & -1.62 & 45.49 \\
\hline 6221 & 0.01 & 0.04 & 0.00 & 0.00 & 0.011 & -0.054 & 0.001 & 0.001 & -2.79 & -1.76 & 1666.96 & 42.48 & & & -1.77 & 43.54 \\
\hline $7 \quad 222$ & 0.04 & 0.07 & -0.01 & 0.00 & 0.045 & -0.095 & 0.015 & 0.004 & -2.87 & -1.23 & 1674.54 & 42.97 & & & -1.24 & 43.97 \\
\hline 8223 & 0.05 & 0.08 & -0.01 & 0.00 & 0.056 & -0.109 & 0.015 & 0.005 & -2.47 & -0.59 & 1683.40 & 42.18 & & & -0.58 & 43.13 \\
\hline 9224 & 0.07 & 0.10 & -0.02 & 0.01 & 0.079 & -0.137 & 0.030 & -0.001 & -3.05 & -0.37 & 1690.99 & 42.67 & & & -0.36 & 43.57 \\
\hline $0 \quad 225$ & 0.09 & 0.10 & -0.03 & 0.01 & 0.100 & -0.138 & 0.043 & 0.001 & -2.85 & 0.06 & 1699.76 & 41.97 & & & 0.08 & 42.84 \\
\hline 1226 & 0.10 & 0.11 & -0.03 & 0.01 & 0.112 & -0.152 & 0.045 & 0.003 & -3.28 & 0.07 & 1707.25 & 42.54 & & & 0.09 & 43.36 \\
\hline 2227 & 0.12 & 0.10 & -0.04 & 0.01 & 0.132 & -0.138 & 0.058 & 0.004 & -2.90 & 0.36 & 171 & 42.01 & & & 0.40 & .81 \\
\hline 3228 & 0.14 & 0.09 & -0.05 & 0.01 & 0.153 & -0.125 & 0.073 & 0.006 & -3.19 & 0.26 & 1723.17 & 42.77 & & & 0.30 & 43.53 \\
\hline $4 \quad 229$ & 0.19 & 0.00 & -0.07 & 0.00 & 0.206 & 0.000 & 0.103 & 0.020 & -3.08 & 0.26 & 1731.77 & 42.24 & & & 0.31 & 42.98 \\
\hline 5230 & 0.18 & 0.00 & -0.08 & 0.00 & 0.195 & 0.000 & 0.114 & 0.022 & -3.97 & -0.12 & 1739.08 & 43.00 & & & -0.06 & 43.71 \\
\hline 6231 & 0.18 & 0.00 & -0.08 & 0.00 & 0.195 & 0.000 & 0.114 & 0.022 & -4.14 & -0.27 & 1747.54 & 42.62 & & & -0.18 & 43.32 \\
\hline 32 & 0.1 & 0.00 & -0.08 & 0.01 & 0.206 & 0.000 & 0.116 & 0.013 & -4.53 & -0.64 & 175 & 43.67 & & & -0.57 & 33 \\
\hline 8233 & 0.19 & 0.00 & -0.08 & 0.01 & 0.206 & 0.000 & 0.116 & 0.013 & -4.64 & -0.86 & 1762.80 & 43.49 & & & -0.77 & 44.14 \\
\hline 9234 & 0.19 & 0.00 & -0.07 & 0.01 & 0.205 & 0.000 & 0.103 & 0.010 & -4.54 & -1.22 & 1769.55 & 44.82 & & & -1.17 & 45.42 \\
\hline $0 \quad 235$ & 0.20 & 0.00 & -0.07 & 0.02 & 0.215 & 0.000 & 0.106 & 0.001 & -4.79 & -1.40 & 1777.48 & 44.96 & & & -1.33 & 45.56 \\
\hline 1236 & 0.20 & 0.00 & -0.07 & 0.02 & 0.215 & 0.000 & 0.106 & 0.001 & -5.21 & -1.78 & 1783.96 & 46.54 & & & -1.71 & 47.12 \\
\hline 37 & 0 & 0.00 & -0.07 & 0 & & & & 01 & & & & & & & -1.79 & \\
\hline 238 & 0.21 & 0.00 & -0.06 & 0.03 & 0.226 & 0.000 & 0.095 & -0.012 & -5.39 & -2.22 & 179 & 48.91 & & & -2.15 & 49.47 \\
\hline $4 \quad 239$ & 0.21 & 0.00 & -0.06 & 0.03 & 0.226 & 0.000 & 0.095 & -0.012 & -5.32 & -2.17 & 1804.91 & 49.81 & & & -2.08 & 50.38 \\
\hline 5240 & 0.22 & 0.00 & -0.06 & 0.04 & 0.236 & 0.000 & 0.098 & -0.021 & -5.86 & -2.53 & 1810.87 & 51.92 & 51.51 & 0.014 & -2.42 & 52.52 \\
\hline 241 & 0.22 & 0.00 & -0.05 & 004 & 0.237 & 0.000 & 0.086 & -0.024 & -5.42 & -2.43 & 1817.73 & 53.14 & 52.94 & & -2.31 & 53.73 \\
\hline 42 & 0 & 0.00 & -0.05 & 0.04 & & & & -0.024 & & -2.71 & & & & & -2.60 & \\
\hline 243 & 0.22 & 0.00 & -0.04 & 0.04 & 0.237 & 0.000 & 0.073 & -0.027 & -5.26 & -2.57 & 182 & 57.09 & 57.18 & & -2.45 & 57.69 \\
\hline 244 & 0.23 & 0.00 & -0.03 & 0.04 & 0.249 & 0.000 & 0.063 & -0.029 & -5.41 & -2.83 & 1835.30 & 59.78 & & & -2.73 & 60.38 \\
\hline 245 & 0.22 & 0.00 & -0.03 & 0.04 & 0.237 & 0.000 & 0.061 & -0.030 & -5.16 & -2.67 & 1841.60 & 61.55 & 61.90 & 0.003 & -2.56 & 62.18 \\
\hline 1246 & 0.23 & 0.00 & -0.02 & 0.04 & 0.249 & 0.000 & 0.051 & -0.032 & -5.35 & -2.94 & 1846.74 & 64.48 & & & -2.84 & 65.11 \\
\hline 247 & 0 & 0.00 & -0.01 & 0.04 & 50 & 0.000 & 39 & -0.035 & -4.94 & -2.67 & 2.70 & 66.60 & & & -2.55 & 67.27 \\
\hline 248 & 0.23 & 0.00 & -0.01 & 0.03 & 0.250 & 0.000 & 0.038 & -0.025 & -4.54 & -2.49 & 1857.16 & 70.20 & & & -2.44 & 70.83 \\
\hline 249 & 0.23 & 0.00 & 0.00 & 0.03 & 0.250 & 0.000 & 0.026 & -0.027 & -4.00 & -2.07 & 1862.72 & 72.72 & & & -2.00 & 73.39 \\
\hline 250 & 0.23 & 0.00 & 0.00 & 0.03 & 0.250 & 0.000 & 0.026 & -0.027 & -3.76 & -1.87 & 1866.94 & 76.57 & & & -1.81 & 77.26 \\
\hline 6251 & 0.22 & 0.00 & 0.00 & 0.02 & 0.239 & 0.000 & 0.023 & -0.018 & -2.98 & -1.34 & 1872.17 & 79.41 & & & -1.32 & 80.10 \\
\hline 252 & 0.22 & 0.00 & 0.01 & 0.02 & 0.240 & 0.000 & 011 & -0.021 & -2.96 & -1.32 & 1876.34 & 83.31 & & & -1.30 & 84.03 \\
\hline 253 & 0.21 & 0.00 & 0.01 & 0.01 & 0.228 & 0.000 & 0.008 & -0.011 & -2.36 & -0.91 & 1881.45 & 86.26 & & & -0.91 & 87.00 \\
\hline 254 & 0.21 & 0.00 & 0.02 & 0.01 & 0.229 & 0.000 & -0.004 & -0.013 & -2.59 & -1.07 & 1885.59 & 90.20 & & & -1.07 & 90.98 \\
\hline $0 \quad 255$ & 0.21 & 0.00 & 0.03 & 0.01 & 0.229 & 0.000 & -0.016 & -0.016 & -2.50 & -0.90 & 1890.73 & 93.13 & & & -0.88 & 93.98 \\
\hline 1256 & 0.21 & 0.00 & 0.04 & 0.00 & 0.230 & 0.000 & -0.030 & -0.009 & -2.82 & -1.11 & 1894.71 & 97.23 & & & -1.10 & 98.11 \\
\hline 257 & 0.21 & 0.00 & 0.04 & 0.00 & 0.230 & 0.000 & -0.030 & -0.009 & -2.64 & -0.93 & 1899.62 & 100.38 & & & -0.91 & 01.33 \\
\hline 258 & 0.20 & 0.00 & 0.04 & 0.00 & 0.219 & 0.000 & -0.031 & -0.008 & -2.60 & -0.96 & 1903.22 & 104.86 & & & -0.95 & 105.87 \\
\hline 259 & 0.19 & 0.00 & 0.04 & 0.00 & 0.207 & 0.000 & -0.033 & -0.008 & -2.04 & -0.51 & 1907.65 & 108.49 & & & -0.49 & 109.57 \\
\hline
\end{tabular}




\section{$Z=95$ (Am)}

\begin{tabular}{|c|c|c|c|c|c|c|c|c|c|c|c|c|c|c|c|}
\hline 165 & 260 & 0.18 & 0.00 & 0.03 & 0.00 & 0.196 & 0.000 & -0.023 & -0.005 & -1.74 & -0.41 & 1910.91 & 113.31 & -0.41 & 114.44 \\
\hline 166 & 261 & 0.16 & 0.00 & 0.02 & 0.00 & 0.173 & 0.000 & -0.013 & -0.003 & -1.12 & -0.09 & 1915.26 & 117.03 & -0.09 & 118.22 \\
\hline 167 & 262 & 0.16 & 0.00 & 0.02 & 0.00 & 0.173 & 0.000 & -0.013 & -0.003 & -1.30 & -0.21 & 1918.54 & 121.82 & -0.22 & 123.08 \\
\hline 88 & 263 & 0.16 & 0.00 & 0.02 & 0.00 & 0.173 & 0.000 & -0.013 & -0.003 & -1.00 & 0.04 & 1922.77 & 125.66 & 0.04 & 127.00 \\
\hline 169 & 264 & 0.13 & 0.00 & 0.00 & 0.01 & 0.140 & 0.000 & 0.008 & -0.010 & -1.11 & -0.28 & 1926.05 & 130.46 & -0.27 & 131.89 \\
\hline 70 & 265 & 0.12 & 0.00 & 0.00 & 0.01 & 0.129 & 0.000 & 0.007 & -0.010 & -0.99 & -0.23 & 1930.28 & 134.30 & -0.22 & 135.82 \\
\hline 71 & 266 & 0.12 & 0.00 & 0.01 & 0.01 & 0.129 & 0.000 & -0.005 & -0.011 & -1.43 & -0.63 & 1933.45 & 139.20 & -0.62 & 140.80 \\
\hline 72 & 267 & -0.17 & 0.00 & 0.01 & -0.01 & -0.176 & 0.000 & 0.001 & 0.010 & -1.83 & -0.62 & 1937.53 & 143.19 & -0.62 & 144.88 \\
\hline J & 268 & -0.13 & 0.00 & 0.00 & -0.01 & -0.135 & 0.000 & 0.007 & 0.009 & -1.87 & -0.97 & 1940.46 & 148.33 & -0.97 & 150.11 \\
\hline 174 & 269 & -0.12 & 0.00 & -0.01 & -0.01 & -0.125 & 0.000 & 0.018 & 0.008 & -1.73 & -0.93 & 1944.31 & 152.55 & -0.92 & 154.44 \\
\hline 13 & 270 & -0.11 & 0.00 & 0.00 & -0.01 & -0.115 & 0.000 & 0.005 & 0.009 & -2.05 & -1.42 & 1947.21 & 157.72 & -1.42 & 159.71 \\
\hline 176 & 271 & -0.10 & 0.00 & 0.01 & -0.01 & -0.105 & 0.000 & -0.007 & 0.011 & -2.08 & -1.56 & 1951.06 & 161.94 & -1.55 & 164.05 \\
\hline 177 & 272 & -0.10 & 0.00 & 0.02 & 0.00 & -0.105 & 0.000 & -0.019 & 0.002 & -2.61 & -2.06 & 1953.78 & 167.30 & -2.05 & 169.51 \\
\hline 78 & 273 & -0.10 & 0.00 & 0.02 & -0.01 & -0.105 & 0.000 & -0.019 & 0.012 & -2.56 & -2.00 & 1957.25 & 171.90 & -1.97 & 174.25 \\
\hline 179 & 274 & -0.08 & 0.00 & 0.01 & 0.00 & -0.084 & 0.000 & -0.009 & 0.001 & -2.47 & -2.01 & 1959.31 & 177.91 & -2.01 & 180.34 \\
\hline 180 & 275 & -0.07 & 0.00 & 0.02 & 0.00 & -0.073 & 0.000 & -0.021 & 0.002 & -2.26 & -1.70 & 1962.35 & 182.94 & -1.69 & 185.51 \\
\hline 181 & 276 & -0.04 & 0.00 & 0.01 & 0.00 & -0.042 & 0.000 & -0.011 & 0.001 & -2.46 & -1.85 & 1964.38 & 188.98 & -1.85 & 191.67 \\
\hline 182 & 277 & 0.01 & 0.00 & 0.00 & 0.00 & 0.011 & 0.000 & 0.000 & 0.000 & -2.28 & -1.65 & 1967.36 & 194.07 & -1.65 & 196.89 \\
\hline 83 & 278 & -0.01 & 0.00 & 0.00 & 0.00 & -0.011 & 0.000 & 0.000 & 0.000 & -2.70 & -2.06 & 1969.47 & 200.03 & -2.06 & 202.98 \\
\hline 184 & 279 & 0.00 & 0.01 & 0.00 & 0.00 & 0.000 & -0.013 & 0.000 & 0.000 & -2.44 & -1.78 & 1972.20 & 205.38 & -1.78 & 208.47 \\
\hline 185 & 280 & 0.01 & 0.05 & 0.00 & 0.00 & 0.012 & -0.067 & 0.001 & 0.002 & -2.17 & -1.14 & 1973.11 & 212.54 & -1.12 & 215.80 \\
\hline 186 & 281 & 0.01 & 0.05 & 0.00 & 0.00 & 0.012 & -0.067 & 0.001 & 0.002 & -1.48 & -0.48 & 1975.28 & 218.43 & -0.46 & 221.85 \\
\hline 187 & 282 & 0.03 & 0.09 & -0.01 & 0.00 & 0.035 & -0.123 & 0.016 & 0.006 & -2.34 & -0.45 & 1976.63 & 225.16 & -0.35 & 228.79 \\
\hline 188 & 283 & 0.05 & 0.10 & -0.02 & 0.01 & 0.057 & -0.136 & 0.029 & -0.002 & -2.38 & -0.12 & 1978.98 & 230.88 & 0.03 & 234.72 \\
\hline 189 & 284 & 0.37 & 0.00 & 0.08 & 0.01 & 0.419 & 0.000 & -0.033 & -0.036 & -3.38 & -0.25 & 1980.33 & 237.60 & -0.17 & 241.53 \\
\hline 190 & 285 & 0.37 & 0.00 & 0.08 & 0.01 & 0.419 & 0.000 & -0.033 & -0.036 & -3.18 & -0.10 & 1982.69 & 243.31 & 0.01 & 247.43 \\
\hline 191 & 286 & 0.37 & 0.00 & 0.09 & 0.00 & 0.419 & 0.000 & -0.047 & -0.032 & -3.58 & -0.28 & 1983.94 & 250.13 & -0.22 & 254.37 \\
\hline 192 & 287 & 0.37 & 0.00 & 0.09 & 0.00 & 0.419 & 0.000 & -0.047 & -0.032 & -3.42 & -0.20 & 1986.21 & 255.93 & -0.11 & 260.37 \\
\hline 193 & 288 & 0.37 & 0.00 & 0.09 & 0.00 & 0.419 & 0.000 & -0.047 & -0.032 & -3.64 & -0.40 & 1987.33 & 262.88 & -0.34 & 267.47 \\
\hline 194 & 289 & 0.36 & 0.00 & 0.09 & 0.00 & 0.408 & 0.000 & -0.050 & -0.031 & -3.47 & -0.22 & 1989.35 & 268.94 & -0.12 & 273.75 \\
\hline 195 & 290 & 0.36 & 0.00 & 0.09 & -0.01 & 0.407 & 0.000 & -0.052 & -0.022 & -3.61 & -0.44 & 1990.33 & 276.02 & -0.41 & 280.94 \\
\hline 196 & 291 & 0.24 & 0.00 & -0.03 & 0.01 & 0.260 & 0.000 & 0.063 & 0.002 & -2.28 & -0.42 & 1992.37 & 282.06 & -0.41 & 287.15 \\
\hline 197 & 292 & 0.24 & 0.00 & -0.02 & 0.01 & 0.261 & 0.000 & 0.051 & -0.001 & -2.40 & -0.69 & 1993.25 & 289.25 & -0.72 & 294.49 \\
\hline 198 & 293 & 0.25 & 0.00 & -0.02 & 0.02 & 0.271 & 0.000 & 0.054 & -0.010 & -2.47 & -0.72 & 1995.18 & 295.39 & -0.69 & 300.88 \\
\hline 199 & 294 & 0.25 & 0.00 & -0.01 & 0.02 & 0.272 & 0.000 & 0.041 & -0.014 & -2.60 & -0.96 & 1995.90 & 302.74 & -0.96 & 308.41 \\
\hline 200 & 295 & 0.27 & 0.00 & 0.01 & 0.02 & 0.296 & 0.000 & 0.022 & -0.019 & -2.55 & -0.90 & 1997.60 & 309.12 & -0.90 & 314.99 \\
\hline 201 & 296 & 0.27 & 0.00 & 0.01 & 0.02 & 0.296 & 0.000 & 0.022 & -0.019 & -2.81 & -1.16 & 1998.19 & 316.59 & -1.17 & 322.66 \\
\hline 202 & 297 & 0.27 & 0.00 & 0.01 & 0.03 & 0.296 & 0.000 & 0.023 & -0.029 & -2.95 & -1.29 & 1999.93 & 322.93 & -1.16 & 329.35 \\
\hline 203 & 298 & 0.26 & 0.00 & 0.00 & 0.03 & 0.284 & 0.000 & 0.033 & -0.026 & -3.17 & -1.59 & 2000.43 & 330.50 & -1.49 & 337.11 \\
\hline 204 & 299 & 0.25 & 0.00 & 0.00 & 0.03 & 0.273 & 0.000 & 0.030 & -0.027 & -2.97 & -1.46 & 2001.77 & 337.23 & -1.33 & 344.10 \\
\hline 205 & 300 & 0.23 & 0.00 & -0.02 & 0.03 & 0.249 & 0.000 & 0.050 & -0.022 & -3.30 & -1.70 & 2002.07 & 345.00 & -1.57 & 352.09 \\
\hline 206 & 301 & 0.20 & 0.00 & -0.05 & 0.03 & 0.215 & 0.000 & 0.081 & -0.016 & -3.74 & -1.77 & 2003.48 & 351.67 & -1.52 & 359.11 \\
\hline 207 & 302 & 0.20 & 0.00 & -0.05 & 0.03 & 0.215 & 0.000 & 0.081 & -0.016 & -4.16 & -2.18 & 2003.81 & 359.40 & -1.93 & 367.07 \\
\hline 208 & 303 & 0.20 & 0.00 & -0.05 & 0.04 & 0.215 & 0.000 & 0.082 & -0.026 & -4.34 & -2.35 & 2005.18 & 366.11 & -1.94 & 374.18 \\
\hline 209 & 304 & 0.20 & 0.00 & -0.05 & 0.04 & 0.215 & 0.000 & 0.082 & -0.026 & -4.73 & -2.72 & 2005.35 & 374.00 & -2.32 & 382.32 \\
\hline 10 & 305 & 0.20 & 0.00 & -0.04 & 0.04 & 0.215 & 0.000 & 0.069 & -0.028 & -4.41 & -2.64 & 2006.33 & 381.10 & -2.26 & 389.64 \\
\hline 211 & 306 & 0.20 & 0.00 & -0.04 & 0.04 & 0.215 & 0.000 & 0.069 & -0.028 & -4.64 & -2.86 & 2006.23 & 389.27 & -2.49 & 398.06 \\
\hline 212 & 307 & 0.20 & 0.00 & -0.03 & 0.04 & 0.215 & 0.000 & 0.057 & -0.031 & -4.18 & -2.60 & 2006.90 & 396.67 & -2.23 & 405.71 \\
\hline 213 & 308 & 0.20 & 0.00 & -0.03 & 0.04 & 0.215 & 0.000 & 0.057 & -0.031 & -4.35 & -2.78 & 2006.62 & 405.02 & -2.42 & 414.31 \\
\hline 214 & 309 & 0.20 & 0.00 & -0.02 & 0.04 & 0.216 & 0.000 & 0.045 & -0.034 & -3.94 & -2.53 & 2007.18 & 412.54 & -2.16 & 422.10 \\
\hline 215 & 310 & 0.20 & 0.00 & -0.02 & 0.04 & 0.216 & 0.000 & 0.045 & -0.034 & -4.18 & -2.78 & 2006.85 & 420.93 & -2.42 & 430.76 \\
\hline 216 & 311 & 0.20 & 0.00 & -0.01 & 0.04 & 0.216 & 0.000 & 0.033 & -0.036 & -3.89 & -2.61 & 2007.36 & 428.50 & -2.23 & 438.61 \\
\hline 217 & 312 & 0.20 & 0.00 & -0.01 & 0.04 & 0.216 & 0.000 & 0.033 & -0.036 & -4.20 & -2.94 & 2006.99 & 436.93 & -2.57 & 447.32 \\
\hline 218 & 313 & 0.21 & 0.00 & 0.00 & 0.04 & 0.228 & 0.000 & 0.023 & -0.038 & -4.11 & -2.92 & 2007.53 & 444.47 & -2.54 & 455.15 \\
\hline 219 & 314 & 0.21 & 0.00 & 0.01 & 0.04 & 0.229 & 0.000 & 0.011 & -0.041 & -4.44 & -3.26 & 2007.05 & 453.02 & -2.85 & 464.02 \\
\hline 220 & 315 & 0.21 & 0.00 & 0.01 & 0.04 & 0.229 & 0.000 & 0.011 & -0.041 & -4.30 & -3.14 & 2007.37 & 460.78 & -2.72 & 472.08 \\
\hline
\end{tabular}




\section{$Z=96(\mathrm{Cm})$}

\begin{tabular}{|c|c|c|c|c|c|c|c|c|c|c|c|c|c|c|c|c|}
\hline 119215 & 0.14 & 0.00 & 0.02 & -0.01 & 0.151 & 0.000 & -0.016 & 0.007 & -1.06 & -0.00 & 1597.16 & 63.07 & & & -0.03 & 64.80 \\
\hline 120216 & -0.15 & 0.00 & 0.02 & -0.01 & -0.156 & 0.000 & -0.014 & 0.012 & -1.08 & 0.08 & 1608.32 & 59.98 & & & 0.05 & 61.63 \\
\hline 1217 & -0.14 & 0.00 & 0.02 & -0.01 & -0.146 & 0.000 & -0.015 & 0.012 & -1.33 & -0.26 & 1617.79 & 58.58 & & & -0.29 & 0.16 \\
\hline 218 & -0.11 & 0.00 & 0.02 & 0.00 & -0.115 & 0.000 & -0.018 & 0.002 & -1.32 & -0.60 & 1629.02 & 55.42 & & & -0.62 & 56.93 \\
\hline 3219 & -0.10 & 0.00 & 0.02 & 0.00 & -0.105 & 0.000 & -0.019 & 0.002 & -1.81 & -1.18 & 1638.39 & 54.12 & & & -1.20 & 55.56 \\
\hline 220 & -0.08 & 0.00 & 0.02 & 0.01 & -0.084 & 0.000 & -0.021 & -0.008 & -1.98 & -1.34 & 1649.11 & 51.47 & & & -1.35 & 52.84 \\
\hline 5221 & 0.02 & 0.03 & 0.00 & 0.00 & 0.022 & -0.040 & 0.001 & 0.001 & -2.36 & -1.53 & 1657.77 & 50.89 & & & -1.53 & 52.20 \\
\hline $6 \quad 222$ & 0.00 & 0.03 & 0.00 & 0.00 & 0.000 & -0.040 & 0.000 & 0.001 & -2.53 & -1.64 & 1668.11 & 48.61 & & & -1.64 & 49.86 \\
\hline 7223 & 0.03 & 0.07 & -0.01 & 0.00 & 0.034 & -0.095 & 0.014 & 0.004 & -2.68 & -1.08 & 1675.70 & 49.10 & & & -1.08 & 50.28 \\
\hline $8 \quad 224$ & 0.04 & 0.08 & -0.01 & 0.00 & 0.045 & -0.109 & 0.015 & 0.005 & -2.24 & -0.38 & 1684.92 & 47.95 & & & -0.37 & 49.09 \\
\hline 225 & 0.06 & 0.09 & -0.02 & 0.01 & 0.067 & -0.123 & 0.029 & -0.003 & -2.32 & -0.09 & 1692.47 & 48.47 & & & -0.08 & 49.55 \\
\hline $0 \quad 226$ & 0.08 & 0.10 & -0.03 & 0.01 & 0.089 & -0.138 & 0.043 & 0.001 & -2.41 & 0.38 & 1701.60 & 47.41 & & & 0.41 & 48.46 \\
\hline 1227 & 0.09 & 0.11 & -0.03 & 0.01 & 0.101 & -0.152 & 0.044 & 0.002 & -2.79 & 0.46 & 1709.06 & 48.02 & & & 0.49 & 49.02 \\
\hline 2228 & 0.14 & 0.08 & -0.05 & 0.01 & 0.152 & -0.111 & 0.072 & 0.005 & -2.10 & 0.80 & 1718.01 & 47.15 & & & 0.85 & 48.11 \\
\hline 3229 & 0.20 & 0.00 & -0.06 & 0.00 & 0.216 & 0.000 & 0.092 & 0.018 & -2.12 & 0.61 & 1725.46 & 47.77 & & & 0.63 & 48.67 \\
\hline 230 & 0.19 & 0.00 & -0.07 & 0.00 & 0.206 & 0.000 & 0.103 & 0.020 & -2.76 & 0.40 & 1734.66 & 46.64 & & & 0.46 & 47.54 \\
\hline 5231 & 0.19 & 0.00 & -0.07 & 0.00 & 0.206 & 0.000 & 0.103 & 0.020 & -3.18 & 0.02 & 1742.00 & 47.37 & & & 0.08 & 48.22 \\
\hline 6232 & 0.19 & 0.00 & -0.07 & 0.01 & 0.205 & 0.000 & 0.103 & 0.010 & -3.23 & -0.11 & 1750.82 & 46.62 & & & -0.04 & 47.44 \\
\hline $7 \quad 233$ & 0.19 & 0.00 & -0.07 & 0.01 & 0.205 & 0.000 & 0.103 & 0.010 & -3.65 & -0.48 & 1757.89 & 47.63 & 47.29 & 0.072 & -0.43 & 48.41 \\
\hline $8 \quad 234$ & 0.19 & 0.00 & -0.07 & 0.01 & 0.205 & 0.000 & 0.103 & 0.010 & -3.80 & -0.61 & 1766.43 & 47.16 & 46.72 & 0.018 & -0.54 & 47.93 \\
\hline 235 & 0.20 & 0.00 & -0.07 & 0.02 & 0.215 & 0.000 & 0.106 & 0.001 & -4.32 & -1.03 & $17^{\prime}$ & 48.39 & & & -0.97 & 49.13 \\
\hline 236 & 0.20 & 0.00 & -0.07 & 0.02 & 0.215 & 0.000 & 0.106 & 0.001 & -4.51 & -1.21 & 1781.57 & 48.15 & & & -1.13 & 48.88 \\
\hline 237 & 0.20 & 0.00 & -0.07 & 0.02 & 0.215 & 0.000 & 0.106 & 0.001 & -4.93 & -1.58 & & 49.71 & & & & 50.41 \\
\hline 2238 & 0.21 & 0.00 & -0.06 & 0.03 & 0.226 & 0.000 & 0.095 & -0.012 & -4.84 & -1.78 & $17 \mathrm{C}$ & 49.72 & 49.40 & 0.037 & -1.69 & 50.42 \\
\hline 3239 & 0.21 & 0.00 & -0.06 & 0.03 & 0.226 & 0.000 & 0.095 & -0.012 & -5.23 & -2.14 & 1802.40 & 51.54 & & & -2.06 & 52.22 \\
\hline 240 & 0.22 & 0.00 & -0.05 & 0.03 & 0.237 & 0.000 & 0.085 & -0.014 & -4.93 & -2.12 & .97 & 52.04 & 51.72 & 02 & -2.04 & 52.71 \\
\hline 241 & 0.22 & 0.00 & -0.05 & 0.04 & 0.237 & 0.000 & 0.086 & -0.024 & -5.48 & -2.55 & .03 & 54.05 & 53.70 & 0.002 & -2.44 & 54.74 \\
\hline 242 & 0.22 & 0.00 & -0.05 & 0.04 & 0.237 & 0.000 & 0.086 & -0.024 & -5.39 & -2.47 & & 87 & & & & .57 \\
\hline 243 & 0.23 & 0.00 & -0.04 & 0.04 & 0.248 & 0.000 & 0.076 & -0.026 & -5.54 & -2.79 & 1828.99 & 57.23 & 57.18 & 0.002 & -2.69 & 57.91 \\
\hline 8244 & 0.23 & 0.00 & -0.03 & 0.04 & 0.249 & 0.000 & 0.063 & -0.029 & -5.20 & -2.67 & 18 & 58.35 & 58.45 & 0.002 & -2.56 & 03 \\
\hline 245 & 0.23 & 0.00 & -0.03 & 0.04 & 0.249 & 0.000 & 0.063 & -0.029 & -5.55 & -3.01 & 1841.42 & 60.94 & 61.01 & 0.002 & -2.90 & 61.63 \\
\hline 246 & 0.23 & 0.00 & -0.02 & 0.04 & 0.249 & 0.000 & 0.051 & -0.032 & -5.28 & -2.92 & & 62.28 & 62.62 & 0.002 & -2.80 & 62.99 \\
\hline 247 & 0.23 & 0.00 & -0.01 & 0.04 & 0.250 & 0.000 & 0.039 & -0.035 & -5.49 & -3.20 & & & & & & 88 \\
\hline 2248 & 0.23 & 0.00 & -0.01 & 0.04 & 0.250 & 0.000 & 0.039 & -0.035 & -5.24 & -2.99 & 1859.72 & 66.86 & 67.39 & 0.005 & -2.86 & 67.60 \\
\hline 3249 & 0.22 & 0.00 & -0.01 & 0.03 & 0.238 & 0.000 & 0.036 & -0.025 & -4.77 & -2.80 & 1864.20 & 70.45 & 70.75 & 0.005 & -2.74 & 71.15 \\
\hline 250 & 0.23 & 0.00 & 0.00 & 0.03 & 0.250 & 0.000 & 0.026 & -0.027 & -4.30 & -2.37 & 1870.12 & 72.60 & 72.99 & 0.011 & -2.30 & 73.33 \\
\hline $5 \quad 251$ & 0.22 & 0.00 & 0.00 & 0.03 & 0.239 & 0.000 & 0.024 & -0.028 & -4.01 & -2.19 & 1874.38 & 76.41 & 76.65 & 0.023 & -2.12 & 77.16 \\
\hline 6252 & 0.22 & 0.00 & 0.01 & 0.02 & 0.240 & 0.000 & 0.011 & -0.021 & -3.32 & -1.71 & 1880.01 & 78.85 & & & -1.67 & 79.60 \\
\hline $57 \quad 253$ & 0.22 & 0.00 & 0.01 & 0.02 & 0.240 & 0.000 & 0.011 & -0.021 & -3.30 & -1.70 & 1884.23 & 82.71 & & & -1.68 & 83.48 \\
\hline $8 \quad 254$ & 0.21 & 0.00 & 0.01 & 0.02 & 0.228 & 0.000 & 0.009 & -0.021 & -2.84 & -1.37 & 1889.78 & 85.23 & & & -1.33 & 86.04 \\
\hline $59 \quad 255$ & 0.21 & 0.00 & 0.02 & 0.01 & 0.229 & 0.000 & -0.004 & -0.013 & -2.94 & -1.43 & 1893.84 & 89.24 & & & -1.42 & 90.06 \\
\hline $60 \quad 256$ & 0.21 & 0.00 & 0.03 & 0.01 & 0.229 & 0.000 & -0.016 & -0.016 & -2.88 & -1.28 & 1899.36 & 91.79 & & & -1.26 & 92.67 \\
\hline 61257 & 0.21 & 0.00 & 0.04 & 0.00 & 0.230 & 0.000 & -0.030 & -0.009 & -3.19 & -1.48 & 1903.35 & 95.88 & & & -1.47 & 96.79 \\
\hline $62 \quad 258$ & 0.21 & 0.00 & 0.04 & 0.00 & 0.230 & 0.000 & -0.030 & -0.009 & -3.02 & -1.30 & 1908.61 & 98.68 & & & -1.28 & 99.65 \\
\hline 163259 & 0.20 & 0.00 & 0.04 & 0.00 & 0.219 & 0.000 & -0.031 & -0.008 & -2.95 & -1.30 & 1912.20 & 103.17 & & & -1.29 & 104.19 \\
\hline $64 \quad 260$ & 0.19 & 0.00 & 0.04 & 0.00 & 0.207 & 0.000 & -0.033 & -0.008 & -2.36 & -0.82 & 1916.94 & 106.49 & & & -0.79 & 107.58 \\
\hline 65261 & 0.18 & 0.00 & 0.04 & 0.00 & 0.196 & 0.000 & -0.034 & -0.007 & -2.18 & -0.69 & 1920.19 & 111.32 & & & -0.67 & 112.46 \\
\hline 166262 & 0.17 & 0.00 & 0.03 & 0.00 & 0.185 & 0.000 & -0.024 & -0.005 & -1.51 & -0.31 & 1924.83 & 114.75 & & & -0.29 & 115.95 \\
\hline 167263 & 0.16 & 0.00 & 0.02 & 0.01 & 0.173 & 0.000 & -0.013 & -0.013 & -1.54 & -0.52 & 1928.22 & 119.43 & & & -0.50 & 120.70 \\
\hline $168 \quad 264$ & 0.16 & 0.00 & 0.02 & 0.00 & 0.173 & 0.000 & -0.013 & -0.003 & -1.18 & -0.14 & 1932.66 & 123.06 & & & -0.14 & 124.39 \\
\hline 169265 & 0.13 & 0.00 & 0.01 & 0.01 & 0.140 & 0.000 & -0.004 & -0.011 & -1.22 & -0.37 & 1935.87 & 127.92 & & & -0.36 & 129.33 \\
\hline $170 \quad 266$ & 0.12 & 0.00 & 0.01 & 0.01 & 0.129 & 0.000 & -0.005 & -0.011 & -1.07 & -0.29 & 1940.41 & 131.45 & & & -0.28 & 132.95 \\
\hline 171267 & 0.12 & 0.00 & 0.01 & 0.01 & 0.129 & 0.000 & -0.005 & -0.011 & -1.49 & -0.70 & 1943.61 & 136.32 & & & -0.69 & 137.89 \\
\hline 72268 & 0.12 & 0.00 & 0.01 & 0.00 & 0.129 & 0.000 & -0.006 & -0.001 & -1.34 & -0.56 & 1947.90 & 140.11 & & & -0.57 & 141.75 \\
\hline 173269 & 0.12 & 0.00 & 0.02 & 0.00 & 0.129 & 0.000 & -0.018 & -0.002 & -1.83 & -0.98 & 1950.91 & 145.17 & & & -0.97 & 146.91 \\
\hline $74 \quad 270$ & 0.12 & 0.00 & 0.03 & -0.01 & 0.129 & 0.000 & -0.031 & 0.006 & -1.79 & -0.84 & 1955.01 & 149.14 & & & -0.81 & 151.01 \\
\hline $75 \quad 271$ & -0.11 & 0.00 & 0.00 & -0.01 & -0.115 & 0.000 & 0.005 & 0.009 & -1.94 & -1.33 & 1957.91 & 154.31 & & & -1.32 & 156.25 \\
\hline
\end{tabular}




\section{$Z=96(\mathrm{Cm})$}

$\begin{array}{llll}176 & 272 & -0.10 & 0.00\end{array}$ $\begin{array}{llll}177 & 273 & -0.10 & 0.00\end{array}$ $\begin{array}{llll}178 & 274 & -0.10 & 0.00\end{array}$ $\begin{array}{llll}179 & 275 & -0.08 & 0.00\end{array}$ $\begin{array}{llll}180 & 276 & -0.07 & 0.00\end{array}$

$\begin{array}{llll}181 & 277 & -0.04 & 0.00\end{array}$

$\begin{array}{llll}182 & 278 & 0.00 & 0.00\end{array}$

$\begin{array}{llll}183 & 279 & 0.00 & 0.00\end{array}$

$\begin{array}{llll}184 & 280 & 0.00 & 0.00\end{array}$

$\begin{array}{lllll}185 & 281 & 0.01 & 0.05 & 0.00\end{array}$

$\begin{array}{lllll}186 & 282 & 0.01 & 0.06 & 0.00\end{array}$

$\begin{array}{llllll}187 & 283 & 0.03 & 0.09 & -0.01\end{array}$

$\begin{array}{llllll}188 & 284 & 0.05 & 0.10 & -0.02\end{array}$

$\begin{array}{lllll}189 & 285 & 0.06 & 0.11 & -0.02\end{array}$

$\begin{array}{lllll}190 & 286 & 0.37 & 0.00 & 0.08\end{array}$

$\begin{array}{lllll}191 & 287 & 0.37 & 0.00 & 0.08\end{array}$

$\begin{array}{lllll}192 & 288 & 0.36 & 0.00 & 0.08\end{array}$

$\begin{array}{llllll}193 & 289 & 0.23 & 0.00 & -0.03\end{array}$

194290

195291

196292

197293

198294

199295

200296

201297

202298

$203 \quad 299$

204300

205301

$\begin{array}{llll}0.23 & 0.00 & -0.03\end{array}$

$\begin{array}{llll}0.23 & 0.00 & -0.03\end{array}$

$\begin{array}{llll}0.23 & 0.00 & -0.03\end{array}$

$\begin{array}{llll}0.23 & 0.00 & -0.03\end{array}$

$\begin{array}{llll}0.24 & 0.00 & -0.02\end{array}$

$\begin{array}{llll}0.25 & 0.00 & -0.01\end{array}$

$\begin{array}{lll}0.26 & 0.00 & 0.00\end{array}$

$\begin{array}{lll}0.26 & 0.00 & 0.01\end{array}$

$\begin{array}{lll}0.26 & 0.00 & 0.01\end{array}$

$\begin{array}{lll}0.25 & 0.00 & 0.00\end{array}$

$\begin{array}{llll}0.24 & 0.00 & -0.01\end{array}$

$\begin{array}{llll}0.23 & 0.00 & -0.02\end{array}$

206302

$\begin{array}{llll}0.22 & 0.00 & -0.03\end{array}$

207303

$\begin{array}{llll}0.21 & 0.00 & -0.04\end{array}$

$208 \quad 304$

$\begin{array}{llll}0.21 & 0.00 & -0.04\end{array}$

209305

210306

$\begin{array}{llll}0.21 & 0.00 & -0.04\end{array}$

$\begin{array}{llll}0.20 & 0.00 & -0.04\end{array}$

211307

$\begin{array}{lll}0.20 & 0.00 & -0.04\end{array}$

212308

213309

$\begin{array}{llll}0.20 & 0.00 & -0.03\end{array}$

$\begin{array}{llll}0.20 & 0.00 & -0.03\end{array}$

$\begin{array}{llll}0.20 & 0.00 & -0.02\end{array}$

214310

215311

216312

$\begin{array}{llll}0.20 & 0.00 & -0.02\end{array}$

$\begin{array}{ll}217 & 313\end{array}$

$\begin{array}{llll}0.20 & 0.00 & -0.01\end{array}$

$218 \quad 314$

$\begin{array}{llll}0.20 & 0.00 & -0.01\end{array}$

$\begin{array}{lll}0.21 & 0.00 & 0.00\end{array}$

219315

$\begin{array}{ll}0.21 & 0.00\end{array}$

0.01

$\begin{array}{lll}0.21 & 0.00 & 0.01\end{array}$

220316

221317

$0.20 \quad 0.00$

222318
$-0.01-0.105$

$0.00-0.105$

$0.000-0.007$

$\begin{array}{lllll}0.011 & -1.97 & -1.45 & 1962.08 & 158.21\end{array}$

$\begin{array}{lllllll}0.000 & -0.019 & 0.002 & -2.49 & -1.94 & 1964.81 & 163.55\end{array}$

$0.000-0.019$

$\begin{array}{lllll}0.012 & -2.43 & -1.87 & 1968.60 & 167.84\end{array}$

$0.00-0.084$

$0.000-0.021$

$\begin{array}{lllll}0.002 & -2.42 & -1.89 & 1970.67 & 173.83\end{array}$

$0.01-0.073$

$0.000-0.021$

$\begin{array}{lllll}-0.008 & -2.14 & -1.57 & 1974.03 & 178.55\end{array}$

$0.00-0.042$

$0.000-0.011$

$\begin{array}{llllll}0.001 & -2.35 & -1.75 & 1976.10 & 184.55\end{array}$

$0.00 \quad 0.000$

$0.000 \quad 0.000$

$\begin{array}{lllll}0.000 & -2.21 & -1.60 & 1979.45 & 189.27\end{array}$

$0.00 \quad 0.000$

$0.000 \quad 0.000$

$\begin{array}{lllll}0.000 & -2.61 & -1.97 & 1981.55 & 195.24\end{array}$

$0.00 \quad 0.000$

0.000

0.000

$\begin{array}{llllll}0.000 & -2.31 & -1.68 & 1984.58 & 200.28\end{array}$

$\begin{array}{lll}0.00 & 0.012 & -0.067\end{array}$

0.001

$\begin{array}{lllll}0.002 & -1.99 & -0.98 & 1985.44 & 207.50\end{array}$

$\begin{array}{llll}0.00 & 0.012 & -0.081\end{array}$

0.002

$\begin{array}{lllll}0.002 & -1.52 & -0.34 & 1987.96 & 213.05\end{array}$

$\begin{array}{llll}0.00 & 0.035 & -0.123\end{array}$

0.016

$\begin{array}{llllll}0.006 & -2.21 & -0.20 & 1989.21 & 219.87\end{array}$

$\begin{array}{lll}0.01 & 0.057 & -0.136\end{array}$

$\begin{array}{llllll}0.029 & -0.002 & -2.20 & 0.06 & 1991.94 & 225.21\end{array}$

$\begin{array}{llllllllll}0.01 & 0.068 & -0.150 & 0.030 & 0.000 & -2.76 & -0.09 & 1993.32 & 231.90\end{array}$

$\begin{array}{lllllllll}0.01 & 0.419 & 0.000 & -0.033 & -0.036 & -2.74 & 0.25 & 1995.81 & 237.48\end{array}$

$0.01 \quad 0.419$

$\begin{array}{lllllll}0.000 & -0.033 & -0.036 & -3.00 & -0.03 & 1997.17 & 244.20\end{array}$

$0.00 \quad 0.406$

$0.000-0.038-0$.

$0.00 \quad 0.249$

$0.01 \quad 0.249$

0.000

0.060

$\begin{array}{lll}0.19 & 1999.61 & 249.82\end{array}$

0.01

0.249

0.000

0.061

0.001

$0.01 \quad 0.249$

$0.01 \quad 0.249$

0.000

0.061

0.001

0.000

0.061

$\begin{array}{llllll}0.001 & -2.14 & -0.39 & 2006.76 & 274.96\end{array}$

$0.02 \quad 0.260$

0.000

0.061

$\begin{array}{lllll}0.001 & -2.46 & -0.70 & 2007.70 & 282.09\end{array}$

$0.02 \quad 0.272$

$0.02 \quad 0.284$

0.000

$\begin{array}{lllllll}-0.011 & -2.33 & -0.68 & 2009.88 & 287.98\end{array}$

0.000

$\begin{array}{llllll}0.041 & -0.014 & -2.57 & -0.96 & 2010.65 & 295.29\end{array}$

$0.02 \quad 0.284$

0.000

$\begin{array}{llllll}0.032 & -0.016 & -2.47 & -0.90 & 2012.64 & 301.36\end{array}$

$0.03 \quad 0.285$

$0.03 \quad 0.273$

0.000

$\begin{array}{llllll}0.020 & -0.020 & -2.76 & -1.21 & 2013.29 & 308.78\end{array}$

$0.03 \quad 0.261$

0.000

$\begin{array}{llllll}0.021 & -0.030 & -2.93 & -1.34 & 2015.33 & 314.82\end{array}$

0.000

$\begin{array}{llllll}0.030 & -0.027 & -3.19 & -1.69 & 2015.88 & 322.34\end{array}$

$\begin{array}{lll}0.03 & 0.249 & 0.000\end{array}$

$\begin{array}{llllll}0.040 & -0.024 & -3.05 & -1.54 & 2017.50 & 328.79\end{array}$

$\begin{array}{lll}0.04 & 0.237 & 0.000\end{array}$

$\begin{array}{llllll}0.050 & -0.022 & -3.39 & -1.81 & 2017.84 & 336.52\end{array}$

$0.04 \quad 0.226$

0.000

$\begin{array}{llllll}0.061 & -0.030 & -3.65 & -1.92 & 2019.58 & 342.85\end{array}$

$0.04 \quad 0.226$

0.000

$\begin{array}{llllll}0.071 & -0.028 & -4.19 & -2.33 & 2019.92 & 350.58\end{array}$

$0.04 \quad 0.226$

0.000

$\begin{array}{llllll}0.071 & -0.028 & -4.19 & -2.36 & 2021.43 & 357.14\end{array}$

$0.04 \quad 0.215$

0.000

$\begin{array}{lllllll}0.071 & -0.028 & -4.55 & -2.71 & 2021.59 & 365.06\end{array}$

$\begin{array}{llllll}0.069 & -0.028 & -4.44 & -2.68 & 2022.91 & 371.80\end{array}$

$0.04 \quad 0.215$

0.000

$\begin{array}{llllll}0.069 & -0.028 & -4.66 & -2.89 & 2022.80 & 379.98\end{array}$

$0.04 \quad 0.215$

0.000

$\begin{array}{llllll}0.057 & -0.031 & -4.25 & -2.67 & 2023.80 & 387.06\end{array}$

0.000

$\begin{array}{llllll}0.057 & -0.031 & -4.42 & -2.85 & 2023.53 & 395.40\end{array}$

$0.04 \quad 0.216$

0.000

$\begin{array}{lllllll}0.045 & -0.034 & -4.05 & -2.64 & 2024.41 & 402.60\end{array}$

$0.04 \quad 0.216$

0.000

$\begin{array}{llllll}0.045 & -0.034 & -4.29 & -2.89 & 2024.08 & 410.99\end{array}$

$0.04 \quad 0.216$

0.000

$0.04 \quad 0.216$

0.000

$\begin{array}{llllll}0.033 & -0.036 & -4.04 & -2.76 & 2024.91 & 418.23\end{array}$

$0.04 \quad 0.228$

0.000

$\begin{array}{llllll}0.033 & -0.036 & -4.35 & -3.10 & 2024.56 & 426.65\end{array}$

$\begin{array}{llllll}0.023 & -0.038 & -4.26 & -3.05 & 2025.35 & 433.94\end{array}$

$\begin{array}{lllllll}0.000 & 0.011 & -0.041 & -4.60 & -3.39 & 2024.87 & 442.49\end{array}$

$0.04 \quad 0.229$

$\begin{array}{llllll}0.011 & -0.041 & -4.45 & -3.27 & 2025.47 & 449.96\end{array}$

$0.04 \quad 0.218$

0.000

$\begin{array}{llllll}0.009 & -0.041 & -4.59 & -3.45 & 2024.71 & 458.79\end{array}$

$\begin{array}{lllllll}0.000 & -0.004 & -0.033 & -4.06 & -2.98 & 2024.83 & 466.74\end{array}$
$-1.44 \quad 160.26$

$-1.94 \quad 165.70$

$-1.84 \quad 170.11$

$-1.88 \quad 176.20$

$-1.54 \quad 181.05$

$-1.75 \quad 187.14$

$-1.60 \quad 191.99$

$\begin{array}{ll}-1.97 & 198.09\end{array}$

$-1.68 \quad 203.26$

$-0.95 \quad 210.64$

$-0.30 \quad 216.35$

$-0.10 \quad 223.37$

$0.21 \quad 228.90$

$0.07 \quad 235.76$

$0.38 \quad 241.47$

$0.08 \quad 248.32$
0.25

$0.25 \quad 254.06$

$0.03 \quad 261.15$

$0.04 \quad 266.90$

$-0.35 \quad 273.99$

$\begin{array}{ll}-0.36 & 279.87\end{array}$

$\begin{array}{ll}-0.69 & 287.18\end{array}$

$-0.64 \quad 293.28$

$-0.95 \quad 300.75$

$-0.89 \quad 307.03$

$-1.20 \quad 314.64$

$-1.19 \quad 321.02$

$-1.56 \quad 328.73$

$-1.40 \quad 335.41$

$-1.67 \quad 343.36$

$-1.59350 .11$

$-1.98 \quad 358.09$
-1.99

$\begin{array}{ll}-1.99 & 364.89\end{array}$

$\begin{array}{ll}-2.35 & 373.04\end{array}$

$-2.29 \quad 380.05$

$\begin{array}{ll}-2.52 & 388.47\end{array}$

$\begin{array}{ll}-2.30 & 395.78\end{array}$

$\begin{array}{ll}-2.49 & 404.37\end{array}$

$-2.27 \quad 411.83$

$\begin{array}{ll}-2.53 & 420.48\end{array}$

$\begin{array}{ll}-2.38 & 428.00\end{array}$

$\begin{array}{ll}-2.73 & 436.69\end{array}$

$-2.67 \quad 444.26$

$-2.98 \quad 453.11$

$\begin{array}{ll}-2.85 & 460.89\end{array}$

$-3.03 \quad 470.01$

$-2.73 \quad 478.08$

\section{$Z=97$ (Bk)}

$\begin{array}{llll}121 & 218 & -0.13 & 0.00\end{array}$

$\begin{array}{llll}122 & 219 & -0.11 & 0.00\end{array}$

$\begin{array}{lll}0.02 & -0.01 & -0.136\end{array}$

$0.000-0.016$

$\begin{array}{llll}0.012 & -1.15 & -0.19 & 1615.53\end{array}$

$0.000-0.018$

$\begin{array}{llll}0.002 & -1.16 & -0.47 & 1626.75\end{array}$

68.13

$\begin{array}{llll}123 & 220 & -0.10 & 0.00\end{array}$

0.02

$0.00-0.115$

$0.000 \quad-0.019$

$\begin{array}{llll}0.002 & -1.62 & -1.01 & 1636.50\end{array}$

$\begin{array}{llll}124 & 221 & -0.09 & 0.00\end{array}$

0.03

$0.00-0.105$

$0.000-0.032$

$\begin{array}{rrrr}0.002 & -1.62 & -1.01 & 1636.50 \\ -0.006 & -1.90 & -1.16 & 1647.25\end{array}$

64.99 


\section{$Z=97$ (Bk)} $\begin{array}{llllll}131 & 228 & 0.09 & 0.11 & -0.03\end{array}$ $\begin{array}{llllll}132 & 229 & 0.21 & 0.00 & -0.05\end{array}$ $\begin{array}{llllll}133 & 230 & 0.21 & 0.00 & -0.05\end{array}$ $\begin{array}{lllll}134 & 231 & 0.20 & 0.00 & -0.06\end{array}$

$\begin{array}{llllll}135 & 232 & 0.20 & 0.00 & -0.06\end{array}$ $\begin{array}{llllll}136 & 233 & 0.20 & 0.00 & -0.06\end{array}$ $\begin{array}{llllll}137 & 234 & 0.20 & 0.00 & -0.06\end{array}$ $\begin{array}{llllll}138 & 235 & 0.20 & 0.00 & -0.06\end{array}$ $\begin{array}{llllll}139 & 236 & 0.20 & 0.00 & -0.06\end{array}$

$\begin{array}{llllll}140 & 237 & 0.21 & 0.00 & -0.06\end{array}$

$\begin{array}{llllll}141 & 238 & 0.22 & 0.00 & -0.05\end{array}$

$\begin{array}{llllll}142 & 239 & 0.21 & 0.00 & -0.06\end{array}$

$\begin{array}{llllll}143 & 240 & 0.22 & 0.00 & -0.05\end{array}$

$\begin{array}{llllll}144 & 241 & 0.22 & 0.00 & -0.05\end{array}$

$\begin{array}{llllll}145 & 242 & 0.22 & 0.00 & -0.05\end{array}$

$\begin{array}{llllll}146 & 243 & 0.22 & 0.00 & -0.04\end{array}$

$\begin{array}{llllll}147 & 244 & 0.22 & 0.00 & -0.04\end{array}$

$\begin{array}{llllll}148 & 245 & 0.23 & 0.00 & -0.03\end{array}$

$\begin{array}{llllll}149 & 246 & 0.23 & 0.00 & -0.02\end{array}$

$\begin{array}{llllll}150 & 247 & 0.23 & 0.00 & -0.02\end{array}$

$\begin{array}{llllll}151 & 248 & 0.23 & 0.00 & -0.01\end{array}$

$\begin{array}{llllll}152 & 249 & 0.23 & 0.00 & -0.01\end{array}$

$\begin{array}{lllll}153 & 250 & 0.23 & 0.00 & 0.00\end{array}$

$\begin{array}{lllll}154 & 251 & 0.23 & 0.00 & 0.00\end{array}$

$155 \quad 252$

$\begin{array}{lll}0.23 & 0.00 & 0.01\end{array}$

156253

0.220 .00

0.01

$157 \quad 254$

$0.22 \quad 0.00$

0.01

$158 \quad 255$

$\begin{array}{lll}0.22 & 0.00 & 0.02\end{array}$

159256

$0.21 \quad 0.00$

0.02

$\begin{array}{ll}160 & 257\end{array}$

$0.21 \quad 0.00$

0.03

161258
162

$\begin{array}{ll}0.21 & 0.00\end{array}$

0.04

$0.21 \quad 0.00$

0.04

163260

$0.20 \quad 0.00$

0.04

164261

$0.20 \quad 0.00$

0.04

$\begin{array}{ll}0.18 & 0.00\end{array}$

0.04

$\begin{array}{ll}165 & 262 \\ 166 & 263\end{array}$

$\begin{array}{lll}0.17 & 0.00 & 0.03\end{array}$

$\begin{array}{ll}167 \quad 264 \\ 168 & 265\end{array}$

$\begin{array}{lll}0.16 & 0.00 & 0.02\end{array}$

$\begin{array}{lll}0.16 & 0.00 & 0.02\end{array}$

169266

0.140 .00

0.01

$\begin{array}{ll}0.13 & 0.00\end{array}$

0.01

$171 \quad 268$

$\begin{array}{ll}0.12 & 0.00\end{array}$

$0.12 \quad 0.00$

$172 \quad 269$

$\begin{array}{ll}0.12 & 0.00\end{array}$

0.01

0.01

0.02

$174 \quad 271$

0.120 .00

0.03

0.00
-0.01

$\begin{array}{lll}0.01 & 0.089 & -0.137\end{array}$

$\begin{array}{llll}0.01 & 0.101 & -0.152\end{array}$

$\begin{array}{lll}0.00 & 0.227 & 0.000\end{array}$

$\begin{array}{lll}0.030 & -0.001 & -2.04\end{array}$

$\begin{array}{lll}0.044 & 0.002 & -2.89\end{array}$

$\begin{array}{ll}0.64 & 1700.97\end{array}$

$\begin{array}{lll}0.081 & 0.016 & -1.87\end{array}$

$0.41 \quad 1709.15$

$\begin{array}{ll}0.39 & 1718.49\end{array}$

$\begin{array}{lll}0.00 & 0.227 & 0.000\end{array}$

0.081

$\begin{array}{ll}0.016 & -2.13\end{array}$

$\begin{array}{ll}0.14 & 1726.39\end{array}$

$0.008-2.40$

$0.20 \quad 1735.35$

0.093

$\begin{array}{llll}0.008 & -2.78 & -0.09 & 1743.00\end{array}$

$\begin{array}{lll}0.01 & 0.216 & 0.000\end{array}$

$\begin{array}{lll}0.01 & 0.216 & 0.000\end{array}$

$\begin{array}{lll}0.01 & 0.216 & 0.000\end{array}$

$\begin{array}{lll}0.02 & 0.215 & 0.000\end{array}$

0.093

$\begin{array}{llll}0.008 & -2.97 & -0.25 & 1751.90\end{array}$

0.093

$\begin{array}{llll}0.008 & -3.39 & -0.63 & 1759.34\end{array}$

$0.02 \quad 0.215$

0.000

$0.093-0$.

$\begin{array}{llll}0.002 & -3.58 & -0.80 & 1767.96\end{array}$

$0.02 \quad 0.226$

$0.03 \quad 0.237$

$0.03 \quad 0.226$

$0.03 \quad 0.237$

0.000

$\begin{array}{llllll}0.093 & -0.002 & -4.05 & -1.23 & 1775.19\end{array}$

0.000

$\begin{array}{llllll}0.095 & -0.001 & -4.38 & -1.46 & 1783.59\end{array}$

0.000

$\begin{array}{llllll}0.085 & -0.014 & -4.58 & -1.83 & 1790.49\end{array}$

$\begin{array}{lllll}0.095 & -0.012 & -5.12 & -2.09 & 1798.63\end{array}$

0.000

$\begin{array}{lllll}0.085 & -0.014 & -5.26 & -2.55 & 1805.36\end{array}$

$\begin{array}{lll}0.04 & 0.237 & 0.000\end{array}$

$\begin{array}{lllll}0.086 & -0.024 & -5.48 & -2.60 & 1813.04\end{array}$

$\begin{array}{lll}0.04 & 0.237 & 0.000\end{array}$

$\begin{array}{lll}0.04 & 0.237 & 0.000\end{array}$

$\begin{array}{lllll}0.086 & -0.024 & -5.85 & -2.94 & 1819.38\end{array}$

$\begin{array}{lll}0.04 & 0.237 & 0.000\end{array}$

$\begin{array}{llllll}0.073 & -0.027 & -5.53 & -2.90 & 1826.70\end{array}$

$\begin{array}{llllll}0.073 & -0.027 & -5.89 & -3.22 & 1832.78\end{array}$

$\begin{array}{lllll}0.063 & -0.029 & -5.69 & -3.18 & 1839.85\end{array}$

$\begin{array}{llllll}0.051 & -0.032 & -5.89 & -3.51 & 1845.68\end{array}$

0.249

0.000

$\begin{array}{llllll}0.051 & -0.032 & -5.83 & -3.47 & 1852.51\end{array}$

$0.04 \quad 0.249$

0.000

0.250

0.000

$\begin{array}{llllll}0.039 & -0.035 & -6.05 & -3.77 & 1858.06\end{array}$

$0.04 \quad 0.250$

0.000

$\begin{array}{llllll}0.039 & -0.035 & -5.81 & -3.56 & 1864.47\end{array}$

0.000

$\begin{array}{llllll}0.026 & -0.027 & -5.36 & -3.38 & 1869.32\end{array}$

0.250

0.000

$\begin{array}{lllll}0.026 & -0.027 & -4.87 & -2.94 & 1875.26\end{array}$

$0.03 \quad 0.251$

$0.03 \quad 0.240$

0.000

$\begin{array}{llllll}0.014 & -0.030 & -4.66 & -2.75 & 1879.87\end{array}$

0.000

$\begin{array}{llllll}0.012 & -0.031 & -4.09 & -2.30 & 1885.56\end{array}$

0.240

0.000

$\begin{array}{lllll}0.011 & -0.021 & -3.85 & -2.26 & 1890.09\end{array}$

$0.02 \quad 0.240$$$
\begin{array}{llllll}
0.004 & -0.013 & -3.45 & -1.93 & 1900.03
\end{array}
$$

$0.01 \quad 0.229$

$\begin{array}{llllll}0.000 & -0.016 & -0.016 & -3.40 & -1.79 & 1905.58\end{array}$

$\begin{array}{llllll}0.000 & -0.028 & -0.018 & -3.77 & -1.97 & 1909.90\end{array}$

$\begin{array}{llllll}0.000 & -0.030 & -0.009 & -3.49 & -1.76 & 1915.16\end{array}$

$0.00-0.219$

$\begin{array}{lllllll}0.000 & -0.031 & -0.008 & -3.42 & -1.76 & 1919.08 & 103.57\end{array}$

$\begin{array}{lllllll}0.000 & -0.031 & -0.008 & -2.85 & -1.24 & 1923.82 & 106.90\end{array}$

$0.00-0.196$

$\begin{array}{lllllll}0.000 & -0.034 & -0.007 & -2.59 & -1.07 & 1927.37 & 111.42\end{array}$

$0.00 \quad 0.185$

$\begin{array}{lllllll}0.000 & -0.024 & -0.005 & -1.86 & -0.64 & 1931.99 & 114.88\end{array}$

$\begin{array}{llllllll}0.000 & -0.013 & -0.013 & -1.86 & -0.81 & 1935.68 & 119.26\end{array}$

0.010 .173

$\begin{array}{lllllll}0.000 & -0.013 & -0.013 & -1.55 & -0.45 & 1940.16 & 122.86\end{array}$

$0.01 \quad 0.151$

$\begin{array}{lllllll}0.000 & -0.003 & -0.011 & -1.47 & -0.57 & 1943.59 & 127.49\end{array}$

$0.01 \quad 0.140$

$\begin{array}{lllllll}0.000 & -0.004 & -0.011 & -1.30 & -0.47 & 1948.14 & 131.02\end{array}$

$0.01 \quad 0.129$

$\begin{array}{lllllll}0.000 & -0.005 & -0.011 & -1.62 & -0.84 & 1951.63 & 135.59\end{array}$

$\begin{array}{llllllll}0.000 & -0.006 & -0.001 & -1.45 & -0.69 & 1955.92 & 139.38\end{array}$

$\begin{array}{lllllll}0.000 & -0.018 & -0.002 & -1.93 & -1.08 & 1959.25 & 144.12\end{array}$

0.129

$0.000-0.031$

$\begin{array}{lllll}0.006 & -1.88 & -0.97 & 1963.38 & 148.06\end{array}$

$\begin{array}{llll}175 & 272 & 0.12 & 0.00\end{array}$

$0.03-0.01 \quad 0.129$

$0.000-0.031$

$\begin{array}{lllll}0.006 & -2.15 & -1.24 & 1966.41 & 153.10\end{array}$

$\begin{array}{llll}176 & 273 & -0.10 & 0.00\end{array}$

$\begin{array}{lll}0.01 & 0.00 & -0.105\end{array}$

$0.000-0.008$

$\begin{array}{lllll}0.001 & -1.87 & -1.38 & 1970.61 & 156.97\end{array}$

$\begin{array}{llll}177 & 274 & -0.10 & 0.00\end{array}$

$\begin{array}{llll}0.02 & 0.00 & -0.105\end{array}$

$0.000-0.019$

$\begin{array}{llllll}0.002 & -2.43 & -1.90 & 1973.69 & 161.96\end{array}$

$\begin{array}{llll}178 & 275 & -0.10 & 0.00\end{array}$

0.02

$0.00-0.105$

$0.000 \quad-0.019$

$\begin{array}{lllll}0.002 & -2.31 & -1.78 & 1977.45 & 166.27\end{array}$

$\begin{array}{llll}179 & 276 & -0.08 & 0.00 \\ 180 & 277 & -0.07 & 0.00\end{array}$

0.02

$0.00-0.084$

$0.000-0.021$

$\begin{array}{lllll}0.002 & -2.30 & -1.78 & 1979.83 & 171.96\end{array}$

$\begin{array}{llll}181 & 278 & -0.05 & 0.00\end{array}$

$\begin{array}{llll}182 & 279 & -0.02 & 0.00\end{array}$

$0.000-0.021$

$\begin{array}{rrrrr}0.002 & -1.99 & -1.44 & 1983.19 & 176.68 \\ -0.008 & -2.25 & -1.61 & 1985.56 & 182.37\end{array}$

$0.01-0.052$

$0.000-0.022$

0.01

$0.00-0.021$

$0.000-0.012$

$\begin{array}{llllll}0.000 & -1.93 & -1.31 & 1988.79 & 187.23\end{array}$

$\begin{array}{lllll}183 & 280 & 0.01 & 0.01 & 0.00\end{array}$

$\begin{array}{lll}0.00 & 0.011 & -0.013\end{array}$

0.000

$\begin{array}{llllll}0.000 & -2.29 & -1.67 & 1991.19 & 192.89\end{array}$

18428

$\begin{array}{lll}0.00 & 0.02 & 0.00\end{array}$

0.00

$0.000-0.027$

0.000

$\begin{array}{llllll}0.000 & -2.03 & -1.37 & 1994.23 & 197.92\end{array}$

$\begin{array}{lllll}185 & 282 & 0.02 & 0.06 & -0.01\end{array}$

$\begin{array}{lll}0.00 & 0.023 & -0.081\end{array}$

0.013

$\begin{array}{llllll}0.003 & -2.09 & -0.88 & 1995.61 & 204.62\end{array}$

$\begin{array}{lll}0.00 & 0.023 & -0.095\end{array}$
0.014 $\begin{array}{llllll}0.004 & -1.72 & -0.30 & 1998.20 & 210.09\end{array}$

$\begin{array}{ll}0.65 & 56.54\end{array}$

$0.42 \quad 56.38$

$0.40 \quad 55.05$

$0.13 \quad 55.17$

$0.22 \quad 54.25$

$-0.08 \quad 54.63$

$-0.23 \quad 53.77$

$-0.61 \quad 54.36$

$-0.76 \quad 53.79$

$-1.20 \quad 54.60$

$-1.42 \quad 54.26$

$\begin{array}{ll}-1.80 & 55.39\end{array}$

$-2.03 \quad 55.33$

$-2.51 \quad 56.63$

$-2.52 \quad 57.06$

$-2.86 \quad 58.76$

$\begin{array}{llll}58.69 & 0.005 & -2.81 & 59.51\end{array}$

$\begin{array}{llll}60.72 & 0.014 & -3.14 & 61.49\end{array}$

$\begin{array}{llll}61.81 & 0.002 & -3.10 & 62.50\end{array}$

$\begin{array}{lllll}63.97 & 0.060 & -3.43 & 64.73\end{array}$

$\begin{array}{llll}65.49 & 0.006 & -3.38 & 66.00\end{array}$

$-3.68 \quad 68.51$

$\begin{array}{llll}69.85 & 0.003 & -3.46 & 70.20\end{array}$

$\begin{array}{llll}72.95 & 0.004 & -3.34 & 73.37\end{array}$

$\begin{array}{llll}75.23 & 0.011 & -2.88 & 75.54\end{array}$

$\begin{array}{ll}-2.70 & 79.01\end{array}$

$-2.24 \quad 81.43$

$-2.25 \quad 84.94$

$-1.90 \quad 87.48$

$-1.94 \quad 91.19$

$\begin{array}{ll}-1.78 \quad 93.77 \\ -1.95 & 97.57\end{array}$

$\begin{array}{ll}-1.95 & 97.57\end{array}$

$-1.76 \quad 100.41$

$-1.75104 .61$

$-1.23 \quad 108.00$ 


\section{$Z=97(\mathrm{Bk})$} $\begin{array}{llllll}188 & 285 & 0.05 & 0.10 & -0.02 & 0.01\end{array}$ $\begin{array}{lllllll}189 & 286 & 0.05 & 0.10 & -0.02 & 0.01\end{array}$ $\begin{array}{llllll}190 & 287 & 0.23 & 0.00 & -0.03 & 0.00\end{array}$ $\begin{array}{lllllll}191 & 288 & 0.23 & 0.00 & -0.03 & 0.00\end{array}$ $\begin{array}{lllllll}192 & 289 & 0.23 & 0.00 & -0.03 & 0.01\end{array}$ $\begin{array}{lllllll}193 & 290 & 0.23 & 0.00 & -0.03 & 0.01\end{array}$ $\begin{array}{lllllll}194 & 291 & 0.23 & 0.00 & -0.03 & 0.01\end{array}$ $\begin{array}{lllllll}195 & 292 & 0.23 & 0.00 & -0.03 & 0.01\end{array}$ $\begin{array}{llllll}196 & 293 & 0.24 & 0.00 & -0.02 & 0.01\end{array}$ $\begin{array}{lllllll}197 & 294 & 0.24 & 0.00 & -0.02 & 0.02\end{array}$ $\begin{array}{llllll}198 & 295 & 0.24 & 0.00 & -0.02 & 0.02\end{array}$ $\begin{array}{llllll}199 & 296 & 0.25 & 0.00 & -0.01 & 0.02\end{array}$ $\begin{array}{llllll}200 & 297 & 0.26 & 0.00 & 0.01 & 0.02\end{array}$ $\begin{array}{llllll}201 & 298 & 0.26 & 0.00 & 0.01 & 0.02\end{array}$ $\begin{array}{lllllll}202 & 299 & 0.26 & 0.00 & 0.01 & 0.03\end{array}$ $\begin{array}{llllll}203 & 300 & 0.25 & 0.00 & 0.00 & 0.03\end{array}$ $\begin{array}{llllll}204 & 301 & 0.24 & 0.00 & -0.01 & 0.03\end{array}$ $\begin{array}{llllll}205 & 302 & 0.23 & 0.00 & -0.02 & 0.04\end{array}$ $\begin{array}{llllll}206 & 303 & 0.23 & 0.00 & -0.02 & 0.04\end{array}$ $\begin{array}{lllllll}207 & 304 & 0.22 & 0.00 & -0.02 & 0.04\end{array}$ $\begin{array}{lllllll}208 & 305 & 0.21 & 0.00 & -0.03 & 0.04\end{array}$ $\begin{array}{llllll}209 & 306 & 0.21 & 0.00 & -0.03 & 0.04\end{array}$ $\begin{array}{llllll}210 & 307 & 0.21 & 0.00 & -0.03 & 0.04\end{array}$ $\begin{array}{llllll}211 & 308 & 0.20 & 0.00 & -0.03 & 0.04\end{array}$

$\begin{array}{lllllll}212 & 309 & 0.20 & 0.00 & -0.03 & 0.04\end{array}$ $\begin{array}{lllllll}213 & 310 & 0.20 & 0.00 & -0.02 & 0.04\end{array}$ $\begin{array}{llllll}214 & 311 & 0.20 & 0.00 & -0.02 & 0.04\end{array}$ $\begin{array}{llllll}215 & 312 & 0.21 & 0.00 & -0.01 & 0.04\end{array}$ $\begin{array}{llllll}216 & 313 & 0.20 & 0.00 & -0.01 & 0.04\end{array}$

$\begin{array}{lllllll}217 & 314 & 0.21 & 0.00 & 0.00 & 0.04\end{array}$ $\begin{array}{llllll}218 & 315 & 0.21 & 0.00 & 0.00 & 0.04\end{array}$ $\begin{array}{lllllll}219 & 316 & 0.21 & 0.00 & 0.01 & 0.04\end{array}$ $\begin{array}{llllll}220 & 317 & 0.21 & 0.00 & 0.01 & 0.04\end{array}$ $\begin{array}{llllll}221 & 318 & 0.21 & 0.00 & 0.02 & 0.03\end{array}$ $\begin{array}{lllllll}222 & 319 & 0.20 & 0.00 & 0.02 & 0.03\end{array}$ $\begin{array}{llllll}223 & 320 & 0.20 & 0.00 & 0.02 & 0.03\end{array}$ 224321 $\begin{array}{llllll}187 & 284 & 0.03 & 0.09 & -0.01 & 0.00\end{array}$
$0.035-0.123$

$0.057-0.136$

$0.057-0.136$

$0.249 \quad 0.000$

$0.249 \quad 0.000$

$0.249 \quad 0.000$

$0.249 \quad 0.000$

$0.249 \quad 0.000$

$0.249 \quad 0.000$

$0.261 \quad 0.000$

$\begin{array}{llllll}0.016 & 0.006 & -2.17 & -0.19 & 1999.80 & 216.57\end{array}$ $\begin{array}{llllll}0.029 & -0.002 & -2.18 & 0.20 & 2002.41 & 222.02\end{array}$ $\begin{array}{llllll}0.029 & -0.002 & -2.38 & 0.07 & 2004.09 & 228.42\end{array}$ $\begin{array}{llllll}0.060 & 0.012 & -1.54 & 0.21 & 2006.79 & 233.79\end{array}$ $\begin{array}{llllll}0.060 & 0.012 & -1.82 & -0.04 & 2008.43 & 240.23\end{array}$ $\begin{array}{lllllll}0.061 & 0.001 & -1.74 & -0.01 & 2011.07 & 245.65\end{array}$ $\begin{array}{llllll}0.061 & 0.001 & -2.03 & -0.27 & 2012.57 & 252.23\end{array}$ $\begin{array}{llllll}0.061 & 0.001 & -2.06 & -0.33 & 2015.14 & 257.72\end{array}$ $\begin{array}{llllllll}0.260 & 0.000 & 0.051 & -0.011 & -2.66 & -0.98 & 2020.17 & 276.91\end{array}$ $\begin{array}{llllllll}0.260 & 0.000 & 0.051 & -0.011 & -2.59 & -0.93 & 2022.33 & 282.82\end{array}$ $\begin{array}{llllllll}0.272 & 0.000 & 0.041 & -0.014 & -2.87 & -1.28 & 2023.47 & 289.75\end{array}$ $\begin{array}{llllllll}0.284 & 0.000 & 0.020 & -0.020 & -2.76 & -1.23 & 2025.48 & 295.82\end{array}$ $\begin{array}{llllllll}0.284 & 0.000 & 0.020 & -0.020 & -3.19 & -1.64 & 2026.54 & 302.82\end{array}$ $\begin{array}{llllllll}0.285 & 0.000 & 0.021 & -0.030 & -3.38 & -1.79 & 2028.60 & 308.84\end{array}$ $\begin{array}{llllllll}0.273 & 0.000 & 0.030 & -0.027 & -3.65 & -2.07 & 2029.39 & 316.12\end{array}$ $\begin{array}{llllllll}0.261 & 0.000 & 0.040 & -0.024 & -3.49 & -1.98 & 2031.06 & 322.51\end{array}$ $\begin{array}{llllllll}0.249 & 0.000 & 0.051 & -0.032 & -4.06 & -2.37 & 2031.82 & 329.83\end{array}$ $\begin{array}{llllllll}0.249 & 0.000 & 0.051 & -0.032 & -3.96 & -2.32 & 2033.40 & 336.32\end{array}$ $\begin{array}{llllllll}0.238 & 0.000 & 0.049 & -0.032 & -4.26 & -2.63 & 2033.93 & 343.86\end{array}$ $\begin{array}{llllllll}0.226 & 0.000 & 0.059 & -0.030 & -4.31 & -2.62 & 2035.42 & 350.45\end{array}$ $\begin{array}{llllllll}0.226 & 0.000 & 0.059 & -0.030 & -4.69 & -2.99 & 2035.88 & 358.05\end{array}$ $\begin{array}{llllllll}0.226 & 0.000 & 0.059 & -0.030 & -4.56 & -2.89 & 2037.14 & 364.86\end{array}$ $\begin{array}{llllllll}0.215 & 0.000 & 0.057 & -0.031 & -4.77 & -3.14 & 2037.35 & 372.73\end{array}$ $\begin{array}{llllllll}0.215 & 0.000 & 0.057 & -0.031 & -4.55 & -2.95 & 2038.39 & 379.76\end{array}$ $\begin{array}{llllllll}0.216 & 0.000 & 0.045 & -0.034 & -4.61 & -3.14 & 2038.42 & 387.80\end{array}$ $\begin{array}{llllllll}0.216 & 0.000 & 0.045 & -0.034 & -4.39 & -2.95 & 2039.32 & 394.97\end{array}$ $\begin{array}{llllllll}0.227 & 0.000 & 0.035 & -0.036 & -4.61 & -3.21 & 2039.29 & 403.07\end{array}$ $\begin{array}{llllllll}0.216 & 0.000 & 0.033 & -0.036 & -4.41 & -3.10 & 2040.14 & 410.29\end{array}$ $\begin{array}{llllllll}0.228 & 0.000 & 0.023 & -0.038 & -4.76 & -3.49 & 2040.12 & 418.39\end{array}$ $\begin{array}{llllllll}0.228 & 0.000 & 0.023 & -0.038 & -4.66 & -3.43 & 2040.89 & 425.69\end{array}$ $\begin{array}{llllllll}0.229 & 0.000 & 0.011 & -0.041 & -5.01 & -3.78 & 2040.71 & 433.94\end{array}$ $\begin{array}{llllllll}0.229 & 0.000 & 0.011 & -0.041 & -4.86 & -3.66 & 2041.30 & 441.42\end{array}$ $\begin{array}{llllllll}0.229 & 0.000 & -0.002 & -0.033 & -4.83 & -3.64 & 2040.63 & 450.16\end{array}$ $\begin{array}{llllllll}0.218 & 0.000 & -0.004 & -0.033 & -4.47 & -3.36 & 2040.94 & 457.93\end{array}$ $\begin{array}{llllllll}0.218 & 0.000 & -0.004 & -0.033 & -4.60 & -3.50 & 2040.31 & 466.62\end{array}$ $\begin{array}{llllllll}0.207 & 0.000 & -0.007 & -0.023 & -3.98 & -2.96 & 2040.24 & 474.76\end{array}$
$-0.10 \quad 219.94$

$0.34 \quad 225.58$

$0.20 \quad 232.12$

$0.24 \quad 237.54$

$-0.02 \quad 244.11$

$0.01 \quad 249.70$

$-0.26 \quad 256.43$

$-0.31 \quad 262.10$

$-0.64 \quad 268.92$
-0.65

$-0.65 \quad 274.80$

$-0.96 \quad 281.81$

$\begin{array}{ll}-0.90 \quad 287.91 \\ -1.28 & 295.00\end{array}$

$\begin{array}{ll}-1.28 & 295.00\end{array}$

$\begin{array}{ll}-1.21 \quad 301.27 \\ -1.64 & 308.46\end{array}$

$-1.64308 .46$

$-1.65 \quad 314.80$

$\begin{array}{ll}-1.96 & 322.27\end{array}$

$-1.86 \quad 328.88$

$\begin{array}{ll}-2.09 & 336.57\end{array}$

$-2.02 \quad 343.29$

$\begin{array}{ll}-2.33 \quad 351.05 \\ -2.29 & 357.89\end{array}$

$\begin{array}{ll}-2.29 & 357.89\end{array}$

$-2.67 \quad 365.72$

$\begin{array}{ll}-2.56 & 372.77\end{array}$

$\begin{array}{ll}-2.80 & 380.88\end{array}$

$-2.60 \quad 388.17$

$-2.81 \quad 396.44$

$-2.61 \quad 403.87$

$\begin{array}{ll}-2.88 & 412.21\end{array}$

$-2.74 \quad 419.72$

$-3.14 \quad 428.07$

$-3.06 \quad 435.65$

$-3.39444 .20$

$-3.26 \quad 451.97$

$-3.43 \quad 460.80$

$\begin{array}{ll}-3.12 \quad 468.87 \\ -3.27 & 477.85\end{array}$

$-3.27 \quad 477.85$

$-2.88 \quad 486.13$

\section{$Z=98$ (Cf)}

$\begin{array}{lllllll}123 & 221 & -0.10 & 0.00 & 0.02 & 0.00 & -0.105\end{array}$

$\begin{array}{llll}124 & 222 & -0.08 & 0.00\end{array}$

$\begin{array}{llll}125 & 223 & 0.03 & 0.04\end{array}$

$\begin{array}{llll}0.02 & 0.01 & -0.084\end{array}$

$\begin{array}{llllll}0.000 & -0.019 & 0.002 & -1.66 & -1.07 & 1636.12\end{array}$ $\begin{array}{llllll}0.000 & -0.021 & -0.008 & -1.82 & -1.21 & 1647.28\end{array}$

$0.00 \quad 0.00$

$0.033-0.054$

0.001

$\begin{array}{llll}0.001 & -2.26 & -1.37 & 1656.36\end{array}$

$0.00 \quad 0.00$

$0.001-0.067$

0.001

$\begin{array}{llll}0.002 & -2.61 & -1.46 & 1667.12\end{array}$

$\begin{array}{lllll}0.014 & 0.004 & -2.47 & -0.92 & 1675.17\end{array}$

70.97

67.89

127225

$\begin{array}{llll}0.03 & 0.07 & -0.01 & 0.00\end{array}$

$0.034-0.095$

$\begin{array}{lllll}0.015 & -0.005 & -1.99 & -0.19 & 1684.80\end{array}$

$0.035-0.108$

$\begin{array}{lllll}0.016 & -0.004 & -1.83 & 0.19 & 1692.70\end{array}$

$\begin{array}{llllllll}0.079 & -0.137 & 0.030 & -0.001 & -1.94 & 0.66 & 1702.26\end{array}$

$0.283 \quad 0.000$

$\begin{array}{lll}0.054 & 0.010 & -1.24\end{array}$

$0.49 \quad 1710.41$

66.88

$128 \quad 226$

$\begin{array}{llll}0.05 & 0.09 & -0.01 & 0.01\end{array}$

0.260

0.000

0.063

$0.002-1.34$

$\begin{array}{ll}0.40 & 1720.22\end{array}$

64.18

64.20

$132 \quad 230$

$\begin{array}{lllll}0.24 & 0.00 & -0.03 & 0.01\end{array}$

$\begin{array}{ll}0.260 \quad 0.000 \\ 0.227 & 0.000\end{array}$

0.063

$0.002-1.60$

$\begin{array}{ll}0.15 & 1728.16\end{array}$

62.65

$\begin{array}{ll}133 & 231 \\ 134 & 232\end{array}$

$\begin{array}{llll}0.24 & 0.00 & -0.03 & 0.01 \\ 0.21 & 0.00 & -0.05 & 0.01\end{array}$

0.227

0.000

0.082

$0.006-2.01$

$0.11 \quad 1737.61$

$0.227 \quad 0.000$

0.082

$\begin{array}{lllll}0.006 & -2.20 & -0.05 & 1745.17\end{array}$

62.82

61.33

61.25

136234

$\begin{array}{lllll}0.21 & 0.00 & -0.05 & 0.01\end{array}$

0.227

0.000

0.082

$\begin{array}{lllll}0.006 & -2.34 & -0.05 & 1754.29\end{array}$

59.51

$0.216 \quad 0.000$

0.093

$\begin{array}{llll}0.008 & -3.07 & -0.41 & 1761.76\end{array}$

59.65

58.26

$137 \quad 235$

$\begin{array}{llll}0.20 & 0.00 & -0.06 & 0.01\end{array}$

0.226

0.000

$\begin{array}{llllll}0.082 & -0.004 & -3.01 & -0.60 & 1770.78\end{array}$

139237

$\begin{array}{lllll}0.21 & 0.00 & -0.05 & 0.02\end{array}$

0.226

0.000

$\begin{array}{llllll}0.082 & -0.004 & -3.48 & -1.04 & 1778.05\end{array}$

$\begin{array}{llllll}0.082 & -0.004 & -3.75 & -1.29 & 1786.85\end{array}$
58.78

57.73

58.33

57.38

58.18

57.46 $\begin{array}{ll}-1.08 & 72.85\end{array}$

$\begin{array}{ll}-1.22 \quad 69.70 \\ -1.38 & 68.61\end{array}$

$-1.38 \quad 68.61$

$-1.47 \quad 65.85$

$-0.92 \quad 65.80$

$-0.19 \quad 64.18$

$0.19 \quad 64.28$

$0.68 \quad 62.75$

$0.44 \quad 62.54$

$0.38 \quad 60.77$

$0.12 \quad 60.85$

$0.12 \quad 59.45$

$-0.05 \quad 59.91$

$-0.04 \quad 58.83$

$-0.39 \quad 59.40$

$-0.58 \quad 58.42$

$-1.02 \quad 59.17$

$-1.26 \quad 58.43$ 


\section{$Z=98(\mathrm{Cf})$}

\section{9}

$\begin{array}{lll}0.22 & 0.00 & -0.05\end{array}$

$\begin{array}{llllll}143 & 241 & 0.22 & 0.00 & -0.05\end{array}$

$\begin{array}{llllll}144 & 242 & 0.22 & 0.00 & -0.04\end{array}$

$\begin{array}{lllll}145 & 243 & 0.22 & 0.00 & -0.04\end{array}$

$\begin{array}{llllll}146 & 244 & 0.23 & 0.00 & -0.03\end{array}$

$147 \quad 245$

148246

149247

150248

151249

$152 \quad 250$

$\begin{array}{lll}153 & 251\end{array}$

$154 \quad 252$

155253

156254

$157 \quad 255$

158256

159257

160258

161259

$162 \quad 260$

163261

164262

165263

166264

167265

168266

169267

170268

$\begin{array}{ll}171 & 269\end{array}$

$172 \quad 270$

$\begin{array}{ll}173 & 271\end{array}$

$174 \quad 272$

$\begin{array}{llll}0.23 & 0.00 & -0.03\end{array}$

$\begin{array}{llll}0.23 & 0.00 & -0.02\end{array}$

$\begin{array}{llll}0.23 & 0.00 & -0.02\end{array}$

$\begin{array}{llll}0.23 & 0.00 & -0.01\end{array}$

$\begin{array}{llll}0.23 & 0.00 & -0.01\end{array}$

$\begin{array}{lll}0.23 & 0.00 & 0.00\end{array}$

$\begin{array}{lll}0.23 & 0.00 & 0.00\end{array}$

$\begin{array}{lll}0.23 & 0.00 & 0.01\end{array}$

$0.22 \quad 0.00$

$0.22 \quad 0.00$

0.01

$0.22 \quad 0.00$

0.01

$\begin{array}{ll}0.22 & 0.00\end{array}$

$\begin{array}{ll}0.21 & 0.00\end{array}$

0.02

0.210 .00

0.03

$0.21 \quad 0.00$

$0.21 \quad 0.00$

$0.20 \quad 0.00$

$0.20 \quad 0.00$

$0.18 \quad 0.00$

$\begin{array}{ll}0.18 & 0.00\end{array}$

$\begin{array}{ll}0.16 & 0.00\end{array}$

$\begin{array}{ll}0.16 & 0.00\end{array}$

$\begin{array}{ll}0.14 & 0.00\end{array}$

$\begin{array}{ll}0.13 & 0.00\end{array}$

0.120 .00

$\begin{array}{ll}0.12 & 0.00\end{array}$

$\begin{array}{ll}0.12 & 0.00\end{array}$

$0.12 \quad 0.00$

$175 \quad 273$

$0.10 \quad 0.00$

$\begin{array}{llll}176 & 274 & -0.10 & 0.00\end{array}$

$\begin{array}{llll}177 & 275 & -0.10 & 0.00\end{array}$

$\begin{array}{llll}178 & 276 & -0.10 & 0.00\end{array}$

$\begin{array}{llll}179 & 277 & -0.08 & 0.00\end{array}$

$\begin{array}{lllll}180 & 278 & -0.07 & 0.00\end{array}$

$\begin{array}{lllll}181 & 279 & -0.04 & 0.00\end{array}$

$182 \quad 280$

$0.00 \quad 0.00$

$183 \quad 281$

$184 \quad 282$

$0.00 \quad 0.00$

185283

$0.00 \quad 0.00$

186284

$0.01 \quad 0.06$

$187 \quad 285$

$\begin{array}{llll}0.02 & 0.07 & 0.00\end{array}$

$\begin{array}{llll}0.03 & 0.09 & -0.01\end{array}$

188286

189287

190288

$\begin{array}{llll}0.04 & 0.10 & -0.01\end{array}$

$\begin{array}{llll}0.05 & 0.10 & -0.02\end{array}$

$\begin{array}{llll}0.23 & 0.00 & -0.03\end{array}$

191289

$\begin{array}{lll}0.23 & 0.00 & -0.03\end{array}$

192290

193291

$\begin{array}{llll}0.23 & 0.00 & -0.03\end{array}$

$\begin{array}{llll}0.23 & 0.00 & -0.03\end{array}$

194292

$\begin{array}{llll}0.23 & 0.00 & -0.03\end{array}$

195293

$\begin{array}{llll}0.24 & 0.00 & -0.02\end{array}$

$\begin{array}{llllll}196 & 294 & 0.24 & 0.00 & -0.02\end{array}$

$\begin{array}{lllll}197 & 295 & 0.24 & 0.00 & -0.02\end{array}$ $\begin{array}{lllllllll}0.03 & 0.237 & 0.000 & 0.085 & -0.014 & -4.40 & -1.82 & 1793.94 & 58.44\end{array}$

$\begin{array}{lllllllll}0.03 & 0.237 & 0.000 & 0.085 & -0.014 & -4.63 & -2.06 & 1802.44 & 58.00\end{array}$

$\begin{array}{llllllll}0.04 & 0.237 & 0.000 & 0.086 & -0.024 & -5.26 & -2.47 & 1809.15\end{array}$

$0.04 \quad 0.237$

$0.04 \quad 0.237$

$\begin{array}{ll}0.04 & 0.249\end{array}$

$0.04 \quad 0.249$

$0.04 \quad 0.249$

$0.04 \quad 0.249$

$0.04 \quad 0.250$

0.000

$\begin{array}{lllll}0.073 & -0.027 & -5.05 & -2.51 & 1817.20\end{array}$

0.000

$\begin{array}{lllll}0.073 & -0.027 & -5.46 & -2.89 & 1823.61\end{array}$

0.000

$\begin{array}{lllll}0.063 & -0.029 & -5.31 & -2.89 & 1831.34\end{array}$

0.000

$\begin{array}{lllll}0.063 & -0.029 & -5.73 & -3.27 & 1837.51\end{array}$

$\begin{array}{lllll}0.051 & -0.032 & -5.51 & -3.22 & 1844.94\end{array}$

0.000

$\begin{array}{llllll}0.051 & -0.032 & -5.94 & -3.63 & 1850.88\end{array}$

$\begin{array}{lllllll}0.000 & 0.039 & -0.035 & -5.81 & -3.59 & 1858.07\end{array}$

$0.04 \quad 0.250$

0.000

$\begin{array}{lllll}0.039 & -0.035 & -6.17 & -3.93 & 1863.71\end{array}$

$0.04 \quad 0.250$

$0.04 \quad 0.250$

0.000

$\begin{array}{lllll}0.027 & -0.037 & -5.93 & -3.75 & 1870.50\end{array}$

$\begin{array}{lllll}0.027 & -0.037 & -5.80 & -3.64 & 1875.46\end{array}$

0.000

$\begin{array}{lllll}0.014 & -0.030 & -5.05 & -3.15 & 1881.69\end{array}$

0.03

0.000

$\begin{array}{lllll}0.012 & -0.031 & -4.81 & -2.99 & 1886.36\end{array}$

$0.03 \quad 0.240$

0.000

$\begin{array}{lllll}0.012 & -0.031 & -4.31 & -2.55 & 1892.42\end{array}$

$\begin{array}{lllllll}0.000 & -0.001 & -0.023 & -4.15 & -2.53 & 1897.00\end{array}$

$\begin{array}{llllll}0.000 & -0.001 & -0.023 & -3.77 & -2.20 & 1902.94\end{array}$

$\begin{array}{llllll}0.000 & -0.016 & -0.016 & -3.84 & -2.31 & 1907.43\end{array}$

$\begin{array}{llllll}0.000 & -0.016 & -0.016 & -3.69 & -2.19 & 1913.35\end{array}$

$\begin{array}{llllll}0.000 & -0.028 & -0.018 & -4.10 & -2.33 & 1917.65\end{array}$

$\begin{array}{llllll}0.000 & -0.041 & -0.011 & -4.06 & -2.14 & 1923.28\end{array}$

$\begin{array}{lllllll}0.000 & -0.043 & -0.010 & -4.01 & -2.14 & 1927.23 & 102.71\end{array}$

$\begin{array}{lllllll}0.000 & -0.043 & -0.010 & -3.44 & -1.62 & 1932.32 & 105.70\end{array}$

$\begin{array}{lllllll}0.000 & -0.034 & -0.007 & -2.90 & -1.39 & 1935.83 & 110.25\end{array}$

$0.00-0.196$

$0.00 \quad 0.196$

0.00

0.174

$\begin{array}{lllllll}0.000 & -0.034 & -0.007 & -2.37 & -0.93 & 1940.76 & 113.40\end{array}$

$\begin{array}{lllllll}0.000 & -0.025 & -0.005 & -2.16 & -1.07 & 1944.43 & 117.80\end{array}$

$\begin{array}{lllllll}0.000 & -0.013 & -0.013 & -1.79 & -0.69 & 1949.24 & 121.06\end{array}$

$\begin{array}{lllllll}0.000 & -0.015 & -0.013 & -1.75 & -0.78 & 1952.67 & 125.70\end{array}$

$0.01 \quad 0.151$

$\begin{array}{lllllll}0.000 & -0.004 & -0.011 & -1.48 & -0.66 & 1957.52 & 128.92\end{array}$

$0.01 \quad 0.129$

$\begin{array}{lllllll}0.000 & -0.005 & -0.011 & -1.80 & -1.03 & 1961.04 & 133.48\end{array}$

$\begin{array}{lllllll}0.000 & -0.018 & -0.002 & -1.71 & -0.92 & 1965.70 & 136.88\end{array}$

$\begin{array}{lllllll}0.000 & -0.018 & -0.002 & -2.09 & -1.29 & 1969.03 & 141.62\end{array}$

$\begin{array}{llllllll}0.000 & -0.031 & 0.006 & -2.07 & -1.16 & 1973.48 & 145.25\end{array}$

$\begin{array}{lllllll}0.000 & -0.044 & 0.005 & -2.32 & -1.32 & 1976.41 & 150.39\end{array}$

$\begin{array}{llllllll}0.000 & -0.007 & 0.011 & -1.94 & -1.44 & 1980.92 & 153.96\end{array}$

$\begin{array}{llllllll}0.000 & -0.019 & 0.002 & -2.45 & -1.92 & 1983.98 & 158.96\end{array}$

$0.000-0.019$

$\begin{array}{lllll}0.012 & -2.38 & -1.83 & 1988.09 & 162.92\end{array}$

$0.000-0.021$

$\begin{array}{lllll}0.002 & -2.33 & -1.80 & 1990.47 & 168.61\end{array}$

$0.000-0.021$

$\begin{array}{lllll}0.002 & -2.00 & -1.45 & 1994.13 & 173.02\end{array}$

$0.00-0.073$

$0.000-0.011$

$\begin{array}{llllll}0.001 & -2.17 & -1.59 & 1996.50 & 178.73\end{array}$

$0.00-0.042$

$0.000 \quad 0.000$

$\begin{array}{llllll}0.000 & -1.98 & -1.41 & 2000.16 & 183.14\end{array}$

$\begin{array}{ll}0.00 & 0.000 \\ 0.00 & 0.000\end{array}$

$0.000 \quad 0.000$

$\begin{array}{lllll}0.000 & -2.31 & -1.71 & 2002.52 & 188.85\end{array}$

$0.00 \quad 0.000$

$0.000 \quad 0.000$

$\begin{array}{lllll}0.000 & -1.96 & -1.38 & 2005.85 & 193.59\end{array}$

$\begin{array}{llll}0.00 & 0.012 & -0.081\end{array}$

0.002

$\begin{array}{lllll}0.002 & -2.01 & -0.83 & 2007.19 & 200.33\end{array}$

$\begin{array}{llll}0.00 & 0.023 & -0.094\end{array}$

$\begin{array}{llll}0.00 & 0.035 & -0.123\end{array}$

0.002

$\begin{array}{lllll}0.003 & -1.63 & -0.24 & 2010.08 & 205.50\end{array}$

$\begin{array}{llll}0.01 & 0.047 & -0.135\end{array}$

0.016

0.006

$0.01 \quad 0.057-0.136$

$0.002-1.92$

$\begin{array}{lll}0.35 & 2014.54 & 217.19\end{array}$

0.01

0.249

0.000

0.061

$0.001-1.33$

$\begin{array}{lll}0.19 & 2016.25 & 223.54\end{array}$

$0.01 \quad 0.249$

0.000

0.061

$0.001-1.61$

$\begin{array}{lll}0.33 & 2019.26 & 228.61\end{array}$

$0.01 \quad 0.249$

0.000

0.061

$0.001-1.59$

$\begin{array}{lll}0.07 & 2020.92 & 235.02\end{array}$

$0.01 \quad 0.249$

0.000

0.061

$\begin{array}{llllll}0.001 & -1.92 & -0.25 & 2025.47 & 246.61\end{array}$

$0.01 \quad 0.26$

0.000

$\begin{array}{lllllll}0.051 & -0.001 & -2.09 & -0.50 & 2029.65 & 258.58\end{array}$

$\begin{array}{lll}0.02 & 0.260 & 0.000\end{array}$

$\begin{array}{llllll}0.051 & -0.011 & -2.17 & -0.58 & 2032.39 & 263.91\end{array}$

0.000

$\begin{array}{lllllll}0.051 & -0.011 & -2.51 & -0.91 & 2033.67 & 270.70\end{array}$

$-1.78 \quad 59.38$

$-2.00 \quad 58.94$

\begin{tabular}{ll}
$-2.40 \quad 60.30$ \\
\hline
\end{tabular}

$\begin{array}{llll}59.34 & 0.037 & -2.43 & 60.31\end{array}$

$-2.82 \quad 61.94$

$\begin{array}{llll}61.48 & 0.003 & -2.81 & 62.28\end{array}$

$\begin{array}{llll}63.39 & 0.003 & -3.19 & 64.16\end{array}$

$\begin{array}{llll}64.09 & 0.002 & -3.13 & 64.81\end{array}$

$\begin{array}{llll}66.14 & 0.008 & -3.55 & 66.93\end{array}$

$\begin{array}{llll}67.24 & 0.005 & -3.49 & 67.83\end{array}$

$\begin{array}{llll}69.73 & 0.002 & -3.84 & 70.25\end{array}$

$\begin{array}{llll}71.17 & 0.002 & -3.64 & 71.55\end{array}$

$\begin{array}{llll}74.14 & 0.004 & -3.54 & 74.67\end{array}$

$\begin{array}{llll}76.03 & 0.005 & -3.08 & 76.48\end{array}$

$\begin{array}{llll}79.30 & 0.006 & -2.93 & 79.89\end{array}$

$\begin{array}{llll}81.34 & 0.012 & -2.48 & 81.93\end{array}$

$-2.50 \quad 85.41$

$-2.17 \quad 87.57$

$-2.30 \quad 91.15$

$-2.17 \quad 93.35$

$-2.30 \quad 97.16$

$-2.10 \quad 99.65$

$-2.11 \quad 103.81$

$-1.59 \quad 106.85$

$-1.38 \quad 111.43$

$-0.91 \quad 114.64$

$-1.06 \quad 119.07$

$-0.67 \quad 122.41$

$-0.77 \quad 127.11$

$-0.65 \quad 130.39$

$\begin{array}{ll}-1.02 \quad 135.02 \\ -0.91 & 138.49\end{array}$

$\begin{array}{ll}-0.91 & 138.49\end{array}$

$-1.29 \quad 143.31$

$\begin{array}{ll}-1.13 & 147.04\end{array}$

$\begin{array}{ll}-1.27 & 152.29\end{array}$

$-1.42 \quad 155.91$

$-1.91 \quad 161.00$

$-1.80 \quad 165.08$

$-1.79 \quad 170.85$ 


\section{$Z=98(\mathbf{C f})$}

$\begin{array}{lllll}198 & 296 & 0.25 & 0.00 & -0.01\end{array}$

$\begin{array}{lllllllll}0.02 & 0.272 & 0.000 & 0.041 & -0.014 & -2.44 & -0.91 & 2036.18 & 276.26\end{array}$

201299 $\begin{array}{lll}0.25 & 0.00-0.00\end{array}$ $0.02 \quad 0.273$ 0.000 $\begin{array}{llllll}0.029 & -0.017 & -2.71 & -1.24 & 2037.31 & 283.20\end{array}$ $\begin{array}{lll}0.26 & 0.00 & 0.01\end{array}$ 0.02 0.000

$0.03 \quad 0.285$

0.000

$\begin{array}{lllllll}0.020 & -0.020 & -2.77 & -1.28 & 2039.71 & 288.88\end{array}$ $\begin{array}{lll}0.26 & 0.00 & 0.01 \\ 0.26 & 0.00 & 0.01\end{array}$

202300 $\begin{array}{lll}0.25 & 0.00 & 0.00\end{array}$

204302

$\begin{array}{lll}0.25 & 0.00 & 0.00\end{array}$

0.03

0.285

0.000

$\begin{array}{llllll}0.021 & -0.030 & -3.43 & -1.83 & 2040.91 & 295.75\end{array}$

$\begin{array}{llllll}0.021 & -0.030 & -3.42 & -1.88 & 2043.17 & 301.55\end{array}$

205303

$\begin{array}{lll}0.24 & 0.00 & -0.01\end{array}$

0.03

0.273

0.000

$\begin{array}{llllll}0.030 & -0.027 & -3.67 & -2.14 & 2043.94 & 308.86\end{array}$

206304

$\begin{array}{lll}0.23 & 0.00 & -0.01\end{array}$

0.261

0.000

$\begin{array}{llllll}0.030 & -0.027 & -3.50 & -1.99 & 2045.86 & 315.01\end{array}$

207305

$\begin{array}{llll}0.22 & 0.00 & -0.02\end{array}$

208306

$\begin{array}{lll}0.21 & 0.00 & -0.03\end{array}$

0.04

0.238

0.000

$\begin{array}{llllll}0.041 & -0.034 & -4.03 & -2.39 & 2046.62 & 322.31\end{array}$

$0.04 \quad 0.226$

0.000

$\begin{array}{llllll}0.039 & -0.035 & -3.85 & -2.30 & 2048.46 & 328.55\end{array}$

$\begin{array}{llllll}0.049 & -0.032 & -4.21 & -2.63 & 2049.03 & 336.06\end{array}$

209307

$\begin{array}{llll}0.21 & 0.00 & -0.03\end{array}$

$0.04 \quad 0.226$

0.000

$\begin{array}{llllll}0.059 & -0.030 & -4.21 & -2.54 & 2050.72 & 342.43\end{array}$

$0.04 \quad 0.226$

0.000

210308

$0.00-0.03$

$0.04 \quad 0.215$

0.000

$\begin{array}{llllll}0.059 & -0.030 & -4.44 & -2.79 & 2052.72 & 356.58\end{array}$

212310

$\begin{array}{lll}0.20 & 0.00 & -0.03\end{array}$

0.04

0.215

0.000

$\begin{array}{llllll}0.057 & -0.031 & -4.66 & -3.04 & 2052.94 & 364.43\end{array}$

$\begin{array}{lll}0.20 & 0.00 & -0.02\end{array}$

$0.04 \quad 0.216$

0.000

$\begin{array}{lllllll}0.057 & -0.031 & -4.44 & -2.85 & 2054.26 & 371.18\end{array}$

$214 \quad 312$

$\begin{array}{lll}0.20 & 0.00 & -0.02\end{array}$

0.04

0.216

0.000

215313

216314

$\begin{array}{lll}0.20 & 0.00 & -0.01\end{array}$

0.04

0.216

0.000

$\begin{array}{llllll}0.045 & -0.034 & -4.32 & -2.90 & 2055.52 & 386.06\end{array}$

$\begin{array}{lll}0.21 & 0.00 & 0.00\end{array}$

0.04
0.04

.228

0.000

$\begin{array}{llllll}0.033 & -0.036 & -4.56 & -3.22 & 2055.55 & 394.10\end{array}$

0.000

$\begin{array}{lllllll}0.023 & -0.038 & -4.43 & -3.12 & 2056.71 & 401.02\end{array}$

$\begin{array}{llllll}0.023 & -0.038 & -4.78 & -3.52 & 2056.69 & 409.11\end{array}$

218316

$\begin{array}{lll}0.21 & 0.00 & 0.01\end{array}$

$\begin{array}{ll}0.04 & 0.229\end{array}$

0.000

$\begin{array}{llllll}0.011 & -0.041 & -4.73 & -3.51 & 2057.80 & 416.06\end{array}$

219317

$\begin{array}{lll}0.21 & 0.00 & 0.01\end{array}$

0.04

0.229

0.000

$\begin{array}{llllll}0.011 & -0.041 & -5.08 & -3.85 & 2057.61 & 424.33\end{array}$

318

$\begin{array}{lll}0.21 & 0.00 & 0.01\end{array}$

$0.04 \quad 0.229$

0.000

$\begin{array}{llllll}0.011 & -0.041 & -4.93 & -3.73 & 2058.48 & 431.53\end{array}$

221319

$\begin{array}{lll}0.21 & 0.00 & 0.02\end{array}$

$0.04 \quad 0.230$

$\begin{array}{lllllll}0.000 & -0.001 & -0.043 & -5.22 & -3.97 & 2058.06 & 440.02\end{array}$

$\begin{array}{lllllll}0.000 & -0.004 & -0.033 & -4.59 & -3.49 & 2058.45 & 447.70\end{array}$

$223 \quad 32$

224322

$0.20+0.00$

0.03

0.218

$\begin{array}{lllllll}0.000 & -0.004 & -0.033 & -4.72 & -3.64 & 2057.83 & 456.39\end{array}$

$\begin{array}{lll}0.19 & 0.00 & 0.03\end{array}$

$\begin{array}{lll}0.18 & 0.00 & 0.03\end{array}$

226324

$\begin{array}{lll}0.18 & 0.00 & 0.03\end{array}$
0.218

$0.01 \quad 0.196$

$0.01 \quad 0.196$

$0.01 \quad 0.197$ $\begin{array}{lllllll}0.000 & -0.019 & -0.025 & -4.24 & -3.19 & 2058.13 & 464.16\end{array}$

$\begin{array}{lllllll}0.000 & -0.022 & -0.015 & -4.26 & -3.23 & 2057.29 & 473.08\end{array}$

$\begin{array}{lllllll}0.000 & -0.022 & -0.015 & -3.98 & -3.00 & 2057.68 & 480.75\end{array}$

$\begin{array}{lllllll}0.000 & -0.034 & -0.017 & -4.28 & -3.20 & 2056.88 & 489.63\end{array}$ 200298

$\begin{array}{llllll}0.059 & -0.030 & -4.57 & -2.90 & 2051.18 & 350.05\end{array}$

$\begin{array}{llllll}0.045 & -0.034 & -4.55 & -3.09 & 2054.33 & 379.18\end{array}$

$\begin{array}{lll}0.18 & 0.00 & 0.04\end{array}$

$-0.88 \quad 281.17$

$-1.23 \quad 288.28$

$-1.25 \quad 294.15$

$-1.70 \quad 301.31$

$-1.74 \quad 307.33$

$-2.01 \quad 314.81$

$-1.85 \quad 321.17$

$-2.10 \quad 328.83$

$-1.99 \quad 335.29$

$-2.33 \quad 343.01$

$-2.20 \quad 349.63$

$-2.57 \quad 357.46$

$-2.45 \quad 364.23$

$-2.70 \quad 372.31$

$-2.50 \quad 379.31$

$-2.74 \quad 387.54$

$-2.55 \quad 394.67$

$-2.87 \quad 402.95$

$-2.76 \quad 410.14$

$-3.16 \quad 418.48$

$-3.12 \quad 425.73$

$-3.46 \quad 434.26$

$-3.32 \quad 441.74$

$\begin{array}{ll}-3.53 & 450.53\end{array}$

$\begin{array}{ll}-3.25 & 458.29\end{array}$

$-3.40 \quad 467.26$

$-3.06 \quad 475.21$

$-3.21 \quad 484.31$

$-2.98 \quad 492.28$
-3.13

$-3.13 \quad 501.50$

\section{$Z=99($ Es)}

$\begin{array}{lllll}125 & 224 & 0.01 & 0.05 & 0.00\end{array}$

$\begin{array}{lllllllllllllll}126 & 225 & 0.00 & 0.06 & 0.00 & 0.00 & 0.002 & -0.081 & 0.002 & 0.002 & -2.74 & -1.42 & 1665.01 & 73.59\end{array}$

$\begin{array}{llllllllllllll}127 & 226 & 0.02 & 0.07 & -0.01 & 0.00 & 0.023 & -0.095 & 0.014 & 0.004 & -2.42 & -0.88 & 1673.46 & 73.20\end{array}$

$\begin{array}{llllllllllllll}128 & 227 & -0.55 & 0.00 & 0.01 & -0.02 & -0.548 & 0.000 & 0.100 & -0.002 & -2.75 & 4.66 & 1678.32 & 76.42\end{array}$

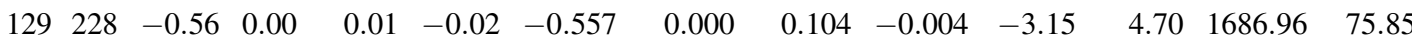

$\begin{array}{llllllllllllll}130 & 229 & 0.25 & 0.00 & -0.02 & 0.00 & 0.272 & 0.000 & 0.052 & 0.010 & -0.97 & 0.52 & 1701.21 & 69.67\end{array}$

132231

$\begin{array}{lll}0.26 & 0.00 & -0.02\end{array}$

$\begin{array}{lll}0.01 & 0.283 & 0.000\end{array}$

$\begin{array}{lll}0.055 & 0.000 & -1.21\end{array}$

$0.40 \quad 1709.70$

69.25

$\begin{array}{lll}0.01 & 0.272 & 0.000\end{array}$

$0.053-0.001-1.18$

$0.36 \quad 1719.50$

67.52

$\begin{array}{llll}0.27 & 0.00 & -0.01\end{array}$

$0.01 \quad 0.294$

0.000

$\begin{array}{lll}0.045 & -0.003 & -1.40\end{array}$

$0.13 \quad 1727.80$

67.29

134233

$\begin{array}{lll}0.27 & 0.00 & -0.01\end{array}$

0.02

0.294

0.000

$0.046-0.013-1.55$

$\begin{array}{ll}0.05 & 1737.34\end{array}$

65.83

$135 \quad 234$

$\begin{array}{lll}0.24 & 0.00 & -0.03\end{array}$

$0.02 \quad 0.260$

0.000

$\begin{array}{lllll}0.064 & -0.008 & -2.01 & -0.19 & 1745.36\end{array}$

65.88

$136 \quad 235$

$\begin{array}{lll}0.24 & 0.00 & -0.03\end{array}$

$0.02-0.260$

0.000

$\begin{array}{lllll}0.064 & -0.008 & -2.19 & -0.31 & 1754.63\end{array}$

64.68

$\begin{array}{lll}0.02 & 0.237 & 0.000\end{array}$

$\begin{array}{lllll}0.072 & -0.007 & -2.70 & -0.62 & 1762.43\end{array}$

64.95

138237

$\begin{array}{lll}0.22 & 0.00 & -0.04\end{array}$

$0.02 \quad 0.237$

0.000

$\begin{array}{lllll}0.072 & -0.007 & -2.97 & -0.87 & 1771.55\end{array}$

63.90

$\begin{array}{lll}0.02 & 0.226 & 0.000\end{array}$

$\begin{array}{lllll}0.082 & -0.004 & -3.65 & -1.26 & 1779.15\end{array}$

64.37

$140 \quad 239$

$\begin{array}{lll}0.22 & 0.00 & -0.04\end{array}$

$\begin{array}{ll}0.03 & 0.237\end{array}$

0.000

$\begin{array}{lllll}0.073 & -0.017 & -3.85 & -1.62 & 1788.10\end{array}$

63.49

$0.03 \quad 0.237$

0.000

$\begin{array}{lllll}0.073 & -0.017 & -4.33 & -2.09 & 1795.51\end{array}$

64.16

$142 \quad 241$

$\begin{array}{lll}0.22 & 0.00 & -0.04\end{array}$

$\begin{array}{ll}0.03 & 0.237\end{array}$

0.000

$\begin{array}{lllll}0.073 & -0.017 & -4.58 & -2.34 & 1804.06\end{array}$

63.68

$\begin{array}{lll}0.03 & 0.237 & 0.000\end{array}$

$\begin{array}{lllll}0.073 & -0.017 & -5.03 & -2.73 & 1811.12\end{array}$

64.68

144243

$\begin{array}{lll}0.22 & 0.00 & -0.04\end{array}$

$\begin{array}{ll}0.04 & 0.237\end{array}$

0.000

$\begin{array}{llllll}0.073 & -0.027 & -5.34 & -2.84 & 1819.27\end{array}$

64.61

$\begin{array}{lllll}145 & 244 & 0.23 & 0.00 & -0.03\end{array}$

$\begin{array}{lll}0.04 & 0.249 & 0.000\end{array}$

$\begin{array}{lllll}0.063 & -0.029 & -5.60 & -3.21 & 1826.05\end{array}$

65.90

$\begin{array}{llllll}146 & 245 & 0.23 & 0.00 & -0.03\end{array}$

$\begin{array}{lll}0.04 & 0.249 & 0.000\end{array}$

$\begin{array}{lllll}0.063 & -0.029 & -5.66 & -3.28 & 1833.88\end{array}$

66.14

$\begin{array}{lll}0.05 & 0.248 & 0.000\end{array}$

$\begin{array}{lllll}0.064 & -0.039 & -6.39 & -3.70 & 1840.46\end{array}$

67.63

$148 \quad 247$

$\begin{array}{lll}0.23 & 0.00 & -0.03 \\ 0.23 & 0.00 & -0.02\end{array}$

$0.04 \quad 0.249$

0.000

$\begin{array}{lllll}0.051 & -0.032 & -5.93 & -3.67 & 1847.93\end{array}$

68.23

149248

$\begin{array}{lll}0.23 & 0.00 & -0.02\end{array}$

$0.04 \quad 0.249$

0.000

$\begin{array}{llllll}0.051 & -0.032 & -6.35 & -4.06 & 1854.22\end{array}$

70.01

$\begin{array}{llllll}0.039 & -0.035 & -6.27 & -4.07 & 1861.49 & 70.81\end{array}$ 


\section{$Z=99$ (Es)}

\begin{tabular}{|c|c|c|c|c|c|c|c|c|c|c|c|c|c|c|c|c|}
\hline 1250 & 0.23 & 0.00 & -0.01 & 0.04 & 0.250 & 0.000 & 0.039 & -0.035 & -6.63 & -4.41 & 1867.49 & 72.89 & & & -4.34 & 73.85 \\
\hline 2251 & 0.23 & 0.00 & 0.00 & 0.04 & 0.250 & 0.000 & 0.027 & -0.037 & -6.42 & -4.26 & 1874.34 & 74.11 & 74.51 & 0.006 & -4.17 & 75.09 \\
\hline 3252 & 0.23 & 0.00 & 0.00 & 0.04 & 0.250 & 0.000 & 0.027 & -0.037 & -6.28 & -4.14 & 1879.64 & 76.88 & 77.29 & 0.050 & -4.06 & 77.85 \\
\hline 253 & 0.23 & 0.00 & 0.01 & 0.03 & 0.251 & 0.000 & 0.014 & -0.030 & -5.54 & -3.65 & 1885.92 & 78.68 & 79.01 & 0.003 & -3.60 & 79.62 \\
\hline 54 & 0.22 & 0.00 & 0.01 & 0.03 & 0.240 & 0.000 & 0.012 & -0.031 & -5.30 & -3.49 & 1890.94 & 81.72 & 81.99 & 0.004 & -3.45 & 32.68 \\
\hline 255 & 0.22 & 0.00 & 0.01 & 0.03 & 0.240 & 0.000 & 0.012 & -0.031 & -4.80 & -3.04 & 97.02 & 71 & .09 & 0.011 & -2.99 & 4.70 \\
\hline 256 & 0.22 & 0.00 & 0.02 & 0.02 & 0.240 & 0.000 & -0.001 & -0.023 & -4.65 & -3.02 & 1901.95 & 86.85 & & & -3.01 & 87.80 \\
\hline 257 & 0.22 & 0.00 & 0.02 & 0.02 & 0.240 & 0.000 & -0.001 & -0.023 & -4.27 & -2.69 & 1907.92 & 88.96 & & & -2.67 & 89.94 \\
\hline 258 & 0.21 & 0.00 & 0.03 & 0.01 & 0.229 & 0.000 & -0.016 & -0.016 & -4.35 & -2.82 & 1912.77 & 92.18 & & & -2.82 & 93.16 \\
\hline 0259 & 0.21 & 0.00 & 0.03 & 0.01 & 0.229 & 0.000 & -0.016 & -0.016 & -4.20 & -2.69 & 1918.71 & 94.31 & & & -2.69 & 95.32 \\
\hline 260 & 0.21 & 0.00 & 0.04 & 0.01 & 0.230 & 0.000 & -0.028 & -0.018 & -4.61 & -2.84 & 1923.37 & 97.72 & & & -2.83 & 8.78 \\
\hline 261 & 0.21 & 0.00 & 0.05 & 0.00 & 0.230 & 0.000 & -0.041 & -0.011 & -4.58 & -2.65 & 1929.03 & 100.14 & & & -2.63 & 01.23 \\
\hline 3262 & 0.20 & 0.00 & 0.05 & 0.00 & 0.219 & 0.000 & -0.043 & -0.010 & -4.52 & -2.64 & 1933.31 & 103.92 & & & -2.63 & 05.05 \\
\hline 4263 & 0.20 & 0.00 & 0.05 & 0.00 & 0.219 & 0.000 & -0.043 & -0.010 & -3.93 & -2.11 & 1938.41 & 106.90 & & & -2.09 & 08.07 \\
\hline 5264 & 0.19 & 0.00 & 0.04 & 0.00 & 0.207 & 0.000 & -0.033 & -0.008 & -3.41 & -1.91 & 1942.29 & 111.08 & & & -1.91 & 12.28 \\
\hline 265 & 0.18 & 0.00 & 0.04 & 0.00 & 0.196 & 0.000 & -0.034 & -0.007 & -2.83 & -1.39 & .18 & 14.26 & & & -1.38 & 15.52 \\
\hline 266 & 0.16 & .00 & 0.03 & 0.01 & 0.174 & 0.000 & -0.024 & -0.015 & -2.63 & -1.47 & .15 & 118.37 & & & -1.46 & 19.68 \\
\hline 267 & 0.16 & 0.00 & 0.02 & 0.01 & 0.173 & 0.000 & -0.013 & -0.013 & -2.18 & -1.23 & 1956.10 & 121.49 & & & -1.22 & 122.85 \\
\hline 268 & 0.16 & 00 & 0.02 & 0.00 & 0.173 & 0.000 & -0.013 & -0.003 & -2.14 & -1.10 & .66 & 126.00 & & & & 127.40 \\
\hline 269 & 0.13 & 0.00 & 0.01 & 0.01 & 0.140 & 0.000 & -0.004 & -0.011 & -1.75 & -0.93 & 1964.48 & 129.25 & & & -0.92 & 130.73 \\
\hline 270 & 0.12 & 0.00 & 0.01 & 0.01 & 29 & 0.000 & -0.005 & -0.011 & -2.03 & -1.26 & 29 & 33.51 & & & -1.25 & 5.05 \\
\hline 271 & 0.12 & 0.00 & 0.02 & 0.00 & 0.129 & 0.000 & -0.018 & -0.002 & -1.93 & -1.14 & .97 & 136.90 & & & -1 & 138.51 \\
\hline 272 & 0.12 & 0.00 & 0.02 & 0.00 & 0.129 & 0.000 & -0.018 & -0.002 & -2.31 & -1.52 & 1976.63 & 141.31 & & & -1.52 & 142.99 \\
\hline 273 & 0.12 & .00 & 0.03 & -0.01 & 0.129 & 0.000 & -0.031 & 0.006 & -2.29 & -1.38 & .09 & 144.93 & & & & 146.70 \\
\hline 274 & 0.12 & 0.00 & 0.03 & 0.01 & 0.129 & 0.000 & -0.031 & 0.006 & -2.54 & -1.64 & 4.45 & 149.63 & & & -1.62 & 151.49 \\
\hline 275 & -0.11 & 0.00 & 0.01 & -0.01 & 5 & 000 & -0.006 & 0.011 & -2.03 & -1.45 & & 48 & & & -1.44 & .41 \\
\hline 276 & 0.10 & 0.00 & 0.02 & 0.00 & 0.105 & 0.000 & -0.019 & 0.002 & -2.48 & -1.95 & & 158.14 & & & & 160.15 \\
\hline $8 \quad 277$ & -0.10 & 0.00 & 0.02 & -0.01 & -0.105 & 0.000 & -0.019 & 0.012 & -2.39 & -1.85 & 1996.21 & 162.10 & & & -1.83 & 164.22 \\
\hline 9278 & -0.08 & 0.00 & 0.02 & 0.00 & -0.084 & 0.000 & -0.021 & 0.002 & -2.31 & -1.80 & .88 & 167.49 & & & & 169.69 \\
\hline 279 & -0.07 & 0.00 & 0.02 & 0.00 & -0.073 & 0.000 & -0.021 & 0.002 & -1.96 & -1.42 & & 171.91 & & & -1.41 & 174.21 \\
\hline 280 & -0.04 & 0.00 & 0.01 & 0.00 & -0.042 & 000 & -0.011 & 0.001 & -2.08 & -1.51 & 5.18 & 177.34 & & & -1.51 & 179.73 \\
\hline 281 & 0.00 & 0.00 & 0.00 & 0.00 & 0.000 & 0.000 & 0.000 & 0.000 & -1.83 & -1.29 & & 181.78 & & & & 184.27 \\
\hline 3282 & 0.00 & 0.01 & 0.00 & 0.00 & 0.000 & -0.013 & 0.000 & 0.000 & -2.19 & -1.61 & .51 & 187.15 & & & -1.61 & 189.76 \\
\hline 283 & 0.00 & 0.04 & 0.00 & 0.00 & 0.001 & -0.054 & 0.001 & 0.001 & -2.10 & -1.27 & .84 & 191.89 & & & -1.25 & 194.62 \\
\hline 84 & 0.01 & 0.07 & 0.00 & 0.00 & 0.013 & -0.094 & 0.002 & 0.003 & -2.23 & -0.81 & .59 & 198.21 & & & -0.77 & 01.09 \\
\hline 285 & 0.02 & 0.08 & 0.01 & 0.00 & 0.024 & -0.109 & 0.015 & 0.005 & -1.97 & -0.27 & & 203.33 & & & & 206.36 \\
\hline 286 & 0.03 & 0.09 & -0.01 & 0.00 & 0.035 & -0.123 & 0.016 & 0.006 & -2.13 & -0.16 & & 209.47 & & & -0.08 & 212.64 \\
\hline $8 \quad 287$ & 0.04 & 0.10 & -0.01 & 0.01 & 0.047 & -0.135 & 0.016 & -0.003 & -2.00 & 0.31 & & 214.68 & & & 0.43 & 218.01 \\
\hline 9288 & 0.23 & 0.00 & -0.03 & 0.01 & 0.249 & 0.000 & 0.061 & 0.001 & -1.51 & 0.14 & .38 & 220.71 & & & 0.16 & 224.07 \\
\hline 0289 & 0.23 & 0.00 & -0.03 & 0.01 & 0.249 & 0.000 & 0.061 & 0.001 & -1.48 & 0.14 & 2029.54 & 225.62 & & & 0.17 & 229.12 \\
\hline 290 & 0.23 & 0.00 & -0.03 & 0.01 & 49 & 0.000 & 0.061 & 0.001 & -1.82 & -0.19 & .58 & 231.65 & & & -0.18 & 235.28 \\
\hline 2291 & 0.23 & 0.00 & -0.02 & 0.00 & 0.250 & 0.000 & 0.048 & 0.008 & -1.63 & -0.18 & 4.57 & 236.73 & & & .19 & 240.49 \\
\hline 3292 & 0.23 & 0.00 & -0.02 & 0.01 & 0.249 & 0.000 & 0.048 & -0.002 & -1.95 & -0.46 & .40 & 242.97 & & & 47 & 246.87 \\
\hline 4293 & 0.23 & 0.00 & -0.02 & 0.01 & 0.249 & 0.000 & 0.048 & -0.002 & -1.94 & -0.45 & 2039.23 & 248.21 & & & 46 & 252.27 \\
\hline $195 \quad 294$ & 0.24 & 0.00 & -0.02 & 0.01 & 0.261 & 0.000 & 0.051 & -0.001 & -2.32 & -0.76 & 2040.94 & 254.58 & & & -0.77 & 258.79 \\
\hline 6295 & 0.24 & 0.00 & -0.02 & 0.02 & 0.260 & 0.000 & 0.051 & -0.011 & -2.41 & -0.83 & 2043.69 & 259.90 & & & -0.80 & 264.32 \\
\hline $97 \quad 296$ & 0.24 & 0.00 & -0.01 & 0.02 & 0.261 & 0.000 & 0.039 & -0.014 & -2.63 & -1.15 & 2045.26 & 266.40 & & & & 270.97 \\
\hline $98 \quad 297$ & 0.24 & 0.00 & -0.01 & 0.02 & 0.261 & 0.000 & 0.039 & -0.014 & -2.61 & -1.11 & 2047.74 & 271.99 & & & -1.09 & 276.73 \\
\hline 199298 & 0.25 & 0.00 & 0.00 & 0.02 & 0.273 & 0.000 & 0.029 & -0.017 & -3.04 & -1.56 & 2049.28 & 278.52 & & & & 283.42 \\
\hline $200 \quad 299$ & 0.25 & 0.00 & 0.00 & 0.03 & 0.273 & 0.000 & 0.030 & -0.027 & -3.27 & -1.71 & 2051.81 & 284.07 & & & -1.59 & 289.26 \\
\hline 1300 & 0.26 & 0.00 & 0.01 & 0.03 & 0.285 & 0.000 & 0.021 & -0.030 & -3.79 & -2.18 & 2053.23 & 290.71 & & & -2.07 & 296.08 \\
\hline 2301 & 0.26 & 0.00 & 0.01 & 0.03 & 0.285 & 0.000 & 0.021 & -0.030 & -3.77 & -2.21 & 2055.48 & 296.54 & & & -2.09 & 302.10 \\
\hline $03 \quad 302$ & 0.25 & 0.00 & 0.01 & 0.03 & 0.273 & 0.000 & 0.018 & -0.030 & -4.05 & -2.51 & 2056.58 & 303.50 & & & -2.39 & 309.25 \\
\hline 94303 & 0.24 & 0.00 & 0.00 & 0.03 & 0.261 & 0.000 & 0.028 & -0.027 & -3.84 & -2.35 & 2058.50 & 309.66 & & & -2.22 & 315.61 \\
\hline 205304 & 0.23 & 0.00 & -0.01 & 0.04 & 0.250 & 0.000 & 0.039 & -0.035 & -4.34 & -2.72 & 2059.53 & 316.70 & & & -2.45 & 322.99 \\
\hline 305 & 0.23 & 0.00 & -0.01 & 0.04 & 0.250 & 0.000 & 0.039 & -0.035 & -4.21 & -2.66 & 2061.40 & 322.90 & & & -2.37 & 329.40 \\
\hline 97306 & 0.22 & 0.00 & -0.02 & 0.04 & 0.238 & 0.000 & 0.049 & -0.032 & -4.51 & -2.93 & 2062.20 & 330.17 & & & -2.64 & 336.89 \\
\hline
\end{tabular}




\section{$Z=99($ Es $)$}

$\begin{array}{lllll}209 & 308 & 0.21 & 0.00 & -0.02\end{array}$

$\begin{array}{lllll}210 & 309 & 0.21 & 0.00 & -0.02\end{array}$

$\begin{array}{lllllllll}0.04 & 0.238 & 0.000 & 0.049 & -0.032 & -4.37 & -2.79 & 2063.84 & 336.60\end{array}$

$\begin{array}{lllllllll}0.04 & 0.227 & 0.000 & 0.047 & -0.033 & -4.66 & -3.10 & 2064.55 & 343.96\end{array}$

$0.04=0.227$

0.000

$\begin{array}{llllll}0.047 & -0.033 & -4.53 & -3.00 & 2066.10 & 350.49\end{array}$

211310

$\begin{array}{llll}0.21 & 0.00 & -0.02\end{array}$

$0.04 \quad 0.227$

0.000

$\begin{array}{llllll}0.047 & -0.033 & -4.80 & -3.26 & 2066.61 & 358.04\end{array}$

212311

$\begin{array}{lll}0.20 & 0.00 & -0.02\end{array}$

0.04

0.216

0.000

$\begin{array}{llllll}0.045 & -0.034 & -4.56 & -3.09 & 2067.96 & 364.77\end{array}$

213312

$\begin{array}{lll}0.20 & 0.00 & -0.02\end{array}$

$0.04 \quad 0.216$

0.000

$\begin{array}{llllll}0.045 & -0.034 & -4.82 & -3.36 & 2068.36 & 372.44\end{array}$

214313

$\begin{array}{llll}0.21 & 0.00 & -0.01\end{array}$

$0.04-0.227$

0.000

$\begin{array}{llllll}0.035 & -0.036 & -4.60 & -3.19 & 2069.57 & 379.30\end{array}$

$\begin{array}{ll}115 & 314\end{array}$

$0.00-0.01$

0.216

0.000

$\begin{array}{llllll}0.033 & -0.036 & -4.88 & -3.53 & 2069.90 & 387.04\end{array}$

217316

$\begin{array}{lll}0.21 & 0.00 & 0.00\end{array}$

0.04

0.228

0.000

$\begin{array}{llllll}0.023 & -0.038 & -4.78 & -3.48 & 2071.11 & 393.90\end{array}$

$0.04 \quad 0.229$

0.000

$\begin{array}{llllll}0.023 & -0.038 & -5.14 & -3.85 & 2071.35 & 401.74\end{array}$

218317

$\begin{array}{lll}0.21 & 0.00 & 0.0\end{array}$

0.04

0.229

0.000

$\begin{array}{llllll}0.011 & -0.041 & -5.11 & -3.87 & 2072.48 & 408.67\end{array}$

220319

$\begin{array}{lll}0.21 & 0.00 & 0.02\end{array}$

$0.04 \quad 0.230$

$\begin{array}{lllllll}0.000 & -0.001 & -0.043 & -5.40 & -4.12 & 2073.48 & 423.82\end{array}$

221320

$\begin{array}{lll}0.21 & 0.00 & 0.02\end{array}$

0.04

0.230

$\begin{array}{lllllll}0.000 & -0.001 & -0.043 & -5.64 & -4.36 & 2073.34 & 432.03\end{array}$

$222 \quad 321$

$\begin{array}{lll}0.20 & 0.00 & 0.02\end{array}$

0.03

0.218

$\begin{array}{lllllll}0.000 & -0.004 & -0.033 & -5.14 & -4.03 & 2073.38 & 448.13\end{array}$

224323

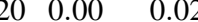

0.02

0.207

225324

$\begin{array}{lll}0.19 & 0.00 & 0.03\end{array}$

0.02

0.207

0.01

0.197

227326

$\begin{array}{lll}0.18 & 0.00 & 0.04\end{array}$

0.00

0.196

229328

$\begin{array}{lll}0.18 & 0.00 & 0.04\end{array}$

0.00

0.197

$\begin{array}{lllllll}0.000 & -0.034 & -0.017 & -4.70 & -3.60 & 2072.99 & 480.80\end{array}$

$\begin{array}{lllllll}0.000 & -0.034 & -0.007 & -4.29 & -3.26 & 2073.16 & 488.71\end{array}$

$\begin{array}{lllllll}0.000 & -0.046 & -0.009 & -4.62 & -3.44 & 2072.50 & 497.44\end{array}$

\section{$Z=100(\mathbf{F m})$}

\begin{tabular}{|c|c|c|c|c|c|c|c|c|c|c|c|c|c|c|c|c|}
\hline $126 \quad 226$ & 0.00 & 0.06 & 0.00 & 0.00 & 0.002 & -0.081 & 0.002 & 0.002 & -2.96 & -1.63 & 1664.81 & 81.07 & & & -1.63 & 83.20 \\
\hline $127 \quad 227$ & 0.01 & 0.07 & -0.01 & 0.00 & 0.013 & -0.095 & 0.014 & 0.003 & -2.55 & -1.00 & 1673.22 & 80.74 & & & -1.00 & 82.79 \\
\hline 228 & -0.56 & 0.00 & 0.01 & -0.02 & -0.557 & 0.000 & 0.104 & -0.004 & -2.69 & 4.70 & 1678.31 & 83.72 & & & 4.26 & 85.25 \\
\hline 9229 & -0.56 & 0.00 & 0.01 & -0.02 & -0.557 & 0.000 & 0.104 & -0.004 & -2.81 & 4.70 & 1687.03 & 83.07 & & & 4.23 & 84.50 \\
\hline 0230 & 0.26 & 0.00 & -0.01 & 0.01 & 0.283 & 0.000 & 0.043 & -0.003 & -0.73 & 0.50 & 1701.69 & 76.48 & & & 0.44 & 78.25 \\
\hline 231 & 0.27 & 0.00 & -0.01 & 0.01 & 0.294 & 0.000 & 0.045 & -0.003 & -1.02 & 0.31 & 1710.30 & 75.94 & & & 0.24 & 77.63 \\
\hline 232 & 0.28 & 0.00 & 0.00 & 0.01 & 0.306 & 0.000 & 0.036 & -0.006 & -1.01 & 0.27 & 1720.49 & 73.82 & & & 0.19 & 75.44 \\
\hline 233 & 0.28 & 0.00 & 0.00 & 0.01 & 0.306 & 0.000 & 0.036 & -0.006 & -1.30 & 0.01 & 1728.86 & 73.52 & & & -0.07 & 75.07 \\
\hline 4234 & 0.28 & 0.00 & 0.00 & 0.02 & 0.306 & 0.000 & 0.037 & -0.016 & -1.49 & -0.09 & 1738.80 & 71.65 & & & -0.14 & 73.17 \\
\hline 5235 & 0.28 & 0.00 & 0.00 & 0.02 & 0.306 & 0.000 & 0.037 & -0.016 & -1.81 & -0.38 & 1746.91 & 71.61 & & & -0.45 & 73.06 \\
\hline 236 & 0.26 & 0.00 & -0.01 & 0.02 & 0.283 & 0.000 & 0.044 & -0.013 & -1.86 & -0.32 & 1756.40 & 70.20 & & & -0.36 & 71.62 \\
\hline $7 \quad 237$ & 0.26 & 0.00 & -0.01 & 0.02 & 0.283 & 0.000 & 0.044 & -0.013 & -2.18 & -0.55 & 1764.15 & 70.51 & & & -0.60 & 71.88 \\
\hline 238 & 0.24 & 0.00 & -0.03 & 0.03 & 0.260 & 0.000 & 0.065 & -0.018 & -2.72 & -0.74 & 1773.59 & 69.15 & & & -0.74 & 70.51 \\
\hline 9239 & 0.24 & 0.00 & -0.03 & 0.03 & 0.260 & 0.000 & 0.065 & -0.018 & -3.14 & -1.12 & 1781.21 & 69.60 & & & -1.13 & 70.90 \\
\hline $0 \quad 240$ & 0.23 & 0.00 & -0.03 & 0.03 & 0.249 & 0.000 & 0.062 & -0.019 & -3.40 & -1.41 & 1790.47 & 68.41 & & & -1.40 & 69.69 \\
\hline 241 & 0.23 & 0.00 & -0.03 & 0.03 & 0.249 & 0.000 & 0.062 & -0.019 & -3.88 & -1.91 & 1797.95 & 69.01 & & & -1.91 & 70.24 \\
\hline 242 & 0.22 & 0.00 & -0.04 & 0.03 & 0.237 & 0.000 & 0.073 & -0.017 & -4.29 & -2.14 & 1806.85 & 68.17 & & & -2.12 & 69.40 \\
\hline 243 & 0.22 & 0.00 & -0.04 & 0.04 & 0.237 & 0.000 & 0.073 & -0.027 & -4.97 & -2.57 & 1813.99 & 69.11 & & & -2.53 & 70.32 \\
\hline 244 & 0.23 & 0.00 & -0.03 & 0.04 & 0.249 & 0.000 & 0.063 & -0.029 & -4.94 & -2.69 & 1822.51 & 68.66 & & & -2.63 & 69.85 \\
\hline 5245 & 0.23 & 0.00 & -0.03 & 0.04 & 0.249 & 0.000 & 0.063 & -0.029 & -5.39 & -3.09 & 1829.35 & 69.88 & & & -3.05 & 71.04 \\
\hline 246 & 0.23 & 0.00 & -0.03 & 0.05 & 0.248 & 0.000 & 0.064 & -0.039 & -5.76 & -3.20 & 1837.60 & 69.71 & 70.14 & 0.039 & -3.09 & 70.90 \\
\hline 247 & 0.23 & 0.00 & -0.02 & 0.04 & 0.249 & 0.000 & 0.051 & -0.032 & -5.75 & -3.57 & 1844.15 & 71.23 & & & -3.51 & 72.35 \\
\hline 248 & 0.23 & 0.00 & -0.02 & 0.04 & 0.249 & 0.000 & 0.051 & -0.032 & -5.79 & -3.60 & 1852.06 & 71.39 & 71.91 & 0.012 & -3.53 & 72.51 \\
\hline 19249 & 0.23 & 0.00 & -0.01 & 0.04 & 0.250 & 0.000 & 0.039 & -0.035 & -6.16 & -4.03 & 1858.42 & 73.10 & & & -3.96 & 74.20 \\
\hline 0250 & 0.23 & 0.00 & -0.01 & 0.04 & 0.250 & 0.000 & 0.039 & -0.035 & -6.21 & -4.08 & 1866.09 & 73.50 & 74.07 & 0.012 & -4.00 & 74.60 \\
\hline 251 & 0.23 & 0.00 & 0.00 & 0.04 & 0.250 & 0.000 & 0.027 & -0.037 & -6.60 & -4.48 & 1872.18 & 75.49 & 75.99 & 0.008 & -4.41 & 76.57 \\
\hline 252 & 0.23 & 0.00 & 0.00 & 0.04 & 0.250 & 0.000 & 0.027 & -0.037 & -6.45 & -4.35 & 1879.42 & 76.32 & 76.82 & 0.006 & -4.26 & 77.41 \\
\hline 253 & 0.23 & 0.00 & 0.01 & 0.04 & 0.251 & 0.000 & 0.015 & -0.040 & -6.37 & -4.24 & 1884.76 & 79.05 & 79.35 & 0.004 & -4.16 & 80.14 \\
\hline 4254 & 0.23 & 0.00 & 0.01 & 0.04 & 0.251 & 0.000 & 0.015 & -0.040 & -5.95 & -3.86 & 1891.50 & 80.38 & 80.90 & 0.003 & -3.76 & 81.49 \\
\hline 5255 & 0.23 & 0.00 & 0.02 & 0.03 & 0.252 & 0.000 & 0.002 & -0.033 & -5.56 & -3.69 & 1896.54 & 83.41 & 83.80 & 0.005 & -3.64 & 84.48 \\
\hline 256 & 0.22 & 0.00 & 0.02 & 0.03 & 0.240 & 0.000 & -0.000 & -0.033 & -5.03 & -3.24 & 1902.98 & 85.05 & 85.49 & 0.007 & -3.18 & 86.14 \\
\hline 257 & 0.22 & 0.00 & 0.03 & 0.02 & 0.241 & 0.000 & -0.013 & -0.026 & -4.95 & -3.24 & 1907.95 & 88.14 & 88.59 & 0.006 & -3.21 & 89.20 \\
\hline 258 & 0.22 & 0.00 & 0.03 & 0.02 & 0.241 & 0.000 & -0.013 & -0.026 & -4.64 & -2.96 & 1914.32 & 89.84 & & & -2.93 & 90.93 \\
\hline 9259 & 0.21 & 0.00 & 0.03 & 0.02 & 0.230 & 0.000 & -0.015 & -0.026 & -4.77 & -3.13 & 1919.24 & 92.99 & & & -3.09 & 94.09 \\
\hline
\end{tabular}




\section{$Z=100(\mathrm{Fm})$}

\begin{tabular}{|c|c|c|c|c|c|c|c|c|c|c|c|c|c|c|}
\hline 160260 & 0.21 & 0.00 & 0.04 & 0.01 & 0.230 & 0.000 & -0.028 & -0.018 & -4.69 & -3.03 & 1925.56 & 94.75 & -3.00 & 95.86 \\
\hline 161261 & 0.21 & 0.00 & 0.04 & 0.01 & 0.230 & 0.000 & -0.028 & -0.018 & -4.93 & -3.18 & 1930.25 & 98.12 & -3.17 & 99.25 \\
\hline $162 \quad 262$ & 0.21 & 0.00 & 0.05 & 0.01 & 0.231 & 0.000 & -0.040 & -0.021 & -5.06 & -3.08 & 1936.34 & 100.11 & -3.04 & 101.30 \\
\hline 63263 & 0.20 & 0.00 & 0.05 & 0.00 & 0.219 & 0.000 & -0.043 & -0.010 & -4.91 & -3.06 & 1940.64 & 103.89 & -3.04 & 105.08 \\
\hline 164264 & 0.20 & 0.00 & 0.05 & 0.00 & 0.219 & 0.000 & -0.043 & -0.010 & -4.33 & -2.53 & 1946.08 & 106.51 & -2.50 & 107.75 \\
\hline $65 \quad 265$ & 0.19 & 0.00 & 0.05 & 0.00 & 0.208 & 0.000 & -0.045 & -0.010 & -4.03 & -2.32 & 1949.98 & 110.69 & -2.29 & 111.96 \\
\hline 66266 & 0.18 & 0.00 & 0.04 & 0.00 & 0.196 & 0.000 & -0.034 & -0.007 & -3.18 & -1.75 & 1955.16 & 113.58 & -1.73 & 114.89 \\
\hline 167267 & 0.16 & 0.00 & 0.03 & 0.01 & 0.174 & 0.000 & -0.024 & -0.015 & -2.95 & -1.79 & 1959.10 & 117.70 & -1.77 & 119.06 \\
\hline $68 \quad 268$ & 0.16 & 0.00 & 0.03 & 0.01 & 0.174 & 0.000 & -0.024 & -0.015 & -2.60 & -1.52 & 1964.37 & 120.50 & -1.49 & 121.92 \\
\hline 169269 & 0.16 & 0.00 & 0.03 & 0.00 & 0.174 & 0.000 & -0.025 & -0.005 & -2.56 & -1.41 & 1967.97 & 124.98 & -1.41 & 126.42 \\
\hline $70 \quad 270$ & 0.14 & 0.00 & 0.02 & 0.01 & 0.151 & 0.000 & -0.015 & -0.013 & -2.14 & -1.21 & 1973.10 & 127.92 & -1.19 & 129.43 \\
\hline 71271 & 0.13 & 0.00 & 0.02 & 0.00 & 0.140 & 0.000 & -0.017 & -0.003 & -2.37 & -1.53 & 1976.92 & 132.17 & -1.53 & 133.73 \\
\hline $172 \quad 272$ & 0.12 & 0.00 & 0.02 & 0.00 & 0.129 & 0.000 & -0.018 & -0.002 & -2.20 & -1.42 & 1981.94 & 135.22 & -1.41 & 136.84 \\
\hline 173273 & 0.12 & 0.00 & 0.02 & 0.00 & 0.129 & 0.000 & -0.018 & -0.002 & -2.57 & -1.78 & 1985.61 & 139.62 & -1.78 & 141.31 \\
\hline 174274 & 0.12 & 0.00 & 0.03 & -0.01 & 0.129 & 0.000 & -0.031 & 0.006 & -2.56 & -1.65 & 1990.41 & 142.89 & -1.63 & 144.67 \\
\hline $175 \quad 275$ & 0.12 & 0.00 & 0.04 & -0.01 & 0.130 & 0.000 & -0.043 & 0.005 & -3.00 & -1.90 & 1993.78 & 147.60 & -1.86 & 149.47 \\
\hline 176276 & -0.10 & 0.00 & 0.01 & -0.01 & -0.105 & 0.000 & -0.007 & 0.011 & -2.13 & -1.63 & 1998.24 & 151.21 & -1.61 & 153.13 \\
\hline 177277 & -0.10 & 0.00 & 0.02 & 0.00 & -0.105 & 0.000 & -0.019 & 0.002 & -2.64 & -2.11 & 2001.65 & 155.87 & -2.10 & 157.86 \\
\hline $178 \quad 278$ & -0.10 & 0.00 & 0.02 & -0.01 & -0.105 & 0.000 & -0.019 & 0.012 & -2.55 & -2.00 & 2006.09 & 159.50 & -1.97 & 161.60 \\
\hline 179279 & -0.08 & 0.00 & 0.02 & 0.00 & -0.084 & 0.000 & -0.021 & 0.002 & -2.49 & -1.96 & 2008.80 & 164.86 & -1.95 & 167.03 \\
\hline 180280 & -0.07 & 0.00 & 0.02 & 0.00 & -0.073 & 0.000 & -0.021 & 0.002 & -2.13 & -1.59 & 2012.78 & 168.95 & -1.58 & 171.22 \\
\hline 181281 & -0.04 & 0.00 & 0.01 & 0.00 & -0.042 & 0.000 & -0.011 & 0.001 & -2.28 & -1.71 & 2015.48 & 174.33 & -1.71 & 176.68 \\
\hline $182 \quad 282$ & 0.00 & 0.00 & 0.00 & 0.00 & 0.000 & 0.000 & 0.000 & 0.000 & -2.01 & -1.46 & 2019.40 & 178.48 & -1.47 & 180.92 \\
\hline 183283 & 0.00 & 0.00 & 0.00 & 0.00 & 0.000 & 0.000 & 0.000 & 0.000 & -2.33 & -1.76 & 2022.09 & 183.85 & -1.77 & 186.40 \\
\hline 184284 & 0.00 & 0.02 & 0.00 & 0.00 & 0.000 & -0.027 & 0.000 & 0.000 & -2.04 & -1.42 & 2025.74 & 188.28 & -1.42 & 190.94 \\
\hline $185 \quad 285$ & 0.01 & 0.06 & 0.00 & 0.00 & 0.012 & -0.081 & 0.002 & 0.002 & -2.07 & -0.90 & 2027.44 & 194.65 & -0.86 & 197.46 \\
\hline 186286 & 0.01 & 0.07 & 0.00 & 0.00 & 0.013 & -0.094 & 0.002 & 0.003 & -1.68 & -0.30 & 2030.66 & 199.50 & -0.25 & 202.44 \\
\hline $187 \quad 287$ & 0.03 & 0.09 & -0.01 & 0.00 & 0.035 & -0.123 & 0.016 & 0.006 & -2.11 & -0.16 & 2032.57 & 205.66 & -0.07 & 208.76 \\
\hline 188288 & 0.03 & 0.09 & -0.01 & 0.00 & 0.035 & -0.123 & 0.016 & 0.006 & -1.57 & 0.33 & 2035.73 & 210.57 & 0.42 & 213.80 \\
\hline 189289 & 0.23 & 0.00 & -0.03 & 0.01 & 0.249 & 0.000 & 0.061 & 0.001 & -1.29 & 0.27 & 2037.68 & 216.70 & 0.29 & 219.98 \\
\hline $190 \quad 290$ & 0.23 & 0.00 & -0.02 & 0.00 & 0.250 & 0.000 & 0.048 & 0.008 & -1.10 & 0.23 & 2041.19 & 221.26 & 0.24 & 224.65 \\
\hline 191291 & 0.23 & 0.00 & -0.02 & 0.00 & 0.250 & 0.000 & 0.048 & 0.008 & -1.46 & -0.13 & 2043.27 & 227.24 & -0.13 & 230.77 \\
\hline $192 \quad 292$ & 0.23 & 0.00 & -0.02 & 0.00 & 0.250 & 0.000 & 0.048 & 0.008 & -1.48 & -0.14 & 2046.59 & 232.00 & -0.13 & 235.67 \\
\hline 193293 & 0.23 & 0.00 & -0.02 & 0.01 & 0.249 & 0.000 & 0.048 & -0.002 & -1.79 & -0.43 & 2048.44 & 238.22 & -0.43 & 242.02 \\
\hline 194294 & 0.23 & 0.00 & -0.02 & 0.01 & 0.249 & 0.000 & 0.048 & -0.002 & -1.78 & -0.35 & 2051.51 & 243.22 & -0.35 & 247.18 \\
\hline $195 \quad 295$ & 0.24 & 0.00 & -0.01 & 0.01 & 0.261 & 0.000 & 0.038 & -0.004 & -2.03 & -0.64 & 2053.21 & 249.59 & -0.67 & 253.67 \\
\hline 196296 & 0.24 & 0.00 & -0.01 & 0.01 & 0.261 & 0.000 & 0.038 & -0.004 & -2.03 & -0.65 & 2056.20 & 254.67 & -0.67 & 258.91 \\
\hline $197 \quad 297$ & 0.24 & 0.00 & -0.01 & 0.02 & 0.261 & 0.000 & 0.039 & -0.014 & -2.49 & -1.04 & 2057.84 & 261.10 & -1.01 & 265.54 \\
\hline 198298 & 0.25 & 0.00 & 0.00 & 0.02 & 0.273 & 0.000 & 0.029 & -0.017 & -2.52 & -1.11 & 2060.74 & 266.28 & -1.08 & 270.88 \\
\hline 199299 & 0.25 & 0.00 & 0.00 & 0.02 & 0.273 & 0.000 & 0.029 & -0.017 & -2.96 & -1.53 & 2062.27 & 272.82 & -1.51 & 277.58 \\
\hline 200300 & 0.26 & 0.00 & 0.01 & 0.03 & 0.285 & 0.000 & 0.021 & -0.030 & -3.30 & -1.76 & 2065.18 & 277.98 & -1.63 & 283.03 \\
\hline 201301 & 0.26 & 0.00 & 0.01 & 0.03 & 0.285 & 0.000 & 0.021 & -0.030 & -3.76 & -2.31 & 2066.68 & 284.56 & -2.18 & 289.77 \\
\hline 202302 & 0.26 & 0.00 & 0.01 & 0.03 & 0.285 & 0.000 & 0.021 & -0.030 & -3.75 & -2.33 & 2069.22 & 290.08 & -2.19 & 295.49 \\
\hline 203303 & 0.25 & 0.00 & 0.01 & 0.03 & 0.273 & 0.000 & 0.018 & -0.030 & -4.06 & -2.67 & 2070.37 & 297.00 & -2.53 & 302.59 \\
\hline 204304 & 0.25 & 0.00 & 0.01 & 0.03 & 0.273 & 0.000 & 0.018 & -0.030 & -3.86 & -2.54 & 2072.61 & 302.83 & -2.39 & 308.61 \\
\hline 205305 & 0.24 & 0.00 & 0.00 & 0.04 & 0.262 & 0.000 & 0.029 & -0.037 & -4.30 & -2.72 & 2073.47 & 310.05 & -2.44 & 316.16 \\
\hline 206306 & 0.23 & 0.00 & -0.01 & 0.04 & 0.250 & 0.000 & 0.039 & -0.035 & -4.11 & -2.67 & 2075.64 & 315.95 & -2.38 & 322.26 \\
\hline 207307 & 0.22 & 0.00 & -0.01 & 0.04 & 0.238 & 0.000 & 0.037 & -0.035 & -4.28 & -2.85 & 2076.35 & 323.31 & -2.56 & 329.82 \\
\hline 208308 & 0.22 & 0.00 & -0.01 & 0.04 & 0.238 & 0.000 & 0.037 & -0.035 & -4.15 & -2.74 & 2078.33 & 329.40 & -2.44 & 336.12 \\
\hline 209309 & 0.21 & 0.00 & -0.02 & 0.04 & 0.227 & 0.000 & 0.047 & -0.033 & -4.48 & -2.96 & 2078.94 & 336.86 & -2.65 & 343.79 \\
\hline 210310 & 0.21 & 0.00 & -0.02 & 0.04 & 0.227 & 0.000 & 0.047 & -0.033 & -4.35 & -2.85 & 2080.77 & 343.10 & -2.53 & 350.26 \\
\hline 211311 & 0.21 & 0.00 & -0.02 & 0.04 & 0.227 & 0.000 & 0.047 & -0.033 & -4.60 & -3.09 & 2081.28 & 350.67 & -2.78 & 358.03 \\
\hline 212312 & 0.21 & 0.00 & -0.01 & 0.04 & 0.227 & 0.000 & 0.035 & -0.036 & -4.36 & -2.95 & 2082.94 & 357.08 & -2.62 & 364.67 \\
\hline 213313 & 0.21 & 0.00 & -0.01 & 0.04 & 0.227 & 0.000 & 0.035 & -0.036 & -4.64 & -3.25 & 2083.36 & 364.73 & -2.93 & 372.54 \\
\hline 214314 & 0.20 & 0.00 & -0.01 & 0.04 & 0.216 & 0.000 & 0.033 & -0.036 & -4.46 & -3.11 & 2084.90 & 371.26 & -2.78 & 379.32 \\
\hline 315 & 0.21 & 0.00 & 0.00 & 0.04 & 0.228 & 0.000 & 0.023 & -0.038 & -4.82 & -3.51 & 2085.29 & 378.93 & -3.17 & 387.23 \\
\hline 216316 & 0.21 & 0.00 & 0.00 & 0.04 & 0.228 & 0.000 & 0.023 & -0.038 & -4.73 & -3.45 & 2086.77 & 385.53 & -3.10 & 394.07 \\
\hline
\end{tabular}




\section{$Z=100(\mathrm{Fm})$}

$\begin{array}{llll}217 & 317 & 0.21 & 0.00\end{array}$

$\begin{array}{llll}218 & 318 & 0.21 & 0.00\end{array}$

$\begin{array}{llll}219 & 319 & 0.21 & 0.00\end{array}$

$\begin{array}{llll}220 & 320 & 0.21 & 0.00\end{array}$

$\begin{array}{llll}221 & 321 & 0.20 & 0.00\end{array}$

$222 \quad 322$

$223 \quad 323$

$\begin{array}{ll}0.20 & 0.00\end{array}$

$\begin{array}{ll}0.20 & 0.00\end{array}$

$224 \quad 324$

$\begin{array}{ll}0.19 & 0.00\end{array}$

$\begin{array}{ll}225 & 325\end{array}$

$\begin{array}{ll}0.19 & 0.00\end{array}$

226326

$0.18 \quad 0.00$

$\begin{array}{ll}227 & 327\end{array}$

$0.18 \quad 0.00$

$\begin{array}{ll}228 & 328\end{array}$

$0.18 \quad 0.00$

229329

$0.18 \quad 0.00$

$230 \quad 330$

$0.18 \quad 0.00$

$0.18 \quad 0.00$

\section{$01-0.04$}

0.01

$\begin{array}{lll}0.04 & 0.229 & 0.000\end{array}$

$\begin{array}{llllll}0.011 & -0.041 & -5.17 & -3.92 & 2087.12 & 393.26\end{array}$

$\begin{array}{llllllllll}0.01 & 0.04 & 0.229 & 0.000 & 0.011 & -0.041 & -5.47 & -4.24 & 2088.58 & 407.94\end{array}$

$\begin{array}{llllllllll}0.02 & 0.04 & 0.230 & 0.000 & -0.001 & -0.043 & -5.47 & -4.20 & 2089.82 & 414.77\end{array}$

$\begin{array}{llllllllll}0.02 & 0.03 & 0.218 & 0.000 & -0.004 & -0.033 & -5.40 & -4.25 & 2089.49 & 423.16\end{array}$

$0.03 \quad 0.03$

$0.03 \quad 0.03$

$\begin{array}{llllllll}0.219 & 0.000 & -0.016 & -0.035 & -5.24 & -4.05 & 2090.44 & 430.29\end{array}$

0.03

0.02

$\begin{array}{llllllll}0.219 & 0.000 & -0.016 & -0.035 & -5.43 & -4.24 & 2090.14 & 438.67\end{array}$

0.03

0.02$$
\begin{array}{llllllll}
0.207 & 0.000 & -0.019 & -0.025 & -4.84 & -3.78 & 2090.70 & 446.17
\end{array}
$$

$0.04 \quad 0.01$

$\begin{array}{llllllll}0.207 & 0.000 & -0.019 & -0.025 & -5.02 & -3.97 & 2090.28 & 454.67\end{array}$

$0.04 \quad 0.01$

$\begin{array}{llllllll}0.197 & 0.000 & -0.034 & -0.017 & -4.76 & -3.63 & 2090.84 & 462.17\end{array}$

$0.04 \quad 0.00$

$\begin{array}{llllllll}0.197 & 0.000 & -0.034 & -0.017 & -4.94 & -3.84 & 2090.32 & 470.77\end{array}$

$0.05 \quad 0.00$$$
\begin{array}{llllllll}
0.196 & 0.000 & -0.034 & -0.007 & -4.52 & -3.49 & 2090.75 & 478.41
\end{array}
$$$$
\begin{array}{llllllll}
0.197 & 0.000 & -0.046 & -0.009 & -4.88 & -3.70 & 2090.12 & 487.11
\end{array}
$$

$\begin{array}{rr}0.06 & -0.01\end{array}$

\section{$Z=101$ (Md)}

$\begin{array}{llll}128 & 229 & -0.56 & 0.00\end{array}$

$\begin{array}{llll}129 & 230 & -0.57 & 0.00\end{array}$

$\begin{array}{llll}130 & 231 & 0.27 & 0.00\end{array}$

$\begin{array}{llll}0.01 & -0.02 & -0.557 & 0.000\end{array}$

$\begin{array}{lllll}0.01 & -0.02 & -0.566 & 0.000\end{array}$

$\begin{array}{lll}0.104 & -0.004 & -2.77 \\ 0.107 & -0.005 & -3.13\end{array}$

$\begin{array}{ll}4.40 & 1676.17\end{array}$

$\begin{array}{llll}0.033 & -0.006 & -0.74\end{array}$

$\begin{array}{ll}4.48 & 1685.20\end{array}$

$\begin{array}{ll}0.36 & 1699.83\end{array}$

$0.033-0.006-1.01$

$\begin{array}{ll}0.16 & 1708.83\end{array}$

$\begin{array}{llll}132 & 233 & 0.28 & 0.00\end{array}$

$\begin{array}{llll}0.00 & 0.01 & 0.295 & 0.000\end{array}$

$\begin{array}{llll}0.023 & -0.009 & -1.08\end{array}$

$\begin{array}{ll}0.08 & 1719.10\end{array}$

$\begin{array}{lllll}0.023 & -0.009 & -1.37 & -0.17 & 1727.84\end{array}$

$\begin{array}{llllllll}133 & 234 & 0.28 & 0.00 & 0.01 & 0.01 & 0.307 & 0.000\end{array}$

$\begin{array}{llllllll}134 & 235 & 0.28 & 0.00 & 0.01 & 0.02 & 0.307 & 0.000\end{array}$

$\begin{array}{llllllll}135 & 236 & 0.28 & 0.00 & 0.01 & 0.02 & 0.307 & 0.000\end{array}$

$\begin{array}{ll}136 & 237\end{array}$

$\begin{array}{lll}0.27 & 0.00 & 0.00\end{array}$

0.02

137238

$\begin{array}{llll}0.26 & 0.00 & -0.01\end{array}$

0.03

$\begin{array}{ll}0.295 & 0.000\end{array}$

$\begin{array}{lllll}0.025 & -0.019 & -1.62 & -0.28 & 1737.83\end{array}$

$\begin{array}{lllll}0.025 & -0.019 & -1.93 & -0.59 & 1746.35\end{array}$

$\begin{array}{lllll}0.034 & -0.016 & -1.94 & -0.48 & 1755.81\end{array}$

$0.283 \quad 0.000$

$\begin{array}{lllll}0.045 & -0.023 & -2.42 & -0.69 & 1763.94\end{array}$

$\begin{array}{llllll}0.045 & -0.023 & -2.54 & -0.79 & 1773.32\end{array}$

$\begin{array}{ll}138 & 239 \\ 139 & 240\end{array}$

$\begin{array}{lll}0.26 & 0.00 & -0.01\end{array}$

0.03

$\begin{array}{ll}0.283 & 0.000\end{array}$

$\begin{array}{ll}140 & 241\end{array}$

$\begin{array}{llll}0.24 & 0.00 & -0.02\end{array}$

0.03

$\begin{array}{ll}0.260 & 0.000\end{array}$

$\begin{array}{lllll}0.052 & -0.021 & -2.95 & -1.11 & 1781.26\end{array}$

$\begin{array}{lllll}0.062 & -0.019 & -3.35 & -1.43 & 1790.58\end{array}$

$\begin{array}{lll}0.03 & 0.237 & 0.000\end{array}$

$\begin{array}{lllll}0.060 & -0.020 & -3.79 & -1.90 & 1798.40\end{array}$

$\begin{array}{llllll}0.060 & -0.020 & -4.03 & -2.18 & 1807.40\end{array}$

142243

$0.22-0.00-0.03$

0.03

$0.237 \quad 0.000$

$\begin{array}{lllll}0.060 & -0.020 & -4.49 & -2.57 & 1814.86\end{array}$

$\begin{array}{lll}0.03 & 0.237 & 0.000\end{array}$

$\begin{array}{llllll}0.061 & -0.030 & -4.84 & -2.69 & 1823.42\end{array}$

$\begin{array}{lllll}0.051 & -0.032 & -5.18 & -3.10 & 1830.64\end{array}$

$\begin{array}{lll}0.04 & 0.249 & 0.000\end{array}$

$\begin{array}{ll}144 & 245 \\ 145 & 246\end{array}$

146247

$\begin{array}{llll}0.22 & 0.00 & -0.03\end{array}$

$\begin{array}{lll}0.23 & 0.00 & -0.02\end{array}$

$\begin{array}{llll}0.23 & 0.00 & -0.02\end{array}$

0.04

$0.249 \quad 0.000$

$\begin{array}{lllll}0.051 & -0.032 & -5.31 & -3.22 & 1838.93\end{array}$

$\begin{array}{llllll}0.051 & -0.032 & -5.77 & -3.64 & 1845.91\end{array}$

$148 \quad 249$

0.04

$0.249 \quad 0.000$

$\begin{array}{lllll}0.039 & -0.035 & -5.77 & -3.72 & 1853.90\end{array}$

$\begin{array}{ll}0.250 & 0.000\end{array}$

$\begin{array}{lllll}0.039 & -0.035 & -6.27 & -4.18 & 1860.65\end{array}$

$0.250 \quad 0.000$

$\begin{array}{llllll}0.027 & -0.037 & -6.36 & -4.30 & 1868.42\end{array}$

$\begin{array}{llllll}0.027 & -0.037 & -6.79 & -4.70 & 1874.87\end{array}$

$\begin{array}{llllll}0.015 & -0.040 & -6.74 & -4.63 & 1882.19\end{array}$

$0.250 \quad 0.000$

$\begin{array}{ll}0.251 & 0.000\end{array}$

$\begin{array}{llllll}0.015 & -0.040 & -6.65 & -4.55 & 1887.92\end{array}$

$\begin{array}{ll}0.251 & 0.000\end{array}$

$\begin{array}{lllll}0.002 & -0.033 & -6.06 & -4.18 & 1894.71\end{array}$

154255

$\begin{array}{ll}0.23 & 0.00\end{array}$

0.01

0.04

$0.252 \quad 0.000$

$\begin{array}{ll}0.22 & 0.00\end{array}$

0.02

0.03

156257

$0.22 \quad 0.00$

0.02

0.03

$\begin{array}{ll}0.240 & 0.000\end{array}$

$\begin{array}{llllll}0.000 & -0.033 & -5.91 & -4.09 & 1900.18\end{array}$

$157 \quad 258$

$0.22 \quad 0.00$

0.03

0.02

$\begin{array}{lllllll}0.241 & 0.000 & -0.013 & -0.026 & -5.44 & -3.74 & 1912.07\end{array}$

$0.22 \quad 0.00$

0.03

0.02

159260

$\begin{array}{ll}0.21 & 0.00\end{array}$

0.04

0.01

$0.241 \quad 0.000$

$-0.0$

$\begin{array}{lllll}0.013 & -0.026 & -5.14 & -3.47 & 1918.48\end{array}$

$\begin{array}{lllllll}0.230 & 0.000 & -0.028 & -0.018 & -5.35 & -3.66 & 1923.78\end{array}$

$\begin{array}{lllllll}0.230 & 0.000 & -0.028 & -0.018 & -5.35 & -3.66 & 1923.78 \\ 0.230 & 0.000 & -0.028 & -0.018 & -5.28 & -3.54 & 1930.10\end{array}$

0.01

0.210 .00

0.05

0.01
0.01

162263

0.210 .00

0.05$$
\begin{array}{llllllll}
0.231 & 0.000 & -0.040 & -0.021 & -5.78 & -3.80 & 1935.24 & 100.43
\end{array}
$$

163264

$\begin{array}{lll}0.21 & 0.00 & 0.06\end{array}$

0.00

164265

165266

$0.20 \quad 0.00$

0.05

0.00

0.2310 .000

$0.19 \quad 0.00$

0.05

0.00

$\begin{array}{llllllll}0.000 & -0.053 & -0.013 & -5.86 & -3.68 & 1946.01 & 105.81\end{array}$

166267

$\begin{array}{lll}0.18 & 0.00 & 0.04\end{array}$

0.00

$\begin{array}{llllllll}0.219 & 0.000 & -0.043 & -0.010 & -4.94 & -3.13 & 1951.44 & 108.44\end{array}$

167268

$\begin{array}{lll}0.17 & 0.00 & 0.04\end{array}$

0.01

$\begin{array}{llllllll}0.208 & 0.000 & -0.045 & -0.010 & -4.60 & -2.88 & 1955.65 & 112.30\end{array}$

$\begin{array}{llllllll}0.196 & 0.000 & -0.034 & -0.007 & -3.71 & -2.26 & 1960.80 & 115.22\end{array}$

168269 $\begin{array}{llllllll}0.185 & 0.000 & -0.035 & -0.017 & -3.64 & -2.23 & 1965.01 & 119.08\end{array}$

$\begin{array}{llllllll}0.174 & 0.000 & -0.024 & -0.015 & -3.03 & -1.92 & 1970.27 & 121.89\end{array}$
$-3.56 \quad 402.05$

$-3.52409 .00$

$-3.86 \quad 417.24$

$-3.78 \quad 424.37$

$-4.03 \quad 432.82$

$-3.77 \quad 440.26$

$-3.97 \quad 448.90$

$\begin{array}{ll}-3.65 & 456.53\end{array}$

$-3.85 \quad 465.29$

$-3.56473 .03$

$-3.77 \quad 481.90$

$\begin{array}{ll}-3.47 \quad 489.78 \\ -3.64 & 498.82\end{array}$

$-3.64 \quad 498.82$

$-3.25 \quad 506.91$

$-3.34 \quad 516.15$

$\begin{array}{ll}3.86 & 94.83\end{array}$

$3.89 \quad 93.74$

$0.26 \quad 87.60$

$0.05 \quad 86.58$

$-0.02 \quad 84.32$

$-0.28 \quad 83.57$

$-0.36 \quad 81.61$

$-0.69 \quad 81.10$

$\begin{array}{ll}-0.55 & 79.67\end{array}$

$-0.75 \quad 79.57$

$-0.83 \quad 78.23$

$-1.14 \quad 78.31$

$-1.44 \quad 77.04$

$-1.91 \quad 77.24$

$-2.18 \quad 76.29$

$\begin{array}{ll}-2.58 & 76.85\end{array}$

$\begin{array}{ll}-2.66 & 76.37\end{array}$

$\begin{array}{ll}-3.08 & 77.18\end{array}$

$-3.18 \quad 76.95$

$-3.61 \quad 78.01$

$\begin{array}{ll}-3.68 & 78.09\end{array}$

$\begin{array}{ll}-4.14 & 79.38\end{array}$

$-4.24 \quad 79.68$

$-4.65 \quad 81.28$

$-4.56 \quad 82.04$

\begin{tabular}{ll}
$-4.49 \quad 84.37$ \\
\hline
\end{tabular}

$\begin{array}{llll}84.84 & 0.007 & -4.15 & 85.63\end{array}$

$\begin{array}{lllll}87.61 & 0.053 & -4.06 & 88.22\end{array}$

$\begin{array}{lllll}89.00 & 0.003 & -3.65 & 89.80\end{array}$

$\begin{array}{llll}91.69 & 0.005 & -3.73 & 92.46\end{array}$

$\begin{array}{ll}-3.45 \quad 94.14 \\ -3.67 & 96.91\end{array}$

$-3.67 \quad 96.91$

$\begin{array}{ll}-3.54 & 98.68\end{array}$

$-3.78 \quad 101.64$

$\begin{array}{ll}-3.65 & 103.65\end{array}$

$\begin{array}{ll}-3.66 & 107.07\end{array}$

$\begin{array}{ll}-3.11 & 109.74\end{array}$

$-2.87 \quad 113.63$

$-2.25 \quad 116.58$

$-2.20120 .50$

\begin{tabular}{ll}
$-1.91 \quad 123.35$ \\
\hline
\end{tabular} 


\section{$Z=101(\mathrm{Md})$}

\begin{tabular}{|c|c|c|c|c|c|c|c|c|c|c|c|c|c|c|}
\hline 169270 & 0.16 & 0.00 & 0.03 & 0.01 & 0.174 & 0.000 & -0.024 & -0.015 & -3.04 & -1.82 & 1974.20 & 126.04 & -1.80 & 127.53 \\
\hline $170 \quad 271$ & 0.14 & 0.00 & 0.02 & 0.01 & 0.151 & 0.000 & -0.015 & -0.013 & -2.49 & -1.54 & 1979.28 & 129.03 & -1.53 & 130.57 \\
\hline 171272 & 0.13 & 0.00 & 0.02 & 0.00 & 0.140 & 0.000 & -0.017 & -0.003 & -2.69 & -1.84 & 1983.42 & 132.96 & -1.85 & 134.54 \\
\hline $172 \quad 273$ & 0.12 & 0.00 & 0.02 & 0.00 & 0.129 & 0.000 & -0.018 & -0.002 & -2.50 & -1.72 & 1988.44 & 136.01 & -1.72 & 137.65 \\
\hline $173 \quad 274$ & 0.12 & 0.00 & 0.02 & 0.00 & 0.129 & 0.000 & -0.018 & -0.002 & -2.87 & -2.08 & 1992.44 & 140.08 & -2.08 & 141.78 \\
\hline $174 \quad 275$ & 0.12 & 0.00 & 0.03 & -0.01 & 0.129 & 0.000 & -0.031 & 0.006 & -2.86 & -1.95 & 1997.27 & 143.33 & -1.93 & 145.12 \\
\hline $175 \quad 276$ & 0.12 & 0.00 & 0.04 & -0.01 & 0.130 & 0.000 & -0.043 & 0.005 & -3.29 & -2.19 & 2000.95 & 147.71 & -2.16 & 149.59 \\
\hline $176 \quad 277$ & 0.12 & 0.00 & 0.04 & -0.02 & 0.130 & 0.000 & -0.043 & 0.014 & -2.96 & -1.85 & 2005.36 & 151.37 & -1.77 & 153.36 \\
\hline $177 \quad 278$ & -0.10 & 0.00 & 0.02 & 0.00 & -0.105 & 0.000 & -0.019 & 0.002 & -2.78 & -2.25 & 2009.03 & 155.78 & -2.25 & 157.77 \\
\hline $178 \quad 279$ & -0.10 & 0.00 & 0.02 & -0.01 & -0.105 & 0.000 & -0.019 & 0.012 & -2.67 & -2.13 & 2013.47 & 159.41 & -2.11 & 161.50 \\
\hline 179280 & -0.08 & 0.00 & 0.02 & 0.00 & -0.084 & 0.000 & -0.021 & 0.002 & -2.59 & -2.07 & 2016.48 & 164.47 & -2.07 & 166.62 \\
\hline $180 \quad 281$ & -0.07 & 0.00 & 0.02 & 0.00 & -0.073 & 0.000 & -0.021 & 0.002 & -2.21 & -1.67 & 2020.46 & 168.56 & -1.67 & 170.81 \\
\hline 181282 & -0.04 & 0.00 & 0.01 & 0.00 & -0.042 & 0.000 & -0.011 & 0.001 & -2.33 & -1.77 & 2023.45 & 173.65 & -1.77 & 175.97 \\
\hline $182 \quad 283$ & 0.00 & 0.03 & 0.00 & 0.00 & 0.000 & -0.040 & 0.000 & 0.001 & -2.18 & -1.49 & 2027.36 & 177.80 & -1.49 & 180.23 \\
\hline $183 \quad 284$ & 0.01 & 0.03 & 0.00 & 0.00 & 0.011 & -0.040 & 0.000 & 0.001 & -2.50 & -1.79 & 2030.37 & 182.86 & -1.79 & 185.39 \\
\hline $184 \quad 285$ & 0.00 & 0.04 & 0.00 & 0.00 & 0.001 & -0.054 & 0.001 & 0.001 & -2.28 & -1.46 & 2034.05 & 187.26 & -1.45 & 189.89 \\
\hline 185286 & 0.01 & 0.07 & 0.00 & 0.00 & 0.013 & -0.094 & 0.002 & 0.003 & -2.43 & -1.02 & 2036.15 & 193.23 & -0.98 & 196.00 \\
\hline 186287 & 0.01 & 0.08 & 0.00 & 0.00 & 0.014 & -0.108 & 0.003 & 0.004 & -2.10 & -0.44 & 2039.40 & 198.05 & -0.38 & 200.95 \\
\hline 187288 & 0.02 & 0.09 & -0.01 & 0.00 & 0.025 & -0.122 & 0.015 & 0.006 & -2.24 & -0.32 & 2041.65 & 203.87 & -0.25 & 206.90 \\
\hline $188 \quad 289$ & 0.03 & 0.10 & -0.01 & 0.01 & 0.036 & -0.135 & 0.016 & -0.003 & -2.02 & 0.20 & 2044.78 & 208.81 & 0.31 & 211.99 \\
\hline 189290 & 0.23 & 0.00 & -0.02 & 0.01 & 0.249 & 0.000 & 0.048 & -0.002 & -1.23 & 0.12 & 2047.07 & 214.60 & 0.11 & 217.78 \\
\hline $190 \quad 291$ & 0.23 & 0.00 & -0.02 & 0.00 & 0.250 & 0.000 & 0.048 & 0.008 & -1.31 & 0.01 & 2050.66 & 219.07 & 0.00 & 222.38 \\
\hline 191292 & 0.23 & 0.00 & -0.02 & 0.00 & 0.250 & 0.000 & 0.048 & 0.008 & -1.63 & -0.33 & 2053.03 & 224.78 & -0.34 & 228.20 \\
\hline $192 \quad 293$ & 0.23 & 0.00 & -0.01 & 0.00 & 0.250 & 0.000 & 0.035 & 0.005 & -1.49 & -0.33 & 2056.35 & 229.53 & -0.35 & 233.07 \\
\hline 193294 & 0.23 & 0.00 & -0.01 & 0.00 & 0.250 & 0.000 & 0.035 & 0.005 & -1.83 & -0.62 & 2058.52 & 235.43 & -0.66 & 239.10 \\
\hline 194295 & 0.23 & 0.00 & -0.01 & 0.01 & 0.250 & 0.000 & 0.036 & -0.005 & -1.83 & -0.62 & 2061.67 & 240.36 & -0.63 & 244.18 \\
\hline 195296 & 0.24 & 0.00 & -0.01 & 0.01 & 0.261 & 0.000 & 0.038 & -0.004 & -2.23 & -0.86 & 2063.63 & 246.46 & -0.90 & 250.42 \\
\hline 196297 & 0.24 & 0.00 & 0.00 & 0.01 & 0.261 & 0.000 & 0.026 & -0.007 & -2.15 & -0.86 & 2066.62 & 251.54 & -0.89 & 255.65 \\
\hline 197298 & 0.24 & 0.00 & 0.00 & 0.01 & 0.261 & 0.000 & 0.026 & -0.007 & -2.54 & -1.34 & 2068.66 & 257.58 & -1.38 & 261.82 \\
\hline 198299 & 0.25 & 0.00 & 0.00 & 0.02 & 0.273 & 0.000 & 0.029 & -0.017 & -2.76 & -1.36 & 2071.52 & 262.79 & -1.35 & 267.25 \\
\hline 199300 & 0.25 & 0.00 & 0.01 & 0.02 & 0.273 & 0.000 & 0.017 & -0.020 & -3.20 & -1.91 & 2073.47 & 268.91 & -1.89 & 273.53 \\
\hline 200301 & 0.25 & 0.00 & 0.01 & 0.02 & 0.273 & 0.000 & 0.017 & -0.020 & -3.32 & -2.02 & 2076.26 & 274.19 & -1.99 & 278.98 \\
\hline 201302 & 0.26 & 0.00 & 0.02 & 0.02 & 0.285 & 0.000 & 0.007 & -0.023 & -3.88 & -2.50 & 2078.00 & 280.52 & -2.48 & 285.48 \\
\hline 202303 & 0.26 & 0.00 & 0.02 & 0.03 & 0.285 & 0.000 & 0.009 & -0.033 & -4.12 & -2.65 & 2080.67 & 285.92 & -2.50 & 291.17 \\
\hline 203304 & 0.25 & 0.00 & 0.01 & 0.03 & 0.273 & 0.000 & 0.018 & -0.030 & -4.34 & -2.95 & 2082.09 & 292.57 & -2.83 & 297.98 \\
\hline 204305 & 0.24 & 0.00 & 0.01 & 0.03 & 0.262 & 0.000 & 0.016 & -0.030 & -4.10 & -2.80 & 2084.31 & 298.42 & -2.66 & 304.02 \\
\hline 205306 & 0.24 & 0.00 & 0.01 & 0.03 & 0.262 & 0.000 & 0.016 & -0.030 & -4.28 & -3.03 & 2085.52 & 305.29 & -2.90 & 311.07 \\
\hline 206307 & 0.23 & 0.00 & 0.00 & 0.03 & 0.250 & 0.000 & 0.026 & -0.027 & -4.06 & -2.79 & 2087.50 & 311.37 & -2.66 & 317.34 \\
\hline 207308 & 0.22 & 0.00 & -0.01 & 0.04 & 0.238 & 0.000 & 0.037 & -0.035 & -4.51 & -3.08 & 2088.62 & 318.33 & -2.80 & 324.63 \\
\hline 208309 & 0.22 & 0.00 & -0.01 & 0.04 & 0.238 & 0.000 & 0.037 & -0.035 & -4.37 & -2.97 & 2090.59 & 324.42 & -2.68 & 330.93 \\
\hline 209310 & 0.21 & 0.00 & -0.02 & 0.04 & 0.227 & 0.000 & 0.047 & -0.033 & -4.64 & -3.20 & 2091.51 & 331.58 & -2.91 & 338.29 \\
\hline 210311 & 0.21 & 0.00 & -0.01 & 0.04 & 0.227 & 0.000 & 0.035 & -0.036 & -4.45 & -3.05 & 2093.31 & 337.85 & -2.75 & 344.77 \\
\hline 211312 & 0.21 & 0.00 & -0.01 & 0.04 & 0.227 & 0.000 & 0.035 & -0.036 & -4.73 & -3.30 & 2094.11 & 345.13 & -3.00 & 352.25 \\
\hline 212313 & 0.21 & 0.00 & -0.01 & 0.04 & 0.227 & 0.000 & 0.035 & -0.036 & -4.59 & -3.17 & 2095.79 & 351.51 & -2.87 & 358.86 \\
\hline 213314 & 0.20 & 0.00 & -0.01 & 0.04 & 0.216 & 0.000 & 0.033 & -0.036 & -4.85 & -3.47 & 2096.50 & 358.88 & -3.16 & 366.44 \\
\hline 214315 & 0.21 & 0.00 & 0.00 & 0.04 & 0.228 & 0.000 & 0.023 & -0.038 & -4.76 & -3.40 & 2098.11 & 365.34 & -3.08 & 373.14 \\
\hline 215316 & 0.20 & 0.00 & 0.00 & 0.04 & 0.217 & 0.000 & 0.021 & -0.038 & -5.09 & -3.80 & 2098.79 & 372.73 & -3.47 & 380.76 \\
\hline 216317 & 0.21 & 0.00 & 0.01 & 0.04 & 0.229 & 0.000 & 0.011 & -0.041 & -5.10 & -3.80 & 2100.32 & 379.27 & -3.44 & 387.55 \\
\hline 217318 & 0.21 & 0.00 & 0.01 & 0.04 & 0.229 & 0.000 & 0.011 & -0.041 & -5.52 & -4.25 & 2100.94 & 386.72 & -3.91 & 395.24 \\
\hline 218319 & 0.21 & 0.00 & 0.02 & 0.04 & 0.230 & 0.000 & -0.001 & -0.043 & -5.57 & -4.26 & 2102.35 & 393.38 & -3.87 & 402.18 \\
\hline 219320 & 0.21 & 0.00 & 0.02 & 0.04 & 0.230 & 0.000 & -0.001 & -0.043 & -5.96 & -4.64 & 2102.76 & 401.04 & -4.25 & 410.08 \\
\hline $220 \quad 321$ & 0.21 & 0.00 & 0.02 & 0.04 & 0.230 & 0.000 & -0.001 & -0.043 & -5.87 & -4.58 & 2103.97 & 407.90 & -4.18 & 417.20 \\
\hline 221322 & 0.21 & 0.00 & 0.03 & 0.03 & 0.230 & 0.000 & -0.014 & -0.035 & -5.97 & -4.69 & 2103.99 & 415.96 & -4.45 & 425.34 \\
\hline 222323 & 0.20 & 0.00 & 0.03 & 0.03 & 0.219 & 0.000 & -0.016 & -0.035 & -5.70 & -4.47 & 2104.92 & 423.10 & -4.22 & 432.75 \\
\hline $223 \quad 324$ & 0.20 & 0.00 & 0.03 & 0.03 & 0.219 & 0.000 & -0.016 & -0.035 & -5.89 & -4.67 & 2104.90 & 431.19 & -4.41 & 441.10 \\
\hline 325 & 0.20 & 0.00 & 0.03 & 0.02 & 0.219 & 0.000 & -0.017 & -0.025 & -5.29 & -4.19 & 2105.44 & 438.72 & -4.08 & 448.74 \\
\hline 25326 & 0.19 & 0.00 & 0.04 & 0.02 & 0.208 & 0.000 & -0.031 & -0.027 & -5.64 & -4.43 & 2105.35 & 446.89 & -4.27 & 457.23 \\
\hline
\end{tabular}


$Z=101$ (Md)

$\begin{array}{rrrrrrrrrrrrrr}226 & 327 & 0.18 & 0.00 & 0.04 & 0.01 & 0.197 & 0.000 & -0.034 & -0.017 & -5.18 & -4.03 & 2105.85 & 454.45 \\ 227 & 328 & 0.18 & 0.00 & 0.04 & 0.01 & 0.197 & 0.000 & -0.034 & -0.017 & -5.36 & -4.24 & 2105.60 & 462.78 \\ 228 & 329 & 0.18 & 0.00 & 0.04 & 0.00 & 0.196 & 0.000 & -0.034 & -0.007 & -4.93 & -3.89 & 2106.03 & 470.41 \\ 229 & 330 & 0.18 & 0.00 & 0.05 & 0.00 & 0.197 & 0.000 & -0.046 & -0.009 & -5.31 & -4.11 & 2105.68 & 478.84 \\ 230 & 331 & 0.18 & 0.00 & 0.05 & -0.01 & 0.197 & 0.000 & -0.047 & 0.000 & -4.89 & -3.63 & 2105.86 & 486.73 \\ 231 & 332 & 0.18 & 0.00 & 0.06 & -0.01 & 0.197 & 0.000 & -0.059 & -0.002 & -5.33 & -3.85 & 2105.40 & 495.26 \\ 232 & 333 & 0.17 & 0.00 & 0.06 & -0.01 & 0.186 & 0.000 & -0.061 & -0.001 & -4.97 & -3.50 & 2105.60 & 503.14 \\ 233 & 334 & 0.17 & 0.00 & 0.07 & -0.02 & 0.186 & 0.000 & -0.074 & 0.007 & -5.48 & -3.75 & 2105.04 & 511.76\end{array}$

\section{$Z=102($ No)}

$\begin{array}{llll}130 & 232 & -0.57 & 0.00\end{array}$

$\begin{array}{llll}0.01 & -0.02 & -0.566 & 0.000\end{array}$

$\begin{array}{llll}132 & 234 & 0.28 & 0.00\end{array}$

$\begin{array}{llll}0.01 & 0.00 & 0.307 & 0.000\end{array}$

$\begin{array}{lll}0.107 & -0.005 & -2.52\end{array}$

$\begin{array}{ll}4.72 & 1695.11\end{array}$

97.63

$\begin{array}{llll}0.01 & 0.01 & 0.307 & 0.000\end{array}$

$\begin{array}{lll}0.022 & 0.001 & -0.64\end{array}$

$\begin{array}{ll}0.34 & 1708.33\end{array}$

$0.27 \quad 1718.98$

92.49

133235

$0.28 \quad 0.00$

0.01

0.01

$\begin{array}{ll}0.307 & 0.000\end{array}$

$\begin{array}{llll}0.023 & -0.009 & -0.77\end{array}$

$0.03 \quad 1727.76$

\section{6}

$0.29 \quad 0.00$

0.02

0.02

$0.319 \quad 0.000$

$-0.009-1.07$

$-0.08 \quad 1738.13$

89.20

$135 \quad 237$

136238

$0.28 \quad 0.00$

$\begin{array}{ll}0.307 & 0.000\end{array}$

$\begin{array}{llllll}0.025 & -0.019 & -1.60 & -0.35 & 1746.64\end{array}$

$\begin{array}{ll}137 & 239\end{array}$

$.28 \quad 0.00$

0.01

0.02

$\begin{array}{ll}0.307 & 0.000\end{array}$

138240

$0.26 \quad 0.00$

0.00

0.02

$0.284 \quad 0.000$

$\begin{array}{lllll}0.025 & -0.019 & -1.62 & -0.38 & 1756.64\end{array}$

139241

$\begin{array}{llll}0.25 & 0.00 & -0.01\end{array}$

0.03

$0.272 \quad 0.000$

$\begin{array}{lllll}0.032 & -0.016 & -1.92 & -0.56 & 1774.43\end{array}$

$\begin{array}{llllll}0.043 & -0.024 & -2.50 & -0.85 & 1782.38\end{array}$

$\begin{array}{lllll}0.050 & -0.022 & -2.79 & -1.14 & 1792.04\end{array}$

$\begin{array}{lllll}0.050 & -0.022 & -3.24 & -1.59 & 1799.88\end{array}$

141243

142244

143245

144246

$\begin{array}{llll}0.23 & 0.00 & -0.02\end{array}$

0.03

$\begin{array}{ll}0.249 & 0.000\end{array}$

$\begin{array}{lll}0.22 & 0.00 & -0.03\end{array}$

0.03

$0.249 \quad 0.000$

$\begin{array}{lllll}0.060 & -0.020 & -3.60 & -1.86 & 1809.23\end{array}$

0.03

$0.237 \quad 0.000$

$\begin{array}{lll}0.22 & 0.00 & -0.03\end{array}$

0.04

$\begin{array}{lllll}0.060 & -0.020 & -4.06 & -2.25 & 1816.73\end{array}$

$\begin{array}{lllll}0.051 & -0.032 & -4.32 & -2.39 & 1825.69\end{array}$

$\begin{array}{lllll}0.051 & -0.032 & -4.79 & -2.81 & 1832.95\end{array}$

146248

$\begin{array}{llll}0.23 & 0.00 & -0.02\end{array}$

0.04

$0.249 \quad 0.000$

$\begin{array}{lllll}0.051 & -0.032 & -4.92 & -2.93 & 1841.60\end{array}$

0.04

$0.250 \quad 0.000$

$\begin{array}{lllll}0.039 & -0.035 & -5.32 & -3.36 & 1848.62\end{array}$

$\begin{array}{lllll}0.039 & -0.035 & -5.44 & -3.48 & 1857.01\end{array}$

$0.250 \quad 0.000$

$148 \quad 250$

$\begin{array}{llll}0.23 & 0.00 & -0.01\end{array}$

0.04

149251

$\begin{array}{lll}0.23 & 0.00 & 0.00\end{array}$

0.04

150252

$0.23 \quad 0.00$

0.00

0.04

$0.250 \quad 0.000$

$\begin{array}{lllll}0.027 & -0.037 & -5.93 & -3.96 & 1863.82\end{array}$

$0.250 \quad 0.000$

$\begin{array}{lllll}0.027 & -0.037 & -6.10 & -4.12 & 1871.99\end{array}$

$152 \quad 254$

$0.251 \quad 0.000$

$\begin{array}{lllll}0.015 & -0.040 & -6.61 & -4.55 & 1878.51\end{array}$

$0.251 \quad 0.000$

$\begin{array}{lllll}0.015 & -0.040 & -6.55 & -4.52 & 1886.23\end{array}$

$0.252 \quad 0.000$

$\begin{array}{lllll}0.002 & -0.033 & -6.28 & -4.44 & 1891.98\end{array}$

$\begin{array}{lllll}0.002 & -0.033 & -5.94 & -4.14 & 1899.19\end{array}$

154256

$0.23 \quad 0.00$

0.02

0.03

155257

$0.22 \quad 0.00$

0.02

0.03

156258

$0.22 \quad 0.00$

0.03

0.02

0.220 .00

0.02

158260

$0.22 \quad 0.00$

0.04

0.02

$0.240 \quad 0.000$

$\begin{array}{lllll}-0.000 & -0.033 & -5.82 & -4.07 & 1904.72\end{array}$

$\begin{array}{lllllll}0.241 & 0.000 & -0.013 & -0.026 & -5.34 & -3.68 & 1911.59\end{array}$

$\begin{array}{lllllll}0.241 & 0.000 & -0.013 & -0.026 & -5.44 & -3.80 & 1917.07\end{array}$

$\begin{array}{lllllll}0.242 & 0.000 & -0.025 & -0.028 & -5.39 & -3.59 & 1923.89\end{array}$

159261

$0.21 \quad 0.00$

0.04

0.01$$
\begin{array}{lllllll}
0.230 & 0.000 & -0.028 & -0.018 & -5.50 & -3.79 & 1929.22
\end{array}
$$

86.90

86.46

84.54

84.52

82.89

83.01

81.42

81.65

80.37

80.94

80.06

80.87

80.29

81.34

81.02

82.28

82.18 83.74 84.09 86.40 87.27

161263

0.210 .00

0.05

0.01

$\begin{array}{llllllll}0.231 & 0.000 & -0.040 & -0.021 & -5.71 & -3.79 & 1936.02 & 98.87\end{array}$

162264

0.210 .00

0.06

0.01$$
\begin{array}{llllllll}
0.231 & 0.000 & -0.040 & -0.021 & -6.01 & -4.06 & 1941.19 & 101.76
\end{array}
$$

163265

164266

$0.20 \quad 0.00$

0.00

$0.231 \quad 0.000$

$\begin{array}{llllll}-0.053 & -0.013 & -6.15 & -4.00 & 1947.70 & 103.33 \\ -0.055 & -0.013 & -6.17 & -4.05 & 1952.44 & 106.66\end{array}$

$0.20 \quad 0.00$

0.06

0.00

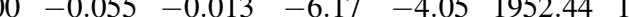

165267

$0.19 \quad 0.00$

0.06

0.00 $0.220 \quad 0.000$

$\begin{array}{llllllll}0.209 & 0.000 & -0.057 & -0.012 & -5.25 & -3.27 & 1962.46 & 112.78\end{array}$ 166268

0.00$$
\begin{array}{llllllll}
0.197 & 0.000 & -0.046 & -0.009 & -4.34 & -2.73 & 1968.05 & 115.27
\end{array}
$$

167269

$0.17 \quad 0.00$

$0.04 \quad 0.00$

$\begin{array}{lllllll}0.185 & 0.000 & -0.036 & -0.007 & -3.93 & -2.61 & 1972.18\end{array}$

168270

$0.16 \quad 0.00$

0.00

$\begin{array}{llllllll}0.174 & 0.000 & -0.037 & -0.007 & -3.50 & -2.29 & 1977.77 & 121.69\end{array}$

169271

$0.16 \quad 0.00$

0.04

0.00

$\begin{array}{llllllll}0.174 & 0.000 & -0.037 & -0.007 & -3.53 & -2.20 & 1981.74 & 125.79\end{array}$

17027

$0.14 \quad 0.00$

0.03

0.00

$\begin{array}{llllllll}0.152 & 0.000 & -0.028 & -0.004 & -2.92 & -1.90 & 1987.13 & 128.47\end{array}$

171273

$0.13 \quad 0.00$

$0.03 \quad 0.00$

$\begin{array}{llllllll}0.141 & 0.000 & -0.029 & -0.004 & -3.19 & -2.21 & 1991.30 & 132.37\end{array}$

173275

174276

0.00

$\begin{array}{llllllll}0.130 & 0.000 & -0.030 & -0.004 & -2.99 & -2.06 & 1996.64 & 135.11\end{array}$

0.120 .00

$0.03 \quad 0.00$

$\begin{array}{llll}0.130 & 0.000 & -0.030 & -0.0\end{array}$

$\begin{array}{llll}0.130 & 0.000 & -0.043\end{array}$

$\begin{array}{lllll}0.005 & -3.41 & -2.30 & 2005.81 & 142.07\end{array}$

$\begin{array}{llll}175 & 277 & 0.12 & 0.00\end{array}$

$0.04-0.01$

$\begin{array}{lll}0.130 & 0.000 & -0.043\end{array}$

$\begin{array}{llllll}0.005 & -3.68 & -2.57 & 2009.55 & 146.41\end{array}$

$\begin{array}{llll}176 & 278 & 0.12 & 0.00\end{array}$

$0.04-0.02$

$\begin{array}{lll}0.130 & 0.000 & -0.043\end{array}$

$\begin{array}{lllll}0.014 & -3.33 & -2.20 & 2014.26 & 149.76\end{array}$

$0.02-0.01$ $\begin{array}{lll}-0.105 & 0.000 & -0.019\end{array}$ $\begin{array}{lllll}0.012 & -3.07 & -2.51 & 2017.85 & 154.25\end{array}$

$-3.97 \quad 464.97$

$-4.18 \quad 473.56$

$-3.88 \quad 481.43$

$\begin{array}{ll}-4.05 & 490.19\end{array}$

$-3.56 \quad 498.37$

$-3.73 \quad 507.25$

$-3.36 \quad 515.44$

$-3.48 \quad 524.49$

$\begin{array}{ll}4.21 & 99.44\end{array}$

$0.23 \quad 94.61$

$0.17 \quad 91.97$

$-0.08 \quad 91.18$

$-0.17 \quad 88.82$

$-0.44 \quad 88.32$

$-0.46 \quad 86.35$

$-0.60 \quad 86.27$

$-0.61 \quad 84.61$

$-0.89 \quad 84.69$

$\begin{array}{ll}-1.15 \quad 83.07 \\ -1.61 & 83.25\end{array}$

$-1.61 \quad 83.25$

$-1.86 \quad 81.94$

$\begin{array}{ll}-2.25 & 82.47\end{array}$

$-2.36 \quad 81.58$

$-2.78 \quad 82.34$

$-2.88 \quad 81.74$

$-3.33 \quad 82.75$

$-3.43 \quad 82.43$

$-3.92 \quad 83.65$

$\begin{array}{llll}82.88 & 0.013 & -4.06 & 83.55\end{array}$

$-4.50 \quad 85.09$

$\begin{array}{llll}84.72 & 0.018 & -4.45 & 85.44\end{array}$

$\begin{array}{llll}86.85 & 0.010 & -4.41 & 87.70\end{array}$

$\begin{array}{llll}87.82 & 0.008 & -4.09 & 88.57\end{array}$

$\begin{array}{llll}90.24 & 0.022 & -4.03 & 91.10\end{array}$

$-3.66 \quad 92.28$

$-3.78 \quad 94.87$

$-3.56 \quad 96.15$

$-3.79 \quad 98.86$

$-3.77 \quad 100.18$

$-4.04 \quad 103.08$ 


\section{$Z=102$ (No)}

$\begin{array}{llll}178 & 280 & -0.10 & 0.00\end{array}$ $\begin{array}{llll}179 & 281 & -0.08 & 0.00\end{array}$ $\begin{array}{llll}180 & 282 & -0.05 & 0.00\end{array}$ $\begin{array}{llll}181 & 283 & -0.04 & 0.00\end{array}$ $\begin{array}{llll}182 & 284 & 0.00 & 0.00\end{array}$

183285 184286 $\begin{array}{ll}185 & 287\end{array}$ 186288 $187 \quad 289$

$188 \quad 290$ 189291 190292

191293

$192 \quad 294$

193295

194296

$195 \quad 297$

196298

197299

198300

199301

200302

201303

202304

203305

204306

205307

206308

207309

208310

209311

210312

211313

212314

213315

214316

$215 \quad 317$

216318

$217 \quad 319$

$218 \quad 320$

219321

$220 \quad 322$

$221 \quad 323$

$222 \quad 324$

$223 \quad 325$

224326

$\begin{array}{ll}225 & 327\end{array}$

226328

$227 \quad 329$

$228 \quad 330$

229331

$230 \quad 332$

$231 \quad 333$

$232 \quad 334$

$233 \quad 335$

234336

$0.00 \quad 0.00$

$\begin{array}{ll}0.00 & 0.03\end{array}$

$0.01 \quad 0.06$

$0.24 \quad 0.00$

$\begin{array}{ll}0.25 & 0.00\end{array}$

0.260 .00

$0.26 \quad 0.00$

$0.26 \quad 0.00$

0.250 .00

$\begin{array}{ll}0.25 & 0.00\end{array}$

$0.24 \quad 0.00$

$0.24 \quad 0.00$

$0.23 \quad 0.00$

$0.20 \quad 0.00$

0.00

$0.20 \quad 0.00$

$0.21 \quad 0.00$
0.20

$0.20 \quad 0.00$

$0.21 \quad 0.00$
0.20

$\begin{array}{ll}0.19 & 0.00\end{array}$

$0.02-0.01-0.105$

$\begin{array}{llll}0.02 & 0.00 & -0.084\end{array}$

$\begin{array}{lll}0.01 & 0.00 & -0.053\end{array}$

$\begin{array}{lll}0.000 & -0.019\end{array}$

$\begin{array}{lllll}0.012 & -2.91 & -2.36 & 2022.59 & 157.58\end{array}$ $0.000-0.021$ $\begin{array}{llllll}0.002 & -2.87 & -2.34 & 2025.66 & 162.58\end{array}$

$\begin{array}{llll}0.01 & 0.00 & -0.042\end{array}$

$0.000-0.011$$$
\begin{array}{lllll}
0.001 & -2.36 & -1.85 & 2029.87 & 166.44
\end{array}
$$

$\begin{array}{lll}0.00 & 0.00 \quad 0.000\end{array}$

$0.000-0.011$

$\begin{array}{lllll}0.001 & -2.65 & -2.09 & 2033.01 & 171.37\end{array}$

0.00

$0.00 \quad 0.000$

$0.000 \quad 0.000$

$\begin{array}{llllll}0.000 & -2.32 & -1.78 & 2037.22 & 175.24\end{array}$

0.00

0.00

$0.000-0.040$

0.000

$\begin{array}{lllll}0.000 & -2.62 & -2.07 & 2040.23 & 180.29\end{array}$

0.00

0.00

$0.012-0.081$

0.002

$\begin{array}{llllll}0.001 & -2.39 & -1.70 & 2044.19 & 184.41\end{array}$

$\begin{array}{lll}0.01 & 0.07 & 0.00\end{array}$

0.00

$0.013-0.094$

0.002

$\begin{array}{lllll}0.002 & -2.38 & -1.21 & 2046.25 & 190.42\end{array}$

$\begin{array}{llll}0.02 & 0.09 & -0.01\end{array}$

0.00

$0.025-0.122$

0.015

$\begin{array}{lllll}0.003 & -1.99 & -0.60 & 2049.79 & 194.95\end{array}$

$\begin{array}{lll}0.02 & 0.09 & -0.01\end{array}$

$\begin{array}{llll}0.01 & 0.025 & -0.122\end{array}$

$\begin{array}{llll}0.015 & -0.004 & -1.71\end{array}$

$\begin{array}{llll}0.16 & 2055.39 & 205.49\end{array}$

$\begin{array}{llll}0.03 & 0.10 & -0.01\end{array}$

0.0

$\begin{array}{llll}0.23 & 0.00 & -0.01\end{array}$

0.00

$0.036-0.135$

$\begin{array}{lll}0.016 & -0.003 & -2.05\end{array}$

$\begin{array}{lll}0.15 & 2057.61 & 211.35\end{array}$

$\begin{array}{lll}0.23 & 0.00 & -0.01\end{array}$

0.00

$0.250 \quad 0.000$

0.035

$0.005-0.91$

$\begin{array}{lll}0.18 & 2061.38 & 215.64\end{array}$

$\begin{array}{llll}0.23 & 0.00 & -0.01\end{array}$

0.00

0.000

0.035

$\begin{array}{lllll}0.005 & -1.23 & -0.15 & 2063.75 & 221.34\end{array}$

$\begin{array}{lll}0.23 & 0.00 & -0.01\end{array}$

$0.00 \quad 0.250$

0.000

0.035

$\begin{array}{llllll}0.005 & -1.62 & -0.49 & 2069.61 & 231.63\end{array}$

$\begin{array}{lll}0.23 & 0.00-0.01\end{array}$

$0.00 \quad 0.250$

0.000

0.035

$\begin{array}{llllll}0.005 & -1.61 & -0.44 & 2073.02 & 236.29\end{array}$

$\begin{array}{lll}0.24 & 0.00 & 0.00\end{array}$

0.01

0.261

0.000

$\begin{array}{llllll}0.026 & -0.007 & -1.91 & -0.74 & 2075.05 & 242.34\end{array}$

$\begin{array}{lll}0.24 & 0.00 & 0.00\end{array}$

0.01

0.261

0.000

$\begin{array}{llllll}0.026 & -0.007 & -1.96 & -0.80 & 2078.41 & 247.04\end{array}$

0.01

0.02

0.261

0.000

$\begin{array}{llllll}0.026 & -0.007 & -2.35 & -1.16 & 2080.34 & 253.19\end{array}$

0.01

0.02

0.273

0.000

$\begin{array}{llllll}0.017 & -0.020 & -2.58 & -1.34 & 2083.66 & 257.94\end{array}$

0.02

0.02

0.000

$\begin{array}{llllll}0.017 & -0.020 & -3.05 & -1.80 & 2085.53 & 264.14\end{array}$

0.02

0.02

$\begin{array}{llllll}0.007 & -0.023 & -3.27 & -1.96 & 2088.67 & 269.07\end{array}$

0.02

0.03

0.285

0.000

$\begin{array}{llllll}0.007 & -0.023 & -3.74 & -2.42 & 2090.40 & 275.41\end{array}$

$\begin{array}{llllll}0.009 & -0.033 & -3.98 & -2.56 & 2093.37 & 280.51\end{array}$

0.02

0.03

0.274

0.000

$\begin{array}{llllll}0.006 & -0.033 & -4.26 & -2.86 & 2094.78 & 287.17\end{array}$

0.01

0.03

0.274

0.000

$\begin{array}{llllll}0.006 & -0.033 & -4.06 & -2.72 & 2097.32 & 292.70\end{array}$

0.000

$\begin{array}{llllll}0.016 & -0.030 & -4.13 & -2.91 & 2098.48 & 299.61\end{array}$

0.01

0.03

0.262

0.000

$\begin{array}{llllll}0.016 & -0.030 & -3.92 & -2.64 & 2100.75 & 305.42\end{array}$

$\begin{array}{lll}0.22 & 0.00 & 0.00\end{array}$

$0.03 \quad 0.239$

0.000

$\begin{array}{lllllll}0.014 & -0.030 & -4.04 & -2.78 & 2101.71 & 312.52\end{array}$

$\begin{array}{lll}0.22 & 0.00 & 0.00\end{array}$

0.03

0.239

0.000

$\begin{array}{llllll}0.024 & -0.028 & -3.86 & -2.66 & 2103.97 & 318.34\end{array}$

$\begin{array}{lll}0.21 & 0.00 & -0.01\end{array}$

0.04

0.227

0.000

$\begin{array}{llllll}0.024 & -0.028 & -4.06 & -2.83 & 2104.83 & 325.55\end{array}$

$\begin{array}{lll}0.21 & 0.00 & -0.01\end{array}$

0.04

0.227

0.000

$\begin{array}{llllll}0.035 & -0.036 & -4.21 & -2.87 & 2107.11 & 331.34\end{array}$

0.000

$\begin{array}{llllll}0.035 & -0.036 & -4.49 & -3.12 & 2107.92 & 338.61\end{array}$

$\begin{array}{lll}0.21 & 0.00 & 0.00\end{array}$

0.03

0.228

0.000

$\begin{array}{llllll}0.022 & -0.028 & -4.08 & -2.88 & 2109.77 & 344.82\end{array}$

0.00

0.03

0.217

0.000

$\begin{array}{llllll}0.020 & -0.028 & -4.37 & -3.19 & 2110.51 & 352.16\end{array}$

0.01

0.03

0.228

0.000

$\begin{array}{llllll}0.010 & -0.031 & -4.37 & -3.19 & 2112.46 & 358.28\end{array}$

0.01

0.03

217

0.000

$\begin{array}{llllll}0.010 & -0.031 & -4.73 & -3.55 & 2113.11 & 365.70\end{array}$

0.01

0.03

0.217

0.000

$\begin{array}{llllll}0.008 & -0.031 & -4.71 & -3.62 & 2115.00 & 371.88\end{array}$

0.02

0.03

0.229

0.02

0.03

0.218

0.000

$\begin{array}{lllllll}0.008 & -0.031 & -5.14 & -4.05 & 2115.59 & 379.36\end{array}$

$\begin{array}{lll}0.20 & 0.00 & 0.03\end{array}$

0.03

0.230

$\begin{array}{lllllll}0.000 & -0.002 & -0.033 & -5.21 & -4.06 & 2117.29 & 385.73\end{array}$

$\begin{array}{llllllll}0.000 & -0.004 & -0.033 & -5.61 & -4.46 & 2117.72 & 393.38\end{array}$

$\begin{array}{lllllll}0.000 & -0.014 & -0.035 & -5.66 & -4.41 & 2119.23 & 399.93\end{array}$

$\begin{array}{lllllll}0.000 & -0.016 & -0.035 & -6.00 & -4.78 & 2119.50 & 407.74\end{array}$

$\begin{array}{lll}0.20 & 0.00 & 0.03\end{array}$

0.03

$\begin{array}{lllllll}0.000 & -0.016 & -0.035 & -5.77 & -4.56 & 2120.71 & 414.60\end{array}$

$\begin{array}{lll}0.20 & 0.00 & 0.04\end{array}$

$0.02 \quad 0.219$

$\begin{array}{lllllll}0.000 & -0.029 & -0.028 & -5.92 & -4.67 & 2120.60 & 422.77\end{array}$

$\begin{array}{lll}0.19 & 0.00 & 0.04\end{array}$

0.02

0.208

$\begin{array}{lllllll}0.000 & -0.031 & -0.027 & -5.62 & -4.41 & 2121.64 & 429.81\end{array}$

0.04

0.01 
$Z=102($ No)

$\begin{array}{llll}235 & 337 & 0.10 & 0.00\end{array}$ $\begin{array}{lllll}236 & 338 & 0.10 & 0.00 & -0.01\end{array}$ $0.02 \quad 0.107$ $\begin{array}{lllllll}0.000 & 0.017 & -0.009 & -4.33 & -3.81 & 2121.96 & 518.28\end{array}$ $\begin{array}{lllllll}0.000 & 0.017 & -0.019 & -4.25 & -3.72 & 2122.45 & 525.86\end{array}$
$-3.80 \quad 530.98$

$-3.61538 .96$

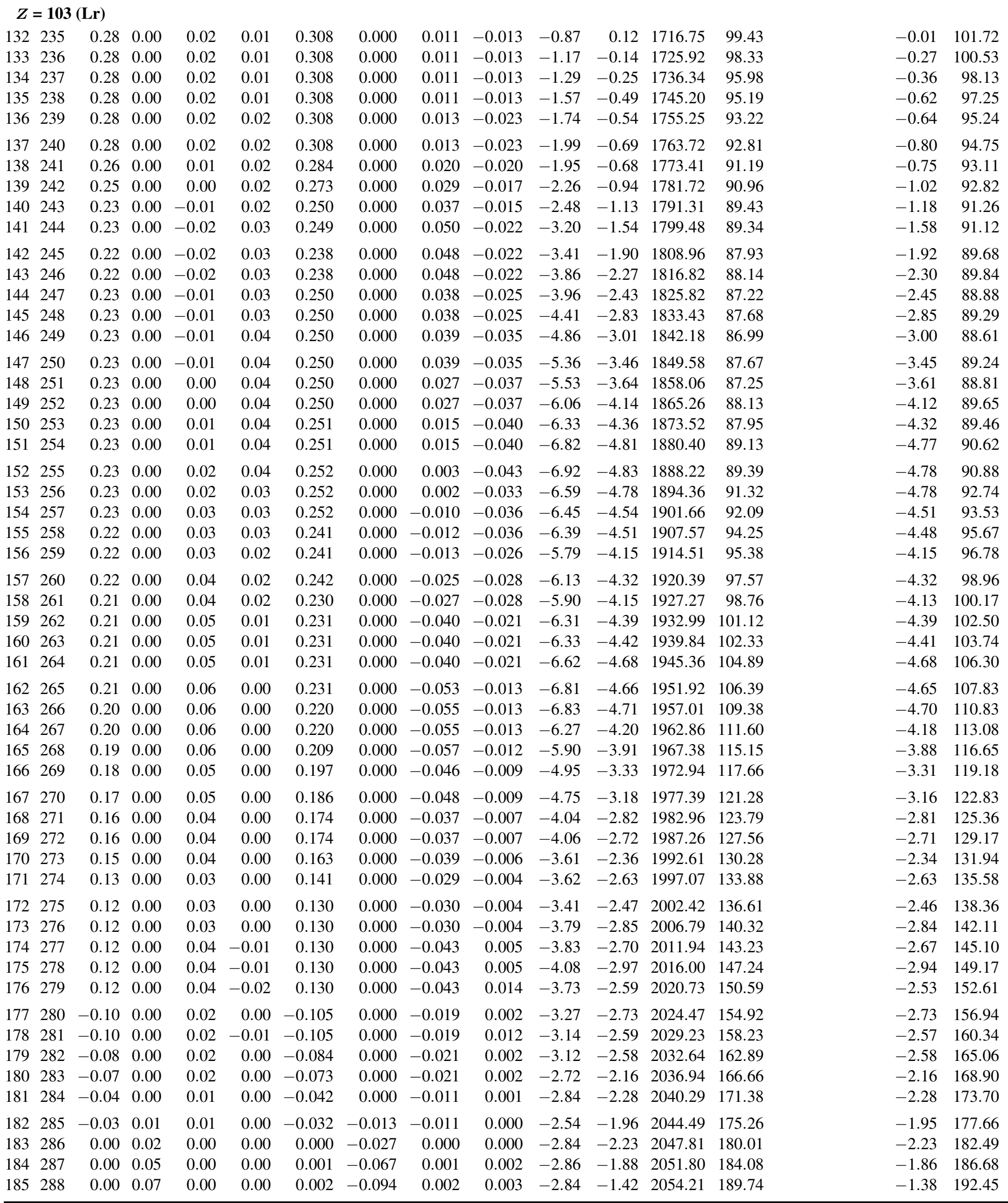


$Z=103(\mathrm{Lr})$

$\begin{array}{rrrrr}186 & 289 & 0.00 & 0.07 & 0.00\end{array}$ $\begin{array}{llllll}187 & 290 & 0.01 & 0.09 & -0.01\end{array}$ $\begin{array}{llllll}188 & 291 & 0.02 & 0.10 & -0.01\end{array}$ $\begin{array}{llllll}189 & 292 & 0.03 & 0.10 & -0.01\end{array}$ $\begin{array}{llllll}190 & 293 & 0.23 & 0.00 & -0.01\end{array}$

$\begin{array}{llllll}191 & 294 & 0.23 & 0.00 & -0.01\end{array}$ $\begin{array}{llllll}192 & 295 & 0.23 & 0.00 & -0.01\end{array}$ $\begin{array}{lllll}193 & 296 & 0.23 & 0.00 & 0.00\end{array}$ $\begin{array}{llllll}194 & 297 & 0.23 & 0.00 & 0.00\end{array}$ $\begin{array}{lllll}195 & 298 & 0.23 & 0.00 & 0.00\end{array}$

$\begin{array}{llll}0.00 & 0.002 & -0.094\end{array}$ $\begin{array}{lll}0.00 & 0.014 & -0.122\end{array}$ $\begin{array}{lll}0.01 & 0.026 & -0.135\end{array}$ $\begin{array}{lll}0.01 & 0.036 & -0.135\end{array}$ $\begin{array}{lll}0.00 & 0.250 & 0.000\end{array}$

$\begin{array}{ll}0.00 & 0.250\end{array}$ $0.00 \quad 0.250$ $\begin{array}{ll}0.00 & 0.250\end{array}$ $\begin{array}{ll}0.00 & 0.250\end{array}$ $0.00 \quad 0.250$ $\begin{array}{llll}196 & 299 & 0.24 & 0.00\end{array}$ $\begin{array}{llll}197 & 300 & 0.24 & 0.00\end{array}$ $\begin{array}{llll}198 & 301 & 0.24 & 0.00\end{array}$ $\begin{array}{llll}199 & 302 & 0.25 & 0.00\end{array}$ $\begin{array}{llll}200 & 303 & 0.25 & 0.00\end{array}$

$\begin{array}{llll}201 & 304 & 0.25 & 0.00\end{array}$ $\begin{array}{llll}202 & 305 & 0.26 & 0.00\end{array}$ $\begin{array}{llll}203 & 306 & 0.25 & 0.00\end{array}$ $\begin{array}{llll}204 & 307 & 0.25 & 0.00\end{array}$ $\begin{array}{llll}205 & 308 & 0.24 & 0.00\end{array}$

$\begin{array}{llll}206 & 309 & 0.24 & 0.00\end{array}$ $\begin{array}{llll}207 & 310 & 0.23 & 0.00\end{array}$ $\begin{array}{llll}208 & 311 & 0.23 & 0.00\end{array}$ $\begin{array}{llll}209 & 312 & 0.22 & 0.00\end{array}$ $\begin{array}{llll}210 & 313 & 0.22 & 0.00\end{array}$

$\begin{array}{llll}211 & 314 & 0.21 & 0.00\end{array}$ $\begin{array}{llll}212 & 315 & 0.21 & 0.00\end{array}$ $\begin{array}{llll}213 & 316 & 0.21 & 0.00\end{array}$ $\begin{array}{llll}214 & 317 & 0.21 & 0.00\end{array}$ $\begin{array}{llll}215 & 318 & 0.21 & 0.00\end{array}$

$\begin{array}{llll}216 & 319 & 0.21 & 0.00\end{array}$ $\begin{array}{llll}217 & 320 & 0.20 & 0.00\end{array}$ $\begin{array}{llll}218 & 321 & 0.21 & 0.00\end{array}$ $\begin{array}{llll}219 & 322 & 0.20 & 0.00\end{array}$ $\begin{array}{llll}220 & 323 & 0.21 & 0.00\end{array}$

$\begin{array}{llll}221 & 324 & 0.20 & 0.00\end{array}$ $\begin{array}{llll}222 & 325 & 0.20 & 0.00\end{array}$ $\begin{array}{llll}223 & 326 & 0.20 & 0.00\end{array}$ $\begin{array}{llll}224 & 327 & 0.19 & 0.00\end{array}$ $\begin{array}{llll}225 & 328 & 0.19 & 0.00\end{array}$ $\begin{array}{llll}226 & 329 & 0.18 & 0.00\end{array}$ $\begin{array}{llll}227 & 330 & 0.18 & 0.00\end{array}$ $\begin{array}{llll}228 & 331 & 0.18 & 0.00\end{array}$ $\begin{array}{llll}229 & 332 & 0.18 & 0.00\end{array}$ $\begin{array}{llll}230 & 333 & 0.18 & 0.00\end{array}$

$\begin{array}{llll}231 & 334 & 0.18 & 0.00\end{array}$ $\begin{array}{llll}232 & 335 & 0.18 & 0.00\end{array}$ $\begin{array}{llll}233 & 336 & 0.17 & 0.00\end{array}$ $\begin{array}{llll}234 & 337 & 0.18 & 0.00\end{array}$ $\begin{array}{llll}235 & 338 & 0.10 & 0.00\end{array}$ $\begin{array}{llll}236 & 339 & 0.10 & 0.00\end{array}$

\section{$Z=104$ (Rf)}

$\begin{array}{llll}134 & 238 & 0.29 & 0.00\end{array}$ $\begin{array}{llll}135 & 239 & 0.29 & 0.00\end{array}$ $\begin{array}{llll}136 & 240 & 0.29 & 0.00\end{array}$ $\begin{array}{llll}137 & 241 & 0.28 & 0.00\end{array}$ $\begin{array}{llll}138 & 242 & 0.26 & 0.00\end{array}$

0.00

0.01

0.01

0.01

0.02

0.02

0.03

0.02

0.02

0.01

0.02

0.01

0.01

0.01

0.01

0.00

0.01

0.01

0.01

0.02

0.02

0.02

0.03

0.02

0.03

0.03

0.03

0.04

0.04

0.04

0.04

0.05

0.05

0.05

0.06

$\begin{array}{ll}0.01 & 0.261\end{array}$

$\begin{array}{ll}0.01 & 0.262\end{array}$

$\begin{array}{ll}0.01 & 0.262\end{array}$

$\begin{array}{ll}0.02 & 0.273\end{array}$

$\begin{array}{ll}0.02 & 0.274\end{array}$

$\begin{array}{ll}0.02 & 0.274\end{array}$

$\begin{array}{ll}0.02 & 0.286\end{array}$

$\begin{array}{ll}0.03 & 0.274\end{array}$

$\begin{array}{lll}0.03 & 0.274\end{array}$

$\begin{array}{ll}0.03 & 0.262\end{array}$

$\begin{array}{lll}0.03 & 0.263\end{array}$

$\begin{array}{ll}0.03 & 0.251\end{array}$

$\begin{array}{ll}0.03 & 0.251\end{array}$

$\begin{array}{ll}0.03 & 0.240\end{array}$

$\begin{array}{ll}0.03 & 0.240\end{array}$

$\begin{array}{ll}0.03 & 0.228\end{array}$

$\begin{array}{lll}0.03 & 0.228\end{array}$

$\begin{array}{ll}0.03 & 0.228\end{array}$

$\begin{array}{ll}0.03 & 0.228\end{array}$

$\begin{array}{ll}0.03 & 0.229\end{array}$

$\begin{array}{ll}0.03 & 0.229\end{array}$

$\begin{array}{ll}0.03 & 0.218\end{array}$

$\begin{array}{ll}0.03 & 0.230\end{array}$

$\begin{array}{lll}0.03 & 0.218\end{array}$

$\begin{array}{ll}0.03 & 0.230\end{array}$

$\begin{array}{ll}0.03 & 0.219\end{array}$

$\begin{array}{ll}0.03 & 0.219\end{array}$

$\begin{array}{ll}0.02 & 0.219\end{array}$

$\begin{array}{ll}0.02 & 0.208\end{array}$

$\begin{array}{ll}0.01 & 0.208\end{array}$

$\begin{array}{ll}0.01 & 0.197\end{array}$

$\begin{array}{ll}0.01 & 0.197\end{array}$

$\begin{array}{ll}0.00 & 0.197\end{array}$

$\begin{array}{ll}0.00 & 0.197\end{array}$

$\begin{array}{ll}0.00 & 0.198\end{array}$

$\begin{array}{lll}0.06 & -0.01 & 0.197\end{array}$

$\begin{array}{llll}0.07 & -0.01 & 0.198\end{array}$

$\begin{array}{llll}0.07 & -0.01 & 0.187\end{array}$

$\begin{array}{lll}0.08 & -0.02 & 0.198\end{array}$

$\begin{array}{lll}-0.01 & 0.01 & 0.107\end{array}$

0.00

$0.01 \quad 0.107$
0.03

0.03

0.03

0.02

0.01 $\begin{array}{ll}0.01 & 0.320\end{array}$ $0.01 \quad 0.320$ $\begin{array}{ll}0.01 & 0.320\end{array}$ $\begin{array}{ll}0.02 & 0.308\end{array}$ $\begin{array}{ll}0.02 & 0.284\end{array}$ $\begin{array}{llllll}0.002 & 0.003 & -2.23 & -0.84 & 2057.80 & 194.23\end{array}$ $\begin{array}{llllll}0.015 & 0.006 & -2.58 & -0.56 & 2060.22 & 199.89\end{array}$ $\begin{array}{llllll}0.016 & -0.003 & -2.30 & -0.07 & 2063.71 & 204.47\end{array}$ $\begin{array}{llllll}0.016 & -0.003 & -2.25 & -0.02 & 2066.18 & 210.06\end{array}$ $\begin{array}{llllll}0.035 & 0.005 & -0.99 & 0.06 & 2069.92 & 214.39\end{array}$ $\begin{array}{lllllll}0.000 & 0.035 & 0.005 & -1.36 & -0.32 & 2072.65 & 219.73\end{array}$ $\begin{array}{llllllll}0.000 & 0.035 & 0.005 & -1.39 & -0.31 & 2076.29 & 224.17\end{array}$ $\begin{array}{lllllll}0.000 & 0.023 & 0.002 & -1.64 & -0.59 & 2078.76 & 229.77\end{array}$ $\begin{array}{llllllll}0.000 & 0.023 & 0.002 & -1.67 & -0.62 & 2082.26 & 234.34\end{array}$ $\begin{array}{llllllll}0.000 & 0.023 & 0.002 & -2.00 & -0.92 & 2084.59 & 240.08\end{array}$ $\begin{array}{lllllll}0.000 & 0.026 & -0.007 & -2.12 & -0.99 & 2087.98 & 244.77\end{array}$ $\begin{array}{lllllll}0.000 & 0.014 & -0.010 & -2.53 & -1.42 & 2090.27 & 250.54\end{array}$ $\begin{array}{lllllll}0.000 & 0.014 & -0.010 & -2.62 & -1.51 & 2093.51 & 255.37\end{array}$ $\begin{array}{lllllll}0.000 & 0.017 & -0.020 & -3.26 & -2.02 & 2095.75 & 261.21\end{array}$ $\begin{array}{lllllll}0.000 & 0.005 & -0.023 & -3.52 & -2.23 & 2098.95 & 266.08\end{array}$ $\begin{array}{lllllll}0.000 & 0.005 & -0.023 & -4.00 & -2.70 & 2100.98 & 272.11\end{array}$ $\begin{array}{lllllll}0.000 & -0.004 & -0.026 & -4.17 & -2.80 & 2103.91 & 277.26\end{array}$ $\begin{array}{lllllll}0.000 & 0.006 & -0.033 & -4.55 & -3.15 & 2105.69 & 283.56\end{array}$ $\begin{array}{lllllll}0.000 & 0.006 & -0.033 & -4.34 & -3.01 & 2108.22 & 289.09\end{array}$ $\begin{array}{lllllll}0.000 & 0.016 & -0.030 & -4.36 & -3.08 & 2109.57 & 295.82\end{array}$ $\begin{array}{lllllll}0.000 & 0.004 & -0.033 & -4.26 & -2.99 & 2112.01 & 301.45\end{array}$ $\begin{array}{lllllll}0.000 & 0.014 & -0.030 & -4.30 & -3.08 & 2113.23 & 308.30\end{array}$ $\begin{array}{lllllll}0.000 & 0.014 & -0.030 & -4.13 & -2.91 & 2115.44 & 314.16\end{array}$ $\begin{array}{lllllll}0.000 & 0.012 & -0.031 & -4.32 & -3.12 & 2116.63 & 321.04\end{array}$ $\begin{array}{lllllll}0.000 & 0.012 & -0.031 & -4.18 & -2.98 & 2118.73 & 327.01\end{array}$ $\begin{array}{lllllll}0.000 & 0.022 & -0.028 & -4.39 & -3.21 & 2119.81 & 334.00\end{array}$ $\begin{array}{lllllll}0.000 & 0.010 & -0.031 & -4.36 & -3.19 & 2121.89 & 339.99\end{array}$ $\begin{array}{lllllll}0.000 & 0.010 & -0.031 & -4.70 & -3.53 & 2122.94 & 347.02\end{array}$ $\begin{array}{lllllll}0.000 & 0.010 & -0.031 & -4.65 & -3.51 & 2124.88 & 353.15\end{array}$ $\begin{array}{lllllll}0.000 & -0.002 & -0.033 & -5.14 & -3.93 & 2125.87 & 360.23\end{array}$ $\begin{array}{lllllll}0.000 & -0.002 & -0.033 & -5.15 & -4.00 & 2127.77 & 366.40\end{array}$ $\begin{array}{lllllll}0.000 & -0.004 & -0.033 & -5.56 & -4.41 & 2128.62 & 373.62\end{array}$ $\begin{array}{lllllll}0.000 & -0.014 & -0.035 & -5.70 & -4.45 & 2130.35 & 379.96\end{array}$ $\begin{array}{lllllll}0.000 & -0.004 & -0.033 & -5.96 & -4.80 & 2131.02 & 387.37\end{array}$ $\begin{array}{lllllll}0.000 & -0.014 & -0.035 & -6.06 & -4.82 & 2132.60 & 393.86\end{array}$ $\begin{array}{lllllll}0.000 & -0.016 & -0.035 & -6.41 & -5.18 & 2133.13 & 401.39\end{array}$ $\begin{array}{lllllll}0.000 & -0.016 & -0.035 & -6.18 & -4.97 & 2134.35 & 408.24\end{array}$ $\begin{array}{lllllll}0.000 & -0.029 & -0.028 & -6.39 & -5.13 & 2134.58 & 416.09\end{array}$ $\begin{array}{llllllll}0.000 & -0.031 & -0.027 & -6.09 & -4.87 & 2135.62 & 423.12\end{array}$ $\begin{array}{lllllll}0.000 & -0.032 & -0.018 & -6.17 & -5.03 & 2135.71 & 431.10\end{array}$ $\begin{array}{llllllll}0.000 & -0.034 & -0.017 & -5.91 & -4.77 & 2136.63 & 438.25\end{array}$ $\begin{array}{lllllll}0.000 & -0.045 & -0.019 & -6.34 & -5.03 & 2136.71 & 446.24\end{array}$ $\begin{array}{lllllll}0.000 & -0.046 & -0.009 & -5.95 & -4.58 & 2137.32 & 453.71\end{array}$ $\begin{array}{lllllll}0.000 & -0.046 & -0.009 & -6.11 & -4.76 & 2137.19 & 461.90\end{array}$ $\begin{array}{lllllll}0.000 & -0.058 & -0.012 & -6.03 & -4.48 & 2137.85 & 469.32\end{array}$ $\begin{array}{lllllll}0.000 & -0.059 & -0.002 & -6.19 & -4.66 & 2137.61 & 477.63\end{array}$ $\begin{array}{lllllll}0.000 & -0.071 & -0.004 & -6.17 & -4.40 & 2138.16 & 485.15\end{array}$ $\begin{array}{lllllll}0.000 & -0.072 & -0.003 & -6.31 & -4.50 & 2137.73 & 493.65\end{array}$ $\begin{array}{lllllll}0.000 & -0.084 & 0.004 & -6.23 & -4.18 & 2138.10 & 501.35\end{array}$ $\begin{array}{lllllll}0.000 & 0.017 & -0.009 & -4.66 & -4.13 & 2137.41 & 510.11\end{array}$ $\begin{array}{lllllll}0.000 & 0.005 & -0.010 & -4.44 & -3.93 & 2137.80 & 517.80\end{array}$

$\begin{array}{lllllll}0.000 & 0.002 & -0.016 & -1.02 & -0.05 & 1735.82 & 103.79\end{array}$ $\begin{array}{lllllll}0.000 & 0.002 & -0.016 & -1.30 & -0.26 & 1744.69 & 102.99\end{array}$ $\begin{array}{lllllll}0.000 & 0.002 & -0.016 & -1.30 & -0.30 & 1755.11 & 100.64\end{array}$ $\begin{array}{lllllll}0.000 & 0.013 & -0.023 & -1.61 & -0.53 & 1763.70 & 100.12\end{array}$ $\begin{array}{lllllll}0.000 & 0.020 & -0.020 & -1.55 & -0.42 & 1773.67 & 98.23\end{array}$

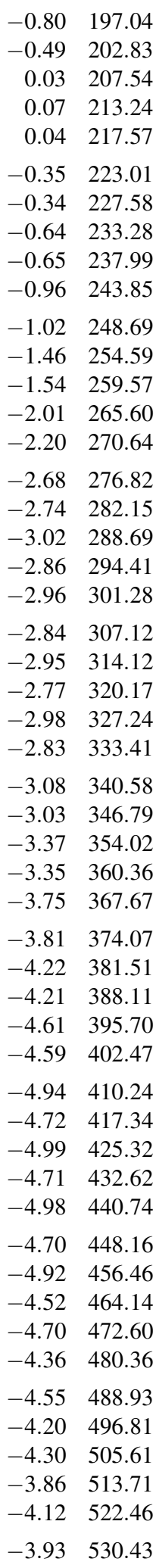

$-0.17 \quad 106.18$

$-0.39 \quad 105.29$

$-0.41 \quad 102.89$

$-0.63 \quad 102.31$

$-0.49 \quad 100.38$ 


\section{$Z=104$ (Rf)}

\section{$\begin{array}{llll}139 & 243 & 0.25 & 0.00\end{array}$}

$\begin{array}{lllll}140 & 244 & 0.23 & 0.00 & -0.01\end{array}$

$\begin{array}{llllll}141 & 245 & 0.23 & 0.00 & -0.01\end{array}$

$\begin{array}{llllll}142 & 246 & 0.23 & 0.00 & -0.01\end{array}$

$\begin{array}{lllll}143 & 247 & 0.22 & 0.00 & -0.02\end{array}$

$\begin{array}{llllll}144 & 248 & 0.22 & 0.00 & -0.01\end{array}$

145249

146250

$\begin{array}{ll}147 & 251\end{array}$

$148 \quad 252$

$\begin{array}{lll}0.22 & 0.00 & -0.01\end{array}$

$\begin{array}{lll}0.23 & 0.00 & 0.00\end{array}$

$\begin{array}{lll}0.23 & 0.00 & 0.00\end{array}$

$\begin{array}{lll}0.23 & 0.00 & 0.01\end{array}$

149253

$150 \quad 254$

151255

$152 \quad 256$

153257

154258

$155 \quad 259$

156260

$157 \quad 261$

158262

159263

160264

161265

162266

163267

$0.23 \quad 0.00$

$\begin{array}{ll}0.23 & 0.00\end{array}$

0.01

$0.23 \quad 0.00$

$\begin{array}{ll}0.23 & 0.00\end{array}$

$0.23 \quad 0.00$

$0.23 \quad 0.00$

$0.22 \quad 0.00$

$0.22 \quad 0.00$

$0.22 \quad 0.00$

0.210 .00

$0.21 \quad 0.00$

$\begin{array}{ll}0.21 & 0.00\end{array}$

$0.21 \quad 0.00$

0.210 .00

$0.21 \quad 0.00$

164268

165269

$0.20 \quad 0.00$

$\begin{array}{ll}0.19 & 0.00\end{array}$

$\begin{array}{ll}0.18 & 0.00\end{array}$

$\begin{array}{ll}0.17 & 0.00\end{array}$

$\begin{array}{ll}167 & 271\end{array}$

$168 \quad 272$

0.160 .00

169273

$0.16 \quad 0.00$

$\begin{array}{ll}170 & 274\end{array}$

$\begin{array}{ll}0.14 & 0.00\end{array}$

$0.13 \quad 0.00$

$0.12 \quad 0.00$
0.12

0.120 .00

$172 \quad 276$

$173 \quad 277$

174278

$0.12 \quad 0.00$

$\begin{array}{ll}175 & 279\end{array}$

$\begin{array}{ll}0.12 & 0.00\end{array}$

176280

$0.12 \quad 0.00$

$\begin{array}{llll}177 & 281 & -0.10 & 0.00\end{array}$

$\begin{array}{llll}178 & 282 & -0.09 & 0.00\end{array}$

$\begin{array}{lllll}179 & 283 & -0.08 & 0.00\end{array}$

$\begin{array}{lllll}180 & 284 & -0.05 & 0.00\end{array}$

$\begin{array}{lllll}181 & 285 & -0.04 & 0.00\end{array}$

$\begin{array}{llll}182 & 286 & 0.00 & 0.00\end{array}$

$183 \quad 287$

$0.00 \quad 0.00$

184288

$\begin{array}{ll}0.00 & 0.02\end{array}$

$185 \quad 289$

186290

$187 \quad 291$

188292

$\begin{array}{ll}0.00 & 0.07\end{array}$

$\begin{array}{lll}0.00 & 0.07 & 0.00\end{array}$

$\begin{array}{lll}0.01 & 0.08 & -0.01\end{array}$

$\begin{array}{llll}0.02 & 0.10 & -0.01 & 0.0\end{array}$

189293

190294

191295

$-0.01$

$\begin{array}{lllll}0.23 & 0.00 & -0.01 & 0.00\end{array}$

192296

193297

$\begin{array}{llll}0.23 & 0.00 & 0.00\end{array}$

0.00

$0.00 \quad 0.00$

$\begin{array}{ll}0.23 & 0.00\end{array}$

194298

195299 $\begin{array}{lll}0.01 & 0.09 & -0.01\end{array}$

$\begin{array}{llll}0.22 & 0.00 & -0.01\end{array}$

$\begin{array}{lllllllll}0.02 & 0.273 & 0.000 & 0.029 & -0.017 & -1.85 & -0.68 & 1782.00 & 97.97\end{array}$

$\begin{array}{lllllllll}0.02 & 0.250 & 0.000 & 0.037 & -0.015 & -2.06 & -0.82 & 1791.93 & 96.11\end{array}$

$\begin{array}{lllllllll}0.02 & 0.250 & 0.000 & 0.037 & -0.015 & -2.47 & -1.19 & 1800.09 & 96.02\end{array}$

$\begin{array}{lllllllll}0.03 & 0.250 & 0.000 & 0.038 & -0.025 & -2.87 & -1.55 & 1809.95 & 94.23\end{array}$

$\begin{array}{lllllllll}0.03 & 0.238 & 0.000 & 0.048 & -0.022 & -3.39 & -1.92 & 1817.83 & 94.42\end{array}$

$\begin{array}{lllllllll}0.03 & 0.238 & 0.000 & 0.036 & -0.025 & -3.49 & -2.08 & 1827.20 & 93.12\end{array}$

$\begin{array}{lllllllll}0.03 & 0.238 & 0.000 & 0.036 & -0.025 & -3.94 & -2.48 & 1834.85 & 93.55\end{array}$

$\begin{array}{lllllllll}0.03 & 0.250 & 0.000 & 0.026 & -0.027 & -4.13 & -2.66 & 1843.96 & 92.50\end{array}$

$\begin{array}{lllllllll}0.03 & 0.250 & 0.000 & 0.026 & -0.027 & -4.65 & -3.13 & 1851.42 & 93.12\end{array}$

$0.03 \quad 0.251$

0.000

$\begin{array}{lllll}0.014 & -0.030 & -4.89 & -3.34 & 1860.29\end{array}$

92.32

$\begin{array}{lllllll}0.000 & 0.014 & -0.030 & -5.44 & -3.85 & 1867.52 & 93.16\end{array}$

$\begin{array}{lllllll}0.000 & 0.002 & -0.033 & -5.80 & -4.10 & 1876.18 & 92.57\end{array}$

$\begin{array}{lllllll}0.000 & 0.002 & -0.033 & -6.33 & -4.59 & 1883.14 & 93.68\end{array}$

$\begin{array}{llllll}0.000 & 0.002 & -0.033 & -6.36 & -4.63 & 1891.32\end{array}$

$\begin{array}{llllll}0.000 & -0.010 & -0.036 & -6.54 & -4.68 & 1897.60\end{array}$

$\begin{array}{llllll}0.000 & -0.010 & -0.036 & -6.29 & -4.44 & 1905.25\end{array}$

$\begin{array}{lllllll}0.000 & -0.025 & -0.028 & -6.22 & -4.43 & 1911.22\end{array}$

$\begin{array}{lllllll}0.000 & -0.025 & -0.028 & -5.93 & -4.18 & 1918.61 & 98.57\end{array}$

$\begin{array}{lllllll}0.000 & -0.025 & -0.028 & -6.10 & -4.35 & 1924.53 & 100.72\end{array}$

$\begin{array}{lllllll}0.000 & -0.040 & -0.021 & -6.03 & -4.21 & 1931.79 & 101.53\end{array}$

$\begin{array}{lllllll}0.000 & -0.040 & -0.021 & -6.42 & -4.58 & 1937.67 & 103.73\end{array}$

$\begin{array}{lllllll}0.000 & -0.052 & -0.023 & -6.76 & -4.59 & 1944.84 & 104.62\end{array}$

$\begin{array}{lllllll}0.000 & -0.053 & -0.013 & -7.01 & -4.92 & 1950.45 & 107.08\end{array}$

$\begin{array}{lllllll}0.000 & -0.065 & -0.015 & -7.39 & -4.95 & 1957.41 & 108.19\end{array}$

$\begin{array}{lllllll}0.000 & -0.065 & -0.015 & -7.46 & -5.02 & 1962.55 & 111.13\end{array}$

$\begin{array}{lllllll}0.000 & -0.067 & -0.015 & -6.90 & -4.52 & 1968.75 & 113.00\end{array}$

$\begin{array}{lllllll}0.000 & -0.057 & -0.012 & -6.17 & -4.20 & 1973.27 & 116.55\end{array}$

$\begin{array}{lllllll}0.000 & -0.058 & -0.012 & -5.54 & -3.60 & 1979.15 & 118.74\end{array}$

$\begin{array}{lllllll}0.000 & -0.048 & -0.009 & -5.01 & -3.45 & 1983.63 & 122.33\end{array}$

$\begin{array}{lllllll}0.000 & -0.049 & -0.009 & -4.53 & -3.09 & 1989.54 & 124.50\end{array}$

$\begin{array}{lllllll}0.000 & -0.049 & -0.009 & -4.54 & -2.98 & 1993.84 & 128.27\end{array}$

$\begin{array}{lllllll}0.000 & -0.040 & -0.006 & -3.88 & -2.68 & 1999.59 & 130.59\end{array}$

$\begin{array}{lllllll}0.000 & -0.029 & -0.004 & -3.94 & -2.96 & 2004.08 & 134.17\end{array}$

$\begin{array}{lllllll}0.000 & -0.030 & -0.004 & -3.76 & -2.82 & 2009.78 & 136.54\end{array}$

$\begin{array}{lllllll}0.000 & -0.030 & -0.004 & -4.14 & -3.20 & 2014.17 & 140.22\end{array}$

$\begin{array}{lllllll}0.000 & -0.043 & 0.005 & -4.22 & -3.09 & 2019.69 & 142.77\end{array}$

$\begin{array}{lllllll}0.000 & -0.043 & 0.005 & -4.46 & -3.35 & 2023.76 & 146.77\end{array}$

$\begin{array}{lllllll}0.000 & -0.055 & 0.013 & -4.37 & -2.97 & 2028.82 & 149.79\end{array}$

$\begin{array}{lllllll}0.000 & -0.019 & 0.002 & -3.60 & -3.06 & 2032.52 & 154.15\end{array}$

$\begin{array}{lllllll}0.000 & -0.020 & 0.012 & -3.47 & -2.92 & 2037.62 & 157.13\end{array}$

$\begin{array}{lllllll}0.000 & -0.021 & 0.002 & -3.49 & -2.93 & 2041.06 & 161.76\end{array}$

$0.000-0.011$

$\begin{array}{llllll}0.001 & -3.01 & -2.50 & 2045.67 & 165.22\end{array}$

$0.000-0.011$

$\begin{array}{lllll}0.001 & -3.28 & -2.71 & 2049.13 & 169.83\end{array}$

$0.000 \quad 0.000$

$\begin{array}{lllll}0.000 & -2.93 & -2.38 & 2053.65 & 173.38\end{array}$

$0.00 \quad 0.000$

$0.000 \quad 0.000$

$\begin{array}{lllll}0.000 & -3.22 & -2.65 & 2056.98 & 178.12\end{array}$

$\begin{array}{llll}0.00 & 0.000 & -0.027 & 0.000\end{array}$

$\begin{array}{lllll}0.000 & -2.85 & -2.24 & 2061.23 & 181.95\end{array}$

$0.002-0.094$

0.002

$\begin{array}{lllll}0.003 & -3.12 & -1.69 & 2063.57 & 187.68\end{array}$

$0.002-0.094$

0.002

$\begin{array}{llllll}0.003 & -2.50 & -1.11 & 2067.47 & 191.85\end{array}$

$0.00 \quad 0.013-0.108$

0.014

$\begin{array}{lllll}0.004 & -2.41 & -0.72 & 2069.78 & 197.60\end{array}$

$0.014-0.121$

$\begin{array}{llllll}0.015 & -0.004 & -2.08 & -0.20 & 2073.57 & 201.90\end{array}$

$0.026-0.135$

$0.239 \quad 0.000$

$\begin{array}{llllll}0.016 & -0.003 & -2.32 & -0.10 & 2076.00 & 207.53\end{array}$

$0.250 \quad 0.000$

0.032

$\begin{array}{lllll}0.015 & -0.76 & 0.22 & 2079.81 & 211.79\end{array}$

$0.250 \quad 0.000$

0.035

$\begin{array}{lllll}0.005 & -1.08 & -0.08 & 2082.48 & 217.20\end{array}$

$0.250 \quad 0.000$

0.023

$\begin{array}{lllll}0.002 & -1.04 & -0.11 & 2086.46 & 221.29\end{array}$

$\begin{array}{lllll}0.002 & -1.40 & -0.42 & 2088.97 & 226.84\end{array}$

$\begin{array}{lllll}0.23 & 0.00 & 0.00 & 0.00 & 0.250\end{array}$

$\begin{array}{lll}0.00 & 0.00 & 0.250\end{array}$

$\begin{array}{lllllll}0.000 & 0.023 & 0.002 & -1.42 & -0.43 & 2092.77 & 231.12\end{array}$

$\begin{array}{lllllll}0.000 & 0.023 & 0.002 & -1.76 & -0.74 & 2095.12 & 236.84\end{array}$

\begin{tabular}{|c|c|c|c|}
\hline & & -0.74 & 100.06 \\
\hline & & -0.86 & 98.17 \\
\hline & & -1.24 & 98.01 \\
\hline & & -1.57 & 96.19 \\
\hline & & -1.94 & 96.33 \\
\hline & & -2.09 & 94.99 \\
\hline & & -2.49 & 95.37 \\
\hline & & -2.66 & 94.29 \\
\hline & & -3.14 & 94.86 \\
\hline & & -3.34 & 94.03 \\
\hline & & -3.85 & 94.83 \\
\hline & & -4.09 & 94.23 \\
\hline & & -4.59 & 95.31 \\
\hline 94.24 & 0.024 & -4.61 & 95.19 \\
\hline & & -4.66 & 96.97 \\
\hline & & -4.41 & 97.38 \\
\hline & & -4.42 & 99.44 \\
\hline & & -4.16 & 100.12 \\
\hline 101.32 & 0.029 & -4.34 & 102.26 \\
\hline & & -4.20 & 103.07 \\
\hline & & -4.57 & 105.25 \\
\hline & & -4.56 & 106.18 \\
\hline & & -4.92 & 108.61 \\
\hline & & -4.91 & 109.77 \\
\hline & & -4.99 & 112.71 \\
\hline & & -4.47 & 114.61 \\
\hline & & -4.18 & 118.15 \\
\hline & & -3.56 & 120.38 \\
\hline & & -3.43 & 123.98 \\
\hline & & -3.07 & 126.18 \\
\hline & & -2.95 & 129.98 \\
\hline & & -2.66 & 132.33 \\
\hline & & -2.95 & 135.94 \\
\hline & & -2.81 & 138.35 \\
\hline & & -3.19 & 142.08 \\
\hline & & -3.06 & 144.70 \\
\hline & & -3.32 & 148.75 \\
\hline & & -2.89 & 151.87 \\
\hline & & -3.06 & 156.22 \\
\hline & & -2.90 & 159.28 \\
\hline & & -2.92 & 163.96 \\
\hline & & -2.50 & 167.48 \\
\hline & & -2.71 & 172.17 \\
\hline & & -2.39 & 175.79 \\
\hline & & -2.65 & 180.61 \\
\hline & & -2.24 & 184.52 \\
\hline & & -1.65 & 190.38 \\
\hline & & -1.07 & 194.65 \\
\hline & & -0.66 & 200.51 \\
\hline & & -0.11 & 204.93 \\
\hline & & -0.00 & 210.68 \\
\hline & & 0.23 & 214.96 \\
\hline & & -0.10 & 220.45 \\
\hline & & -0.13 & 224.65 \\
\hline & & -0.45 & 230.31 \\
\hline & & -0.46 & 234.71 \\
\hline & & -0.77 & 240.55 \\
\hline
\end{tabular}

$-0.74 \quad 100.06$

$-0.86 \quad 98.17$

$-1.57 \quad 96.19$

$-1.94 \quad 96.33$

$-2.49-95.37$

$-2.66 \quad 94.29$

$-3.14 \quad 94.86$

$-3.85 \quad 94.83$

$-4.09 \quad 94.23$

$-4.61 \quad 95.19$

$-4.41 \quad 97.38$

$-4.42 \quad 99.44$

$\begin{array}{ll}-4.34 & 102.26\end{array}$

$-4.56106 .18$

$-4.92 \quad 108.61$

$-4.91 \quad 109.77$

$-4.47 \quad 114.61$

$-4.18118 .15$

$-3.43 \quad 123.98$

$-3.07 \quad 126.18$

$\begin{array}{ll}-2.95 & 129.98\end{array}$

$-2.66132 .33$

$-2.95 \quad 135.94$

$\begin{array}{rr}-2.81 & 138.35 \\ -3.19 & 142.08\end{array}$

$-3.06 \quad 144.70$

$-3.32 \quad 148.75$

$-2.89 \quad 151.87$

$-3.06 \quad 156.22$

$\begin{array}{lll}-2.90 & 159.28\end{array}$

$\begin{array}{ll}-2.71 & 172.17\end{array}$

$\begin{array}{ll}-2.39 & 175.79\end{array}$

$-2.65180 .61$

$-2.24 \quad 184.52$

$-1.65190 .38$

204.93

$0.23 \quad 214.96$

$-0.10 \quad 220.45$

$-0.13 \quad 224.65$

$-0.46 \quad 234.71$ 


\section{$Z=104$ (Rf)}

$\begin{array}{llll}196 & 300 & 0.24 & 0.00\end{array}$ $\begin{array}{llll}197 & 301 & 0.24 & 0.00\end{array}$ $\begin{array}{llll}198 & 302 & 0.25 & 0.00\end{array}$ $\begin{array}{llll}199 & 303 & 0.25 & 0.00\end{array}$ $\begin{array}{llll}200 & 304 & 0.25 & 0.00\end{array}$

$\begin{array}{llll}201 & 305 & 0.26 & 0.00\end{array}$ $\begin{array}{llll}202 & 306 & 0.26 & 0.00\end{array}$ $\begin{array}{llll}203 & 307 & 0.25 & 0.00\end{array}$ $\begin{array}{llll}204 & 308 & 0.25 & 0.00\end{array}$ $\begin{array}{llll}205 & 309 & 0.25 & 0.00\end{array}$

$\begin{array}{llll}206 & 310 & 0.24 & 0.00\end{array}$ $\begin{array}{llll}207 & 311 & 0.24 & 0.00\end{array}$ $\begin{array}{llll}208 & 312 & 0.23 & 0.00\end{array}$ $\begin{array}{llll}209 & 313 & 0.23 & 0.00\end{array}$ $\begin{array}{llll}210 & 314 & 0.22 & 0.00\end{array}$ $\begin{array}{llll}211 & 315 & 0.22 & 0.00\end{array}$ $\begin{array}{llll}212 & 316 & 0.22 & 0.00\end{array}$ $\begin{array}{llll}213 & 317 & 0.21 & 0.00\end{array}$ $\begin{array}{llll}214 & 318 & 0.21 & 0.00\end{array}$ $\begin{array}{llll}215 & 319 & 0.21 & 0.00\end{array}$

$\begin{array}{llll}216 & 320 & 0.21 & 0.00\end{array}$ $\begin{array}{llll}217 & 321 & 0.21 & 0.00\end{array}$ $\begin{array}{llll}218 & 322 & 0.21 & 0.00\end{array}$ $\begin{array}{llll}219 & 323 & 0.20 & 0.00\end{array}$ $\begin{array}{lllll}220 & 324 & 0.20 & 0.00\end{array}$

$\begin{array}{llll}221 & 325 & 0.20 & 0.00\end{array}$ $\begin{array}{llll}222 & 326 & 0.20 & 0.00\end{array}$ $\begin{array}{llll}223 & 327 & 0.20 & 0.00\end{array}$ $\begin{array}{llll}224 & 328 & 0.19 & 0.00\end{array}$ $\begin{array}{llll}225 & 329 & 0.19 & 0.00\end{array}$

$\begin{array}{llll}226 & 330 & 0.18 & 0.00\end{array}$ $\begin{array}{llll}227 & 331 & 0.18 & 0.00\end{array}$ $\begin{array}{llll}228 & 332 & 0.18 & 0.00\end{array}$ $\begin{array}{llll}229 & 333 & 0.18 & 0.00\end{array}$ $\begin{array}{llll}230 & 334 & 0.18 & 0.00\end{array}$

$\begin{array}{llll}231 & 335 & 0.18 & 0.00\end{array}$ $\begin{array}{llll}232 & 336 & 0.18 & 0.00\end{array}$ $\begin{array}{llll}233 & 337 & 0.18 & 0.00\end{array}$ $\begin{array}{llll}234 & 338 & 0.17 & 0.00\end{array}$ $\begin{array}{llll}235 & 339 & 0.09 & 0.00\end{array}$

\section{$Z=105(\mathrm{Db})$}

$\begin{array}{llll}136 & 241 & 0.28 & 0.00\end{array}$ $\begin{array}{llll}137 & 242 & 0.28 & 0.00\end{array}$ $\begin{array}{llll}138 & 243 & 0.27 & 0.00\end{array}$ $\begin{array}{llll}139 & 244 & 0.26 & 0.00\end{array}$ $\begin{array}{llll}140 & 245 & 0.23 & 0.00\end{array}$

$\begin{array}{llll}141 & 246 & 0.23 & 0.00\end{array}$

$\begin{array}{lllll}142 & 247 & 0.23 & 0.00 & 0.00\end{array}$ $\begin{array}{llllll}143 & 248 & 0.22 & 0.00 & -0.01\end{array}$

$\begin{array}{lllll}144 & 249 & 0.22 & 0.00 & 0.00\end{array}$ $\begin{array}{lllll}145 & 250 & 0.22 & 0.00 & 0.00\end{array}$

0.03

0.03

0.03

0.02

0.00

0.00

$\begin{array}{llll}146 & 251 & 0.22 & 0.00\end{array}$ $\begin{array}{llll}147 & 252 & 0.23 & 0.00\end{array}$ $\begin{array}{llll}148 & 253 & 0.23 & 0.00\end{array}$ $\begin{array}{llll}149 & 254 & 0.23 & 0.00\end{array}$ $\begin{array}{llll}150 & 255 & 0.23 & 0.00\end{array}$ $\begin{array}{llll}151 & 256 & 0.23 & 0.00\end{array}$ $\begin{array}{lll}0.01 & 0.262 & 0.000\end{array}$

$\begin{array}{llllll}0.014 & -0.010 & -1.88 & -0.83 & 2098.82 & 241.21\end{array}$ $\begin{array}{lllllllll}0.01 & 0.262 & 0.000 & 0.014 & -0.010 & -2.31 & -1.26 & 2101.14 & 246.96\end{array}$ $\begin{array}{lll}0.01 & 0.274 & 0.000\end{array}$ $\begin{array}{llllll}0.004 & -0.013 & -2.50 & -1.39 & 2104.72 & 251.45\end{array}$ $\begin{array}{lll}0.01 & 0.274 & 0.000\end{array}$ $\begin{array}{llllll}0.004 & -0.013 & -2.98 & -1.85 & 2106.91 & 257.34\end{array}$ $\begin{array}{lll}0.02 & 0.274 & 0.000\end{array}$ $\begin{array}{llllll}0.005 & -0.023 & -3.33 & -2.11 & 2110.46 & 261.86\end{array}$ $\begin{array}{lllllllll}0.02 & 0.286 & 0.000 & -0.004 & -0.026 & -3.96 & -2.62 & 2112.54 & 267.85\end{array}$ $\begin{array}{lllllllll}0.02 & 0.286 & 0.000 & -0.004 & -0.026 & -4.02 & -2.71 & 2115.77 & 272.69\end{array}$ $\begin{array}{lllllllll}0.02 & 0.275 & 0.000 & -0.007 & -0.026 & -4.26 & -2.94 & 2117.43 & 279.10\end{array}$ $\begin{array}{lllllllll}0.02 & 0.275 & 0.000 & -0.007 & -0.026 & -4.07 & -2.79 & 2120.25 & 284.35\end{array}$ $\begin{array}{lllllllll}0.02 & 0.275 & 0.000 & -0.007 & -0.026 & -4.19 & -2.92 & 2121.66 & 291.01\end{array}$ $\begin{array}{lllllllll}0.02 & 0.263 & 0.000 & -0.009 & -0.026 & -3.96 & -2.75 & 2124.32 & 296.42\end{array}$ $\begin{array}{lllllllll}0.02 & 0.263 & 0.000 & -0.009 & -0.026 & -4.13 & -2.95 & 2125.65 & 303.17\end{array}$ $\begin{array}{lllllllll}0.03 & 0.252 & 0.000 & 0.002 & -0.033 & -4.04 & -2.84 & 2128.22 & 308.66\end{array}$ $\begin{array}{lllllllll}0.03 & 0.252 & 0.000 & 0.002 & -0.033 & -4.24 & -3.01 & 2129.38 & 315.58\end{array}$ $\begin{array}{lllllllll}0.03 & 0.240 & 0.000 & -0.000 & -0.033 & -4.09 & -2.97 & 2131.88 & 321.15\end{array}$ $\begin{array}{lllllllll}0.03 & 0.240 & 0.000 & -0.000 & -0.033 & -4.35 & -3.16 & 2132.91 & 328.19\end{array}$ $\begin{array}{lllllllll}0.02 & 0.241 & 0.000 & -0.013 & -0.026 & -4.17 & -3.02 & 2135.16 & 334.01\end{array}$ $\begin{array}{lllllllll}0.03 & 0.229 & 0.000 & -0.002 & -0.033 & -4.60 & -3.40 & 2136.25 & 340.99\end{array}$ $\begin{array}{lllllllll}0.03 & 0.229 & 0.000 & -0.002 & -0.033 & -4.59 & -3.46 & 2138.56 & 346.75\end{array}$ $\begin{array}{lllllllll}0.03 & 0.229 & 0.000 & -0.002 & -0.033 & -5.00 & -3.86 & 2139.54 & 353.85\end{array}$ $\begin{array}{lllllllll}0.03 & 0.230 & 0.000 & -0.014 & -0.035 & -5.15 & -3.92 & 2141.72 & 359.74\end{array}$ $\begin{array}{lllllllll}0.03 & 0.230 & 0.000 & -0.014 & -0.035 & -5.60 & -4.37 & 2142.60 & 366.93\end{array}$ $\begin{array}{lllllllll}0.03 & 0.230 & 0.000 & -0.014 & -0.035 & -5.61 & -4.40 & 2144.61 & 372.99\end{array}$ $\begin{array}{lllllllll}0.03 & 0.219 & 0.000 & -0.016 & -0.035 & -6.01 & -4.80 & 2145.32 & 380.35\end{array}$ $\begin{array}{lllllllll}0.03 & 0.219 & 0.000 & -0.016 & -0.035 & -5.97 & -4.76 & 2147.13 & 386.61\end{array}$ $\begin{array}{lllllllll}0.03 & 0.219 & 0.000 & -0.016 & -0.035 & -6.33 & -5.12 & 2147.67 & 394.14\end{array}$ $\begin{array}{lllllllll}0.03 & 0.220 & 0.000 & -0.028 & -0.038 & -6.35 & -5.01 & 2149.27 & 400.62\end{array}$ $\begin{array}{lllllllll}0.02 & 0.219 & 0.000 & -0.029 & -0.028 & -6.38 & -5.15 & 2149.47 & 408.49\end{array}$ $\begin{array}{lllllllll}0.02 & 0.208 & 0.000 & -0.031 & -0.027 & -6.11 & -4.90 & 2150.80 & 415.22\end{array}$ $\begin{array}{lllllllll}0.01 & 0.208 & 0.000 & -0.044 & -0.020 & -6.43 & -5.12 & 2150.96 & 423.14\end{array}$ $\begin{array}{lllllllll}0.01 & 0.197 & 0.000 & -0.045 & -0.019 & -6.20 & -4.88 & 2152.17 & 430.00\end{array}$ $\begin{array}{lllllllll}0.00 & 0.197 & 0.000 & -0.046 & -0.009 & -6.35 & -4.94 & 2152.05 & 438.19\end{array}$ $\begin{array}{lllllllll}0.00 & 0.197 & 0.000 & -0.046 & -0.009 & -6.05 & -4.69 & 2153.13 & 445.19\end{array}$ $\begin{array}{lllllllll}0.00 & 0.198 & 0.000 & -0.058 & -0.012 & -6.53 & -4.94 & 2153.07 & 453.31\end{array}$ $\begin{array}{llllllllll}0.06 & -0.01 & 0.197 & 0.000 & -0.059 & -0.002 & -6.15 & -4.62 & 2153.96 & 460.50\end{array}$ $\begin{array}{llllllllll}0.07 & -0.01 & 0.198 & 0.000 & -0.071 & -0.004 & -6.69 & -4.88 & 2153.80 & 468.73\end{array}$ $\begin{array}{lllllllllll}0.07 & -0.01 & 0.198 & 0.000 & -0.071 & -0.004 & -6.35 & -4.58 & 2154.58 & 476.02\end{array}$ $\begin{array}{llllllllll}0.08 & -0.02 & 0.198 & 0.000 & -0.084 & 0.004 & -6.93 & -4.82 & 2154.29 & 484.38\end{array}$ $\begin{array}{llllllllll}0.08 & -0.02 & 0.187 & 0.000 & -0.085 & 0.005 & -6.50 & -4.39 & 2154.82 & 491.92\end{array}$ $\begin{array}{lllllllll}0.01 & 0.096 & 0.000 & 0.016 & -0.009 & -4.92 & -4.38 & 2154.16 & 500.65\end{array}$

$\begin{array}{lllllllll}0.01 & 0.308 & 0.000 & -0.001 & -0.016 & -1.45 & -0.48 & 1752.95 & 110.09\end{array}$ $\begin{array}{lllllllll}0.01 & 0.308 & 0.000 & -0.001 & -0.016 & -1.68 & -0.67 & 1761.88 & 109.24\end{array}$ $\begin{array}{lllllllll}0.02 & 0.297 & 0.000 & -0.002 & -0.026 & -1.83 & -0.63 & 1771.95 & 107.24\end{array}$ $\begin{array}{lllllllll}0.02 & 0.285 & 0.000 & 0.007 & -0.023 & -1.98 & -0.81 & 1780.59 & 106.67\end{array}$ $\begin{array}{lllllllll}0.02 & 0.250 & 0.000 & 0.025 & -0.018 & -2.08 & -1.03 & 1790.62 & 104.70\end{array}$ $\begin{array}{lllllllll}0.02 & 0.250 & 0.000 & 0.025 & -0.018 & -2.46 & -1.44 & 1799.19 & 104.20\end{array}$ $\begin{array}{lllllllll}0.02 & 0.250 & 0.000 & 0.025 & -0.018 & -2.69 & -1.65 & 1808.94 & 102.53\end{array}$ $\begin{array}{lllllllll}0.02 & 0.238 & 0.000 & 0.035 & -0.015 & -3.15 & -2.00 & 1817.17 & 102.37\end{array}$ $\begin{array}{llllllllll}0.02 & 0.239 & 0.000 & 0.023 & -0.018 & -3.31 & -2.17 & 1826.58 & 101.03\end{array}$ $\begin{array}{lllllllll}0.03 & 0.239 & 0.000 & 0.024 & -0.028 & -3.97 & -2.59 & 1834.61 & 101.07\end{array}$

$\begin{array}{lllllllllll}0.00 & 0.03 & 0.239 & 0.000 & 0.024 & -0.028 & -4.21 & -2.82 & 1843.81 & 99.94\end{array}$ $\begin{array}{llllllllll}0.01 & 0.03 & 0.251 & 0.000 & 0.014 & -0.030 & -4.79 & -3.31 & 1851.65 & 100.17\end{array}$ $\begin{array}{llllllllll}0.01 & 0.03 & 0.251 & 0.000 & 0.014 & -0.030 & -5.06 & -3.55 & 1860.59 & 99.31\end{array}$ $\begin{array}{llllllllll}0.02 & 0.03 & 0.252 & 0.000 & 0.002 & -0.033 & -5.69 & -4.06 & 1868.18 & 99.79\end{array}$ $\begin{array}{llllllllll}0.02 & 0.03 & 0.252 & 0.000 & 0.002 & -0.033 & -6.06 & -4.40 & 1876.96 & 99.08\end{array}$ $\begin{array}{llllllllll}0.02 & 0.03 & 0.252 & 0.000 & 0.002 & -0.033 & -6.58 & -4.88 & 1884.27 & 99.84\end{array}$
$-0.85 \quad 245.07$

$-1.29 \quad 250.94$

$-1.41 \quad 255.58$

$-1.87 \quad 261.60$

$-2.06 \quad 266.34$

$-2.56 \quad 272.48$

$-2.64 \quad 277.49$

$-2.88 \quad 284.05$

$-2.71 \quad 289.47$

$\begin{array}{ll}-2.86 & 296.28\end{array}$

$\begin{array}{ll}-2.67 & 301.88\end{array}$

$\begin{array}{ll}-2.88 & 308.79\end{array}$

$-2.67 \quad 314.56$

$-2.85 \quad 321.65$

$-2.79 \quad 327.42$

$-2.98 \quad 334.64$

$\begin{array}{ll}-2.93 & 340.57\end{array}$

$-3.22 \quad 347.83$

$-3.26 \quad 353.81$

$-3.67 \quad 361.10$

$-3.69 \quad 367.25$

$-4.14 \quad 374.64$

$-4.17 \quad 380.92$

$-4.56 \quad 388.51$

$-4.52 \quad 395.00$

$-4.88 \quad 402.75$

$\begin{array}{ll}-4.71 \quad 409.52 \\ -5.00 & 417.47\end{array}$

$\begin{array}{ll}-5.00 & 417.47\end{array}$

$-4.74 \quad 424.46$

$-5.01 \quad 432.57$

$-4.76 \quad 439.69$

$-4.88 \quad 448.08$

$-4.62 \quad 455.34$

$-4.81 \quad 463.78$

$-4.49 \quad 471.23$

$\begin{array}{ll}-4.69 & 479.79\end{array}$

$-4.38 \quad 487.36$

$-4.50 \quad 496.12$

$-4.05 \quad 503.96$

$-4.36 \quad 512.66$

$\begin{array}{ll}-0.61 & 112.58\end{array}$

$-0.81 \quad 111.64$

$-0.73 \quad 109.60$

$-0.92 \quad 108.97$

$-1.09106 .98$

$\begin{array}{ll}-1.51 & 106.41\end{array}$

$-1.71 \quad 104.69$

$\begin{array}{ll}-2.05 & 104.47\end{array}$

$\begin{array}{ll}-2.21 \quad 103.09 \\ -2.62 & 103.08\end{array}$

\begin{tabular}{ll}
$-2.62 \quad 103.08$ \\
\hline
\end{tabular}

$-2.84 \quad 101.92$

$-3.35 \quad 102.10$

$-3.57 \quad 101.20$

$-4.08 \quad 101.64$

$-4.41 \quad 100.91$

$-4.90 \quad 101.64$ 


\section{$Z=105(\mathrm{Db})$}

\begin{tabular}{|c|c|c|c|c|c|c|c|c|c|c|c|c|c|c|}
\hline $152 \quad 257$ & 0.23 & 0.00 & 0.03 & 0.03 & 0.252 & 0.000 & -0.010 & -0.036 & -6.82 & -4.99 & 1892.55 & 99.63 & -4.99 & 101.41 \\
\hline 153258 & 0.23 & 0.00 & 0.03 & 0.03 & 0.252 & 0.000 & -0.010 & -0.036 & -6.88 & -5.05 & 1899.19 & 101.07 & -5.05 & 102.82 \\
\hline 154259 & 0.23 & 0.00 & 0.04 & 0.03 & 0.253 & 0.000 & -0.022 & -0.038 & -6.87 & -4.86 & 1906.93 & 101.40 & -4.85 & 103.15 \\
\hline 5260 & 0.22 & 0.00 & 0.04 & 0.02 & 0.242 & 0.000 & -0.025 & -0.028 & -6.70 & -4.93 & 1913.32 & 103.08 & -4.94 & 104.78 \\
\hline 156261 & 0.22 & 0.00 & 0.04 & 0.02 & 0.242 & 0.000 & -0.025 & -0.028 & -6.41 & -4.68 & 1920.75 & 103.72 & -4.68 & 105.42 \\
\hline $7 \quad 262$ & 0.22 & 0.00 & 0.05 & 0.01 & 0.242 & 0.000 & -0.038 & -0.021 & -6.70 & -4.86 & 1927.02 & 105.52 & -4.89 & 107.18 \\
\hline $8 \quad 263$ & 0.21 & 0.00 & 0.05 & 0.01 & 0.231 & 0.000 & -0.040 & -0.021 & -6.61 & -4.81 & 1934.40 & 106.21 & -4.82 & 107.88 \\
\hline 9264 & 0.21 & 0.00 & 0.06 & 0.01 & 0.232 & 0.000 & -0.052 & -0.023 & -7.31 & -5.18 & 1940.63 & 108.05 & -5.18 & 109.72 \\
\hline $60 \quad 265$ & 0.21 & 0.00 & 0.06 & 0.01 & 0.232 & 0.000 & -0.052 & -0.023 & -7.40 & -5.23 & 1947.87 & 108.88 & -5.22 & 110.56 \\
\hline 161266 & 0.21 & 0.00 & 0.06 & 0.00 & 0.231 & 0.000 & -0.053 & -0.013 & -7.65 & -5.57 & 1953.83 & 110.99 & -5.58 & 112.64 \\
\hline $62 \quad 267$ & 0.21 & 0.00 & 0.07 & 0.00 & 0.232 & 0.000 & -0.065 & -0.015 & -8.08 & -5.64 & 1960.86 & 112.03 & -5.62 & 113.72 \\
\hline 3268 & 0.21 & 0.00 & 0.07 & 0.00 & 0.232 & 0.000 & -0.065 & -0.015 & -8.15 & -5.71 & 1966.34 & 114.63 & -5.70 & 116.31 \\
\hline $64 \quad 269$ & 0.20 & 0.00 & 0.07 & 0.00 & 0.221 & 0.000 & -0.067 & -0.015 & -7.59 & -5.21 & 1972.56 & 116.48 & -5.18 & 118.19 \\
\hline 165270 & 0.19 & 0.00 & 0.07 & -0.01 & 0.209 & 0.000 & -0.070 & -0.004 & -7.15 & -4.86 & 1977.39 & 119.72 & -4.84 & 121.44 \\
\hline 166271 & 0.19 & 0.00 & 0.07 & -0.01 & 0.209 & 0.000 & -0.070 & -0.004 & -6.52 & -4.24 & 1983.28 & 121.90 & -4.20 & 123.65 \\
\hline $167 \quad 272$ & 0.17 & 0.00 & 0.06 & 0.00 & 0.186 & 0.000 & -0.060 & -0.011 & -5.89 & -4.03 & 1988.03 & 125.22 & -4.01 & 126.97 \\
\hline 168273 & 0.16 & 0.00 & 0.05 & 0.00 & 0.175 & 0.000 & -0.049 & -0.009 & -5.08 & -3.62 & 1993.91 & 127.41 & -3.60 & 129.19 \\
\hline 169274 & 0.16 & 0.00 & 0.05 & 0.00 & 0.175 & 0.000 & -0.049 & -0.009 & -5.08 & -3.51 & 1998.55 & 130.85 & -3.49 & 132.64 \\
\hline $170 \quad 275$ & 0.14 & 0.00 & 0.04 & 0.00 & 0.152 & 0.000 & -0.040 & -0.006 & -4.39 & -3.18 & 2004.29 & 133.18 & -3.17 & 135.00 \\
\hline 171276 & 0.13 & 0.00 & 0.04 & 0.00 & 0.141 & 0.000 & -0.041 & -0.006 & -4.65 & -3.47 & 2009.13 & 136.41 & -3.46 & 138.27 \\
\hline 2277 & 0.12 & 0.00 & 0.04 & 0.00 & 0.130 & 0.000 & -0.042 & -0.005 & -4.47 & -3.34 & 2014.85 & 138.76 & -3.32 & 140.66 \\
\hline $73 \quad 278$ & 0.12 & 0.00 & 0.04 & -0.01 & 0.130 & 0.000 & -0.043 & 0.005 & -4.86 & -3.71 & 2019.57 & 142.11 & -3.69 & 144.05 \\
\hline 4279 & 0.12 & 0.00 & 0.04 & -0.01 & 0.130 & 0.000 & -0.043 & 0.005 & -4.73 & -3.60 & 2025.11 & 144.64 & -3.58 & 146.63 \\
\hline 5280 & 0.12 & 0.00 & 0.05 & -0.01 & 0.130 & 0.000 & -0.054 & 0.003 & -5.23 & -3.85 & 2029.51 & 148.32 & -3.81 & 150.37 \\
\hline 76281 & 0.12 & 0.00 & 0.05 & -0.02 & 0.130 & 0.000 & -0.055 & 0.013 & -4.89 & -3.48 & 2034.59 & 151.31 & -3.41 & 153.44 \\
\hline $77 \quad 282$ & 0.09 & 0.00 & 0.04 & -0.01 & 0.097 & 0.000 & -0.045 & 0.006 & -4.34 & -3.29 & 2038.34 & 155.62 & -3.26 & 157.77 \\
\hline $78 \quad 283$ & -0.09 & 0.01 & 0.02 & -0.01 & -0.094 & -0.013 & -0.020 & 0.012 & -3.82 & -3.24 & 2043.54 & 158.50 & -3.22 & 160.68 \\
\hline 179284 & -0.07 & 0.00 & 0.01 & 0.00 & -0.073 & 0.000 & -0.010 & 0.001 & -3.73 & -3.26 & 2047.32 & 162.79 & -3.27 & 165.02 \\
\hline $\begin{array}{ll}30 & 285\end{array}$ & -0.05 & 0.00 & 0.01 & 0.00 & -0.053 & 0.000 & -0.011 & 0.001 & -3.37 & -2.85 & 2051.97 & 166.21 & -2.86 & 168.51 \\
\hline 181286 & -0.04 & 0.00 & 0.01 & 0.00 & -0.042 & 0.000 & -0.011 & 0.001 & -3.62 & -3.05 & 2055.73 & 170.52 & -3.05 & 172.88 \\
\hline $182 \quad 287$ & -0.02 & 0.00 & 0.01 & 0.00 & -0.021 & 0.000 & -0.012 & 0.000 & -3.27 & -2.68 & 2060.23 & 174.10 & -2.68 & 176.53 \\
\hline 3288 & 0.01 & 0.00 & 0.00 & 0.00 & 0.011 & 0.000 & 0.000 & 0.000 & -3.46 & -2.90 & 2063.82 & 178.57 & -2.90 & 181.08 \\
\hline $34 \quad 289$ & 0.00 & 0.03 & 0.00 & 0.00 & 0.000 & -0.040 & 0.000 & 0.001 & -3.18 & -2.49 & 2068.09 & 182.37 & -2.48 & 184.96 \\
\hline 5290 & 0.00 & 0.07 & 0.00 & 0.00 & 0.002 & -0.094 & 0.002 & 0.003 & -3.40 & -1.97 & 2070.78 & 187.76 & -1.94 & 190.46 \\
\hline 186291 & 0.00 & 0.08 & 0.00 & 0.00 & 0.003 & -0.108 & 0.003 & 0.004 & -3.08 & -1.40 & 2074.70 & 191.91 & -1.35 & 194.72 \\
\hline 187292 & -0.62 & 0.00 & -0.02 & -0.02 & -0.610 & 0.000 & 0.156 & -0.028 & -3.39 & 6.88 & 2069.45 & 205.23 & 6.57 & 207.77 \\
\hline $188 \quad 293$ & 0.01 & 0.09 & -0.01 & 0.01 & 0.014 & -0.121 & 0.015 & -0.004 & -2.37 & -0.53 & 2081.17 & 201.58 & -0.45 & 204.60 \\
\hline 189294 & 0.02 & 0.10 & -0.01 & 0.01 & 0.026 & -0.135 & 0.016 & -0.003 & -2.58 & -0.38 & 2083.87 & 206.95 & -0.29 & 210.07 \\
\hline $190 \quad 295$ & 0.22 & 0.00 & 0.00 & -0.01 & 0.239 & 0.000 & 0.020 & 0.012 & -0.76 & 0.07 & 2087.56 & 211.33 & 0.05 & 214.46 \\
\hline 191296 & 0.23 & 0.00 & 0.00 & -0.01 & 0.250 & 0.000 & 0.022 & 0.012 & -1.14 & -0.22 & 2090.53 & 216.43 & -0.24 & 219.66 \\
\hline $192 \quad 297$ & 0.22 & 0.00 & 0.00 & -0.01 & 0.239 & 0.000 & 0.020 & 0.012 & -1.18 & -0.27 & 2094.54 & 220.49 & -0.28 & 223.83 \\
\hline 193298 & 0.23 & 0.00 & 0.00 & 0.00 & 0.250 & 0.000 & 0.023 & 0.002 & -1.51 & -0.56 & 2097.34 & 225.76 & -0.60 & 229.18 \\
\hline 194299 & 0.23 & 0.00 & 0.01 & 0.00 & 0.250 & 0.000 & 0.011 & -0.001 & -1.52 & -0.58 & 2101.16 & 230.02 & -0.62 & 233.55 \\
\hline 195300 & 0.23 & 0.00 & 0.01 & 0.00 & 0.250 & 0.000 & 0.011 & -0.001 & -1.89 & -0.92 & 2103.84 & 235.40 & -0.96 & 239.05 \\
\hline 196301 & 0.24 & 0.00 & 0.01 & 0.01 & 0.262 & 0.000 & 0.014 & -0.010 & -2.02 & -1.04 & 2107.60 & 239.72 & -1.07 & 243.51 \\
\hline 197302 & 0.24 & 0.00 & 0.02 & 0.01 & 0.262 & 0.000 & 0.002 & -0.013 & -2.53 & -1.46 & 2110.20 & 245.19 & -1.49 & 249.10 \\
\hline 198303 & 0.24 & 0.00 & 0.02 & 0.01 & 0.262 & 0.000 & 0.002 & -0.013 & -2.67 & -1.60 & 2113.80 & 249.66 & -1.62 & 253.71 \\
\hline 199304 & 0.25 & 0.00 & 0.03 & 0.01 & 0.274 & 0.000 & -0.008 & -0.016 & -3.28 & -2.08 & 2116.31 & 255.22 & -2.10 & 259.41 \\
\hline $200 \quad 305$ & 0.25 & 0.00 & 0.03 & 0.01 & 0.274 & 0.000 & -0.008 & -0.016 & -3.50 & -2.28 & 2119.82 & 259.79 & -2.30 & 264.12 \\
\hline 201306 & 0.25 & 0.00 & 0.03 & 0.02 & 0.275 & 0.000 & -0.007 & -0.026 & -4.16 & -2.82 & 2122.23 & 265.45 & -2.77 & 269.98 \\
\hline 202307 & 0.25 & 0.00 & 0.03 & 0.02 & 0.275 & 0.000 & -0.007 & -0.026 & -4.23 & -2.91 & 2125.46 & 270.29 & -2.85 & 274.98 \\
\hline 203308 & 0.25 & 0.00 & 0.03 & 0.02 & 0.275 & 0.000 & -0.007 & -0.026 & -4.49 & -3.18 & 2127.46 & 276.36 & -3.14 & 281.20 \\
\hline 204309 & 0.25 & 0.00 & 0.03 & 0.02 & 0.275 & 0.000 & -0.007 & -0.026 & -4.30 & -3.03 & 2130.29 & 281.60 & -2.97 & 286.59 \\
\hline 310 & 0.25 & 0.00 & 0.04 & 0.02 & 0.276 & 0.000 & -0.019 & -0.029 & -4.62 & -3.21 & 2132.05 & 287.92 & -3.13 & 293.09 \\
\hline 206311 & 0.24 & 0.00 & 0.03 & 0.02 & 0.263 & 0.000 & -0.009 & -0.026 & -4.22 & -3.02 & 2134.69 & 293.34 & -2.96 & 298.66 \\
\hline 207312 & 0.24 & 0.00 & 0.03 & 0.02 & 0.263 & 0.000 & -0.009 & -0.026 & -4.39 & -3.21 & 2136.31 & 299.80 & -3.16 & 305.27 \\
\hline 208313 & 0.24 & 0.00 & 0.04 & 0.02 & 0.264 & 0.000 & -0.021 & -0.029 & -4.40 & -3.07 & 2138.85 & 305.32 & -2.97 & 311.02 \\
\hline
\end{tabular}


$Z=105(\mathrm{Db})$

$\begin{array}{lllll}209 & 314 & 0.24 & 0.00 & 0.04\end{array}$ $\begin{array}{lllll}210 & 315 & 0.23 & 0.00 & 0.04\end{array}$ $\begin{array}{lllll}211 & 316 & 0.24 & 0.00 & 0.05\end{array}$ $\begin{array}{lllll}212 & 317 & 0.23 & 0.00 & 0.04\end{array}$ $\begin{array}{lllll}213 & 318 & 0.23 & 0.00 & 0.05\end{array}$ $\begin{array}{lllll}214 & 319 & 0.23 & 0.00 & 0.05\end{array}$ $\begin{array}{lllll}215 & 320 & 0.22 & 0.00 & 0.04\end{array}$ $\begin{array}{lllll}216 & 321 & 0.21 & 0.00 & 0.03\end{array}$ $\begin{array}{lllll}217 & 322 & 0.21 & 0.00 & 0.03\end{array}$ $\begin{array}{lllll}218 & 323 & 0.21 & 0.00 & 0.04\end{array}$ $\begin{array}{llllll}219 & 324 & 0.21 & 0.00 & 0.04\end{array}$ $\begin{array}{llllll}220 & 325 & 0.21 & 0.00 & 0.04\end{array}$ $\begin{array}{lllll}221 & 326 & 0.20 & 0.00 & 0.04\end{array}$ $\begin{array}{lllll}222 & 327 & 0.20 & 0.00 & 0.04\end{array}$ $\begin{array}{llllll}223 & 328 & 0.20 & 0.00 & 0.04\end{array}$ $\begin{array}{lllll}224 & 329 & 0.19 & 0.00 & 0.05\end{array}$ $\begin{array}{lllll}225 & 330 & 0.19 & 0.00 & 0.05\end{array}$ $\begin{array}{lllll}226 & 331 & 0.18 & 0.00 & 0.05\end{array}$ $\begin{array}{lllll}227 & 332 & 0.18 & 0.00 & 0.05\end{array}$ $\begin{array}{lllll}228 & 333 & 0.18 & 0.00 & 0.06\end{array}$

$\begin{array}{lllll}229 & 334 & 0.18 & 0.00 & 0.06\end{array}$ $\begin{array}{llllll}230 & 335 & 0.18 & 0.00 & 0.07\end{array}$ $\begin{array}{llllll}231 & 336 & 0.18 & 0.00 & 0.07 & -0 .\end{array}$ $\begin{array}{llllll}232 & 337 & 0.18 & 0.00 & 0.07 & -0 \\ 233 & 338 & 0.18 & 0.00 & 0.08\end{array}$ $\begin{array}{lllll}234 & 339 & 0.17 & 0.00 & 0.08\end{array}$

\section{$Z=106(\mathrm{Sg})$}

$\begin{array}{lllll}138 & 244 & 0.28 & 0.00 & 0.04\end{array}$ $\begin{array}{lllll}139 & 245 & 0.26 & 0.00 & 0.03\end{array}$ $\begin{array}{llllll}140 & 246 & 0.24 & 0.00 & 0.01\end{array}$ $\begin{array}{lllll}141 & 247 & 0.23 & 0.00 & 0.00\end{array}$ $\begin{array}{lllll}142 & 248 & 0.22 & 0.00 & 0.00\end{array}$

$\begin{array}{llllll}143 & 249 & 0.22 & 0.00 & 0.00\end{array}$ $\begin{array}{lllll}144 & 250 & 0.22 & 0.00 & 0.00\end{array}$ $\begin{array}{lllll}145 & 251 & 0.22 & 0.00 & 0.00\end{array}$ $\begin{array}{lllll}146 & 252 & 0.23 & 0.00 & 0.01\end{array}$ $\begin{array}{lllll}147 & 253 & 0.23 & 0.00 & 0.01\end{array}$

$\begin{array}{llllll}148 & 254 & 0.23 & 0.00 & 0.02\end{array}$ $\begin{array}{lllll}149 & 255 & 0.23 & 0.00 & 0.02\end{array}$ $\begin{array}{lllll}150 & 256 & 0.23 & 0.00 & 0.03\end{array}$ $\begin{array}{llllll}151 & 257 & 0.23 & 0.00 & 0.03\end{array}$ $\begin{array}{lllll}152 & 258 & 0.23 & 0.00 & 0.03\end{array}$ $\begin{array}{lllll}153 & 259 & 0.23 & 0.00 & 0.04\end{array}$ $\begin{array}{llllll}154 & 260 & 0.22 & 0.00 & 0.04\end{array}$ $\begin{array}{lllll}155 & 261 & 0.22 & 0.00 & 0.04\end{array}$ $\begin{array}{lllll}156 & 262 & 0.22 & 0.00 & 0.05\end{array}$ $\begin{array}{lllll}157 & 263 & 0.22 & 0.00 & 0.05\end{array}$

$\begin{array}{llllll}158 & 264 & 0.21 & 0.00 & 0.06\end{array}$ $\begin{array}{lllll}159 & 265 & 0.21 & 0.00 & 0.06\end{array}$ $\begin{array}{lllll}160 & 266 & 0.21 & 0.00 & 0.06\end{array}$ $\begin{array}{lllll}161 & 267 & 0.21 & 0.00 & 0.07\end{array}$ $\begin{array}{lllll}162 & 268 & 0.21 & 0.00 & 0.07\end{array}$

$\begin{array}{lllll}163 & 269 & 0.21 & 0.00 & 0.07\end{array}$ $\begin{array}{lllll}164 & 270 & 0.20 & 0.00 & 0.08\end{array}$ $\begin{array}{llllll}165 & 271 & 0.20 & 0.00 & 0.08 & -0 .\end{array}$ $\begin{array}{llllll}166 & 272 & 0.19 & 0.00 & 0.07 & -0 .\end{array}$ $\begin{array}{llllll}167 & 273 & 0.18 & 0.00 & 0.07\end{array}$ $\begin{array}{lllllllll}0.02 & 0.264 & 0.000 & -0.021 & -0.029 & -4.62 & -3.31 & 2140.37 & 311.88\end{array}$ $\begin{array}{lllllllll}0.02 & 0.253 & 0.000 & -0.023 & -0.029 & -4.49 & -3.23 & 2142.83 & 317.49\end{array}$ $\begin{array}{lllllllll}0.02 & 0.265 & 0.000 & -0.033 & -0.031 & -5.03 & -3.59 & 2144.33 & 324.06\end{array}$ $\begin{array}{lllllllll}0.02 & 0.253 & 0.000 & -0.023 & -0.029 & -4.71 & -3.41 & 2146.54 & 329.92\end{array}$ $\begin{array}{lllllllll}0.02 & 0.254 & 0.000 & -0.035 & -0.031 & -5.30 & -3.79 & 2147.93 & 336.61\end{array}$ $\begin{array}{lllllllll}0.02 & 0.254 & 0.000 & -0.035 & -0.031 & -5.26 & -3.79 & 2150.18 & 342.43\end{array}$ $\begin{array}{lllllllll}0.02 & 0.242 & 0.000 & -0.025 & -0.028 & -5.40 & -4.11 & 2151.36 & 349.31\end{array}$ $\begin{array}{lllllllll}0.03 & 0.230 & 0.000 & -0.014 & -0.035 & -5.45 & -4.21 & 2153.58 & 355.17\end{array}$ $\begin{array}{lllllllll}0.03 & 0.230 & 0.000 & -0.014 & -0.035 & -5.88 & -4.68 & 2154.77 & 362.05\end{array}$ $\begin{array}{lllllllll}0.03 & 0.231 & 0.000 & -0.026 & -0.038 & -6.09 & -4.72 & 2156.79 & 368.09\end{array}$ $\begin{array}{lllllllll}0.03 & 0.231 & 0.000 & -0.026 & -0.038 & -6.51 & -5.13 & 2157.80 & 375.16\end{array}$ $\begin{array}{lllllllll}0.03 & 0.231 & 0.000 & -0.026 & -0.038 & -6.48 & -5.11 & 2159.62 & 381.41\end{array}$ $\begin{array}{lllllllll}0.03 & 0.220 & 0.000 & -0.028 & -0.038 & -6.88 & -5.50 & 2160.48 & 388.62\end{array}$ $\begin{array}{lllllllll}0.03 & 0.220 & 0.000 & -0.028 & -0.038 & -6.70 & -5.35 & 2162.03 & 395.14\end{array}$ $\begin{array}{lllllllll}0.02 & 0.219 & 0.000 & -0.029 & -0.028 & -6.73 & -5.49 & 2162.52 & 402.73\end{array}$ $\begin{array}{lllllllll}0.02 & 0.209 & 0.000 & -0.043 & -0.030 & -6.71 & -5.30 & 2163.91 & 409.41\end{array}$ $\begin{array}{lllllllll}0.01 & 0.208 & 0.000 & -0.044 & -0.020 & -6.84 & -5.53 & 2164.35 & 417.03\end{array}$ $\begin{array}{lllllllll}0.01 & 0.197 & 0.000 & -0.045 & -0.019 & -6.60 & -5.13 & 2165.40 & 424.06\end{array}$ $\begin{array}{lllllllll}0.01 & 0.197 & 0.000 & -0.045 & -0.019 & -6.83 & -5.38 & 2165.74 & 431.79\end{array}$ $\begin{array}{lllllllll}0.00 & 0.198 & 0.000 & -0.058 & -0.012 & -6.74 & -5.12 & 2166.81 & 438.79\end{array}$ $\begin{array}{lllllllll}0.00 & 0.198 & 0.000 & -0.058 & -0.012 & -6.96 & -5.36 & 2167.02 & 446.65\end{array}$ $\begin{array}{lllllllll}-0.01 & 0.198 & 0.000 & -0.071 & -0.004 & -6.92 & -5.09 & 2167.95 & 453.79\end{array}$ $\begin{array}{lllllllll}-0.01 & 0.198 & 0.000 & -0.071 & -0.004 & -7.17 & -5.34 & 2168.05 & 461.76\end{array}$ $\begin{array}{lllllllll}-0.01 & 0.198 & 0.000 & -0.071 & -0.004 & -6.84 & -5.03 & 2168.83 & 469.05\end{array}$ $\begin{array}{lllllllll}-0.02 & 0.198 & 0.000 & -0.084 & 0.004 & -7.45 & -5.31 & 2168.84 & 477.12\end{array}$ $\begin{array}{lllll}0.005 & -7.01 & -4.87 & 2169.36 & 484.67\end{array}$

$\begin{array}{lllllllll}0.01 & 0.309 & 0.000 & -0.013 & -0.020 & -1.47 & -0.48 & 1771.51 & 114.96\end{array}$ $\begin{array}{lllllllll}0.01 & 0.286 & 0.000 & -0.006 & -0.016 & -1.59 & -0.65 & 1780.16 & 114.38\end{array}$ $\begin{array}{lllllllll}0.02 & 0.262 & 0.000 & 0.015 & -0.020 & -1.74 & -0.79 & 1790.49 & 112.12\end{array}$ $\begin{array}{lllllllll}0.02 & 0.250 & 0.000 & 0.025 & -0.018 & -2.08 & -1.09 & 1799.00 & 111.69\end{array}$ $\begin{array}{lllllllll}0.02 & 0.239 & 0.000 & 0.023 & -0.018 & -2.30 & -1.37 & 1809.18 & 109.58\end{array}$ $\begin{array}{llllllllll}0.02 & 0.239 & 0.000 & 0.023 & -0.018 & -2.71 & -1.71 & 1817.43 & 109.39\end{array}$ $\begin{array}{lllllllll}0.02 & 0.239 & 0.000 & 0.023 & -0.018 & -2.94 & -1.90 & 1827.24 & 107.66\end{array}$ $\begin{array}{lllllllll}0.03 & 0.239 & 0.000 & 0.024 & -0.028 & -3.59 & -2.33 & 1835.31 & 107.66\end{array}$ $\begin{array}{llllllllll}0.03 & 0.251 & 0.000 & 0.014 & -0.030 & -3.90 & -2.57 & 1844.89 & 106.15\end{array}$ $\begin{array}{llllllllll}0.03 & 0.251 & 0.000 & 0.014 & -0.030 & -4.44 & -3.06 & 1852.76 & 106.36\end{array}$ $\begin{array}{lllllllll}0.03 & 0.252 & 0.000 & 0.002 & -0.033 & -4.85 & -3.35 & 1862.11 & 105.08\end{array}$ $\begin{array}{lllllllll}0.03 & 0.252 & 0.000 & 0.002 & -0.033 & -5.40 & -3.86 & 1869.73 & 105.52\end{array}$ $\begin{array}{lllllllll}0.03 & 0.252 & 0.000 & -0.010 & -0.036 & -5.94 & -4.23 & 1878.90 & 104.43\end{array}$ $\begin{array}{lllllllll}0.03 & 0.252 & 0.000 & -0.010 & -0.036 & -6.50 & -4.75 & 1886.28 & 105.12\end{array}$ $\begin{array}{lllllllll}0.03 & 0.252 & 0.000 & -0.010 & -0.036 & -6.61 & -4.86 & 1894.91 & 104.56\end{array}$ $\begin{array}{llllllllll}0.03 & 0.253 & 0.000 & -0.022 & -0.038 & -6.92 & -4.97 & 1901.64 & 105.90\end{array}$ $\begin{array}{lllllllll}0.03 & 0.242 & 0.000 & -0.024 & -0.038 & -6.72 & -4.79 & 1909.73 & 105.88 \\ 0.02 & 0.242 & 0.000 & -0.025 & -0.028 & -6.56 & -4.87 & 1916.17 & 107.51\end{array}$ $\begin{array}{lllllllll}0.02 & 0.243 & 0.000 & -0.037 & -0.031 & -6.59 & -4.66 & 1923.99 & 107.76\end{array}$ $\begin{array}{lllllllll}0.01 & 0.242 & 0.000 & -0.038 & -0.021 & -6.65 & -4.88 & 1930.33 & 109.50\end{array}$

$\begin{array}{lllllllll}0.01 & 0.232 & 0.000 & -0.052 & -0.023 & -6.89 & -4.84 & 1938.07 & 109.83\end{array}$ $\begin{array}{lllllllll}0.01 & 0.232 & 0.000 & -0.052 & -0.023 & -7.34 & -5.27 & 1944.38 & 111.59\end{array}$ $\begin{array}{lllllllll}0.01 & 0.232 & 0.000 & -0.052 & -0.023 & -7.43 & -5.32 & 1951.97 & 112.07\end{array}$ $\begin{array}{lllllllll}0.00 & 0.232 & 0.000 & -0.065 & -0.015 & -8.11 & -5.73 & 1958.03 & 114.08\end{array}$ $\begin{array}{lllllllll}0.00 & 0.232 & 0.000 & -0.065 & -0.015 & -8.18 & -5.79 & 1965.39 & 114.79\end{array}$

$\begin{array}{lllllllll}0.00 & 0.232 & 0.000 & -0.065 & -0.015 & -8.25 & -5.86 & 1970.89 & 117.36\end{array}$ $\begin{array}{lllllllll}-0.01 & 0.221 & 0.000 & -0.080 & -0.007 & -8.10 & -5.39 & 1977.49 & 118.84\end{array}$ $\begin{array}{lllllllll}-0.01 & 0.221 & 0.000 & -0.080 & -0.007 & -7.73 & -5.06 & 1982.37 & 122.03\end{array}$ $\begin{array}{lllllllll}-0.01 & 0.209 & 0.000 & -0.070 & -0.004 & -6.66 & -4.42 & 1988.57 & 123.90\end{array}$ $\begin{array}{lllllllll}-0.01 & 0.198 & 0.000 & -0.071 & -0.004 & -6.37 & -4.20 & 1993.34 & 127.20\end{array}$
$-3.21 \quad 317.73$

$-3.12 \quad 323.54$

$-3.45 \quad 330.32$

$-3.30 \quad 336.33$

$-3.64 \quad 343.25$

$-3.63 \quad 349.28$

$-4.00 \quad 356.31$

$-4.00 \quad 362.47$

$-4.47 \quad 369.55$

$-4.46 \quad 375.86$

$-4.87 \quad 383.13$

$-4.84 \quad 389.61$

$-5.23 \quad 397.04$

$-5.07 \quad 403.80$

$-5.36 \quad 411.46$

$-5.10 \quad 418.45$

$\begin{array}{ll}-5.44 & 426.20\end{array}$

$-5.02 \quad 433.48$

$-5.27 \quad 441.45$

$-5.00 \quad 448.72$

$\begin{array}{ll}-5.25 & 456.83\end{array}$

$-4.91 \quad 464.29$

$-5.16 \quad 472.52$

$-4.85 \quad 480.08$

$-5.01 \quad 488.53$

$-4.54 \quad 496.38$

$-0.60 \quad 117.57$

$-0.75 \quad 116.93$

$-0.85 \quad 114.64$

$-1.16 \quad 114.14$

$-1.42 \quad 111.98$

$-1.76 \quad 111.73$

$\begin{array}{ll}-1.94 & 109.95\end{array}$

$-2.36 \quad 109.90$

$\begin{array}{ll}-2.59 & 108.35\end{array}$

$-3.09 \quad 108.50$

$-3.37 \quad 107.19$

$-3.88 \quad 107.58$

$\begin{array}{ll}-4.24 & 106.47\end{array}$

$-4.76 \quad 107.12$

$-4.85 \quad 106.54$

$-4.97 \quad 107.85$

$\begin{array}{ll}-4.76 & 107.82\end{array}$

$\begin{array}{ll}-4.88 & 109.39\end{array}$

$-4.65 \quad 109.64$

$-4.90 \quad 111.33$

$-4.83 \quad 111.68$

$-5.27 \quad 113.42$

$\begin{array}{ll}-5.31 & 113.90\end{array}$

$-5.72 \quad 115.90$

$-5.77 \quad 116.62$

$\begin{array}{ll}-5.85 & 119.19\end{array}$

$-5.34 \quad 120.70$

$\begin{array}{ll}-5.03 & 123.89\end{array}$

$\begin{array}{ll}-4.38 & 125.77\end{array}$

$-4.16 \quad 129.09$ 


\section{$Z=106(\mathrm{Sg})$}

$\begin{array}{llll}169 & 275 & 0.16 & 0.00\end{array}$

$\begin{array}{lllll}170 & 276 & 0.14 & 0.00\end{array}$

$\begin{array}{llll}171 & 277 & 0.13 & 0.00\end{array}$

$\begin{array}{llll}172 & 278 & 0.12 & 0.00\end{array}$

$173 \quad 279$

$\begin{array}{ll}0.12 \quad 0.00 \\ 0.12 & 0.00\end{array}$

$174 \quad 280$

$\begin{array}{ll}0.12 & 0.00\end{array}$

$\begin{array}{ll}175 & 281\end{array}$

0.120 .00

176282

$\begin{array}{ll}0.10 & 0.00\end{array}$

$177 \quad 283$

$0.09 \quad 0.00$

$\begin{array}{llll}178 & 284 & -0.09 & 0.00\end{array}$

$\begin{array}{llll}179 & 285 & -0.07 & 0.00\end{array}$

$\begin{array}{llll}180 & 286 & -0.05 & 0.00\end{array}$

$\begin{array}{llll}181 & 287 & -0.03 & 0.00\end{array}$

$\begin{array}{llll}182 & 288 & 0.00 & 0.00\end{array}$

$\begin{array}{llll}183 & 289 & 0.00 & 0.00\end{array}$

$\begin{array}{llll}184 & 290 & 0.00 & 0.00\end{array}$

$\begin{array}{lllll}185 & 291 & 0.00 & 0.06\end{array}$

186292

$\begin{array}{ll}0.00 & 0.07\end{array}$

$187 \quad 293$

$0.00 \quad 0.08$

$188 \quad 294$

189295

190296

191297

192298

19329

194300

195301

196302

197303

$\begin{array}{ll}0.01 & 0.09\end{array}$

$\begin{array}{llll}0.01 & 0.10 & -0.0\end{array}$

$\begin{array}{llll}0.02 & 0.10 & -0.01\end{array}$

$0.22 \quad 0.00$

$0.22 \quad 0.00$

$\begin{array}{ll}0.23 & 0.00\end{array}$

$\begin{array}{ll}0.23 & 0.00\end{array}$

$0.23 \quad 0.00$

$0.24 \quad 0.00$

$0.24 \quad 0.00$

198304

$0.25 \quad 0.00$

199305

200306

201307

202308

$\begin{array}{ll}0.25 & 0.00\end{array}$

$\begin{array}{ll}0.25 & 0.00\end{array}$

$\begin{array}{ll}0.25 & 0.00\end{array}$

0.260 .00

203309

$\begin{array}{ll}0.25 & 0.00\end{array}$

204310

205311

$0.25 \quad 0.00$

$0.25 \quad 0.00$

206312

207313

$208 \quad 314$

209315

210316

211317

212318

$213 \quad 319$

214320

$\begin{array}{ll}215 & 321\end{array}$

$216 \quad 322$

$217 \quad 323$

$218 \quad 324$

219325

$0.24 \quad 0.00$

$0.24 \quad 0.00$

$0.24 \quad 0.00$

$0.25 \quad 0.00$

$0.25 \quad 0.00$

$0.25 \quad 0.00$

0.250 .00

$0.25 \quad 0.00$

$0.24 \quad 0.00$

$\begin{array}{ll}0.23 & 0.00\end{array}$

$\begin{array}{ll}0.22 & 0.00\end{array}$

$0.21 \quad 0.00$

$\begin{array}{ll}0.21 & 0.00\end{array}$

$\begin{array}{ll}0.21 & 0.00\end{array}$

$0.21 \quad 0.00$

$0.20 \quad 0.00$

$\begin{array}{ll}221 & 327\end{array}$

$222 \quad 328$

$0.20 \quad 0.00$

$\begin{array}{ll}223 & 329\end{array}$

$0.20 \quad 0.00$

224330

0.00 $\begin{array}{lll}0.05 & 0.00 & 0.175\end{array}$

$\begin{array}{lll}0.06 & -0.01 & 0.175\end{array}$

$\begin{array}{lll}0.05 & 0.00 & 0.153\end{array}$

$\begin{array}{lll}0.04 & 0.00 & 0.141\end{array}$

$\begin{array}{lll}0.04 & 0.00 & 0.130\end{array}$

$0.05-0.01$

$0.05-0.01$

0.130

$0.05-0.02$

0.130

$\begin{array}{ll}0.05 & -0.02\end{array}$

0.130

$0.04-0.01 \quad 0.097$

$0.02-0.01-0.094$

$\begin{array}{llll}0.01 & 0.00 & -0.073\end{array}$

$\begin{array}{llll}0.01 & 0.00 & -0.053\end{array}$

$\begin{array}{llll}0.01 & 0.00 & -0.032\end{array}$

0.00

$0.00 \quad 0.000$

$0.00 \quad 0.000$

0.00

$\begin{array}{llllllll}0.000 & 0.000 & 0.000 & 0.000 & -3.58 & -3.02 & 2076.86 & 180.89 \\ 0.002 & -0.081 & 0.002 & 0.002 & -3.55 & -2.35 & 2079.41 & 186.41\end{array}$

$\begin{array}{lllllll}0.000 & -0.049 & -0.009 & -5.18 & -3.75 & 1999.51 & 129.10\end{array}$

$\begin{array}{lllllll}0.000 & -0.062 & -0.001 & -5.47 & -3.66 & 2004.19 & 132.49\end{array}$

$\begin{array}{lllllll}0.000 & -0.052 & -0.007 & -4.85 & -3.41 & 2010.35 & 134.40\end{array}$

$\begin{array}{lllllll}0.000 & -0.041 & -0.006 & -4.90 & -3.74 & 2015.24 & 137.58\end{array}$

$\begin{array}{lllllll}0.000 & -0.042 & -0.005 & -4.80 & -3.67 & 2021.37 & 139.53\end{array}$

$0.000-0.054$

$\begin{array}{lllll}0.003 & -5.45 & -4.07 & 2026.13 & 142.84\end{array}$

$0.000-0.054$

$\begin{array}{lllll}0.003 & -5.32 & -3.95 & 2032.00 & 145.04\end{array}$

$0.000-0.055$

$\begin{array}{lllll}0.013 & -5.69 & -4.26 & 2036.47 & 148.65\end{array}$

$0.000-0.057$

$\begin{array}{lllll}0.014 & -5.19 & -3.79 & 2041.77 & 151.41\end{array}$

$0.000-0.045$

$\begin{array}{lllll}0.006 & -4.82 & -3.76 & 2045.71 & 155.54\end{array}$

$0.000-0.020$

$\begin{array}{lllll}0.012 & -4.24 & -3.67 & 2051.20 & 158.13\end{array}$

$0.000-0.010$

$\begin{array}{lllll}0.001 & -4.22 & -3.73 & 2055.03 & 162.37\end{array}$

$\begin{array}{lllllll}0.000 & -0.011 & 0.001 & -3.91 & -3.37 & 2060.05 & 165.42\end{array}$

$\begin{array}{lllllll}0.000 & -0.011 & 0.000 & -4.17 & -3.57 & 2063.83 & 169.71\end{array}$

$0.000 \quad 0.000$

$\begin{array}{lllll}0.000 & -3.83 & -3.24 & 2068.69 & 172.92\end{array}$

$\begin{array}{lllllll}0.000 & 0.000 & 0.000 & -4.07 & -3.48 & 2072.32 & 177.36\end{array}$

$\begin{array}{llllllll}0.002 & -0.081 & 0.002 & 0.002 & -3.55 & -2.35 & 2079.41 & 186.41\end{array}$

$\begin{array}{llllllllll}0.00 & 0.00 & 0.002 & -0.094 & 0.002 & 0.003 & -3.14 & -1.73 & 2083.61 & 190.29\end{array}$

$\begin{array}{llllllllll}0.00 & 0.00 & 0.003 & -0.108 & 0.003 & 0.004 & -2.95 & -1.29 & 2086.20 & 195.77\end{array}$

$\begin{array}{llllllllll}0.00 & 0.01 & 0.014 & -0.121 & 0.003 & -0.005 & -2.54 & -0.62 & 2090.16 & 199.88\end{array}$

0.01

$0.015-0.135$

$\begin{array}{lllllll}0.016 & -0.003 & -2.70 & -0.51 & 2092.92 & 205.19\end{array}$

0.01

$0.026-0.135$

$\begin{array}{llllll}0.016 & -0.003 & -2.17 & -0.01 & 2096.87 & 209.31\end{array}$

$0.00-0.01$

$0.239 \quad 0.000$

$\begin{array}{llllll}0.020 & 0.012 & -0.80 & 0.01 & 2099.53 & 214.72\end{array}$

$0.00-0.01$

0.239

0.000

0.020

$\begin{array}{llllll}0.012 & -0.87 & -0.03 & 2103.85 & 218.47\end{array}$

0.250

0.000

$\begin{array}{llllll}0.010 & 0.009 & -1.21 & -0.33 & 2106.68 & 223.72\end{array}$

$0.01 \quad 0.00$

0.250

0.000

$\begin{array}{llllll}0.011 & -0.001 & -1.21 & -0.35 & 2110.80 & 227.66\end{array}$

$\begin{array}{lll}0.01 & 0.00 & 0.250\end{array}$

0.000

$\begin{array}{llllll}0.011 & -0.001 & -1.59 & -0.67 & 2113.48 & 233.06\end{array}$

$\begin{array}{lll}0.02 & 0.00 & 0.262\end{array}$

0.000

$\begin{array}{llllll}0.001 & -0.004 & -1.74 & -0.83 & 2117.57 & 237.04\end{array}$

$0.02-0.00$

\subsection{3}

$0.01 \quad 0.274$

0.000

0.00

$\begin{array}{lllllll}0.000 & -0.008 & -0.016 & -2.53 & -1.43 & 2124.14 & 246.61\end{array}$

$\begin{array}{lllllll}0.000 & -0.008 & -0.016 & -3.06 & -1.91 & 2126.66 & 252.17\end{array}$

$0.03-0.01-0.274$

$\begin{array}{lllllll}0.000 & -0.008 & -0.016 & -3.28 & -2.13 & 2130.48 & 256.41\end{array}$

$\begin{array}{lll}0.03 & 0.01 & 0.274\end{array}$

$\begin{array}{lll}0.04 & 0.01 & 0.287\end{array}$

$\begin{array}{lllllll}0.000 & -0.008 & -0.016 & -3.77 & -2.60 & 2132.83 & 262.14\end{array}$

$\begin{array}{lllllll}0.000 & -0.018 & -0.019 & -4.01 & -2.72 & 2136.39 & 266.64\end{array}$

$\begin{array}{lll}0.03 & 0.02 & 0.275\end{array}$

$\begin{array}{lllllll}0.000 & -0.007 & -0.026 & -4.26 & -3.01 & 2138.42 & 272.69\end{array}$

$\begin{array}{lll}0.04 & 0.02 & 0.276\end{array}$

$\begin{array}{lll}0.04 & 0.02 & 0.276\end{array}$

$\begin{array}{lllllll}0.000 & -0.019 & -0.029 & -4.28 & -2.91 & 2141.60 & 277.58\end{array}$

$\begin{array}{lllllll}0.000 & -0.019 & -0.029 & -4.45 & -3.10 & 2143.36 & 283.89\end{array}$

$\begin{array}{lll}0.04 & 0.02 & 0.264\end{array}$

$\begin{array}{lllllll}0.000 & -0.021 & -0.029 & -4.19 & -2.85 & 2146.25 & 289.08\end{array}$

$\begin{array}{lll}0.04 & 0.02 & 0.264\end{array}$

$\begin{array}{lllllll}0.000 & -0.021 & -0.029 & -4.41 & -3.07 & 2147.90 & 295.49\end{array}$

$\begin{array}{lll}0.04 & 0.02 & 0.264\end{array}$

$\begin{array}{lll}0.06 & 0.01 & 0.277\end{array}$

$\begin{array}{lll}0.06 & 0.01 & 0.277\end{array}$

$\begin{array}{lll}0.07 & 0.01 & 0.278\end{array}$

$\begin{array}{lllllll}0.000 & -0.021 & -0.029 & -4.22 & -2.94 & 2150.75 & 300.72\end{array}$

$\begin{array}{lllllll}0.000 & -0.044 & -0.025 & -4.84 & -3.24 & 2152.34 & 307.20\end{array}$

$\begin{array}{lllllll}0.000 & -0.044 & -0.025 & -4.72 & -3.18 & 2155.11 & 312.50\end{array}$

$\begin{array}{lllllll}0.000 & -0.056 & -0.028 & -5.41 & -3.60 & 2156.67 & 319.01\end{array}$

$\begin{array}{lll}0.07 & 0.01 & 0.278\end{array}$

$\begin{array}{lllllll}0.000 & -0.056 & -0.028 & -5.32 & -3.57 & 2159.32 & 324.43\end{array}$

$\begin{array}{lll}0.07 & 0.01 & 0.278\end{array}$

$\begin{array}{lllllll}0.000 & -0.056 & -0.028 & -5.64 & -3.84 & 2160.60 & 331.22\end{array}$

$\begin{array}{lll}0.06 & 0.02 & 0.266\end{array}$

$\begin{array}{lllllll}0.000 & -0.045 & -0.034 & -5.45 & -3.78 & 2163.08 & 336.81\end{array}$

$\begin{array}{lll}0.06 & 0.02 & 0.255\end{array}$

$\begin{array}{lllllll}0.000 & -0.047 & -0.034 & -5.80 & -4.11 & 2164.27 & 343.69\end{array}$

$\begin{array}{lll}0.05 & 0.02 & 0.243\end{array}$

$\begin{array}{lllllll}0.000 & -0.037 & -0.031 & -5.45 & -4.06 & 2166.63 & 349.41\end{array}$

$\begin{array}{lllllll}0.000 & -0.026 & -0.038 & -5.86 & -4.53 & 2167.83 & 356.28\end{array}$

$0.04 \quad 0.03 \quad 0.231$

$\begin{array}{lllllll}0.000 & -0.026 & -0.038 & -5.88 & -4.56 & 2170.12 & 362.05\end{array}$

$\begin{array}{lll}0.04 & 0.03 & 0.231\end{array}$

$\begin{array}{lllllll}0.000 & -0.026 & -0.038 & -6.30 & -4.95 & 2171.11 & 369.14\end{array}$

$\begin{array}{lll}0.04 & 0.03 & 0.231\end{array}$

$\begin{array}{lllllll}0.000 & -0.026 & -0.038 & -6.26 & -4.93 & 2173.21 & 375.11\end{array}$

$\begin{array}{lll}0.04 & 0.03 & 0.220\end{array}$

$\begin{array}{lllllll}0.000 & -0.028 & -0.038 & -6.66 & -5.33 & 2174.08 & 382.32\end{array}$

$\begin{array}{lll}0.04 & 0.03 & 0.220\end{array}$

$\begin{array}{lllllll}0.000 & -0.028 & -0.038 & -6.49 & -5.17 & 2175.91 & 388.55\end{array}$

$\begin{array}{lll}0.05 & 0.02 & 0.220\end{array}$

$\begin{array}{lllllll}0.000 & -0.041 & -0.030 & -6.81 & -5.41 & 2176.49 & 396.04\end{array}$

$\begin{array}{lllllll}0.000 & -0.043 & -0.030 & -6.58 & -5.22 & 2178.16 & 402.45\end{array}$

$-3.73130 .99$

$-3.62134 .41$

$-3.38 \quad 136.35$

$-3.72139 .54$

$-3.65141 .52$

$\begin{array}{ll}-4.03 & 144.89\end{array}$

$-3.91 \quad 147.13$

$-4.19 \quad 150.80$

$-3.71 \quad 153.62$

$-3.73157 .76$

$-3.66160 .38$

$-3.73 \quad 164.65$

$-3.37167 .76$

$\begin{array}{ll}-3.57 & 172.12\end{array}$

$-3.24 \quad 175.39$

$-3.48 \quad 179.90$

$-3.02 \quad 183.51$

$-2.33 \quad 189.13$

$-1.69193 .10$

$-1.24198 .67$

\begin{tabular}{ll}
$-0.55 \quad 202.90$ \\
\hline
\end{tabular}

$-0.42 \quad 208.32$

$\begin{array}{ll}0.08 & 212.54\end{array}$

$0.00 \quad 217.94$

$-0.03 \quad 221.80$

$-0.35 \quad 227.13$

$-0.38 \quad 231.18$

$-0.71 \quad 236.68$

$-0.86 \quad 240.78$

$-1.28 \quad 246.35$

$-1.43 \quad 250.64$

$-1.92 \quad 256.31$

$-2.13 \quad 260.70$

$-2.61 \quad 266.54$

$\begin{array}{ll}-2.70 & 271.22\end{array}$

$\begin{array}{ll}-2.96 & 277.45\end{array}$

$\begin{array}{ll}-2.81 & 282.52\end{array}$

$-3.01 \quad 288.98$ 
$Z=106(\mathrm{Sg})$

$\begin{array}{lllll}225 & 331 & 0.19 & 0.00 & 0.05\end{array}$ $\begin{array}{lllll}226 & 332 & 0.18 & 0.00 & 0.05\end{array}$ $\begin{array}{lllll}227 & 333 & 0.18 & 0.00 & 0.06\end{array}$ $\begin{array}{lllll}228 & 334 & 0.18 & 0.00 & 0.06\end{array}$ $\begin{array}{lllll}229 & 335 & 0.18 & 0.00 & 0.06\end{array}$

0.01 0.197 $0.00 \quad 0.198$ $0.00 \quad 0.198$ 0.00

$\begin{array}{llllll}230 & 336 & 0.18 & 0.00 & 0.07 & -0.01\end{array}$ $\begin{array}{llllll}231 & 337 & 0.18 & 0.00 & 0.07 & -0.01\end{array}$ $\begin{array}{llllll}232 & 338 & 0.18 & 0.00 & 0.08 & -0.02\end{array}$ $233 \quad 339$ $\begin{array}{llll}0.18 & 0.00 & 0.08 & -0.02\end{array}$
0.198
0.198

0.198

0.198 $\begin{array}{lllllll}0.000 & -0.044 & -0.020 & -6.72 & -5.27 & 2178.43 & 410.25 \\ 0.000 & -0.045 & -0.019 & -6.51 & -5.07 & 2179.95 & 416.80 \\ 0.000 & -0.058 & -0.012 & -6.95 & -5.32 & 2180.29 & 424.52 \\ 0.000 & -0.058 & -0.012 & -6.70 & -5.11 & 2181.69 & 431.21 \\ 0.000 & -0.058 & -0.012 & -6.93 & -5.34 & 2181.89 & 439.07 \\ 0.000 & -0.071 & -0.004 & -6.93 & -5.11 & 2183.13 & 445.90 \\ 0.000 & -0.071 & -0.004 & -7.18 & -5.36 & 2183.23 & 453.88 \\ 0.000 & -0.084 & 0.004 & -7.30 & -5.16 & 2184.39 & 460.79 \\ 0.000 & -0.084 & 0.004 & -7.50 & -5.36 & 2184.31 & 468.93\end{array}$
$0.01 \quad 0.262$

0.02

0.02

0.02

0.02

0.02

0.02

0.02

0.02

0.03

0.03

0.03

0.03

0.02

0.02

154261

$155 \quad 262$

156263

$157 \quad 264$

$158 \quad 265$

159266

$\begin{array}{ll}160 & 267\end{array}$

161268

$\begin{array}{ll}162 & 269\end{array}$

$163 \quad 270$

164271

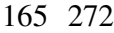

$166 \quad 273$

$\begin{array}{ll}167 & 274\end{array}$

168275

169276

$\begin{array}{ll}170 & 277\end{array}$

$\begin{array}{ll}171 & 278\end{array}$

$172 \quad 279$

$173 \quad 280$

$174 \quad 281$

$175 \quad 282$

$176 \quad 283$

$177 \quad 284$

$178 \quad 285$

179286

$\begin{array}{lllll}180 & 287 & -0.05 & 0.00 & 0.01\end{array}$

$\begin{array}{lllll}181 & 288 & 0.00 & 0.00 & 0.00\end{array}$

$\begin{array}{lllll}182 & 289 & 0.00 & 0.00 & 0.00\end{array}$

$\begin{array}{lllll}183 & 290 & 0.00 & 0.00 & 0.00\end{array}$

$\begin{array}{lllll}184 & 291 & 0.00 & 0.00 & 0.00\end{array}$

$\begin{array}{lllll}185 & 292 & 0.00 & 0.06 & 0.00\end{array}$

$\begin{array}{lllll}186 & 293 & -0.01 & 0.07 & 0.00\end{array}$ $\begin{array}{llllllll}0.262 & 0.000 & 0.002 & -0.013 & -1.73 & -0.92 & 1788.32 & 121.58\end{array}$ $\begin{array}{llllllll}0.251 & 0.000 & 0.013 & -0.021 & -2.14 & -1.21 & 1797.18 & 120.80\end{array}$ $\begin{array}{llllllll}0.251 & 0.000 & 0.013 & -0.021 & -2.38 & -1.42 & 1807.33 & 118.72\end{array}$ $\begin{array}{llllllll}0.251 & 0.000 & 0.013 & -0.021 & -2.78 & -1.85 & 1816.04 & 118.08\end{array}$ $\begin{array}{llllllll}0.240 & 0.000 & 0.011 & -0.021 & -3.03 & -2.06 & 1825.90 & 116.29\end{array}$

$\begin{array}{llllllll}0.240 & 0.000 & 0.011 & -0.021 & -3.49 & -2.45 & 1834.30 & 115.96\end{array}$ $\begin{array}{llllllll}0.251 & 0.000 & 0.001 & -0.023 & -3.85 & -2.71 & 1843.93 & 114.40\end{array}$ $\begin{array}{llllllll}0.251 & 0.000 & 0.001 & -0.023 & -4.38 & -3.19 & 1852.15 & 114.25\end{array}$ $\begin{array}{llllllll}0.252 & 0.000 & -0.011 & -0.026 & -4.88 & -3.52 & 1861.57 & 112.90\end{array}$ $\begin{array}{llllllll}0.252 & 0.000 & -0.010 & -0.036 & -5.65 & -4.05 & 1869.57 & 112.97\end{array}$ $\begin{array}{llllllll}0.252 & 0.000 & -0.010 & -0.036 & -6.10 & -4.46 & 1878.81 & 111.81\end{array}$ $\begin{array}{llllllll}0.252 & 0.000 & -0.010 & -0.036 & -6.65 & -4.95 & 1886.52 & 112.17\end{array}$ $\begin{array}{llllllll}0.253 & 0.000 & -0.022 & -0.038 & -7.02 & -5.13 & 1895.26 & 111.50\end{array}$ $\begin{array}{llllllll}0.253 & 0.000 & -0.023 & -0.029 & -6.90 & -5.23 & 1902.32 & 112.51\end{array}$ $\begin{array}{llllllll}0.254 & 0.000 & -0.035 & -0.031 & -7.03 & -5.13 & 1910.52 & 112.38\end{array}$ $\begin{array}{lllllllll}0.02 & 0.243 & 0.000 & -0.037 & -0.031 & -7.19 & -5.26 & 1917.37 & 113.60\end{array}$ $\begin{array}{lllllllll}0.01 & 0.242 & 0.000 & -0.038 & -0.021 & -6.79 & -5.07 & 1925.23 & 113.82\end{array}$ $\begin{array}{lllllllll}0.01 & 0.243 & 0.000 & -0.050 & -0.023 & -7.41 & -5.38 & 1932.01 & 115.11\end{array}$ $\begin{array}{lllllllll}0.01 & 0.232 & 0.000 & -0.052 & -0.023 & -7.39 & -5.37 & 1939.81 & 115.38\end{array}$ $\begin{array}{lllllllll}0.01 & 0.232 & 0.000 & -0.052 & -0.023 & -7.84 & -5.79 & 1946.46 & 116.80\end{array}$ $\begin{array}{lllllllll}0.00 & 0.232 & 0.000 & -0.065 & -0.015 & -8.23 & -5.93 & 1954.16 & 117.18\end{array}$ $\begin{array}{lllllllll}0.00 & 0.232 & 0.000 & -0.065 & -0.015 & -8.67 & -6.36 & 1960.58 & 118.82\end{array}$ $\begin{array}{lllllllll}0.00 & 0.232 & 0.000 & -0.065 & -0.015 & -8.74 & -6.37 & 1967.93 & 119.54\end{array}$ $\begin{array}{lllllllll}0.00 & 0.222 & 0.000 & -0.079 & -0.017 & -9.27 & -6.48 & 1973.81 & 121.74\end{array}$ $\begin{array}{lllllllll}-0.01 & 0.221 & 0.000 & -0.080 & -0.007 & -8.73 & -6.03 & 1980.45 & 123.17\end{array}$ $\begin{array}{llllllll}0.221 & 0.000 & -0.081 & 0.002 & -8.38 & -5.70 & 1985.66 & 126.03\end{array}$ $\begin{array}{llllllll}0.210 & 0.000 & -0.082 & -0.006 & -7.67 & -5.03 & 1991.87 & 127.89\end{array}$ $\begin{array}{llllllll}0.198 & 0.000 & -0.071 & -0.004 & -6.95 & -4.78 & 1996.94 & 130.89\end{array}$ $\begin{array}{llllllll}0.175 & 0.000 & -0.062 & -0.001 & -5.99 & -4.15 & 2002.96 & 132.95\end{array}$ $\begin{array}{llllllll}0.175 & 0.000 & -0.062 & -0.001 & -5.98 & -4.17 & 2008.08 & 135.89\end{array}$ $\begin{array}{llllllll}0.152 & 0.000 & -0.052 & 0.002 & -5.40 & -3.97 & 2014.31 & 137.74\end{array}$ $\begin{array}{llllllll}0.130 & 0.000 & -0.042 & -0.005 & -5.47 & -4.32 & 2019.56 & 140.55\end{array}$ $\begin{array}{llllllll}0.130 & 0.000 & -0.043 & 0.005 & -5.39 & -4.25 & 2025.70 & 142.49\end{array}$ $\begin{array}{llllllll}0.130 & 0.000 & -0.054 & 0.003 & -6.05 & -4.65 & 2030.79 & 145.47\end{array}$ $\begin{array}{lllllllll}0.130 & 0.000 & -0.054 & 0.003 & -5.91 & -4.53 & 2036.67 & 147.66\end{array}$ $\begin{array}{llllllll}0.130 & 0.000 & -0.055 & 0.013 & -6.27 & -4.83 & 2041.46 & 150.94\end{array}$ $\begin{array}{llllllll}0.130 & 0.000 & -0.055 & 0.013 & -5.84 & -4.44 & 2046.87 & 153.60\end{array}$ $\begin{array}{llllllll}0.097 & 0.000 & -0.045 & 0.006 & -5.39 & -4.32 & 2051.04 & 157.50\end{array}$ $\begin{array}{lllllllll}0.00 & -0.084 & 0.000 & -0.021 & 0.002 & -4.66 & -4.11 & 2056.43 & 160.18\end{array}$ $\begin{array}{lllllllll}0.00 & -0.073 & 0.000 & -0.010 & 0.001 & -4.70 & -4.22 & 2060.63 & 164.05\end{array}$ $\begin{array}{lllllllll}0.00 & -0.053 & 0.000 & -0.011 & 0.001 & -4.40 & -3.87 & 2065.68 & 167.08\end{array}$ $\begin{array}{lllllllll}0.00 & 0.000 & 0.000 & 0.000 & 0.000 & -4.62 & -4.02 & 2069.74 & 171.09\end{array}$ $\begin{array}{lllllllll}0.00 & 0.000 & 0.000 & 0.000 & 0.000 & -4.34 & -3.74 & 2074.67 & 174.23\end{array}$ $\begin{array}{lllllllll}0.00 & 0.000 & 0.000 & 0.000 & 0.000 & -4.56 & -3.96 & 2078.60 & 178.37\end{array}$ $\begin{array}{lllllllll}0.00 & 0.000 & 0.000 & 0.000 & 0.000 & -4.06 & -3.49 & 2083.14 & 181.90\end{array}$ $\begin{array}{lllllllll}0.00 & 0.002 & -0.081 & 0.002 & 0.002 & -4.00 & -2.80 & 2085.99 & 187.13\end{array}$ $\begin{array}{lllllllll}0.00 & -0.008 & -0.094 & 0.002 & 0.003 & -3.60 & -2.19 & 2090.21 & 190.98\end{array}$
$-5.17 \quad 419.19$

$-4.95 \quad 425.99$

$-5.20 \quad 433.95$

$-4.98 \quad 440.88$

$\begin{array}{ll}-5.23 & 448.99\end{array}$

$-4.92 \quad 456.14$

$-5.18 \quad 464.36$

$-4.86 \quad 471.65$

$-5.06 \quad 480.05$

$\begin{array}{ll}-1.01 & 124.33\end{array}$

$\begin{array}{ll}-1.29 & 123.49\end{array}$

$-1.50 \quad 121.35$

$-1.93 \quad 120.64$

$-2.12118 .81$

$-2.52 \quad 118.41$

$-2.77 \quad 116.80$

$-3.26116 .59$

$-3.58 \quad 115.20$

$-4.09 \quad 115.24$

$-4.48 \quad 114.04$

$-4.99 \quad 114.35$

$-5.14113 .67$

$-5.27114 .61$

$-5.15 \quad 114.47$

$-5.29115 .66$

$-5.10 \quad 115.85$

$-5.41 \quad 117.11$

$-5.38 \quad 117.39$

$-5.81 \quad 118.78$

$-5.93 \quad 119.15$

$-6.37120 .78$

$-6.37121 .51$

$-6.47 \quad 123.72$

$-6.00 \quad 125.15$

$-5.67128 .01$

$-4.99 \quad 129.90$

$\begin{array}{ll}-4.76 & 132.89\end{array}$

$-4.13 \quad 134.96$

$-4.15 \quad 137.92$

$\begin{array}{ll}-3.95 & 139.79\end{array}$

$-4.31 \quad 142.62$

$-4.23 \quad 144.58$

$-4.62147 .60$

$-4.49 \quad 149.83$

$\begin{array}{ll}-4.77 & 153.17\end{array}$

$-4.38 \quad 155.88$

$\begin{array}{ll}-4.29 & 159.79\end{array}$

$\begin{array}{ll}-4.11 & 162.49\end{array}$

$-4.22 \quad 166.41$

$\begin{array}{ll}-3.87 & 169.49\end{array}$

$\begin{array}{ll}-4.02 & 173.55\end{array}$

$-3.75 \quad 176.76$

$-3.96180 .96$

$-3.49 \quad 184.56$

$-2.78 \quad 189.88$

$-2.16 \quad 193.82$ 


\section{$Z=107(B h)$}

\begin{tabular}{|c|c|c|c|c|c|c|c|c|c|c|c|c|}
\hline 94 & 00 & 8 & & & & & & & 5.00 & 71 & & \\
\hline 95 & 0.00 & .08 & 0.00 & 000 & & -0.108 & & 004 & -2.66 & -1.02 & & \\
\hline 96 & 0.64 & 00 & 0.01 & -0.02 & .630 & 0.000 & 54 & 0.026 & -3.13 & 6.96 & & \\
\hline 97 & 0.01 & 10 & 0.00 & 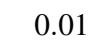 & & & & & -2.44 & & & \\
\hline 08 & 02 & 2. & -0.01 & 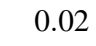 & 26 & & & -0.012 & -2.78 & -0.20 & & \\
\hline 299 & & & & & & & & & & & & \\
\hline 00 & 23 & 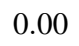 & 1 & 0.01 & & & & & -1.26 & & & \\
\hline 01 & 23 & 00 & 2 & 0.01 & & & 03 & & -1.31 & & & \\
\hline 02 & 24 & 00 & 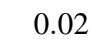 & 000 & & & & & -1.67 & & & \\
\hline 03 & 24 & 00 & 2 & 0.0 & 62 & & & -0 . & -1.79 & 87 & & 36.53 \\
\hline 04 & 24 & 00 & 2 & 0.0 & & & & & -2.24 & & & \\
\hline 05 & 4 & 00 & 3 & 0 & & & & -0 & -2.50 & & & \\
\hline 06 & 25 & 00 & 3 & 0.0 & & & 08 & -0 & -3.11 & -2.01 & & \\
\hline 07 & 5 & 00 & & & & & & & -3.33 & & & \\
\hline 08 & 6 & 00 & 4 & 1 & & & 18 & 19 & -4.00 & -2.73 & & 260.62 \\
\hline 309 & & 00 & & & & & 5 & - & & & & \\
\hline 10 & & 00 & & & & & 20 & & -4 & & & \\
\hline 2 & & 0 & & 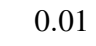 & & & 0 & -0 & -4 & & & \\
\hline 12 & & 0 & & & & & & & -4 & & & \\
\hline 13 & 5 & 00 & C & c & & & 32 & & -4.38 & & & \\
\hline & & 000 & & & & & 4 & & 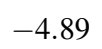 & & & \\
\hline- & & 00 & & & & & 44 & & -4 & & & \\
\hline 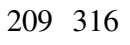 & & 0 & & & & & 4 & - & 5 & & & \\
\hline 17 & & 0 & & & & & & & & & & \\
\hline & & & & & & & & & & & & \\
\hline & & 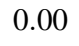 & & & & & & & & & & \\
\hline P & & & & & & & 58 & & & & & \\
\hline 21 & 4 & 00 & & & & & 58 & 27 & -5 & & & \\
\hline 22 & 4 & 00 & & & & & 58 & & & & & \\
\hline 23 & 3 & .00 & 0 & 0 & & & -0.047 & -0 & -5.95 & & & $3^{2}$ \\
\hline 24 & 2 & 0 & & & & & 0 & & -6 & & & 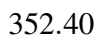 \\
\hline 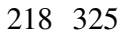 & & & & & & & & & & & & \\
\hline 20 & & & & & & & 39 & & 2 & & & \\
\hline בר & & & & & & & -0 . & & & & & \\
\hline 328 & 0.20 & 0.00 & 0.04 & 0.1 & 20 & & -0.028 & -0.038 & -6.81 & & & 311.0 \\
\hline 329 & 20 & 00 & 0.05 & 0.02 & 20 & 0 & -0.041 & -0.030 & -6.70 & 34 & & 4.05 \\
\hline 200 & & & & & & & & & & & & \\
\hline I & & 0 & & & & & & & & & & \\
\hline 32 & & 0 & & & & & -0 & & & & & 40 \\
\hline 333 & 0.18 & 0.00 & 0.0 & 0.0 & & & -0.045 & -0.019 & -6.81 & & & 411.60 \\
\hline 234 & 0.18 & 000 & 006 & 000 & & & -0. & 2 & -7 & - & 99 & 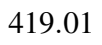 \\
\hline & & & & & & & & & & & & \\
\hline ( & & .0 & & & & & & & & & & 433.2 \\
\hline ( & & 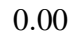 & & & & & -0 & & & & & 440.0 \\
\hline - & 0.18 & 0.00 & 0.0 & - & & & -0.071 & -0.004 & -7.58 & -5.75 & 2196.62 & 447.7 \\
\hline (2) & .1 & .00 & .00 & 0.02 & .198 & 0.000 & -0.084 & 0.00 & 7 & 5 & 2191.02 & 454.6 \\
\hline
\end{tabular}

$Z=108(\mathrm{Hs})$

\begin{tabular}{|c|c|c|c|c|c|c|c|c|c|c|c|c|c|c|}
\hline $142 \quad 250$ & 0.23 & 0.00 & 0.02 & 0.01 & 0.251 & 0.000 & -0.001 & -0.013 & -1.79 & -1.05 & 1806.69 & 126.65 & -1.13 & 129.54 \\
\hline $143 \quad 251$ & 0.22 & 0.00 & 0.01 & 0.02 & 0.240 & 0.000 & 0.011 & -0.021 & -2.26 & -1.36 & 1815.32 & 126.09 & -1.43 & 128.92 \\
\hline $144 \quad 252$ & 0.22 & 0.00 & 0.01 & 0.02 & 0.240 & 0.000 & 0.011 & -0.021 & -2.53 & -1.60 & 1825.57 & 123.91 & -1.66 & 126.69 \\
\hline $45 \quad 253$ & 0.22 & 0.00 & 0.01 & 0.02 & 0.240 & 0.000 & 0.011 & -0.021 & -2.98 & -2.06 & 1834.07 & 123.48 & -2.12 & 126.18 \\
\hline $146 \quad 254$ & 0.22 & 0.00 & 0.02 & 0.02 & 0.240 & 0.000 & -0.001 & -0.023 & -3.36 & -2.33 & 1844.07 & 121.55 & -2.38 & 124.20 \\
\hline 255 & 0.22 & 0.00 & 0.02 & 0.02 & 0.240 & 0.000 & -0.001 & -0.023 & -3.89 & -2.81 & 1852.32 & 121.37 & -2.86 & 123.96 \\
\hline 256 & 0.22 & 0.00 & 0.03 & 0.02 & 0.241 & 0.000 & -0.013 & -0.026 & -4.39 & -3.16 & 1862.13 & 119.63 & -3.21 & 122.18 \\
\hline $9 \quad 257$ & 0.23 & 0.00 & 0.04 & 0.02 & 0.253 & 0.000 & -0.023 & -0.029 & -5.12 & -3.67 & 1870.14 & 119.69 & -3.72 & 122.17 \\
\hline 258 & 0.23 & 0.00 & 0.04 & 0.02 & 0.253 & 0.000 & -0.023 & -0.029 & -5.57 & -4.07 & 1879.72 & 118.18 & -4.11 & 120.63 \\
\hline 1259 & 0.23 & 0.00 & 0.04 & 0.02 & 0.253 & 0.000 & -0.023 & -0.029 & -6.11 & -4.56 & 1887.46 & 118.52 & -4.61 & 120.91 \\
\hline
\end{tabular}

$-1.67 \quad 199.10$
-0.98

$\begin{array}{ll}-0.98 & 203.31\end{array}$

$\begin{array}{ll}6.56 & 215.82\end{array}$

$-0.25 \quad 212.71$

$-0.08 \quad 218.04$

$-0.14 \quad 221.86$

$-0.46 \quad 226.88$

$\begin{array}{ll}-0.46 \quad 230.95 \\ -0.81 & 236.11\end{array}$

$-0.81 \quad 236.11$

$-0.91 \quad 240.24$

$\begin{array}{ll}-1.39 & 245.45\end{array}$

$\begin{array}{ll}-1.52 \quad 249.73 \\ -1.03 & 255.07\end{array}$

$\begin{array}{ll}-2.03 & 255.07\end{array}$

$-2.24 \quad 259.45$

$-2.74 \quad 264.97$

$\begin{array}{ll}-2.83 & 269.62\end{array}$

$-3.04 \quad 275.61$

$-2.94 \quad 280.61$

$\begin{array}{ll}-3.13 & 286.77\end{array}$

$-2.94 \quad 292.04$

$-3.18 \quad 298.30$

$-3.08 \quad 303.64$

$-3.40 \quad 309.98$

$-3.35 \quad 315.42$

$\begin{array}{ll}-3.69 & 321.89\end{array}$

$-3.62 \quad 327.51$

$-4.01 \quad 334.08$

$\begin{array}{ll}-3.92 \quad 339.87 \\ -4.17 & 346.73\end{array}$

$-4.17 \quad 346.73$

$-4.12 \quad 352.63$

$-4.49 \quad 359.51$

$-4.44 \quad 365.56$

$-4.86 \quad 372.52$

$-4.82 \quad 378.71$

$-5.22 \quad 385.84$

$\begin{array}{ll}-5.16 & 392.20\end{array}$

$-5.49 \quad 399.54$

$-5.45 \quad 413.80$

$-5.27 \quad 420.55$

$-5.56 \quad 428.20$

$-5.34 \quad 435.12$

$-5.57 \quad 442.96$

$\begin{array}{ll}-5.33 & 450.04\end{array}$

$-5.58 \quad 457.98$

$-5.31465 .23$

$\begin{array}{ll}-1.13 & 129.54\end{array}$

$-1.43 \quad 128.92$

$\begin{array}{ll}66 & 126.69\end{array}$

126.18

$-2.86123 .96$

$-3.21 \quad 122.18$

$-4.11 \quad 120.63$
$-5.12406 .34$ 


\section{$Z=108(\mathrm{Hs})$}

\begin{tabular}{|c|c|c|c|c|c|c|c|c|c|c|c|c|c|c|c|c|}
\hline $52 \quad 260$ & 0.23 & 0.00 & 0.04 & 0.02 & 0.253 & 0.000 & -0.023 & -0.029 & -6.28 & -4.73 & 1896.55 & 117.50 & & & -4.76 & 119.86 \\
\hline 153261 & 0.23 & 0.00 & 0.05 & 0.02 & 0.254 & 0.000 & -0.035 & -0.031 & -6.73 & -4.92 & 1903.73 & 118.39 & & & -4.95 & 120.71 \\
\hline 4262 & 0.22 & 0.00 & 0.05 & 0.02 & 0.243 & 0.000 & -0.037 & -0.031 & -6.67 & -4.85 & 1912.32 & 117.88 & & & -4.86 & 120.18 \\
\hline 263 & 0.22 & 0.00 & 0.05 & 0.01 & 0.242 & 0.000 & -0.038 & -0.021 & -6.65 & -4.99 & 1919.21 & 119.06 & & & -5.03 & 121.30 \\
\hline 6264 & 0.22 & 0.00 & 0.06 & 0.01 & 0.243 & 0.000 & -0.050 & -0.023 & -6.83 & -4.90 & 1927.52 & 118.82 & 119.60 & 0.044 & -4.92 & 121.05 \\
\hline 265 & 0.22 & 0.00 & 0.06 & 0.01 & 0.243 & 0.000 & -0.050 & -0.023 & -7.16 & -5.22 & 1934.33 & 120.08 & & & -5.24 & 122.28 \\
\hline 266 & 0.21 & 0.00 & 0.06 & 0.01 & 0.232 & 0.000 & -0.052 & -0.023 & -7.19 & -5.25 & 1942.52 & 119.96 & & & -5.26 & 122.15 \\
\hline 267 & 0.21 & 0.00 & 0.07 & 0.00 & 0.232 & 0.000 & -0.065 & -0.015 & -7.94 & -5.70 & 1949.22 & 121.32 & & & -5.72 & 123.49 \\
\hline 268 & 0.21 & 0.00 & 0.07 & 0.00 & 0.232 & 0.000 & -0.065 & -0.015 & -8.13 & -5.90 & 1957.33 & 121.29 & & & -5.90 & 123.44 \\
\hline 12269 & 0.21 & 0.00 & 0.07 & 0.00 & 0.232 & 0.000 & -0.065 & -0.015 & -8.57 & -6.27 & 1963.73 & 122.96 & & & -6.28 & 125.10 \\
\hline 270 & 0.20 & 0.00 & 0.08 & 0.00 & 0.222 & 0.000 & -0.079 & -0.017 & -9.07 & -6.35 & 1971.48 & 123.28 & & & -6.32 & 125.44 \\
\hline 3271 & 0.20 & 0.00 & 0.08 & -0.01 & 0.221 & 0.000 & -0.080 & -0.007 & -9.24 & -6.56 & 1977.48 & 125.35 & & & -6.55 & 127.49 \\
\hline 4272 & 0.20 & 0.00 & 0.08 & -0.01 & 0.221 & 0.000 & -0.080 & -0.007 & -8.76 & -6.12 & 1984.48 & 126.43 & & & -6.09 & 128.57 \\
\hline 5273 & 0.20 & 0.00 & 0.08 & -0.02 & 0.221 & 0.000 & -0.081 & 0.002 & -8.42 & -5.79 & 1989.72 & 129.26 & & & -5.76 & 131.40 \\
\hline $6 \quad 274$ & 0.19 & 0.00 & 0.08 & -0.02 & 0.209 & 0.000 & -0.083 & 0.003 & -7.75 & -5.11 & 1996.24 & 130.80 & & & -5.06 & 132.97 \\
\hline 275 & 0.18 & 0.00 & 0.08 & -0.02 & 0.198 & 0.000 & -0.084 & 0.004 & -7.41 & -4.84 & 2001.32 & 133.80 & & & -4.79 & 135.97 \\
\hline $8 \quad 276$ & 0.16 & 0.00 & 0.06 & -0.01 & 0.175 & 0.000 & -0.062 & -0.001 & -6.01 & -4.22 & 2007.68 & 135.51 & & & -4.19 & 137.67 \\
\hline 9277 & 0.15 & 0.00 & 0.06 & -0.01 & 0.164 & 0.000 & -0.063 & 0.000 & -6.05 & -4.29 & 2012.89 & 138.38 & & & -4.26 & 140.54 \\
\hline $\begin{array}{ll}0 & 278\end{array}$ & 0.12 & 0.00 & 0.04 & 0.00 & 0.130 & 0.000 & -0.042 & -0.005 & -5.32 & -4.18 & 2019.54 & 139.79 & & & -4.17 & 141.96 \\
\hline 1279 & 0.12 & 0.00 & 0.04 & 0.00 & 0.130 & 0.000 & -0.042 & -0.005 & -5.74 & -4.59 & 2024.87 & 142.53 & & & -4.59 & 144.71 \\
\hline 280 & 0.12 & 0.00 & 0.04 & -0.01 & 0.130 & 0.000 & -0.043 & 0.005 & -5.68 & -4.54 & 2031.37 & 144.11 & & & -4.53 & 6.32 \\
\hline 281 & 0.12 & 0.00 & 0.05 & -0.01 & 0.130 & 0.000 & -0.054 & 0.003 & -6.35 & -4.95 & 2036.49 & 147.06 & & & -4.92 & 149.31 \\
\hline 282 & 0.12 & 0.00 & 0.05 & -0.02 & 0.130 & 0.000 & -0.055 & 0.013 & -6.31 & -4.86 & 2042.73 & & & & -4.80 & 151.19 \\
\hline 283 & 0.10 & 0.00 & 0.05 & -0.02 & 0.108 & 0.000 & -0.057 & 0.014 & -6.59 & -5.13 & 2047.51 & 152.18 & & & -5.07 & 154.52 \\
\hline 6284 & 0.09 & 0.00 & 0.05 & -0.02 & 0.097 & 0.000 & -0.057 & 0.015 & -6.28 & -4.84 & 2053.34 & 154.42 & & & -4.77 & 156.80 \\
\hline 285 & 0.08 & 0.00 & 0.04 & -0.02 & 0.086 & 0.000 & -0.046 & 0.016 & -6.04 & -4.88 & 2057.69 & 158.14 & & & -4.82 & 0.55 \\
\hline 286 & -0.08 & 0.00 & 0.01 & -0.01 & -0.084 & 0.000 & -0.009 & 0.010 & -5.13 & -4.63 & 2063.36 & 160.54 & & & -4.62 & 162.94 \\
\hline 287 & -0.05 & 0.00 & 0.01 & 0.00 & -0.053 & 0.000 & -0.011 & 0.001 & -5.28 & -4.74 & 2067.58 & 164.39 & & & -4.74 & 166.83 \\
\hline 288 & -0.04 & 0.00 & 0.01 & 0.00 & -0.042 & 0.000 & -0.011 & 0.001 & -5.04 & -4.45 & 2073.01 & 167.03 & & & -4.45 & 169.51 \\
\hline 1289 & -0.03 & 0.00 & 0.01 & 0.00 & -0.032 & 0.000 & -0.011 & 0.000 & -5.31 & -4.67 & 2077.16 & 170.96 & & & -4.67 & 17 \\
\hline 290 & 0.00 & 0.00 & 0.00 & 0.00 & 0.000 & 0.000 & 0.000 & 0.000 & -4.95 & -4.33 & 2082.34 & 173.85 & & & -4.33 & 176.43 \\
\hline 291 & 0.00 & 0.00 & 0.00 & 0.00 & 0.000 & 0.000 & 0.000 & 0.000 & -5.16 & -4.53 & 2086.27 & 177.99 & & & -4.53 & 180.64 \\
\hline 292 & 0.00 & 0.00 & 0.00 & 0.00 & 0.000 & 0.000 & 0.000 & 0.000 & -4.64 & -4.04 & 2091.11 & & & & -4.04 & 183.93 \\
\hline 293 & 0.00 & 0.04 & 0.00 & 0.00 & 0.001 & -0.054 & 0.001 & 0.001 & -4.00 & -3.16 & 2093.79 & 186.61 & & & -3.16 & 189.40 \\
\hline 294 & 0.00 & 0.04 & 0.00 & 0.00 & 0.001 & -0.054 & 0.001 & 0.001 & -3.31 & -2.51 & 2098.28 & 190.20 & & & -2.50 & 193.05 \\
\hline 295 & 0.00 & 0.07 & 0.00 & 0.00 & 0.002 & -0.094 & 0.002 & 0.003 & -3.30 & -1.91 & 2101.05 & 195.50 & & & -1.88 & 198.45 \\
\hline 296 & 0.00 & 0.08 & 0.00 & 0.00 & 0.003 & -0.108 & 0.003 & 0.004 & -2.81 & -1.17 & 2105.27 & 199.35 & & & -1.12 & 202.39 \\
\hline 297 & -0.64 & 0.00 & -0.01 & -0.02 & -0.630 & 0.000 & 0.154 & -0.026 & -2.85 & 6.93 & 2100.36 & 212.33 & & & 6.58 & 215.06 \\
\hline $0 \quad 298$ & -0.64 & 0.00 & -0.01 & -0.03 & -0.629 & 0.000 & 0.156 & -0.019 & -2.23 & 7.33 & 2104.73 & 216.03 & & & 7.05 & 218.91 \\
\hline 1299 & 0.02 & 0.10 & -0.01 & 0.01 & 0.026 & -0.135 & 0.016 & -0.003 & -2.37 & -0.18 & 2115.26 & 213.57 & & & -0.10 & 216.90 \\
\hline 2300 & 0.23 & 0.00 & 0.01 & -0.01 & 0.250 & 0.000 & 0.010 & 0.009 & -0.51 & 0.17 & 2119.50 & 217.40 & & & 0.16 & 220.73 \\
\hline 301 & 0.23 & 0.00 & 0.01 & -0.01 & 0.250 & 0.000 & 0.010 & 0.009 & -0.88 & -0.14 & 2122.66 & 222.32 & & & -0.16 & 225.73 \\
\hline 94302 & 0.23 & 0.00 & 0.01 & -0.01 & 0.250 & 0.000 & 0.010 & 0.009 & -0.92 & -0.15 & 2127.09 & 225.96 & & & -0.17 & 229.47 \\
\hline 303 & 0.23 & 0.00 & 0.02 & -0.01 & 0.251 & 0.000 & -0.003 & 0.006 & -1.31 & -0.47 & 2130.09 & 231.02 & & & -0.50 & 234.63 \\
\hline 196304 & 0.24 & 0.00 & 0.02 & 0.00 & 0.262 & 0.000 & 0.001 & -0.004 & -1.39 & -0.62 & 2134.48 & 234.71 & & & -0.65 & 238.41 \\
\hline $97 \quad 305$ & 0.24 & 0.00 & 0.02 & 0.00 & 0.262 & 0.000 & 0.001 & -0.004 & -1.85 & -1.03 & 2137.41 & 239.85 & & & -1.07 & 243.65 \\
\hline 98306 & 0.25 & 0.00 & 0.03 & 0.00 & 0.274 & 0.000 & -0.009 & -0.006 & -2.10 & -1.18 & 2141.64 & 243.69 & & & -1.21 & 247.62 \\
\hline 199307 & 0.25 & 0.00 & 0.03 & 0.00 & 0.274 & 0.000 & -0.009 & -0.006 & -2.61 & -1.66 & 2144.47 & 248.93 & & & -1.70 & 252.97 \\
\hline 0308 & 0.26 & 0.00 & 0.03 & 0.01 & 0.286 & 0.000 & -0.006 & -0.016 & -2.91 & -1.91 & 2148.64 & 252.84 & & & -1.92 & 257.02 \\
\hline 201309 & 0.26 & 0.00 & 0.03 & 0.01 & 0.286 & 0.000 & -0.006 & -0.016 & -3.42 & -2.40 & 2151.31 & 258.23 & & & -2.41 & 262.53 \\
\hline 2310 & 0.26 & 0.00 & 0.04 & 0.01 & 0.287 & 0.000 & -0.018 & -0.019 & -3.67 & -2.51 & 2155.18 & 262.44 & & & -2.50 & 266.90 \\
\hline 311 & 0.26 & 0.00 & 0.04 & 0.01 & 0.287 & 0.000 & -0.018 & -0.019 & -3.87 & -2.72 & 2157.43 & 268.26 & & & -2.72 & 272.84 \\
\hline 4312 & 0.25 & 0.00 & 0.04 & 0.01 & 0.275 & 0.000 & -0.020 & -0.019 & -3.74 & -2.60 & 2160.89 & 272.87 & & & -2.58 & 277.60 \\
\hline 313 & 0.25 & 0.00 & 0.05 & 0.01 & 0.276 & 0.000 & -0.032 & -0.022 & -4.16 & -2.80 & 2162.98 & 278.85 & & & -2.76 & 283.74 \\
\hline 06314 & 0.25 & 0.00 & 0.05 & 0.01 & 0.276 & 0.000 & -0.032 & -0.022 & -3.95 & -2.63 & 2166.24 & 283.66 & & & -2.58 & 288.70 \\
\hline 315 & 0.25 & 0.00 & 0.06 & 0.01 & 0.277 & 0.000 & -0.044 & -0.025 & -4.48 & -2.91 & 2168.25 & 289.72 & & & -2.83 & 294.94 \\
\hline 316 & 0.25 & 0.00 & 0.06 & 0.01 & 0.277 & 0.000 & -0.044 & -0.025 & -4.34 & -2.82 & 2171.44 & 294.60 & & & -2.72 & 299.98 \\
\hline
\end{tabular}


$Z=108(\mathrm{Hs})$

$\begin{array}{lllll}209 & 317 & 0.25 & 0.00 & 0.07\end{array}$ $\begin{array}{lllll}210 & 318 & 0.25 & 0.00 & 0.07\end{array}$ $\begin{array}{lllll}211 & 319 & 0.25 & 0.00 & 0.08\end{array}$ $\begin{array}{lllll}212 & 320 & 0.25 & 0.00 & 0.08\end{array}$ $\begin{array}{lllll}213 & 321 & 0.25 & 0.00 & 0.08\end{array}$ $\begin{array}{llllll}214 & 322 & 0.24 & 0.00 & 0.07\end{array}$ $\begin{array}{lllll}215 & 323 & 0.23 & 0.00 & 0.07\end{array}$ $\begin{array}{lllll}216 & 324 & 0.22 & 0.00 & 0.06\end{array}$ $\begin{array}{lllll}217 & 325 & 0.22 & 0.00 & 0.06\end{array}$ $\begin{array}{lllll}218 & 326 & 0.21 & 0.00 & 0.05\end{array}$ $\begin{array}{llllll}219 & 327 & 0.21 & 0.00 & 0.05\end{array}$ $\begin{array}{lllll}220 & 328 & 0.20 & 0.00 & 0.05\end{array}$ $\begin{array}{lllll}221 & 329 & 0.20 & 0.00 & 0.05\end{array}$ $\begin{array}{lllll}222 & 330 & 0.20 & 0.00 & 0.05\end{array}$ $\begin{array}{lllll}223 & 331 & 0.19 & 0.00 & 0.05\end{array}$ $\begin{array}{llllll}224 & 332 & 0.19 & 0.00 & 0.05\end{array}$ $\begin{array}{lllll}225 & 333 & 0.18 & 0.00 & 0.05\end{array}$ $\begin{array}{lllll}226 & 334 & 0.18 & 0.00 & 0.05\end{array}$ $\begin{array}{lllll}227 & 335 & 0.18 & 0.00 & 0.06\end{array}$ $\begin{array}{lllll}228 & 336 & 0.00 & 0.00 & 0.00\end{array}$ $\begin{array}{lllll}229 & 337 & -0.01 & 0.00 & 0.00\end{array}$ $\begin{array}{lllll}230 & 338 & 0.00 & 0.00 & 0.00\end{array}$ $\begin{array}{lllll}231 & 339 & 0.01 & 0.00 & 0.00\end{array}$ $\begin{array}{lllllllll}0.00 & 0.278 & 0.000 & -0.057 & -0.018 & -4.88 & -3.12 & 2173.32 & 300.79\end{array}$ $\begin{array}{lllllllll}0.00 & 0.278 & 0.000 & -0.057 & -0.018 & -4.80 & -3.08 & 2176.41 & 305.77\end{array}$ $\begin{array}{lllllllll}0.00 & 0.279 & 0.000 & -0.069 & -0.021 & -5.57 & -3.51 & 2178.28 & 311.98\end{array}$ $\begin{array}{lllllllll}0.00 & 0.279 & 0.000 & -0.069 & -0.021 & -5.49 & -3.48 & 2181.23 & 317.09\end{array}$ $\begin{array}{lllllllll}0.01 & 0.279 & 0.000 & -0.068 & -0.031 & -5.99 & -3.90 & 2182.95 & 323.45\end{array}$ $\begin{array}{lllllllll}0.01 & 0.267 & 0.000 & -0.058 & -0.027 & -5.47 & -3.77 & 2185.65 & 328.82\end{array}$ $\begin{array}{lllllllll}0.01 & 0.255 & 0.000 & -0.060 & -0.026 & -5.81 & -4.00 & 2187.04 & 335.50\end{array}$ $\begin{array}{lllllllll}0.02 & 0.244 & 0.000 & -0.049 & -0.033 & -5.57 & -3.99 & 2189.72 & 340.90\end{array}$ $\begin{array}{lllllllll}0.02 & 0.244 & 0.000 & -0.049 & -0.033 & -5.95 & -4.36 & 2191.11 & 347.58\end{array}$ $\begin{array}{lllllllll}0.02 & 0.231 & 0.000 & -0.039 & -0.030 & -5.60 & -4.28 & 2193.57 & 353.18\end{array}$ $\begin{array}{lllllllll}0.02 & 0.231 & 0.000 & -0.039 & -0.030 & -6.02 & -4.69 & 2194.87 & 359.96\end{array}$ $\begin{array}{lllllllll}0.02 & 0.220 & 0.000 & -0.041 & -0.030 & -6.00 & -4.67 & 2197.25 & 365.64\end{array}$ $\begin{array}{lllllllll}0.02 & 0.220 & 0.000 & -0.041 & -0.030 & -6.43 & -5.08 & 2198.42 & 372.55\end{array}$ $\begin{array}{lllllllll}0.02 & 0.220 & 0.000 & -0.041 & -0.030 & -6.35 & -5.04 & 2200.66 & 378.39\end{array}$ $\begin{array}{lllllllll}0.02 & 0.209 & 0.000 & -0.043 & -0.030 & -6.70 & -5.20 & 2201.43 & 385.68\end{array}$ $\begin{array}{lllllllll}0.01 & 0.208 & 0.000 & -0.044 & -0.020 & -6.38 & -5.00 & 2203.37 & 391.82\end{array}$ $\begin{array}{lllllllll}0.01 & 0.197 & 0.000 & -0.045 & -0.019 & -6.77 & -5.34 & 2204.20 & 399.06\end{array}$ $\begin{array}{lllllllll}0.00 & 0.197 & 0.000 & -0.046 & -0.009 & -6.53 & -5.18 & 2206.05 & 405.27\end{array}$ $\begin{array}{lllllllll}0.00 & 0.198 & 0.000 & -0.058 & -0.012 & -7.14 & -5.54 & 2206.77 & 412.62\end{array}$ $\begin{array}{lllllllll}0.00 & 0.000 & 0.000 & 0.000 & 0.000 & -6.41 & -5.87 & 2208.98 & 418.49\end{array}$ $\begin{array}{lllllllll}0.00 & -0.011 & 0.000 & 0.000 & 0.000 & -6.56 & -6.02 & 2209.37 & 426.17\end{array}$ $\begin{array}{lllllllll}0.00 & 0.000 & 0.000 & 0.000 & 0.000 & -6.36 & -5.85 & 2210.95 & 432.66\end{array}$ $\begin{array}{lllllllll}0.00 & 0.011 & 0.000 & 0.000 & 0.000 & -6.39 & -5.90 & 2211.12 & 440.57\end{array}$

$\begin{array}{ll}-3.03 & 306.32 \\ -2.98 & 311.47 \\ -3.37 & 317.87 \\ -3.33 & 323.17 \\ -3.70 & 329.75 \\ -3.60 & 335.25 \\ -3.84 & 342.11 \\ -3.77 & 347.74 \\ -4.15 & 354.60 \\ -4.10 & 360.36 \\ -4.52 & 367.32 \\ -4.48 & 373.21 \\ -4.90 & 380.31 \\ -4.86 & 386.36 \\ -5.02 & 393.86 \\ -4.89 & 400.13 \\ -5.23 & 407.59 \\ -5.12 & 413.98 \\ -5.43 & 421.60 \\ -5.87 & 427.58 \\ -6.02 & 435.49 \\ -5.85 & 442.22 \\ -5.90 & 450.37\end{array}$

\section{$Z=109$ (Mt)}

$\begin{array}{lllll}144 & 253 & 0.22 & 0.00 & 0.02\end{array}$ $\begin{array}{lllll}145 & 254 & 0.22 & 0.00 & 0.02\end{array}$ $\begin{array}{lllll}146 & 255 & 0.22 & 0.00 & 0.03\end{array}$ $\begin{array}{lllll}147 & 256 & 0.22 & 0.00 & 0.03\end{array}$ $\begin{array}{lllll}148 & 257 & 0.22 & 0.00 & 0.03\end{array}$

0.01

$\begin{array}{llllllll}0.240 & 0.000 & -0.002 & -0.013 & -2.33 & -1.55 & 1823.24 & 133.52\end{array}$ $\begin{array}{lllllllll}0.01 & 0.240 & 0.000 & -0.002 & -0.013 & -2.78 & -1.96 & 1832.05 & 132.79\end{array}$ $\begin{array}{lllllllll}0.01 & 0.241 & 0.000 & -0.014 & -0.016 & -3.22 & -2.35 & 1842.20 & 130.71\end{array}$ $\begin{array}{lllllllll}0.01 & 0.241 & 0.000 & -0.014 & -0.016 & -3.72 & -2.78 & 1850.77 & 130.21\end{array}$ $\begin{array}{lllllllll}0.01 & 0.241 & 0.000 & -0.014 & -0.016 & -4.12 & -3.13 & 1860.61 & 128.44\end{array}$ $\begin{array}{lllll}149 & 258 & 0.22 & 0.00 & 0.04\end{array}$ $\begin{array}{lllll}150 & 259 & 0.22 & 0.00 & 0.04\end{array}$ $\begin{array}{lllll}151 & 260 & 0.22 & 0.00 & 0.04\end{array}$ $\begin{array}{lllll}152 & 261 & 0.22 & 0.00 & 0.04\end{array}$ $\begin{array}{lllll}153 & 262 & 0.22 & 0.00 & 0.05\end{array}$

$\begin{array}{lllll}154 & 263 & 0.22 & 0.00 & 0.05\end{array}$ $\begin{array}{lllll}155 & 264 & 0.22 & 0.00 & 0.06\end{array}$ $\begin{array}{lllll}156 & 265 & 0.22 & 0.00 & 0.06\end{array}$ $\begin{array}{lllll}157 & 266 & 0.21 & 0.00 & 0.06\end{array}$ $\begin{array}{lllll}158 & 267 & 0.21 & 0.00 & 0.07\end{array}$ 0.01 0.02

\subsection{2}

0.02

0.02

0.01

0.01

0.00

$$
0.00
$$

0.00

159268 $\begin{array}{llll}0.21 & 0.00 & 0.07\end{array}$ 0.00 160269 $\begin{array}{ll}161 & 270\end{array}$

$\begin{array}{ll}162 & 271\end{array}$

163272

$\begin{array}{lll}0.21 & 0.00 & 0.07\end{array}$

0.00 $\begin{array}{lllll}0.21 & 0.00 & 0.07 & -0.01\end{array}$ $\begin{array}{lllll}0.20 & 0.00 & 0.08 & -0.01\end{array}$

164273 $165 \quad 274$ 166275 167276

168277

169278

$\begin{array}{ll}170 & 279\end{array}$

171280

$\begin{array}{ll}172 & 281\end{array}$

173282 $\begin{array}{lll}0.20 & 0.00 & 0.08\end{array}$

$\begin{array}{llll}0.20 & 0.00 & 0.08 & -0.01\end{array}$ $\begin{array}{lllll}0.20 & 0.00 & 0.09 & -0.02\end{array}$ $\begin{array}{lllll}0.19 & 0.00 & 0.08 & -0.02\end{array}$ $\begin{array}{lllll}0.18 & 0.00 & 0.08 & -0.02\end{array}$ $\begin{array}{lllll}0.16 & 0.00 & 0.06 & -0.01\end{array}$

$\begin{array}{llll}0.14 & 0.00 & 0.05 & 0.00\end{array}$ $\begin{array}{rrrr}0.12 & 0.00 & 0.04 & 0.00\end{array}$ $\begin{array}{lllll}0.12 & 0.00 & 0.04 & -0.01\end{array}$ $\begin{array}{llll}0.12 & 0.00 & 0.05 & -0.01\end{array}$ $\begin{array}{llllll}175 & 284 & 0.10 & 0.00 & 0.05 & -0.02\end{array}$ 176285 $\begin{array}{llllllll}0.241 & 0.000 & -0.026 & -0.019 & -4.83 & -3.62 & 1868.95 & 128.17\end{array}$ $\begin{array}{llllllll}0.242 & 0.000 & -0.025 & -0.028 & -5.48 & -4.04 & 1878.60 & 126.60\end{array}$ $\begin{array}{llllllll}0.242 & 0.000 & -0.025 & -0.028 & -6.03 & -4.54 & 1886.70 & 126.57\end{array}$ $\begin{array}{llllllll}0.242 & 0.000 & -0.025 & -0.028 & -6.22 & -4.73 & 1895.83 & 125.51\end{array}$ $\begin{array}{llllllll}0.243 & 0.000 & -0.037 & -0.031 & -6.70 & -4.93 & 1903.38 & 126.03\end{array}$ $\begin{array}{llllllll}0.242 & 0.000 & -0.038 & -0.021 & -6.48 & -4.90 & 1912.04 & 125.44\end{array}$ $\begin{array}{llllllll}0.243 & 0.000 & -0.050 & -0.023 & -7.01 & -5.12 & 1919.34 & 126.21\end{array}$ $\begin{array}{llllllll}0.243 & 0.000 & -0.052 & -0.014 & -6.81 & -5.05 & 1927.70 & 125.92\end{array}$ $\begin{array}{llllllll}0.231 & 0.000 & -0.053 & -0.013 & -7.21 & -5.41 & 1934.92 & 126.78\end{array}$ $\begin{array}{llllllll}0.232 & 0.000 & -0.065 & -0.015 & -7.67 & -5.48 & 1943.16 & 126.60\end{array}$ $\begin{array}{llllllll}0.232 & 0.000 & -0.065 & -0.015 & -8.22 & -5.99 & 1950.28 & 127.56\end{array}$ $\begin{array}{llllllll}0.232 & 0.000 & -0.065 & -0.015 & -8.40 & -6.16 & 1958.39 & 127.52\end{array}$ $\begin{array}{llllllll}0.232 & 0.000 & -0.066 & -0.006 & -8.81 & -6.59 & 1965.19 & 128.79\end{array}$ $\begin{array}{llllllll}0.221 & 0.000 & -0.080 & -0.007 & -9.42 & -6.77 & 1973.06 & 128.99\end{array}$ $\begin{array}{llllllll}0.221 & 0.000 & -0.080 & -0.007 & -9.66 & -7.00 & 1979.43 & 130.70\end{array}$ $\begin{array}{llllllll}0.221 & 0.000 & -0.080 & -0.007 & -9.18 & -6.57 & 1986.45 & 131.74\end{array}$ $\begin{array}{llllllll}0.222 & 0.000 & -0.093 & 0.000 & -9.37 & -6.30 & 1992.10 & 134.17\end{array}$ $\begin{array}{llllllll}0.209 & 0.000 & -0.083 & 0.003 & -8.23 & -5.61 & 1998.63 & 135.70\end{array}$ $\begin{array}{llllllll}0.198 & 0.000 & -0.084 & 0.004 & -7.90 & -5.33 & 2004.03 & 138.38\end{array}$ $\begin{array}{llllllll}0.175 & 0.000 & -0.062 & -0.001 & -6.47 & -4.69 & 2010.40 & 140.07\end{array}$ $\begin{array}{llllllll}0.153 & 0.000 & -0.052 & -0.007 & -6.26 & -4.82 & 2016.00 & 142.55\end{array}$ $\begin{array}{llllllll}0.130 & 0.000 & -0.042 & -0.005 & -5.91 & -4.77 & 2022.73 & 143.89\end{array}$ $\begin{array}{llllllll}0.130 & 0.000 & -0.043 & 0.005 & -6.34 & -5.20 & 2028.41 & 146.29\end{array}$ $\begin{array}{llllllll}0.130 & 0.000 & -0.054 & 0.003 & -6.54 & -5.14 & 2034.92 & 147.84\end{array}$ $\begin{array}{llllllll}0.130 & 0.000 & -0.054 & 0.003 & -6.94 & -5.53 & 2040.35 & 150.49\end{array}$ $\begin{array}{lllllllll}0.130 & 0.000 & -0.055 & 0.013 & -6.90 & -5.45 & 2046.62 & 152.29\end{array}$ $\begin{array}{llllllll}0.108 & 0.000 & -0.057 & 0.014 & -7.21 & -5.72 & 2051.74 & 155.24\end{array}$ $\begin{array}{llllllll}0.108 & 0.000 & -0.057 & 0.014 & -6.85 & -5.40 & 2057.55 & 157.50\end{array}$
$-1.64 \quad 136.54$

$-2.04 \quad 135.74$

$-2.42 \quad 133.60$

$-2.87 \quad 133.03$

$-3.21 \quad 131.21$

$-3.69130 .87$

$-4.10 \quad 129.27$

$-4.60 \quad 129.18$

$-4.77 \quad 128.09$

$-4.98 \quad 128.56$

$\begin{array}{ll}-4.95 & 127.93\end{array}$

$-5.17 \quad 128.66$

$-5.10 \quad 128.33$

$-5.46 \quad 129.16$

$-5.51 \quad 128.98$

$-6.03 \quad 129.90$

$-6.18 \quad 129.85$

$-6.63 \quad 131.09$

$-6.77 \quad 131.30$

$\begin{array}{ll}-7.01 & 132.99\end{array}$

$-6.56 \quad 134.04$

$-6.28 \quad 136.47$

$-5.58 \quad 138.01$

$\begin{array}{ll}-5.30 & 140.68\end{array}$

$-4.68 \quad 142.37$

$-4.82 \quad 144.84$

$\begin{array}{ll}-4.76 & 146.19\end{array}$

$\begin{array}{ll}-5.19 & 148.60\end{array}$

$\begin{array}{ll}-5.12 & 150.19\end{array}$

$-5.51 \quad 152.85$

$-5.40 \quad 154.70$

$\begin{array}{ll}-5.67 & 157.69\end{array}$

$-5.34 \quad 159.98$ 


\section{$Z=109$ (Mt)}

$\begin{array}{lllllll}177 & 286 & 0.08 & 0.00 & 0.04 & -0.01 & 0.086\end{array}$ $\begin{array}{lllll}178 & 287 & -0.08 & 0.00 & 0.02\end{array}$ $\begin{array}{lllll}179 & 288 & -0.04 & 0.00 & 0.00\end{array}$ $\begin{array}{lllll}180 & 289 & -0.03 & 0.00 & 0.00\end{array}$ $\begin{array}{lllll}181 & 290 & -0.01 & 0.00 & 0.00\end{array}$

$\begin{array}{lllll}182 & 291 & 0.00 & 0.00 & 0.00\end{array}$ $\begin{array}{lllll}183 & 292 & 0.00 & 0.00 & 0.00\end{array}$ $\begin{array}{lllll}184 & 293 & 0.00 & 0.00 & 0.00\end{array}$ $\begin{array}{lllll}185 & 294 & 0.00 & 0.01 & 0.00\end{array}$ $\begin{array}{lllll}186 & 295 & 0.00 & 0.03 & 0.00\end{array}$ $\begin{array}{lllll}187 & 296 & 0.00 & 0.06 & 0.00\end{array}$ $\begin{array}{lllll}188 & 297 & 0.00 & 0.07 & 0.00\end{array}$ $\begin{array}{lllll}189 & 298 & 0.01 & 0.08 & 0.00\end{array}$ $\begin{array}{lllll}190 & 299 & 0.01 & 0.09 & 0.00\end{array}$ $\begin{array}{lllll}191 & 300 & 0.02 & 0.09 & 0.00\end{array}$ $0.00-0.084$ $0.00-0.042$ $0.00-0.032$ $0.00-0.011$

$0.00 \quad 0.000$ $0.00 \quad 0.000$ $0.00 \quad 0.000$ $\begin{array}{llll}0.00 & 0.000 & -0.040\end{array}$ $\begin{array}{lllllll}0.000 & -0.045 & 0.007 & -6.49 & -5.39 & 2062.18 & 160.95\end{array}$ $\begin{array}{lllllll}0.000 & -0.021 & 0.002 & -5.78 & -5.21 & 2067.94 & 163.25\end{array}$ $\begin{array}{lllllll}0.000 & 0.001 & 0.000 & -5.89 & -5.32 & 2072.49 & 166.77\end{array}$ $\begin{array}{lllllll}0.000 & 0.000 & 0.000 & -5.65 & -5.05 & 2077.95 & 169.38\end{array}$ $\begin{array}{lllllll}0.000 & 0.000 & 0.000 & -5.95 & -5.30 & 2082.45 & 172.96\end{array}$ $\begin{array}{lll}0.00 & 0.002 & -0.081\end{array}$ $\begin{array}{lll}0.00 & 0.002-0.094\end{array}$ $0.01 \quad 0.014-0.107$ $\begin{array}{lll}0.01 & 0.014 & -0.121\end{array}$ $0.01 \quad 0.025-0.121$

192301 193302 $\begin{array}{lll}0.02 & 0.10 & 0.00\end{array}$ 0.02 194303 195304 196305 $\begin{array}{llll}0.23 & 0.00 & 0.01 & -0.01\end{array}$ $0.025-0.133$ $0.250 \quad 0.000$ $\begin{array}{llllllll}0.000 & 0.000 & 0.000 & -5.63 & -4.99 & 2087.68 & 175.80 \\ 0.000 & 0.000 & 0.000 & -5.81 & -5.17 & 2091.91 & 179.64\end{array}$ $\begin{array}{lllllll}0.000 & 0.000 & 0.000 & -5.63 & -4.99 & 2087.68 & 175.80 \\ 0.000 & 0.000 & 0.000 & -5.81 & -5.17 & 2091.91 & 179.64\end{array}$ $\begin{array}{llll}0.23 & 0.00 & 0.01 & -0.01\end{array}$ $\begin{array}{llll}0.24 & 0.00 & 0.02 & -0.01\end{array}$ $0.250 \quad 0.000$ 0.000 $\begin{array}{lllll}0.000 & -5.28 & -4.66 & 2096.75 & 182.87\end{array}$ $\begin{array}{llllll}0.000 & 0.000 & -4.34 & -3.76 & 2099.71 & 187.98\end{array}$ $\begin{array}{lllllll}0.000 & 0.001 & -3.79 & -3.10 & 2104.21 & 191.56\end{array}$ $\begin{array}{lllllll}0.002 & 0.002 & -3.57 & -2.41 & 2107.20 & 196.63\end{array}$ $\begin{array}{lllllll}0.002 & 0.003 & -3.02 & -1.65 & 2111.42 & 200.49\end{array}$ $\begin{array}{llllll}0.003 & -0.006 & -2.84 & -1.23 & 2114.50 & 205.48\end{array}$ $\begin{array}{lllllll}0.003 & -0.005 & -2.50 & -0.62 & 2118.67 & 209.38\end{array}$ $\begin{array}{lllllll}0.003 & -0.005 & -2.23 & -0.47 & 2121.85 & 214.27\end{array}$ $\begin{array}{llllll}0.004 & -0.013 & -2.12 & 0.02 & 2125.97 & 218.22\end{array}$ $\begin{array}{llllll}0.010 & 0.009 & -0.83 & -0.15 & 2129.29 & 222.97\end{array}$ $\begin{array}{llllllll}0.262 & 0.000 & -0.001 & 0.006 & -1.27 & -0.56 & 2137.12 & 231.29\end{array}$ $\begin{array}{llllllll}0.262 & 0.000 & -0.001 & 0.006 & -1.35 & -0.63 & 2141.45 & 235.02\end{array}$

197306 $\begin{array}{llll}0.24 & 0.00 & 0.02 & -0.01\end{array}$ $\begin{array}{lll}0.25 & 0.00 & 0.02\end{array}$ 198307 $\begin{array}{lll}0.26 & 0.00 & 0.03\end{array}$ 199308 $\begin{array}{lll}0.25 & 0.00 & 0.03\end{array}$ 200309 $\begin{array}{lll}0.26 & 0.00 & 0.03\end{array}$ $\begin{array}{lllll}201 & 310 & 0.26 & 0.00 & 0.03\end{array}$

0.00 0.273 $0.00 \quad 0.285$ $0.00 \quad 0.274$ $0.00 \quad 0.285$ $0.01 \quad 0.286$

$\begin{array}{lllllll}0.000 & 0.003 & -0.003 & -1.78 & -1.03 & 2144.67 & 239.88\end{array}$ $\begin{array}{lllllll}0.000 & -0.007 & -0.007 & -2.03 & -1.20 & 2148.93 & 243.69\end{array}$ $\begin{array}{lllllll}0.000 & -0.009 & -0.006 & -2.54 & -1.65 & 2152.03 & 248.66\end{array}$ $\begin{array}{lllllll}0.000 & -0.007 & -0.007 & -2.75 & -1.89 & 2156.19 & 252.57\end{array}$ $\begin{array}{lllllll}0.000 & -0.006 & -0.016 & -3.34 & -2.39 & 2159.18 & 257.65\end{array}$ 202311 $\begin{array}{lll}0.26 & 0.00 & 0.04\end{array}$ 203312 $\begin{array}{lll}0.26 & 0.00 & 0.04\end{array}$ 204313 $205 \quad 314$ $\begin{array}{lll}0.26 & 0.00 & 0.04\end{array}$ $\begin{array}{lll}0.25 & 0.00 & 0.05\end{array}$ 206315 $\begin{array}{lll}0.25 & 0.00 & 0.06\end{array}$

207316 $\begin{array}{lll}0.25 & 0.00 & 0.06\end{array}$ $208 \quad 317$ $\begin{array}{lll}0.25 & 0.00 & 0.07\end{array}$ 209318 $\begin{array}{llll}0.25 & 0.00 & 0.07\end{array}$ $\begin{array}{lllll}210 & 319 & 0.25 & 0.00 & 0.08\end{array}$ $\begin{array}{lllll}211 & 320 & 0.25 & 0.00 & 0.08\end{array}$

$\begin{array}{lllll}212 & 321 & 0.25 & 0.00 & 0.08\end{array}$

$\begin{array}{lllll}213 & 322 & 0.25 & 0.00 & 0.08\end{array}$ $\begin{array}{lllll}214 & 323 & 0.24 & 0.00 & 0.08\end{array}$ $\begin{array}{llllll}215 & 324 & 0.23 & 0.00 & 0.07\end{array}$ $\begin{array}{lllll}216 & 325 & 0.22 & 0.00 & 0.06\end{array}$ $\begin{array}{lllll}217 & 326 & 0.22 & 0.00 & 0.06\end{array}$ $\begin{array}{llllll}218 & 327 & 0.20 & 0.00 & 0.04\end{array}$ $\begin{array}{lllll}219 & 328 & 0.20 & 0.00 & 0.05\end{array}$ $\begin{array}{lllll}220 & 329 & 0.20 & 0.00 & 0.05\end{array}$ $\begin{array}{lllll}221 & 330 & 0.20 & 0.00 & 0.05\end{array}$ $\begin{array}{llllll}222 & 331 & 0.19 & 0.00 & 0.05\end{array}$ $\begin{array}{lllll}223 & 332 & 0.19 & 0.00 & 0.05\end{array}$ $\begin{array}{llllll}224 & 333 & 0.18 & 0.00 & 0.05\end{array}$ $\begin{array}{lllll}225 & 334 & 0.18 & 0.00 & 0.05\end{array}$ $\begin{array}{lllll}226 & 335 & 0.18 & 0.00 & 0.06\end{array}$ $\begin{array}{llllll}227 & 336 & 0.18 & 0.00 & 0.06\end{array}$ $\begin{array}{lllll}228 & 337 & 0.00 & 0.00 & 0.00\end{array}$ $\begin{array}{lllll}229 & 338 & -0.01 & 0.00 & 0.00\end{array}$ $\begin{array}{lllll}230 & 339 & 0.00 & 0.00 & 0.00\end{array}$
$0.01 \quad 0.287$

$0.01 \quad 0.287$

$0.01 \quad 0.287$

$0.00 \quad 0.276$

$\begin{array}{ll}0.00 & 0.277\end{array}$

$\begin{array}{ll}0.00 & 0.277\end{array}$

$0.00 \quad 0.278$

$0.00 \quad 0.278$

$\begin{array}{ll}0.00 & 0.279\end{array}$

$0.00 \quad 0.279$

$\begin{array}{ll}0.00 & 0.279\end{array}$

$\begin{array}{ll}0.00 & 0.279\end{array}$

$0.00 \quad 0.267$

$0.01 \quad 0.255$

$0.01 \quad 0.243$

$\begin{array}{lllllll}0.000 & -0.018 & -0.019 & -3.56 & -2.46 & 2163.02 & 261.89\end{array}$

$\begin{array}{lllllll}0.000 & -0.018 & -0.019 & -3.77 & -2.69 & 2165.57 & 267.40\end{array}$

$\begin{array}{lllllll}0.000 & -0.018 & -0.019 & -3.57 & -2.52 & 2169.01 & 272.04\end{array}$

$\begin{array}{lllllll}0.000 & -0.033 & -0.012 & -3.89 & -2.67 & 2171.33 & 277.79\end{array}$

$\begin{array}{lllllll}0.000 & -0.045 & -0.015 & -3.99 & -2.56 & 2174.66 & 282.53\end{array}$

$\begin{array}{lllllll}0.000 & -0.045 & -0.015 & -4.28 & -2.84 & 2176.97 & 288.29\end{array}$ $\begin{array}{lllllll}0.000 & -0.057 & -0.018 & -4.49 & -2.80 & 2180.22 & 293.11\end{array}$ $\begin{array}{lllllll}0.000 & -0.057 & -0.018 & -4.84 & -3.11 & 2182.40 & 299.00\end{array}$ $\begin{array}{lllllll}0.000 & -0.069 & -0.021 & -5.16 & -3.12 & 2185.55 & 303.92\end{array}$ $\begin{array}{lllllll}0.000 & -0.069 & -0.021 & -5.56 & -3.53 & 2187.69 & 309.86\end{array}$ $\begin{array}{lllllll}0.000 & -0.069 & -0.021 & -5.47 & -3.50 & 2190.64 & 314.98\end{array}$ $\begin{array}{lllllll}0.000 & -0.069 & -0.021 & -5.81 & -3.86 & 2192.58 & 321.10\end{array}$ $\begin{array}{lllllll}0.000 & -0.071 & -0.020 & -5.69 & -3.69 & 2195.25 & 326.51\end{array}$ $\begin{array}{lllllll}0.000 & -0.060 & -0.026 & -5.80 & -4.02 & 2197.03 & 332.80\end{array}$ $0.01 \quad 0.243$ $\begin{array}{lllllll}0.000 & -0.050 & -0.023 & -5.37 & -3.92 & 2199.62 & 338.28\end{array}$ $0.02 \quad 0.219$ $0.02 \quad 0.220$ $0.02 \quad 0.220$ $\begin{array}{lllllll}0.000 & -0.050 & -0.023 & -5.70 & -4.23 & 2201.24 & 344.74\end{array}$ $\begin{array}{lllllll}0.000 & -0.029 & -0.028 & -5.34 & -4.25 & 2203.81 & 350.23\end{array}$ $\begin{array}{lllllll}0.000 & -0.041 & -0.030 & -6.04 & -4.72 & 2205.45 & 356.67\end{array}$ $\begin{array}{lllllll}0.000 & -0.041 & -0.030 & -6.05 & -4.77 & 2207.91 & 362.28\end{array}$ $\begin{array}{lllllllll}0.02 & 0.220 & 0.000 & -0.041 & -0.030 & -6.47 & -5.19 & 2209.35 & 368.91\end{array}$ $\begin{array}{lllllllll}0.02 & 0.209 & 0.000 & -0.043 & -0.030 & -6.45 & -4.99 & 2211.43 & 374.90\end{array}$ $\begin{array}{lllllllll}0.01 & 0.208 & 0.000 & -0.044 & -0.020 & -6.67 & -5.30 & 2212.64 & 381.76\end{array}$ $\begin{array}{lllllllll}0.01 & 0.197 & 0.000 & -0.045 & -0.019 & -6.61 & -5.20 & 2214.68 & 387.79\end{array}$ $\begin{array}{lllllllll}0.01 & 0.197 & 0.000 & -0.045 & -0.019 & -7.01 & -5.60 & 2215.85 & 394.70\end{array}$ $\begin{array}{lllllllll}0.00 & 0.198 & 0.000 & -0.058 & -0.012 & -7.10 & -5.51 & 2217.77 & 400.85\end{array}$ $\begin{array}{lllllllll}0.00 & 0.198 & 0.000 & -0.058 & -0.012 & -7.42 & -5.84 & 2218.73 & 407.95\end{array}$ $\begin{array}{lllllllll}0.00 & 0.000 & 0.000 & 0.000 & 0.000 & -7.21 & -6.65 & 2221.42 & 413.33\end{array}$ $\begin{array}{lllllllll}0.00 & -0.011 & 0.000 & 0.000 & 0.000 & -7.33 & -6.77 & 2222.06 & 420.77\end{array}$ $\begin{array}{lllllllll}0.00 & 0.000 & 0.000 & 0.000 & 0.000 & -7.12 & -6.59 & 2223.62 & 427.28\end{array}$
$-5.36 \quad 163.43$

$-5.21 \quad 165.74$

$-5.33 \quad 169.30$

$-5.05 \quad 171.96$

$-5.31 \quad 175.58$

$\begin{array}{ll}-4.99 & 178.47\end{array}$

$-5.17 \quad 182.36$

$-4.67 \quad 185.65$

$-3.76 \quad 190.82$

$-3.09194 .46$

$-2.39 \quad 199.62$

$-1.62 \quad 203.56$

$-1.19 \quad 208.64$

$-0.56 \quad 212.63$

$-0.41 \quad 217.61$

$0.13 \quad 221.69$

$-0.19 \quad 226.38$

$-0.20 \quad 230.10$

$\begin{array}{ll}-0.60 & 234.88\end{array}$

$-0.67 \quad 238.73$

$-1.09 \quad 243.66$

$\begin{array}{ll}-1.25 \quad 247.59 \\ -1.71 & 252.66\end{array}$

$-1.71 \quad 252.66$

$-1.93 \quad 256.70$

$-2.42 \quad 261.91$

$-2.46 \quad 266.30$

$\begin{array}{ll}-2.70 & 271.92\end{array}$

$\begin{array}{ll}-2.52 & 276.70\end{array}$

$\begin{array}{ll}-2.68 & 282.57\end{array}$

$\begin{array}{ll}-2.53 & 287.49\end{array}$

\begin{tabular}{ll}
$-2.82 \quad 293.38$ \\
\hline
\end{tabular}

$-2.73 \quad 298.40$

$-3.04 \quad 304.43$

$-3.00 \quad 309.56$

$-3.41 \quad 315.64$

\begin{tabular}{ll}
$-3.37 \quad 320.94$ \\
\hline
\end{tabular}

$-3.74 \quad 327.21$

$\begin{array}{ll}-3.54 & 332.81\end{array}$

$-3.88 \quad 339.27$

$-3.81 \quad 344.90$

$-4.13 \quad 351.52$

$-4.13 \quad 357.22$

$-4.57 \quad 363.88$

$\begin{array}{ll}-4.60 & 369.69\end{array}$

$-5.02 \quad 376.50$

$-4.81 \quad 382.71$

$\begin{array}{ll}-5.22 & 389.67\end{array}$

$-5.10 \quad 395.92$

\begin{tabular}{ll}
$-5.51 \quad 403.03$ \\
\hline
\end{tabular}

$-5.41409 .41$

$-5.74 \quad 416.73$

$\begin{array}{ll}-6.65 & 422.23\end{array}$

$-6.78429 .89$

$-6.59436 .62$

\section{$Z=110$ (Ds)}

$\begin{array}{llllllllllllll}146 & 256 & 0.21 & 0.00 & 0.02 & 0.01 & 0.229 & 0.000 & -0.004 & -0.013 & -2.51 & -1.72 & 1841.32 & 138.88\end{array}$

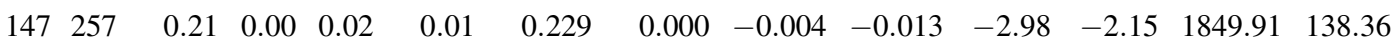




\section{$Z=110$ (Ds)}

\begin{tabular}{|c|c|c|c|c|c|c|c|c|c|c|c|c|c|c|}
\hline $148 \quad 258$ & 0.22 & 0.00 & 0.03 & 0.01 & 0.241 & 0.000 & -0.014 & -0.016 & -3.47 & -2.62 & 1860.22 & 136.12 & -2.68 & 139.15 \\
\hline 149259 & 0.21 & 0.00 & 0.03 & 0.01 & 0.229 & 0.000 & -0.016 & -0.016 & -4.03 & -3.10 & 1868.60 & 135.81 & -3.17 & 138.78 \\
\hline $50 \quad 260$ & 0.22 & 0.00 & 0.04 & 0.01 & 0.241 & 0.000 & -0.026 & -0.019 & -4.62 & -3.47 & 1878.54 & 133.94 & -3.53 & 136.86 \\
\hline 1261 & 0.22 & 0.00 & 0.04 & 0.02 & 0.242 & 0.000 & -0.025 & -0.028 & -5.33 & -3.97 & 1886.67 & 133.88 & -4.03 & 136.75 \\
\hline $52 \quad 262$ & 0.22 & 0.00 & 0.04 & 0.02 & 0.242 & 0.000 & -0.025 & -0.028 & -5.53 & -4.15 & 1896.16 & 132.47 & -4.19 & 135.30 \\
\hline 3263 & 0.22 & 0.00 & 0.05 & 0.01 & 0.242 & 0.000 & -0.038 & -0.021 & -5.84 & -4.36 & 1903.74 & 132.96 & -4.42 & 135.72 \\
\hline 4264 & 0.22 & 0.00 & 0.05 & 0.01 & 0.242 & 0.000 & -0.038 & -0.021 & -5.82 & -4.36 & 1912.77 & 132.00 & -4.40 & 134.72 \\
\hline 265 & 0.22 & 0.00 & 0.06 & 0.00 & 0.243 & 0.000 & -0.052 & -0.014 & -6.26 & -4.59 & 1920.13 & 132.71 & -4.65 & 135.38 \\
\hline 6266 & 0.21 & 0.00 & 0.06 & 0.00 & 0.231 & 0.000 & -0.053 & -0.013 & -6.27 & -4.59 & 1928.91 & 132.01 & -4.63 & 134.65 \\
\hline $72 \quad 267$ & 0.21 & 0.00 & 0.06 & 0.00 & 0.231 & 0.000 & -0.053 & -0.013 & -6.67 & -4.92 & 1936.12 & 132.87 & -4.97 & 135.47 \\
\hline $8 \quad 268$ & 0.21 & 0.00 & 0.07 & 0.00 & 0.232 & 0.000 & -0.065 & -0.015 & -7.17 & -5.07 & 1944.79 & 132.26 & -5.10 & 134.85 \\
\hline 9269 & 0.21 & 0.00 & 0.07 & -0.01 & 0.232 & 0.000 & -0.066 & -0.006 & -7.67 & -5.58 & 1951.94 & 133.19 & -5.62 & 135.74 \\
\hline $60 \quad 270$ & 0.21 & 0.00 & 0.07 & -0.01 & 0.232 & 0.000 & -0.066 & -0.006 & -7.88 & -5.77 & 1960.40 & 132.79 & -5.79 & 135.33 \\
\hline 161271 & 0.20 & 0.00 & 0.08 & -0.01 & 0.221 & 0.000 & -0.080 & -0.007 & -8.86 & -6.30 & 1967.33 & 133.94 & -6.32 & 136.46 \\
\hline $162 \quad 272$ & 0.20 & 0.00 & 0.08 & -0.01 & 0.221 & 0.000 & -0.080 & -0.007 & -9.07 & -6.50 & 1975.56 & 133.77 & -6.50 & 136.29 \\
\hline $63 \quad 273$ & 0.20 & 0.00 & 0.08 & -0.01 & 0.221 & 0.000 & -0.080 & -0.007 & -9.31 & -6.73 & 1981.96 & 135.45 & -6.74 & 137.94 \\
\hline $4 \quad 274$ & 0.20 & 0.00 & 0.09 & -0.02 & 0.222 & 0.000 & -0.093 & 0.000 & -9.40 & -6.38 & 1989.40 & 136.08 & -6.35 & 138.59 \\
\hline $165 \quad 275$ & 0.20 & 0.00 & 0.09 & -0.02 & 0.222 & 0.000 & -0.093 & 0.000 & -9.09 & -6.05 & 1995.01 & 138.55 & -6.03 & 141.03 \\
\hline 166276 & 0.19 & 0.00 & 0.09 & -0.02 & 0.210 & 0.000 & -0.094 & 0.001 & -8.47 & -5.46 & 2001.98 & 139.64 & -5.41 & 142.14 \\
\hline 167277 & 0.18 & 0.00 & 0.08 & -0.02 & 0.198 & 0.000 & -0.084 & 0.004 & -7.72 & -5.21 & 2007.44 & 142.25 & -5.18 & 144.73 \\
\hline $168 \quad 278$ & 0.16 & 0.00 & 0.06 & -0.01 & 0.175 & 0.000 & -0.062 & -0.001 & -6.39 & -4.66 & 2014.24 & 143.53 & -4.65 & 145.99 \\
\hline 169279 & 0.12 & 0.00 & 0.04 & 0.00 & 0.130 & 0.000 & -0.042 & -0.005 & -6.11 & -4.98 & 2020.04 & 145.80 & -4.97 & 148.25 \\
\hline $170 \quad 280$ & 0.12 & 0.00 & 0.04 & 0.00 & 0.130 & 0.000 & -0.042 & -0.005 & -6.11 & -4.98 & 2027.16 & 146.76 & -4.97 & 149.21 \\
\hline 171281 & 0.12 & 0.00 & 0.04 & 0.00 & 0.130 & 0.000 & -0.042 & -0.005 & -6.52 & -5.38 & 2032.83 & 149.15 & -5.38 & 151.61 \\
\hline $172 \quad 282$ & 0.12 & 0.00 & 0.04 & -0.01 & 0.130 & 0.000 & -0.043 & 0.005 & -6.46 & -5.33 & 2039.68 & 150.37 & -5.32 & 152.86 \\
\hline $173 \quad 283$ & 0.10 & 0.00 & 0.04 & -0.01 & 0.108 & 0.000 & -0.044 & 0.005 & -6.91 & -5.75 & 2045.17 & 152.96 & -5.74 & 155.46 \\
\hline $174 \quad 284$ & 0.09 & 0.00 & 0.04 & -0.01 & 0.097 & 0.000 & -0.045 & 0.006 & -6.89 & -5.74 & 2051.83 & 154.37 & -5.72 & 156.89 \\
\hline $175 \quad 285$ & 0.09 & 0.00 & 0.05 & -0.01 & 0.097 & 0.000 & -0.057 & 0.005 & -7.54 & -6.11 & 2057.05 & 157.21 & -6.07 & 159.77 \\
\hline 176286 & 0.09 & 0.00 & 0.05 & -0.02 & 0.097 & 0.000 & -0.057 & 0.015 & -7.34 & -5.85 & 2063.27 & 159.06 & -5.79 & 161.67 \\
\hline $177 \quad 287$ & 0.07 & 0.00 & 0.04 & -0.01 & 0.075 & 0.000 & -0.046 & 0.007 & -7.06 & -5.92 & 2068.00 & 162.41 & -5.89 & 165.01 \\
\hline $178 \quad 288$ & -0.07 & 0.00 & 0.01 & 0.00 & -0.073 & 0.000 & -0.010 & 0.001 & -6.28 & -5.77 & 2074.10 & 164.38 & -5.77 & 166.98 \\
\hline 179289 & -0.03 & 0.00 & 0.00 & 0.00 & -0.032 & 0.000 & 0.000 & 0.000 & -6.61 & -5.99 & 2078.78 & 167.77 & -5.99 & 170.41 \\
\hline $180 \quad 290$ & -0.03 & 0.00 & 0.01 & 0.00 & -0.032 & 0.000 & -0.011 & 0.000 & -6.41 & -5.76 & 2084.61 & 170.01 & -5.76 & 172.69 \\
\hline 181291 & 0.00 & 0.00 & 0.00 & 0.00 & 0.000 & 0.000 & 0.000 & 0.000 & -6.66 & -5.98 & 2089.09 & 173.60 & -5.98 & 176.32 \\
\hline $182 \quad 292$ & 0.00 & 0.00 & 0.00 & 0.00 & 0.000 & 0.000 & 0.000 & 0.000 & -6.32 & -5.65 & 2094.62 & 176.14 & -5.66 & 178.90 \\
\hline 183293 & 0.00 & 0.00 & 0.00 & 0.00 & 0.000 & 0.000 & 0.000 & 0.000 & -6.49 & -5.81 & 2098.85 & 179.98 & -5.81 & 182.79 \\
\hline $184 \quad 294$ & 0.00 & 0.00 & 0.00 & 0.00 & 0.000 & 0.000 & 0.000 & 0.000 & -5.94 & -5.29 & 2104.00 & 182.91 & -5.30 & 185.77 \\
\hline $185 \quad 295$ & 0.00 & 0.01 & 0.00 & 0.00 & 0.000 & -0.013 & 0.000 & 0.000 & -4.98 & -4.37 & 2106.96 & 188.02 & -4.37 & 190.94 \\
\hline 186296 & 0.00 & 0.00 & 0.00 & 0.00 & 0.000 & 0.000 & 0.000 & 0.000 & -4.23 & -3.66 & 2111.73 & 191.33 & -3.67 & 194.30 \\
\hline $187 \quad 297$ & 0.00 & 0.05 & 0.00 & 0.00 & 0.001 & -0.067 & 0.001 & 0.002 & -3.70 & -2.74 & 2114.50 & 196.63 & -2.72 & 199.68 \\
\hline 188298 & 0.00 & 0.05 & 0.00 & 0.00 & 0.001 & -0.067 & 0.001 & 0.002 & -2.85 & -1.93 & 2118.97 & 200.22 & -1.91 & 203.34 \\
\hline 189299 & 0.01 & 0.07 & 0.00 & 0.01 & 0.013 & -0.094 & 0.002 & -0.007 & -2.70 & -1.36 & 2121.92 & 205.35 & -1.32 & 208.55 \\
\hline 190300 & 0.01 & 0.08 & 0.00 & 0.01 & 0.014 & -0.107 & 0.003 & -0.006 & -2.26 & -0.68 & 2126.34 & 208.99 & -0.63 & 212.29 \\
\hline 191301 & 0.03 & 0.09 & -0.01 & 0.01 & 0.035 & -0.122 & 0.015 & -0.004 & -2.34 & -0.51 & 2129.51 & 213.90 & -0.45 & 217.28 \\
\hline 192302 & 0.03 & 0.10 & -0.01 & 0.02 & 0.036 & -0.134 & 0.016 & -0.013 & -2.16 & 0.02 & 2133.90 & 217.58 & 0.13 & 221.09 \\
\hline 193303 & 0.34 & 0.00 & 0.07 & -0.01 & 0.381 & 0.000 & -0.035 & -0.013 & -0.97 & 0.04 & 2137.05 & 222.50 & -0.02 & 225.93 \\
\hline 194304 & 0.23 & 0.00 & 0.01 & -0.01 & 0.250 & 0.000 & 0.010 & 0.009 & -0.48 & 0.05 & 2141.78 & 225.85 & 0.03 & 229.40 \\
\hline 195305 & 0.24 & 0.00 & 0.01 & -0.01 & 0.262 & 0.000 & 0.012 & 0.009 & -0.86 & -0.28 & 2145.10 & 230.60 & -0.31 & 234.22 \\
\hline 196306 & 0.24 & 0.00 & 0.02 & -0.01 & 0.262 & 0.000 & -0.001 & 0.006 & -0.93 & -0.31 & 2149.69 & 234.07 & -0.33 & 237.80 \\
\hline 197307 & 0.24 & 0.00 & 0.02 & -0.01 & 0.262 & 0.000 & -0.001 & 0.006 & -1.37 & -0.70 & 2152.91 & 238.93 & -0.73 & 242.74 \\
\hline 198308 & 0.26 & 0.00 & 0.03 & 0.00 & 0.285 & 0.000 & -0.007 & -0.007 & -1.58 & -0.87 & 2157.47 & 242.44 & -0.90 & 246.35 \\
\hline 199309 & 0.26 & 0.00 & 0.03 & 0.00 & 0.285 & 0.000 & -0.007 & -0.007 & -2.08 & -1.37 & 2160.64 & 247.34 & -1.41 & 251.35 \\
\hline 200310 & 0.26 & 0.00 & 0.03 & 0.00 & 0.285 & 0.000 & -0.007 & -0.007 & -2.31 & -1.55 & 2165.05 & 251.00 & -1.59 & 255.13 \\
\hline 311 & 0.26 & 0.00 & 0.03 & 0.00 & 0.285 & 0.000 & -0.007 & -0.007 & -2.80 & -2.01 & 2168.01 & 256.11 & -2.06 & 260.34 \\
\hline 202312 & 0.26 & 0.00 & 0.04 & 0.00 & 0.286 & 0.000 & -0.019 & -0.010 & -2.97 & -2.05 & 2172.10 & 260.09 & -2.07 & 264.46 \\
\hline 313 & 0.26 & 0.00 & 0.04 & 0.01 & 0.287 & 0.000 & -0.018 & -0.019 & -3.29 & -2.30 & 2174.70 & 265.57 & -2.30 & 270.07 \\
\hline 204314 & 0.26 & 0.00 & 0.04 & 0.01 & 0.287 & 0.000 & -0.018 & -0.019 & -3.09 & -2.13 & 2178.43 & 269.91 & -2.12 & 274.55 \\
\hline
\end{tabular}


$Z=110(\mathrm{Ds})$

$\begin{array}{lllll}205 & 315 & 0.26 & 0.00 & 0.05\end{array}$ $\begin{array}{lllll}206 & 316 & 0.25 & 0.00 & 0.05\end{array}$ $\begin{array}{llllll}207 & 317 & 0.25 & 0.00 & 0.06\end{array}$ $\begin{array}{llllll}208 & 318 & 0.25 & 0.00 & 0.07\end{array}$ $\begin{array}{lllll}209 & 319 & 0.25 & 0.00 & 0.07\end{array}$ $\begin{array}{llllll}210 & 320 & 0.25 & 0.00 & 0.08\end{array}$ $\begin{array}{lllll}211 & 321 & 0.25 & 0.00 & 0.08\end{array}$ $\begin{array}{lllll}212 & 322 & 0.25 & 0.00 & 0.08\end{array}$ $\begin{array}{lllll}213 & 323 & 0.25 & 0.00 & 0.08\end{array}$ $\begin{array}{lllll}214 & 324 & 0.24 & 0.00 & 0.08\end{array}$ $\begin{array}{llllll}215 & 325 & 0.23 & 0.00 & 0.07\end{array}$ $\begin{array}{llllll}216 & 326 & 0.19 & 0.00 & 0.03\end{array}$ $\begin{array}{llllll}217 & 327 & 0.19 & 0.00 & 0.03\end{array}$ $\begin{array}{lllll}218 & 328 & 0.18 & 0.00 & 0.03\end{array}$ $\begin{array}{lllll}219 & 329 & 0.19 & 0.00 & 0.04\end{array}$ $\begin{array}{llllll}220 & 330 & 0.19 & 0.00 & 0.04\end{array}$ $\begin{array}{lllll}221 & 331 & 0.19 & 0.00 & 0.04\end{array}$ $\begin{array}{llllll}222 & 332 & 0.18 & 0.00 & 0.04\end{array}$ $\begin{array}{lllll}223 & 333 & 0.18 & 0.00 & 0.05\end{array}$ $\begin{array}{lllll}224 & 334 & 0.18 & 0.00 & 0.05\end{array}$ $\begin{array}{lllll}225 & 335 & 0.18 & 0.00 & 0.05\end{array}$ $\begin{array}{lllll}226 & 336 & 0.17 & 0.00 & 0.05\end{array}$ $\begin{array}{lllll}227 & 337 & 0.02 & 0.00 & 0.01\end{array}$ $\begin{array}{lllll}228 & 338 & 0.00 & 0.00 & 0.00\end{array}$ $\begin{array}{lllll}229 & 339 & -0.01 & 0.00 & 0.00\end{array}$ $\begin{array}{lllllllll}0.00 & 0.287 & 0.000 & -0.031 & -0.013 & -3.39 & -2.32 & 2180.80 & 275.61\end{array}$ $\begin{array}{lllllllll}0.00 & 0.276 & 0.000 & -0.033 & -0.012 & -3.18 & -2.09 & 2184.30 & 280.17\end{array}$ $\begin{array}{lllllllll}0.00 & 0.277 & 0.000 & -0.045 & -0.015 & -3.73 & -2.39 & 2186.64 & 285.91\end{array}$ $\begin{array}{lllllllll}0.00 & 0.278 & 0.000 & -0.057 & -0.018 & -3.93 & -2.33 & 2190.16 & 290.46\end{array}$ $\begin{array}{lllllllll}0.00 & 0.278 & 0.000 & -0.057 & -0.018 & -4.28 & -2.63 & 2192.35 & 296.35\end{array}$ $\begin{array}{lllllllll}0.00 & 0.279 & 0.000 & -0.069 & -0.021 & -4.59 & -2.63 & 2195.77 & 301.00\end{array}$ $\begin{array}{lllllllll}0.00 & 0.279 & 0.000 & -0.069 & -0.021 & -4.99 & -3.03 & 2197.91 & 306.93\end{array}$ $\begin{array}{lllllllll}0.00 & 0.279 & 0.000 & -0.069 & -0.021 & -4.89 & -2.99 & 2201.14 & 311.76\end{array}$ $\begin{array}{lllllllll}0.00 & 0.279 & 0.000 & -0.069 & -0.021 & -5.23 & -3.35 & 2203.09 & 317.89\end{array}$ $\begin{array}{lllllllll}0.00 & 0.267 & 0.000 & -0.071 & -0.020 & -5.17 & -3.21 & 2206.08 & 322.97\end{array}$ $\begin{array}{lllllllll}0.01 & 0.255 & 0.000 & -0.060 & -0.026 & -5.32 & -3.62 & 2207.93 & 329.19\end{array}$ $\begin{array}{lllllllll}0.02 & 0.207 & 0.000 & -0.019 & -0.025 & -4.35 & -3.45 & 2210.74 & 334.45\end{array}$ $\begin{array}{lllllllll}0.02 & 0.207 & 0.000 & -0.019 & -0.025 & -4.75 & -3.82 & 2212.42 & 340.84\end{array}$ $\begin{array}{lllllllll}0.02 & 0.196 & 0.000 & -0.021 & -0.025 & -4.76 & -3.90 & 2215.33 & 346.00\end{array}$ $\begin{array}{lllllllll}0.02 & 0.208 & 0.000 & -0.031 & -0.027 & -5.40 & -4.35 & 2216.96 & 352.45\end{array}$ $\begin{array}{lllllllll}0.02 & 0.208 & 0.000 & -0.031 & -0.027 & -5.40 & -4.36 & 2219.66 & 357.82\end{array}$ $\begin{array}{lllllllll}0.02 & 0.208 & 0.000 & -0.031 & -0.027 & -5.83 & -4.64 & 2220.97 & 364.58\end{array}$ $\begin{array}{lllllllll}0.02 & 0.197 & 0.000 & -0.032 & -0.027 & -5.82 & -4.58 & 2223.47 & 370.15\end{array}$ $\begin{array}{lllllllll}0.01 & 0.197 & 0.000 & -0.045 & -0.019 & -6.36 & -5.00 & 2224.79 & 376.90\end{array}$ $\begin{array}{lllllllll}0.01 & 0.197 & 0.000 & -0.045 & -0.019 & -6.34 & -4.99 & 2227.19 & 382.57\end{array}$ $\begin{array}{lllllllll}0.00 & 0.197 & 0.000 & -0.046 & -0.009 & -6.64 & -5.34 & 2228.32 & 389.52\end{array}$ $\begin{array}{lllllllll}0.00 & 0.186 & 0.000 & -0.048 & -0.009 & -6.58 & -5.27 & 2230.52 & 395.38\end{array}$ $\begin{array}{lllllllll}0.00 & 0.021 & 0.000 & -0.012 & -0.000 & -6.98 & -6.45 & 2232.35 & 401.63\end{array}$ $\begin{array}{lllllllll}0.00 & 0.000 & 0.000 & 0.000 & 0.000 & -7.88 & -7.28 & 2235.33 & 406.71\end{array}$ $\begin{array}{lllllllll}0.00 & -0.011 & 0.000 & 0.000 & 0.000 & -7.98 & -7.39 & 2235.95 & 414.17\end{array}$

$\begin{array}{ll}-2.33 & 280.36 \\ -2.08 & 285.07 \\ -2.36 & 290.96 \\ -2.25 & 295.70 \\ -2.56 & 301.72 \\ -2.49 & 306.58 \\ -2.91 & 312.65 \\ -2.85 & 317.65 \\ -3.22 & 323.92 \\ -3.06 & 329.19 \\ -3.47 & 335.57 \\ -3.36 & 340.94 \\ -3.74 & 347.50 \\ -3.80 & 352.84 \\ -4.23 & 359.50 \\ -4.23 & 365.06 \\ -4.51 & 372.00 \\ -4.44 & 377.77 \\ -4.91 & 384.67 \\ -4.89 & 390.55 \\ -5.29 & 397.65 \\ -5.20 & 403.73 \\ -6.44 & 410.13 \\ -7.29 & 415.42 \\ -7.39 & 423.09\end{array}$

\section{$Z=111(\mathbf{R g})$}

$\begin{array}{lllll}148 & 259 & 0.20 & 0.00 & 0.02\end{array}$ $\begin{array}{lllll}149 & 260 & 0.20 & 0.00 & 0.02\end{array}$

0.01 0.02 0.02 150261 $\begin{array}{lll}0.20 & 0.00 & 0.03\end{array}$ $\begin{array}{llllll}151 & 262 & 0.21 & 0.00 & 0.03\end{array}$ $\begin{array}{lllll}152 & 263 & 0.21 & 0.00 & 0.04\end{array}$

$\begin{array}{lllll}153 & 264 & 0.20 & 0.00 & 0.04\end{array}$ $\begin{array}{lllll}154 & 265 & 0.20 & 0.00 & 0.04\end{array}$ $\begin{array}{lllll}155 & 266 & 0.20 & 0.00 & 0.05\end{array}$ $\begin{array}{lllll}156 & 267 & 0.20 & 0.00 & 0.05\end{array}$ $\begin{array}{lllll}157 & 268 & 0.20 & 0.00 & 0.06\end{array}$

$\begin{array}{ll}158 & 269\end{array}$

159270

$\begin{array}{ll}160 & 271\end{array}$

$\begin{array}{ll}161 & 272\end{array}$

162273

163274

$164 \quad 275$

165276

$\begin{array}{lll}166 & 277\end{array}$

167278

168279

169280

$\begin{array}{ll}170 & 281\end{array}$

$\begin{array}{ll}171 & 282\end{array}$

$172 \quad 283$

$173 \quad 284$

174285

175286

$176 \quad 287$

$177 \quad 288$

177288 $\begin{array}{lllll}0.20 & 0.00 & 0.06 & 0.00\end{array}$ $\begin{array}{llll}0.20 & 0.00 & 0.07 & -0.01\end{array}$ $\begin{array}{lllll}0.20 & 0.00 & 0.07 & -0.01\end{array}$ $\begin{array}{llll}0.20 & 0.00 & 0.08 & -0.01\end{array}$ $\begin{array}{lllll}0.20 & 0.00 & 0.08 & -0.01\end{array}$

$\begin{array}{lllll}0.20 & 0.00 & 0.09 & -0.02\end{array}$ $\begin{array}{lllll}0.20 & 0.00 & 0.09 & -0.02\end{array}$ $\begin{array}{lllll}0.18 & 0.00 & 0.08 & -0.02\end{array}$ $\begin{array}{lllll}0.18 & 0.00 & 0.08 & -0.02\end{array}$ $\begin{array}{lllll}0.16 & 0.00 & 0.06 & -0.01\end{array}$ $\begin{array}{lllll}0.15 & 0.00 & 0.06 & -0.01\end{array}$ $\begin{array}{llll}0.12 & 0.00 & 0.03 & 0.00\end{array}$ $\begin{array}{lllll}0.12 & 0.00 & 0.04 & 0.00\end{array}$ $\begin{array}{llll}0.10 & 0.00 & 0.03 & 0.00\end{array}$ $\begin{array}{lllll}0.09 & 0.00 & 0.03 & 0.00\end{array}$ $\begin{array}{lllll}0.09 & 0.00 & 0.03 & 0.00\end{array}$ $\begin{array}{lllll}0.08 & 0.00 & 0.04 & -0.01\end{array}$ $\begin{array}{lllll}0.08 & 0.00 & 0.04 & -0.01\end{array}$ $\begin{array}{llll}0.08 & 0.00 & 0.04 & -0.01\end{array}$ $\begin{array}{lllll}0.07 & 0.00 & 0.04 & -0.01\end{array}$ $\begin{array}{llllllll}0.218 & 0.000 & -0.006 & -0.013 & -3.21 & -2.43 & 1857.78 & 145.85\end{array}$ $\begin{array}{llllllll}0.218 & 0.000 & -0.005 & -0.023 & -3.87 & -2.91 & 1866.50 & 145.20\end{array}$ $\begin{array}{llllllll}0.219 & 0.000 & -0.017 & -0.025 & -4.32 & -3.20 & 1876.40 & 143.38\end{array}$ $\begin{array}{llllllll}0.230 & 0.000 & -0.015 & -0.026 & -4.81 & -3.73 & 1884.91 & 142.94\end{array}$ $\begin{array}{llllllll}0.230 & 0.000 & -0.027 & -0.028 & -5.16 & -3.81 & 1894.32 & 141.59\end{array}$ $\begin{array}{llllllll}0.219 & 0.000 & -0.030 & -0.018 & -5.24 & -4.02 & 1902.26 & 141.73\end{array}$ $\begin{array}{llllllll}0.219 & 0.000 & -0.030 & -0.018 & -5.29 & -4.09 & 1911.40 & 140.66\end{array}$ $\begin{array}{llllllll}0.220 & 0.000 & -0.042 & -0.020 & -5.85 & -4.39 & 1919.16 & 140.97\end{array}$ $\begin{array}{llllllll}0.219 & 0.000 & -0.043 & -0.010 & -5.77 & -4.41 & 1927.99 & 140.21\end{array}$ $\begin{array}{llllllll}0.220 & 0.000 & -0.055 & -0.013 & -6.49 & -4.81 & 1935.61 & 140.66\end{array}$ $\begin{array}{llllllll}0.220 & 0.000 & -0.055 & -0.013 & -6.63 & -4.96 & 1944.33 & 140.02\end{array}$ $\begin{array}{llllllll}0.220 & 0.000 & -0.068 & -0.005 & -7.53 & -5.50 & 1951.83 & 140.58\end{array}$ $\begin{array}{llllllll}0.220 & 0.000 & -0.068 & -0.005 & -7.75 & -5.71 & 1960.35 & 140.13\end{array}$ $\begin{array}{llllllll}0.221 & 0.000 & -0.080 & -0.007 & -8.68 & -6.20 & 1967.58 & 140.98\end{array}$ $\begin{array}{llllllll}0.221 & 0.000 & -0.080 & -0.007 & -8.89 & -6.40 & 1975.85 & 140.78\end{array}$ $\begin{array}{llllllll}0.222 & 0.000 & -0.093 & 0.000 & -9.71 & -6.71 & 1982.66 & 142.04\end{array}$ $\begin{array}{llllllll}0.222 & 0.000 & -0.093 & 0.000 & -9.32 & -6.30 & 1990.07 & 142.71\end{array}$ $\begin{array}{llllllll}0.198 & 0.000 & -0.084 & 0.004 & -8.68 & -6.12 & 1996.16 & 144.68\end{array}$ $\begin{array}{llllllll}0.198 & 0.000 & -0.084 & 0.004 & -8.14 & -5.63 & 2003.26 & 145.65\end{array}$ $\begin{array}{llllllll}0.175 & 0.000 & -0.062 & -0.001 & -7.17 & -5.46 & 2009.13 & 147.85\end{array}$ $\begin{array}{llllllll}0.164 & 0.000 & -0.063 & 0.000 & -6.79 & -5.11 & 2016.14 & 148.91\end{array}$ $\begin{array}{llllllll}0.130 & 0.000 & -0.030 & -0.004 & -6.42 & -5.49 & 2022.35 & 150.77\end{array}$ $\begin{array}{llllllll}0.130 & 0.000 & -0.042 & -0.005 & -6.62 & -5.49 & 2029.49 & 151.71\end{array}$ $\begin{array}{llllllll}0.108 & 0.000 & -0.032 & -0.003 & -6.85 & -5.91 & 2035.51 & 153.76\end{array}$ $\begin{array}{llllllll}0.097 & 0.000 & -0.032 & -0.003 & -6.87 & -5.93 & 2042.45 & 154.89\end{array}$ $\begin{array}{llllllll}0.097 & 0.000 & -0.032 & -0.003 & -7.34 & -6.38 & 2048.29 & 157.12\end{array}$ $\begin{array}{llllllll}0.086 & 0.000 & -0.045 & 0.007 & -7.57 & -6.39 & 2055.00 & 158.48\end{array}$ $\begin{array}{lllllllll}0.086 & 0.000 & -0.045 & 0.007 & -7.96 & -6.76 & 2060.56 & 161.00\end{array}$ $\begin{array}{llllllll}0.086 & 0.000 & -0.045 & 0.007 & -7.66 & -6.48 & 2066.77 & 162.86\end{array}$ $\begin{array}{llllllll}0.075 & 0.000 & -0.046 & 0.007 & -7.85 & -6.67 & 2071.94 & 165.76\end{array}$ $\begin{array}{ll}-2.50 & 149.16\end{array}$

$-2.97 \quad 148.45$

$-3.26 \quad 146.57$

$-3.79 \quad 146.05$

$-3.86144 .66$

$-4.08 \quad 144.74$

$-4.14 \quad 143.63$

$-4.44 \quad 143.88$

$-4.46 \quad 143.08$

$-4.86143 .48$

$-5.01 \quad 142.82$

$\begin{array}{ll}-5.55 & 143.34\end{array}$

$-5.74 \quad 142.87$

$-6.24143 .68$

$-6.42 \quad 143.48$

$-6.73 \quad 144.72$

$-6.29 \quad 145.38$

$-6.12 \quad 147.34$

$-5.61 \quad 148.31$

$-5.47 \quad 150.48$

$-5.10 \quad 151.54$

$-5.51 \quad 153.38$

$-5.49 \quad 154.33$

$-5.91 \quad 156.38$

$-5.93 \quad 157.52$

$-6.38159 .76$

$-6.37 \quad 161.15$

$-6.74163 .68$

$-6.46 \quad 165.57$

$-6.65168 .49$

$-6.50 \quad 170.45$ 


\section{$Z=111(\mathrm{Rg})$}

$\begin{array}{llll}179 & 290 & -0.03 & 0.00\end{array}$ $\begin{array}{llll}180 & 291 & -0.02 & 0.00\end{array}$ $\begin{array}{llll}181 & 292 & -0.01 & 0.00\end{array}$ $\begin{array}{llll}182 & 293 & 0.00 & 0.00\end{array}$ $\begin{array}{llll}183 & 294 & 0.00 & 0.00\end{array}$

184295 $185 \quad 296$ 186297 $0.00 \quad 0.00$

0.00 0.00

\subsection{0}

0.00

0.00

0.00 $\begin{array}{lll}0.00 & 0.01\end{array}$ $0.00 \quad 0.00$

$187 \quad 298$ $\begin{array}{ll}0.00 & 0.04\end{array}$

$188 \quad 299$

$0.00 \quad 0.03$

189300

190301

191302

192303

193304

194305

195306

196307

197308

199310

200311

201312

202313

203314

204315

205316

206317

$207 \quad 318$

$208 \quad 319$

209320

$210 \quad 321$

211322

$212 \quad 323$

$213 \quad 324$

$214 \quad 325$

$215 \quad 326$

216327

$\begin{array}{ll}217 & 328\end{array}$

$218 \quad 329$

219330

$220 \quad 331$

221332

$222 \quad 333$

$223 \quad 334$

224335

226337

$227 \quad 338$

228339

0.00

0.00

0.00

0.010 .06

0.00

$\begin{array}{lll}0.01 & 0.06 & 0.00\end{array}$

$\begin{array}{llll}0.03 & 0.08 & -0.01\end{array}$

$\begin{array}{llll}0.03 & 0.08 & -0.01\end{array}$

$\begin{array}{lll}0.03 & 0.10 & 0.00\end{array}$

$\begin{array}{ll}0.34 & 0.00\end{array}$

$0.24 \quad 0.00$

$\begin{array}{ll}0.25 & 0.00\end{array}$

$\begin{array}{lll}0.26 & 0.00 \\ 0.27 & 0.00\end{array}$

$\begin{array}{ll}0.26 & 0.00\end{array}$

$0.26 \quad 0.00$

$0.26 \quad 0.00$

$0.26 \quad 0.00$

$\begin{array}{ll}0.26 & 0.00\end{array}$

$0.26 \quad 0.00$

$\begin{array}{ll}0.25 & 0.00\end{array}$

$\begin{array}{ll}0.26 & 0.00\end{array}$

$0.26 \quad 0.00$

$0.26 \quad 0.00$

$\begin{array}{ll}0.26 & 0.00\end{array}$

$\begin{array}{ll}0.26 & 0.00\end{array}$

$0.26 \quad 0.00$

$0.25 \quad 0.00$

$\begin{array}{ll}0.19 & 0.00\end{array}$

$0.19 \quad 0.00$

$\begin{array}{ll}0.18 & 0.00\end{array}$

$\begin{array}{ll}0.18 & 0.00\end{array}$

$0.18 \quad 0.00$

$0.18 \quad 0.00$

$0.18 \quad 0.00$

$\begin{array}{ll}0.18 & 0.00\end{array}$

$\begin{array}{ll}0.18 & 0.00\end{array}$

$0.18 \quad 0.00$

$0.05 \quad 0.00$

$\begin{array}{ll}0.05 & 0.00\end{array}$

$-0.01 \quad 0.00$

$0.02 \quad 0.00$

$0.00 \quad 0.00$

0.03

0.04
198309

225336

$0.00-0.032$

$\begin{array}{lll}0.00 & -0.021\end{array}$

$0.000 \quad 0.000$

$\begin{array}{lllll}0.000 & -7.47 & -6.82 & 2083.15 & 170.70\end{array}$

$0.00 \quad-0.011$

$0.000 \quad 0.000$

$\begin{array}{llllll}0.000 & -7.24 & -6.57 & 2088.97 & 172.94\end{array}$

$0.00 \quad 0.000$

$0.000 \quad 0.000$

$\begin{array}{lllll}0.000 & -7.50 & -6.80 & 2093.78 & 176.21\end{array}$

$0.00 \quad 0.000$

0.000

0.000

$\begin{array}{llllll}0.000 & -7.14 & -6.45 & 2099.31 & 178.74\end{array}$

$\begin{array}{lll}0.00 & 0.000 & 0.000\end{array}$

0.000

$\begin{array}{llllll}0.000 & -7.25 & -6.56 & 2103.81 & 182.32\end{array}$

0.00

$0.000-0.013$

0.000

$\begin{array}{llllll}0.000 & -6.70 & -6.03 & 2108.96 & 185.23\end{array}$

0.00

$0.000 \quad 0.000$

0.000

$\begin{array}{lllll}0.000 & -5.78 & -5.14 & 2112.27 & 190.00\end{array}$

0.00

$0.001-0.054$

$\begin{array}{llllll}0.000 & -5.01 & -4.42 & 2117.03 & 193.31\end{array}$

$\begin{array}{llllll}0.001 & -4.26 & -3.44 & 2120.07 & 198.35\end{array}$

0.00

$0.000-0.040$

0.000

$\begin{array}{lllll}0.001 & -3.27 & -2.62 & 2124.55 & 201.93\end{array}$

0.00

$0.012-0.081$

0.002

$\begin{array}{lllll}0.002 & -3.05 & -1.93 & 2127.68 & 206.87\end{array}$

0.01

$0.012-0.081$

0.002

$\begin{array}{llllll}0.002 & -2.27 & -1.19 & 2132.06 & 210.57\end{array}$

0.01

$\begin{array}{ll}0.035 & -0.108 \\ 0.035 & -0.108\end{array}$

$\begin{array}{llllll}0.015 & -0.005 & -2.44 & -0.82 & 2135.33 & 215.36\end{array}$

0.02

$0.036-0.134$

0.004

\subsection{1}

$0.07-0.0$

0.262

0.000

$-0.035$

$0.02-0.01$

0.262

0.000

0.012

$0.000-0.001$

$0.02-0.01$

0.273

0.285

$0.03-0.01$

0.000

$0.000-0.008$

0.297

$\begin{array}{lllllll}0.000 & -0.005 & -0.007 & -1.95 & -1.36 & 2167.41 & 247.86\end{array}$
$0.00 \quad 0.285$

$\begin{array}{lllllll}0.000 & -0.007 & -0.007 & -2.18 & -1.54 & 2171.83 & 251.51\end{array}$

$\begin{array}{lllllll}0.000 & -0.007 & -0.007 & -2.68 & -2.00 & 2175.09 & 256.32\end{array}$

$\begin{array}{lllllll}0.000 & -0.019 & -0.010 & -2.84 & -2.02 & 2179.17 & 260.31\end{array}$

$\begin{array}{lllllll}0.000 & -0.019 & -0.010 & -3.05 & -2.20 & 2182.00 & 265.55\end{array}$

$\begin{array}{lll}0.04 & 0.00 & 0.286\end{array}$

$\begin{array}{lllllll}0.000 & -0.019 & -0.010 & -2.85 & -2.04 & 2185.74 & 269.88\end{array}$

$\begin{array}{lllllll}0.000 & -0.031 & -0.013 & -3.25 & -2.24 & 2188.43 & 275.27\end{array}$

$\begin{array}{lllllll}0.000 & -0.033 & -0.012 & -3.00 & -1.97 & 2191.90 & 279.86\end{array}$

$\begin{array}{lllllll}0.000 & -0.043 & -0.016 & -3.50 & -2.26 & 2194.52 & 285.32\end{array}$

$\begin{array}{lllllll}0.000 & -0.056 & -0.009 & -3.61 & -2.16 & 2198.00 & 289.91\end{array}$

$\begin{array}{llllllllll}0.07 & -0.01 & 0.289 & 0.000 & -0.056 & -0.009 & -3.95 & -2.51 & 2200.53 & 295.45\end{array}$

$\begin{array}{llllllllll}0.08 & -0.01 & 0.289 & 0.000 & -0.068 & -0.012 & -4.21 & -2.41 & 2203.86 & 300.19\end{array}$

$\begin{array}{llllllllll}0.08 & -0.01 & 0.289 & 0.000 & -0.068 & -0.012 & -4.58 & -2.79 & 2206.26 & 305.86\end{array}$

$\begin{array}{llllllllll}0.08 & -0.01 & 0.289 & 0.000 & -0.068 & -0.012 & -4.49 & -2.72 & 2209.48 & 310.71\end{array}$

$\begin{array}{llllllllll}0.08 & 0.00 & 0.279 & 0.000 & -0.069 & -0.021 & -4.95 & -3.12 & 2211.75 & 316.52\end{array}$

$\begin{array}{lll}0.03 & 0.01 & 0.207\end{array}$

$\begin{array}{lllllll}0.000 & -0.020 & -0.015 & -3.75 & -2.94 & 2214.70 & 321.64\end{array}$

$\begin{array}{lll}0.03 & 0.01 & 0.207\end{array}$

$\begin{array}{lll}0.02 & 0.02 & 0.196\end{array}$

$\begin{array}{lllllll}0.000 & -0.020 & -0.015 & -4.11 & -3.30 & 2216.79 & 327.62\end{array}$

$\begin{array}{lllllll}0.000 & -0.009 & -0.023 & -4.19 & -3.41 & 2219.89 & 332.59\end{array}$

$\begin{array}{lll}0.03 & 0.02 & 0.196\end{array}$

$\begin{array}{lllllll}0.000 & -0.021 & -0.025 & -4.76 & -3.85 & 2221.92 & 338.64\end{array}$

$\begin{array}{lll}0.03 & 0.02 & 0.196\end{array}$

$\begin{array}{lllllll}0.000 & -0.021 & -0.025 & -4.80 & -3.88 & 2224.79 & 343.83\end{array}$

$\begin{array}{lll}0.03 & 0.02 & 0.196\end{array}$

$\begin{array}{lllllll}0.000 & -0.021 & -0.025 & -5.22 & -4.29 & 2226.65 & 350.05\end{array}$

0.01

0.197

$\begin{array}{lllllll}0.000 & -0.034 & -0.017 & -5.24 & -4.16 & 2229.22 & 355.55\end{array}$

$\begin{array}{lll}0.04 & 0.01 & 0.197\end{array}$

$\begin{array}{lllllll}0.000 & -0.034 & -0.017 & -5.69 & -4.58 & 2230.96 & 361.88\end{array}$

$\begin{array}{lllllll}0.000 & -0.045 & -0.019 & -5.96 & -4.66 & 2233.59 & 367.32\end{array}$

0.01

$\begin{array}{lllllll}0.000 & -0.045 & -0.019 & -6.45 & -5.13 & 2235.24 & 373.74\end{array}$

$\begin{array}{lll}0.02 & 0.00 & 0.054\end{array}$

$\begin{array}{lllllll}0.000 & -0.023 & -0.001 & -4.97 & -4.54 & 2237.07 & 379.98\end{array}$

$\begin{array}{lll}0.02 & -0.01 & 0.053\end{array}$

$0.000-0.023$

$\begin{array}{lllll}0.009 & -5.86 & -5.36 & 2238.94 & 386.18\end{array}$

$\begin{array}{lll}0.00 & 0.00 & -0.011\end{array}$

$0.000 \quad 0.000$

$\begin{array}{lllll}0.000 & -6.72 & -6.16 & 2242.03 & 391.17\end{array}$

$\begin{array}{lll}0.00 & 0.00 & 0.021\end{array}$

$0.000 \quad 0.000$

$\begin{array}{lllll}0.000 & -7.83 & -7.26 & 2244.05 & 397.22\end{array}$

$\begin{array}{lll}0.00 & 0.00 \quad 0.000\end{array}$

0.000

0.000

$\begin{array}{lllll}0.000 & -8.78 & -8.16 & 2247.09 & 402.25\end{array}$

\section{$Z=112(\mathrm{Cn})$}

\section{$150262-0.20-0.00$}

$\begin{array}{llll}151 & 263 & 0.20 & 0.00\end{array}$

$\begin{array}{llll}152 & 264 & 0.22 & 0.00\end{array}$

0.02

$0.02 \quad 0.218$

$\begin{array}{lllllll}0.000 & -0.005 & -0.023 & -3.53 & -2.66 & 1875.59 & 151.47\end{array}$

$\begin{array}{lll}0.03 & 0.02 & 0.219\end{array}$$$
0.000
$$

$\begin{array}{lllllll}0.000 & -0.017 & -0.025 & -4.14 & -3.15 & 1884.10 & 151.04\end{array}$ $\begin{array}{lll}0.04 & 0.01 & 0.24\end{array}$

$\begin{array}{lllllll}0.000 & -0.026 & -0.019 & -4.22 & -3.23 & 1893.86 & 149.34\end{array}$

153265

$\begin{array}{ll}0.20 & 0.00\end{array}$

0.04

$0.01 \quad 0.219$

$\begin{array}{lllllll}0.000 & -0.030 & -0.018 & -4.54 & -3.44 & 1901.83 & 149.45\end{array}$

$\begin{array}{lllllll}0.000 & -0.030 & -0.018 & -4.61 & -3.52 & 1911.32 & 148.02\end{array}$

\begin{tabular}{lllllll}
155 & 267 & 0.19 & 0.00 & 0.04 & 0.01 & 0.208 \\
\hline
\end{tabular}

$-6.82 \quad 173.45$

$-6.57 \quad 175.74$

$-6.80179 .03$

$-6.45181 .61$

$-6.56185 .23$

$-6.04188 .19$

$-5.14 \quad 193.01$

$-4.42196 .36$

$-3.44 \quad 201.47$

$-2.62 \quad 205.11$

$-1.91 \quad 210.13$

$-1.17 \quad 213.89$

$-0.77 \quad 218.78$

$\begin{array}{ll}-0.20 & 222.57\end{array}$

$-0.21 \quad 227.24$

\begin{tabular}{ll}
$-0.11 \quad 230.74$ \\
\hline
\end{tabular}

$-0.39 \quad 235.31$

$-0.40 \quad 238.89$

$-0.77 \quad 243.55$

$-0.89 \quad 247.18$

$-1.42 \quad 251.86$

$-1.59 \quad 255.63$

$-2.06 \quad 260.54$ 


\section{$Z=112(\mathrm{Cn})$}

$\begin{array}{llll}156 & 268 & 0.19 & 0.00\end{array}$ $\begin{array}{llll}157 & 269 & 0.19 & 0.00\end{array}$ $\begin{array}{llll}158 & 270 & 0.19 & 0.00\end{array}$ $\begin{array}{lllll}159 & 271 & 0.19 & 0.00\end{array}$ $\begin{array}{llll}160 & 272 & 0.19 & 0.00\end{array}$

$\begin{array}{lllll}161 & 273 & 0.20 & 0.00\end{array}$

$\begin{array}{llll}162 & 274 & 0.20 & 0.00\end{array}$

$\begin{array}{llll}163 & 275 & 0.20 & 0.00\end{array}$

$\begin{array}{llll}164 & 276 & 0.19 & 0.00\end{array}$

165277

$\begin{array}{ll}0.18 & 0.00\end{array}$

166278

$\begin{array}{lll}0.17 & 0.00\end{array}$

167279

168280

$\begin{array}{ll}0.16 & 0.00\end{array}$

$\begin{array}{ll}0.13 & 0.00\end{array}$

169281

170282

$\begin{array}{lll}0.08 & 0.00\end{array}$

$\begin{array}{ll}0.08 & 0.00\end{array}$

$171 \quad 283$
172

$172 \quad 284$

173285

$174 \quad 286$

$175 \quad 287$

$176 \quad 288$

$177 \quad 289$

178290

17929

$180 \quad 292$

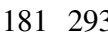

$182 \quad 294$

183295

184296

$185 \quad 297$

$186 \quad 298$

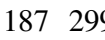

$188 \quad 300$

189301

190302

191303

192304

193305

194306

195307

196308

197309

$198 \quad 310$

199311

200312

201313

$202 \quad 314$

$203 \quad 315$

204316

$205 \quad 317$

206318

$207 \quad 319$

$208 \quad 320$

$209 \quad 321$

$210 \quad 322$

211323

$212 \quad 324$

$\begin{array}{ll}0.08 & 0.00\end{array}$

$0.08 \quad 0.00$

$0.08 \quad 0.00$

$\begin{array}{ll}0.07 & 0.00\end{array}$

$0.07 \quad 0.00$
0.06

$\begin{array}{ll}0.06 & 0.00\end{array}$

$\begin{array}{ll}0.04 & 0.00\end{array}$

$\begin{array}{ll}0.00 & 0.00\end{array}$

$\begin{array}{ll}0.00 & 0.00\end{array}$

$\begin{array}{ll}0.00 & 0.00\end{array}$

$\begin{array}{ll}0.00 & 0.00\end{array}$

$\begin{array}{ll}0.00 & 0.00\end{array}$

$\begin{array}{lll}0.00 & 0.00\end{array}$

$\begin{array}{ll}0.00 & 0.01\end{array}$

$\begin{array}{ll}0.00 & 0.00\end{array}$

$\begin{array}{ll}0.01 & 0.02\end{array}$

$\begin{array}{ll}0.00 & 0.00\end{array}$

$0.01 \quad 0.03$

$\begin{array}{lll}0.00 & 0.01\end{array}$

$\begin{array}{ll}0.26 & 0.00\end{array}$

$\begin{array}{ll}0.26 & 0.00\end{array}$

$\begin{array}{ll}0.26 & 0.00\end{array}$

$0.27 \quad 0.00$

$0.27 \quad 0.00$

$\begin{array}{ll}0.27 \quad 0.00 \\ 0.27 & 0.00\end{array}$

$\begin{array}{ll}0.27 & 0.00\end{array}$

0.260 .00

$0.26 \quad 0.00$

0.260 .00

0.260 .00

0.260 .00

0.260 .00

0.260 .00

$0.26 \quad 0.00$ $\begin{array}{llllllllll}0.05 & 0.00 & 0.208 & 0.000 & -0.045 & -0.010 & -5.13 & -3.86 & 1928.33 & 147.16\end{array}$

$\begin{array}{llllllllll}0.05 & 0.00 & 0.208 & 0.000 & -0.045 & -0.010 & -5.55 & -4.27 & 1935.98 & 147.58\end{array}$

$\begin{array}{llllllllll}0.06 & 0.00 & 0.209 & 0.000 & -0.057 & -0.012 & -6.01 & -4.44 & 1945.05 & 146.58\end{array}$

$\begin{array}{lll}0.06 & 0.00 & 0.209\end{array}$

$\begin{array}{lll}0.07 & -0.01 & 0.209\end{array}$

$0.08-0.01$

$0.09-0.02$

$0.09-0.02$

$0.09-0.02$

$0.08-0.02$

$0.07-0.01$

$0.06-0.01$

$0.04 \quad 0.00$

$0.00 \quad 0.01$

$\begin{array}{ll}0.01 & 0.01\end{array}$

0.221

0.222

0.222

0.210

0.198

$\begin{array}{lllllll}0.000 & -0.057 & -0.012 & -6.56 & -4.89 & 1952.51 & 147.20\end{array}$

$\begin{array}{lllllll}0.000 & -0.070 & -0.004 & -7.13 & -5.15 & 1961.42 & 146.35\end{array}$

$\begin{array}{lllllll}0.000 & -0.080 & -0.007 & -8.03 & -5.64 & 1968.67 & 147.18\end{array}$

$\begin{array}{lllllll}0.000 & -0.093 & 0.000 & -8.75 & -5.85 & 1977.29 & 146.63\end{array}$

$\begin{array}{lllllll}0.000 & -0.093 & 0.000 & -9.07 & -6.11 & 1984.07 & 147.92\end{array}$

$\begin{array}{lllllll}0.000 & -0.094 & 0.001 & -8.80 & -5.84 & 1991.96 & 148.10\end{array}$

$\begin{array}{lllllll}0.000 & -0.084 & 0.004 & -8.18 & -5.71 & 1998.13 & 150.00\end{array}$

$\begin{array}{llllllll}0.187 & 0.000 & -0.072 & -0.003 & -7.22 & -5.19 & 2005.54 & 150.67\end{array}$

0.175

0.141

$\begin{array}{lllllll}0.000 & -0.062 & -0.001 & -6.86 & -5.24 & 2011.65 & 152.62\end{array}$

$\begin{array}{lllllll}0.000 & -0.041 & -0.006 & -6.16 & -5.09 & 2019.20 & 153.15\end{array}$

$\begin{array}{lllllll}0.000 & 0.003 & -0.010 & -6.26 & -5.54 & 2025.50 & 154.92\end{array}$

$\begin{array}{lllllll}0.000 & -0.009 & -0.011 & -6.43 & -5.68 & 2033.11 & 155.38\end{array}$

$0.01 \quad 0.01$

0.086

$-0.01 \quad 0.00$

$0.02 \quad 0.00$

0.086

$\begin{array}{llllllll}0.000 & -0.009 & -0.011 & -6.93 & -6.15 & 2039.21 & 157.35\end{array}$

$\begin{array}{ll}0.03 \quad 0.00 \\ 0.03 & -0.01\end{array}$

$0.03-0.01$

$0.04-0.01$

0.086

$\begin{array}{lllllll}0.000 & -0.021 & -0.002 & -7.00 & -6.19 & 2046.49 & 158.14\end{array}$

$0.03-0.01$

$0.01 \quad 0.00$

$0.00 \quad 0.00$

$\begin{array}{lll}0.00 & 0.00 & 0.000\end{array}$

$0.000-0.033-0.4$

$\begin{array}{lllll}-0.003 & -7.61 & -6.63 & 2052.34 & 160.36\end{array}$

0.075

$0.000-0.034$

$\begin{array}{lllll}0.008 & -7.61 & -6.62 & 2059.36 & 161.41\end{array}$

0.075

$0.000-0.046$

$\begin{array}{llllll}0.007 & -8.20 & -6.99 & 2064.93 & 163.91\end{array}$

0.064

$0.000-0.034$

$\begin{array}{llllll}0.008 & -7.75 & -6.78 & 2071.54 & 165.38\end{array}$

$\begin{array}{lllllll}0.000 & -0.011 & -0.000 & -7.82 & -7.10 & 2076.86 & 168.12\end{array}$

$\begin{array}{lllllll}0.000 & 0.000 & 0.000 & -7.83 & -7.14 & 2083.50 & 169.55\end{array}$

$\begin{array}{lllllll}0.000 & 0.000 & 0.000 & -8.21 & -7.49 & 2088.66 & 172.48\end{array}$

$\begin{array}{lllllll}0.000 & 0.000 & 0.000 & -8.00 & -7.27 & 2094.83 & 174.37\end{array}$

$\begin{array}{lll}0.00 & 0.00 & 0.000\end{array}$

0.000

0.000

$\begin{array}{llllll}0.000 & -8.29 & -7.55 & 2099.70 & 177.57\end{array}$

$0.00 \quad 0.00$

$\begin{array}{ll}0.00 & 0.00\end{array}$

0.000

0.000

0.000

$\begin{array}{lllll}0.000 & -7.92 & -7.19 & 2105.55 & 179.80\end{array}$

$\begin{array}{lll}0.00 & 0.00\end{array}$

$0.00 \quad 0.00$
0.00

0.000

0.000

0.000

$\begin{array}{llllll}0.000 & -8.01 & -7.28 & 2110.04 & 183.37\end{array}$

$0.00 \quad 0.00$

0.000

$\begin{array}{llllll}0.000 & -7.45 & -6.74 & 2115.50 & 185.99\end{array}$

$\begin{array}{lll}0.000 & 0.000 & 0.000\end{array}$

$\begin{array}{lllll}-6.50 & -5.82 & 2118.80 & 190.76\end{array}$

$\begin{array}{ll}0.00 \quad 0.00 \\ 0.00 & 0.00\end{array}$

$\begin{array}{lll}0.011 & -0.027\end{array}$

0.000

$\begin{array}{llllll}0.000 & -5.74 & -5.11 & 2123.89 & 193.74\end{array}$

$0.00 \quad 0.00$

$\begin{array}{ll}0.00 & 0.00\end{array}$

0.00

0.00

$\begin{array}{llll}0.03 & 0.07 & -0.01\end{array}$

0.01

$\begin{array}{llll}0.03 & 0.07 & -0.01 & 0.01\end{array}$

$\begin{array}{llll}0.04 & 0.09 & -0.01 & 0.02\end{array}$

$\begin{array}{ll}0.000 & 0.000\end{array}$

0.000

$\begin{array}{llllll}0.000 & -4.63 & -3.98 & 2126.78 & 198.92\end{array}$

$0.011-0.040$

0.000

$\begin{array}{llllll}0.000 & -3.78 & -3.25 & 2131.67 & 202.11\end{array}$

$0.000-0.013$

0.000

$\begin{array}{lllll}0.001 & -2.95 & -2.30 & 2134.56 & 207.28\end{array}$

$0.034-0.094$

$\begin{array}{lllllll}0.014 & -0.006 & -2.34 & -0.98 & 2142.33 & 215.66\end{array}$

$\begin{array}{llll}0.08 & 0.00 & -0.04 & -0.02\end{array}$

$\begin{array}{rrrr}0.49 & 0.00 & 0.00 & 0.02\end{array}$

$0.01-0.01$

$0.034-0.094$

$\begin{array}{llllll}0.014 & -0.006 & -1.70 & -0.39 & 2146.97 & 219.09\end{array}$

$0.046-0.121$

$\begin{array}{llllll}0.016 & -0.014 & -2.00 & -0.08 & 2150.15 & 223.98\end{array}$

$0.086 \quad 0.000$

0.05

$\begin{array}{llllll}0.025 & -0.53 & 0.40 & 2154.73 & 227.47\end{array}$

$0.551 \quad 0.000$
0.000

0.116

$\begin{array}{llllll}0.007 & -1.91 & -2.53 & 2160.97 & 229.30\end{array}$

$0.02-0.01$

0.284

0.000

0.016

$\begin{array}{llllll}0.010 & -0.54 & -0.23 & 2163.56 & 234.79\end{array}$

$0.28 \quad 0.00$

$0.02-0.01$

0.285

$0.000 \quad 0.004$

$\begin{array}{llllll}0.007 & -0.98 & -0.61 & 2167.07 & 239.34\end{array}$

0.285

$0.000 \quad 0.004$

$\begin{array}{lllll}0.007 & -1.10 & -0.71 & 2171.88 & 242.61\end{array}$

$0.03 \quad 0.00$

0.308

$\begin{array}{lllllll}0.000 & -0.002 & -0.006 & -1.58 & -1.17 & 2175.32 & 247.23\end{array}$

$\begin{array}{lll}0.03 & 0.00\end{array}$

0.297

0.000

$\begin{array}{lllllll}0.000 & -0.005 & -0.007 & -2.29 & -1.76 & 2183.26 & 255.44\end{array}$

0.297

$\begin{array}{lllllll}0.000 & -0.005 & -0.007 & -2.30 & -1.78 & 2187.65 & 259.13\end{array}$

$\begin{array}{llll}0.04 & 0.00 & 0.298\end{array}$

$\begin{array}{lllllll}0.000 & -0.017 & -0.010 & -2.60 & -1.92 & 2190.44 & 264.40\end{array}$

$0.04 \quad 0.00$

0.286

$\begin{array}{lllllll}0.000 & -0.019 & -0.010 & -2.42 & -1.73 & 2194.44 & 268.48\end{array}$

$0.05 \quad 0.00$

0.287

$\begin{array}{lllllll}0.000 & -0.031 & -0.013 & -2.48 & -1.63 & 2200.87 & 278.18 \\ 0.000 & -0.045 & -0.006 & -2.95 & -1.84 & 2203.42 & 283.71\end{array}$

$0.06-0.01$

0.288

$0.07-0.01$

0.289

$\begin{array}{lllllll}0.000 & -0.056 & -0.009 & -3.07 & -1.72 & 2207.18 & 288.02\end{array}$

$0.07-0.01$

0.289

$\begin{array}{lllllll}0.000 & -0.056 & -0.009 & -3.42 & -2.07 & 2209.71 & 293.56\end{array}$

$0.08-0.01$

0.289

$\begin{array}{lllllll}0.000 & -0.068 & -0.012 & -3.67 & -1.96 & 2213.32 & 298.02\end{array}$

$0.08-0.01$

0.289

$\begin{array}{lllllll}0.000 & -0.068 & -0.012 & -4.04 & -2.34 & 2215.73 & 303.68\end{array}$

0.289

$\begin{array}{lllllll}0.000 & -0.068 & -0.012 & -3.96 & -2.22 & 2219.19 & 308.29\end{array}$

$\begin{array}{ll}-3.91 & 150.29\end{array}$

$-4.32 \quad 150.66$

$\begin{array}{ll}-4.47 & 149.63\end{array}$

$\begin{array}{ll}-4.93 & 150.20\end{array}$

$\begin{array}{ll}-5.18 & 149.33\end{array}$

$-5.68 \quad 150.11$ 
$\boldsymbol{Z}=\mathbf{1 1 2}(\mathrm{Cn})$

\section{$\begin{array}{llll}213 & 325 & 0.19 & 0.00\end{array}$}

214326 $0.18 \quad 0.00$

$\begin{array}{ll}215 & 327\end{array}$

$0.18 \quad 0.00$

$\begin{array}{ll}0.17 & 0.00\end{array}$

$0.17 \quad 0.00$

217329

218330

219331

$0.17 \quad 0.00$

$\begin{array}{ll}0.17 & 0.00\end{array}$

220332

221333

$222 \quad 334$

$223 \quad 335$

224336

225337

226338

$227 \quad 339$

$\begin{array}{ll}0.17 & 0.00\end{array}$

$0.17 \quad 0.00$

$\begin{array}{ll}0.05 & 0.00\end{array}$

$\begin{array}{ll}0.05 & 0.00\end{array}$

$0.02 \quad 0.00$

$\begin{array}{ll}0.00 & 0.00\end{array}$

$0.01 \quad 0.00$

\subsection{2}

0.03

0.02

0.02

0.03

0.03

$\begin{array}{lll}0.17 & 0.00 & 0.03\end{array}$

0.04

0.04

0.02

$Z=113$

153266

154267

$155 \quad 268$

$\begin{array}{ll}156 & 269\end{array}$

$157 \quad 270$

$\begin{array}{ll}158 & 271\end{array}$

$159 \quad 272$

$\begin{array}{lll}160 & 273\end{array}$

$161 \quad 274$

$162 \quad 275$

163276

$\begin{array}{lll}164 & 277\end{array}$

$\begin{array}{ll}165 & 278\end{array}$

$\begin{array}{lll}166 & 279\end{array}$

$167 \quad 280$

$\begin{array}{ll}0.19 & 0.00\end{array}$

$\begin{array}{ll}0.18 & 0.00\end{array}$

$\begin{array}{ll}0.19 & 0.00\end{array}$

$0.18 \quad 0.00$

$0.18 \quad 0.00$

$0.18 \quad 0.00$

$0.18 \quad 0.00$

$\begin{array}{ll}0.19 & 0.00\end{array}$

$\begin{array}{ll}0.19 & 0.00\end{array}$

0.190 .00

$\begin{array}{ll}0.19 & 0.00\end{array}$

$0.18 \quad 0.00$

$\begin{array}{ll}0.18 & 0.00\end{array}$

0.01

0.00

0.00

0.01
$0.01 \quad 0.207$

$0.01 \quad 0.195$

$0.01 \quad 0.196$

$0.01 \quad 0.184$

$0.01 \quad 0.184$

$0.01 \quad 0.185$

$0.01 \quad 0.185$

$0.01 \quad 0.185$

$0.01 \quad 0.185$

$0.01 \quad 0.185$

$0.00 \quad 0.054$

$0.00 \quad 0.053$

$0.00 \quad 0.021$

$0.00 \quad 0.000$

$0.00 \quad 0.011$ $\begin{array}{lllllll}0.000 & -0.020 & -0.015 & -3.29 & -2.50 & 2221.35 & 314.21\end{array}$

$\begin{array}{lllllll}0.000 & -0.010 & -0.013 & -3.16 & -2.50 & 2224.76 & 318.86\end{array}$

$\begin{array}{lllllll}0.000 & -0.022 & -0.015 & -3.69 & -2.90 & 2226.91 & 324.79\end{array}$

$\begin{array}{lllllll}0.000 & -0.011 & -0.013 & -3.70 & -3.05 & 2230.33 & 329.44\end{array}$

$\begin{array}{lllllll}0.000 & -0.011 & -0.013 & -4.10 & -3.43 & 2232.31 & 335.53\end{array}$

$\begin{array}{lllllll}0.000 & -0.023 & -0.015 & -4.28 & -3.53 & 2235.52 & 340.39\end{array}$

$\begin{array}{lllllll}0.000 & -0.023 & -0.015 & -4.69 & -3.78 & 2237.23 & 346.76\end{array}$

$\begin{array}{lllllll}0.000 & -0.023 & -0.015 & -4.68 & -3.78 & 2240.21 & 351.84\end{array}$

$\begin{array}{lllllll}0.000 & -0.035 & -0.017 & -5.33 & -4.24 & 2241.99 & 358.14\end{array}$

$\begin{array}{lllllll}0.000 & -0.035 & -0.017 & -5.36 & -4.28 & 2244.87 & 363.33\end{array}$

$\begin{array}{lllllll}0.000 & -0.023 & -0.001 & -4.93 & -4.49 & 2246.26 & 370.01\end{array}$

$\begin{array}{lllllll}0.000 & -0.011 & -0.001 & -5.45 & -5.01 & 2249.48 & 374.86\end{array}$

$\begin{array}{lllllll}0.000 & 0.000 & 0.000 & -6.38 & -5.87 & 2251.39 & 381.02\end{array}$

$\begin{array}{llllllll}0.000 & 0.000 & 0.000 & -7.42 & -6.84 & 2254.92 & 385.57\end{array}$

$\begin{array}{lllllll}0.000 & -0.012 & -0.000 & -8.56 & -7.95 & 2256.95 & 391.61\end{array}$
$-2.48 \quad 320.00$

$\begin{array}{ll}-2.49 & 324.79\end{array}$

$-2.88 \quad 330.89$

$-3.05 \quad 335.68$

$-3.43 \quad 341.93$

$-3.50 \quad 346.97$

$-3.75 \quad 353.50$

$-3.76 \quad 358.76$

$-4.18 \quad 365.26$

$-4.22 \quad 370.63$

$-4.48 \quad 377.44$

$-5.01 \quad 382.47$

$-5.88 \quad 388.82$

$-6.84 \quad 393.56$

$-7.95 \quad 399.80$

$-3.16 \quad 162.53$

$-3.25 \quad 161.01$

$-3.54 \quad 160.88$

$-3.66159 .58$

$-4.10 \quad 159.58$

$-4.25158 .52$

$-4.77158 .68$

$-5.00 \quad 157.80$

$-5.49 \quad 158.24$

$-5.69157 .64$

$-6.04 \quad 158.47$

$-5.82 \quad 158.53$

$\begin{array}{ll}-5.71 & 160.07\end{array}$

$-5.82160 .05$

$-5.93161 .58$

$-6.01 \quad 161.84$

$\begin{array}{ll}-6.44 & 163.29\end{array}$

$-6.52163 .78$

$-6.96 \quad 165.45$

$-6.97166 .23$

$\begin{array}{ll}-7.40 & 168.13\end{array}$

$\begin{array}{ll}-7.37 & 169.18\end{array}$

$-7.74 \quad 171.37$

$-7.64 \quad 172.71$

$-8.08 \quad 175.03$

$-8.06 \quad 176.52$

$-8.36179 .18$

$-8.08 \quad 181.14$

$-8.28 \quad 184.12$

$\begin{array}{ll}-7.89 & 186.40\end{array}$

$\begin{array}{ll}-7.95 & 189.72\end{array}$

$\begin{array}{ll}-7.40 & 192.37\end{array}$

$-6.48 \quad 196.87$

\begin{tabular}{ll}
$-5.77 \quad 199.87$ \\
\hline
\end{tabular}

$-4.64 \quad 204.78$

$-3.91 \quad 208.00$

$-2.93 \quad 212.95$

$-2.23 \quad 216.34$

$-1.48 \quad 221.25$

$-0.84 \quad 224.76$

$-0.47 \quad 229.48$ 


\section{$Z=113$}

194307 195308 $\begin{array}{lllll}0.08 & 0.00 & -0.04 & -0.02\end{array}$ 196309 197310 $\begin{array}{lllll}0.09 & 0.00 & -0.04 & -0.02\end{array}$ $\begin{array}{lllll}0.10 & 0.00 & -0.04 & -0.01\end{array}$ 198311 $\begin{array}{llll}0.48 & 0.00 & 0.02 & 0.01\end{array}$ $\begin{array}{lllll}0.27 & 0.00 & 0.02 & -0.01\end{array}$

199312 $\begin{array}{ll}0.28 & 0.00\end{array}$

$0.03 \quad 0.00$

200313

201314

$0.27 \quad 0.00$

$0.02 \quad 0.00$

202315

$0.27 \quad 0.00$

$0.27 \quad 0.00$

0.03

0.00

$0.27 \quad 0.00$

0.04

0.00

$203 \quad 316$

204317

$0.27 \quad 0.00$

0.04

0.00

205318

$\begin{array}{ll}0.26 & 0.00\end{array}$

0.04

0.00
$0.26 \quad 0.00$

206319

$\begin{array}{ll}0.26 & 0.00\end{array}$

$208 \quad 321$

$0.26 \quad 0.00$

$209 \quad 322$

$\begin{array}{ll}0.26 & 0.00\end{array}$

210323

$\begin{array}{ll}0.26 & 0.00\end{array}$

211324

$212 \quad 325$

$\begin{array}{ll}0.26 & 0.00\end{array}$

$\begin{array}{ll}0.26 & 0.00\end{array}$

$213 \quad 326$

$\begin{array}{ll}0.17 & 0.00\end{array}$

214327

215328

216329

$217 \quad 330$

218331

219332

$\begin{array}{ll}0.16 & 0.00\end{array}$

$\begin{array}{ll}0.16 & 0.00\end{array}$

$\begin{array}{ll}0.16 & 0.00\end{array}$

$\begin{array}{ll}0.16 & 0.00\end{array}$

$\begin{array}{ll}0.16 & 0.00\end{array}$

220333

$\begin{array}{ll}0.16 & 0.00\end{array}$

$\begin{array}{ll}0.16 & 0.00\end{array}$

$221 \quad 334$

$\begin{array}{ll}0.16 & 0.00\end{array}$

$222 \quad 335$

$223 \quad 336$

$\begin{array}{ll}0.07 & 0.00\end{array}$

$0.05 \quad 0.00$

224337

225338

$\begin{array}{ll}0.05 \quad 0.00 \\ 0.02 & 0.00\end{array}$

$0.02 \quad 0.00$

$\begin{array}{ll}0.01 & 0.00\end{array}$

$0.05 \quad 0.00$

$\begin{array}{lll}0.06 & -0.01\end{array}$

$\begin{array}{lll}0.06 & -0.01\end{array}$

$\begin{array}{lll}0.07 & -0.01\end{array}$

$0.07-0.01$

$\begin{array}{ll}0.08 & -0.01\end{array}$

$\begin{array}{ll}0.08 & -0.01\end{array}$

$0.01 \quad 0.02$

$\begin{array}{ll}0.01 & 0.02\end{array}$

$\begin{array}{ll}0.02 & 0.01\end{array}$

$0.02 \quad 0.01$

$0.02 \quad 0.01$

$\begin{array}{ll}0.02 & 0.01\end{array}$

$0.02 \quad 0.01$

$\begin{array}{ll}0.03 & 0.01\end{array}$

$0.03 \quad 0.01$

$\begin{array}{ll}0.03 & -0.01\end{array}$

$0.02 \quad 0.00$

226339

\section{$Z=114(\mathrm{Fl})$}

$\begin{array}{llll}155 & 269 & 0.18 & 0.00\end{array}$

$\begin{array}{llll}156 & 270 & 0.17 & 0.00\end{array}$

$\begin{array}{llll}157 & 271 & 0.17 & 0.00\end{array}$

$\begin{array}{llll}158 & 272 & 0.17 & 0.00\end{array}$

$\begin{array}{ll}0.01 & 0.00\end{array}$

$0.00 \quad 0.00$

$0.00 \quad 0.00$ $\begin{array}{llllllll}0.086 & 0.000 & 0.051 & 0.025 & -1.17 & -0.19 & 2160.40 & 229.09\end{array}$ $\begin{array}{llllllll}0.097 & 0.000 & 0.052 & 0.026 & -1.09 & -0.08 & 2163.90 & 233.67\end{array}$ $\begin{array}{llllllll}0.107 & 0.000 & 0.053 & 0.016 & -0.75 & 0.15 & 2168.57 & 237.07\end{array}$ $\begin{array}{llllllll}0.541 & 0.000 & 0.085 & 0.001 & -2.14 & -3.17 & 2175.32 & 238.38\end{array}$ $\begin{array}{llllllll}0.296 & 0.000 & 0.006 & 0.007 & -1.04 & -0.78 & 2177.65 & 244.12\end{array}$ $\begin{array}{llllllll}0.308 & 0.000 & -0.002 & -0.006 & -1.55 & -1.24 & 2181.39 & 248.46\end{array}$ $\begin{array}{llllllll}0.296 & 0.000 & 0.007 & -0.003 & -1.66 & -1.36 & 2186.06 & 251.86\end{array}$ $\begin{array}{llllllll}0.297 & 0.000 & -0.005 & -0.007 & -2.21 & -1.77 & 2189.58 & 256.41\end{array}$ $\begin{array}{llllllll}0.297 & 0.000 & -0.005 & -0.007 & -2.23 & -1.80 & 2193.98 & 260.08\end{array}$ $\begin{array}{llllllll}0.298 & 0.000 & -0.017 & -0.010 & -2.52 & -1.92 & 2197.05 & 265.08\end{array}$ $\begin{array}{llllllll}0.298 & 0.000 & -0.017 & -0.010 & -2.26 & -1.71 & 2201.05 & 269.15\end{array}$ $\begin{array}{llllllll}0.286 & 0.000 & -0.019 & -0.010 & -2.41 & -1.81 & 2203.94 & 274.34\end{array}$ $\begin{array}{llllllll}0.287 & 0.000 & -0.031 & -0.013 & -2.33 & -1.55 & 2207.73 & 278.61\end{array}$ $\begin{array}{llllllll}0.288 & 0.000 & -0.045 & -0.006 & -2.77 & -1.74 & 2210.54 & 283.87\end{array}$ $\begin{array}{llllllll}0.288 & 0.000 & -0.045 & -0.006 & -2.57 & -1.59 & 2214.28 & 288.21\end{array}$ $\begin{array}{llllllll}0.289 & 0.000 & -0.056 & -0.009 & -3.21 & -1.91 & 2217.08 & 293.48\end{array}$ $\begin{array}{llllllll}0.289 & 0.000 & -0.056 & -0.009 & -3.06 & -1.72 & 2220.62 & 298.01\end{array}$ $\begin{array}{llllllll}0.289 & 0.000 & -0.068 & -0.012 & -3.82 & -2.12 & 2223.34 & 303.37\end{array}$ $\begin{array}{llllllll}0.289 & 0.000 & -0.068 & -0.012 & -3.85 & -2.14 & 2226.94 & 307.84\end{array}$ $\begin{array}{llllllll}0.184 & 0.000 & 0.002 & -0.021 & -3.14 & -2.44 & 2229.40 & 313.44\end{array}$ $\begin{array}{llllllll}0.173 & 0.000 & 0.000 & -0.021 & -3.10 & -2.44 & 2232.83 & 318.09\end{array}$ $\begin{array}{llllllll}0.173 & 0.000 & -0.013 & -0.013 & -3.44 & -2.79 & 2235.20 & 323.78\end{array}$ $\begin{array}{llllllll}0.173 & 0.000 & -0.013 & -0.013 & -3.57 & -2.88 & 2238.57 & 328.49\end{array}$ $\begin{array}{llllllll}0.173 & 0.000 & -0.013 & -0.013 & -3.98 & -3.28 & 2240.84 & 334.29\end{array}$ $\begin{array}{llllllll}0.173 & 0.000 & -0.013 & -0.013 & -4.00 & -3.33 & 2244.03 & 339.17\end{array}$ $\begin{array}{llllllll}0.173 & 0.000 & -0.013 & -0.013 & -4.35 & -3.68 & 2246.12 & 345.16\end{array}$ $\begin{array}{llllllll}0.174 & 0.000 & -0.024 & -0.015 & -4.52 & -3.75 & 2249.17 & 350.18\end{array}$ $\begin{array}{llllllll}0.174 & 0.000 & -0.024 & -0.015 & -4.94 & -4.17 & 2251.19 & 356.23\end{array}$ $\begin{array}{llllllll}0.075 & 0.000 & -0.034 & 0.008 & -5.01 & -4.38 & 2254.23 & 361.26\end{array}$ $\begin{array}{llllllll}0.054 & 0.000 & -0.023 & -0.001 & -5.70 & -5.22 & 2256.53 & 367.02\end{array}$ $\begin{array}{llllllll}0.053 & 0.000 & -0.011 & -0.001 & -6.23 & -5.76 & 2259.78 & 371.85\end{array}$ $\begin{array}{llllllll}0.021 & 0.000 & 0.000 & 0.000 & -7.18 & -6.63 & 2261.97 & 377.73\end{array}$ $\begin{array}{llllllll}0.011 & 0.000 & 0.000 & 0.000 & -8.18 & -7.58 & 2265.49 & 382.29\end{array}$
$-0.10 \quad 232.92$

$0.02 \quad 237.57$

$0.21 \quad 241.01$

$-3.42 \quad 242.09$

$-0.83 \quad 248.12$

$-1.31 \quad 252.52$

$-1.42 \quad 256.02$

$-1.84 \quad 260.66$

$-1.85 \quad 264.44$

$-1.98 \quad 269.55$

$-1.75 \quad 273.73$

$-1.86 \quad 279.02$

$-1.57 \quad 283.44$

$-1.75 \quad 288.82$

$-1.59 \quad 293.29$

$-1.90 \quad 298.70$

$-1.69303 .38$

$\begin{array}{ll}-2.06 & 308.89\end{array}$

$-2.07 \quad 313.51$

$-2.40 \quad 319.22$

$\begin{array}{ll}-2.39 & 324.02\end{array}$

$\begin{array}{ll}-2.79 & 329.81\end{array}$

$-2.87 \quad 334.67$

$-3.28 \quad 340.61$

$-3.33 \quad 345.66$

$-3.68 \quad 351.80$

$-3.72 \quad 357.01$

$-4.15 \quad 363.23$

$-4.33 \quad 368.45$

$-5.21 \quad 374.36$

$\begin{array}{ll}-5.76 & 379.35\end{array}$

$-6.64 \quad 385.41$

$-7.58 \quad 390.15$

$\begin{array}{ll}-3.09 & 169.14\end{array}$

$-3.20 \quad 167.51$

$-3.60 \quad 167.50$

$-3.74 \quad 166.10$

$-4.23 \quad 166.27$

$-4.42 \quad 165.08$

$-4.92 \quad 165.47$

$\begin{array}{ll}-5.06 & 164.59\end{array}$

$-5.46 \quad 165.33$

$-6.15 \quad 164.15$

$-6.44165 .25$

$-6.59164 .85$

$-6.81 \quad 166.25$

$-6.78166 .28$

$-6.83168 .08$

$\begin{array}{ll}-6.86 & 168.29\end{array}$

$-7.26169 .96$

$-7.24 \quad 170.45$

$-7.65 \quad 172.34$

$-7.78 \quad 172.90$

$\begin{array}{ll}-8.19 & 175.01\end{array}$

$-8.28175 .84$

$\begin{array}{ll}-8.59 & 178.27\end{array}$ 


\section{$Z=114(\mathrm{Fl})$}

\begin{tabular}{|c|c|c|c|c|c|c|c|c|c|c|c|c|c|c|c|}
\hline & & & & & & & & & & & & & & & \\
\hline 178 & 292 & 0.00 & 0.00 & 0.00 & 0.00 & 0.000 & 0.000 & 0.000 & 0.000 & -9.45 & -8.68 & 2091.52 & 176.12 & -8.69 & 179.31 \\
\hline 179 & 293 & 0.00 & 0.00 & 0.00 & 0.00 & 0.000 & 0.000 & 0.000 & 0.000 & -9.71 & -8.93 & 2096.90 & 178.81 & -8.93 & 182.01 \\
\hline 180 & 294 & 0.00 & 0.00 & 0.00 & 0.00 & 0.000 & 0.000 & 0.000 & 0.000 & -9.44 & -8.66 & 2103.36 & 180.41 & -8.66 & 183.63 \\
\hline 1 & 295 & 0.00 & 0.00 & 0.00 & 0.00 & 0.000 & 0.000 & 0.000 & 0.000 & -9.66 & -8.87 & 2108.51 & 183.34 & -8.87 & 186.58 \\
\hline 182 & 296 & 0.00 & 0.00 & 0.00 & 0.00 & 0.000 & 0.000 & 0.000 & 0.000 & -9.26 & -8.48 & 2114.66 & 185.26 & -8.49 & 188.53 \\
\hline 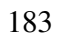 & 297 & 0.00 & 0.00 & 0.00 & 0.00 & 0.000 & 0.000 & 0.000 & 0.000 & -9.29 & -8.51 & 2119.43 & 188.57 & -8.52 & 191.86 \\
\hline & 298 & 0.00 & 0.00 & 0.00 & 0.00 & 0.000 & 0.000 & 0.000 & 0.000 & -8.69 & -7.94 & 2125.19 & 190.88 & -7.95 & 194.20 \\
\hline 18 & 299 & 0.00 & 0.01 & 0.00 & 0.00 & 0.000 & -0.013 & 0.000 & 0.000 & -7.73 & -7.01 & 2128.80 & 195.34 & -7.01 & 198.69 \\
\hline & 300 & 0.00 & 0.00 & 0.00 & 0.00 & 0.000 & 0.000 & 0.000 & 0.000 & -6.99 & -6.31 & 2134.23 & 197.98 & -6.31 & 201.37 \\
\hline 87 & 301 & 0.01 & 0.01 & 0.00 & 0.00 & 0.011 & -0.013 & 0.000 & 0.000 & -5.77 & -5.11 & 2137.39 & 202.89 & -5.11 & 206.32 \\
\hline 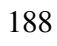 & 302 & 0.00 & 0.00 & 0.00 & 0.00 & 0.000 & 0.000 & 0.000 & 0.000 & -5.03 & -4.43 & 2142.65 & 205.70 & -4.44 & 209.17 \\
\hline ניב & 303 & 0.01 & 0.01 & -0.01 & -0.01 & 0.011 & -0.014 & 0.012 & 0.010 & -4.01 & -3.40 & 2145.78 & 210.64 & -3.38 & 214.18 \\
\hline 90 & 304 & 0.00 & 0.00 & 0.00 & 0.00 & 0.000 & 0.000 & 0.000 & 0.000 & -3.23 & -2.73 & 2150.87 & 213.63 & -2.73 & 217.20 \\
\hline . & 305 & 0.02 & 0.02 & -0.01 & 0.00 & 0.021 & -0.027 & 0.012 & 0.001 & -2.40 & -1.85 & 2153.98 & 218.59 & -1.85 & 222.22 \\
\hline ? & 306 & 0.00 & 0.00 & 0.00 & 0.00 & 0.000 & 0.000 & 0.000 & 0.000 & -1.65 & -1.25 & 2158.94 & 221.70 & -1.25 & 225.38 \\
\hline 193 & 307 & -0.06 & 0.00 & -0.02 & 0.00 & -0.063 & 0.000 & 0.025 & -0.001 & -1.04 & -0.62 & 2162.11 & 226.59 & -0.62 & 230.35 \\
\hline 194 & 308 & 0.07 & 0.02 & -0.04 & -0.01 & 0.075 & -0.028 & 0.051 & 0.014 & -1.28 & -0.38 & 2167.25 & 229.52 & -0.33 & 233.40 \\
\hline & 309 & 0.08 & 0.00 & -0.05 & -0.01 & 0.086 & 0.000 & 0.064 & 0.016 & -1.38 & -0.21 & 2170.72 & 234.13 & -0.14 & 238.10 \\
\hline . & 310 & 0.09 & 0.00 & -0.05 & -0.01 & 0.097 & 0.000 & 0.064 & 0.017 & -1.16 & 0.03 & 2175.67 & 237.26 & 0.12 & 241.30 \\
\hline 197 & 311 & 0.48 & 0.00 & 0.02 & 0.01 & 0.541 & 0.000 & 0.085 & 0.001 & -1.85 & -3.22 & 2182.37 & 238.62 & -3.43 & 242.44 \\
\hline 198 & 312 & 0.49 & 0.00 & 0.02 & 0.01 & 0.553 & 0.000 & 0.090 & 0.003 & -1.62 & -3.15 & 2187.33 & 241.73 & -3.34 & 245.65 \\
\hline 199 & 313 & 0.28 & 0.00 & 0.02 & 0.00 & 0.308 & 0.000 & 0.010 & -0.003 & -1.23 & -1.12 & 2188.59 & 248.55 & -1.19 & 252.67 \\
\hline 200 & 314 & 0.27 & 0.00 & 0.02 & 0.00 & 0.296 & 0.000 & 0.007 & -0.003 & -1.40 & -1.21 & 2193.52 & 251.69 & -1.26 & 255.91 \\
\hline 201 & 315 & 0.27 & 0.00 & 0.02 & 0.00 & 0.296 & 0.000 & 0.007 & -0.003 & -1.86 & -1.62 & 2197.06 & 256.22 & -1.68 & 260.53 \\
\hline 202 & 316 & 0.27 & 0.00 & 0.03 & 0.00 & 0.297 & 0.000 & -0.005 & -0.007 & -1.95 & -1.62 & 2201.73 & 259.62 & -1.66 & 264.04 \\
\hline 03 & 317 & 0.27 & 0.00 & 0.03 & 0.00 & 0.297 & 0.000 & -0.005 & -0.007 & -2.08 & -1.74 & 2204.80 & 264.62 & -1.79 & 269.12 \\
\hline 14 & 318 & 0.27 & 0.00 & 0.04 & 0.00 & 0.298 & 0.000 & -0.017 & -0.010 & -1.93 & -1.47 & 2209.04 & 268.45 & -1.50 & 273.07 \\
\hline 205 & 319 & 0.27 & 0.00 & 0.04 & 0.00 & 0.298 & 0.000 & -0.017 & -0.010 & -2.01 & -1.56 & 2211.93 & 273.63 & -1.61 & 278.34 \\
\hline 206 & 320 & 0.27 & 0.00 & 0.05 & -0.01 & 0.298 & 0.000 & -0.030 & -0.003 & -1.84 & -1.26 & 2215.97 & 277.66 & -1.29 & 282.50 \\
\hline 207 & 321 & 0.27 & 0.00 & 0.06 & -0.01 & 0.299 & 0.000 & -0.042 & -0.007 & -2.29 & -1.43 & 2218.77 & 282.93 & -1.44 & 287.89 \\
\hline 208 & 322 & 0.26 & 0.00 & 0.06 & -0.01 & 0.288 & 0.000 & -0.045 & -0.006 & -2.13 & -1.24 & 2222.77 & 287.01 & -1.24 & 292.10 \\
\hline 209 & 323 & 0.27 & 0.00 & 0.07 & -0.01 & 0.300 & 0.000 & -0.054 & -0.010 & -2.68 & -1.49 & 2225.50 & 292.35 & -1.48 & 297.57 \\
\hline 210 & 324 & 0.27 & 0.00 & 0.07 & -0.01 & 0.300 & 0.000 & -0.054 & -0.010 & -2.53 & -1.34 & 2229.37 & 296.55 & -1.31 & 301.91 \\
\hline 1 & 325 & 0.27 & 0.00 & 0.08 & -0.01 & 0.301 & 0.000 & -0.066 & -0.013 & -3.27 & -1.70 & 2232.06 & 301.93 & -1.65 & 307.44 \\
\hline 212 & 326 & 0.14 & 0.00 & 0.00 & 0.02 & 0.151 & 0.000 & 0.009 & -0.020 & -2.32 & -1.69 & 2235.91 & 306.15 & -1.64 & 311.78 \\
\hline 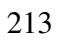 & 327 & 0.16 & 0.00 & 0.00 & 0.02 & 0.172 & 0.000 & 0.012 & -0.019 & -2.69 & -2.06 & 2238.46 & 311.67 & -2.02 & 317.43 \\
\hline 214 & 328 & 0.14 & 0.00 & 0.01 & 0.02 & 0.151 & 0.000 & -0.002 & -0.021 & -2.72 & -2.04 & 2242.15 & 316.05 & -1.99 & 321.96 \\
\hline 15 & 329 & 0.14 & 0.00 & 0.01 & 0.01 & 0.151 & 0.000 & -0.003 & -0.011 & -3.00 & -2.41 & 2244.55 & 321.72 & -2.41 & 327.71 \\
\hline 16 & 330 & 0.16 & 0.00 & 0.01 & 0.01 & 0.173 & 0.000 & -0.001 & -0.011 & -3.07 & -2.53 & 2248.23 & 326.12 & -2.53 & 332.24 \\
\hline 217 & 331 & 0.16 & 0.00 & 0.02 & 0.01 & 0.173 & 0.000 & -0.013 & -0.013 & -3.56 & -2.93 & 2250.52 & 331.90 & -2.92 & 338.18 \\
\hline 0 & 332 & 0.16 & 0.00 & 0.02 & 0.01 & 0.173 & 0.000 & -0.013 & -0.013 & -3.58 & -2.98 & 2253.98 & 336.51 & -2.97 & 342.95 \\
\hline 219 & 333 & 0.16 & 0.00 & 0.02 & 0.01 & 0.173 & 0.000 & -0.013 & -0.013 & -3.93 & -3.33 & 2256.07 & 342.49 & -3.33 & 349.08 \\
\hline 20 & 334 & 0.11 & 0.00 & 0.03 & -0.01 & 0.119 & 0.000 & -0.032 & 0.006 & -4.21 & -3.52 & 2259.52 & 347.11 & -3.48 & 353.89 \\
\hline 21 & 335 & 0.09 & 0.00 & 0.03 & -0.01 & 0.097 & 0.000 & -0.033 & 0.007 & -4.77 & -4.09 & 2261.70 & 353.00 & -4.06 & 359.94 \\
\hline 20 & 336 & -0.01 & 0.00 & 0.00 & 0.00 & -0.011 & 0.000 & 0.000 & 0.000 & -4.90 & -4.44 & 2265.16 & 357.61 & -4.44 & 364.68 \\
\hline & 337 & 0.05 & 0.00 & 0.02 & 0.00 & 0.054 & 0.000 & -0.023 & -0.001 & -5.91 & -5.40 & 2267.59 & 363.26 & -5.39 & 370.50 \\
\hline 224 & 338 & -0.01 & 0.00 & 0.00 & 0.00 & -0.011 & 0.000 & 0.000 & 0.000 & -6.62 & -6.07 & 2271.24 & 367.68 & -6.08 & 375.08 \\
\hline 225 & 339 & 0.01 & 0.00 & 0.00 & 0.00 & 0.011 & 0.000 & 0.000 & 0.000 & -7.61 & -7.03 & 2273.53 & 373.46 & -7.03 & 381.04 \\
\hline & $=115$ & & & & & & & & & & & & & & \\
\hline 157 & 272 & 0.15 & 0.00 & 0.03 & 0.00 & 0.162 & 0.000 & -0.027 & -0.005 & -4.14 & -3.43 & 1932.22 & 173.21 & -3.48 & 177.11 \\
\hline 158 & 273 & 0.15 & 0.00 & 0.04 & 0.00 & 0.163 & 0.000 & -0.039 & -0.006 & -4.47 & -3.56 & 1941.65 & 171.85 & -3.60 & 175.70 \\
\hline 159 & 274 & 0.17 & 0.00 & 0.06 & -0.01 & 0.186 & 0.000 & -0.061 & -0.001 & -5.43 & -3.99 & 1949.79 & 171.78 & -4.05 & 175.55 \\
\hline 160 & 275 & 0.18 & 0.00 & 0.07 & -0.02 & 0.198 & 0.000 & -0.072 & 0.006 & -6.03 & -4.20 & 1959.05 & 170.59 & -4.25 & 174.32 \\
\hline 161 & 276 & 0.18 & 0.00 & 0.08 & -0.02 & 0.198 & 0.000 & -0.084 & 0.004 & -6.99 & -4.70 & 1967.02 & 170.70 & -4.75 & 174.37 \\
\hline 162 & 277 & 0.18 & 0.00 & 0.08 & -0.02 & 0.198 & 0.000 & -0.084 & 0.004 & -7.25 & -4.91 & 1976.03 & 169.76 & -4.95 & 173.40 \\
\hline 163 & 278 & 0.03 & 0.00 & 0.00 & 0.00 & 0.032 & 0.000 & 0.000 & 0.000 & -6.12 & -5.58 & 1983.92 & 169.94 & -5.58 & 173.57 \\
\hline 164 & 279 & -0.01 & 0.00 & 0.00 & 0.00 & -0.011 & 0.000 & 0.000 & 0.000 & -6.72 & -6.20 & 1993.09 & 168.83 & -6.20 & 172.42 \\
\hline
\end{tabular}




\section{$Z=115$}

165280

166281

$\begin{array}{lll}0.02 & 0.00 & -0.01\end{array}$ $\begin{array}{lll}0.03 & 0.00 & -0.01\end{array}$

$0.00 \quad 0.021$

$167 \quad 282$

$\begin{array}{lll}0.06 & 0.00 & -0.02\end{array}$

$0.00 \quad 0.032$

0.000

0.012

$\begin{array}{llllll}0.000 & -7.11 & -6.48 & 2000.36 & 169.64\end{array}$

$\begin{array}{llllllll}0.000 & 0.012 & 0.000 & -7.21 & -6.54 & 2008.73 & 169.34\end{array}$

168283

$\begin{array}{lll}0.06 & 0.00 & -0.02\end{array}$

$0.01 \quad 0.064$

0.000

$\begin{array}{llllll}0.026 & -0.008 & -7.83 & -6.94 & 2023.97 & 170.24\end{array}$

169284

$\begin{array}{lll}0.06 & 0.00 & -0.01\end{array}$

0.01

0.064

0.000

$\begin{array}{llllll}0.014 & -0.009 & -8.18 & -7.38 & 2030.94 & 171.35\end{array}$

170285

$\begin{array}{lll}0.06 & 0.00 & -0.01\end{array}$

$0.01 \quad 0.064$

0.000

$\begin{array}{llllll}0.014 & -0.009 & -8.20 & -7.40 & 2038.81 & 171.55\end{array}$

171286

$172 \quad 287$

$\begin{array}{lll}0.07 & 0.00 & 0.00\end{array}$

$0.01 \quad 0.075$

0.000

$\begin{array}{llllll}0.002 & -0.010 & -8.58 & -7.79 & 2045.51 & 172.92\end{array}$

173288

$\begin{array}{lll}0.06 & 0.00 & 0.00\end{array}$

$0.01 \quad 0.064$

0.000

$\begin{array}{llllll}0.002 & -0.010 & -8.52 & -7.74 & 2053.07 & 173.43\end{array}$

174289

$0.06 \quad 0.00$

0.01

$\begin{array}{lllllll}0.000 & -0.010 & -0.001 & -8.90 & -8.12 & 2059.55 & 175.02\end{array}$

$\begin{array}{llll}175 & 290 & 0.05 & 0.00\end{array}$

0.01

0.00

0.064

$\begin{array}{llll}176 & 291 & -0.04 & 0.00\end{array}$

0.00

$0.00 \quad 0.053$

0.000

$0.00-0.042$

0.000

$-0.011$

0.001

$0.00-0.042$

0.000

0.001$$
\begin{array}{llll}
-9.19 & -8.40 & 2073.10 & 177.61
\end{array}
$$

$\begin{array}{llll}177 & 292 & -0.04 & 0.00 \\ 178 & 293 & -0.02 & 0.00\end{array}$

$\begin{array}{llll}179 & 294 & -0.02 & 0.00\end{array}$

0.00

$0.00-0.021$

0.000

0.001

$\begin{array}{lllll}0.000 & -9.14 & -8.55 & 2080.43 & 178.36\end{array}$

$0.00-0.021$

0.000

0.000

$\begin{array}{llllll}0.000 & -9.55 & -8.92 & 2086.46 & 180.40\end{array}$

$\begin{array}{llll}180 & 295 & -0.02 & 0.00\end{array}$

$\begin{array}{llll}181 & 296 & -0.01 & 0.00\end{array}$

0.00

$0.00-0.021$

$0.000 \quad 0.000$

$\begin{array}{llll}182 & 297 & -0.01 & 0.00\end{array}$

0.00

$0.00-0.011$

0.000

0.000

$\begin{array}{llllll}0.000 & -9.61 & -8.85 & 2105.57 & 185.50\end{array}$

$\begin{array}{llll}183 & 298 & -0.01 & 0.00\end{array}$

0.00

$0.00-0.011$

0.000

$\begin{array}{lllll}0.000 & -9.80 & -9.02 & 2110.99 & 188.15\end{array}$

$0.00-0.011$

0.000

0.000

$\begin{array}{llllll}0.000 & -9.37 & -8.60 & 2117.12 & 190.09\end{array}$

$\begin{array}{llll}184 & 299 & 0.00 & 0.00\end{array}$

0.00

$0.00 \quad 0.000$

0.000

0.000

$\begin{array}{lllll}0.000 & -9.41 & -8.64 & 2122.22 & 193.06\end{array}$

185300

18630

$187 \quad 302$

$\begin{array}{lll}0.00 & 0.01 & 0.00\end{array}$

0.00

$0.000-0.013$

0.000

$\begin{array}{llllll}0.000 & -8.79 & -8.04 & 2127.97 & 195.39\end{array}$

$\begin{array}{lll}0.00 & 0.000 & 0.000\end{array}$

0.000

$\begin{array}{lllll}0.000 & -7.83 & -7.11 & 2131.90 & 199.53\end{array}$

188303

$\begin{array}{lll}0.01 & 0.01 & 0.00\end{array}$

$\begin{array}{llll}0.00 & 0.011 & -0.013\end{array}$

0.000

$\begin{array}{llllll}0.000 & -7.08 & -6.41 & 2137.34 & 202.16\end{array}$

189304

$0.02 \quad 0.01-0.01$

$\begin{array}{lllll}190 & 305 & -0.01 & 0.00 & 0.00\end{array}$

$\begin{array}{llllll}191 & 306 & 0.04 & 0.05 & -0.02\end{array}$

192307

193308

194309

$\begin{array}{lll}0.05 & 0.05 & -0.02\end{array}$

$\begin{array}{lll}0.00 & 0.011 & 0.000\end{array}$

0.000

$\begin{array}{llllll}0.000 & -5.91 & -5.25 & 2140.85 & 206.71\end{array}$

$\begin{array}{lll}0.00 & 0.021 & -0.014\end{array}$

0.012

$\begin{array}{llll}0.00 & -0.011 & 0.000\end{array}$

0.000

$\begin{array}{lllll}0.000 & -4.16 & -3.56 & 2149.59 & 214.12\end{array}$

$\begin{array}{llll}0.00 & 0.044 & -0.068\end{array}$

0.026

$\begin{array}{lllll}0.000 & -3.36 & -2.87 & 2154.67 & 217.12\end{array}$

$\begin{array}{llll}0.00 & 0.054 & -0.068\end{array}$

0.026

$\begin{array}{llllll}0.003 & -3.20 & -2.14 & 2158.23 & 221.62\end{array}$

$\begin{array}{lll}0.00 & 0.065 & -0.082\end{array}$

0.027

$\begin{array}{llllll}0.003 & -2.61 & -1.60 & 2163.27 & 224.66\end{array}$

$\begin{array}{lll}0.06 & 0.06 & -0.02\end{array}$

0.00

$0.076-0.069$

0.039

$\begin{array}{lllll}0.004 & -2.45 & -1.26 & 2167.04 & 228.96\end{array}$

$\begin{array}{lllll}0.08 & 0.00 & -0.05 & -0.01\end{array}$

19631

$\begin{array}{llll}0.09 & 0.00 & -0.05\end{array}$

$0.086 \quad 0.000$

0.064

19731

$\begin{array}{ll}0.49 & 0.00\end{array}$

0.01

0.01

0.097

0.000

0.064

$\begin{array}{lllll}0.016 & -1.88 & -0.68 & 2175.79 & 236.35\end{array}$

$\begin{array}{ll}0.552 & 0.000\end{array}$

0.102

$\begin{array}{llll}-1.64 & -0.41 & 2180.73 & 239.48\end{array}$

198313

$0.49 \quad 0.00$

0.01

0.01

0.552

0.000

0.102

$\begin{array}{llllll}0.009 & -1.92 & -3.56 & 2187.64 & 240.65\end{array}$

199314

$0.29 \quad 0.00$

0.02

0.00

0.319

0.000

200315

201316

202317

$0.28 \quad 0.00$

0.02

0.00

0.308

0.000

$\begin{array}{llllll}0.012 & -0.003 & -1.26 & -1.33 & 2194.03 & 250.39\end{array}$

0.00

0.296

0.000

$\begin{array}{llllll}0.010 & -0.003 & -1.42 & -1.39 & 2198.95 & 253.55\end{array}$

$203 \quad 318$

0.03

0.00

204319

$0.27 \quad 0.00$

0.03

0.00

0.297

205320

$0.27 \quad 0.00$

$0.04 \quad 0.00$

0.298

$\begin{array}{lllllll}0.000 & -0.005 & -0.007 & -2.04 & -1.78 & 2210.70 & 266.01\end{array}$

$\begin{array}{lllllll}0.000 & -0.005 & -0.007 & -1.72 & -1.52 & 2214.95 & 269.83\end{array}$

206321

$0.27 \quad 0.00$

$0.05-0.01$

0.298

$\begin{array}{lllllll}0.000 & -0.017 & -0.010 & -1.94 & -1.57 & 2218.09 & 274.76\end{array}$

$0.27 \quad 0.00$

$0.05-0.01$

0.298

$\begin{array}{lllllll}0.000 & -0.030 & -0.003 & -1.75 & -1.25 & 2222.12 & 278.80\end{array}$

$\begin{array}{lllllll}0.000 & -0.030 & -0.003 & -1.91 & -1.43 & 2225.24 & 283.76\end{array}$

208323

$0.27 \quad 0.00$

$0.06-0.01$

0.299

$\begin{array}{lllllll}0.000 & -0.042 & -0.007 & -1.91 & -1.18 & 2229.18 & 287.89\end{array}$

209324

$0.27 \quad 0.00$

$0.06-0.01$

0.299

$\begin{array}{lllllll}0.000 & -0.042 & -0.007 & -2.16 & -1.35 & 2232.11 & 293.02\end{array}$

210325

$\begin{array}{lll}0.14 & 0.00 & -0.01\end{array}$

0.02

0.150

$\begin{array}{lllllll}0.000 & 0.022 & -0.018 & -1.86 & -1.23 & 2236.02 & 297.19\end{array}$

211326

$\begin{array}{lll}0.14 & 0.00 & -0.01\end{array}$

0.02

0.150

0.000

$\begin{array}{llllll}0.022 & -0.018 & -2.22 & -1.57 & 2238.98 & 302.30\end{array}$

212327

$0.14 \quad 0.00$

$0.00 \quad 0.02$

0.151

0.000

$\begin{array}{llllll}0.009 & -0.020 & -2.26 & -1.63 & 2242.92 & 306.43\end{array}$

213328

$0.14 \quad 0.00$

$0.00-0.02$

0.151

0.000

$\begin{array}{llllll}0.009 & -0.020 & -2.65 & -2.00 & 2245.75 & 311.67\end{array}$

215330

$0.14 \quad 0.00$

$0.01-0.02$

0.151

$\begin{array}{lllllll}0.000 & -0.002 & -0.021 & -2.79 & -2.15 & 2249.61 & 315.88\end{array}$

216331

0.110 .00

0.020 .01

0.119

$\begin{array}{lllllll}0.000 & -0.002 & -0.021 & -3.20 & -2.54 & 2252.32 & 321.25\end{array}$

217332

0.110 .00

$0.02 \quad 0.01$

0.119

$\begin{array}{lllllll}0.000 & -0.018 & -0.012 & -3.23 & -2.66 & 2256.00 & 325.63\end{array}$

218333

0.110 .00

$\begin{array}{lll}0.03 & 0.00\end{array}$

0.119

$\begin{array}{lllllll}0.000 & -0.018 & -0.012 & -3.65 & -3.06 & 2258.57 & 331.13\end{array}$

0.110 .00

$0.03 \quad 0.00$

0.119

$\begin{array}{lllllll}0.000 & -0.031 & -0.004 & -3.91 & -3.25 & 2262.18 & 335.60\end{array}$

$\begin{array}{lllllll}0.000 & -0.031 & -0.004 & -4.37 & -3.69 & 2264.64 & 341.21\end{array}$

220335

$0.09 \quad 0.00$ 


\section{$Z=115$}

222337

0.01

$0.00 \quad 0.053$

$\begin{array}{lllllll}0.000 & -0.011 & -0.001 & -5.41 & -4.96 & 2274.19 & 355.88\end{array}$

$\begin{array}{llll}224 & 339 & -0.01 & 0.00\end{array}$

\section{$Z=116(\mathrm{Lv})$}

$\begin{array}{llll}159 & 275 & 0.16 & 0.00\end{array}$

160276

$\begin{array}{ll}0.16 & 0.00 \\ 0.17 & 0.00\end{array}$

$\begin{array}{lll}0.05 & -0.01\end{array}$

0.174

$0.000-0.023-0$.

$\begin{array}{llll}0.00 & 0.00 & -0.011\end{array}$

$0.000 \quad 0.000$

0.000

$0.06-0.01$

$0.000-0.050$

$0.000-0.061-$

$\begin{array}{lllll}0.001 & -4.56 & -3.44 & 1948.99 & 179.87\end{array}$
$-4.96 \quad 362.88$

$\begin{array}{ll}-5.80 & 368.53\end{array}$

$-6.35 \quad 373.24$ $\begin{array}{ll}161 & 277\end{array}$

$162 \quad 278$

$\begin{array}{ll}0.17 & 0.00\end{array}$ $0.00 \quad 0.00$

$0.07-0.02$

163279

$0.03 \quad 0.00$

0.00

0.186

0.000

164280

$\begin{array}{lll}0.00 & 0.00 & 0.00\end{array}$

165281

166282

$167 \quad 283$

168284

$\begin{array}{llll}0.03 & 0.00 & -0.01\end{array}$

$\begin{array}{llll}0.05 & 0.00 & -0.02\end{array}$

$\begin{array}{llll}0.06 & 0.00 & -0.02\end{array}$

$\begin{array}{llll}0.06 & 0.00 & -0.02\end{array}$

169285

170286

$\begin{array}{lll}0.07 & 0.00 & -0.01\end{array}$

$\begin{array}{ll}171 & 287\end{array}$

$\begin{array}{lll}0.07 & 0.00 & -0.01\end{array}$

$\begin{array}{lll}0.07 & 0.00 & 0.00\end{array}$

$\begin{array}{ll}0.07 & 0.00\end{array}$

0.00

0.00

0.032

$0.00 \quad 0.000$

0.00

0.00

0.01

0.032

0.053

0.01

0.064

$0.01-0.075$

$0.02 \quad 0.075$

$0.01 \quad 0.075$

$0.01 \quad 0.075$

$173 \quad 289$

$0.07 \quad 0.00$

174290

$175 \quad 29$

$\begin{array}{ll}0.06 & 0.00\end{array}$

0.01

0.00

0.075

$0.00 \quad 0.064$

$0.00 \quad 0.064$

$0.00-0.073$

$0.00-0.063$

$\begin{array}{llll}177 & 293 & -0.06 & 0.00\end{array}$

$0.00-0.042$

$\begin{array}{llll}178 & 294 & -0.04 & 0.00\end{array}$

$\begin{array}{llll}179 & 295 & -0.02 & 0.00\end{array}$

0.00

$0.00-0.021$

0.00

$0.00-0.011$

$\begin{array}{lllll}180 & 296 & -0.01 & 0.00\end{array}$

$\begin{array}{ll}181 & 297\end{array}$

182298

183299

$0.00 \quad 0.00$

0.00

0.00

$0.00 \quad 0.00$

0.00

0.00

184300

185301

$0.00 \quad 0.00$

0.00

$\begin{array}{lll}0.00 & 0.01 & 0.00\end{array}$

0.00

0.000

0.000

$0.00 \quad 0.000$

$\begin{array}{lllllll}0.000 & -0.074 & 0.007 & -5.97 & -4.15 & 1966.58 & 178.42\end{array}$

$\begin{array}{lllllll}0.000 & 0.000 & 0.000 & -5.21 & -4.74 & 1976.31 & 176.77\end{array}$

$0.000 \quad 0.000$

$\begin{array}{lllll}0.000 & -5.76 & -5.22 & 1984.04 & 177.11\end{array}$

$\begin{array}{lllllll}0.000 & 0.000 & 0.000 & -6.35 & -5.83 & 1993.53 & 175.68\end{array}$

$\begin{array}{llllllll}0.000 & 0.012 & 0.000 & -6.73 & -6.08 & 2000.80 & 176.49\end{array}$

$\begin{array}{lllllll}0.000 & 0.025 & 0.001 & -6.97 & -6.15 & 2009.51 & 175.85\end{array}$

$\begin{array}{lllllll}0.000 & 0.026 & -0.008 & -7.51 & -6.63 & 2016.78 & 176.65\end{array}$

$\begin{array}{lllllll}0.000 & 0.026 & -0.008 & -7.61 & -6.73 & 2025.29 & 176.21\end{array}$

$\begin{array}{lllllll}0.000 & 0.014 & -0.009 & -7.98 & -7.19 & 2032.30 & 177.27\end{array}$

$\begin{array}{lllllll}0.000 & 0.014 & -0.019 & -8.13 & -7.21 & 2040.50 & 177.15\end{array}$

$\begin{array}{lllllll}0.000 & 0.002 & -0.010 & -8.38 & -7.60 & 2047.22 & 178.49\end{array}$

$\begin{array}{lllllll}0.000 & 0.002 & -0.010 & -8.29 & -7.51 & 2055.08 & 178.71\end{array}$

$\begin{array}{lllllll}0.000 & -0.010 & -0.001 & -8.65 & -7.88 & 2061.56 & 180.30\end{array}$

$\begin{array}{llllllll}0.000 & -0.022 & -0.001 & -8.63 & -7.77 & 2069.17 & 180.76\end{array}$

$\begin{array}{llllllll}0.000 & -0.022 & -0.001 & -8.97 & -8.09 & 2075.39 & 182.61\end{array}$

$\begin{array}{lllllll}0.000 & 0.002 & -0.000 & -8.77 & -8.30 & 2083.10 & 182.97\end{array}$

$\begin{array}{lllllll}0.000 & 0.001 & 0.000 & -9.14 & -8.62 & 2089.10 & 185.04\end{array}$

$\begin{array}{lllllll}0.000 & 0.001 & 0.000 & -9.08 & -8.45 & 2096.22 & 186.00\end{array}$

$\begin{array}{lllllll}0.000 & 0.000 & 0.000 & -9.39 & -8.65 & 2101.90 & 188.39\end{array}$

$\begin{array}{lllllll}0.000 & 0.000 & 0.000 & -9.08 & -8.32 & 2108.64 & 189.72\end{array}$

$\begin{array}{lllllll}0.000 & 0.000 & 0.000 & -9.27 & -8.48 & 2114.08 & 192.35\end{array}$

$\begin{array}{lllllll}0.000 & 0.000 & 0.000 & -8.84 & -8.07 & 2120.53 & 193.97\end{array}$

$0.000 \quad 0.000$

$\begin{array}{lllll}0.000 & -8.86 & -8.10 & 2125.63 & 196.94\end{array}$

186302

187303

188304

$0.00 \quad 0.00$

0.00

0.00

$0.000 \quad 0.000$

$\begin{array}{lllll}0.000 & -8.24 & -7.50 & 2131.70 & 198.94\end{array}$

$\begin{array}{lll}0.01 & 0.02 & 0.00\end{array}$

0.00

0.000

$\begin{array}{llllll}0.000 & -7.30 & -6.59 & 2135.66 & 203.05\end{array}$

0.00

0.000

$\begin{array}{llllll}0.000 & -6.53 & -5.87 & 2141.41 & 205.38\end{array}$

0.00

$0.011-0.027$

0.000

$\begin{array}{lllll}0.000 & -5.40 & -4.70 & 2144.92 & 209.94\end{array}$

189305

$\begin{array}{lll}0.02 & 0.03 & -0.01\end{array}$

190306

$\begin{array}{lll}0.04 & 0.05 & -0.02\end{array}$

$\begin{array}{lllll}191 & 307 & -0.04 & 0.00 & -0.01\end{array}$

$\begin{array}{lllll}192 & 308 & 0.05 & 0.07 & -0.02\end{array}$

$\begin{array}{lll}0.00 & 0.022 & -0.041\end{array}$

$\begin{array}{lll}0.00 & 0.044 & -0.068\end{array}$

0.000

$\begin{array}{llllll}0.000 & -4.59 & -4.02 & 2150.50 & 212.43\end{array}$

$\begin{array}{lll}0.00 & -0.042 & 0.000\end{array}$

0.013

$\begin{array}{lllll}0.001 & -3.73 & -3.00 & 2153.98 & 217.02\end{array}$

$\begin{array}{lll}0.01 & 0.055 & -0.095\end{array}$

0.026

$\begin{array}{lllll}0.003 & -3.42 & -2.34 & 2159.39 & 219.68\end{array}$

193309

$\begin{array}{llll}0.06 & 0.07 & -0.02\end{array}$

$\begin{array}{lll}0.01 & 0.066 & -0.095\end{array}$

$\begin{array}{llllll}0.012 & -0.001 & -2.01 & -1.63 & 2163.00 & 224.15\end{array}$

$\begin{array}{lllllll}0.027 & -0.005 & -2.87 & -1.39 & 2168.64 & 226.58\end{array}$

$\begin{array}{llllll}0.027 & -0.005 & -2.59 & -1.14 & 2172.51 & 230.77\end{array}$

194310

$\begin{array}{llll}0.07 & 0.06 & -0.03\end{array}$

0.00

$0.076-0.083$

0.040

$\begin{array}{lllll}0.006 & -2.03 & -0.73 & 2177.80 & 233.56\end{array}$

195311

$\begin{array}{lllll}0.08 & 0.00 & -0.05 & -0.01\end{array}$

196312

$\begin{array}{lllll}0.09 & 0.00 & -0.05 & -0.01\end{array}$

$0.086 \quad 0.000$

0.064

$\begin{array}{lllll}0.016 & -1.61 & -0.44 & 2181.46 & 237.97\end{array}$

197313

$\begin{array}{llll}0.10 & 0.00 & -0.05 & -0.01\end{array}$

0.097

0.000

0.064

$\begin{array}{lllll}0.017 & -1.40 & -0.20 & 2186.73 & 240.77\end{array}$

$198 \quad 314$

$\begin{array}{llll}0.10 & 0.00 & -0.05\end{array}$

199315

$\begin{array}{lll}0.49 & 0.00 & 0.02\end{array}$

0.00

108

0.065

$\begin{array}{lllll}0.018 & -1.50 & -0.23 & 2190.54 & 245.03\end{array}$

0.000

0.065

$\begin{array}{llllll}0.007 & -1.26 & -0.10 & 2195.74 & 247.91\end{array}$

200316

201317

202318

203319

204320

205321

206322

207323

$208 \quad 324$

209325

210326

211327

\section{$0.28 \quad 0.00$}

0.01

0.553

0.000

$\begin{array}{llllll}0.090 & 0.003 & -1.75 & -3.85 & 2203.08 & 248.63\end{array}$

$0.28 \quad 0.00$

0.02

0.000

$\begin{array}{lllllll}0.010 & -0.003 & -1.27 & -1.33 & 2205.73 & 254.06\end{array}$

$0.28 \quad 0.00$

0.02

0.00

0.308

0.000

$\begin{array}{llllll}0.010 & -0.003 & -1.66 & -1.68 & 2209.51 & 258.35\end{array}$

$0.28 \quad 0.00$

0.03

0.00

0.308

0.000

$\begin{array}{llllll}0.010 & -0.003 & -1.64 & -1.66 & 2214.47 & 261.46\end{array}$

$\begin{array}{lllllll}0.000 & -0.002 & -0.006 & -1.82 & -1.75 & 2217.82 & 266.18\end{array}$

$0.28 \quad 0.00$

0.03

$0.00 \quad 0.308$

$\begin{array}{lllllll}0.000 & -0.002 & -0.006 & -1.46 & -1.42 & 2222.30 & 269.77\end{array}$

$0.27 \quad 0.00$

$0.00 \quad 0.298$

$\begin{array}{lllllll}0.000 & -0.017 & -0.010 & -1.68 & -1.41 & 2225.39 & 274.75\end{array}$

$\begin{array}{lllllll}0.000 & -0.017 & -0.010 & -1.29 & -1.09 & 2229.72 & 278.49\end{array}$

$\begin{array}{llllll}0.27 & 0.00 & 0.05 & -0.01 & 0.298\end{array}$

$\begin{array}{lllllll}0.000 & -0.030 & -0.003 & -1.63 & -1.24 & 2232.81 & 283.47\end{array}$

$\begin{array}{lllll}0.27 & 0.00 & 0.06 & -0.01 & 0.299\end{array}$

$\begin{array}{lllllll}0.000 & -0.042 & -0.007 & -1.61 & -0.97 & 2237.02 & 287.34\end{array}$

$0.27 \quad 0.00$

$0.06-0.01$

0.299

$\begin{array}{lllllll}0.000 & -0.042 & -0.007 & -1.87 & -1.15 & 2239.98 & 292.45\end{array}$

$\begin{array}{lll}0.22 & 0.00 & 0.02\end{array}$

0.01

0.240 $\begin{array}{lllllll}0.000 & -0.002 & -0.013 & -1.32 & -0.98 & 2244.13 & 296.37\end{array}$

$\begin{array}{lllllll}0.000 & 0.020 & -0.018 & -1.88 & -1.32 & 2247.09 & 301.48\end{array}$

$-3.49 \quad 183.94$ 
$Z=116(\mathrm{Lv})$

$\begin{array}{rrrrrrrrrrrrrr}212 & 328 & 0.13 & 0.00 & 0.00 & 0.02 & 0.140 & 0.000 & 0.008 & -0.020 & -1.88 & -1.27 & 2251.20 & 305.44 \\ 213 & 329 & 0.13 & 0.00 & 0.00 & 0.02 & 0.140 & 0.000 & 0.008 & -0.020 & -2.26 & -1.62 & 2254.02 & 310.69 \\ 214 & 330 & 0.11 & 0.00 & 0.01 & 0.01 & 0.118 & 0.000 & -0.007 & -0.011 & -2.30 & -1.83 & 2258.23 & 314.55 \\ 215 & 331 & 0.11 & 0.00 & 0.02 & 0.01 & 0.119 & 0.000 & -0.018 & -0.012 & -2.79 & -2.24 & 2260.98 & 319.88 \\ 216 & 332 & 0.11 & 0.00 & 0.02 & 0.01 & 0.119 & 0.000 & -0.018 & -0.012 & -2.97 & -2.42 & 2265.00 & 323.93 \\ 217 & 333 & 0.11 & 0.00 & 0.02 & 0.01 & 0.119 & 0.000 & -0.018 & -0.012 & -3.40 & -2.83 & 2267.58 & 329.41 \\ 218 & 334 & 0.11 & 0.00 & 0.03 & 0.00 & 0.119 & 0.000 & -0.031 & -0.004 & -3.65 & -3.01 & 2271.46 & 333.61 \\ 219 & 335 & 0.11 & 0.00 & 0.03 & 0.00 & 0.119 & 0.000 & -0.031 & -0.004 & -4.11 & -3.45 & 2273.93 & 339.21 \\ 220 & 336 & 0.09 & 0.00 & 0.03 & -0.01 & 0.097 & 0.000 & -0.033 & 0.007 & -4.42 & -3.77 & 2277.80 & 343.41 \\ 221 & 337 & 0.09 & 0.00 & 0.03 & -0.01 & 0.097 & 0.000 & -0.033 & 0.007 & -5.00 & -4.32 & 2280.24 & 349.04 \\ 222 & 338 & 0.05 & 0.00 & 0.01 & 0.00 & 0.053 & 0.000 & -0.011 & -0.001 & -5.09 & -4.65 & 2283.97 & 353.38 \\ 223 & 339 & 0.05 & 0.00 & 0.02 & 0.00 & 0.054 & 0.000 & -0.023 & -0.001 & -6.04 & -5.51 & 2286.58 & 358.84\end{array}$

$Z=117$

161278

$162 \quad 279$

163280

$\begin{array}{lll}0.07 & 0.00\end{array}$

$0.04 \quad 0.00$

0.00

$0.00 \quad 0.075$

0.000

0.002

$\begin{array}{llllll}0.000 & -4.76 & -4.25 & 1964.76 & 187.54\end{array}$ $\begin{array}{lll}0.04 & 0.00 & 0.00\end{array}$

0.00

0.043

0.000

0.001

$\begin{array}{llllll}0.000 & -5.24 & -4.71 & 1974.38 & 185.98\end{array}$

164281

$\begin{array}{lll}0.00 & 0.00 & 0.00\end{array}$

$0.000 \quad 0.001$

$\begin{array}{lllll}0.000 & -5.83 & -5.27 & 1982.53 & 185.90\end{array}$

165282

$\begin{array}{lll}0.04 & 0.00 & -0.01\end{array}$

0.00

0.000

0.000

$\begin{array}{llllll}0.000 & -6.36 & -5.84 & 1992.01 & 184.49\end{array}$

0.000

0.013

$\begin{array}{lllll}0.001 & -6.79 & -6.11 & 1999.63 & 184.95\end{array}$

167284

$\begin{array}{lll}0.06 & 0.00 & -0.01\end{array}$

$0.00 \quad 0.064$

0.000

$\begin{array}{llllll}0.014 & 0.001 & -6.93 & -6.24 & 2008.43 & 184.22\end{array}$

$0.01 \quad 0.075$

0.000

$\begin{array}{llllll}0.014 & -0.009 & -7.46 & -6.70 & 2016.01 & 184.71\end{array}$

168285

$0.07 \quad 0.00-0.01$

$0.01 \quad 0.075$

0.000

$\begin{array}{llllll}0.014 & -0.009 & -7.61 & -6.84 & 2024.58 & 184.21\end{array}$

169286

170287

$\begin{array}{lll}0.07 & 0.00 & -0.01\end{array}$

$0.01 \quad 0.075$

0.000

$\begin{array}{llllll}0.014 & -0.009 & -8.07 & -7.29 & 2031.91 & 184.95\end{array}$

$\begin{array}{lllllll}0.000 & 0.002 & -0.010 & -8.09 & -7.34 & 2040.16 & 184.77\end{array}$

171288

$0.08 \quad 0.00$

0.01

$0.01 \quad 0.086$

$\begin{array}{lllllll}0.000 & -0.009 & -0.011 & -8.54 & -7.75 & 2047.23 & 185.78\end{array}$

172289

$0.07 \quad 0.00$

0.01

0.01

0.075

$\begin{array}{lllllll}0.000 & -0.010 & -0.011 & -8.49 & -7.68 & 2055.13 & 185.95\end{array}$

173290

$0.07 \quad 0.00$

$\begin{array}{ll}0.02 & 0.00\end{array}$

0.075

$0.000-0.022$

$\begin{array}{lllll}0.002 & -8.94 & -8.07 & 2061.96 & 187.19\end{array}$

17429

$0.07 \quad 0.00$

$0.03-0.01$

0.075

$0.000-0.034$

$\begin{array}{lllll}0.008 & -9.03 & -7.99 & 2069.62 & 187.60\end{array}$

$\begin{array}{llll}175 & 292 & 0.07 & 0.00\end{array}$

$\begin{array}{lll}0.03 & -0.01 & 0.075\end{array}$

$0.000-0.034$

$\begin{array}{lllll}0.008 & -9.34 & -8.29 & 2076.14 & 189.15\end{array}$

$\begin{array}{llll}176 & 293 & -0.07 & 0.00\end{array}$

$\begin{array}{lll}0.00 & 0.00 & -0.073\end{array}$

$\begin{array}{lllllll}0.000 & 0.002 & -0.000 & -8.84 & -8.40 & 2083.77 & 189.59\end{array}$

$\begin{array}{llll}177 & 294 & -0.07 & 0.00\end{array}$

0.0

$0.00-0.073$

$0.000-0.010$

$\begin{array}{lllll}0.001 & -9.23 & -8.73 & 2090.11 & 191.33\end{array}$

$\begin{array}{llll}179 & 296 & -0.03 & 0.00\end{array}$

$0.00-0.052$

$0.000 \quad 0.001$

$\begin{array}{lllll}0.000 & -9.07 & -8.50 & 2097.18 & 192.32\end{array}$

$\begin{array}{llll}180 & 297 & -0.03 & 0.00\end{array}$

$0.00-0.032$

$0.000 \quad 0.000$

$\begin{array}{lllll}0.000 & -9.33 & -8.64 & 2103.12 & 194.46\end{array}$

$0.000-0.011$

$\begin{array}{lllll}0.000 & -9.04 & -8.31 & 2109.89 & 195.76\end{array}$

181298

182299

$0.00 \quad 0.00$

0.00

$0.00 \quad 0.000$

$0.000 \quad 0.000$

$\begin{array}{lllll}0.000 & -9.16 & -8.38 & 2115.55 & 198.17\end{array}$

183300

$0.00 \quad 0.00$

0.00

0.00

0.000

0.000

0.000

$\begin{array}{llllll}0.000 & -8.72 & -7.95 & 2122.01 & 199.78\end{array}$

18430

$\begin{array}{lll}0.00 & 0.00 \\ 0.00 & 0.00\end{array}$

0.00

$0.00 \quad 0.000$

0.000

0.000

$\begin{array}{lllll}0.000 & -8.73 & -7.97 & 2127.41 & 202.45\end{array}$

185302

186303 $0.00 \quad 0.01$

0.00

0.00

0.000

0.000

$\begin{array}{llllll}0.000 & -8.10 & -7.37 & 2133.49 & 204.45\end{array}$

$\begin{array}{llllll}0.000 & -7.17 & -6.47 & 2137.77 & 208.23\end{array}$

187304

$\begin{array}{lll}0.00 & 0.00 & 0.00\end{array}$

$\begin{array}{lll}0.00 & 0.000 & 0.000\end{array}$

0.000

$\begin{array}{lllll}0.000 & -6.40 & -5.74 & 2143.53 & 210.54\end{array}$

$\begin{array}{lll}0.00 & 0.011 & -0.027\end{array}$

0.000

$\begin{array}{lllll}0.000 & -5.29 & -4.60 & 2147.38 & 214.77\end{array}$

$\begin{array}{lllll}188 & 305 & 0.00 & 0.00 & 0.00\end{array}$

$0.00 \quad 0.000$

0.000

0.000

$\begin{array}{llllll}0.000 & -4.45 & -3.89 & 2152.95 & 217.27\end{array}$

$0.00-0.032$

0.000

$\begin{array}{llllll}0.012 & -0.000 & -3.41 & -2.88 & 2156.75 & 221.54\end{array}$

$\begin{array}{lllll}190 & 307 & 0.00 & 0.00 & 0.00\end{array}$

$0.00 \quad 0.000$

0.000

0.000

$\begin{array}{lllll}0.000 & -2.67 & -2.20 & 2162.15 & 224.21\end{array}$

$\begin{array}{rrr}-0.01 & -0.397 & 0.000\end{array}$

$\begin{array}{llllll}0.004 & 0.020 & -3.99 & -1.83 & 2166.40 & 228.03\end{array}$

$\begin{array}{lllll}192 & 309 & 0.06 & 0.08 & -0.01\end{array}$

$\begin{array}{lllll}193 & 310 & -0.40 & 0.00 & 0.05\end{array}$

$\begin{array}{lll}0.01 & 0.066 & -0.108\end{array}$

$\begin{array}{llllll}0.016 & -0.005 & -3.26 & -1.70 & 2172.17 & 230.33\end{array}$

$\begin{array}{lll}0.00 & -0.407 & 0.000\end{array}$$$
\begin{array}{llllll}
0.011 & -3.34 & -1.26 & 2176.16 & 234.41
\end{array}
$$

$\begin{array}{llll}194 & 311 & -0.40 & 0.00\end{array}$

$\begin{array}{llll}195 & 312 & -0.40 & 0.00\end{array}$

0.04

$0.00-0.406$

0.000

0.016

$\begin{array}{lllll}0.007 & -2.79 & -0.81 & 2181.42 & 237.23\end{array}$

$\begin{array}{llll}196 & 313 & -0.41 & 0.00\end{array}$

$\begin{array}{llll}197 & 314 & 0.48 & 0.00\end{array}$

0.04

$0.00-0.406$

0.000

0.016

$\begin{array}{lllll}0.007 & -2.70 & -0.69 & 2185.56 & 241.16\end{array}$

$\begin{array}{lll}0.00 & 0.01 & 0.539\end{array}$

0.000

0.020

$\begin{array}{lllll}0.007 & -2.37 & -0.38 & 2190.77 & 244.01\end{array}$

$\begin{array}{llll}198 & 315 & 0.49 & 0.00\end{array}$

0.01

0.01

$\begin{array}{llll}199 & 316 & 0.49 & 0.00\end{array}$

$\begin{array}{llll}200 & 317 & 0.29 & 0.00\end{array}$

0.0

0.552

0.000

0.109

$\begin{array}{lllll}0.014 & -2.10 & -4.07 & 2198.53 & 244.33\end{array}$

0.000

0.102

$\begin{array}{llllll}0.009 & -1.61 & -3.95 & 2203.76 & 247.17\end{array}$

$\begin{array}{llll}201 & 318 & 0.28 & 0.00\end{array}$

$\begin{array}{llll}202 & 319 & 0.28 & 0.00\end{array}$

$\begin{array}{llll}203 & 320 & 0.28 & 0.00\end{array}$

0.02

$0.00-0.319$

0.000

0.102

$\begin{array}{lllll}0.009 & -1.89 & -4.17 & 2207.88 & 251.13\end{array}$

$0.00 \quad 0.308$

0.000

0.308

0.000

$\begin{array}{llllll}0.010 & -0.003 & -1.76 & -1.86 & 2214.47 & 260.67\end{array}$

$0.00 \quad 0.308$

$0.000-0.002-0.006-1.87 \quad-1.88-2223.04 \quad 268.24$

$\begin{array}{llllllll}0.000 & -0.002 & -0.006 & -1.51 & -1.57 & 2227.56 & 271.80\end{array}$
$-1.22 \quad 311.07$

$-1.57 \quad 316.44$

$\begin{array}{ll}-1.82 & 320.39\end{array}$

$-2.23 \quad 325.85$

$-2.40 \quad 330.04$

$-2.81 \quad 335.65$

$-2.99 \quad 339.98$

$\begin{array}{ll}-3.43 & 345.73\end{array}$

$\begin{array}{ll}-3.73 & 350.09\end{array}$

$-4.29 \quad 355.87$

$-4.65 \quad 360.33$

$-5.50 \quad 365.96$

$-4.26 \quad 191.82$

$\begin{array}{ll}-4.71 & 190.21\end{array}$

$-5.28 \quad 190.08$

$-5.84188 .62$

$\begin{array}{ll}-6.11 & 189.03\end{array}$

$-6.24188 .26$

$-6.71 \quad 188.70$

$-6.84188 .17$

$\begin{array}{ll}-7.30 & 188.88\end{array}$

$-7.34 \quad 188.67$

$-7.76 \quad 189.64$

$-7.68 \quad 189.79$

$-8.08 \quad 191.01$

$-7.98 \quad 191.41$

$-8.29192 .95$

$-8.41 \quad 193.37$

$-8.74 \quad 195.09$

$-8.50 \quad 196.09$

$-8.65198 .22$

$-8.31 \quad 199.53$

$-8.39 \quad 201.95$

$-7.96203 .57$

$\begin{array}{ll}-7.97 & 206.25\end{array}$

$\begin{array}{ll}-7.37 & 208.25\end{array}$

$-6.47 \quad 212.06$

$-5.75 \quad 214.40$

$-4.60 \quad 218.64$

$\begin{array}{ll}-3.89 & 221.17\end{array}$

$-2.88 \quad 225.48$

$\begin{array}{ll}-2.20 & 228.18\end{array}$

$-2.02 \quad 231.85$

$-1.67 \quad 234.42$

$\begin{array}{ll}-1.48 \quad 238.29 \\ -1.01 & 241.16\end{array}$

$-1.01 \quad 241.16$

$-0.92 \quad 245.13$

$-0.60 \quad 248.05$

$-4.29 \quad 248.42$

$-4.17 \quad 251.32$

$-4.43 \quad 255.30$

$-1.71 \quad 260.99$

$-1.95 \quad 265.17$

$-1.91 \quad 268.36$

$-1.97 \quad 272.90$

$-1.64 \quad 276.55$ 


\section{$Z=117$}

$\begin{array}{rrrrrrrrrrrrrr}205 & 322 & 0.28 & 0.00 & 0.04 & -0.01 & 0.309 & 0.000 & -0.016 & -0.000 & -1.63 & -1.53 & 2230.91 & 276.52 \\ 206 & 323 & -0.45 & 0.02 & 0.04 & 0.00 & -0.454 & -0.021 & 0.032 & 0.005 & -2.25 & 0.10 & 2233.93 & 281.57 \\ 207 & 324 & 0.28 & 0.01 & 0.05 & -0.01 & 0.310 & -0.013 & -0.028 & -0.004 & -1.52 & -1.28 & 2238.54 & 285.03 \\ 208 & 325 & 0.28 & 0.00 & 0.06 & -0.01 & 0.310 & 0.000 & -0.040 & -0.007 & -1.47 & -1.00 & 2242.76 & 288.89 \\ 209 & 326 & 0.28 & 0.00 & 0.06 & -0.01 & 0.310 & 0.000 & -0.040 & -0.007 & -1.71 & -1.19 & 2246.01 & 293.70 \\ 210 & 327 & 0.28 & 0.00 & 0.07 & -0.01 & 0.311 & 0.000 & -0.052 & -0.010 & -1.86 & -1.01 & 2250.17 & 297.62 \\ 211 & 328 & 0.28 & 0.00 & 0.07 & -0.01 & 0.311 & 0.000 & -0.052 & -0.010 & -2.17 & -1.30 & 2253.37 & 302.49 \\ 212 & 329 & 0.11 & 0.00 & 0.01 & 0.02 & 0.118 & 0.000 & -0.006 & -0.021 & -1.94 & -1.41 & 2257.65 & 306.28 \\ 213 & 330 & 0.11 & 0.00 & 0.01 & 0.01 & 0.118 & 0.000 & -0.007 & -0.011 & -2.17 & -1.72 & 2260.71 & 311.29 \\ 214 & 331 & 0.37 & 0.00 & -0.02 & 0.01 & 0.408 & 0.000 & 0.088 & 0.010 & -1.71 & -2.20 & 2265.20 & 314.87 \\ 215 & 332 & 0.11 & 0.00 & 0.02 & 0.01 & 0.119 & 0.000 & -0.018 & -0.012 & -2.90 & -2.36 & 2267.97 & 320.17 \\ 216 & 333 & 0.11 & 0.00 & 0.02 & 0.01 & 0.119 & 0.000 & -0.018 & -0.012 & -3.07 & -2.53 & 2272.00 & 324.22 \\ 217 & 334 & 0.11 & 0.00 & 0.03 & 0.00 & 0.119 & 0.000 & -0.031 & -0.004 & -3.62 & -2.98 & 2274.91 & 329.38 \\ 218 & 335 & 0.11 & 0.00 & 0.03 & 0.00 & 0.119 & 0.000 & -0.031 & -0.004 & -3.81 & -3.17 & 2278.80 & 333.56 \\ 219 & 336 & 0.10 & 0.00 & 0.03 & 0.00 & 0.108 & 0.000 & -0.032 & -0.003 & -4.30 & -3.66 & 2281.59 & 338.83 \\ 220 & 337 & 0.09 & 0.00 & 0.03 & -0.01 & 0.097 & 0.000 & -0.033 & 0.007 & -4.68 & -4.01 & 2285.51 & 342.99 \\ 221 & 338 & 0.09 & 0.00 & 0.04 & -0.01 & 0.097 & 0.000 & -0.045 & 0.006 & -5.48 & -4.61 & 2288.27 & 348.30 \\ 222 & 339 & 0.07 & 0.00 & 0.03 & -0.01 & 0.075 & 0.000 & -0.034 & 0.008 & -5.54 & -4.86 & 2291.93 & 352.71\end{array}$

$-1.61 \quad 281.35$

$-0.16 \quad 286.30$

$\begin{array}{ll}-1.35 \quad 290.05 \\ -1.04 & 294.04\end{array}$

$-1.04 \quad 294.04$

$-1.24 \quad 298.94$

$-1.03 \quad 303.00$
-1.33

$-1.33 \quad 307.96$

$-1.36 \quad 311.94$

$-1.72 \quad 317.02$

$-2.27 \quad 320.64$

$\begin{array}{ll}-2.35 & 326.15\end{array}$

$-2.51 \quad 330.33$

$-2.97 \quad 335.61$

$-3.16 \quad 339.92$

$-3.64345 .33$

$-3.99 \quad 349.65$

$-4.56 \quad 355.12$

$-4.82 \quad 359.67$

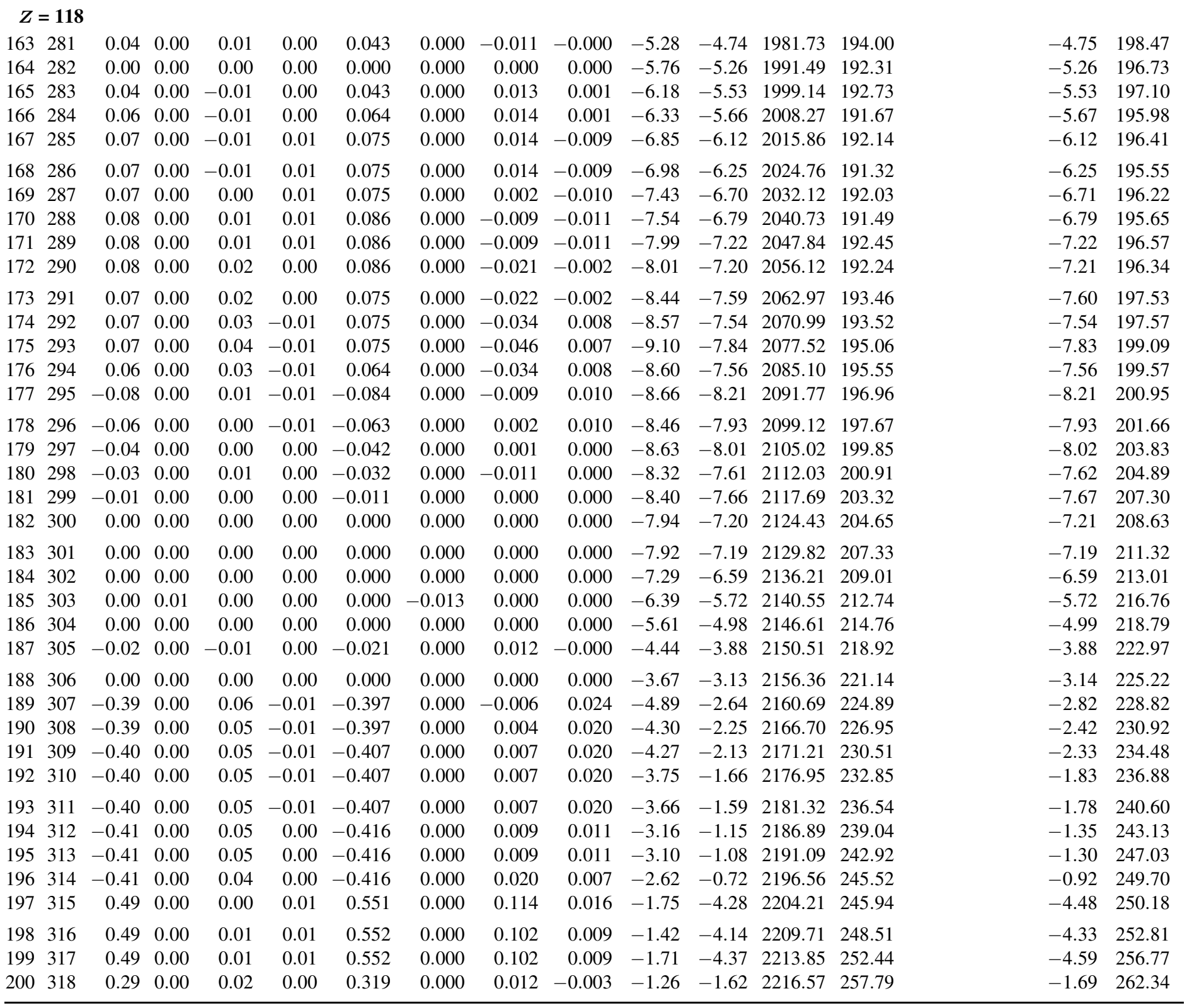




\begin{tabular}{|c|c|c|c|c|c|c|c|c|c|c|c|c|c|c|}
\hline \multicolumn{15}{|l|}{$Z=118$} \\
\hline 201319 & 0.28 & 0.00 & 0.02 & 0.00 & 0.308 & 0.000 & 0.010 & -0.003 & -1.65 & -1.84 & 2220.53 & 261.90 & -1.91 & 266.51 \\
\hline 202320 & 0.28 & 0.00 & 0.02 & 0.00 & 0.308 & 0.000 & 0.010 & -0.003 & -1.63 & -1.82 & 2225.80 & 264.70 & -1.88 & 269.39 \\
\hline $203 \quad 321$ & 0.28 & 0.00 & 0.03 & 0.00 & 0.308 & 0.000 & -0.002 & -0.006 & -1.75 & -1.85 & 2229.40 & 269.18 & -1.92 & 273.93 \\
\hline 204322 & 0.28 & 0.00 & 0.03 & 0.00 & 0.308 & 0.000 & -0.002 & -0.006 & -1.39 & -1.53 & 2234.21 & 272.44 & -1.60 & 277.29 \\
\hline $205 \quad 323$ & -0.45 & 0.00 & 0.05 & 0.00 & -0.455 & 0.000 & 0.022 & 0.010 & -2.32 & -0.14 & 2236.22 & 278.50 & -0.41 & 283.23 \\
\hline $206 \quad 324$ & -0.46 & 0.00 & 0.05 & 0.00 & -0.465 & 0.000 & 0.025 & 0.009 & -2.19 & 0.04 & 2240.98 & 281.81 & -0.21 & 286.63 \\
\hline $207 \quad 325$ & -0.46 & 0.00 & 0.05 & 0.00 & -0.465 & 0.000 & 0.025 & 0.009 & -2.42 & -0.16 & 2244.42 & 286.44 & -0.43 & 291.33 \\
\hline 208326 & -0.47 & 0.00 & 0.05 & -0.01 & -0.474 & 0.000 & 0.030 & 0.017 & -2.32 & 0.05 & 2248.99 & 289.94 & -0.18 & 294.96 \\
\hline 209327 & 0.28 & 0.00 & 0.06 & -0.01 & 0.310 & 0.000 & -0.040 & -0.007 & -1.50 & -1.09 & 2253.22 & 293.79 & -1.13 & 299.10 \\
\hline 210328 & 0.22 & 0.00 & 0.01 & 0.01 & 0.239 & 0.000 & 0.010 & -0.011 & -0.93 & -0.78 & 2257.52 & 297.55 & -0.81 & 302.97 \\
\hline 211329 & 0.16 & 0.00 & -0.03 & 0.02 & 0.172 & 0.000 & 0.049 & -0.013 & -1.85 & -1.14 & 2260.81 & 302.34 & -1.10 & 307.93 \\
\hline 212330 & 0.28 & 0.01 & 0.08 & -0.01 & 0.313 & -0.014 & -0.064 & -0.013 & -2.22 & -1.14 & 2265.25 & 305.96 & -1.11 & 311.64 \\
\hline $213 \quad 331$ & 0.37 & 0.00 & -0.02 & 0.01 & 0.408 & 0.000 & 0.088 & 0.010 & -1.90 & -2.54 & 2269.42 & 309.87 & -2.61 & 315.56 \\
\hline 214332 & 0.37 & 0.00 & -0.02 & 0.01 & 0.408 & 0.000 & 0.088 & 0.010 & -1.77 & -2.43 & 2273.62 & 313.74 & -2.48 & 319.57 \\
\hline 215333 & 0.37 & 0.00 & -0.02 & 0.01 & 0.408 & 0.000 & 0.088 & 0.010 & -1.96 & -2.58 & 2276.38 & 319.05 & -2.65 & 324.97 \\
\hline 216334 & 0.37 & 0.00 & -0.02 & 0.01 & 0.408 & 0.000 & 0.088 & 0.010 & -1.80 & -2.44 & 2280.38 & 323.13 & -2.49 & 329.18 \\
\hline 217335 & 0.11 & 0.00 & 0.03 & 0.00 & 0.119 & 0.000 & -0.031 & -0.004 & -3.14 & -2.53 & 2282.93 & 328.65 & -2.52 & 334.89 \\
\hline 218336 & 0.11 & 0.00 & 0.03 & 0.00 & 0.119 & 0.000 & -0.031 & -0.004 & -3.33 & -2.71 & 2287.10 & 332.55 & -2.69 & 338.92 \\
\hline 219337 & 0.09 & 0.00 & 0.04 & -0.01 & 0.097 & 0.000 & -0.045 & 0.006 & -4.11 & -3.30 & 2290.01 & 337.71 & -3.25 & 344.25 \\
\hline 220338 & 0.09 & 0.00 & 0.04 & -0.01 & 0.097 & 0.000 & -0.045 & 0.006 & -4.49 & -3.66 & 2294.20 & 341.58 & -3.61 & 348.26 \\
\hline 221339 & 0.09 & 0.00 & 0.04 & -0.01 & 0.097 & 0.000 & -0.045 & 0.006 & -5.09 & -4.24 & 2296.95 & 346.91 & -4.18 & 353.72 \\
\hline \multicolumn{15}{|l|}{$Z=119$} \\
\hline 165284 & 0.03 & 0.00 & -0.01 & 0.00 & 0.032 & 0.000 & 0.012 & 0.000 & -6.11 & -5.49 & 1997.16 & 201.99 & -5.50 & 206.66 \\
\hline 166285 & 0.06 & 0.00 & 0.00 & 0.00 & 0.064 & 0.000 & 0.002 & 0.000 & -6.17 & -5.56 & 2006.26 & 200.97 & -5.57 & 205.58 \\
\hline 167286 & 0.06 & 0.00 & 0.00 & 0.00 & 0.064 & 0.000 & 0.002 & 0.000 & -6.64 & -6.01 & 2014.18 & 201.12 & -6.02 & 205.68 \\
\hline 168287 & -0.08 & 0.00 & -0.01 & 0.00 & -0.084 & 0.000 & 0.014 & -0.001 & -6.82 & -6.41 & 2023.36 & 200.01 & -6.43 & 204.51 \\
\hline 169288 & 0.07 & 0.00 & 0.00 & 0.01 & 0.075 & 0.000 & 0.002 & -0.010 & -7.27 & -6.56 & 2030.74 & 200.70 & -6.57 & 205.16 \\
\hline $170 \quad 289$ & 0.08 & 0.00 & 0.01 & 0.01 & 0.086 & 0.000 & -0.009 & -0.011 & -7.41 & -6.67 & 2039.41 & 200.10 & -6.68 & 204.53 \\
\hline 171290 & 0.08 & 0.00 & 0.02 & 0.00 & 0.086 & 0.000 & -0.021 & -0.002 & -7.96 & -7.16 & 2046.90 & 200.68 & -7.17 & 205.07 \\
\hline 172291 & 0.08 & 0.00 & 0.03 & 0.00 & 0.086 & 0.000 & -0.033 & -0.003 & -8.13 & -7.16 & 2055.22 & 200.43 & -7.17 & 204.79 \\
\hline 173292 & 0.08 & 0.00 & 0.03 & -0.01 & 0.086 & 0.000 & -0.034 & 0.007 & -8.60 & -7.60 & 2062.45 & 201.28 & -7.61 & 205.61 \\
\hline 174293 & 0.07 & 0.00 & 0.04 & -0.01 & 0.075 & 0.000 & -0.046 & 0.007 & -8.83 & -7.57 & 2070.51 & 201.29 & -7.57 & 205.60 \\
\hline $175 \quad 294$ & 0.07 & 0.00 & 0.04 & -0.02 & 0.075 & 0.000 & -0.046 & 0.017 & -9.27 & -7.91 & 2077.41 & 202.46 & -7.90 & 206.75 \\
\hline 176295 & 0.07 & 0.00 & 0.04 & -0.02 & 0.075 & 0.000 & -0.046 & 0.017 & -8.96 & -7.62 & 2084.98 & 202.96 & -7.60 & 207.23 \\
\hline 177296 & 0.06 & 0.00 & 0.03 & -0.01 & 0.064 & 0.000 & -0.034 & 0.008 & -8.80 & -7.75 & 2091.46 & 204.55 & -7.75 & 208.79 \\
\hline $178 \quad 297$ & -0.06 & 0.00 & 0.00 & -0.01 & -0.063 & 0.000 & 0.002 & 0.010 & -8.32 & -7.81 & 2099.17 & 204.91 & -7.81 & 209.14 \\
\hline 179298 & -0.03 & 0.00 & 0.00 & 0.00 & -0.032 & 0.000 & 0.000 & 0.000 & -8.49 & -7.83 & 2105.32 & 206.84 & -7.83 & 211.05 \\
\hline 180299 & -0.03 & 0.00 & 0.01 & 0.00 & -0.032 & 0.000 & -0.011 & 0.000 & -8.17 & -7.49 & 2112.41 & 207.82 & -7.49 & 212.02 \\
\hline 181300 & -0.02 & 0.00 & 0.01 & 0.00 & -0.021 & 0.000 & -0.012 & 0.000 & -8.27 & -7.54 & 2118.38 & 209.91 & -7.54 & 214.11 \\
\hline 182301 & 0.00 & 0.00 & 0.00 & 0.00 & 0.000 & 0.000 & 0.000 & 0.000 & -7.76 & -7.04 & 2125.10 & 211.26 & -7.04 & 215.46 \\
\hline $183 \quad 302$ & 0.00 & 0.00 & 0.00 & 0.00 & 0.000 & 0.000 & 0.000 & 0.000 & -7.73 & -7.01 & 2130.80 & 213.64 & -7.01 & 217.84 \\
\hline 184303 & 0.00 & 0.00 & 0.00 & 0.00 & 0.000 & 0.000 & 0.000 & 0.000 & -7.08 & -6.40 & 2137.20 & 215.31 & -6.40 & 219.51 \\
\hline 185304 & 0.00 & 0.01 & 0.00 & 0.00 & 0.000 & -0.013 & 0.000 & 0.000 & -6.19 & -5.54 & 2141.85 & 218.73 & -5.54 & 222.94 \\
\hline 186305 & 0.00 & 0.00 & 0.00 & 0.00 & 0.000 & 0.000 & 0.000 & 0.000 & -5.40 & -4.80 & 2147.93 & 220.73 & -4.80 & 224.95 \\
\hline 187306 & -0.01 & 0.00 & -0.01 & 0.00 & -0.010 & 0.000 & 0.012 & -0.000 & -4.25 & -3.70 & 2152.15 & 224.58 & -3.70 & 228.82 \\
\hline 188307 & -0.39 & 0.00 & 0.05 & -0.01 & -0.397 & 0.000 & 0.004 & 0.020 & -5.39 & -3.39 & 2158.45 & 226.35 & -3.60 & 230.40 \\
\hline 189308 & -0.40 & 0.00 & 0.06 & -0.01 & -0.407 & 0.000 & -0.003 & 0.024 & -5.59 & -3.33 & 2163.51 & 229.35 & -3.56 & 233.41 \\
\hline $190 \quad 309$ & -0.40 & 0.00 & 0.06 & -0.01 & -0.407 & 0.000 & -0.003 & 0.024 & -5.17 & -2.95 & 2169.55 & 231.39 & -3.15 & 235.49 \\
\hline 191310 & -0.40 & 0.00 & 0.05 & -0.01 & -0.407 & 0.000 & 0.007 & 0.020 & -4.92 & -2.85 & 2174.38 & 234.63 & -3.08 & 238.73 \\
\hline 192311 & -0.40 & 0.00 & 0.05 & -0.01 & -0.407 & 0.000 & 0.007 & 0.020 & -4.40 & -2.38 & 2180.14 & 236.95 & -2.58 & 241.10 \\
\hline 193312 & -0.40 & 0.00 & 0.04 & -0.01 & -0.406 & 0.000 & 0.018 & 0.015 & -4.20 & -2.30 & 2184.80 & 240.35 & -2.53 & 244.51 \\
\hline 194313 & -0.41 & 0.00 & 0.05 & 0.00 & -0.416 & 0.000 & 0.009 & 0.011 & -3.83 & -1.88 & 2190.41 & 242.81 & -2.11 & 247.01 \\
\hline $195 \quad 314$ & -0.41 & 0.00 & 0.04 & -0.01 & -0.416 & 0.000 & 0.021 & 0.015 & -3.77 & -1.81 & 2194.91 & 246.38 & -2.05 & 250.62 \\
\hline 196315 & -0.42 & 0.00 & 0.05 & 0.00 & -0.426 & 0.000 & 0.012 & 0.011 & -3.40 & -1.41 & 2200.36 & 249.01 & -1.65 & 253.29 \\
\hline 197316 & -0.42 & 0.00 & 0.05 & 0.00 & -0.426 & 0.000 & 0.012 & 0.011 & -3.32 & -1.32 & 2204.66 & 252.78 & -1.59 & 257.08 \\
\hline 198317 & -0.42 & 0.00 & 0.04 & 0.00 & -0.426 & 0.000 & 0.023 & 0.006 & -2.91 & -1.02 & 2210.01 & 255.50 & -1.26 & 259.87 \\
\hline 199318 & -0.42 & 0.00 & 0.04 & 0.00 & -0.426 & 0.000 & 0.023 & 0.006 & -2.85 & -0.93 & 2214.14 & 259.44 & -1.20 & 263.85 \\
\hline
\end{tabular}




\section{$Z=119$}

$\begin{array}{llll}200 & 319 & -0.42 & 0.00\end{array}$ $\begin{array}{lllll}201 & 320 & -0.43 & 0.00\end{array}$ $\begin{array}{llll}202 & 321 & -0.43 & 0.00\end{array}$ $\begin{array}{llll}203 & 322 & -0.43 & 0.00\end{array}$ $\begin{array}{llll}204 & 323 & -0.44 & 0.00\end{array}$ $\begin{array}{llll}205 & 324 & -0.44 & 0.00\end{array}$ $\begin{array}{llll}206 & 325 & -0.45 & 0.00\end{array}$ $\begin{array}{llll}207 & 326 & -0.45 & 0.00\end{array}$ $\begin{array}{llll}208 & 327 & -0.45 & 0.00\end{array}$ $\begin{array}{llll}209 & 328 & -0.47 & 0.01\end{array}$

$\begin{array}{lllll}210 & 329 & 0.29 & 0.00\end{array}$

211330 $0.28 \quad 0.00$ $\begin{array}{llll}0.37 & 0.00 & -0.01\end{array}$

212331

$\begin{array}{llll}0.37 & 0.00 & -0.01\end{array}$

$0.04-0.01-0.426$

$\begin{array}{lll}0.04 & -0.01 & -0.435\end{array}$

$\begin{array}{lll}0.04 & -0.01 & -0.435\end{array}$

$\begin{array}{lll}0.04 & -0.01 & -0.435\end{array}$

$\begin{array}{llll}0.04 & -0.01 & -0.445\end{array}$

$\begin{array}{llll}0.04 & -0.01 & -0.445\end{array}$

$\begin{array}{llll}0.04 & -0.01 & -0.455\end{array}$

$\begin{array}{llll}0.04 & -0.01 & -0.455\end{array}$

$\begin{array}{llll}0.04 & -0.01 & -0.455\end{array}$

$\begin{array}{lll}0.05 & 0.00 & -0.474\end{array}$

$\begin{array}{llll}0.07 & -0.01 & 0.323\end{array}$

$0.07-0.01$

0.311

0.00

0.409

214333

0.0

0.408

0.00

0.409

$0.00 \quad 0.409$

$\begin{array}{lll}0.37 & 0.00 & -0.01\end{array}$

0.00

0.409

0.00

0.409

$217 \quad 336$

$\begin{array}{llll}0.37 & 0.00 & -0.01\end{array}$

0.00

0.421

219338

$0.09 \quad 0.00$

$0.04-0.01$

0.097

220339

$\begin{array}{lll}0.04 & -0.01 & 0.097\end{array}$ $\begin{array}{llll}0.37 & 0.00 & -0.02\end{array}$
0.000

0.000

0.000

0.000

0.000

0.000

0.000

0.000

$-0.009$

$0.000-0.052$

$0.000 \quad 0.074$

$0.000 \quad 0.088$

$0.000 \quad 0.074$

$0.000 \quad 0.074$

$0.000 \quad 0.074$

$0.000 \quad 0.074$

$0.000 \quad 0.065$

$0.000-0.045$

$0.000-0.045$
$0.000-0.049$

$\begin{array}{lllll}0.015 & -2.57 & -0.63 & 2219.32 & 262.33\end{array}$ $\begin{array}{llllll}0.014 & -2.79 & -0.87 & 2223.59 & 266.13\end{array}$ $\begin{array}{lllll}0.014 & -2.47 & -0.61 & 2228.64 & 269.15\end{array}$ $\begin{array}{lllll}0.014 & -2.62 & -0.72 & 2232.61 & 273.26\end{array}$ $\begin{array}{lllll}0.014 & -2.43 & -0.48 & 2237.50 & 276.43\end{array}$ $\begin{array}{lllll}0.014 & -2.56 & -0.59 & 2241.30 & 280.71\end{array}$ $\begin{array}{lllll}0.013 & -2.45 & -0.39 & 2246.07 & 284.01\end{array}$ $\begin{array}{lllll}0.013 & -2.65 & -0.57 & 2249.78 & 288.37\end{array}$ $\begin{array}{lllll}0.013 & -2.39 & -0.36 & 2254.36 & 291.86\end{array}$ $\begin{array}{llllll}0.009 & -2.61 & -0.36 & 2257.73 & 296.57\end{array}$

\section{$Z=120$}

$\begin{array}{lllllll}167 & 287 & -0.08 & 0.00 & -0.01 & 0.00 & -0.084\end{array}$ $\begin{array}{llllllll}168 & 288 & -0.10 & 0.00 & -0.01 & -0.01 & -0.104\end{array}$ $\begin{array}{lllllll}169 & 289 & -0.10 & 0.00 & -0.01 & -0.01 & -0.104\end{array}$ $\begin{array}{llllllll}170 & 290 & -0.12 & 0.00 & -0.01 & -0.01 & -0.125\end{array}$ $\begin{array}{lllllll}171 & 291 & 0.08 & 0.00 & 0.03 & 0.00 & 0.086\end{array}$ $\begin{array}{lllll}172 & 292 & 0.08 & 0.00\end{array}$ $\begin{array}{llll}173 & 293 & 0.08 & 0.00\end{array}$ $\begin{array}{llll}174 & 294 & 0.08 & 0.00\end{array}$ $\begin{array}{llll}175 & 295 & 0.07 & 0.00\end{array}$ $\begin{array}{llll}176 & 296 & 0.07 & 0.00\end{array}$

$\begin{array}{lllll}177 & 297 & 0.06 & 0.00\end{array}$ $\begin{array}{llll}178 & 298 & -0.06 & 0.00\end{array}$ $\begin{array}{llll}179 & 299 & -0.03 & 0.00\end{array}$ $\begin{array}{llll}180 & 300 & -0.03 & 0.00\end{array}$ $\begin{array}{llll}181 & 301 & 0.00 & 0.00\end{array}$ $\begin{array}{lllll}182 & 302 & 0.00 & 0.00\end{array}$ $\begin{array}{llll}183 & 303 & 0.00 & 0.00\end{array}$ $\begin{array}{llll}184 & 304 & 0.00 & 0.00\end{array}$ $\begin{array}{llll}185 & 305 & 0.00 & 0.01\end{array}$ $\begin{array}{llll}186 & 306 & 0.00 & 0.00\end{array}$

$\begin{array}{lllll}187 & 307 & -0.39 & 0.00\end{array}$ $\begin{array}{llll}188 & 308 & -0.40 & 0.00\end{array}$ $\begin{array}{llll}189 & 309 & -0.40 & 0.00\end{array}$ $\begin{array}{llll}190 & 310 & -0.40 & 0.00\end{array}$ $\begin{array}{llll}191 & 311 & -0.40 & 0.00\end{array}$

$\begin{array}{llll}192 & 312 & -0.41 & 0.00\end{array}$ $\begin{array}{llll}193 & 313 & -0.41 & 0.00\end{array}$ $\begin{array}{llll}194 & 314 & -0.42 & 0.00\end{array}$ $\begin{array}{lllll}195 & 315 & -0.42 & 0.00\end{array}$ $\begin{array}{llll}196 & 316 & -0.42 & 0.00\end{array}$ $\begin{array}{llll}197 & 317 & -0.42 & 0.00\end{array}$ $\begin{array}{llll}198 & 318 & -0.43 & 0.00\end{array}$ $\begin{array}{llll}199 & 319 & -0.43 & 0.00\end{array}$ $\begin{array}{lllll}200 & 320 & -0.43 & 0.00\end{array}$ $\begin{array}{llll}201 & 321 & -0.43 & 0.00\end{array}$ $\begin{array}{lll}0.03 & -0.01 \quad 0.086\end{array}$ $\begin{array}{lll}0.04 & -0.01 & 0.086\end{array}$ $\begin{array}{lll}0.04 & -0.02 & 0.086\end{array}$ $\begin{array}{llll}0.04 & -0.02 & 0.075\end{array}$ $\begin{array}{lll}0.04 & -0.02 \quad 0.075\end{array}$ $\begin{array}{llll}0.04 & -0.02 & 0.064\end{array}$ $\begin{array}{lll}0.00 & -0.01 & -0.063\end{array}$ $\begin{array}{lll}0.00 & 0.00 & -0.032\end{array}$ $\begin{array}{lll}0.01 & 0.00 & -0.032\end{array}$ $\begin{array}{lll}0.00 & 0.00 \quad 0.000\end{array}$

$\begin{array}{lll}0.00 & 0.00 & 0.000\end{array}$ $0.00 \quad 0.00 \quad 0.000$ $\begin{array}{lll}0.00 & 0.00 & 0.000\end{array}$

$\begin{array}{lllllll}0.000 & 0.014 & -0.001 & -5.99 & -5.61 & 2013.47 & 209.11\end{array}$ $\begin{array}{lllllll}0.000 & 0.016 & 0.008 & -6.19 & -5.74 & 2022.72 & 207.94\end{array}$ $\begin{array}{llllllll}0.000 & 0.016 & 0.008 & -6.65 & -6.19 & 2030.42 & 208.31\end{array}$ $\begin{array}{llllllll}0.000 & 0.018 & 0.008 & -6.69 & -6.20 & 2039.31 & 207.49\end{array}$ $\begin{array}{lllllll}0.000 & -0.033 & -0.003 & -7.34 & -6.41 & 2046.55 & 208.32\end{array}$ $\begin{array}{llllllll}0.000 & -0.034 & 0.007 & -7.42 & -6.47 & 2055.26 & 207.69\end{array}$ $\begin{array}{llllllll}0.000 & -0.045 & 0.007 & -8.10 & -6.90 & 2062.49 & 208.52\end{array}$ $\begin{array}{lllllll}0.000 & -0.046 & 0.016 & -8.22 & -6.93 & 2070.93 & 208.15\end{array}$ $\begin{array}{lllllll}0.000 & -0.046 & 0.017 & -8.57 & -7.23 & 2077.82 & 209.34\end{array}$ $\begin{array}{llllllll}0.000 & -0.046 & 0.017 & -8.24 & -6.92 & 2085.70 & 209.53\end{array}$ $0.000-0.047$ $0.000 \quad 0.002$ $0.000 \quad 0.000$ $0.000-0.011$ $0.000 \quad 0.000$

$0.000 \quad 0.000$ $0.000 \quad 0.000$ $0.000 \quad 0.000$ $\begin{array}{lllll}0.00 & 0.00 & 0.000 & -0.013 & 0.000\end{array}$ $\begin{array}{lllll}0.00 & 0.00 & 0.000 & 0.000 & 0.000\end{array}$ $\begin{array}{llll}0.05 & -0.01 & -0.397\end{array}$ $\begin{array}{llll}0.06 & -0.01 & -0.407\end{array}$ $\begin{array}{lll}0.06 & -0.01 & -0.407\end{array}$ $\begin{array}{llll}0.06 & -0.01 & -0.407\end{array}$ $\begin{array}{llll}0.05 & -0.01 & -0.407\end{array}$

$\begin{array}{ll}0.000 \quad 0.004 \\ 0.000 & -0.003\end{array}$ $0.000-0.003$

$0.000-0.003$ $0.000-0.003$ $0.000 \quad 0.007$

$\begin{array}{llll}0.05 & -0.01 & -0.416\end{array}$

$0.000 \quad 0.010$ $\begin{array}{llllll}0.017 & -8.37 & -7.03 & 2092.17 & 211.13\end{array}$ $\begin{array}{lllll}0.010 & -7.52 & -7.05 & 2100.16 & 211.21\end{array}$ $\begin{array}{lllll}0.000 & -7.70 & -7.07 & 2106.33 & 213.11\end{array}$ $\begin{array}{lllll}0.000 & -7.38 & -6.72 & 2113.73 & 213.79\end{array}$ $\begin{array}{llllll}0.000 & -7.46 & -6.74 & 2119.70 & 215.89\end{array}$ $\begin{array}{lllll}0.000 & -6.98 & -6.29 & 2126.77 & 216.88\end{array}$ $\begin{array}{llllll}0.000 & -6.93 & -6.25 & 2132.47 & 219.25\end{array}$ $\begin{array}{lllll}0.000 & -6.28 & -5.62 & 2139.18 & 220.62\end{array}$ $\begin{array}{lllll}0.000 & -5.40 & -4.78 & 2143.86 & 224.01\end{array}$ $\begin{array}{lllll}0.000 & -4.59 & -4.02 & 2150.24 & 225.71\end{array}$ $\begin{array}{llllll}0.020 & -5.56 & -3.68 & 2155.23 & 228.78\end{array}$ $\begin{array}{llllll}0.024 & -5.57 & -3.47 & 2161.94 & 230.14\end{array}$ $\begin{array}{lllll}0.024 & -5.55 & -3.43 & 2167.05 & 233.11\end{array}$ $\begin{array}{lllll}0.024 & -5.12 & -3.05 & 2173.38 & 234.85\end{array}$ $\begin{array}{lllll}0.020 & -4.87 & -2.94 & 2178.23 & 238.07\end{array}$ $0.05-0.01-0.416$ $0.000 \quad 0.010$ $\begin{array}{lllll}0.019 & -4.43 & -2.48 & 2184.29 & 240.08\end{array}$ $\begin{array}{lllll}0.019 & -4.37 & -2.43 & 2189.01 & 243.43\end{array}$ $\begin{array}{llllllllll}0.05 & 0.00 & -0.426 & 0.000 & 0.012 & 0.011 & -3.88 & -2.00 & 2194.91 & 245.60\end{array}$ $\begin{array}{llllllllll}0.05 & 0.00 & -0.426 & 0.000 & 0.012 & 0.011 & -3.86 & -1.97 & 2199.46 & 249.12\end{array}$ $\begin{array}{llllllllll}0.05 & 0.00 & -0.426 & 0.000 & 0.012 & 0.011 & -3.45 & -1.62 & 2205.26 & 251.40\end{array}$ $\begin{array}{llllllllll}0.04 & -0.01 & -0.426 & 0.000 & 0.024 & 0.015 & -3.39 & -1.53 & 2209.56 & 255.16\end{array}$ $\begin{array}{llllllllll}0.05 & 0.00 & -0.436 & 0.000 & 0.015 & 0.010 & -3.10 & -1.20 & 2215.20 & 257.60\end{array}$ $\begin{array}{llllllllll}0.05 & 0.00 & -0.436 & 0.000 & 0.015 & 0.010 & -3.05 & -1.13 & 2219.35 & 261.51\end{array}$ $\begin{array}{llllllllll}0.04 & -0.01 & -0.435 & 0.000 & 0.027 & 0.014 & -2.75 & -0.86 & 2224.85 & 264.08\end{array}$ $\begin{array}{llllllllll}0.04 & -0.01 & -0.435 & 0.000 & 0.027 & 0.014 & -2.76 & -1.02 & 2229.07 & 267.94\end{array}$
$-0.86 \quad 266.85$

$-1.13 \quad 270.67$

$-0.85 \quad 273.78$

$-0.97 \quad 277.93$

$-0.73 \quad 281.19$

$-0.86 \quad 285.52$

$-0.65 \quad 288.91$

$-0.86 \quad 293.33$

$-0.62 \quad 296.93$

$-0.69 \quad 301.65$

$-0.98 \quad 304.90$

$-1.22 \quad 309.62$

$-2.72 \quad 311.82$

$-2.94 \quad 316.73$

$-2.80 \quad 320.74$

$-3.00 \quad 325.82$

$\begin{array}{ll}-2.87 & 329.99\end{array}$

$\begin{array}{ll}-3.08 & 335.23\end{array}$

$\begin{array}{ll}-3.02 & 339.49\end{array}$

$\begin{array}{ll}-3.29 & 344.81\end{array}$

$-3.65348 .81$

$\begin{array}{ll}-5.63 & 213.97\end{array}$

$-5.76 \quad 212.74$

$-6.20 \quad 213.06$

$-6.22 \quad 212.19$

$-6.42 \quad 212.99$

$-6.48 \quad 212.32$

$-6.91 \quad 213.13$

$-6.92 \quad 212.74$

$-7.22 \quad 213.90$

$-6.91214 .06$

$-7.01 \quad 215.65$

$\begin{array}{ll}-7.05 & 215.69\end{array}$

$\begin{array}{ll}-7.08 & 217.56\end{array}$

$-6.72 \quad 218.23$

$-6.75 \quad 220.32$

$-6.29 \quad 221.30$

$\begin{array}{ll}-6.25 & 223.68\end{array}$

$-5.63 \quad 225.04$

$-4.78 \quad 228.43$

$-4.03 \quad 230.14$

$-3.90 \quad 233.00$

$-3.68 \quad 234.40$

$-3.65 \quad 237.36$

$-3.24 \quad 239.15$

$-3.16 \quad 242.36$

$-2.68 \quad 244.41$

$\begin{array}{ll}-2.66 & 247.78\end{array}$

$-2.24 \quad 249.96$

$-2.22 \quad 253.51$

$-1.85 \quad 255.85$

$-1.76 \quad 259.65$

$-1.44 \quad 262.13$

$\begin{array}{ll}-1.39 & 266.07\end{array}$

$-1.08 \quad 268.74$

$-1.26 \quad 272.63$ 


\section{$Z=120$}

$\begin{array}{llll}202 & 322 & -0.44 & 0.00\end{array}$ $\begin{array}{llll}203 & 323 & -0.44 & 0.00\end{array}$ $\begin{array}{llll}204 & 324 & -0.44 & 0.00\end{array}$ $\begin{array}{llll}205 & 325 & -0.45 & 0.00\end{array}$ $\begin{array}{llll}206 & 326 & -0.45 & 0.00\end{array}$

$\begin{array}{lll}0.04 & 0.00 & -0.445\end{array}$ $\begin{array}{lll}0.04 & -0.01 & -0.445\end{array}$ $\begin{array}{llll}0.04 & -0.01 & -0.445\end{array}$ $\begin{array}{lll}0.04 & -0.01 & -0.455\end{array}$ $\begin{array}{lll}0.04 & -0.01 & -0.455\end{array}$

$\begin{array}{llll}207 & 327 & -0.46 & 0.00\end{array}$ $\begin{array}{llll}208 & 328 & -0.46 & 0.00\end{array}$ $\begin{array}{llll}209 & 329 & -0.46 & 0.00\end{array}$ $\begin{array}{llll}210 & 330 & -0.46 & 0.00\end{array}$ $\begin{array}{lll}0.05 & 0.00 & -0.465\end{array}$ $\begin{array}{llll}0.04 & -0.01 & -0.464\end{array}$ $\begin{array}{llll}0.04 & -0.01 & -0.464\end{array}$ $\begin{array}{lll}0.04 & -0.01 & -0.464\end{array}$ $\begin{array}{lllllll}211 & 331 & 0.37 & 0.00 & -0.01 & 0.00 & 0.409\end{array}$

212332 $\begin{array}{lll}0.37 & 0.00 & -0.01\end{array}$ $0.00 \quad 0.409$ $213 \quad 333$ $\begin{array}{llll}0.37 & 0.00 & -0.01\end{array}$

$\begin{array}{ll}0.00 & 0.409\end{array}$

$\begin{array}{ll}0.00 & 0.409\end{array}$

214334

$\begin{array}{llll}0.37 & 0.00 & -0.01\end{array}$

$\begin{array}{llll}0.37 & 0.00 & -0.01\end{array}$

$0.00 \quad 0.409$

216336

$\begin{array}{llll}0.37 & 0.00 & -0.01\end{array}$

0.00

0.409

$217 \quad 337$

$0.38 \quad 0.00$

0.00

$\begin{array}{ll}0.00 & 0.421\end{array}$

218338

$\begin{array}{ll}0.38 & 0.00\end{array}$

0.00

0.00

0.421

$\begin{array}{lll}0.38 & 0.00 & 0.00\end{array}$

$0.00 \quad 0.421$ $\begin{array}{lllllll}0.000 & 0.029 & 0.006 & -2.47 & -0.75 & 2234.40 & 270.69\end{array}$ $\begin{array}{llllllll}0.000 & 0.030 & 0.014 & -2.58 & -0.77 & 2238.30 & 274.86\end{array}$ $\begin{array}{lllllll}0.000 & 0.030 & 0.014 & -2.30 & -0.38 & 2243.32 & 277.90\end{array}$ $\begin{array}{lllllll}0.000 & 0.033 & 0.013 & -2.51 & -0.64 & 2247.29 & 282.00\end{array}$ $\begin{array}{lllllll}0.000 & 0.033 & 0.013 & -2.32 & -0.32 & 2252.23 & 285.14\end{array}$ $\begin{array}{lllllll}0.000 & 0.025 & 0.009 & -2.55 & -0.59 & 2256.04 & 289.40\end{array}$ $\begin{array}{lllllll}0.000 & 0.036 & 0.013 & -2.41 & -0.34 & 2260.88 & 292.63\end{array}$ $\begin{array}{lllllll}0.000 & 0.036 & 0.013 & -2.62 & -0.55 & 2264.46 & 297.12\end{array}$ $\begin{array}{lllllll}0.000 & 0.036 & 0.013 & -2.51 & -0.47 & 2269.30 & 300.35\end{array}$ $\begin{array}{lllllll}0.000 & 0.074 & 0.015 & -1.47 & -2.60 & 2274.64 & 303.08\end{array}$ $\begin{array}{lllllll}0.000 & 0.074 & 0.015 & -1.38 & -2.53 & 2279.33 & 306.47\end{array}$ $\begin{array}{lllllll}0.000 & 0.074 & 0.015 & -1.62 & -2.75 & 2282.60 & 311.26\end{array}$ $\begin{array}{lllllll}0.000 & 0.074 & 0.015 & -1.50 & -2.64 & 2287.08 & 314.85\end{array}$ $\begin{array}{lllllll}0.000 & 0.074 & 0.015 & -1.72 & -2.81 & 2290.17 & 319.85\end{array}$ $\begin{array}{lllllll}0.000 & 0.074 & 0.015 & -1.58 & -2.69 & 2294.47 & 323.61\end{array}$ $\begin{array}{llllllll}0.000 & 0.065 & 0.011 & -1.51 & -2.90 & 2297.43 & 328.72\end{array}$ $\begin{array}{lllllll}0.000 & 0.065 & 0.011 & -1.43 & -2.83 & 2301.64 & 332.58\end{array}$ $\begin{array}{lllllll}0.000 & 0.065 & 0.011 & -1.67 & -3.04 & 2304.46 & 337.84\end{array}$

\section{$Z=121$}

$\begin{array}{lllllll}169 & 290 & -0.12 & 0.00 & -0.01 & -0.01 & -0.125\end{array}$

$\begin{array}{lllllll}170 & 291 & -0.12 & 0.00 & -0.01 & -0.01 & -0.125\end{array}$

$\begin{array}{llllllll}171 & 292 & -0.12 & 0.00 & -0.01 & -0.01 & -0.125\end{array}$

$\begin{array}{lllllll}172 & 293 & -0.12 & 0.00 & -0.02 & -0.01 & -0.124\end{array}$

$\begin{array}{lllllll}173 & 294 & 0.07 & 0.00 & 0.03 & -0.01 & 0.075\end{array}$

$\begin{array}{llllllll}174 & 295 & 0.07 & 0.00 & 0.04 & -0.02 & 0.075\end{array}$

$\begin{array}{llll}175 & 296 & 0.07 & 0.00\end{array}$

$\begin{array}{llll}176 & 297 & 0.07 & 0.00\end{array}$

$\begin{array}{lll}0.04 & -0.02 & 0.075\end{array}$

$\begin{array}{lll}0.04 & -0.02 & 0.075\end{array}$

$\begin{array}{llll}177 & 298 & 0.06 & 0.00\end{array}$

$\begin{array}{llll}178 & 299 & -0.02 & 0.00\end{array}$

$0.03-0.01 \quad 0.064$

$\begin{array}{lll}0.00 & 0.00 & -0.021\end{array}$

$\begin{array}{llll}179 & 300 & -0.02 & 0.00\end{array}$

$\begin{array}{llll}180 & 301 & -0.02 & 0.00\end{array}$

$\begin{array}{llll}181 & 302 & -0.02 & 0.00\end{array}$

$\begin{array}{llll}182 & 303 & 0.00 & 0.00\end{array}$

$\begin{array}{lll}0.01 & 0.00 & -0.021\end{array}$

$\begin{array}{lll}0.01 & 0.00 & -0.021\end{array}$

$\begin{array}{lll}0.01 & 0.00 & -0.021\end{array}$

$\begin{array}{lll}0.00 & 0.00 & 0.000\end{array}$

$\begin{array}{lll}0.00 & 0.00 & 0.000\end{array}$

0.000

$\begin{array}{llllll}0.018 & 0.008 & -6.50 & -6.04 & 2028.31 & 217.71\end{array}$

0.000

$\begin{array}{llllll}0.018 & 0.008 & -6.61 & -6.14 & 2037.31 & 216.78\end{array}$

0.000

$\begin{array}{llllll}0.018 & 0.008 & -6.97 & -6.50 & 2045.03 & 217.13\end{array}$

$\begin{array}{llllllll}0.000 & 0.029 & 0.007 & -7.01 & -6.46 & 2053.65 & 216.58\end{array}$

$\begin{array}{lllllll}0.000 & -0.034 & 0.008 & -7.45 & -6.46 & 2060.78 & 217.52\end{array}$

$\begin{array}{lllllll}0.000 & -0.046 & 0.017 & -7.77 & -6.47 & 2069.22 & 217.15\end{array}$

$\begin{array}{lllllll}0.000 & -0.046 & 0.017 & -8.11 & -6.78 & 2076.44 & 218.00\end{array}$

$\begin{array}{lllllll}0.000 & -0.046 & 0.017 & -7.78 & -6.47 & 2084.34 & 218.18\end{array}$

$\begin{array}{lllllll}0.000 & -0.034 & 0.008 & -7.64 & -6.63 & 2091.19 & 219.40\end{array}$

$\begin{array}{lllllll}0.000 & 0.000 & 0.000 & -7.20 & -6.57 & 2099.12 & 219.54\end{array}$

$\begin{array}{lllllll}0.000 & -0.012 & 0.000 & -7.48 & -6.80 & 2105.81 & 220.92\end{array}$ $\begin{array}{lllllll}0.000 & -0.012 & 0.000 & -7.12 & -6.44 & 2113.22 & 221.58\end{array}$ $\begin{array}{lllllll}0.000 & -0.012 & 0.000 & -7.20 & -6.51 & 2119.55 & 223.32\end{array}$ $\begin{array}{lllllll}0.000 & 0.000 & 0.000 & -6.67 & -6.00 & 2126.59 & 224.35\end{array}$ $\begin{array}{lllllll}0.000 & 0.000 & 0.000 & -6.60 & -5.94 & 2132.59 & 226.43\end{array}$ $\begin{array}{lllll}184 & 305 & 0.00 & 0.00\end{array}$ $\begin{array}{llll}185 & 306 & 0.00 & 0.01\end{array}$ $\begin{array}{llll}186 & 307 & 0.00 & 0.00\end{array}$ $\begin{array}{llll}187 & 308 & -0.40 & 0.00\end{array}$ $\begin{array}{llll}188 & 309 & -0.40 & 0.00\end{array}$

$\begin{array}{lllll}189 & 310 & -0.40 & 0.00\end{array}$ $\begin{array}{llll}190 & 311 & -0.40 & 0.00\end{array}$ $\begin{array}{llll}191 & 312 & -0.41 & 0.00\end{array}$ $\begin{array}{llll}192 & 313 & -0.41 & 0.00\end{array}$ $\begin{array}{llll}193 & 314 & -0.41 & 0.00\end{array}$

$\begin{array}{llll}194 & 315 & -0.42 & 0.00\end{array}$ $\begin{array}{llll}195 & 316 & -0.42 & 0.00\end{array}$ $\begin{array}{llll}196 & 317 & -0.43 & 0.00\end{array}$ $\begin{array}{lllll}197 & 318 & -0.43 & 0.00\end{array}$ $\begin{array}{lllll}198 & 319 & -0.43 & 0.00\end{array}$

$\begin{array}{llll}199 & 320 & -0.43 & 0.00\end{array}$ $\begin{array}{llll}200 & 321 & -0.43 & 0.00\end{array}$ $\begin{array}{lllll}201 & 322 & -0.44 & 0.00\end{array}$ $\begin{array}{llll}202 & 323 & -0.44 & 0.00\end{array}$ $\begin{array}{llll}203 & 324 & -0.45 & 0.00\end{array}$

$\begin{array}{llll}204 & 325 & -0.45 & 0.00\end{array}$ $\begin{array}{llll}205 & 326 & -0.45 & 0.00\end{array}$ $\begin{array}{llll}206 & 327 & -0.46 & 0.00\end{array}$ $\begin{array}{llllllllll}0.00 & 0.00 & 0.000 & 0.000 & 0.000 & 0.000 & -5.94 & -5.31 & 2139.30 & 227.79\end{array}$

$\begin{array}{llllllllll}0.00 & 0.00 & 0.000 & -0.013 & 0.000 & 0.000 & -5.07 & -4.48 & 2144.32 & 230.84\end{array}$

$\begin{array}{llllllllll}0.00 & 0.00 & 0.000 & 0.000 & 0.000 & 0.000 & -4.26 & -3.72 & 2150.70 & 232.54\end{array}$ $\begin{array}{llllllllll}0.06 & -0.01 & -0.407 & 0.000 & -0.003 & 0.024 & -5.79 & -3.79 & 2156.41 & 234.89\end{array}$ $\begin{array}{llllllllll}0.06 & -0.01 & -0.407 & 0.000 & -0.003 & 0.024 & -5.58 & -3.60 & 2163.16 & 236.21\end{array}$ $\begin{array}{llllllllll}0.06 & -0.01 & -0.407 & 0.000 & -0.003 & 0.024 & -5.56 & -3.57 & 2168.57 & 238.87\end{array}$ $\begin{array}{llllllllll}0.06 & -0.01 & -0.407 & 0.000 & -0.003 & 0.024 & -5.14 & -3.18 & 2174.93 & 240.59\end{array}$ $\begin{array}{llllllllll}0.06 & -0.01 & -0.417 & 0.000 & -0.000 & 0.024 & -5.11 & -3.08 & 2180.08 & 243.51\end{array}$ $\begin{array}{llllllllll}0.05 & -0.01 & -0.416 & 0.000 & 0.010 & 0.019 & -4.48 & -2.65 & 2186.20 & 245.46\end{array}$ $\begin{array}{llllllllll}0.05 & -0.01 & -0.416 & 0.000 & 0.010 & 0.019 & -4.42 & -2.60 & 2191.22 & 248.51\end{array}$ $\begin{array}{lllllllllll}0.05 & 0.00 & -0.426 & 0.000 & 0.012 & 0.011 & -3.96 & -2.20 & 2197.16 & 250.64\end{array}$ $\begin{array}{llllllllll}0.05 & 0.00 & -0.426 & 0.000 & 0.012 & 0.011 & -3.95 & -2.17 & 2202.02 & 253.85\end{array}$ $\begin{array}{llllllllll}0.05 & 0.00 & -0.436 & 0.000 & 0.015 & 0.010 & -3.63 & -1.84 & 2207.85 & 256.10\end{array}$ $\begin{array}{llllllllll}0.05 & 0.00 & -0.436 & 0.000 & 0.015 & 0.010 & -3.59 & -1.77 & 2212.48 & 259.54\end{array}$ $\begin{array}{llllllllll}0.05 & 0.00 & -0.436 & 0.000 & 0.015 & 0.010 & -3.26 & -1.48 & 2218.17 & 261.92\end{array}$ $\begin{array}{llllllllll}0.04 & 0.00 & -0.435 & 0.000 & 0.025 & 0.006 & -3.17 & -1.42 & 2222.62 & 265.54\end{array}$ $\begin{array}{llllllllll}0.04 & -0.01 & -0.435 & 0.000 & 0.027 & 0.014 & -2.93 & -1.16 & 2228.15 & 268.08\end{array}$ $\begin{array}{llllllllll}0.05 & 0.00 & -0.445 & 0.000 & 0.018 & 0.010 & -2.97 & -1.12 & 2232.46 & 271.84\end{array}$ $\begin{array}{llllllllll}0.04 & 0.00 & -0.445 & 0.000 & 0.029 & 0.006 & -2.67 & -0.91 & 2237.86 & 274.51\end{array}$ $\begin{array}{llllllllll}0.05 & 0.00 & -0.455 & 0.000 & 0.022 & 0.010 & -2.83 & -0.90 & 2242.02 & 278.42\end{array}$ $\begin{array}{lllllllllll}0.05 & 0.00 & -0.455 & 0.000 & 0.022 & 0.010 & -2.62 & -0.75 & 2247.31 & 281.21\end{array}$ $\begin{array}{llllllllll}0.04 & -0.01 & -0.455 & 0.000 & 0.033 & 0.013 & -2.78 & -0.88 & 2251.43 & 285.15\end{array}$ $\begin{array}{llllllllll}0.05 & 0.00 & -0.465 & 0.000 & 0.025 & 0.009 & -2.67 & -0.74 & 2256.56 & 288.09\end{array}$
$-1.00 \quad 275.43$

$-1.02 \quad 279.66$

$-0.60 \quad 282.79$

$-0.90 \quad 286.92$

$-0.56 \quad 290.16$

$-0.88 \quad 294.45$

$-0.60 \quad 297.80$

$-0.83 \quad 302.34$

$-0.72 \quad 305.69$

$\begin{array}{ll}-2.68 & 308.68\end{array}$

$-2.59 \quad 312.18$

$-2.83 \quad 317.05$

$-2.70 \quad 320.76$

$-2.90 \quad 325.84$

$-2.76 \quad 329.73$

$-3.02 \quad 334.89$

$\begin{array}{ll}-2.93 & 338.89\end{array}$

$-3.17 \quad 344.24$

$\begin{array}{ll}-6.07 & 222.76\end{array}$

$\begin{array}{ll}-6.17 & 221.77\end{array}$

$\begin{array}{ll}-6.53 & 222.08\end{array}$

$-6.48 \quad 221.49$

$-6.47 \quad 222.40$
-6.46

$-6.46 \quad 222.01$

$-6.78 \quad 222.83$

$-6.46 \quad 222.98$

$-6.64 \quad 224.16$

$-6.58 \quad 224.27$

\begin{tabular}{ll}
$-6.81 \quad 225.63$ \\
\hline
\end{tabular}

$\begin{array}{ll}-6.44 & 226.27\end{array}$

$-6.52 \quad 228.00$

$\begin{array}{ll}-6.00 & 229.01\end{array}$

$-5.95 \quad 231.09$

$\begin{array}{ll}-5.31 & 232.44\end{array}$

$\begin{array}{ll}-4.48 & 235.49\end{array}$

$-3.72 \quad 237.19$

$\begin{array}{ll}-4.06 & 239.28\end{array}$

$-3.85 \quad 240.63$

$-3.82 \quad 243.29$

$-3.41 \quad 245.05$

$-3.34 \quad 247.95$

$-2.90 \quad 249.94$

$-2.87 \quad 253.00$

$-2.47 \quad 255.15$

\begin{tabular}{ll}
$-2.47 \quad 258.37$ \\
\hline
\end{tabular}

$-2.12 \quad 260.66$

$-2.08 \quad 264.12$

$-1.76 \quad 266.57$

\begin{tabular}{ll}
$-1.72 \quad 270.20$ \\
\hline
\end{tabular}

\begin{tabular}{ll}
$-1.42 \quad 272.84$ \\
\hline
\end{tabular}

$-1.43 \quad 276.60$

$-1.20 \quad 279.35$

$-1.23 \quad 283.28$

$-1.05 \quad 286.15$

$-1.18 \quad 290.16$

$-1.05 \quad 293.16$ 
$Z=121$

$\begin{array}{llll}207 & 328 & -0.46 & 0.00\end{array}$

$\begin{array}{lllll}208 & 329 & -0.46 & 0.00\end{array}$

$\begin{array}{llll}209 & 330 & -0.47 & 0.00\end{array}$

$\begin{array}{llllllll}210 & 331 & 0.39 & 0.01 & 0.00 & 0.00 & 0.433 & -0.013\end{array}$

211332

$\begin{array}{lll}0.38 & 0.01 & -0.01\end{array}$

$\begin{array}{lll}0.00 & 0.421 & -0.013\end{array}$

212333

$213 \quad 334$

$\begin{array}{lll}0.37 & 0.01 & -0.01\end{array}$

$\begin{array}{lll}0.00 & 0.409 & -0.013\end{array}$

214335

$\begin{array}{lll}0.37 & 0.01 & -0.01\end{array}$

215336

$\begin{array}{lll}0.38 & 0.01 & 0.00\end{array}$

0.00

$0.409-0.013$

216337

$\begin{array}{ll}0.38 & 0.01\end{array}$

0.00

0.00

$0.421-0.013$

$0.38 \quad 0.00$

0.00

0.00

0.421

217338

$\begin{array}{ll}0.37 & 0.00\end{array}$

0.00

0.00

0.410

0.000

218339

\section{$Z=122$}

$\begin{array}{lllllll}172 & 294 & -0.12 & 0.00 & -0.02 & -0.01 & -0.124\end{array}$

$\begin{array}{lllllll}173 & 295 & -0.12 & 0.00 & -0.01 & -0.01 & -0.125\end{array}$

$\begin{array}{lllllll}174 & 296 & 0.07 & 0.00 & 0.04 & -0.01 & 0.075\end{array}$

$\begin{array}{llllllll}175 & 297 & 0.07 & 0.00 & 0.04 & -0.02 & 0.075\end{array}$

$\begin{array}{llll}176 & 298 & 0.07 & 0.00\end{array}$

$\begin{array}{lll}0.04 & -0.02 & 0.075\end{array}$

$\begin{array}{llll}177 & 299 & -0.08 & 0.00\end{array}$

$\begin{array}{llll}178 & 300 & 0.00 & 0.00\end{array}$

$\begin{array}{lll}0.00 & -0.01 & -0.084\end{array}$

$\begin{array}{lll}0.00 & 0.00 & 0.000\end{array}$

$\begin{array}{llll}0.00 & 0.00 & -0.011\end{array}$

$\begin{array}{rrrr}179 & 301 & -0.01 & 0.00 \\ 180 & 302 & 0.00 & 0.00\end{array}$

0.00

$0.00 \quad 0.000$

$\begin{array}{llll}181 & 303 & 0.00 & 0.00\end{array}$

0.00

0.0

$\begin{array}{llll}182 & 304 & 0.00 & 0.00\end{array}$

$\begin{array}{lllll}183 & 305 & 0.00 & 0.00\end{array}$

$\begin{array}{llll}184 & 306 & 0.00 & 0.00\end{array}$

$\begin{array}{llll}185 & 307 & 0.00 & 0.01\end{array}$

$\begin{array}{llll}186 & 308 & 0.00 & 0.00\end{array}$

$\begin{array}{llll}187 & 309 & -0.40 & 0.00\end{array}$

$\begin{array}{lllll}188 & 310 & -0.40 & 0.00\end{array}$

$\begin{array}{llll}189 & 311 & -0.40 & 0.00\end{array}$

$\begin{array}{llll}190 & 312 & -0.40 & 0.00\end{array}$

$\begin{array}{llll}191 & 313 & -0.41 & 0.00\end{array}$

$\begin{array}{llll}192 & 314 & -0.41 & 0.00\end{array}$

$\begin{array}{llll}193 & 315 & -0.42 & 0.00\end{array}$

$\begin{array}{llll}194 & 316 & -0.42 & 0.00\end{array}$

$\begin{array}{llll}195 & 317 & -0.43 & 0.00\end{array}$

$\begin{array}{llll}196 & 318 & -0.43 & 0.00\end{array}$

$\begin{array}{llll}197 & 319 & -0.43 & 0.00\end{array}$

$\begin{array}{llll}198 & 320 & -0.43 & 0.00\end{array}$

$\begin{array}{llll}199 & 321 & -0.44 & 0.00\end{array}$

$\begin{array}{llll}200 & 322 & -0.44 & 0.00\end{array}$

$\begin{array}{lllll}201 & 323 & -0.44 & 0.00\end{array}$

$\begin{array}{llll}202 & 324 & -0.44 & 0.00\end{array}$

$\begin{array}{llll}203 & 325 & 0.29 & 0.00\end{array}$

$\begin{array}{llll}204 & 326 & -0.46 & 0.00\end{array}$

$\begin{array}{lllll}205 & 327 & -0.46 & 0.00\end{array}$

$\begin{array}{llll}206 & 328 & -0.46 & 0.00\end{array}$

$\begin{array}{lllll}207 & 329 & -0.47 & 0.00\end{array}$

$\begin{array}{llll}208 & 330 & -0.47 & 0.00\end{array}$

$\begin{array}{llll}209 & 331 & 0.29 & 0.00\end{array}$

$\begin{array}{llll}210 & 332 & 0.40 & 0.00\end{array}$

$\begin{array}{llll}211 & 333 & 0.39 & 0.00\end{array}$

$\begin{array}{lllll}212 & 334 & 0.38 & 0.00\end{array}$

$\begin{array}{lllll}213 & 335 & 0.37 & 0.00\end{array}$

$\begin{array}{llll}214 & 336 & 0.38 & 0.00\end{array}$

215337 $\begin{array}{lllll}0.00 & 0.00 & 0.000 & 0.000 & 0.000\end{array}$

$\begin{array}{lllll}0.00 & 0.00 & 0.000 & 0.000 & 0.000\end{array}$

$\begin{array}{rrrrr}0.00 & 0.00 & 0.000 & 0.000 & 0.000\end{array}$

$\begin{array}{lllll}0.00 & 0.00 & 0.000 & -0.013 & 0.000\end{array}$

$\begin{array}{lllll}0.00 & 0.00 & 0.000 & 0.000 & 0.000\end{array}$

$\begin{array}{lll}0.06 & -0.01 & -0.407\end{array}$

$\begin{array}{llll}0.06 & -0.01 & -0.407\end{array}$

$\begin{array}{lll}0.06 & -0.01 & -0.407\end{array}$

$\begin{array}{llll}0.06 & -0.01 & -0.407\end{array}$

$\begin{array}{llll}0.06 & -0.01 & -0.417\end{array}$

$0.000-0.003$

$0.000-0.003$

$0.000-0.003$

$0.000-0.003$

$0.000-0.000$

$\begin{array}{lll}0.05 & -0.01 & -0.416\end{array}$

$0.000 \quad 0.010$

$\begin{array}{llll}0.06 & 0.00 & -0.426\end{array}$

$\begin{array}{llll}0.05 & 0.00 & -0.426\end{array}$

0.000

0.002

$\begin{array}{lll}0.05 & 0.00 & -0.436\end{array}$

0.000

0.012

$\begin{array}{llll}0.05 & 0.00 & -0.436\end{array}$

0.000

0.015

$\begin{array}{llll}0.05 & 0.00 & -0.436\end{array}$

0.000

0.015

$\begin{array}{llll}0.04 & 0.00 & -0.435\end{array}$

0.000

0.015

$\begin{array}{llll}0.05 & 0.00 & -0.445\end{array}$

0.000

0.025

0.04

$0.00 \quad-0.445$

0.000

0.018

$\begin{array}{llll}0.04 & 0.00 & -0.445\end{array}$

0.000

0.029

0.04

$\begin{array}{ll}0.00 & -0.445\end{array}$

$\begin{array}{lll}0.02 & 0.01 & 0.319\end{array}$

$\begin{array}{llll}0.05 & 0.00 & -0.465\end{array}$

0.000

0.029

0.000

0.029

$\begin{array}{llll}0.05 & 0.00 & -0.465\end{array}$

$\begin{array}{llll}0.05 & 0.00 & -0.465\end{array}$

0.000

0.014

0.025

$0.000 \quad 0.025$

$0.000 \quad 0.025$

$\begin{array}{llll}0.05 & 0.00 & -0.474\end{array}$

$\begin{array}{llll}0.05 & 0.00 & -0.474\end{array}$

$\begin{array}{lll}0.06 & -0.01 & 0.322\end{array}$

$\begin{array}{lll}0.00 & 0.00 & 0.445\end{array}$

0.000

0.028

$\begin{array}{ll}0.000 \quad 0.028 \\ 0.000 & -0.037\end{array}$

$\begin{array}{lll}0.000 & -0.037\end{array}$

$0.000 \quad 0.073$

$\begin{array}{lll}0.00 & 0.00 & 0.433\end{array}$

0.000

0.069

$\begin{array}{ll}0.00 & 0.421\end{array}$

$0.000 \quad 0.065$

$0.000 \quad 0.074$

$0.000 \quad 0.065$

$0.000 \quad 0.065$ $\begin{array}{lllll}0.013 & -2.85 & -0.85 & 2260.50 & 292.22\end{array}$

$\begin{array}{lllll}0.013 & -2.75 & -0.83 & 2265.57 & 295.23\end{array}$

$\begin{array}{llllll}0.017 & -3.10 & -1.01 & 2269.42 & 299.45\end{array}$

$\begin{array}{lllll}0.012 & -0.87 & -2.63 & 2275.97 & 300.97\end{array}$

$\begin{array}{lllll}0.016 & -1.39 & -2.81 & 2279.66 & 305.36\end{array}$

$\begin{array}{lllll}0.015 & -1.43 & -2.73 & 2284.33 & 308.75\end{array}$

$\begin{array}{lllll}0.015 & -1.66 & -2.94 & 2287.89 & 313.27\end{array}$

$\begin{array}{llllll}0.011 & -1.23 & -2.84 & 2292.39 & 316.84\end{array}$

$\begin{array}{lllll}0.011 & -1.35 & -2.92 & 2300.10 & 325.27\end{array}$

$\begin{array}{lllll}0.010 & -1.72 & -3.09 & 2303.30 & 330.14\end{array}$

$\begin{array}{lllll}0.006 & -1.42 & -3.06 & 2307.55 & 333.96\end{array}$ $\begin{array}{llllll}0.011 & -1.46 & -3.01 & 2295.75 & 321.55\end{array}$

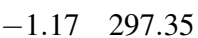

$\begin{array}{ll}-1.12 \quad 300.46 \\ -1.33 & 304.73\end{array}$

$-1.33 \quad 304.73$

$-2.76 \quad 306.53$

$-2.93 \quad 311.01$

$-2.82 \quad 314.52$

$\begin{array}{ll}-3.05 & 319.11\end{array}$

$-2.96 \quad 322.76$

$-3.16 \quad 327.55$

$\begin{array}{ll}-3.05 & 331.39\end{array}$

$-3.23 \quad 336.35$

$-3.21 \quad 340.28$

$\begin{array}{lllll}0.007 & -6.44 & -5.91 & 2053.09 & 224.43\end{array}$

$\begin{array}{llllll}0.008 & -6.44 & -6.03 & 2060.36 & 225.23\end{array}$

$\begin{array}{llllll}0.007 & -6.70 & -5.53 & 2068.61 & 225.05\end{array}$

$\begin{array}{llllll}0.017 & -7.14 & -5.85 & 2075.86 & 225.87\end{array}$

$\begin{array}{llllll}0.017 & -6.80 & -5.53 & 2084.07 & 225.73\end{array}$

$\begin{array}{llllll}0.010 & -6.50 & -6.25 & 2091.49 & 226.39\end{array}$

$\begin{array}{lllll}0.000 & -6.36 & -5.73 & 2099.28 & 226.67\end{array}$

$\begin{array}{llllll}0.000 & -6.60 & -5.96 & 2106.00 & 228.02\end{array}$

$\begin{array}{lllll}0.000 & -6.22 & -5.57 & 2113.69 & 228.40\end{array}$

$\begin{array}{llllll}0.000 & -6.31 & -5.66 & 2120.06 & 230.10\end{array}$

$\begin{array}{lllll}0.000 & -5.80 & -5.17 & 2127.44 & 230.79\end{array}$

$\begin{array}{lllll}0.000 & -5.72 & -5.10 & 2133.44 & 232.86\end{array}$

$\begin{array}{llllll}0.000 & -5.04 & -4.46 & 2140.46 & 233.92\end{array}$

$\begin{array}{lllll}0.000 & -4.19 & -3.64 & 2145.50 & 236.94\end{array}$

$\begin{array}{llllll}0.000 & -3.36 & -2.87 & 2152.19 & 238.33\end{array}$

$\begin{array}{lllll}0.024 & -5.18 & -3.38 & 2158.36 & 240.23\end{array}$

$\begin{array}{lllll}0.024 & -4.98 & -3.20 & 2165.42 & 241.24\end{array}$

$\begin{array}{llllll}0.024 & -4.97 & -3.16 & 2170.85 & 243.88\end{array}$

$\begin{array}{llllll}0.024 & -4.54 & -2.77 & 2177.50 & 245.30\end{array}$

$\begin{array}{lllll}0.024 & -4.50 & -2.66 & 2182.67 & 248.20\end{array}$

$\begin{array}{llllll}0.019 & -3.85 & -2.25 & 2189.10 & 249.84\end{array}$

$\begin{array}{llllll}0.015 & -3.87 & -2.16 & 2194.10 & 252.92\end{array}$

$\begin{array}{llllll}0.011 & -3.36 & -1.81 & 2200.40 & 254.69\end{array}$

$\begin{array}{llllll}0.010 & -3.41 & -1.77 & 2205.26 & 257.90\end{array}$

$\begin{array}{llllll}0.010 & -3.07 & -1.48 & 2211.43 & 259.80\end{array}$

$\begin{array}{llllll}0.010 & -3.03 & -1.43 & 2216.09 & 263.21\end{array}$

$\begin{array}{llllll}0.006 & -2.66 & -1.15 & 2222.09 & 265.29\end{array}$

$\begin{array}{llllll}0.010 & -2.78 & -1.12 & 2226.60 & 268.85\end{array}$

$\begin{array}{lllll}0.006 & -2.46 & -1.06 & 2232.62 & 270.90\end{array}$

$\begin{array}{lllll}0.006 & -2.71 & -1.28 & 2237.19 & 274.40\end{array}$

$\begin{array}{lllll}0.006 & -2.43 & -0.99 & 2242.81 & 276.85\end{array}$

$\begin{array}{llllll}-0.013 & -1.91 & -2.47 & 2248.48 & 279.26\end{array}$

$\begin{array}{llllll}0.009 & -2.45 & -0.84 & 2252.57 & 283.23\end{array}$

$\begin{array}{llllll}0.009 & -2.52 & -0.88 & 2256.63 & 287.24\end{array}$

$\begin{array}{lllll}0.009 & -2.30 & -0.56 & 2261.86 & 290.09\end{array}$

$\begin{array}{llllll}0.009 & -2.63 & -0.88 & 2266.02 & 294.00\end{array}$

$\begin{array}{llllll}0.009 & -2.45 & -0.68 & 2271.20 & 296.89\end{array}$

$\begin{array}{lllll}-0.007 & -1.32 & -1.31 & 2275.52 & 300.64\end{array}$

$\begin{array}{lllll}0.013 & -0.33 & -2.52 & 2281.94 & 302.30\end{array}$

$\begin{array}{llllll}0.012 & -0.65 & -2.64 & 2285.58 & 306.72\end{array}$

$\begin{array}{lllll}0.011 & -0.70 & -2.55 & 2290.53 & 309.84\end{array}$

$\begin{array}{llllll}0.015 & -1.26 & -2.76 & 2294.10 & 314.35\end{array}$

$\begin{array}{llllll}0.011 & -0.83 & -2.67 & 2298.89 & 317.62\end{array}$

$\begin{array}{lllll}0.011 & -1.05 & -2.87 & 2302.29 & 322.30\end{array}$ $\begin{array}{ll}-5.93 & 229.65\end{array}$

$\begin{array}{ll}-6.06 & 230.40\end{array}$

$\begin{array}{ll}-5.53 & 230.20\end{array}$

$\begin{array}{ll}-5.85 & 230.99\end{array}$

$-5.53230 .81$

$-6.26 \quad 231.42$

$-5.73 \quad 231.68$

$-5.97 \quad 233.00$

$\begin{array}{ll}-5.57 & 233.35\end{array}$

$-5.66 \quad 235.04$

$-5.17 \quad 235.71$

$\begin{array}{ll}-5.10 & 237.77\end{array}$

$-4.46 \quad 238.82$

$-3.64 \quad 241.83$

$-2.88 \quad 243.22$

$-3.64 \quad 244.86$

$-3.43 \quad 245.90$

$-3.41 \quad 248.53$

$\begin{array}{ll}-2.99 & 249.99\end{array}$

$\begin{array}{ll}-2.92 & 252.87\end{array}$

$-2.49 \quad 254.54$

$\begin{array}{ll}-2.44 & 257.59\end{array}$

$-2.07 \quad 259.40$

$-2.07 \quad 262.60$

$-1.75 \quad 264.56$

$-1.72 \quad 267.99$

\begin{tabular}{ll}
$-1.41 \quad 270.12$ \\
\hline
\end{tabular}

$\begin{array}{ll}-1.43 & 273.69\end{array}$

$-1.33 \quad 275.81$

$\begin{array}{ll}-1.57 & 279.33\end{array}$

$-1.26 \quad 281.85$

$-2.55 \quad 284.51$

$-1.13 \quad 288.32$

$\begin{array}{ll}-1.20 & 292.37\end{array}$

$-0.85 \quad 295.30$

$\begin{array}{ll}-1.21 \quad 299.24 \\ -0.98 & 302.23\end{array}$

$\begin{array}{ll}-0.98 & 302.23\end{array}$

$\begin{array}{ll}-1.38 & 306.29\end{array}$

$-2.77 \quad 312.47$

$\begin{array}{ll}-2.65 & 315.70\end{array}$

$-2.85 \quad 320.30$

$-2.77 \quad 323.66$

$-2.99 \quad 328.40$ $\begin{array}{ll}-2.62 & 307.98\end{array}$ 
$Z=122$

216338

$\begin{array}{ll}0.38 & 0.00\end{array}$

$0.00 \quad 0.421$

0.000

0.065

$\begin{array}{lllll}0.011 & -0.97 & -2.80 & 2306.95 & 325.71\end{array}$

$\begin{array}{lllll}0.010 & -1.35 & -2.96 & 2310.15 & 330.58\end{array}$

$Z=123$

$\begin{array}{lllllll}174 & 297 & -0.16 & 0.00 & -0.02 & -0.01 & -0.165\end{array}$

$\begin{array}{lllllll}175 & 298 & -0.11 & 0.00 & -0.01 & -0.01 & -0.115\end{array}$

$\begin{array}{lllllll}176 & 299 & -0.10 & 0.00 & -0.01 & -0.01 & -0.104\end{array}$

$0.000 \quad 0.033$

$\begin{array}{lllll}0.005 & -6.16 & -5.68 & 2066.79 & 234.16\end{array}$

$\begin{array}{lllllll}0.000 & 0.017 & 0.008 & -6.09 & -5.83 & 2074.19 & 234.84\end{array}$

$\begin{array}{lllllll}0.000 & 0.016 & 0.008 & -5.82 & -5.62 & 2082.53 & 234.56\end{array}$

$\begin{array}{lllllll}177 & 300 & -0.08 & 0.00 & 0.00 & -0.01 & -0.084\end{array}$

$0.000 \quad 0.003$

$\begin{array}{lllll}0.008 & -5.82 & -5.62 & 2082.53 & 234.56 \\ 0.010 & -6.05 & -5.83 & 2089.76 & 235.40\end{array}$

$\begin{array}{llll}178 & 301 & -0.01 & 0.00\end{array}$

$\begin{array}{lll}0.00 & 0.00 & -0.011\end{array}$

0.000$$
\begin{array}{lllll}
0.000 & -5.95 & -5.38 & 2097.64 & 235.60
\end{array}
$$

$\begin{array}{llll}179 & 302 & -0.01 & 0.00\end{array}$

$\begin{array}{llll}180 & 303 & -0.02 & 0.00\end{array}$

$\begin{array}{llll}0.00 & 0.00 & -0.011\end{array}$

$0.000 \quad 0.000$

$\begin{array}{lllll}0.000 & -6.18 & -5.57 & 2104.64 & 236.67\end{array}$

$\begin{array}{llll}181 & 304 & 0.00 & 0.00\end{array}$

$\begin{array}{llll}182 & 305 & 0.00 & 0.00\end{array}$

$\begin{array}{llll}0.01 & 0.00 & -0.02\end{array}$

$0.000-0.012$$$
\begin{array}{lllll}
0.000 & -5.81 & -5.20 & 2112.37 & 237.01
\end{array}
$$

$\begin{array}{llllll}0.000 & -5.86 & -5.24 & 2119.00 & 238.45\end{array}$

$\begin{array}{lllll}0.000 & -5.33 & -4.74 & 2126.39 & 239.13\end{array}$

$\begin{array}{llll}183 & 306 & 0.00 & 0.00\end{array}$

$\begin{array}{lll}0.00 & 0.00 & 0.000\end{array}$

$0.000 \quad 0.000$

$\begin{array}{llll}184 & 307 & 0.00 & 0.00\end{array}$

$\begin{array}{llll}185 & 308 & 0.00 & 0.01\end{array}$

$\begin{array}{llll}186 & 309 & 0.00 & 0.00\end{array}$

$\begin{array}{llll}187 & 310 & -0.40 & 0.00\end{array}$

$\begin{array}{llll}188 & 311 & -0.40 & 0.00\end{array}$

$\begin{array}{lll}0.00 & 0.00 & 0.000\end{array}$

0.000

0.000

$\begin{array}{lllll}0.00 & 0.00 & 0.000 & 0.000 & 0.000\end{array}$

$\begin{array}{lllll}0.00 & 0.00 & 0.000 & -0.013 & 0.000\end{array}$

$\begin{array}{rrrrr}0.00 & 0.00 & 0.000 & 0.000 & 0.000\end{array}$

$\begin{array}{llll}0.06 & -0.01 & -0.407\end{array}$

$0.000-0.003$

$\begin{array}{llll}0.07 & -0.01 & -0.407\end{array}$

$0.000-0.014$

$\begin{array}{llll}189 & 312 & -0.40 & 0.00\end{array}$

$\begin{array}{llll}190 & 313 & -0.41 & 0.00\end{array}$

$\begin{array}{lll}0.06 & -0.01 & -0.407\end{array}$

$0.000-0.003$

$\begin{array}{llll}0.06 & -0.01 & -0.417\end{array}$

$0.000-0.000$

$\begin{array}{llll}191 & 314 & -0.41 & 0.00\end{array}$

$\begin{array}{lll}0.06 & 0.00 & -0.417\end{array}$

$0.000-0.001$

$\begin{array}{llll}192 & 315 & -0.42 & 0.00\end{array}$

$\begin{array}{llll}193 & 316 & -0.42 & 0.00\end{array}$

$\begin{array}{lll}0.06 & 0.00 & -0.426\end{array}$

$0.000 \quad 0.002$

$\begin{array}{llllll}0.05 & 0.00 & -0.426 & 0.000 & 0.012\end{array}$

$\begin{array}{lllll}0.000 & -5.23 & -4.65 & 2132.69 & 240.91\end{array}$

$\begin{array}{lllll}0.000 & -4.54 & -4.00 & 2139.71 & 241.95\end{array}$

$\begin{array}{lllll}0.000 & -3.70 & -3.20 & 2145.08 & 244.65\end{array}$

$\begin{array}{lllll}0.000 & -2.86 & -2.43 & 2151.78 & 246.03\end{array}$

$\begin{array}{lllll}0.024 & -4.88 & -3.22 & 2158.55 & 247.33\end{array}$

$\begin{array}{lllll}0.028 & -4.92 & -3.05 & 2165.64 & 248.32\end{array}$

$\begin{array}{llllll}0.024 & -4.67 & -3.01 & 2171.37 & 250.65\end{array}$

$\begin{array}{llllll}0.024 & -4.31 & -2.64 & 2178.06 & 252.04\end{array}$

$\begin{array}{lllll}0.015 & -4.11 & -2.55 & 2183.55 & 254.61\end{array}$

$\begin{array}{lllll}0.015 & -3.70 & -2.15 & 2190.01 & 256.22\end{array}$

$\begin{array}{llllll}0.011 & -3.54 & -2.10 & 2195.35 & 258.96\end{array}$

$\begin{array}{llll}194 & 317 & -0.42 & 0.00\end{array}$

$\begin{array}{llll}195 & 318 & -0.43 & 0.00\end{array}$

$\begin{array}{lll}0.05 & 0.00 & -0.426\end{array}$

$0.000 \quad 0.012$

$\begin{array}{llllll}0.011 & -3.17 & -1.77 & 2201.69 & 260.69\end{array}$

$\begin{array}{llll}196 & 319 & -0.43 & 0.00\end{array}$

$\begin{array}{llll}197 & 320 & -0.43 & 0.00\end{array}$

$\begin{array}{lll}0.05 & 0.00 & -0.436\end{array}$

$0.000 \quad 0.015$

$\begin{array}{lll}0.05 & 0.00 & -0.436\end{array}$

$0.000 \quad 0.015$

$\begin{array}{llllll}0.04 & 0.00 & -0.435 & 0.000 & 0.025\end{array}$

$\begin{array}{llll}198 & 321 & -0.44 & 0.00\end{array}$

0.05

0.000

0.018

$\begin{array}{llll}199 & 322 & -0.44 & 0.00\end{array}$

0.04

$0.00-0.445$

$0.000 \quad 0.029$

$\begin{array}{llll}200 & 323 & -0.44 & 0.00\end{array}$

$\begin{array}{llll}201 & 324 & -0.45 & 0.00\end{array}$

$\begin{array}{llll}202 & 325 & -0.45 & 0.00\end{array}$

$\begin{array}{llll}203 & 326 & -0.46 & 0.00\end{array}$

0.04

$0.00-0.445$

$0.000 \quad 0.029$

$0.00-0.445$

$0.000 \quad 0.032$

$\begin{array}{llll}0.04 & 0.00 & -0.454\end{array}$

0.000

0.032

$\begin{array}{llll}0.04 & 0.00 & -0.464\end{array}$

0.000

0.035

$\begin{array}{llll}204 & 327 & -0.46 & 0.00\end{array}$

$\begin{array}{lllll}205 & 328 & -0.46 & 0.00\end{array}$

$\begin{array}{llll}0.04 & 0.00 & -0.464\end{array}$

0.000

0.035

$\begin{array}{llll}0.04 & 0.00 & -0.464\end{array}$

0.000

0.035

$\begin{array}{llll}206 & 329 & -0.47 & 0.00\end{array}$

0.05

$0.00 \quad-0.474$

0.000

0.028

$\begin{array}{llll}207 & 330 & -0.47 & 0.00\end{array}$

$\begin{array}{llll}208 & 331 & 0.30 & 0.00\end{array}$

$0.00-0.474$

$\begin{array}{ll}0.000 \quad 0.028 \\ 0.000 & -0.021\end{array}$

$\begin{array}{lll}0.000 & -0.021\end{array}$

$\begin{array}{llll}209 & 332 & 0.29 & 0.00\end{array}$

$\begin{array}{llll}210 & 333 & -0.47 & 0.00\end{array}$

$\begin{array}{llll}0.06 & -0.01 \quad 0.322\end{array}$

0.000

$\begin{array}{llll}0.04 & -0.01 & -0.474\end{array}$

0.000

$-0.037$

$0.000-0.060$

$\begin{array}{lllll}0.010 & -3.26 & -1.80 & 2206.92 & 263.54\end{array}$

$\begin{array}{llllll}0.010 & -2.92 & -1.49 & 2213.08 & 265.44\end{array}$

$\begin{array}{lllll}0.006 & -2.85 & -1.45 & 2218.05 & 268.54\end{array}$

$\begin{array}{llllll}0.010 & -2.68 & -1.20 & 2224.09 & 270.58\end{array}$

$\begin{array}{lllll}0.006 & -2.65 & -1.20 & 2228.92 & 273.82\end{array}$

$\begin{array}{lllll}0.006 & -2.49 & -1.18 & 2235.00 & 275.81\end{array}$

$\begin{array}{llllll}0.005 & -2.87 & -1.46 & 2239.93 & 278.95\end{array}$

$\begin{array}{llllll}0.005 & -2.68 & -1.30 & 2245.70 & 281.25\end{array}$

$\begin{array}{llllll}0.005 & -2.92 & -1.43 & 2250.30 & 284.72\end{array}$

$\begin{array}{lllll}0.005 & -2.60 & -1.16 & 2255.78 & 287.32\end{array}$

$\begin{array}{llllll}0.005 & -2.67 & -1.15 & 2260.06 & 291.10\end{array}$

$\begin{array}{llllll}0.009 & -2.59 & -1.00 & 2265.48 & 293.75\end{array}$

$\begin{array}{llllll}0.009 & -2.88 & -1.25 & 2269.87 & 297.44\end{array}$

$\begin{array}{llll}211 & 334 & 0.40 & 0.00\end{array}$

$\begin{array}{lll}0.01 & 0.00 & 0.445\end{array}$

0.000

0.060

212335

$0.39 \quad 0.00$

$0.01-0.01$

0.434

0.000

0.055

213336

$0.38 \quad 0.00$

0.00

0.00

0.421

0.000

0.065

0.065

$\begin{array}{ll}214 & 337 \\ 215 & 338\end{array}$

$\begin{array}{ll}0.38 & 0.00 \\ 0.38 & 0.00\end{array}$

0.00

$0.00 \quad 0.421$

0.000

0.065

216339

$0.38 \quad 0.00$

0.000

0.053

$\begin{array}{lllll}-0.014 & -0.99 & -1.42 & 2275.43 & 299.95\end{array}$

$Z=124$

$\begin{array}{lllllll}176 & 300 & -0.22 & 0.00 & -0.02 & -0.01 & -0.226\end{array}$

$\begin{array}{llllllll}177 & 301 & -0.22 & 0.00 & -0.03 & -0.02 & -0.225\end{array}$

$0.000 \quad 0.042$

$\begin{array}{lllll}0.003 & -5.82 & -5.07 & 2081.92 & 242.46\end{array}$

$0.000 \quad 0.054$

$\begin{array}{llllll}0.010 & -6.42 & -5.40 & 2089.29 & 243.16\end{array}$

$\begin{array}{llllllll}178 & 302 & -0.23 & 0.00 & -0.03 & -0.02 & -0.235\end{array}$

0.000

0.056

$\begin{array}{lllllll}179 & 303 & -0.25 & 0.00 & -0.02 & -0.02 & -0.255\end{array}$

$0.000 \quad 0.048$

$\begin{array}{lllll}0.009 & -6.42 & -5.36 & 2097.90 & 242.63\end{array}$

$\begin{array}{lllllll}180 & 304 & 0.00 & 0.00 & 0.00 & 0.00 & 0.000\end{array}$

$0.000 \quad 0.000$

$\begin{array}{lllll}0.011 & -6.34 & -5.43 & 2104.79 & 243.81\end{array}$

$\begin{array}{lllllllll}181 & 305 & 0.00 & 0.00 & 0.00 & 0.00 & 0.000 & 0.000 & 0.000\end{array}$

$\begin{array}{lllll}0.000 & -4.81 & -4.24 & 2112.02 & 244.65\end{array}$

$\begin{array}{lllllllll}182 & 306 & 0.00 & 0.00 & 0.00 & 0.00 & 0.000 & 0.000 & 0.000\end{array}$

$\begin{array}{lllll}0.000 & -4.87 & -4.30 & 2118.70 & 246.05\end{array}$

$\begin{array}{lllllll}183 & 307 & 0.00 & 0.00 & 0.00 & 0.00 & 0.000\end{array}$

0.000 


\section{$Z=124$}

$\begin{array}{llll}185 & 309 & -0.16 & 0.00\end{array}$ $\begin{array}{lllll}186 & 310 & -0.39 & 0.00\end{array}$ $\begin{array}{llll}187 & 311 & -0.40 & 0.00\end{array}$ $\begin{array}{llll}188 & 312 & -0.40 & 0.00\end{array}$ $\begin{array}{llll}189 & 313 & 0.39 & 0.00\end{array}$

$\begin{array}{llll}190 & 314 & 0.39 & 0.00\end{array}$ $\begin{array}{llll}191 & 315 & 0.38 & 0.00\end{array}$ $\begin{array}{llll}192 & 316 & -0.42 & 0.00\end{array}$ $\begin{array}{llll}193 & 317 & -0.42 & 0.00\end{array}$ $\begin{array}{llll}194 & 318 & -0.43 & 0.00\end{array}$ $\begin{array}{llll}195 & 319 & -0.43 & 0.00\end{array}$ $\begin{array}{llll}196 & 320 & -0.43 & 0.00\end{array}$ $\begin{array}{llll}197 & 321 & 0.48 & 0.00\end{array}$ $\begin{array}{llll}198 & 322 & -0.44 & 0.00\end{array}$ $\begin{array}{llll}199 & 323 & -0.45 & 0.00\end{array}$

$\begin{array}{llll}200 & 324 & -0.45 & 0.00\end{array}$ $\begin{array}{llll}201 & 325 & -0.45 & 0.00\end{array}$ $\begin{array}{llll}202 & 326 & 0.29 & 0.00\end{array}$ $\begin{array}{llll}203 & 327 & 0.29 & 0.00\end{array}$ $\begin{array}{llll}204 & 328 & 0.29 & 0.00\end{array}$

$\begin{array}{llll}205 & 329 & 0.29 & 0.00\end{array}$

$\begin{array}{llll}206 & 330 & 0.30 & 0.00\end{array}$

$\begin{array}{llll}207 & 331 & 0.30 & 0.00\end{array}$

$\begin{array}{llll}208 & 332 & 0.30 & 0.00\end{array}$

$\begin{array}{llll}209 & 333 & 0.30 & 0.00\end{array}$

210334

$0.30 \quad 0.00$

$\begin{array}{llll}211 & 335 & 0.40 & 0.00\end{array}$

$\begin{array}{llll}212 & 336 & 0.40 & 0.00\end{array}$

$\begin{array}{llll}213 & 337 & 0.30 & 0.00\end{array}$

$\begin{array}{llll}214 & 338 & 0.40 & 0.00\end{array}$

215339

$$
-0.02-0 .
$$

$\begin{array}{lllll}0.00 & -0.398 & 0.000 & -0.028\end{array}$

$0.00-0.407-0.000-0.025$

$\begin{array}{lllll}0.07 & 0.00 & -0.407 & 0.000 & -0.015\end{array}$

$\begin{array}{lllll}0.005 & -3.64 & -3.35 & 2146.53 & 250.49\end{array}$ $\begin{array}{lllll}0.024 & -4.45 & -2.66 & 2153.61 & 251.49\end{array}$ $\begin{array}{lllll}0.024 & -4.61 & -2.77 & 2159.71 & 253.46\end{array}$ $\begin{array}{lllll}0.020 & -4.13 & -2.59 & 2167.11 & 254.13\end{array}$ $\begin{array}{lllllllll}0.02 & 0.441 & 0.000 & -0.011 & -0.041 & -5.12 & -6.13 & 2176.43 & 252.88\end{array}$ $\begin{array}{llllllllll}0.07 & 0.02 & 0.441 & 0.000 & -0.011 & -0.041 & -4.75 & -5.80 & 2183.47 & 253.91\end{array}$ $\begin{array}{llllllllll}0.07 & 0.01 & 0.429 & 0.000 & -0.017 & -0.032 & -4.60 & -5.64 & 2188.91 & 256.55\end{array}$ $\begin{array}{llllllllll}0.06 & 0.00 & -0.426 & 0.000 & 0.002 & 0.015 & -2.97 & -1.68 & 2192.11 & 261.42\end{array}$ $\begin{array}{llllllllll}0.06 & 0.00 & -0.426 & 0.000 & 0.002 & 0.015 & -2.94 & -1.62 & 2197.46 & 264.14\end{array}$ $\begin{array}{llllllllll}0.05 & 0.00 & -0.436 & 0.000 & 0.015 & 0.010 & -2.46 & -1.26 & 2204.06 & 265.60\end{array}$ $\begin{array}{llllllllll}0.05 & 0.00 & -0.436 & 0.000 & 0.015 & 0.010 & -2.52 & -1.29 & 2209.30 & 268.43\end{array}$ $\begin{array}{llllllllll}0.05 & 0.00 & -0.436 & 0.000 & 0.015 & 0.010 & -2.18 & -1.01 & 2215.80 & 270.01\end{array}$ $\begin{array}{llllllllll}0.00 & 0.01 & 0.539 & 0.000 & 0.109 & 0.014 & -2.08 & -6.45 & 2226.27 & 267.61\end{array}$ $\begin{array}{llllllllll}0.04 & 0.00 & -0.445 & 0.000 & 0.029 & 0.006 & -1.96 & -0.93 & 2227.33 & 274.62\end{array}$ $\begin{array}{llllllllll}0.05 & 0.01 & -0.455 & 0.000 & 0.020 & 0.002 & -2.41 & -1.26 & 2232.51 & 277.52\end{array}$ $\begin{array}{llllllllll}0.04 & 0.01 & -0.454 & 0.000 & 0.031 & -0.003 & -2.34 & -1.19 & 2238.84 & 279.26\end{array}$ $\begin{array}{llllllllll}0.04 & 0.00 & -0.454 & 0.000 & 0.032 & 0.005 & -2.69 & -1.50 & 2243.81 & 282.36\end{array}$ $\begin{array}{llllllllll}0.02 & 0.01 & 0.319 & 0.000 & 0.014 & -0.013 & -2.09 & -2.89 & 2251.42 & 282.82\end{array}$ $\begin{array}{llllllllll}0.02 & 0.01 & 0.319 & 0.000 & 0.014 & -0.013 & -2.14 & -2.89 & 2255.91 & 286.40\end{array}$ $\begin{array}{llllllllll}0.02 & 0.01 & 0.319 & 0.000 & 0.014 & -0.013 & -1.70 & -2.50 & 2261.55 & 288.83\end{array}$ $\begin{array}{llllllllll}0.03 & 0.01 & 0.320 & 0.000 & 0.002 & -0.016 & -1.67 & -2.36 & 2265.73 & 292.73\end{array}$ $\begin{array}{llllllllll}0.04 & 0.00 & 0.332 & 0.000 & -0.009 & -0.010 & -1.14 & -1.91 & 2271.14 & 295.39\end{array}$ $\begin{array}{llllllllll}0.05 & 0.00 & 0.333 & 0.000 & -0.021 & -0.014 & -1.34 & -1.83 & 2275.20 & 299.39\end{array}$ $\begin{array}{llllllllll}0.05 & 0.00 & 0.333 & 0.000 & -0.021 & -0.014 & -0.99 & -1.52 & 2280.57 & 302.09\end{array}$ $\begin{array}{llllllllll}0.06 & 0.00 & 0.334 & 0.000 & -0.033 & -0.017 & -1.40 & -1.65 & 2284.68 & 306.06\end{array}$ $\begin{array}{llllllllll}0.07 & -0.01 & 0.335 & 0.000 & -0.047 & -0.011 & -1.40 & -1.40 & 2289.94 & 308.87\end{array}$ $\begin{array}{llllllllll}0.00 & 0.00 & 0.445 & 0.000 & 0.073 & 0.013 & -0.24 & -2.86 & 2295.21 & 311.67\end{array}$ $\begin{array}{llllllllll}0.01 & 0.00 & 0.445 & 0.000 & 0.060 & 0.008 & 0.01 & -2.73 & 2300.42 & 314.53\end{array}$ $\begin{array}{llllllllll}0.08 & -0.01 & 0.336 & 0.000 & -0.058 & -0.015 & -1.66 & -1.35 & 2302.69 & 320.33\end{array}$ $\begin{array}{llllllllll}0.02 & 0.00 & 0.446 & 0.000 & 0.048 & 0.002 & -0.03 & -2.81 & 2309.32 & 321.77\end{array}$ $Z=125$

$\begin{array}{llllllll}178 & 303 & -0.24 & 0.00 & -0.02 & -0.02 & -0.245 & 0.000\end{array}$ $\begin{array}{lllllllll}179 & 304 & -0.24 & 0.00 & -0.02 & -0.02 & -0.245 & 0.000\end{array}$ $\begin{array}{lllllllll}180 & 305 & -0.25 & 0.00 & -0.02 & -0.02 & -0.255 & 0.000\end{array}$ $\begin{array}{llllllll}181 & 306 & -0.26 & 0.00 & -0.01 & -0.03 & -0.266 & 0.000\end{array}$ $\begin{array}{lllllllll}182 & 307 & -0.26 & 0.00 & -0.01 & -0.02 & -0.266 & 0.000\end{array}$

183308

184309

185310 $0.00 \quad 0.00$ $\begin{array}{lll}0.00 & 0.00\end{array}$ $0.39 \quad 0.00$ $186 \quad 31$ 0.390 .00 $\begin{array}{llll}187 & 312 & 0.39 & 0.00\end{array}$

188313 189314 $0.39 \quad 0.00$ 191316 $\begin{array}{ll}0.39 & 0.00 \\ 0.39 & 0.00\end{array}$ $\begin{array}{ll}0.38 & 0.00\end{array}$ $\begin{array}{llll}192 & 317 & 0.38 & 0.00\end{array}$

$\begin{array}{llll}193 & 318 & 0.38 & 0.00\end{array}$ $\begin{array}{llll}194 & 319 & -0.43 & 0.00\end{array}$ $\begin{array}{llll}195 & 320 & -0.43 & 0.00\end{array}$ $\begin{array}{lllll}196 & 321 & 0.48 & 0.00 & -0.01\end{array}$ $\begin{array}{lllll}197 & 322 & 0.48 & 0.00 & 0.00\end{array}$

$\begin{array}{llll}198 & 323 & -0.45 & 0.00\end{array}$ $\begin{array}{llll}199 & 324 & -0.45 & 0.00\end{array}$ $\begin{array}{llll}200 & 325 & -0.45 & 0.00\end{array}$ $\begin{array}{llll}201 & 326 & -0.46 & 0.00\end{array}$ $\begin{array}{llll}202 & 327 & -0.46 & 0.00\end{array}$ $\begin{array}{llll}0.00 & 0.00 & 0.000 & 0.000\end{array}$ $\begin{array}{llll}0.00 & 0.00 & 0.000 & 0.000\end{array}$

$\begin{array}{llll}0.06 & 0.02 & 0.440 & 0.000\end{array}$

$\begin{array}{lllll}0.06 & 0.02 & 0.440 & 0.000\end{array}$

$\begin{array}{llll}0.06 & 0.02 & 0.440 & 0.000\end{array}$

0.043

$\begin{array}{lllll}0.011 & -0.50 & -2.98 & 2313.00 & 326.17\end{array}$

$\begin{array}{llllll}0.046 & 0.011 & -6.40 & -5.56 & 2096.09 & 251.72\end{array}$ $\begin{array}{llllll}0.046 & 0.011 & -6.48 & -5.62 & 2103.29 & 252.59\end{array}$ $\begin{array}{lllllll}0.048 & 0.011 & -6.01 & -5.16 & 2111.27 & 252.69\end{array}$ $\begin{array}{llllll}0.039 & 0.022 & -5.74 & -4.86 & 2117.89 & 254.14\end{array}$ $\begin{array}{lllllll}0.038 & 0.013 & -5.02 & -4.30 & 2125.55 & 254.55\end{array}$ $\begin{array}{lllllll}0.000 & 0.000 & -3.49 & -3.02 & 2130.99 & 257.18\end{array}$ $\begin{array}{lllllll}0.000 & 0.000 & -2.78 & -2.37 & 2138.34 & 257.90\end{array}$ $\begin{array}{lllllll}0.000 & -0.037 & -5.63 & -7.16 & 2149.64 & 254.68\end{array}$ $\begin{array}{llllll}0.000 & -0.037 & -5.31 & -6.83 & 2157.10 & 255.28\end{array}$ $\begin{array}{llllll}0.000 & -0.037 & -5.47 & -6.96 & 2163.53 & 256.93\end{array}$ $\begin{array}{llllllllll}0.07 & 0.02 & 0.441 & 0.000 & -0.011 & -0.041 & -5.48 & -6.69 & 2170.84 & 257.69\end{array}$ $\begin{array}{llllllllll}0.07 & 0.02 & 0.441 & 0.000 & -0.011 & -0.041 & -5.63 & -6.80 & 2177.05 & 259.55\end{array}$ $\begin{array}{llllllllll}0.07 & 0.02 & 0.441 & 0.000 & -0.011 & -0.041 & -5.27 & -6.48 & 2184.11 & 260.56\end{array}$ $\begin{array}{llllllllll}0.07 & 0.02 & 0.429 & 0.000 & -0.015 & -0.041 & -5.39 & -6.35 & 2189.88 & 262.86\end{array}$ $\begin{array}{llllllllll}0.07 & 0.01 & 0.429 & 0.000 & -0.017 & -0.032 & -4.57 & -5.84 & 2196.55 & 264.26\end{array}$ $\begin{array}{llllllllll}0.07 & 0.01 & 0.429 & 0.000 & -0.017 & -0.032 & -4.33 & -5.55 & 2201.97 & 266.92\end{array}$ $\begin{array}{llllllllll}0.06 & 0.00 & -0.436 & 0.000 & 0.005 & 0.015 & -2.36 & -1.21 & 2204.62 & 272.34\end{array}$ $\begin{array}{llllllllll}0.05 & 0.00 & -0.436 & 0.000 & 0.015 & 0.010 & -2.21 & -1.19 & 2210.11 & 274.92\end{array}$ $\begin{array}{lllllllll}0.01 & 0.538 & 0.000 & 0.122 & 0.021 & -2.23 & -6.84 & 2222.54 & 270.56\end{array}$ $\begin{array}{llllllllll}0.05 & 0.01 & -0.455 & 0.000 & 0.020 & 0.002 & -2.08 & -1.08 & 2228.71 & 280.53\end{array}$ $\begin{array}{llllllllll}0.04 & 0.01 & -0.454 & 0.000 & 0.031 & -0.003 & -2.45 & -1.48 & 2234.25 & 283.06\end{array}$ $\begin{array}{llllllllll}0.04 & 0.01 & -0.454 & 0.000 & 0.031 & -0.003 & -2.46 & -1.47 & 2240.66 & 284.73\end{array}$ $\begin{array}{llllllllll}0.04 & 0.01 & -0.464 & 0.000 & 0.034 & -0.003 & -2.88 & -1.77 & 2245.92 & 287.54\end{array}$ $\begin{array}{llllllllll}0.04 & 0.01 & -0.464 & 0.000 & 0.034 & -0.003 & -2.70 & -1.62 & 2252.00 & 289.53\end{array}$
$-3.39 \quad 255.87$

$-2.92 \quad 256.62$

$-3.07 \quad 258.54$

$\begin{array}{ll}-2.87 & 259.23\end{array}$

$-6.33 \quad 258.05$

$-5.97 \quad 259.11$

$-5.85 \quad 261.71$

$-1.97 \quad 266.51$

$\begin{array}{ll}-1.94 & 269.22\end{array}$

$-1.57 \quad 270.70$

\begin{tabular}{ll}
$-1.61 \quad 273.53$ \\
\hline
\end{tabular}

$-1.30 \quad 275.15$

$\begin{array}{ll}-6.70 & 272.83\end{array}$

$-1.24 \quad 279.80$

$\begin{array}{ll}-1.60 & 282.69\end{array}$

$-1.50 \quad 284.50$

$-1.84 \quad 287.60$

$-2.97 \quad 288.37$

$-2.97 \quad 291.98$

$-2.57 \quad 294.47$

$-2.44 \quad 298.40$

$-2.00 \quad 301.11$

$-1.93 \quad 305.16$

$-1.61 \quad 307.94$

$-1.74311 .96$

$-1.47 \quad 314.86$

$-2.99317 .66$

$\begin{array}{ll}-2.87 & 320.59\end{array}$

$-1.40 \quad 326.55$

$-2.97 \quad 327.96$

$-3.14 \quad 332.45$

$\begin{array}{ll}-5.66 & 257.54\end{array}$

$-5.74 \quad 258.36$

$-5.26 \quad 258.42$

$-4.97 \quad 259.83$

$-4.41 \quad 260.20$

\begin{tabular}{ll}
$-3.03 \quad 262.92$ \\
\hline
\end{tabular}

$-2.37 \quad 263.61$

$\begin{array}{ll}-7.45 & 260.09\end{array}$

$\begin{array}{ll}-7.09 & 260.70\end{array}$

$\begin{array}{ll}-7.23 & 262.32\end{array}$

$-6.92 \quad 263.10$

\begin{tabular}{ll}
$-7.06 \quad 264.93$ \\
\hline
\end{tabular}

$-6.70 \quad 265.97$

$\begin{array}{ll}-6.57 & 268.27\end{array}$

$-6.07 \quad 269.66$

$-5.79 \quad 272.31$

$-1.56 \quad 277.63$

$-1.56 \quad 280.20$

$\begin{array}{ll}-7.06 & 276.00\end{array}$

$\begin{array}{ll}-7.33 & 278.49\end{array}$

$-1.44 \quad 285.87$

$-1.87 \quad 288.40$

$\begin{array}{ll}-1.83 & 290.12\end{array}$

$-2.17 \quad 292.93$

$-1.99 \quad 294.98$ 


\section{$Z=125$}

203328

204329

$0.29 \quad 0.00$

0.02

$\begin{array}{lll}0.01 & 0.319 & 0.000\end{array}$

$\begin{array}{llllll}0.014 & -0.013 & -2.41 & -3.27 & 2258.43 & 291.17\end{array}$

205330

$\begin{array}{ll}.29 & 0.00 \\ 0.29 & 0.00\end{array}$

0.01

0.3190 .000

$\begin{array}{lllllll}0.014 & -0.013 & -1.96 & -2.85 & 2264.05 & 293.61\end{array}$

206331

$0.30 \quad 0.00$

0.04

0.01

$0.320 \quad 0.000$

$\begin{array}{lllllll}0.002 & -0.016 & -1.95 & -2.71 & 2268.52 & 297.22\end{array}$

207332

$0.30 \quad 0.00$

0.05

0.00

0.3320 .000

$-0.008-0.020$

$-1.52$

$\begin{array}{lll}2.22 & 2273.90 & 299.91\end{array}$

208333

$0.31 \quad 0.00$

0.06

0.00

209334

$\begin{array}{ll}0.30 & 0.00\end{array}$

0.06

$0.345 \quad 0.000-0.0$

$021-0.014$$$
\begin{array}{llllllll}
0.334 & 0.000 & -0.033 & -0.017 & -1.66 & -1.98 & 2288.05 & 309.97
\end{array}
$$

11 335

$\begin{array}{llll}0.31 & 0.00 & 0.07 & 0.00\end{array}$

$\begin{array}{llllllll}0.347 & 0.000 & -0.042 & -0.021 & -1.57 & -1.76 & 2293.35 & 312.75\end{array}$

211336

212337

$0.30 \quad 0.00$

$0.07-0.01$$$
\begin{array}{llllllll}
0.335 & 0.000 & -0.047 & -0.011 & -1.82 & -1.89 & 2297.57 & 316.60
\end{array}
$$$$
\begin{array}{llllllll}
0.347 & 0.000 & -0.056 & -0.015 & -1.80 & -1.67 & 2302.72 & 319.52
\end{array}
$$

213338

$\begin{array}{lllll}0.40 & 0.00 & 0.02 & -0.01\end{array}$

$\begin{array}{lll}0.446 & 0.000\end{array}$

$\begin{array}{lllllll}0.046 & 0.012 & -0.28 & -3.16 & 2308.14 & 322.18\end{array}$

214339
$0.193 \quad 0.000$ $\begin{array}{lllllll}0.052 & -0.012 & -1.22 & -0.81 & 2310.97 & 327.42\end{array}$ $\begin{array}{ll}-3.38 & 296.92\end{array}$

$\begin{array}{ll}-2.95 & 299.42\end{array}$

$\begin{array}{ll}-2.82 & 303.05\end{array}$

$\begin{array}{ll}-2.32 \quad 305.80 \\ -2.29 & 309.52\end{array}$

$-2.29 \quad 309.52$

\begin{tabular}{ll}
$-1.97 \quad 312.27$ \\
\hline
\end{tabular}

$\begin{array}{ll}-2.09 & 316.01\end{array}$

$\begin{array}{ll}-1.85 & 318.87\end{array}$

$-1.99 \quad 322.76$

$-1.76 \quad 325.78$
-3.36

$-3.36 \quad 328.38$

$-0.78 \quad 333.92$

\section{$Z=126$}

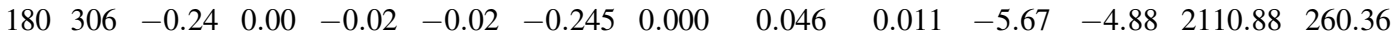
$\begin{array}{llllllllllllll}181 & 307 & -0.26 & 0.00 & -0.01 & -0.03 & -0.266 & 0.000 & 0.039 & 0.022 & -5.42 & -4.61 & 2117.56 & 261.76\end{array}$ $\begin{array}{llllllllllllll}182 & 308 & -0.26 & 0.00 & -0.01 & -0.03 & -0.266 & 0.000 & 0.039 & 0.022 & -4.86 & -4.07 & 2125.55 & 261.84\end{array}$

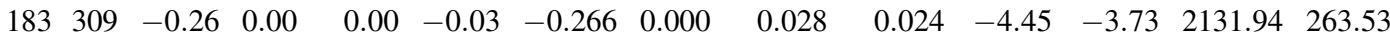

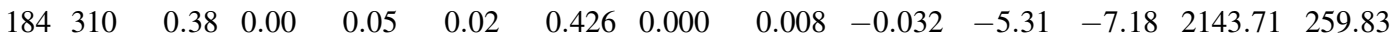
$\begin{array}{llllllllllllll}185 & 311 & 0.39 & 0.00 & 0.06 & 0.02 & 0.440 & 0.000 & 0.000 & -0.037 & -5.50 & -7.26 & 2150.31 & 261.30\end{array}$ $\begin{array}{llllllllllllll}186 & 312 & 0.39 & 0.00 & 0.06 & 0.02 & 0.440 & 0.000 & 0.000 & -0.037 & -5.18 & -6.96 & 2158.11 & 261.57\end{array}$ $\begin{array}{llllllllllllll}187 & 313 & 0.39 & 0.00 & 0.06 & 0.02 & 0.440 & 0.000 & 0.000 & -0.037 & -5.34 & -7.12 & 2164.58 & 263.16\end{array}$ $\begin{array}{llllllllllllll}188 & 314 & 0.39 & 0.00 & 0.07 & 0.02 & 0.441 & 0.000 & -0.011 & -0.041 & -5.42 & -6.88 & 2172.23 & 263.58\end{array}$ $\begin{array}{llllllllllllll}189 & 315 & 0.39 & 0.00 & 0.07 & 0.02 & 0.441 & 0.000 & -0.011 & -0.041 & -5.57 & -6.99 & 2178.47 & 265.42\end{array}$ $\begin{array}{llllllllllllll}190 & 316 & 0.39 & 0.00 & 0.07 & 0.02 & 0.441 & 0.000 & -0.011 & -0.041 & -5.20 & -6.66 & 2185.82 & 266.14\end{array}$ $\begin{array}{lllllllllllllll}191 & 317 & 0.38 & 0.00 & 0.07 & 0.02 & 0.429 & 0.000 & -0.015 & -0.041 & -5.36 & -6.55 & 2191.62 & 268.41\end{array}$ $\begin{array}{llllllll}0.429 & 0.000 & -0.017 & -0.032 & -4.53 & -5.99 & 2198.55 & 269.55\end{array}$ $\begin{array}{llllllllllllll}193 & 319 & 0.38 & 0.00 & 0.07 & 0.01 & 0.429 & 0.000 & -0.017 & -0.032 & -4.28 & -5.73 & 2204.01 & 272.17\end{array}$

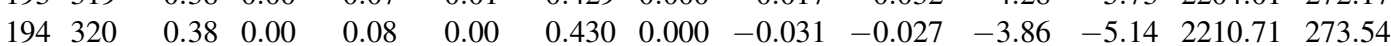
$\begin{array}{llllllllllllll}195 & 321 & 0.48 & 0.00 & -0.01 & 0.01 & 0.538 & 0.000 & 0.122 & 0.021 & -2.29 & -7.30 & 2218.39 & 273.93\end{array}$ $\begin{array}{llllllllllllll}196 & 322 & 0.48 & 0.00 & -0.01 & 0.02 & 0.538 & 0.000 & 0.124 & 0.011 & -2.11 & -7.16 & 2225.35 & 275.04\end{array}$ $\begin{array}{llllllllllllll}197 & 323 & 0.48 & 0.00 & 0.00 & 0.01 & 0.539 & 0.000 & 0.109 & 0.014 & -2.30 & -7.42 & 2230.94 & 277.52\end{array}$ $\begin{array}{lllllllllllllll}198 & 324 & 0.48 & 0.00 & 0.00 & 0.01 & 0.539 & 0.000 & 0.109 & 0.014 & -2.10 & -7.34 & 2237.76 & 278.77\end{array}$ $\begin{array}{llllllllllllll}199 & 325 & 0.49 & 0.00 & 0.01 & 0.01 & 0.552 & 0.000 & 0.102 & 0.009 & -1.93 & -7.53 & 2243.10 & 281.50\end{array}$ $\begin{array}{llllllllllllll}200 & 326 & -0.46 & 0.00 & 0.04 & 0.01 & -0.464 & 0.000 & 0.034 & -0.003 & -2.38 & -1.56 & 2243.84 & 288.84\end{array}$ $\begin{array}{lllllllllllllll}201 & 327 & -0.46 & 0.00 & 0.04 & 0.01 & -0.464 & 0.000 & 0.034 & -0.003 & -2.75 & -1.87 & 2249.13 & 291.62\end{array}$

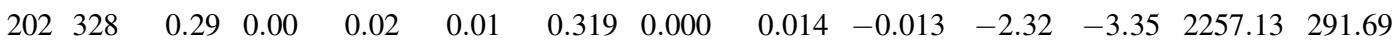
$\begin{array}{llllllllllllll}203 & 329 & 0.29 & 0.00 & 0.02 & 0.01 & 0.319 & 0.000 & 0.014 & -0.013 & -2.38 & -3.38 & 2261.95 & 294.94\end{array}$

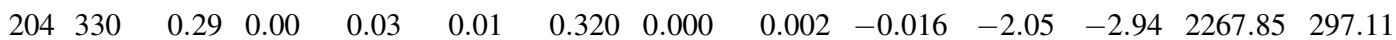
$\begin{array}{llllllllllllll}205 & 331 & 0.29 & 0.00 & 0.03 & 0.01 & 0.320 & 0.000 & 0.002 & -0.016 & -1.94 & -2.75 & 2272.28 & 300.75\end{array}$ $\begin{array}{llllllllllllll}206 & 332 & 0.30 & 0.00 & 0.04 & 0.01 & 0.332 & 0.000 & -0.008 & -0.020 & -1.50 & -2.34 & 2278.03 & 303.08\end{array}$

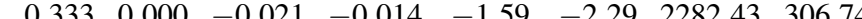
$\begin{array}{llllllllllllll}208 & 334 & 0.30 & 0.00 & 0.05 & 0.00 & 0.333 & 0.000 & -0.021 & -0.014 & -1.23 & -1.98 & 2288.10 & 309.15\end{array}$

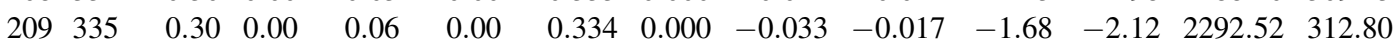
$\begin{array}{llllllllllllll}210 & 336 & 0.31 & 0.00 & 0.07 & 0.00 & 0.347 & 0.000 & -0.042 & -0.021 & -1.60 & -1.90 & 2298.10 & 315.28\end{array}$ $\begin{array}{llllllllllllll}211 & 337 & 0.30 & 0.00 & 0.07 & 0.00 & 0.335 & 0.000 & -0.045 & -0.021 & -1.93 & -2.05 & 2302.36 & 319.10\end{array}$ $\begin{array}{llllllllllllll}212 & 338 & 0.31 & 0.00 & 0.08 & -0.01 & 0.347 & 0.000 & -0.056 & -0.015 & -1.83 & -1.81 & 2307.76 & 321.77\end{array}$

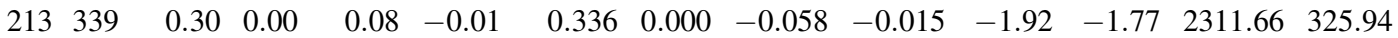

$$
Z=127
$$

$\begin{array}{llll}183 & 310 & -0.26 & 0.00\end{array}$ $\begin{array}{llll}184 & 311 & 0.39 & 0.00\end{array}$ $\begin{array}{llll}185 & 312 & 0.39 & 0.00\end{array}$ $\begin{array}{llll}186 & 313 & 0.39 & 0.00\end{array}$

$\begin{array}{llll}0.00 & -0.03 & -0.266 & 0.000\end{array}$

$\begin{array}{llll}0.06 & 0.02 & 0.440 & 0.000\end{array}$

$\begin{array}{llllll}0.028 & 0.024 & -4.60 & -3.90 & 2130.37 & 272.38\end{array}$ $\begin{array}{lllll}0.06 & 0.02 & 0.440 & 0.000\end{array}$

$\begin{array}{llllll}0.000 & -0.037 & -5.62 & -7.62 & 2142.42 & 268.40\end{array}$ $\begin{array}{llll}187 & 314 & 0.39 & 0.00\end{array}$

$\begin{array}{lllll}0.06 & 0.02 & 0.440 & 0.000\end{array}$

$\begin{array}{llllll}0.000 & -0.037 & -5.80 & -7.76 & 2149.39 & 269.51\end{array}$

$\begin{array}{lllll}188 & 315 & 0.39 & 0.00\end{array}$

$0.07 \quad 0.02$

$\begin{array}{llll}189 & 316 & 0.39 & 0.00\end{array}$

0.07

0.02

$\begin{array}{llllll}-0.011 & -0.041 & -6.04 & -7.65 & 2164.03 & 271.01\end{array}$

$\begin{array}{llllllll}0.441 & 0.000 & -0.011 & -0.041 & -5.80 & -7.44 & 2171.73 & 271.38\end{array}$

$\begin{array}{llllllll}0.429 & 0.000 & -0.015 & -0.041 & -5.86 & -7.23 & 2185.64 & 273.61\end{array}$

$-4.98 \quad 266.43$

$-4.72 \quad 267.77$

$-4.17 \quad 267.82$

$-3.83 \quad 269.47$

$-7.42 \quad 265.60$

$-7.53 \quad 267.01$

$-7.20 \quad 267.29$

$-7.38 \quad 268.85$

$\begin{array}{ll}-7.10 & 269.29\end{array}$

$\begin{array}{ll}-7.24 & 271.09\end{array}$

$-6.87 \quad 271.83$

$\begin{array}{ll}-6.76 & 274.09\end{array}$

$\begin{array}{ll}-6.21 & 275.23\end{array}$

$\begin{array}{ll}-5.96 & 277.82\end{array}$

$\begin{array}{ll}-5.37 & 279.20\end{array}$

$\begin{array}{ll}-7.53 & 279.59\end{array}$

\begin{tabular}{ll}
$-7.37 \quad 280.74$ \\
\hline
\end{tabular}

$\begin{array}{ll}-7.68 & 283.17\end{array}$

$-7.56 \quad 284.48$

$-7.84 \quad 287.14$

$-1.91 \quad 294.45$

$\begin{array}{ll}-2.25 & 297.24\end{array}$

$-3.44 \quad 297.63$

$-3.47 \quad 300.90$

$-3.03 \quad 303.11$

$-2.85 \quad 306.78$

$-2.42 \quad 309.16$

$-2.40 \quad 312.85$

$-2.08 \quad 315.32$

$-2.22 \quad 319.02$

$-1.98 \quad 321.58$

$-2.13 \quad 325.45$

$\begin{array}{ll}-1.89 & 328.19\end{array}$

$-1.84 \quad 332.43$

$\begin{array}{ll}-4.03 & 278.62\end{array}$

\begin{tabular}{ll}
$-7.93 \quad 274.42$ \\
\hline
\end{tabular}

$-8.08 \quad 275.48$

$\begin{array}{ll}-7.79 & 275.68\end{array}$

$\begin{array}{ll}-7.96 & 276.94\end{array}$

$\begin{array}{ll}-7.72 & 277.32\end{array}$

$\begin{array}{ll}-7.84 & 278.83\end{array}$

$-7.47 \quad 279.55$ 


\section{$Z=127$}

191318

$\begin{array}{lll}0.38 & 0.00 & 0.07\end{array}$ $\begin{array}{lll}0.38 & 0.00 & 0.07\end{array}$ $192 \quad 319$ $\begin{array}{lll}0.38 & 0.00 & 0.08\end{array}$ $193 \quad 320$ $\begin{array}{lll}0.38 & 0.00 & 0.08\end{array}$

19432 $\begin{array}{lllll}195 & 322 & 0.38 & 0.00 & 0.08\end{array}$

$\begin{array}{lllll}196 & 323 & 0.38 & 0.00 & 0.08\end{array}$ $\begin{array}{lllll}197 & 324 & 0.48 & 0.00 & 0.00\end{array}$ $\begin{array}{lllll}198 & 325 & 0.49 & 0.00 & 0.01\end{array}$ $\begin{array}{lllll}199 & 326 & 0.49 & 0.00 & 0.01\end{array}$ $\begin{array}{lllll}200 & 327 & -0.46 & 0.00 & 0.04\end{array}$

$\begin{array}{lllll}201 & 328 & -0.47 & 0.00 & 0.04\end{array}$ $\begin{array}{lllll}202 & 329 & 0.29 & 0.00 & 0.02\end{array}$ $\begin{array}{llllll}203 & 330 & 0.29 & 0.00 & 0.02\end{array}$

$\begin{array}{lllll}204 & 331 & 0.29 & 0.00 & 0.03\end{array}$ $\begin{array}{lllll}205 & 332 & 0.29 & 0.00 & 0.03\end{array}$

206333

$207 \quad 334$

$\begin{array}{lll}0.30 & 0.00 & 0.04\end{array}$

208335

209336

210337

211338

212339

$\begin{array}{lll}0.30 & 0.00 & 0.05\end{array}$

$\begin{array}{lll}0.31 & 0.00 & 0.06\end{array}$

$\begin{array}{lll}0.30 & 0.00 & 0.06\end{array}$

$\begin{array}{lll}0.31 & 0.00 & 0.07\end{array}$

$\begin{array}{lll}0.30 & 0.00 & 0.07\end{array}$

$\begin{array}{lll}0.31 & 0.00 & 0.08\end{array}$

\section{$Z=128$}

185313

186314

$\begin{array}{lll}0.39 & 0.00 & 0.06\end{array}$ $\begin{array}{llll}0.39 & 0.00 & 0.07\end{array}$

$187 \quad 315$

188316

$\begin{array}{llll}0.39 & 0.00 & 0.07\end{array}$

$\begin{array}{llll}0.39 & 0.00 & 0.07\end{array}$

189317

$\begin{array}{llll}0.39 & 0.00 & 0.07\end{array}$

190318

$\begin{array}{lll}0.39 & 0.00 & 0.08\end{array}$

191319

$192 \quad 320$

$\begin{array}{lll}0.39 & 0.00 & 0.08\end{array}$

$193 \quad 321$

$\begin{array}{lll}0.38 & 0.00 & 0.08\end{array}$

194322

$\begin{array}{lll}0.38 & 0.00 & 0.08\end{array}$

$\begin{array}{llll}0.38 & 0.00 & 0.08\end{array}$

195323

$\begin{array}{lll}0.38 & 0.00 & 0.08\end{array}$

196324

$197 \quad 325$

198326

$\begin{array}{lll}0.38 & 0.00 & 0.09\end{array}$

$\begin{array}{llll}0.38 & 0.00 & 0.09\end{array}$

199327

$\begin{array}{lll}0.49 & 0.00 & 0.01\end{array}$

$\begin{array}{lllll}200 & 328 & 0.49 & 0.00 & 0.02\end{array}$

$\begin{array}{lllll}201 & 329 & -0.47 & 0.00 & 0.04\end{array}$

$\begin{array}{lllll}202 & 330 & 0.29 & 0.00 & 0.02\end{array}$

$\begin{array}{lllll}203 & 331 & 0.29 & 0.00 & 0.02\end{array}$

$\begin{array}{lllll}204 & 332 & 0.29 & 0.00 & 0.03\end{array}$

$\begin{array}{lllll}205 & 333 & 0.29 & 0.00 & 0.03\end{array}$

206334

$207 \quad 335$

208336

209337

210338

211339

$\begin{array}{llll}0.29 & 0.00 & 0.04\end{array}$ $\begin{array}{lll}0.29 & 0.00 & 0.04\end{array}$ $\begin{array}{lll}0.30 & 0.00 & 0.06\end{array}$ $\begin{array}{lll}0.30 & 0.00 & 0.06\end{array}$

$\begin{array}{lll}0.31 & 0.00 & 0.07\end{array}$ $\begin{array}{lll}0.30 & 0.00 & 0.07\end{array}$

$Z=129$

187316

$\begin{array}{ll}188 & 317\end{array}$

$\begin{array}{lll}0.39 & 0.00 & 0.07\end{array}$ $\begin{array}{lll}0.39 & 0.00 & 0.08\end{array}$ $\begin{array}{lllll}189 & 318 & 0.39 & 0.00 & 0.08\end{array}$ $\begin{array}{lllll}190 & 319 & 0.39 & 0.00 & 0.08\end{array}$ $\begin{array}{llllll}191 & 320 & 0.38 & 0.00 & 0.08\end{array}$ $\begin{array}{lllllllll}0.02 & 0.429 & 0.000 & -0.015 & -0.041 & -5.77 & -7.09 & 2191.72 & 275.60\end{array}$ $\begin{array}{lllllllll}0.01 & 0.429 & 0.000 & -0.017 & -0.032 & -4.93 & -6.57 & 2198.70 & 276.69\end{array}$ $\begin{array}{lllllllll}0.01 & 0.430 & 0.000 & -0.029 & -0.037 & -5.10 & -6.30 & 2204.45 & 279.01\end{array}$ $\begin{array}{lllllllll}0.00 & 0.430 & 0.000 & -0.031 & -0.027 & -4.29 & -5.74 & 2211.19 & 280.35\end{array}$ $\begin{array}{lllllllll}0.00 & 0.430 & 0.000 & -0.031 & -0.027 & -4.03 & -5.49 & 2216.76 & 282.84\end{array}$

$\begin{array}{lllllllll}0.00 & 0.430 & 0.000 & -0.031 & -0.027 & -3.48 & -4.98 & 2223.36 & 284.32\end{array}$ $\begin{array}{lllllllll}0.01 & 0.539 & 0.000 & 0.109 & 0.014 & -2.51 & -7.96 & 2231.98 & 283.77\end{array}$ $\begin{array}{lllllllll}0.01 & 0.552 & 0.000 & 0.102 & 0.009 & -1.87 & -7.80 & 2238.74 & 285.08\end{array}$ $\begin{array}{lllllllll}0.01 & 0.552 & 0.000 & 0.102 & 0.009 & -2.16 & -8.10 & 2244.48 & 287.41\end{array}$ $\begin{array}{lllllllll}0.01 & -0.464 & 0.000 & 0.034 & -0.003 & -2.53 & -1.88 & 2244.99 & 294.98\end{array}$ $\begin{array}{lllllllll}0.01 & -0.474 & 0.000 & 0.037 & -0.004 & -2.94 & -2.17 & 2250.54 & 297.49\end{array}$ $\begin{array}{lllllllll}0.01 & 0.319 & 0.000 & 0.014 & -0.013 & -2.60 & -3.74 & 2258.65 & 297.46\end{array}$ $\begin{array}{lllllllll}0.02 & 0.319 & 0.000 & 0.015 & -0.022 & -2.84 & -3.78 & 2263.77 & 300.40\end{array}$ $\begin{array}{lllllllll}0.01 & 0.320 & 0.000 & 0.002 & -0.016 & -2.34 & -3.24 & 2269.58 & 302.66\end{array}$ $\begin{array}{lllllllll}0.01 & 0.320 & 0.000 & 0.002 & -0.016 & -2.24 & -3.14 & 2274.40 & 305.92\end{array}$ $\begin{array}{lllllllll}0.01 & 0.332 & 0.000 & -0.008 & -0.020 & -1.79 & -2.73 & 2280.15 & 308.24\end{array}$ $\begin{array}{lllllllll}0.01 & 0.333 & 0.000 & -0.020 & -0.023 & -2.03 & -2.70 & 2284.86 & 311.60\end{array}$ $\begin{array}{lllllllll}0.00 & 0.345 & 0.000 & -0.030 & -0.018 & -1.64 & -2.38 & 2290.53 & 314.01\end{array}$ $\begin{array}{lllllllll}0.00 & 0.334 & 0.000 & -0.033 & -0.017 & -1.98 & -2.50 & 2295.22 & 317.39\end{array}$ $\begin{array}{lllllllll}0.00 & 0.347 & 0.000 & -0.042 & -0.021 & -1.92 & -2.31 & 2300.84 & 319.83\end{array}$ $\begin{array}{lllllllll}0.00 & 0.335 & 0.000 & -0.045 & -0.021 & -2.24 & -2.43 & 2305.36 & 323.39\end{array}$ $\begin{array}{lllllllll}-0.01 & 0.347 & 0.000 & -0.056 & -0.015 & -2.15 & -2.21 & 2310.79 & 326.03\end{array}$

$\begin{array}{lllllllll}0.02 & 0.440 & 0.000 & 0.000 & -0.037 & -5.52 & -7.80 & 2149.28 & 276.90\end{array}$ $\begin{array}{lllllllll}0.02 & 0.441 & 0.000 & -0.011 & -0.041 & -5.59 & -7.52 & 2157.44 & 276.82\end{array}$ $\begin{array}{lllllllll}0.02 & 0.441 & 0.000 & -0.011 & -0.041 & -5.84 & -7.72 & 2164.28 & 278.05\end{array}$ $\begin{array}{lllllllll}0.02 & 0.441 & 0.000 & -0.011 & -0.041 & -5.61 & -7.51 & 2172.28 & 278.11\end{array}$ $\begin{array}{lllllllll}0.02 & 0.441 & 0.000 & -0.011 & -0.041 & -5.75 & -7.62 & 2178.83 & 279.64\end{array}$ $\begin{array}{lllllllll}0.01 & 0.442 & 0.000 & -0.025 & -0.037 & -5.51 & -7.29 & 2186.50 & 280.04\end{array}$ $\begin{array}{lllllllll}0.01 & 0.442 & 0.000 & -0.025 & -0.037 & -5.43 & -7.15 & 2192.60 & 282.01\end{array}$ $\begin{array}{lllllllll}0.01 & 0.430 & 0.000 & -0.029 & -0.037 & -5.19 & -6.67 & 2199.92 & 282.76\end{array}$ $\begin{array}{lllllllll}0.01 & 0.430 & 0.000 & -0.029 & -0.037 & -4.97 & -6.42 & 2205.71 & 285.04\end{array}$ $\begin{array}{lllllllll}0.01 & 0.430 & 0.000 & -0.029 & -0.037 & -4.36 & -5.85 & 2212.74 & 286.08\end{array}$ $\begin{array}{lllllllll}0.00 & 0.430 & 0.000 & -0.031 & -0.027 & -3.89 & -5.59 & 2218.32 & 288.58\end{array}$ $\begin{array}{lllllllll}0.00 & 0.431 & 0.000 & -0.043 & -0.032 & -3.78 & -5.09 & 2225.23 & 289.74\end{array}$ $\begin{array}{lllllllll}0.00 & 0.431 & 0.000 & -0.043 & -0.032 & -3.46 & -4.74 & 2230.52 & 292.51\end{array}$ $\begin{array}{lllllllll}0.01 & 0.552 & 0.000 & 0.102 & 0.009 & -1.86 & -8.32 & 2241.32 & 289.79\end{array}$ $\begin{array}{lllllllll}0.01 & 0.552 & 0.000 & 0.102 & 0.009 & -2.16 & -8.54 & 2247.00 & 292.18\end{array}$ $\begin{array}{lllllllll}0.00 & 0.553 & 0.000 & 0.087 & 0.012 & -1.90 & -8.31 & 2253.79 & 293.46\end{array}$ $\begin{array}{lllllllll}0.01 & -0.474 & 0.000 & 0.037 & -0.004 & -2.80 & -2.28 & 2253.04 & 302.28\end{array}$ $\begin{array}{lllllllll}0.02 & 0.319 & 0.000 & 0.015 & -0.022 & -2.75 & -3.79 & 2261.38 & 302.01\end{array}$ $\begin{array}{lllllllll}0.02 & 0.319 & 0.000 & 0.015 & -0.022 & -2.82 & -3.81 & 2266.50 & 304.96\end{array}$ $\begin{array}{lllllllll}0.01 & 0.320 & 0.000 & 0.002 & -0.016 & -2.33 & -3.36 & 2272.69 & 306.85\end{array}$

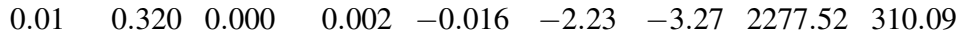
$\begin{array}{lllllllll}0.01 & 0.321 & 0.000 & -0.010 & -0.020 & -1.97 & -2.84 & 2283.55 & 312.12\end{array}$ $\begin{array}{lllllllll}0.01 & 0.321 & 0.000 & -0.010 & -0.020 & -1.97 & -2.85 & 2288.31 & 315.44\end{array}$ $\begin{array}{lllllllll}0.00 & 0.334 & 0.000 & -0.033 & -0.017 & -1.84 & -2.51 & 2294.25 & 317.58\end{array}$ $\begin{array}{lllllllll}0.00 & 0.334 & 0.000 & -0.033 & -0.017 & -2.02 & -2.68 & 2299.00 & 320.90\end{array}$ $\begin{array}{lllllllll}0.00 & 0.347 & 0.000 & -0.042 & -0.021 & -1.94 & -2.47 & 2304.89 & 323.07\end{array}$ $\begin{array}{lllllllll}0.00 & 0.335 & 0.000 & -0.045 & -0.021 & -2.31 & -2.63 & 2309.45 & 326.58\end{array}$

$\begin{array}{lllllllll}0.02 & 0.441 & 0.000 & -0.011 & -0.041 & -5.90 & -7.99 & 2162.75 & 286.86\end{array}$ $\begin{array}{lllllllll}0.02 & 0.443 & 0.000 & -0.023 & -0.046 & -6.08 & -7.77 & 2170.77 & 286.92\end{array}$ $\begin{array}{lllllllll}0.02 & 0.443 & 0.000 & -0.023 & -0.046 & -6.30 & -7.95 & 2177.69 & 288.07\end{array}$ $\begin{array}{lllllllll}0.02 & 0.443 & 0.000 & -0.023 & -0.046 & -5.98 & -7.66 & 2185.43 & 288.40\end{array}$ $\begin{array}{lllllllll}0.01 & 0.430 & 0.000 & -0.029 & -0.037 & -5.91 & -7.53 & 2191.82 & 290.08\end{array}$
$-7.35 \quad 281.51$

$-6.83 \quad 282.60$

$-6.58 \quad 284.89$

$-6.01 \quad 286.23$

$-5.77 \quad 288.71$

$\begin{array}{ll}-5.24 & 290.22\end{array}$

$-8.29 \quad 289.61$

$-8.14 \quad 290.92$

$-8.47 \quad 293.23$

$-2.28 \quad 300.78$

$\begin{array}{ll}-2.62 & 303.27\end{array}$

$-3.85 \quad 303.60$

$-3.88 \quad 306.58$

$-3.34 \quad 308.86$

$-3.26 \quad 312.15$

$-2.84314 .50$

$-2.82 \quad 317.90$

$\begin{array}{ll}-2.50 & 320.34\end{array}$

$\begin{array}{ll}-2.62 & 323.77\end{array}$

$-2.42 \quad 326.28$

$\begin{array}{ll}-2.54 & 329.89\end{array}$

$-2.32 \quad 332.59$

$\begin{array}{ll}-8.11 \quad 283.20 \\ -7.81 & 283.12\end{array}$

$-7.81 \quad 283.12$

$\begin{array}{ll}-8.02 & 284.30\end{array}$

\begin{tabular}{ll}
$-7.78 \quad 284.37$ \\
\hline
\end{tabular}

$-7.91 \quad 285.86$

$\begin{array}{ll}-7.57 & 286.24\end{array}$

$-7.45 \quad 288.17$

$-6.91 \quad 288.96$

$-6.69291 .21$

$-6.09 \quad 292.28$

$-5.86 \quad 294.72$

$-5.34295 .92$

$\begin{array}{ll}-5.00 & 298.67\end{array}$

$-8.63 \quad 295.91$

$-8.88 \quad 298.28$

$-8.64 \quad 299.59$

$-2.71 \quad 308.32$

$-3.87 \quad 308.42$

$-3.90 \quad 311.38$

$-3.46 \quad 313.28$

$-3.37 \quad 316.54$

$-2.93 \quad 318.62$

$-2.95 \quad 321.96$

$-2.61 \quad 324.14$

$\begin{array}{ll}-2.79 & 327.49\end{array}$

$-2.57 \quad 329.72$

$-2.73 \quad 333.28$

$\begin{array}{ll}-8.34 & 293.39\end{array}$

$-8.09 \quad 293.45$

$\begin{array}{ll}-8.29 & 294.55\end{array}$

$-7.97 \quad 294.89$

$-7.85 \quad 296.52$ 


\section{$Z=129$}

192321

$193 \quad 322$

$\begin{array}{lll}0.38 & 0.00 & 0.08\end{array}$ $\begin{array}{lll}0.38 & 0.00 & 0.08\end{array}$

194323

$\begin{array}{lll}0.38 & 0.00 & 0.09\end{array}$

$\begin{array}{llllll}195 & 324 & 0.38 & 0.00 & 0.09\end{array}$

$\begin{array}{lllllllll}0.01 & 0.430 & 0.000 & -0.029 & -0.037 & -5.39 & -7.03 & 2199.15 & 290.82\end{array}$

$\begin{array}{lllllllll}0.01 & 0.430 & 0.000 & -0.029 & -0.037 & -5.20 & -6.83 & 2205.28 & 292.76\end{array}$

$\begin{array}{lllllllll}0.00 & 0.431 & 0.000 & -0.043 & -0.032 & -4.89 & -6.33 & 2212.41 & 293.71\end{array}$

196325

$\begin{array}{lll}0.38 & 0.00 & 0.09\end{array}$

0.00

$\begin{array}{llllllll}0.431 & 0.000 & -0.043 & -0.032 & -4.59 & -6.03 & 2218.24 & 295.95\end{array}$

0.00

197326

198327

$\begin{array}{llll}0.38 & 0.00 & 0.10 & -0.01\end{array}$

199328

$\begin{array}{llll}0.38 & 0.00 & 0.10 & -0.01\end{array}$

200329

$\begin{array}{lll}0.49 & 0.00 & 0.01\end{array}$

0.01

$\begin{array}{llllllll}0.431 & 0.000 & -0.043 & -0.032 & -4.09 & -5.58 & 2225.21 & 297.05\end{array}$

$\begin{array}{llllllll}0.432 & 0.000 & -0.057 & -0.027 & -4.09 & -5.17 & 2230.75 & 299.57\end{array}$

$\begin{array}{llllllll}0.432 & 0.000 & -0.057 & -0.027 & -3.59 & -4.72 & 2237.52 & 300.88\end{array}$

$\begin{array}{llllllll}0.552 & 0.000 & 0.102 & 0.009 & -2.44 & -9.17 & 2247.73 & 298.74\end{array}$

$\begin{array}{lllllllll}0.00 & 0.553 & 0.000 & 0.087 & 0.012 & -2.20 & -8.96 & 2254.56 & 299.98\end{array}$

$\begin{array}{lllll}201 & 330 & -0.48 & 0.00 & 0.04\end{array}$

$\begin{array}{lll}0.02 & -0.483 & 0.000\end{array}$

$\begin{array}{llllll}0.039 & -0.012 & -3.10 & -2.63 & 2253.80 & 308.81\end{array}$

202331

$\begin{array}{lll}0.29 & 0.00 & 0.02\end{array}$

203332

$\begin{array}{lll}0.29 & 0.00 & 0.03\end{array}$

0.02

$0.319 \quad 0.000$

$\begin{array}{llllll}0.015 & -0.022 & -3.02 & -4.15 & 2262.16 & 308.52\end{array}$

204333

$\begin{array}{llll}0.29 & 0.00 & 0.03\end{array}$

205334

$\begin{array}{llll}0.29 & 0.00 & 0.03\end{array}$

206335

$\begin{array}{llll}0.29 & 0.00 & 0.04\end{array}$

207336

$208 \quad 337$

209338

$\begin{array}{lll}0.29 & 0.00 & 0.04\end{array}$

$\begin{array}{lll}0.30 & 0.00 & 0.06\end{array}$

$\begin{array}{lll}0.30 & 0.00 & 0.06\end{array}$

210339

$\begin{array}{llll}0.30 & 0.00 & 0.07\end{array}$

$Z=130$

189319

$\begin{array}{lll}0.39 & 0.00 & 0.08\end{array}$

$\begin{array}{llll}0.39 & 0.00 & 0.08\end{array}$

0.01

0.01

0.01

$\begin{array}{ll}0.320 & 0.000\end{array}$

$\begin{array}{llllll}0.002 & -0.016 & -3.04 & -4.14 & 2267.55 & 311.21\end{array}$

$0.320 \quad 0.000$

$\begin{array}{llllll}0.002 & -0.016 & -2.63 & -3.77 & 2273.82 & 313.00\end{array}$

0.01

$320 \quad 0.000$

$\begin{array}{llllll}0.002 & -0.016 & -2.53 & -3.67 & 2278.94 & 315.96\end{array}$

0.00

$\begin{array}{llllllll}0.321 & 0.000 & -0.010 & -0.020 & -2.30 & -3.27 & 2290.05 & 320.99\end{array}$

0.00

$\begin{array}{llllllll}0.334 & 0.000 & -0.033 & -0.017 & -2.15 & -2.91 & 2295.98 & 323.13\end{array}$

0.00

$\begin{array}{llllllll}0.334 & 0.000 & -0.033 & -0.017 & -2.34 & -3.08 & 2301.01 & 326.17\end{array}$

$\begin{array}{llllllll}0.335 & 0.000 & -0.045 & -0.021 & -2.47 & -2.87 & 2306.92 & 328.33\end{array}$

$\begin{array}{lllllllll}0.02 & 0.443 & 0.000 & -0.023 & -0.046 & -5.96 & -7.89 & 2177.42 & 295.62\end{array}$ $\begin{array}{lllllllll}0.01 & 0.442 & 0.000 & -0.025 & -0.037 & -5.34 & -7.59 & 2185.45 & 295.67\end{array}$ 191321 $\begin{array}{lll}0.38 & 0.00 & 0.08\end{array}$ 0.01 $\begin{array}{llllllll}0.430 & 0.000 & -0.029 & -0.037 & -5.61 & -7.53 & 2191.94 & 297.25\end{array}$ $\begin{array}{lllll}192 & 322 & 0.38 & 0.00 & 0.08\end{array}$ $\begin{array}{lllllllll}0.01 & 0.430 & 0.000 & -0.029 & -0.037 & -5.09 & -6.99 & 2199.52 & 297.74\end{array}$ 193323 $\begin{array}{lll}0.38 & 0.00 & 0.09\end{array}$

0.00

194324

195325

196326

$197 \quad 327$

198328

199329

$\begin{array}{lll}0.38 & 0.00 & 0.09\end{array}$

0.00

$\begin{array}{lll}0.38 & 0.00 & 0.09\end{array}$

0.00

$\begin{array}{lllll}0.38 & 0.00 & 0.10 & -0.01\end{array}$

$\begin{array}{lllll}0.38 & 0.00 & 0.10 & -0.01\end{array}$

$\begin{array}{lllll}0.38 & 0.00 & 0.10 & -0.01\end{array}$

200330

$\begin{array}{lllll}0.38 & 0.00 & 0.11 & -0.02\end{array}$

201331

$202 \quad 332$

$\begin{array}{llll}0.38 & 0.00 & 0.11\end{array}$

$\begin{array}{lll}0.29 & 0.00 & 0.02\end{array}$

$\begin{array}{lll}0.29 & 0.00 & 0.02\end{array}$

203333

$\begin{array}{llll}0.29 & 0.00 & 0.03\end{array}$

204334

$\begin{array}{lll}0.29 & 0.00 & 0.03\end{array}$

$205 \quad 335$

206336

$207 \quad 337$

$\begin{array}{lll}0.29 & 0.00 & 0.04\end{array}$

$\begin{array}{lll}0.29 & 0.00 & 0.04\end{array}$

$\begin{array}{lll}0.29 & 0.00 & 0.05\end{array}$

208338

$\begin{array}{lll}0.29 & 0.00 & 0.05\end{array}$

209339

$\begin{array}{lll}0.29 & 0.00 & 0.06\end{array}$

$Z=131$

192323

193324

$\begin{array}{lll}0.38 & 0.00 & 0.09\end{array}$

$\begin{array}{lll}0.38 & 0.00 & 0.09\end{array}$

0.00

$\begin{array}{llllllll}0.431 & 0.000 & -0.043 & -0.032 & -5.13 & -6.76 & 2205.64 & 299.69\end{array}$

$\begin{array}{llllllll}0.431 & 0.000 & -0.043 & -0.032 & -4.58 & -6.29 & 2213.09 & 300.32\end{array}$

$\begin{array}{llllllll}0.431 & 0.000 & -0.043 & -0.032 & -4.28 & -5.97 & 2218.93 & 302.54\end{array}$

$\begin{array}{llllllll}0.432 & 0.000 & -0.057 & -0.027 & -4.11 & -5.45 & 2226.12 & 303.42\end{array}$

$\begin{array}{llllllll}0.432 & 0.000 & -0.057 & -0.027 & -3.93 & -5.25 & 2231.89 & 305.73\end{array}$

$\begin{array}{llllllll}0.432 & 0.000 & -0.057 & -0.027 & -3.42 & -4.78 & 2238.94 & 306.74\end{array}$

$\begin{array}{llllllll}0.433 & 0.000 & -0.071 & -0.023 & -3.71 & -4.60 & 2244.53 & 309.23\end{array}$

$\begin{array}{llllllll}0.433 & 0.000 & -0.071 & -0.023 & -3.29 & -4.22 & 2251.48 & 310.35\end{array}$

$\begin{array}{llllllll}0.319 & 0.000 & 0.015 & -0.022 & -3.12 & -4.42 & 2257.26 & 312.64\end{array}$

$\begin{array}{llllllll}0.319 & 0.000 & 0.015 & -0.022 & -2.99 & -4.26 & 2264.24 & 313.73\end{array}$

$\begin{array}{llllllll}0.320 & 0.000 & 0.003 & -0.026 & -3.21 & -4.29 & 2269.67 & 316.37\end{array}$

$\begin{array}{lllllllll}0.02 & 0.320 & 0.000 & 0.003 & -0.026 & -2.81 & -3.90 & 2276.24 & 317.88\end{array}$

$\begin{array}{lllllllll}0.01 & 0.321 & 0.000 & -0.010 & -0.020 & -2.72 & -3.78 & 2281.33 & 320.85\end{array}$

$\begin{array}{lllllllll}0.01 & 0.321 & 0.000 & -0.010 & -0.020 & -2.32 & -3.42 & 2287.74 & 322.52\end{array}$

$\begin{array}{lllllllll}0.01 & 0.322 & 0.000 & -0.022 & -0.023 & -2.60 & -3.42 & 2292.78 & 325.55\end{array}$

$\begin{array}{lllllllll}0.01 & 0.322 & 0.000 & -0.022 & -0.023 & -2.25 & -3.12 & 2299.06 & 327.34\end{array}$

0.00

$\begin{array}{llllllll}0.322 & 0.000 & -0.036 & -0.017 & -2.60 & -3.26 & 2304.07 & 330.40\end{array}$

194325

195326

196327

$\begin{array}{lll}0.38 & 0.00 & 0.09\end{array}$

0.00

$\begin{array}{llllllll}0.431 & 0.000 & -0.043 & -0.032 & -5.46 & -7.34 & 2198.04 & 306.51\end{array}$

$\begin{array}{llllllll}0.431 & 0.000 & -0.043 & -0.032 & -5.34 & -7.15 & 2204.50 & 308.12\end{array}$

$\begin{array}{llllllll}0.431 & 0.000 & -0.043 & -0.032 & -4.77 & -6.62 & 2211.91 & 308.78\end{array}$

$\begin{array}{llllllll}0.432 & 0.000 & -0.057 & -0.027 & -4.84 & -6.30 & 2218.04 & 310.72\end{array}$

$\begin{array}{llllllll}0.432 & 0.000 & -0.057 & -0.027 & -4.39 & -5.91 & 2225.38 & 311.45\end{array}$

$\begin{array}{llllllll}0.432 & 0.000 & -0.057 & -0.027 & -4.21 & -5.70 & 2231.44 & 313.47\end{array}$

$\begin{array}{llllllll}0.433 & 0.000 & -0.071 & -0.023 & -4.16 & -5.22 & 2238.49 & 314.49\end{array}$

$\begin{array}{llllllll}0.433 & 0.000 & -0.071 & -0.023 & -4.07 & -5.12 & 2244.45 & 316.59\end{array}$

$\begin{array}{llllllll}0.433 & 0.000 & -0.071 & -0.023 & -3.65 & -4.66 & 2251.33 & 317.79\end{array}$

$\begin{array}{llllllll}0.435 & 0.000 & -0.083 & -0.027 & -4.15 & -4.53 & 2257.08 & 320.11\end{array}$

202333

$\begin{array}{llll}0.38 & 0.00 & 0.12 & -0.02\end{array}$

203334

$\begin{array}{lll}0.28 & 0.00 & 0.02\end{array}$

0.02

204335

$\begin{array}{lll}0.29 & 0.00 & 0.03\end{array}$

0.02

$\begin{array}{llllllll}0.308 & 0.000 & 0.013 & -0.023 & -3.44 & -4.58 & 2264.28 & 320.98\end{array}$

$\begin{array}{lll}0.29 & 0.00 & 0.03\end{array}$

0.02

$\begin{array}{ll}0.320 & 0.000\end{array}$

$\begin{array}{llllll}0.003 & -0.026 & -3.52 & -4.70 & 2270.09 & 323.24\end{array}$

205336

$\begin{array}{llll}0.29 & 0.00 & 0.04 & 0.01\end{array}$ $\begin{array}{ll}-7.33 & 297.27\end{array}$

$\begin{array}{ll}-7.14 & 299.18\end{array}$

$\begin{array}{ll}-6.64 & 300.12\end{array}$

$\begin{array}{ll}-6.35 & 302.34\end{array}$

$-5.87 \quad 303.46$

$-5.50 \quad 305.95$

$-5.01 \quad 307.28$

$-9.57 \quad 305.04$

$-9.35 \quad 306.31$

$-3.11 \quad 315.05$

$-4.25 \quad 315.16$

$-4.28 \quad 317.83$

$-3.88 \quad 319.65$

$-3.79 \quad 322.63$

$-3.38 \quad 324.67$

$\begin{array}{ll}-3.39 & 327.72\end{array}$

$-3.04 \quad 329.88$

$-3.22 \quad 332.95$

$-2.98 \quad 335.18$

$\begin{array}{ll}-8.22 & 302.44\end{array}$

$-7.91 \quad 302.47$

$\begin{array}{ll}-7.85 & 304.02\end{array}$

$-7.28 \quad 304.52$

$-7.08 \quad 306.41$

$-6.58 \quad 307.05$

$-6.29 \quad 309.24$

$-5.75310 .12$

$-5.56 \quad 312.40$

$-5.07 \quad 313.45$

$-4.91 \quad 315.90$

$-4.49 \quad 317.06$

$-4.52 \quad 319.52$

$-4.35 \quad 320.64$

$-4.39 \quad 323.28$

$-3.99 \quad 324.82$

$\begin{array}{ll}-3.90 & 327.77\end{array}$

$-3.52 \quad 329.48$

$-3.53 \quad 332.52$

$-3.21 \quad 334.36$

$-3.38 \quad 337.43$

$\begin{array}{ll}-7.70 & 313.55\end{array}$

$-7.53 \quad 315.11$

$-6.97315 .78$

$-6.68 \quad 317.67$

$-6.25318 .42$

$-6.07 \quad 320.40$

$-5.56 \quad 321.43$

$-5.48 \quad 323.51$

$-4.99 \quad 324.73$

$-4.87 \quad 327.04$

$-4.68 \quad 328.16$

$-4.82 \quad 330.40$

$-4.42 \quad 331.92$

$-4.35 \quad 334.56$ 


\section{$Z=131$}

$\begin{array}{lllll}206 & 337 & 0.29 & 0.00 & 0.04\end{array}$ $\begin{array}{lllll}207 & 338 & 0.29 & 0.00 & 0.05\end{array}$ $\begin{array}{lllll}208 & 339 & 0.29 & 0.00 & 0.05\end{array}$ $\begin{array}{lllllllll}0.01 & 0.321 & 0.000 & -0.010 & -0.020 & -2.66 & -3.85 & 2288.50 & 329.05\end{array}$ $\begin{array}{lllllllll}0.01 & 0.322 & 0.000 & -0.022 & -0.023 & -2.96 & -3.87 & 2293.84 & 331.77\end{array}$ $\begin{array}{lllllllll}0.01 & 0.322 & 0.000 & -0.022 & -0.023 & -2.61 & -3.56 & 2300.14 & 333.55\end{array}$
$-3.98 \quad 336.25$

$-4.00 \quad 338.98$

$-3.68 \quad 340.80$

\section{$Z=132$}

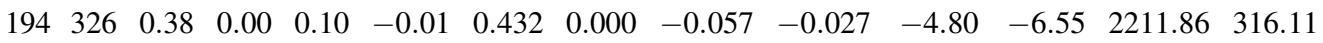
$\begin{array}{llllllllllllll}195 & 327 & 0.38 & 0.00 & 0.10 & -0.01 & 0.432 & 0.000 & -0.057 & -0.027 & -4.60 & -6.35 & 2218.13 & 317.92\end{array}$ $\begin{array}{llllllllllllll}196 & 328 & 0.38 & 0.00 & 0.10 & -0.01 & 0.432 & 0.000 & -0.057 & -0.027 & -4.15 & -5.91 & 2225.73 & 318.40\end{array}$ $\begin{array}{llllllllllllll}197 & 329 & 0.38 & 0.00 & 0.11 & -0.02 & 0.433 & 0.000 & -0.071 & -0.023 & -4.44 & -5.72 & 2231.80 & 320.39\end{array}$

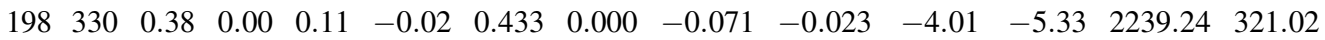
$\begin{array}{llllllllllllll}199 & 331 & 0.38 & 0.00 & 0.11 & -0.02 & 0.433 & 0.000 & -0.071 & -0.023 & -3.91 & -5.14 & 2245.14 & 323.20\end{array}$ $\begin{array}{llllllllllllll}200 & 332 & 0.38 & 0.00 & 0.12 & -0.02 & 0.435 & 0.000 & -0.083 & -0.027 & -4.08 & -4.72 & 2252.35 & 324.05\end{array}$ $\begin{array}{llllllllllllll}201 & 333 & 0.38 & 0.00 & 0.12 & -0.03 & 0.434 & 0.000 & -0.085 & -0.018 & -4.08 & -4.69 & 2258.21 & 326.27\end{array}$ $\begin{array}{llllllllllllll}202 & 334 & 0.28 & 0.00 & 0.02 & 0.02 & 0.308 & 0.000 & 0.013 & -0.023 & -3.47 & -4.74 & 2265.70 & 326.85\end{array}$

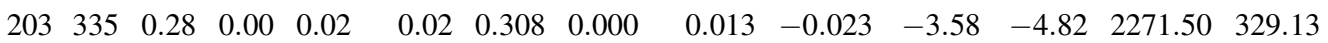

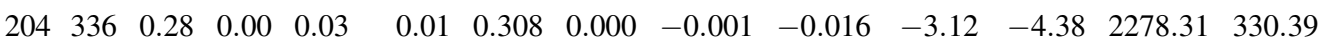
$\begin{array}{llllllllllllll}205 & 337 & 0.29 & 0.00 & 0.04 & 0.01 & 0.321 & 0.000 & -0.010 & -0.020 & -3.08 & -4.36 & 2283.81 & 332.95\end{array}$ $\begin{array}{llllllllllllll}206 & 338 & 0.29 & 0.00 & 0.04 & 0.01 & 0.321 & 0.000 & -0.010 & -0.020 & -2.67 & -4.00 & 2290.51 & 334.33\end{array}$ $\begin{array}{llllllllllllll}207 & 339 & 0.29 & 0.00 & 0.05 & 0.01 & 0.322 & 0.000 & -0.022 & -0.023 & -3.02 & -4.05 & 2295.92 & 336.99\end{array}$

\section{$Z=133$}

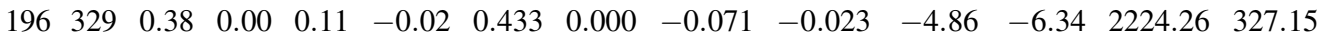
$\begin{array}{llllllllllllll}197 & 330 & 0.38 & 0.00 & 0.11 & -0.02 & 0.433 & 0.000 & -0.071 & -0.023 & -4.77 & -6.22 & 2230.71 & 328.77\end{array}$ $\begin{array}{llllllllllllll}198 & 331 & 0.38 & 0.00 & 0.11 & -0.02 & 0.433 & 0.000 & -0.071 & -0.023 & -4.33 & -5.74 & 2238.07 & 329.48\end{array}$ $\begin{array}{llllllllllllll}199 & 332 & 0.38 & 0.00 & 0.12 & -0.02 & 0.435 & 0.000 & -0.083 & -0.027 & -4.83 & -5.61 & 2244.32 & 331.31\end{array}$ $\begin{array}{llllllllllllll}200 & 333 & 0.38 & 0.00 & 0.12 & -0.02 & 0.435 & 0.000 & -0.083 & -0.027 & -4.47 & -5.28 & 2251.63 & 332.06\end{array}$ $\begin{array}{llllllllllllll}201 & 334 & 0.38 & 0.00 & 0.12 & -0.03 & 0.434 & 0.000 & -0.085 & -0.018 & -4.47 & -5.24 & 2257.77 & 333.99\end{array}$ $\begin{array}{llllllllllllll}202 & 335 & 0.38 & 0.00 & 0.12 & -0.03 & 0.434 & 0.000 & -0.085 & -0.018 & -4.14 & -4.94 & 2264.94 & 334.90\end{array}$

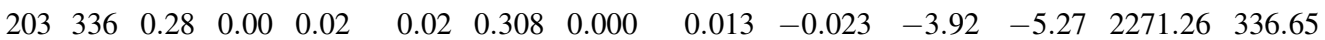
$\begin{array}{llllllllllllll}204 & 337 & 0.28 & 0.00 & 0.03 & 0.01 & 0.308 & 0.000 & -0.001 & -0.016 & -3.47 & -4.83 & 2278.09 & 337.89\end{array}$ $\begin{array}{llllllllllllll}205 & 338 & 0.37 & 0.00 & 0.12 & -0.03 & 0.422 & 0.000 & -0.089 & -0.017 & -4.20 & -4.61 & 2283.68 & 340.38\end{array}$

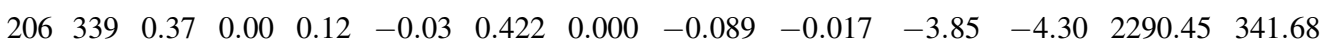

$$
Z=134
$$

$\begin{array}{llllllllllllll}198 & 332 & 0.38 & 0.00 & 0.12 & -0.02 & 0.435 & 0.000 & -0.083 & -0.027 & -4.72 & -5.77 & 2238.06 & 336.78\end{array}$ $\begin{array}{llllllllllllll}199 & 333 & 0.38 & 0.00 & 0.12 & -0.02 & 0.435 & 0.000 & -0.083 & -0.027 & -4.71 & -5.73 & 2244.41 & 338.50\end{array}$ $\begin{array}{llllllllllllll}200 & 334 & 0.38 & 0.00 & 0.12 & -0.03 & 0.434 & 0.000 & -0.085 & -0.018 & -4.36 & -5.40 & 2252.01 & 338.97\end{array}$ $\begin{array}{llllllllllllll}201 & 335 & 0.38 & 0.00 & 0.12 & -0.03 & 0.434 & 0.000 & -0.085 & -0.018 & -4.36 & -5.37 & 2258.18 & 340.87\end{array}$ $\begin{array}{llllllllllllll}202 & 336 & 0.38 & 0.00 & 0.12 & -0.03 & 0.434 & 0.000 & -0.085 & -0.018 & -4.03 & -5.07 & 2265.63 & 341.50\end{array}$

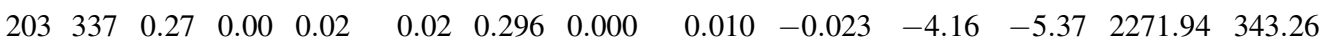

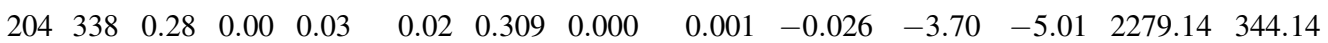
$\begin{array}{llllllllllllll}205 & 339 & 0.28 & 0.00 & 0.04 & 0.01 & 0.309 & 0.000 & -0.013 & -0.020 & -3.67 & -4.92 & 2284.88 & 346.47\end{array}$

$$
Z=135
$$

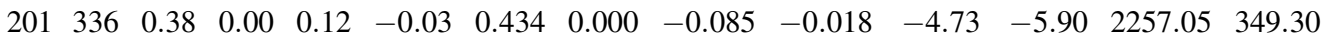
$\begin{array}{llllllllllllll}202 & 337 & 0.38 & 0.00 & 0.12 & -0.03 & 0.434 & 0.000 & -0.085 & -0.018 & -4.40 & -5.61 & 2264.51 & 349.90\end{array}$

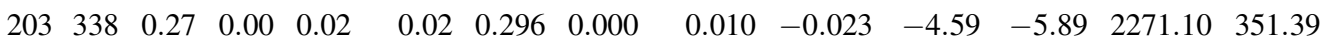
$\begin{array}{llllllllllllll}204 & 339 & 0.37 & 0.00 & 0.12 & -0.03 & 0.422 & 0.000 & -0.089 & -0.017 & -4.49 & -5.30 & 2278.08 & 352.48\end{array}$ $Z=136$

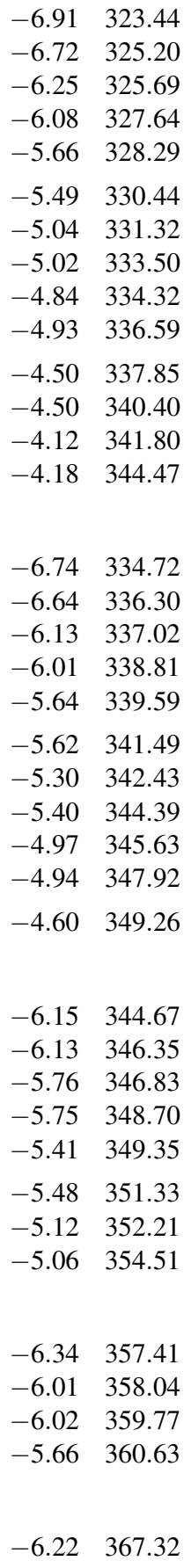

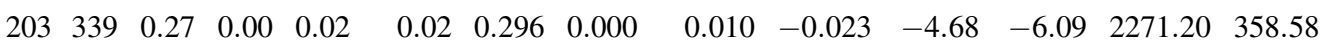

